\title{
ISOPHOTOMETRY OF BRIGHTEST ELLIPTICAL GALAXIES IN RICH CLUSTERS
}

\author{
Thesis by \\ Alain C. Porter \\ In Partial Fulfillment of the Requirements \\ for the Degree of \\ Doctor of Philosophy
}

California Institute of Technology

Pasadena, California

1988

(Submitted December 9, 1987) 
-ii-

(C) 1988

Alain C. Porter

All Rights Reserved 
I wander away from the midnight VAX:

Renounce approximation for a time, And, since no one is waiting, I relax, Find the stairs that lead to the roof, and climb.

Canopus shimmers over subdued palms, The first time it has ever touched my eyes. It adds an oddness to familiar calm, Reminding me of changes, and I sigh, Remembering when Orion was cold, but clear. Neither he nor I needed anyone near, And the things that I didn't know, couldn't see Did not then matter as much to me as now.

I have gained much, but I'm not sure how. And for all the time and effort I've spent, I'm - not more unhappy, but less content. 


\section{ACKNOWLEDGMENTS}

Since acknowledgments are for thanking those who helped you finish a work, I can begin only by listing the people who have done so much to save my neck since 1983: Mark Gutchen, and Drs. Morris Furman, Cary Presant, Robert McKenna, Sam Halpern, Michael Kadin, Jeffrey Hahn, Robert Neer, and my advisor, J. B. Oke. I long to elaborate, and name those who have played crucial supporting roles or just rallied to my support, but will say only: my most heartfelt thanks.

Fortunately, I have many more mundane acknowledgments to make as well.

Most obviously, I am indebted to several groups and people for generous financial support. I thank those in the astronomy department who have looked after my stipend, and Gerry Neugebauer and Bob Brucato at Palomar, for an RA and for giving me an interesting assignment in the Hartmann tests. Maarten Schmidt helped send me to a meeting in Trieste in 1983 and asked nothing in return. I owe extra special thanks to $\mathrm{Al}$ Moffet and Arden Albee for getting me an ARCS fellowship, which got me out of a deep hole. I am equally grateful to ARCS for their generosity.

I still have fond memories of Rocky Hill, though I have been long time gone and took almost no science there. Things Kathy Geiersbach, Steve Russey, Hugh Campbell, and others taught me have seen me through the times since. Forsan et haec olim meminisse iuvabit.

My friends from Sector HV have remained with me in spirit, wherever we have gone. Amiel Sternberg has been a fast friend through too many misfortunes. I only wish he could have come to Cal. And I still thank him for Pink, even though I 
have been through Generator and Joy Division by now. I thank Sallie Baliunas for making my extravagant summer back in Cambridge both possible and profitable. The support that Christine Jones and Bill Forman, my undergrad advisers, have always given me without question, I value more than I can say. I am still indebted to Alex Dalgarno and Josh Grindlay for the help they gave me. Business has often brought Curt to town, but I miss the company of Mishev, Phil and Dean, Neil, Dr. J., and Carriage Return, among others.

Still, it was at Caltech that I first belonged to, and enjoyed, a. large peer group. The mere mention of Alex Filippenko's name triggers an incoherent jumble of hilarious memories: milk, strawberries, Westwood, rabid dogs, astronomical collaborators, Jorgé. I thank him for activating my latent interest in supernovae. Don Schneider gave me a private vote of confidence in April 1983, which I treasure, and I have always admired his taste, which is not all in his mouth. John Biretta I had already met, one karmic day in the Flatirons. I have paid him his donuts and forgiven him a wild drive. I also thank him for PAMELA and shared insights into chromodynamics. Mike Rich helped me out on several occasions, and we mulled over the vagaries of fate (and of other things) on many a late night run to the Salt Shaker. Among the other Dimetrodons were Nancy (thanks for Huntington and Haagen-Dazs), Michael Ashley back in $\mathrm{Oz}$ (where he and his family were my generous hosts during the vacation of my life), Mary Barsony (FUN), Bruce (Our Lips Are Sealed) Berriman, James Fillmore (a green stop sign?!), John Hoessel (control P, John), Keith Horne ("avernge, I guess!"), Dave Hough ("A fair-haired young grad from Caltech/. . ."), Pawan Kumar (who delighted me with how he picked up American slang), Kevin Lind and Rick Edelson (matter and antimatter), Roger Linfield (strong-fingered rock climber), Matt Malkan ("But I was there! I 
could have stopped him!"), Jim McCarthy (forget tall, man!), Georg Pauls ("I Want the Future Now"), Jeff Pier (who will remain unrelated to Alex), Abi Saha (for new perspectives on romanticism), Fernando Selman (who dares look even fainter than I did), and Richard Wade (whose good advice I came dangerously close to taking). Post '83 gap friends have included la belle Gigi Adelman, Blaise Canzian, Steve Myers (no squid he), Debbie Padgett, Alain Picard (Jean-Luc), Rich Rand (earthquake!), Karl Stapelfeldt, and Chuck Steidel (the Funstigator). I hope those I've omitted will forgive my short memory.

A lot of people have made nontrivial contributions to my graduate career by giving me lots of time as TAC nembers (I've spent over 100 nights at Palomar), taking observations for me themselves (Judy Cohen, George Djorgovski, Shri Kulkarni, and Mike Rich), being a big help on or about observing runs (Larry Blakée, Bill Qualls, Bob Thicksten and Merle Sweet, Skip Staples, Roger Higson, Dave Tennant, Juan Carrasco, and Ardith, Wally, Linda, and Alicia at the Monastery), building useful things or writing clever software (John Biretta, Todd Boroson, Fred Harris, Steve Kent, Tim Pearson, Keith Shortridge, and John Tonry), arranging funds or cookies (Lorna, Jill, and Lilo), guiding me through the stacks and databases (Helen Knudsen; sorry about the Yamamoto Circulars!), teaching good physics courses (Peter Goldreich and Kip Thorne), or being generally inspiring. Chief among these not already mentioned have been Jesse Greenstein (utor, fruor, fungor, potior, vescor!), HMP Wal Sargent, and Nick Scoville.

As I could begin in only one way, there is only one in which I can end. My parents have given me invaluable material and moral support throughout, and I cannot thank them enough. And to Ardith El-Kareh, for helping with my theoretical 
work, for being the kindred spirit and collaborator I had despaired of finding, I owe the greatest gratitude of all. 


\begin{abstract}
This thesis presents ellipse-fitting isophotometry of 175 brightest elliptical galaxies in Abell clusters (BCEs). Galaxy structure is traced to an average major axis of $50 \mathrm{kpc}$, and some galaxies are traced beyond $100 \mathrm{kpc}$. The data consist not only of major- and minor-axis surface brightness profiles but also of isophote ellipticity, major axis position angle, and centroid position profiles.
\end{abstract}

Almost all BCEs have some local structure that does not show in an azimuthally averaged brightness profile. However, local structure shows no correlation with global parameters such as galaxy luminosity or size. These results echo similar earlier findings for field ellipticals.

The average ellipticity of a BCE is a strongly increasing function of physical radius as are most individual ellipticity profiles. This distinguishes BCEs as a class from other elliptical galaxies and explains why $\mathrm{cD}$ halos as a class are highly flattened. The deficit of large round isophotes is so marked that the isoluminosity surfaces of BCEs at large radii cannot be drawn from a population of randomly oriented oblate spheroids. This is the first sample of galaxies for which such an exclusion has been possible.

The distinction of the isophotes from an oblate population becomes possible over the same range of radii $(20-30 \mathrm{kpc})$ at which the isophotes become significantly aligned with the global position angle of the cluster. These results confirm and further quantify the long-suspected origin of BCEs in cluster material. The detection of nonconcentric light in $20 \%$ of the BCEs in the sample suggests that some galaxy construction continues to the present day. 
Particular attention has been paid to the morphology of $\mathrm{cD}$ halos. The inner edges of these halos range from sharp to very gradual, are almost always associated with sudden ellipticity increases, and often also with isophote twists or nonconcentric light. This indicates that they have some dynamically significant identity, perhaps as material accreted from the cluster.

An analytic method for separating the light distributions of overlapping binary galaxies has been derived and applied to images of 12 such systems. It is based solely on the assumption that each galaxy in the pair has point-reflection symmetry about its own center. Nonconcentric light is a probe of interactions between the members of the pair. 


\section{TABLE OF CONTENTS}

Acknowledgments

Abstract

viii

Chapter 1

Isophotometry of 175 Brightest Elliptical

Galaxies in Abell Clusters

Chapter 2

Isophotometry of $\mathrm{cD}$ Halos

Chapter 3

An Analytic Method for Separating the

Surface Brightness Profiles of Multiple Nuclei

Appendix

Figure 1 of Chapter 1 


\title{
CHAPTER I
}

\section{ISOPHOTOMETRY OF 175 BRIGHTEST ELLIPTICAL GALAXIES IN ABELL CLUSTERS*}

\author{
Alain Porter \\ Palomar Observatory, California Institute of Technology \\ Pasadena, CA 91125 \\ Donald P. Schneider \\ Institute for Advanced Study \\ Princeton, NJ 08540 \\ John G. Hoessel \\ Washburn Observatory, University of Wisconsin \\ Madison, WI 53706
}

to be submitted to The Astrophysical Journal (Supplements)

* Observations obtained in part at the Palomar 1.5-meter telescope, operated jointly by the California Institute of Technology and the Carnegie Institute of Washington. 
In nova fert animus mutatas dicere formas Corpora.

Ovid, Metamorphoses 


\begin{abstract}
Deep isophotometry of a sample of 175 brightest cluster ellipticals (BCEs) is presented and discussed. Over half the sample is measured out to a distance along the major axis of $50 \mathrm{kpc}\left(H_{0}=60 \mathrm{~km} \mathrm{sec}^{-1} \mathrm{kpc}^{-1}, q_{0}=\frac{1}{2}\right)$. Profiles of isophote major and minor axes, ellipticity, position angle, and centroid are derived at intervals of $0.25 \mathrm{mag} \mathrm{\textrm {sec } ^ { - 2 }}$ in $r$ surface brightness. The average ellipticity of $\mathrm{BCEs}$ is a strongly increasing function of metric radius. This behavior is rarely seen in field ellipticals. The shape (local) parameters of BCEs are not correlated with their global properties or the global properties of the clusters they inhabit. Almost all individual $\mathrm{BCEs}$ have significantly increasing ellipticity profiles, and almost all show local structure that is not reflected in azimuthally averaged (one-dimensional) surface brightness profiles. Fewer than 39 galaxies in the present sample show significant amounts of ellipticity decrease with increasing radius anywhere in their surface brightness profiles. Seventeen have roughly constant ellipticities, and only 2 have steadily decreasing ellipticity profiles. About $20 \%$ of the galaxies in the sample show isophote twists greater than $40^{\circ}$. A comparable number show significant nonconcentricity. Thirteen BCEs, primarily multiple-nucleus systems, show both. At radii greater than about $30 \mathrm{kpc}, \mathrm{BCEs}$ become strongly aligned with the distribution of galaxies and X-ray gas in clusters, and become so predominantly flattened that they cannot represent a randomly oriented population of oblate spheroids. These results are evidence that at least the outer regions of BCEs are dynamically strongly coupled to their clusters, which have recently been shown to be very prolate or stringy. BCEs may have been formed this way simultaneously with their clusters, but the presence of significant amounts of nonconcentric light
\end{abstract}


in 15 to $20 \%$ of them suggests that at least some galaxy growth continues to the present day. 


\section{INTRODUCTION}

Our knowledge of the surface brightness profiles of elliptical galaxies has increased dramatically in recent years. A decade ago it was generally believed that all ellipticals were oblate spheroids and that the very luminous galaxies in the centers of rich clusters were all "cD galaxies," supergiant, superluminous cannibals qualitatively different from common field ellipticals in some way that was never very clearly defined.

The 1970s saw the beginning of extended photometric studies of elliptical galaxies, both with photographic plates (Oemler 1976; King 1978; Strom and Strom 1978a, b, c, 1979; Schombert 1986, 1987) and especially with the new CCD cameras (e.g., Leach 1981; Hoessel, Thuan, and Gunn 1980; Schneider, Gunn, and Hoessel 1983a, 1983b; Hoessel and Schneider 1985; Kormendy 1985; Lauer 1985; and others). Among the results particularly relevant to the present work are the quantification of the $\mathrm{cD}$ classification (Tonry 1987, Schombert 1988) and the realization, based on dynamical observations as well as the discovery of changing axial ratios and isophote twisting, that many ellipticals are probably triaxial. Most recently it has been demonstrated that ellipticals commonly show dust patches and lanes (e.g., Ebneter and Balick 1985; Ebneter, Djorgovski, and Davis 1988), nonelliptical isophotes (Jedrzejewski 1987), and other irregularities, sometimes even in the absence of obvious perturbing influences. The most comprehensive isophotometric surveys to date have been those of Kent (1984, 1985), Djorgovski (1985b), Michard (1985), and Lauer (1985).

The new detector technology also fostered studies of elliptical galaxy dynamics (v. de Zeeuw 1987). Among the most interesting observations was the 
discovery by Dressler (1979) that the velocity dispersion profile of the brightest cluster elliptical (henceforth BCE) in A2029 continues to increase out to a radius of $100 \mathrm{kpc}$. Some doubts about the strength of this conclusion persist, but it has been confirmed in other galaxies by Carter et al. (1981, 1985), and it is at least clear that the velocity dispersion profiles in many BCEs show no decrease with radius, as they do in field ellipticals (v., for instance, Davies et al. 1983, Tonry 1985). In the past year two groups have published generalizations of the Faber-Jackson (1976) relation, showing that to within observational errors, ellipticals lie on a plane in their parameter space (Djorgovski and Davis 1987, Dressler et al. 1987). Hoessel, Oegerle, and Schneider (1987) have shown that BCEs lie on the same plane.

The new discoveries have not been restricted to optical wavelengths. The success of the Einstein Observatory revealed facts about elliptical galaxies, and BCEs in particular, too numerous to reference completely. These include the discovery of correlations between the sizes and luminosities of BCEs and the Xray luminosities of the clusters in which they occur (Porter 1984), the discovery that not all dynamically evolved clusters form dynamically dominant BCEs (in other words, cDs: Jones and Forman 1984), and the detection of cooling-gas inflows in the centers of the densest clusters (v. Fabian, Nulsen, and Canizares 1984 for a review, and Bertschinger and Meiksin 1986 for a dissenting opinion).

Meanwhile, advances in computing hardware and numerical techniques have fostered simulations of elliptical galaxy formation and dynamics, and of galaxy mergers. The work most relevant to the systems in this paper includes van Albada (1982); Merritt (1983, 1984, 1985); Villumsen (1982, 1983); Farouki, Shapiro, and Duncan (1983); Duncan, Farouki, and Shapiro (1983); and Cavaliere et al. (1986). Van Albada showed that violent relaxation could produce structures with $r^{\frac{1}{4}}$ law 
surface brightness profiles and velocity dispersions that are extremely radially biased in the outer regions. Merritt's work questioned the conclusion of ongoing galaxy growth that many workers had drawn from the existence of multiple nuclei in BCEs, pointing out that many of these systems are probably not bound and suggesting that $\mathrm{BCE}$ structure is determined quickly, early in a cluster's history. Farouki and coworkers found evidence that mergers are not homologous, and Cavaliere et al. emphasized the importance of subclustering in delaying the final relaxation of a cluster. These studies allow a more detailed consideration of the morphology of merger products than did the pioneering work of Ostriker, Hausman, and coworkers (Ostriker and Tremaine 1975, Ostriker and Hausman 1977, Hausman and Ostriker 1978, McGlynn and Ostriker 1980).

Earlier papers of this series (Schneider, Gunn, and Hoessel 1983a, 1983b; Hoessel and Schneider 1985, papers I, II, and IV, respectively) dealt with observations of $\mathrm{BCEs}$ in a large sample of Abell clusters. Paper IV presented uniform photometry of BCEs in 175 clusters, including 16-kpc aperture magnitudes $\left(H_{o}=60, q_{0}=\frac{1}{2}\right)$, model fits to the azimuthally averaged light distribution within that radius, and an estimate of the frequency of occurrence of multiple nuclei. Since the chief goal of that paper was to evaluate the usefulness of BCEs as standard candles for cosmology, the light distributions of the multiple-nucleus galaxies were not cleaned or separated, since such an operation would be difficult or impossible to perform on clusters at very high redshift.

In this paper we take a different point of view, concentrating on the galaxies themselves. We have removed from the images of the BCEs in the sample of Paper IV all stars, neighboring galaxies, and secondary nuclei and present isophotometry of the BCEs in the form of ellipses fit to the galaxy isophotes. Our primary purpose 
is to examine the two-dimensional information in the surface brightness distribution. The motivation is twofold. The main hope is that shape parameters, particularly ellipticity profiles, will provide more clues than brightness profiles to the dynamics of these galaxies and their relation to their clusters. It is also of interest to establish to what extent BCEs are structurally similar to fainter and smaller ellipticals. The discussion in this paper will be limited mostly to the ellipse fits. A future paper in the series will discuss in more detail the departures from ellipticity (boxiness, dust lanes, asymmetries) in the sample.

In Section II we describe the procedure that was used to clean the images, and the results are presented in Section III. The isophotometric properties of BCEs are compared with those of field ellipticals in Section IV. Section V comprises a discussion of the results, and the conclusions are summarized in Section VI. Throughout this paper we assume $H_{0}=60 \mathrm{~km} \mathrm{sec}^{-1} \mathrm{Mpc}^{-1}$ and $q_{0}=\frac{1}{2}$. 


\section{OBSERVATIONS AND REDUCTIONS}

The data analyzed in this paper are the CCD images of the 175 BCEs of Paper IV. These images typically have a field of view of 4.5 with a scale of 0.548 per pixel, and exposure times 5-30 minutes. All were taken through the $r$ filter of the Thuan-Gunn (1976) system. Most were taken in periods of 1 " to $2^{\prime \prime}$ seeing on the Palomar 1.5-meter reflector. Table 1 lists basic data on the exposures. The clusters are listed by Abell number in column 1, and their redshifts are given in column 2 . Column 3 lists the date of the observation, in a 6-digit format: for instance, 091879 stands for UT September 18, 1979. Column 4 lists the exposure in seconds, column 5 the image scale in arc seconds per pixel, and column 6 the photometric calibration of the image in magnitudes per data number. Column 7 lists the full width at half maximum of the seeing, which was fit with a double Gaussian in each image. A more detailed description of the data may be found in Paper IV.

The data were reduced on the Space Telescope Wide Field/Planetary Camera VAX $11 / 780$ with the interactive software package CASSANDRA. The effects of bright stars were the first to be corrected. Saturated pixels were flagged by being reset to a large positive number: 32767 . Bad columns were repaired by interpolation from their neighbors. The removal of foreign objects could then begin in earnest. To isolate the light distribution of the $\mathrm{BCE}$, stars and other galaxies were edited out of the image with a polynomial interpolation routine. A rectangular box or mask was drawn around them, and the interpolation was based on the pixels surrounding the box. This was done while viewing the image at maximum contrast on a display system to ensure complete removal of the halos of neighboring galaxies wherever possible. The interpolation was done independently in both dimensions, and the 
results at each pixel, averaged. Shot noise appropriate to the intensity level at each pixel was then added. The parameters of the interpolation (the box size, the degree of the polynomial, and the frame size) could be varied within certain ranges to produce the best possible results.

Large objects in regions of high gradient in the BCE profile could not always be perfectly cleaned. Most of these fall into one of two categories:

1) galaxies or nuclei in regions of large intensity gradient (star images in these areas are generally small enough to be well removed even so), or

2) systems of two or more nuclei of comparable strength and small separation.

Fits from such regions are identified in the comments accompanying Figure 1 and can usually be identified by the breaks, clearly anomalous values, and high residuals they produce in the profiles. Multiple-nucleus galaxies can usually be adequately treated by the simultaneous ellipse-fitting technique of Lauer (1986, 1988) or the analytic method of Porter (1988b), but this has not been attempted here because of the small proportion of really intractable clusters in this sample.

This interpolation of signal and reconstruction of noise carries with it certain risks, and many workers in the past have chosen the more conservative approach of simply discarding contaminated regions of a picture. The greatest danger is that a galaxy in the neighborhood of the $\mathrm{BCE}$ may have a large, low surface brightness envelope of its own that will bias the interpolation if it is not, or cannot be, completely removed. We have been confident in proceeding as described because of the speed and intensity resolution of the imaging systems used. A picture can quickly be examined at a wide range of contrasts, and so any features that contaminate the fits can easily be seen and noted, if not perfectly cleaned. By 
the same token, the sky background could be brought into high relief, allowing the limiting magnitude of the photometry to be intelligently chosen. Systematic errors surely remain at a few percent of the sky brightness, but these are common to all photometry.

After the galaxies were cleaned as well as possible, ellipses were fit to the isophotes at intervals of 0.25 magnitudes. The software allows further picture editing at this stage by presenting for approval the pixels to which an isophote is to be fit, allowing any of them to be discarded. For each isophote of a galaxy, the following properties were derived: $\mu_{r}$, the Thuan-Gunn $r$ surface brightness in magnitudes per square arc second (using standard photometric calibration procedures); a and $\mathrm{b}$, the major and minor axes; $\epsilon=1-\frac{b}{a}$, the ellipticity; $\theta$, the position angle of the major axis; and $\mathrm{x}_{0}$ and $\mathrm{y}_{0}$, the coordinates of the centroid. The fits to each isophote are all independent of one another. Some studies have used the parameters of a given isophote as initial guesses for the fit to a neighboring one, and the fitted isophote parameters may thus be artificially correlated at low light levels. This should not be a problem with our method.

The use of surface brightness as the independent variable for isophotometry is also an approach different from that taken by most previous studies. Usually the major axis of an isophote with a fixed center is given, and the best-fitting values of the ellipticity, position angle, and surface brightness are found by standard $\chi^{2}$ minimization techniques. Since most elliptical galaxies have a very high degree of central symmetry (Lauer 1985), this procedure is usually satisfactory. The BCEs in Abell clusters have several important differences from field ellipticals, however. Many have complex multiple or obviously disturbed nuclei, and even some that appear relatively smooth are known to have significant large-scale asymmetries. 
A2634 and the broadest nucleus of A1185 are fine examples of such lopsided galaxies. Under these circumstances use of surface brightness as the independent variable provides the advantage that the ellipse centroids can naturally be varied as easily as the other shape parameters. It will be seen that this freedom gives further insight into the dynamics of a number of interesting BCEs.

In the best images isophotometry could be done reproducibly (not necessarily accurately; v. next paragraph) to a level of $27 \mathrm{mag} \operatorname{arcsec}^{-2}$. This is $0.4 \%$ of typical sky brightness in the Gunn $r$ passband and is consistent with the expected limit, as the following argument shows. The intensity scale in most of the images is approximately 29.3 magnitudes per data number, and in the deepest pictures, is 30.9. The radius of a $\mu_{r}=27$ isophote is at least 50 pixels and is usually larger, depending on redshift and galaxy profile. Such isophotes therefore contained over 2500 counts and should therefore, by a crude estimate, be determined to better than $2 \%$. To monitor the quality of the isophote fits, approximate $1 \sigma$ deviations were calculated at each brightness level. These are root mean squares of the distances from each pixel in an isophote to the fitted ellipse, not perpendicular to the ellipse, but along the line connecting the working pixel to the ellipse centroid. The difference between these two measures is significant only for the most eccentric isophotes, and the adopted definition of $\sigma$ in such cases is conservative.

The reproducibility of the photometry to this level is also demonstrated by independent analysis of four images of A2175 that were obtained under a variety of conditions with different cameras and telescopes. The fits to these data are shown in Figure 2. The ellipticity is reproduced to within \pm 0.05 and the major-axis angle to within $\pm 5^{\circ}$ between $a=2.5$ (about 3 times the seeing half width) and $\mu_{r}=26$. The ellipse centroids are reproduced to $2-3^{\prime \prime}$ at the same surface brightness, and 
the brightness profiles are essentially identical as faint as $\mu_{r}=27.5$. This is at a level where the "isophotes" of this galaxy are no longer even approximately elliptical and are probably dominated by systematic errors and scattered light in the field (v., e.g., King 1971 and Piccirillo 1973).

In fact, most of the isophotometry is cut off at levels between $23<\mu_{r}<26$ by other factors, the two worst being severely contaminated isophotes and imperfect flat fielding. These limitations have the advantage that they are grossly obvious and cause the quality of the isophotometry to deteriorate suddenly below some threshold brightness, rather than infecting it with subtle systematic errors. Poor sky subtraction due to the limited field of view is also a problem with some of the nearer clusters, but since the focus of the present study will be on shape parameters rather than photometry, this is not a serious concern.

Near the centers of the galaxies the isophotometry is seriously affected by seeing, which decreases the apparent ellipticity of isophotes; pixellation, which tends to increase it; and tracking quality, which, if poor, can completely mask true ellipticities and major-axis angles and noticeably degrade the surface brightness profiles at small radii. Rather than attempt fitting of seeing convolved ellipses image by image, we have conducted experiments on simulated and real data for a variety of true ellipticities and seeing conditions. This gives a rough idea of the accuracy of good data.

Two types of experiments were run. In the first, CASSANDRA was used to simulate images of galaxies with a variety of brightness profiles and constant ellipticities, blurred by various amounts of seeing. These artificial data were then reduced in the same way as the real pictures. Aside from showing the extent of seeing 
effects, these reductions allowed estimation of the precision to which major-axis position angles were determined as a function of ellipticity and surface brightness.

Over two dozen simulations were run, of galaxies with ellipticities from 0 to 0.6. Seeing was modelled with double Gaussians, whose power was split equally between components having $\frac{\sigma_{2}}{\sigma_{1}}=2$. Full-width half maxima ranged from $0 . \prime 8$ to $3{ }^{\prime \prime} 0$ (half energy radii 0"'9 to 3".5). Most of the models had surface brightness following the usual approximation to a King profile:

$$
\frac{I_{0}}{\left(1+\left(\frac{r}{a}\right)^{2}\right)}
$$

with "core radius" $a=1$ !' 6 . Effects of differences in central concentration were explored with King profiles having $a=0.5$ and de Vaucouleurs models having $r_{e}$ $=5.5$ and 13."0. All of the simulated images had scales of 0.'548 per pixel, 29.3 magnitudes per data number, and a sky level of $\mu_{r}=21$, to match the majority of the real images.

Reductions of some of the simulated pictures are shown in Figure 3. They suggest a good rule of thumb for estimating the effects even of relatively poor seeing: the ellipticity of a galaxy with a King profile is unaffected at radii larger than four or five times the full width at half maximum of the seeing disk, and even at the radius of the seeing disk, the apparent ellipticity is generally within 0.1 of its true value. These are conservative guidelines for systems in the range E4 to E5. If a galaxy has a steeper central brightness profile, the effect of seeing on the central isophotes is greater: a concentrated E6 de Vaucouleurs galaxy may have E4 isophotes at the radius of the seeing disk. Nevertheless the ellipticity regains its true value outside $r=5 \sigma$, where it can be determined with precisions ranging from \pm 0.02 in an E2 
galaxy, \pm 0.01 in an E3, and even better in flatter galaxies. The simulations suggest that for galaxies flatter than E6, systematic underestimation of ellipticity begins.

Rounder galaxies, in the range E2 to E3, show no effects of seeing outside two or three times the seeing radius. The effect of seeing on E1 galaxies is barely detectable. In this regime the primary limitation is the inability of the software to see a perfect circle in a finitely sampled image. When a model E0 galaxy was built and the resulting image reduced, the measured ellipticities of its isophotes scattered up to 0.05 .

Figure 3 also shows how ellipticity and signal strength govern the precision with which an isophote's major axis angle can be measured. Surprisingly, even the position angle of an E0.2 isophote can be measured, though only at the brightest levels, and only to within 10 or 20 degrees. Surface brightness ceases to have as strong an effect on the precision of position angle measurements when isophotes become as flat as $\mathrm{E} 2$, by which point the internal scatter is $\pm 3^{\circ}$. Ideally, position angles of isophotes flatter than E6 can be measured with a precision of better than a degree.

Centroid positions are very precisely measurable for all models and are completely unaffected by seeing. Figure 3 indicates that for isophotes brighter than $\mu_{r}=25$, the noise in the measurements is less than 0.'5 along the major axis of an isophote, and less than 0!'25 along the minor axis. However, the centroid positions are sensitive to telescope guiding errors, and many galaxies showed small amplitude $\left(<1^{\prime \prime}\right)$ centroid jogs in right ascension due to such errors. These are easily distinguished by inspection of Figure 1 and are usually noted in the accompanying comments. 
All of these guidelines are for the surface brightness range $\mu_{r} \leq 25$, i.e., for isophotes containing at least several dozen DN per pixel and not significantly contaminated by light from other objects or poor flat fielding. At fainter levels systematic errors once again dominate.

The simulated data had two major limitations: they are inevitably cleaner than real data taken with imperfect detectors, and CASSANDRA is not presently capable of simulating variable-ellipticity galaxies. A second experiment was therefore also run. Six images with good (1!2 or better FWHM) seeing and a variety of ellipticity profiles were chosen from the sample, degraded by smoothing with Gaussians, and rereduced. Three of the images were on the 1.5-meter scale (0.'548 per pixel), and three were Hale 5-meter images with scales of 0.415 per pixel. Gaussians of 1.55 and 3.1 pixels FWHM were used for the smoothing, producing images of approximately $2^{\prime \prime}$ and $3^{\prime \prime}$ seeing.

The degraded profiles are compared to the original results for the same galaxies in Figure 5. The plot for A1081 shows that if the ellipticity of a galaxy's isophotes is large, but also changes substantially at small radii, seeing may decrease the measured $\epsilon$ by as much as 0.25 at $1^{\prime \prime}$. Encouragingly, however, the tests on both A1081 and A1413 suggest that large errors are confined to within about 3 times the seeing radius, even for 3 " seeing. Two other points are worth noting. First, the detailed effects of the smoothing on A1809 are matched very well by an image of that cluster taken in poorer seeing (Porter 1988a). Second, the tests on A1413 show that its central ellipticity minimum is not a seeing artifact. This is an important verification of a type of feature that is visible in many of the galaxies in Figure 1.

Another important test of the results is comparison to published isophotometry of some of the same galaxies in the sample. Surface brightness profiles 
are available for many of the galaxies in our sample, but ellipse fits are much rarer. Most ellipticals with available isophotometry are at much smaller redshifts than our galaxies, and in many cases the ellipse centroids were fixed rather than being free parameters. Nevertheless there are two studies to which we can compare our results quantitatively. We have an overlap of 10 galaxies with the sample of Malumuth and Kirshner (1985). Djorgovski's (1985b) restricted sample (i.e., of ellipticals only) included NGC 2832 in A779, NGC 4889 in Coma (A1656), NGC 6173 in A2197, and NGC 6166 in A2199.

Our surface brightness, ellipticity, and position angle profiles are plotted against those of these two studies in Figure 4. The abscissa, instead of being $r$ surface brightness as in Figure 1 , is the independent variable used by the other study: the major axis for Djorgovski's 4 galaxies, and the mean radius $\sqrt{a b}$ for those of MK. Overall the agreement is quite good. Djorgovski's profiles are uniformly brighter than ours, because his r passband was broader than ours, and no zero-point correction has been included in the plots (v. Djorgovski 1985a). But the shapes of his brightness profiles are reproduced quite well, allowing for the difficulty of correcting for the complex structure of NGC 6166 (the BCE in A2199). The only significant disagreement between our shape profiles is in the ellipticity of A779, which Djorgovski finds to be half an ellipticity class rounder than we do. The seeing in Djorgovski's image was relatively poor, and this may explain the difference, although it extends to larger radii than our tests suggest it should.

The agreement of our fits with those of MK is satisfactory, though not perfect. The ellipticity profiles of A2107, A2124, and A2147 agree at all values between 0.0 and 0.6. In A2052 a large isophote twist at very small radii and ellipticities ( $\mathrm{r}<3^{\prime \prime}$, $\epsilon<0.1$ ) is reproduced remarkably well. Of the disagreements that exist, some are 
easily understood. The profiles of A2670 are an extreme example of the limits MK placed on their attempts to derive ellipticities and position angles for very round and very faint isophotes: they have set $\epsilon$ and major axis PA to reasonable constants for the entire profile of this nearly round galaxy. The variations in our profiles, especially the two ellipticity minima, may be primarily due to improperly cleaned companion galaxies.

Four disagreements, however, are not as easily explained. These are in the position angle profiles of A401 and A2124 and the surface brightness profiles of A401 and A496. The position angle profiles of A2124 agree over most of their range, but ours shows a sharp twist of $70^{\circ}$, from east-west to PA $110^{\circ}$, before returning to $150^{\circ}$ at $\mathrm{r}=10^{\prime \prime}$, where it is once again in fair agreement with MK's profile. One might speculate that their algorithm is not good at detecting sharp position angle gradients, had it not done so, in agreement with our results, in A2052. The disagreement in the position angle profiles of A401 is even worse: MK observe a twist of $50^{\circ}$ in the inner $5^{\prime \prime}$, but we do not. Equally curious is the disagreement in the surface brightness profiles of A401 and A496: our profiles are significantly steeper than MK's. Yet their seeing was not significantly worse than ours, and one would not expect typical errors in sky subtraction to affect profiles at the $\mu_{r}=22$ level.

The only other isophotometric studies we have found of galaxies in the present sample are in Carter (1978) and DiTullio (1979). DiTullio (1979) observed NGC 4889, and Carter (1978) observed NGC 6173 and 6166. Their profiles are in satisfactory agreement with ours and Djorgovski's. Carter's ellipticity profiles are up to 0.05 higher than ours in some places, but the disagreements are much less significant than those discussed above. 


\section{ISOPHOTOMETRY}

As stated in II, ellipses were fitted to the galaxy isophotes at intervals of 0.25 magnitude. The results are shown in Figure 1, in order of Abell number. Plots for some second-brightest cluster galaxies are also shown, usually for clusters in which the first and second galaxies have comparable magnitudes or in which there is reason to believe the second-ranked galaxy may have some unusual dynamical significance. Nine profiles are shown for each galaxy, all as a function of surface brightness. These are the major and minor axes, and their geometric mean, the ellipticity, eccentricity, and major-axis position angle, the isophote centroid coordinates $x_{0}$ and $y_{0}$ (relative to the nucleus), and the $1 \sigma$ deviation of the fit from the pixels used. For systems with multiple nuclei, different symbols are used for the inner regions of the (usually 2) different nuclei and for the outer envelope. The ellipticity and major-axis angle profiles are often the most interesting, but the others also warrant inspection. As stated above, the $1 \sigma$ value is the mean distance from a pixel in an isophote to the fitted ellipse, not perpendicular to the ellipse, but along the line connecting the pixel to the center of the ellipse. It should be referred to in evaluating the reliability of the surface brightness profiles, and especially the centroid displacement profiles. The surface brightness, centroid displacement, and $1 \sigma$ plots are scaled to the variation of those quantities. The ellipticity, eccentricity, and position angle profiles are shown at the same scales for all galaxies: 0.0 to 0.7 for ellipticity, 0.0 to 1.0 for eccentricity, and covering $180^{\circ}$ about the average value for the major-axis angle. This is best to display the variations in the sample. Because of its length, Figure 1 is presented as an appendix to the thesis. Comments on individual clusters are given with the figure. In this section we will discuss the properties of the sample as a whole. 
There are two ways to approach the mass of data in Figure 1. The first is more quantitative and objective: one can, for example, plot the distribution of ellipticities at a fixed radius, or the amount of isophote twisting between two chosen radii. The second is more qualitative and subjective. One can classify galaxies by the morphology of their ellipticity profiles, or look for significant drift or breaks in isophote centroid positions. Each approach provides some insight into the data and can correct overinterpretations made using the other approach. We will describe our more quantitative experiments first, and the qualitative ones second.

Given this first large body of isophotometry of BCEs, one of the most obvious questions to ask is: what is the distribution of ellipticities of $\mathrm{BCEs}$ as a function of radius? It is important to know whether it varies significantly and whether it resembles the classical distribution of ellipticities of field galaxies (Sandage, Freeman, and Stokes 1970; Binney 1978). The comparison to field ellipticals is reserved for the next section. We now concentrate on the ellipticities of BCEs.

Since isophotes were fit at 0.25 magnitude intervals and each choice of inner isophote was somewhat arbitrarily made for convenience, the measured isophotes corresponded to different physical radii for different galaxies. To facilitate the analysis, a simple weighting scheme was devised to estimate the ellipticity of a galaxy at a series of chosen semimajor axes $a_{k}$. The weighting was by a Gaussian function of $\log$ a:

$$
\epsilon\left(a_{k}\right)=\frac{\sum_{i} \epsilon\left(a_{i}\right) \exp \left(\frac{-\log \left(\frac{a_{k}}{a_{i}}\right)}{\sigma^{2}}\right)}{\sum_{i} \exp \left(\frac{-\log \left(\frac{a_{k}}{a_{i}}\right)}{\sigma^{2}}\right)}
$$


where $a_{i}$ are the major axes of the measured isophotes. The dispersion $\sigma$ was chosen to be 0.1 , which usually resulted in significant contributions from 3 to 5 measured isophotes. This was enough to suppress some noise, but not significant large-scale variations in the profiles. The major axis angle profiles were treated in the same way. Data obviously contaminated by poor fits in the regions of overlap among multiple nuclei were edited out by hand.

An important additional filter applied to the data was the exclusion of all isophotes with semimajor axes less than 9 times the seeing FWHM in the image. This was done in consideration of the results of the seeing tests described above. The fiducial semimajor axes chosen were $2,4,8,16,32,64$, and $128 \mathrm{kpc}$. These points have equal separations of 0.3 in the logarithm, and represent the physical radii sampled by the data between seeing disks and the outer limits of the galaxies. The sample sizes at those radii were $47,152,167,158,139,67$, and 9 , respectively.

Figure 6 is a plot of the average ellipticity of the sample detected at each of these radii. The error bars are root mean square deviations from the means. There is a clear increase in the average $\epsilon$, from 0.14 at $2 \mathrm{kpc}$ to 0.45 at $128 \mathrm{kpc}$. The increase is also clearly visible in the histograms in Figure 11 . Within $32 \mathrm{kpc}$, this increase is very well represented by $\epsilon=0.08+0.13 \log (a)$. The figure suggests that the increase accelerates beyond $32 \mathrm{kpc}$, but the sample size is becoming smaller, and the error bars drawn are likely to be underestimates of the true uncertainties.

We now show that the average ellipticity increases with radius because individual galaxies grow flatter toward the outside, and not because round galaxies are intrinsically smaller, or drop out of the sample because of some selection effects. Figure 14 histograms the change in ellipticity from $4 \mathrm{kpc}$ to 16,32 , and $64 \mathrm{kpc}$ in galaxies present in the sample at both radii. Only 4 out of $63(6 \%)$ of the BCEs 
have $\Delta \epsilon<0$ between 4 and $64 \mathrm{kpc}$, and the percentage for the 16 and $32 \mathrm{kpc}$ histograms is less than $10 \%$. Conversely, the $4 \mathrm{kpc}$ ellipticity histogram of those BCEs that drop out of the sample at larger radii looks no different from $n(\epsilon)$ for the entire sample at $4 \mathrm{kpc}$.

We have studied the behavior of $\mathrm{BCE}$ ellipticities at multiples of their de Vaucouleurs effective radii and core radii as well as at fixed metric radii. The parametric sizes were taken from Paper IV, and ellipticities were again measured at intervals of 0.3 in the logarithm, but this time covering the range $\frac{1}{16} r_{e}$ to $4 r_{e}$ for the de Vaucouleurs fits, and $0.8 a$ to $51.2 a$ for the modified Hubble fits. Since the average effective radius of the sample is $32 \mathrm{kpc}$ and the average core radius is 2.4 $\mathrm{kpc}$, this should cover approximately the same range $(2-128 \mathrm{kpc})$ studied in Figures 6,11 , and 14 .

For these experiments an additional precaution had to be taken: galaxies not well fit by a particular model were not used to study the behavior of ellipticity at multiples of the scale length of that model. This was necessary because of the fitting procedure used in Paper IV. At that time, "galaxy centers" were determined in a completely unbiased way: they were defined as the position giving maximum luminosity within a circular aperture with a radius of $16 \mathrm{kpc}$. The scale lengths in Paper IV were determined from azimuthally symmetric model fits using that center. There are enough double and multiple BCEs in the sample to mask correlations sought using all the resulting fits. Table I in Paper IV indicated which BCEs were well fit by which model, if either, and only such good fits were used in the present work. This results in a $50 \%$ decrease in the sample size, but is necessary for the present. We intend to produce improved fits to the cleaned minor- and major-axis profiles in a future paper. 
Figure 7 is the plot of $\epsilon\left(\log \frac{a}{r_{e}}\right)$. The trend of the first 5 points is identical to that visible in Figure 6, but there is not as marked an ellipticity increase in the last two points. The sample sizes for the points in this figure are $22,47,79,88,81$, 58 , and 21. This suggests that the reason the trend is less pronounced is that there is some "mixing" of physical radii in the conversion to scale lengths. This is not surprising, since $\mathrm{BCEs}$ have a range of effective radii, but it is perhaps significant that ellipticity depends a bit more strongly on physical radius than on scale length. Comparison of the ellipticity histograms at multiples of $r_{e}$ in Figure 12 to those at fixed radii in Figure 11 also suggests that the increase in ellipticity is slighter as a function of $\frac{a}{r_{e}}$. But the small sample sizes at large radii make this conclusion tentative.

Despite the conservative corrections for seeing, multiple nuclei, and poor quality fits, the plot of ellipticity at various multiples of a galaxy's core radius in Figure 8 shows almost no trend. This is perhaps not surprising, since the average core radius, $2 \mathrm{kpc}$, is an order of magnitude smaller than the radii at which the changes in ellipticity are being observed. The sample sizes for the points in this figure are $34,52,78,80,64,42$, and 12 . One might expect uncertainties in the core radius fits to cause blurring of any trend at large radii, but Figure 8 shows no convincing trend even at scales of 1-10 core radii. Since there have been numerous indications that the interiors of $\mathrm{BCEs}$ are causally related to their cluster environment (Paper I and references therein, Porter 1984), and since other results presented in this paper strengthen the evidence for physical connections between a cluster and its $\mathrm{BCE}$, this is somewhat disturbing and requires further study.

Isophote twisting was also studied in an objective fashion. Figure 16 shows histograms of isophote twists between $4 \mathrm{kpc}$ and 16,32, and $64 \mathrm{kpc}$. Once again an 
additional restriction had to be made to the sample: this time only those galaxies that had $\epsilon \geq 0.1$ at both the radii in question were included. This was done to eliminate the scatter in PA measurements of nearly round isophotes. Nevertheless the figure shows that about a third of the BCEs studied show isophote twists of more than $10^{\circ}$, and that this figure is more or less independent of the major-axis baseline chosen for the measurement.

We searched for correlations among ellipticity, ellipticity gradient, and isophote twisting, as well as for correlations between any of those properties and global properties such as core radius, effective radius, growth parameter, reduced absolute magnitude (v. Paper IV), and cluster X-ray luminosity. These searches were performed over the full range of fiducial radii at which the shape parameters were measured. No correlations were found other than can be explained by the increase of ellipticity with radius and various selection effects. For instance, it could be said that "faint BCEs are rounder than average," but that is because they have smaller parametric radii (Paper IV) and are therefore observed only at small physical radii, where isophotes are rounder than at $128 \mathrm{kpc}$. Samples of the scatter plots found are shown as Figures $18\left(\epsilon\right.$ at $8 \mathrm{kpc}$ vs. $\left.\log r_{e}\right), 19(\Delta \epsilon$ between 4 and $32 \mathrm{kpc}$ vs. $\log r_{e}$ ), and 20 (isophote twisting between 4 and $32 \mathrm{kpc}$ vs. $\log r_{e}$ ).

We turn now to the less quantitative, but most instructive and not entirely subjective exercise of looking at the profiles.

Undoubtedly the most striking property of the sample is the ubiquity of increasing ellipticity profiles. Furthermore most ellipticity profiles are quite simple in that they show at most one change of slope. Seventy-two show none, and only 15 show more than one. As for the increases, they may be shallow, they may occupy only part of a profile that is elsewhere constant, and they may compete 
with decreases in other parts of the profile, but there are only $24 \mathrm{BCEs}$ in the entire sample of 175 whose ellipticity profiles are nowhere increasing, and 19 of these are constant: only 5 show decrease but no increase. There are 39 galaxies in which some ellipticity decrease is present, but these have a greater increase in other parts of the profile. BCEs with significant ellipticity decreases are listeci in Table II, and the 19 with constant ellipticity are listed in Table III. Variations over less than about 0.5 magnitudes in surface brightness ( 3 fitted isophotes) are disregarded as local perturbations.

The listing in Table II is fairly objective, as closer examination of several of these galaxies (listed in parentheses) shows they are not typical ellipticals. A1474, for instance, is obviously an edge-on disk galaxy, and A193, A389, A1123, and A1904 have unresolved complex central structure. The ellipticity decrease in A2283 is so slight that only A2328 and A2382 are convincing candidates for steady ellipticity decrease, and even these images have some tracking error and poor seeing $\left(2^{\prime \prime}\right.$ and 1:!7, respectively). The amplitudes of the ellipticity decreases are very small. The ellipticity of A2328 goes from 0.2 to 0.1 , and that of A2382 from 0.2 ( 0.3 , if one accepts the innermost point) to essentially 0. A2328 has a small companion $2^{\prime \prime}$ from its center, and A2382 has a compact companion 18" to the south. Further comments acompany Figure 1.

The most interesting fact about galaxies with self-similar isophotes is that they are much rounder than other ellipticals. The average ellipticity of the 17 galaxies in this class is 0.12 , and all are at least as round as E2. Anticipating the discussion below, we note that Djorgovski's sample of ellipticals gives the same results. In other words, no ellipticals that are anywhere flatter than E2 have selfsimilar isophotes. 
DiTullio (1979) has concluded that decreasing ellipticity profiles are predominant among isolated galaxies, occurring less frequently among members of groups and clusters, and never among BCEs. The present data strongly support the last part of her claim.

Ellipticity variations in $\mathrm{BCEs}$ have another interesting property. When they are large enough, they are clearly visible as disturbances in the surface brightness profiles, or differences between the major- and minor-axis brightness profiles. While inspecting Figure 1, we noticed that ellipticity disturbances are almost never reflected by the minor-axis surface brightness profile alone. In other words, there is a tendency for the surface brightness profiles to be smoother along the minor axis. This statement must be interpreted cautiously, for it is certainly true that there are many galaxies with inflections or bumps on both axes, and many examples of structure in ellipticity profiles that cannot be attributed to disturbances along only one axis. Nevertheless we have identified 27 examples of an ellipticity change due to a change on a major-axis profile, and only 4 ellipticity changes arising from a minor-axis brightness profile.

Similarly we have searched for galaxies with bright "halos," i.e., those whose surface brightness profiles have significant decreases of slope. By the definition of Schombert (1988), these are $\mathrm{cD}$ galaxies, and many of them have associated disturbances in their ellipticity and position angle profiles to suggest that they have two dynamical components. However the breaks are to be interpreted, they too seem to show a preference for major-axis profiles. Further discussion of these galaxies is given in Porter (1988a, chapter II of this thesis).

Figure 1 shows a large percentage of galaxies with strikingly large isophote twists. A naive tabulation gives 70 galaxies with total twist amplitudes of $40^{\circ}$ 
or more! However, 41 of these involve isophotes with ellipticities of less than 0.1 and must be regarded with considerable skepticism. We have compiled a list of 33 galaxies with what we feel have credible twist amplitudes of at least $40^{\circ}$ and examined their surface brightness and ellipticity profiles in more detail. All have two component surface brightness profiles, and many have structure in their ellipticity profiles which is clearly associated with the isophote twisting. The list is presented in Table IV.

One particularly interesting type of behavior observed in a few galaxies is the coincidence of a sharp rotation in the isophotes with a pronounced local circularization of the isophotes. The most convincing examples of this are A978 and A1228. This behavior is almost certainly indicative of superposed but misaligned components of the light distribution. In the regime where two such components have similar surface brightness, the ellipticity of each is suppressed. Within this region the apparent major axis is due primarily to the light of the smaller, brighter component; outside it is dominated by the light of the larger component. In between lies the transition.

A quantity of interest is the maximum observed major-axis rotation over a range of surface brightness in which the intensity, ellipticity, and centroid profiles show no evidence of complex structure. A2061 has a PA profile that rotates through $40^{\circ}$ between $4^{\prime \prime}<a<20^{\prime \prime}$, where the ellipticity increases steadily from 0.07 to 0.4 . The profile shows even larger axis twists at smaller radii, but since the isophotes involved have $\epsilon<0.05$, these are not trustworthy. Outside 20"the PA stabilizes, and there is a slight indication of a halo.

Since it is possible to model isophote twists of much greater than $40^{\circ}$ with triaxial figures (Williams [1981] modelled a $60^{\circ}$ twist in NGC 596 this 
way), and since many smaller isophote twists are probably the result of external perturbations (e.g., the two galaxies in A154 have twists of $10^{\circ}$ and $20^{\circ}$ ), it is not possible to separate intrinsic and projected twists on the basis of photometry alone. Spectroscopic studies of the BCEs in Table IV should greatly further our understanding of elliptical galaxy dynamics.

Table $\mathrm{V}$ lists 34 galaxies found to be significantly asymmetric, as shown by isophote centroids displaced from their nuclei. Most of these centroid drifts have been verified by antisymmetrizing the images: rotating the frame by $180^{\circ}$ about the $\mathrm{BCE}$ nucleus and subtracting it from itself. Antisymmetries show up as dipole residuals when this is done (Porter 1988b). The tables show that antisymmetries are not limited to obviously multiple galaxies: the drifts in A43 and A1630 are as strong as those in A42 and A910, though perhaps weaker than the powerful disturbance in A1185. They also show that while the proportions of isophote twisting and asymmetry in the sample are about equal, they are not strongly associated. There are only 13 galaxies in common between Tables IV and V. Most of these do have multiple nuclei or close companions. 


\section{PAST ISOPHOTOMETRY OF ELLIPTICALS}

The first ellipticity profiles date from the early 1960s (e.g., Liller 1960, 1966), but only recently have such observations become common, thanks to CCDs. King (1978) published isophotometry of elliptical galaxies based on photographic plates. DiTullio $(1978,1979)$ published ellipticity profiles of NGC 4889 and numerous isolated and cluster ellipticals. The most extensive isophotometric study of elliptical galaxies has been the thesis of Djorgovski (1985b), and the only extensive isophotometric study of BCEs so far has been that of Malumuth and Kirshner (1985).

These studies have shown that ellipticals exhibit a great variety of flattening profiles: some constant, some increasing, some decreasing, some mixed, some apparently completely irregular. Often irregularities in the brightness and flattening profiles are correlated (e.g., Davis et al. 1985), a phenomenon that the present study and that of Djorgovski show is quite general. However, there are two simple types of flattening curve that account for most galaxies so far observed. These are (1) a continuous, steady (as opposed to accelerating) increase in $\epsilon$, and (2) an increase in $\epsilon$ followed by a turnover at some intermediate radius. Few galaxies have truly constant flattening profiles, and even fewer have decreasing ones. These are results that have been borne out by the present study.

To compare the properties of BCEs with field ellipticals, we shall use the primary sample of Djorgovski (1985b). This is a complete magnitude-limited set of 122 ellipticals drawn from the CfA redshift survey. We have calculated the average ellipticity of the field ellipticals at the same fiducial radii with the same technique used for the BCEs. Distances to the galaxies were calculated by 
Djorgovski according to a Virgocentric flow model with a local group infall velocity of $300 \mathrm{~km} \mathrm{sec}^{-1}$.

Figures $9,10,13,15$, and 17 show the same data for Djorgovski's galaxies as Figures 6, 7, 11, 14, and 16, respectively, for the BCEs: ellipticity plotted as a function of radius, ellipticity at multiples of $r_{e}$, histograms of ellipticity at fixed radii, ellipticity changes between fixed radii, and isophote twists between fixed radii. The sample sizes for the points in Figure 9 are 121, 117, 93, 41, and 12, and for Figure 10 , are $5,18,51,86,93,84$, and 48 . The $\mathrm{BCE}$ sample extends to larger radii than the field sample, partly because the field galaxies are intrinsically smaller, and partly because the nearest field galaxies were not imaged as far out as $128 \mathrm{kpc}$. Note also that the field ellipticals have much smaller effective radii, so that while Figures 6 and 7 sample the same region of space in the BCEs, Figures 9 and 10 sample different regimes in the field galaxies.

At any rate it is clear that field ellipticals do not show nearly as strong an increase of ellipticity with radius as do BCEs. This is true whether one plots ellipticity as a function of physical radius or as a function of $\frac{a}{r_{e}}$. Due to small sample sizes, it is not possible to state that the field galaxies are rounder than BCEs at large radii, but they do seem to be more elliptical at small radii: their average ellipticity is about 0.2 everywhere, whereas the BCEs have an average ellipticity of 0.13 at $2 \mathrm{kpc}$, with a root mean square deviation of 0.04 . Nowhere within $32 \mathrm{kpc}$ are field ellipticals uniformly very flattened, as BCEs are at large radii.

Four other remarks about the sample of field ellipticals can be made. First, despite the absence of a strong trend in Figure 9, there is some evidence that these galaxies are flatter at large radii. Indications of this effect are visible in Figures 13 and 15. Isophotometry of any field galaxies that can be found to extend to radii 
of $50 \mathrm{kpc}$ should be done to investigate this possibility. Large-format CCDs that would make this project feasible are now available.

Second, Figure 17 suggests strongly that field ellipticals show more isophote twisting than BCEs. Over the longest baseline shown, half the sample has rotated through more than $20^{\circ}$. This result must be interpreted cautiously for several reasons. Although galaxies with $\epsilon<0.1$ have again been excluded from consideration, we are looking at radii that are not only physically different (the histograms for the field ellipticals are referred to $2 \mathrm{kpc}$, not $4 \mathrm{kpc}$ ) but also different relative to the galaxy structure (i.e., $r_{e}$ ). A more careful comparison of isophote twisting in field ellipticals and BCEs should prove enlightening, but is outside the immediate scope of this paper.

Third, we remarked above that only BCEs rounder than E2 have similar isophotes. The same is true of Djorgovski's sample of field ellipticals.

Fourth, the absence of any correlation between shape parameters at fixed radius and global galaxy parameters in BCEs is the same as the result found for the field ellipticals by Djorgovski. 


\section{DISCUSSION}

\section{The intrinsic shapes of BCEs}

The most striking behavior of the galaxies in the present sample is the uniform tendency for their flattening profiles to increase. One consequence of this is that there are very few $\mathrm{BCEs}$ that are as round as $\mathrm{E} 0$ or $\mathrm{E} 1$ at radii greater than $32 \mathrm{kpc}$. It is therefore relatively easy to show that the shapes of $B C E_{s}$ at large radii cannot be drawn from a reasonable population of oblate spheroids. This is the first sample of ellipticals for which a choice between a prolate and oblate distribution has been possible on the grounds of shape observations.

The projection of intrinsic ellipticity distributions of oblate and prolate spheroids to apparent distributions, with the assumption of random orientations, is a well-studied problem (Sandage, Freeman, and Stokes 1970; Binney 1978). If $f(q)$ is the intrinsic distribution of axial ratios $\left(\epsilon=1-\frac{b}{a}=1-q\right)$, the apparent distribution is

$$
n(\beta)=\int_{0}^{\beta} \frac{\beta f(q) d q}{\sqrt{\left(1-q^{2}\right)\left(\beta^{2}-q^{2}\right)}}
$$

for an oblate population, and

$$
n(\beta)=\int_{0}^{\beta} \frac{\frac{q^{2}}{\beta^{2}} f(q) d q}{\sqrt{\left(1-q^{2}\right)\left(\beta^{2}-q^{2}\right)}}
$$

for a prolate population. The kernel in the integrand for the oblate distribution has the effect of producing a significant proportion of apparently round galaxies even 
for a population of disks. Thus large numbers of spiral disks look circular. This is not so for the population of prolate spheroids.

These projection formulae were applied to Gaussian distributions of spheroids

$$
f(q)=\exp \left(\frac{\left(q-q_{0}\right)^{2}}{2 \sigma^{2}}\right)
$$

with mean axial ratios $q_{0}$ ranging from 0.9 to 0.4 , in steps of 0.05 , and dispersions $\sigma$ of $0.05,0.1,0.15$, and 0.2 . In Figure 16 we display the resulting distributions of apparent ellipticities for the models with $\sigma=0.1$ and $q_{0}=0.65,0.6,0.55$, and 0.5 . The prolate population provides a much better match to the observations than the oblate one. Populations with other values of $q_{0}$ and $\sigma$ do not fit the observations at large radii well at all. At smaller radii (say, $a<16 \mathrm{kpc}$ ), the apparent ellipticity distribution is indistinguishable from that of samples that have been studied before, and no choice between oblate and prolate populations is possible.

The potential of the data to inform us of the intrinsic shapes of BCEs at large radii has probably not been exhausted. One might try to invert the observed ellipticity distribution using Lucy's iterative method, as did Binney and de Vaucouleurs (1981). In fact, the small sample size combined with the high likelihood that $\mathrm{BCEs}$ are triaxial, rather than exactly prolate, would probably make this an exercise in futility. A more constructive question to put to the data would be whether or not the isophotes are still elliptical, and the level surfaces therefore presumably ellipsoidal, at $64 \mathrm{kpc}$. The residuals to the ellipse fits do not give an obvious answer. The 64-kpc isophotes of some galaxies appear to be elliptical to within the measurement errors. A2029 is an obvious example. Other galaxies are 
clearly irregular at this radius. A more careful study of this question, to include derivations of higher order Fourier coefficients of isophote fits is planned.

\section{Ellipticity profiles and dynamics}

The uniform tendency for the ellipticity of the BCEs in the present sample to be monotonically increasing is especially interesting in contrast to the frequency of turnover in $\epsilon(\mathrm{a})$ often observed in Djorgovski's sample of field galaxies. In this section we explore the dynamical reasons for these different behaviors.

First, note that the ellipticity profiles observed are likely to be stable in most cases. The two-body relaxation timescale for an elliptical galaxy is vastly longer than the Hubble time even at fairly small radii, and the crossing time and violent relaxation timescales much shorter than the Hubble time, even at fairly large radii. In other words, the observed flattening profiles are not due to differences in the degree of relaxation at different radii. (This is the opposite of the situation in globular clusters. See Shapiro and Marchant 1976 and references cited therein.) Therefore, one can conclude from the rarity of profiles with $\epsilon^{\prime}(a)<0$ in BCEs either that the formation of such profiles is suppressed or that they are unstable, or perhaps both.

Binney (1980) has made an important study of the relation between the flattening profile of a galaxy and its rotation curve. First studying galaxies with isotropic velocity dispersion, he found a remarkable difference between those that have increasing flattening profiles and those in which $\epsilon(a)$ turns over or decreases. The rotation curves of the latter are flat after an initial rise, while those of the latter peak and then decay. 
This model gives a clue to understanding the flattening profiles of BCEs. As Binney was among the first to appreciate, real luminous ellipticals are not isotropic rotators. Some or all of their support against the force of gravity may be provided by dispersion pressure rather than ordered rotation. A galaxy's total kinetic energy is the sum of its streaming and dispersion energies. Binney's model suggests that if one introduces even a moderate amount of anisotropy into a model whose rotation curve is already low, a negative streaming kinetic energy - an unphysical situation - will result. In more conventional terms, a dispersion-supported galaxy with decreasing ellipticity is unstable. To the best of our knowledge, this is an unproven conjecture, but it is supported by a great deal of circumstantial evidence.

There are many good reasons to believe that massive elliptical galaxies have anisotropic dispersion, at least in their outer regions. These include the frequent observation of low rotation velocities (e.g., Davies et al. 1983, Tonry 1985), minoraxis rotation and isophote twisting, which are signs of triaxiality (Binney 1985), and the results of numerical simulations (e.g., van Albada 1982) which show that violent relaxation tends to produce velocity ellipsoids that are radially elongated far from a galaxy's center. This can therefore be viewed as a partial explanation of the almost total absence of decreasing ellipticity curves among BCEs and of their rarity even among field ellipticals.

One would like to use Binney's models to derive kinematic information from observed flattening profiles. Unfortunately the models are at least as sensitive to the anisotropy that they can accommodate as they are to $\epsilon(a)$. Random motion can drastically alter the shape of a rotation curve, obscuring any effects due to the mass distribution. This is illustrated partly by the three galaxies to which Binney applied the algorithm and even more dramatically by the application of the technique to 
NGC 5813 by Efstathiou, Ellis, and Carter (1982). Although the fainter isophotes of this galaxy flatten in a very typical manner, its rotation curve shows a sharp peak $(80 \mathrm{~km} / \mathrm{sec})$ at small radii $\left(3^{\prime \prime}\right)$ and drops to only $8 \mathrm{~km} / \mathrm{sec}$ beyond $10^{\prime \prime}$. This is in fact the lowest rotation velocity reported in the compilation of Davies et al. (1983). Efstathiou et al. enjoyed tolerable success in reproducing this rotation curve by making the model suitably anisotropic.

Ideally one can make limited guesses about the peak rotation velocity on the basis of the central ellipticity. Binney's work shows that a galaxy with a round core cannot be prevented from having a high rotation velocity at some, usually small, radius. This is the cause of the observed peak in the rotation curve of NGC 5813, whose central ellipticity is only 0.1 . Therefore one expects a galaxy like A189, whose ellipticity climbs from essentially 0 in a bright nucleus to 0.6 at $50 \mathrm{kpc}$, to have significant rotation, and indeed it does: $250 \mathrm{~km} \mathrm{sec}^{-1}$ (Tonry 1985). On the other hand, Dressler (1979) found no significant rotation peak in A2029, which the present study shows to have a similar rise in ellipticity. One should also be very cautious in applying models built for 100-pc ellipticals to kpc sized giants, especially since seeing makes it impossible to observe the inner kpc of all but the nearest BCEs.

Finally, a flattening profile of the form $\epsilon^{\prime}(a)>0$ does not rule out rotational support of a galaxy. A counterexample is NGC 4742 in the Virgo cluster, observed by Djorgovski (1985b) to have $\epsilon(\mathrm{a})$ rising from 0.2 to 0.45 , and by Davies et al. (1983) to be fully supported by a flat rotation curve of $80 \mathrm{~km} / \mathrm{sec}$. Still, this is a faint $\left(\mathrm{M}_{B}=-19.4\right)$ galaxy among many others in a cluster, quite different from a BCE. Most kinematic observations of BCEs to date (of A2029 by Dressler [1979], of IC 2082 by Carter et al. [1981, 1985], of NGC 4889 in Coma by Davies et al. [1983b], 
and of A401 by Faber, Burstein, and Dressler [1977]) show them to have essentially zero rotation. The largest kinematic study of BCEs to date is that of Tonry (1985), who observed a complete sample of nearby multiple nuclei, and found significant rotation in less than 5 of 14 BCEs. Of the 5 that show rotation, one (A193) had unresolved central structure, one (A189) has an unusually bright nucleus, one (A2151) is in a dynamically young cluster, and two (A1228, A2199) have rotation velocities less than $50 \mathrm{~km} / \mathrm{sec}$, and complex structure besides. More kinematical observations are desirable, but there is not yet any strong reason to doubt that most $\mathrm{BCEs}$ have negligible rotation. Binney's work suggests that galaxies whose ellipticity profiles turn over are the most promising candidates for BCEs which do rotate.

There are also sound theoretical reasons to expect BCEs to have low or zero rotation. They are likely to have more extensive merger and accretion histories than common ellipticals, and the mixing that occurs during mergers will suppress the proportion of ordered to random kinetic energy supporting a galaxy against rotation (Davies et al. 1983).

\section{Alignment of BCEs and Clusters}

It has been known for some time that BCEs are aligned with the clusters in which they are found. Early reports of the alignment include Matthews, Morgan, and Schmidt (1964) and Sastry (1968). Noonan (1972) and Austin and Peach (1974) found that the cluster extension and alignment with the BCE in A1413 was a strong function of galaxy luminosity. More recently, Dressler (1978) and Carter and Metcalfe (1980) confirmed these results in a larger sample of rich clusters, and Binggeli (1982) introduced an objective quantification both of cluster orientation and ellipticity. Studies of a much larger sample of clusters have recently been 
carried out by Struble and Peebles (1985) and Argyres et al. (1986). These and other large-scale surveys have made it clear that clusters are not spherical, but prolate or stringlike.

Most studies of alignment to date have relied on estimates of the ellipticity and orientation of galaxy and cluster that are both relatively subjective and global. Isophotometry of large numbers of galaxies has become available only recently, and cluster isopleths are difficult to draw accurately because of luminosity effects and background uncertainties. The isophotometry presented here can be used to examine the question of galaxy-cluster alignment in more detail than has been done before. In the future we plan to derive our own galaxy count maps in the centers of some of the richer clusters in the sample.

We have made our comparison to the work of Binggeli (1982), Struble and Peebles (1985), and Argyres et al. (1986). Binggeli's sample is small but better controlled, and has a large overlap with our sample: 36 objects, if we use the $32-$ $\mathrm{kpc}$ isophotes. The more recent work extends the sample to fully 115 of our clusters. This is illustrated in Figure 23, which shows the absolute values of the differences between the galaxy PA measurements in the cited papers (solid circles represent Binggeli data, open circles data from the later papers) and the PAs measured for our 32-kpc isophotes. These differences are plotted as a function of the ellipticity of our $32-\mathrm{kpc}$ isophote, on which, as expected, they show some dependence. The important feature of this plot is the good agreement between our galaxy orientations and Binggeli's. It is clear from a detailed comparison of the data sets that most of Binggeli's effective isophotes had surface brightnesses near $\mu_{r}=23 \mathrm{mag} \mathrm{sec}^{-2}$, but only a couple of these galaxies have significant isophote twists at radii larger than a few arc seconds. 
Figure 22 plots the relative angle between our $32-\mathrm{kpc}$ isophotes and the clusters from the above studies in bins of $15^{\circ}$. The solid lines represent the sample of Binggeli, and the dashed lines the combined samples of all 3 papers. The alignment is quite convincing. It is not strongly correlated with galaxy ellipticity, as is demonstrated by Figure 24. It is a feature of the outer regions of BCEs. Analogs of Figure 22 were plotted using the 16-kpc and 8-kpc isophotes, and the alignment was much weaker, although it was still visible.

Finally, the PAs of the 32-kpc isophotes were also compared to visual estimates of the orientation of the cluster X-ray contours in the atlas of Jones and Forman (1988). The total overlap of our 2 samples was 63 clusters. The Xray contours are lumpy and irregular, but orientations good to about $15^{\circ}$ could be obtained for 41 of them. The rest were discarded because of lack of signal, complex subtructure, or circularity of the X-ray contours. The alignment of the 41 with the galaxy isophotes is histogrammed in Figure 25. This correlation is also very convincing.

\section{Nonconcentric Light: Evidence for Modern Mergers}

The observations that the outer regions of $\mathrm{BCEs}$ are both prolate as a class and strongly aligned with the prolate clusters in which they lie is strong new evidence of a physical connection between the two structures, but the sequence of events that led to the present situation is not firmly established. Two extreme scenarios are possible: first, that the outer regions of BCEs are formed with the rest of the cluster at early times and evolve little or not at all after cluster collapse, and second, that BCEs are originally similar to normal ellipticals and grow prolate outer regions by accreting matter along the cluster axis. 
The idea that BCEs are actively growing as we watch has been prevalent in the literature of the past decade, as referenced in the introduction. But it has been known for some time that if the low-energy members of a cluster are consumed rapidly, the high-velocity survivors can resist accretion into a single merger product almost indefinitely. Thus Merritt $(1983,1984,1985)$ has reminded us that scenarios in which evolution occurs mostly in the distant past are not easily ruled out by indirect evidence, and Cavaliere et al. (1986) have demonstrated again that strong subclustering can significantly delay cluster virialization. The observation that Abell clusters have an unusually high proportion of multiple nuclei (Paper IV), once considered proof of ongoing mergers, has been weakened by the observation that many multiple-nucleus systems have high relative velocities (Tonry 1985; Smith et al 1985; Hoessel, Borne, and Schneider 1985).

There is, however, some firmer evidence for ongoing galaxy growth: significant amounts of nonconcentric light in 15 to $20 \%$ of the BCEs in the present sample. This was found in the form of nonconcentric isophotes and examined more closely by extracting the antisymmetric parts of the images of the galaxies in question, as described in Section III. Large amounts of nonconcentric light, usually in the form of diffuse patches, are found not only in multiple-nucleus systems (e.g., A21, A42, A910, A1185, and A1291), but in single-nucleus BCEs (e.g., A43, A496, A957, A1630, A1767, and A2079). Significant asymmetries are found in the surface brightness profiles of over 30 BCEs. Two examples are illustrated in Figure 26. The distribution of the "loose light" in A957 is especially striking: in addition to a faint patch of light $1^{\prime}$ to the west of the nucleus, there is a luminous patch with an extended tail in the inner $15^{\prime \prime}$ on the east side. This is probably a small companion being tidally disrupted by the BCE. 
These patches of light are unlike any isolated structures found during the cleaning of the pictures, and it is therefore likely that they are intrinsic features of the BCEs. This means that they are subject to net gravitational and dynamical friction forces and cannot be very long lived. Simulations by Villumsen (1982) and study of V Zw 311 (Schneider and Gunn 1982) suggest that the lifetimes of such features are no longer than $10^{9}$ years, since complete mergers take place on timescales of $5 \times 10^{8}$ to $2 \times 10^{9}$ years. Such a short timescale, combined with the fact that at least one out of 6 BCEs has asymmetric light, and the presumption that Abell clusters have ages close to a Hubble time, are strong evidence that significant mass accretion has continued after cluster collapse in many BCEs. Whether "significant" means growth of $25 \%$ or $100 \%$ is a question for future study and probably depends on individual circumstances. Kinematic study of the galaxies in Table V would be a good approach to this question. Searches for asymmetries in field ellipticals should also be conducted as a control experiment.

Summarizing the results of this section: there is good evidence that aside from simply being larger than field ellipticals, BCEs have significantly different shape behavior. At radii of $\sim 30 \mathrm{kpc}$, they line up with the clusters in which they are found, as traced by both the galaxy distribution and X-ray gas. At the same radii their shapes become distinguishable from a population of oblate spheroids. This is consistent with a scenario in which they are formed from, or accreted a significant amount of mass from, their clusters, which have recently been shown to be very elongated objects, either prolate or stringlike. The prevalence of asymmetric patches of light suggests that a significant amount of BCE growth occurs after cluster collapse, right up to the present. If $\mathrm{BCEs}$ originally had significant rotation 
and/or decreasing ellipticity profiles, these features were suppressed during the evolution of the galaxy to its present state. 


\section{CONCLUSIONS}

Isophotometry of 175 brightest elliptical galaxies in Abell clusters has produced the following results:

1) The ellipticity, position angle, and centroid profiles of many BCEs show complex structure which may or may not be reflected in their surface brightness profiles. One-dimensional parametric models such as the King and de Vaucouleurs laws are therefore of limited use in probing the dynamics of these systems. In this respect $\mathrm{BCEs}$ are no different from any other elliptical galaxies.

2) Disturbances and changes of slope in ellipticity profiles may more often be correlated with changes along the major-axis surface brightness profile than changes along the minor-axis surface brightness profile.

3) Approximately half of all BCEs in rich clusters have monotonically increasing ellipticity profiles. Only about $1 \%$ have monotonically decreasing ellipticity. Galaxies with self-similar isophotes are never flatter than E2. In these respects BCEs resemble field ellipticals as represented by the sample of Djorgovski (1985b).

4) Turnover in the flattening profile is relatively rare in BCEs: only 16 galaxies in the present sample $(10 \%)$ show this behavior. One quarter of the field ellipticals studied by Djorgovski (1985) show it. The maximum ellipticities of these galaxies are also smaller in the BCE sample, exceeding E3 only in A1921 and A2218. In Djorgovski's sample the maximum ellipticities of these galaxies are often E4 and E5. 
5) According to current dynamical models, the above results are consistent with the observed absence of rotation in very luminous ellipticals. BCEs with decreasing ellipticity are good candidates for a search for rotation in cluster galaxies.

6) The average ellipticity of $\mathrm{BCEs}$ is a strongly increasing function of radius as far out as $128 \mathrm{kpc}$. Data available for field ellipticals out to $32 \mathrm{kpc}$ show only weak evidence that their average ellipticity is a function of radius.

7) There is evidence that field ellipticals show more isophote twisting than do BCEs.

8) There is no evident correlation between the ellipticity of a BCE at fixed radius and its size as measured by an effective or core radius, the slope of its light distribution at intermediate or large radii, or the X-ray luminosity of the BCE's cluster. The sizes and shapes of BCEs are not related other than as cited in conclusion 6 .

9) Isophote twisting shows no obvious correlation with the behavior of the ellipticity profile. Large isophote twists are invariably associated with other complex structure such as kinks in the surface brightness, ellipticity, or centroid profiles. The reverse is not true.

10) There is no obvious systematic difference between the shape parameter profiles of single BCEs and those with multiple or satellite nuclei.

11) The observed ellipticity distribution of $\mathrm{BCEs}$ at radii greater than about $30 \mathrm{kpc}$ cannot be modelled by a reasonable population of oblate spheroids. Although the light distribution at large radii is probably still ellipsoidal, it must be at best prolate, and more likely triaxial. 
12) Isophotes of BCEs with semimajor axes greater than about $30 \mathrm{kpc}$ tend strongly to be aligned with the distribution of galaxies in their clusters and with the isophotes of the million degree X-ray gas filling the clusters.

13) The presence of nonconcentric isophotes, due mostly to asymmetric patches of light, in $20 \%$ of $\mathrm{BCEs}$, is evidence that some galaxy growth has continued between cluster collapse and the present day.

We are grateful to S. Djorgovski for sharing his data and experience with us. This work represents part of ACP's Ph.D. thesis at the California Institute of Technology. ACP gratefully acknowledges support from Palomar Observatory, an ARCS Fellowship, and an NSF grant to J. B. Oke. DPS acknowledges the support of NASA Grant NAS5-29225 and an Exxon Fellowship. At Wisconsin this research is partially supported by a grant from the Research Corporation and by a subcontract to JPL NASA contract NAS7-918. We thank J. A. Westphal and J. E. Gunn for their support of the instrumentation used in this work. The ellipse fitting routines, as well as all of the device dependent software, were written by Robert Deverill. 


\section{REFERENCES}

Austin, T. B., and Peach, J. V. 1974, M.N.R.A.S. 168591.

Bertschinger, E., and Meiksin, A. 1986, Ap.J.Lett. 306 L1.

Binggeli, B. 1982, Astr.Ap. 107338.

Binney, J. 1978, M.N.R.A.S. 183501.

Binney, J. 1980, M.N.R.A.S. 190421.

Binney, J. 1985, M.N.R.A.S. 212767.

Binney, J., and de Vaucouleurs, G. 1981, M.N.R.A.S. 194679.

Carter, D. 1978, M.N.R.A.S. 182797.

Carter, D., and Metcalfe, N. 1980, M.N.R.A.S. 191325.

Carter, D.; Efstathiou, G.; Ellis, R. S.; Inglis, I.; and Godwin, J. 1981, M.N.R.A.S. $19215 \mathrm{P}$.

Carter, D.; Inglis, I.; Ellis, R. S.; Efstathiou, G.; and Godwin, J. 1985, M.N.R.A.S. 212471.

Cavaliere, A.; Santangelo, D.; Tarquini, G.; and Vittorio, N. 1986, Ap.J. 305651. 
Contopoulos, G. 1956, Zs.f.Ap. 39126.

Davies, R. L.; Efstathiou, G.; Fall, S. M.; Illingworth, G.; and Schechter, P. L. 1983, Ap.J. 26641.

Davis, L.; Cawson, M.; Davies, R. L.; and Illingworth, G. 1985, A.J. 90169.

de Zeeuw, P. T. 1987, in Dynamics of Elliptical Galaxies, ed. P. T. de Zeeuw (Dordrecht: Reidel), p. 271.

diTullio, G. 1978, Astr.Ap.Lett. 62 L17.

diTullio, G. 1979, Astr.Ap.Suppl. 37591.

Djorgovski, S. 1985a, P.A.S.P. 971119.

Djorgovski, S. 1985b, Ph.D. thesis, University of California at Berkeley.

Djorgovski, S., and Davis, M. 1987, Ap.J. 31359.

Dressler, A. 1978, Ap.J. 22655.

Dressler, A. 1979, Ap.J. 231659.

Dressler, A., et al. 1987, Ap.J. 31342.

Duncan, M. J.; Farouki, R. T.; and Shapiro, S. L. 1983, Ap.J. 27122. 
Ebneter, K., and Balick, B. 1985, A.J. 90183.

Ebneter, K.; Djorgovski, S.; and Davis, M. 1988, A.J. 95422.

Efstathiou, G.; Ellis, R. S.; and Carter, D. 1982, M.N.R.A.S. 201975.

Faber, S. M.; Burstein, D.; and Dressler, A. 1977, A.J. 82941.

Faber, S. M., and Jackson, R. E. 1976, Ap.J. 204668.

Fabian, A. C.; Nulsen, P. E. J.; and Canizares, C. R. 1984, Nature 310733.

Farouki, R. T.; Shapiro, S. L.; and Duncan, M. J. 1983, Ap. J. 265597.

Fillmore, J. A. 1986, A.J. 911096.

Hausman, M. A., and Ostriker, J. P. 1978, Ap.J. 224320.

Hoessel, J. G. 1980, Ap.J. 241493.

Hoessel, J. G.; Borne, K. D.; and Schneider, D. P. 1985, Ap.J. 29394.

Hoessel, J. G.; Oegerle, W. R.; and Schneider, D. P. 1987, A.J. 941111.

Hoessel, J. G., and Schneider, D. P. 1985, A.J. 901648 (Paper IV).

Hoessel, J. G.; Thuan, T. X.; and Gunn, J. E. 1980, Ap.J. 241486. 
Jedrzejewski, R. I. 1987, M.N.R.A.S. 226747.

Jones, C., and Forman, W. R. 1984, Ap.J. 27628.

Jones, C., and Forman, W. R. 1988, in preparation.

Kent, S. M. 1984, Ap.J.Suppl. 56105.

Kent, S. M. 1985, Ap.J.Suppl. 59115.

King, I. R. 1971, P.A.S.P. 83199.

King, I. R. 1978, Ap.J. 2221.

Kormendy, J. 1985, Ap.J. 29573.

Lauer, T. 1985, Ap.J. 295104.

Lauer, T. 1986, Ap.J. 31134.

Lauer, T. 1988, Ap.J. 32549.

Leach, R. 1981, Ap.J. 248485.

Liller, M. H. 1960, Ap.J. 132306.

Liller, M. H. 1966, Ap.J. 14628. 
Malumuth, E., and Kirshner, R. P. 1985, Ap.J. 2918.

Matthews, T. A.; Morgan, W. W.; and Schmidt, M. 1964, Ap.J. 14035.

McGlynn, T. A., and Ostriker, J. P. 1980, Ap.J. 241915.

Merritt, D. 1983, Ap.J. 26424.

Merritt, D. 1984, Ap.J. 27626.

Merritt, D. 1985, Ap.J. 28918.

Michard, R. 1985, Astr.Ap.Suppl. 59205.

Morbey, C. 1983, Ap.J. 274502.

Noonan, T. W. 1972, A.J. 779.

Oemler, A. 1976, Ap.J. 209693.

Ostriker, J. P., and Hausman, M. 1977, Ap.J.Lett. 217 L125.

Ostriker, J. P., and Tremaine, S. D. 1975, Ap.J.Lett. 202 L113.

Piccirillo, J. 1973, P.A.S.P. 85278. 
Porter, A. C. 1984, in Clusters and Groups of Galaxies, ed. F. Mardirossian, G. Giuricin, and M. Mezzetti. (Dordrecht: Reidel), p. 351.

Porter, A. C. $1988 \mathrm{a}$, in preparation (chapter II).

Porter, A. C. $1988 \mathrm{~b}$, in preparation (chapter III).

Porter, A. C., Schneider, D. P., and Hoessel, J. G. 1988, in preparation.

Sandage, A. R., Freeman, K., and Stokes, N. R. 1970, Ap.J. 160831.

Sarazin, C. L., and O'Connell, R. W. 1983, Ap.J. 268552.

Sastry, G. 1968, P.A.S.P. 80252.

Schneider, D. P., and Gunn, J. E. 1982, Ap.J. 26314.

Schneider, D. P., Gunn, J. E., and Hoessel, J. G. 1983a, Ap.J. 264337 (Paper I)

Schneider, D. P., Gunn, J. E., and Hoessel, J. G. 1983b, Ap.J. 268476 (Paper II).

Schombert, J. 1986, Ap.J.Suppl. 60303.

Schombert, J. 1987, Ap.J.Suppl. 64643.

Schombert, J. 1988, Ap.J. 328475. 
Shapiro, S. L., and Marchant, A. B. 1976, Ap.J. 210757.

Smith, R. M.; Efstathiou, G.; Ellis, R. S.; Frenk, C. S.; and Valentijn, E. A. 1985, M.N.R.A.S. 216 71P.

Stewart, G. C.; Fabian, A. C.; Jones, C.; and Forman, W. R. 1984, Ap.J. 2851.

Strom, S., and Strom, K. 1978a, A.J. 8373.

Strom, S., and Strom, K. 1978b, A.J. 83732.

Strom, S., and Strom, K. 1978c, A.J. 831293.

Strom, S., and Strom, K. 1979, A.J. 841091.

Struble, M. F., and Peebles, P. J. E. A.J. 90582.

Thuan, T. X., and Gunn, J. E. 1976, P.A.S.P. 88543.

Thuan, T. X., and Romanishin, W. 1981, Ap.J. 248439.

Tonry, J. 1985, A.J. 902431.

Tonry, J. 1987, in Dynamics of Elliptical Galaxies, ed. P. T. de Zeeuw (Dordrecht: Reidel), p. 89.

van Albada, T. S. 1982, M.N.R.A.S. 201939. 
Villumsen, J. V. 1982, M.N.R.A.S. 199493.

Villumsen, J. V. 1983, M.N.R.A.S. 204219.

Williams, T. B. 1981, Ap. J. 244458. 
TABLE I. BCEs Observed.

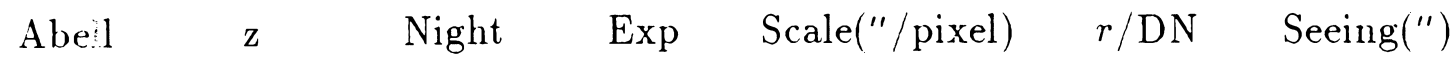

\begin{tabular}{|c|c|c|c|c|c|c|}
\hline 21 & 0.0948 & 081880 & 500 & 0.548 & 29.36 & 1.40 \\
\hline 22 & 0.1432 & 081680 & 500 & 0.548 & 29.25 & 1.69 \\
\hline 42 & 0.1087 & 081780 & 500 & 0.548 & 29.28 & 1.44 \\
\hline 43 & 0.1114 & 081880 & 500 & 0.548 & 29.38 & 1.70 \\
\hline$T 7$ & 0.0719 & 081880 & 500 & 0.548 & 29.37 & 1.37 \\
\hline 35 & 0.0556 & 081780 & 1500 & 0.548 & 30.51 & 1.51 \\
\hline 98 & 0.1033 & 082679 & 300 & 0.575 & 28.37 & 1.65 \\
\hline 104 & 0.0822 & 081880 & 500 & 0.548 & 29.37 & 1.40 \\
\hline 119 & 0.0446 & 082479 & 300 & 0.548 & 28.28 & 1.43 \\
\hline $13 \hat{0}$ & 0.1569 & 082679 & 300 & 0.575 & 28.38 & 1.68 \\
\hline 140 & 0.1591 & 081680 & 500 & 0.548 & 29.26 & 1.71 \\
\hline 151 & 0.0526 & 081680 & 500 & 0.548 & 29.29 & 1.64 \\
\hline 154 & 0.0612 & 082479 & 300 & 0.548 & 28.22 & 1.56 \\
\hline 160 & 0.0442 & 082679 & 300 & 0.575 & 28.36 & 1.71 \\
\hline 163 & 0.0457 & 083179 & 300 & 0.575 & 28.34 & 1.37 \\
\hline 186 & 0.1066 & 081780 & 500 & 0.548 & 29.32 & 1.26 \\
\hline 189 & 0.0349 & 081780 & 500 & 0.548 & 29.34 & 1.37 \\
\hline 193 & 0.0478 & 092179 & 300 & 0.548 & 28.29 & 1.71 \\
\hline 225 & 0.0692 & 102381 & 400 & 0.442 & 30.58 & 1.49 \\
\hline 246 & 0.0753 & 102381 & 480 & 0.442 & 30.57 & 1.59 \\
\hline 274 & 0.1289 & 091879 & 500 & 0.548 & 28.62 & 1.40 \\
\hline 277 & 0.0947 & 091879 & 500 & 0.548 & 28.61 & 1.43 \\
\hline 279 & 0.0797 & 081780 & 500 & 0.548 & 29.34 & 1.28 \\
\hline 326 & 0.0558 & 082979 & 300 & 0.575 & 28.34 & 1.36 \\
\hline 339 & 0.1160 & 092079 & 500 & 0.548 & 28.17 & 1.97 \\
\hline 399 & 0.0725 & 102581 & 600 & 0.442 & 30.79 & 1.79 \\
\hline 400 & 0.0231 & 091979 & 300 & 0.548 & 28.06 & 1.16 \\
\hline 401 & 0.0472 & 091979 & 600 & 0.548 & 28.79 & 2.06 \\
\hline 410 & 0.0897 & 101180 & 300 & 0.422 & 31.54 & 1.29 \\
\hline 496 & 0.0326 & 092079 & 500 & 0.548 & 28.29 & 1.49 \\
\hline 6.34 & 0.0266 & 012180 & 500 & 0.548 & 29.28 & 1.51 \\
\hline 65.5 & 0.1245 & 032279 & 300 & 0.575 & 29.02 & 1.57 \\
\hline 66.5 & 0.1832 & 022080 & 500 & 0.548 & 29.02 & 1.67 \\
\hline 671 & 0.0497 & 041480 & 500 & 0.548 & 29.35 & 1.30 \\
\hline 733 & 0.1159 & 032279 & 300 & 0.575 & 29.02 & 1.53 \\
\hline 777 & 0.2240 & 092179 & 120 & 0.415 & 30.34 & 1.34 \\
\hline 779 & 0.0201 & 041480 & 500 & 0.548 & 29.27 & 1.41 \\
\hline
\end{tabular}




\begin{tabular}{|c|c|c|c|c|c|c|}
\hline Abell & z & Night & $\operatorname{Exp}$ & Scale("/pixel) & $r / \mathrm{DN}$ & Seeing(") \\
\hline 787 & 0.1355 & 041480 & 500 & 0.548 & 29.21 & 1.46 \\
\hline 819 & 0.0759 & 032279 & 300 & 0.575 & 29.00 & 1.86 \\
\hline 858 & 0.0881 & 032179 & 300 & 0.575 & 29.00 & 1.63 \\
\hline 882 & 0.1408 & 032279 & 300 & 0.575 & 29.01 & 1.53 \\
\hline 910 & 0.2055 & 041982 & 300 & 0.415 & 31.90 & 1.04 \\
\hline 9.57 & 0.0437 & 041980 & 500 & 0.548 & 29.31 & 1.14 \\
\hline 978 & 0.0527 & 041980 & 500 & 0.548 & 29.30 & 1.25 \\
\hline 993 & 0.0530 & 041980 & 500 & 0.548 & 29.30 & 1.56 \\
\hline 1020 & 0.0650 & 041980 & 500 & 0.548 & 29.32 & 1.17 \\
\hline 1035 & 0.0799 & 041480 & 500 & 0.548 & 29.28 & 1.36 \\
\hline 1081 & 0.1588 & 041380 & 500 & 0.548 & 29.46 & 1.11 \\
\hline 1123 & 0.1235 & 032279 & 300 & 0.575 & 28.99 & 1.77 \\
\hline 1126 & 0.0828 & 041980 & 500 & 0.548 & 29.35 & 1.33 \\
\hline 1149 & 0.0710 & 032279 & 300 & 0.575 & 29.01 & 1.77 \\
\hline 1155 & 0.0738 & 032179 & 300 & 0.575 & 29.01 & 1.57 \\
\hline 1169 & 0.0582 & 041380 & 500 & 0.548 & 29.44 & 1.36 \\
\hline 1170 & 0.1620 & 032279 & 300 & 0.575 & 29.01 & 1.71 \\
\hline 1185 & 0.0349 & 042080 & 500 & 0.548 & 29.33 & 1.55 \\
\hline 1187 & 0.0791 & 042080 & 500 & 0.548 & 29.33 & 1.51 \\
\hline 1190 & 0.0794 & 041380 & 500 & 0.548 & 29.37 & 1.30 \\
\hline 1213 & 0.0484 & 042080 & 500 & 0.548 & 29.33 & 1.56 \\
\hline 1216 & 0.0524 & 042080 & 500 & 0.548 & 29.31 & 1.49 \\
\hline 1224 & 0.2897 & 022080 & 500 & 0.548 & 29.15 & 1.44 \\
\hline $122 \bar{\imath}$ & 0.1117 & 041480 & 500 & 0.548 & 29.42 & 1.31 \\
\hline 1228 & 0.0344 & 042080 & 500 & 0.548 & 29.32 & 1.24 \\
\hline 12.38 & 0.0716 & 042080 & 500 & 0.548 & 29.31 & 1.47 \\
\hline 1264 & 0.1267 & 041480 & 500 & 0.548 & 29.46 & 1.38 \\
\hline 1291 & 0.0586 & 050580 & 500 & 0.548 & 29.31 & 1.75 \\
\hline 1346 & 0.0970 & 042080 & 500 & 0.548 & 29.32 & 1.41 \\
\hline 1364 & 0.1070 & 042080 & 500 & 0.548 & 29.32 & 1.35 \\
\hline 1365 & 0.0763 & 050580 & 500 & 0.548 & 29.28 & 1.55 \\
\hline 1373 & 0.1314 & 042080 & 500 & 0.548 & 29.32 & 1.52 \\
\hline 1377 & 0.0509 & 050580 & 500 & 0.548 & 29.29 & 1.81 \\
\hline 1382 & 0.1046 & 050680 & 500 & 0.548 & 28.97 & 1.26 \\
\hline 1383 & 0.0598 & 050580 & 500 & 0.548 & 29.39 & 1.86 \\
\hline 1399 & 0.0913 & 041480 & 500 & 0.548 & 29.12 & 1.29 \\
\hline 1401 & 0.1670 & 041480 & 500 & 0.548 & 29.31 & 1.55 \\
\hline
\end{tabular}




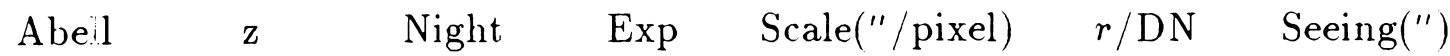

\begin{tabular}{|c|c|c|c|c|c|c|}
\hline 1412 & 0.0839 & 050680 & 500 & 0.548 & 29.25 & 1.29 \\
\hline 1413 & 0.1431 & 022282 & 300 & 0.415 & 31.28 & 1.04 \\
\hline 1436 & 0.0646 & 050580 & 500 & 0.548 & 29.34 & 1.82 \\
\hline 1461 & 0.0538 & 041480 & 500 & 0.548 & 29.39 & 1.16 \\
\hline 1468 & 0.0853 & 050580 & 500 & 0.548 & 29.44 & 2.34 \\
\hline 1474 & 0.0778 & 032480 & 500 & 0.548 & 29.47 & 1.78 \\
\hline $15 \mathrm{~L} 4$ & 0.1995 & 041980 & 1500 & 0.548 & 30.55 & 1.16 \\
\hline 1541 & 0.0892 & 032480 & 500 & 0.548 & 29.46 & 1.64 \\
\hline 1548 & 0.1608 & 022282 & 300 & 0.415 & 31.51 & 1.16 \\
\hline 1559 & 0.1042 & 032279 & 300 & 0.575 & 29.00 & 1.47 \\
\hline 1589 & 0.0699 & 032179 & 300 & 0.575 & 29.01 & 1.86 \\
\hline 16.30 & 0.0649 & 032179 & 300 & 0.575 & 28.99 & 1.84 \\
\hline 1644 & 0.0456 & 032480 & 500 & 0.548 & 29.45 & 1.90 \\
\hline 1651 & 0.0842 & 050680 & 500 & 0.548 & 29.31 & 1.32 \\
\hline 1656 & 0.0230 & 032480 & 500 & 0.548 & 29.33 & 1.97 \\
\hline 1674 & 0.1055 & 041380 & 500 & 0.548 & 29.42 & 1.39 \\
\hline 1689 & 0.1784 & 061780 & 300 & 0.422 & 31.23 & 1.25 \\
\hline 1691 & 0.0722 & 041980 & 500 & 0.548 & 29.33 & 1.31 \\
\hline $17: 38$ & 0.1146 & 041380 & 500 & 0.548 & 29.44 & 1.32 \\
\hline 1749 & 0.0562 & 042080 & 500 & 0.548 & 29.33 & 1.21 \\
\hline 1767 & 0.0712 & 042080 & 500 & 0.548 & 29.33 & 1.17 \\
\hline 1773 & 0.0776 & 032480 & 500 & 0.548 & 29.37 & 1.39 \\
\hline 1775 & 0.0718 & 032480 & 500 & 0.548 & 29.34 & 1.52 \\
\hline 1735 & 0.0792 & 041480 & 500 & 0.548 & 29.45 & 1.46 \\
\hline 1793 & 0.0849 & 042080 & 500 & 0.548 & 29.33 & 1.56 \\
\hline 1795 & 0.0631 & 042080 & 500 & 0.548 & 29.32 & 1.22 \\
\hline 1809 & 0.0788 & 042080 & 500 & 0.548 & 29.33 & 1.10 \\
\hline 1825 & 0.0632 & 032480 & 500 & 0.548 & 29.47 & 1.23 \\
\hline 1827 & 0.0668 & 070680 & 500 & 0.548 & 29.37 & 1.23 \\
\hline 1831 & 0.0749 & 042080 & 500 & 0.548 & 29.33 & 1.16 \\
\hline 1837 & 0.0376 & 041980 & 500 & 0.548 & 29.30 & 1.30 \\
\hline 1880 & 0.1413 & 022080 & 500 & 0.548 & 29.15 & 1.44 \\
\hline 1904 & 0.0719 & 042080 & 500 & 0.548 & 29.32 & 1.32 \\
\hline 1913 & 0.0533 & 032580 & 500 & 0.548 & 29.05 & 2.60 \\
\hline 1913 & 0.1415 & 070680 & 500 & 0.548 & 29.35 & 1.26 \\
\hline 1921 & 0.1352 & 022282 & 300 & 0.415 & 31.45 & 1.20 \\
\hline 1927 & 0.0740 & 042080 & 500 & 0.548 & 29.34 & 1.19 \\
\hline
\end{tabular}


Abell $\mathrm{z} \quad$ Night Exposure Scale("/pixel) $r / \mathrm{DN} \quad$ Seeing(")

\begin{tabular}{|c|c|c|c|c|c|c|}
\hline 1934 & 0.2195 & 092179 & 300 & 0.415 & 31.36 & 1.43 \\
\hline 1940 & 0.1393 & 022282 & 300 & 0.415 & 31.33 & 1.14 \\
\hline 198.3 & 0.0458 & 032580 & 500 & 0.548 & 29.24 & 2.48 \\
\hline 1984 & 0.1231 & 070680 & 500 & 0.548 & 29.37 & 1.24 \\
\hline 1991 & 0.0589 & 032580 & 500 & 0.548 & 29.27 & 2.53 \\
\hline 1999 & 0.1032 & 042080 & 500 & 0.548 & 29.32 & 1.28 \\
\hline 2005 & 0.1251 & 032480 & 500 & 0.548 & 29.42 & 1.24 \\
\hline 2022 & 0.0565 & 032480 & 500 & 0.548 & 29.37 & 1.34 \\
\hline 2028 & 0.0772 & 032480 & 500 & 0.548 & 29.30 & 1.72 \\
\hline 2029 & 0.0777 & 032480 & 1000 & 0.548 & 30.08 & 1.54 \\
\hline $203 i j$ & 0.1163 & 070680 & 500 & 0.548 & 29.45 & 1.27 \\
\hline 2040 & 0.0456 & 032580 & 500 & 0.548 & 29.38 & 2.79 \\
\hline 2048 & 0.0945 & 041980 & 500 & 0.548 & 29.33 & 1.19 \\
\hline 2052 & 0.0351 & 042080 & 500 & 0.548 & 29.32 & 1.37 \\
\hline 2061 & 0.0782 & 041480 & 500 & 0.548 & 29.38 & 1.39 \\
\hline 2063 & 0.0337 & 032580 & 500 & 0.548 & 29.35 & 2.54 \\
\hline 2065 & 0.0722 & 041480 & 500 & 0.548 & 29.20 & 1.36 \\
\hline 2067 & 0.0726 & 041980 & 500 & 0.548 & 29.33 & 1.43 \\
\hline 2079 & 0.0657 & 042080 & 500 & 0.548 & 29.33 & 1.40 \\
\hline 2089 & 0.0743 & 042080 & 500 & 0.548 & 29.34 & 1.49 \\
\hline 2092 & 0.0669 & 041980 & 500 & 0.548 & 29.32 & 1.32 \\
\hline 2107 & 0.0421 & 032580 & 500 & 0.548 & 29.29 & 2.63 \\
\hline 2110 & 0.0978 & 070680 & 500 & 0.548 & 29.38 & 1.43 \\
\hline 2124 & 0.0671 & 041980 & 500 & 0.548 & 29.33 & 1.24 \\
\hline $212 !$ & 0.2465 & 061580 & 300 & 0.422 & 31.43 & 1.27 \\
\hline 2142 & 0.0911 & 041980 & 500 & 0.548 & 29.33 & 1.31 \\
\hline 2147 & 0.0377 & 032580 & 500 & 0.548 & 29.30 & 2.35 \\
\hline 2151 & 0.0360 & 032580 & 500 & 0.548 & 29.41 & 2.36 \\
\hline 2152 & 0.0444 & 041980 & 500 & 0.548 & 29.32 & 1.26 \\
\hline 2175 & 0.0978 & 032480 & 600 & 0.548 & 29.60 & 1.64 \\
\hline 2197 & 0.0303 & 050680 & 500 & 0.548 & 29.46 & 2.32 \\
\hline 2199 & 0.0312 & 083179 & 500 & 0.575 & 28.84 & 1.91 \\
\hline 2218 & 0.1710 & 022282 & 300 & 0.415 & 30.71 & 1.22 \\
\hline 2244 & 0.0970 & 070680 & 500 & 0.548 & 29.39 & 1.36 \\
\hline 2246 & 0.2250 & 070480 & 1500 & 0.548 & 30.62 & 1.60 \\
\hline $225 !$ & 0.0747 & 070580 & 1500 & 0.548 & 30.57 & 1.53 \\
\hline 2256 & 0.0747 & 070580 & 1500 & 0.548 & 30.55 & 2.01 \\
\hline
\end{tabular}




\begin{tabular}{ccccccc} 
Abell & z & Night & Exp & Scale("/pixel) & $r / D N$ & Seeing(") \\
& & & & & & \\
\hline 2263 & 0.1051 & 070680 & 500 & 0.548 & 29.46 & 1.31 \\
2283 & 0.1830 & 070680 & 500 & 0.548 & 30.61 & 1.71 \\
2329 & 0.1470 & 083079 & 500 & 0.575 & 28.85 & 2.07 \\
2347 & 0.1196 & 083079 & 500 & 0.575 & 28.83 & 2.27 \\
2377 & 0.0808 & 070680 & 500 & 0.548 & 29.33 & 1.37 \\
2382 & 0.0648 & 082979 & 400 & 0.548 & 28.65 & 1.65 \\
2384 & 0.0943 & 083179 & 500 & 0.575 & 28.85 & 1.25 \\
2388 & 0.0615 & 070680 & 500 & 0.548 & 29.36 & 1.36 \\
2399 & 0.0587 & 083079 & 500 & 0.575 & 28.87 & 1.78 \\
2400 & 0.0881 & 070680 & 500 & 0.548 & 29.35 & 1.35 \\
2420 & 0.0838 & 070680 & 500 & 0.548 & 29.30 & 1.63 \\
2440 & 0.0904 & 070680 & 500 & 0.548 & 29.40 & 1.44 \\
2457 & 0.0597 & 082979 & 500 & 0.548 & 28.91 & 2.04 \\
2459 & 0.0736 & 070680 & 500 & 0.548 & 29.31 & 1.56 \\
2462 & 0.0755 & 070680 & 500 & 0.548 & 29.33 & 1.66 \\
2469 & 0.0656 & 070680 & 500 & 0.548 & 29.35 & 1.38 \\
2496 & 0.1233 & 070680 & 500 & 0.548 & 29.35 & 1.44 \\
2521 & 0.1359 & 070680 & 500 & 0.548 & 29.32 & 1.41 \\
2554 & 0.1060 & 081680 & 500 & 0.548 & 29.26 & 1.65 \\
2559 & 0.0796 & 070680 & 500 & 0.548 & 29.35 & 1.52 \\
2597 & 0.0826 & 081680 & 500 & 0.548 & 29.30 & 1.68 \\
2622 & 0.0621 & 070680 & 500 & 0.548 & 29.41 & 1.26 \\
2645 & 0.2460 & 050580 & 400 & 0.415 & 32.38 & 1.45 \\
2670 & 0.0774 & 081680 & 500 & 0.548 & 29.30 & 1.48
\end{tabular}


TABLE II. BCEs with Significant Ellipticity Decreases.

\begin{tabular}{cccc}
$\epsilon^{\prime \prime} \sim 0$ & $\epsilon^{\prime \prime}<0$ & $\epsilon^{\prime \prime}>0$ & more complex \\
\hline & & & \\
$(\mathrm{A} 193)$ & $\mathrm{A} 634$ & $(\mathrm{~A} 277)$ & $\mathrm{A} 104$ \\
$(\mathrm{~A} 389)$ & $(\mathrm{A} 910)$ & $\mathrm{A} 655$ & $\mathrm{~A} 1169$ \\
$\mathrm{~A} 1123$ & $\mathrm{~A} 1213$ & $\mathrm{~A} 787$ & $\mathrm{~A} 1185$ \\
$\mathrm{~A} 2283$ & $\mathrm{~A} 1377$ & $(\mathrm{~A} 1081)$ & $\mathrm{A} 1364$ \\
$\mathrm{~A} 2328$ & $\mathrm{~A} 1383$ & $\mathrm{~A} 1228$ & $\mathrm{~A} 1691$ \\
$\mathrm{~A} 2382$ & $\mathrm{~A} 1461$ & $\mathrm{~A} 1382$ & $\mathrm{~A} 1749$ \\
& (A1474) & $\mathrm{A} 1904$ & $\mathrm{~A} 1809$ \\
& $\mathrm{~A} 1913$ & $\mathrm{~A} 2005$ & $\mathrm{~A} 2028$ \\
& $\mathrm{~A} 1921$ & & $\mathrm{~A} 2079$ \\
& $\mathrm{~A} 1984$ & & $\mathrm{~A} 2554$ \\
& $\mathrm{~A} 2022$ & & \\
& $\mathrm{~A} 2065$ & & \\
& $\mathrm{~A} 2218$ & & \\
& $\mathrm{~A} 2440$ & &
\end{tabular}

TABLE III. BCEs with Constant Ellipticity.

$\begin{array}{cccccc}\text { A193 } & \text { A225 } & \text { A777 } & \text { A882 } & \text { A957 } & \text { A1155 } \\ \text { A1365 } & \text { A1401 } & \text { A1436 } & \text { A1548 } & \text { A1689 } & \text { A1827 } \\ \text { A1880 } & \text { A1999 } & \text { A2125 } & \text { A2152 } & \text { A2244 } & \text { A2377 } \\ \text { A2670 } & & & & & \end{array}$


TABLE IV. BCEs with Isophote Twists Greater than $40^{\circ}$.

\begin{tabular}{cccccc}
\hline A42 & A43 & A225 & A277 & A279 & A389 \\
A655 & A957 & A1169 & A1228 & A1291 & A1674 \\
A1767 & A1785 & A1793 & A1825 & A1827 & A1904 \\
A1913 & A 1927 & A1934 & A1983 & A2005 & A2036 \\
A2048 & A2061 & A2067 & A2125 & A2142 & A2283 \\
A2382 & A2440 & A2597 & & &
\end{tabular}

TABLE V. BCEs with Significant Asymmetries.

$\begin{array}{cccccc}\text { A } 42 & \text { A43 } & \text { A140 } & \text { A401 } & \text { A496 } & \text { A910 } \\ \text { A } 957 & \text { A } 993 & \text { A1126 } & \text { A1185 } & \text { A1291 } & \text { A1364 } \\ \text { A } 1377 & \text { A1630 } & \text { A1767 } & \text { A1773 } & \text { A1793 } & \text { A1825 } \\ \text { A } 1904 & \text { A1921 } & \text { A2052 } & \text { A2061 } & \text { A2063 } & \text { A2067 } \\ \text { A2079 } & \text { A2142 } & \text { A2244 } & \text { A2283 } & \text { A2382 } & \text { A2400 } \\ \text { A2420 } & \text { A2440 } & \text { A2462 } & \text { A2559 } & & \end{array}$




\section{FIGURE CAPTIONS.}

FIGURE 1. Brightness and shape parameter profiles for all galaxies in the sample. All parameters are presented as functions of $r$ surface brightness. Each page presents data on a single galaxy. For multiple nucleus systems, different plotting symbols are used for the individual nuclei and for their common envelope. A selection of profiles of second-brightest cluster galaxies are also presented. The profiles are: in the top row, from left to right, the major and minor axes, and their geometric mean; in the middle row, the ellipticity, eccentricity, and major-axis position angle; in the bottom row, the isophote centroid coordinates $x_{0}$ and $y_{0}$, and the $1 \sigma$ deviation of the fit from the pixels used.

FIGURE 2. Isophotometric profiles for 4 separately reduced images of A2175, taken with 3 different optical configurations. Plotting conventions are as in Figure 1 , except that the abscissa in the second and third rows of panels is $\log b$ in $\operatorname{arc}$ seconds.

FIGURE 3. Isophotometric profiles of artificial galaxies convolved with double Gaussian seeing. All galaxies shown here are modified Hubble laws, with core radii of 1.'6. Plotting conventions are as in Figure 1, except that the abscissa in the second and third rows of panels is $\log b$ in arc seconds. The first two sheets show isophote fits to E1 and E4 models with seeing FWHM of $0.7,1.3$, and 2"6. The third panel shows isophote fits to E0, E1, and E2 models with 1!"3 seeing.

FIGURE 4. Comparison of results of present study to those of Malumuth and Kirshner (1981) and Djorgovski (1985). The abscissa for the galaxies in Djorgovski's sample (A779, A1656, A2197, and A2199) is $\log \mathrm{r}=\log \sqrt{a b}$ in arc seconds; for the 
remaining galaxies, it is $\log$ a. On each page the panels show, from left to right, red surface brightness, ellipticity, and major-axis position angle.

FIGURE 5. Results from smoothing images with good (1") seeing by Gaussians to degrade their resolution to approximately $2^{\prime \prime}$ and $3^{\prime \prime}$, followed by repetition of the reductions. The fits to the original data are shown by solid circles, to the lesssmoothed image by crosses, and to the more smoothed data by asterisks. Plotting conventions are as in Figure 1, except that the abscissa in the second and third rows of panels is $\log b$ in arc seconds.

FIGURE 6. The average ellipticity of galaxies in the BCE sample at 2, 4, 8, 16, 32, 64 , and $128 \mathrm{kpc}$, plotted as a function of $\log$ a. The sample sizes at these radii are $47,152,167,158,139$, and 67 . The error bars are the root mean square deviation from the average. No isophotes with major axis less than 3 times the seeing FWHM were used in the construction of this figure.

FIGURE 7. The average ellipticity of galaxies in the BCE sample at $\frac{1}{16} r_{e}, \frac{1}{8} r_{e}, \frac{1}{4} r_{e}$, $\frac{1}{2} r_{e}, r_{e}, 2 r_{e}$, and $4 r_{e}$, where $r_{e}$ is the effective radius of the best one-dimensional de Vaucouleurs fit to a galaxy. The sample sizes at these radii are $22,47,79,88,81$, 58 , and 21 .

FIGURE 8. The average ellipticity of galaxies in the BCE sample at $0.8 \mathrm{a}, 1.6 \mathrm{a}, 3.2 \mathrm{a}$, $6.4 \mathrm{a}, 12.8 \mathrm{a}, 25.6 \mathrm{a}$, and $51.2 \mathrm{a}$, where $\mathrm{a}$ is the core radius of the best one-dimensional modified Hubble fit to a galaxy. The sample sizes at these radii are $34,52,78,80$, 64,42 , and 12.

FIGURE 9. The average ellipticity of galaxies in Djorgovski's field sample at 2, 4, 8,16 , and $32 \mathrm{kpc}$, plotted as a function of $\log \mathrm{a}$. The sample sizes at these radii are $121,117,93,41$, and 12 . 
FIGURE 10. The average ellipticity of galaxies in Djorgovski's field sample at $\frac{1}{16} r_{e}$, $\frac{1}{8} r_{e}, \frac{1}{4} r_{e}, \frac{1}{2} r_{e}, r_{e}, 2 r_{e}$, and $4 r_{e}$, where $r_{e}$ is the effective radius of the best onedimensional de Vaucouleurs fit to a galaxy. The sample sizes at these radii are 5 , $18,51,86,93,84$, and 48 .

FIGURE 11. Histogram of ellipticities in the BCE sample at 4, 16, 32, and $64 \mathrm{kpc}$. FIGURE 12. Histogram of ellipticities in the BCE sample at $\frac{1}{8} r_{e}, \frac{1}{2} r_{e}, r_{e}$, and $2 r_{e}$. FIGURE 13. Histogram of ellipticities in Djorgovski's field sample at 2, 8, 16, and $32 \mathrm{kpc}$.

FIGURE 14. Histogram of the ellipticity changes in the BCE sample from $4 \mathrm{kpc}$ to 16,32 , and $64 \mathrm{kpc}$.

FIGURE 15. Histogram of the ellipticity changes in Djorgovski's field sample from $2 \mathrm{kpc}$ to 8,16 , and $32 \mathrm{kpc}$.

FIGURE 16. Histogram of the amount of isophote twisting in the BCE sample from $4 \mathrm{kpc}$ to 16,32 , and $64 \mathrm{kpc}$.

FIGURE 17. Histogram of the amount of isophote twisting in Djorgovski's field sample from $2 \mathrm{kpc}$ to 8,16 , and $32 \mathrm{kpc}$.

FIGURE 18. Scatter plot of the ellipticity of $8 \mathrm{kpc}$ isophotes vs. $\log r_{e}$ in the BCE sample.

FIGURE 19. Scatter plot of the change in ellipticity between the 4 and $32 \mathrm{kpc}$ isophotes vs. $\log r_{e}$ in the $\mathrm{BCE}$ sample.

FIGURE 20. Scatter plot of the isophote twisting between 4 and $32 \mathrm{kpc}$ vs. $\log r_{e}$ in the $\mathrm{BCE}$ sample. 
FIGURE 21. Comparison of the observed ellipticity distributions of populations of oblate and prolate spheroids. The intrinsic ellipticity distributions of all populations shown are Gaussians with $\sigma$ of 0.1 . From top to bottom, the mean intrinsic ellipticities are $0.35,0.4,0.45$, and 0.5 . These figures are to be compared with the histograms in Figure 7.

FIGURE 22. Histogram of the alignment of the $32-\mathrm{kpc}$ isophote of a BCE with the host cluster. The abscissa is the position angle difference in degrees, referred to the range 0-90. The solid lines refer to the data of Binggeli (1982), the dashed lines to the combined sample of that paper, Struble and Peebles (1985), and Argyres et al. (1986).

FIGURE 23. Comparison of galaxy orientations measured by Binggeli (1982, solid circles) and Struble and Peebles (1985) and Argyres et al. (1986) (open circles) to the orientations of the $32-\mathrm{kpc}$ isophotes of the present study, plotted against the ellipticity of those isophotes.

FIGURE 24. Plot of the galaxy-cluster alignments histogrammed in Figure 22, against the ellipticity of the $32-\mathrm{kpc}$ isophote.

FIGURE 25. Histogram of the alignment of the 32-kpc galaxy isophotes with the approximate position angles of the cluster X-ray isophotes.

FIGURE 26. Antisymmetric parts of the raw images of A496 and A957. These images are negatives, and the field of view is $2^{\prime}$ in both cases. In A957, note the faint luminous patch $1^{\prime}$ west of the center and the prominent swath of light in the inner $15^{\prime \prime}$ on the east side. 
$-65-$

FIGURE 1

is presented as an appendix to the thesis, due to its length 


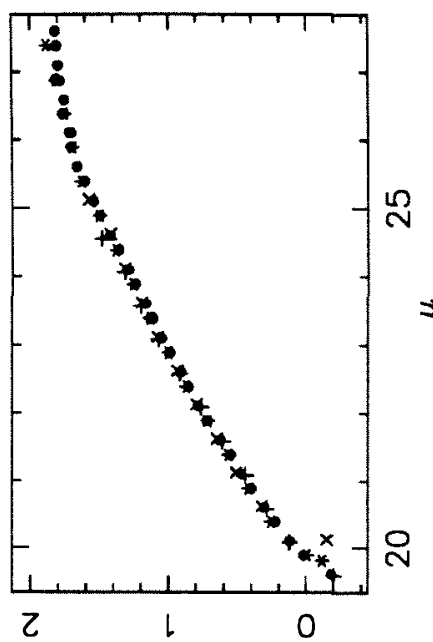

(.1)sn!pod 6ol

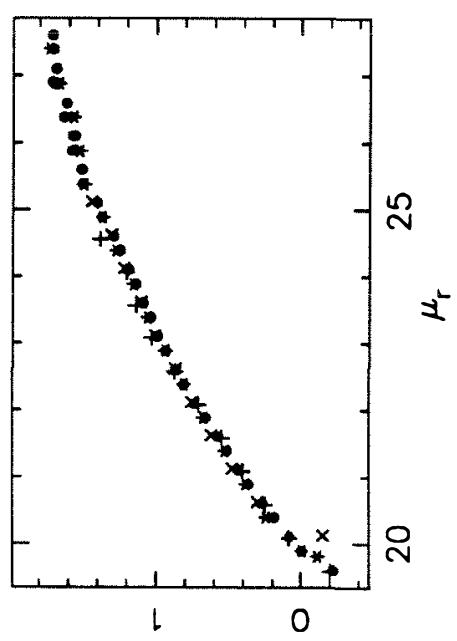

(.1) 9601

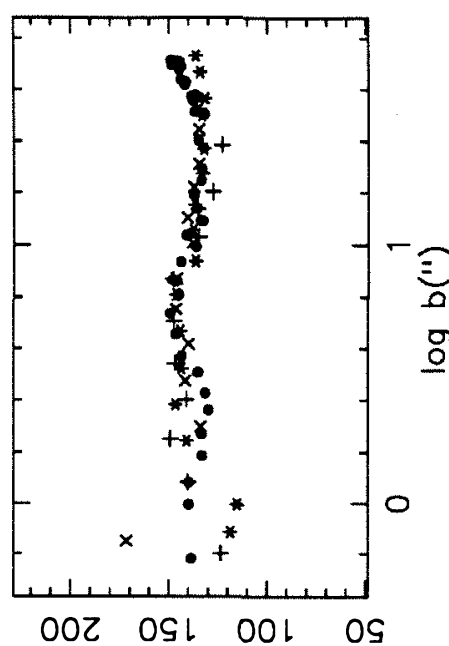

$\forall d$ s!xo so!

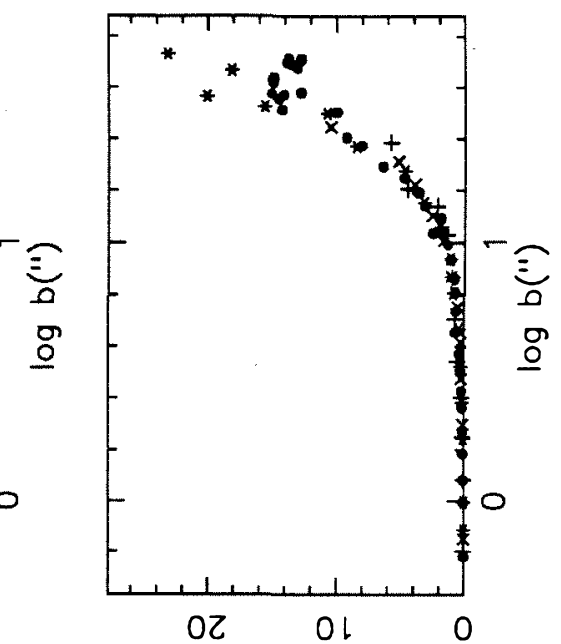

(11) $7 !+10$ Dub!s auo

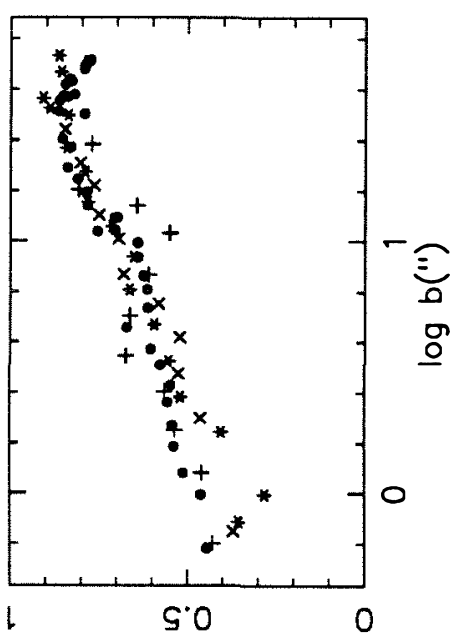

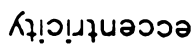

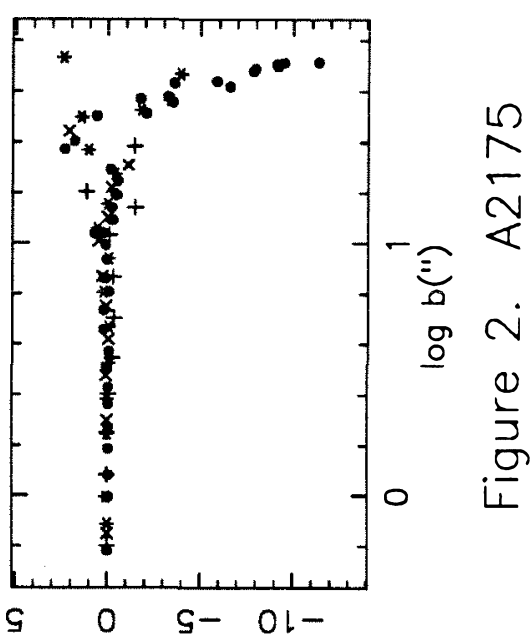

(.1) $725+10 \kappa$

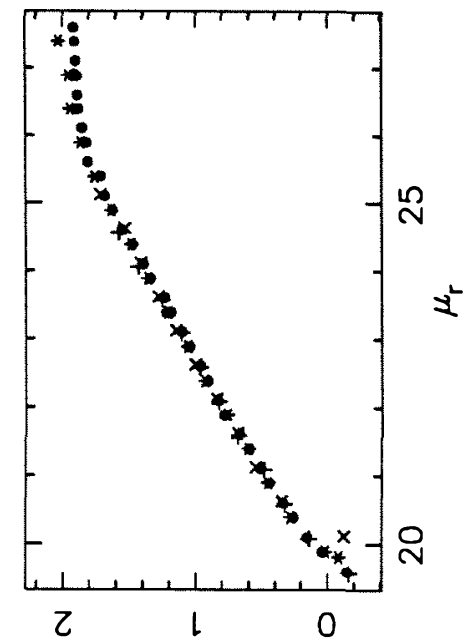

(.1) $0 \mathrm{6ol}$
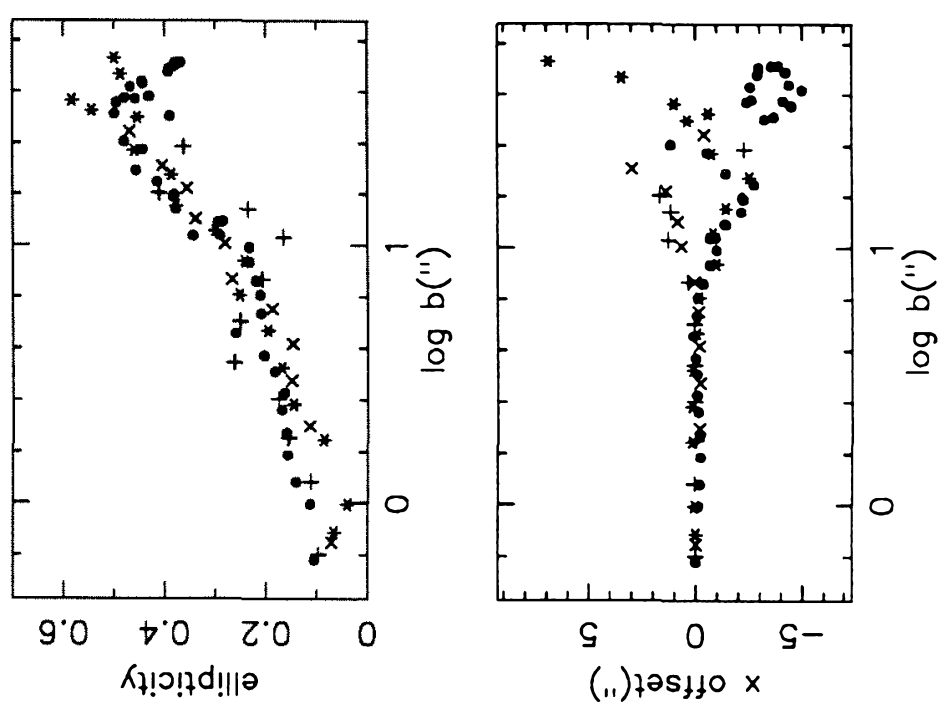


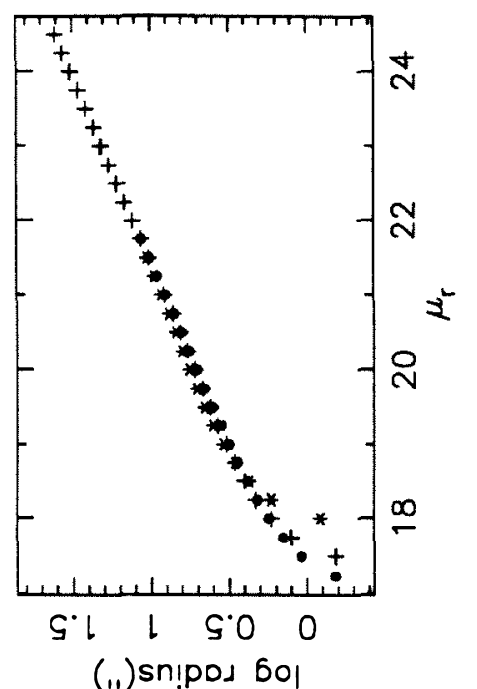

(i1)sn!pod bol
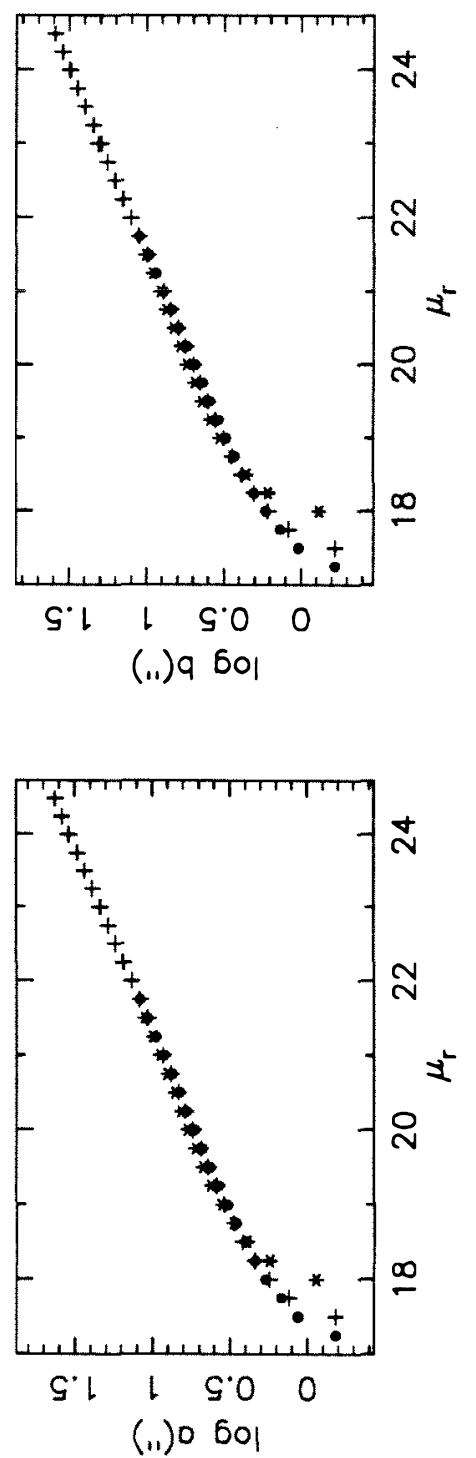

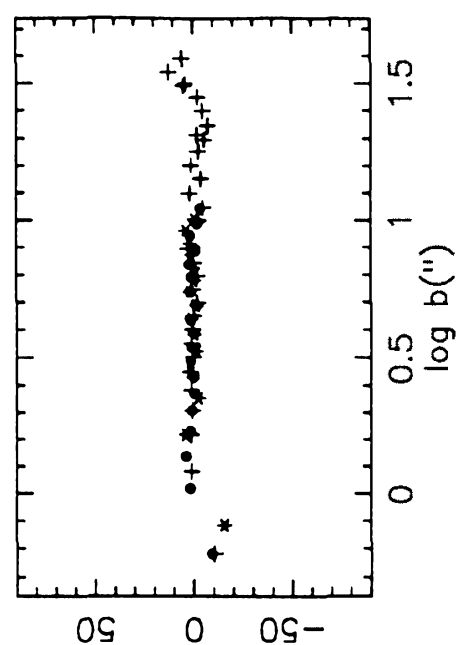

$\forall d$ s!Xo so!̣om

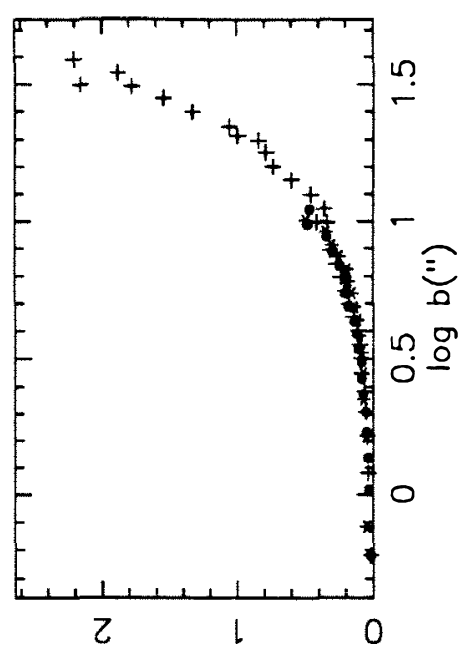

(11) + ! t to oub!s aน०

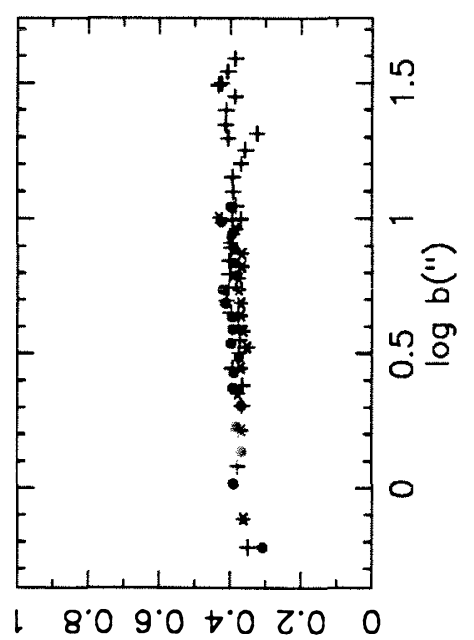

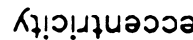

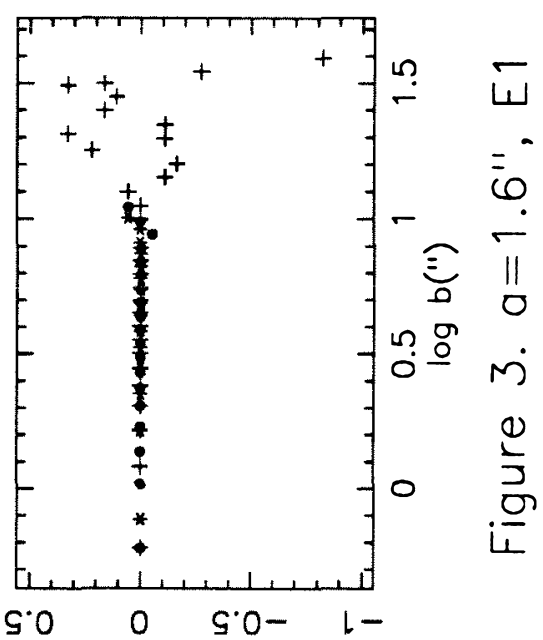

(.1) zast
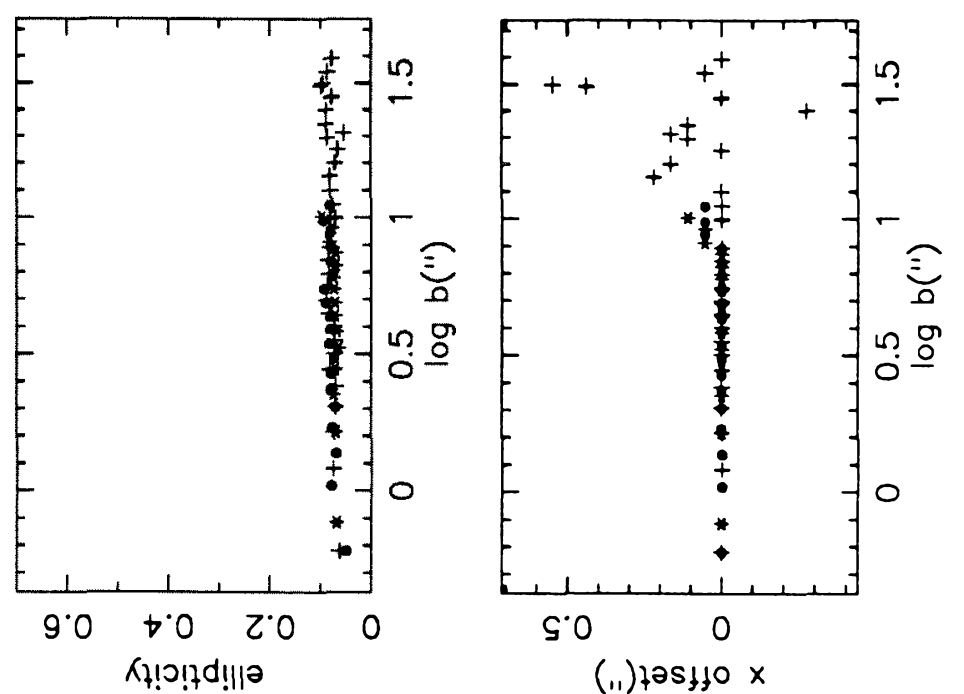

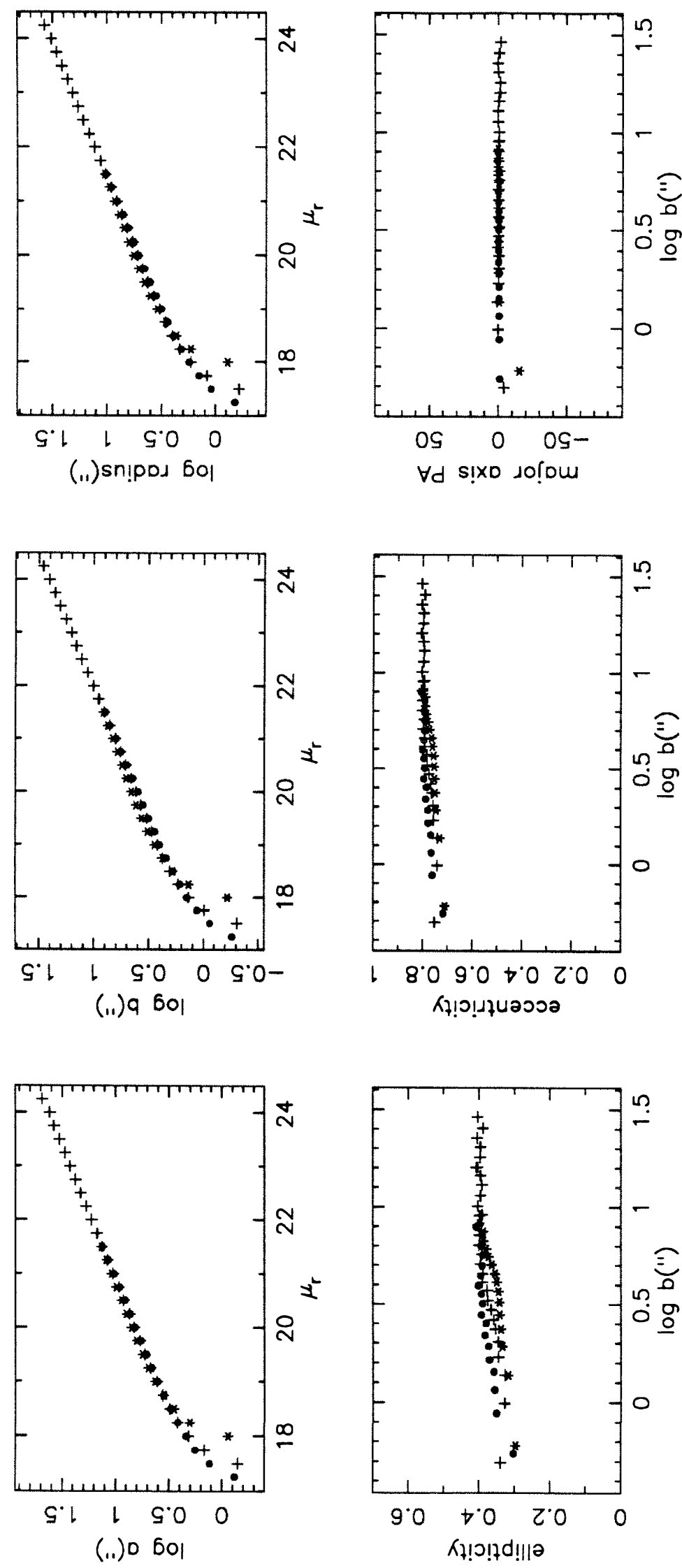

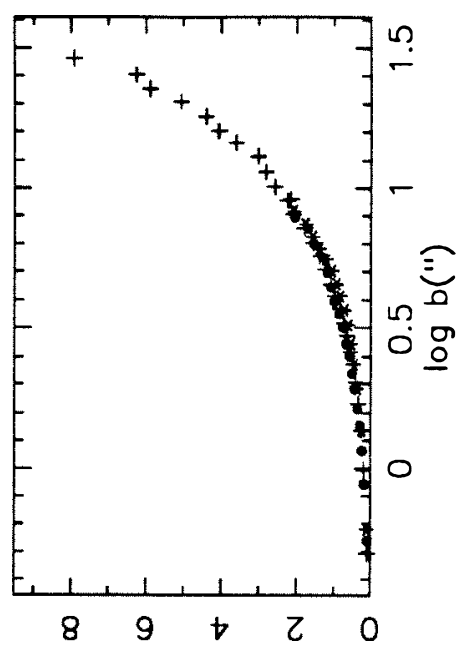

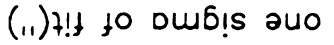
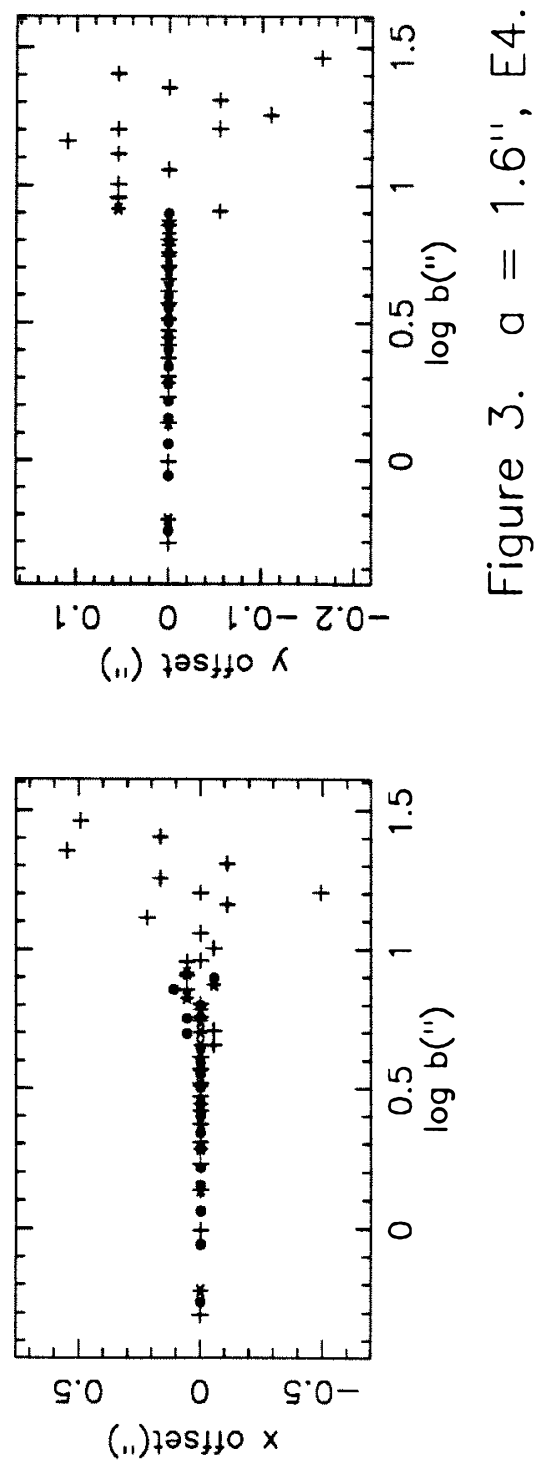


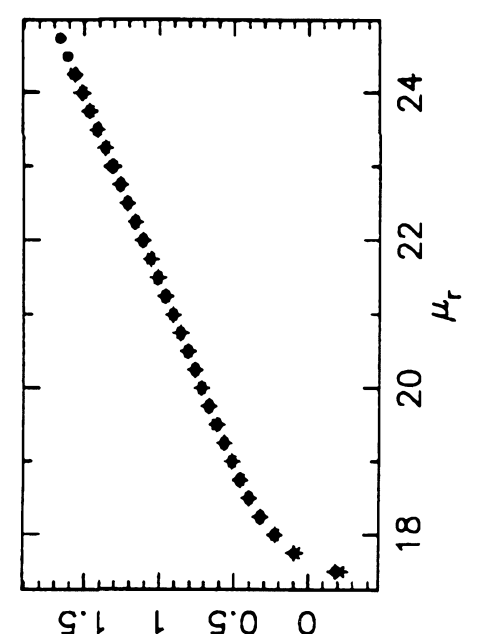

(.1)sn!pos 6ol

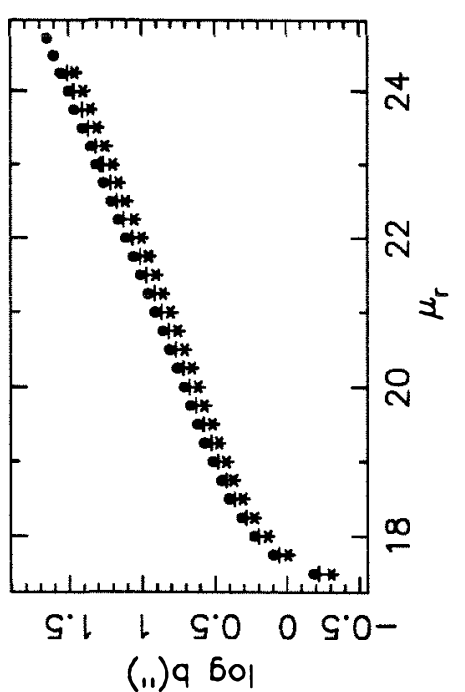

(.1) 9 bol

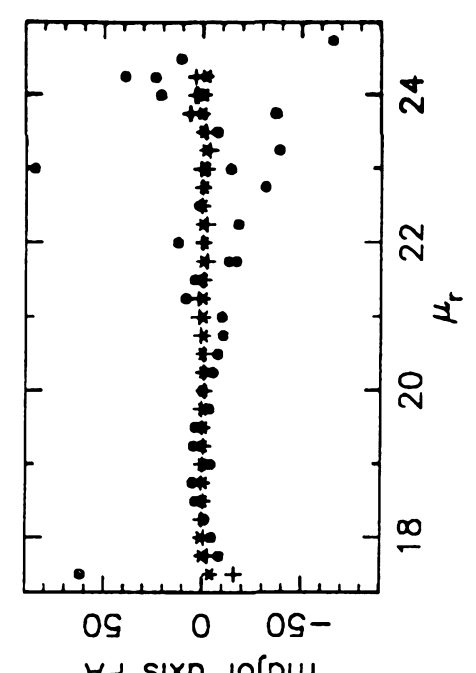

$\forall d$ s! Xo دo! ow

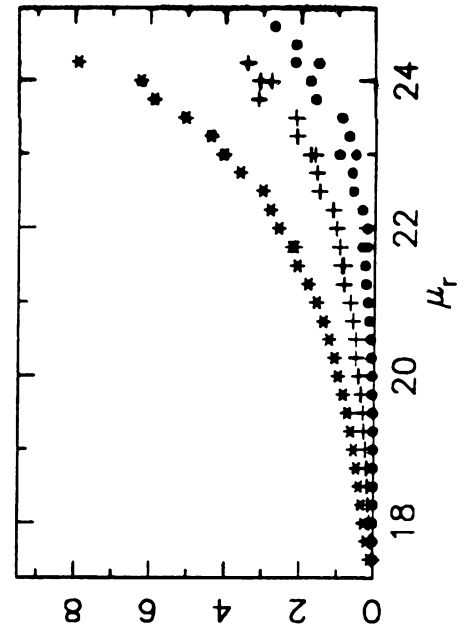

(11) 4 !t to Du6!s auo

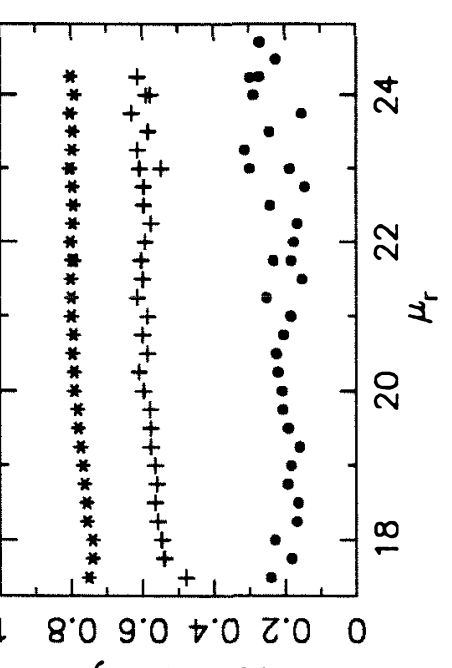

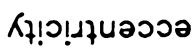
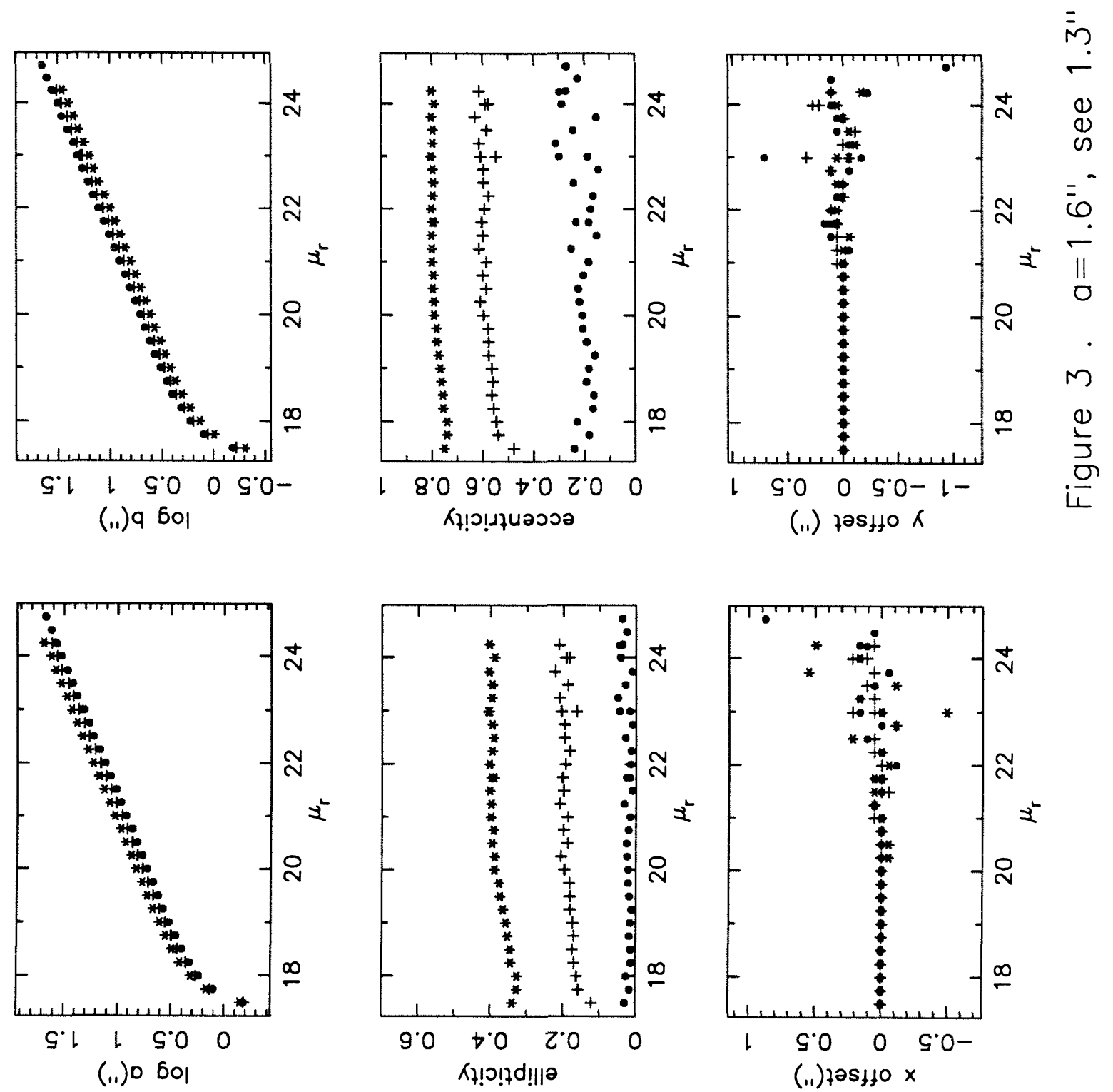
$-70-$
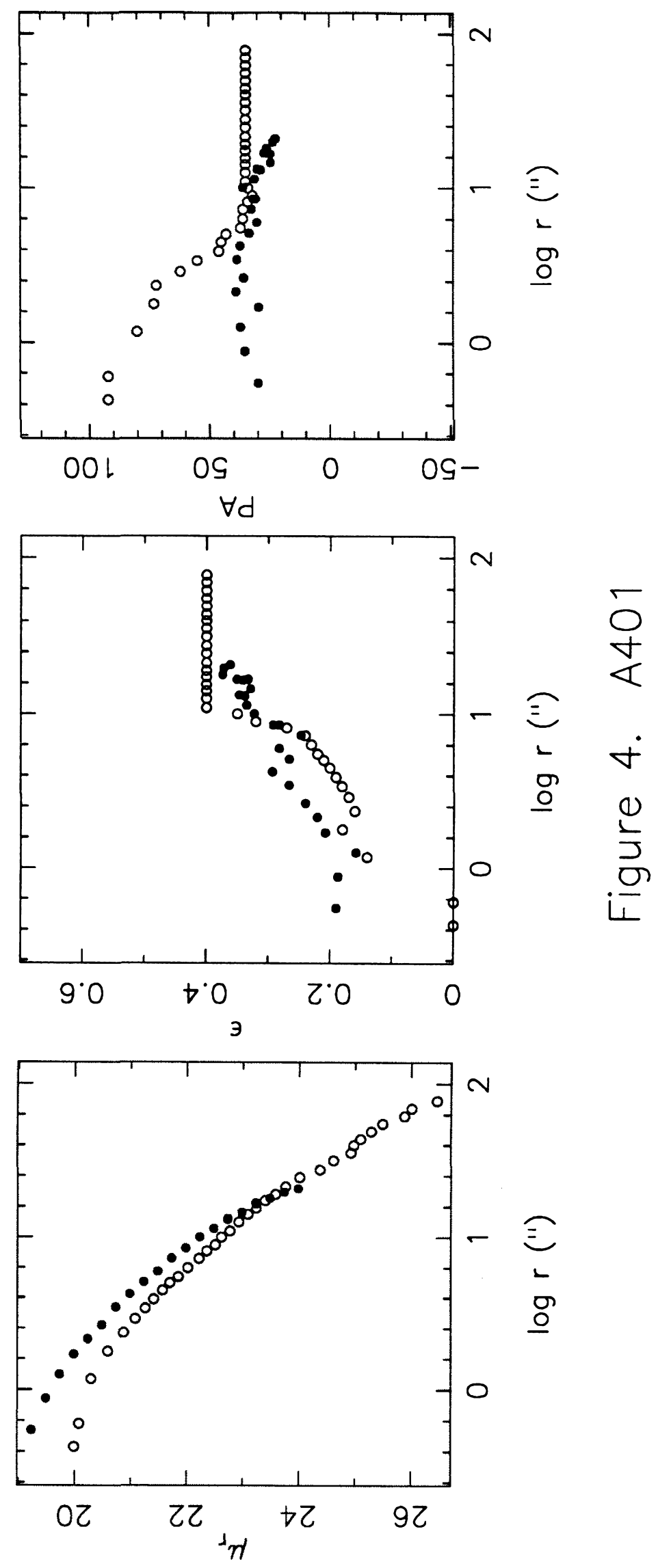
$-71-$
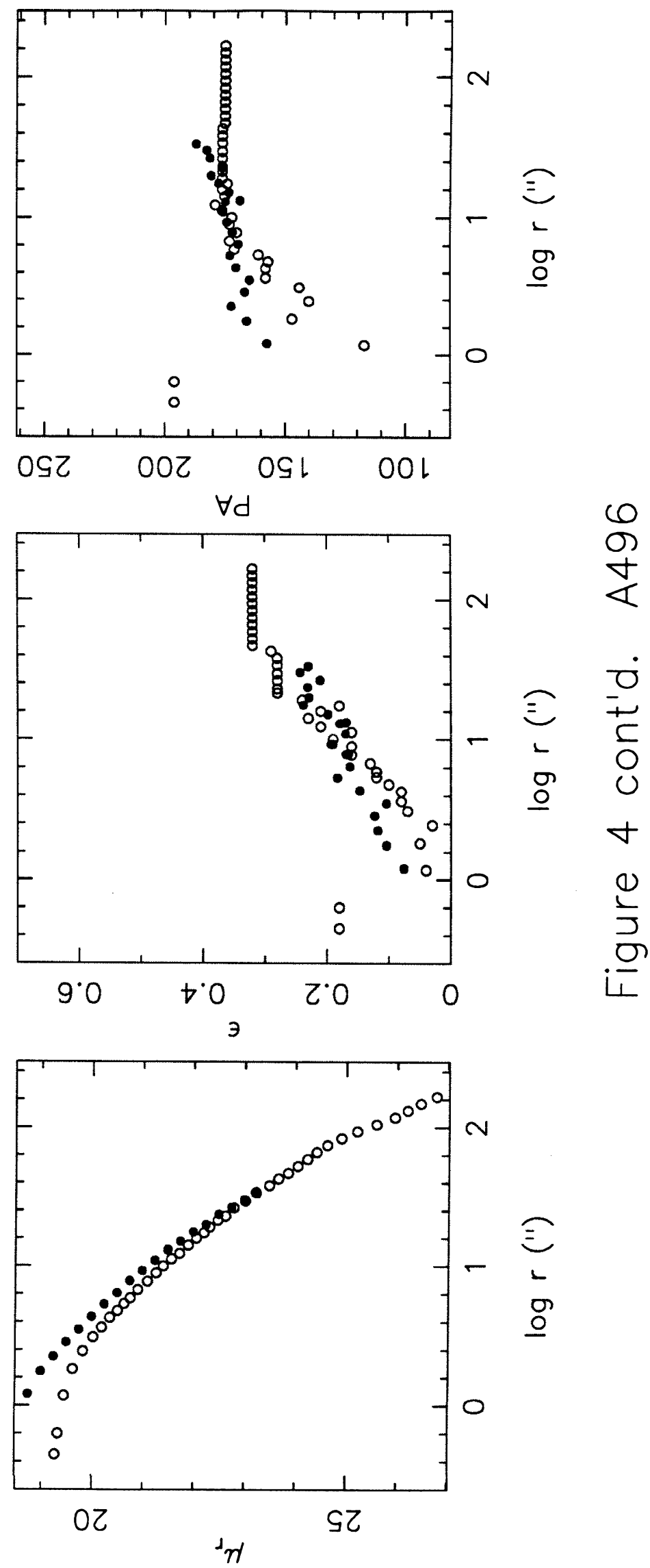
$-72-$
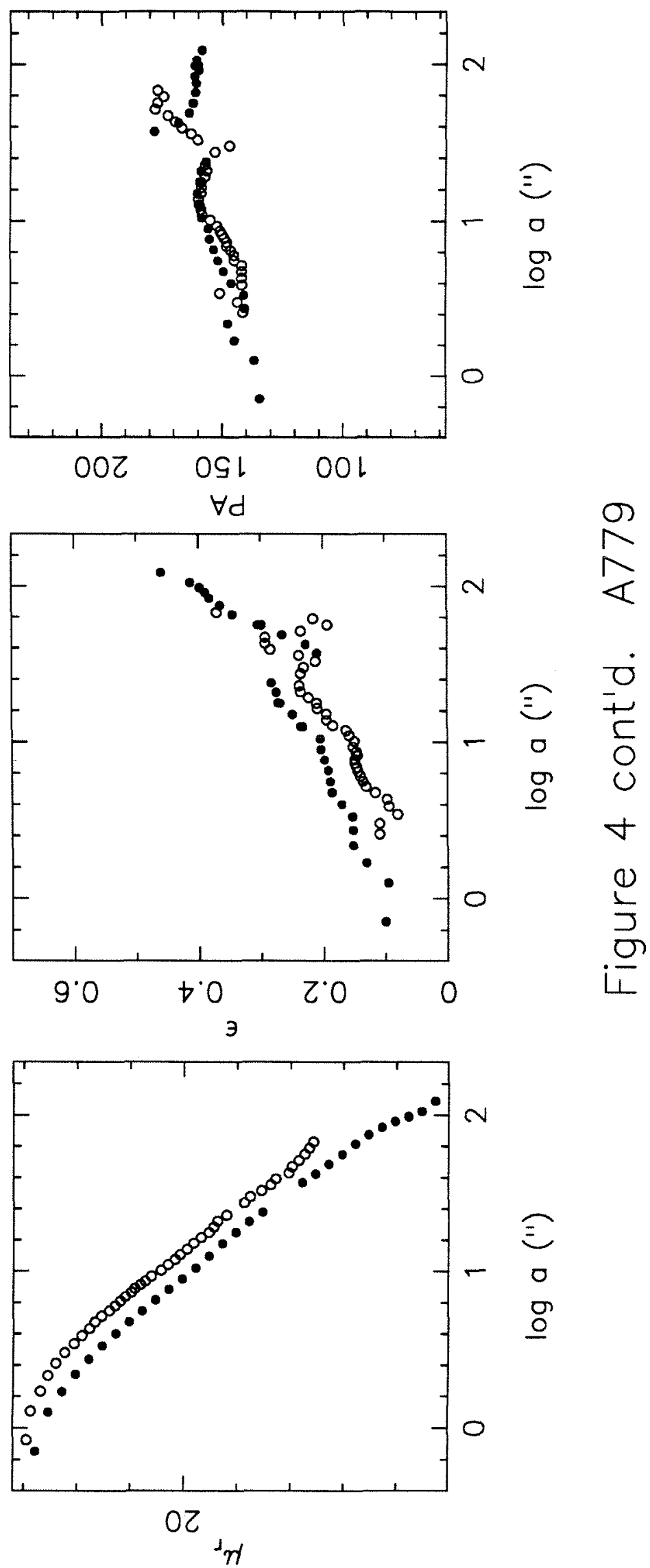
$-73-$
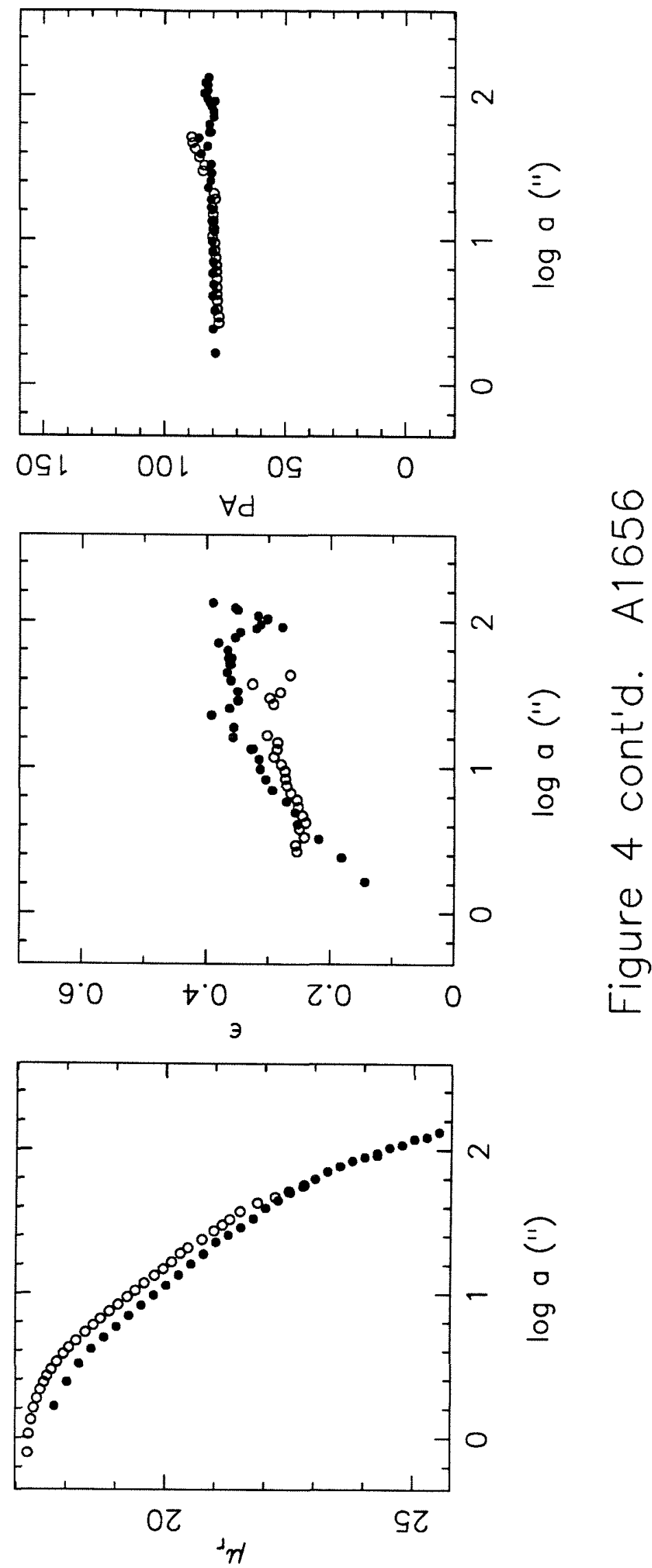

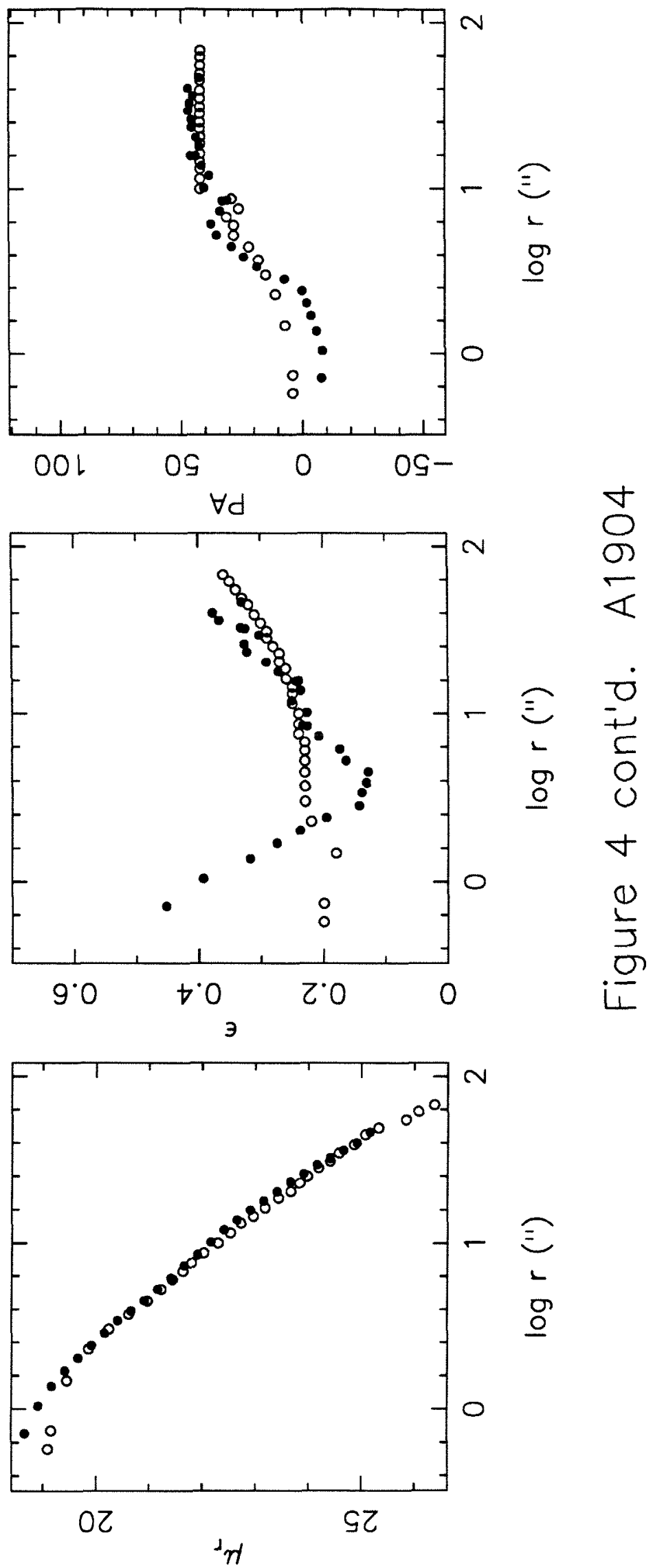

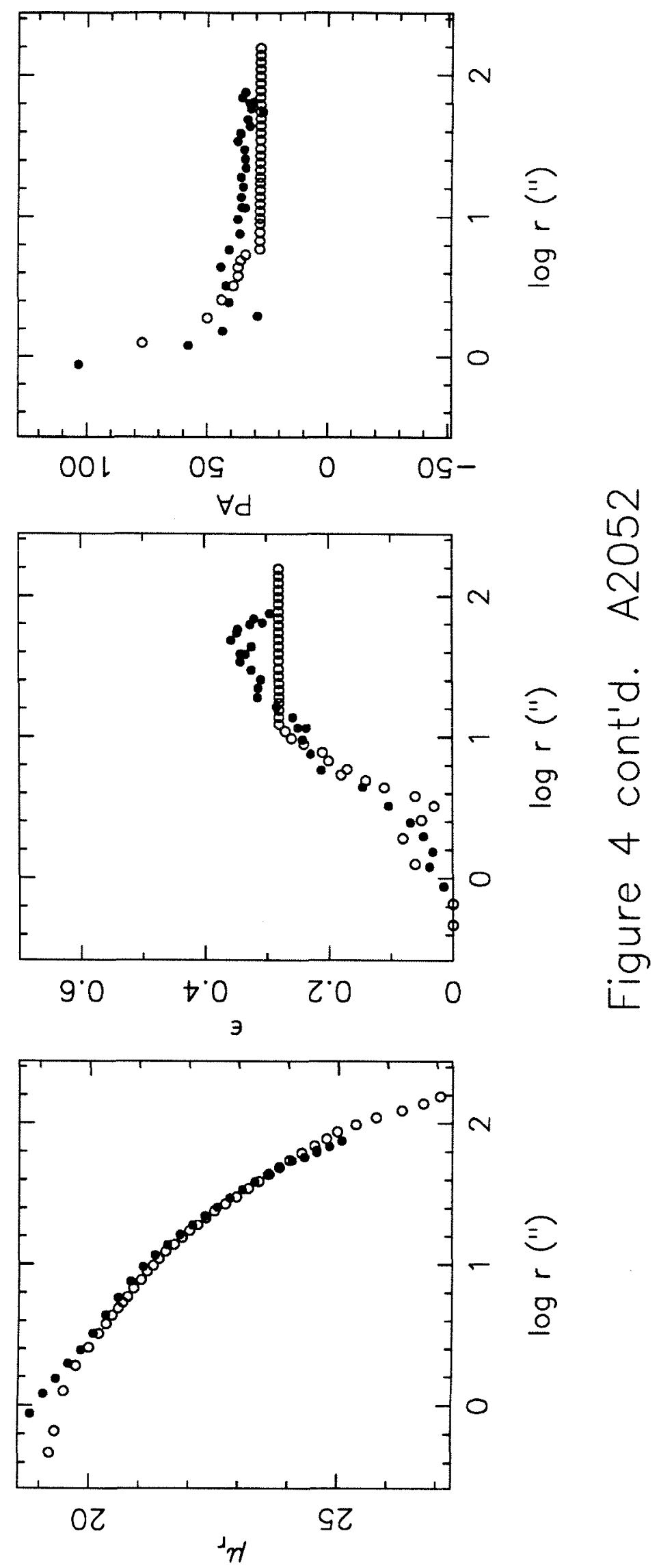

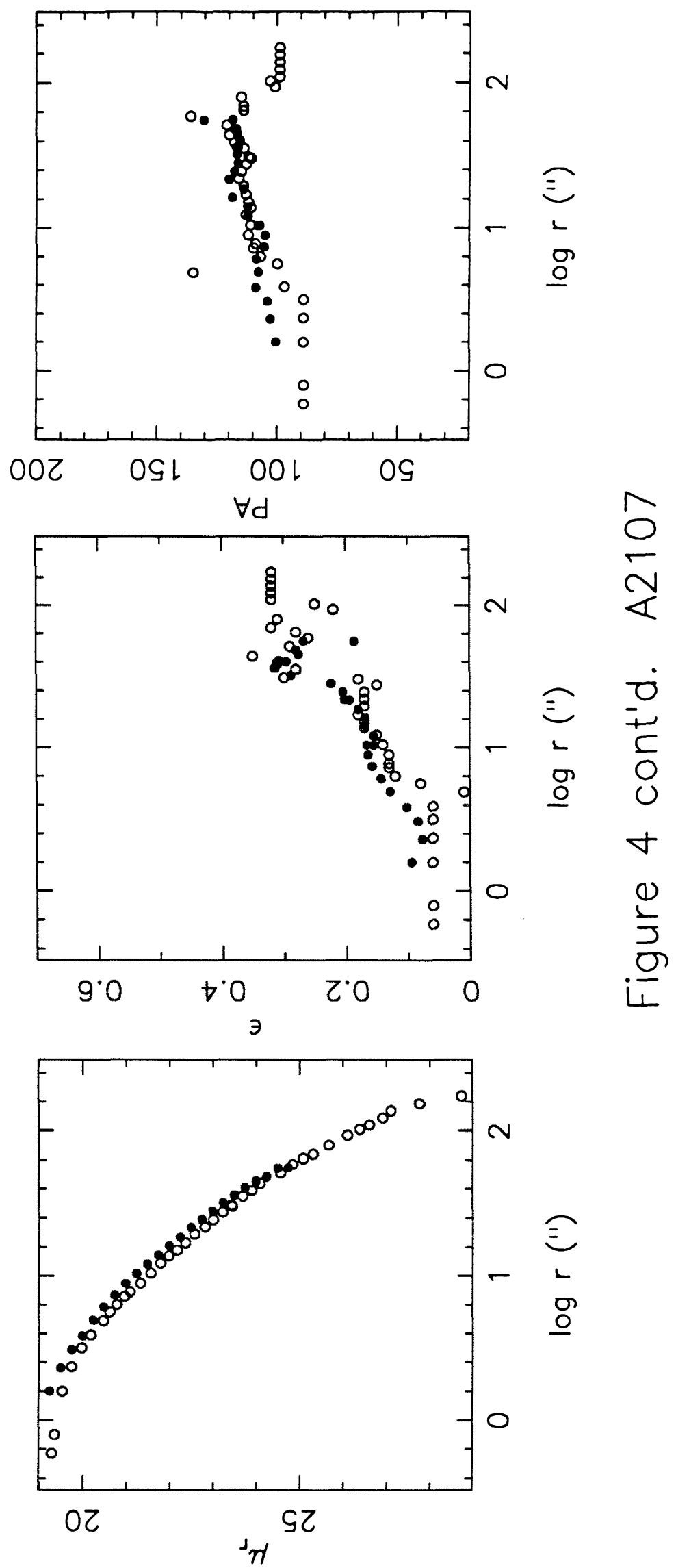
$-77-$
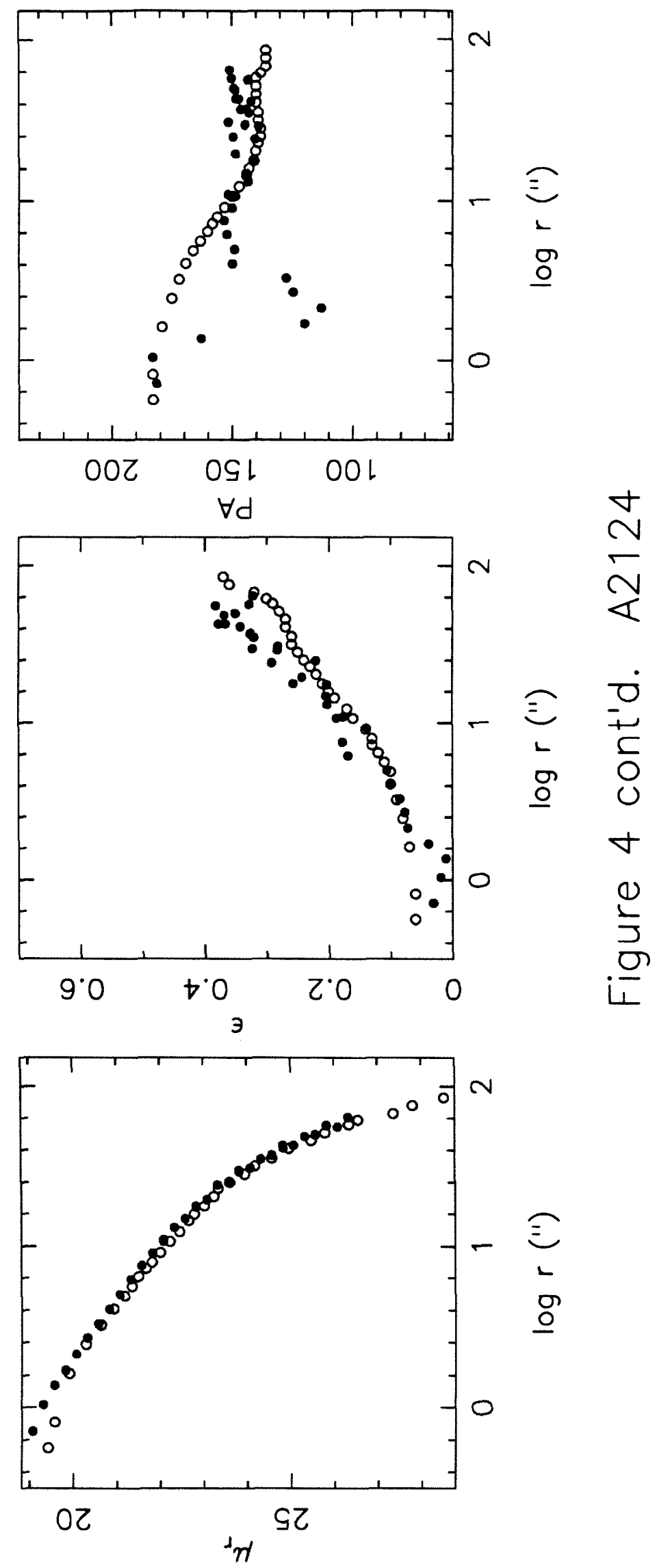
$-78-$
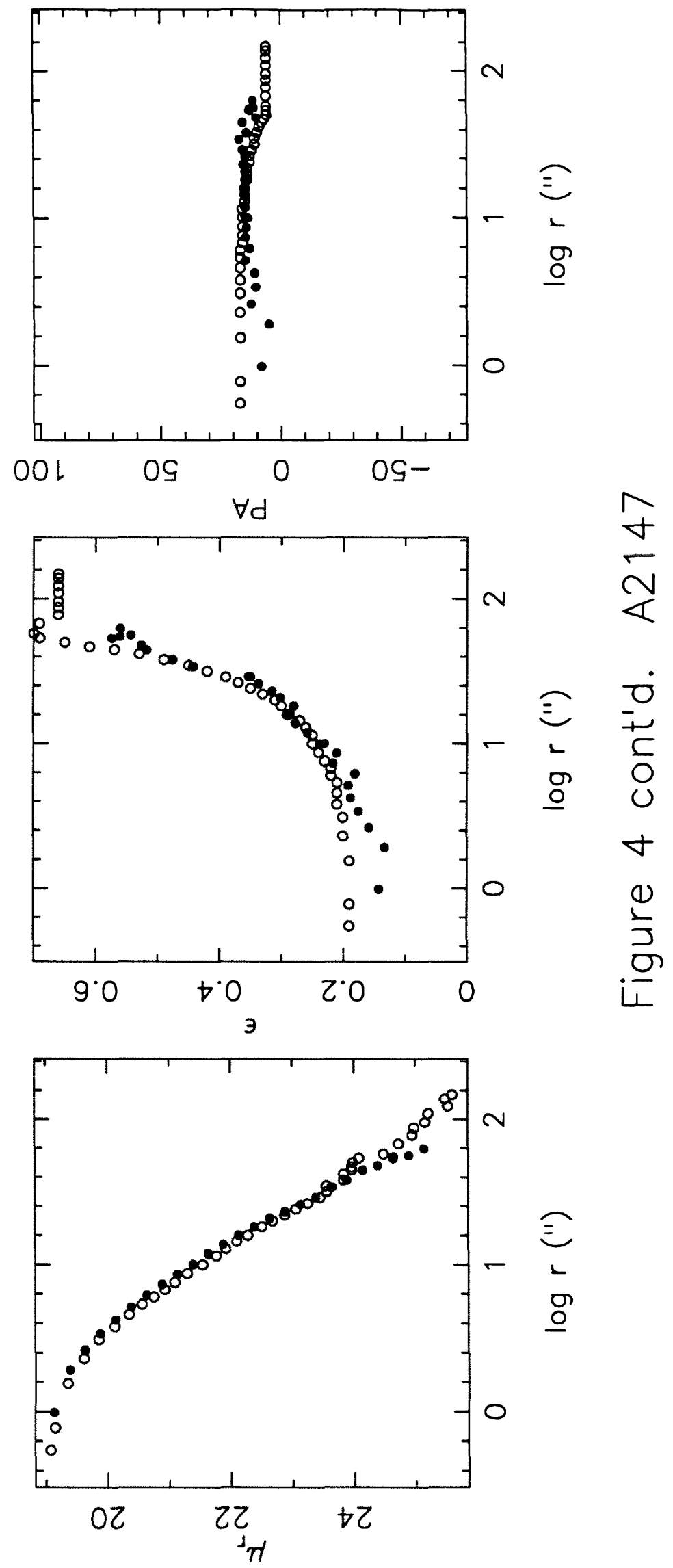
-79-
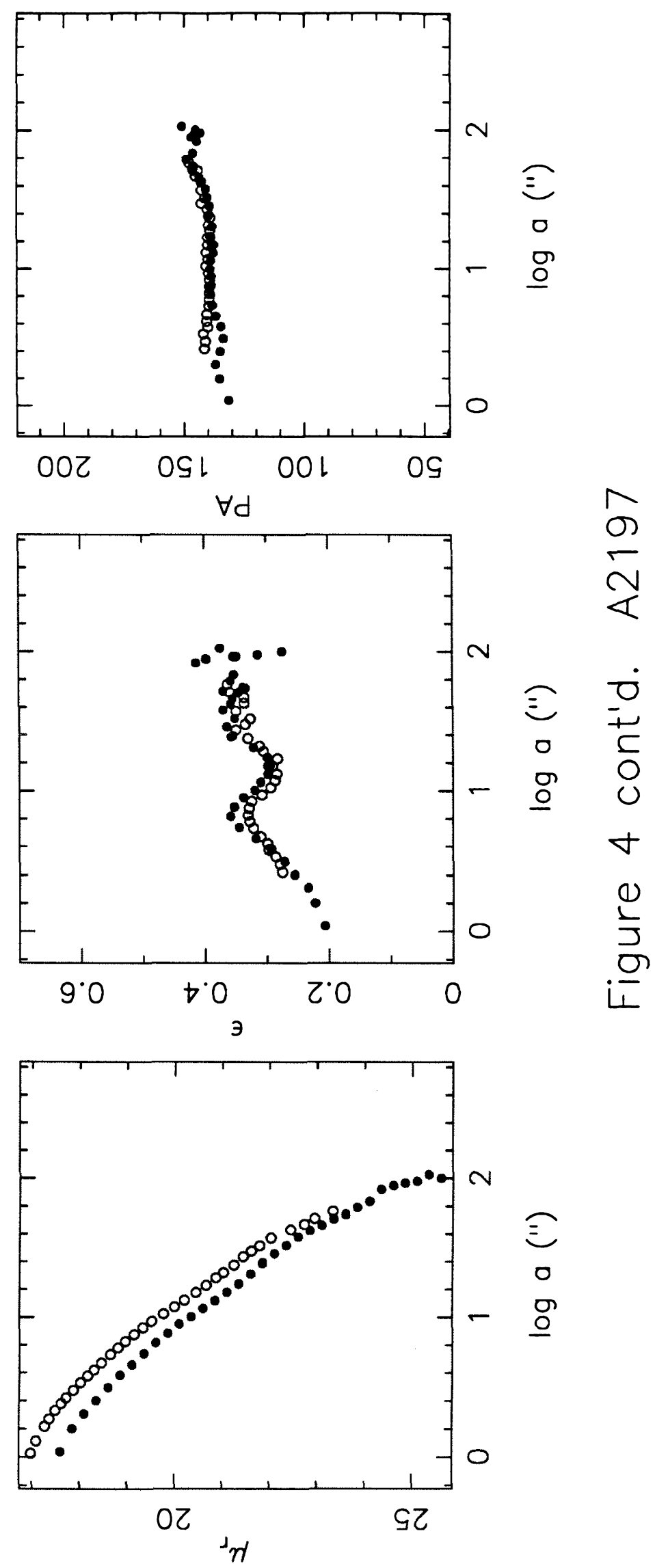
$-80-$
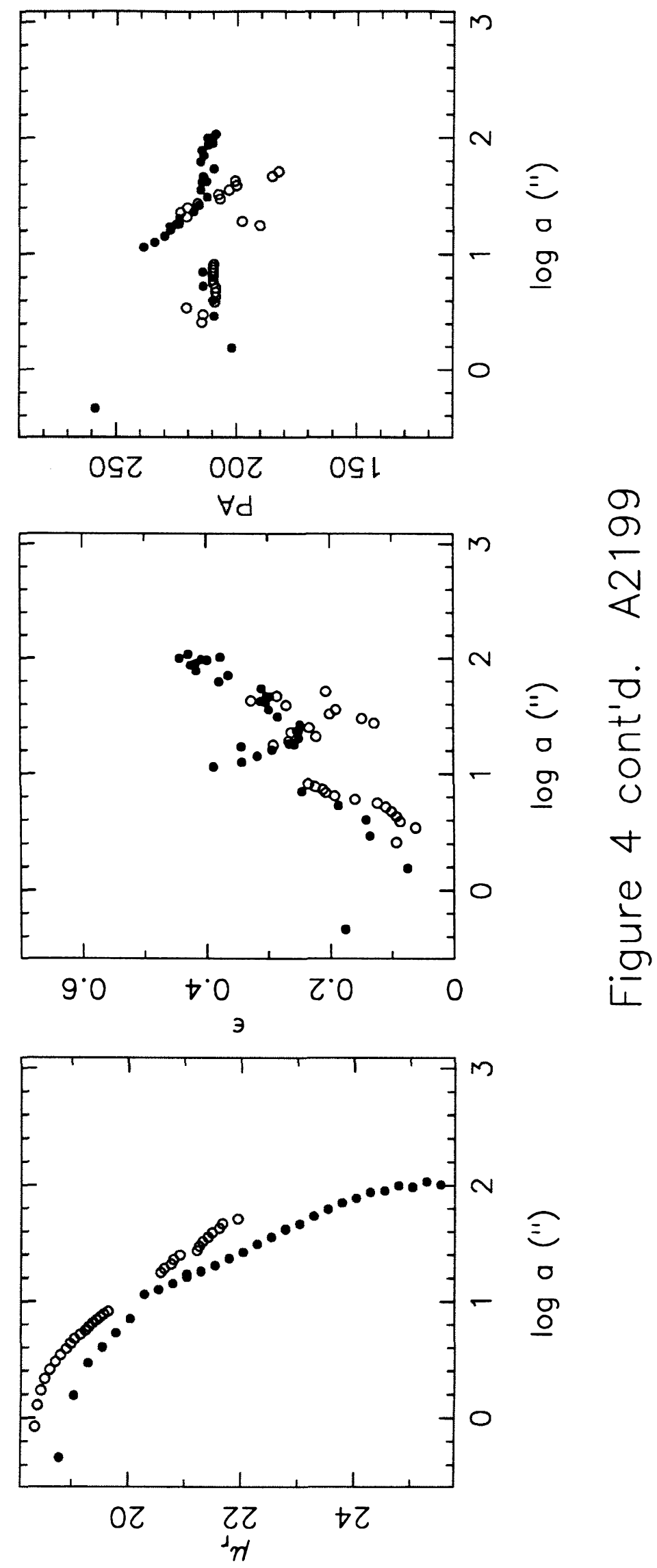
$-81-$
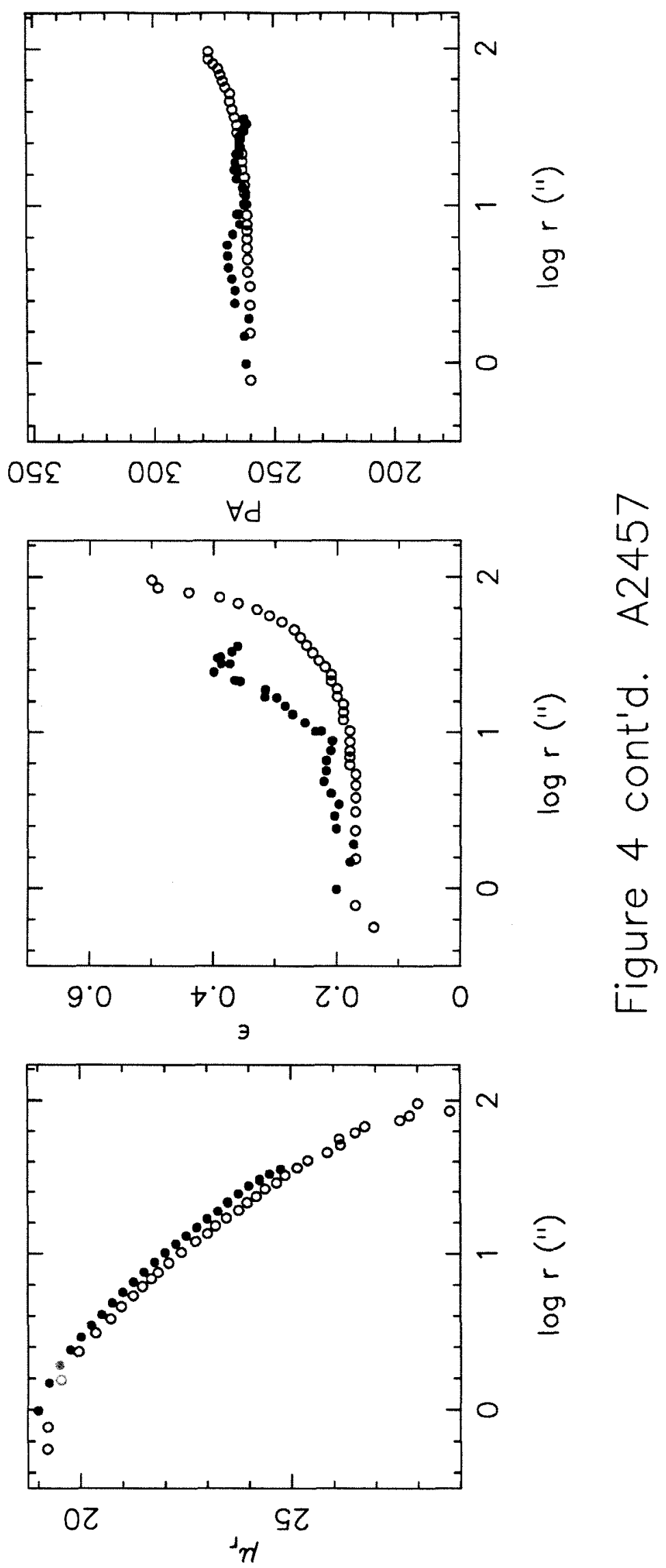

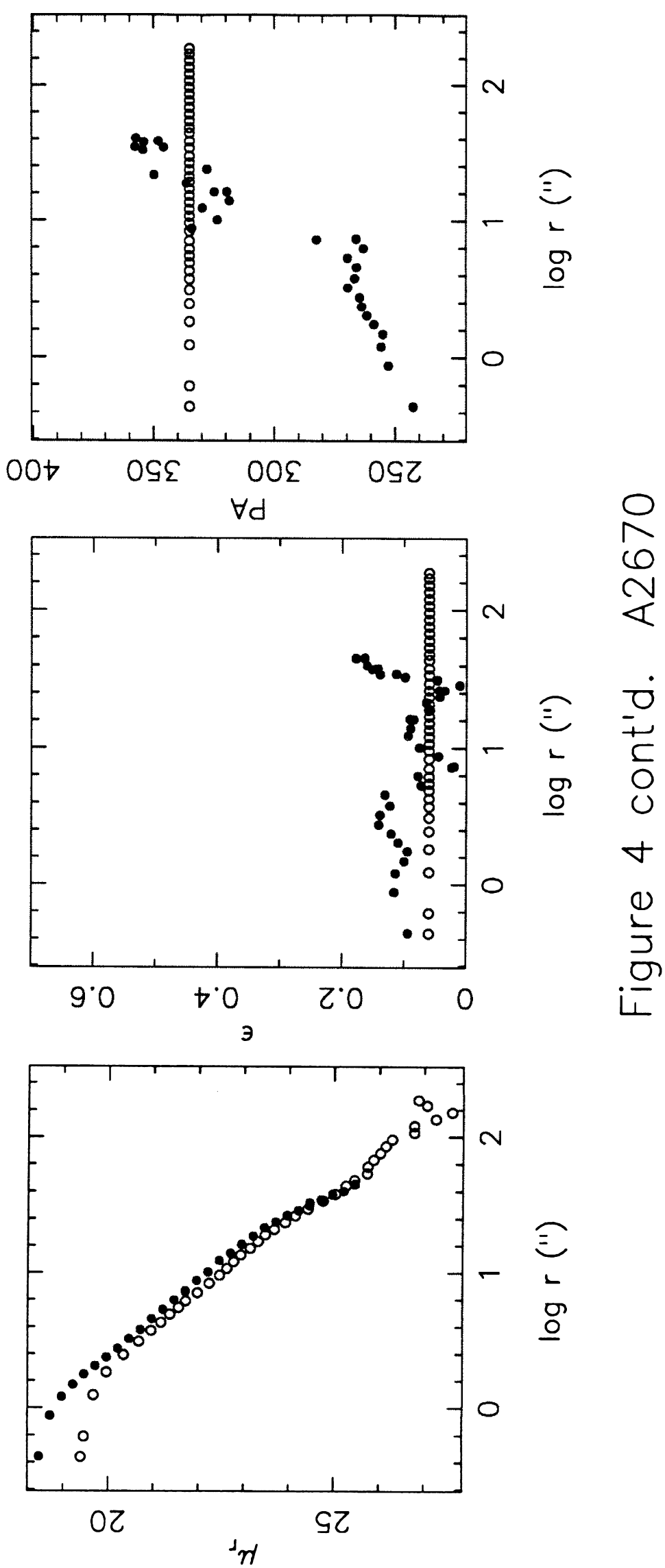

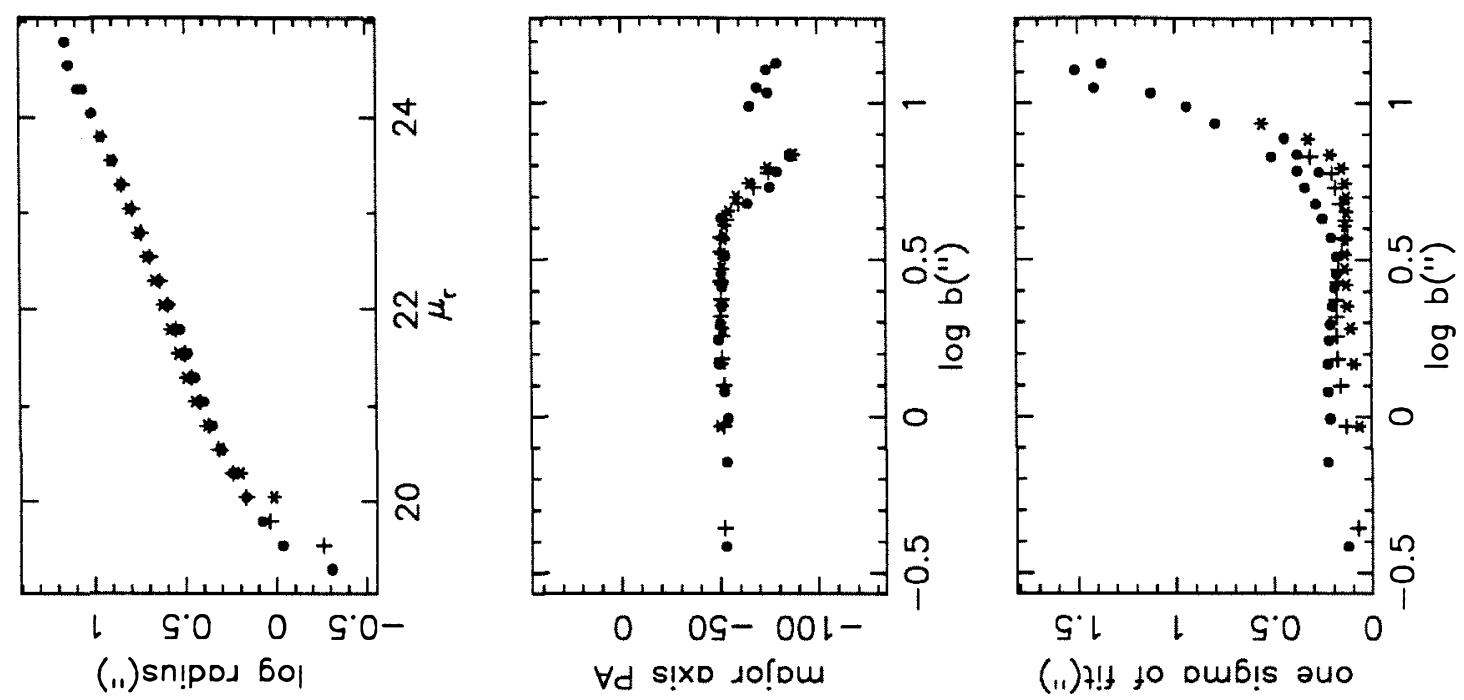

(11) $7 !+10$ oub!s auo
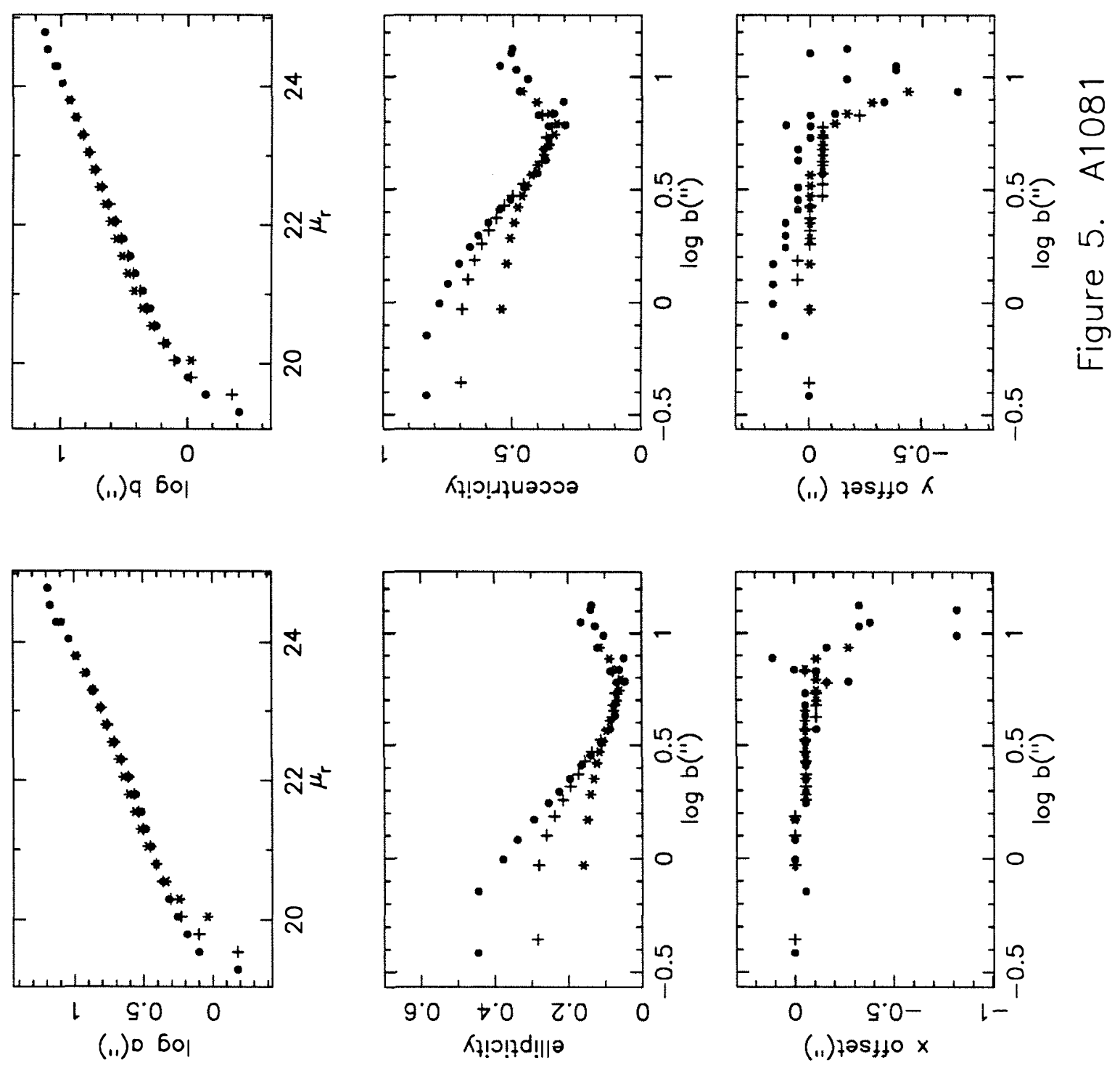


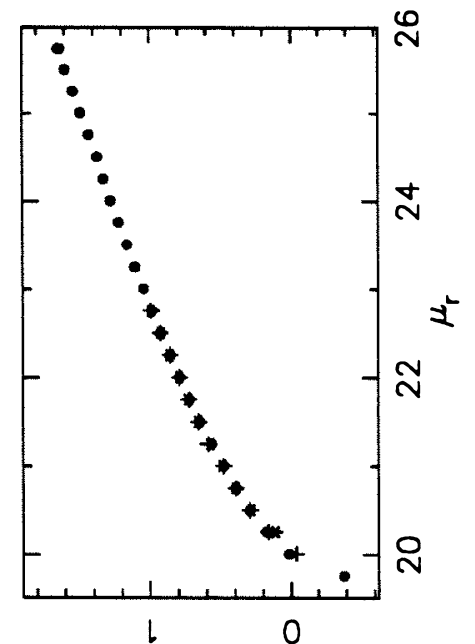

(.1) sn!pod 6ol
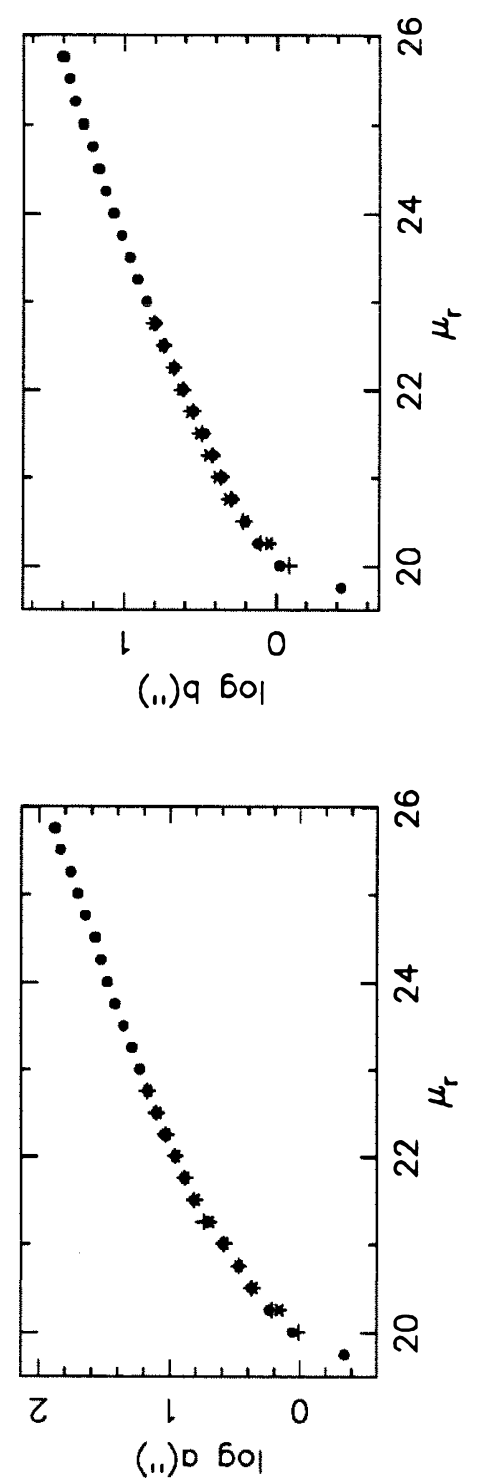

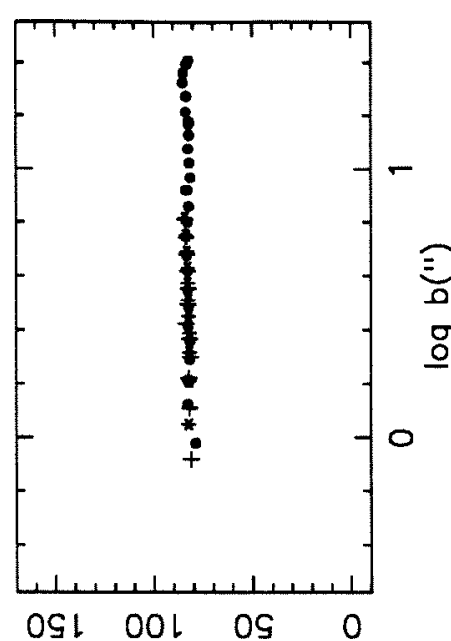

$\forall d$ s!xo dorom
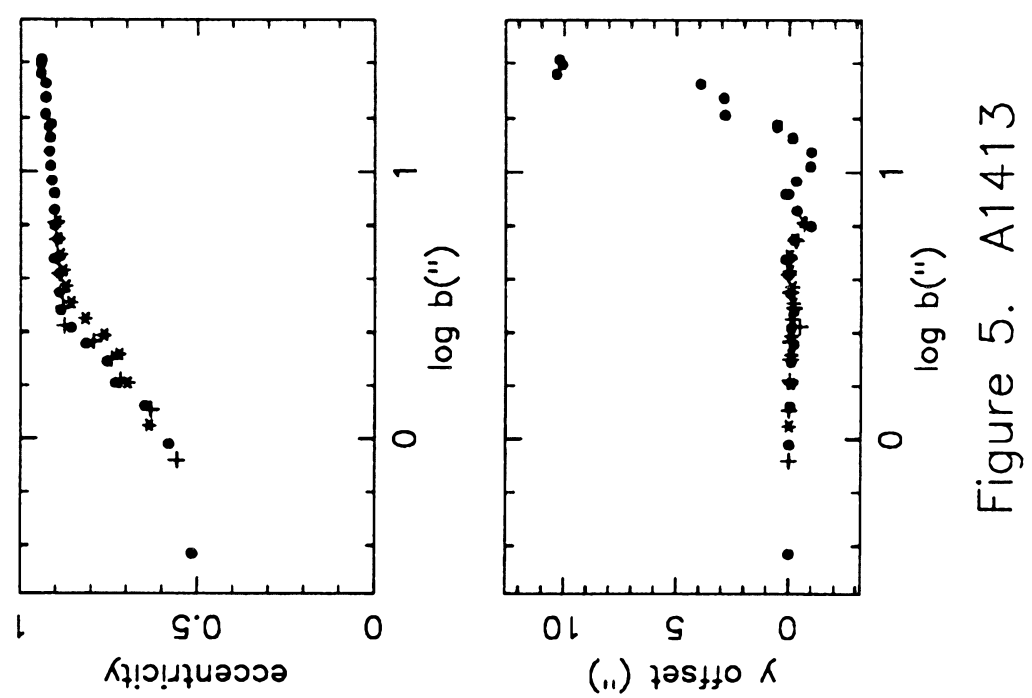
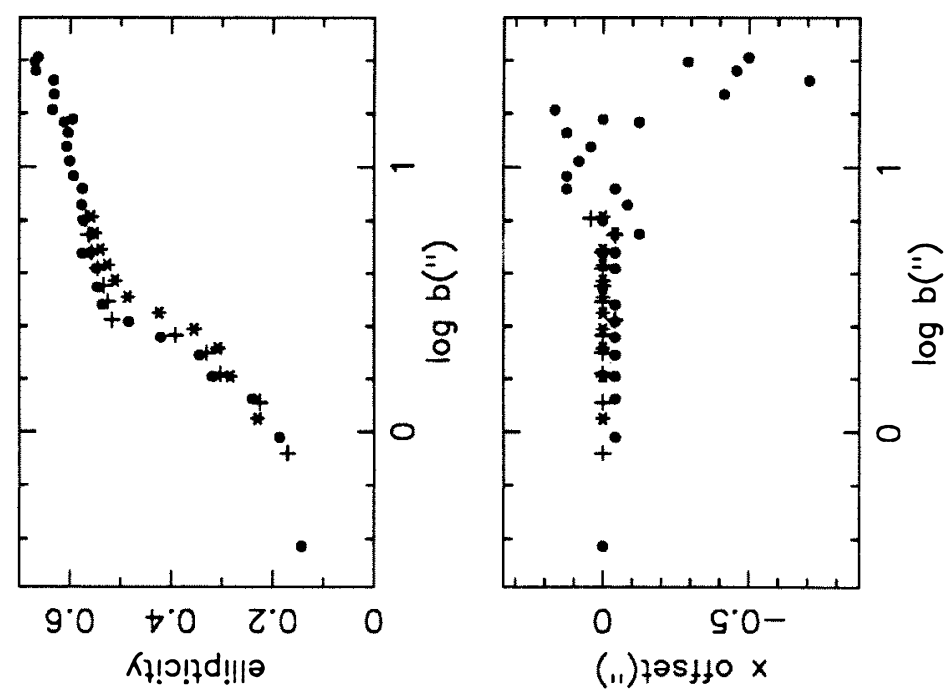


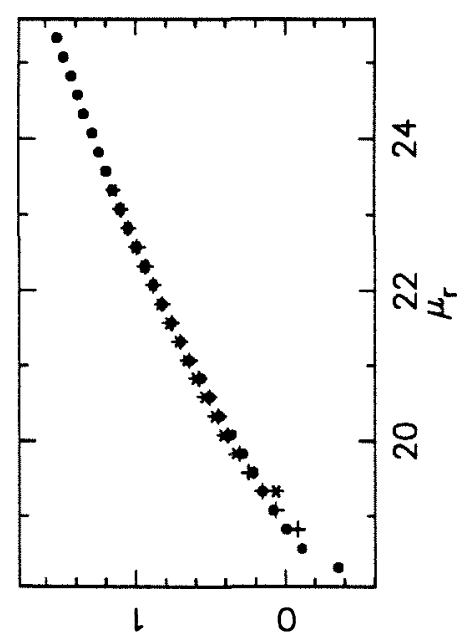

(.1) sn!pos 6ol
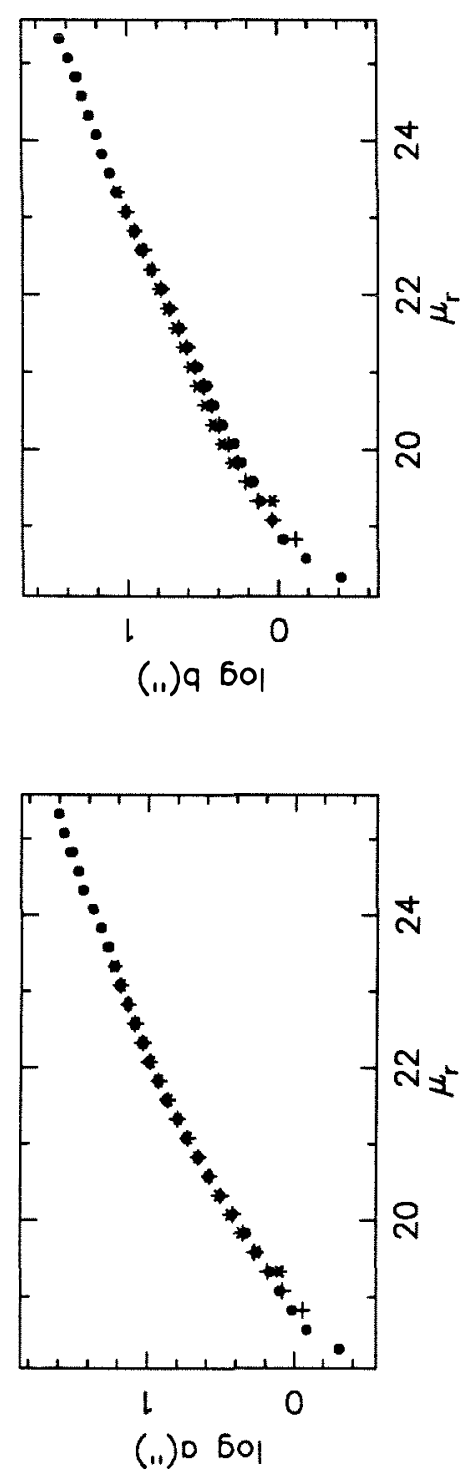

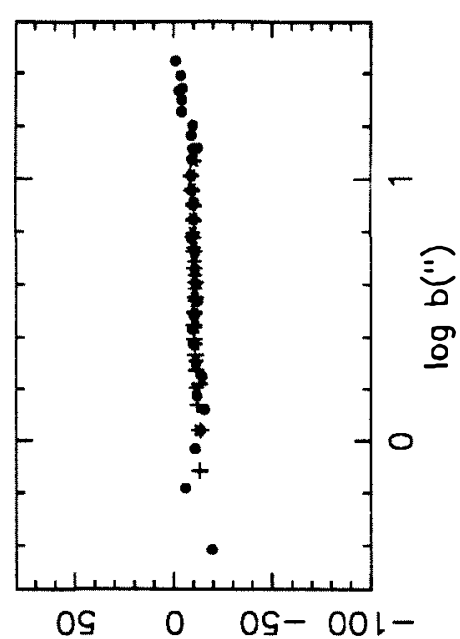

$\forall d s ! \times 0$ Jo!̣m
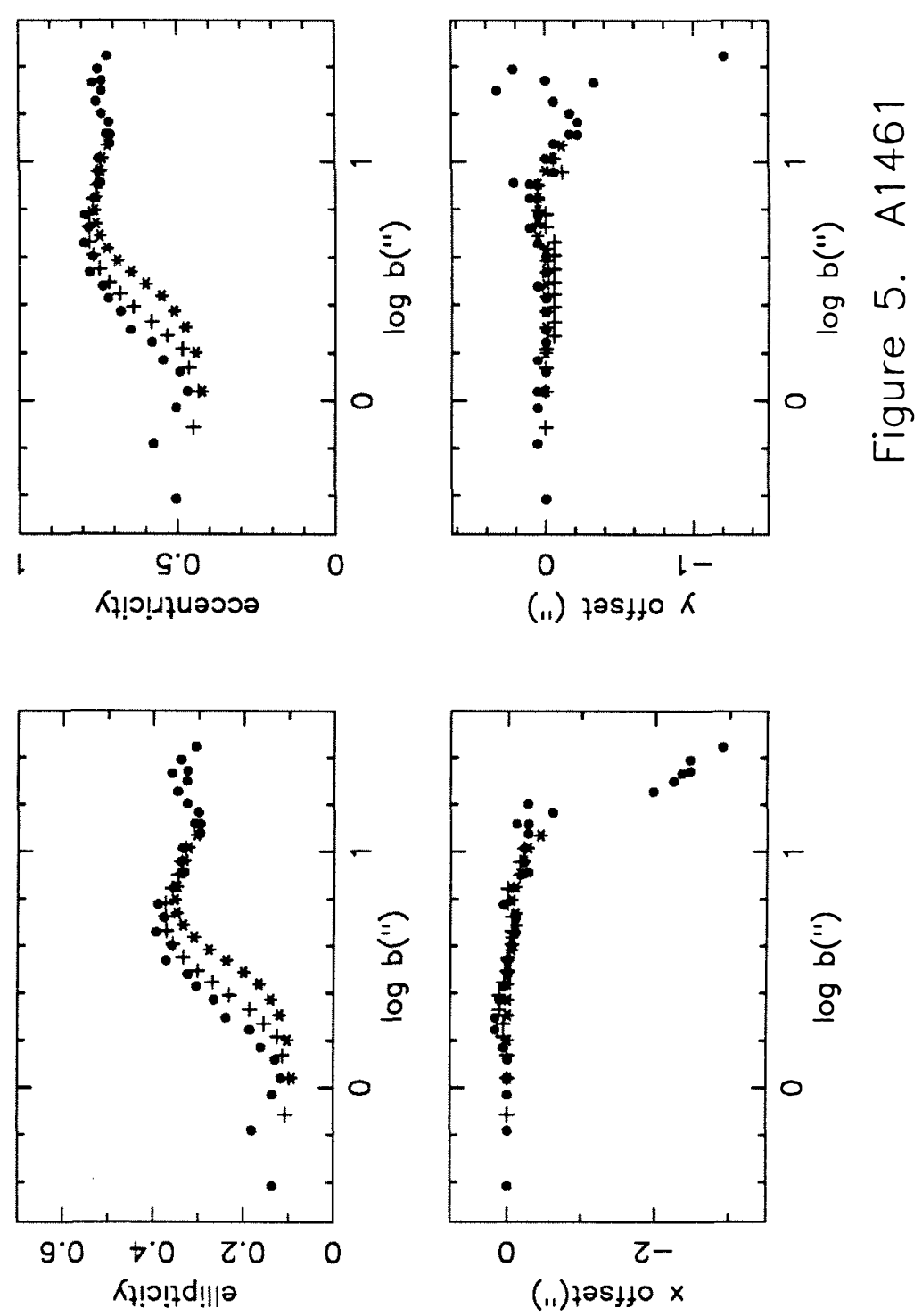


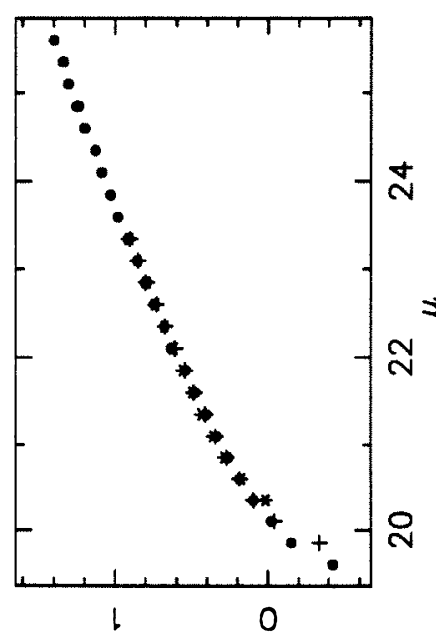

(11)sn!pod 60।

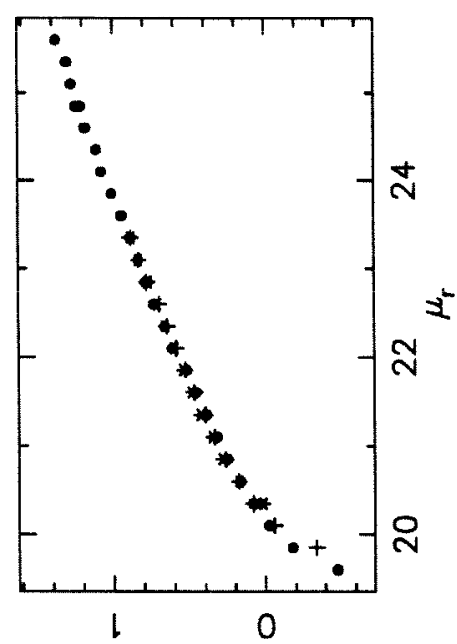

(.1) 9 601

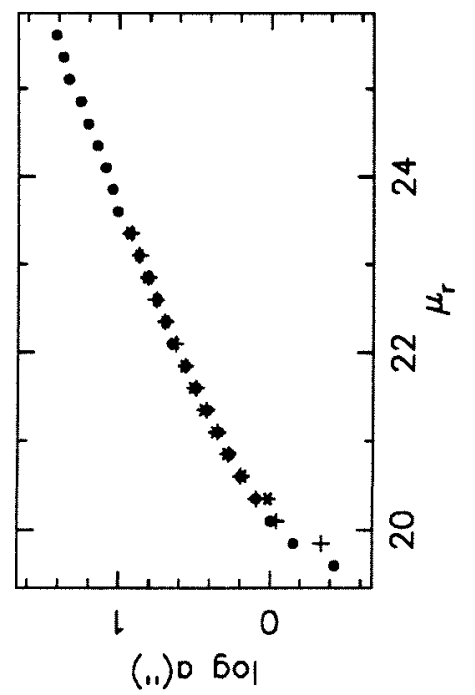

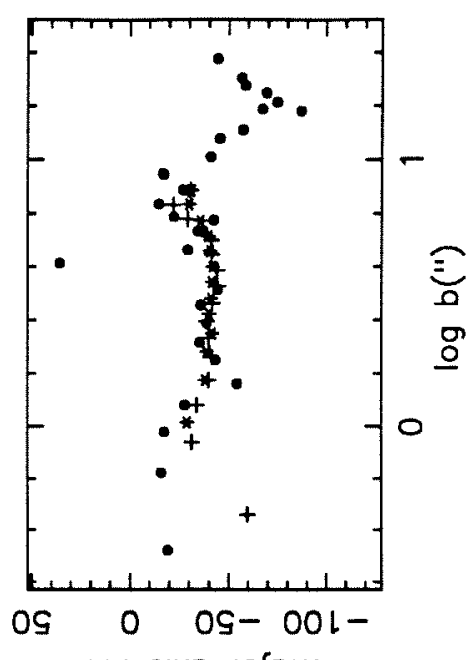

$\forall d$ s!xo so!
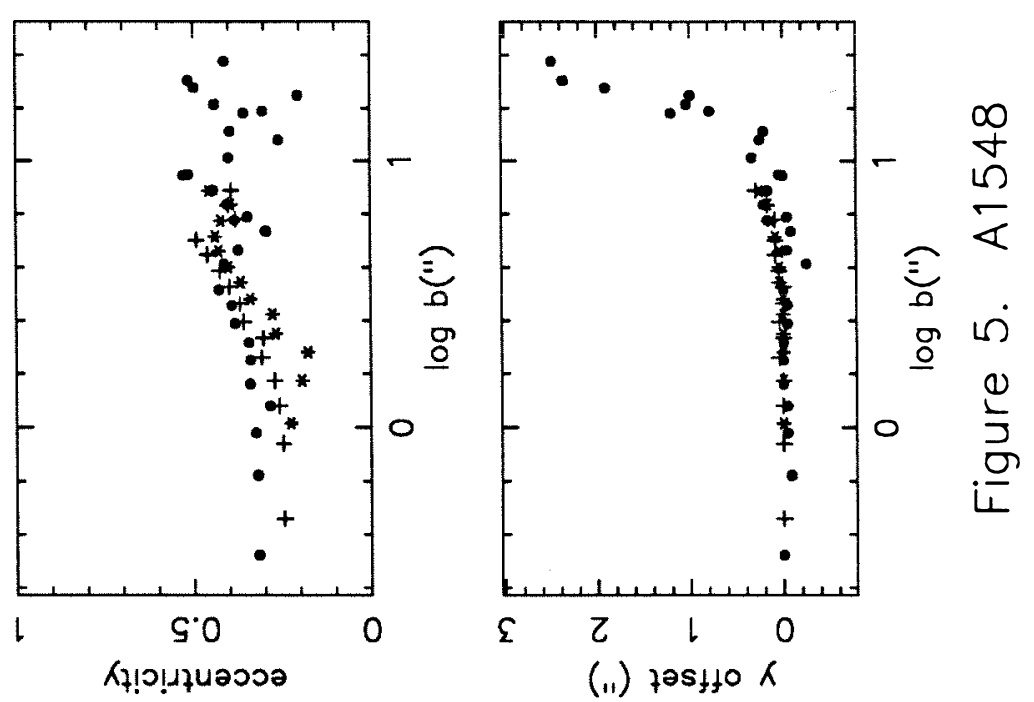

อ 음 (i1) $)$ ! t to oub! auo

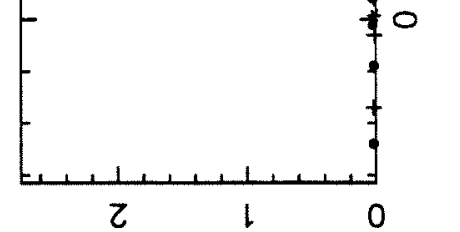



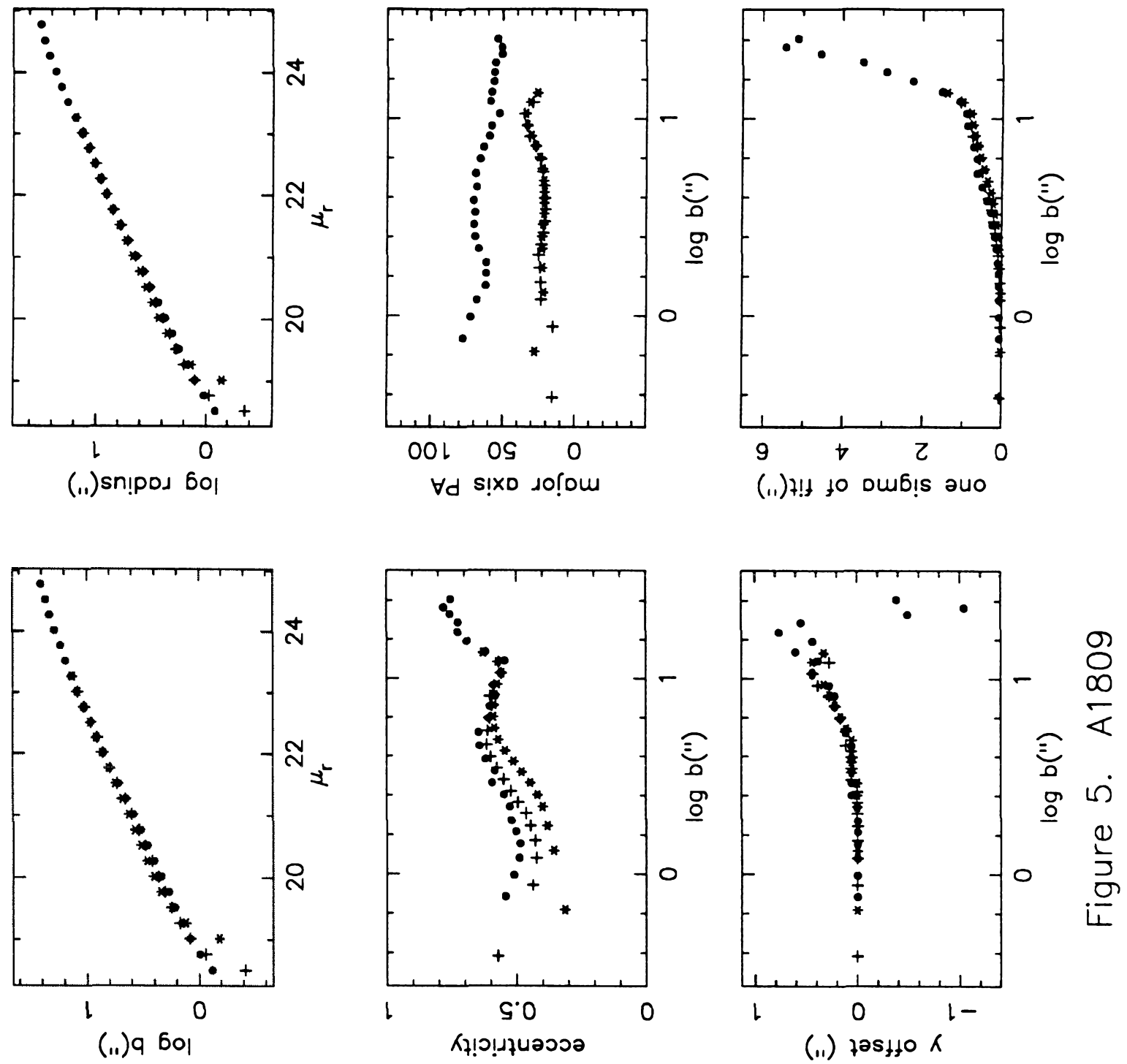

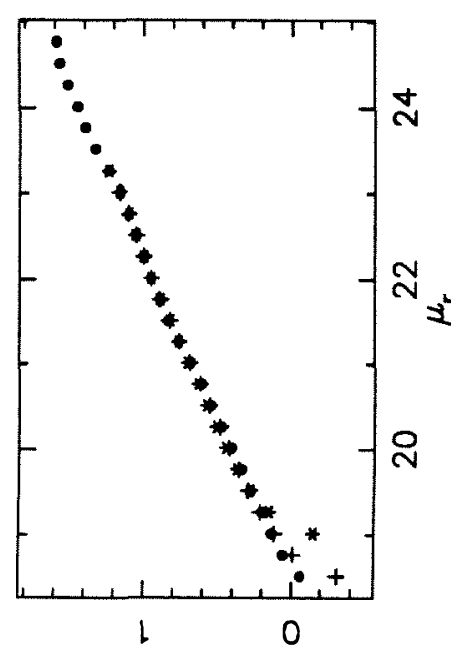

(.1) 0 bol

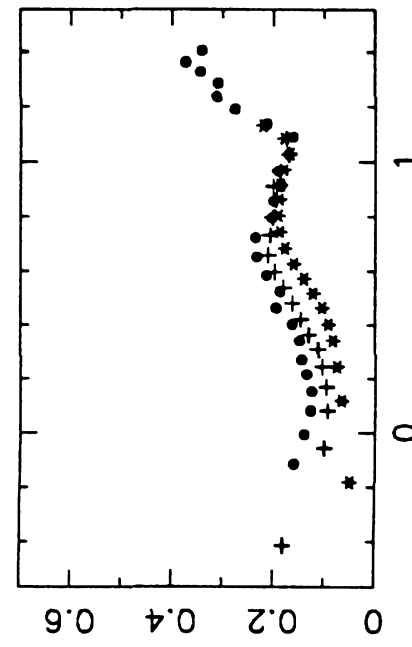

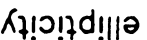

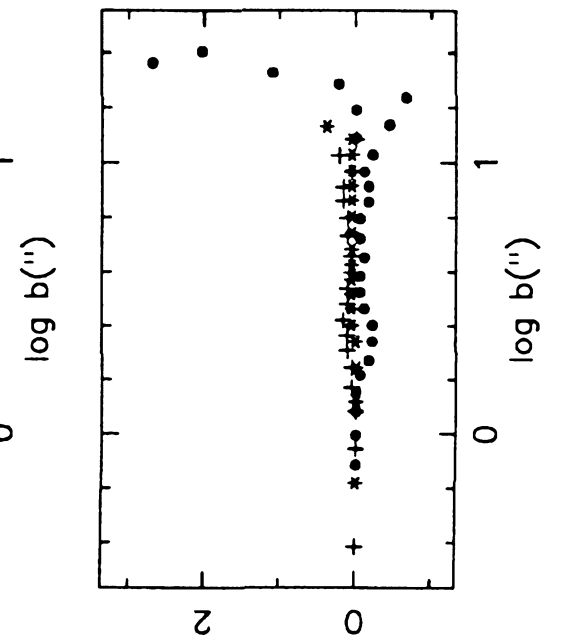

(.1) $125 \$ 0 \times$ 


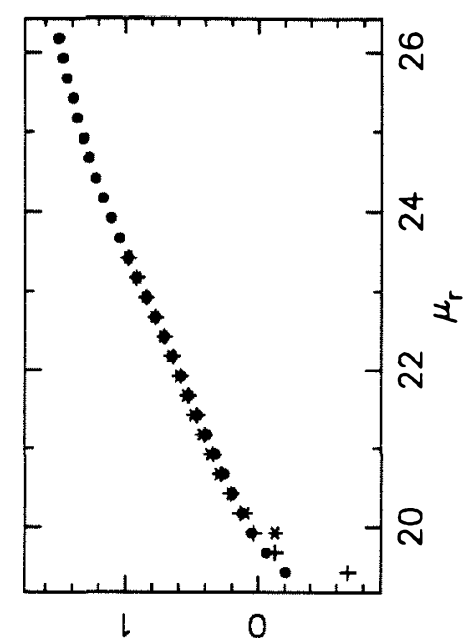

(11)sn!pod 5ol

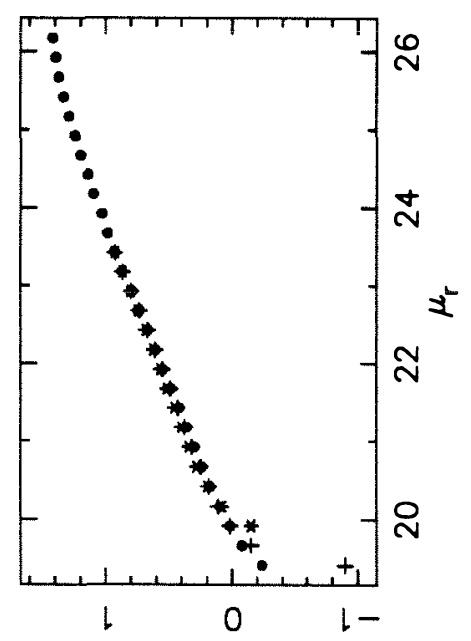

(11) 9601

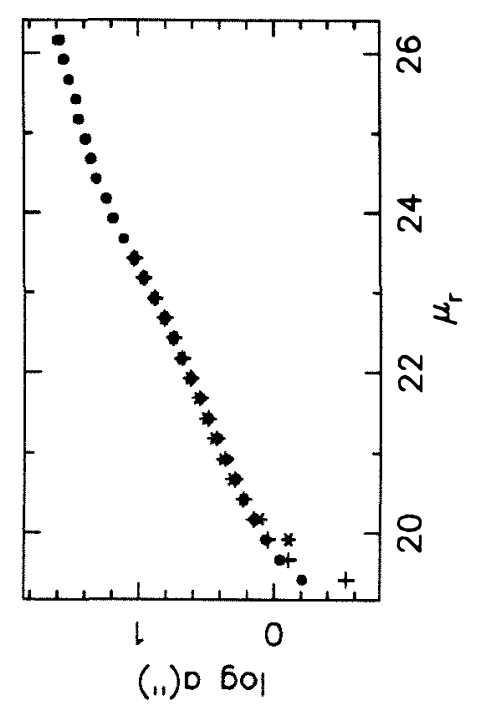

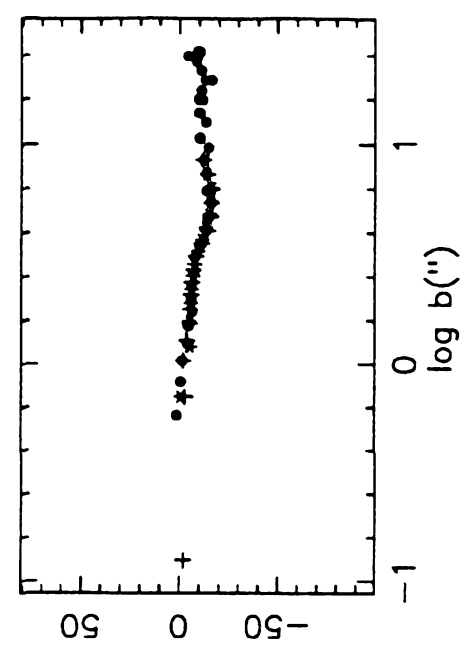

$\forall d$ s!xo so! lom

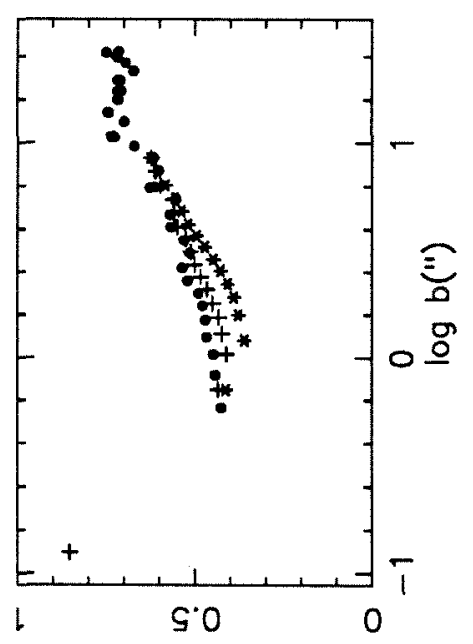

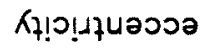

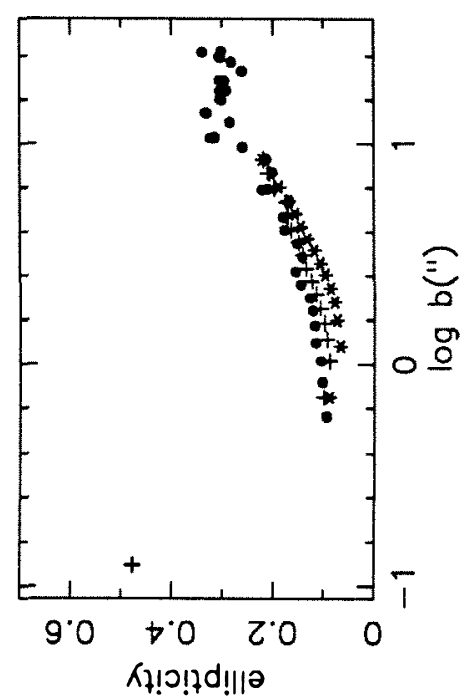

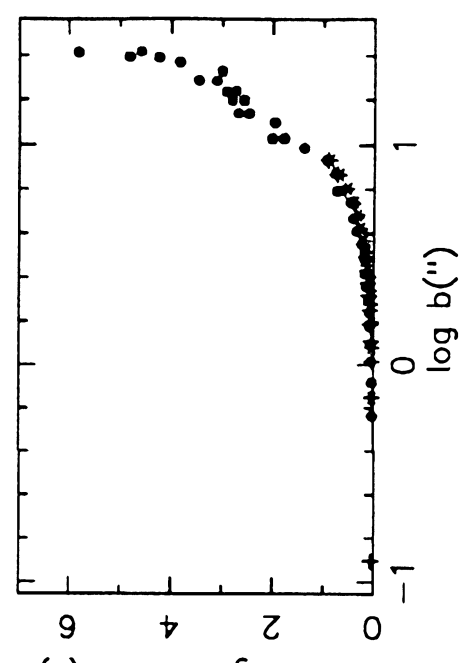

(11) 7 ! t to Dub! au०
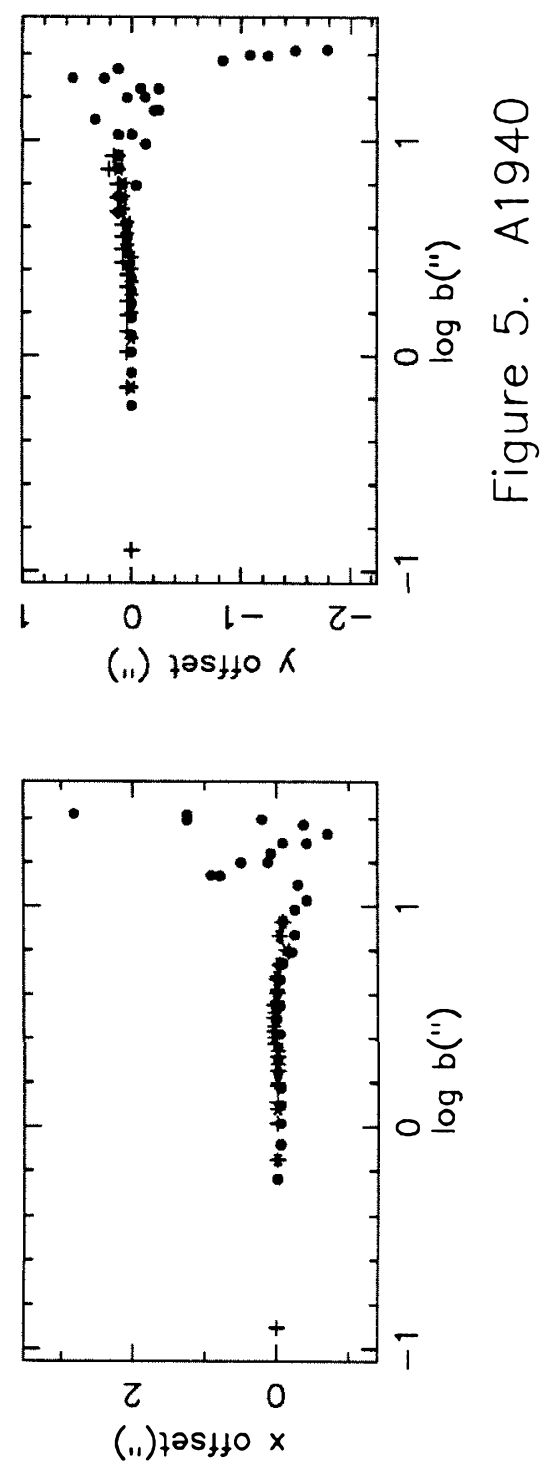


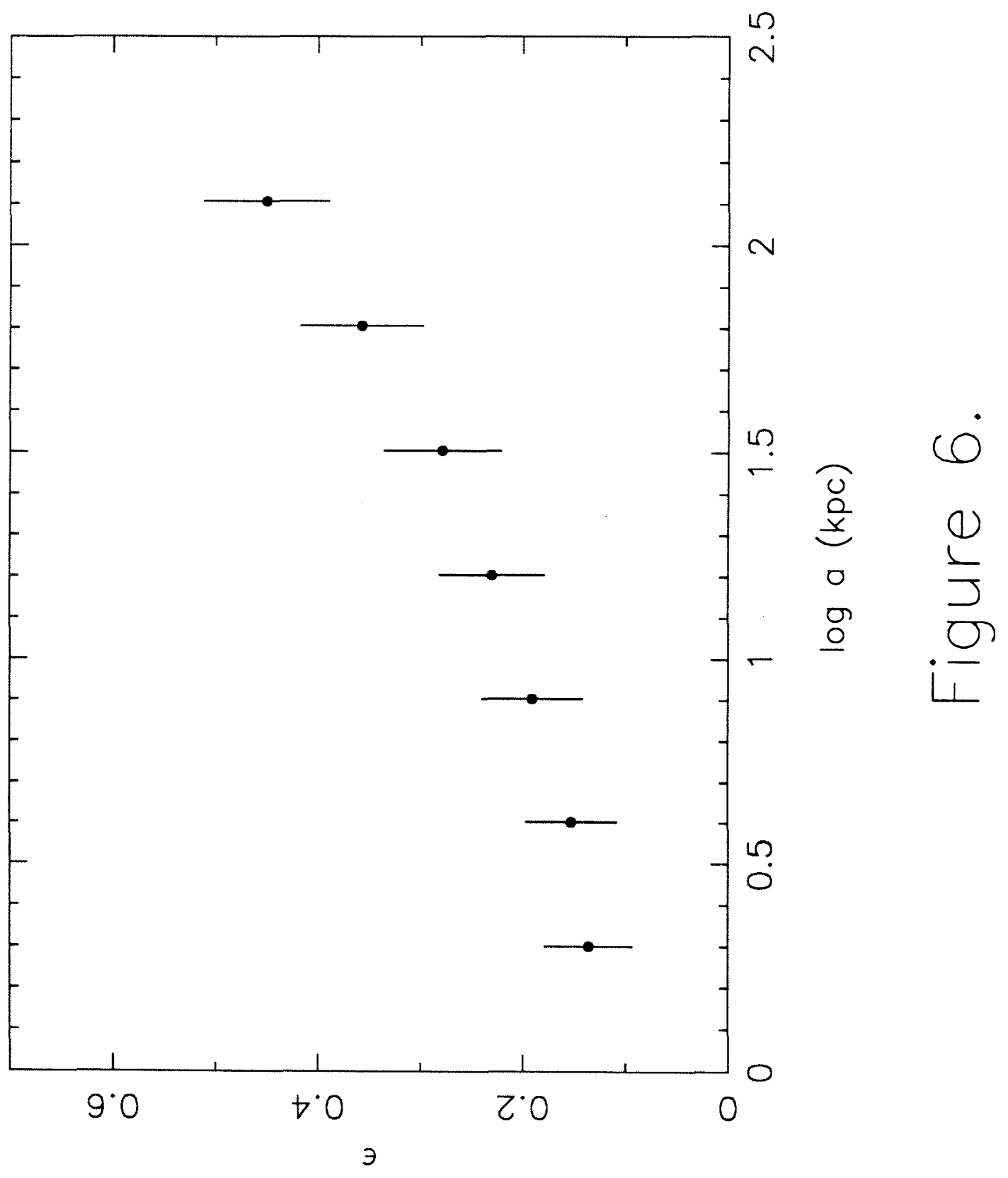




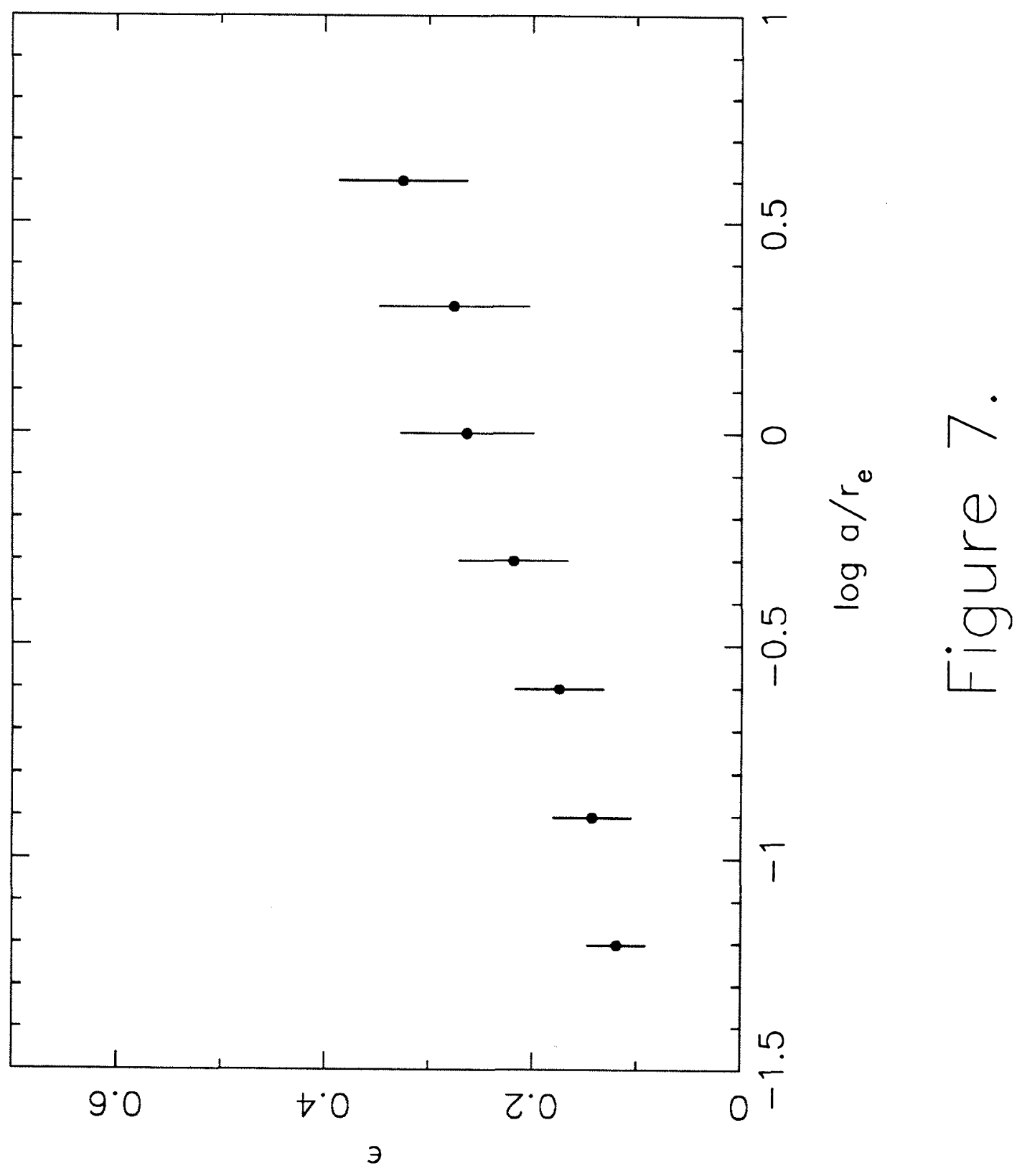




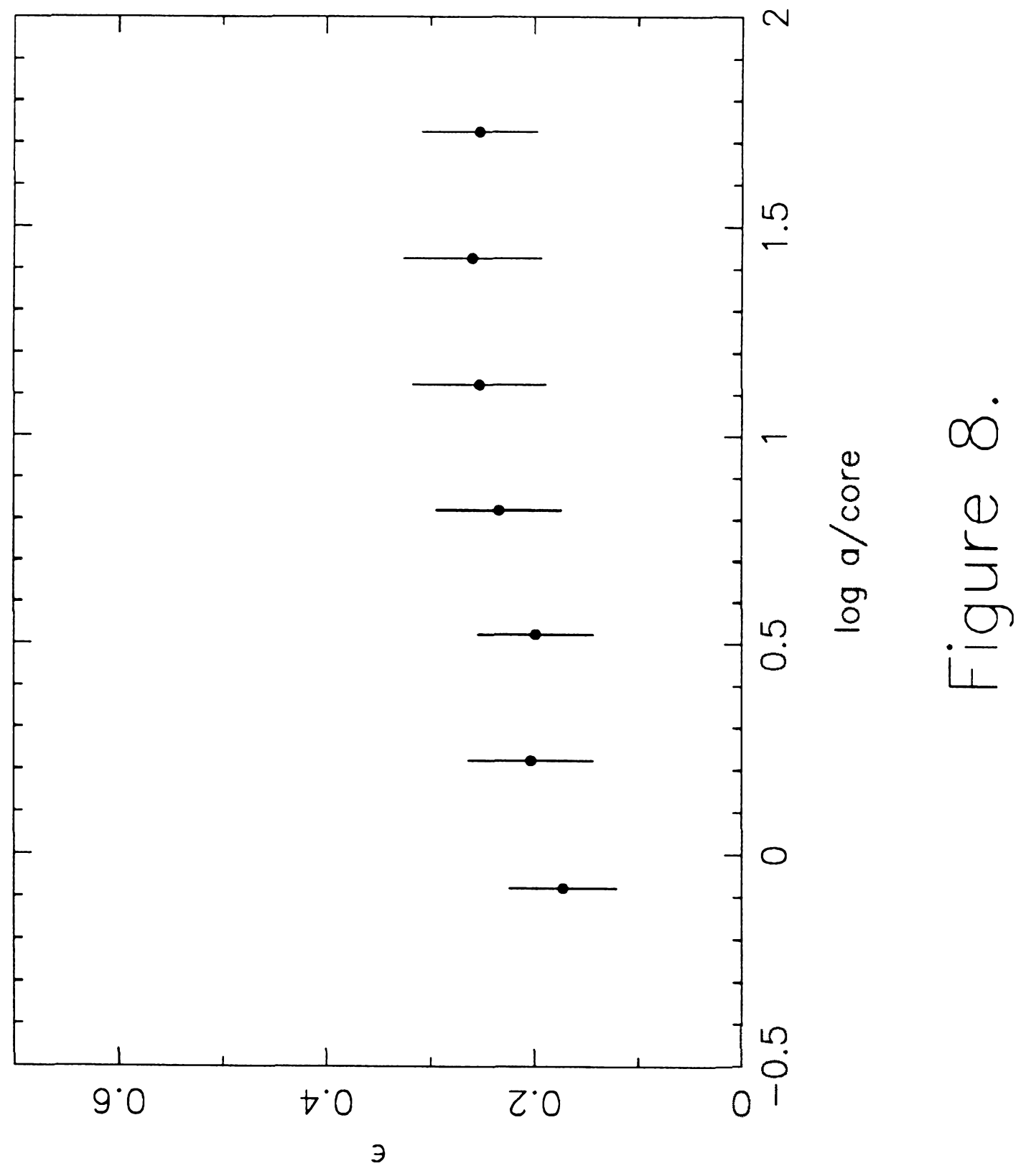




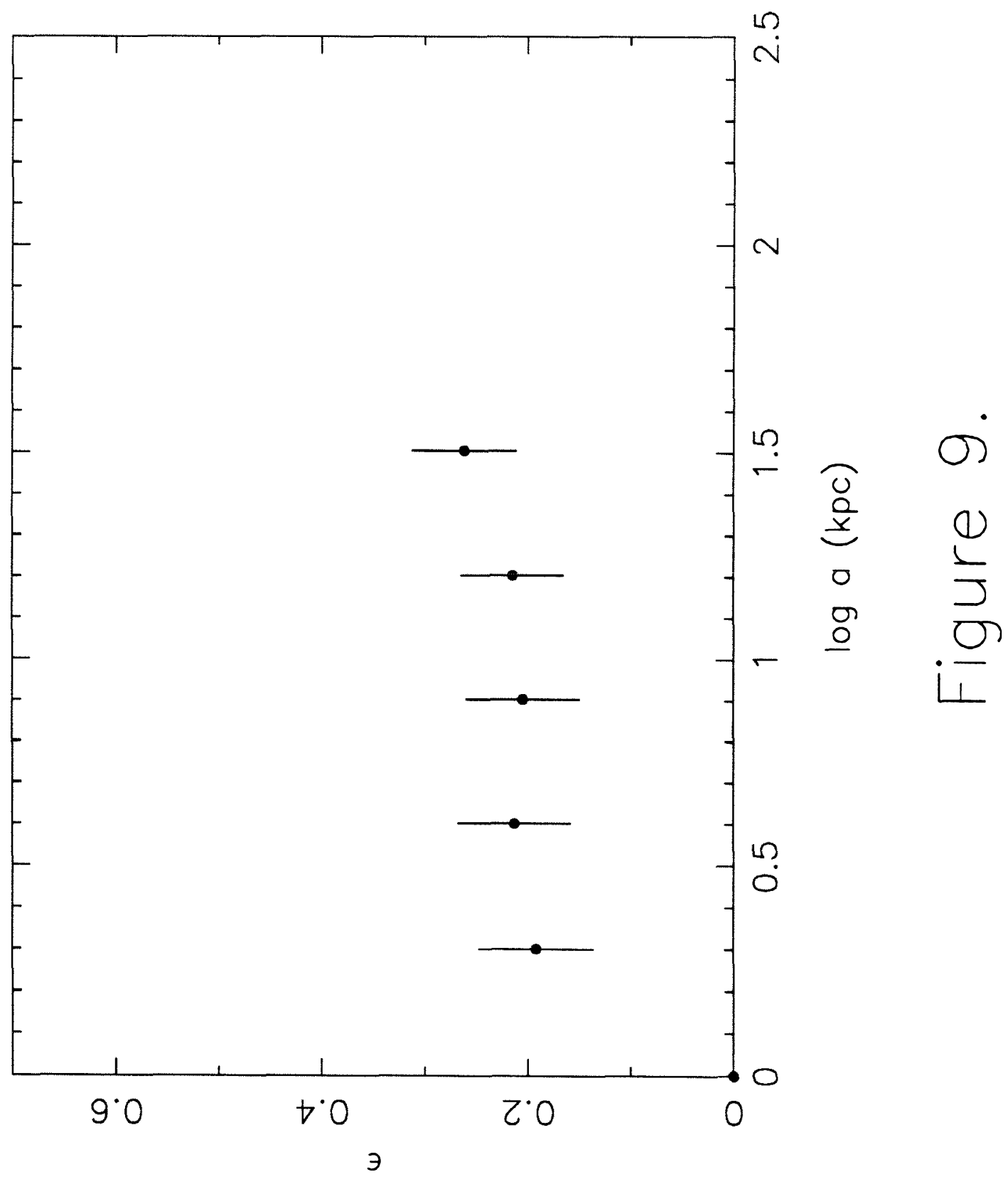




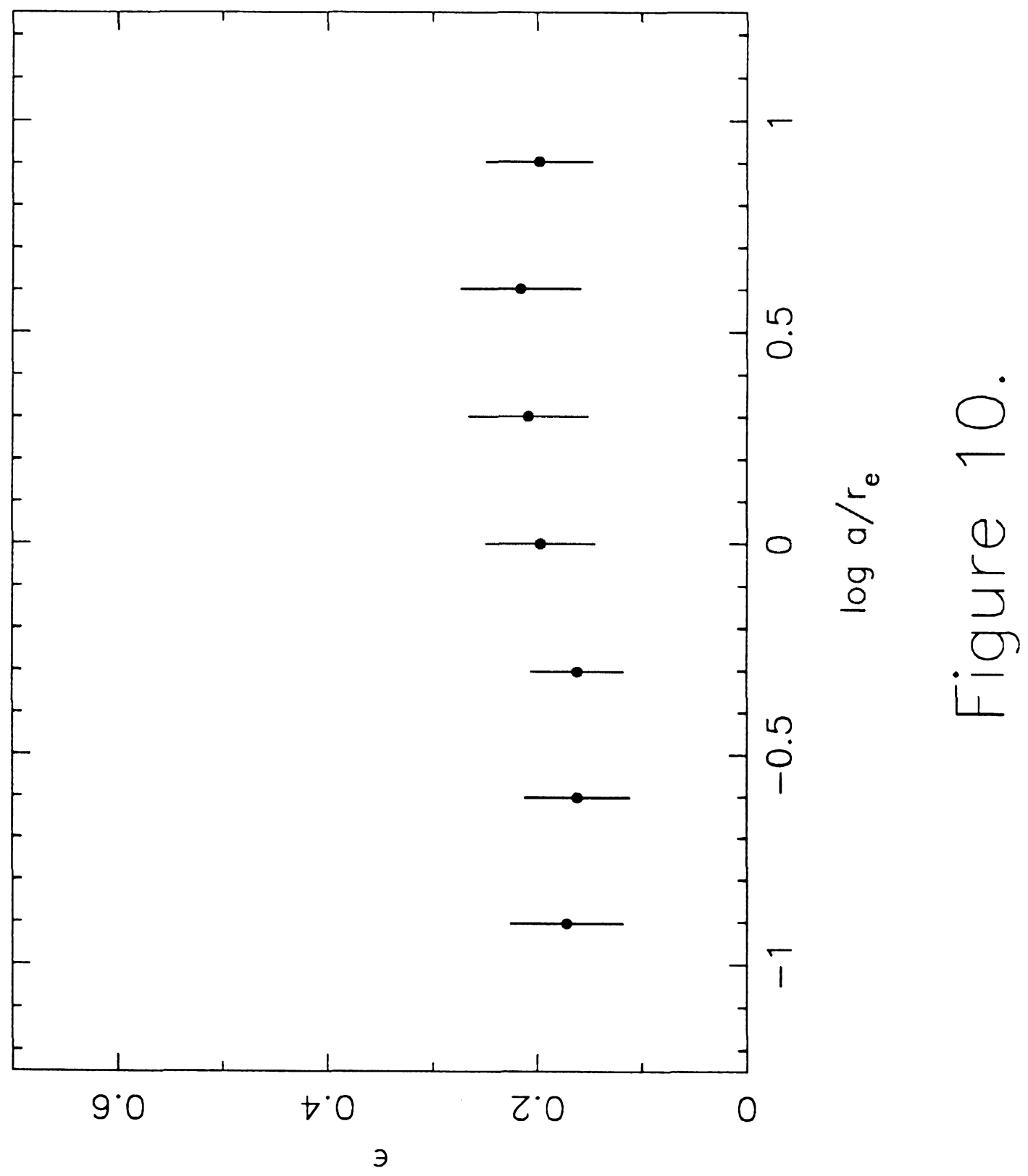



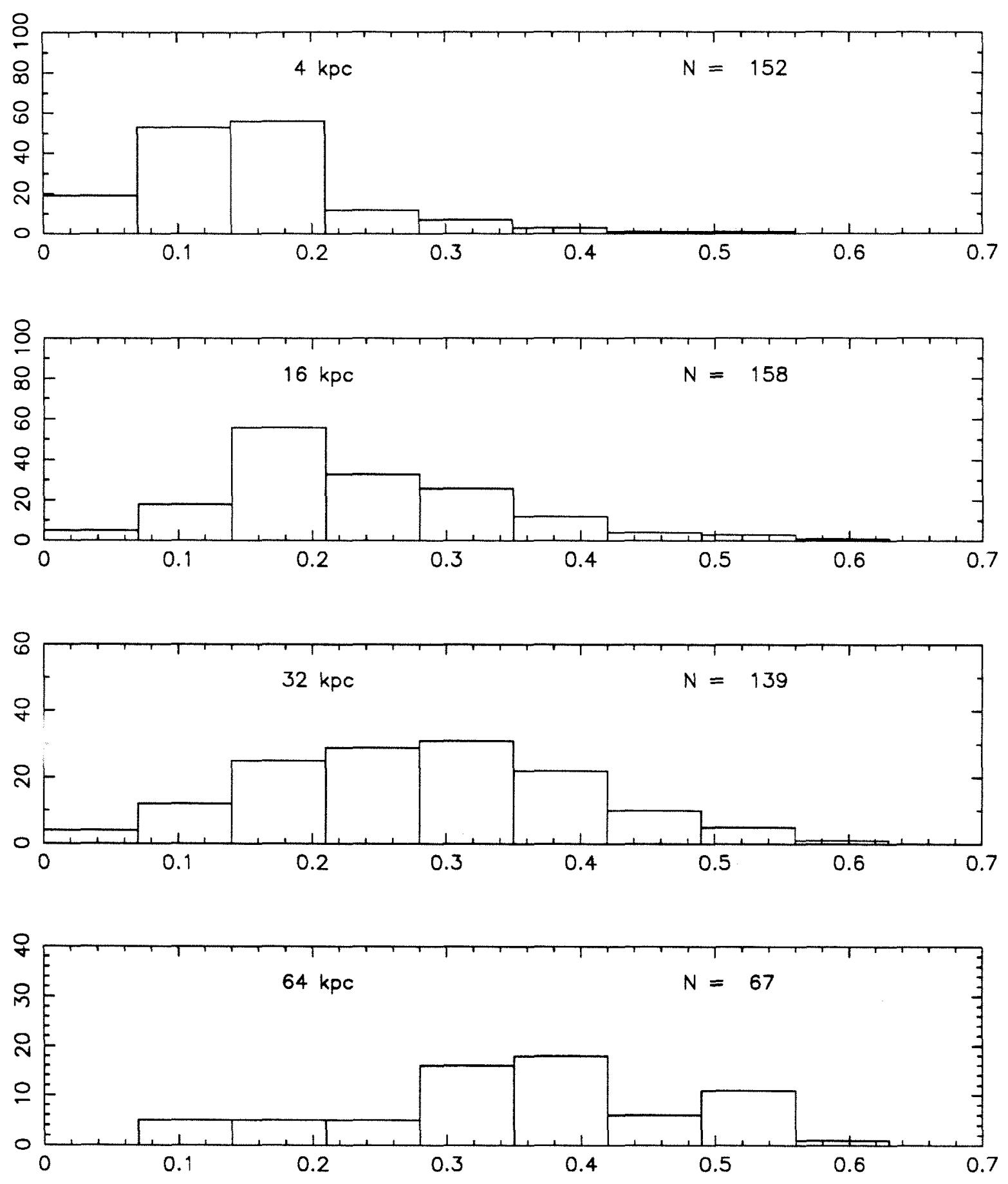

Figure 11. $B C E \in$ histograms. 

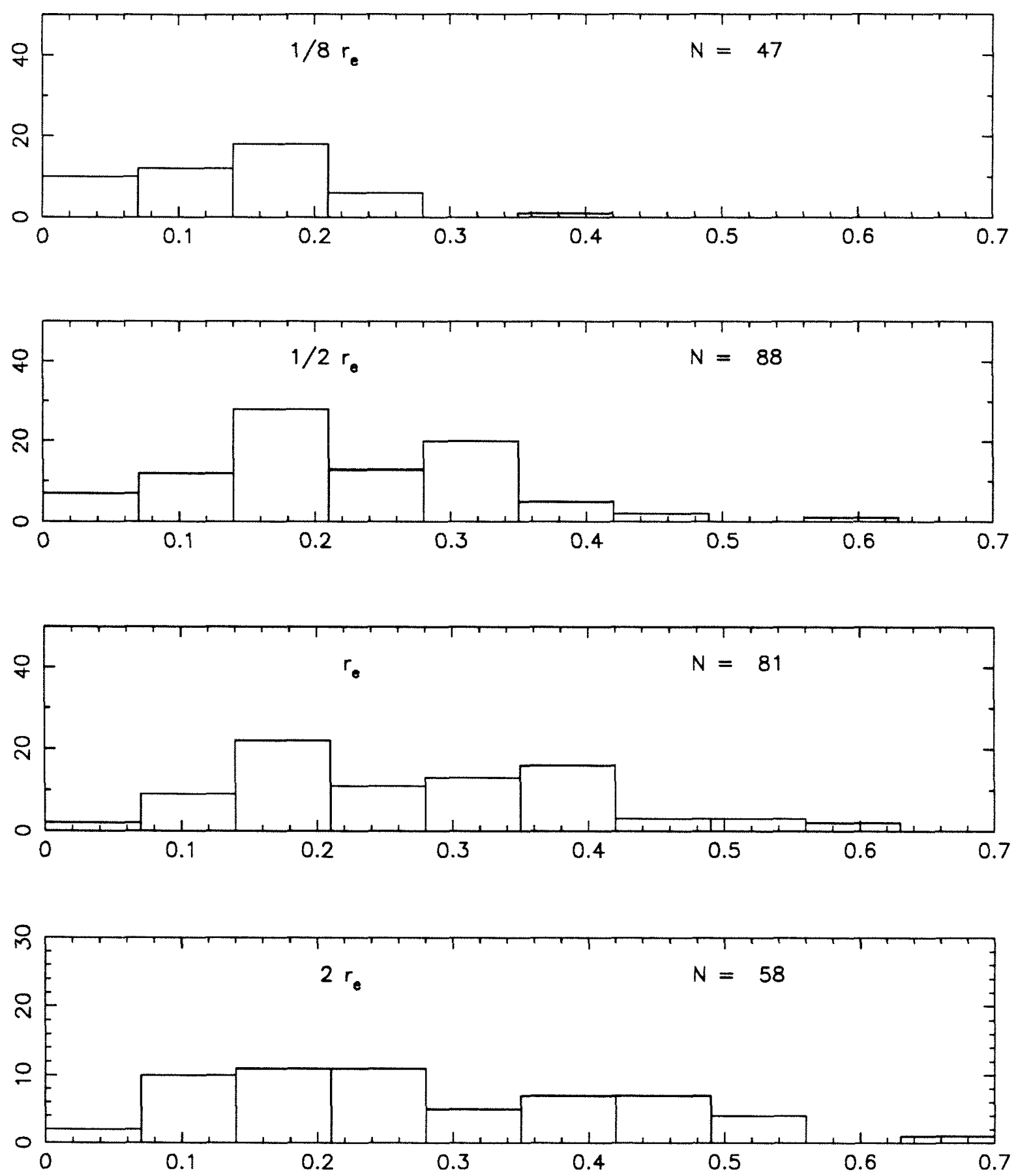

Figure 12. $B C E \in$ histograms. 

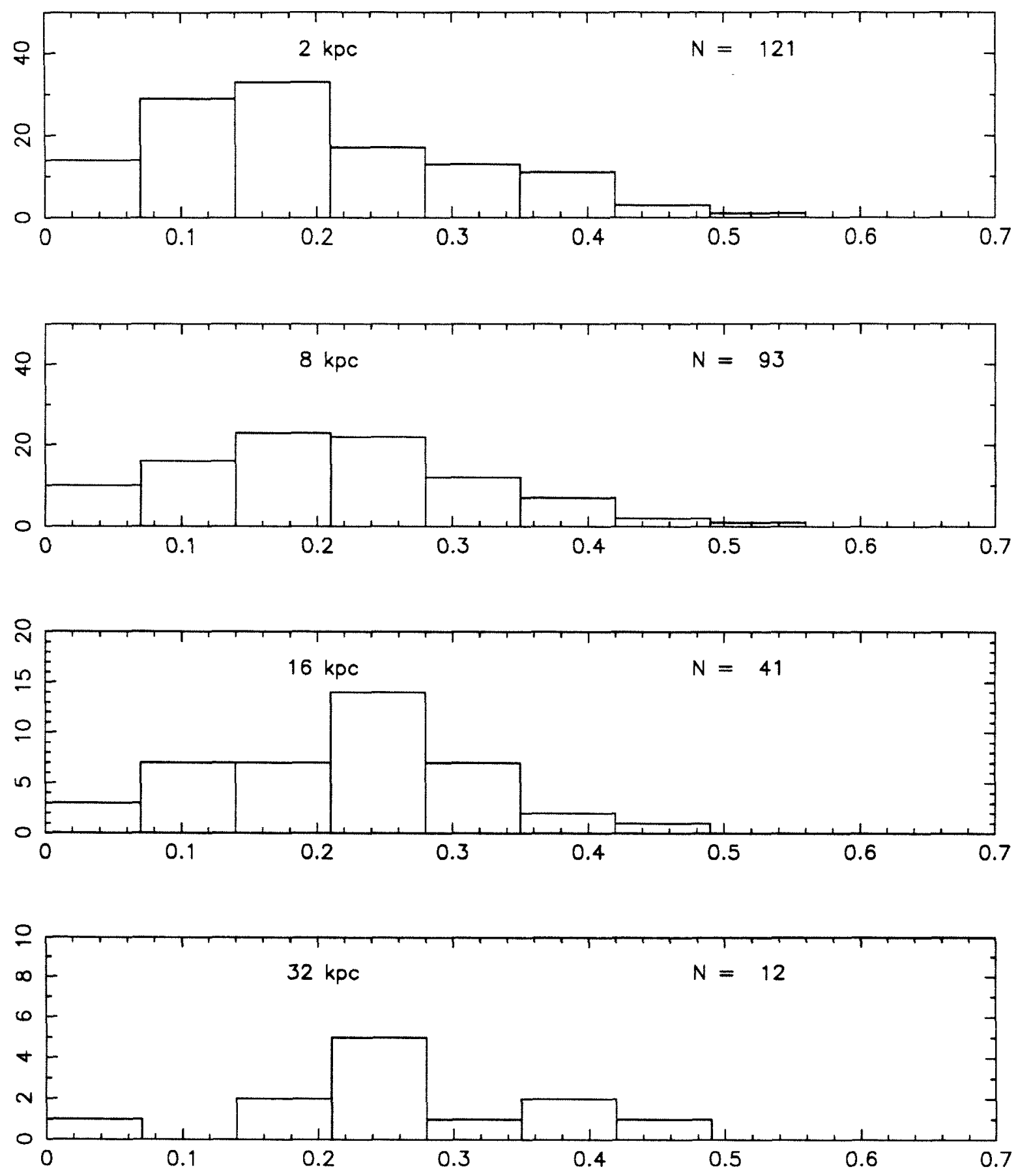

Figure 13. Field $\epsilon$ histograms. 

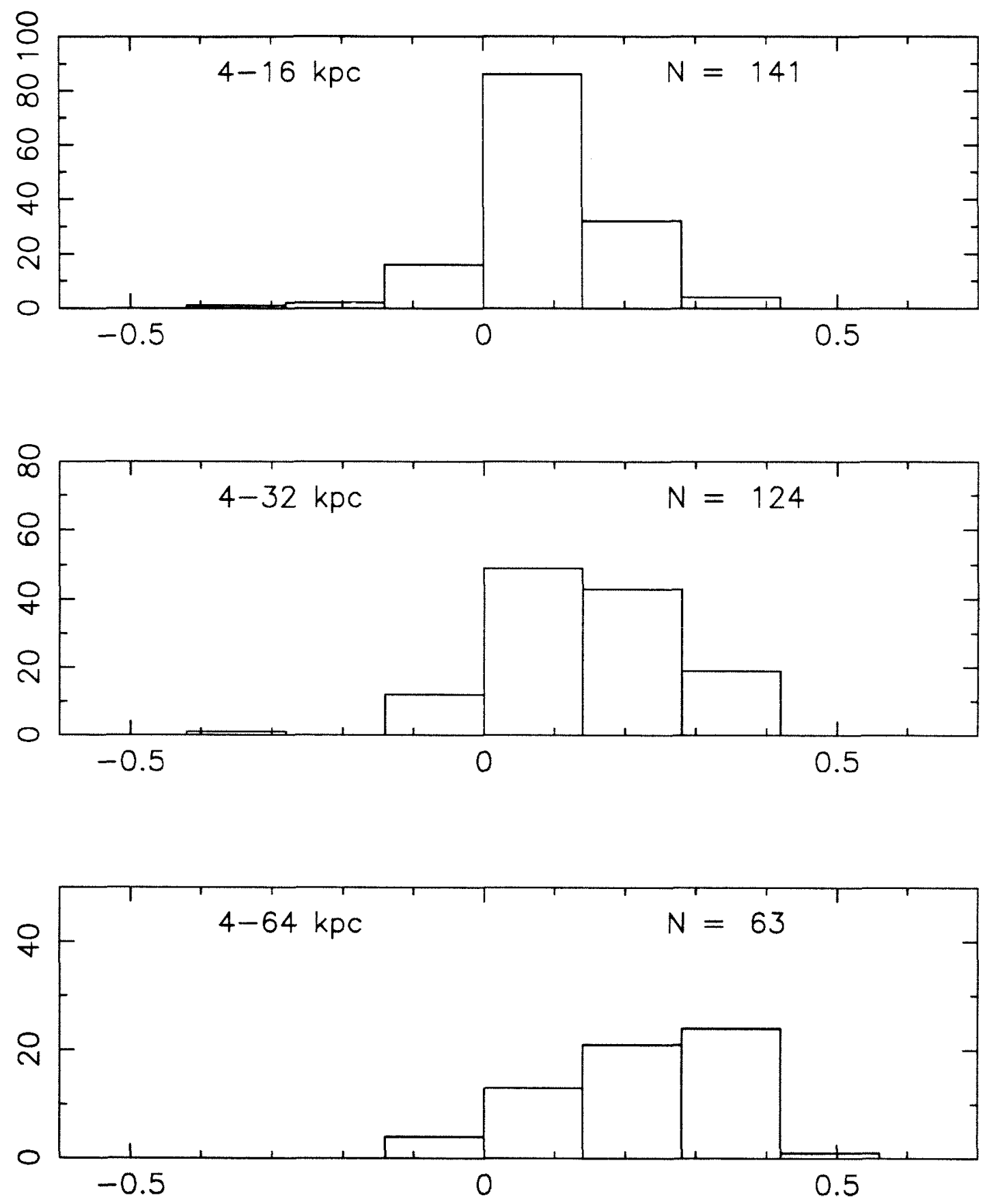

Figure 14. BCE $\triangle e$ histograms. 

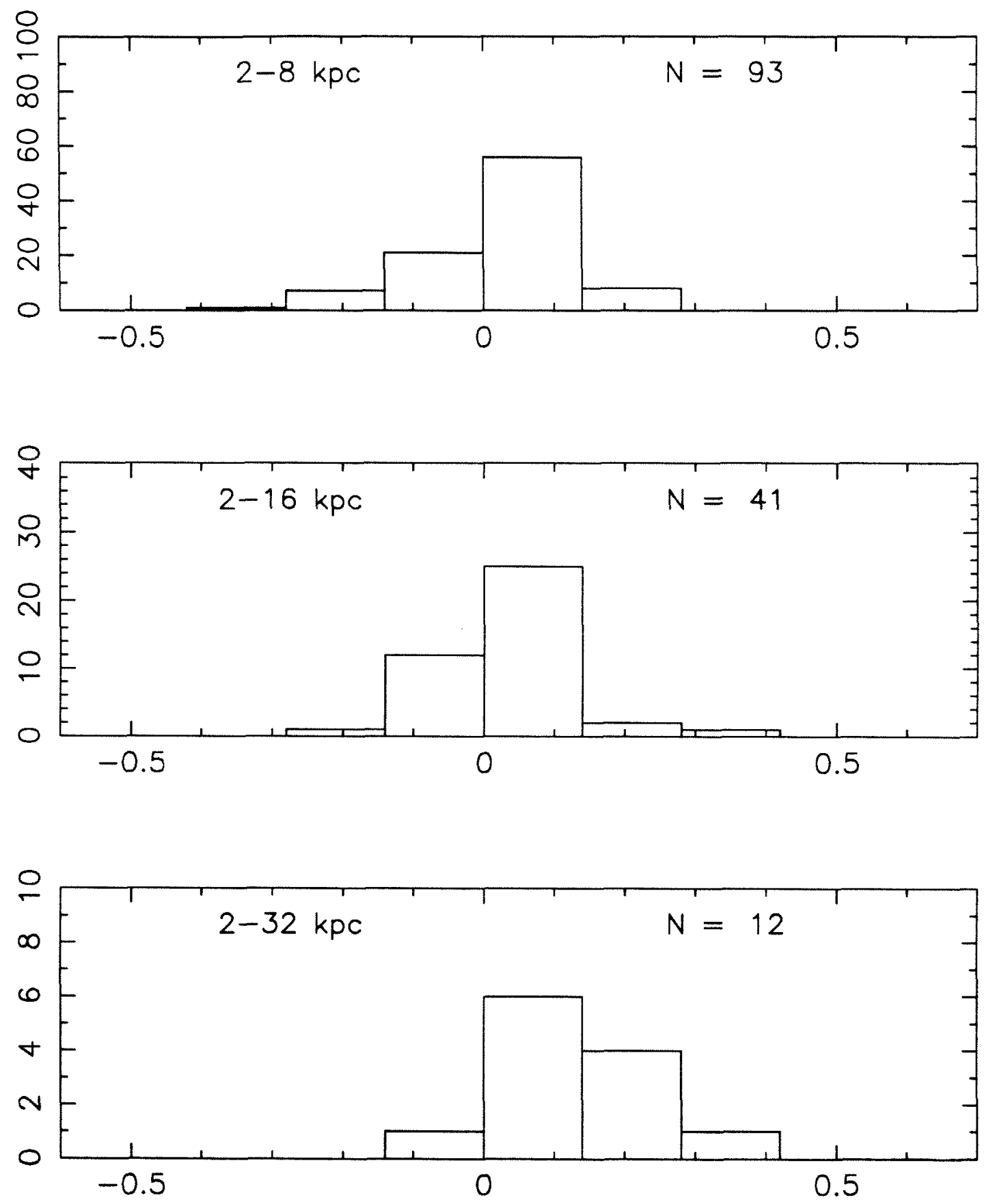

Figure 15. Field $\Delta \epsilon$ histograms. 

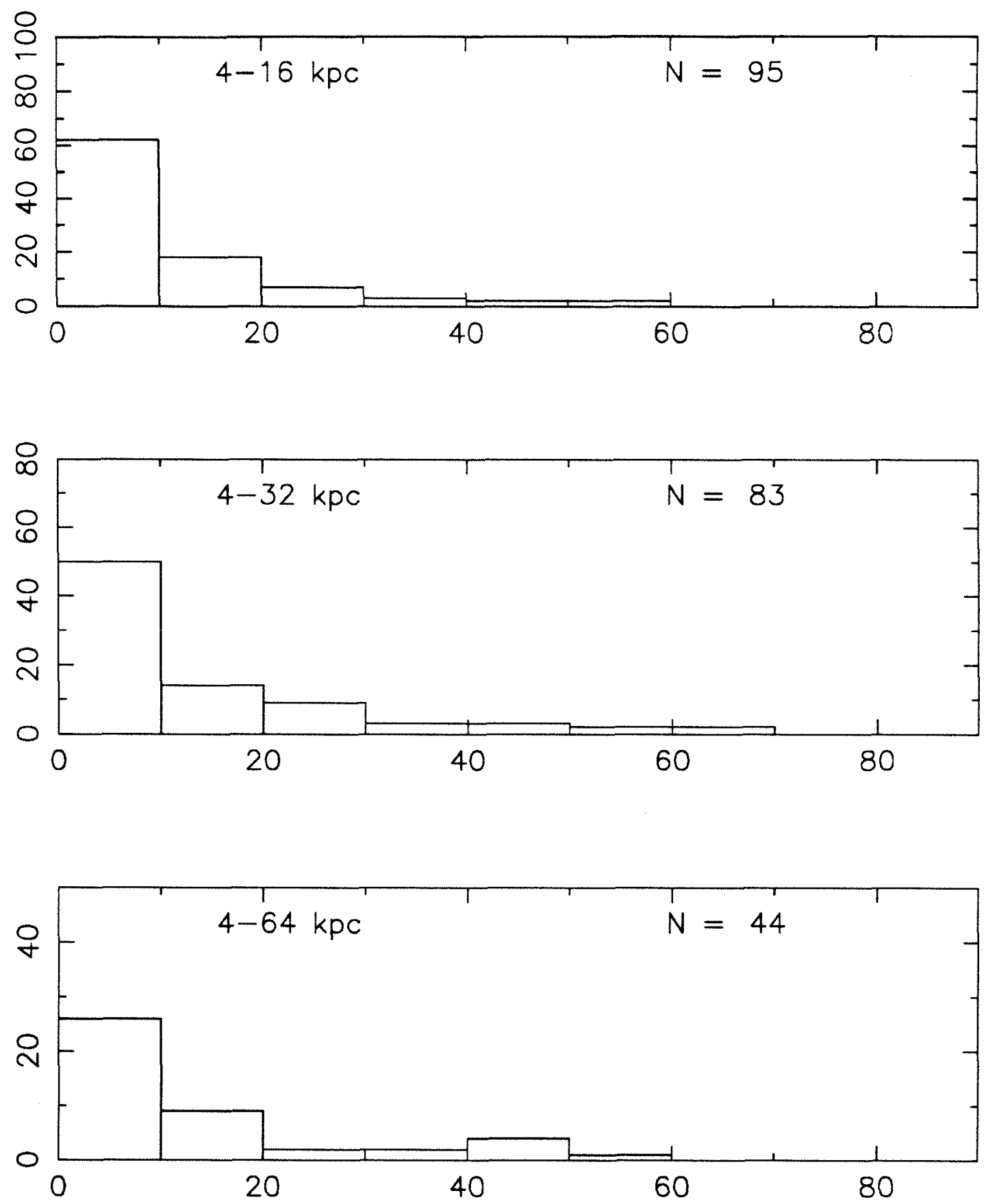

Figure 16. BCE PA twist histograms. 

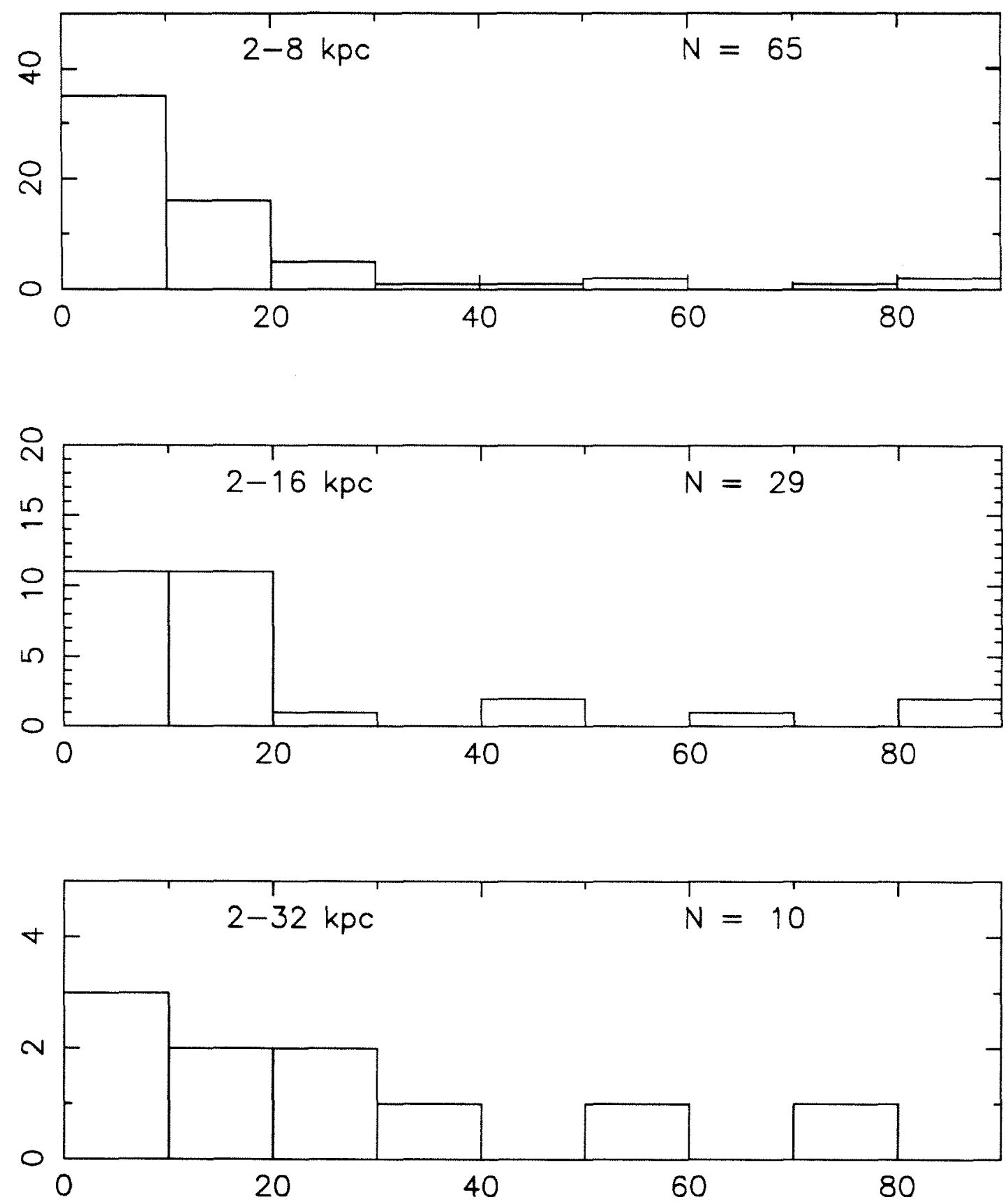

Figure 17. Field PA twist histograms. 
$-101-$

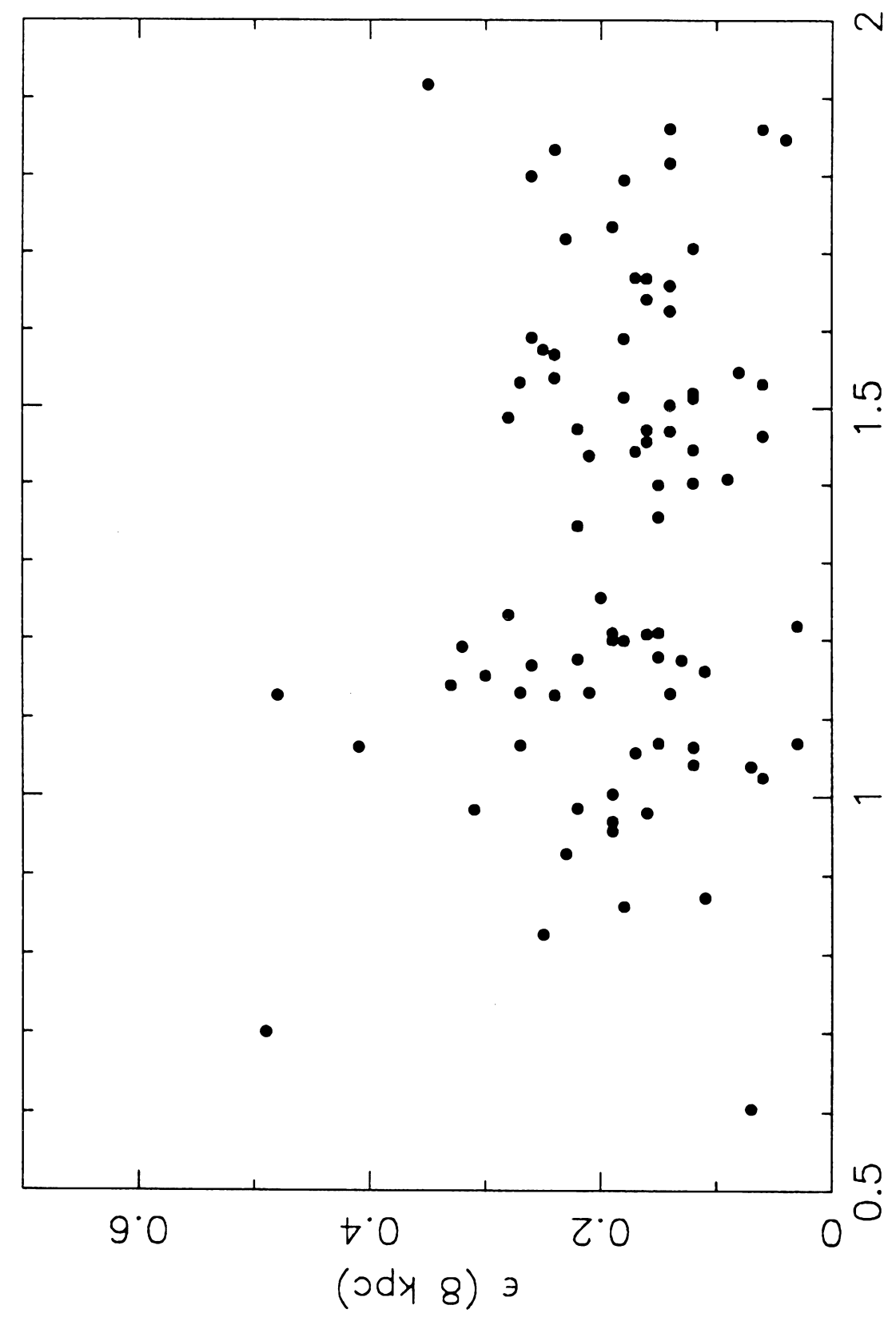




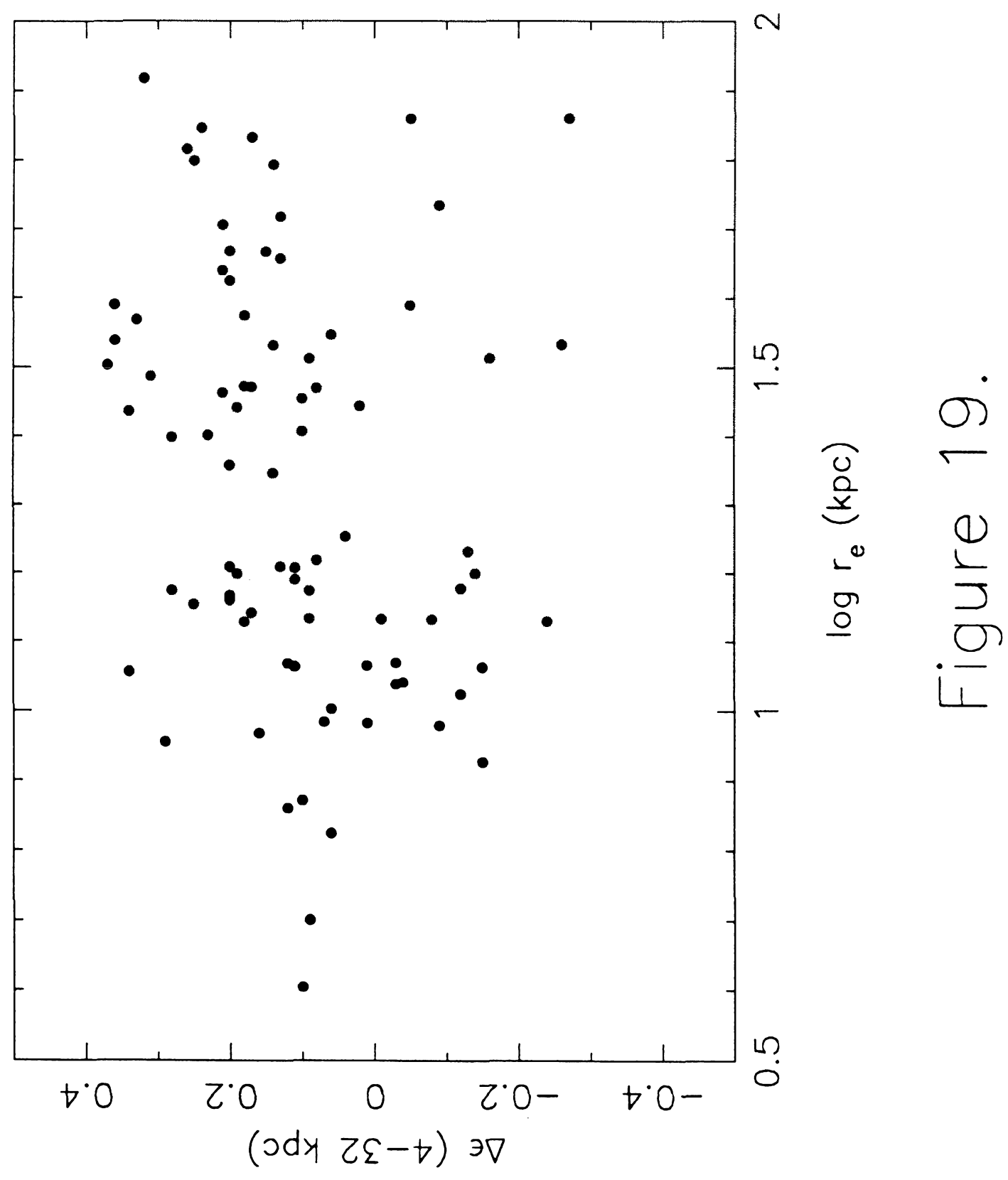




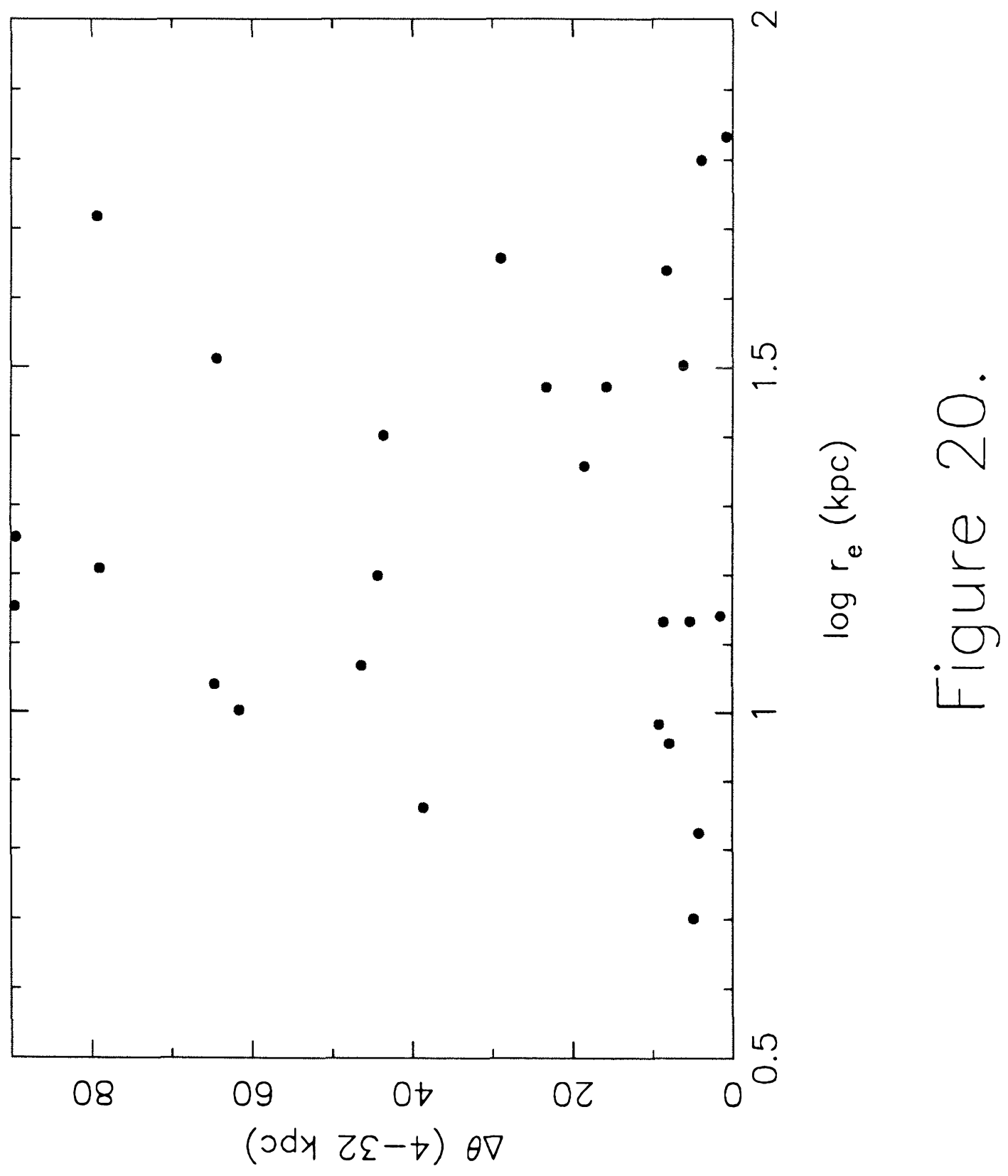



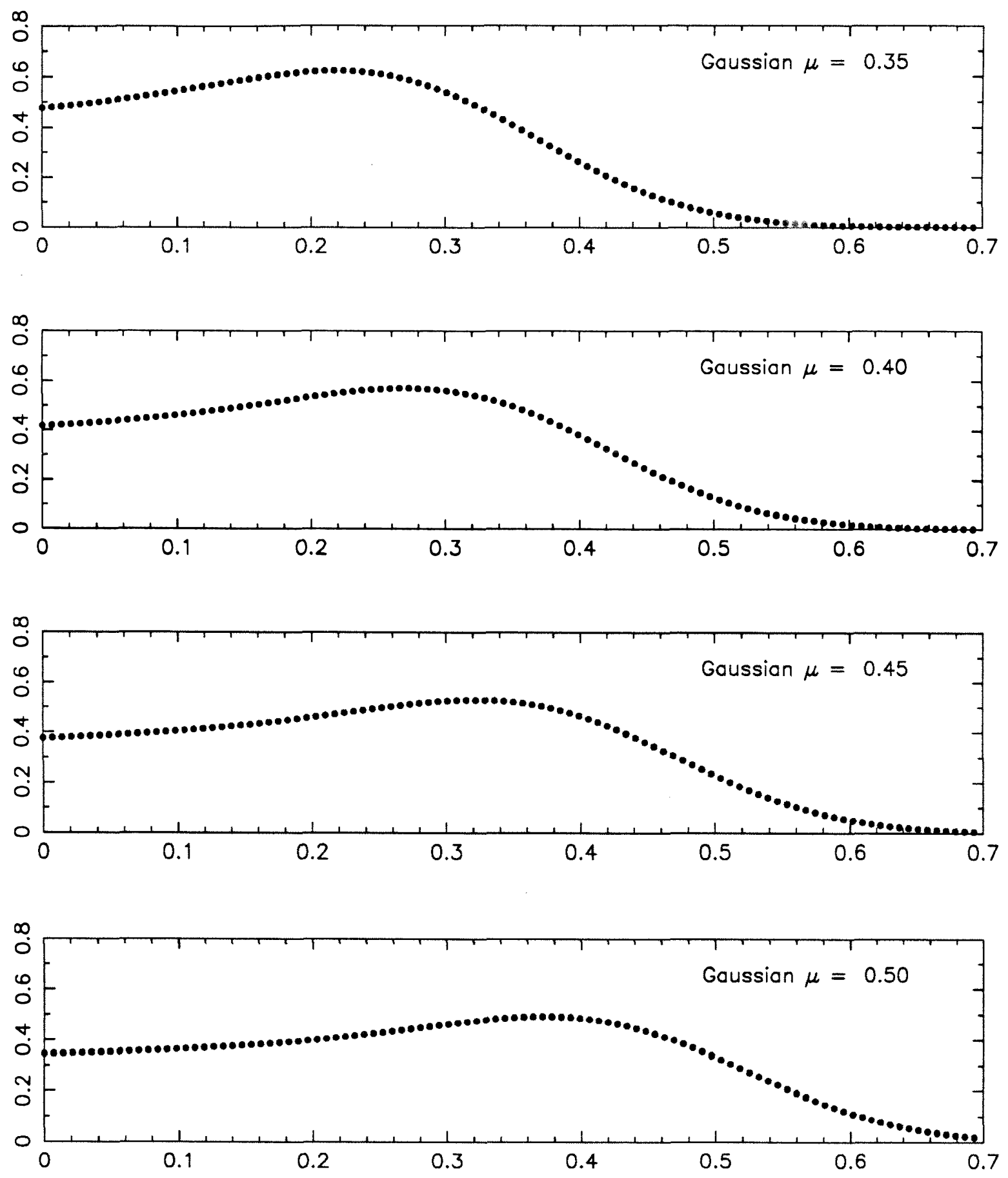

Figure 21. $n(\epsilon)$, oblate spheroids. 

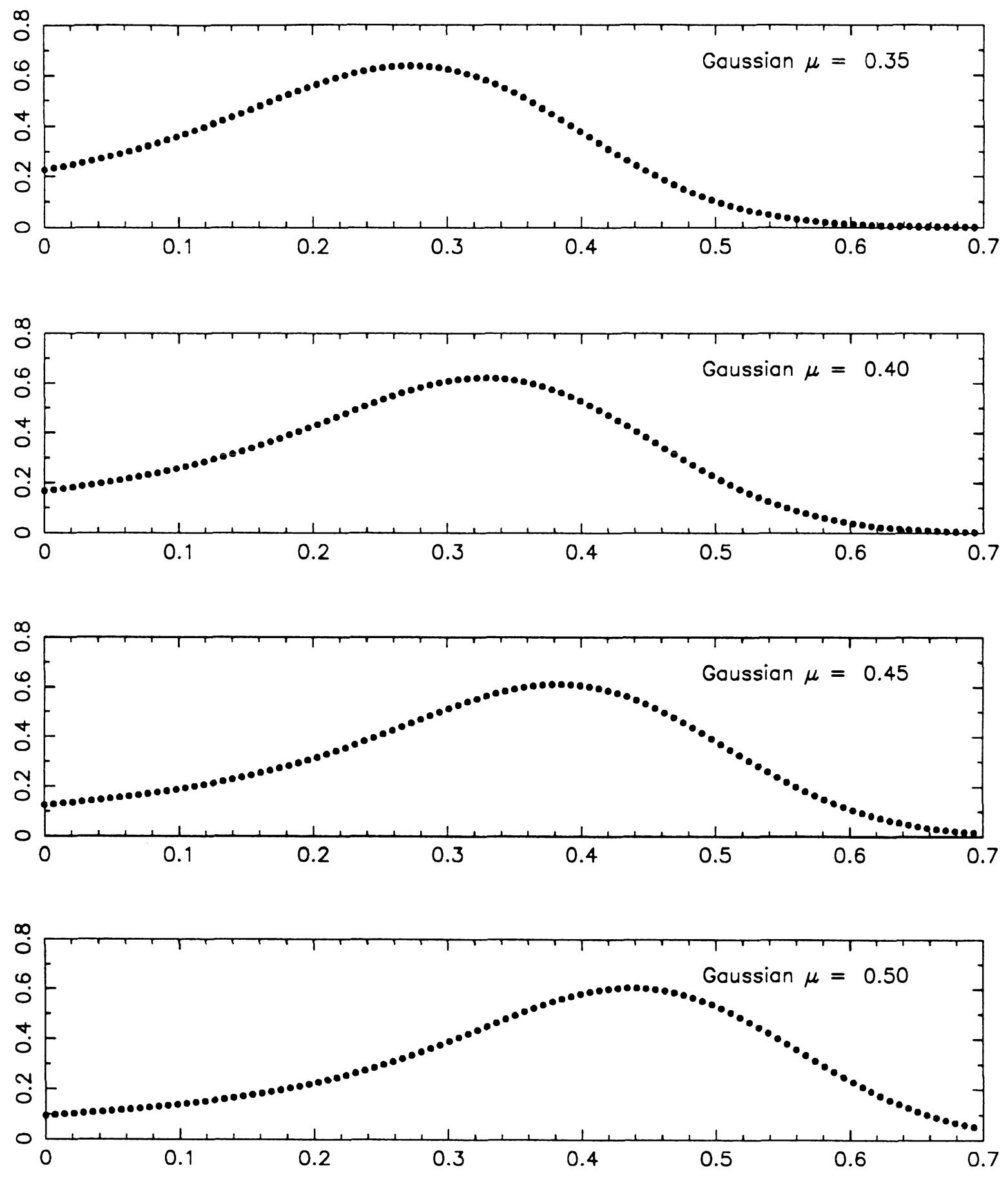

Figure 21. $n(\epsilon)$, prolate spheroids. 


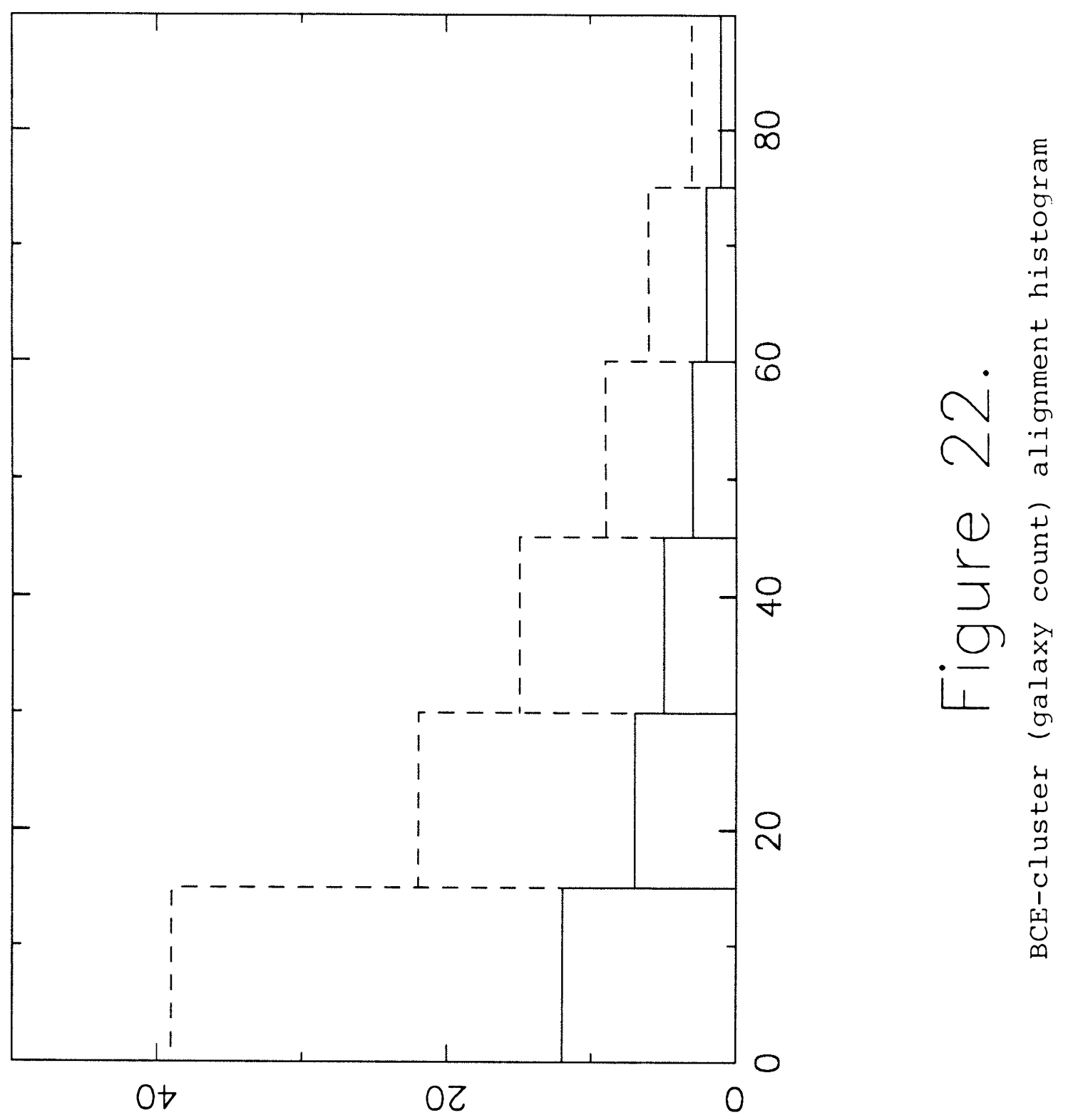




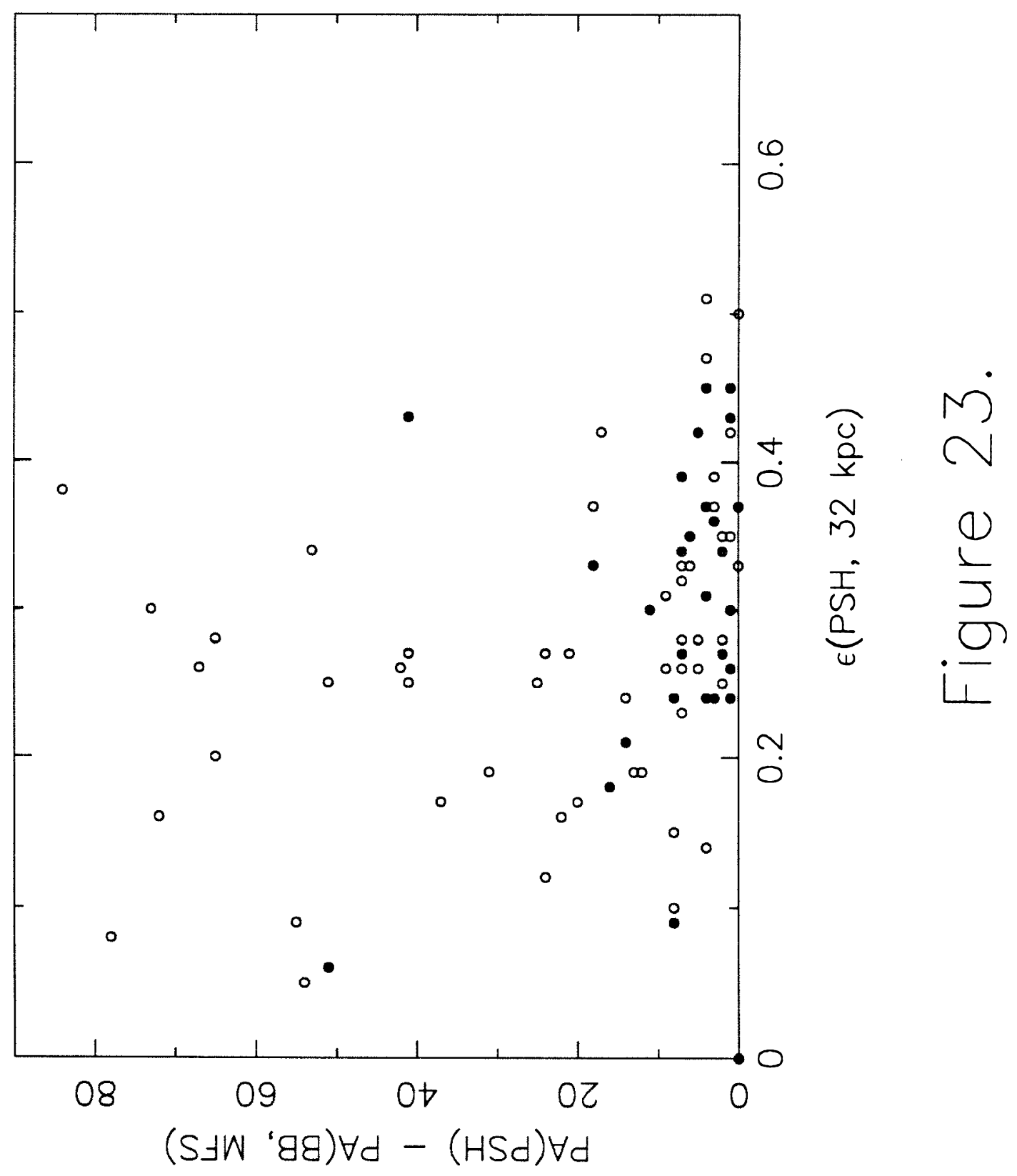




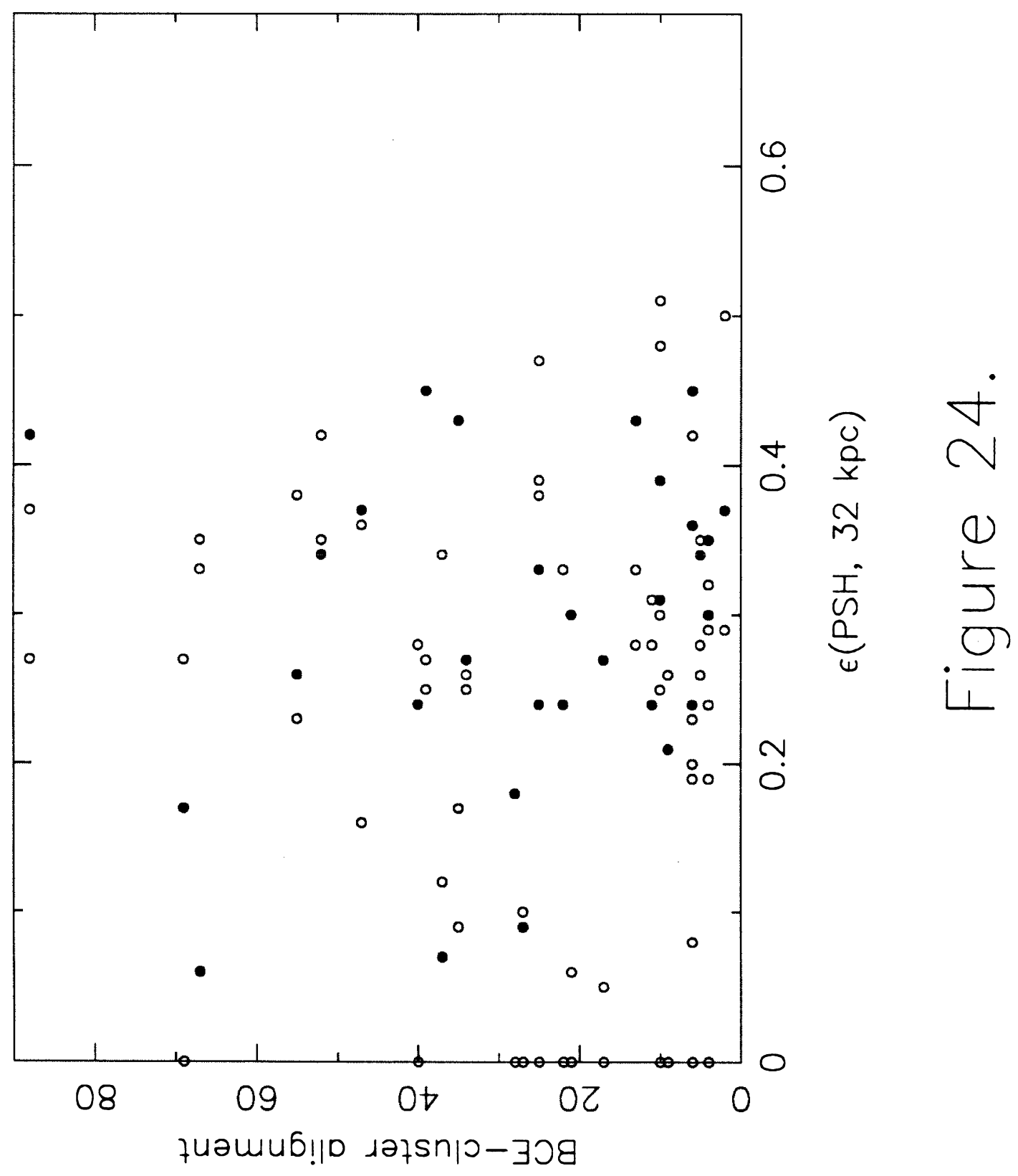




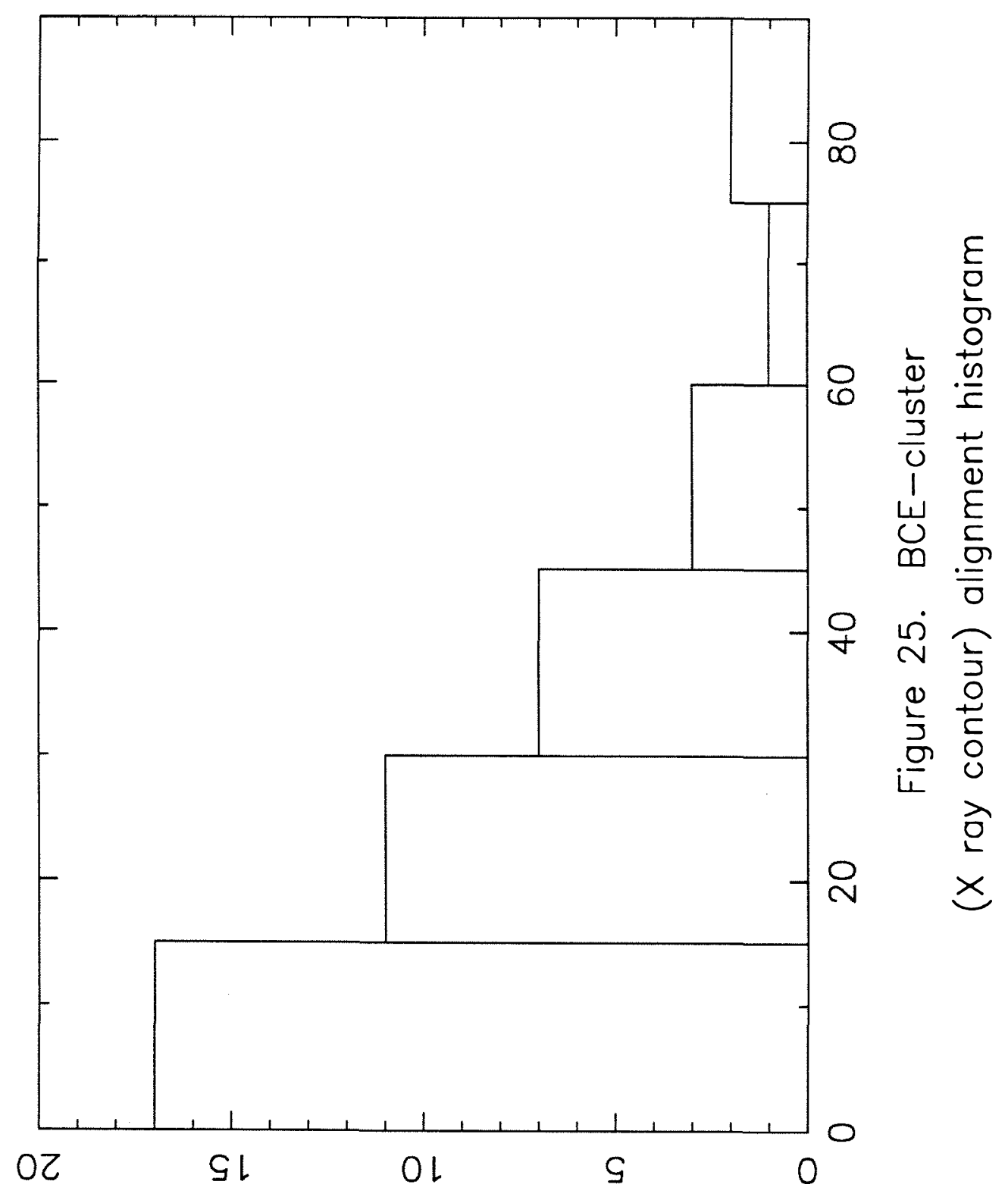




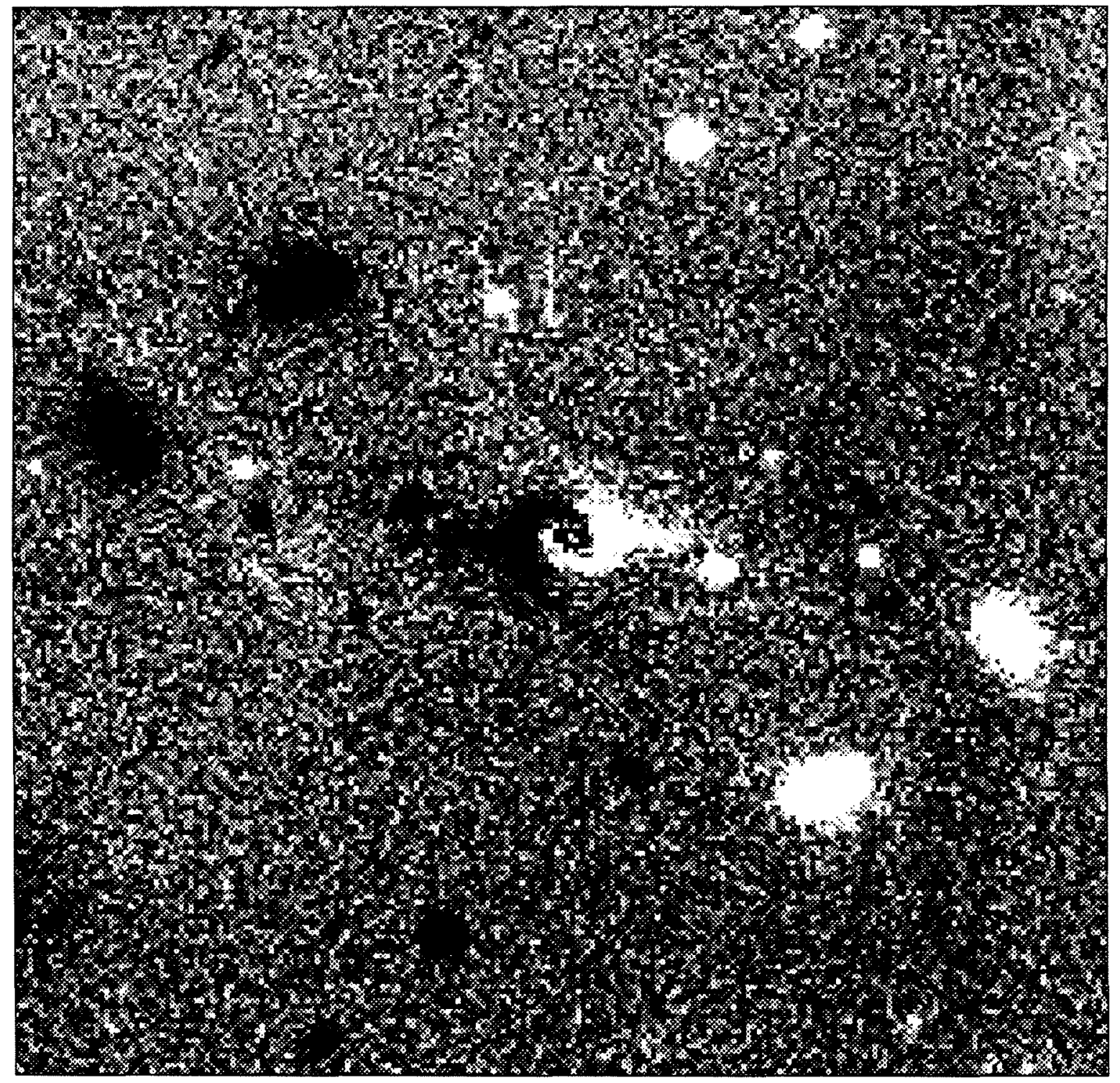

'Figure 26. A957' 


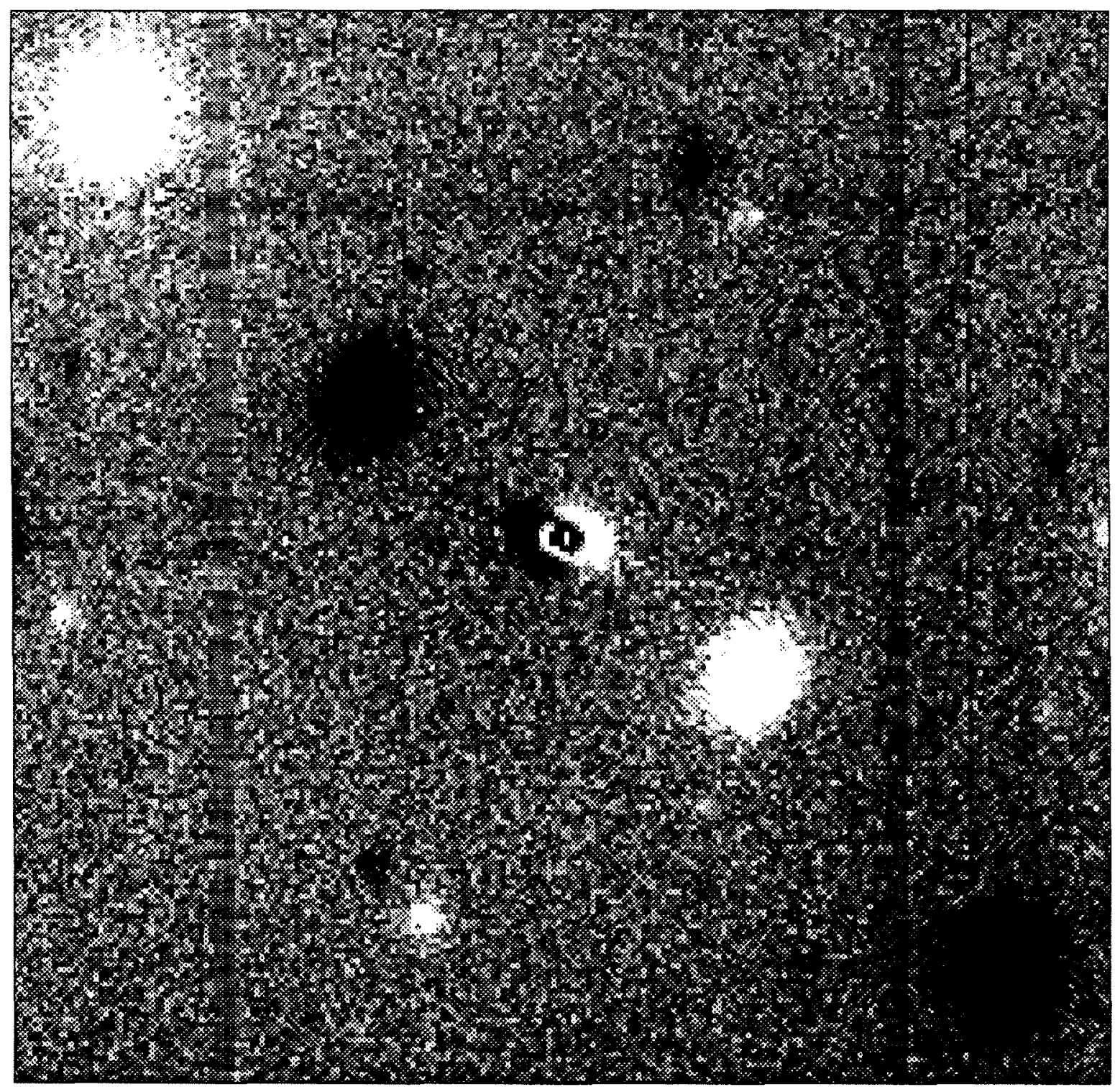

'Figure 26. A1377' 
$-112-$

CHAPTER II

\title{
ISOPHOTOMETRY OF cD HALOS
}

\author{
Alain Porter \\ to be submitted to The Astronomical Journal
}


$-113-$

Things that never had names before are now easily described.

It makes conversation easy.

David Byrne 


\begin{abstract}
A cD halo is that part of a giant (D) elliptical galaxy outside a relatively abrupt increase in the slope of its surface brightness profile. Isophotometry of $23 \mathrm{cD}$ galaxies from the samples of Schombert (1988) and Porter, Schneider, and Hoessel (1988; chapter 1 of this thesis) shows that the sharpness of onset of the halos of galaxies which have been called $\mathrm{cDs}$ varies considerably. Almost all halos are associated with increases in the ellipticity profile, which are sometimes more prominent than the break in the surface brightness profile. Breaks in the position angle profile and centroid displacements (nonconcentric light) are also commonly associated with halos. This strengthens the argument that $\mathrm{cD}$ halos are dynamically distinct components of a galaxy's light distribution. The largest, lowest surface brightness halos may have a primordial origin, while some smaller ones may be fed by tidal stripping, merger events, and gas accretion. A qualitative difference in the origin of different halos is also suggested by the fact that many of those with higher surface brightness steepen again at large radii. The presence of an inflection in the surface brightness associated with slope breaks in the shape profiles is at present the most useful definition of a $\mathrm{cD}$ halo, but it should be exercised with caution. NGC 5044 is an undisputable $\mathrm{cD}$ galaxy in a poor group.
\end{abstract}




\section{INTRODUCTION}

Once upon a time, elliptical galaxies contained no dust. Hubble had practically defined them this way (1936), and therefore, for many years, any elliptical that grew spots was banished to the realm of the S0s (cf. Schweizer 1987). But as astronomers became sharper and sharper eyed (often by unsharp masking) and saw more and more S0s, they began to fear that soon there would be no ellipticals left. Eventually they realized that a little dust could be an integral and interesting a property of an elliptical galaxy (Hawarden et al. 1981).

The above fable is of an originally useful (Humason, Mayall, and Sandage 1956) classification scheme that had to be relaxed when observational techniques improved to the point that it became unprofitably restrictive. This paper deals with a set of objects whose original classification has been too relaxed: the $\mathrm{cD}$ galaxies. They have traditionally been viewed as hybrids of giant elliptical galaxies and rich clusters of the same. This view dates from the first description of Matthews, Morgan, and Schmidt (1964) of them as "dominating, large, centrally located [galaxies]." The impression was strengthened by the deep photometry of Oemler (1976), who discovered that the most luminous galaxies in the richest clusters have envelopes of light in clear excess of the surface brightness profile that would have been extrapolated from the inner regions. There has even been speculation that the final evolutionary state of a rich cluster of galaxies is a giant galaxy which has consumed all of the gas and other galaxies that once surrounded it.

In the past 20 years, however, confusion has arisen as to the proper definition of a "cD," which galaxies satisfy it, and in what environments they occur. Originally location at the center of a rich cluster was part of the definition, but outlying 
galaxies with halos have been found in the Coma and Hercules clusters (Oemler 1976, Schombert 1988), and Morgan, Kayser, and White (1975) and Albert, White, and Morgan (1977) reported the existence of $\mathrm{cD}$ galaxies in clusters too poor to have been included in the catalog of Abell (1958). Morbey and Morris (1983) and Malumuth and Kirshner (1985) have confirmed the existence of halos around some of these AWM and MKW galaxies, similar to those found by Oemler in richer clusters. These observations have remained controversial, however, being contradicted by Thuan and Romanishin (1981) and Schombert (1988).

Conversely, there are some extremely luminous, distended galaxies in very rich clusters (e.g., A85, A2029) that have often been called cDs but which turn out not to have any obvious break in their surface brightness profiles, as do galaxies like those in A1413 and A2670. The picture was further complicated by a discovery of the Einstein Observatory. Jones and Forman (1984) found that the presence of a "cD galaxy" in a cluster did not imply the presence of a hot, luminous well of X-ray gas (e.g., A2634), nor vice versa (A2256).

Tonry (1987) has recently reviewed the taxonomy and properties of these galaxies. The essence of his classification scheme is to call unusually distended galaxies (those with logarithmic slope $\sim-2.0$ at $\mu_{V}=24$ ) $\mathrm{D}$ galaxies, and to reserve the classification " $\mathrm{cD}$ " for those whose surface brightness profiles show a "break" or an inflection point: an abrupt decrease to lower slope at large radii, implying the existence of two components. Schombert (1988) recently applied this definition in an objective way to study a sample of $\mathrm{cD}$ brightness profiles, focusing on their interaction with the clusters that contain them.

The present paper has two main goals. The first is to find additional clues to the nature and origin of such "excess light" in the shape parameter 
profiles of galaxies that have them. To this end I have adopted the same strict morphological definition as Tonry and Schombert, referring to the inner component (which resembles an E or D galaxy) as the galaxy, and the outer component of "excess" light as the halo. I combine new isophotometry with data from Porter, Schneider, and Hoessel (1988, and chapter 1 of this thesis, henceforth PSH) to examine quantitatively the sharpness of the edges of $\mathrm{cD}$ halos and to search for breaks (changes in slope that occur over less than 0.5 magnitudes of surface brightness) in ellipticity, major-axis position angle, and isophote centroid position profiles that correspond to the edges of halos. I consider what bearing such structure has on models of halo origin.

The second goal is to evaluate the success of the morphological definition of a halo and consider an alternative more closely grounded in cluster dynamics: namely, that a halo is a body of light bound less tightly by any galaxy it envelops than the cluster (or group) it fills. This is not a new concept, but it has not yet been used to define a large sample for detailed study, and its consideration is encouraged by the discovery ( $v$. III below) that some halos have rather ill-defined edges. The sample so defined would consist of the largest and lowest surface brightness halos in the present sample (Schombert's "bright halos"), as well as intracluster light like that in Coma (Hoessel, Melnick, and White 1977) and clusters presently being studied by Selman (1988). I will conclude that while this proposed alternative is interesting, it is, because of observational limitations, not yet practical, and may not be free of its own ambiguities. The morphological definition suffices if used with proper caution.

The observations and reduction methods are briefly reviewed in Section II, having been presented in detail in PSH. The results are presented in Section III, and discussed in Section IV. Possible alternative definitions of halos and models 
of their formation are evaluated in Section V. The conclusions are summarized in Section VI. The values $H_{0}=60 \mathrm{~km} \mathrm{sec}^{-1} \mathrm{kpc}^{-1}$ and $q_{0}=\frac{1}{2}$ are used throughout.

\section{OBSERVATIONS AND REDUCTIONS}

A list of the galaxies in the present study is presented in Table 1. All are brightest cluster ellipticals unless otherwise noted. All the images studied were taken in the Thuan-Gunn (1976) $r$ passband. Most were taken with one of 2 CCD cameras on the Palomar 1.5-meter reflector. The new images for this study were taken in 1984 and 1985 with the TI 800 x 800 chip normally used in the blue camera of the Double Spectrograph on the Hale 5-meter reflector. It was coupled to reimaging optics giving an image scale of $0 \prime^{\prime} 657$ pixel $^{-1}$ and a field of view of $9^{\prime}$. To improve the flatness of the images and guard against scattered light in the optics, multiple exposures of some of the galaxies were taken, with the nucleus centered in different quadrants in the different exposures. These "dodged" frames were aligned and coadded before reduction. Since the exposures were consecutive, no rotation was necessary to align individual frames. Galaxies known to have faint but very extended halos were preferentially observed. It should be stressed that these images are not well calibrated photometrically. The intensity zero points are probably good to 0.5 magnitude, but not 0.1 magnitude. Since the primary goal of the study was to study shape parameters, this was not regarded as a serious problem.

This sample was extended by borrowing frames of $\mathrm{cDs}$ with relatively bright halos from the sample of PSH. Choices for the extension included galaxies in the sample of Schombert (1988) for which PSH had good images and other galaxies in their sample that showed evidence of halos in their surface brightness profiles as a 
function of $r=\sqrt{a b}$, where $\mathrm{a}$ and $\mathrm{b}$ are the major and minor axes of an isophote. This criterion was made because of the fact that most past surface photometry of ellipticals has been one-dimensional. These are pictures taken with a $500 \times 500$ TI CCD and reimaging optics that gave a scale of $0{ }^{\prime \prime} 548$ pixel $^{-1}$. These data are described most fully in Hoessel and Schneider (1985). Most of these were also taken on the Palomar 1.5-meter telecope, but one (A1904) was taken with the 5-meter at an image scale of $0 . \prime 415$ pixel $^{-1}$. The resulting combined sample is not complete in any sense but is the first isophotometric study specifically of $\mathrm{cD}$ halos.

The image of NGC 6034, a cD in a subcluster of Hercules, was kindly taken by S. Kulkarni with the 4-Shooter (Gunn et al. 1987) on the Hale 5-meter reflector.

The images were reduced in exactly the same way as in the study of PSH: first, all blemishes, stars, and galaxies other than the $\mathrm{cD}$ were removed from the image by interpolation across a mask from the neighboring pixels, and second, ellipses were fit to the isophotes of the cleaned galaxy at intervals of 0.25 magnitude in surface brightness. A complete discussion of this reduction process, its capabilities, and its limitations is given in that paper. The images of A400 and A779, which have bright nearby companions, were cleaned of those companions using the analytic method of Porter (1988, chapter 3 of this thesis).

\section{RESULTS}

In Table 1 the objects studied are identified by Abell cluster number in the first column. The optical system used is given in column 2, 1 standing for the 800 x $800 \mathrm{TI}, 2$ for 1.5 -meter $500 \times 500 \mathrm{TI}$ data from PSH, 3 for 5 -meter $500 \times 500$ data, and 4 for the 4-Shooter image. Column 3 lists the exposure time in seconds. In column 4 the number of individual frames coadded is given, with a " $\mathrm{d}$ " added if 
the frames were dodged as described above. Column 5 gives the seeing FWHM in the coadded image. Column 6 lists the major axis in arc seconds at which the halo appears in the surface brightness profile. Columns 7, 8, and 9 indicate whether there are breaks at this position in the ellipticity, position angle, and centroid position profiles, respectively. Columns 10,11 , and 12 indicate, respectively, whether the halo occurs on the major axis, minor axis, and whether it was observed to steepen at large radii.

The results of the ellipse fitting are plotted in Figure 1. The six panels on each page of the figure show smoothed first derivatives of the shape profiles of the galaxy named at the bottom of that page. To obtain these plots, the raw surface brightness was first smoothed as described in Section III of PSH. The value of the derivative at a given isophote was then calculated using that isophote and its outer neighbor. The smoothing was done to minimize noise in the data, so that the structure remaining visible is real. The observed scatter in a quantity may then be accepted as intrinsic and used to gauge the significance of a break or bend. A detailed discussion of uncertainties in the fitted isophote parameters is given in PSH.

The abscissae of all six panels of Figure 1 are $\mu_{r}$, the Gunn $r$ surface brightness in magnitudes per square arc second. The top two panels show the logarithmic slope of the surface brightness profile along the major and minor axes. As expected, all the galaxies in this sample are $\mathrm{D}$ galaxies. The slopes plotted may be compared directly to the $\beta$ values in Schombert (1988); the agreement is good except for A779, whose halo is very weak, and for A2147.

The lower four panels show the derivatives of isophote ellipticity, major axis position angle, and centroid in right ascension and declination, taken with respect to 
$\mu_{r}$. Use of surface brightness instead of radius as the reference variable is a matter of convention and is done for consistency with PSH. The important point is that plotting derivatives highlights the local changes and breaks that are masked by the global structure of the galaxy in conventional plots.

A comment on the detectability of centroid displacements is in order. At the centers of galaxies these are masked, partly by seeing, but mostly by even small tracking errors. At large radii, where a galaxy's surface brightness is only a fraction of the sky background and its slope is very low, the uncertainty in the centroid position grows very large (approximately as $\left.\left(\Delta \mu_{r}\right)^{2}\right)$, and even moderate amounts of nonconcentric light could not be detected. Thus there is a very strong bias, which I have not attempted to model, against discovery of centroid displacements outside a narrow range of radii, and this should be kept in mind when interpreting the results below.

In Figures 2 and 3, images of NGC 5044 and A1767, two particularly interesting members of the sample, are reproduced. When a galaxy was also observed by PSH, their photometry is compared to ours in Figure 4. The format of this figure is identical to that of Figure 1 of PSH, except that the ellipticity, eccentricity, major-axis angle, centroid positions, and fit error are plotted as functions of $\log b$ to facilitate comparison in case of zero point offsets and sky subtraction errors in the photometric calibrations.

Notes on individual galaxies follow and are themselves followed by some more general observations. 
This cluster is problematical in several ways: the central "galaxy" is a dumbbell system, a close, almost equal pair, making detection of any halo suspect, and it is 1.5 northeast of the X-ray center of the cluster (Forman and Jones, in preparation). Nevertheless Schombert's (1986) strong halo signal has been confirmed in the analytic decomposition of the two galaxy profiles by Porter (1988, chapter 3 of this thesis). Further discussion of this system may be found in that paper.

\section{A496}

The $800 \times 800$ image covers about a magnitude of the halo of this galaxy. The halo is a major-axis feature and is close to a sharp increase in the ellipticity (though not as close as in most other $\mathrm{cDs}$ ). The position angle remains relatively stable. It is within about $30^{\circ}$ of alignment with the X-ray isophotes (Forman and Jones, in preparation) but almost at right angles to the galaxy distribution in the cluster, according to Binggeli (1982). The photometry of PSH suggested that the outer isophotes (in the region 30" $<a<50^{\prime \prime}$ ) were displaced several arc seconds to the north, and the present photometry confirms this. The quality of the ellipse fits to those isophotes is poor, but that is due partly to their boxiness. The nonconcentricity is clearly visible out to $50^{\prime \prime}$ if the image is antisymmetrized about the galaxy nucleus (i.e., the picture is rotated $180^{\circ}$ and subtracted from itself). Outside this radius, on the actual halo, the centroid errors are larger, and the offset is not significant.

\section{A779}

This system, also separated into its two components by Porter (1988), has one of the weakest halos in the sample. The break, at $\mu_{r}=20$, seems well defined, 
especially on the major axis, but the outer part of the profile steepens again, and there is no ellipticity jump at the surface brightness break.

\section{A858}

The only significant break in the profiles is at $\mu_{r}=21.5$, where there are sharp decreases in the profile slope and ellipticity, and a northward centroid displacement. Antisymmetrization of the image shows that this latter is due to a small patch of excess light there. The inner part of the galaxy is itself rich in structure, showing a rapid ellipticity increase and isophote twisting.

\section{$\mathbf{A 9 7 8}$}

The very small ellipticity of this galaxy makes evaluation of the majoraxis position angle profile difficult. The image also has some tracking error, but antisymmetrization suggests that some of the centroid displacement is real.

\section{A1169}

The break in the surface brightness profile consists mostly of a truncation along the minor axis at $\mu_{r}=23$. This is most clearly visible in the ellipticity profile, because the scatter in the major-and minor-axis surface brightness profiles is correlated. The westward extension of the halo is easily visible upon inspection of the image.

\section{$\mathbf{A 1 2 2 4}$}

This is a very distant cluster: $\mathrm{z}=0.29$. The halo appears to be real, but a deeper image is needed to improve the quality of the isophotometry.

\section{$\mathbf{A} 1238$}


This galaxy is closely surrounded by half a dozen companions. The halo begins at $5^{\prime \prime}$, however, and cannot be entirely due to contamination from these galaxies. It is, however, a good candidate for a tidal distension.

\section{A1382, A1383}

These remarkably similar galaxies have bright, compact nuclei off-centered in "halos" with very large ellipticity breaks. The ellipticity of the halo of A1383 shows strong, small scale variations. It is questionable whether galaxies whose "halos" start at such small radii truly deserve to be classified as $\mathrm{cDs}$. One suspects they simply have compact nuclei and disks. The X-ray center of A1382 lies about $2^{\prime}$ east of the BCE.

\section{$\mathbf{A 1 6 3 0}$}

The interior of this galaxy is not smooth, showing instead a great deal of poorly resolved patchy structure.

\section{A1650}

The structural profile of this galaxy shows two breaks, at about $\mu_{r}=21.3$ 22.0 and 23.3-24.0. At the first break, only $5^{\prime \prime}-10^{\prime \prime}$ from the center, the ellipticity, which has been increasing rapidly, first accelerates and then stabilizes at E5.5. This activity is visible as a dimple on the major-axis surface brightness profile, but affects the minor axis very little if at all. At $a=50^{\prime \prime}$, the ellipticity again increases, to E6.5. This change is visible in both the major- and minor-axis profiles. The large centroid drifts are artifacts of a sky gradient in the image.

The second structural break requires confirmation. It would be interesting to learn whether A1650 has a Mpc scale halo like those of A1413 and A2670. 


\section{NGC 4874 (A1656)}

The edge of this halo is marked by an ellipticity increase, but the galaxy then seems to get round again. Baum, Thomsen, and Morgan (1986) made a detailed study of the magnesium gradient in this galaxy. The metallicity shows no strong break at the edge of the halo. They also stated that the ellipticity of the profile continues to increase at fainter levels. In view of the emphasis that has been placed on the great ellipticity of $\mathrm{cD}$ halos and the prevalence of ellipticity increases found in the present work, a deciding observation is in order.

\section{$\mathbf{A 1 7 6 7}$}

The profiles in Figure 1 and the contour map in Figure 2 should be considered side by side. The "halo" begins as a shoulder extending northwest, pulling the centroid about 1 " from the nucleus, but the excess light then shifts to the southeast, and the isophotes become very boxy. These disturbances occupy the region between $21<\mu_{r}<23$. Inside, the isophotes are elongated east-west; outside, north-south. Schombert (1988) found that the disturbed regions are about 0.1 magnitude bluer in $B-V$ than the center. Despite the disturbances, there is no visible companion in the area. It is tempting to speculate that this system is in the final stages of a merger or is somehow being disturbed by the pair of large neighbors $1^{\prime}$ to the south. It would certainly repay dynamical study.

\section{$\mathbf{A 1 7 9 5}$}

A1795 attracted attention recently when Heckman (1981) discovered a large amount of cooling gas in its central regions. The present image, from PSH, shows a richly structured object. The halo begins fairly close to the center, at $a=8^{\prime \prime}$, and is associated with breaks in the ellipticity and major-axis angle profiles and a drift 
of the centroid $2^{\prime \prime}$ to the east. The reality of this structure has been confirmed by a model subtraction (Porter, Schneider, and Hoessel, in preparation), and some of it is due to patches of light coincident with the emission-line regions observed by van Bruegel, Heckman, and Miley (1984). It steepens again quickly, although the slope remains more than shallow enough for the galaxy to be called a D.

\section{A1809}

The identification of this $\mathrm{cD}$ is somewhat complicated by the presence nearby of a bright and unusual foreground galaxy, NGC 5338. This is the galaxy identified as the $\mathrm{cD}$ in this cluster by Schombert (1988), but unpublished spectroscopy (Porter, in preparation) shows that it has zero redshift emission and is probably a foreground So.

The brightness profile of the real $\mathrm{A} 1809 \mathrm{cD}$ resembles that of $\mathrm{A} 1650$. It shows a bump of finite extent, mostly on the major axis, just above $\mu_{r}=24$ $\left(a=40^{\prime \prime}\right)$, rather than an indefinitely extended envelope. Between the center (at $\mu_{r}=19.5$ ) and this bump, the isophotes grow steadily flatter and rotate at a constant rate through $25^{\circ}$. They also show a significant centroid drift of $1 . \prime 5$ to the northeast. The luminosity bump is associated with a sudden change in the shape parameters. The ellipticity increases, the isophotes rotate back to their central PA, the centroid drift stops, and the fit error increases suddenly, suggesting, since there is no obvious contamination from large companions, that the isophotes are now significantly nonelliptical.

The panel of Figure 4 showing A1809 is particularly interesting. The isophote fits from this work are shown as solid circles, and those from PSH as asterisks. First of all, there is a significant discrepancy in the ellipticities of the isophotes in the 
inner $5^{\prime \prime}$. This is caused by the superior seeing in the image of PSH: 1"'1, as opposed to $2 ! 5$ in the $800 \times 800$ picture. This also causes a visible difference in the surface brightness profiles. As part of their seeing tests, PSH blurred their image of A1809 with 1" and 2" Gaussians and reproduced the ellipticity profile of this paper almost exactly.

Other points to note in Figure 4 are that the bump in the surface brightness profile is reproduced despite a clear discrepancy in the sky level subtraction and that the reversal in the isophote twist at large radii is also well reproduced. This makes it more credible than the reversal of the centroid shift, which is masked by scatter.

\section{A1921}

This galaxy has considerable central structure and a very small inner halo radius, which makes its classification as a $\mathrm{cD}$ suspect. If the halo is real, it is unusual in becoming rounder at large radii.

\section{A2029}

This galaxy is included in Figure 1 as an example of a luminous D galaxy that is clearly not a $\mathrm{cD}$ by the present definition. Note the steady decrease of the profile slope to below -2. Despite this, the galaxy still dominates the cluster: it is the galaxy in which Dressler (1979) found a radially increasing velocity dispersion profile, and the small cluster members line up visibly along the major axis.

\section{A2065}

The classification of this galaxy as a $\mathrm{cD}$ is suspect for two reasons. First, its ellipticity profile decreases at large radii, since the "halo," the increase in profile slope, is on the minor axis. This makes it unlike almost all other first-ranked cluster 
ellipticals (PSH). Furthermore, the X-ray center of the cluster lies $1^{\prime}$ to the east of this galaxy, near 2 smaller ellipticals.

\section{A2142}

This galaxy does appear to have a well-defined halo whose onset is not contaminated by the companion $27 "$ away along the major axis, for the brightness profile bends, and the strong inner isophote rotation stops, at $6^{\prime \prime}$. There is another bright galaxy $1: 8$ away in position angle $300^{\circ}$, within $10^{\circ}$ of the halo axis.

\section{A2147}

This galaxy has a clear major-axis halo associated with a marked ellipticity increase outside $a=40^{\prime \prime}$. The fit errors are somewhat large, but the ellipticity increase is confirmed by Malumuth and Kirshner (1985). They observed an isophote twist of $15^{\circ}$, but the present data do not confirm it.

\section{A2151}

NGC 6034 is a celebrated noncentral cD galaxy (Schombert 1986, 1987). Figure 1 shows that the onset of the halo (at $\left.\mathrm{a}=20^{\prime \prime}\right)$ is extremely gentle in the major-axis surface brightness profile. However, there is a fairly sharp decrease in the minor-axis profile slope at $\mu_{r}=22.5$, which is closely associated with an ellipticity increase and westward centroid offset. Curiously, a relatively strong isophote twist ( $20^{\circ}$ over 5 magnitudes) continues smoothly across this region. The extreme flatness of this halo is reflected in the difference between the logarithmic slopes of the profile along the major and minor axes: -1.2 and -1.9. The isophotes with major axes greater than about $25^{\prime \prime}$ appear to be boxy, especially on the east side. Study of the halo at fainter levels is unfortunately prevented by a bright star $40^{\prime \prime}$ south of the nucleus. 
A2420

The ellipticity profile of this galaxy increases more rapidly outside $20^{\prime \prime}$ than within that radius, and the change is manifested as a major-axis halo. It is rather

a faint one; Schombert (1988) found it has only $\frac{1}{3}$ the luminosity of the underlying galaxy. The centroid offset appears to be real; at any rate, a broad, low surface brightness dipole residual is visible when the image is antisymmetrized about the $\mathrm{BCE}$ center. This could easily be confirmed with a deeper exposure.

\section{NGC 5044 (WP23)}

This galaxy is a member of a sparse group near the Virgo cluster. It is not particularly well known but was found by the Einstein Observatory to be an unusually luminous X-ray source. Unpublished photometry by Porter and Jones revealed that it has an extended halo, and spectroscopy by Porter (in preparation) shows it to have strong $\mathrm{H} \alpha$ emission. These observations make it a good candidate for cooling-gas inflow and prompted its inclusion in the present sample.

The surface brightness profiles show a shallow interior and a gradual upturn at $\mu_{r}=22$. This feature is much stronger on the major axis than on the minor axis. The only associated shape parameter breaks are in the position angle and centroid profiles. But the position angle profile shows stronger activity at brighter magnitudes, and the centroid change is only marginally significant. The eccentricity increases rapidly only below $\mu_{r}=22.5$, where the position angle stabilizes after having rotated through $40^{\circ}$ in just 2 magnitudes.

This galaxy is a strong example of a $\mathrm{cD}$ galaxy in a poor group.

\section{Remarks on Other Galaxies}


There are notable omissions from Table 1. The largest and most luminous halos include those in A1413, A2199, and A2670. These halos begin at very low surface brightnesses, and CCD images deep and flat enough to perform isophotometry on them have not yet been obtained. An additional problem with the A2670 field is the presence of a bright star $80^{\prime}$ north-northeast of the galaxy. This star is at least 13th magnitude (it is saturated in all available images), and lies well within the halo reported by Oemler (1976): its projected distance from the galaxy at the redshift of the cluster is only $130 \mathrm{kpc}$. The image of PSH, and another taken with the $800 \times 800 \mathrm{CCD}$, almost reach the surface brightness at which Oemler's halo begins but do not actually show it. The work of King (1971) and Piccirillo (1973) on the wings of stellar point-spread functions suggest that the halo of A2670 is not an aureole of the star: it is several magnitudes too bright. Nevertheless careful CCD isophotometry of this halo is most desirable, not merely to confirm its existence, but to determine its ellipticity. The inner part of A2670 is one of the roundest ellipticals known. Is its halo also round, or does it reflect the ellipticity of the cluster?

Two galaxies included in both the samples of Schombert (1988) and PSH have been omitted here. The inflection point in the surface brightness profile of A1904 is entirely due to its complex central structure, and the bulge in the profile of A1913 is due to an obvious thick bar or disk. This is interesting in its own right, but certainly not what one wants to call a $\mathrm{cD}$ halo. Besides, neither of these galaxies is at the $\mathrm{X}$-ray center of its cluster, and residence near a density maximum is a well-established necessary condition for a galaxy to be a true $\mathrm{cD}$ (Schombert 1988). Another galaxy, A151, was omitted because adequate photometry could not be done on the PSH image. 
Some other galaxies worthy of passing mention are the multiple-nucleus systems whose surface brightness profiles were separated by Lauer $(1986,1988)$ and Porter (1988). In several of these systems (A194, A1185, A1291, A2634), the A component profile shows a definite upturn or knee. Again, the morphologies of the galaxies in that sample probably have a variety of causes. The knee of A1185 is a good candidate for a tidal heating phenomenon. The galaxy in A2634 is well known to have a high velocity relative to the cluster, and its halo is markedly asymmetric, suggesting that it has been distorted by a recent encounter with another galaxy. The shape profile derivatives of these galaxies ought to be compared to those of the galaxies in Table 1, but suitable data are not presently at hand.

\section{DISCUSSION}

Plotting derivatives instead of profiles is one of the major innovations of this study. It allows the first objective classification of the strength and sharpness of the edges of $\mathrm{cD}$ halos. Until this year the only quantitative measurements of halos have been luminosities, which are uncertain due to their low surface brightness and questionable radial extent. Schombert (1988) measured their scale lengths, but did not examine their inner edges, nor did he derive their shape profiles.

Figure 1 shows a wide variation in the suddenness of halo onset. A near discontinuity in the profile slope, the type of behavior one might consider "classical," is displayed by A7790, A1383, and A1795. Smoother transitions-"bends," as opposed to "breaks" in the slope-are more common: A496, A978, A2147, and NGC 5044 are examples. In some D galaxies, such as A858 and A1169, there is enough scatter in the profile slope that one could question whether the slope increase 
is a local instead of a global phenomenon, i.e., whether the galaxy is a $\mathrm{cD}$ rather than just a $\mathrm{D}$. In at least one system that is widely accepted to be a cD, NGC 6034 , there is no clear break in the major-axis profile slope at all, despite the fact that the scatter is very small and that the slope increases steadily to -1.1 at $\mu_{r}=24.5$. On the other hand, there is a sharp decrease in the minor-axis slope of this galaxy. Note that this galaxy is an outlying member of the cluster, situated at a local density maximum.

Another variation in halo morphology is immediately apparent from Figure 1. $\mathrm{cD}$ halos have traditionally been thought of as having roughly power law structure (i.e., $\mathrm{I}(\mathrm{r}) \propto r^{-\beta}$, where $\beta \sim 1.5$ ) and indefinite extent. This impression was created largely by Oemler's (1976) profiles of A1413 and A2670, and similar behavior is visible along the major axes of A496, A978, A2147, and A2420 in the current data (which admittedly do not reach as deep). But Figure 1 shows that many other halos steepen again at large radii. Prime examples are A1650, NGC 4839 in Coma (A1656), and A1809, whose major-axis logarithmic slopes decrease to -2 or less. Other galaxies show intermediate behavior: the major-axis profile of A1795, for example, has a brief interval of slope -1.1, outside of which the slope stabilizes quickly at -1.4. One might almost call this a collar rather than a halo. Oemler also observed this type of behavior in some galaxies, such as A2199, but this is less well remembered.

The third observation to be made from the profile slope plots in Figure 1 is that $c D$ halos occur significantly more frequently along major axes than along minor axes. Of the galaxies observed, only A2065 has only a minor axis halo, and its membership in this sample might be questioned. But half these $\mathrm{cDs}$ have no particular halo along the minor axis. The effect is most striking in A496, 
A2147, NGC 6034 in A2151, A2420, and NGC 5044, the slopes of whose minoraxis profiles continue to decrease to the limit of the observations. Even many halos that extend onto the minor axis, such as those in NGC 4839 in Coma, and A1795, are significantly weaker there than on the major axis.

It is clear, then, that there is as wide a variety in the slope and sharpness of $\mathrm{cD}$ halos as there is in their luminosity and extent (Schombert 1988). I have not attempted to quantify this variety, partly because the spatial resolution of the isophotometry is uncertain (especially after the smoothing process), but mostly because the variety is inherently qualitative. There is not an overwhelming correlation between the sharpness or slope of a halo and its size or luminosity. For instance, the halo of NGC 6034 is extremely shallow, and that of A779 quite sharp edged, but neither is extremely luminous (Schombert 1988). However, there are clues that some such correlation might appear in a more complete sample. The halos of A2142 and A2147, which Schombert finds to be very luminous, are relatively sharp-edged. It is necessary to extend the present work to include the larger, lower surface brightness halos before a firm conclusion can be made on this point.

Another important result of this study is that the edge of a halo is almost always accompanied by an ellipticity break. The change may be small, but there are only 4 galaxies that lack it: A400 and A779, whose halos are very faint, A1238, and NGC 5044, which does have an ellipticity increase at larger radii. A858 and A2065 show ellipticity decreases. They could be viewed as having minor-axis halos, or it could be questioned whether they truly deserve to be called $\mathrm{cD}$ galaxies.

These ellipticity changes are of added interest because, about half the time, they are more abrupt than the change in the surface brightness profile. This is most remarkable in A1656, A1809, and A1921. In at least one galaxy (A1169), 
the sharpness of the ellipticity break appears to be a result of correlated scatter in the major- and minor-axis surface brightness profiles. In most, however, its greater sharpness is clearly genuine. Such a break therefore has the potential to locate halo edges more precisely than the brightness profile.

There are also numerous clear cases of isophote twists (e.g., A978, A1809, A2142) and nonconcentric light (A1656, A1767, A2147) associated with halo edges. Sometimes an isophote twist stabilizes outside a halo edge, as in A858, A2142, and NGC 5044. Breaks in these profiles can be difficult to detect, for position angles of round isophotes are indeterminate, and centroid uncertainties grow rapidly with an isophote's magnitude (v. III above).

The correlation of breaks in the ellipticity and surface brightness profiles greatly reinforces the interpretation of halos as populations of stars dynamically distinct from the underlying galaxies. Since a surface brightness or shape profile is a projection of a distribution function out of 6 dimensions into one, and projections can only smooth discontinuities, a discontinuous derivative in two or more independent profiles is good evidence of some very sharp edge in the original function. The isophotometry of field ellipticals by Djorgovski (1985) and of cluster ellipticals by PSH shows that dynamical complexity is characteristic of ellipticals. Halos are just one form of it, one that occurs in clusters.

Complexity need not imply instability. An extreme example of a stable twocomponent system is our own galaxy, and we know enough about M87 and other ellipticals to be confident that ellipticity breaks can persist in relatively relaxed systems. Nonconcentric light, however, is another matter, for it implies a net gravitational force between different parts of the galaxy. It cannot be static and strongly suggests that a system that has it has recently interacted with a companion, 
whether that be the cluster potential or a consumed satellite. A similar argument can probably be made about strong isophote twisting, invoking torques instead of forces. This leads to the testable prediction that halos with nonconcentric light or strong isophote twisting are not relaxed configurations and should show significant kinematical activity. It would be surprising if spectroscopy of A1767 revealed no streaming velocities in the very nonelliptical, displaced isophotes. Conversely, halos that show only ellipticity breaks (or none) are likely to be at rest with respect to their parent galaxies. Such observations would be feasible with a large telescope. They would be difficult, but rewarding.

Another important result of the present study is the discovery of a strong $\mathrm{cD}$ halo in a poor group galaxy: NGC 5044. The existence of the halos reported by Morbey and Morris (1983) have been questioned by other observers, and at any rate, they have much lower surface brightnesses. Thanks to its low redshift, the edge of the halo in NGC 5044 occurs above $\mu_{r}=23 \mathrm{mag} \mathrm{sec}^{-2}$.

In the following section I will first briefly review the formation models and consider what bearing, if any, the new data have on them. Next I will consider some of the successes and limitations of the morphological definition of halos and, finally, assess an alternative definition more directly motivated by what we believe about different kinds of halos.

\section{MODELS AND DEFINITIONS OF cD HALOS}

Schombert (1988) has recently confronted four favored models of halo formation with new observations of halos. His most relevant observations were: 
1) that optical halo luminosity is weakly correlated with the optical luminosity of the underlying galaxy, suggesting "parallel formation processes" for the two components,

2) that optical halo luminosity is correlated somewhat more strongly with cluster X-ray luminosity, as $L_{e n v} \propto L_{x}^{1.06 \pm 0.18}$, connecting the halo to the global evolution of the cluster,

3) that optical halo luminosity is correlated with cluster richness as $L_{e n v} \propto$ $N^{1.62 \pm 0.44}$

4) that the average optical halo profile, $\rho_{e n v} \propto r^{-2.6 \pm 0.3}$, is the same as that of the galaxy counts of West et al. (1987), and

5) that there was little or no evidence of a $(B-V)$ gradient on the halos of A1413, A1767, and NGC 6034.

The four models currently favored are mergers (e.g., Villumsen 1982), tidal stripping (Malumuth and Richstone 1984), cooling-gas accretion (Fabian and Nulsen 1977, Cowie and Binney 1977, Stewart et al. 1984), and primordial origin (Miller 1983; Merritt 1984, 1985). Of these, Schombert favored some combination of stripping and primordial origin. Stripping is suggested by the agreement of his data with the prediction of Malumuth and Richstone (1984) that $L_{e n v} \propto N^{2}$ and the similarity of the halo and galaxy count profiles but may be constrained by the lack of a color gradient on the halos. NGC 5044, a cD galaxy in a relatively poor group, could conceivably pose a problem to this model, but its halo luminosity has not been measured yet. Doubt is also cast on the sufficiency of the stripping model alone by the existence of very luminous non- $\mathrm{cD}$ galaxies in rich clusters such as $\mathrm{A} 85$ and A2029. 
In the models of Malumuth and Richstone and Thuan and Romanishin (1981), the seed galaxy itself is a merger product. Mergers are less favored as a method for building halos on the grounds that they generate insufficient energy to explain the scales and luminosities of halos (e.g., Villumsen 1982), and gas accretion has the problem that in most systems the mass-flow rates are too low to have built up massive halos, especially since the stagnation radii for gas flows appear to be relatively small. Note that the images of optical emission line gas in, for instance, A496, A1795, and A2199 in Cowie et al. (1983) show a much stronger radial gradient than do $\mathrm{cD}$ halos, and what emission those galaxies do show outside the halo edge radii listed in Table 1 is quite patchy. Another problem with explaining many halos as products of accreted gas is apparent from the data in Stewart et al. (1984). A1795 has a derived mass-flow rate of $400 M_{\odot} y r^{-1}$, but A1809 and A2670 have no flows, since the cooling times in those clusters is longer than a Hubble time. Conversely, A2029, with no halo at all, has a flow rate $\mathrm{M}=250 \mathrm{M}_{o} y r^{-1}$. Finally, the debate about whether cooling gas flows actually exist on the scales modelled is still raging.

Isophotometry is not a direct key to halo origins. Any of the currently favored models can accommodate the observed correlation of ellipticity and other shape breaks with halo edges, especially if some of the small-scale halos can be transient phenomena. Nor can any of them, including primordial origin, be certainly ruled out by the absence of strong isophote twists or nonconcentric light.

Is is true, however, that the shape behavior of some individual halos could be due to processes suggested by other data. The clearest example of this is A1795, whose halo is at least partly composed of a line emission region. Distinct patches of light also contribute to the halos of A496, A1809, and other galaxies suspected 
of gas inflow. In other galaxies one has reasons to suspect dynamical activity. The halos of A400, A779, and other galaxies with close companions are likely to involve tidal distension and stripping in amounts depending on the encounter velocity. The distortion of the isophotes of A1767 is so marked that, in the absence of nuclear activity, it is difficult to attribute to anything other than collision with its two neighbors to the south. As expected, halos of this type are of finite extent, in that they steepen again at large radii.

A primordial origin is perhaps most likely for those halos that have the smoothest edges and largest size scales. Many of the best candidates for this could not be observed in the present sample, but objects such as NGC 5044, NGC 6034, and even A496 may represent them.

By now the reader has probably sensed that a new ambiguity has crept into the definition of a $\mathrm{cD}$ galaxy. The vague canonical lore, that $\mathrm{cD}$ halos are extremely massive, low-surface-brightness structures with characteristic sizes of hundreds of kiloparsecs, and that as such they are only loosely bound to the galaxies they envelop, has been replaced by a more formal definition based on breaks in the slope of the surface brightness profile. This explicit exclusion of the D galaxies (e.g., A85) and "X-ray dominant" galaxies (e.g., A2634) which many people call cDs has made possible the conclusions of Schombert (1988) and this paper. It is an approach that emphasizes the elliptical-halo-cluster sequence by assigning to the halo its own special identity.

But it has also blurred the distinction between $\mathrm{cDs}$ and other complex ellipticals, allowing new, different interlopers such as A1382, A1904, and perhaps A1238, A2065, and others to dehomogenize the sample. Some of these galaxies probably just have weak disks. Even more subtly, it has included in the class 
galaxies such as A1767 and A1809, which, while they definitely have "extra light" in some sense closely related to the original concept, have it on a completely different scale from prototypes such as A1413 and A2670. Schombert acknowledged this by his distinction between "faint envelopes" and "bright envelopes."

To preserve the original intent of the definition, it is tempting to try to restrict the use of the term $\mathrm{cD}$. One could restrict it to a halo distributed on a cluster scale, i.e., to material that is bound less strongly to any galaxy it envelops than to the cluster potential. This criterion would reliably reject most tidally distended galaxies and transient merger products, such as we conjecture A1767 to be. But it would also reject A1795, which has one of the smallest "effective radii" in Schombert's sample but is generally agreed to be a classical $\mathrm{cD}$. Furthermore there is no obvious bimodality in the distribution of effective radii Schombert derived for the halos in his sample. Alternatively, one could attempt to restrict the definition to halos that are at rest with respect to the underlying galaxy, i.e., to equilibrium configurations. The problem with this definition is that such faint distended objects are impossible to observe spectroscopically. It might not turn out to be very restrictive, either.

Another point is that PSH have recently shown that structural breaks of the sort examined in this paper abound in BCEs, and have advanced new, strong arguments that the outer regions $(a \geq 30 \mathrm{kpc})$ of BCEs are structurally closely related to their clusters. Both of these findings hold even for galaxies without "halos." In the end one is forced to admit that the primary motivation for restricting the definition of a halo is to make it easier to decide between the primordialorigin and stripping-formation models and that this urge is too post hoc to be legitimate. Any definition will have its limitations and exceptions and must be applied thoughtfully. This was the moral of the story in the introduction. In 
this context it is also constructive to consider the situation with wide-angle tail (WAT) radio sources, which are governed by different processes on different size scales (Burns 1986). There is no a priori reason all $\mathrm{cD}$ halos or structural breaks in ellipticals should be formed the same way. If and when the search for intracluster light reveals "halos without galaxies," it may be time to reopen the discussion.

\section{CONCLUSIONS}

I have adopted a formal morphological definition of a $\mathrm{cD}$ halo, observed a sample of $23 \mathrm{cD}$ galaxies, and reached the following conclusions:

1) The sharpness of halo edges varies greatly, with no strict dependence on halo luminosity or size. The same is true of the halos' logarithmic slopes. The slopes often vary with radius, and many halos steepen again at large radii. The distinction between $\mathrm{D}$ and $\mathrm{cD}$ galaxies is therefore not completely unambiguous.

2) The edge of a halo is almost always associated with a break in the ellipticity profile. About half the time these breaks are sharper than the halo edge and help define it better than the surface brightness profile. Isophote twists and nonconcentric light are also common features of halo edges. The existence and sharpness of such breaks is strong evidence that the halos and the galaxies within them are dynamically distinct entities.

3) Small, faint, and richly structured halos are probably due to a variety of recent phenomena, namely, gas inflow and galactic encounters. They may therefore show interesting kinematic structure, and should be searched for it. 
4) Larger, smoother halos of higher total luminosity are more likely to have primordial origins. These will require further observation to complete the present study properly.

5) NGC 5044 is a true cD galaxy in a poor group. Measurement of its luminosity could constrain the model of halo construction by stripping, which should be restricted to rich clusters.

6) The adopted halo definition emphasizes the continuity in the galaxy-halocluster sequence. An alternative definition of a $\mathrm{cD}$ halo as a component of cluster light not bound to any galaxy that it envelops is not at present a very practical one. All definitions should be used thoughtfully and carefully.

I am grateful to J. Schombert for enlightening discussions of his work and to S. Djorgovski for urging me to plot derivatives. Thanks go to the Palomar time allocation committee for its generosity, S. Kulkarni for the image of NGC 6034, and to S. Staples and J. Phinney for assistance at the telescope. Financial support was provided by an NSF grant to J. B. Oke and an ARCS Fellowship. 


\section{REFERENCES}

Abell, G. A. 1958, Astrophys. J. Supp. Ser. 3211.

Albert, C. E.; White, R. A.; and Morgan, W. W. 1977, Astrophys. J. 211309.

Baum, W. A.; Thomsen, B., and Morgan, B. L. 1986, Astrophys. J. 30183.

Binggeli, B. 1982, Astron. Astrophys. 107338.

Burns, J. O. 1986, Can. J. Phys. 64373.

Boroson, T. A.; Thompson, I. B.; and Shectman, S. 1983, Astron. J. 881707.

Cowie, L. L., and Binney, J. 1977, Astrophys. J. 215723.

Cowie, L. L.; Hu. E. M.; Jenkins, E. B.; and York, D. G. 1983, Astrophys. J. 272 29.

Djorgovski, S. G. 1985, Ph.D. thesis, University of California at Berkeley.

Dressler, A. 1979, Astrophys. J. 231659.

Fabian, A. C., and Nulsen, P. E. J. 1977, Mon. Not. Royal Ast. Soc. 180479.

Forman, W. R., and Jones, C. 1988, in preparation. 
Gunn, J. E., et al. 1987, Opt. Eng. 26779.

Hawarden, T. G.; Elson, R. A. W.; Longmore, A. J.; Tritton, S. B.; and Corwin, H. G., Jr. 1981, Mon. Not. Royal Ast. Soc. 196747.

Heckman, T. A. 1981, Astrophys. J. Lett. 250 L59.

Hoessel, J. G.; Melnick, J.; and White, S. D. M. 1977, Mon. Not. Royal Ast. Soc. 180207.

Hoessel, J. G., and Schneider, D. P. 1985, Astron. J. 901648.

Hubble, E. P. 1936, The Realm of the Nebulae. (New York: Dover).

Humason, M. L.; Mayall, N. U.; and Sandage, A. R. 1956, Astron. J. 6197.

Jones, C., and Forman, W. R. 1984, Astrophys. J. 27638.

King, I. R. 1971, Pub. Ast. Soc. Pacific 83199.

Lauer, T. R. 1986, Astrophys. J. 31134.

Lauer, T. R. 1988, Astrophys. J. 32549.

Malumuth, E. M., and Kirshner, R. P. 1985, Astrophys. J. 2918.

Malumuth, E. M., and Richstone, D. O. 1984, Astrophys. J. 276413. 
Matthews, T. A.; Morgan, W. W.; and Schmidt, M. 1964, Astrophys. J. 14035.

Merritt, D. 1984, Astrophys. J. 27626.

Merritt, D. 1985, Astrophys. J. 28918.

Miller, G. E. 1983, Astrophys. J. 268495.

Morbey, C. L., and Morris, S. 1983, Astrophys. J. 274502.

Morgan, W. W.; Kayser, S.; and White, R. A. 1975, Astrophys. J. 199545.

Oemler, A. 1976, Astrophys. J. 209693.

Piccirillo, J. 1973, Pub. Ast. Soc. Pacific 85278.

Porter, A. C. 1988, in preparation (chapter III).

Porter, A. C.; Schneider, D. P.; and Hoessel, J. G. 1988, in preparation.

Schombert, J. 1986, Astrophys. J. Supp. Ser. 60603.

Schombert, J. 1987, Astrophys. J. Supp. Ser. 64643.

Schombert, J. 1988, Astrophys. J. 328475. 
Schweizer, F. 1987, in Dynamics of Elliptical Galaxies, de Zeeuw, P. T., ed. (Dordrecht: Reidel), p. 109.

Selman, F. J. 1988, in preparation.

Stewart, G. C.; Fabian, A. C.; Jones, C.; and Forman, W. R. 1984, Astrophys. J. 2851.

Struble, M. F. 1987, Astrophys. J. 317338.

Thuan, T. X., and Gunn, J. E. 1976, Pub. Ast. Soc. Pacific 88543.

Thuan, T. X., and Romanishin, W. R. 1981, Astrophys. J. 248439.

Tonry, J. 1987, in Dynamics of Elliptical Galaxies, ed. P. T. de Zeeuw (Dordrecht: Reidel), p. 89.

van Bruegel, W.; Heckman, T.; and Miley, G. 1984, Astrophys. J. 27679.

Villumsen, J. V. 1982, Mon. Not. Royal Ast. Soc. 199493.

West, M. J.; Dekel, A.; and Oemler, A. 1987, Astrophys. J. 3161. 
TABLE I. cD Galaxies Observed.

\begin{tabular}{rrrllllllllll} 
Cluster & Opt & Exp. & $\mathrm{n}$ & see & $\mathrm{a}(\mathrm{h})$ & $\Delta \epsilon$ & $\Delta \theta$ & $\Delta x_{0}$ & $\operatorname{maj}$ ax? & $\min$ ax? & steepen? \\
\hline & & & & & & & & & & & \\
$\mathrm{A} 400$ & 2 & 300 & 2 & $1.2 "$ & $4 "$ & $\mathrm{~N}$ & $?$ & $\mathrm{Y} ?$ & $\mathrm{Y}$ & $\mathrm{Y}$ & $\mathrm{Y}$ \\
$\mathrm{A} 496$ & 1 & 700 & $2 \mathrm{~d}$ & 1.0 & 25 & $(\mathrm{Y})$ & $\mathrm{N} ?$ & $\mathrm{~N}$ & $\mathrm{Y}$ & $\mathrm{N}$ & $\mathrm{N}$ \\
& 2 & 500 & 1 & 1.5 & & & & & & & \\
$\mathrm{~A} 779$ & 2 & 500 & 1 & 1.4 & 10 & $\mathrm{~N}$ & $\mathrm{Y} ?$ & $\mathrm{Y} ?$ & $\mathrm{Y}$ & $\mathrm{Y}$ & $\mathrm{Y}$ \\
$\mathrm{A} 858$ & 2 & 300 & 1 & 1.6 & 5 & $\mathrm{Y}$ & $\mathrm{N}$ & $\mathrm{Y}$ & $\mathrm{Y}$ & $\mathrm{Y}$ & $\mathrm{N}$ \\
$\mathrm{A} 978$ & 2 & 500 & 1 & 1.3 & 5 & $\mathrm{Y}$ & $\mathrm{Y} ?$ & $\mathrm{Y} ?$ & $\mathrm{Y}$ & $\mathrm{N}$ & $\mathrm{Y}$ \\
$\mathrm{A} 1169$ & 2 & 500 & 1 & 1.4 & 10 & $\mathrm{Y}$ & $\mathrm{Y}$ & $\mathrm{Y}$ & $\mathrm{Y}$ & $\mathrm{N}$ & $\mathrm{N}$ \\
$\mathrm{A} 1224$ & 2 & 500 & 1 & 1.4 & 3 & $\mathrm{Y}$ & $\mathrm{Y} ?$ & $\mathrm{~N} ?$ & $\mathrm{Y}$ & $\mathrm{Y}$ & $\mathrm{Y}$ \\
$\mathrm{A} 1238$ & 2 & 500 & 1 & 1.5 & 5 & $\mathrm{~N} ?$ & $\mathrm{Y}$ & $\mathrm{N}$ & $\mathrm{Y}$ & $\mathrm{Y}$ & $\mathrm{N}$ \\
$\mathrm{A} 1382$ & 2 & 500 & 1 & 1.3 & 3 & $\mathrm{Y}$ & $\mathrm{Y}$ & $\mathrm{Y}$ & $\mathrm{Y}$ & $\mathrm{Y}$ & $\mathrm{Y}$ \\
$\mathrm{A} 1383$ & 2 & 500 & 1 & 1.9 & 5 & $\mathrm{Y}$ & $\mathrm{Y}$ & $\mathrm{Y}$ & $\mathrm{Y}$ & $\mathrm{Y}$ & $\mathrm{Y} ?$ \\
$\mathrm{~A} 1630$ & 2 & 300 & 1 & 1.8 & 8 & $\mathrm{Y}$ & $\mathrm{Y}$ & $\mathrm{Y}$ & $\mathrm{Y}$ & $\mathrm{Y}$ & $\mathrm{Y}$ \\
$\mathrm{A} 1650$ & 1 & 1440 & $4 \mathrm{~d}$ & 2.5 & 8 & $\mathrm{Y}$ & $\mathrm{Y}$ & $\mathrm{N}$ & $\mathrm{Y}$ & $\mathrm{N}$ & $\mathrm{Y}$ \\
& & & & & & $\mathrm{Y}$ & $\mathrm{Y}$ & $\mathrm{Y} ?$ & $\mathrm{Y}$ & $\mathrm{Y}$ & $\mathrm{Y}$ \\
$\mathrm{A} 1656$ & 2 & 300 & 1 & 2.0 & 50 & $\mathrm{Y}$ & $?$ & $\mathrm{Y}$ & $\mathrm{Y}$ & $\mathrm{Y}$ & $\mathrm{Y}$ \\
$\mathrm{A} 1767$ & 2 & 500 & 1 & 1.2 & 5 & $\mathrm{Y}$ & $\mathrm{Y}$ & $\mathrm{Y}$ & $\mathrm{Y}$ & $\mathrm{Y}$ & $\mathrm{Y}$ \\
$\mathrm{A} 1795$ & 2 & 500 & 1 & 1.2 & 8 & $\mathrm{Y}$ & $\mathrm{Y} ?$ & $\mathrm{Y}$ & $\mathrm{Y}$ & $\mathrm{Y}$ & $\mathrm{Y}$ \\
$\mathrm{A} 1809$ & 1 & 720 & $2 \mathrm{~d}$ & 2.5 & 16 & $\mathrm{Y}$ & $\mathrm{Y}$ & $\mathrm{Y}$ & $\mathrm{Y}$ & $\mathrm{N}$ & $\mathrm{Y}$ \\
& 2 & 500 & 1 & 1.1 & & & & & & & \\
$\mathrm{~A} 1921$ & 4 & 300 & 1 & 1.2 & 4 & $\mathrm{Y}$ & $\mathrm{Y}$ & $\mathrm{Y}$ & $\mathrm{Y}$ & $\mathrm{Y}$ & $\mathrm{N}$ \\
$\mathrm{A} 2065$ & 2 & 500 & 1 & 1.4 & 5 & $\mathrm{Y}$ & $\mathrm{Y}$ & $\mathrm{N}$ & $\mathrm{N}$ & $\mathrm{Y}$ & $\mathrm{N}$ \\
$\mathrm{A} 2142$ & 2 & 500 & 1 & 1.3 & 6 & $\mathrm{Y}$ & $\mathrm{Y}$ & $\mathrm{Y}$ & $\mathrm{Y}$ & $\mathrm{N}$ & $\mathrm{N}$ \\
$\mathrm{A} 2147$ & 2 & 500 & 1 & 2.4 & 32 & $\mathrm{Y}$ & $\mathrm{N}$ & $\mathrm{Y}$ & $\mathrm{Y}$ & $\mathrm{N}$ & $\mathrm{N}$ \\
$\mathrm{A} 2151$ & 3 & 600 & 1 & 1.5 & 25 & $\mathrm{Y}$ & $\mathrm{N}$ & $\mathrm{Y}$ & $\mathrm{Y}$ & $\mathrm{N}$ & $\mathrm{N}$ \\
$\mathrm{A} 2420$ & 2 & 500 & 1 & 1.6 & 10 & $\mathrm{Y}$ & $\mathrm{Y}$ & $\mathrm{Y}$ & $\mathrm{Y}$ & $\mathrm{N}$ & $\mathrm{N}$ \\
$\mathrm{WP} 23$ & 1 & 1440 & $4 \mathrm{~d}$ & 3.0 & 50 & $(\mathrm{~N})$ & $\mathrm{Y}$ & $\mathrm{Y}$ & $\mathrm{Y}$ & $\mathrm{N}$ & $\mathrm{N}$
\end{tabular}

Notes to Table I. A779: NGC 2832. A1656: NGC 4874. A2151: NGC 6034. WP23: NGC 5044. See text for explanation of entries. 


\section{FIGURE CAPTIONS.}

FIGURE 1. Smoothed first derivatives of the shape profiles of all the galaxies in the sample. The abscissae of all six panels on each page are $\mu_{r}$, the Gunn $r$ surface brightness in magnitudes per square arc second. The top two panels show the logarithmic slope of the surface brightness profile along the major and minor axes. The lower four panels show the derivatives of isophote ellipticity, major axis position angle, and centroid in right ascension and declination, taken with respect to $\mu_{r}$.

FIGURE 2. Contour plot of NGC 5044, a cD galaxy in a small group. The contours are drawn at intervals of 1 magnitude $\operatorname{arcsec}^{-2}$ in $r$. The field of view is $5^{\prime}$ on a side.

FIGURE 3. Contour plot of A1767. The contours are drawn at intervals of 1 magnitude $\operatorname{arcsec}^{-2}$ in $r$. The field of view is 4.5 on a side.

FIGURE 4. Comparison of isophotometry of $800 \times 800$ TI CCD data with 500 x 500 TI CCD data. The panels correspond to those in figure 1, except that the independent variable in the second two rows is $\log b$, to facilitate comparison of the data. 

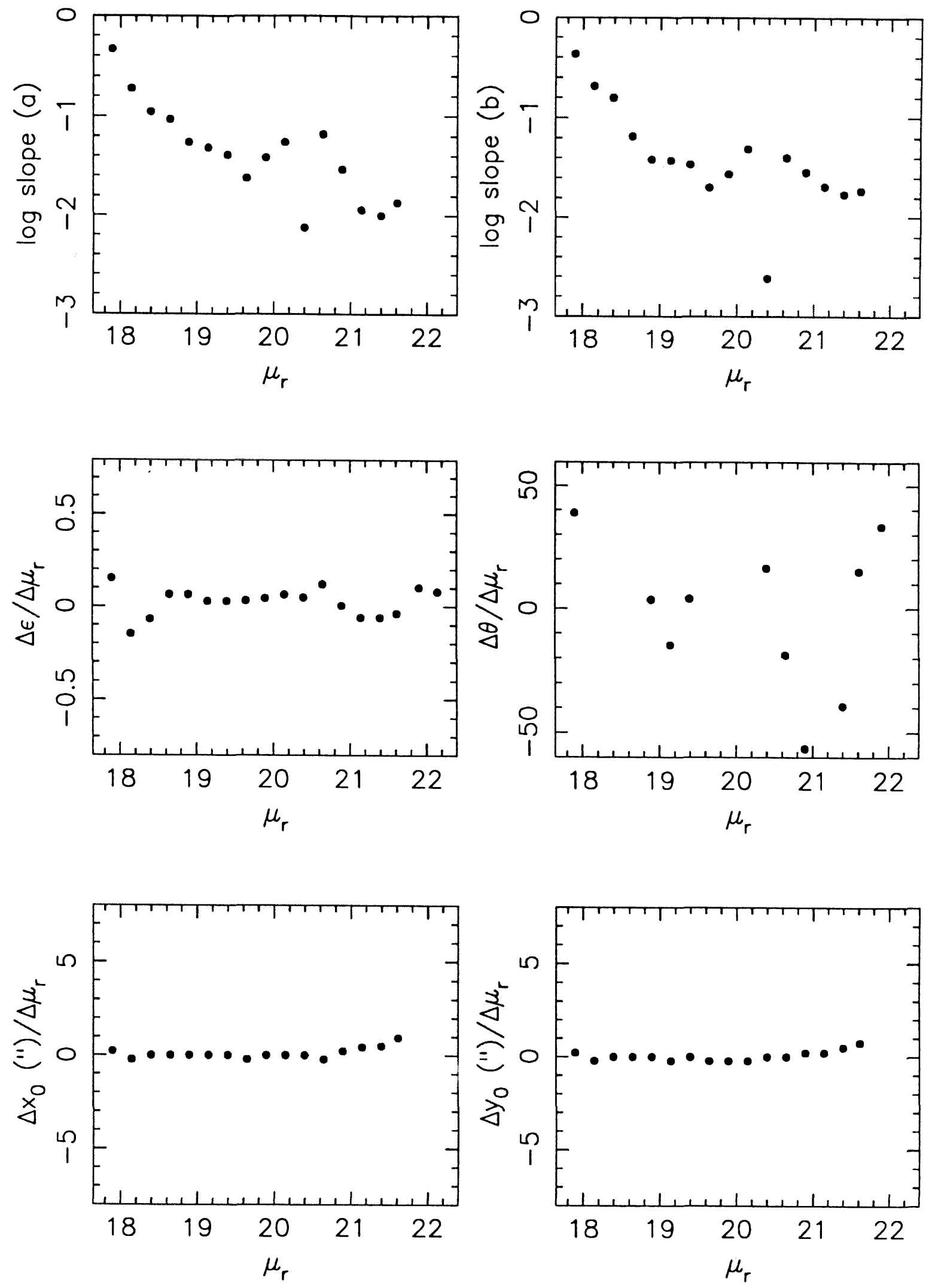

Figure 1. A400 s 

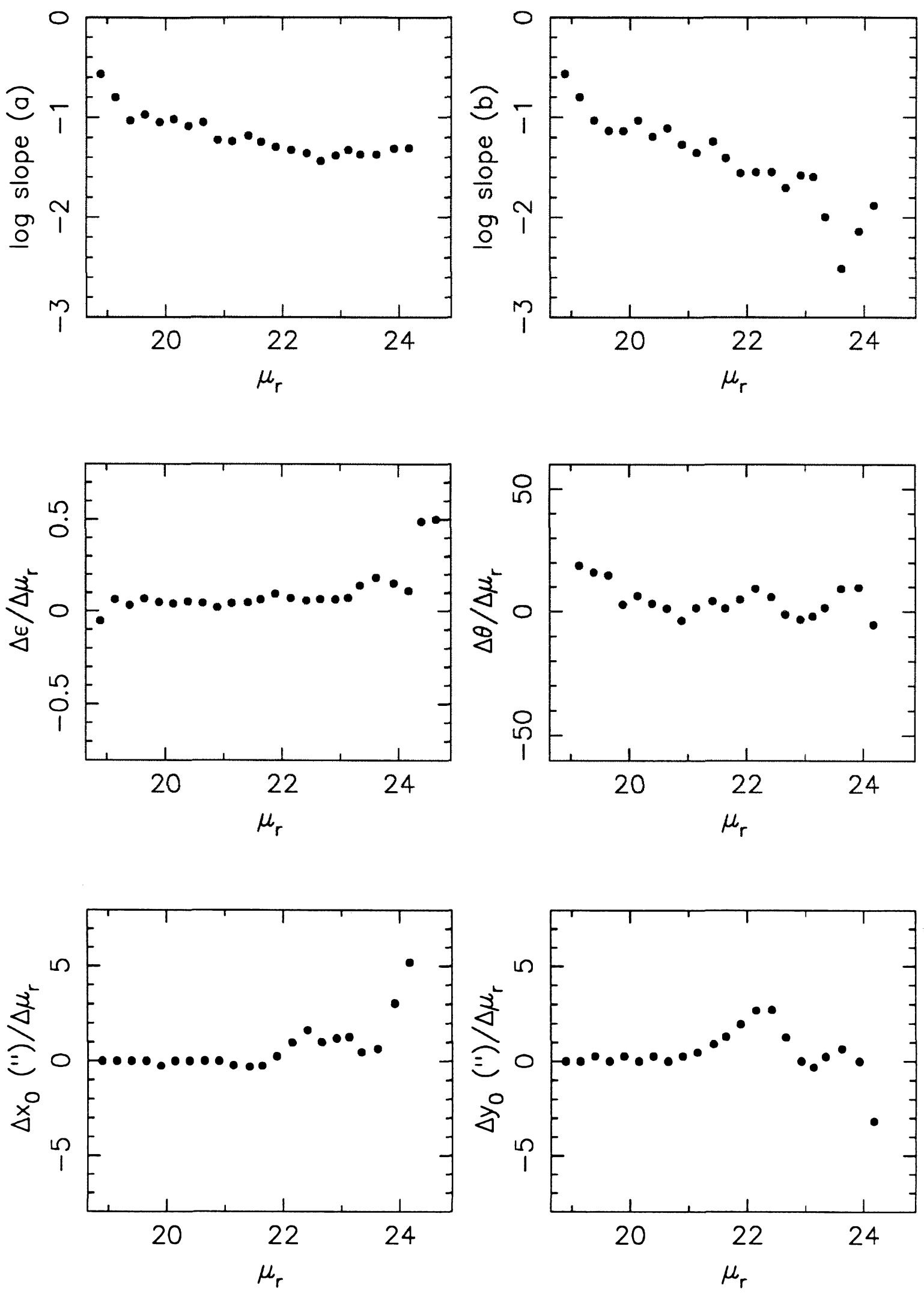

Figure 1 cont'd. A496 

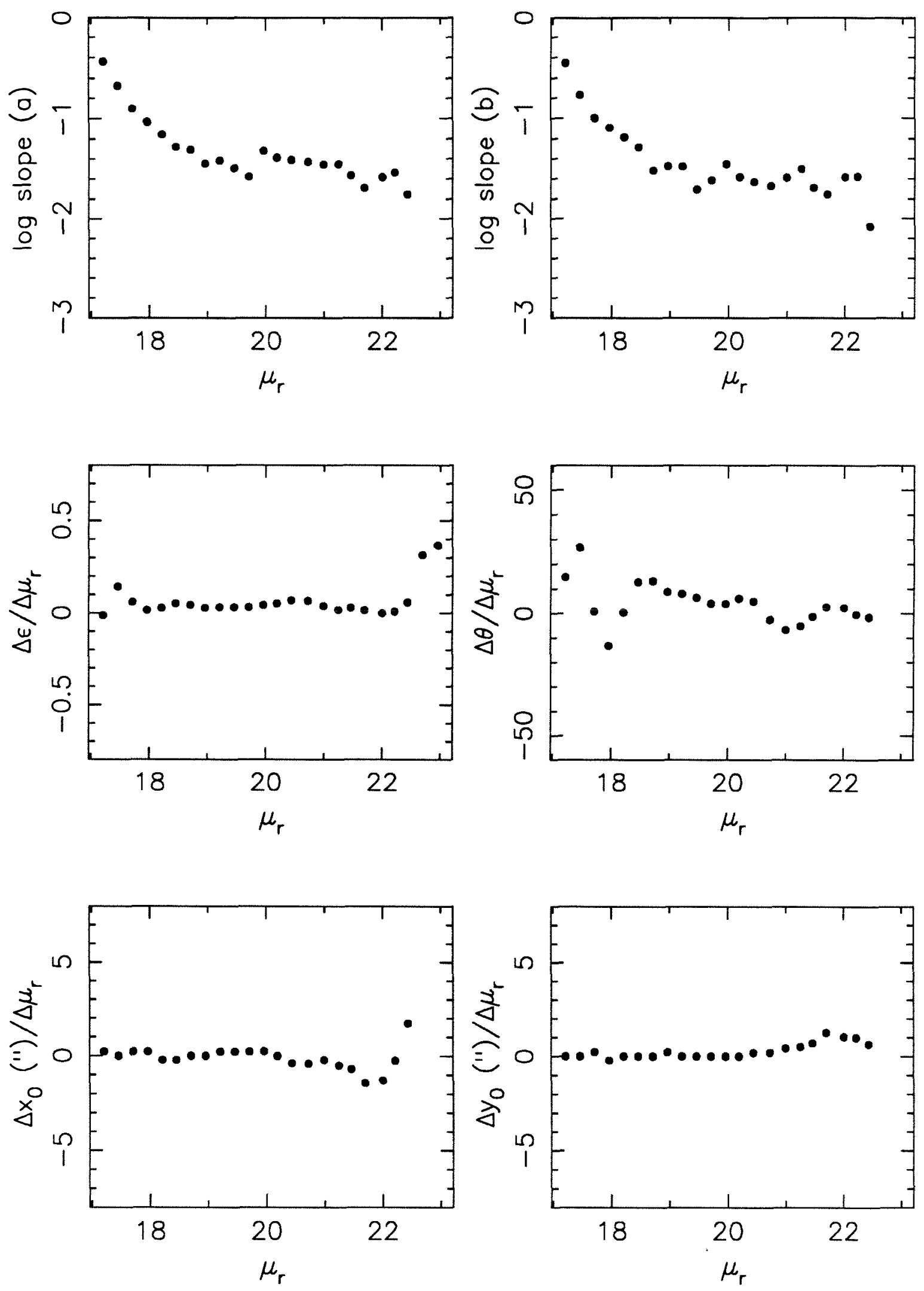

Figure 1 cont'd. A779 A 

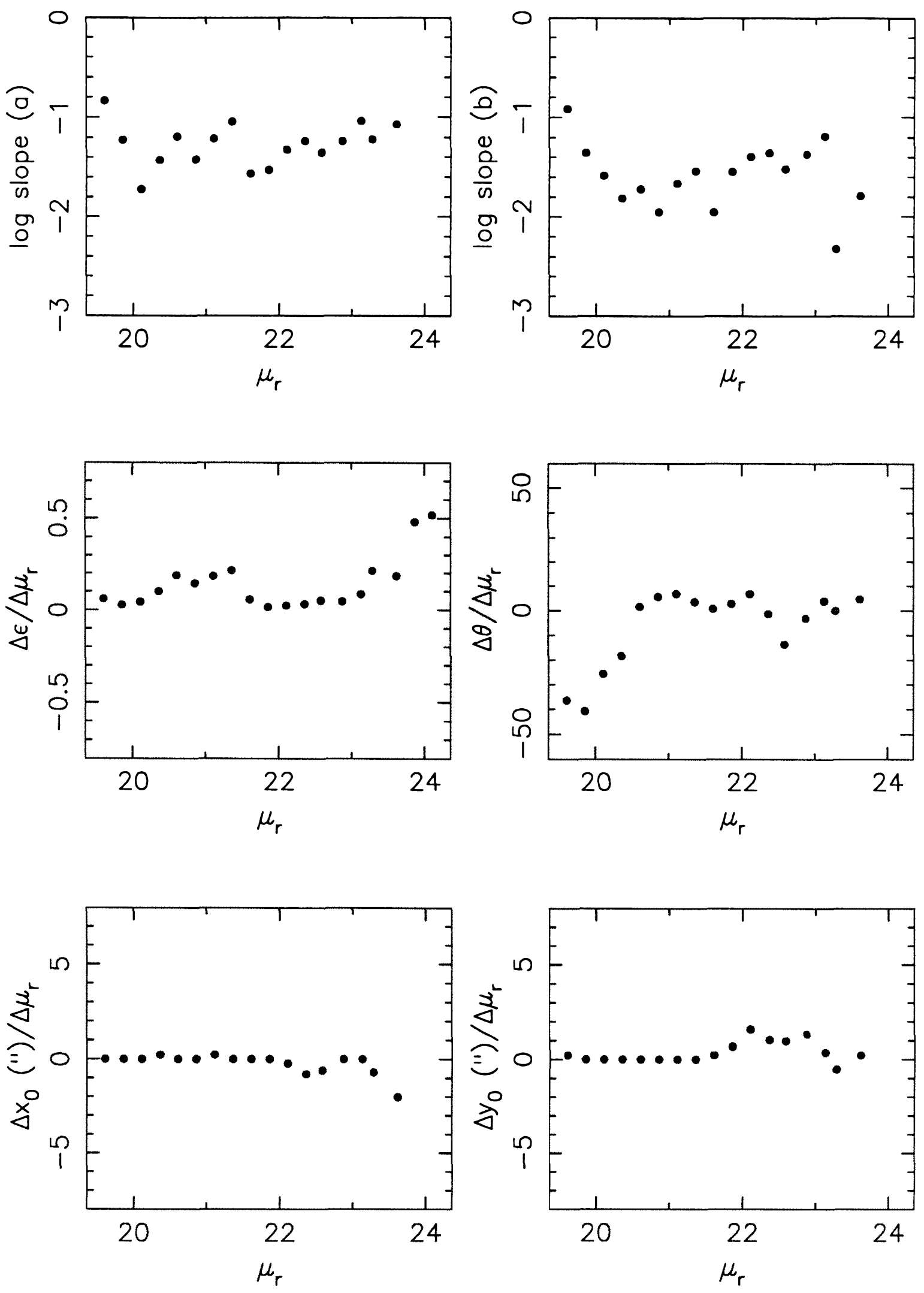

Figure 1 cont'd. A858 

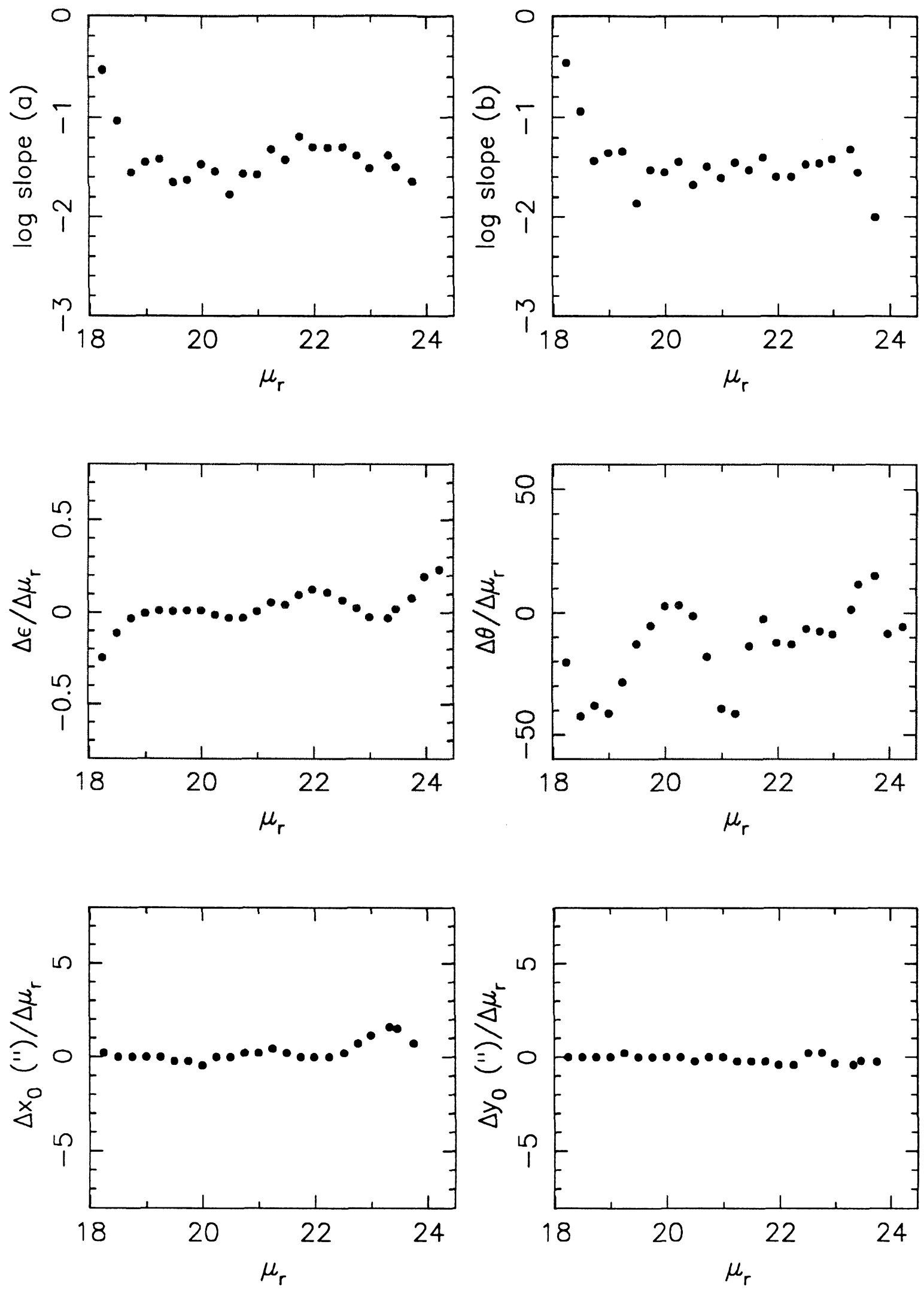

Figure 1 cont'd. A978 

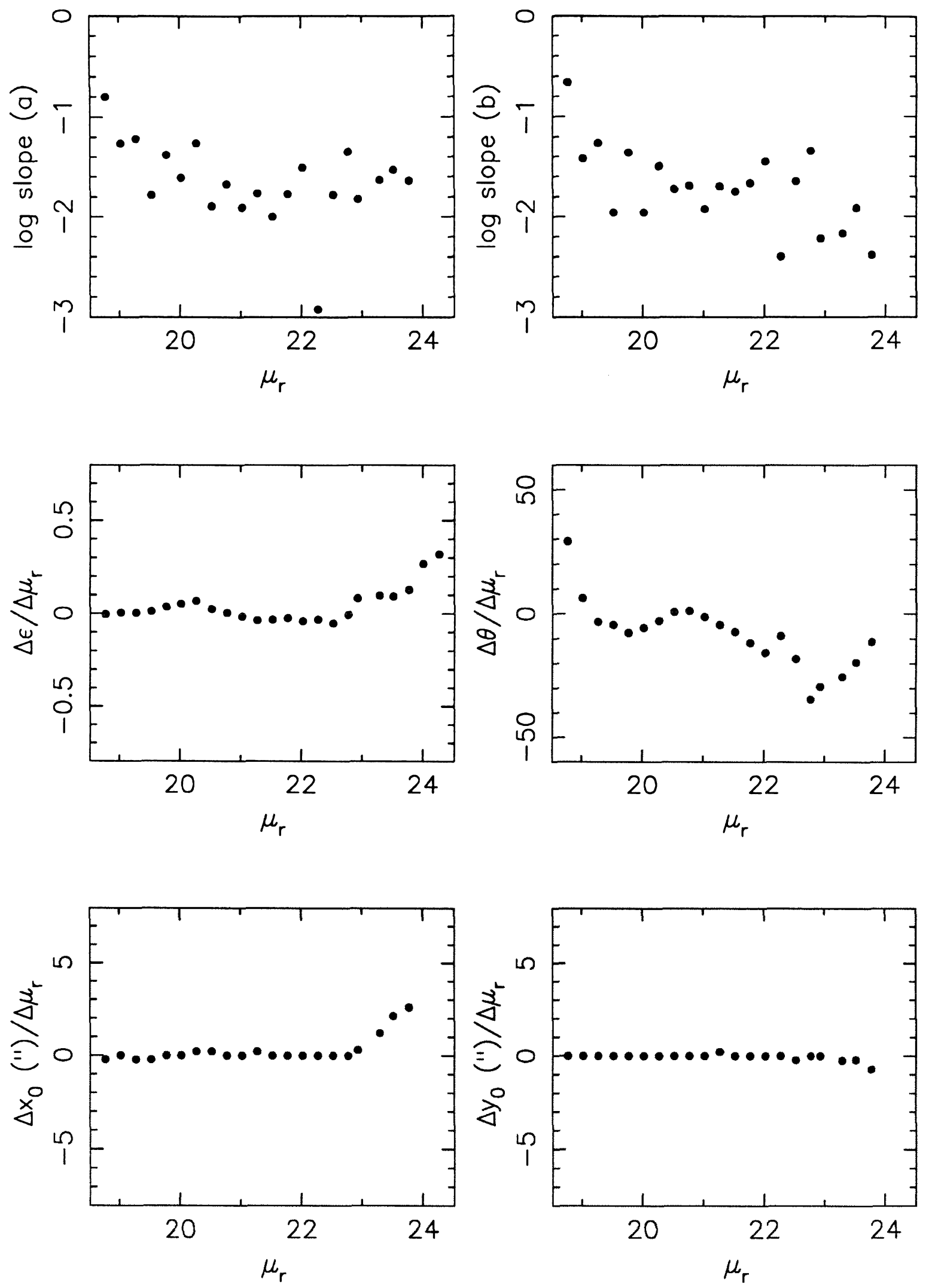

Figure 1 cont'd. A1169 

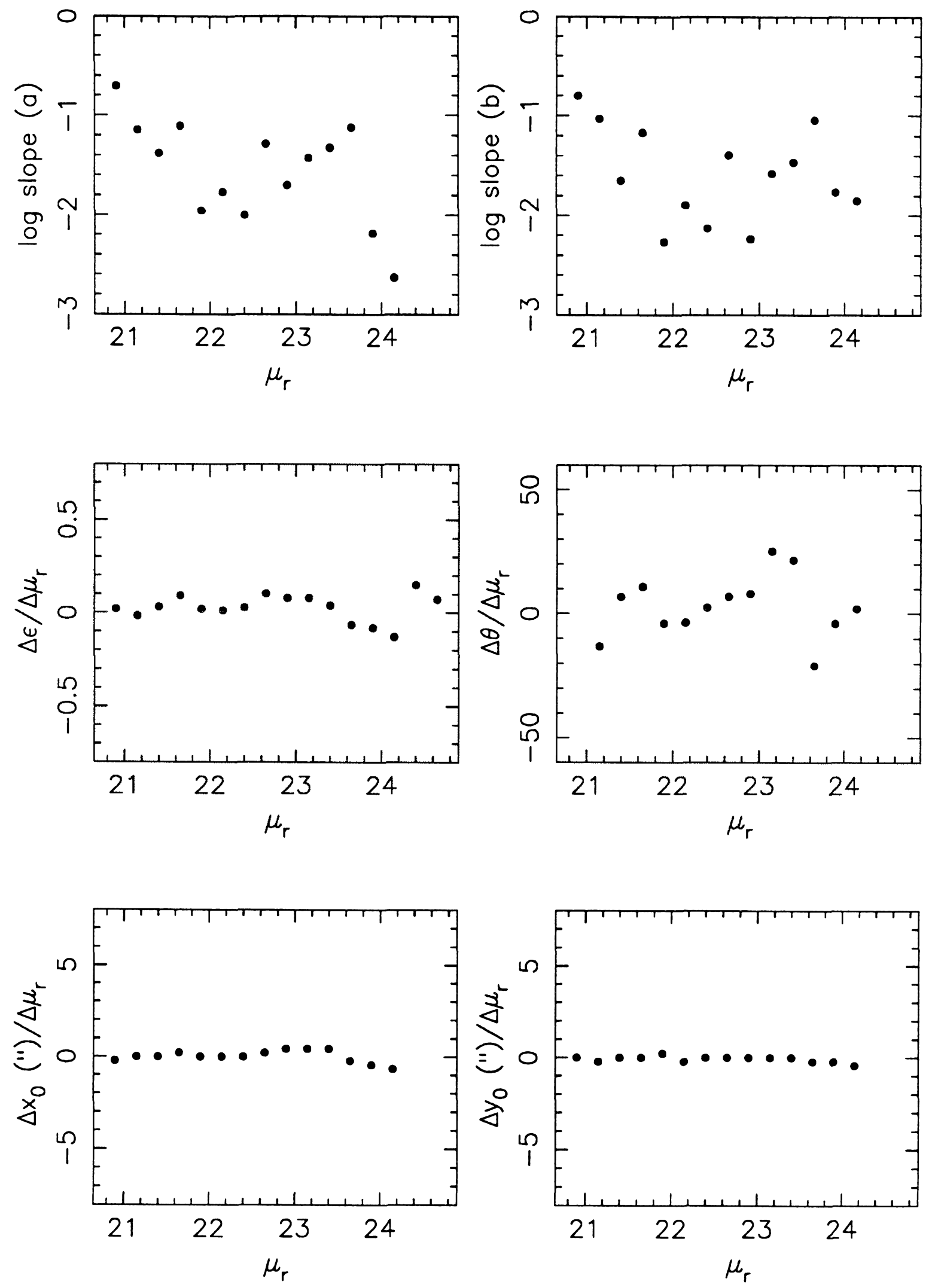

Figure 1 cont'd. A1224 

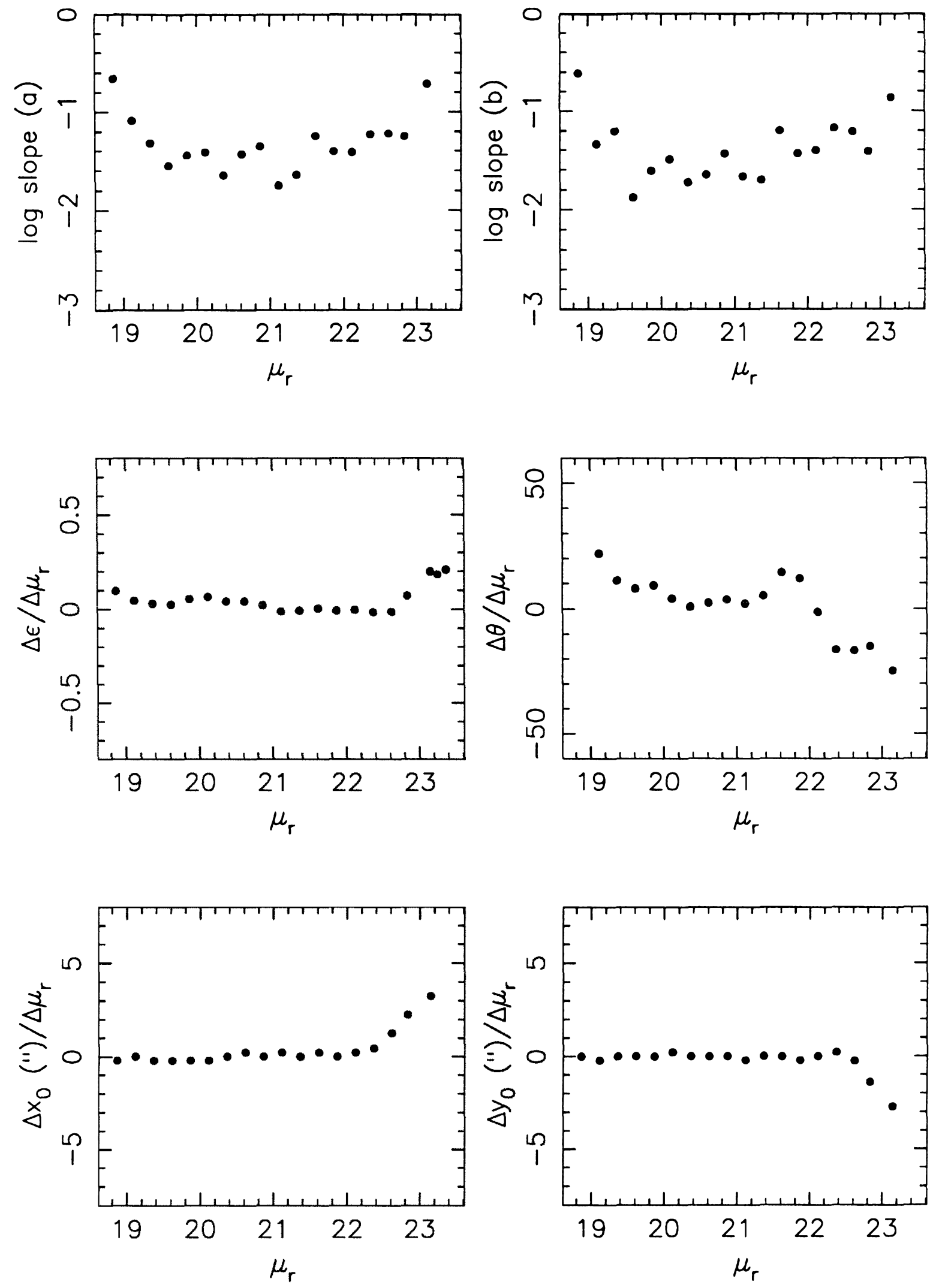

Figure 1 cont'd. A1238 

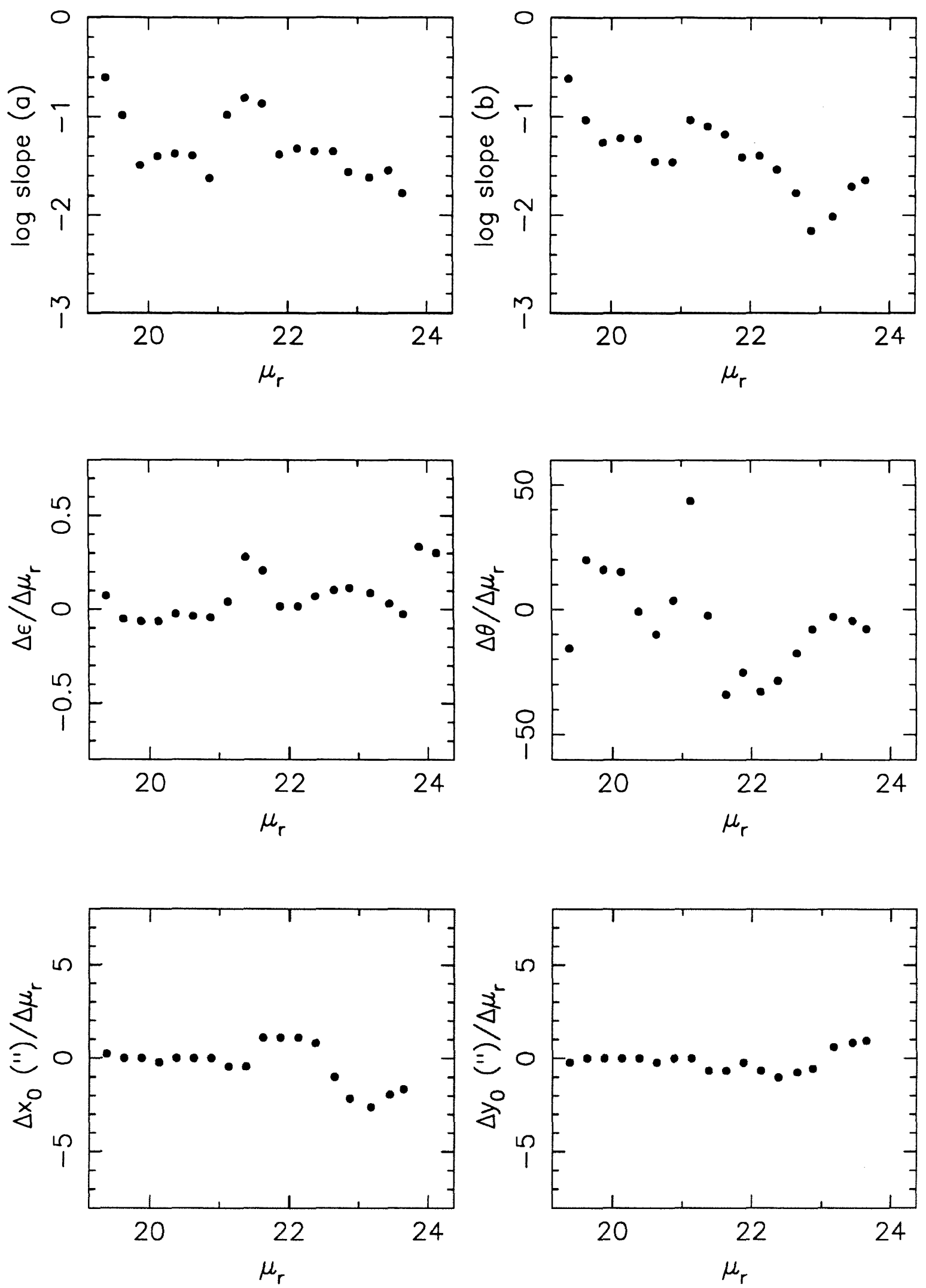

Figure 1 cont'd. A1382 

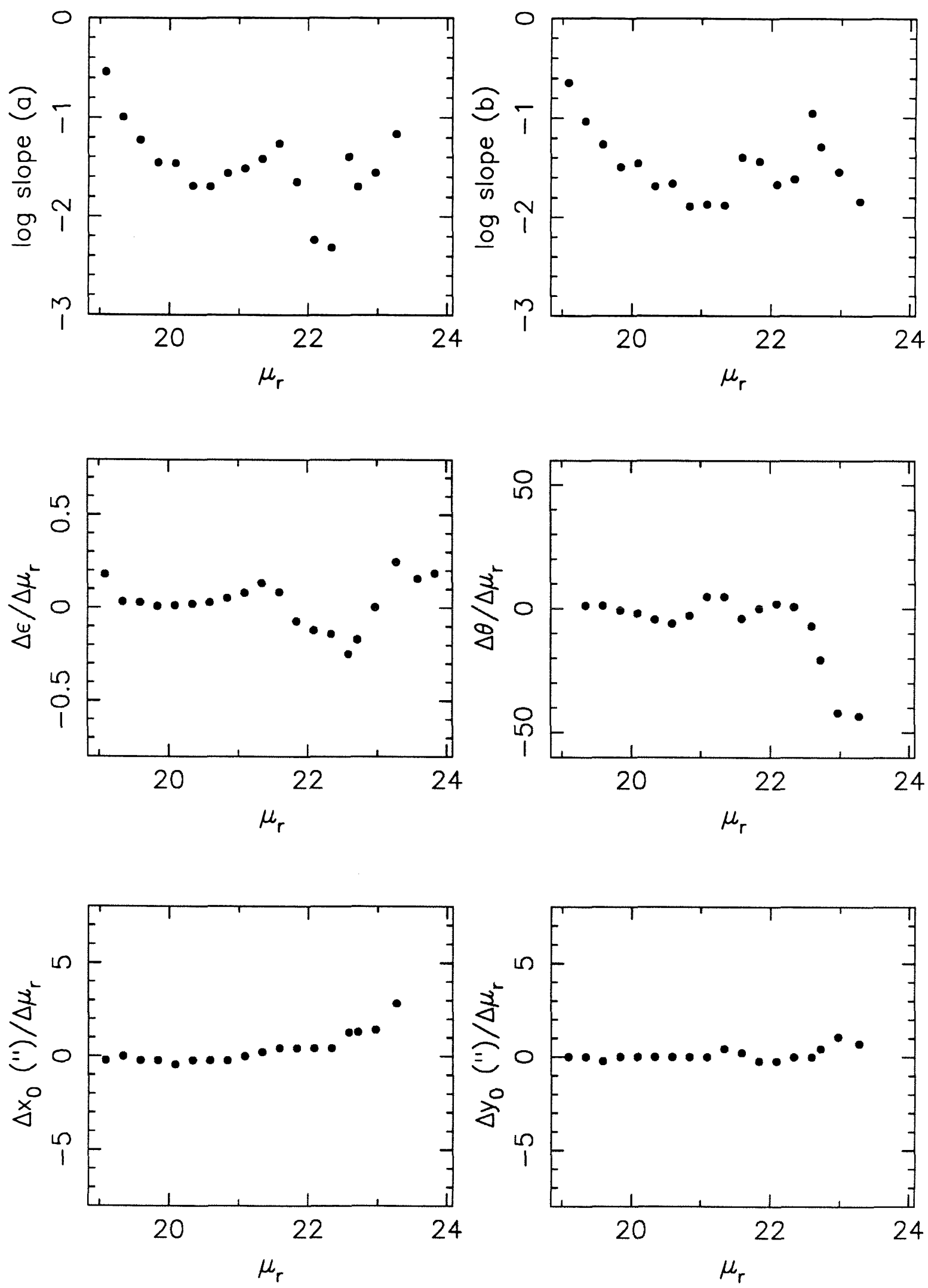

Figure 1 cont'd. A1383 

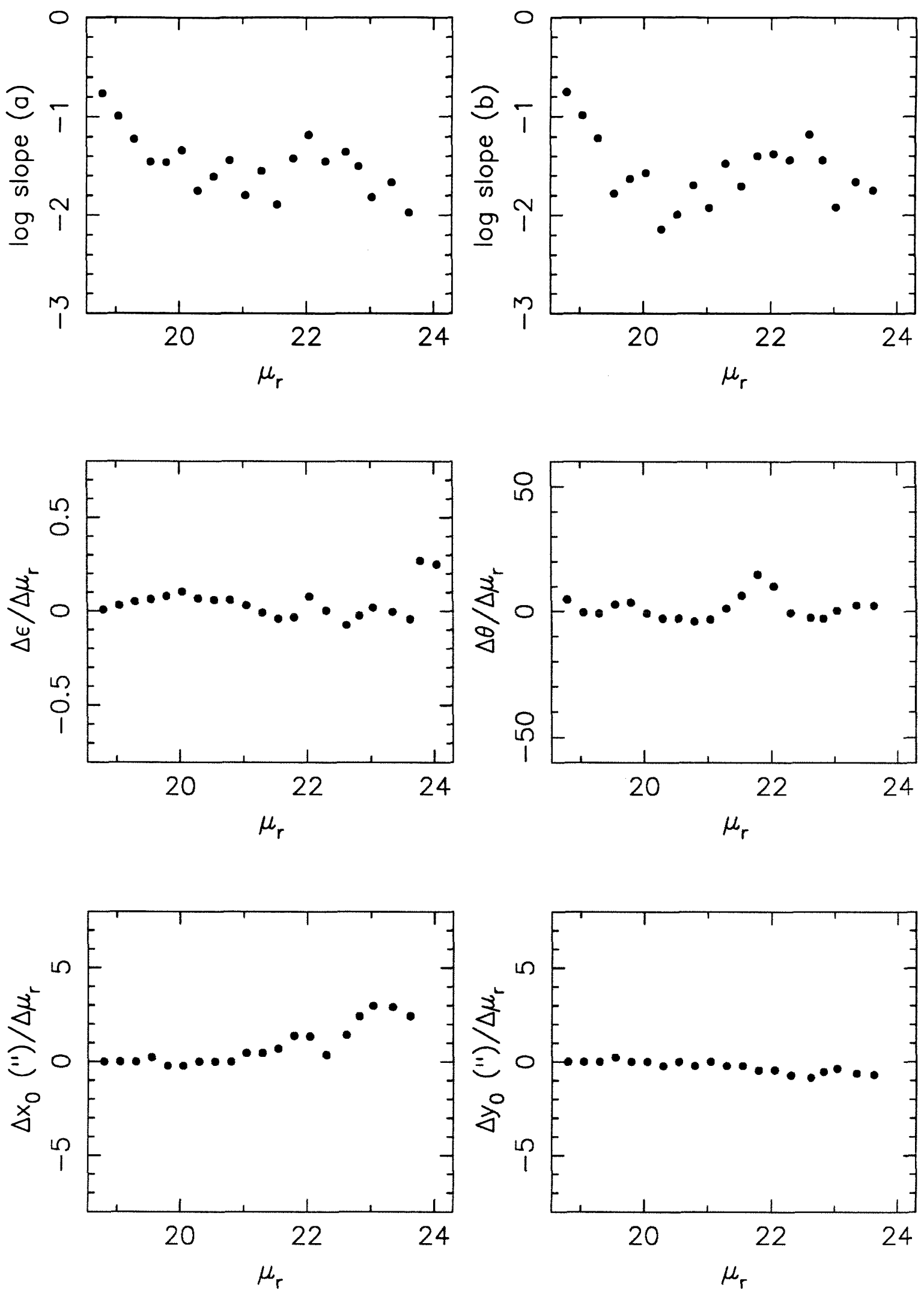

Figure 1 cont'd. A1630 

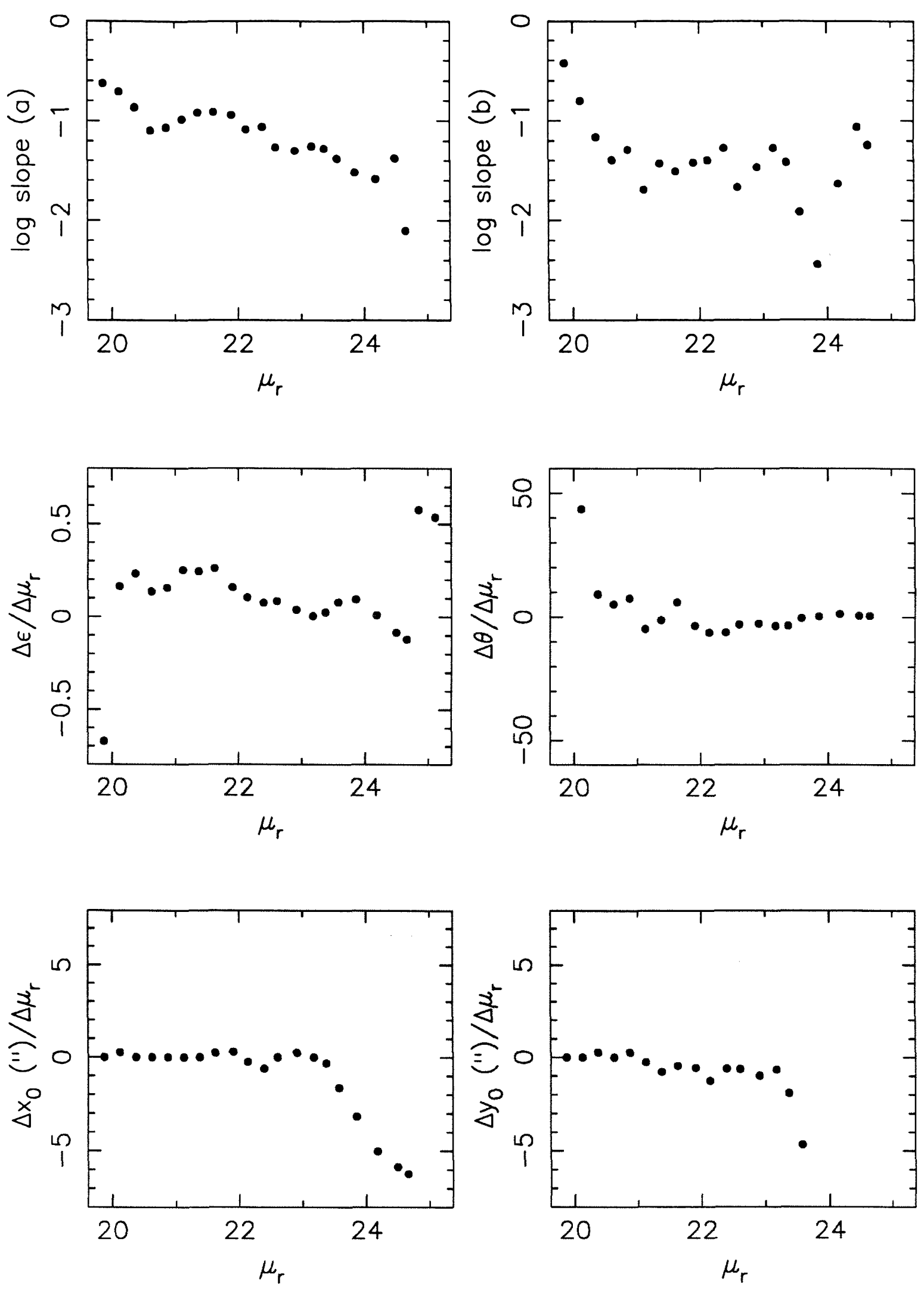

Figure 1 cont'd. A1650 

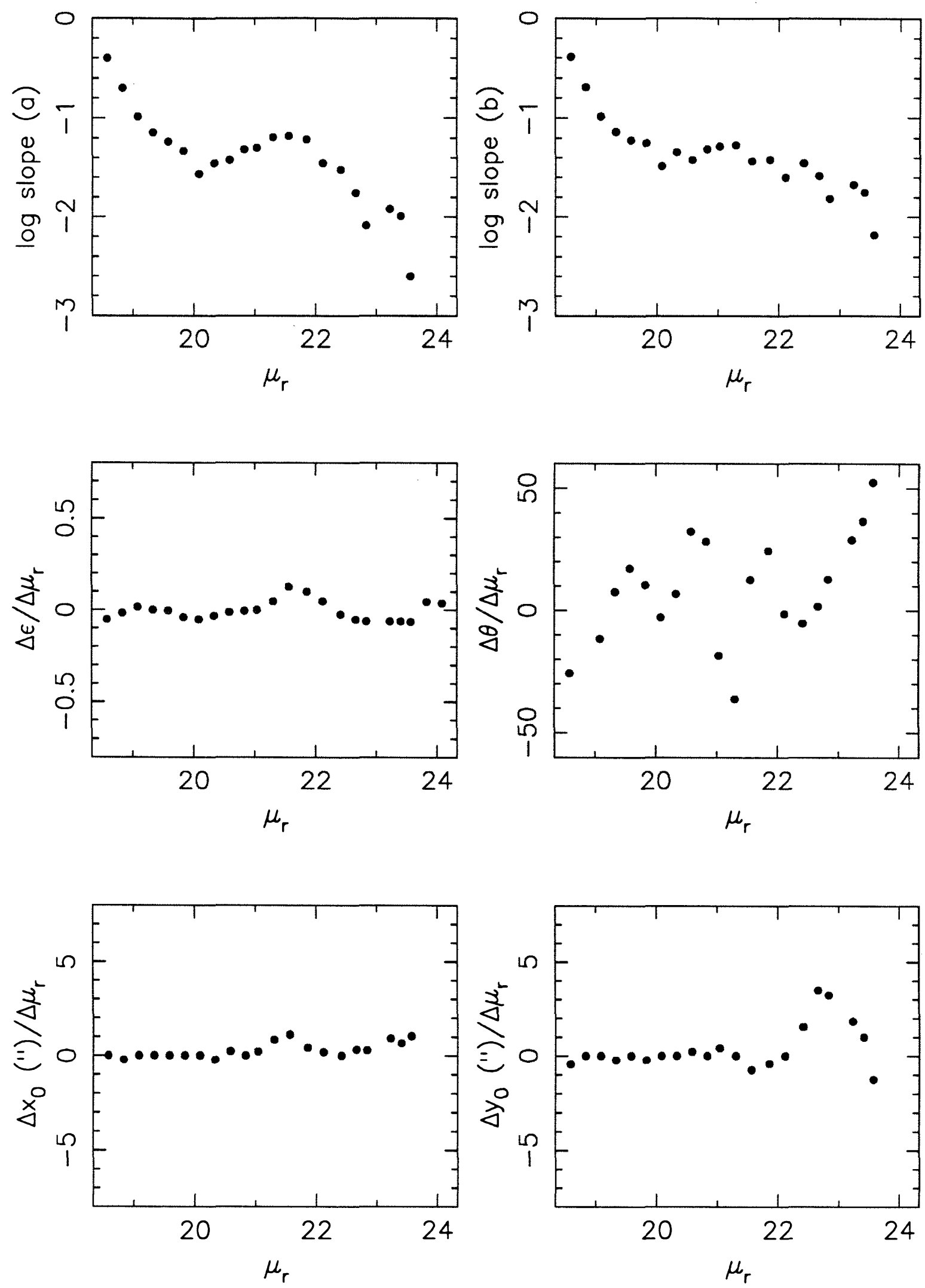

Figure 1 cont'd. A1656 B 

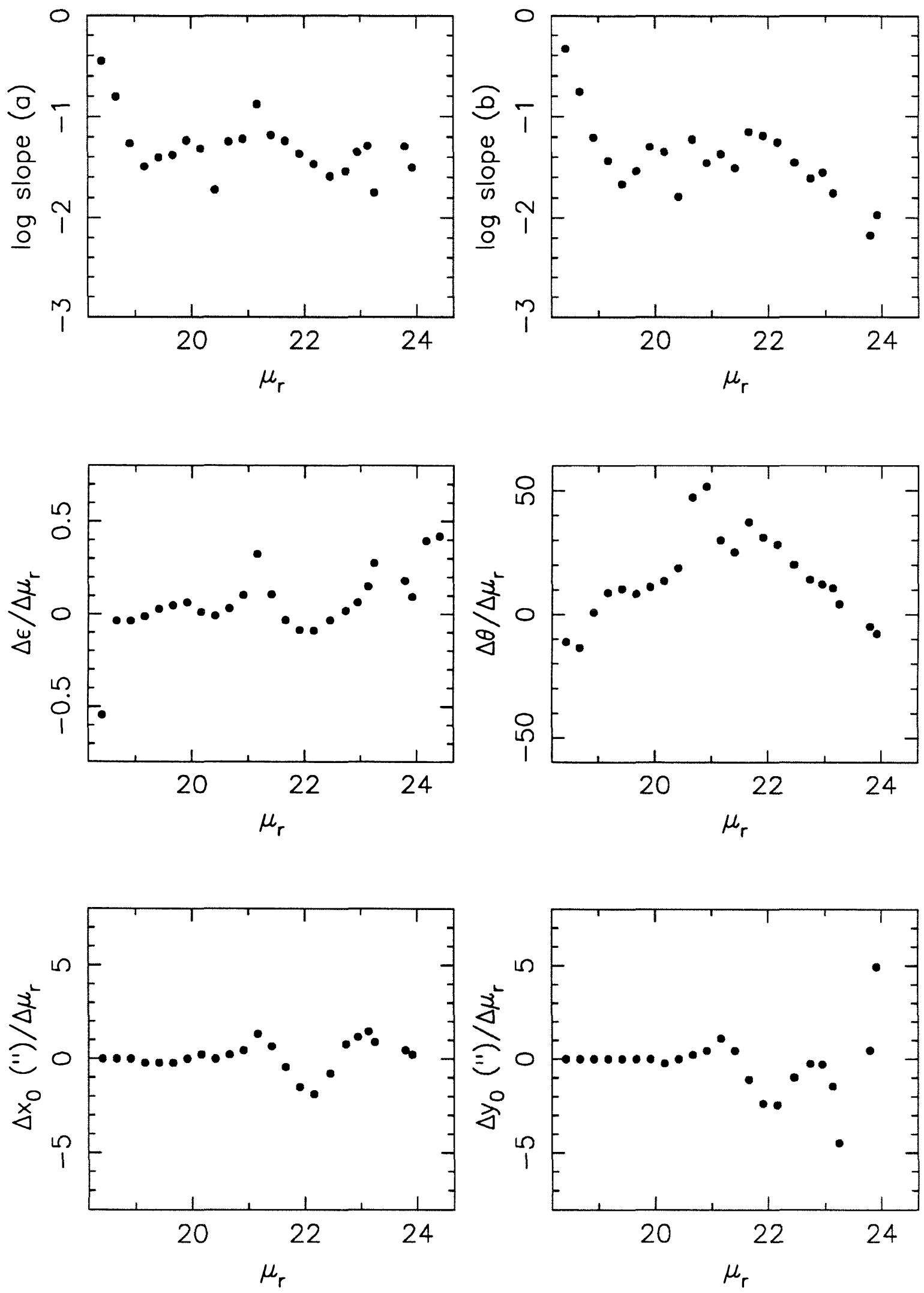

Figure 1 cont'd. A1767 

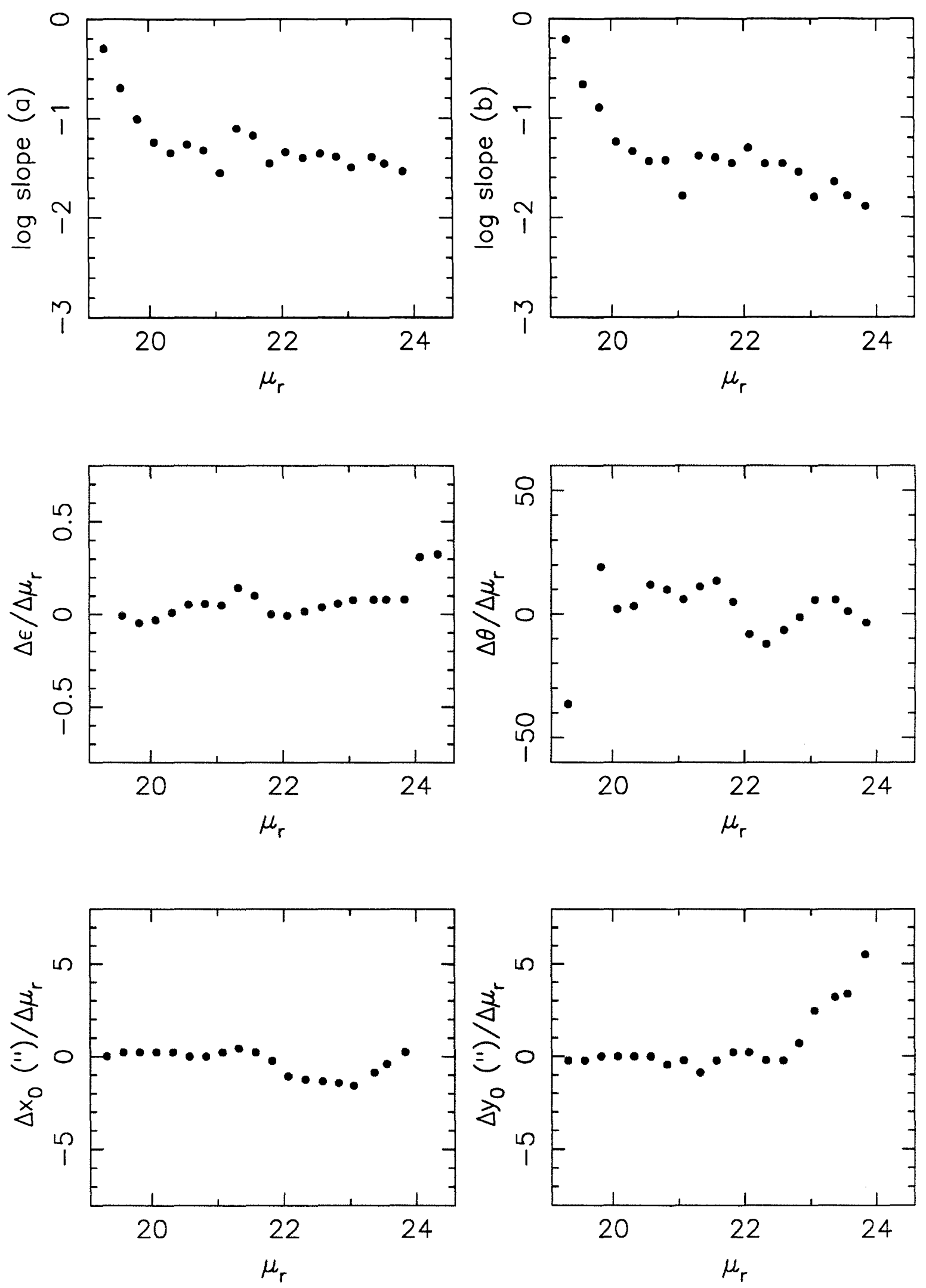

Figure 1 cont'd. A1795 

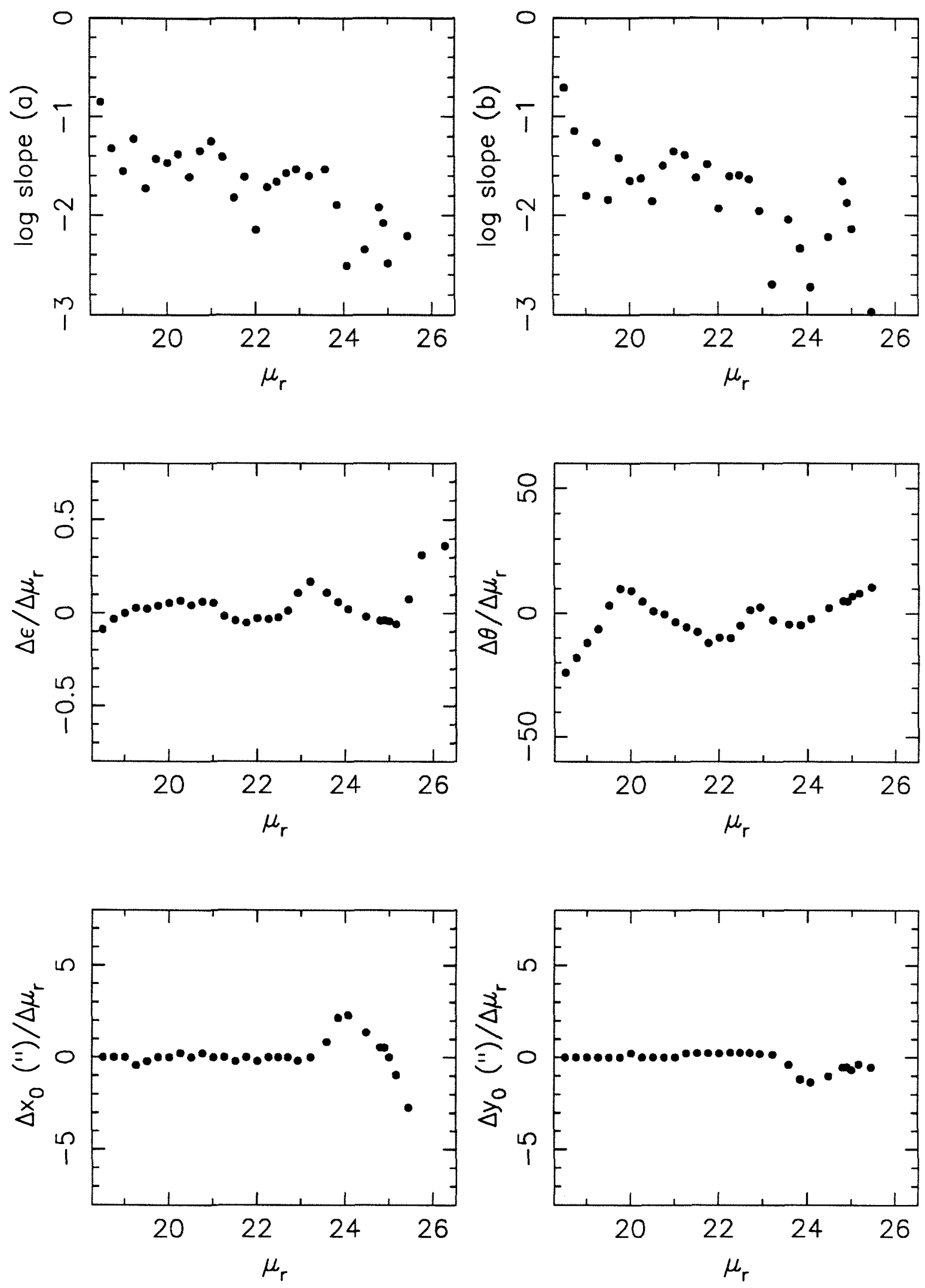

Figure 1 cont'd. A1809 

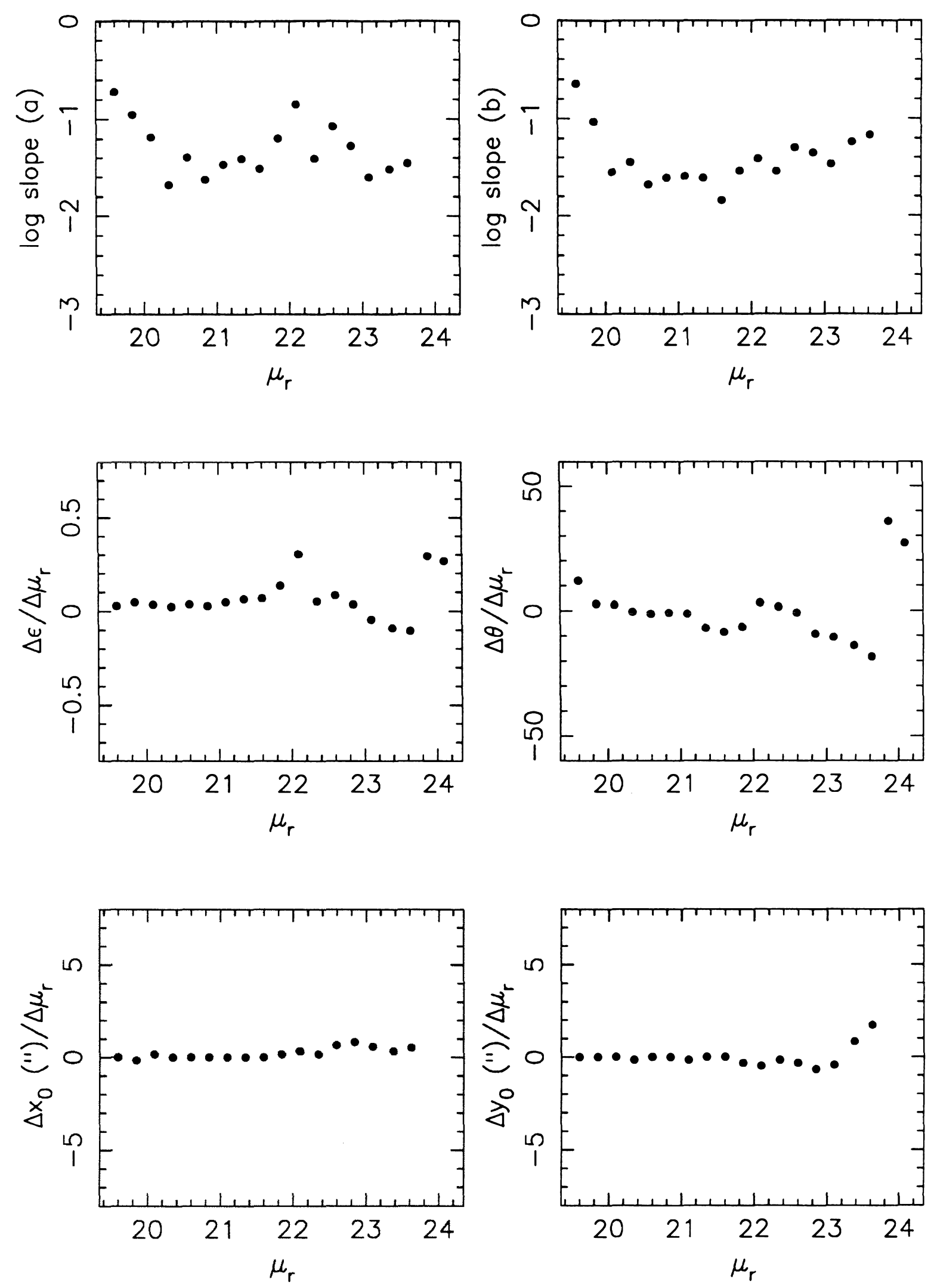

Figure 1 cont'd. A1921 

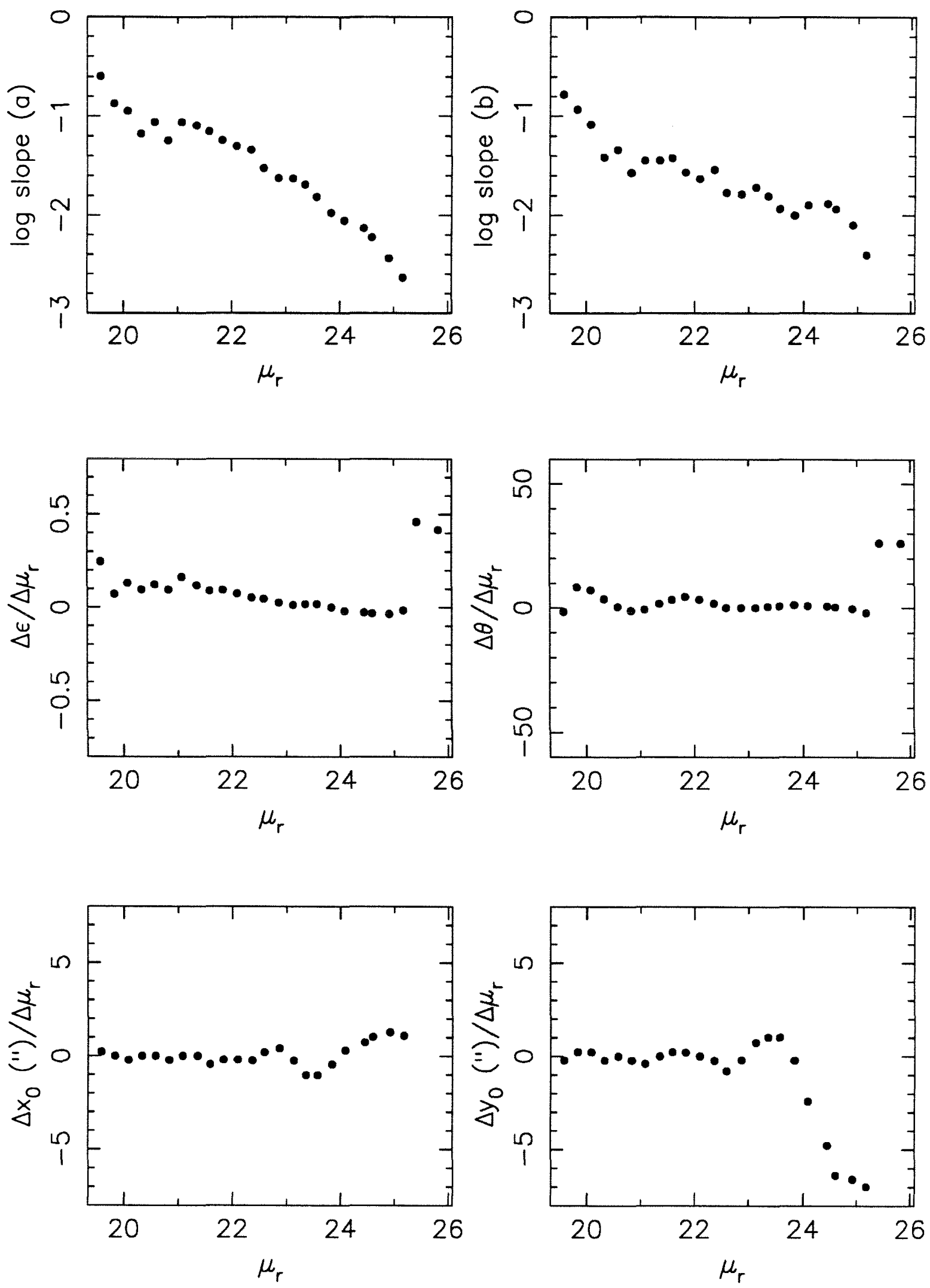

Figure 1 cont'd. A2029 

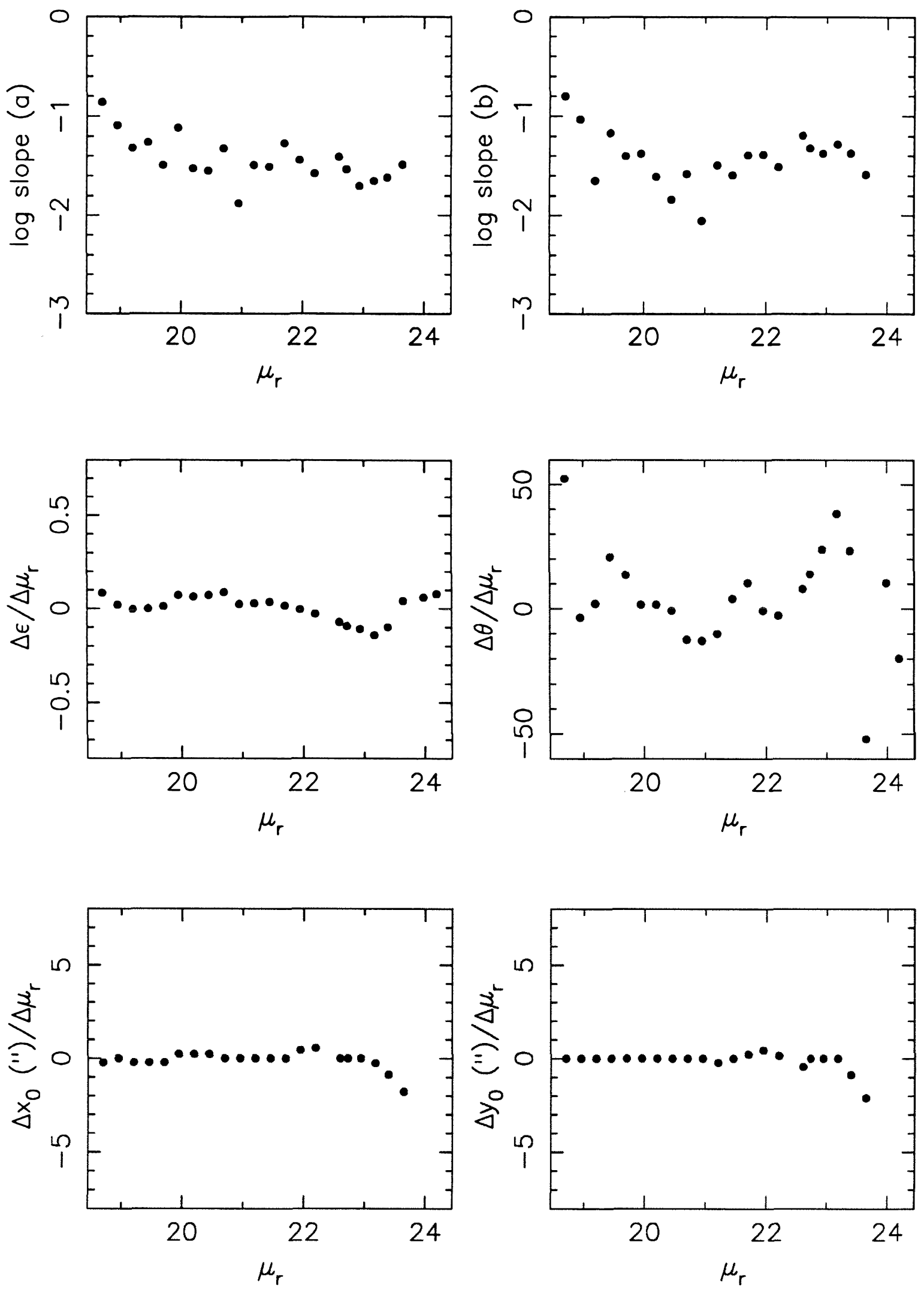

Figure 1 cont'd. A2065 

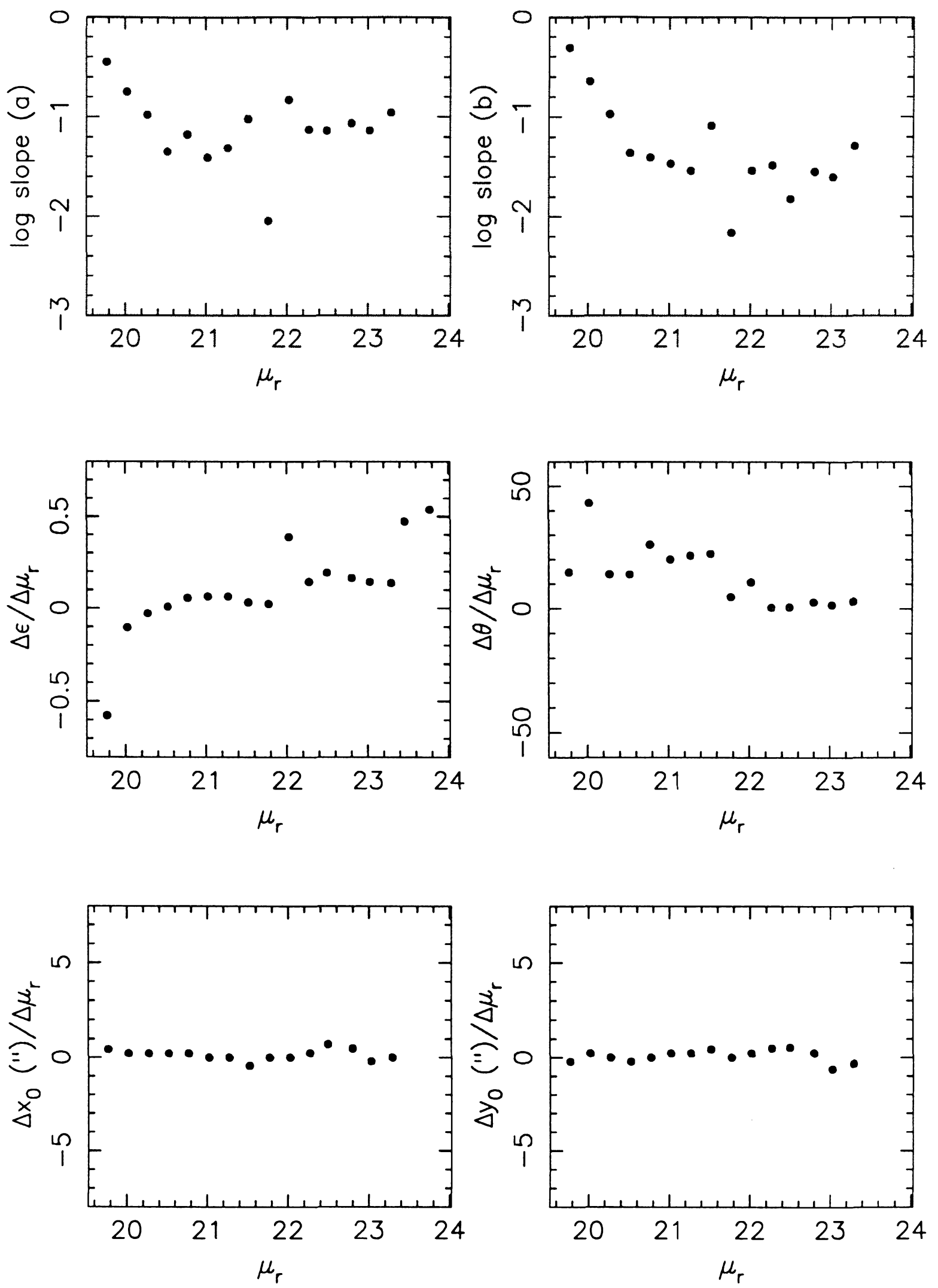

Figure 1 cont'd. A2142 A 

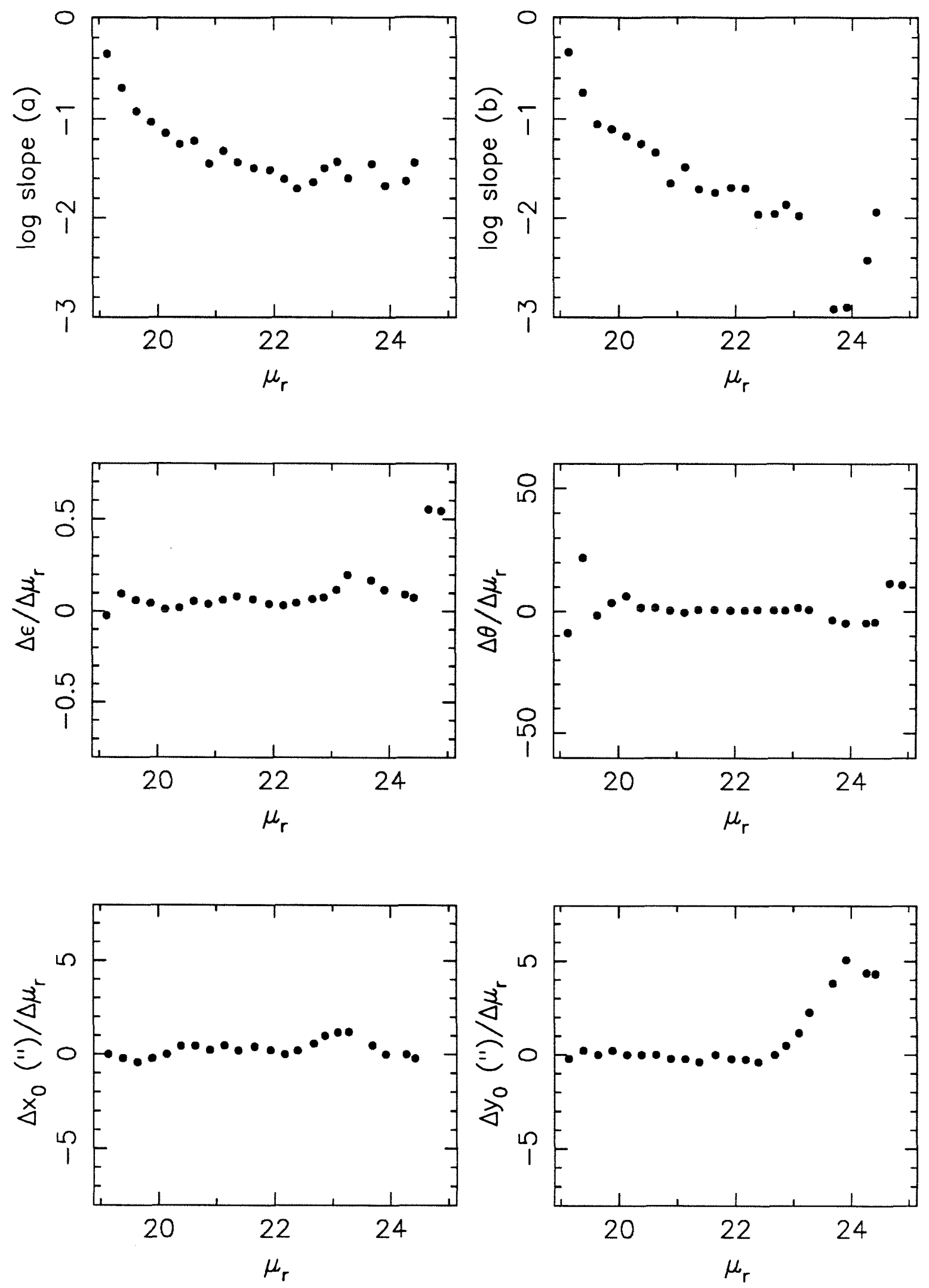

Figure 1 cont'd. A2147 

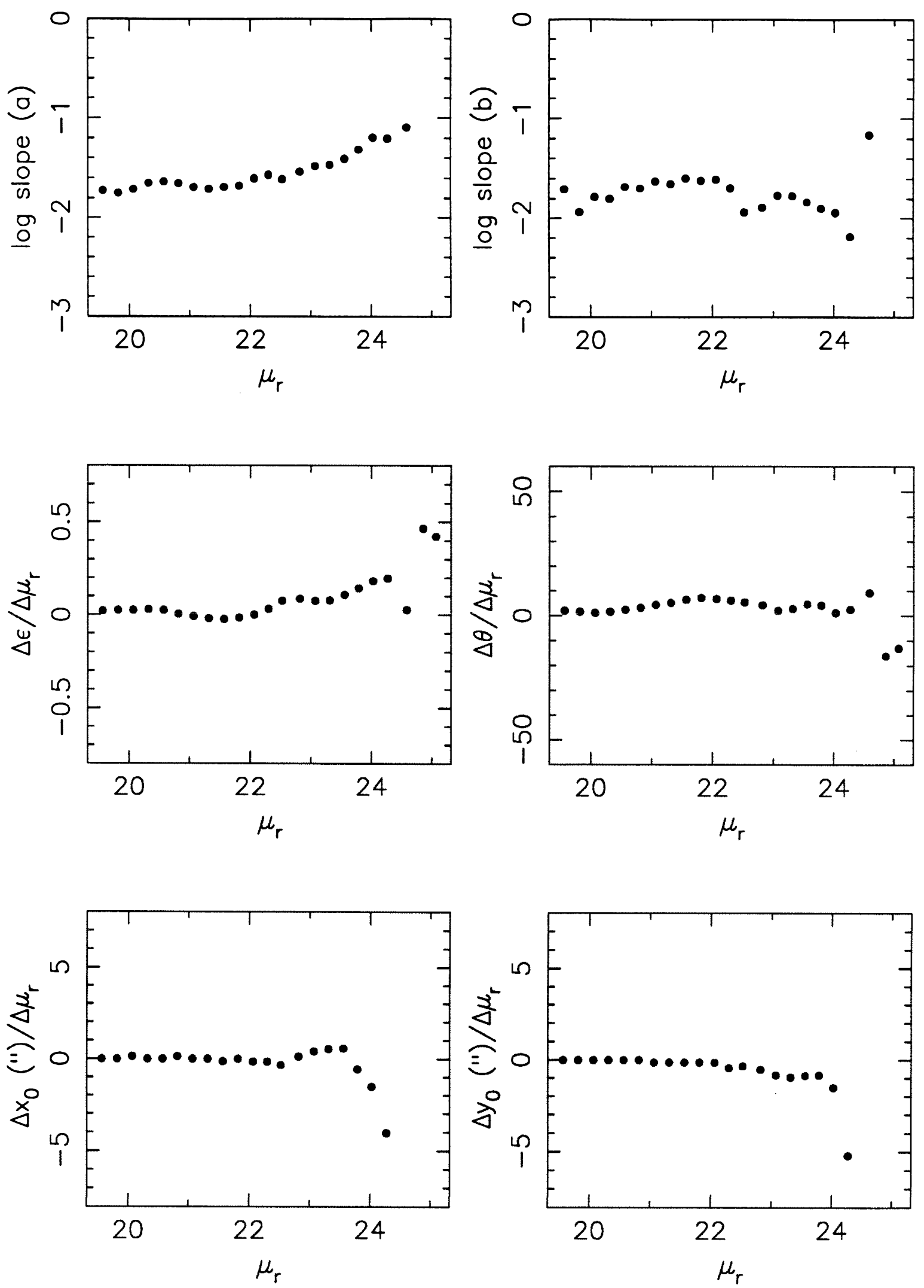

Figure 1 cont'd. NGC 6034 

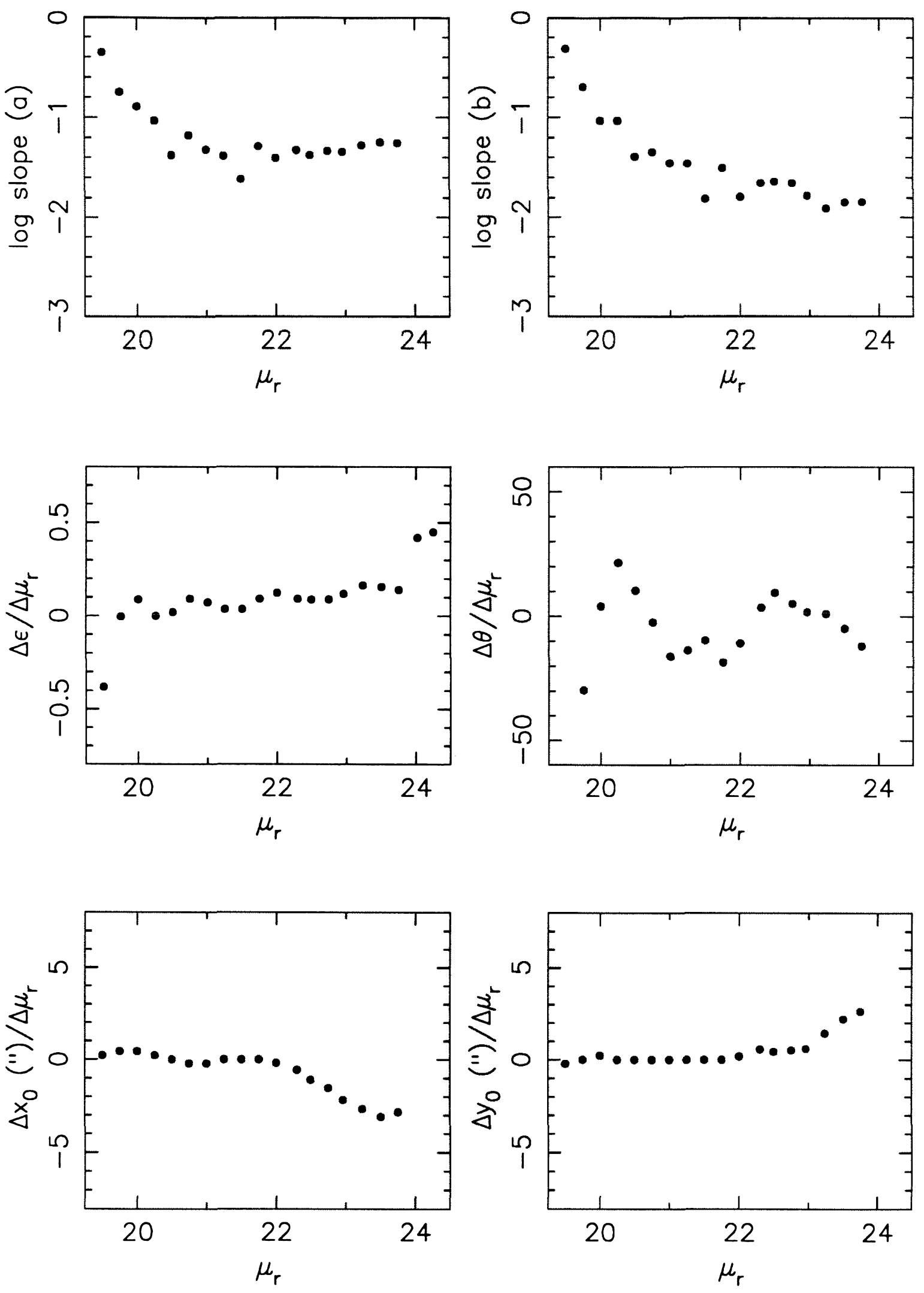

Figure 1 cont'd. A2420 

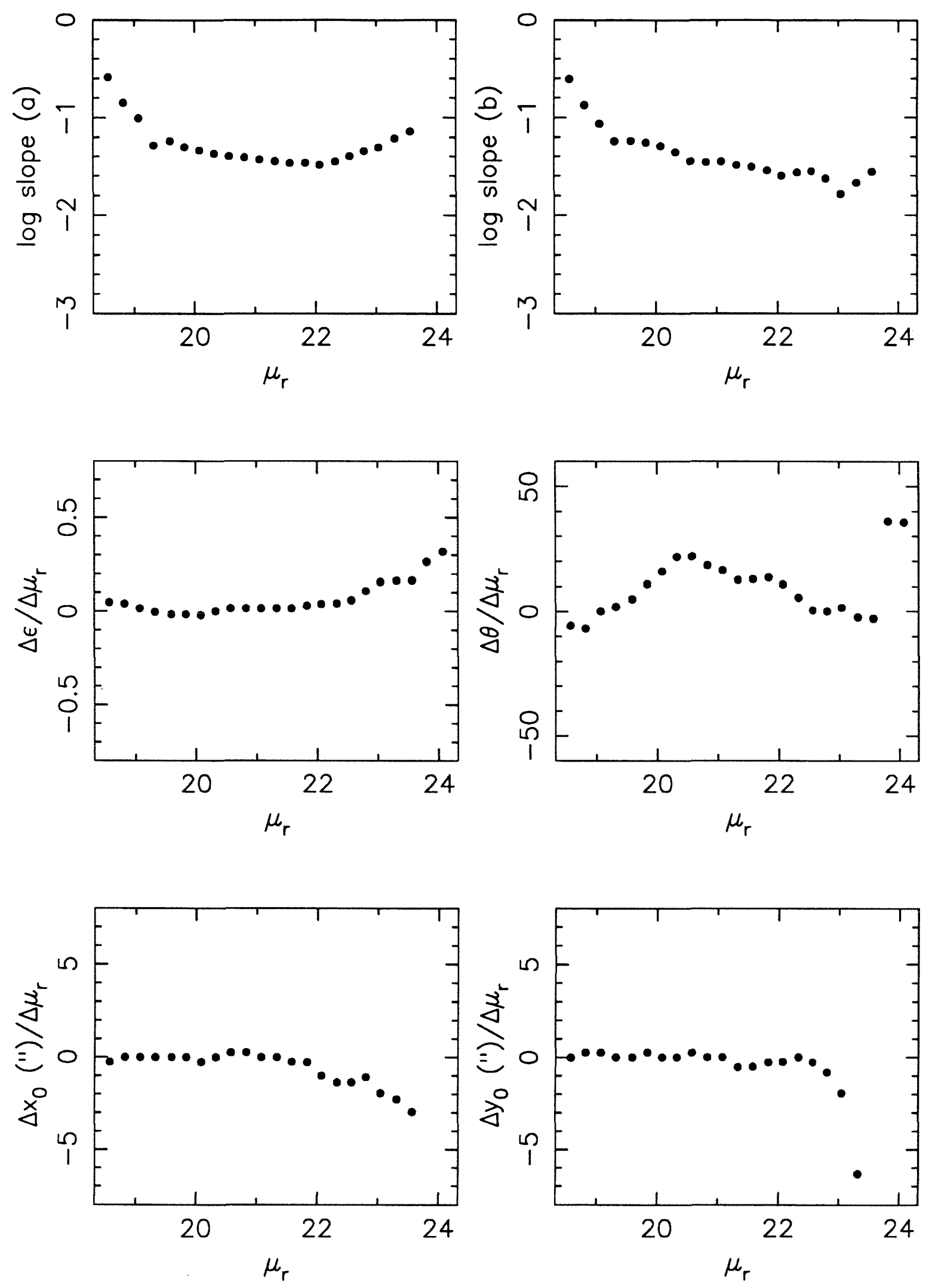

Figure 1 cont'd. NGC 5044 


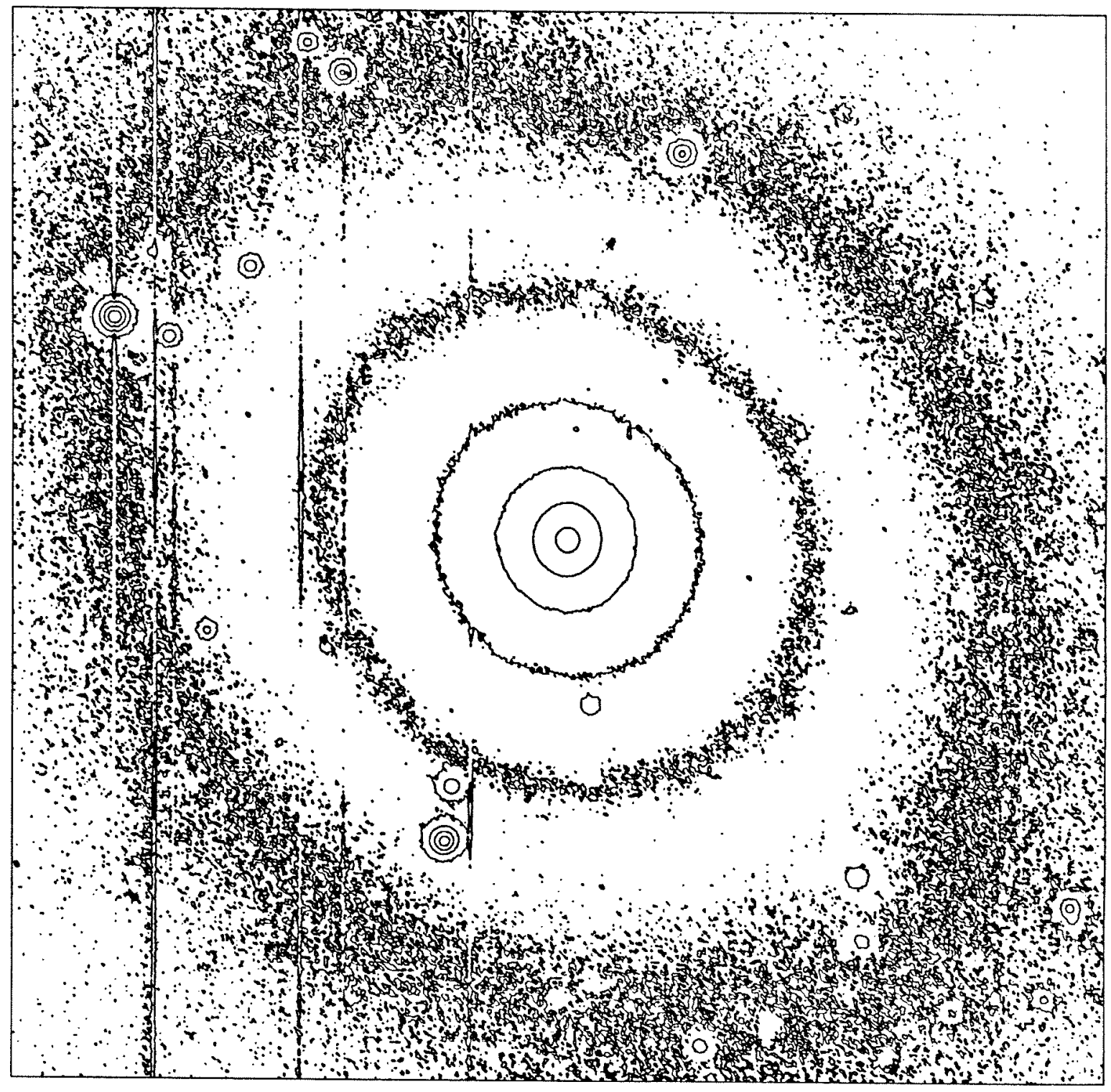

Figure 2. NGC 5044 


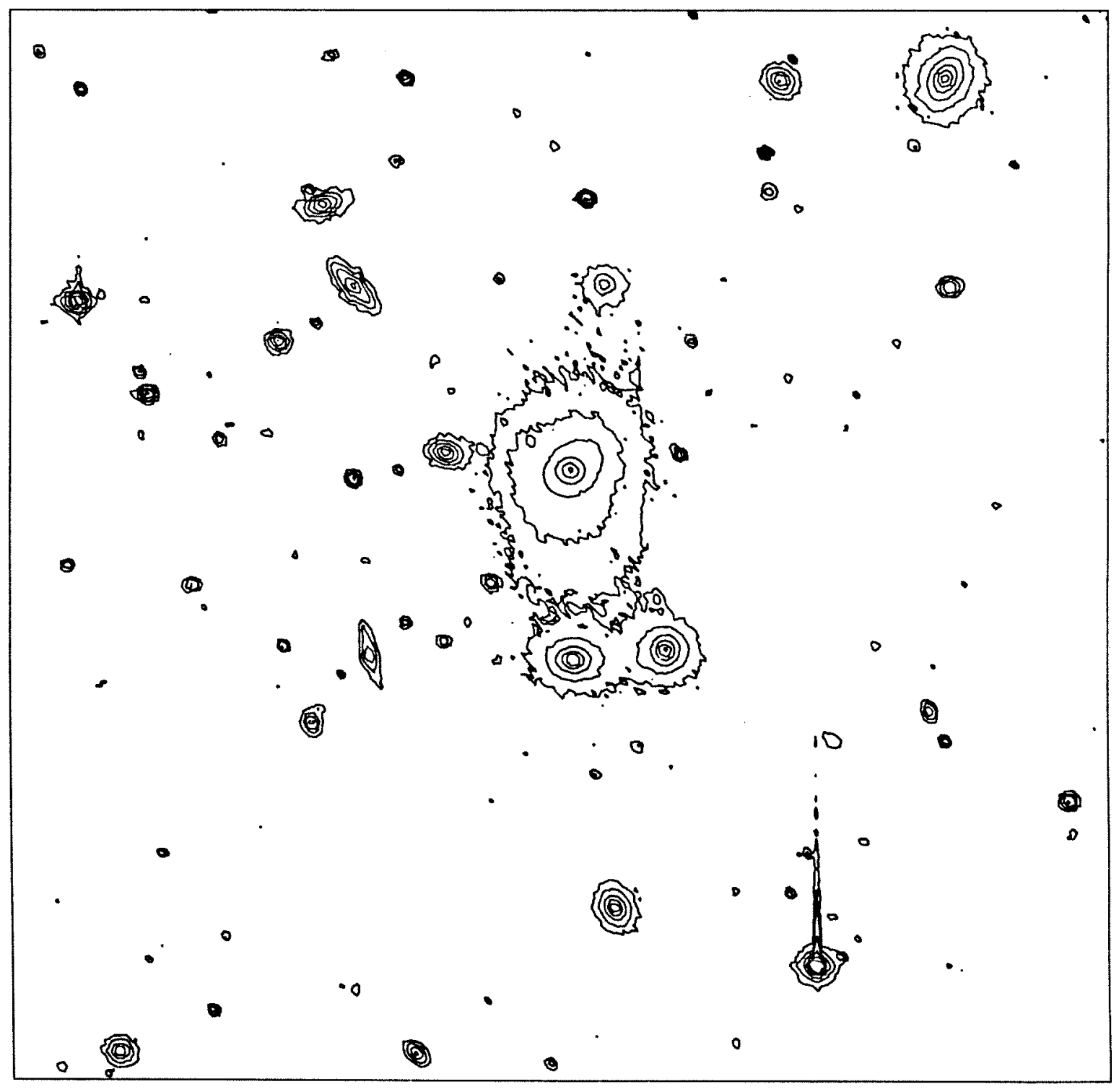

Figure 3. A1767 


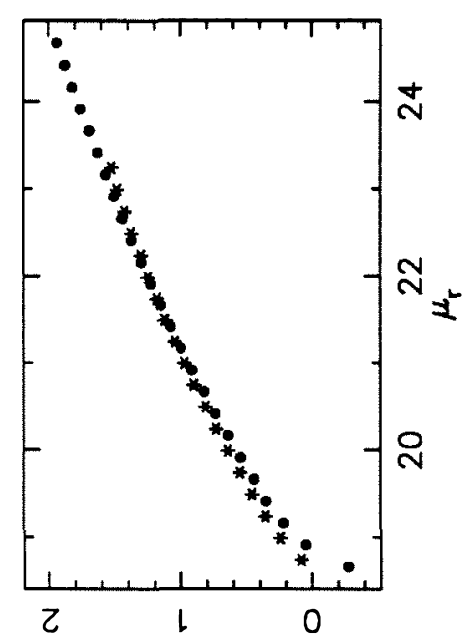

(..)sn!pod 6ol

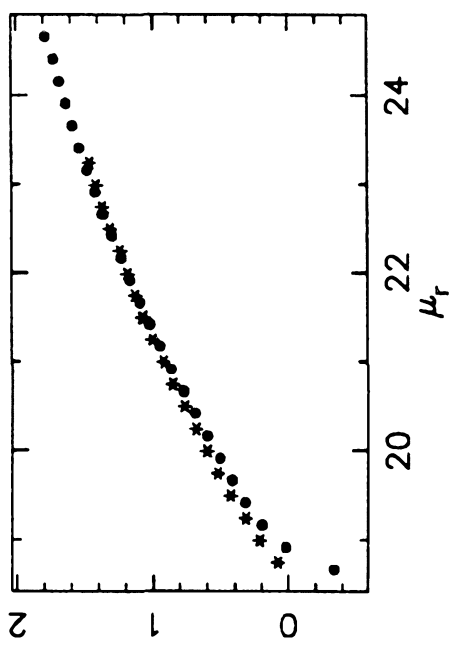

(.1) 9601

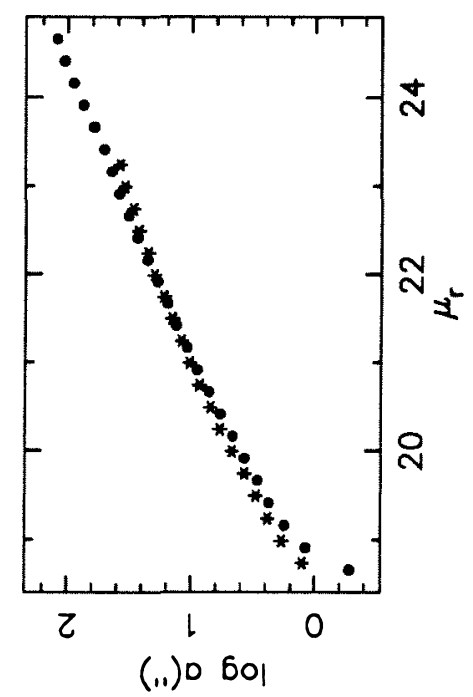

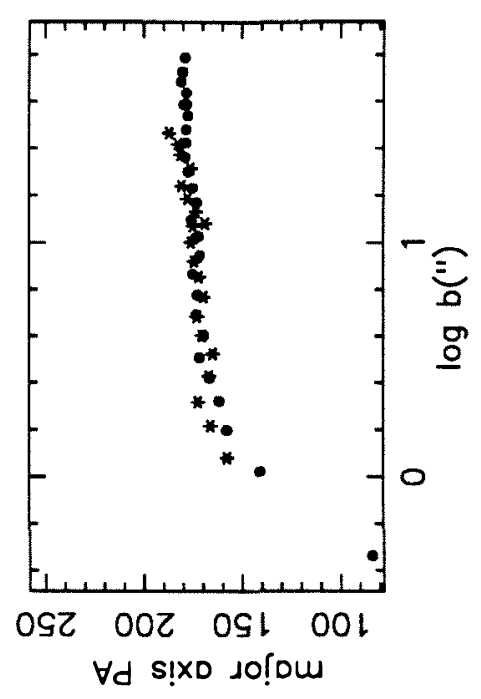

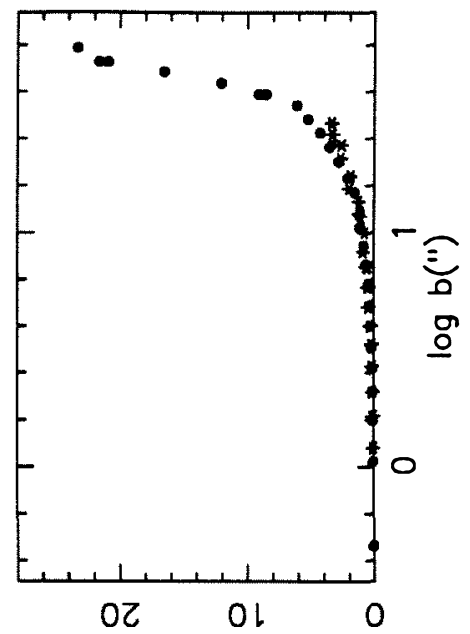

(11) $7 !+10$ Dub!s auo
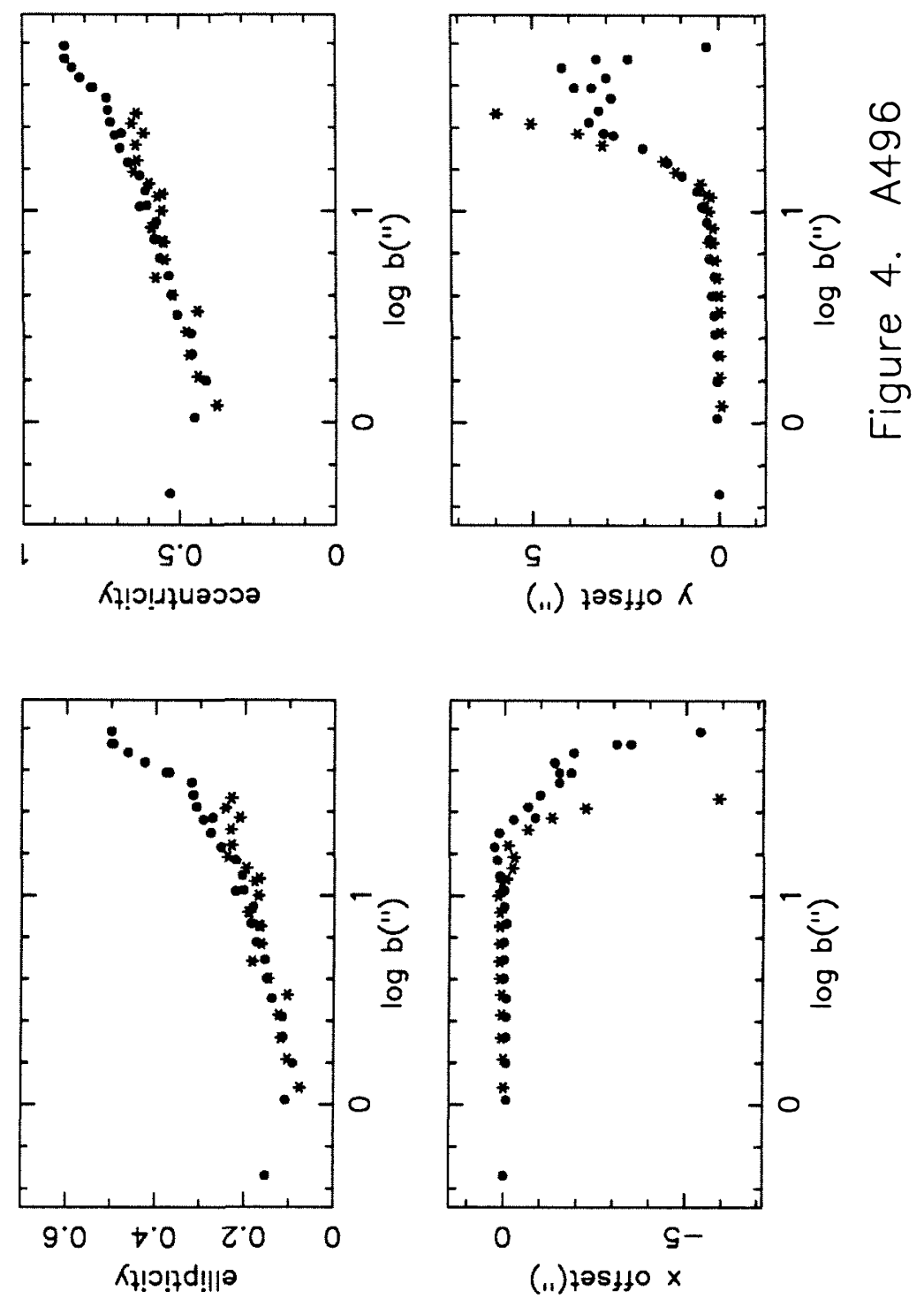
-175-
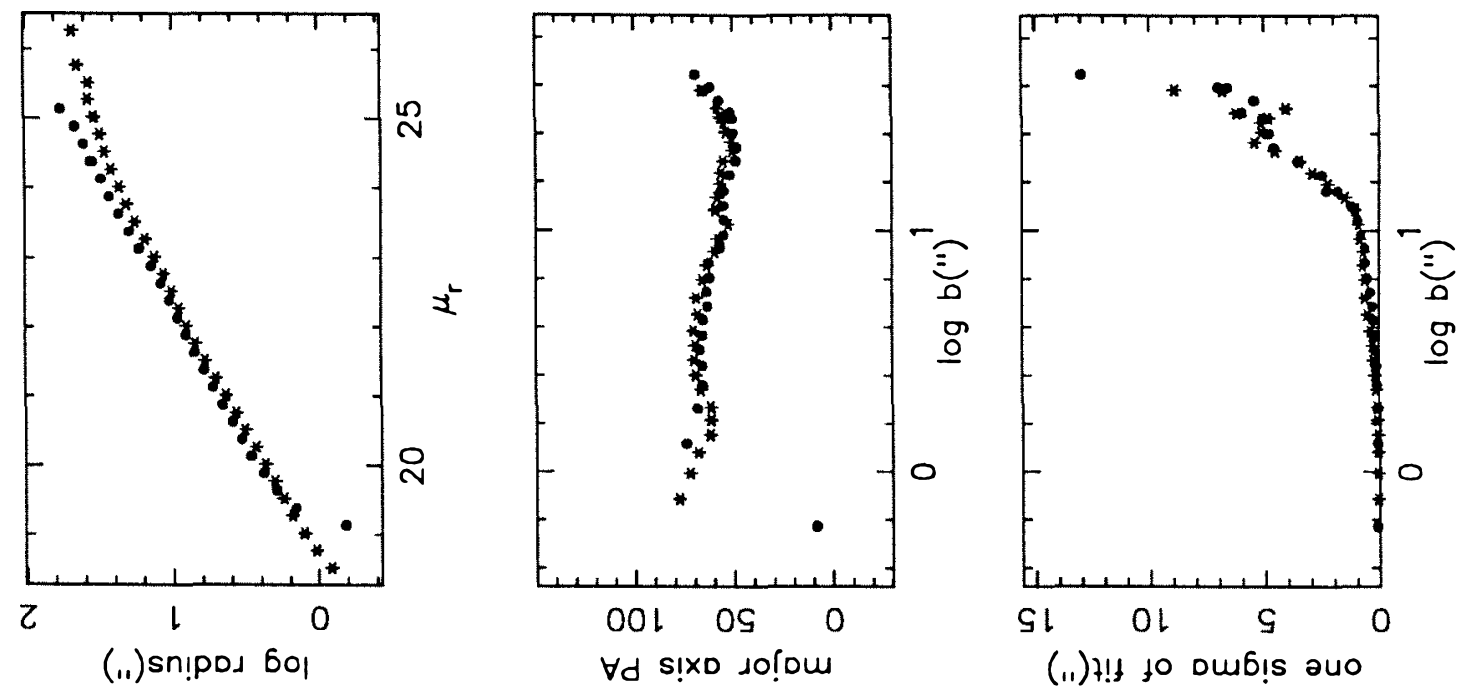

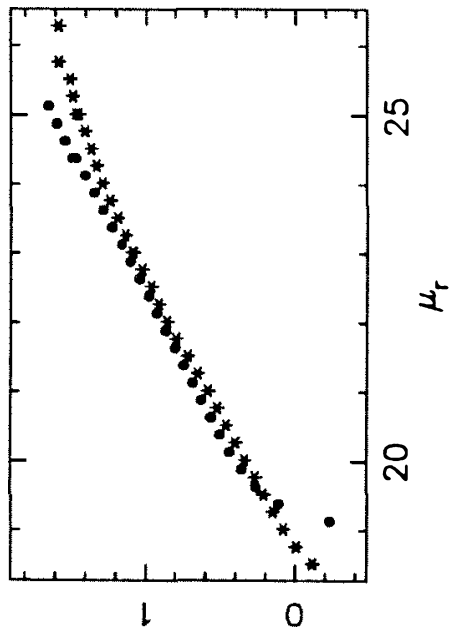

(.1) 9 60이

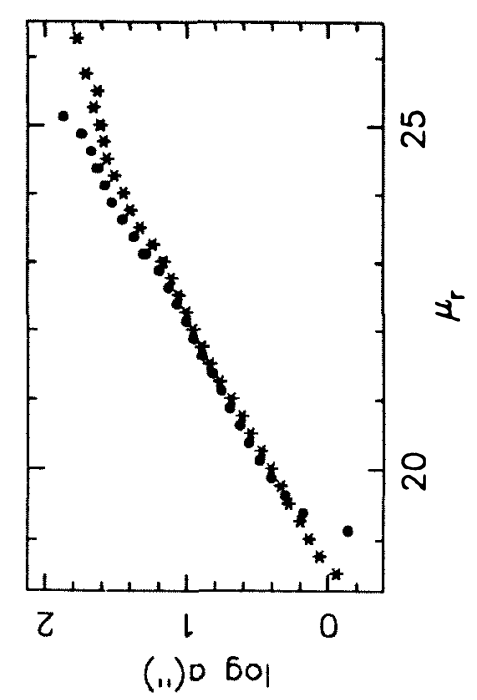

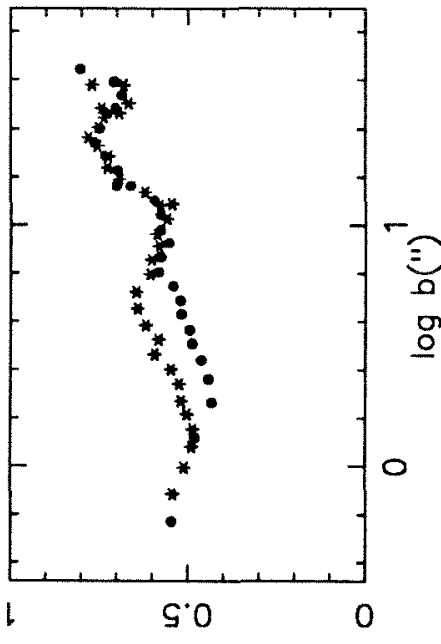

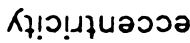

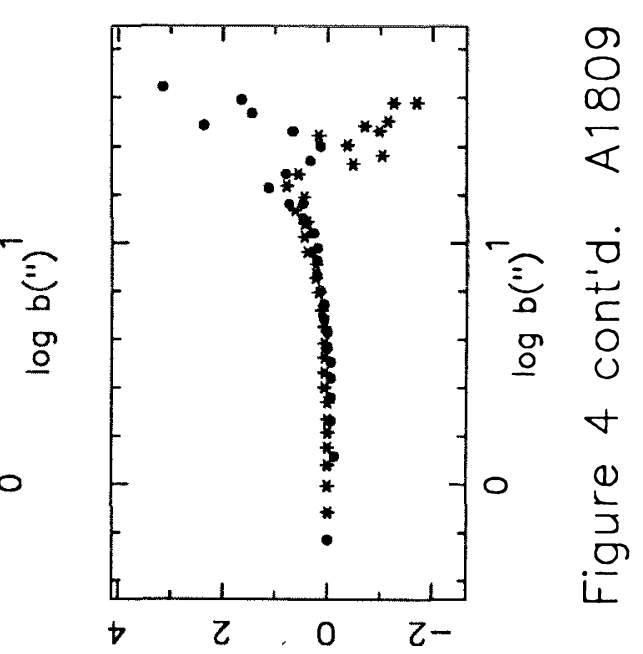

(.1) $205150 \mathrm{~K}$

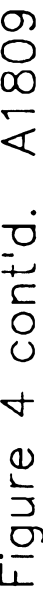
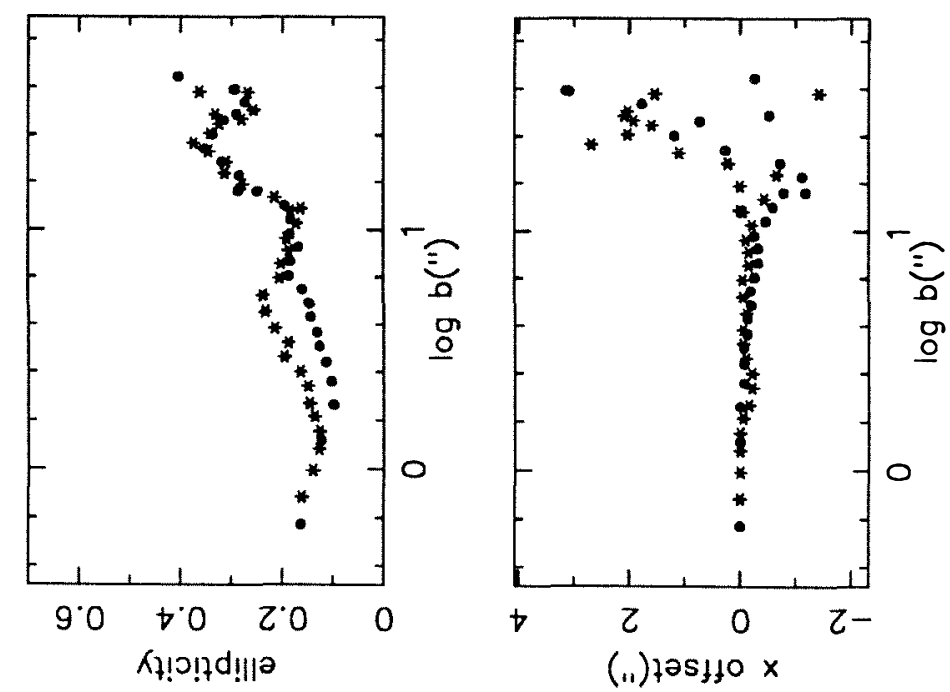
-176-

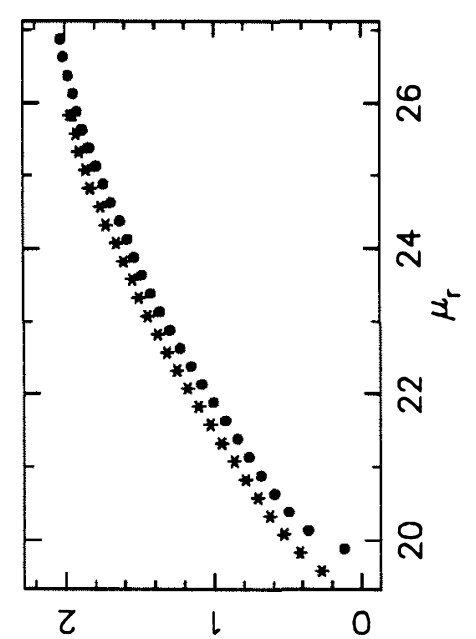

(.1) sn!pod 601

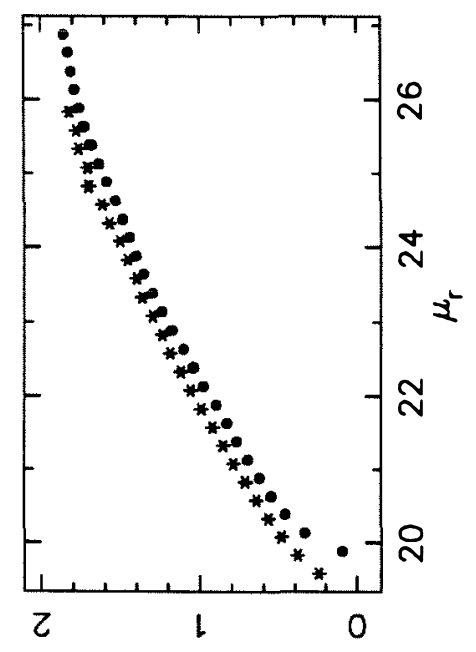

(.1) $q \mathrm{bol}$

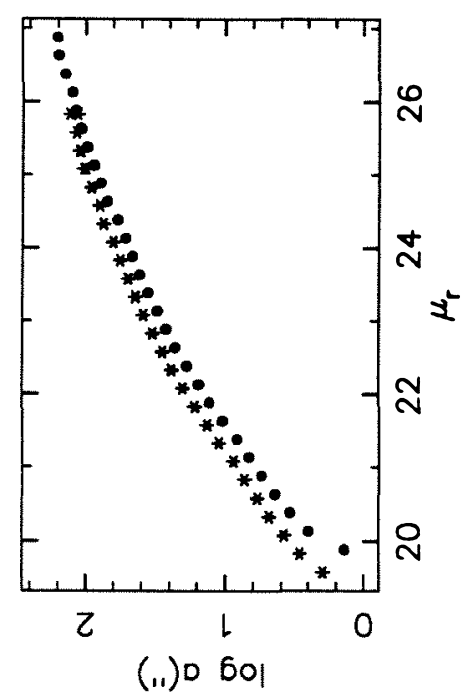

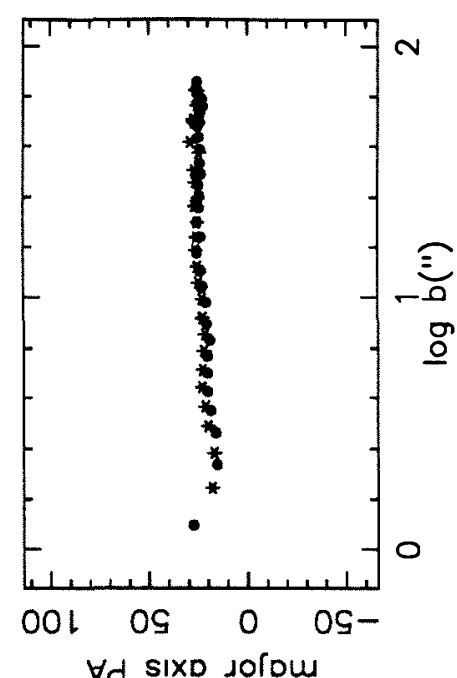

$\forall d$ s!xD
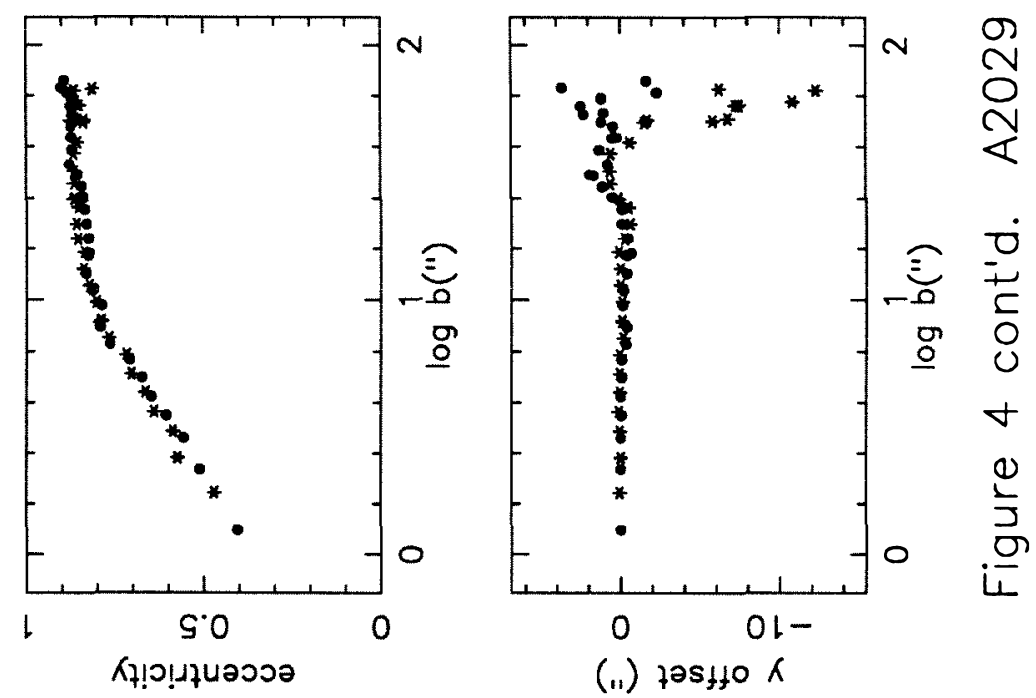

(ii) tos $40 \mathrm{~K}$

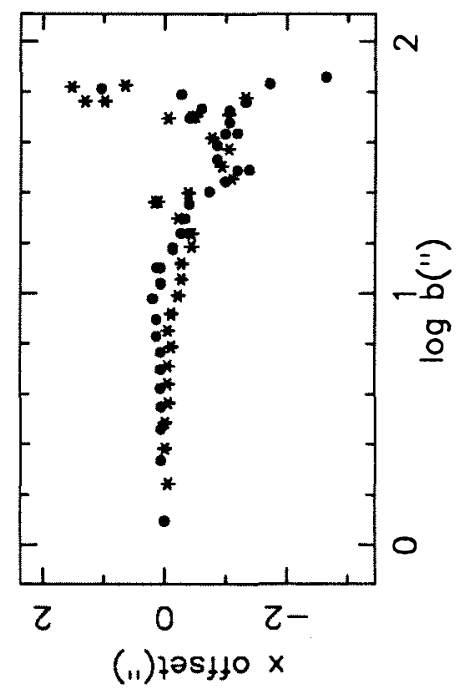




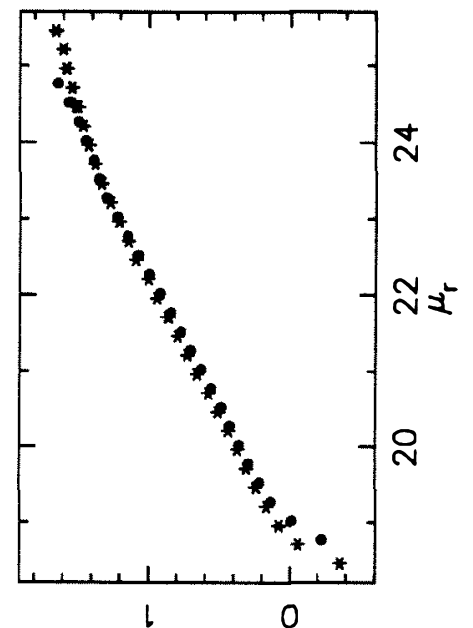

(.1)sn!pod 6ol

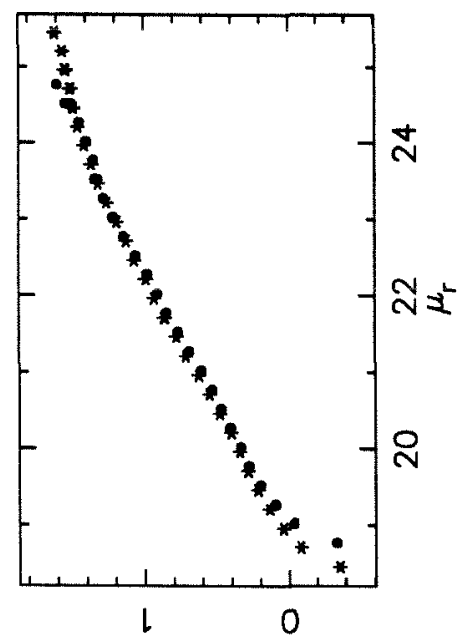

(11) 9601

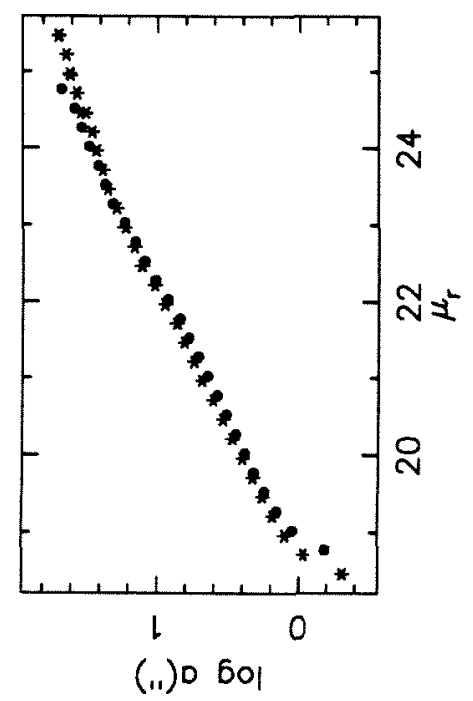

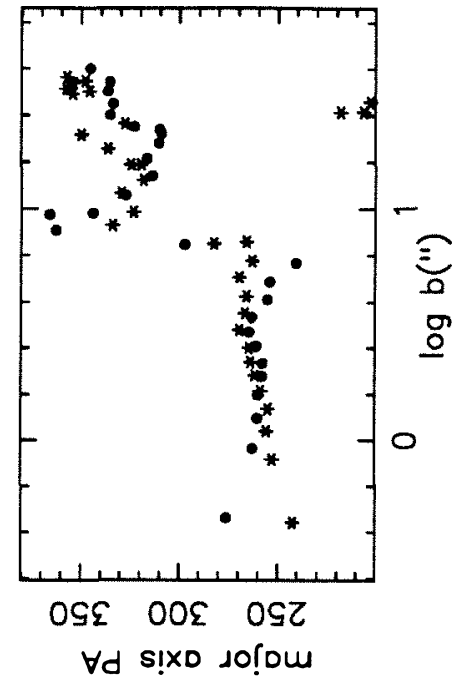
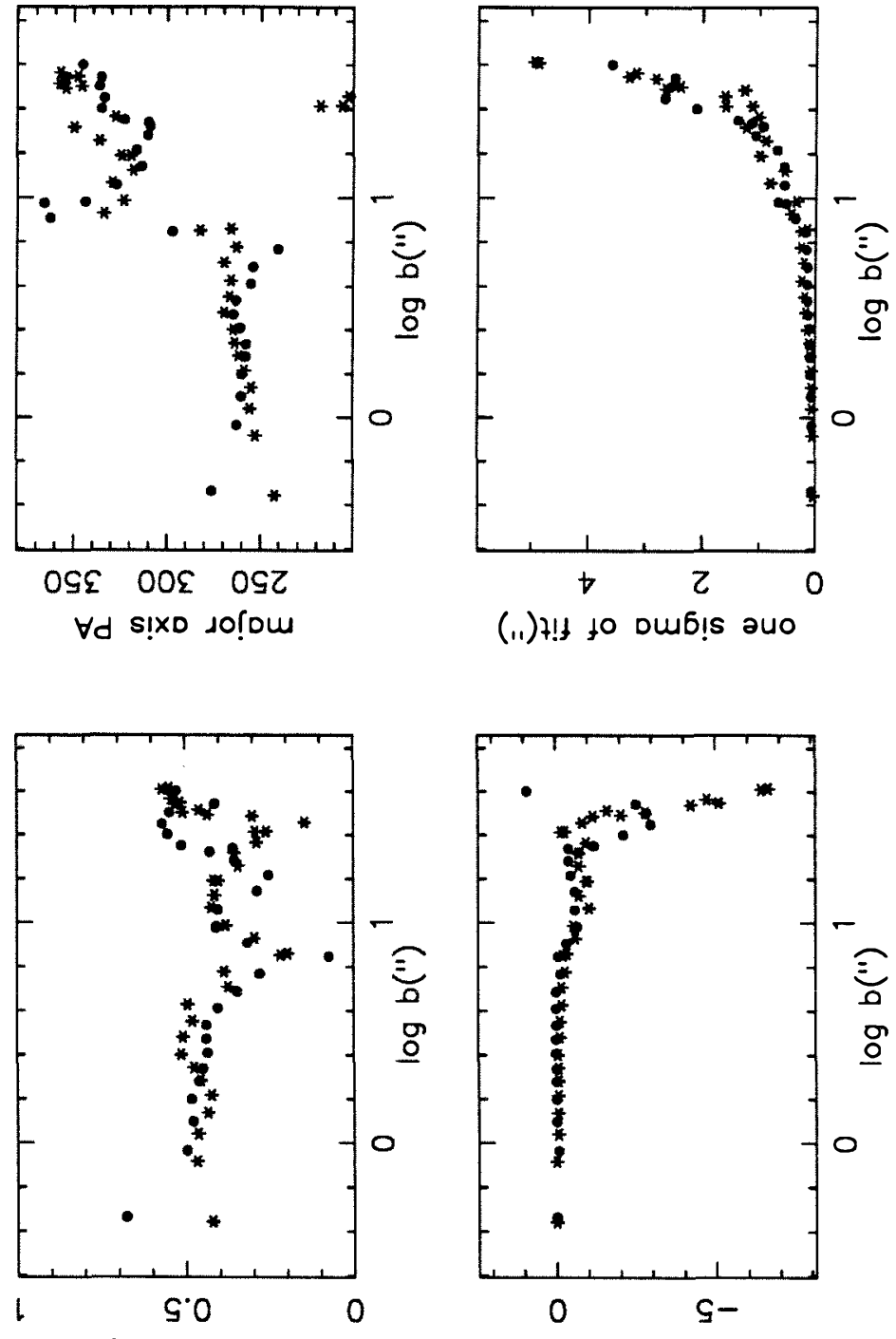

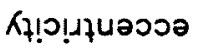

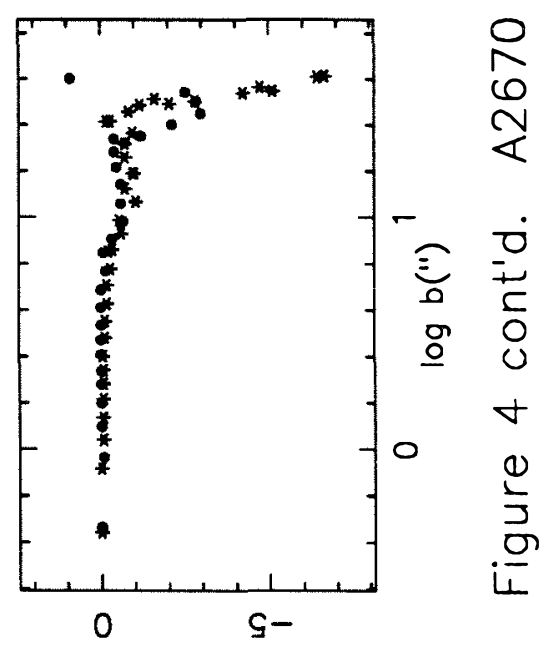

(ii) $725+10 \kappa$

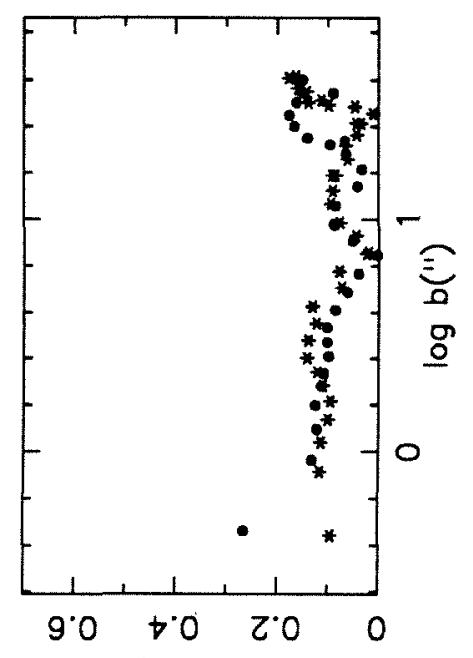

रł!ว!†d!||ә

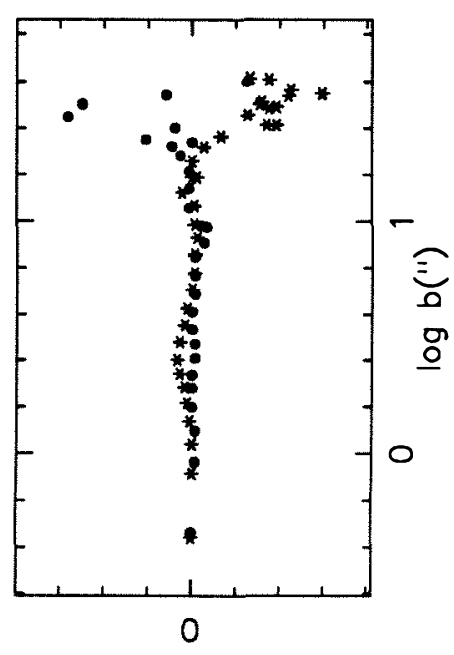

(.1) $725110 \times$ 
CHAPTER III

\author{
AN ANALYTIC METHOD FOR SEPARATING THE \\ SURFACE BRIGHTNESS PROFILES OF MULTIPLE NUCLEI
}

\author{
Alain Porter
}

to be submitted to The Astronomical Journal 
Let us make computations of the stars.

Peter Hammill 


\begin{abstract}
An analytic decomposition of the components of a binary galaxy is derived and applied to 12 double nuclei of brightest cluster ellipticals. The sole assumption necessary is that each component of the light distribution has point reflection symmetry about a unique center. The method is competitive with the least squares decomposition developed by Lauer $(1986,1988)$ under a variety of conditions and can also faithfully reproduce limited amounts of asymmetry in a surface brightness profile.
\end{abstract}




\section{INTRODUCTION: DEALING WITH MULTIPLE NUCLEI}

Multiple nuclei are one of the most characteristic features of the brightest ellipticals in rich clusters (henceforth BCEs; v. Schneider, Gunn, and Hoessel 1983; Hoessel and Schneider 1985 [HS]), and at the same time one of the most difficult to study. The first question that arises with respect to a compact group of galaxy nuclei is that of association: are they bound, or even physically close? The high galaxy density in the core of a rich cluster makes it plausible that such a group could be a chance projection. Traditionally the only approach to this problem (in the absence of obvious interactions) has been to measure the relative radial velocities of the nuclei. Many such observations have been made (Jenner 1974; Tonry 1985; Hoessel, Borne, and Schneider 1985; Smith et al. 1985), and enough large-velocity differences have been found to be somewhat embarrassing to standard merger scenarios (Merritt 1985).

The abundance of multiple nuclei among BCEs also complicates attempts to use these galaxies as standard candles for cosmology. Since multiple nuclei are progressively less well resolved at larger redshifts, attempts to separate out the light of the brightest or dynamically dominant component to reduce scatter in the Hubble diagram may just introduce systematic errors at least as large as those eliminated. It was for this reason that HS adopted a strictly formal definition of the center and metric luminosity of a $\mathrm{BCE}$ which could be applied to both single and multiple nuclei in an unbiased way.

Such an approach, although optimal for the study of large samples, tells less than nothing about individual physically interesting BCEs such as NGC 6166 . In their isophotometry of the HS sample, Porter, Schneider, and Hoessel (1988, 
$\mathrm{PSH}$ ) followed the profiles of individual nuclei as far as possible but still had to concede data in large regions where they overlap. By far the best decomposition of multiple nuclei to date has been performed by Lauer $(1986,1988)$. His program fits concentric ellipses to the isophotes of up to four overlapping galaxies simultaneously. This generates separate images of each component and, equally importantly, images of the residuals or departures from the assumption, which are likely to represent interactions among the galaxies fitted. He has applied this technique to 16 multiplenucleus systems with great success.

Lauer's is a least-squares algorithm. Guesses at the isophote parameters are tested by:

1) generating a model picture by logarithmic interpolation between isophotes,

2) summing the squares of the differences between the model and observed images, pixel by pixel, and

3) using the local gradient of this $\chi^{2}$ to generate the next-best guess. While this produces satisfactory results, it is computationally expensive, and formal uncertainties in the numerous varied parameters are not recorded. In the analytic spirit I have derived a simple algorithm for the exact decomposition of a pair of overlapping galaxies. The sole assumption made about the light distribution of each component is that it has point-reflection symmetry about a unique center.

The main advantages of this method over Lauer's are two. The first is that the underlying assumption is much more general, allowing the method to be applied to spirals, bipolar jets, or any other objects with point reflection symmetry. In fact, as will be shown, even asymmetric light distributions are faithfully reproduced if they are sufficiently localized. The second advantage is that the algorithm is 
fast, cheap, and exact. There is no iteration and no danger that the solution is nonunique. All of the computations described in this paper took less than an hour of CPU time on a VAX 11/780.

The main advantages of Lauer's method over the one described here are also two. The first is that his approach is trivially extended to more than two components. In its present form the analytic method cannot separate more than two galaxies. Four could in principle be handled with the introduction of a second symmetry assumption, but the execution would, for practical reasons, be limited to very special cases. Lauer's second advantage is that his procedure can be modified, without too much trouble, to follow nonconcentric isophotes. The analytic method presented here succeeds precisely because of the assumption of a single center of symmetry for each member of a pair.

Each method, then, has its preferred uses, and I will show that each can be used to check the results of the other. Between them they remove the difficulty from the study of almost all multiple nuclei.

Section II presents the algorithm. Section III describes the results of its application to images from the HS sample, and Section IV evaluates its strengths and weaknesses. A summary is presented in Section V. Questions of the extension of the model are treated in an appendix. Dynamical interpretation of the separated images will be outside the scope of this paper, awaiting improved implementation of the algorithm (as explained in Section III) and its application to higher-quality data. 


\section{THE ALGORITHM}

Let

$$
I(x, y)=f_{1}(x, y)+f_{2}(x, y)
$$

where $\mathrm{I}(\mathrm{x}, \mathrm{y})$ represents the data in hand and $f_{1}$ and $f_{2}$ are the distinct contributions of the two galaxies. The assumption is that

$$
\begin{aligned}
& f_{1}(x, y)=f_{1}\left(2 a_{1}-x, 2 b_{1}-y\right) \\
& f_{2}(x, y)=f_{2}\left(2 a_{2}-x, 2 b_{2}-y\right),
\end{aligned}
$$

i.e., that $f_{1}$ is symmetric about the center $\left(a_{1}, b_{1}\right)$, and $f_{2}$ is symmetric about the center $\left(a_{2}, b_{2}\right)$. Note that this is a weaker constraint than the assumption that the isophotes are concentric ellipses. For instance, it encompasses boxiness.

Now consider the antisymmetric sum

$$
A_{1}(x, y)=I(x, y)-I\left(2 a_{1}-x, 2 b_{1}-y\right) .
$$

By our assumption concerning the symmetry of $f_{1}$,

$$
A_{1}(x, y)=f_{2}(x, y)-f_{2}\left(2 a_{1}-x, 2 b_{1}-y\right) .
$$

By the symmetry of $f_{2}$, 


$$
f_{2}\left(2 a_{1}-x, 2 b_{1}-y\right)=f_{2}\left(x+2\left(a_{2}-a_{1}\right), y+2\left(b_{2}-b_{1}\right)\right) .
$$

Therefore

$$
\begin{gathered}
A_{1}\left(x+2\left(a_{2}-a_{1}\right), y+2\left(b_{2}-b_{1}\right)\right)=f_{2}\left(x+2\left(a_{2}-a_{1}\right), y+2\left(b_{2}-b_{1}\right)\right. \\
-f_{2}\left(2 a_{1}-x-2\left(a_{2}-a_{1}\right), 2 b_{1}-y-2\left(b_{2}-b_{1}\right)\right) \\
=f_{2}\left(2 a_{1}-x, 2 b_{1}-y\right)-f_{2}\left(x+4\left(a_{2}-a_{1}\right), y+4\left(b_{2}-b_{1}\right)\right) .
\end{gathered}
$$

This may be used to substitute for $f_{2}\left(2 a_{1}-x, 2 b_{1}-y\right)$ in the antisymmetric sum. It is clear that $\mathrm{N}$ iterations of this procedure will give the expression

$$
\begin{gathered}
\sum_{n=0}^{N} A_{1}\left(x+2 n\left(a_{2}-a_{1}\right), y+2 n\left(b_{2}-b_{1}\right)\right)=f_{2}(x, y)- \\
f_{2}\left(x+2 N\left(a_{2}-a_{1}\right), y+2 N\left(b_{2}-b_{1}\right)\right) .
\end{gathered}
$$

For sufficiently large $\mathrm{N}$, the second term on the right becomes the sky level. Taking this to be zero recovers the light distribution of the second galaxy alone:

$$
f_{2}(x, y)=\sum_{n=0}^{N} A_{1}\left(x+2 n\left(a_{2}-a_{1}\right), y+2 n\left(b_{2}-b_{1}\right)\right) .
$$

Similarly, for the first galaxy, using the definition

$$
A_{2}(x, y)=I(x, y)-I\left(2 a_{2}-x, 2 b_{2}-y\right)
$$

gives 


$$
f_{1}(x, y)=\sum_{n=0}^{N} A_{2}\left(x+2 n\left(a_{1}-a_{2}\right), y+2 n\left(b_{1}-b_{2}\right)\right)
$$

Formally these expressions for $f_{1}$ and $f_{2}$ should be infinite sums, but in practice the limited sensitivity of the data and finite extent of the galaxies make it feasible to truncate the sum at finite $\mathrm{N}$, and indeed the finite field of view makes it necessary.

An intuitive understanding of the algorithm is easily to achieve. Taking the antisymmetric sum $A(x, y)$ is the same as rotating the inage $180^{\circ}$ about the center of one of the galaxies and subtracting it from itself. If the galaxy whose center was used is symmetric, it and the sky vanish, leaving information only on the other galaxy. This is illustrated by Figure 2. If the remaining galaxy is not very extended, this may be all that is necessary. The little spiral in Figure 2 is this kind of easy target. Its positive and negative images do not overlap. One is able to reproduce the image of the entire galaxy with $N=0$ in the sum given above. 'This also illustrates how the algorithm is able to reproduce faithfully limited amounts of asymmetric light. Even if the little spiral in Figure 2 had been completely irregular, one could have generated a picture of it by calculating $A$ as long as its partner was symmetric.

For galaxies that extend beyond the centers of their companions, however, the sum must be taken to $N \geq 1$ to unscramble the overlapping positive and negative images. To reconstruct any pixel, one takes the sum of the values of $A$ at that pixel and at vector intervals equal to twice the center separation. This sum can be taken until it is cut off by the edges of the frame, or until it contributes more noise than signal. This explains why the algorithm is capable of recording asymmetries despite the assumption that the galaxies are symmetric: the only thing that can 
mask them is asymmetries in the other galaxy $\left(2 n\left(a_{1}-a_{2}\right), 2 n\left(b_{1}-b_{2}\right)\right)$ pixels away in $A$. It also explains the trains of reflections that show up in the separated images (v. Figure 1). 


\section{APPLICATION TO THE DATA}

The algorithm has been encoded and applied to images of 12 BCEs from the sample of HS that are binary or have nearby companions. It should be emphasized that the quality of these images is not state-of-the-art-certainly not competitive with Lauer's sample of 4 Shooter images. Though sufficient to the purposes of HS and PSH, these are old, mostly short (500-second) exposures taken with a $500 \mathrm{x}$ 500 Texas Instruments CCD of mediocre cosmetic quality on a 1.5-meter telescope. The data are described in more detail in HS. The success of the algorithm on such a nonideal data set bodes very well for its future usefulness.

Object centers were first determined to a fraction of a pixel using a standard centroiding routine. The positions were input to the algorithm, and a simple areaweighting technique was used to account for the fact that objects are not exactly centered within pixels. The sample covers a wide range of component separations and relative brightnesses, as well as image qualities.

For this initial study I have arbitrarily truncated all summations at $N=4$. As indicated above, a smaller $\mathrm{N}$ may sometimes be sufficient or even desirable to reduce noise in the output images. The noise generated by the summation is nonnegligible and often reduces the magnitude of the faintest fittable isophotes by 2. This seems a high price to pay, and indeed is what is presently limiting the sample of clusters to which the algorithm can profitably be applied. But Figure 4 indicates that it still allows photometry to depths comparable to Lauer's. This may be because for the outermost isophotes of a complex system, a 2-component fit is not significantly better than a single-component one. 
The original and separated images of the systems studied are presented in Figure 1 , in the following format. In the upper left corner, called panel $a$, is the original image, with its intensity scaled to best display both the sky structure and the overlap of the galaxy envelopes. This is the image that is input to the algorithm after it has been cleaned of all stars and galaxies other than the main pair, in the way described by PSH. The output images of the brighter and fainter components are shown in the upper right and lower left panels, called $b$ and $c$, respectively. These panels are scaled in the same way as the first, except for the subtraction of the sky level. Ellipses are fit to the isophotes of these images using standard techniques (PSH), and these fits used to subtract models of the two galaxies as described by Lauer (1986). The residual image is the one displayed in the lower right panel $(d)$, again at the same brightness scale as the original. The images of A42, A160, A787, A1827, A1984, and A2521 have 1' fields of view. Those of A400, A779, A1185, A1775, and A2022 have 2' fields of view. That of A2079 has a $3{ }^{\prime}$ field. The surface brightness and isophote shape profiles of the galaxy pairs are presented in Figure 3.

In the comments on the individual systems that follow, I will refer to the brighter and larger member of a pair as "A," and the smaller and fainter member as "B." If it is not obvious which is which, the identifications will be explicitly stated.

A42.

This is a system with a significant amount of light not centered on either galaxy nucleus, as indicated by the isophotometry of PSH and confirmed by the light and dark streaks in the component images. Therefore, as Figure 5 shows, the separated images could not be accurately photometered to levels much fainter than 
the original data. However, the same figure shows indications that the photometry, especially of $\mathrm{B}$, has been improved by the separation. It is now clearer that $\mathrm{B}$ has a truncated brightness profile. In the outermost isophotes, this is partly an artifact of the residual light, as is shown by the anomalous eastward centroid drift. But in the inner $3^{\prime \prime}$, the truncation is probably real. There is considerable excess light to the southeast of the two nuclei, and Figure 5 strongly suggests that it is not bound to $\mathrm{B}$. Whether this light is a skew component of $\mathrm{A}$, a loose patch of stars, or a complete third component of very low surface brightness could be tested by Lauer's method.

A160.

This system indicates the potential of the algorithm much better than does A42. Figure 5 shows that after the separation, A could be photometered 4 times further out than in PSH, and B twice as far out. The increase of the ellipticity of A to 0.2 outside $10^{\prime \prime}$ suspected by PSH is confirmed, for B has a truncated brightness profile. This makes this system a good candidate for a tidal encounter. Indeed Tonry (1985) found a velocity difference of only $104 \mathrm{~km} \mathrm{sec}^{-1}$ for this pair.

A400.

Poor flat fielding of this image caused an east-west gradient in the sky level of the component pictures, which is clearly visible in Figure 1. Nevertheless the separation of this pair was an unqualified success: the components can now be photometered to radii comparable to their separation. The southern component is clearly A, for its scale length is larger, and the northern galaxy has a decreasing ellipticity profile, which distinguishes it from almost all brightest cluster ellipticals 
(PSH). The residuals visible in panels $b$ and $c$ are primarily due to imperfect cleaning of small objects near the nuclei.

Lauer (1988) found that the isophotes of B are nonconcentric, and both this work and PSH have confirmed this: Lauer's dipole residuals are echoed by our centroid drifts. Tonry (1985) found a velocity difference of $426 \mathrm{~km} \mathrm{sec}^{-1}$ for this pair. He found no rotation in B, but his slit was aligned north-south, and B may be an east-west aligned oblate rotator. A400 is the site of $3 \mathrm{C} 75$, a spectacular pair of jets emerging from the nuclei of A and B (Yokosama and Inoue 1985, Owen et al. 1986). Yokosama and Inoue argue that A and B are orbiting each other. Whether they are bound is an open question.

\section{A779.}

This is probably the sample member best suited to illustrating the powers of the algorithm. The members of this strongly overlapping pair are cleanly separated, and Figure 4 shows that Lauer's observations of isophote rotation in both A and B, as well as the truncation of $\mathrm{B}$, are confirmed. These have already been suggested by the photometry of PSH, but the photometry of $\mathrm{B}$ from the present image has now been extended by 3 magnitudes. The outer isophotes of $B$ appear also to have been circularized and are significantly offset away from A. This is confirmation of Lauer's claim of differential tidal truncation of B. Note from Figure 5 that in the primitive photometry of $\mathrm{PSH}$, the isophote centroids of $\mathrm{B}$ were drawn toward A by contamination from that galaxy.

The reflections of A's antisymmetries, visible in panels $b$ and $c$, are nearly as interesting as the galaxies themselves. The outer east-west dipole ring is probably a tracking error artifact, since it also appears in B. The bright arc about $3^{\prime \prime}$ east of 
the nucleus of $\mathrm{A}$ appears to be real, however, since it also appears when the image is unsharp masked, and may be present in Lauer's residual map. It is certainly worthy of further study.

Faint patches and small arcs are elusive things, however, and I do not confirm Lauer's report of a patch of diffuse light about $\frac{1}{3}$ of the way from B to A. All that is visible in this region of panel $d$ is the overlap of faint rings caused by interpolation errors in the model subtraction routine. Note that such a ring is also barely visible in Lauer's residual map. This, plus the reflection of nucleus A which plagues his image, is probably the source of his detection.

\section{A787.}

This pair is well separated and appears fairly typical: B has constant ellipticity and a truncated light profile, and $\mathrm{A}$ may have a $\mathrm{cD}$ halo. A deeper exposure could easily confirm or refute this claim. Jenner (1974) found a velocity difference of $799 \mathrm{~km} \mathrm{sec}^{-1}$ between this pair.

\section{A1185.}

This system is presented as a test of the algorithm under extremely adverse conditions. It is a triple system and is known to have significant amounts of asymmetric light (PSH, Lauer 1988) and a low relative radial velocity between the two nuclei (Hoessel, Borne, and Schneider 1985; Tonry 1985; Smith et al. 1985).

To run the algorithm, the compact nucleus to the southwest was boxed out as described in PSH, and the program given the two brightest nuclei to separate. In view of the problems presented by this system, it did surprisingly well. Figure 4 shows that the surface brightness profile of $B$ is depressed relative to Lauer's, because this nucleus sits in a region of negative residual caused by the loose light. 
But Lauer's profile of $\mathrm{A}$ is reproduced as faint as $r=22 \mathrm{mag} \mathrm{sec}^{-2}$. The centroid displacement he reported is not reproduced as well. Those shown in Figure 5 move east instead of west, as it is obvious from inspection of the original image that they should. A slightly better picture of A can be generated by subtracting panel $b$, the separated image of $B$, from the original data. A comparison of photometry on such an image to the standard procedure is shown in Figure 6. The false centroid drift in right ascension has been suppressed, but the correct drift is still not revealed. This illustrates the sensitivity of the algorithm to asymmetric light, even more vividly than did the analysis of A42.

\section{A1775.}

This galaxy is a well known example of a high-velocity encounter (Jenner 1974, Hintzen 1979). The separation improves the photometry, due to the considerable overlap between $\mathrm{A}$ and $\mathrm{B}$, and suggests that $\mathrm{B}$ is truncated along its minor axis. There is a residual asymmetry in A that could be simulating such a truncation, however, and confirmation is necessary.

\section{A1827.}

The structure in the first few arc seconds of these profiles is probably caused by telescope tracking error. However, the isophote rotation detected by PSH at larger radii in both components is confirmed: the outer isophotes line up with the chord connecting the nuclei.

\section{A1984.}

The northern component of this almost equal pair has been designated $\mathrm{A}$, since it was labelled the brightest cluster member by HS. The data, however, suggest that it is less extended than the southern member and confirm the discovery by 
PSH that it has a decreasing ellipticity profile. It also appears to have a relatively complex central strucure. The southern galaxy may be the better choice for first-ranked cluster member.

\section{A2022.}

This pair is more widely separated and more unequal in brightness than the others in the sample, but the separation still improves on the photometry of PSH. Both $\mathrm{A}$ and $\mathrm{B}$ have constant, small, perhaps even decreasing, ellipticity profiles. The is sphotes of $\mathrm{B}$ are aligned in the direction to $\mathrm{A}$. Those of $\mathrm{A}$ do not point to $\mathrm{B}$, but they are significantly displaced in that direction.

\section{A2079.}

This cluster was noted by PSH to have a great deal of activity. There is a bright central galaxy with a strongly increasing ellipticity profile, boxy outer isophotes, and an obvious swath of excess light $30^{\prime \prime}$ to the north. There is a large companion 40 (60 kpc for $H_{0}=60 \mathrm{~km} \mathrm{sec}^{-1} \mathrm{kpc}^{-1}$ ) away in PA $195^{\circ}$ and a general appearance of a band of light overlapping the two. The isophotes of both $\mathrm{A}$ and $\mathrm{B}$ are losely aligned with this axis. Galaxy A shows strong centroid jogs of up to $10^{\prime \prime}$ which are at least partly attributable to this excess light. The jump at $10^{\prime \prime}$ on the minor axis is visible as a ring in panel $b$ for this cluster. This is as large as the $1 \sigma$ value for the same isophotes, even though that is dominated by the boxiness of the light distribution in this system. If any further evidence of strong interaction in this system were required, it would be provided by the observation of Smith et al. that $\mathrm{A}$ and $\mathrm{B}$ have effectively identical radial velocities.

$\mathrm{A}$ and $\mathrm{B}$ are well enough separated that the present algorithm adds nothing to our knowledge of this cluster. This image is presented instead as another test of 
the method's performance under adverse conditions, viz., large amounts of "loose light." The centroid displacement was so strong in this case that the residual panel $d$ could not be adequately constructed until isophotes were measured at intervals of 0.1 magnitudes, instead of the usual 0.25 . Nor has the range of the photometry been extended by use of the symmetry assumption. Still, the light distribution of the two galaxies has been adequately modelled within a certain radius.

\section{A2521.}

The photometry of PSH on this close, aligned, faint pair was improved enough to show that the ellipticity of A decreases outside of $3^{\prime \prime}$. The stability of the centroid profiles suggest that this decrease is not caused by contamination from residuals along the major axis. The central structure of this galaxy is somewhat complicated: Figure 1 shows it is peanut-shaped. It does not, however, appear to be an unresolved double: such an object would have a large central ellipticity. The oversubtraction in panel $d$ of Figure 1 is caused by asymmetric residuals. 


\section{CONCLUSIONS}

The above descriptions already give a detailed picture of the strengths and weaknesses of the algorithm, both in an absolute sense and relative to the alternative least-squares approach. The program was also run on about two dozen other binarynucleus or double galaxies from the HS sample. In most of these images the pair was distant enough that the algorithm did not produce photometry any deeper than that of PSH. As stated above, the summation enhances sky noise considerably, sometimes by two magnitudes. For the reductions in this paper, the truncation index $\mathrm{N}$ of the sum was simply set to 4 . It may be possible to improve the algorithm by adjusting $\mathrm{N}$ to maximize signal to noise. This would allow its application to more widely separated pairs of galaxies, such as those in A154, A1377, and A2152.

Some pairs on which the algorithm was tried proved to be insufficiently resolved. What constitutes sufficiency in this context is a strong function of the relative brightnesses, separations, and degree of symmetry of the pair members, as well as image quality, seeing, and sky flatness. For instance, A1126 and A2645, with center separations of $5^{\prime \prime}$ and $4^{\prime \prime}$, respectively, could not be reduced, but A160, with a separation of $7^{\prime \prime}$, was easy, and A42 and A1185, with separations of $9^{\prime \prime}$ and $7^{\prime \prime}$, could be attempted despite large amounts of asymmetric light.

It is clear from the tests on A42 and A1185 that the algorithm cannot tolerate extended, high signal-to-noise asymmetries. Attempts to apply it to systems like the quadruple nucleus of A1291 would certainly have failed. Fortunately, such systems are rare. A1185 was the only one in Lauer's sample of 14, and there are only 8 in the PSH sample of 175 BCEs. 
The algorithm is also sensitive to large scale variations in the sky background. The effects of flattening failure are visible in the separated images of A400. Fortunately, the decomposition on a scale comparable to the component separation is not seriously affected. The algorithm is robust in the face of poorly cleaned images (A400, A1775) and low signal to noise (A160). This increases its potential value in rich and high-redshift clusters.

Finally, there are many cases in which application of the full algorithm is simply unnecessary. These include many very unequal pairs, such as A85, A671, and even A1656. All that is necessary to study these systems is the initial asymmetrization: rotation and subtraction. This simple step is a powerful tool even for the study of isolated ellipticals and disk systems and any other image that possesses even an approximate central symmetry. 


\section{REFERENCES}

Hintzen, P. 1979, Pub. Astron. Soc. Pacific 91426.

Hoessel, J. G.; Borne, K. D.; and Schneider, D. P. 1985, Astrophys. J. 29394.

Hoessel, J. G., and Schneider, D. P. 1985, Astron.J. 90 1648. (HS)

Jenner, D. C. 1974, Astrophys. J. 19155.

Lauer, T. R. 1986, Astrophys. J. 31134.

Lauer, T. R. 1988, Astrophys. J. 32549.

Merritt, D. 1985, Astrophys. J. 28918.

Owen, F. N.; O'Dea, C. P.; Inoue, M.; and Eilek, J. A. 1986, Astrophys. J. Lett. 294 L85.

Porter, A. C.; Schneider, D. P.; and Hoessel, J. G. 1988, in preparation. (PSH)

Schneider, D. P.; Gunn, J. E.; and Hoessel, J. G. 1983, Astrophys. J. 268476.

Smith, R. M.; Efstathiou, G.; Ellis, R. S.; Frenk, C. S.; and Valentijn, E. A. 1985, Mon. Not. Royal Astron. Soc. 216 71P.

Tonry, J. L. 1985, Astron. J. 902431.

Yokosawa, M., and Inoue, M. 1985, Proc. Astron. Soc. Japan 37655. 


\section{APPENDIX: ATTEMPTS TO DERIVE AN ANALYTIC SEPARATION FORMULA FOR MORE THAN TWO GALAXIES}

First we will show that the algorithm based on point reflection symmetry cannot be extended to more than two galaxies. The derivation is best done in Fourier space.

The light distribution is

$$
I(x, y)=\sum_{i}^{G} f_{i}(x, y),
$$

with symmetries about centers $\left(a_{i}, b_{i}\right)$,

$$
\begin{gathered}
f_{i}(x, y)=f_{i}\left(2 a_{i}-x, 2 b_{i}-y\right), \\
f_{i}\left(2 a_{j}-x, 2 b_{j}-y\right)=f_{i}\left(x+2\left(a_{i}-a_{j}\right), y+2\left(b_{i}-b_{j}\right)\right) .
\end{gathered}
$$

Here $\mathrm{G}$ is used for the number of galaxies because $\mathrm{N}$ has already been used for the sum truncation. Define the general antisymmetric sums

$$
A_{i}(x, y)=I(x, y)-\sum_{j} c_{i j} I\left(2 a_{j}-x, 2 b_{j}-y\right)
$$

This definition seems intuitively sensible as a generalization of the approach of cancelling out the light of the other galaxy. Substitution of the assumed form of $\mathrm{I}(\mathrm{x}, \mathrm{y})$ and application of the symmetries of its addends show that 


$$
A_{i}(x, y)=I(x, y)-\sum_{j} c_{i j} f_{j}\left(x+2\left(a_{i}-a_{j}\right), y+2\left(b_{i}-b_{j}\right)\right)
$$

The Fourier transform

$$
\hat{f}(\zeta, \eta)=\frac{1}{\sqrt{2 \pi}} \int_{-\infty}^{+\infty} e^{-i(x \zeta+y \eta)} f(x, y) d x d y
$$

of this is

$$
\hat{A}_{i}(\zeta, \eta)=\sum_{j} 1-\sum_{k} \frac{c_{i k} u_{j}}{u_{k}} \hat{f}_{j}(\zeta, \eta)
$$

where I have adopted the notation

$$
u_{i} \equiv e^{-2 i\left(a_{i} \zeta+b_{i} \eta\right)}
$$

Further defining

$$
M_{i} \equiv \sum_{k} \frac{c_{i k}}{u_{k}}
$$

shows that

$$
\hat{A}_{i}(\zeta, \eta)=\sum_{j} 1-M_{i} u_{j} \hat{f}_{j}(\zeta, \eta)
$$

This is a fairly simple linear system, with transformation matrix given by

$$
T_{i j}=1-M_{i} u_{j}
$$


But such a simple matrix can be factored in the following way:

$$
T_{i j}=R_{i k} S_{k j}
$$

with

$$
\begin{aligned}
& R_{i k}=\operatorname{delta}_{k 1}+M_{i} \delta_{k G} \\
& S_{k j}=\operatorname{delta}_{k 1}+u_{j} \delta_{k G} .
\end{aligned}
$$

Since both these matrices clearly have zero determinant when $G>2$, the solution for the $\hat{f}_{i}$ does not exist.

This failure can be understood as the result of trying to extract more than $2 \frac{n^{2}}{2}$ bits of information from a picture that contains only $n^{2}$ pixels. This suggests that in principle the method could be generalized to as many as 4 galaxies if the assumption of point-reflection symmetry were replaced by the assumption of two plane-reflection symmetries. In practice the following serious difficulty would arise.

The centers of galaxies are not strongly masked even by nearby neighbors and can therefore be determined a priori to within a fraction of a pixel. The algorithm requires these as input and succeeds because they can be provided. The suggested generalization would require a priori determination of the major and minor axes of all the components. But major-axis angles are not so easily determined even in isolated systems, especially those with low ellipticity. Their specification in overlapping multiple galaxies would be that much more difficult. Furthermore many of the systems to which the generalized method would be applied (e.g., A1185, A1291, A2199, A2256) are physically interacting and therefore have nonconcentric 
and twisting isophotes, not to mention loose light. There is at present no analytic method that can compete with simultaneous isophote fitting for systems with more than 2 nuclei. 


\section{FIGURE CAPTIONS.}

FIGURE 1. Images of the galaxy pairs before and after separation, together with residuals of models fit to the separated images. A detailed explanation of the panels and scales is given in the text.

FIGURE 2. Illustration of the first step of the algorithm using A2670. The original image is at top. It has been rotated about the centers of the two galaxies and subtracted from itself to produce the panels at the bottom.

FIGURE 3. Brightness and shape parameter profiles for all pairs of galaxies in the sample. Each page presents data on a single pair. Profiles of the A components are plotted with solid circles, and profiles of the $\mathrm{B}$ components are plotted with asterisks. The profiles are: in the top row, from left to right, the major and minor axis surface brightness profiles, and the profile of their geometric mean $\sqrt{a b}$; in the middle row, the ellipticity, eccentricity, and major-axis position angle, as functions of $\log \mathrm{b}$; in the bottom row, the isophote centroid coordinates $x_{0}$ and $y_{0}$, and the $1 \sigma$ deviation of the fit from the pixels used, as functions of $\log \mathrm{b}$.

FIGURE 4. Comparison of profiles of separated components to the results of Lauer (1988) for galaxies also in his sample. The isophote twisting in the components of A779 is shown in the bottom left panel; the top profile is of the A component. The remaining panels are surface brightness profiles plotted as functions of log a. All observations are in the Thuan-Gunn $r$ passband, and no zero-point corrections have been applied. The disagreement in the A400 profiles is due to different seeing: 1" in the HS image, and 5" in Lauer's. 
FIGURE 5. Comparison of isophote fits to the separated images in this study (solid circles) to the fits attempted by PSH (asterisks). The plot format is as in Figure 3, save that each page of this figure represents a single galaxy, not a pair.

FIGURE 6. Comparison of fits to the separated image of A1185A (filled circles) to fits to the difference of the original image and the separated image of A1185B. The plot format is as in Figure 3. 


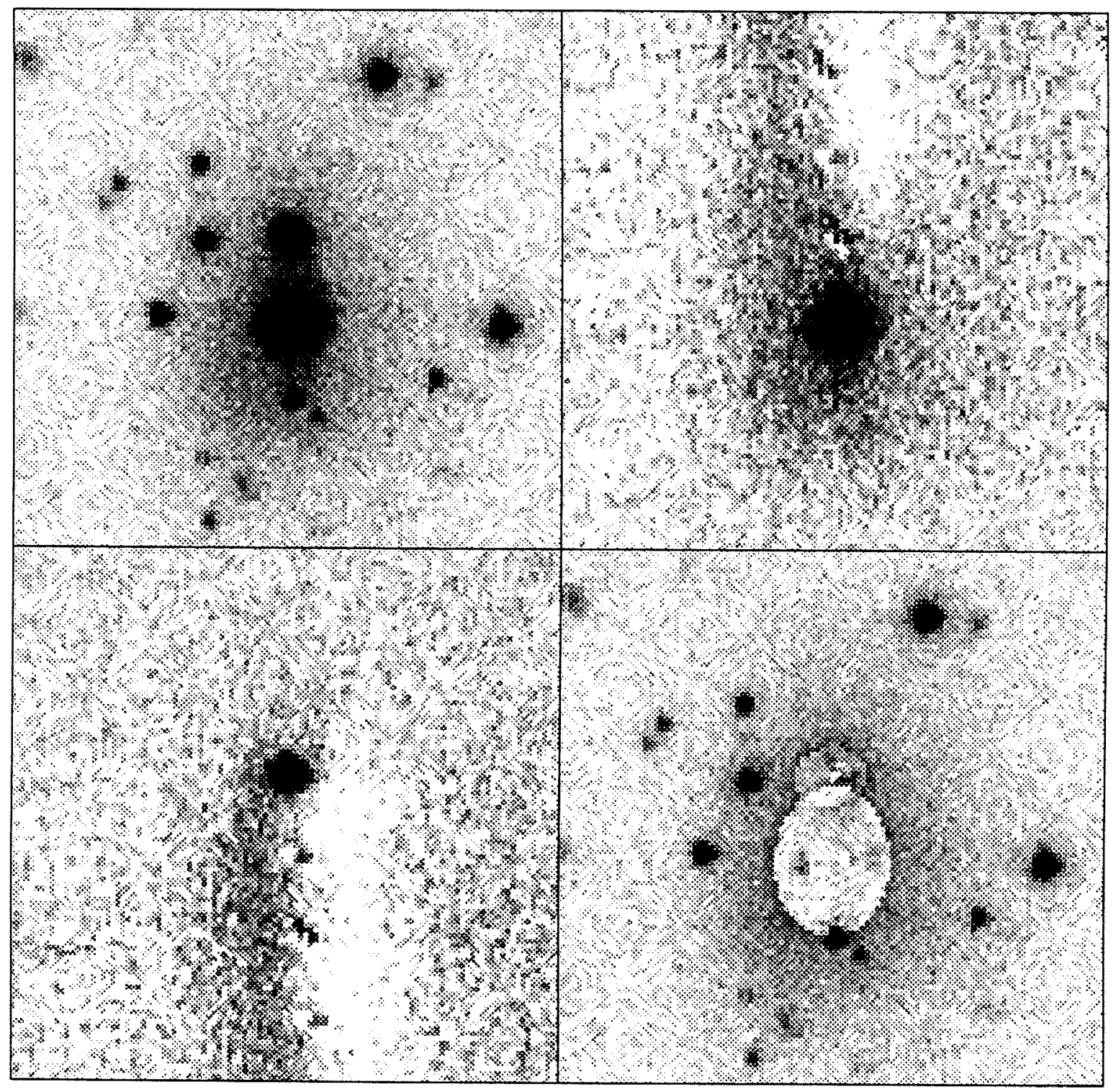

Figure 1. A42 


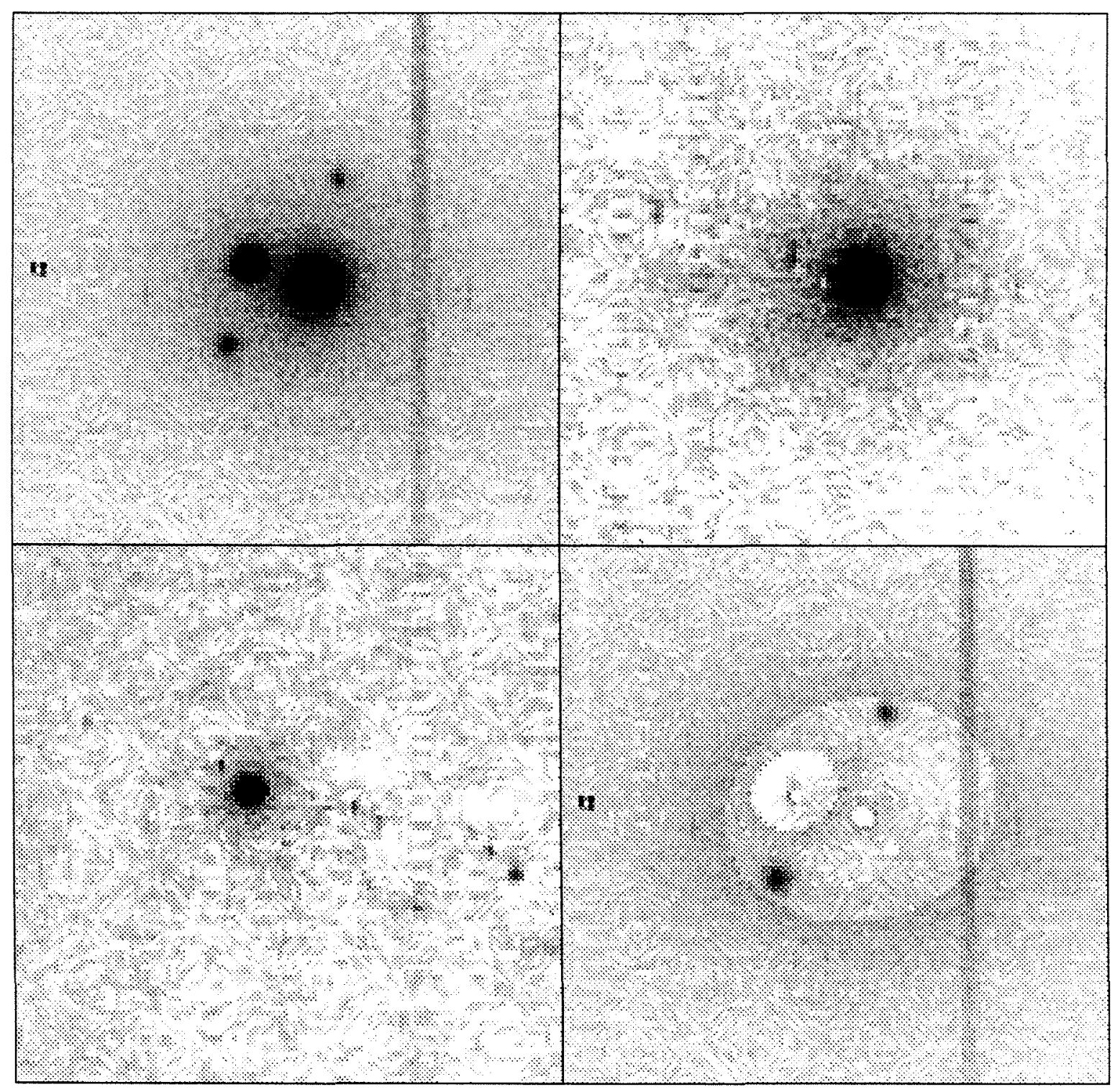

Figure 1 cont'd. A160 


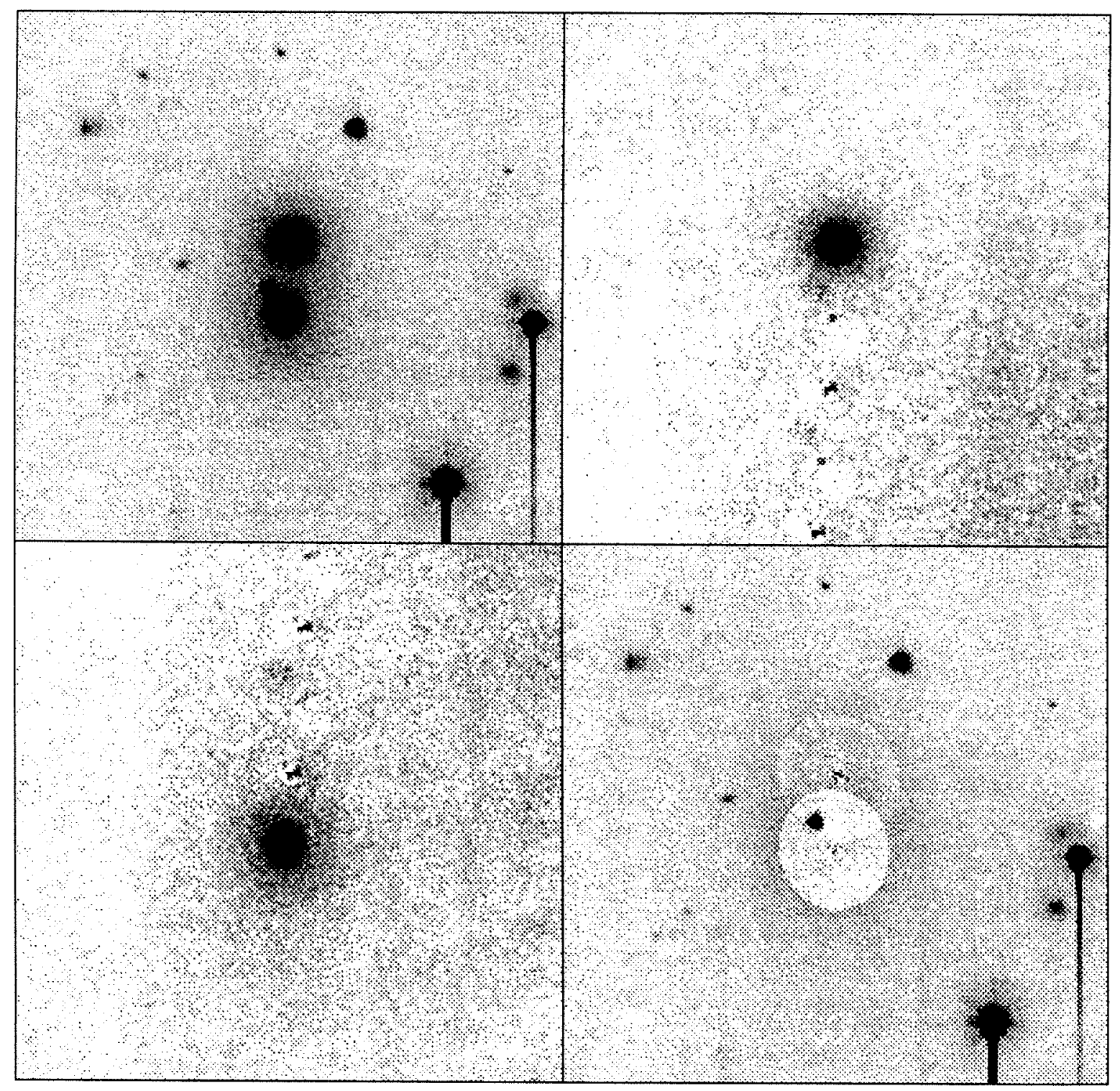

Figure 1 cont'd. A400 


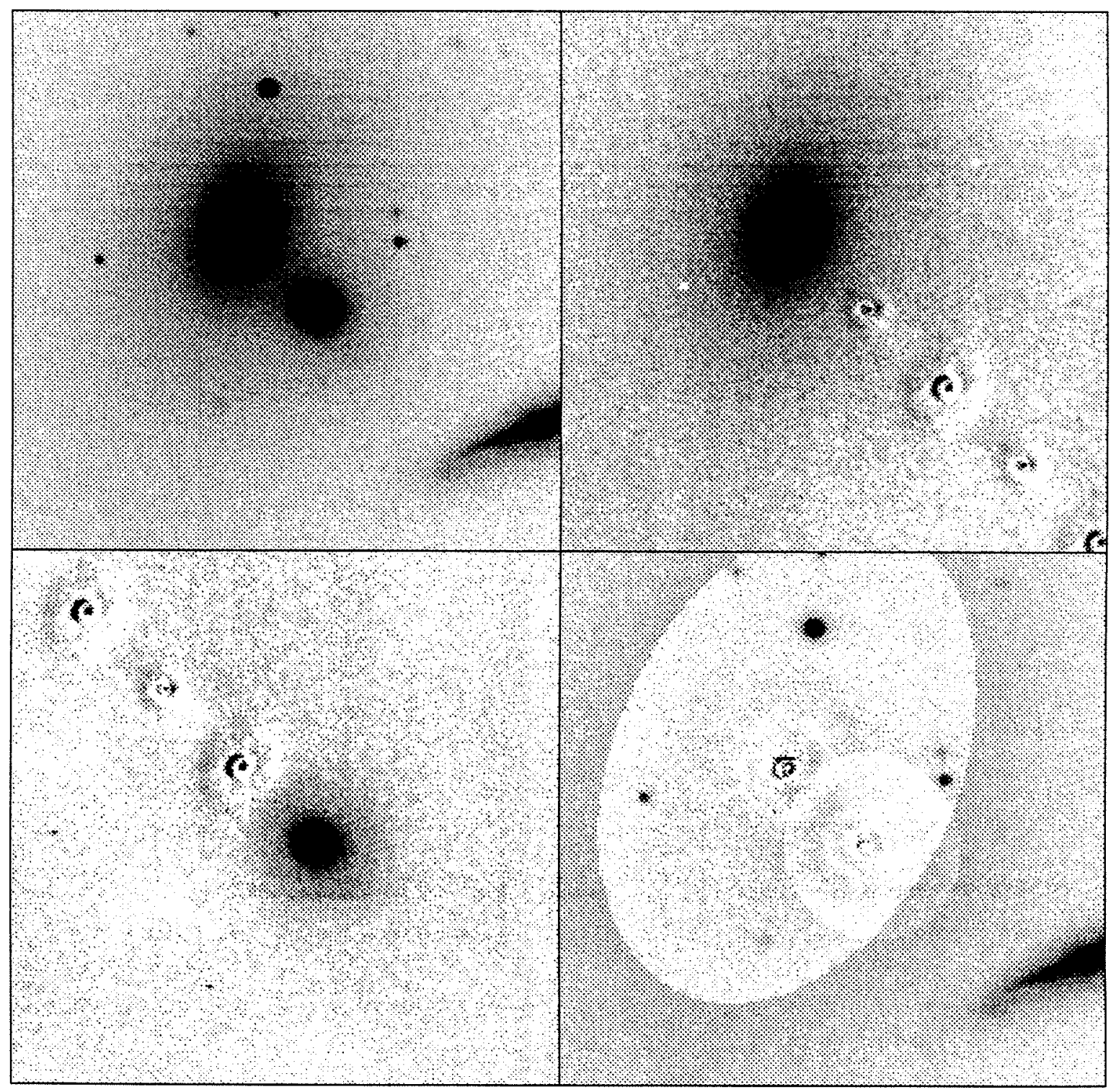

Figure 1 cont'd. A779 


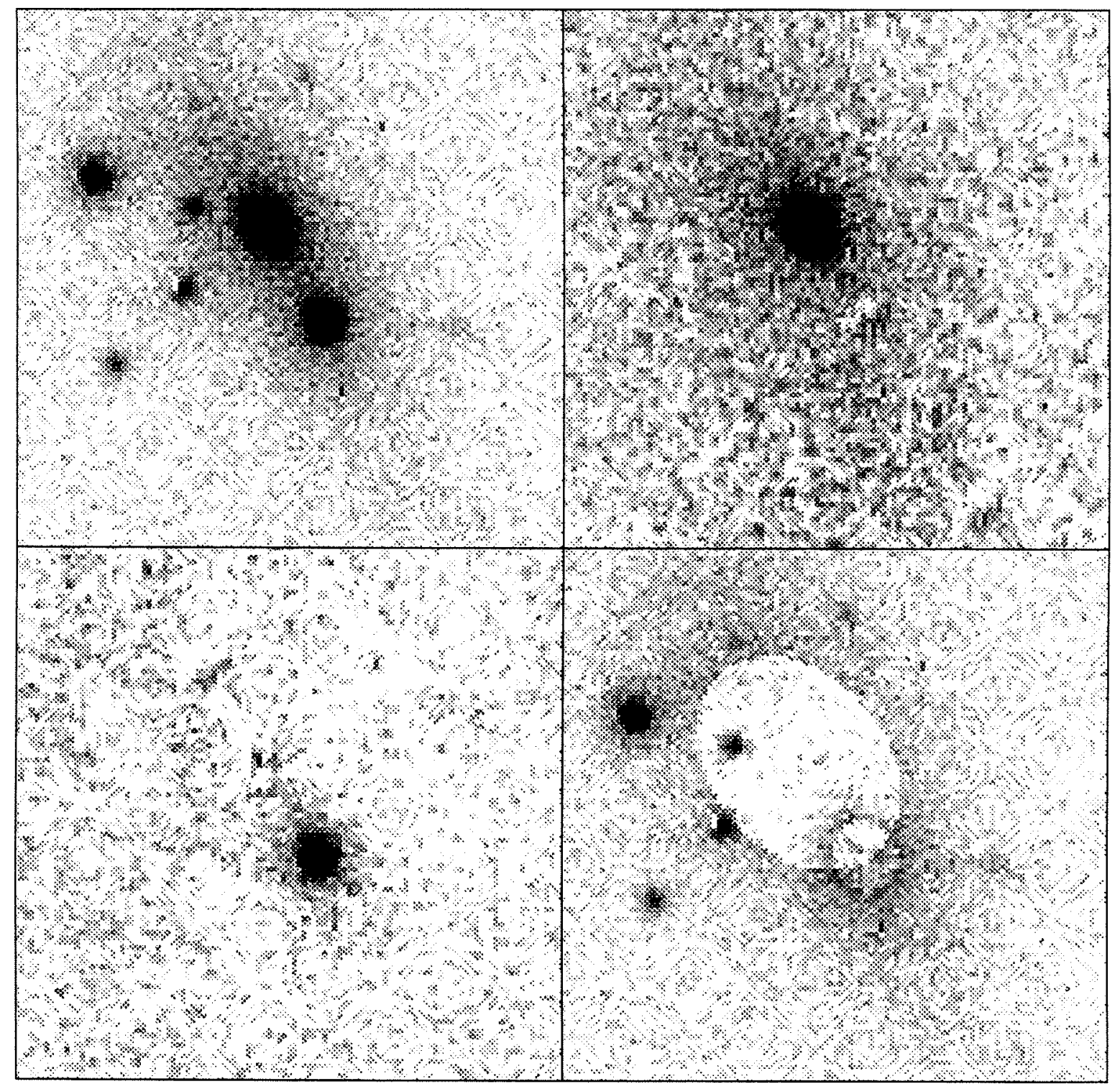

Figure 1 cont'd. A787 


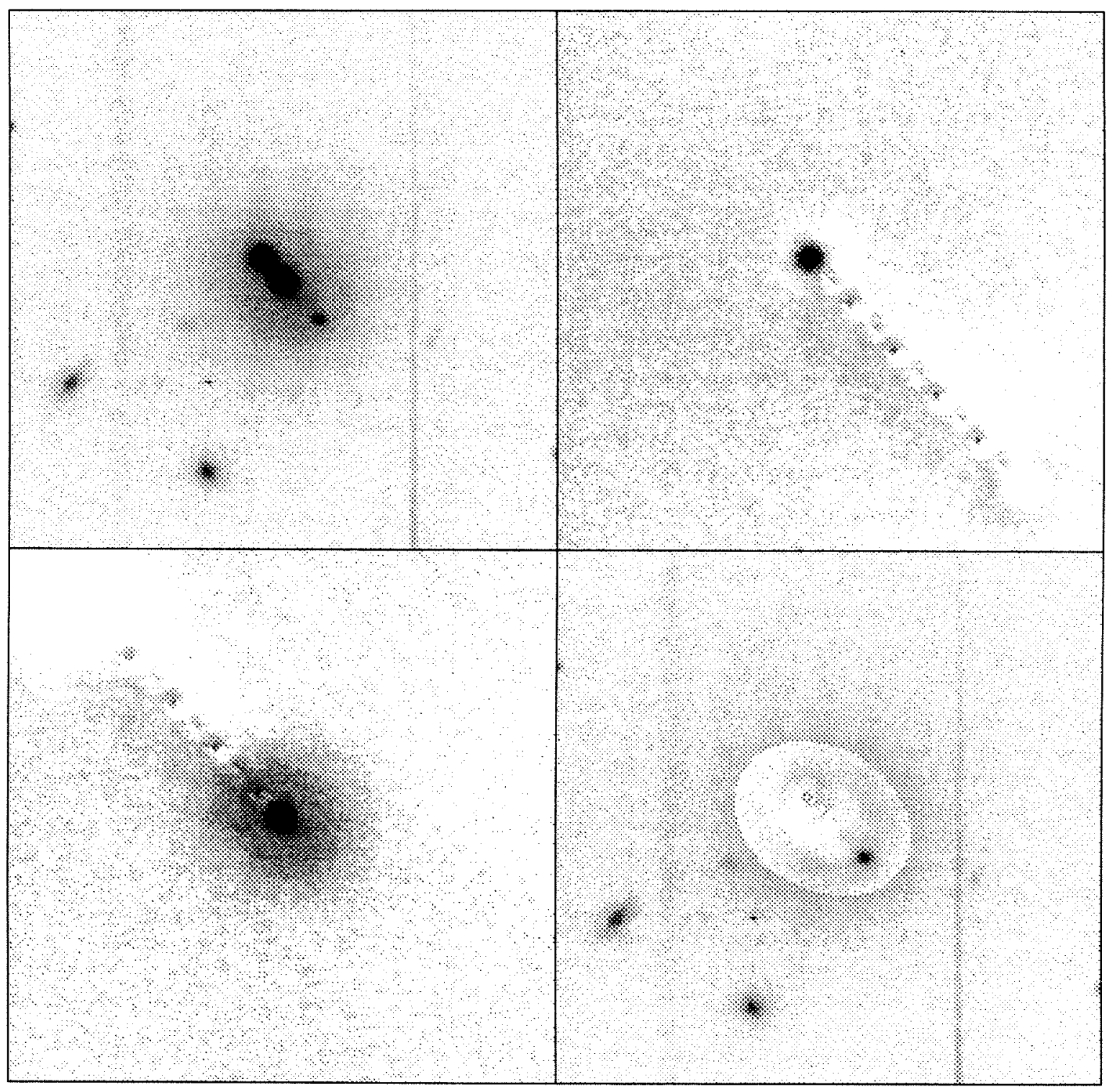

Figure 1 cont'd. A1185 


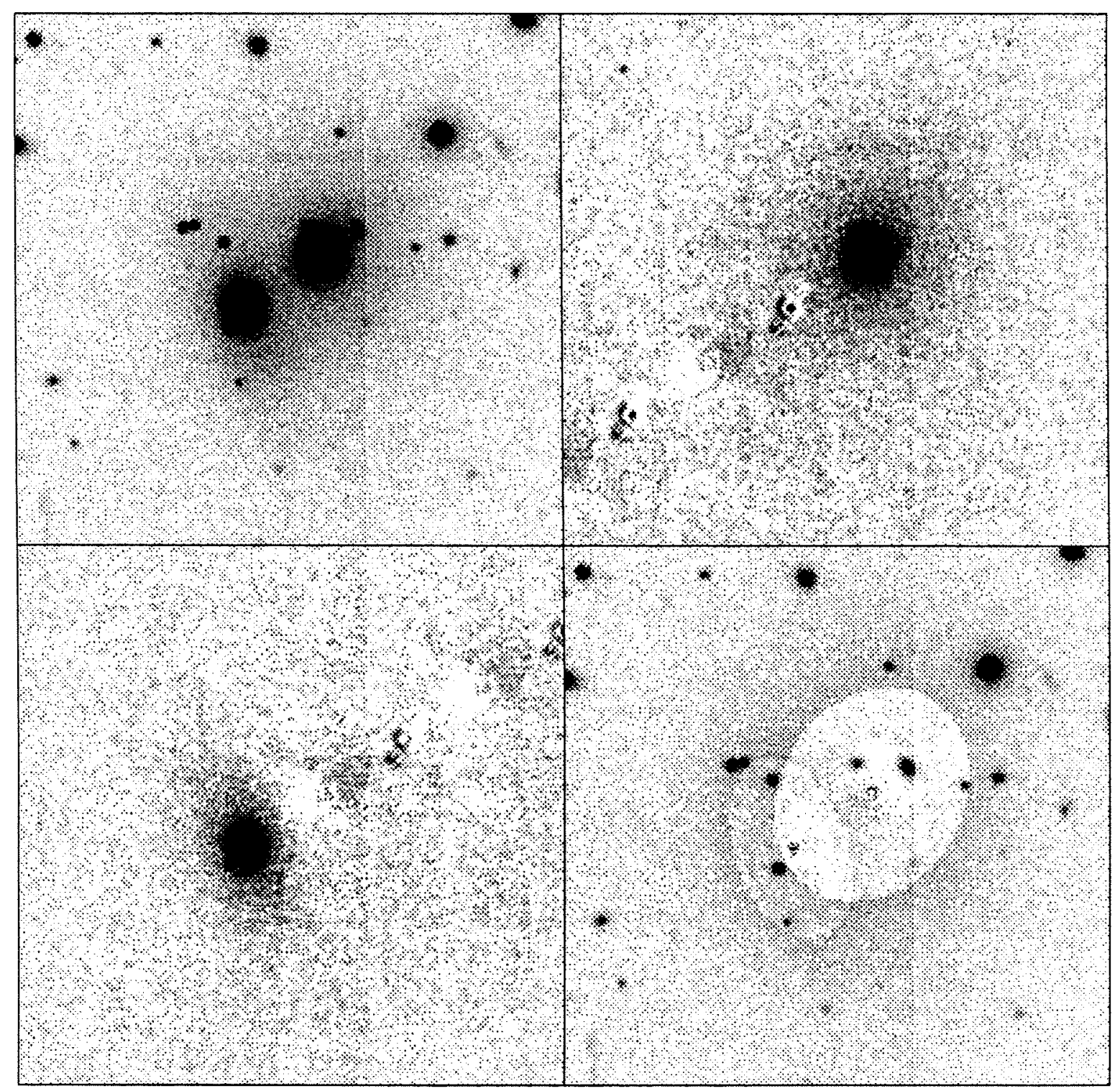

Figure 1 cont'd. A1775 


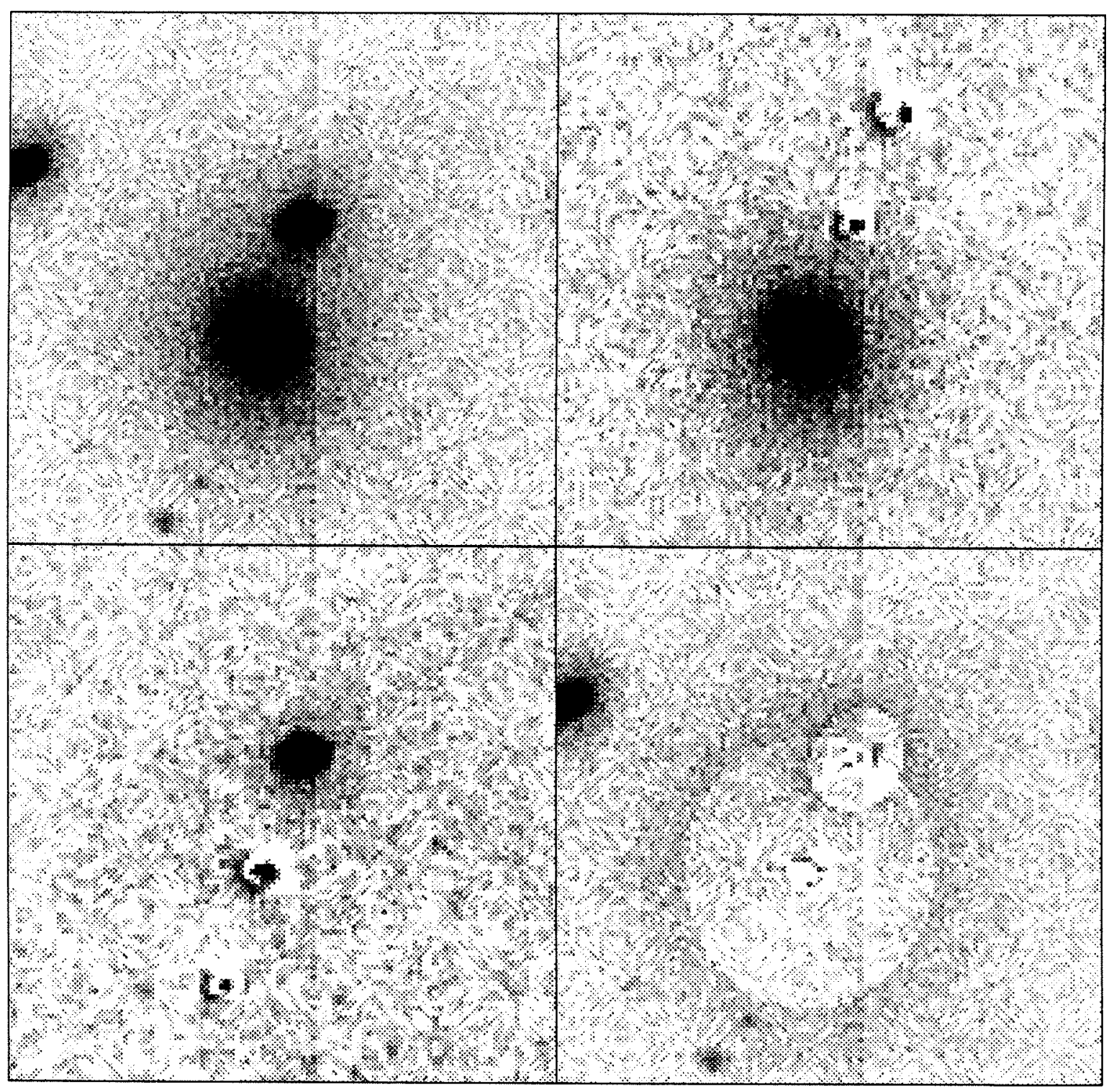

Figure 1 cont'd. A1827 


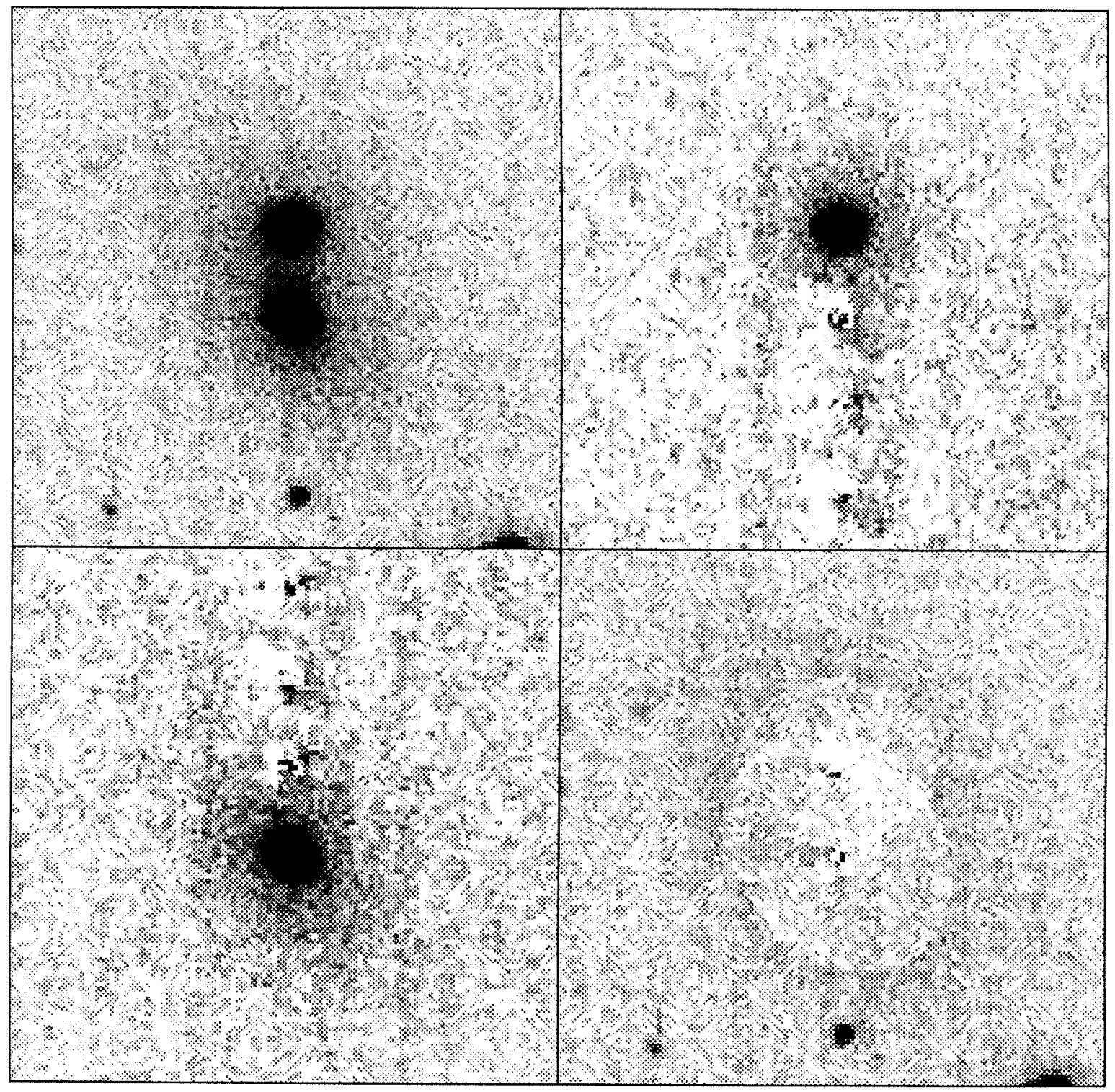

Figure 1 cont'd. A1984 


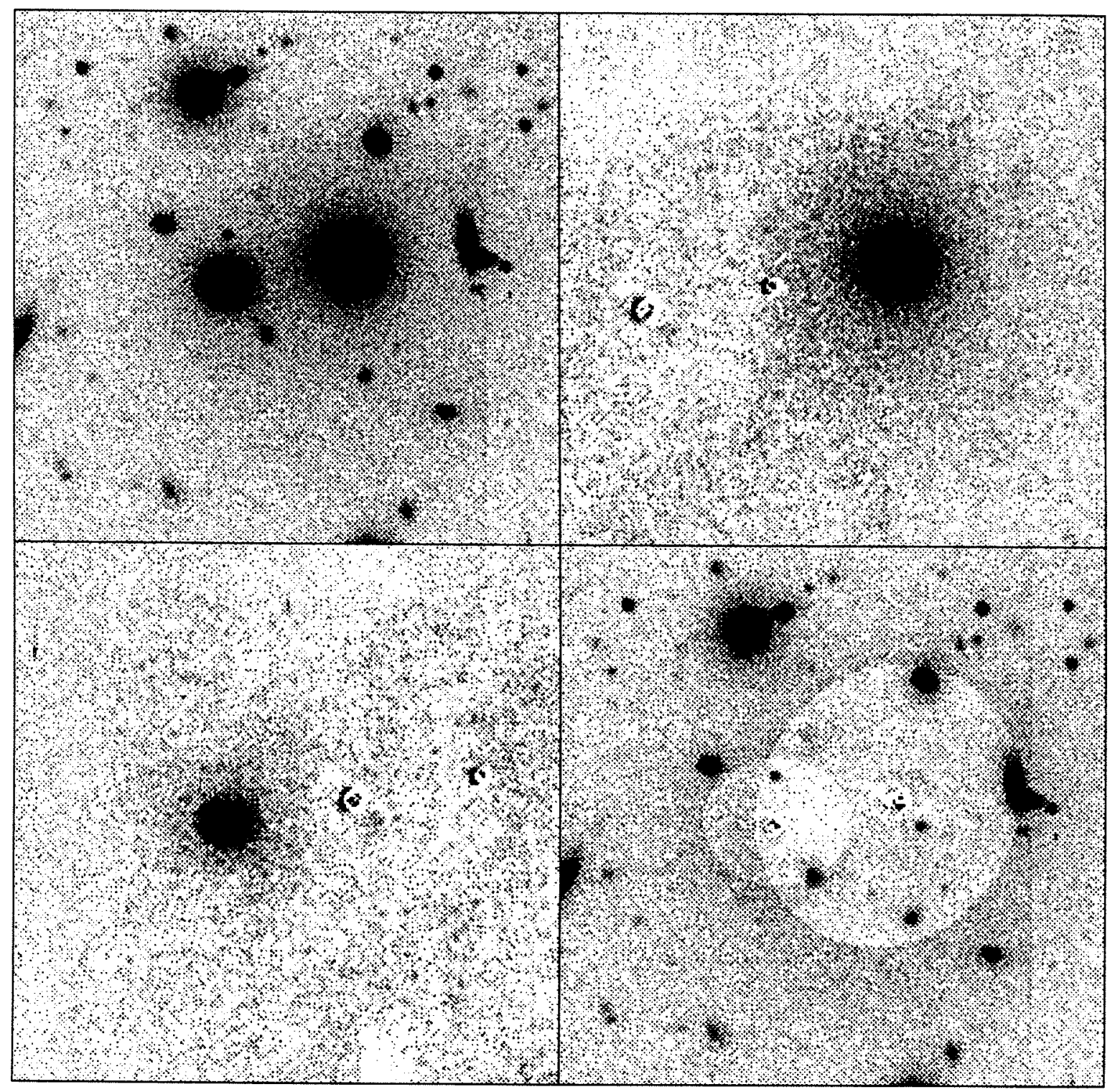

Figure 1 cont'd. A2022 


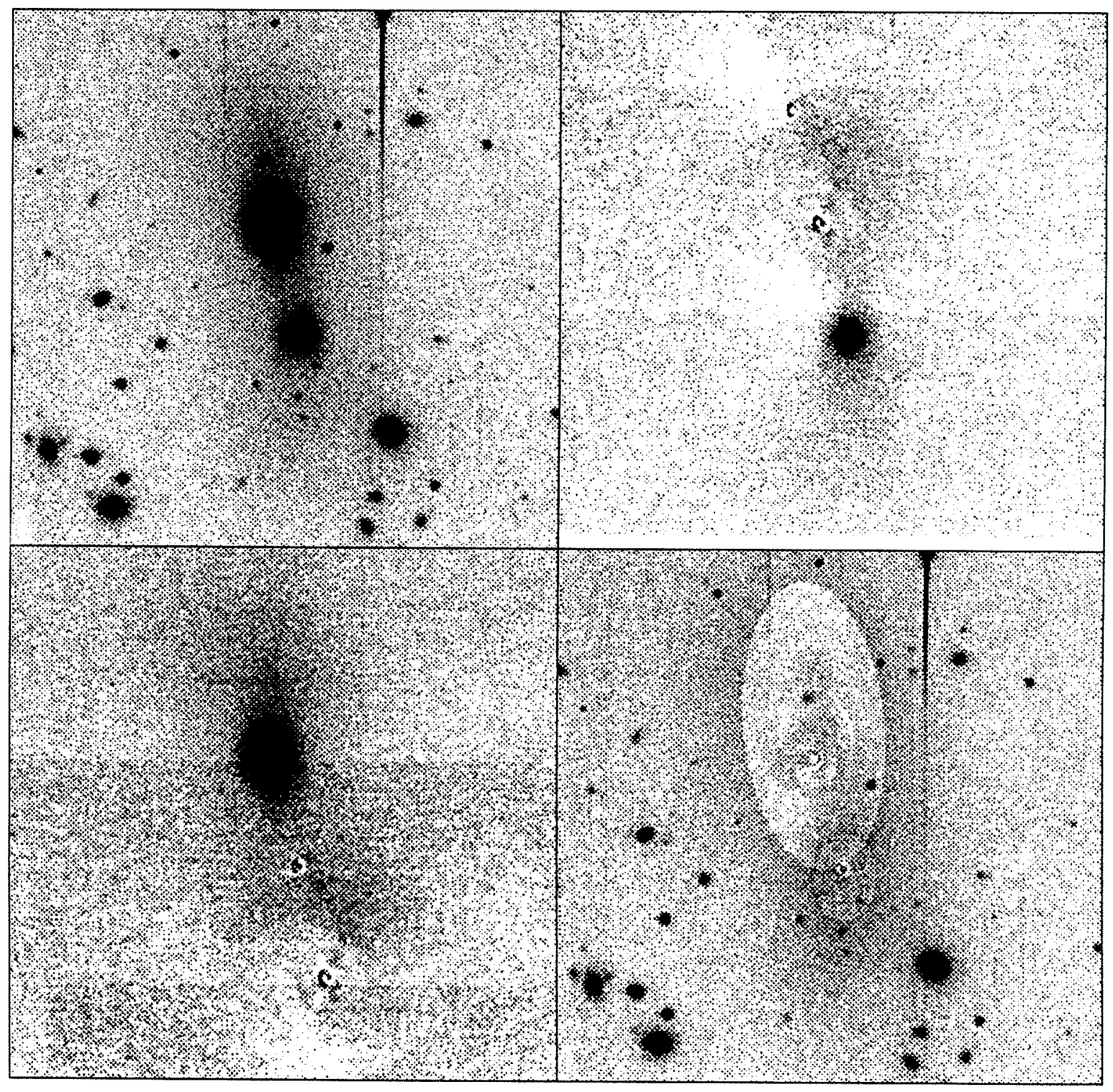

Figure 1 cont'd. A2079 


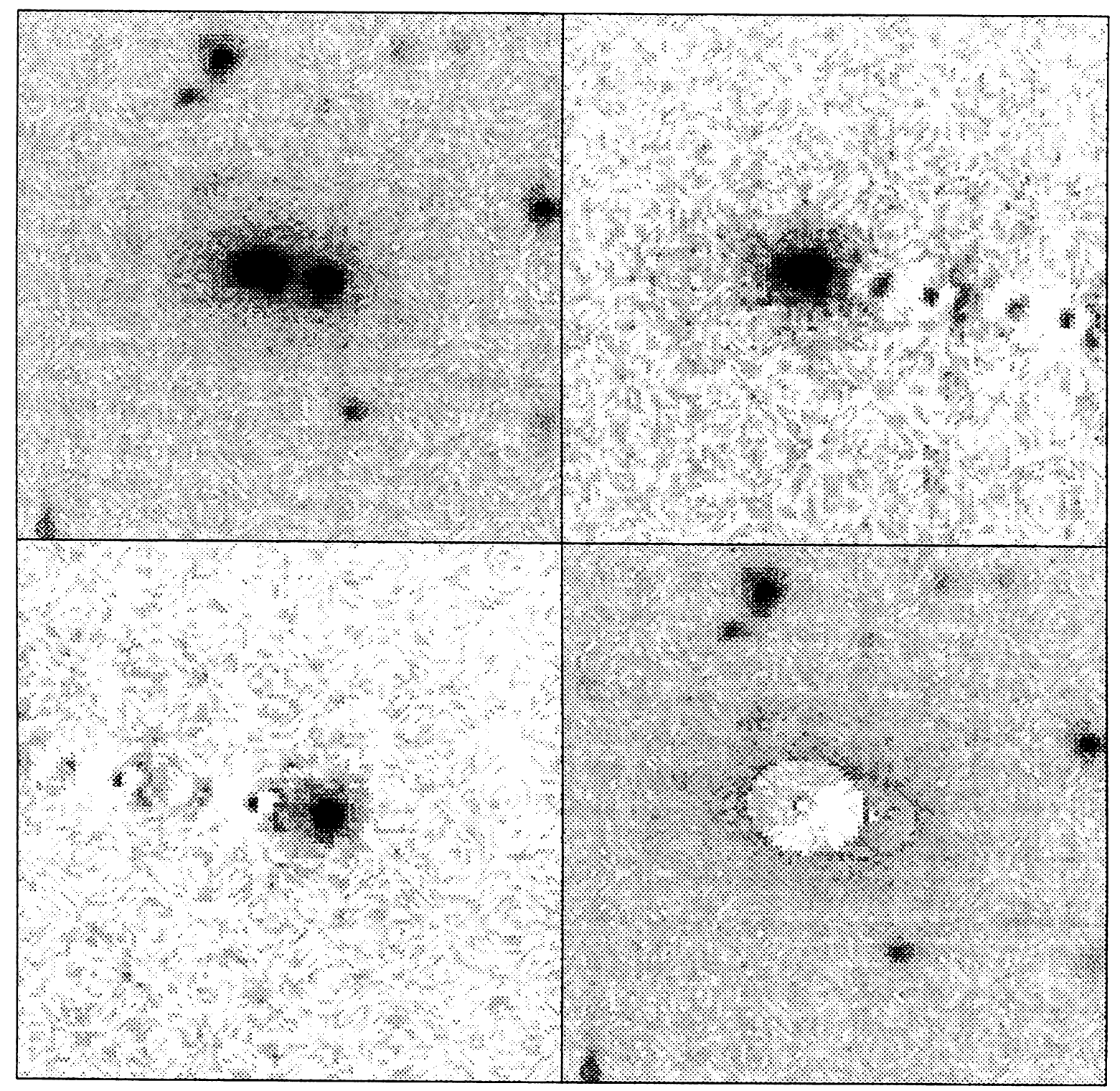

Figure 1 cont'd. A2521 


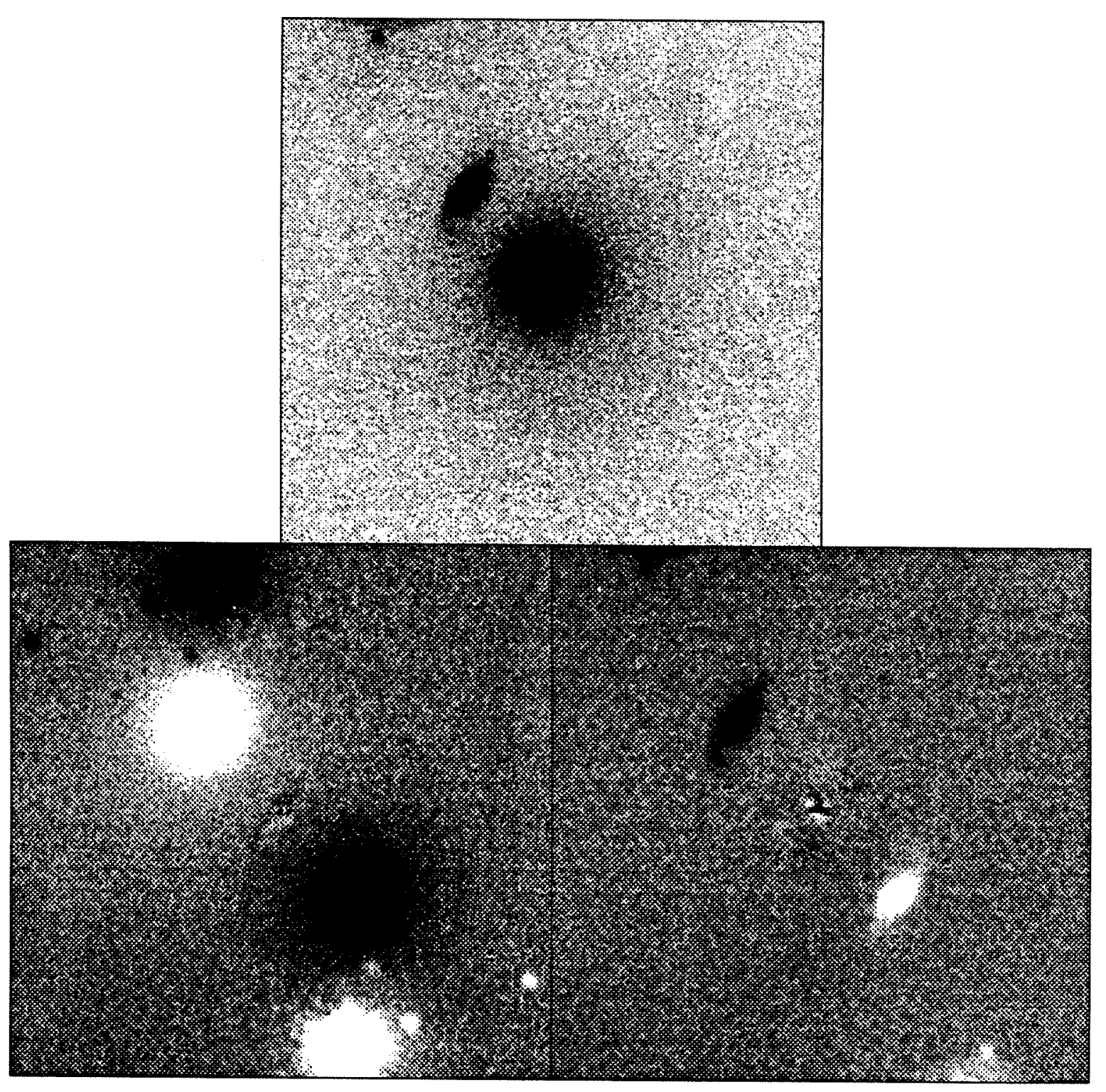

Figure 2. A2670 

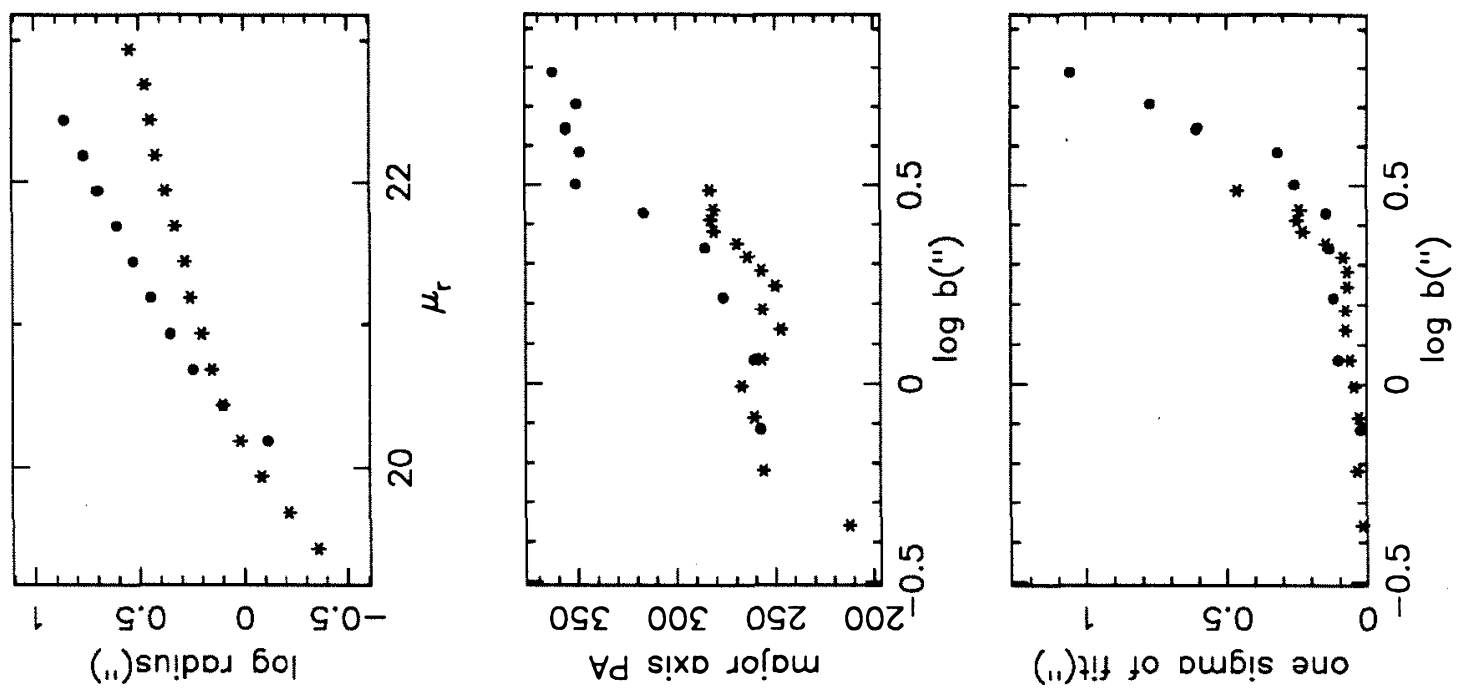

(i1) $+! \$$ to oub!s 2u०

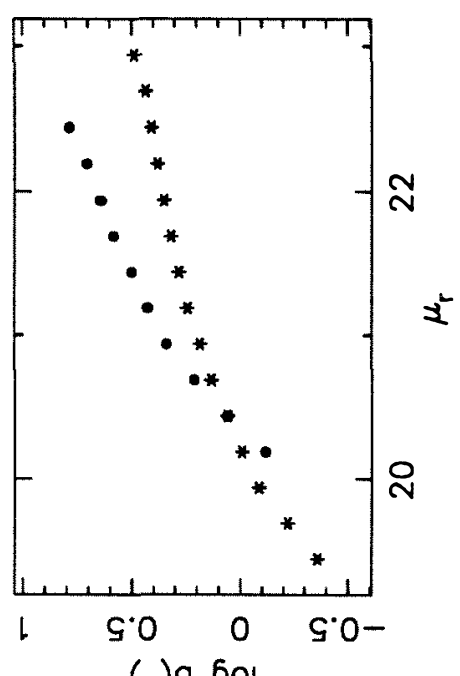

(.1) 9 6이

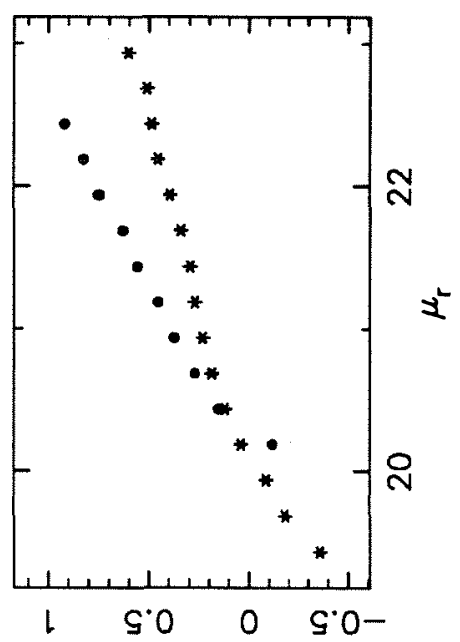

(ii) $\mathrm{D}$ 60।
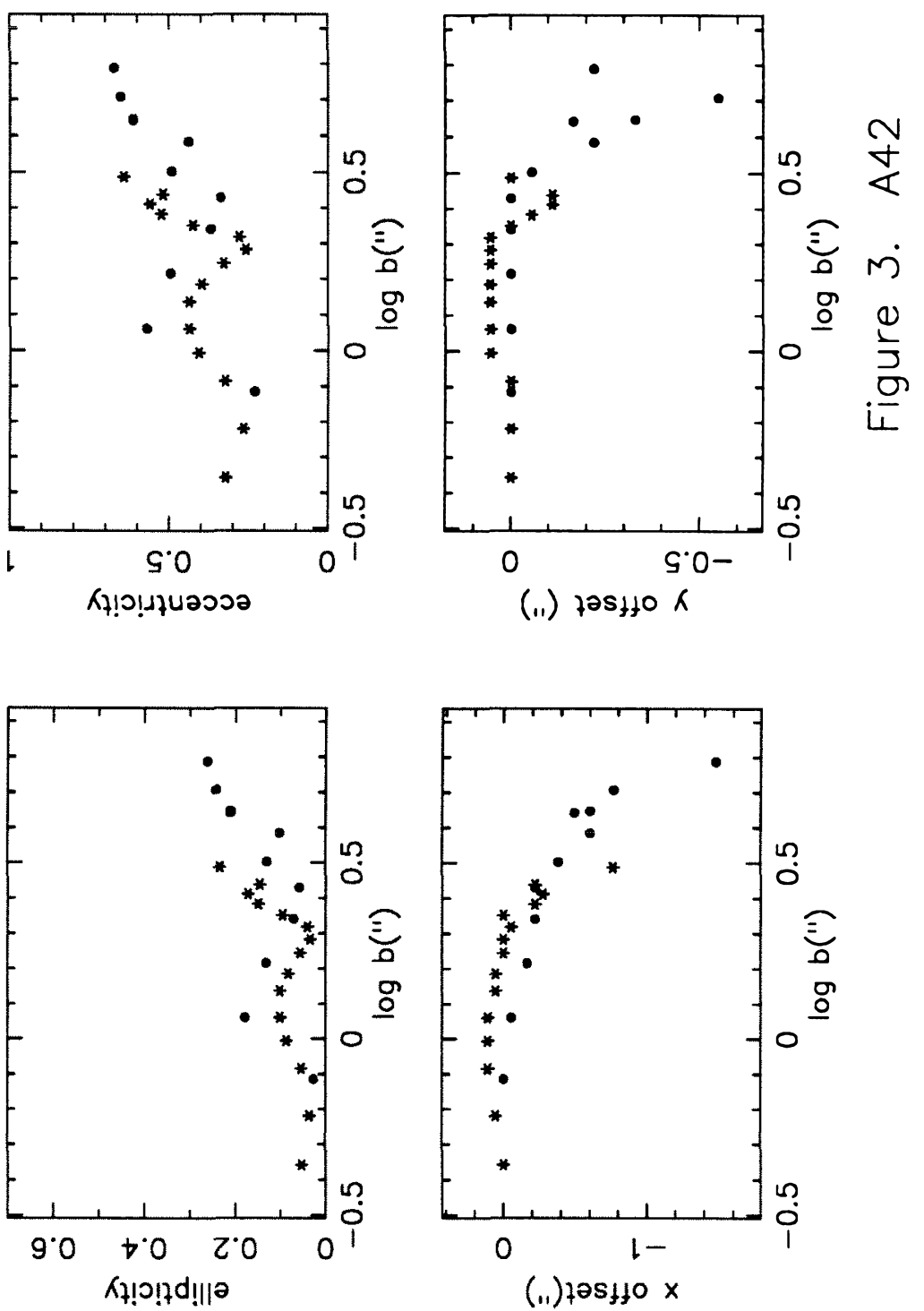


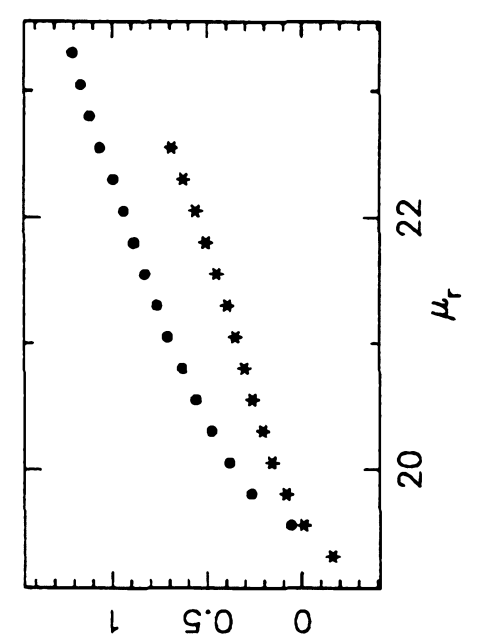

(.1)sn!pos 60।
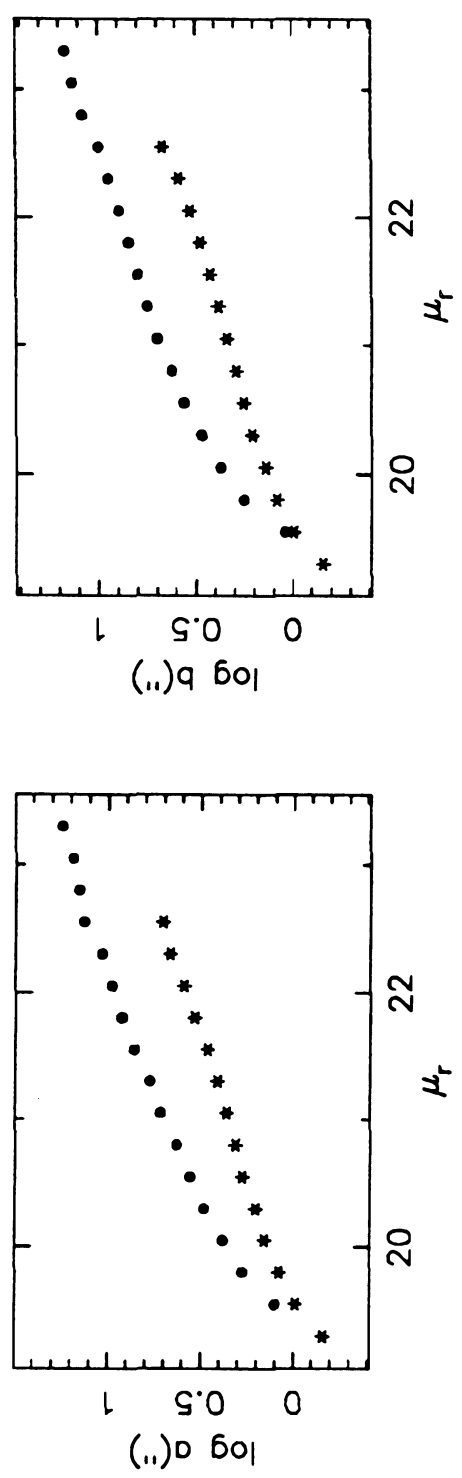

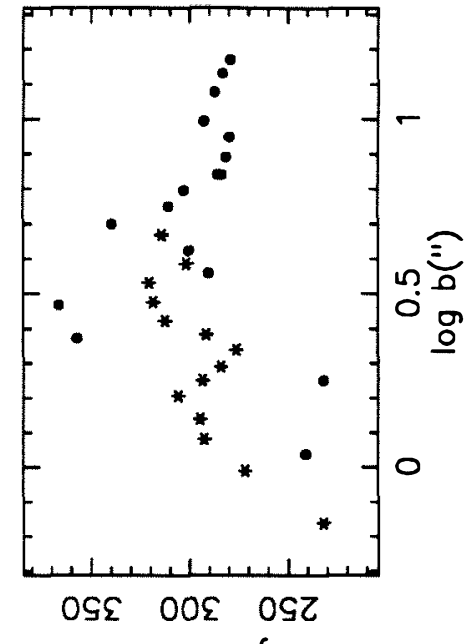

$\forall d$ s!xo do!now
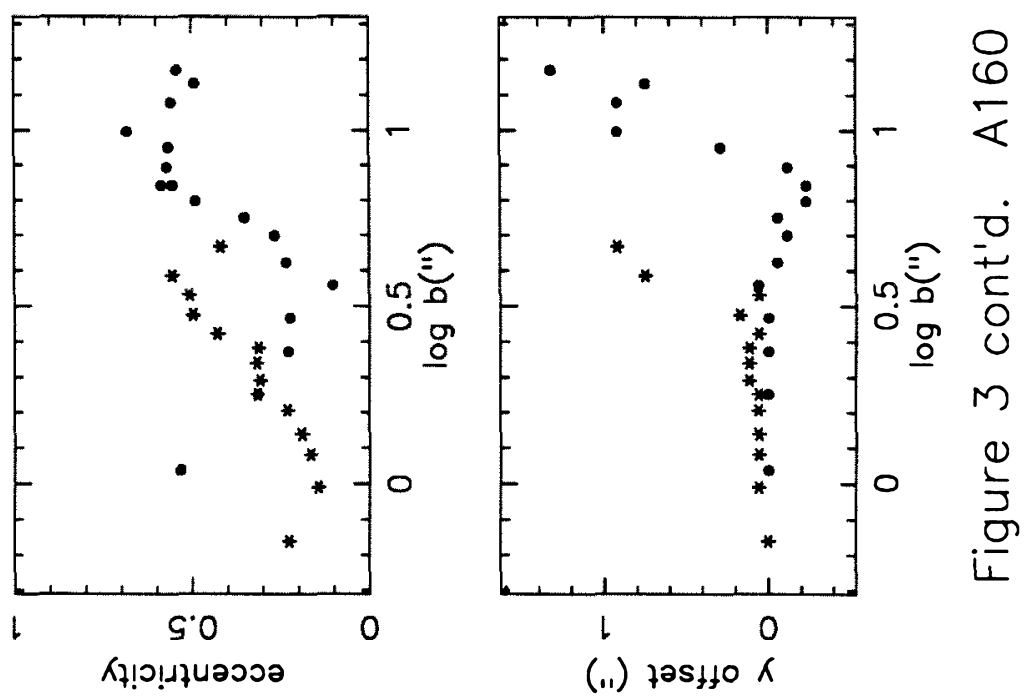
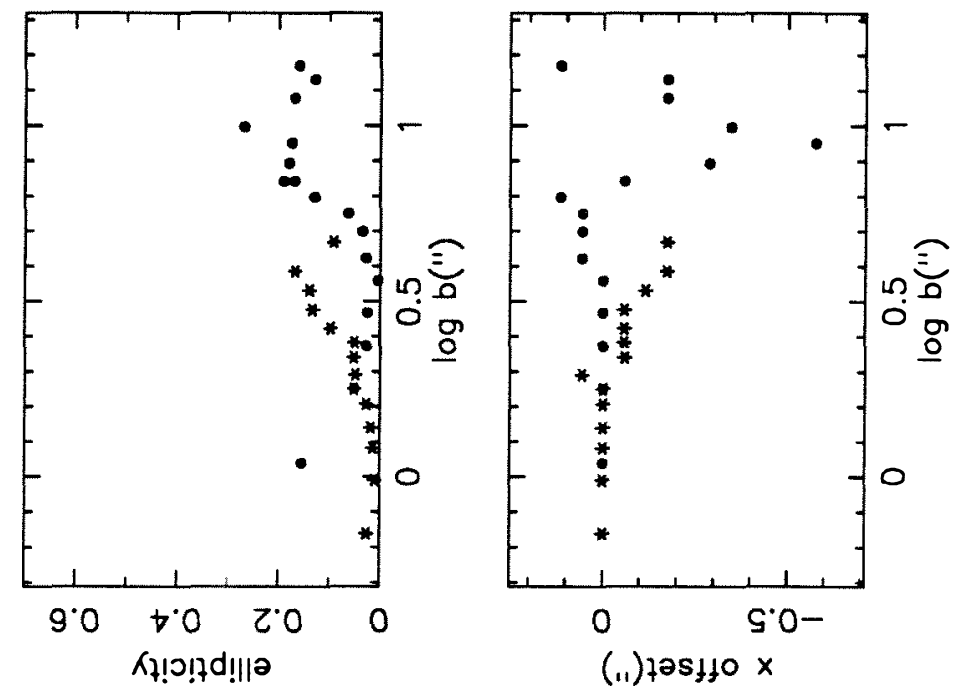

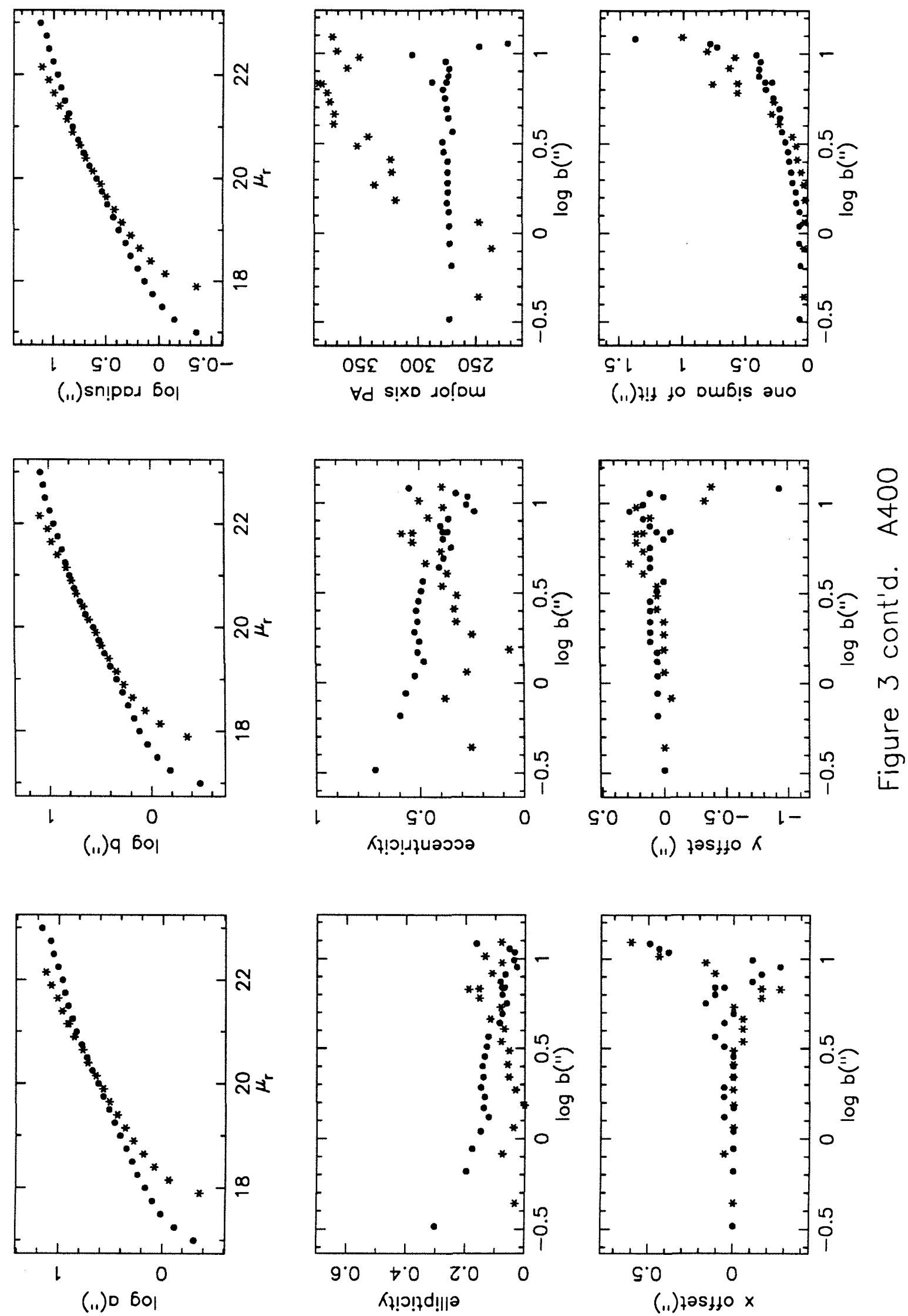

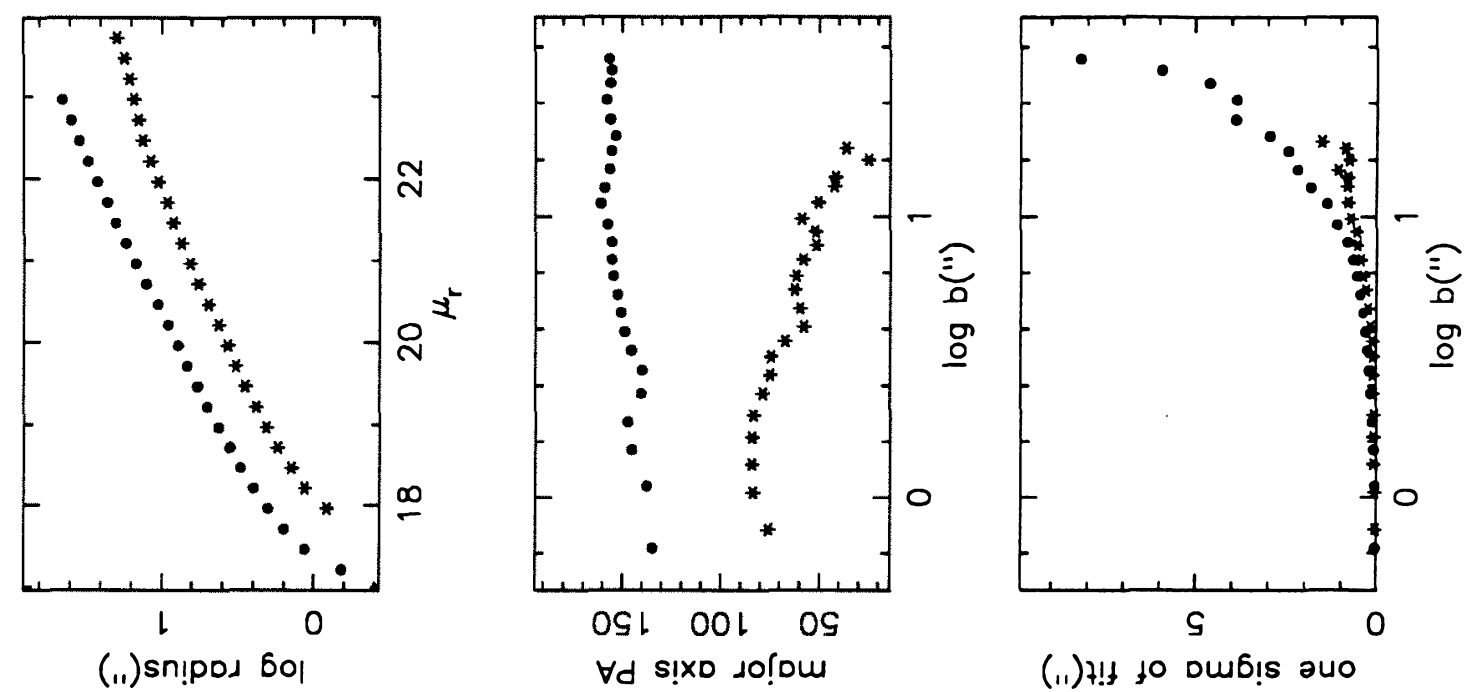

(11) $4 !+$ to Du6!s aนo

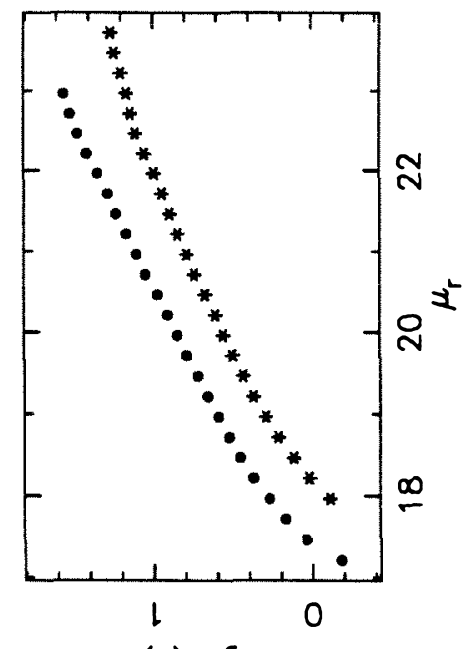

(i1) 9 50।
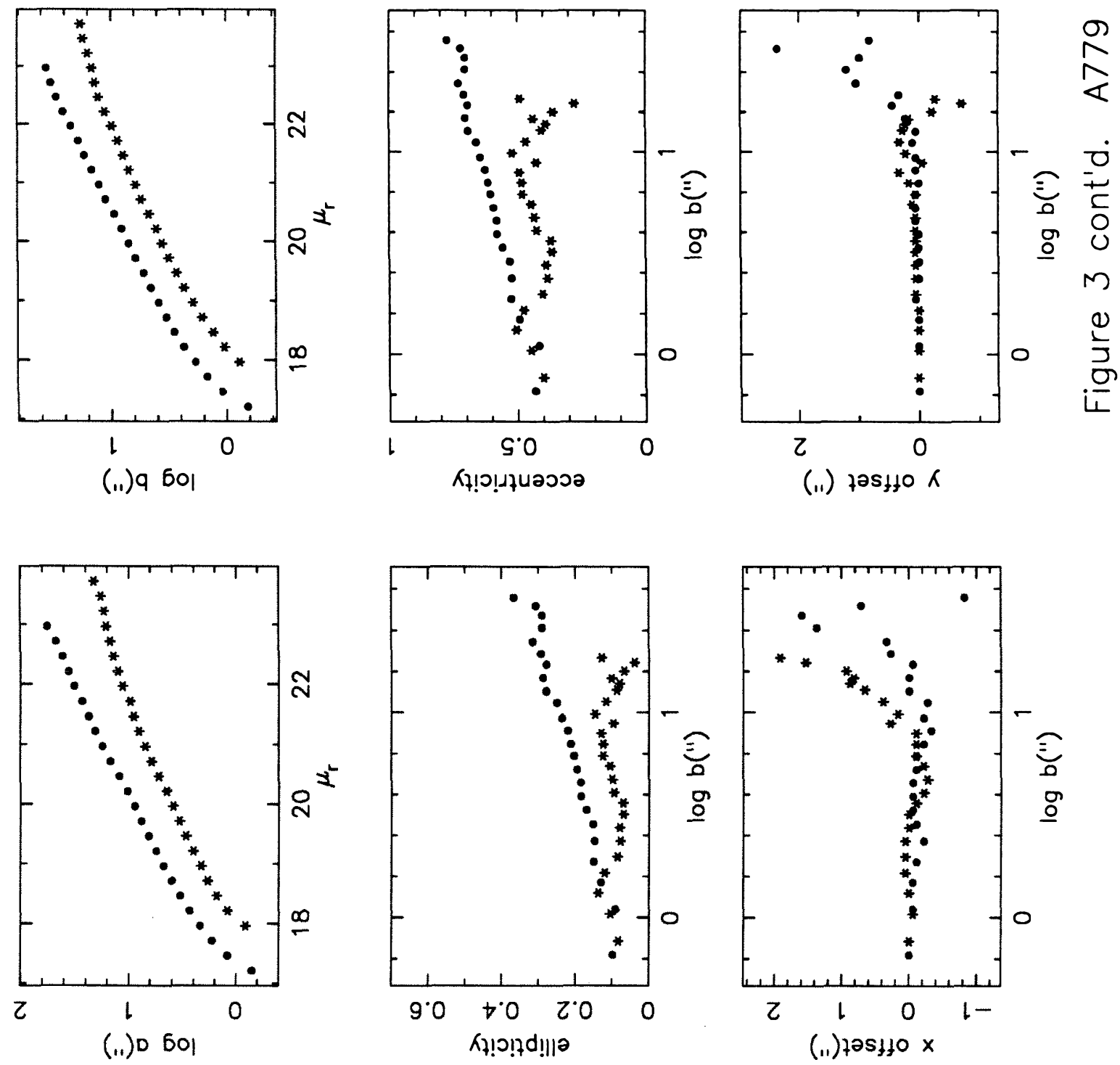

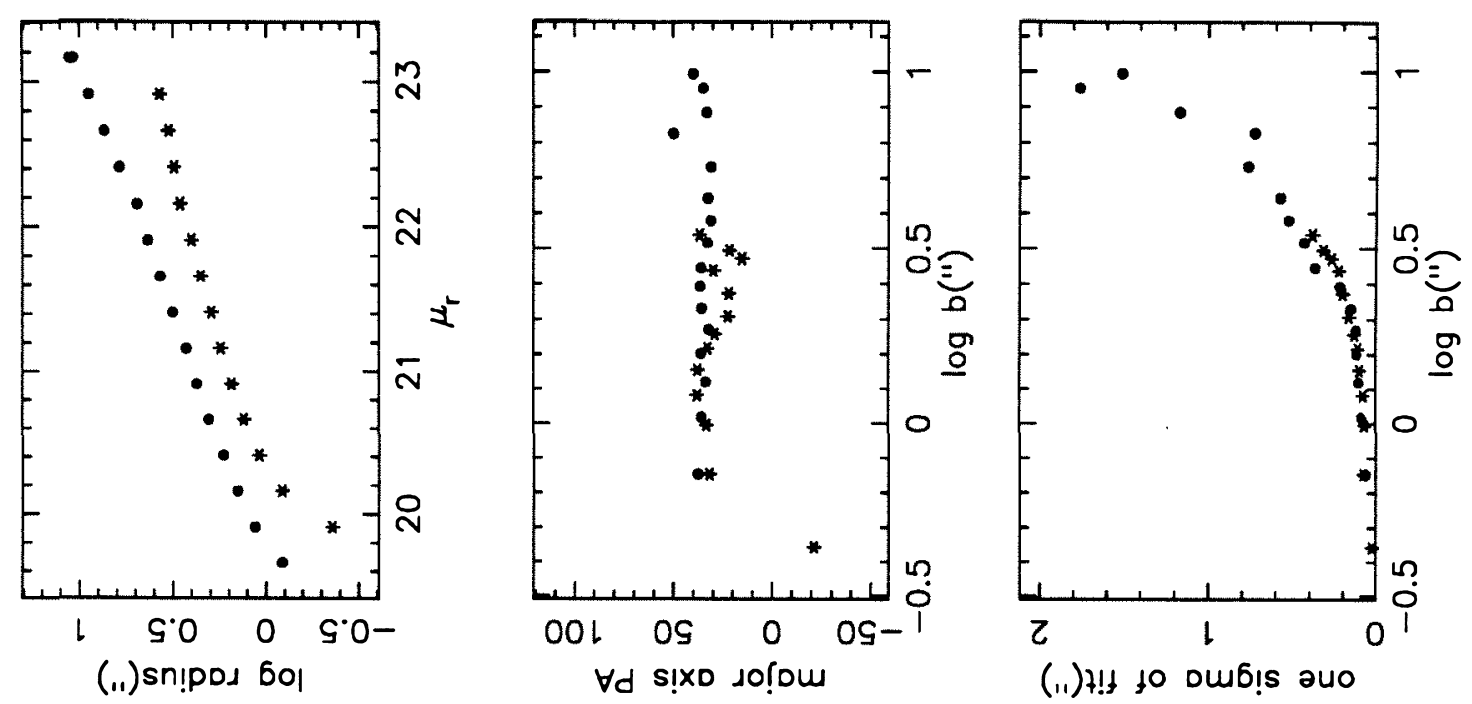

(11) 4 ! 10 Du6!s auo
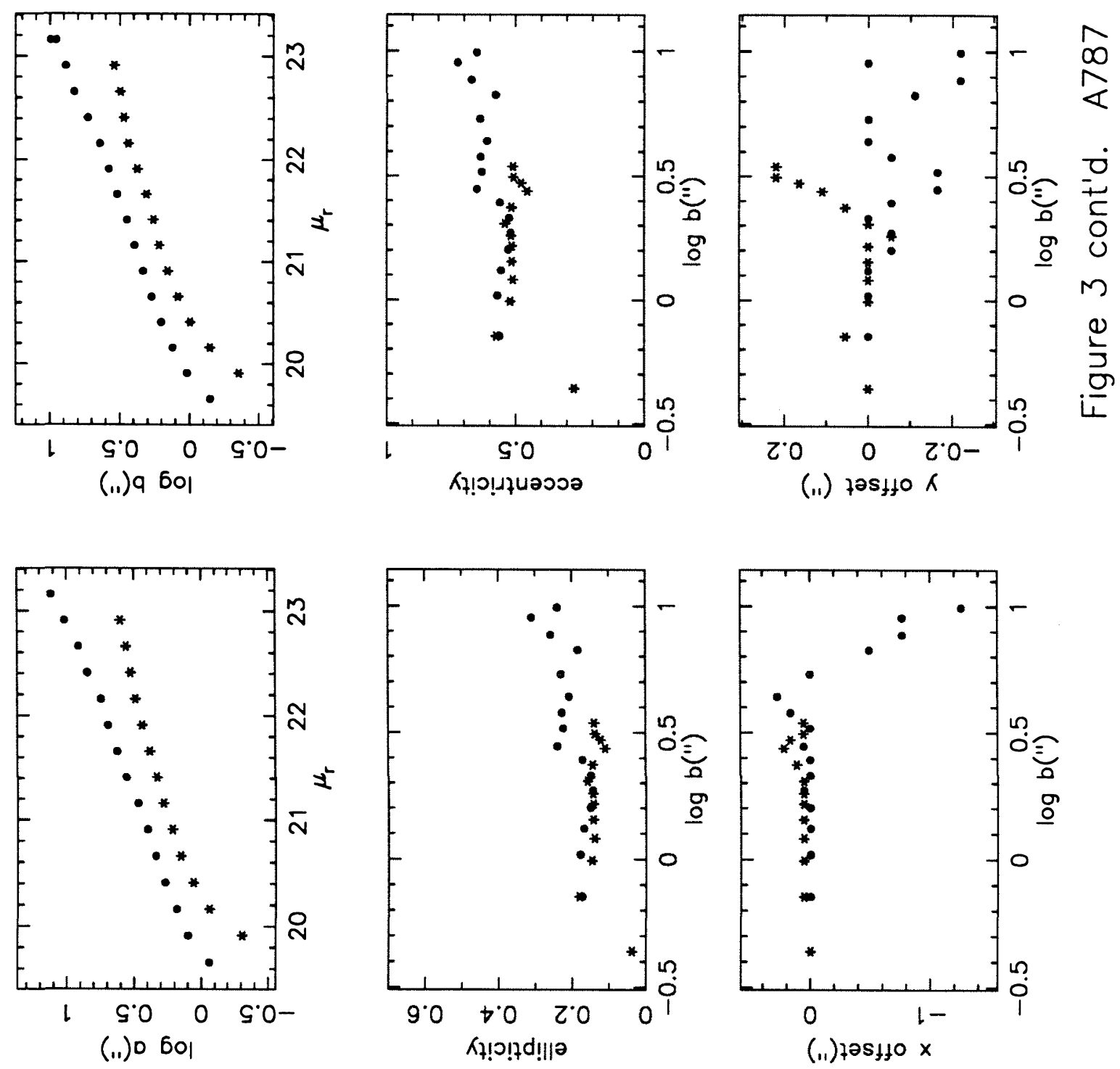

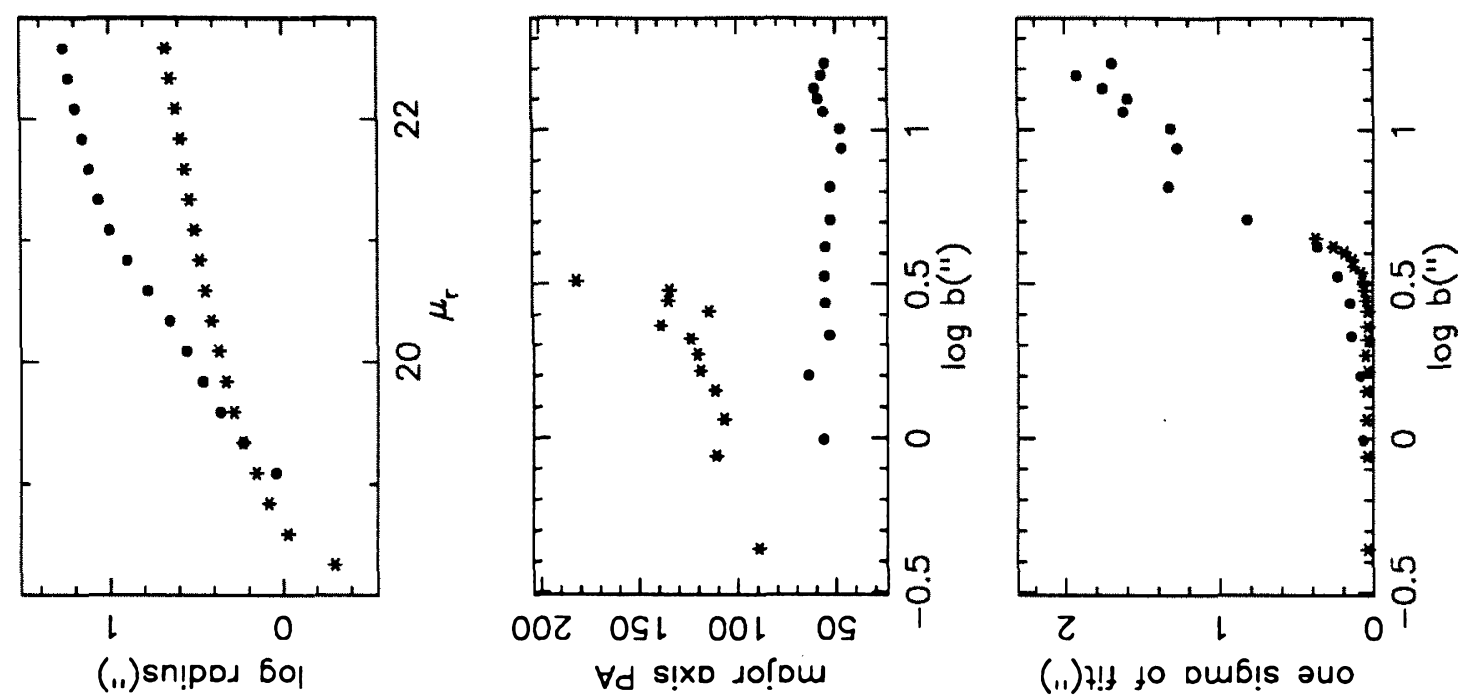

(.1) $+! 1 ~ t 0$ Dub! a a
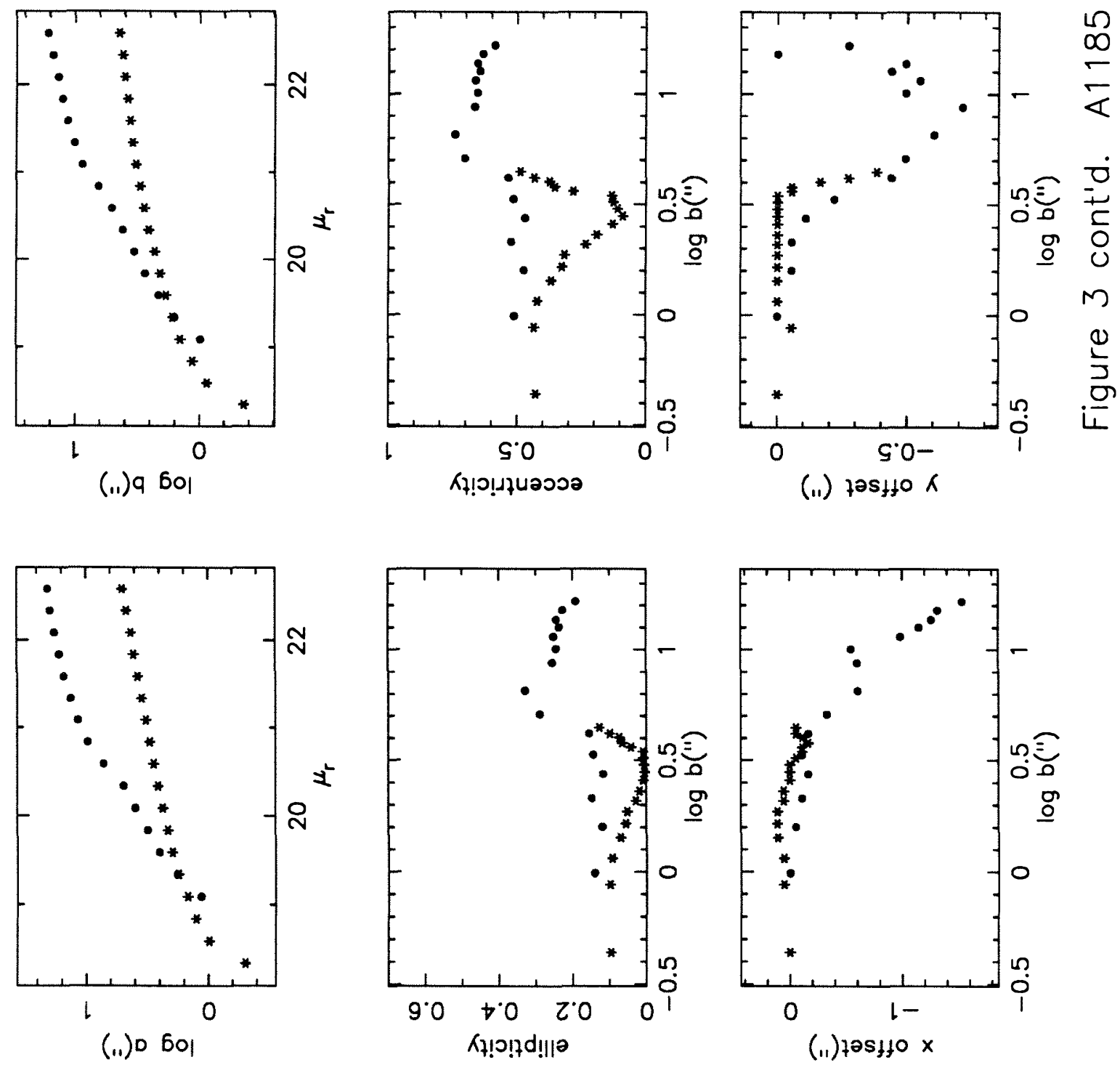


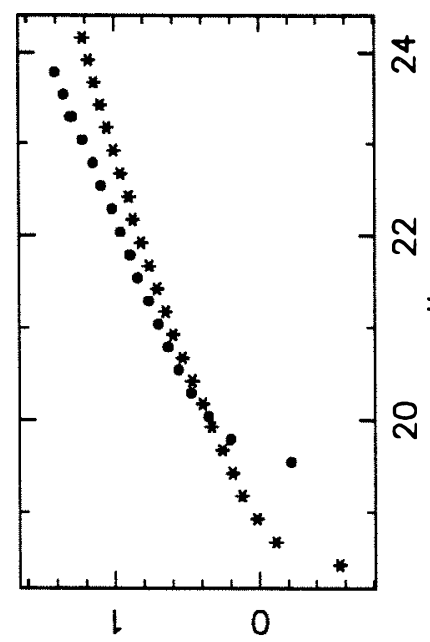

(.1) sn!pod 6ol

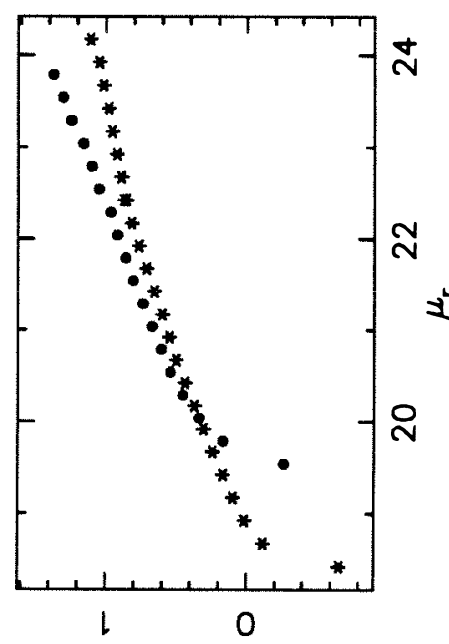

(.1) 960

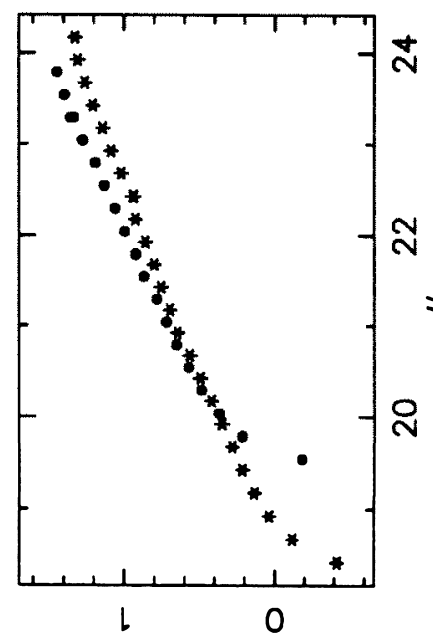

(.1) 0601

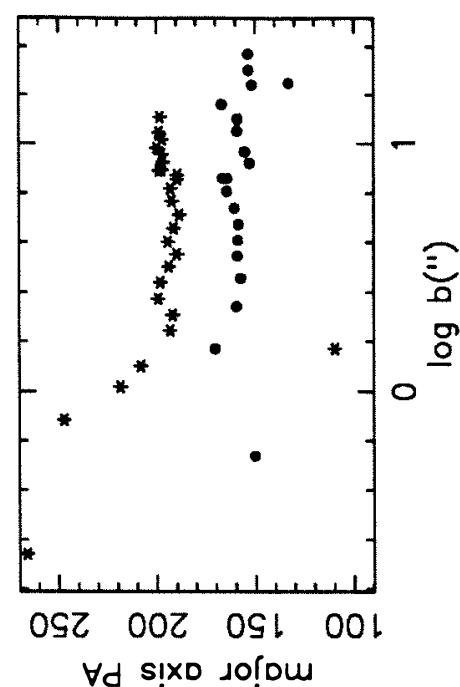

$\forall d$ s!xo jo! om

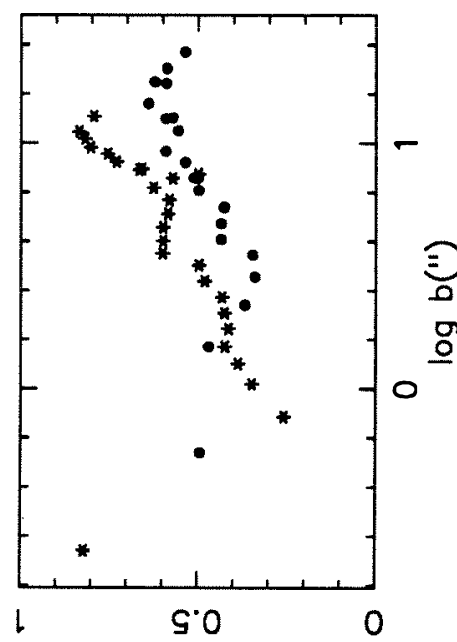

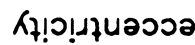
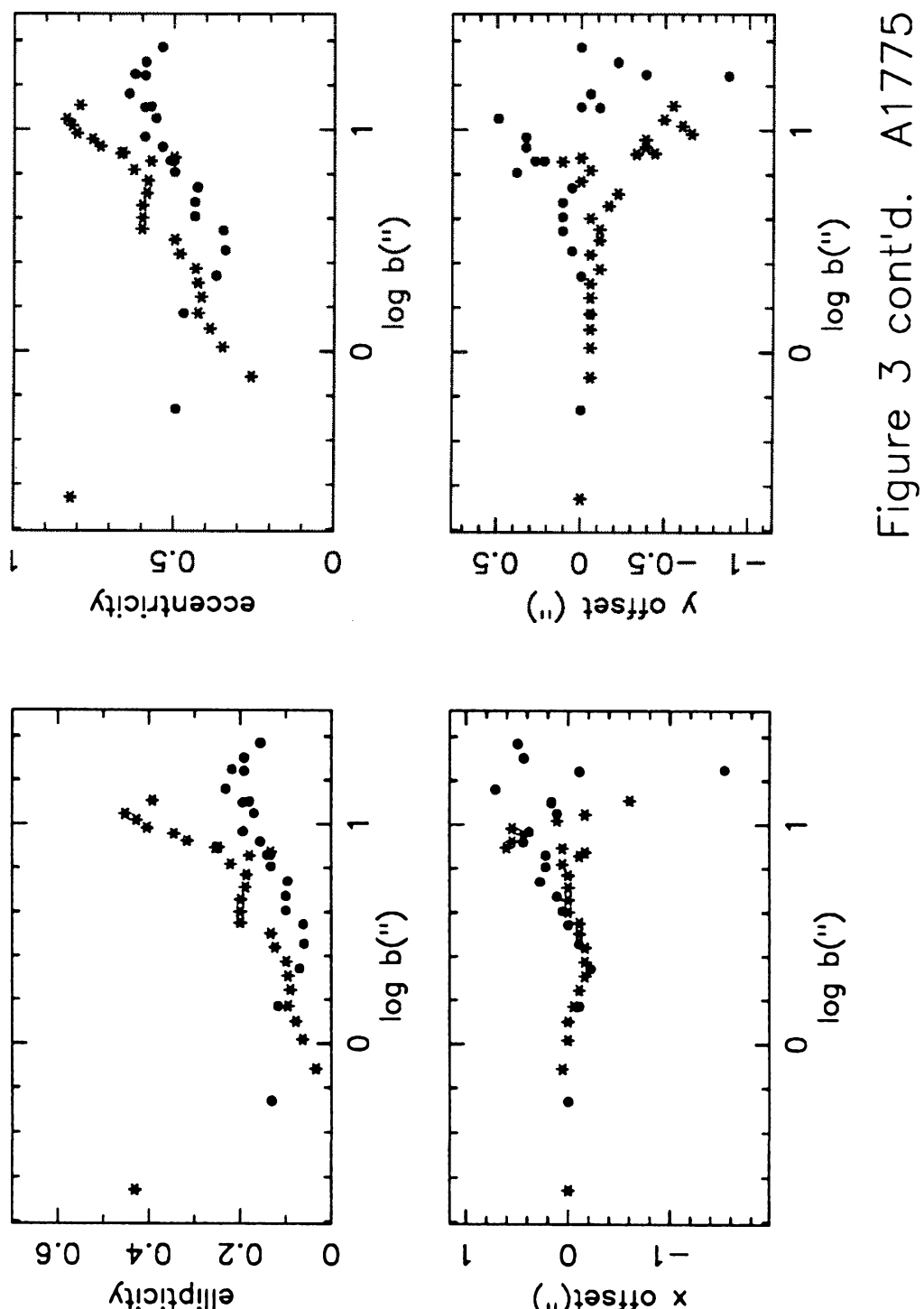

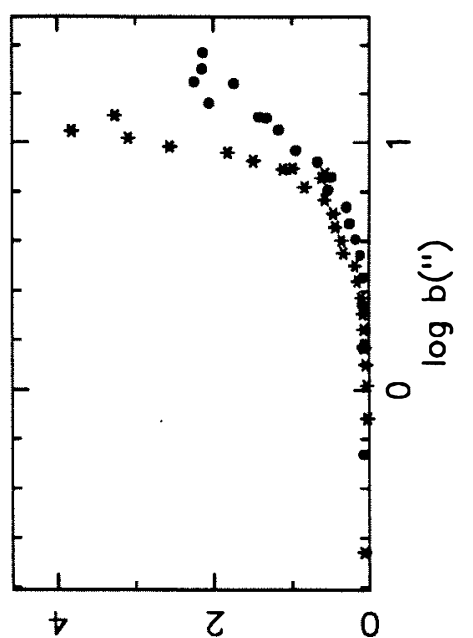

(11) $+! !$ to oub!s auo

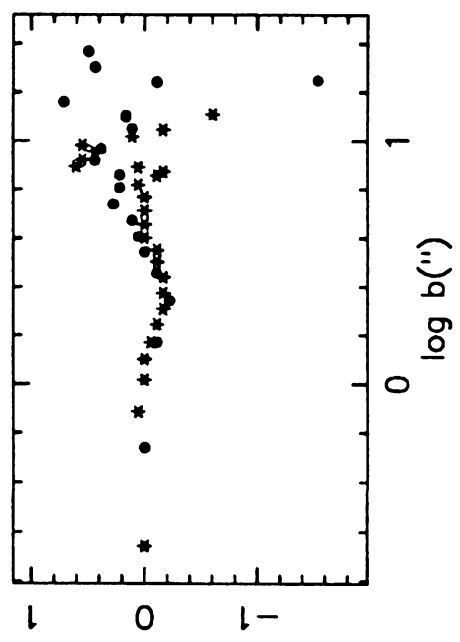

(11) $+205150 \times$ 

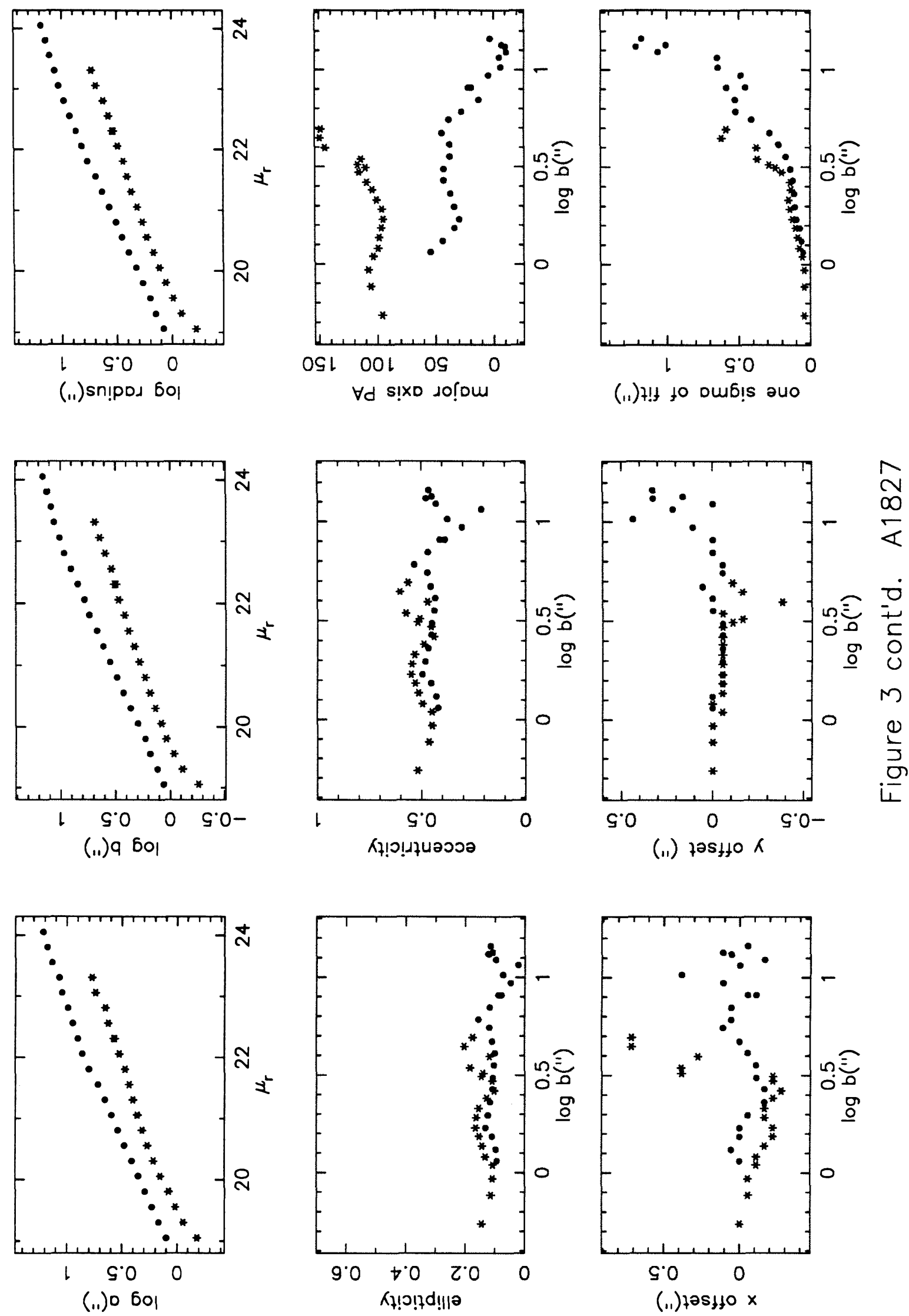


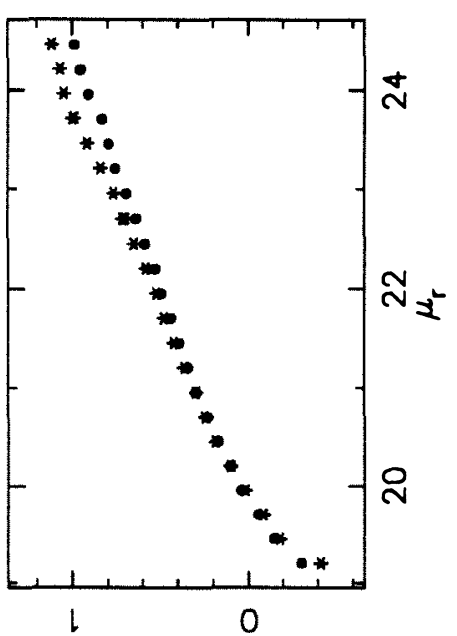

(.1) sn!pod 60।
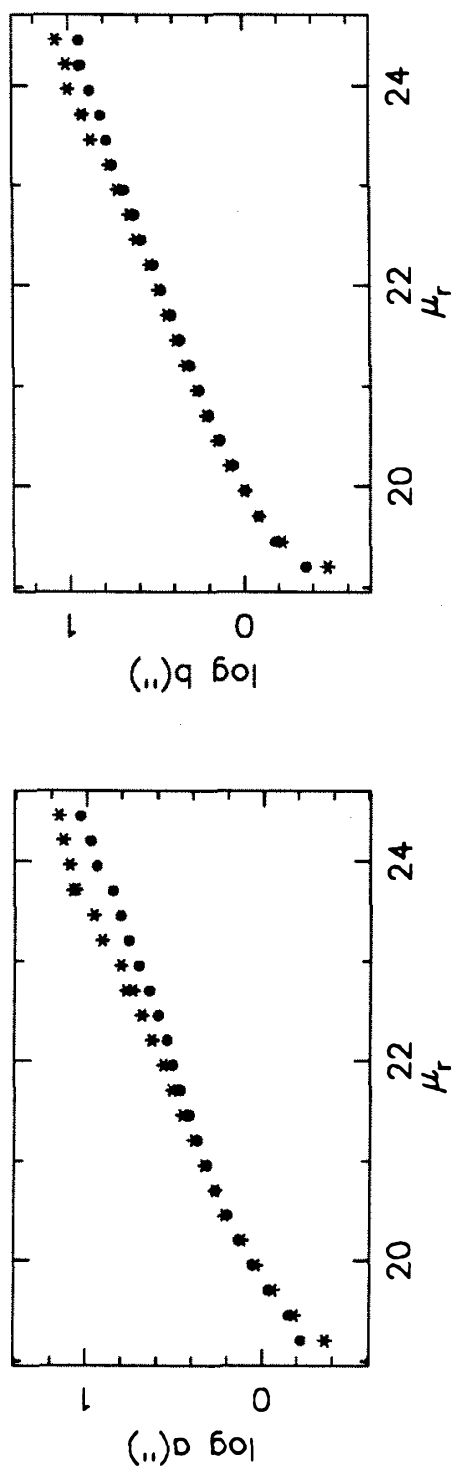
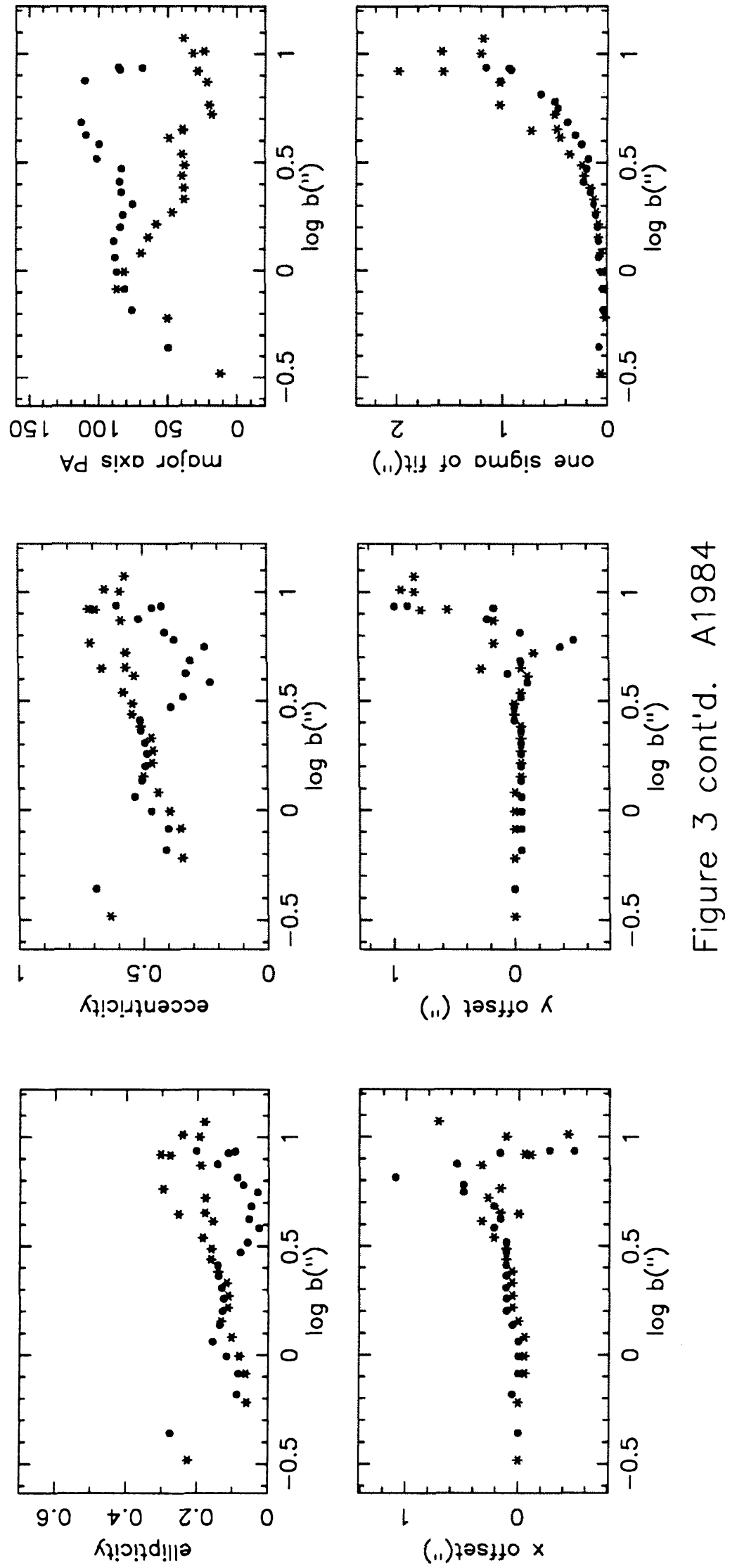


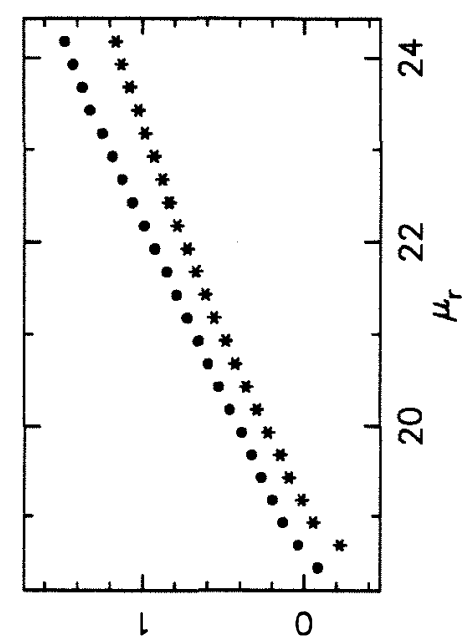

(.1) sn!pod 6ol

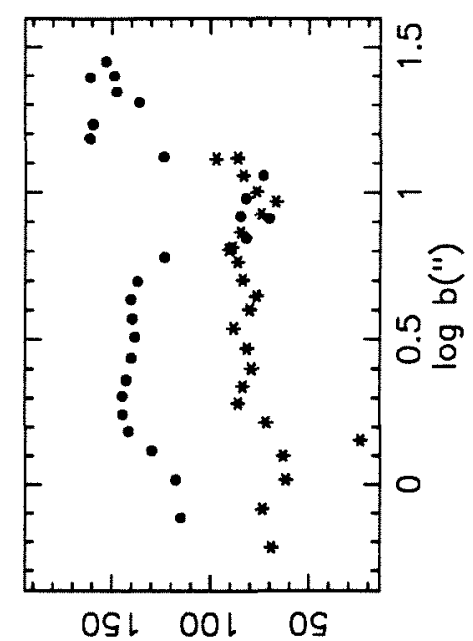

$\forall d$ s!xD

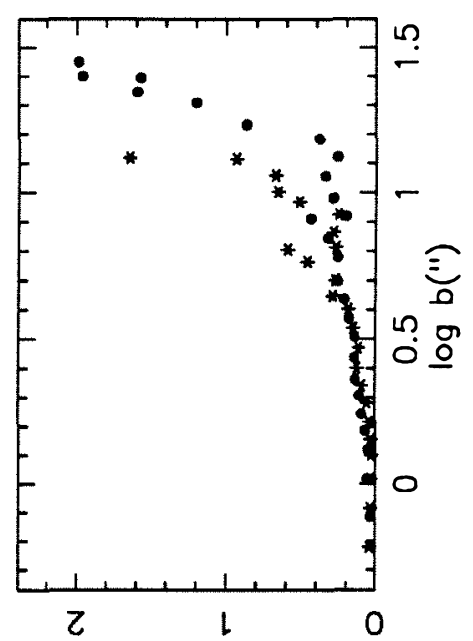

(11) 4 !t to ow6!s auo

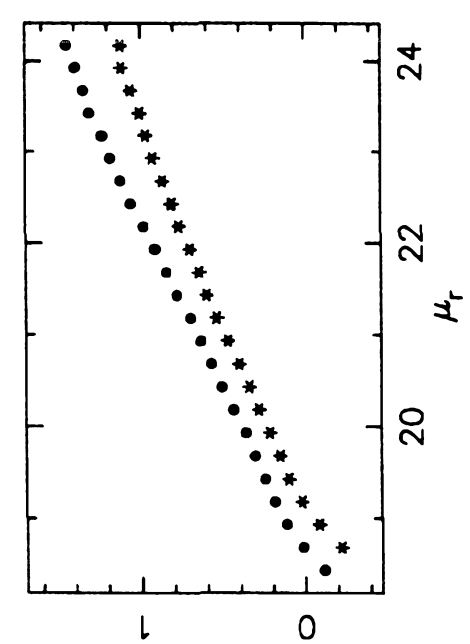

(i1) 9601

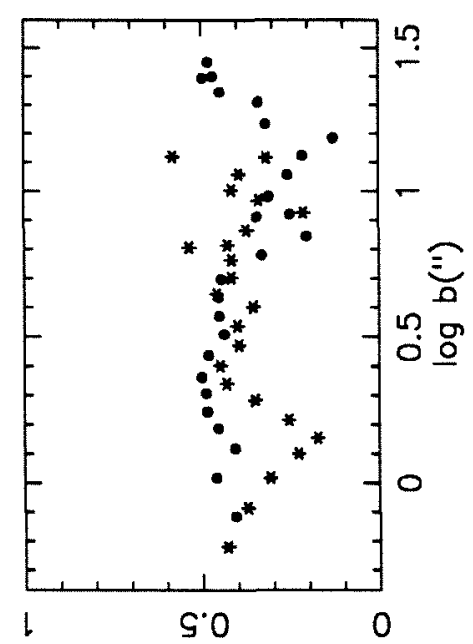

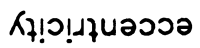

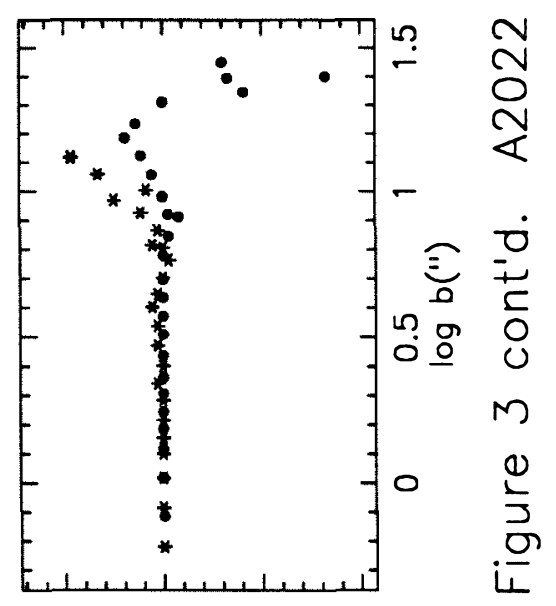

(ii) $725+10 K$

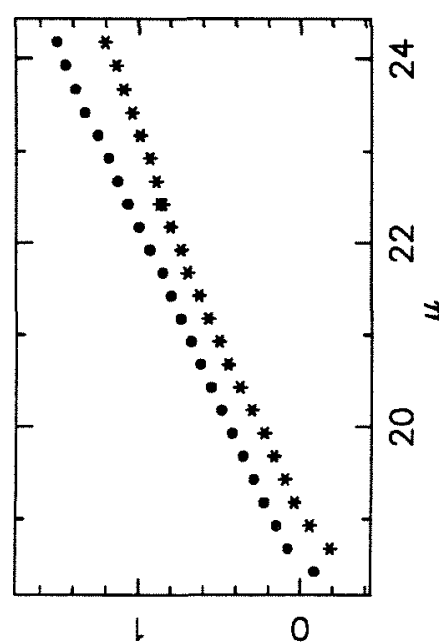

(.1) $0 \mathrm{60}$
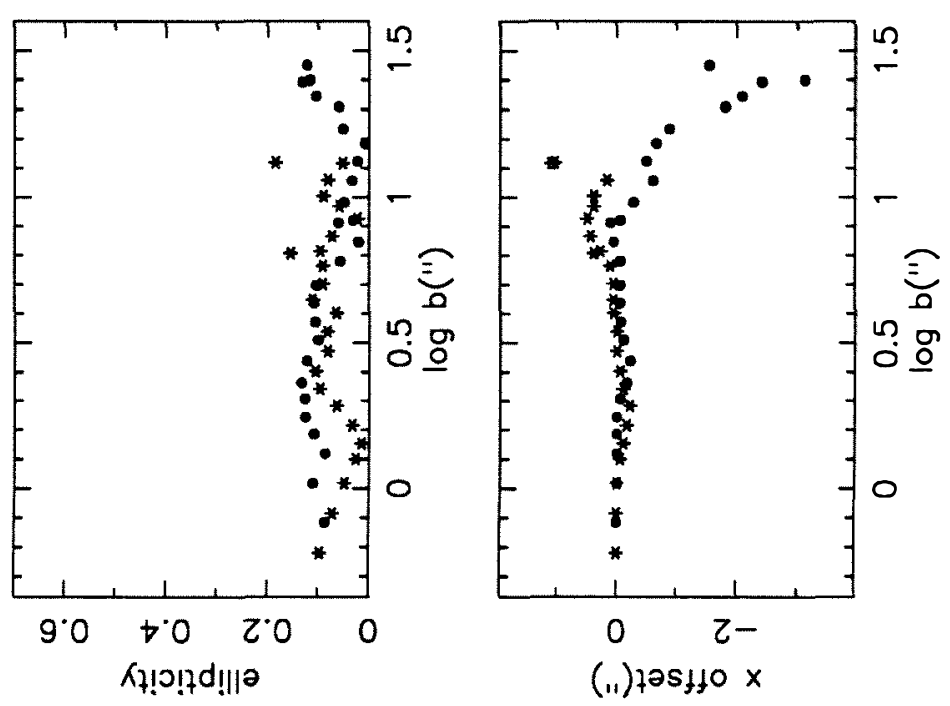


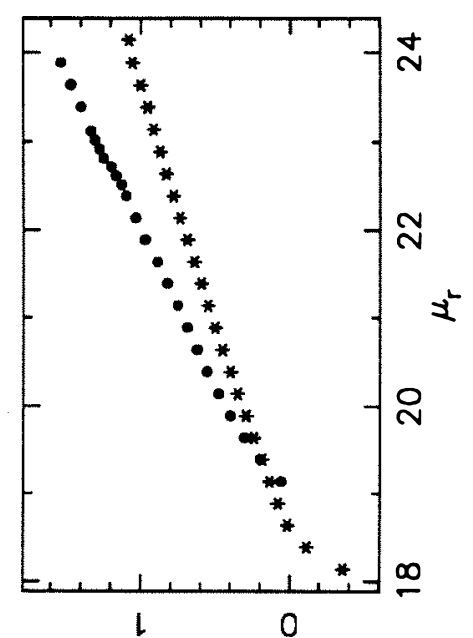

(..) sn!pod 6ol
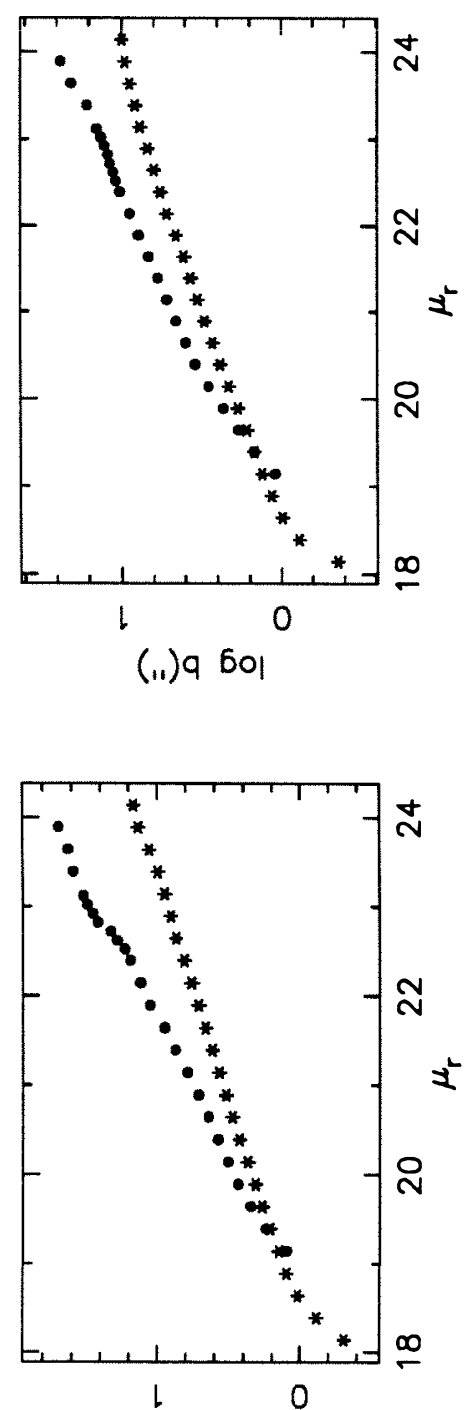

(.1) 0 601

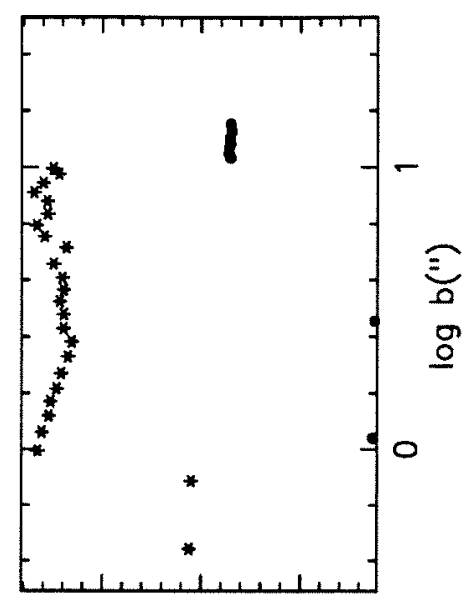

OSL 001 OS

$\forall d$ s!xo so!̣om

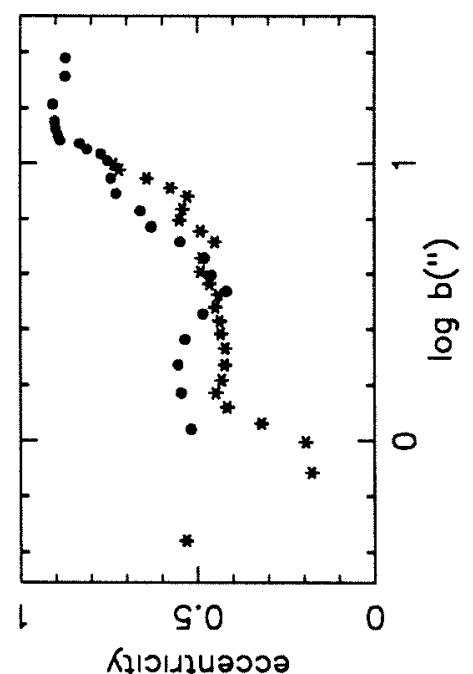

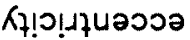

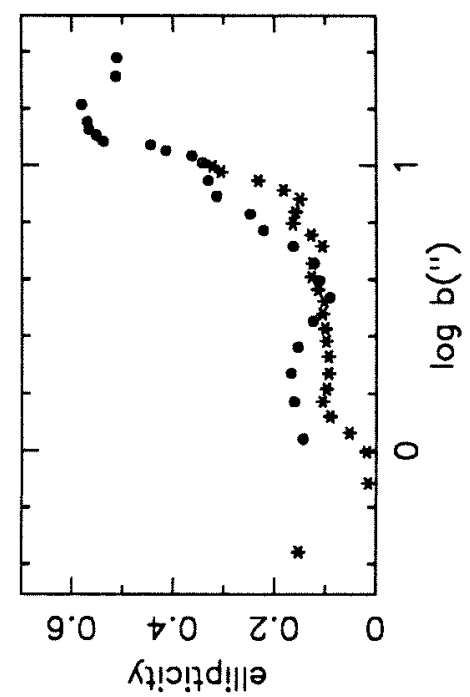

Ð

응

(11) $7 !+$ to Du6!s auo

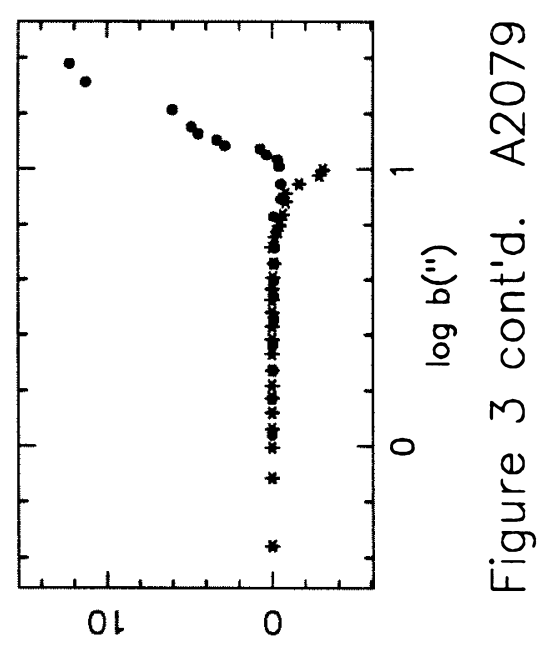

(ii) $725410 \mathrm{~K}$

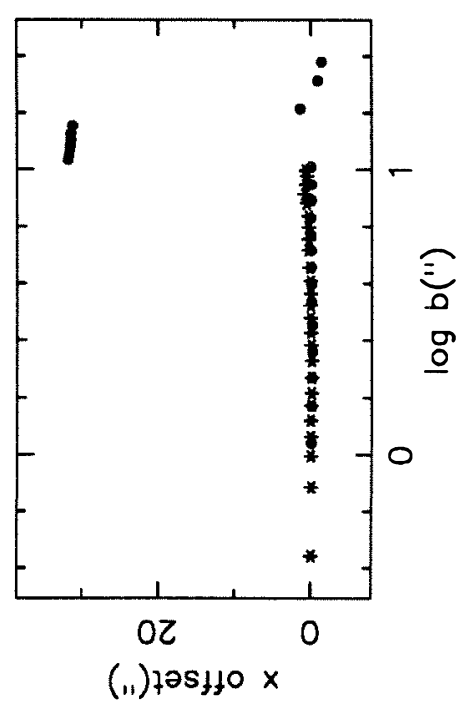




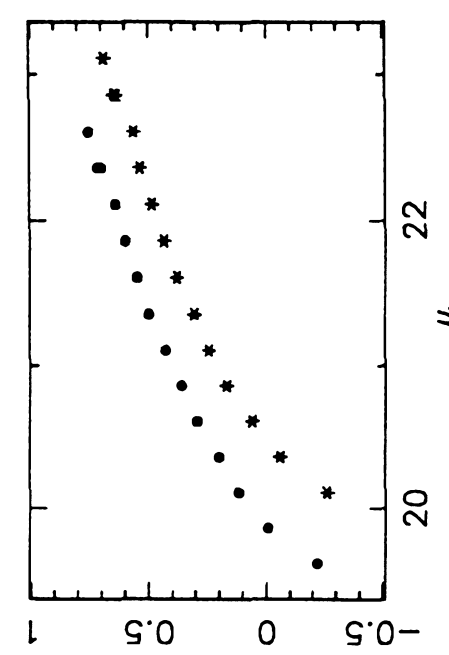

(..)sn!pos 6ol
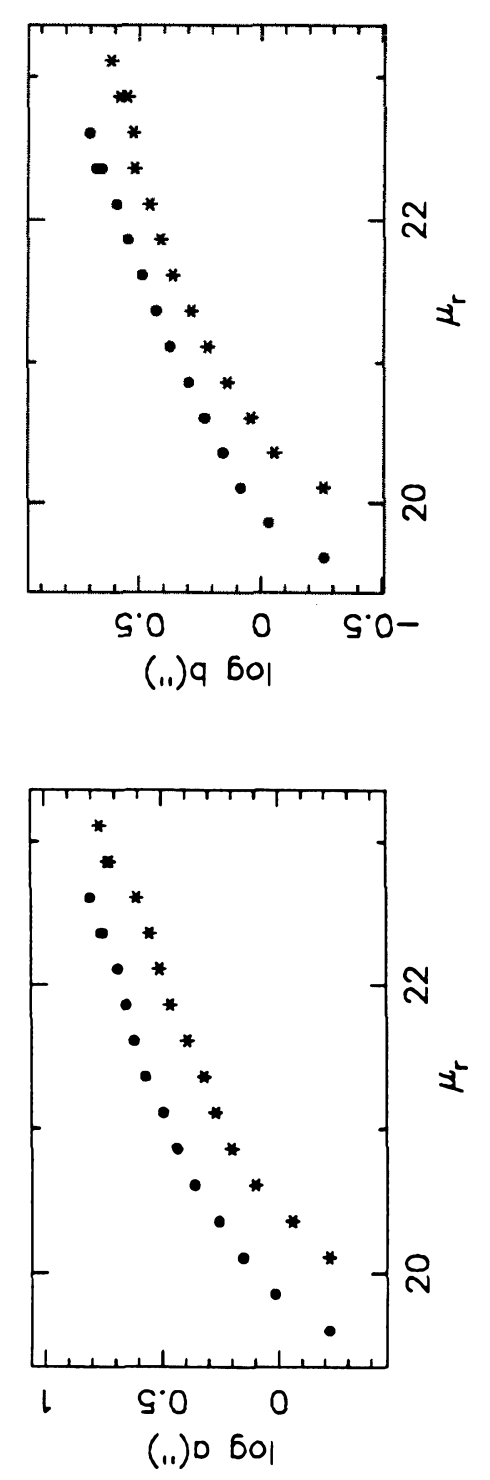

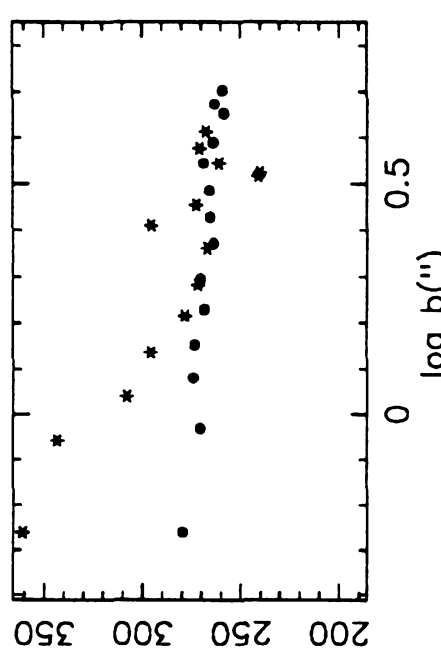

$\forall d$ S!Xo
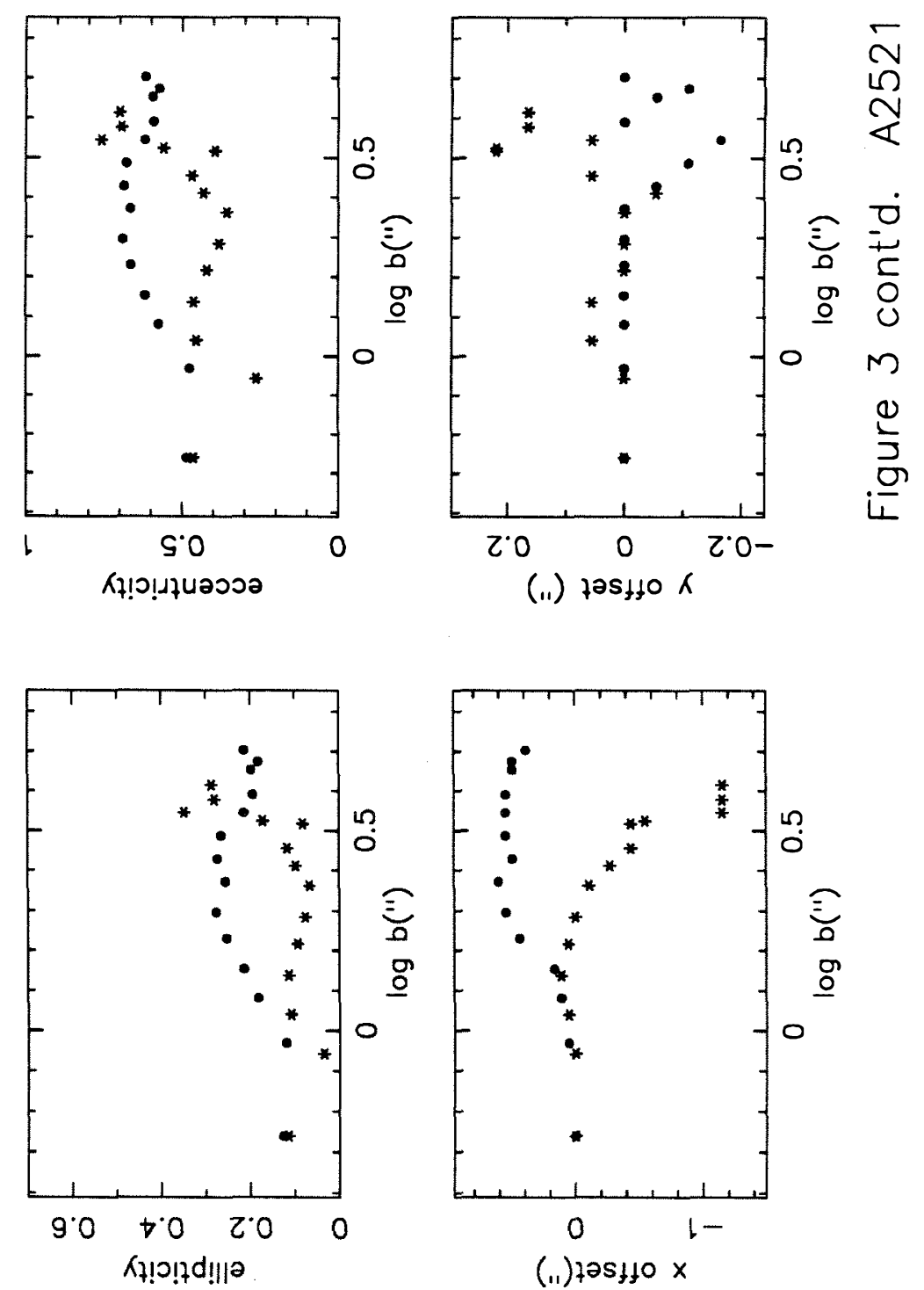
A400

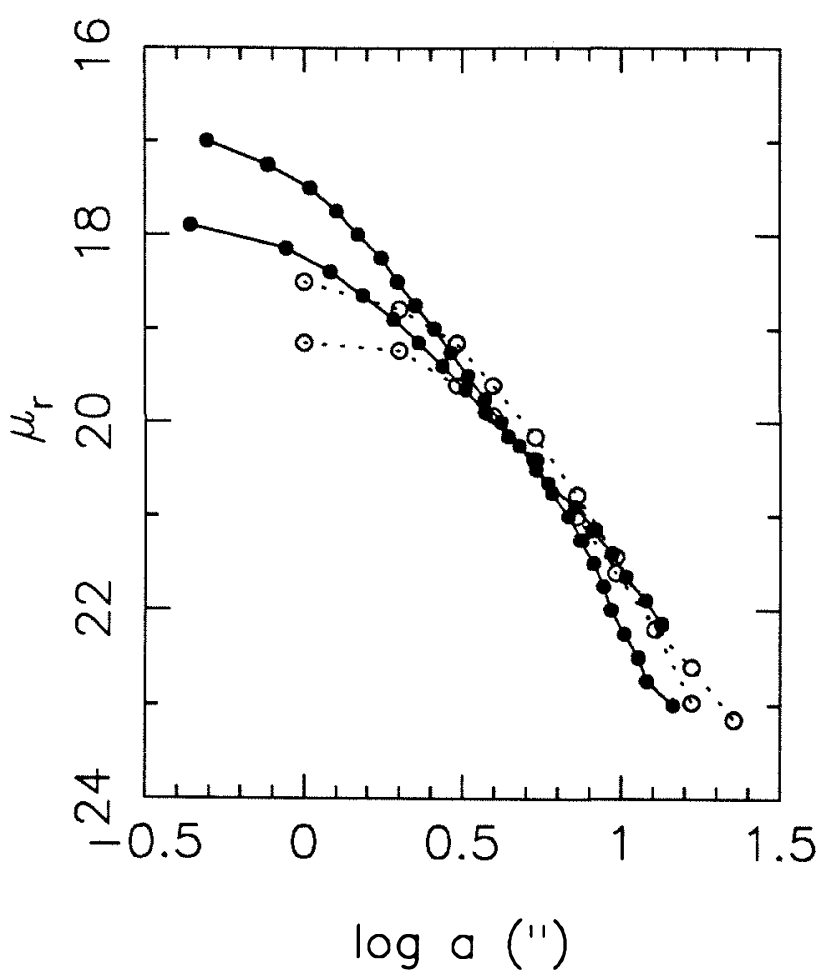

A1185

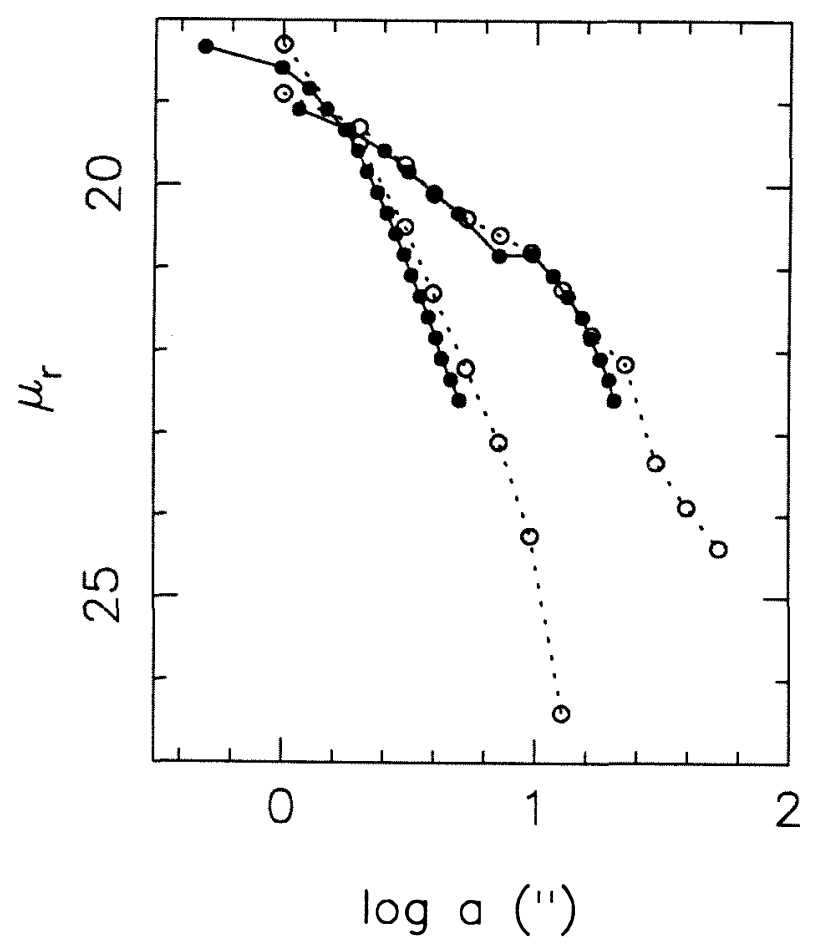

A779

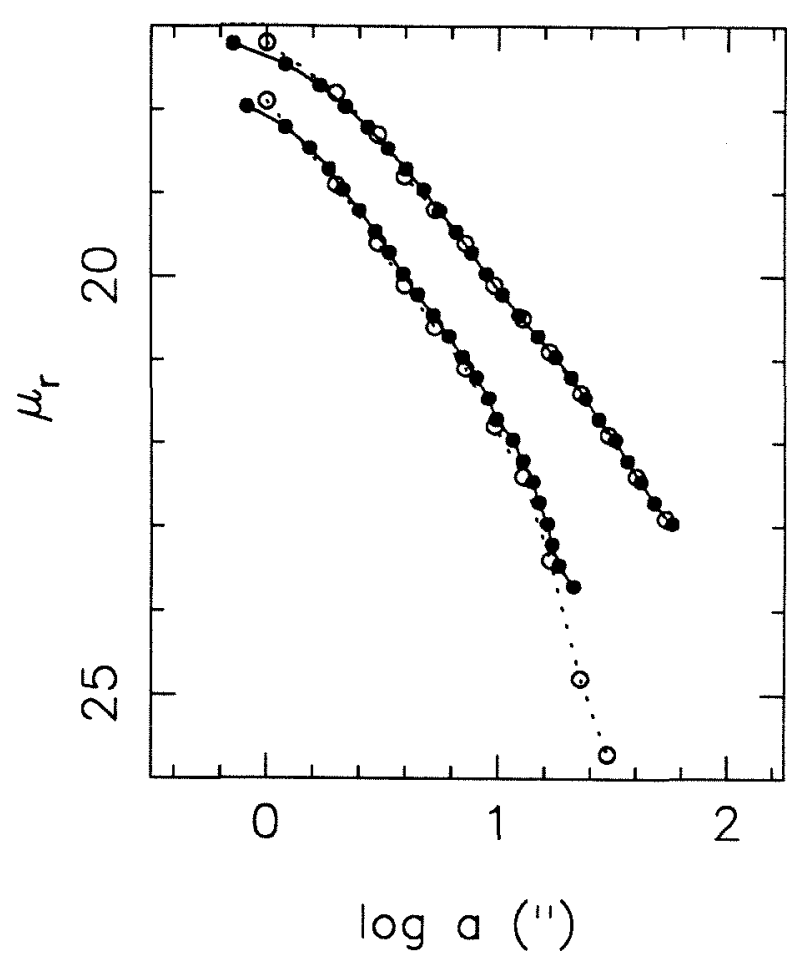

A779

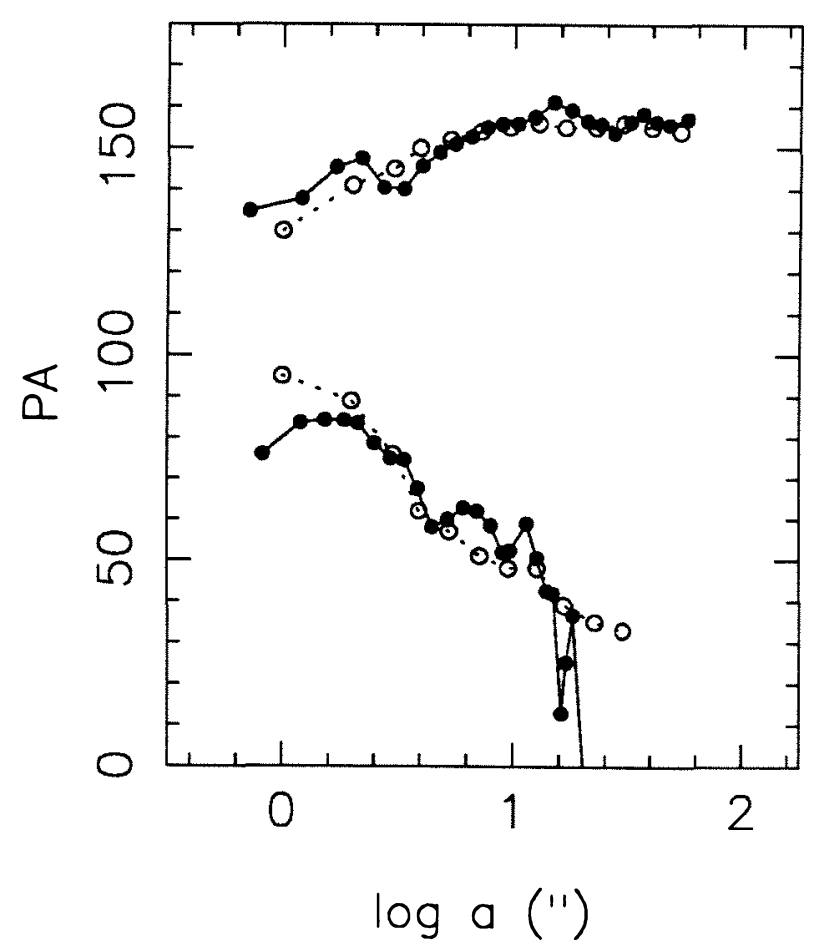

Figure 4. 

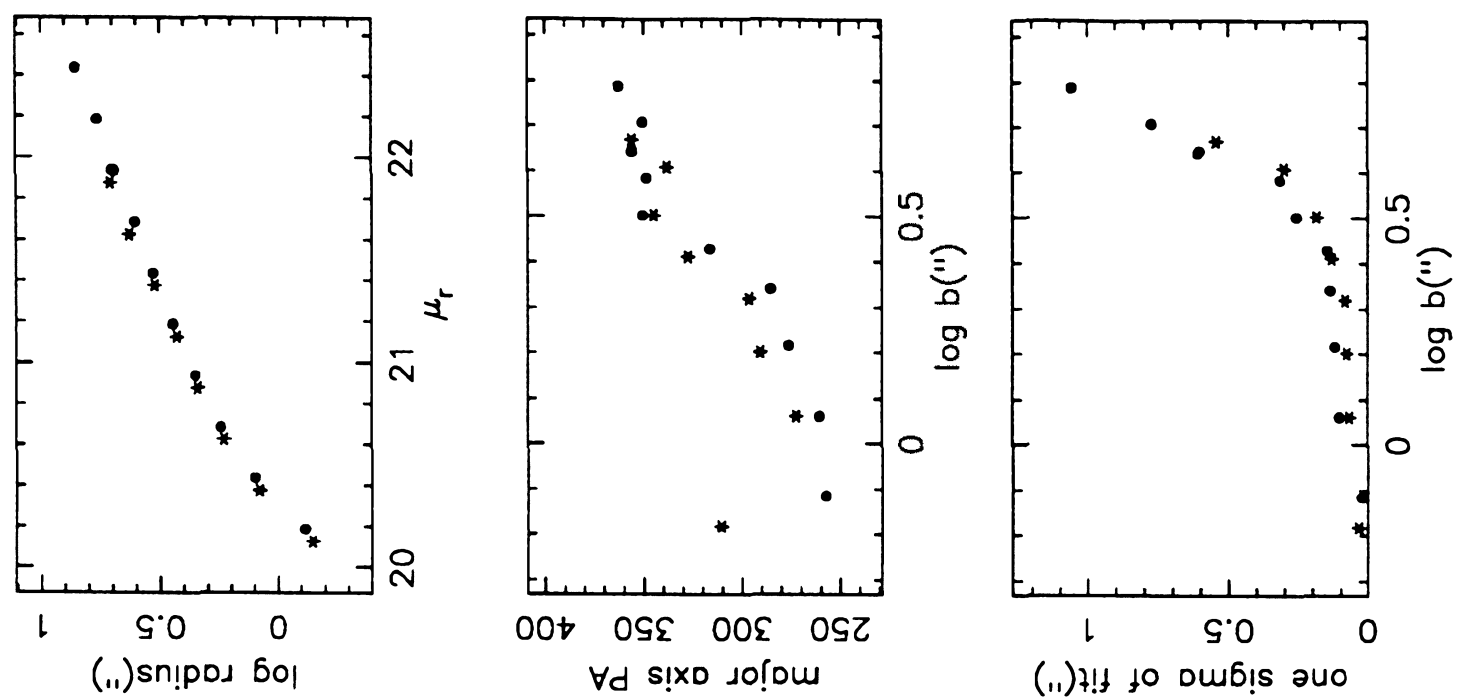

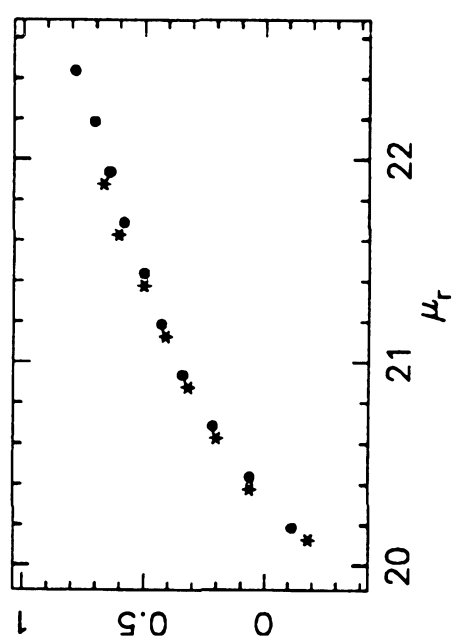

(.1) $q \mathrm{bol}$

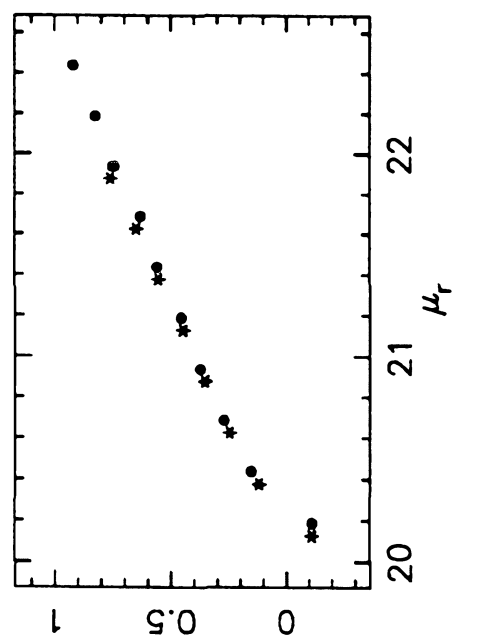

(i.) 0 6ol

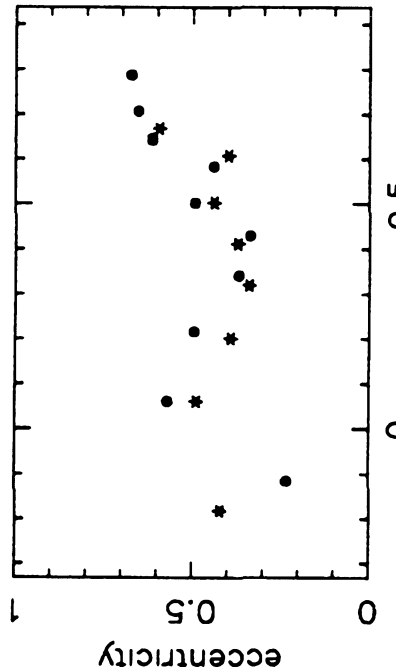

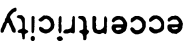

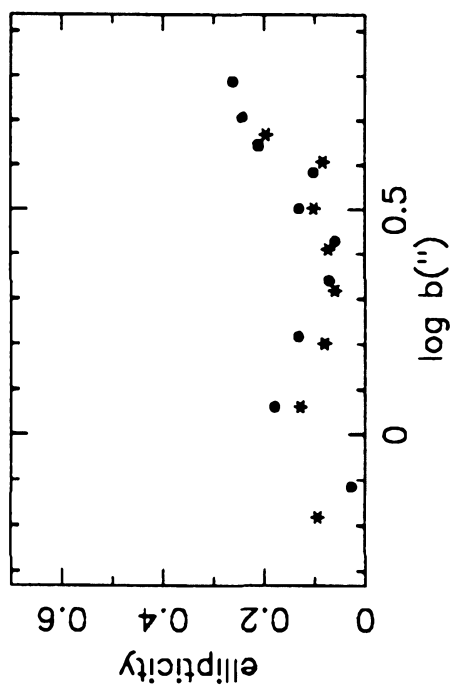

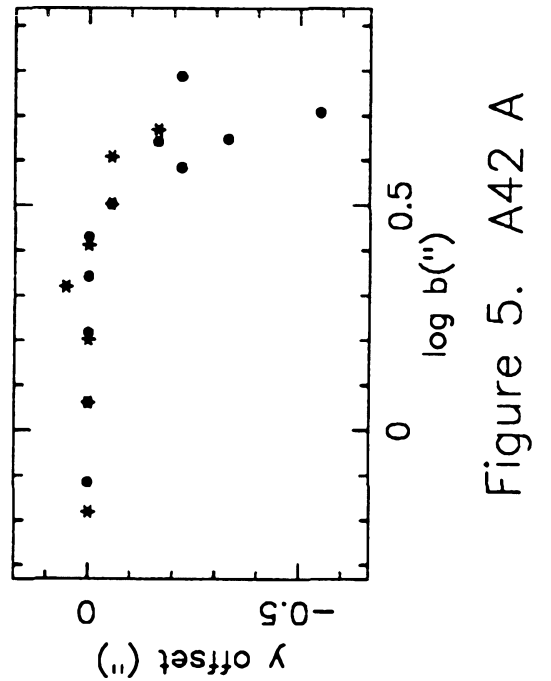

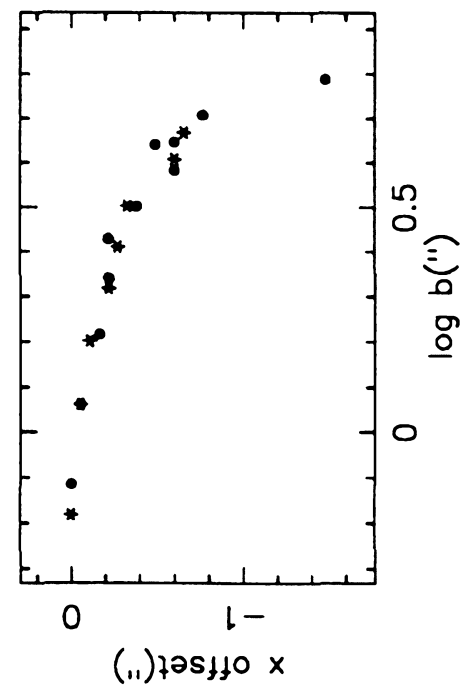


$-232-$
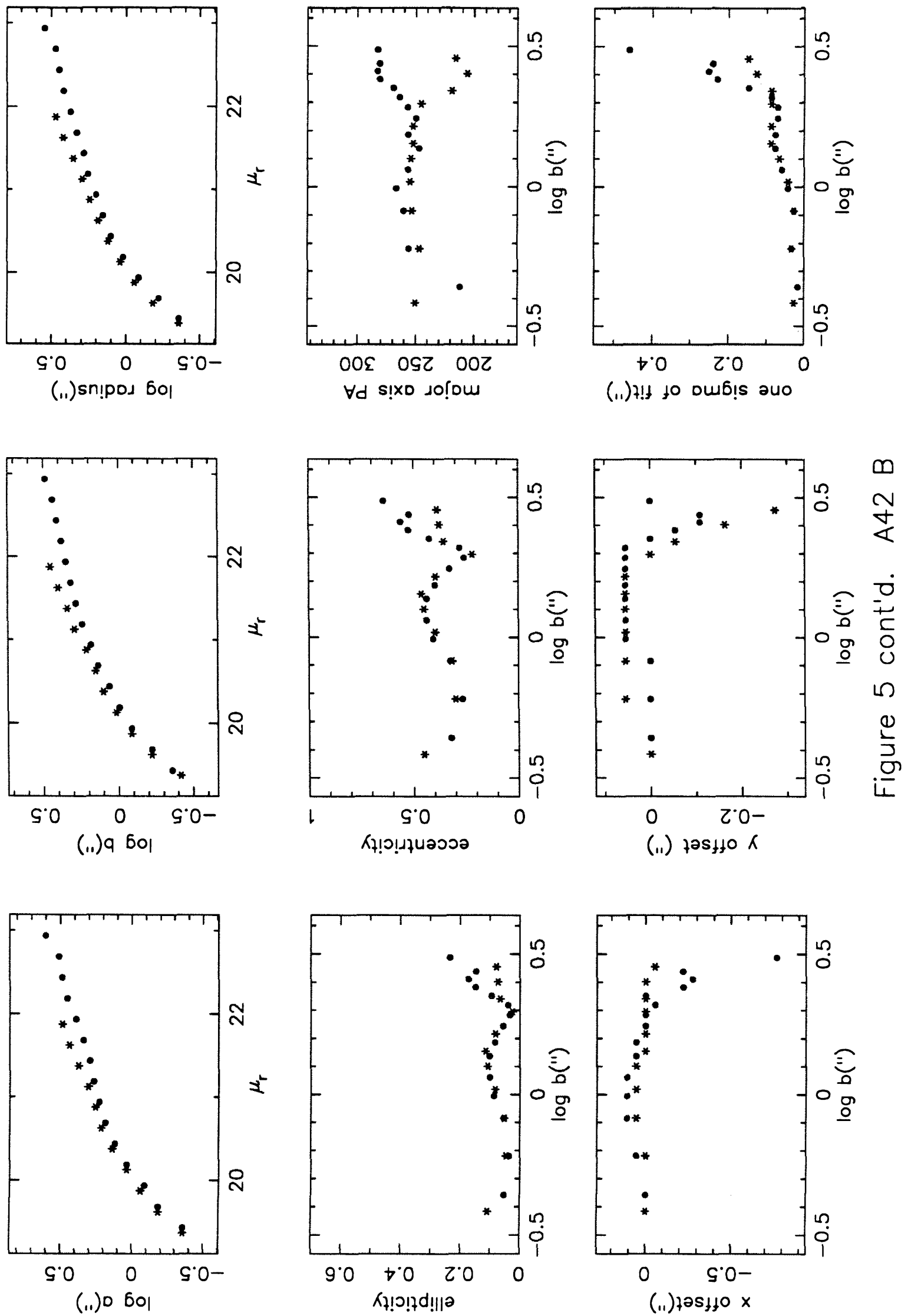


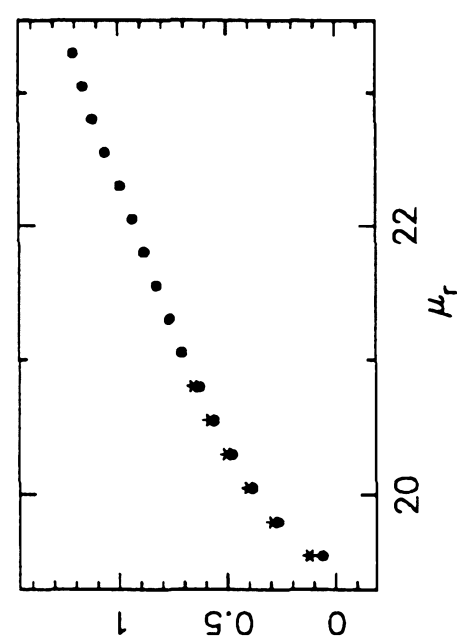

(II)sn!pDs 6ol

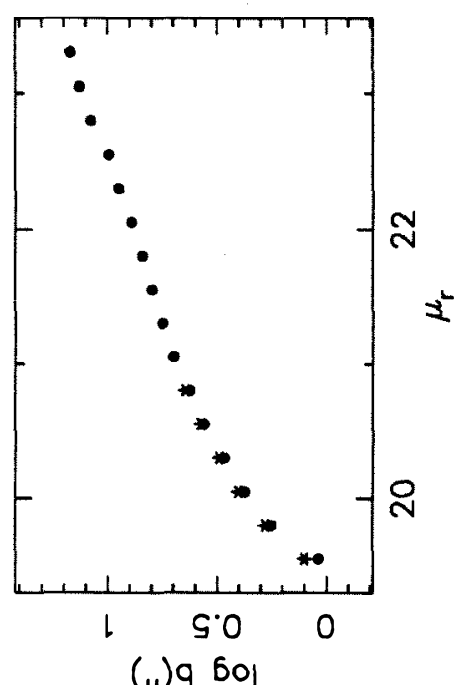

(.1) 960

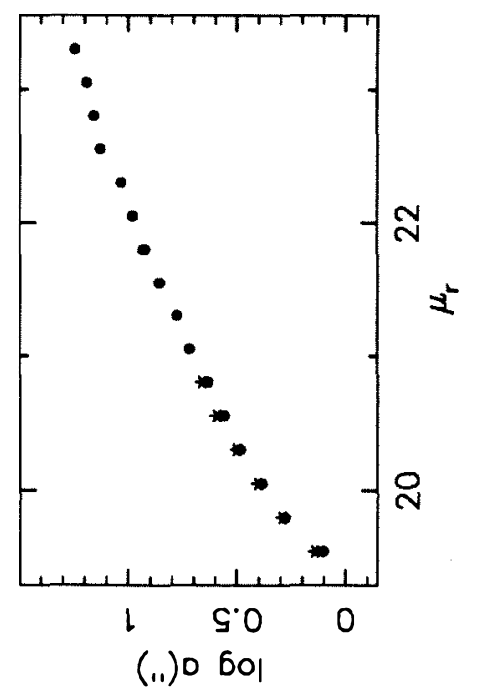

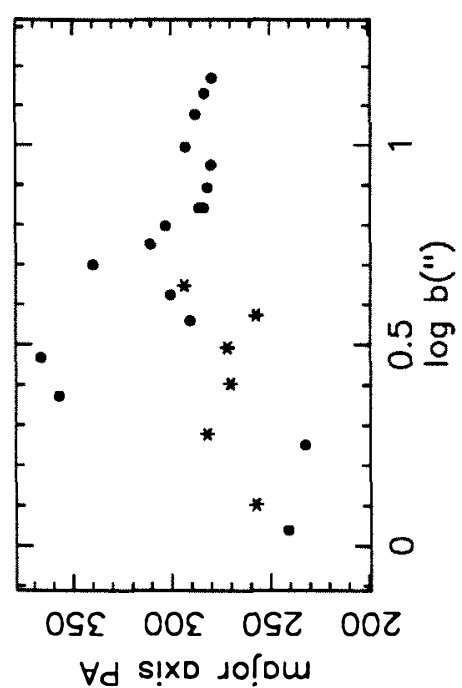

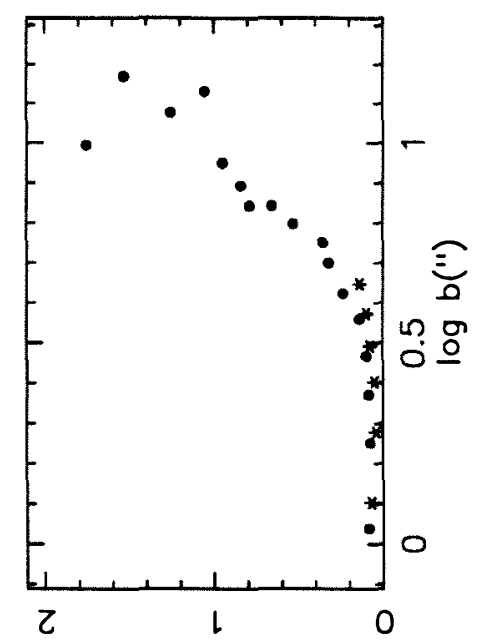

(11) + !! to Dub! ano
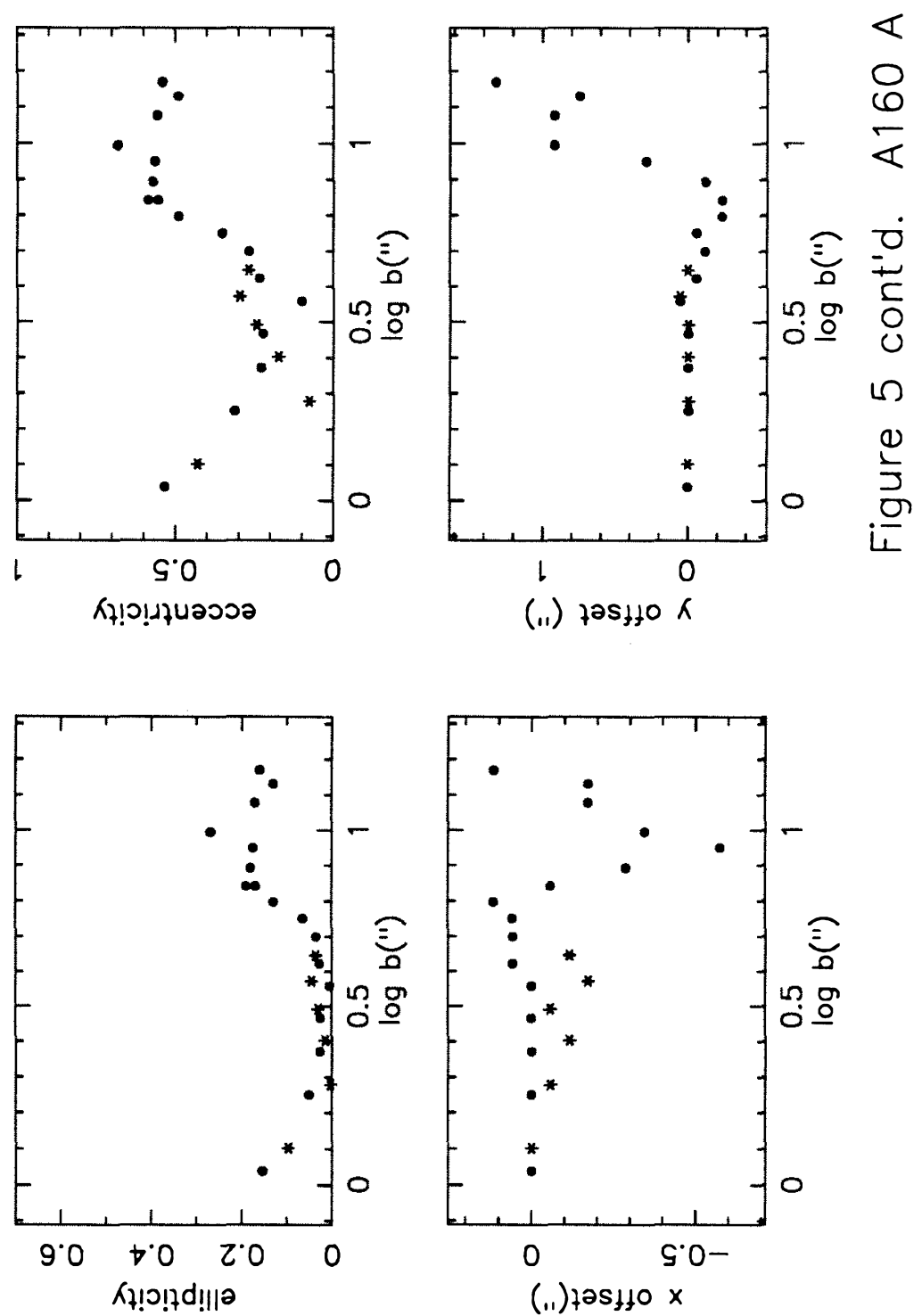


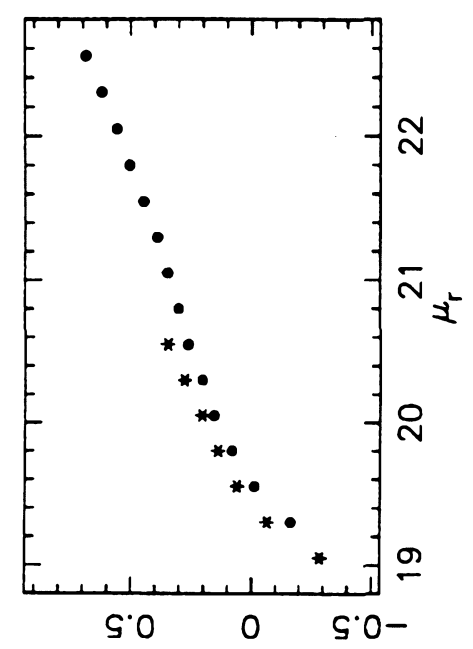

(.1) sn!pod 6ol

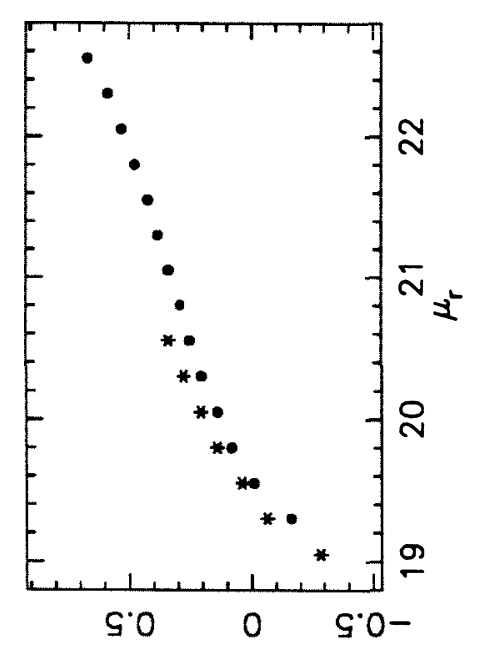

(.1) 9601

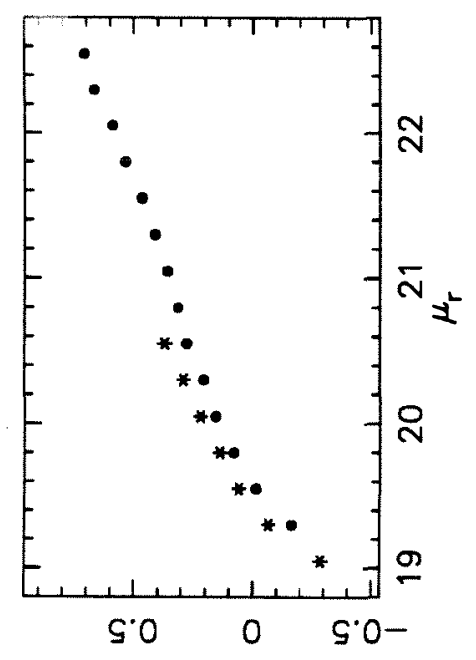

(.1) 0601

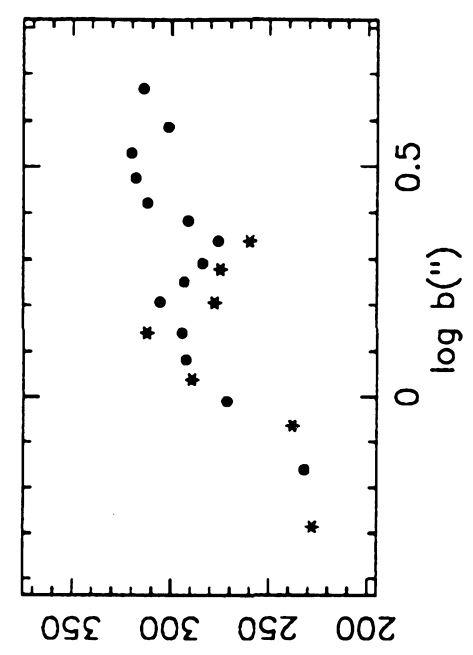

$\forall d$ s!xo

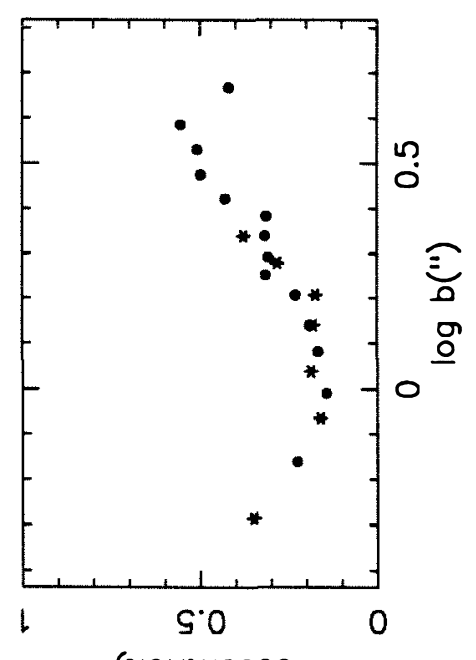

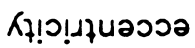

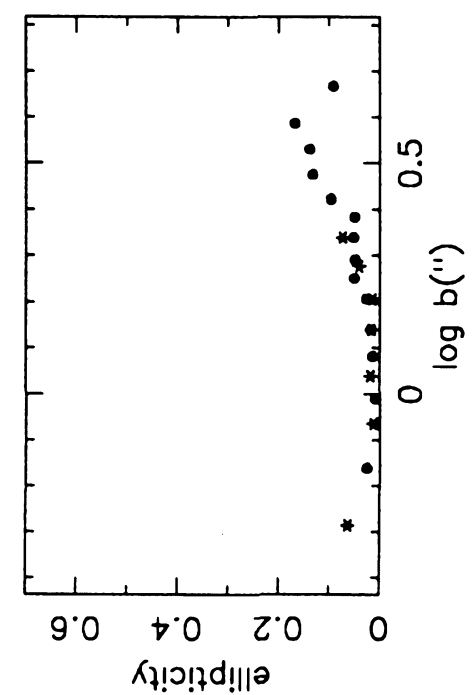

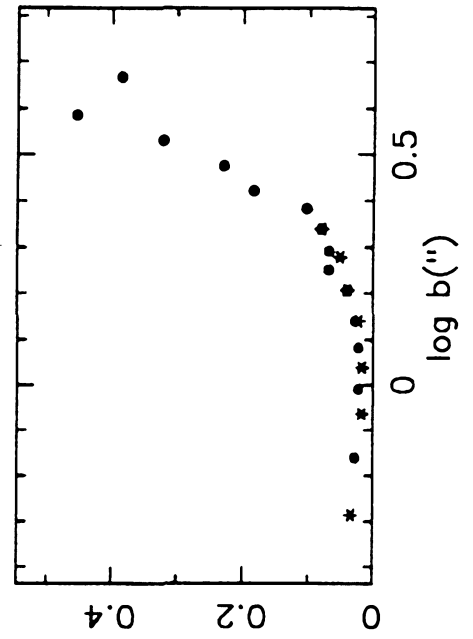

(.1) $+!+10$ oub!s auo

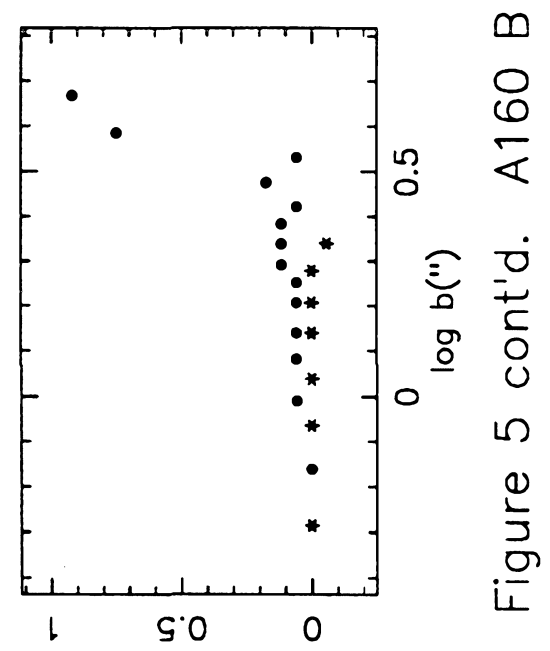

(i1) $725+10 \mathrm{~K}$

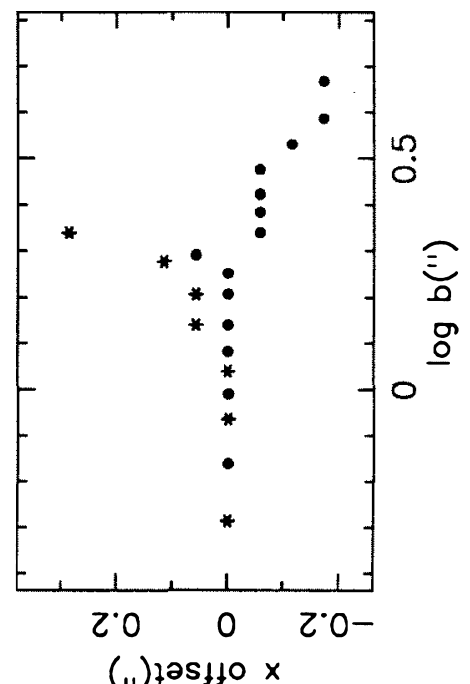



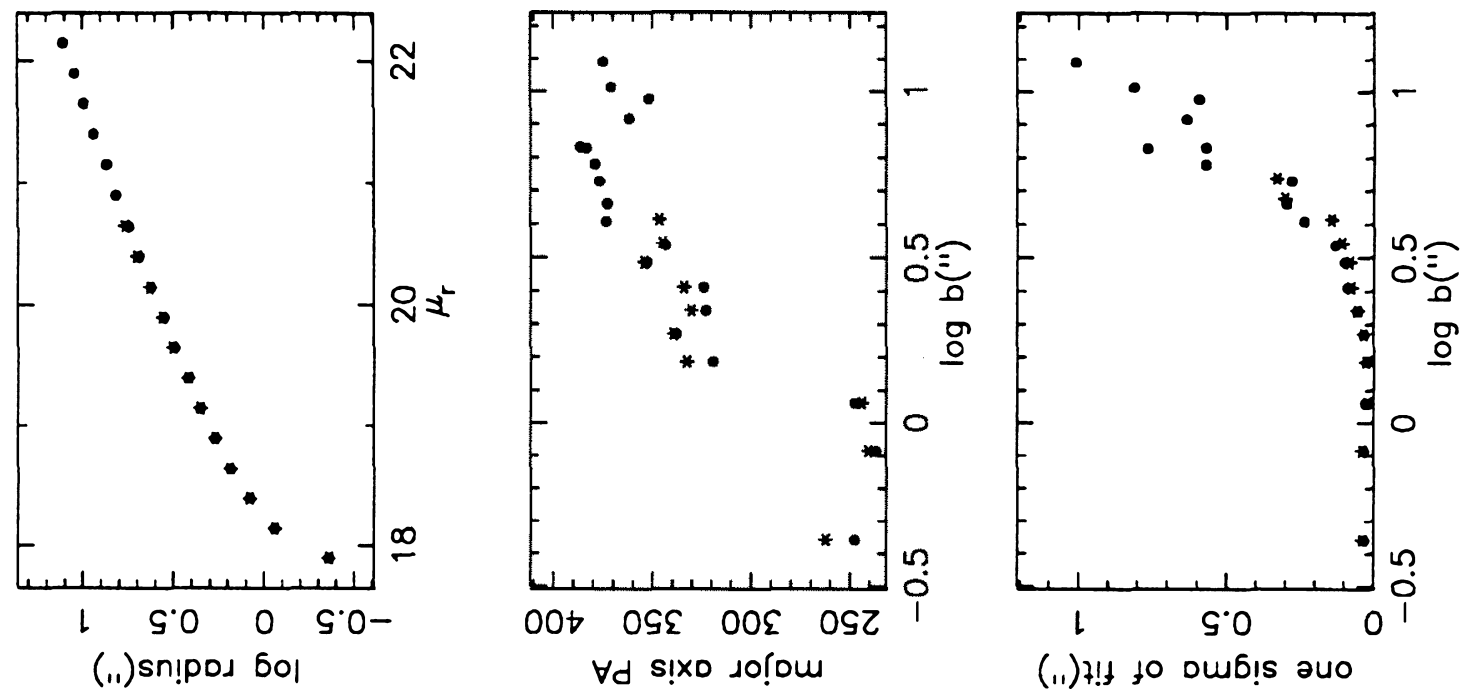

$\forall d$ s!xo 10 ! om

(11) $7 ! t$ to oub!s auo
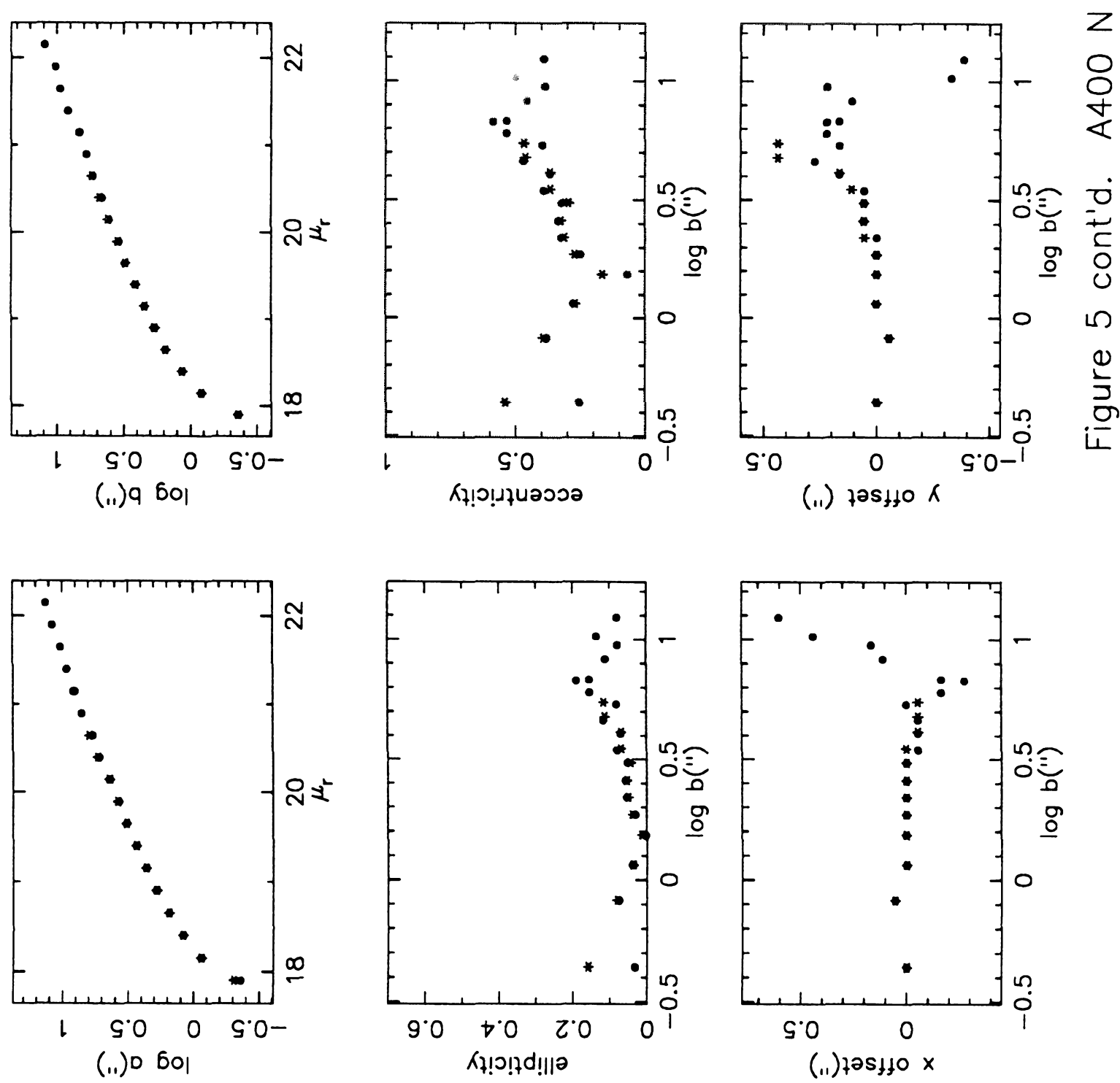

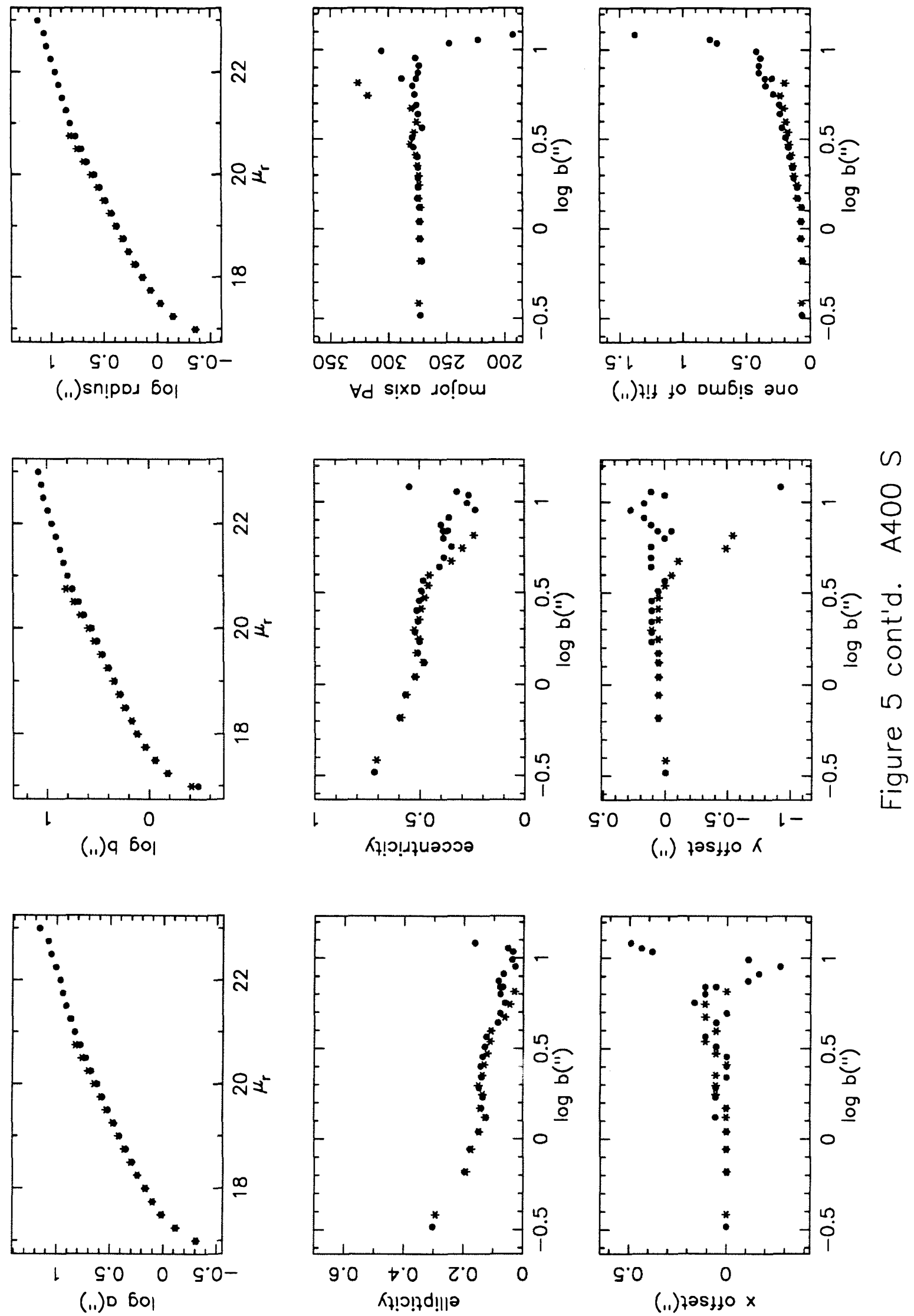


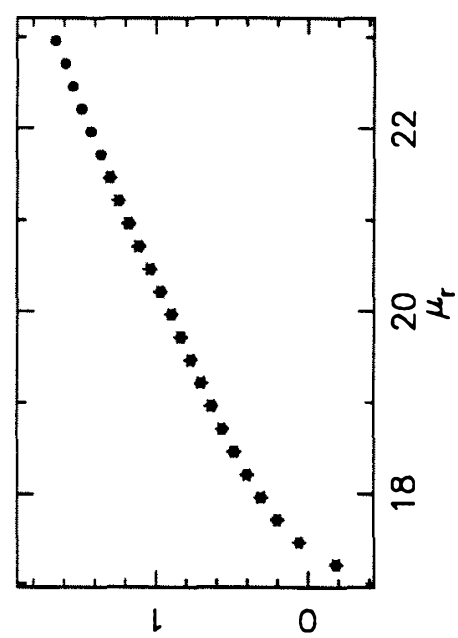

(..)sn!pod 6ol

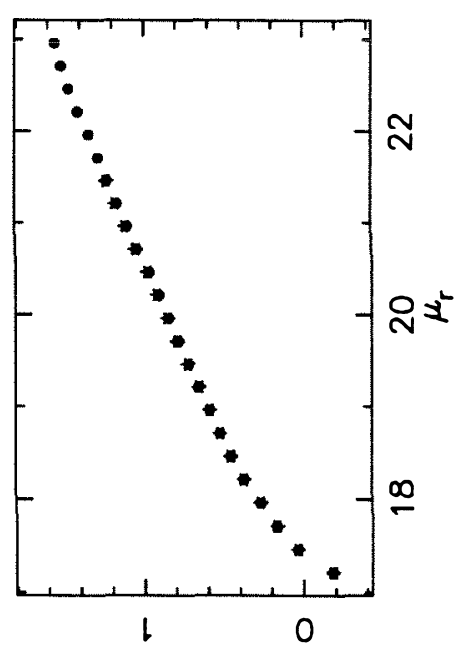

(.1) 9 6ol

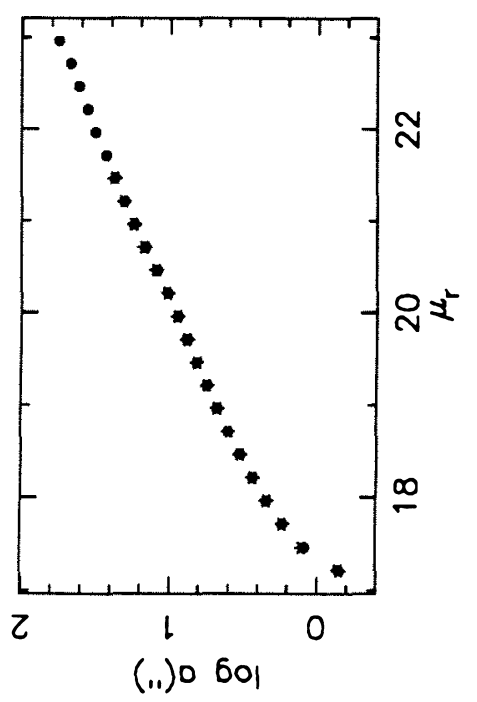

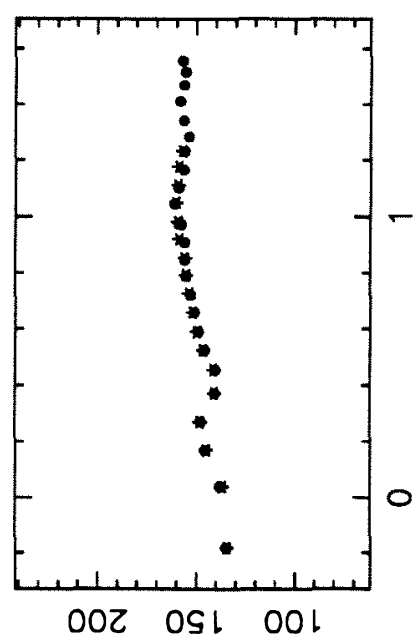

$\forall d$ s!xD so!ou

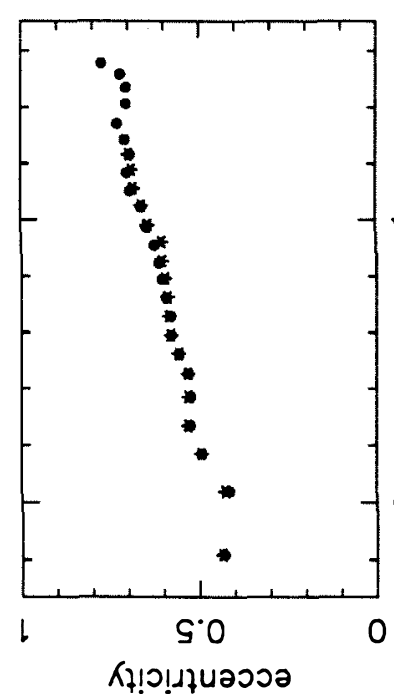

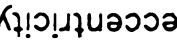

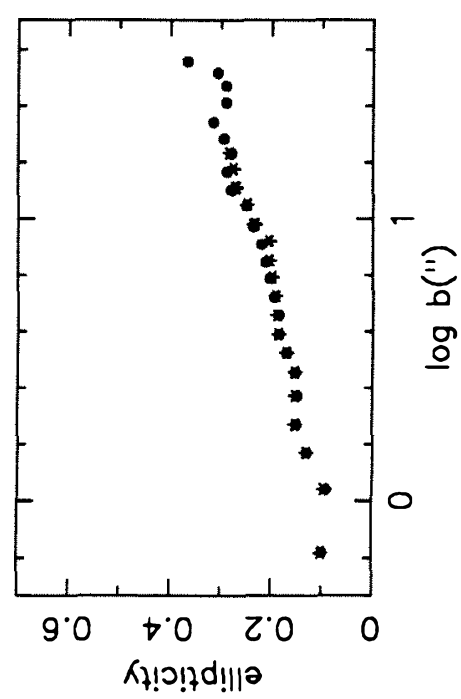

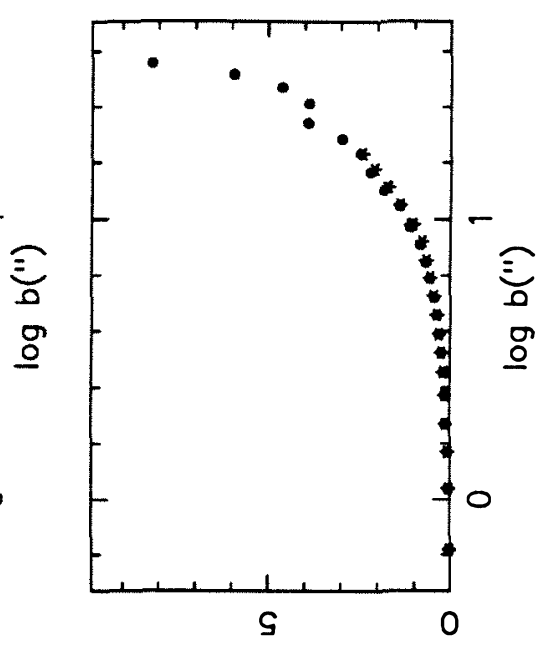

(.1) )!! to oub! ano

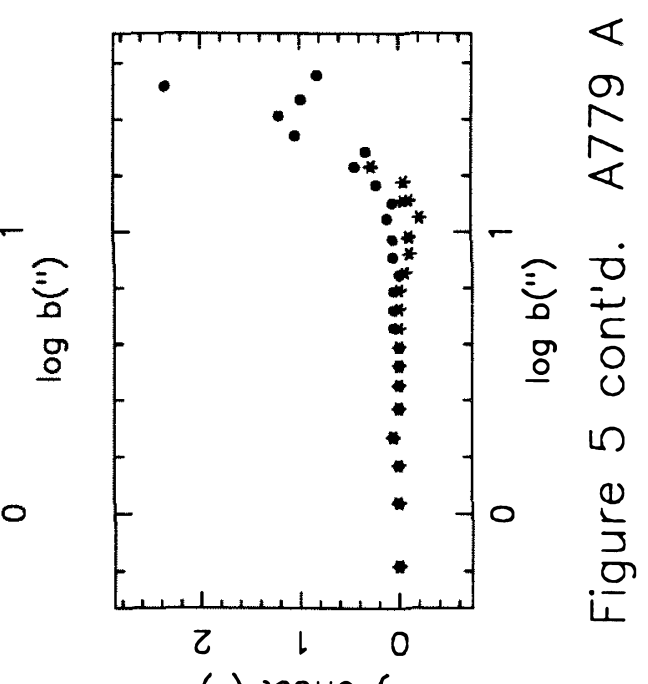

(11) $125 \% 10 \mathrm{~K}$

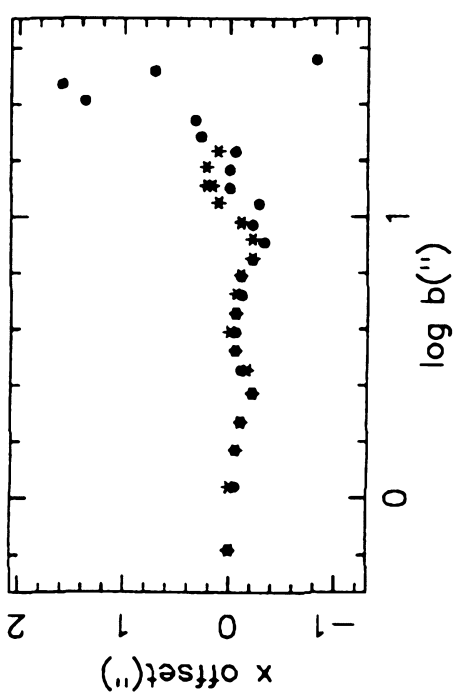


$-238-$
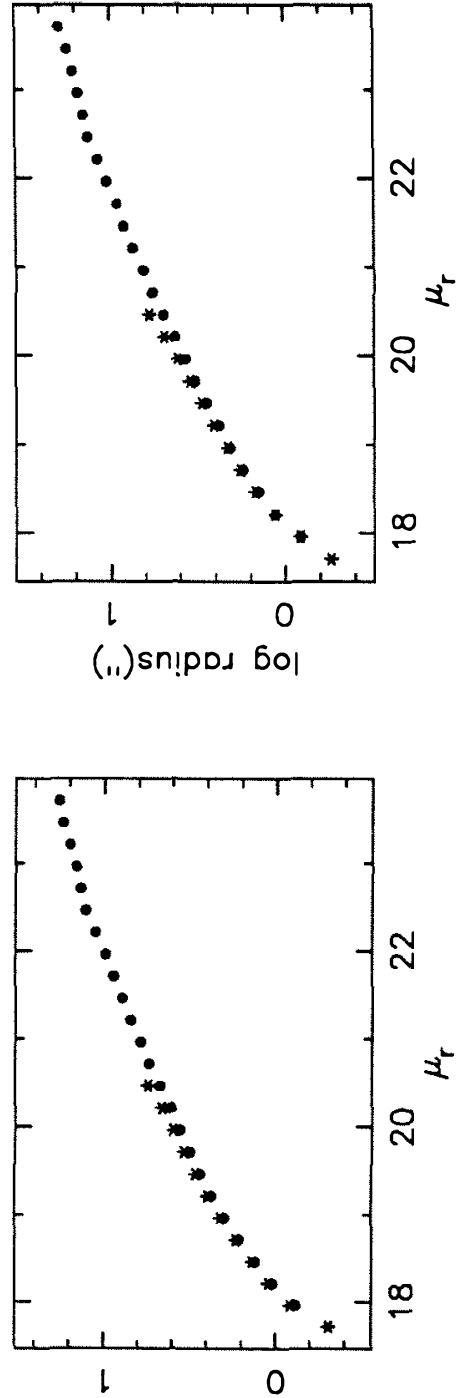

(.1) $9 \mathrm{bol}$

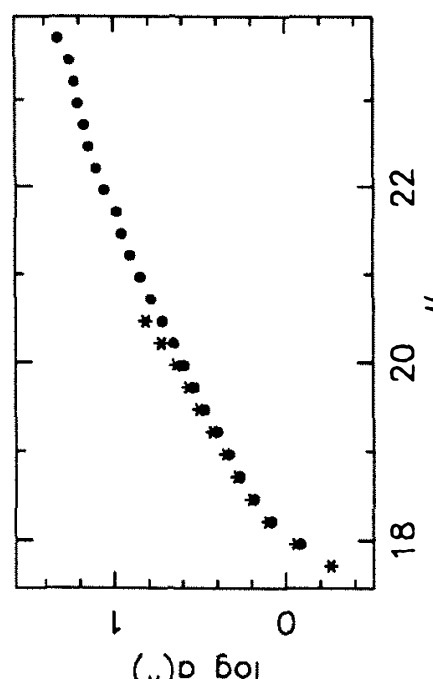

(.1) $\mathrm{O} \mathrm{BO}$
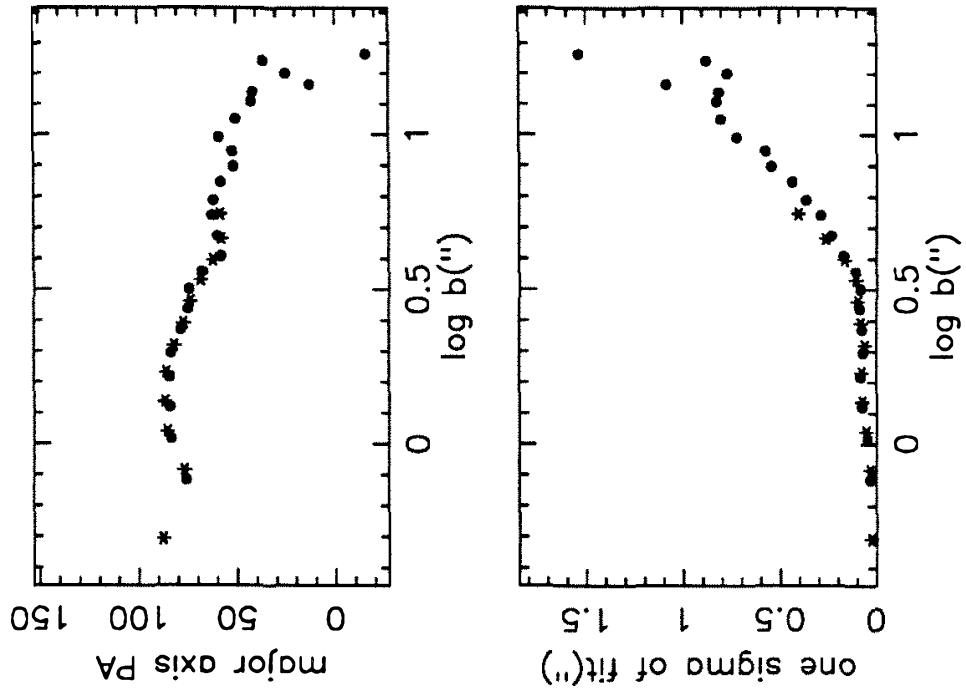

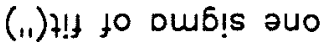

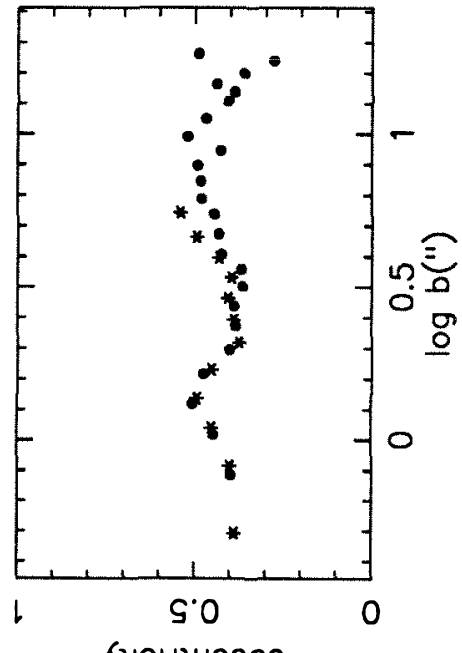

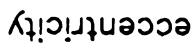

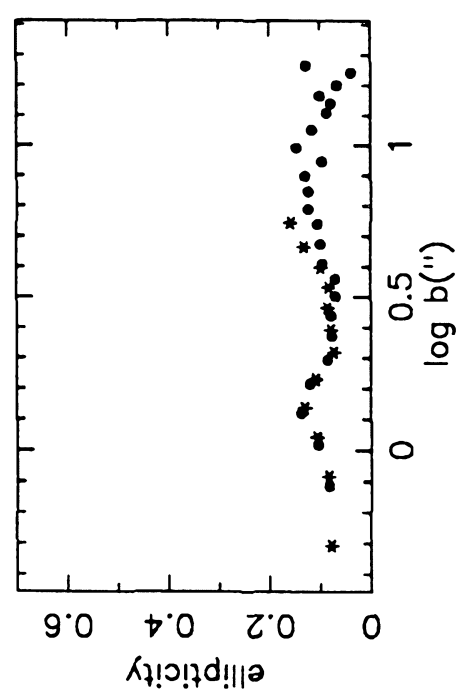

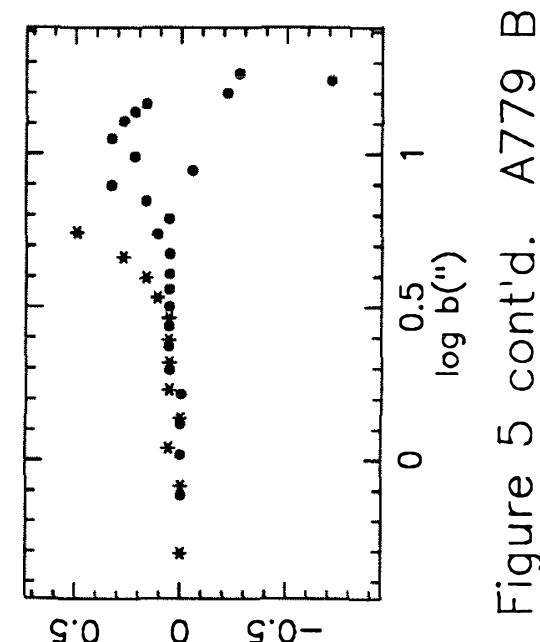

(ii) tast $10 \mathrm{~K}$

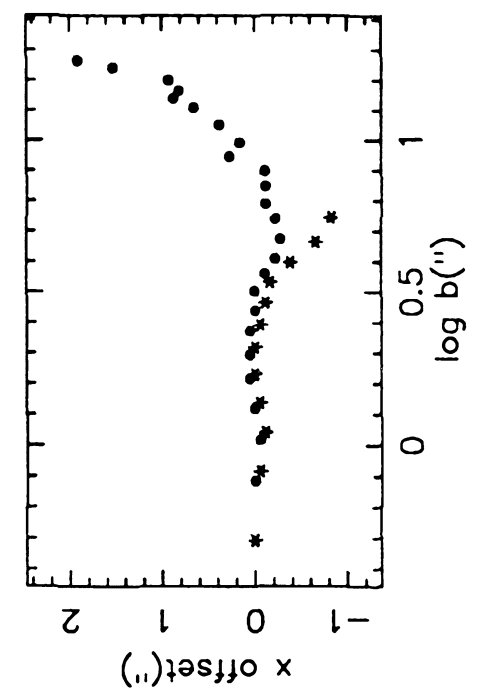



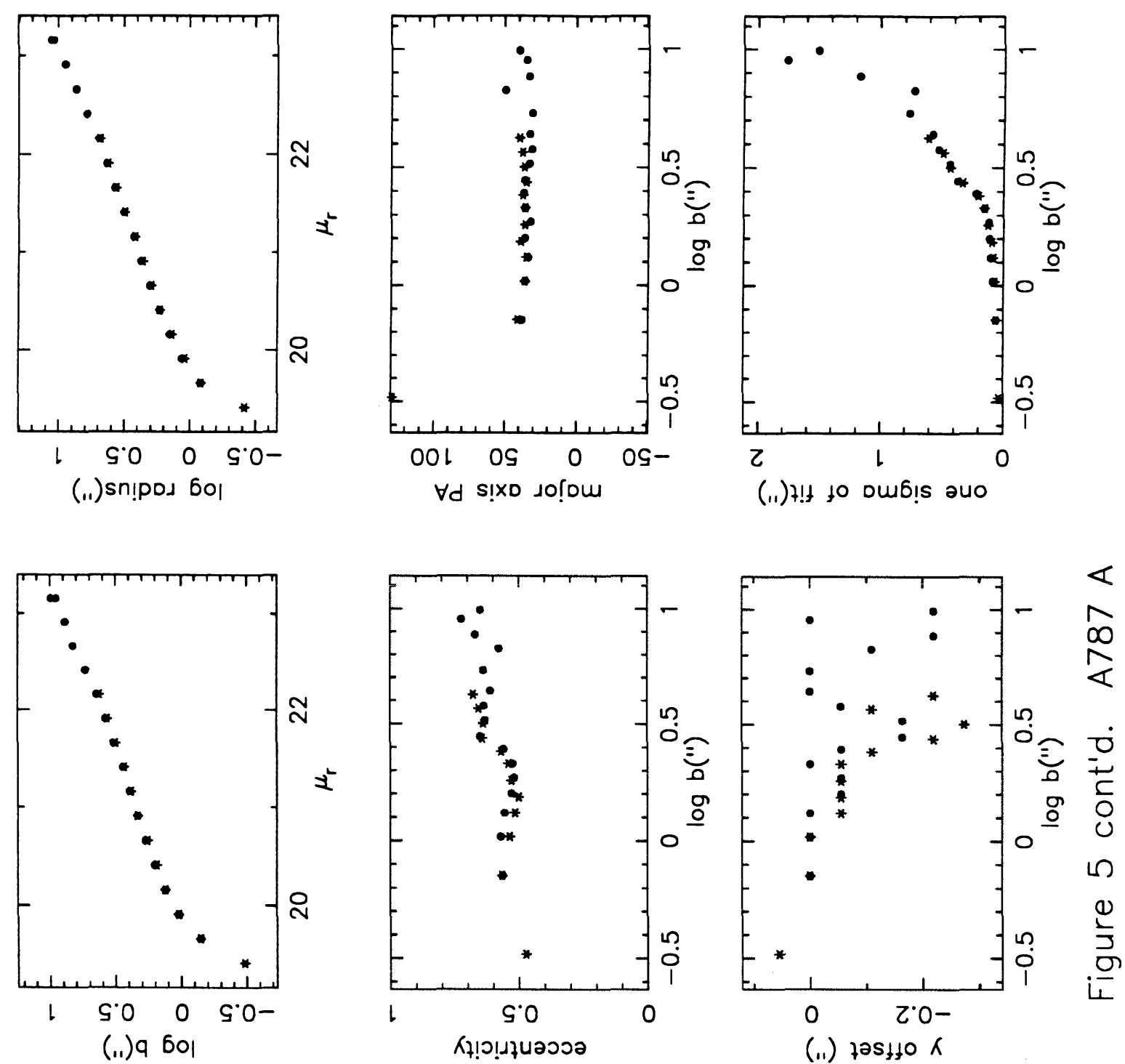

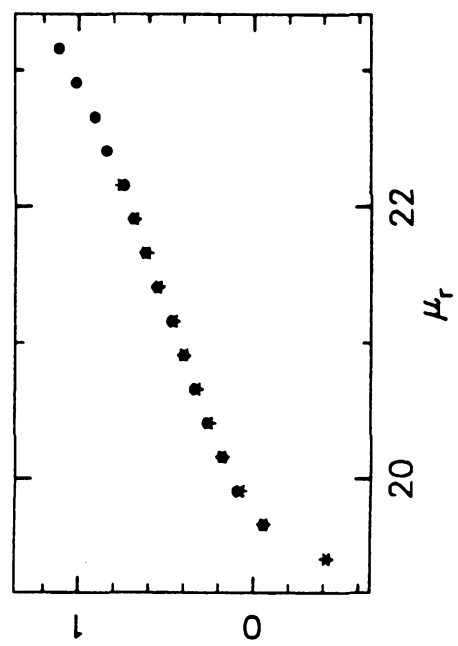

(.1) 0601

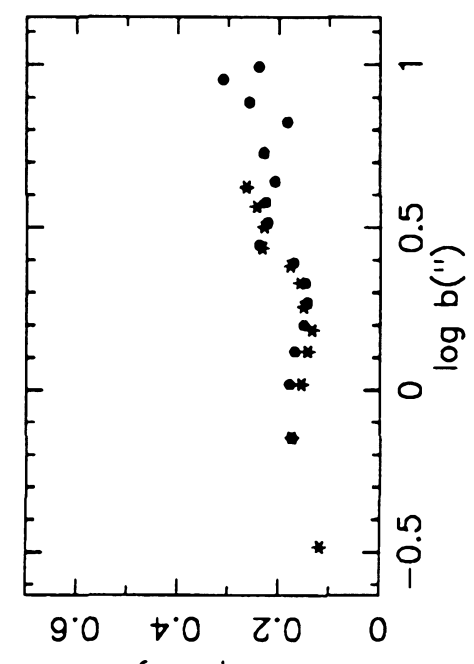

र \{!o!?d!॥|

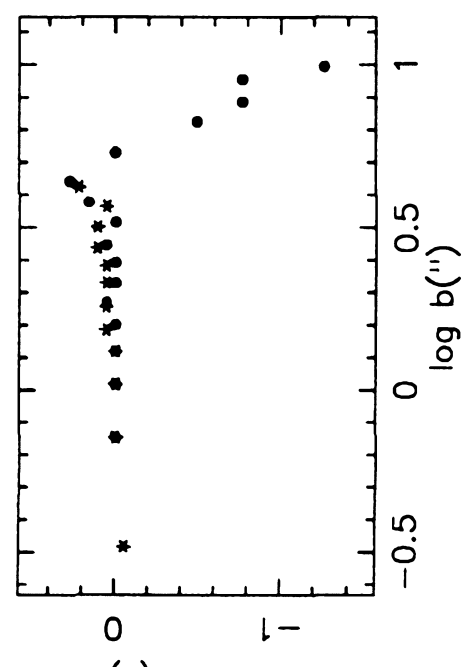

(.1) $725+150 \times$ 


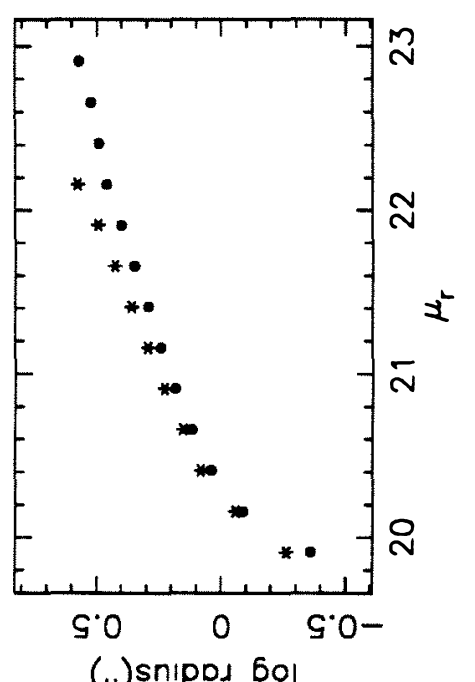

(.1) sn!pod 6ol

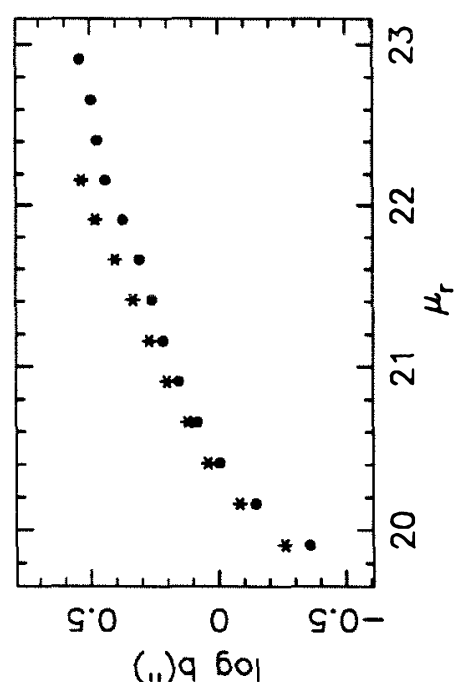

(.1) 960

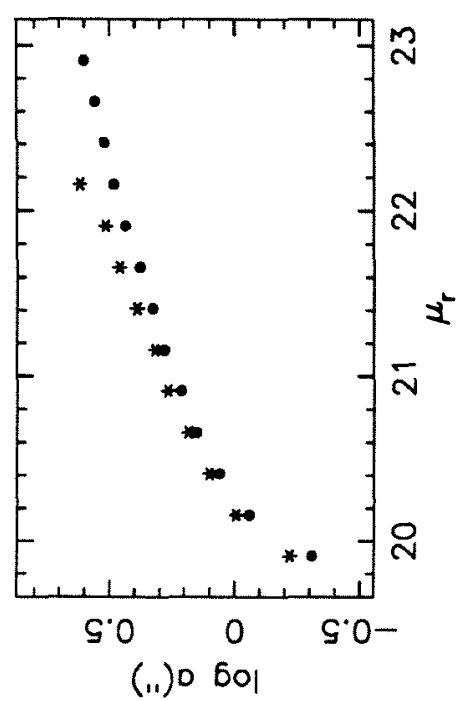

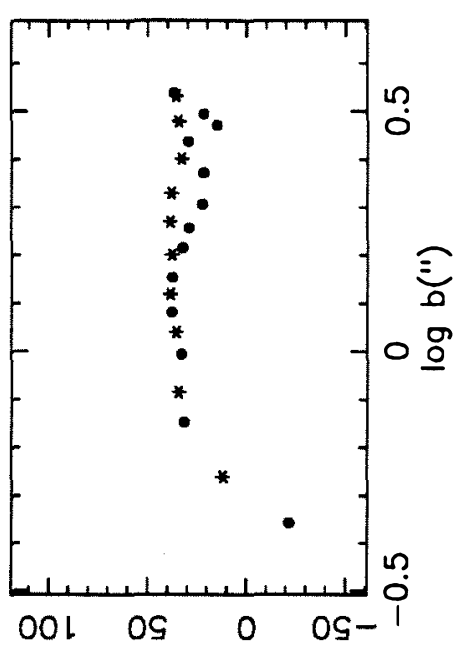

$\forall d$ S!xD so!̣om

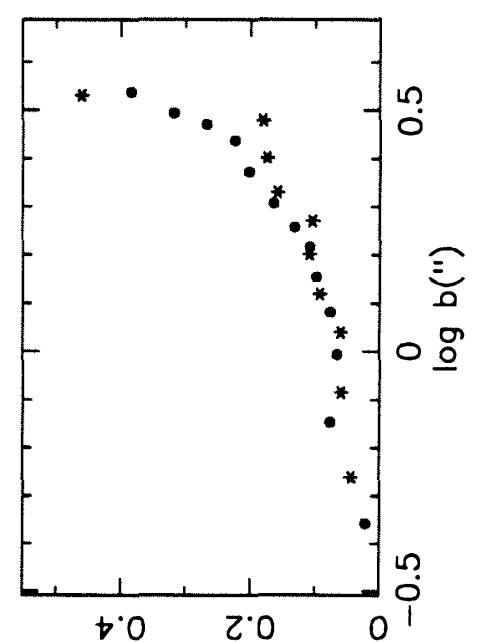

(11) $7 !+$ to Dub!s auo

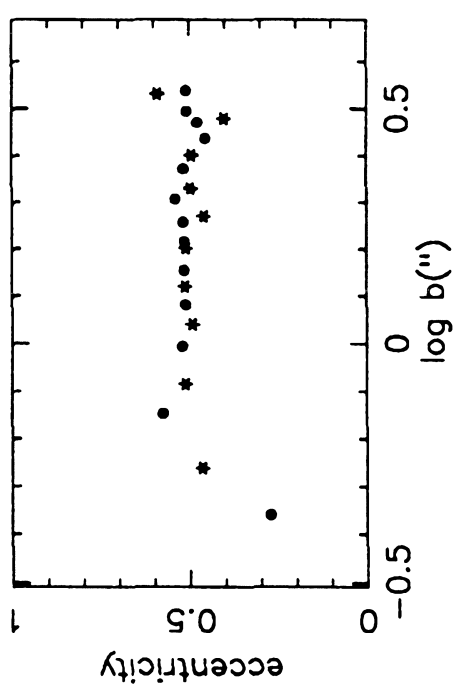

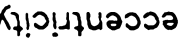

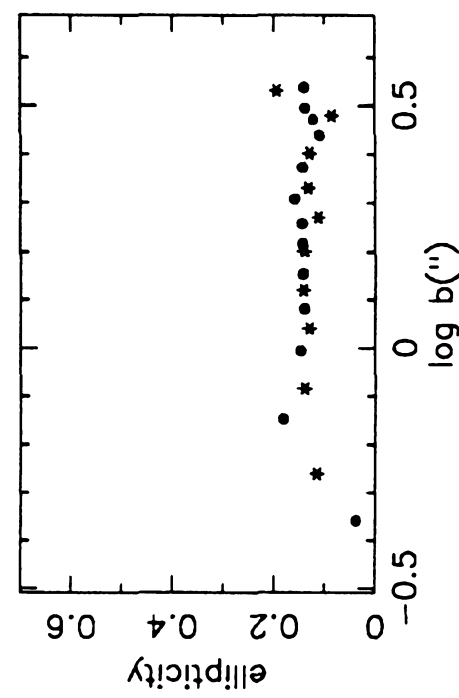

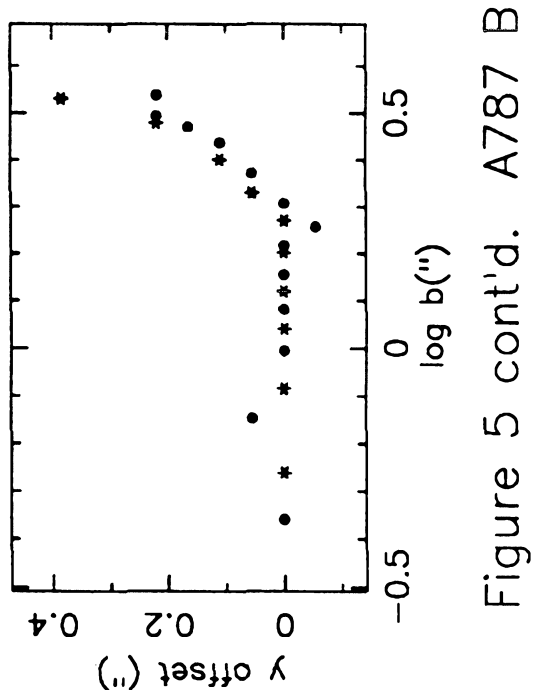

(.1) $125+10 \mathrm{~K}$

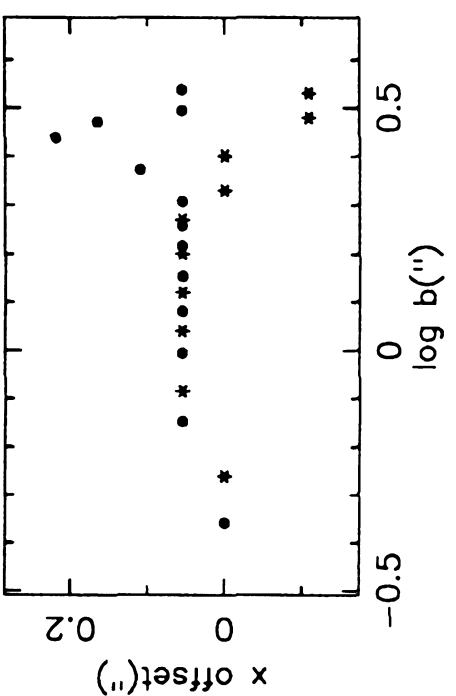




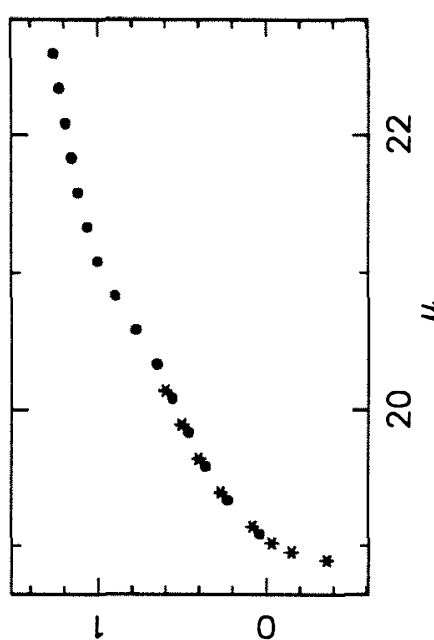

(.1)sn!pos 6ol

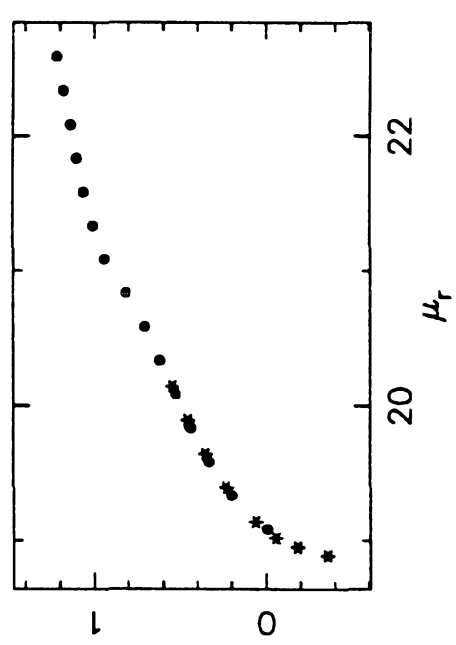

(i1) 9 6ol

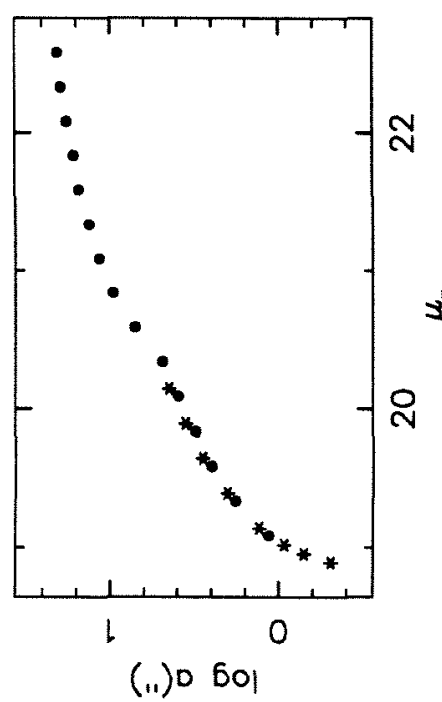

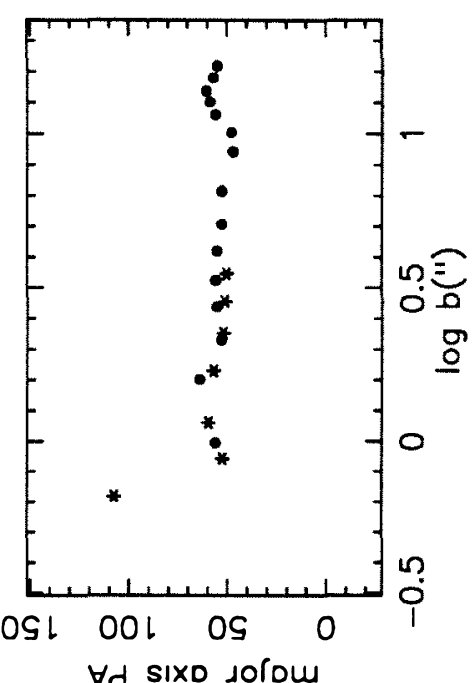

$\forall d$ s!xo so!lom

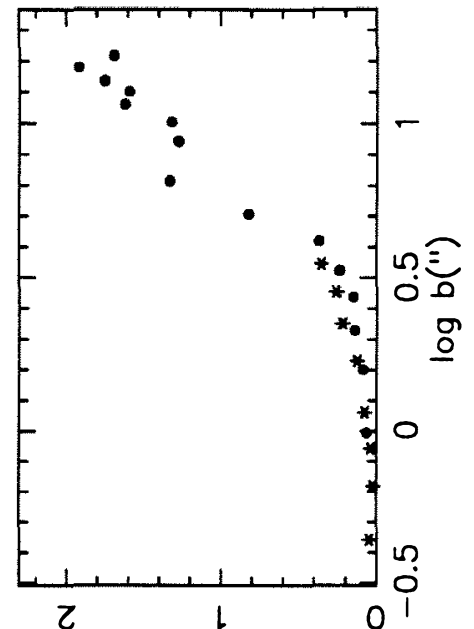

(11) H!! to Dub!s auo
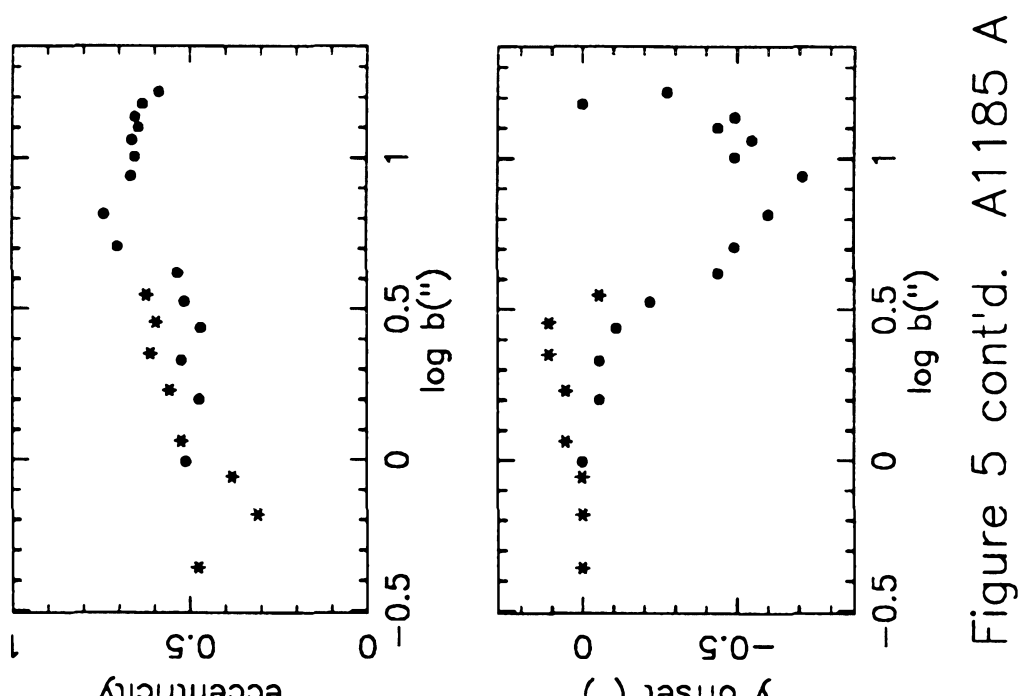

(i1) 7os $150 \mathrm{~K}$
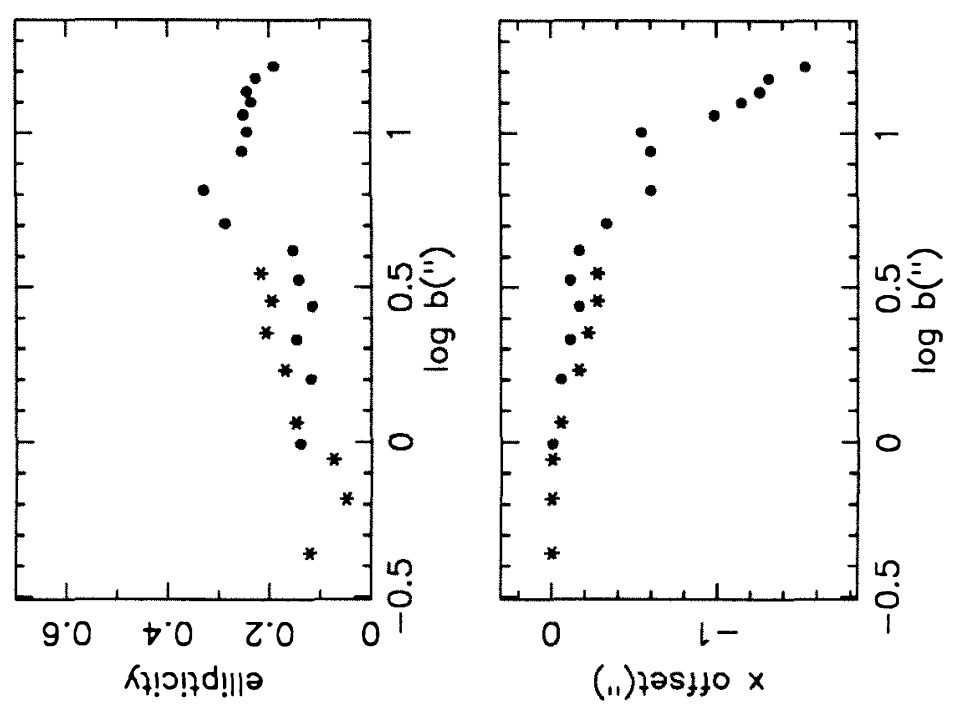
$-242-$

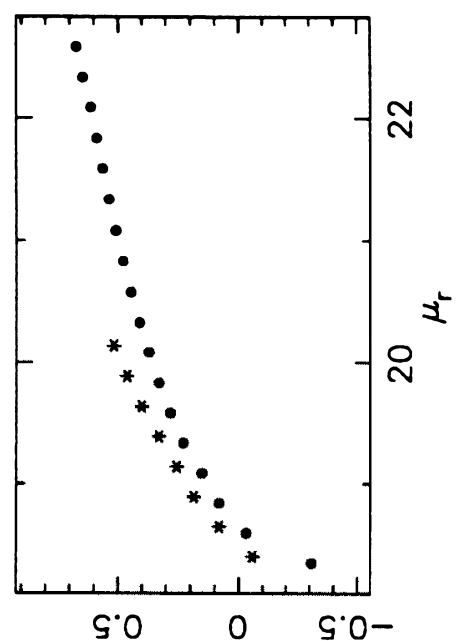

(.1)sn!pos 6ol

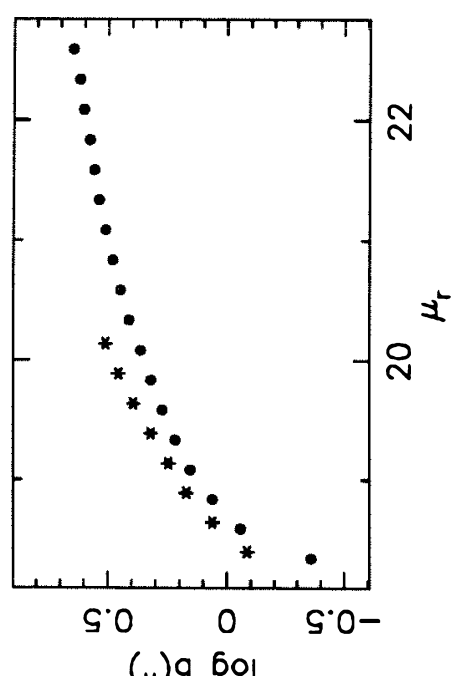

(.1) 960

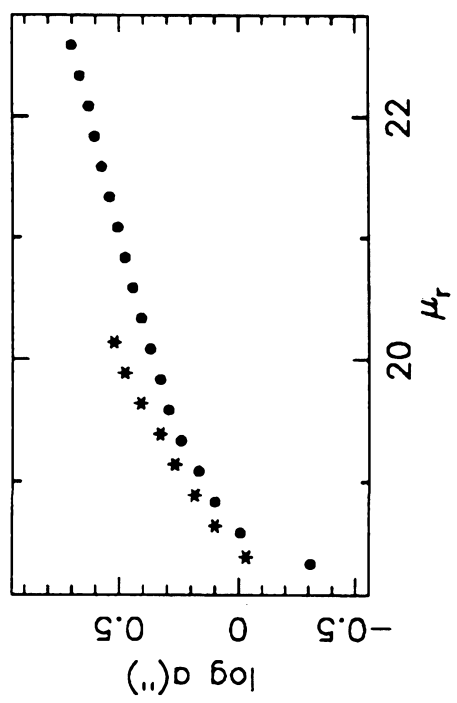

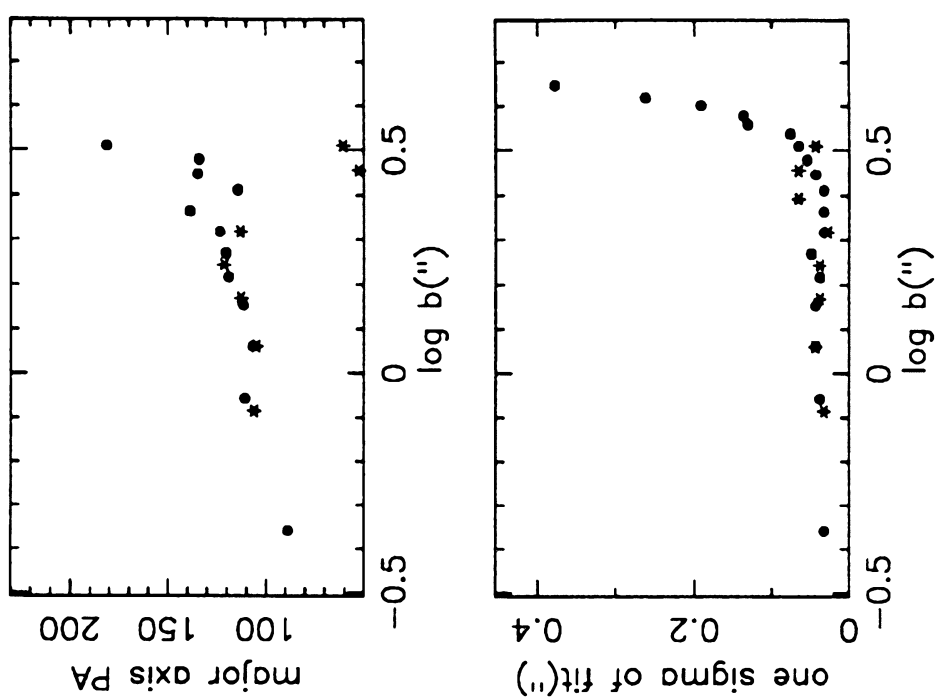

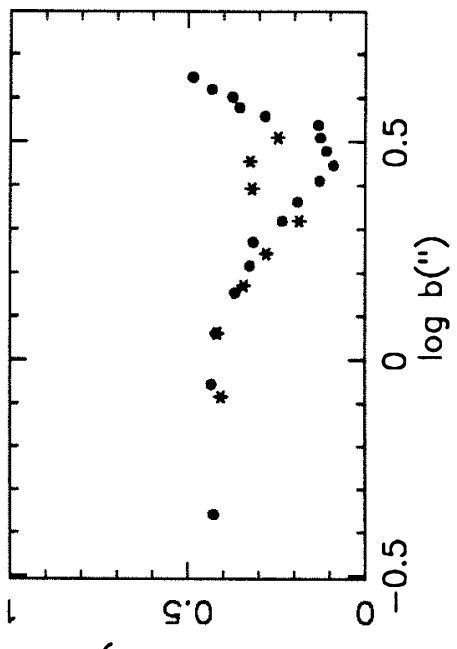

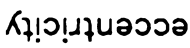

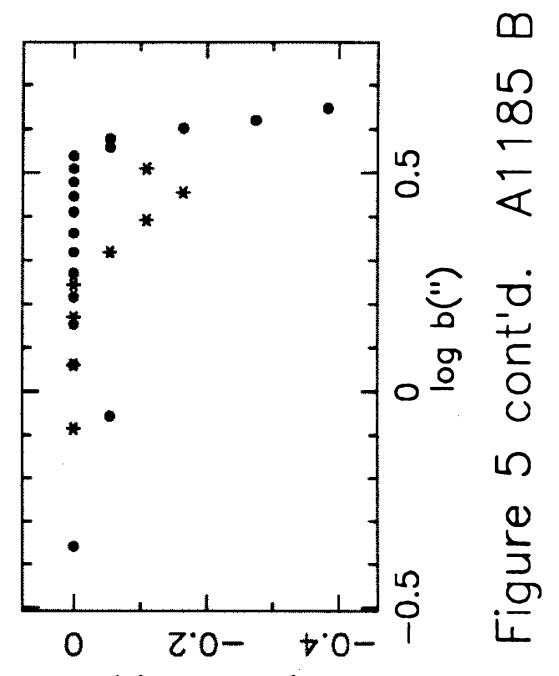

(ii) tast

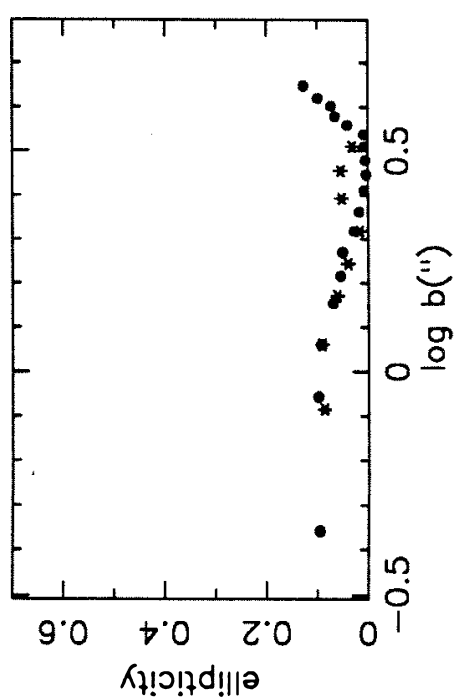

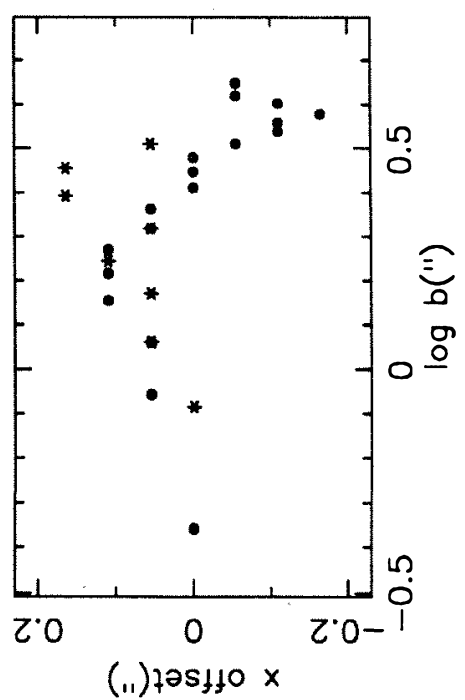




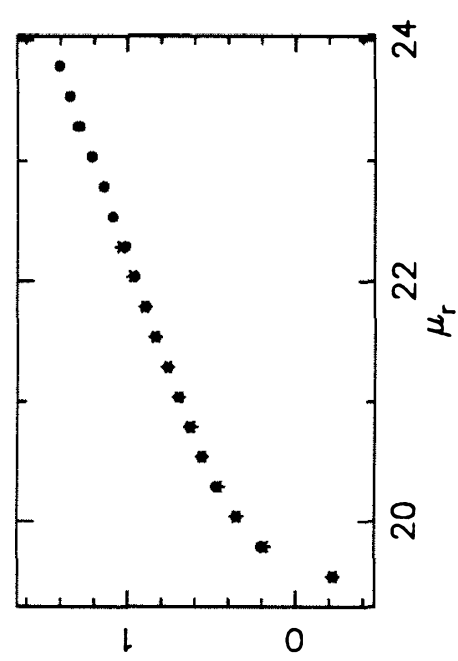

(.1) sn!pos 6ol
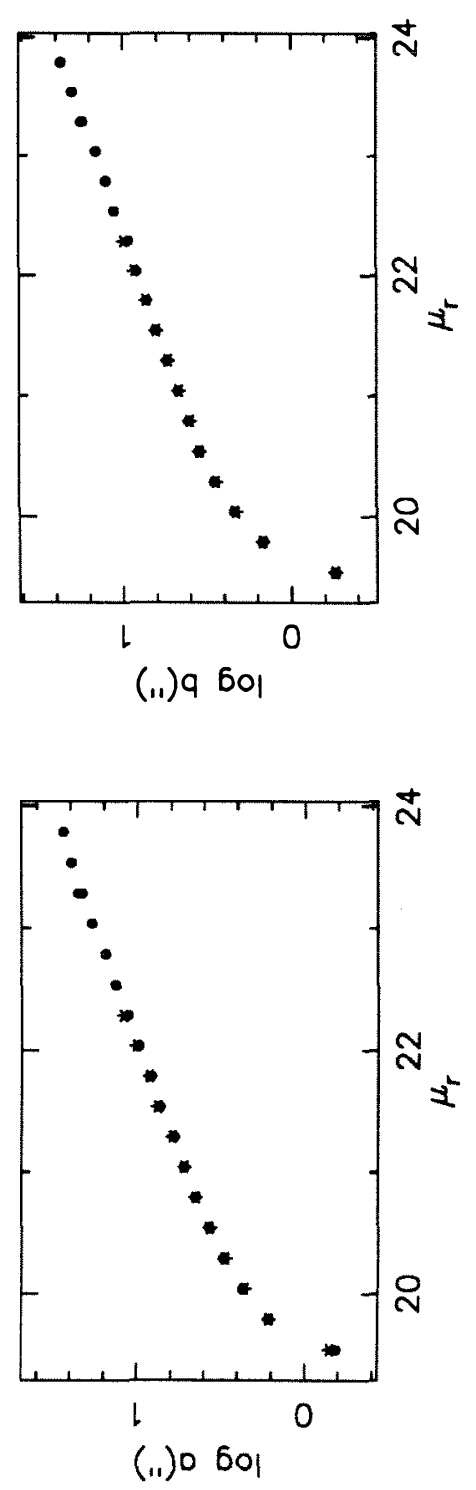

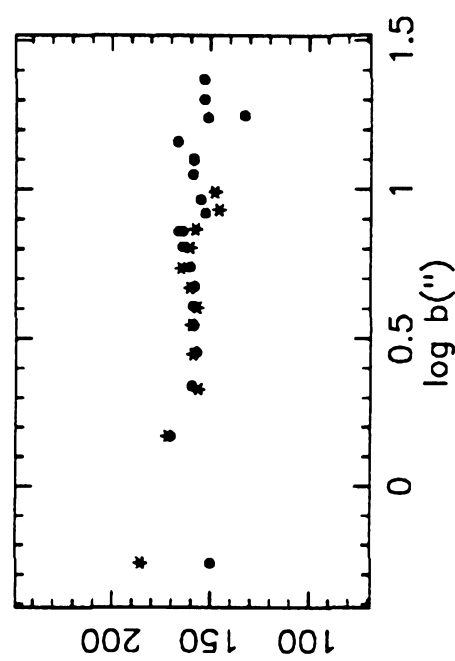

$\forall d$ s!xo so!̣om

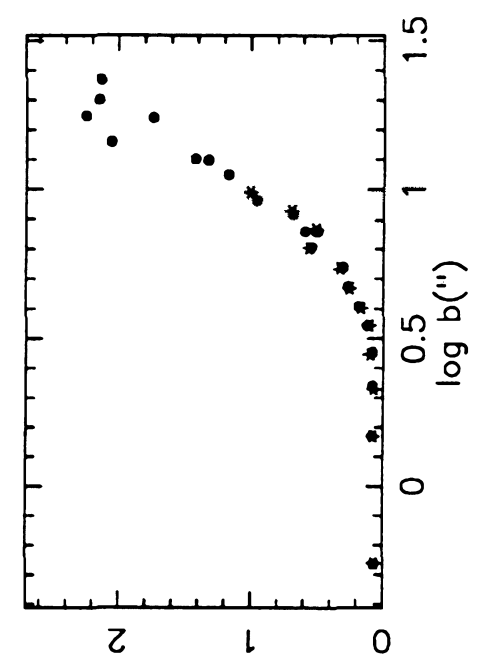

(.1) $+! t ~ j 0$ oub! !s avo
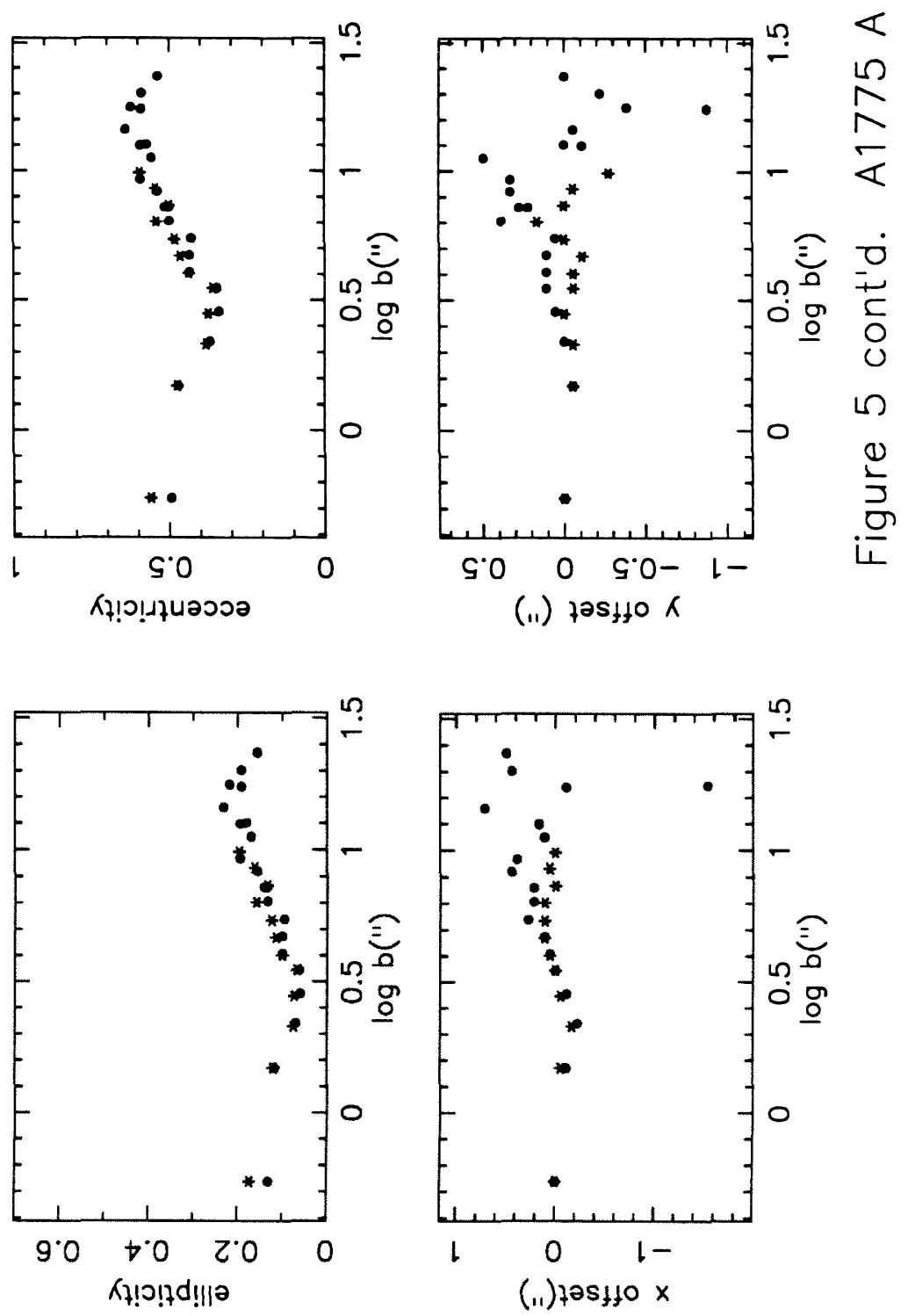


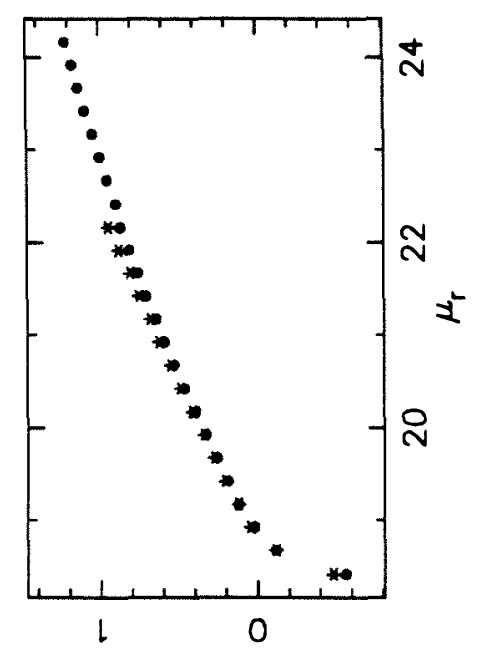

(.1) sn!pod 6ol
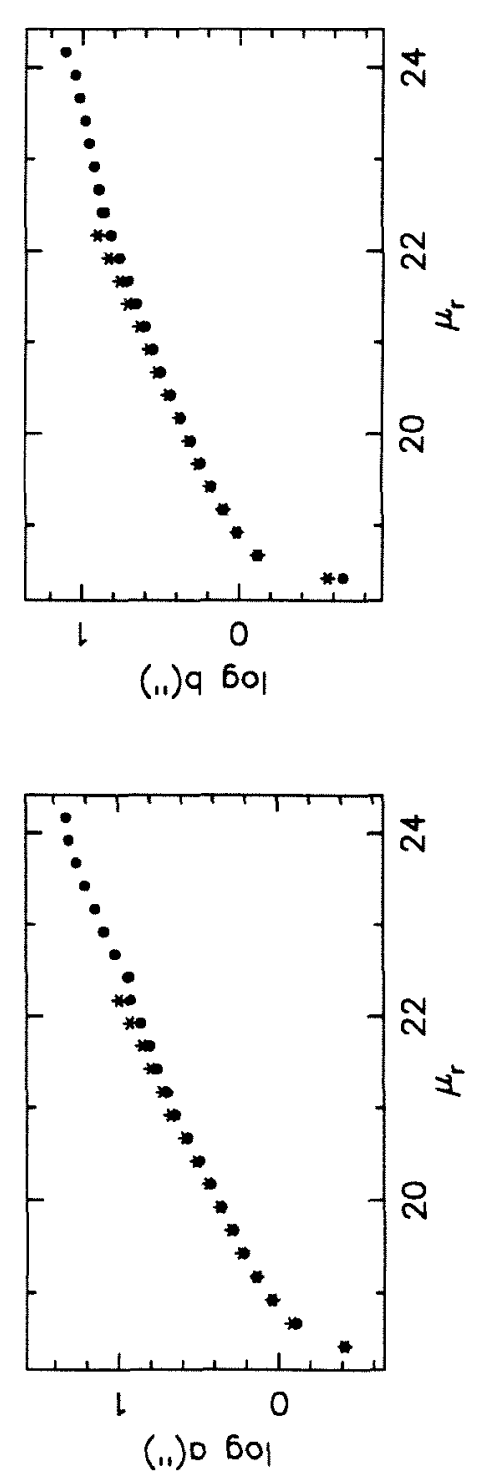
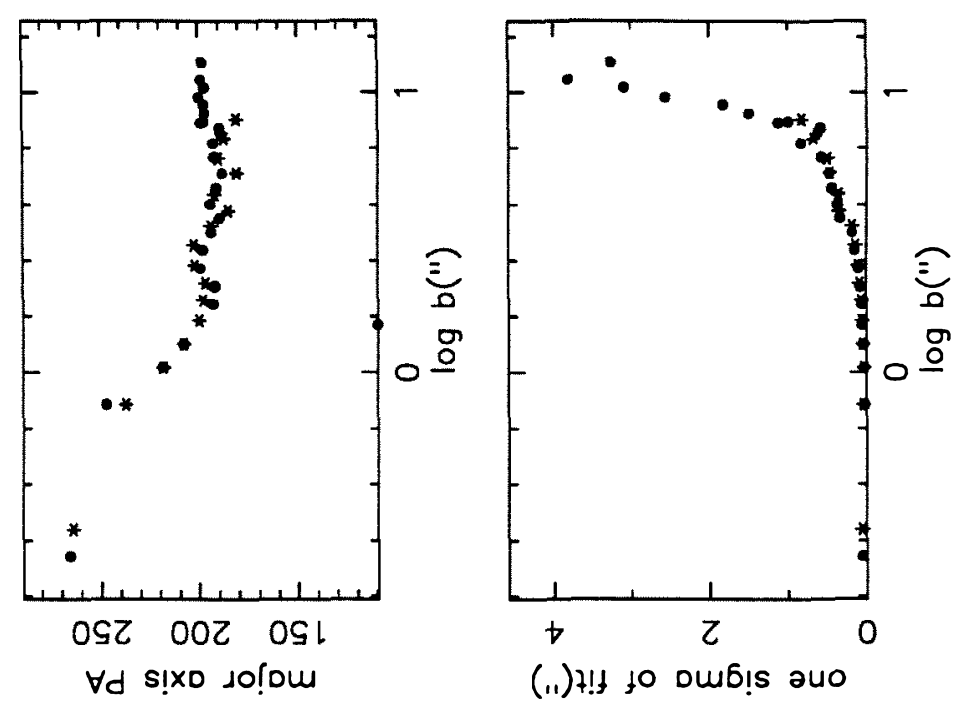

(11) $7 !+$ to oub!s auo

\section{三}
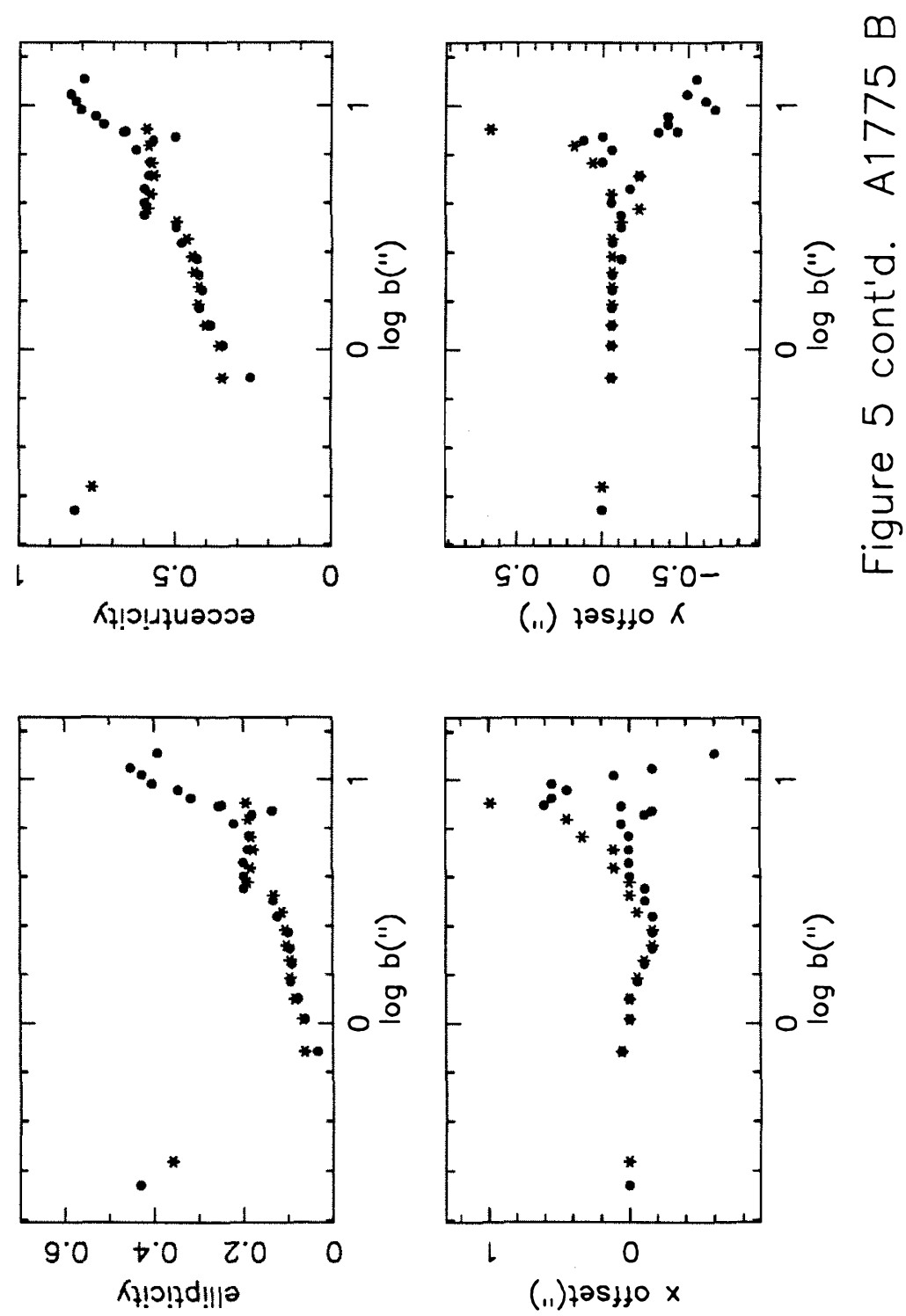

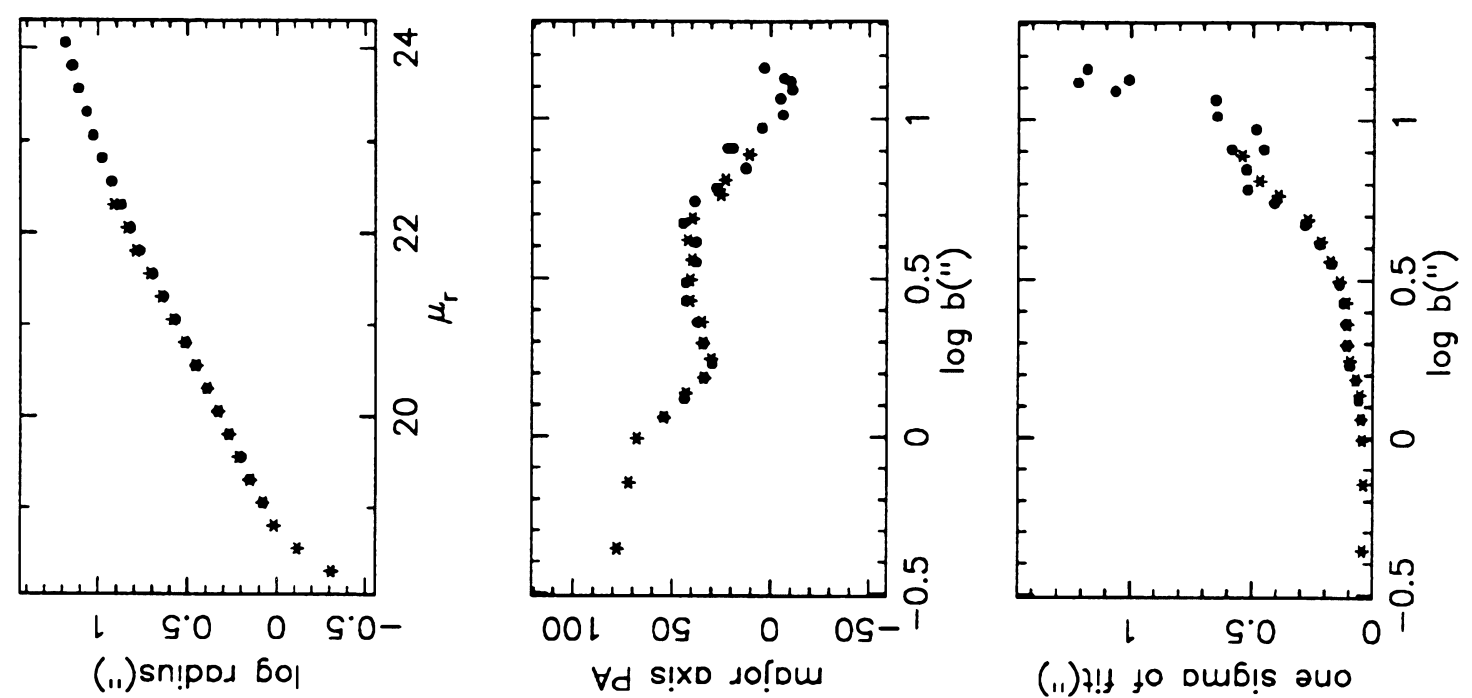

(11) 4 !! to Dub!s auo

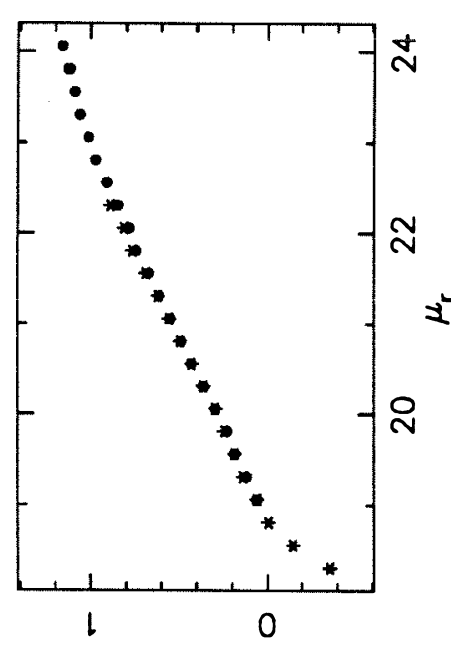

(.1) 9 6ol

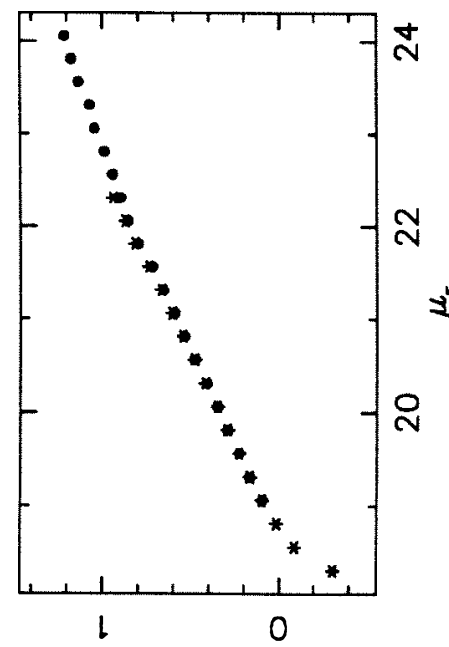

(..) 0601

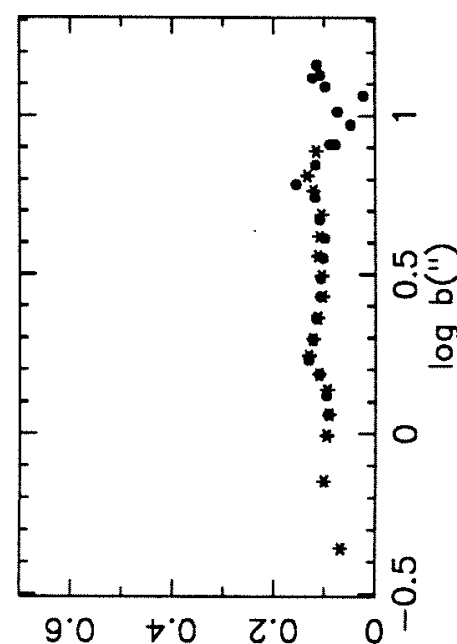

Kฟ!!!?!!||ә

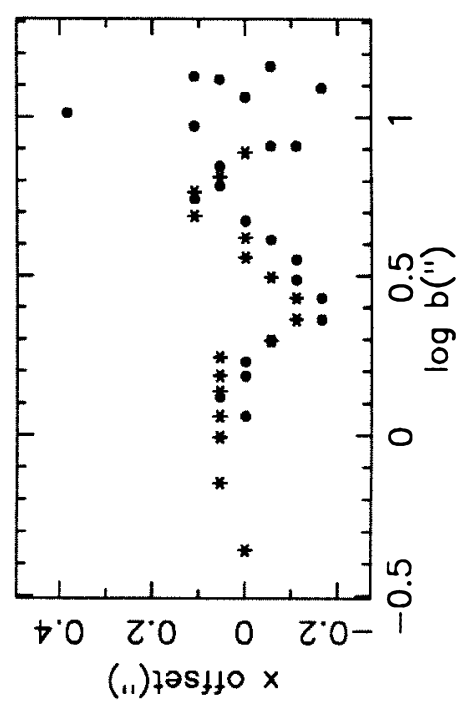




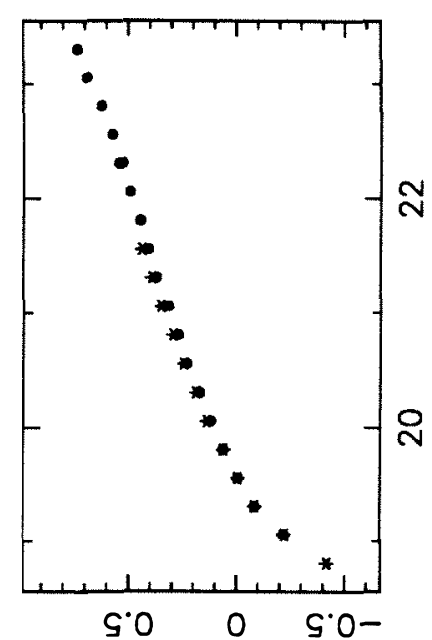

(.1)sn!pos 601

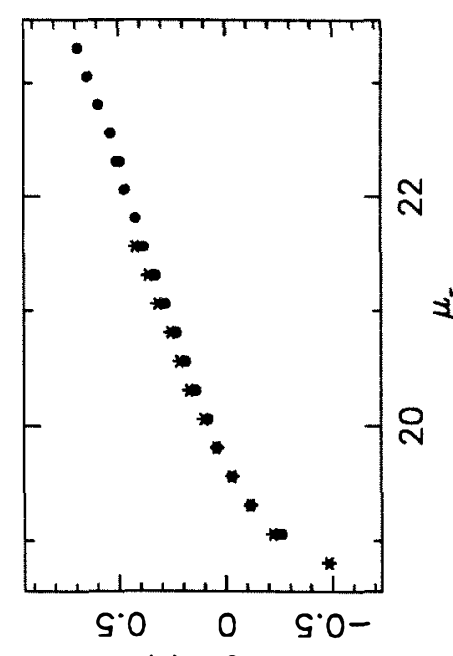

(.1) 9 bol

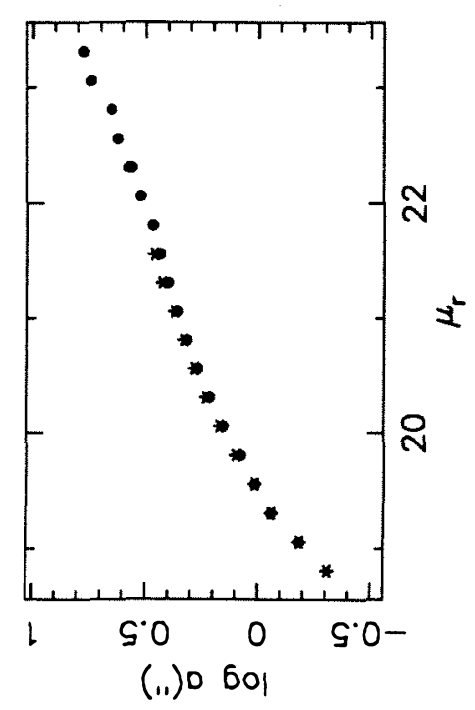

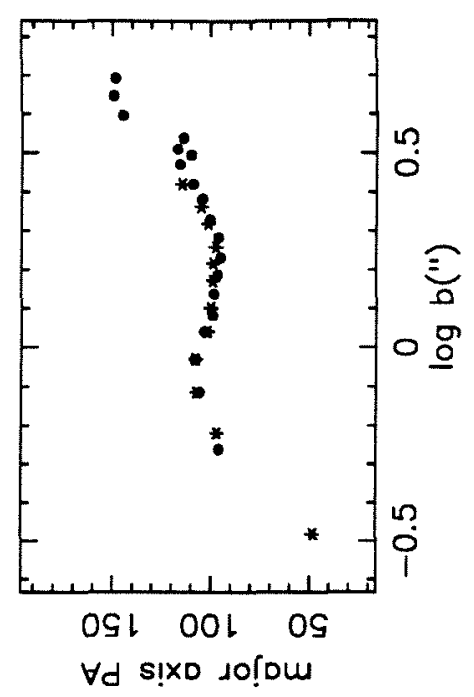

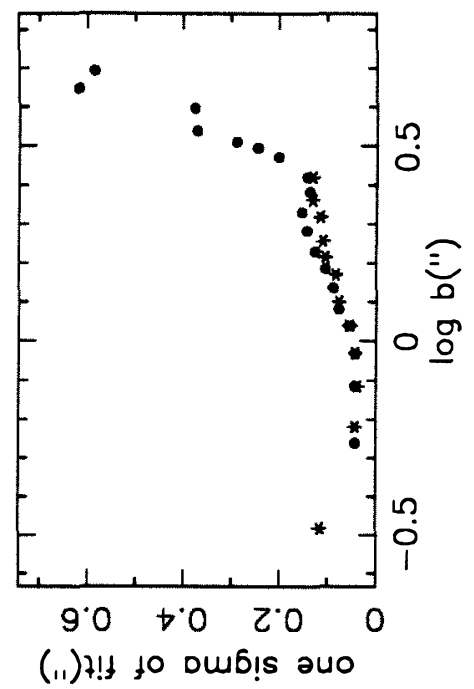

(11) + !! to Dub! ano

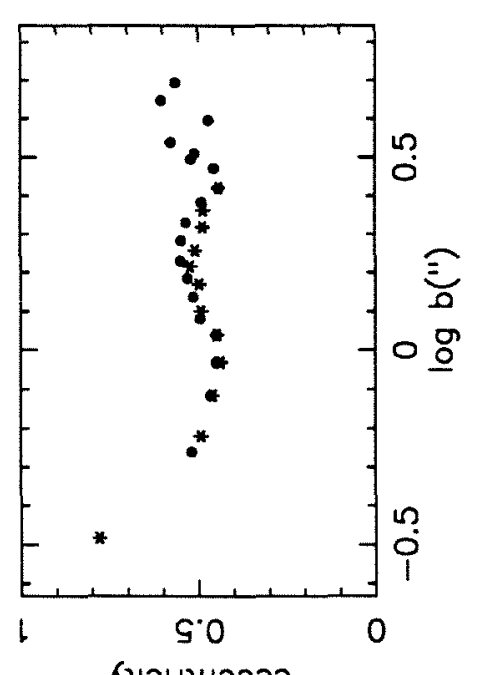

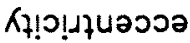
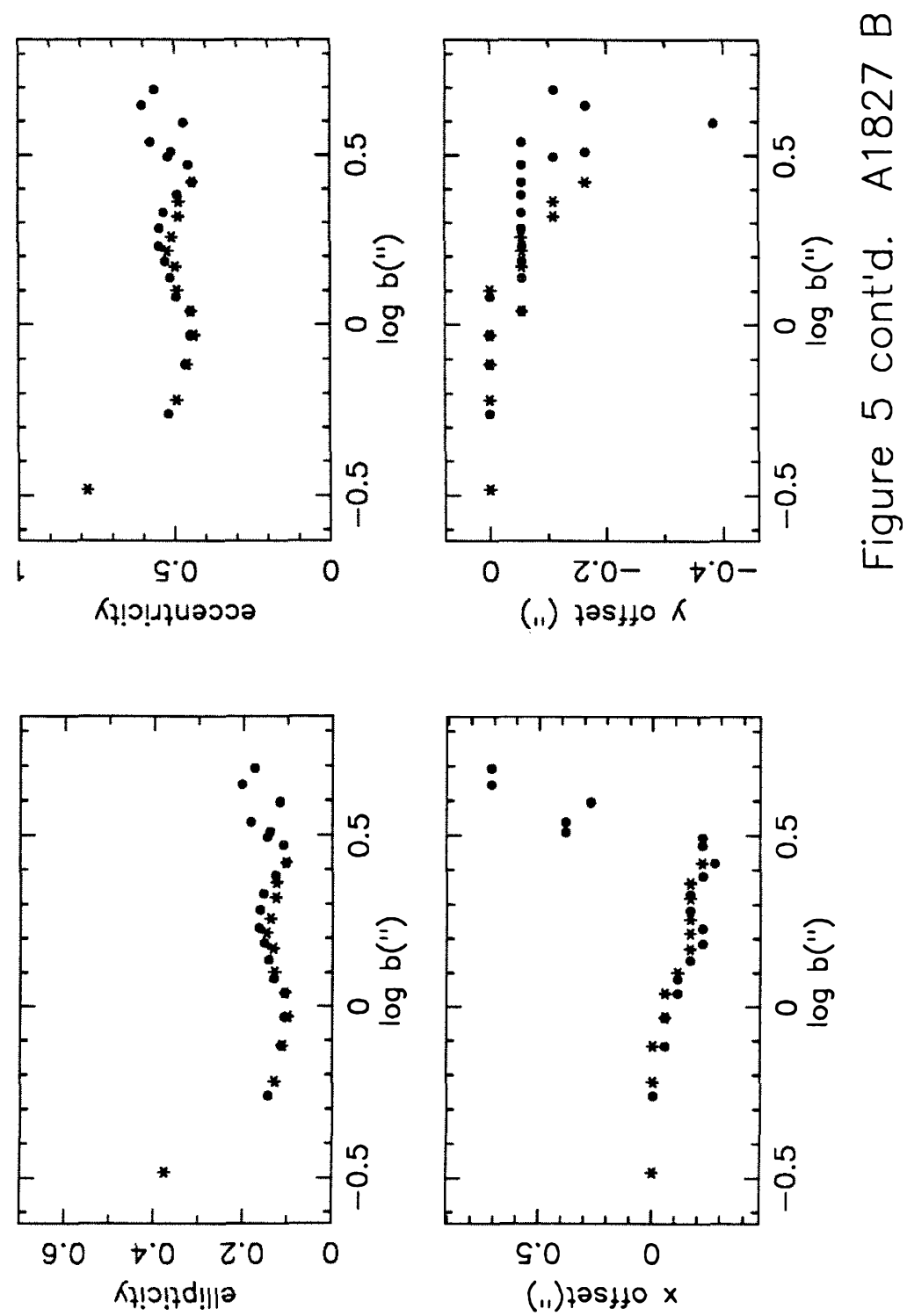

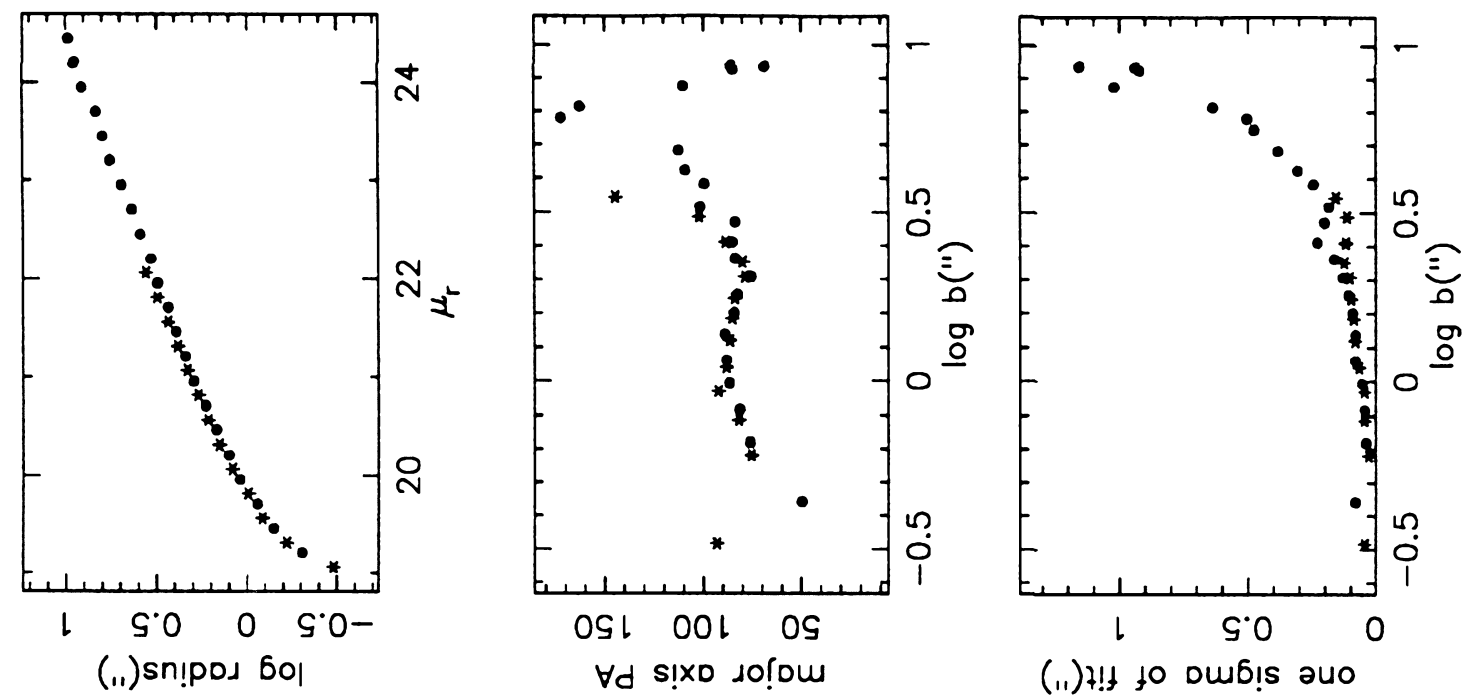

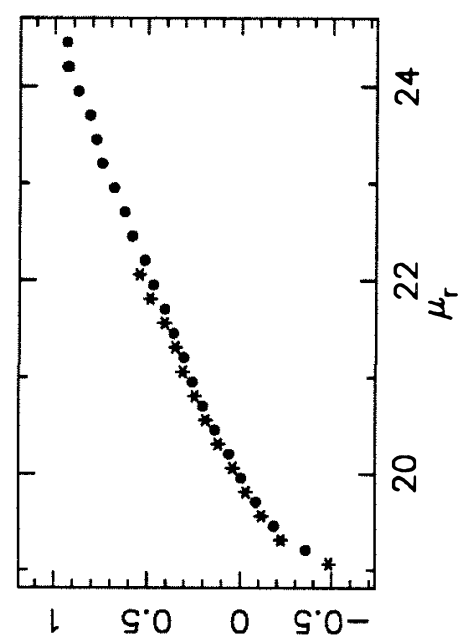

(.1) 9 6ol

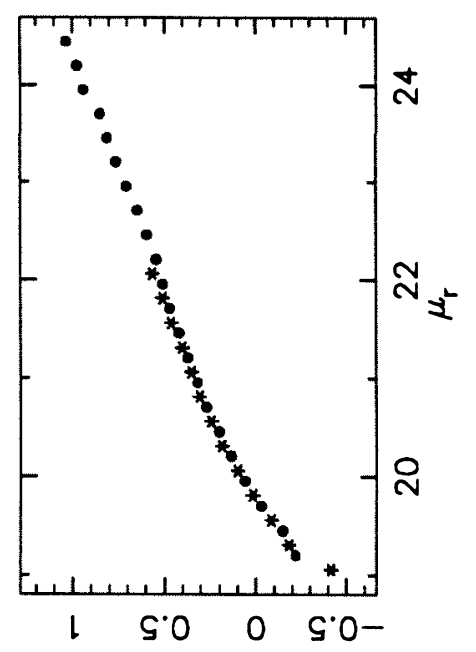

(.1) 0001
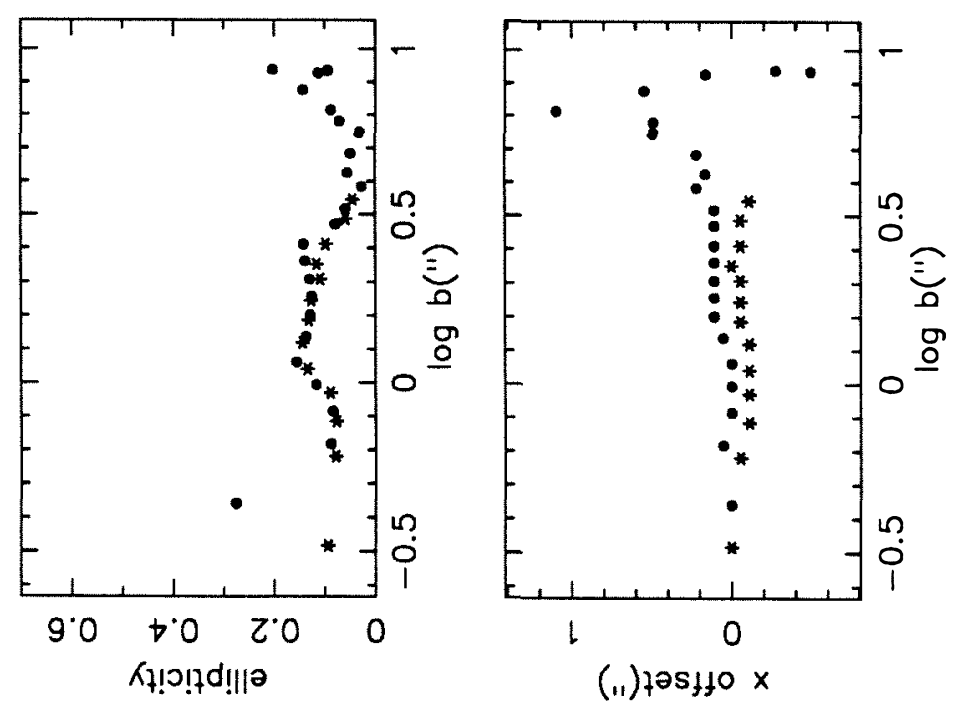


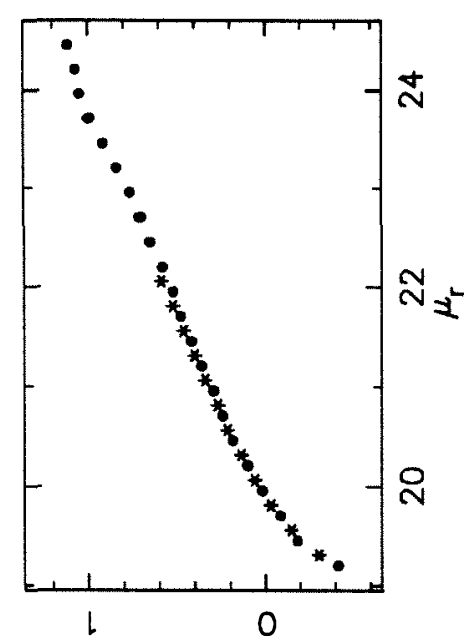

(.1)sn!pos 601

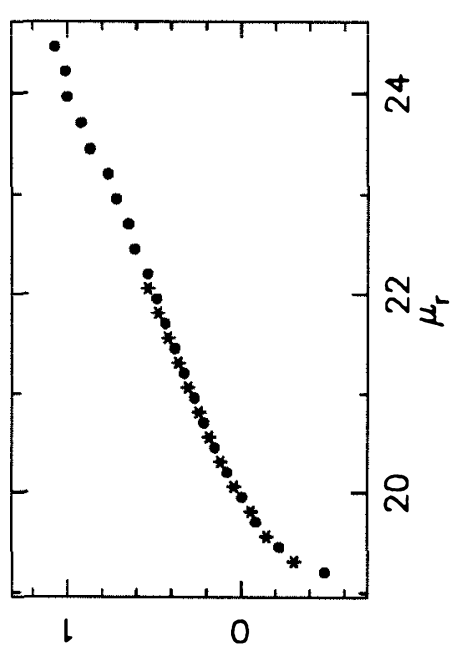

(.1) 960

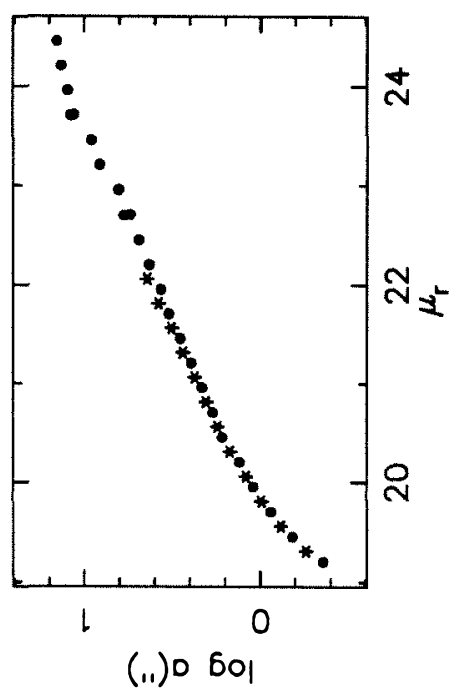

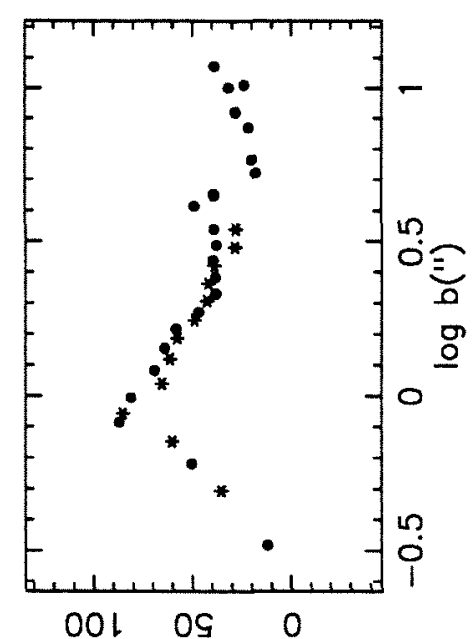

$\forall d$ s!xo $10 !$ ow
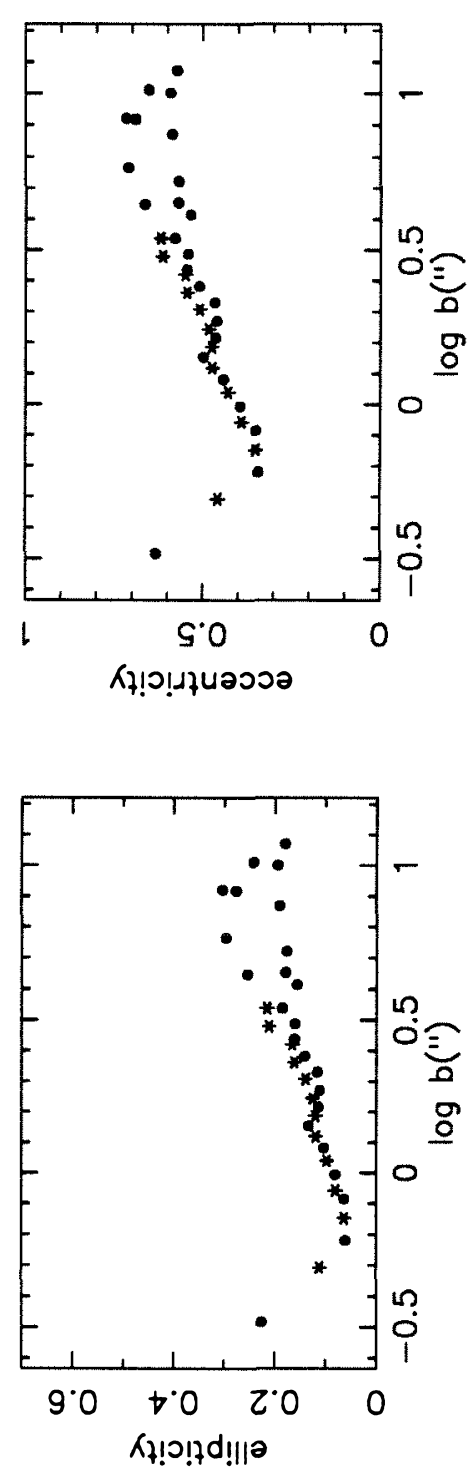

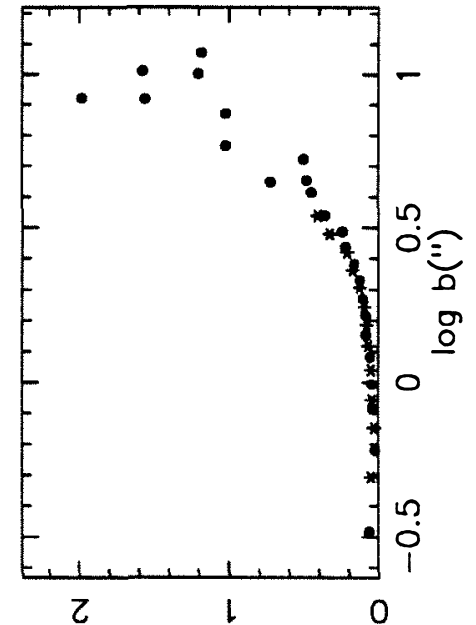

(11) 7 !t to Dub!s au०

$\infty$
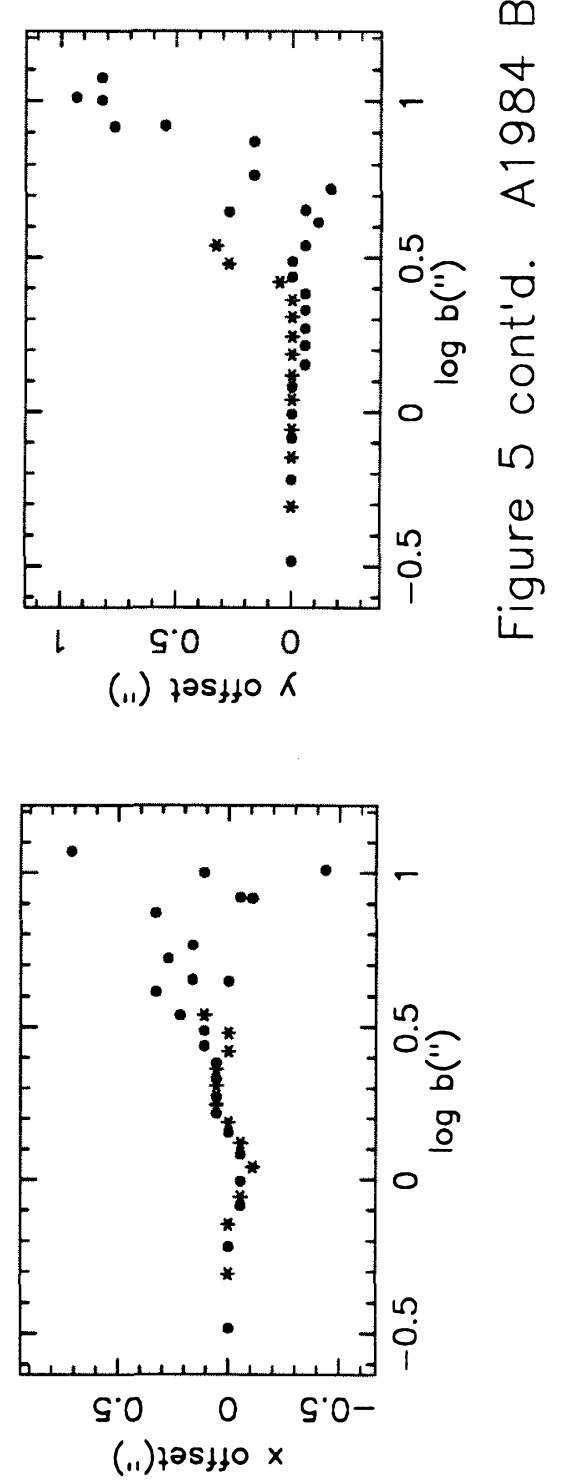


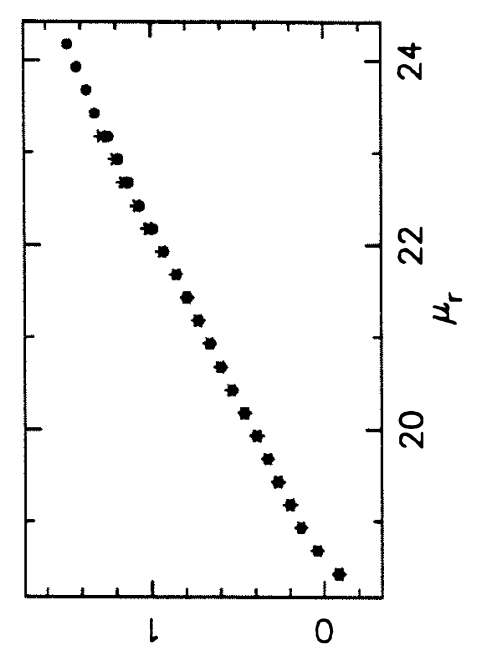

(11) sn!pos 6ol

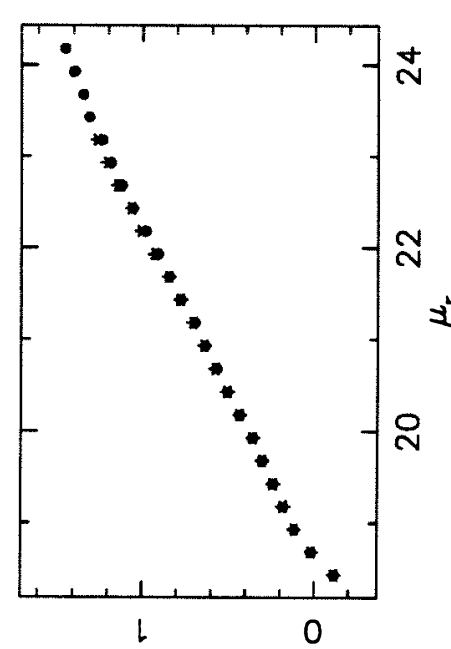

(.1) 9 bol

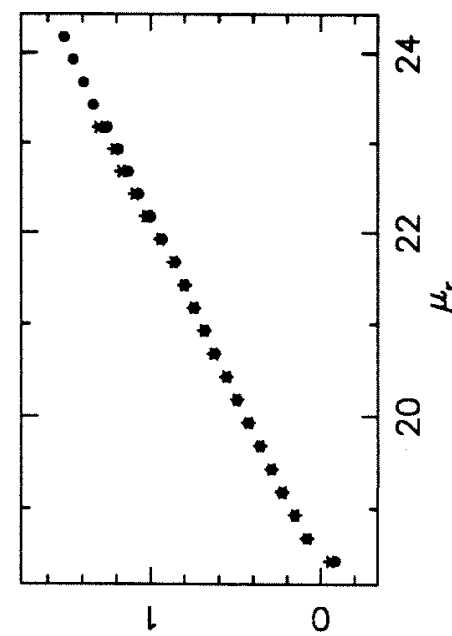

(.1) $0 \mathrm{bol}$

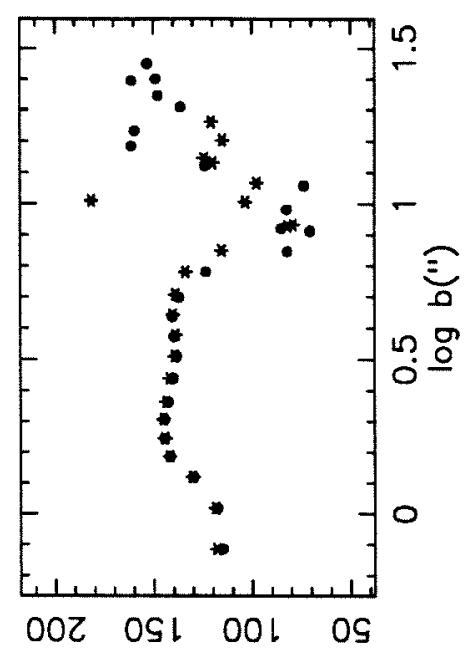

$\forall d$ s!xo do!

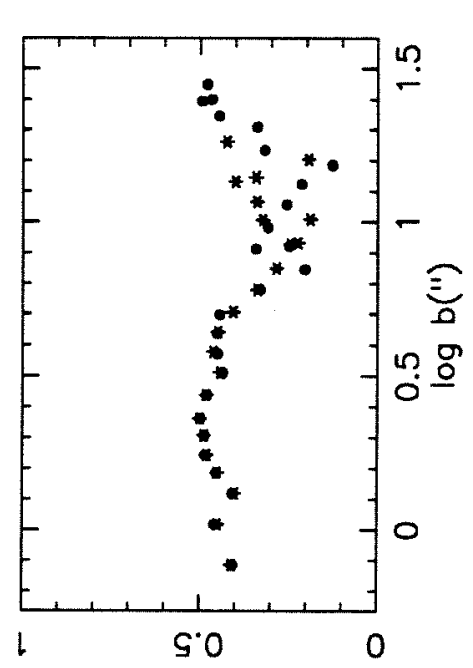

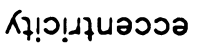

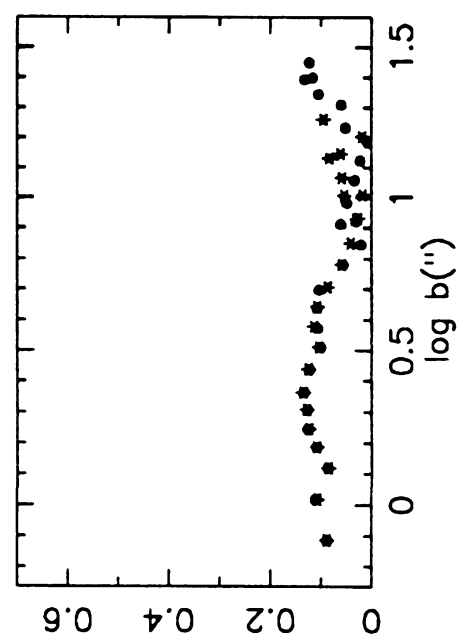

रł! !! d!||ə

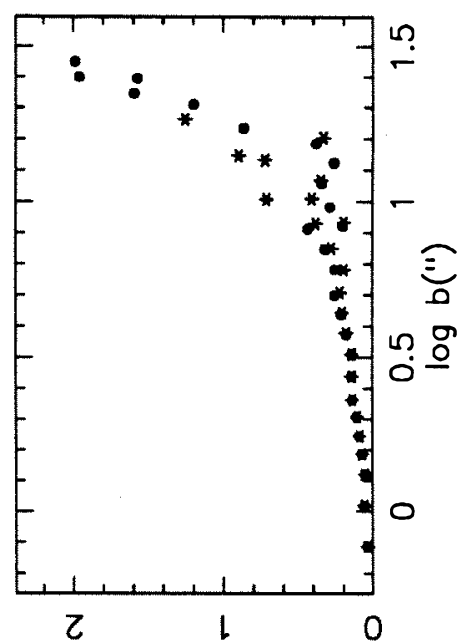

(i) 7 !! to oub!s auo
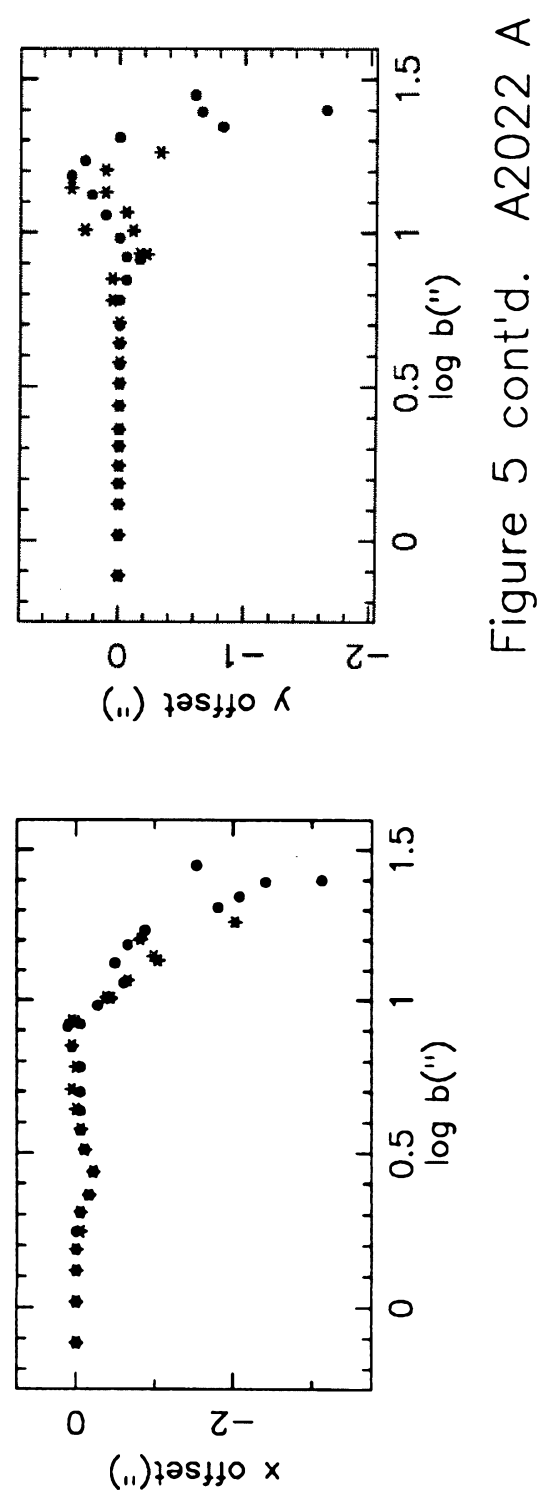

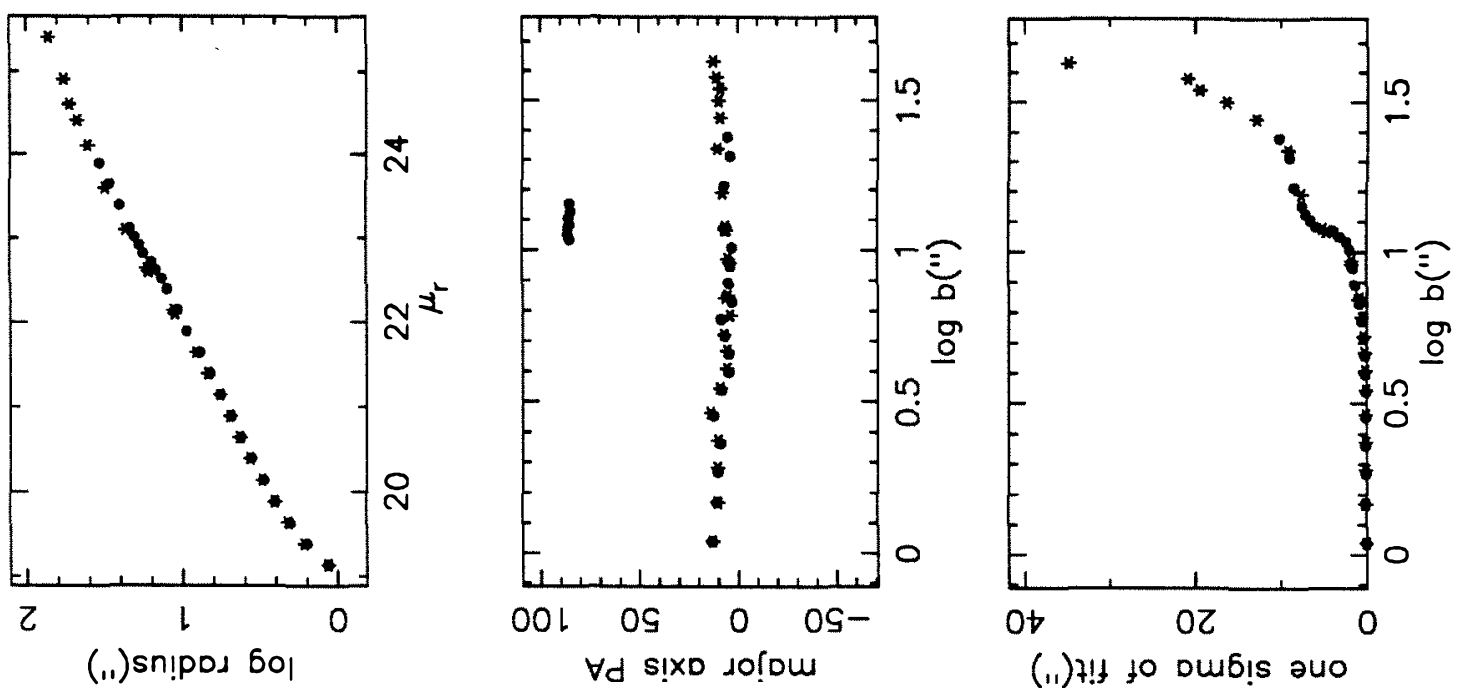

(.1) $7 ! !$ to Du6!s aน०

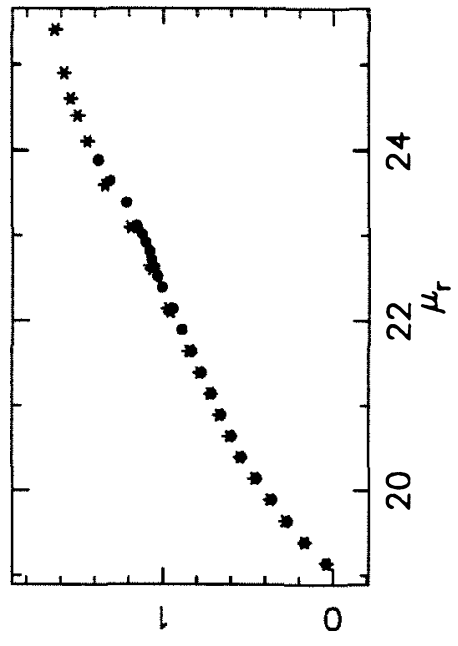

(.1) 9601

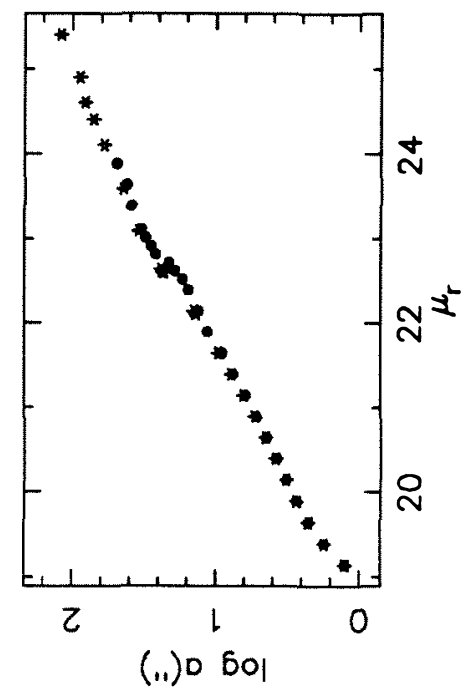

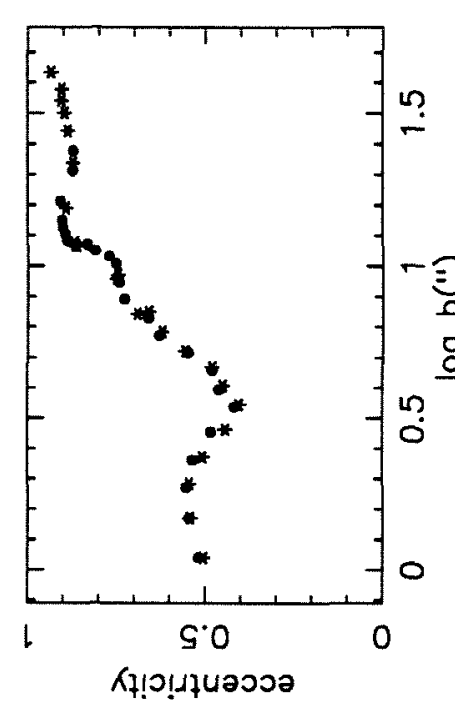

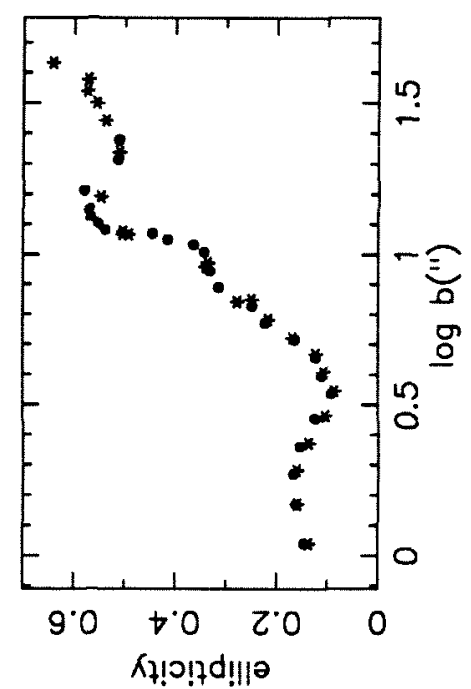

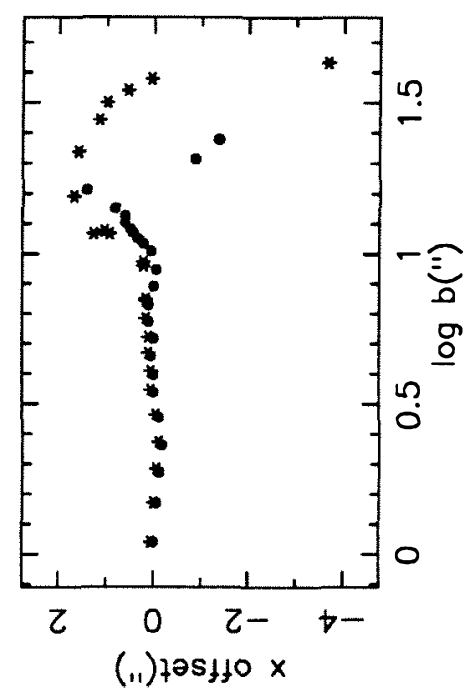



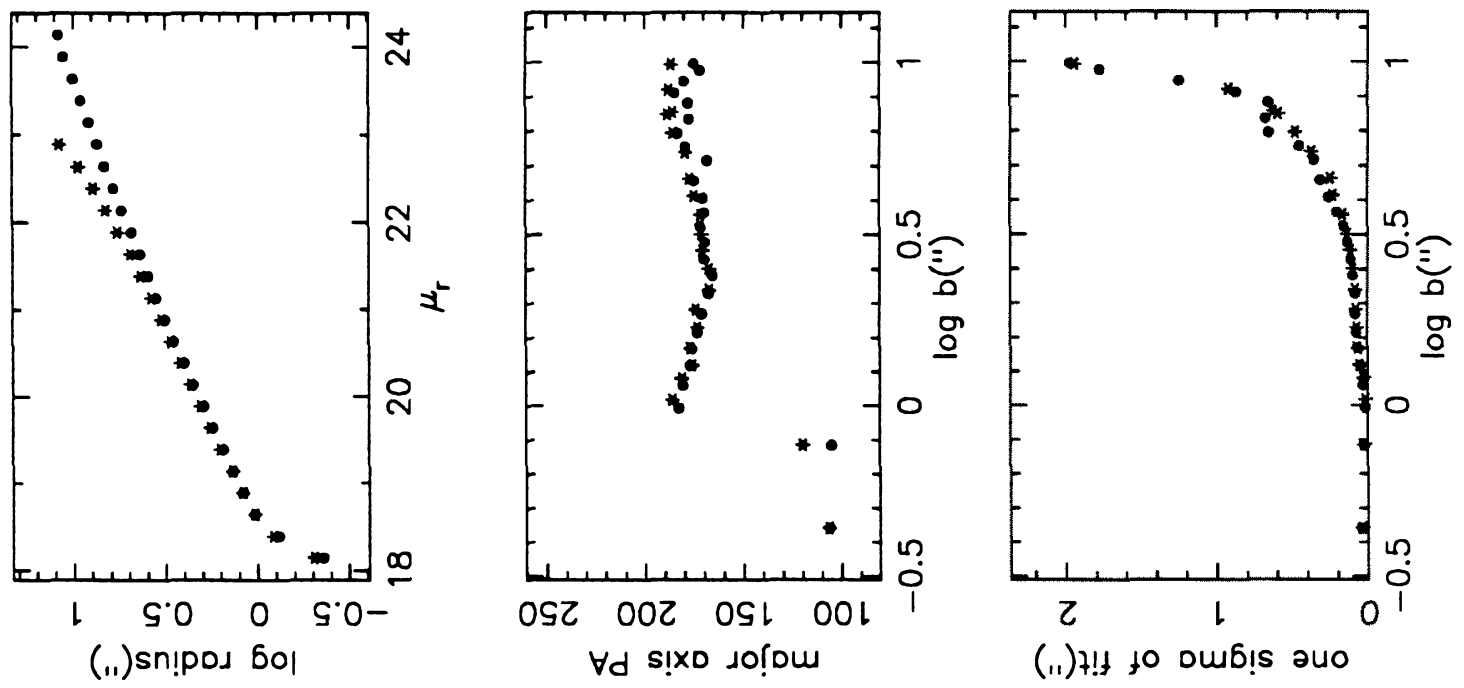

(11) + !! to oub!s 2u०

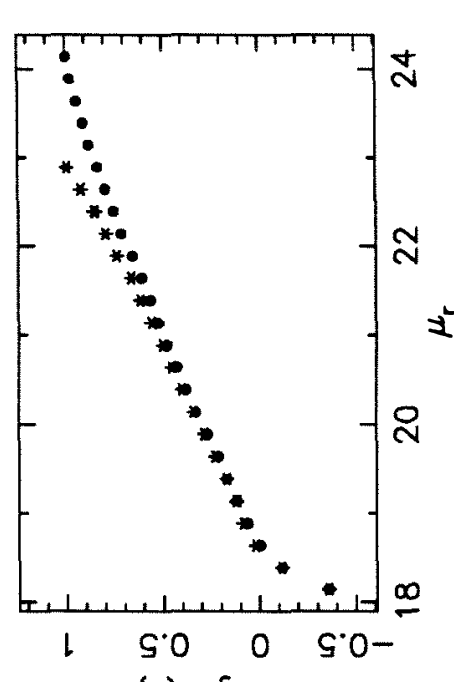

(.1) $\mathrm{q}$ bol

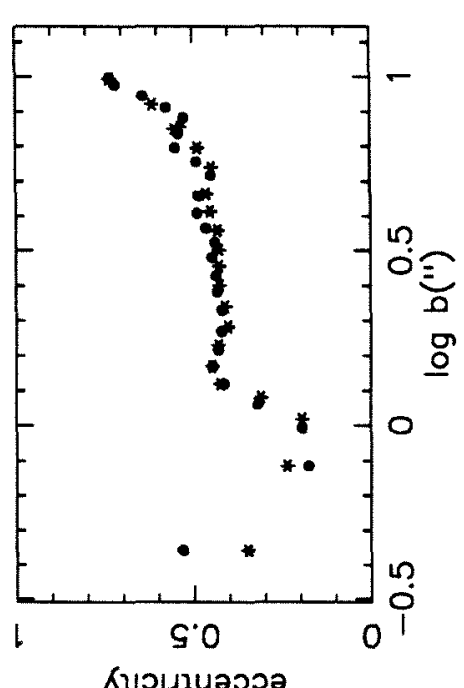

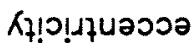
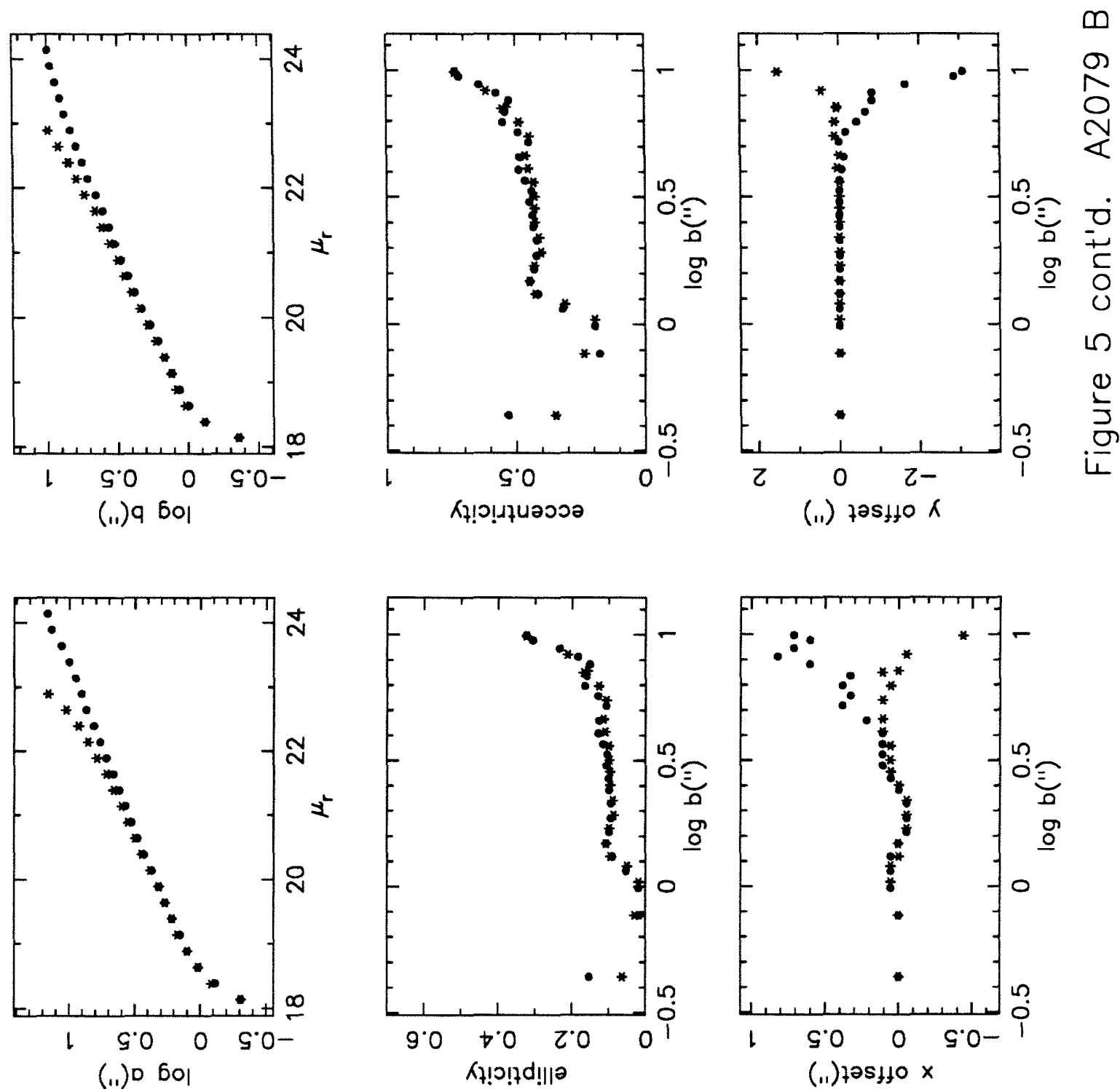


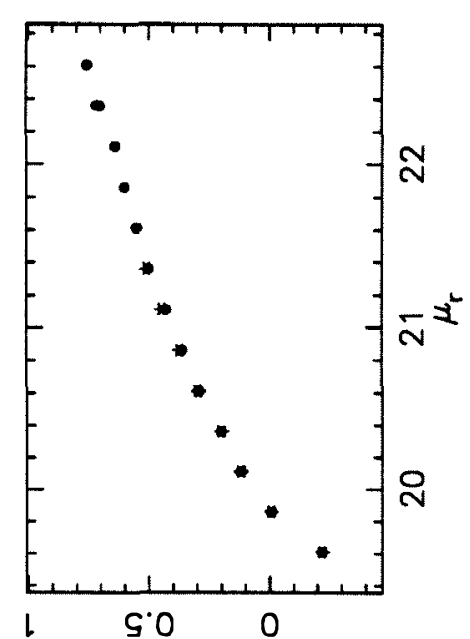

(.1)sn!pod 60।

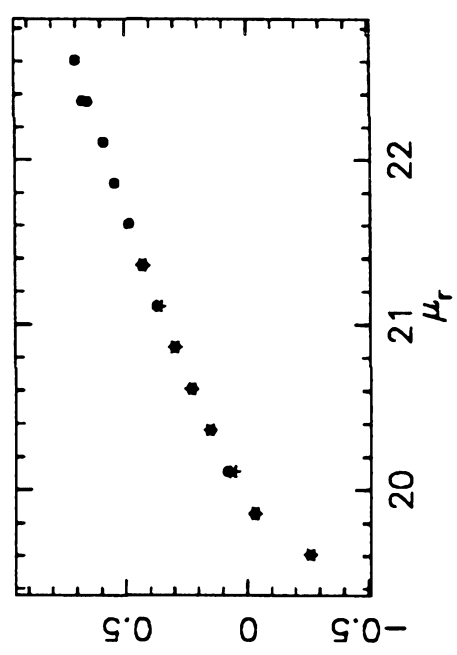

(.1) 9 50।

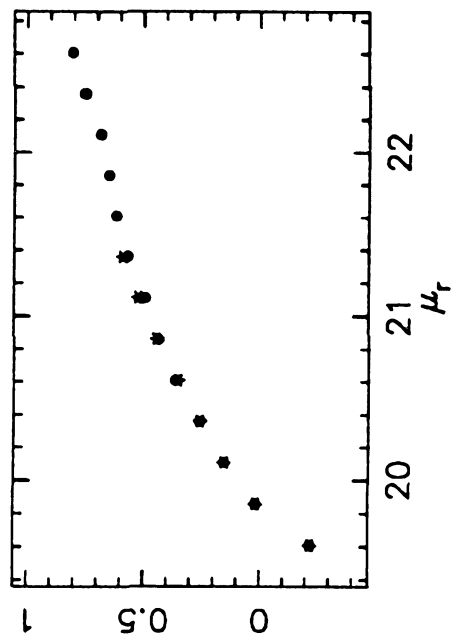

(.1) 0 6ol

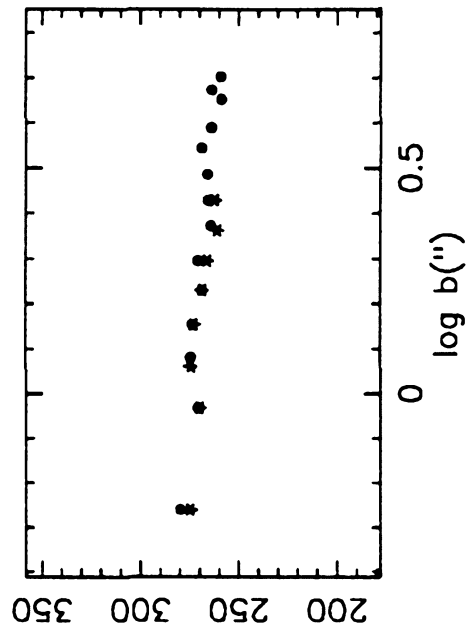

$\forall d$ s!xo so!́m

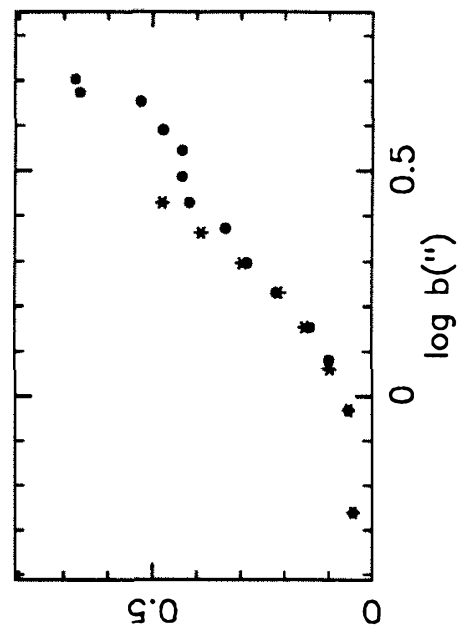

(11) $+!+10$ oub!s auo

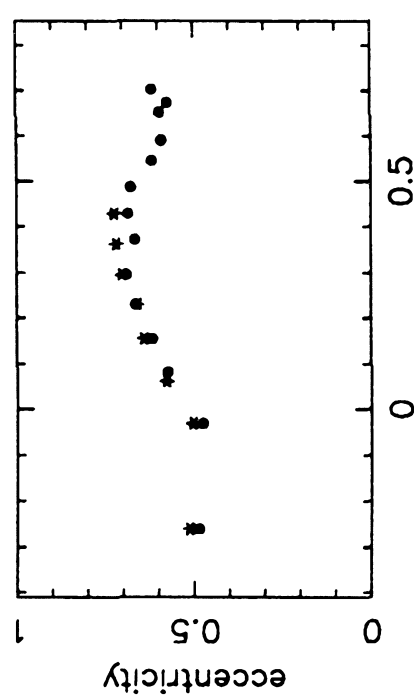

$<$
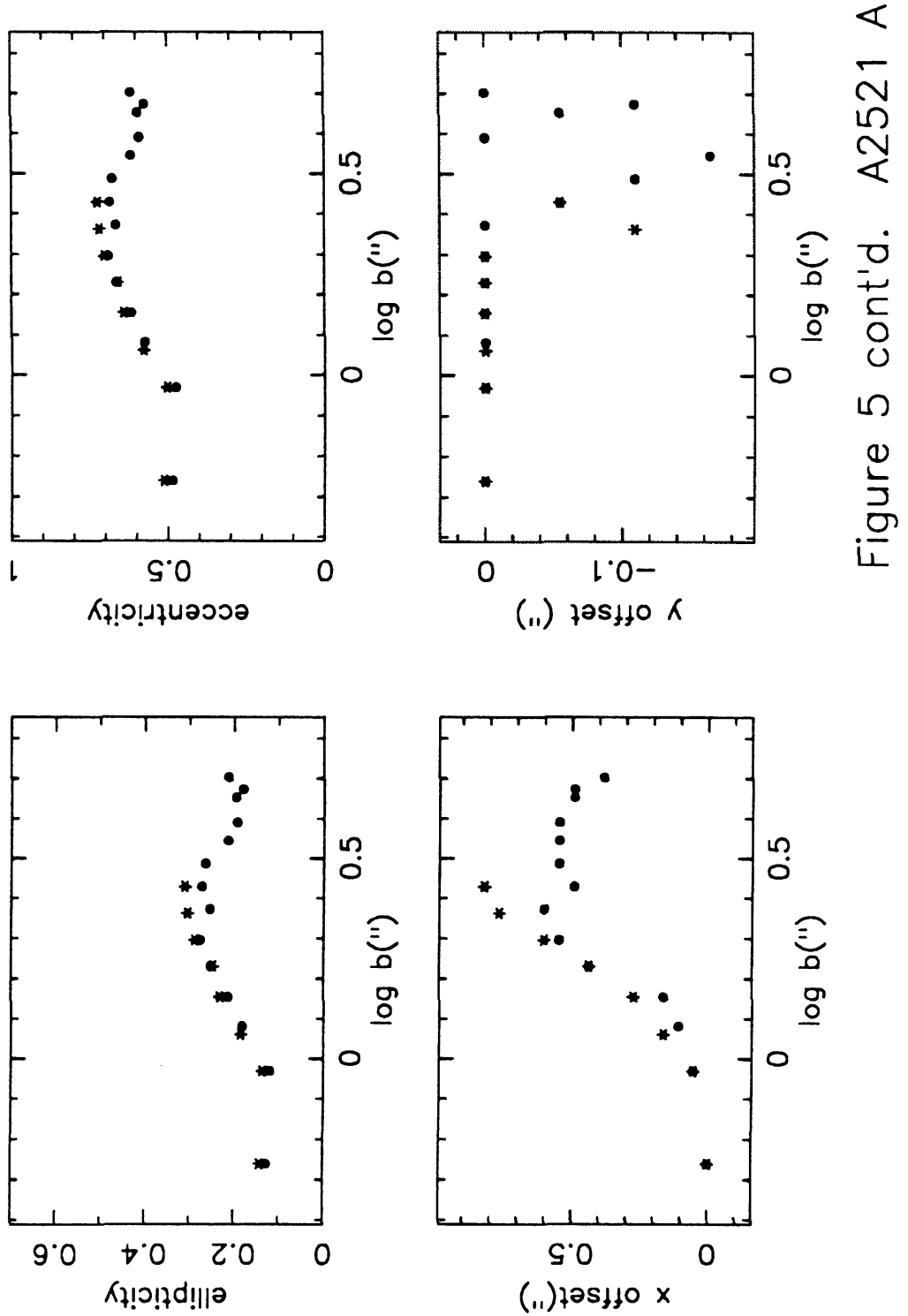


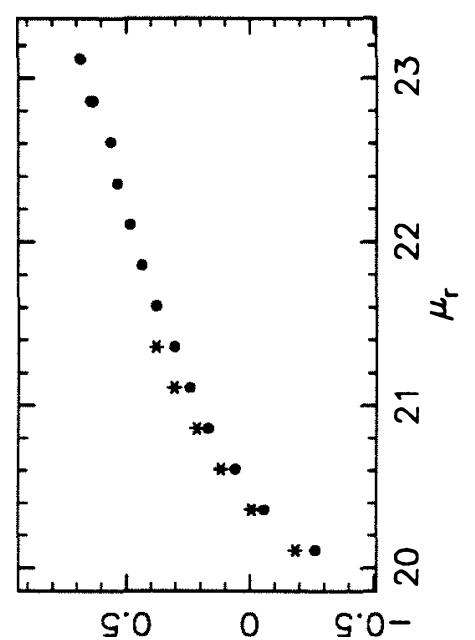

(.1) sn!pos 6ol

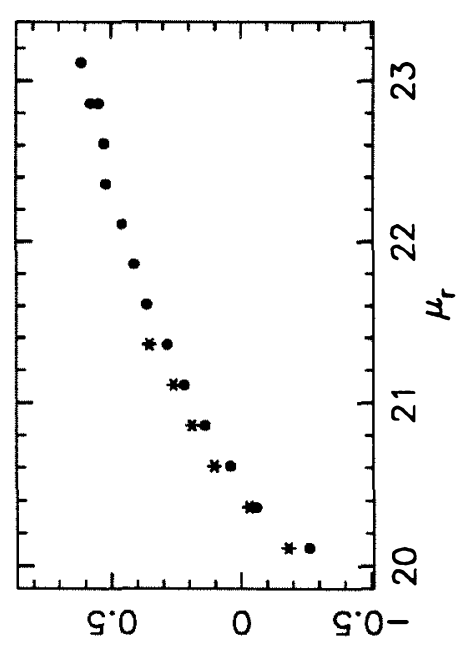

(.1) $q \mathrm{bo}$

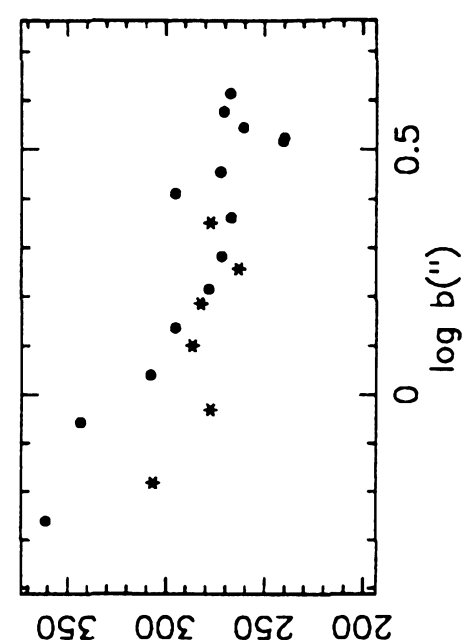

$\forall d s$ !xo so!́m

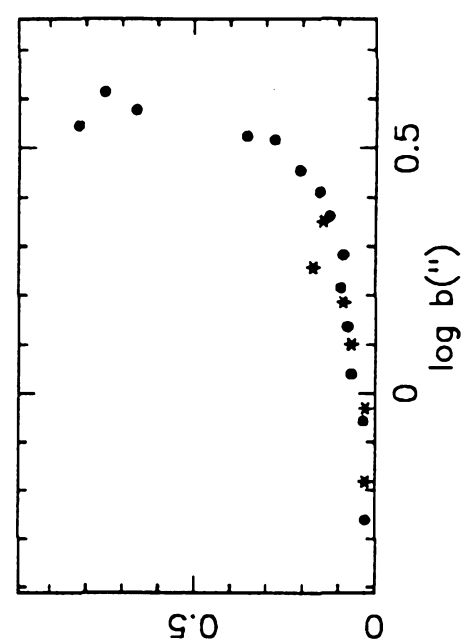

(11) + !! to Dub! au०

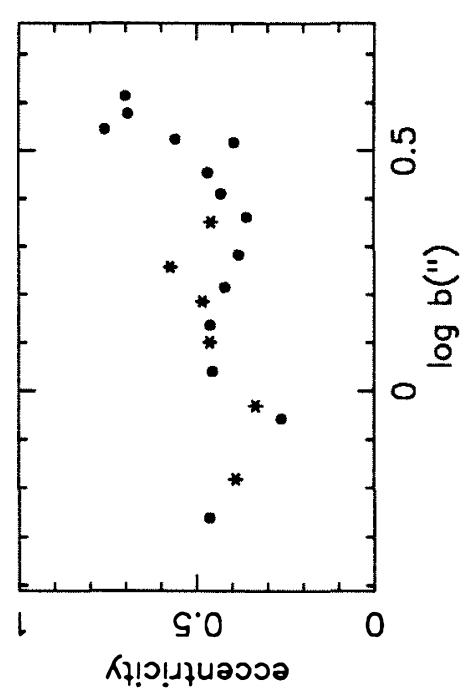

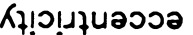
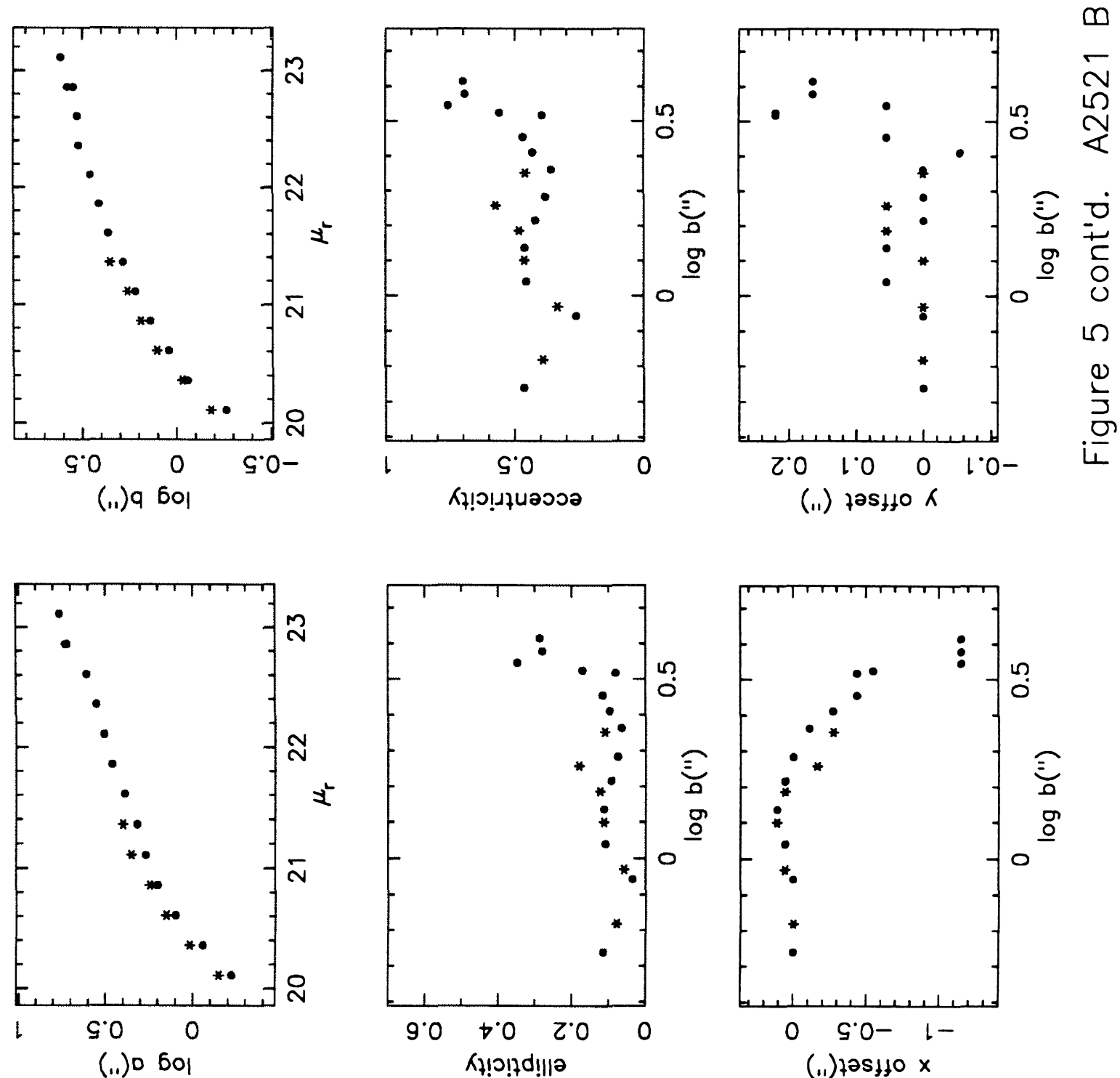

Kł!อ!!d!!|ə

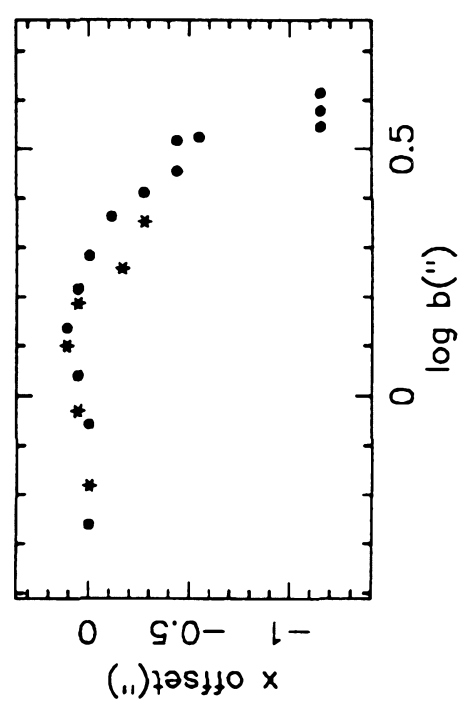



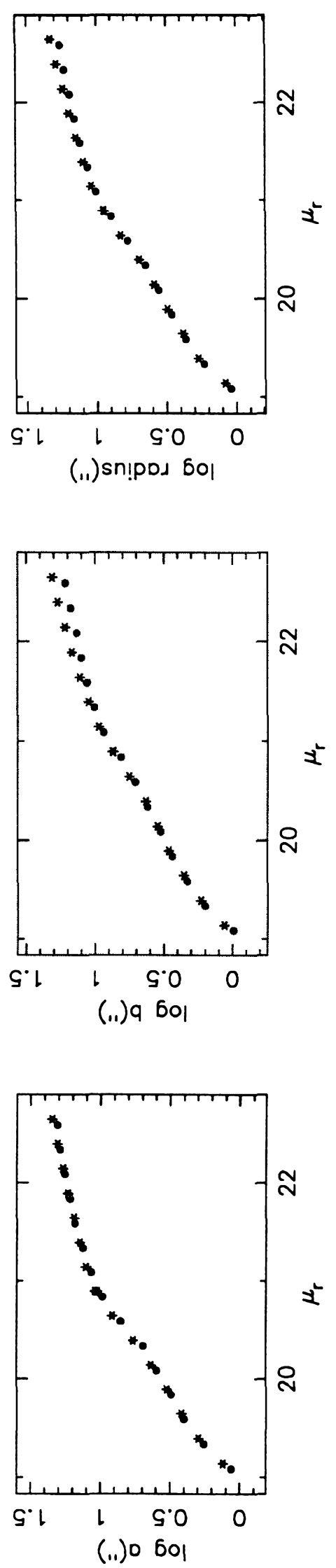
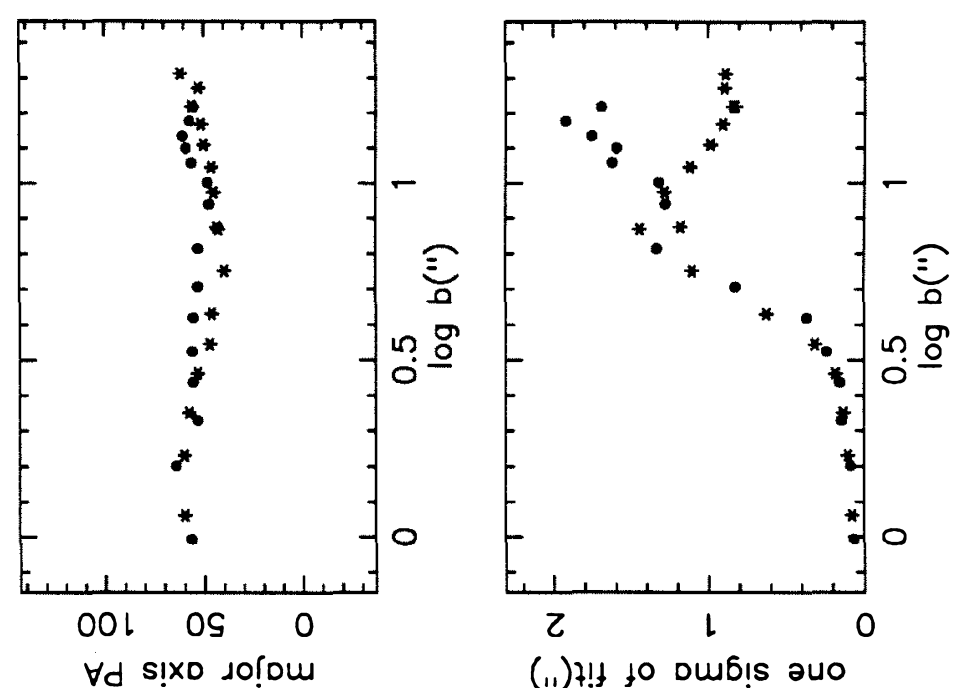

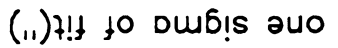
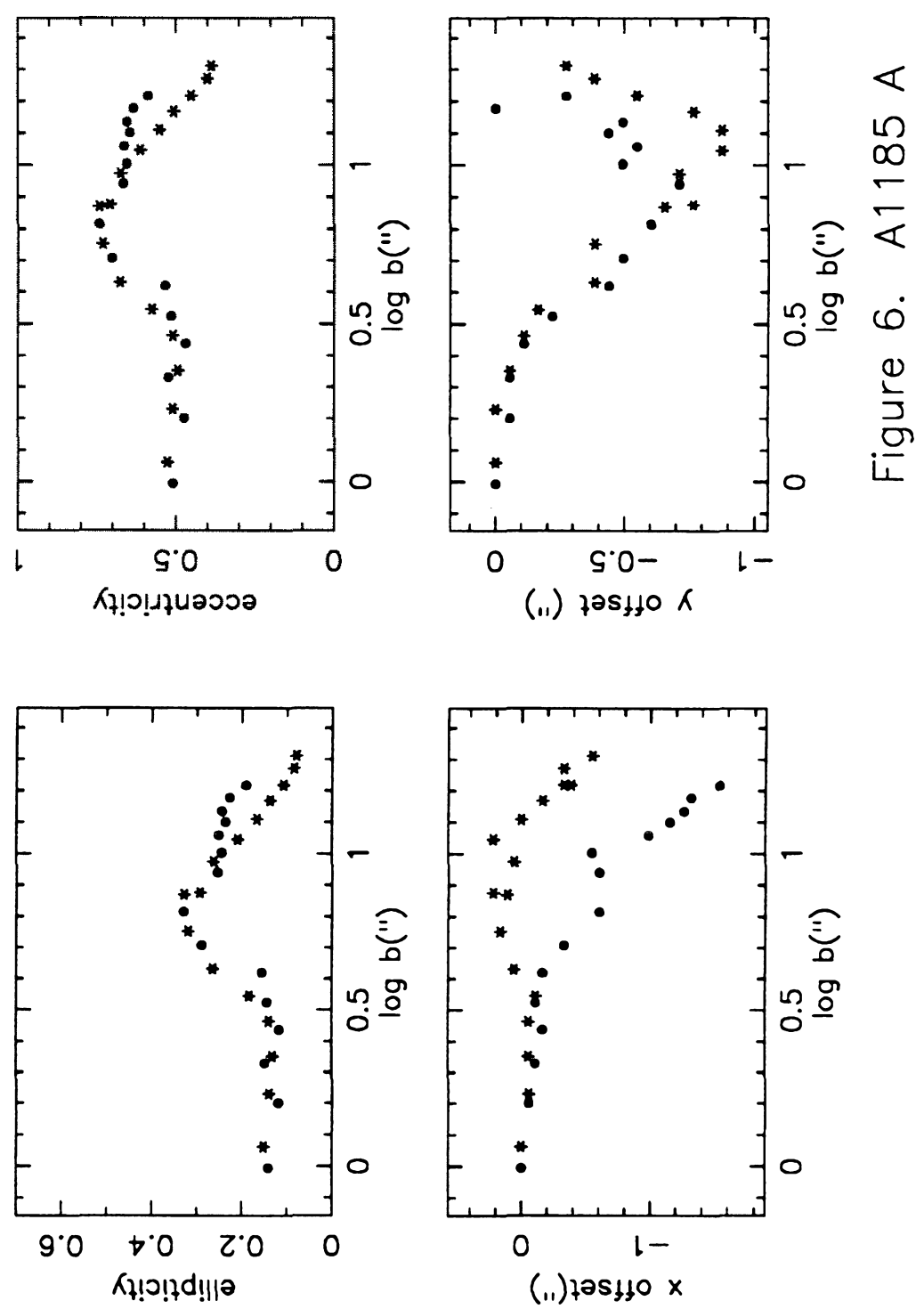
$-255-$

APPENDIX

Chapter 1, Figure 1

Notes for Chapter I 


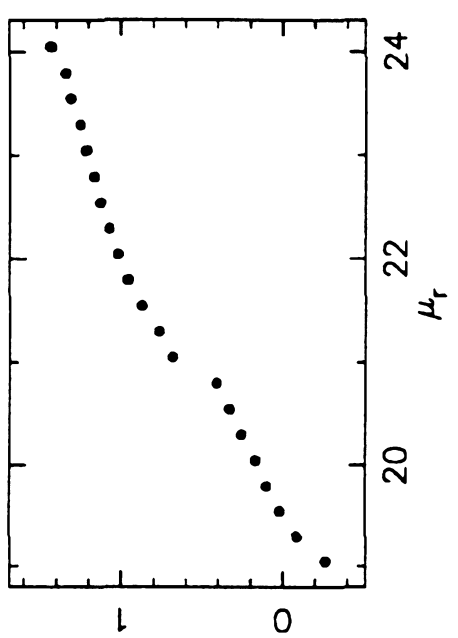

(.1) sn!pod 6ol

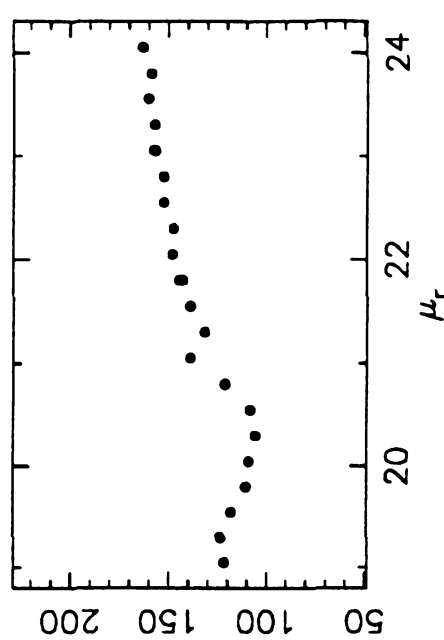

$\forall d$ s!xo so!̣m

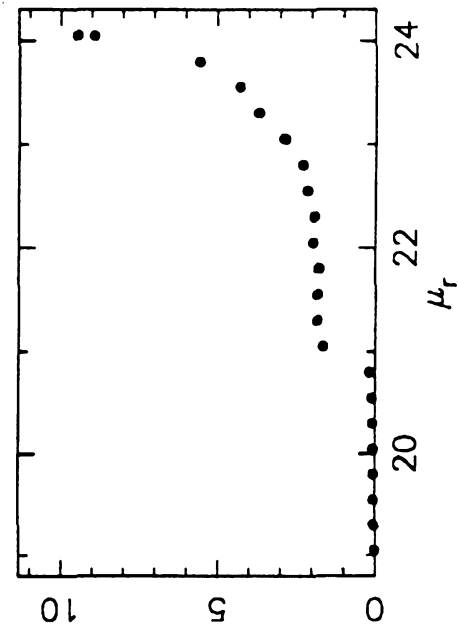

(.1) $7 ! 1$ to oub! avo

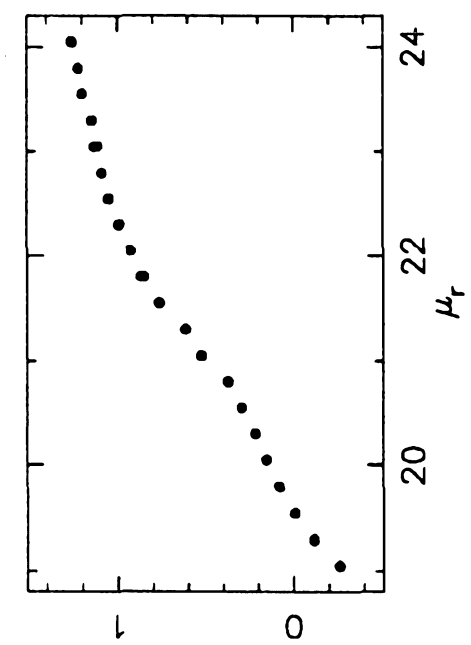

(i1) 9601

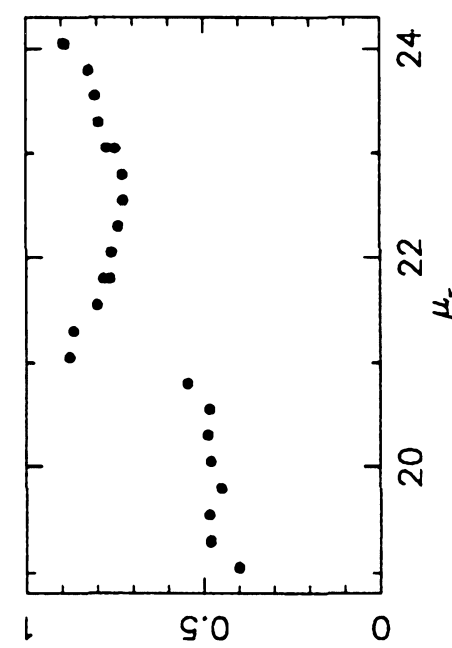

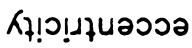

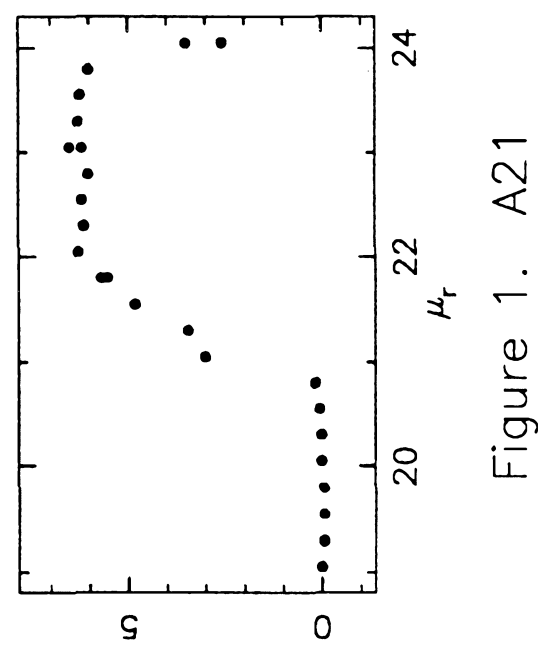

(ii) $725+10 K$

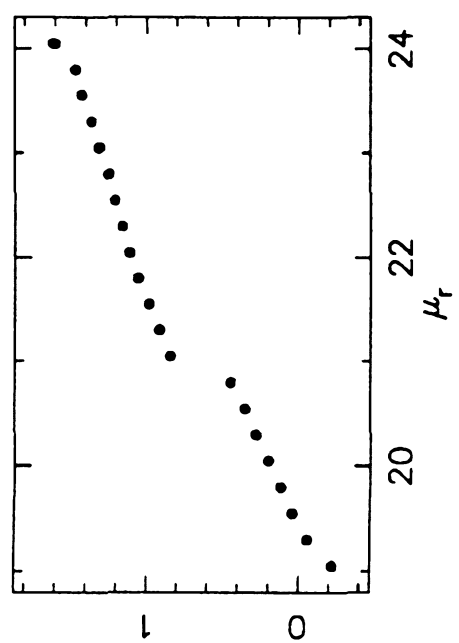

(11) 0601

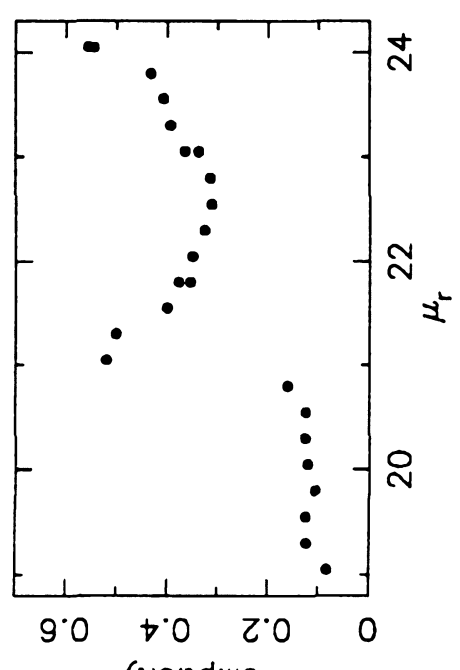

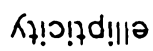

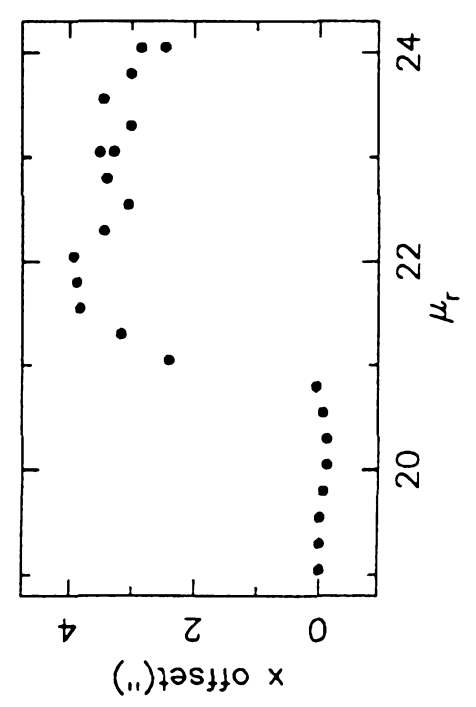




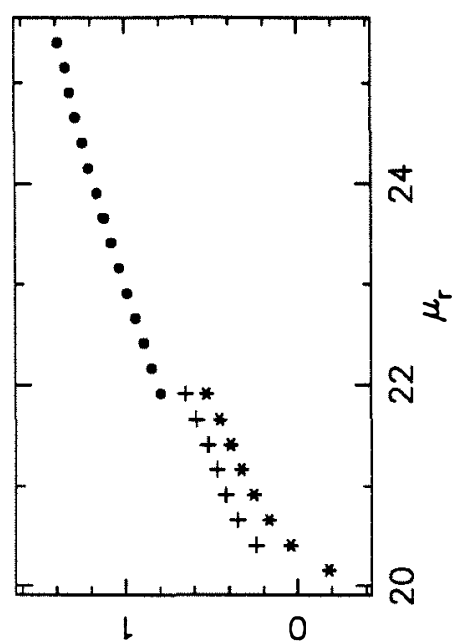

(.1) sn!pos 6ol
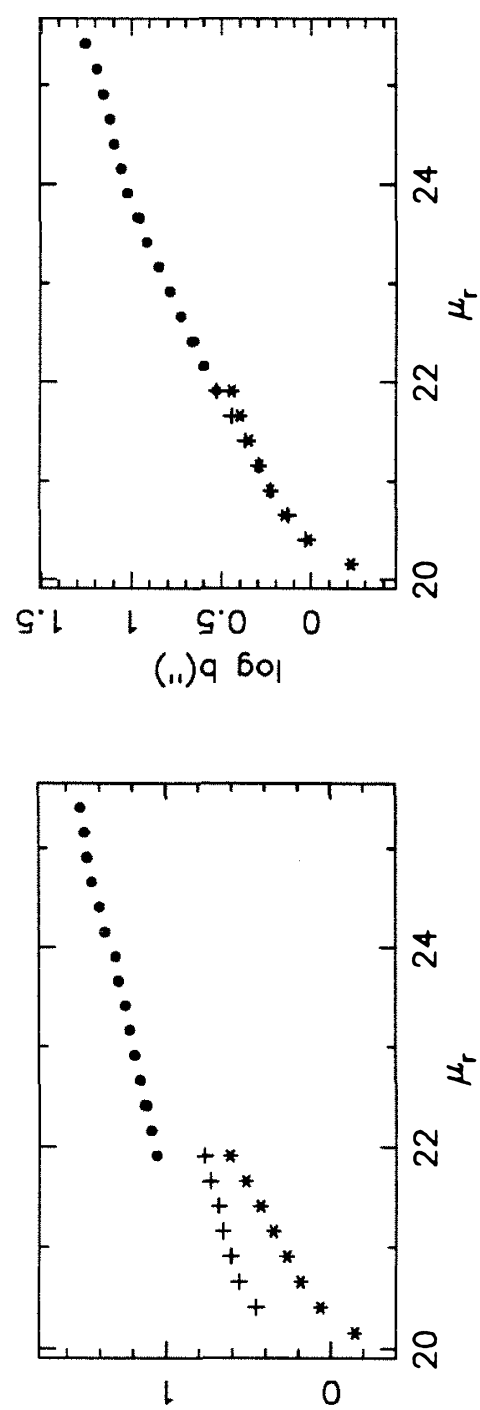

(.1) 0 601
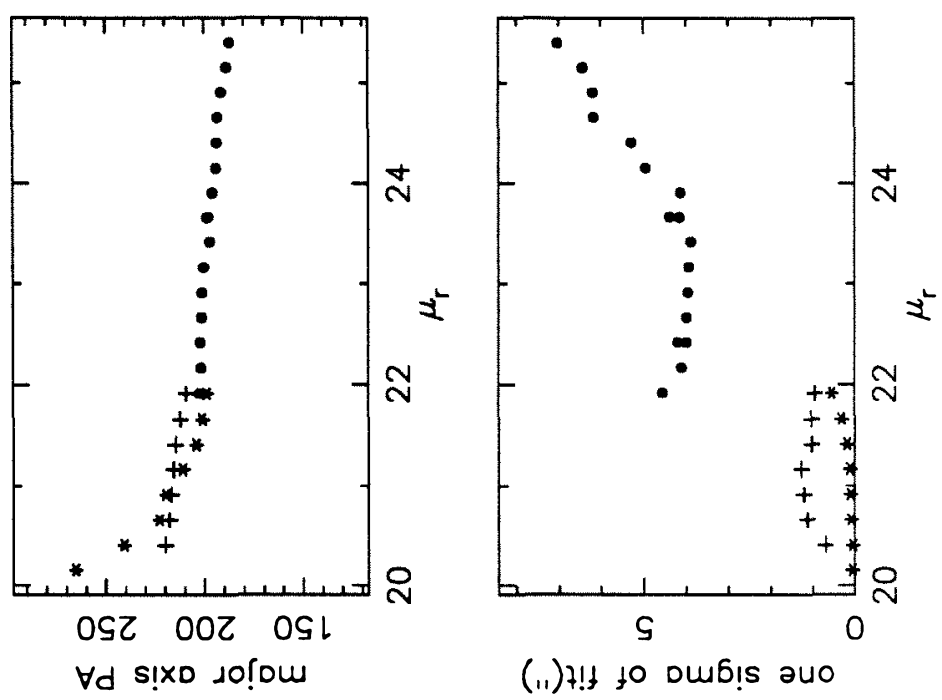

(11) $7 !+$ to oub! ano
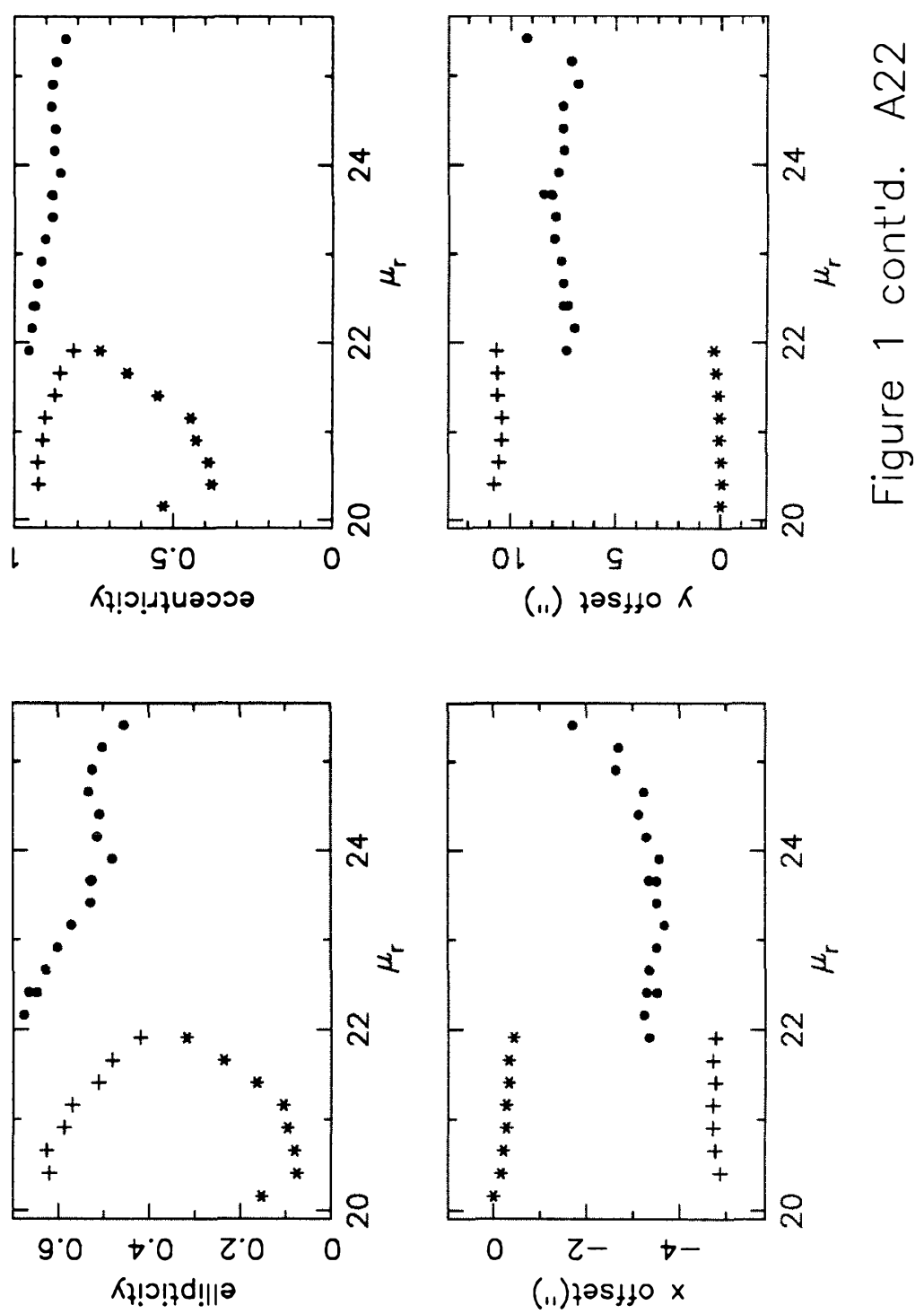


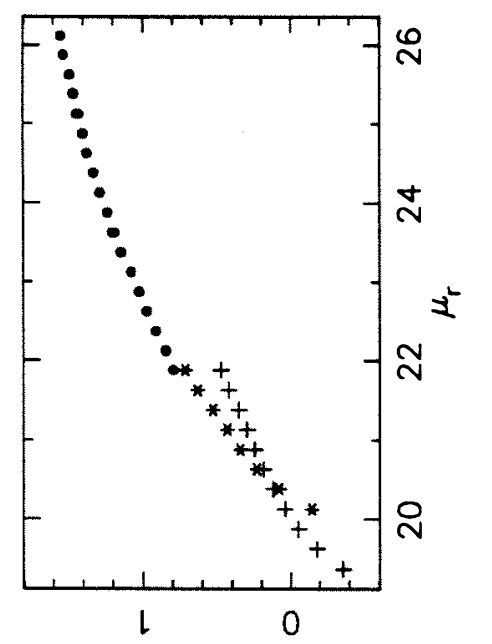

(.1)sn!pod 60।
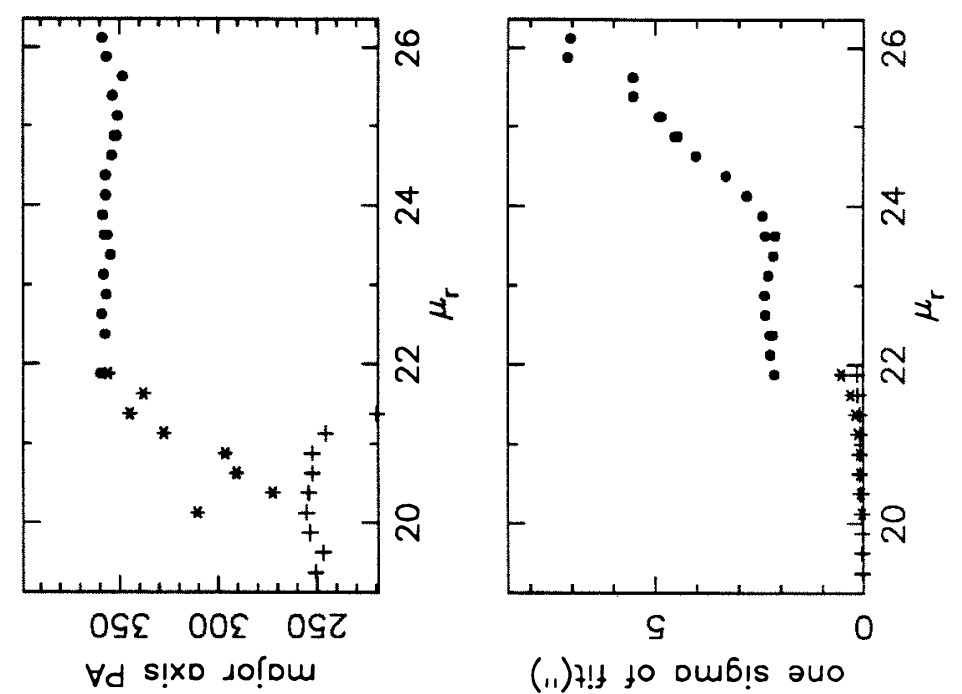

(11) $7 !+$ to ou6!s aиo

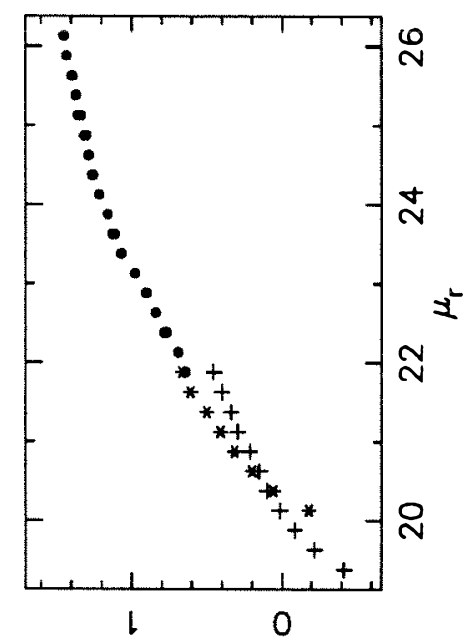

(.1) 9 6ol

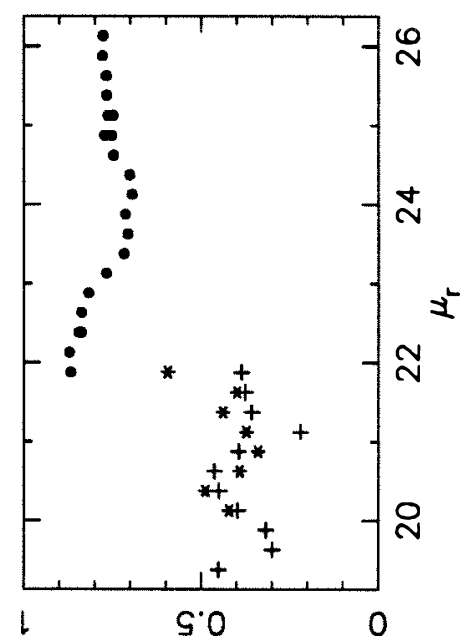

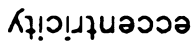

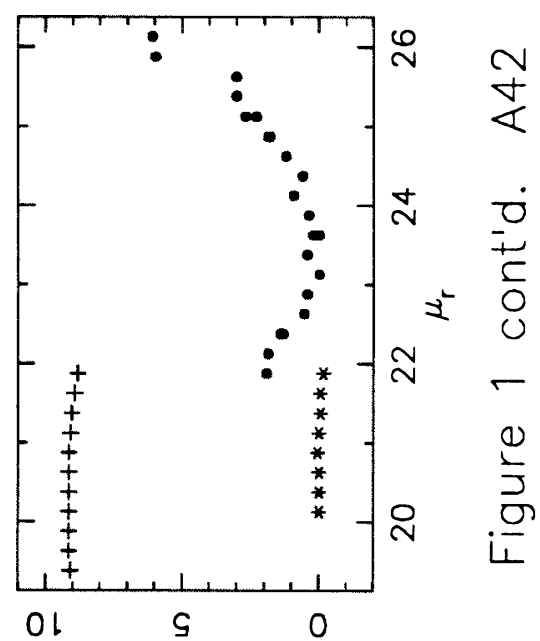

(ii) $725+10 K$
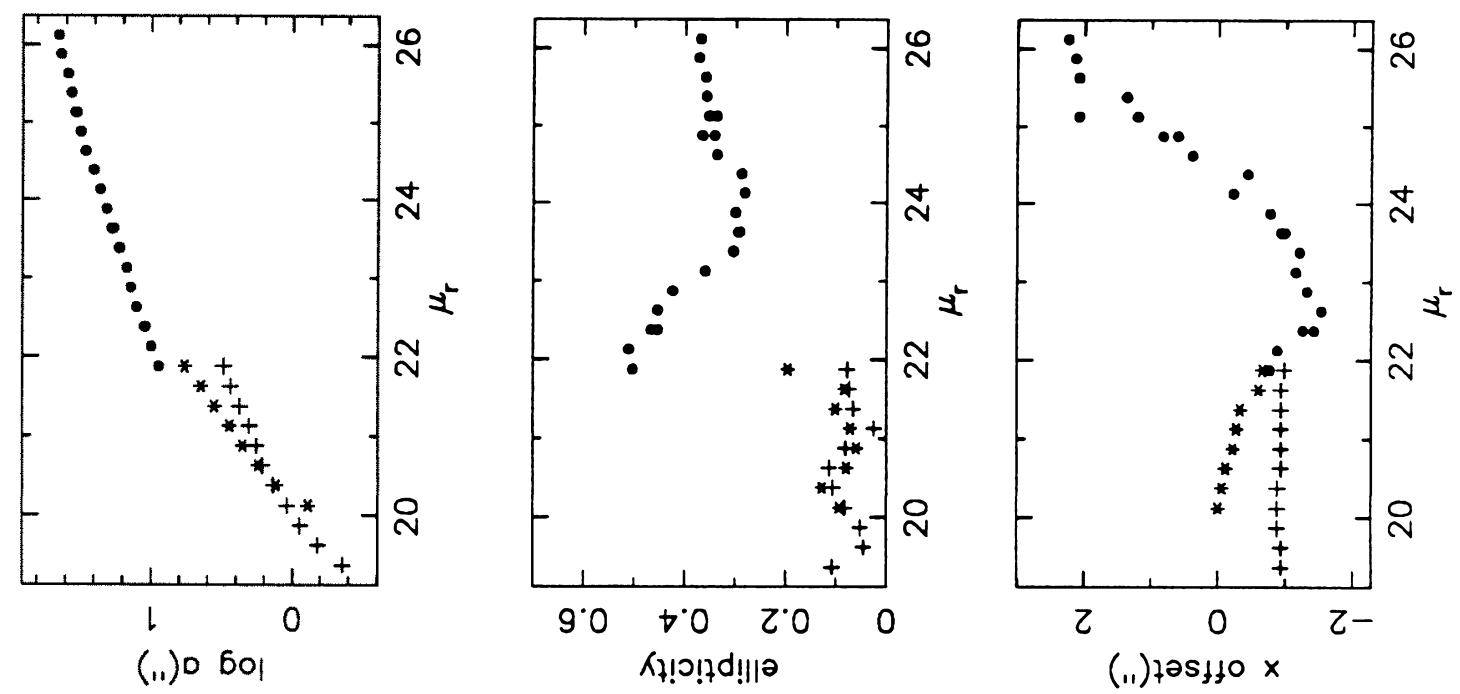


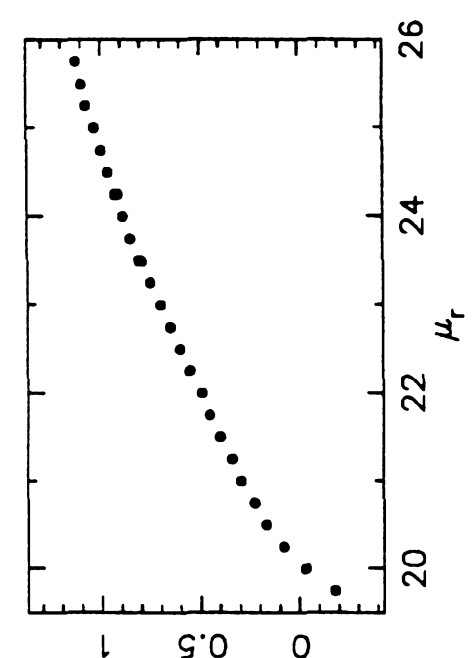

(.1) sn!pos 60।
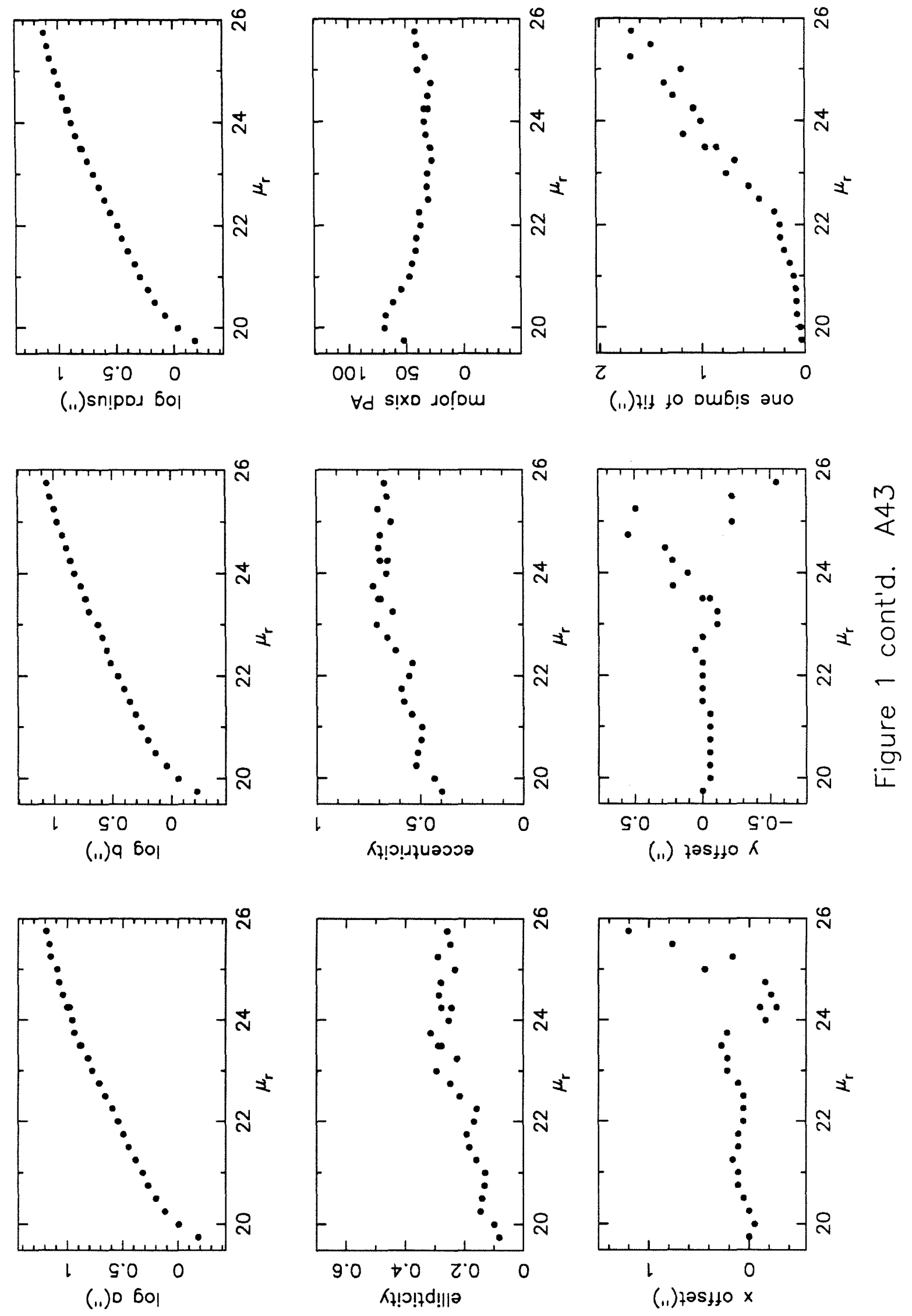

(11) $+!+10$ Dub!s auo

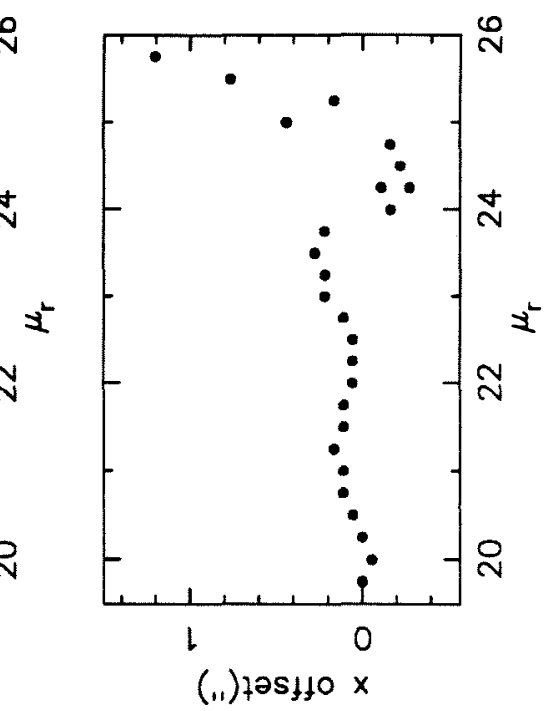



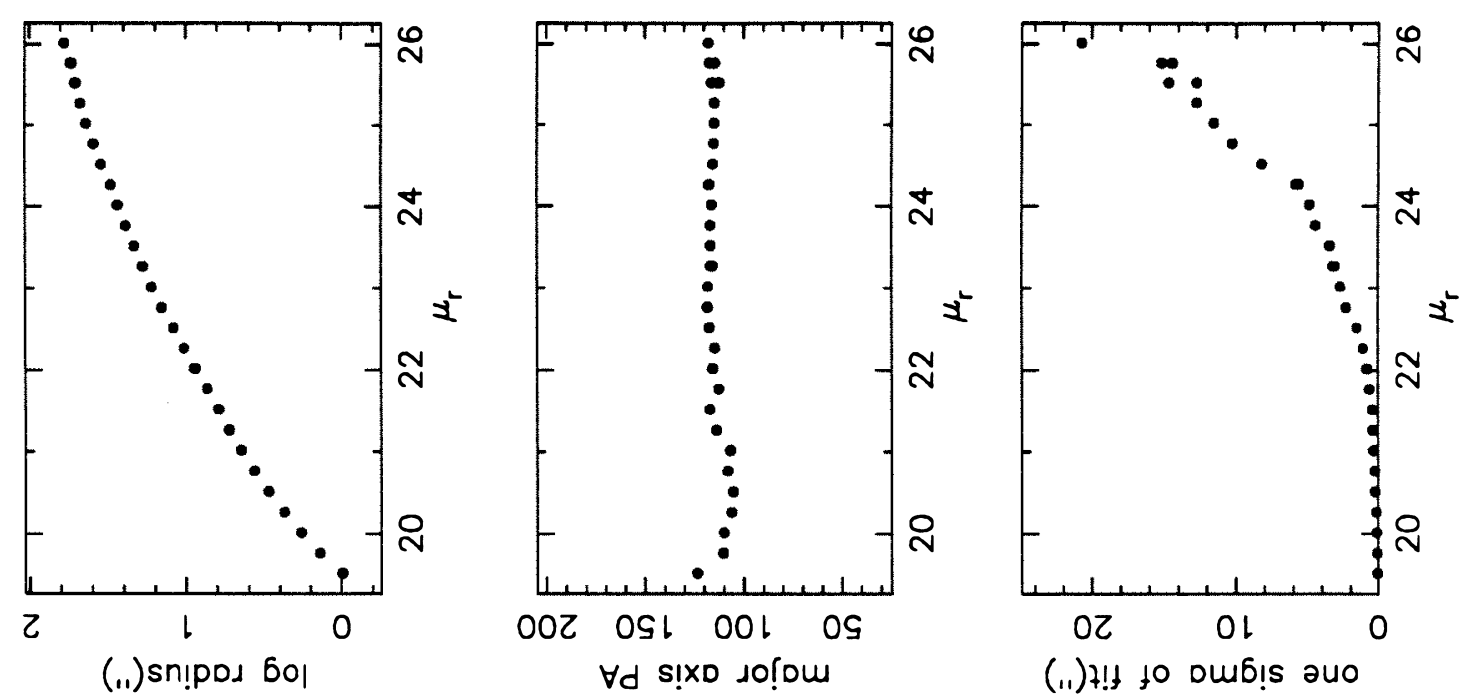

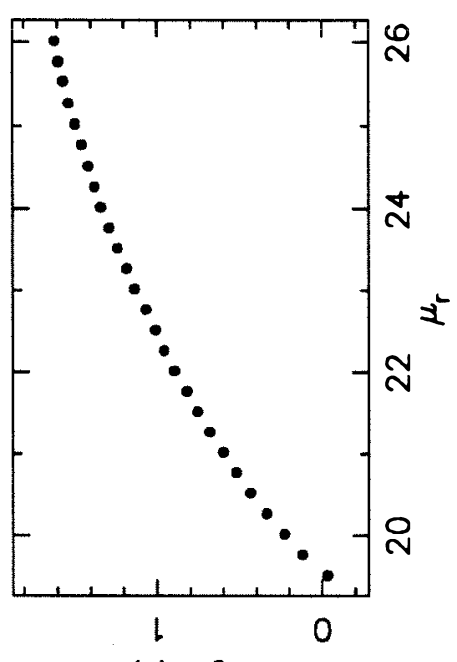

(.1) 9 6ol
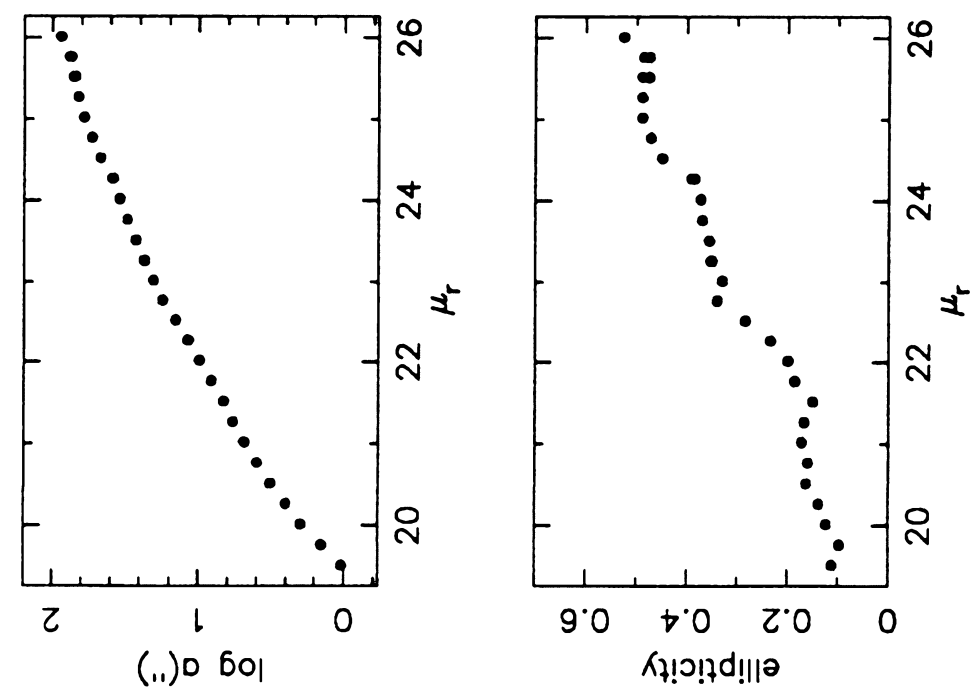

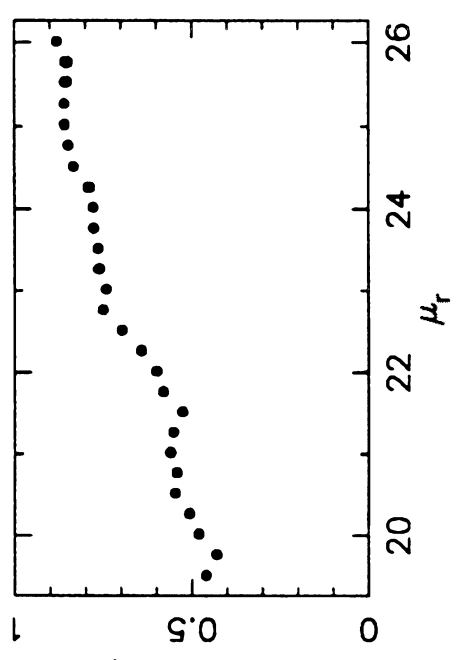

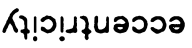

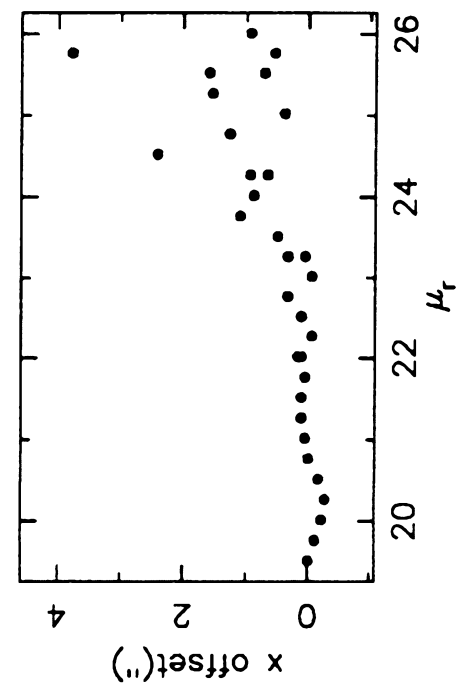

(ii) $725410 \mathrm{~K}$

$\hat{\gamma}$

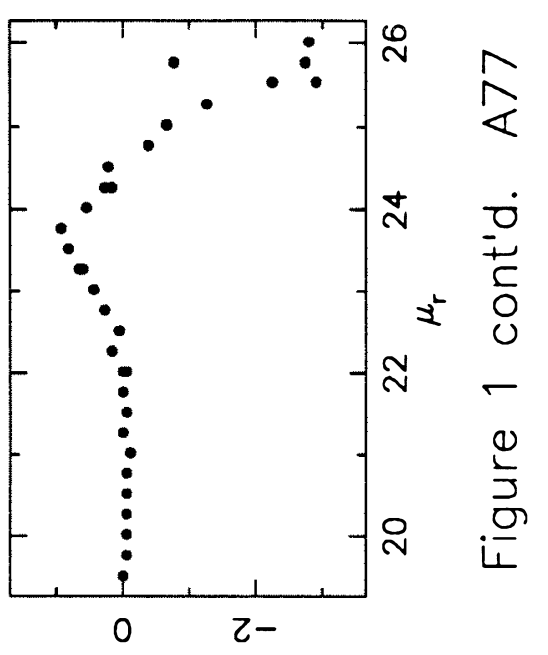




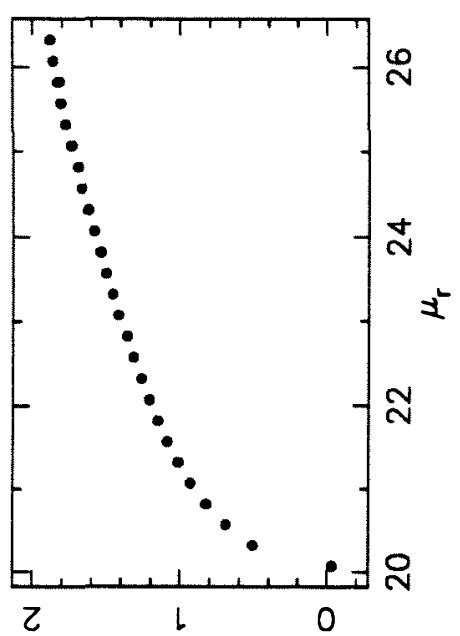

(.1) sn!pod 60।
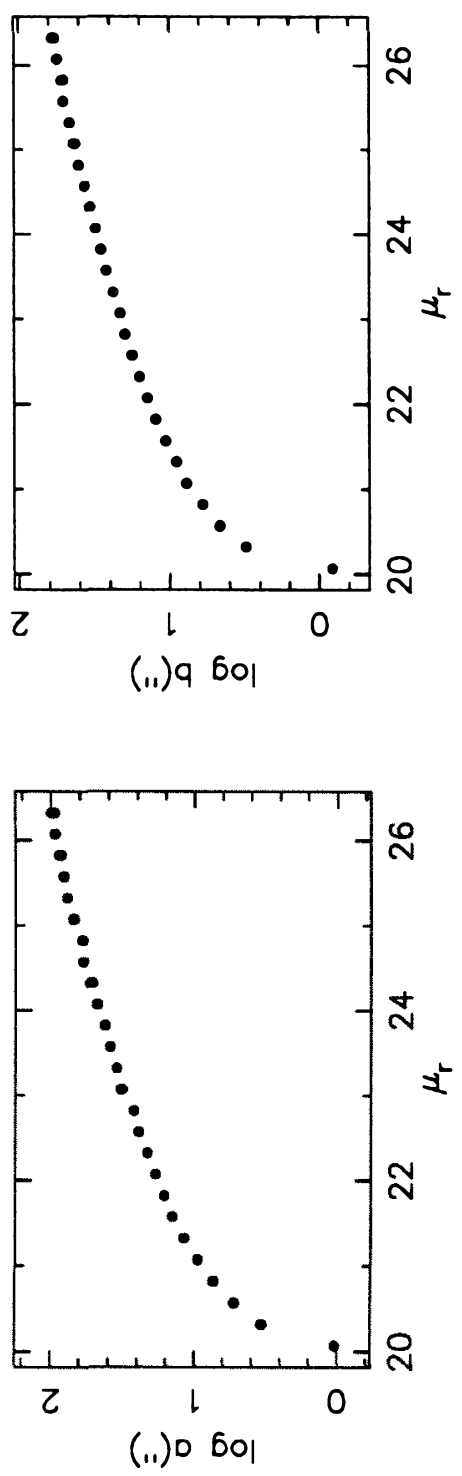

(.1) $\mathrm{D} \mathrm{bo}$

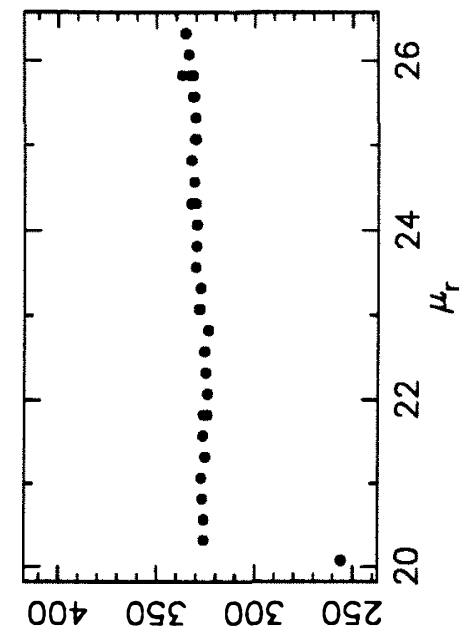

$\forall d$ s!xo دo! om

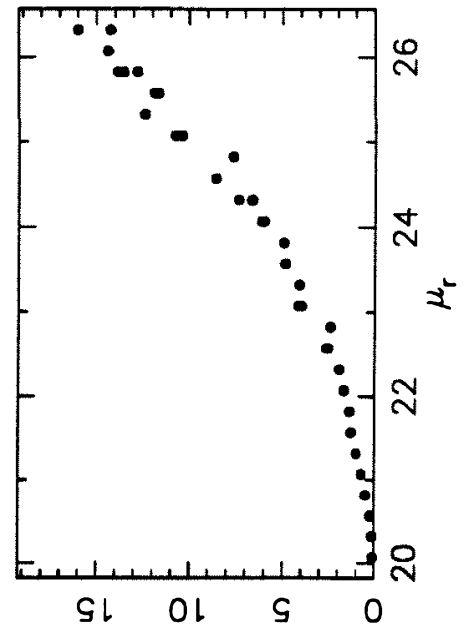

(11) 7 ! t 10 Dub! ano
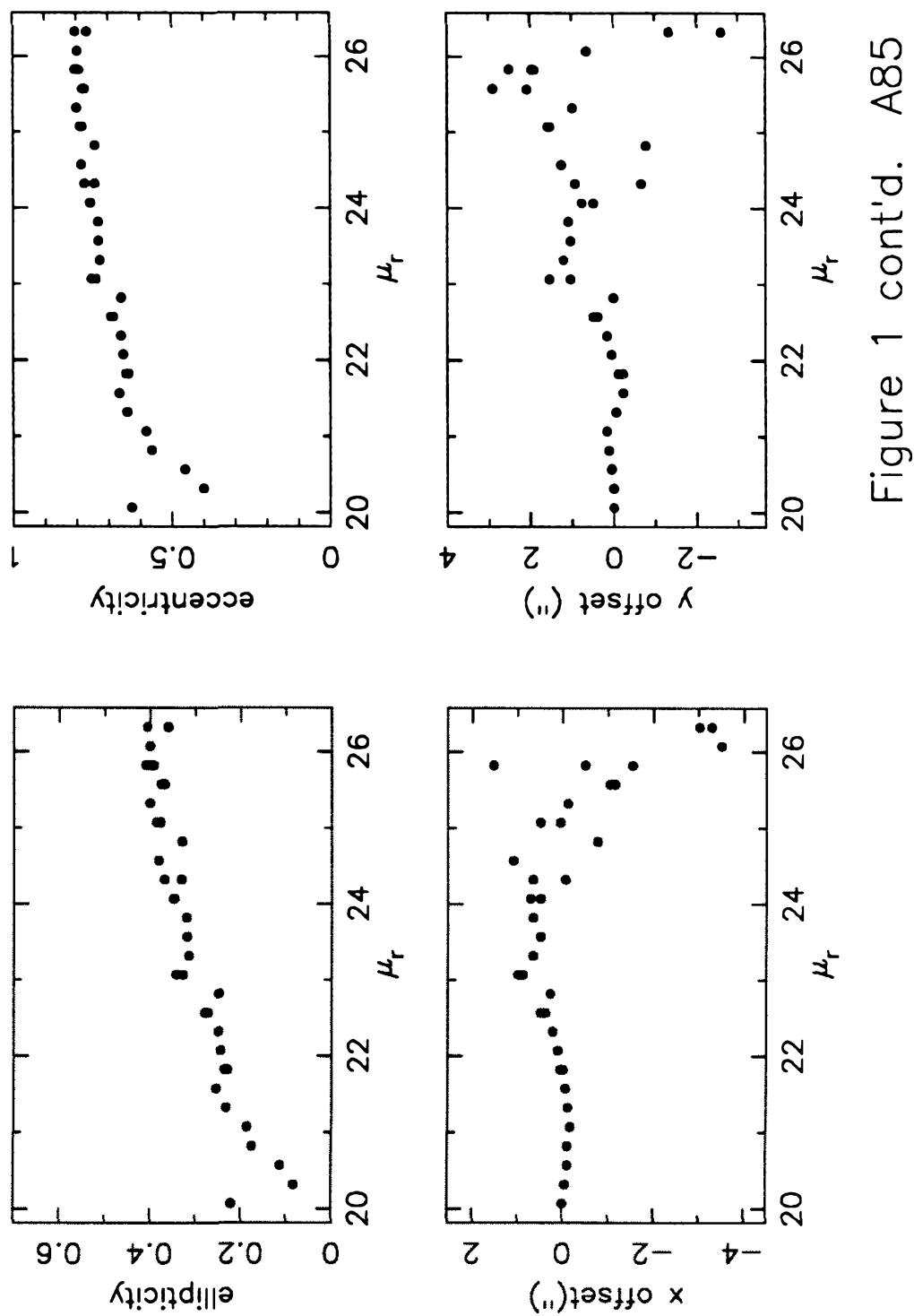


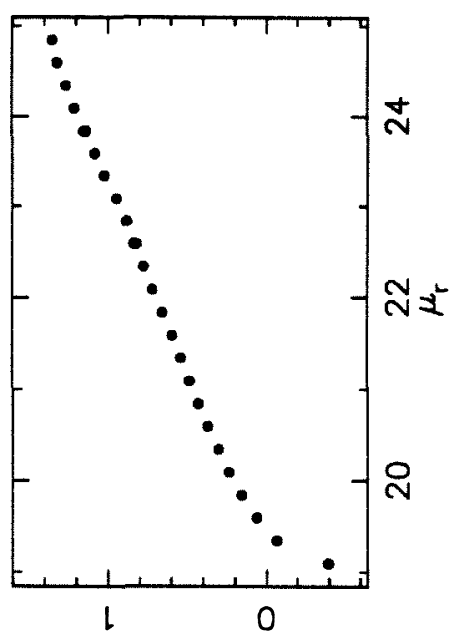

(.1) sn!pod 50

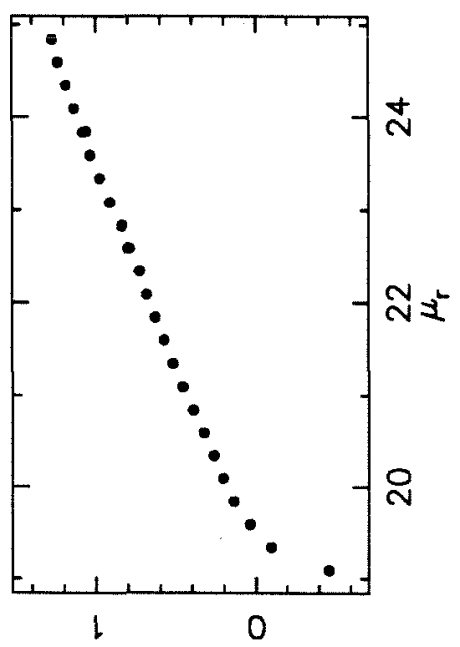

(.1) 960

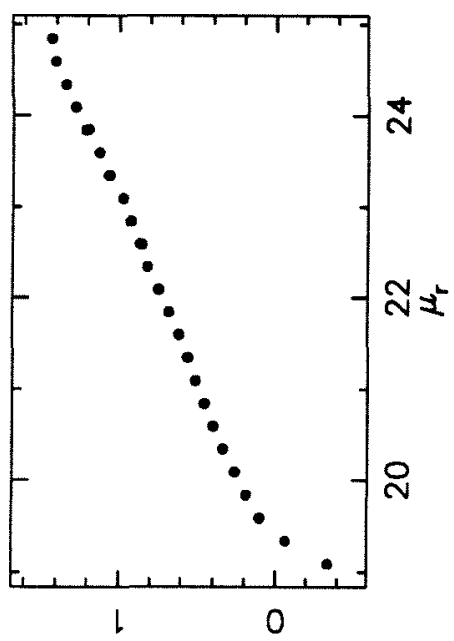

(i.) 0 6ol

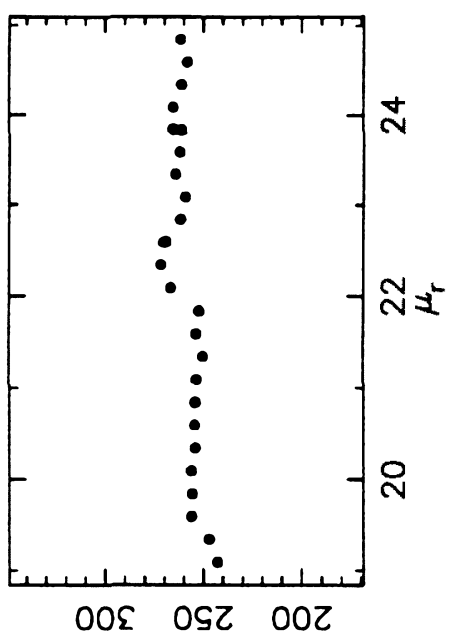

$\forall d$ s!xo دo!̣om

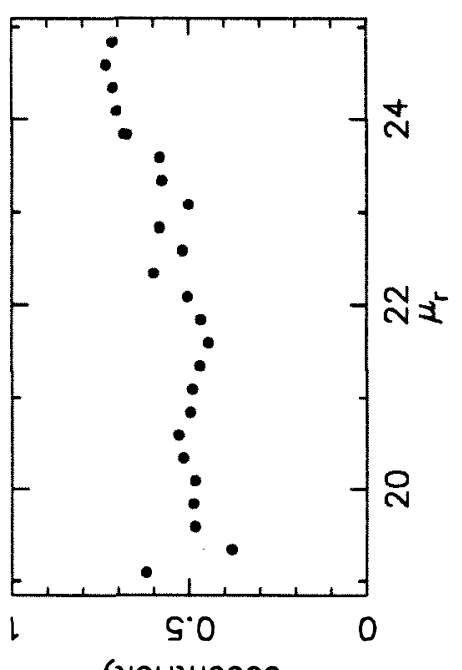

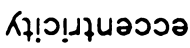

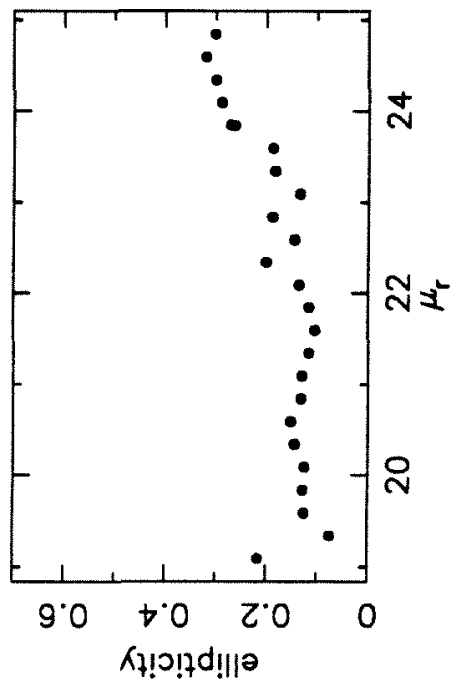

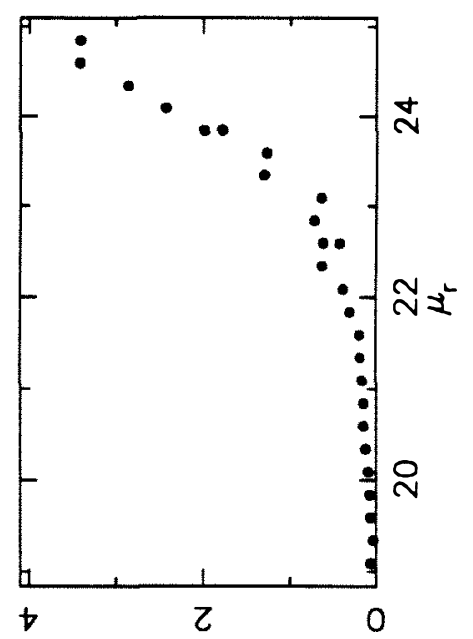

(11) $7 ! t$ to oub!s auo
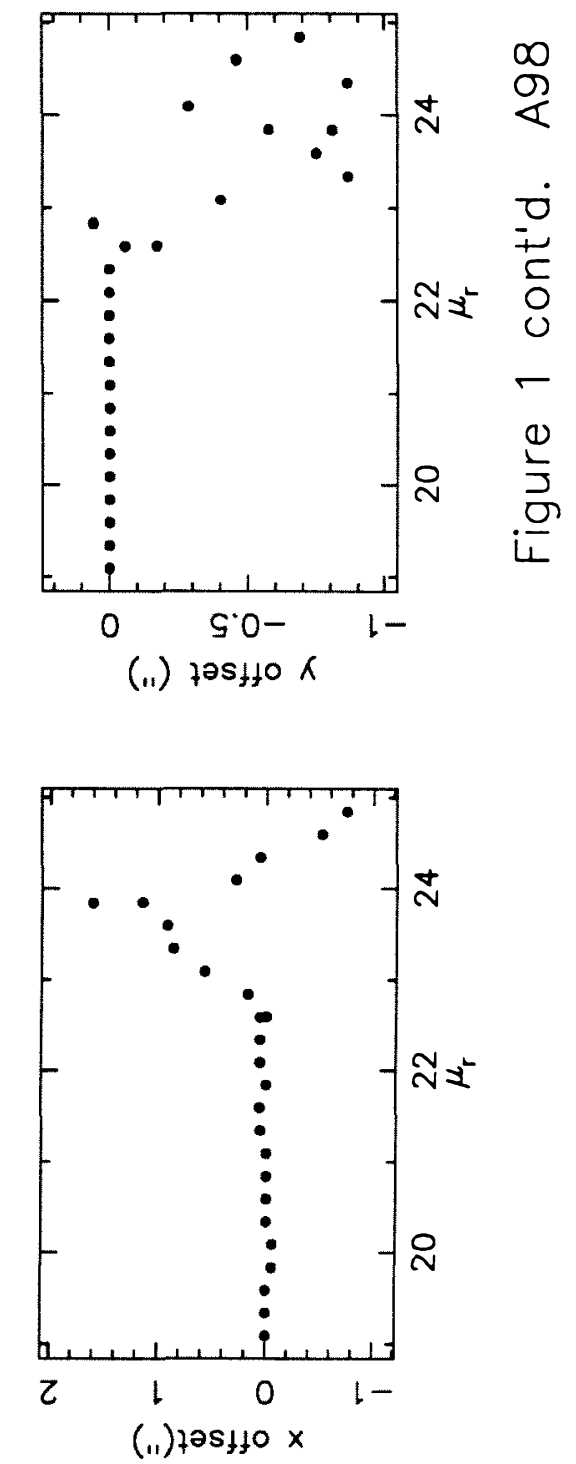


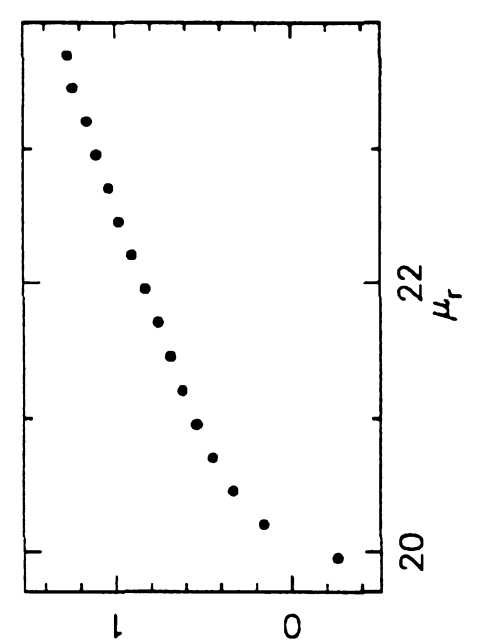

(.1)sn!pos 6ol

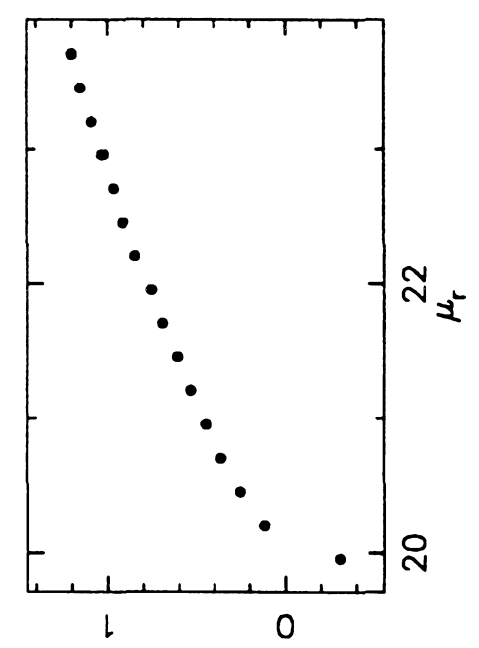

(i1) 960

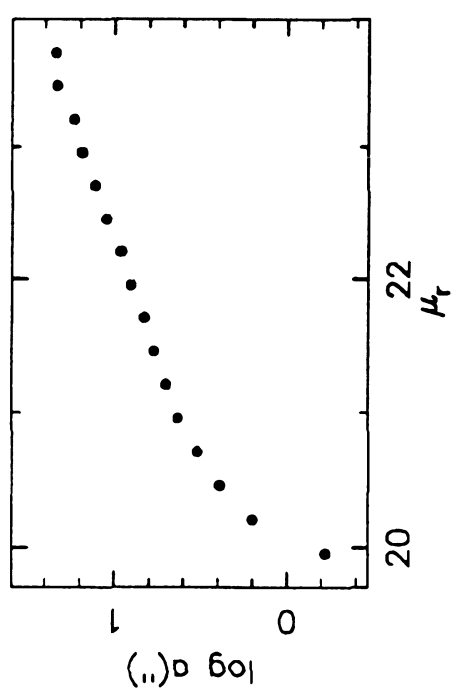

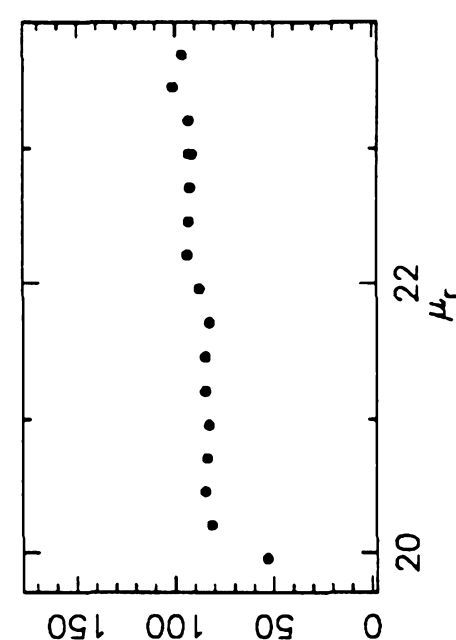

$\forall d$ s!xo so!̣u
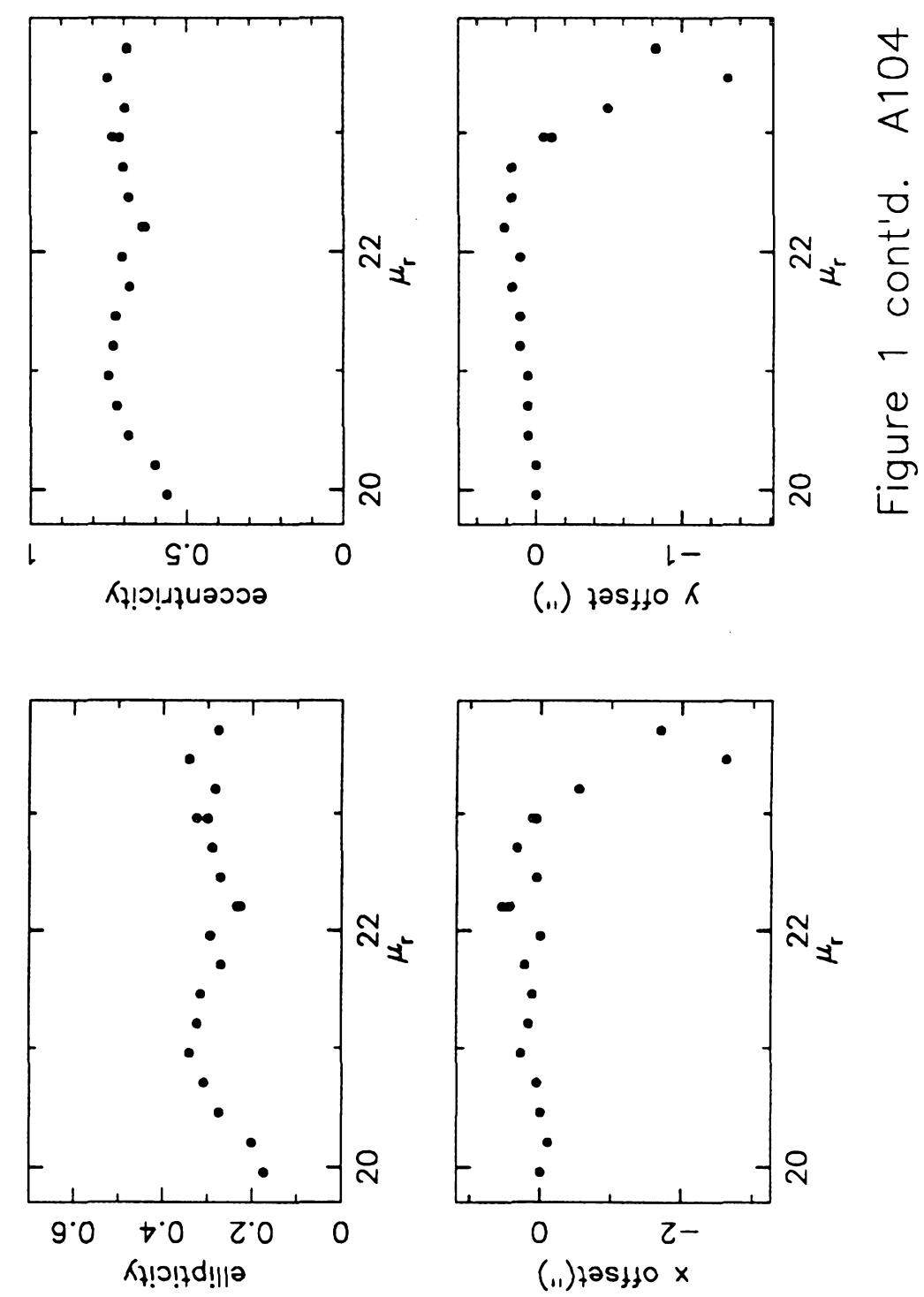

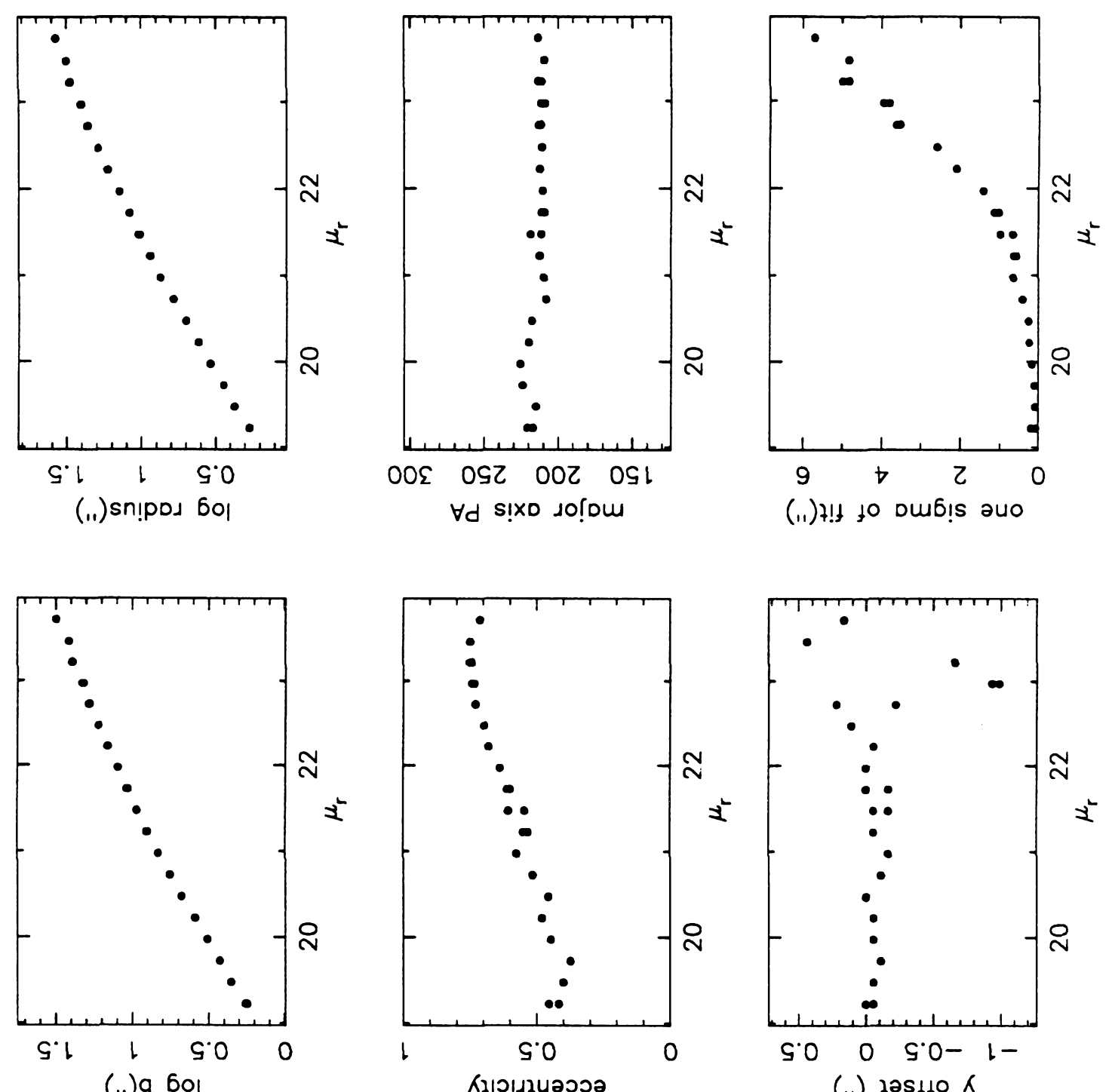

र์!ว!นาบวัวа

(11) $7 !$ t to oub!s auo
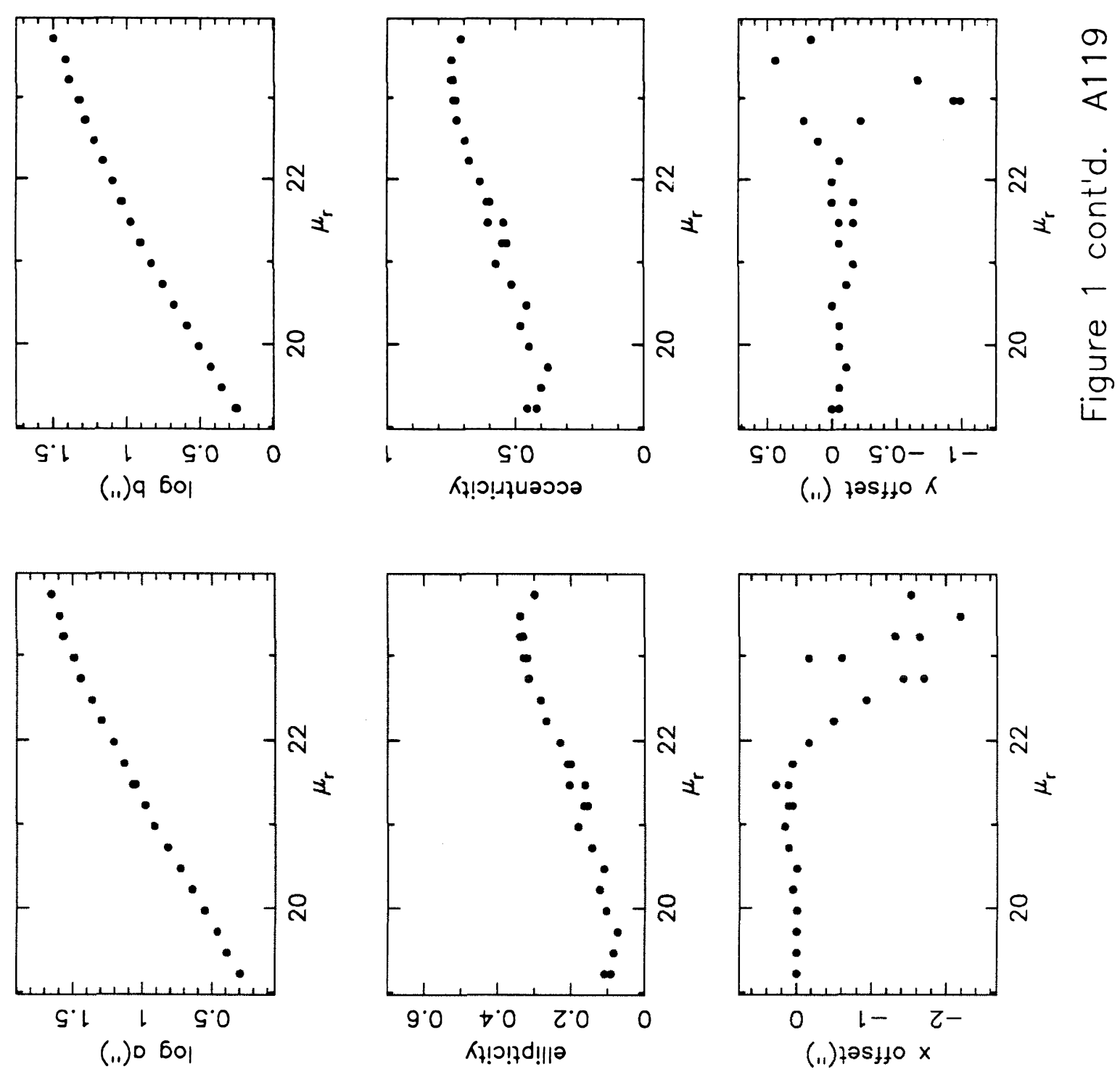

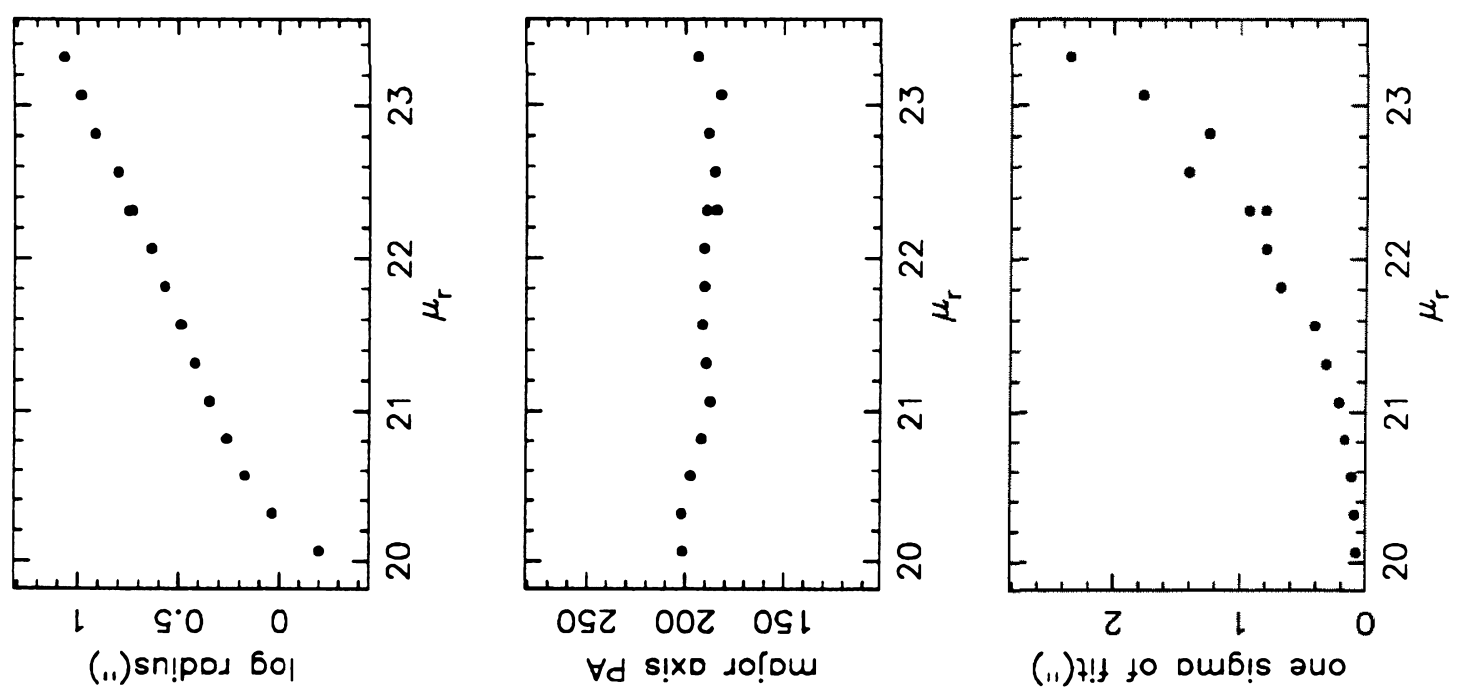

(i1) $+! t$ to oub!s auo

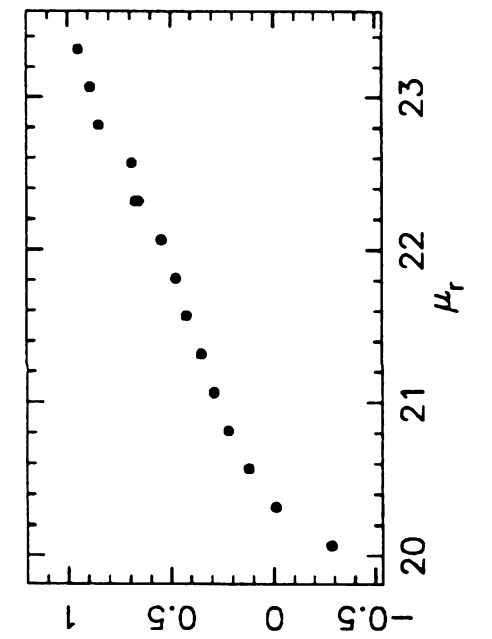

(.1) 960
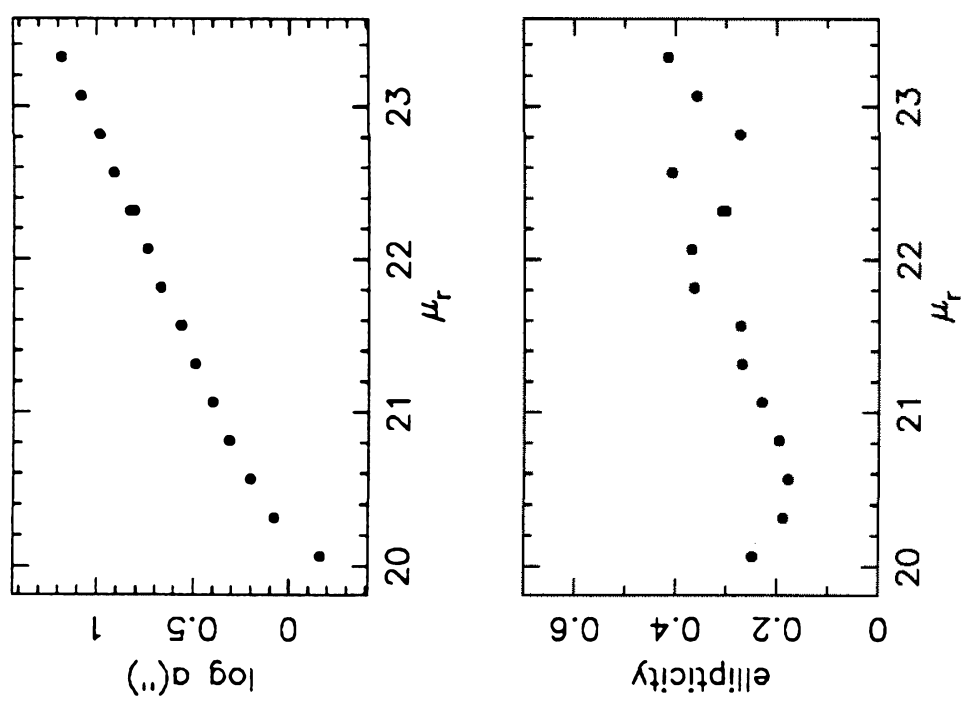

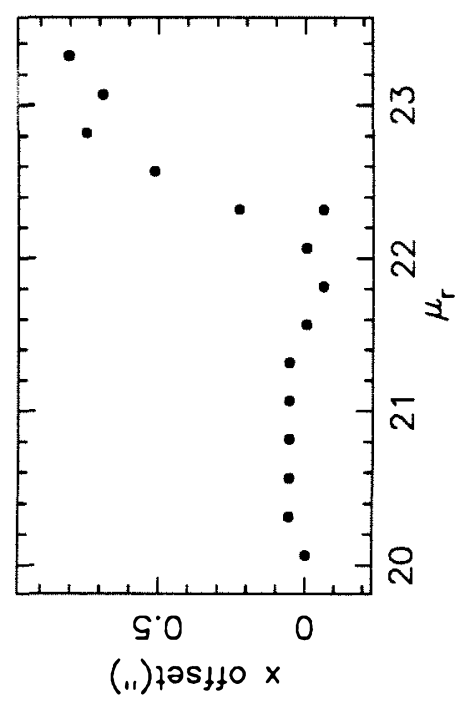



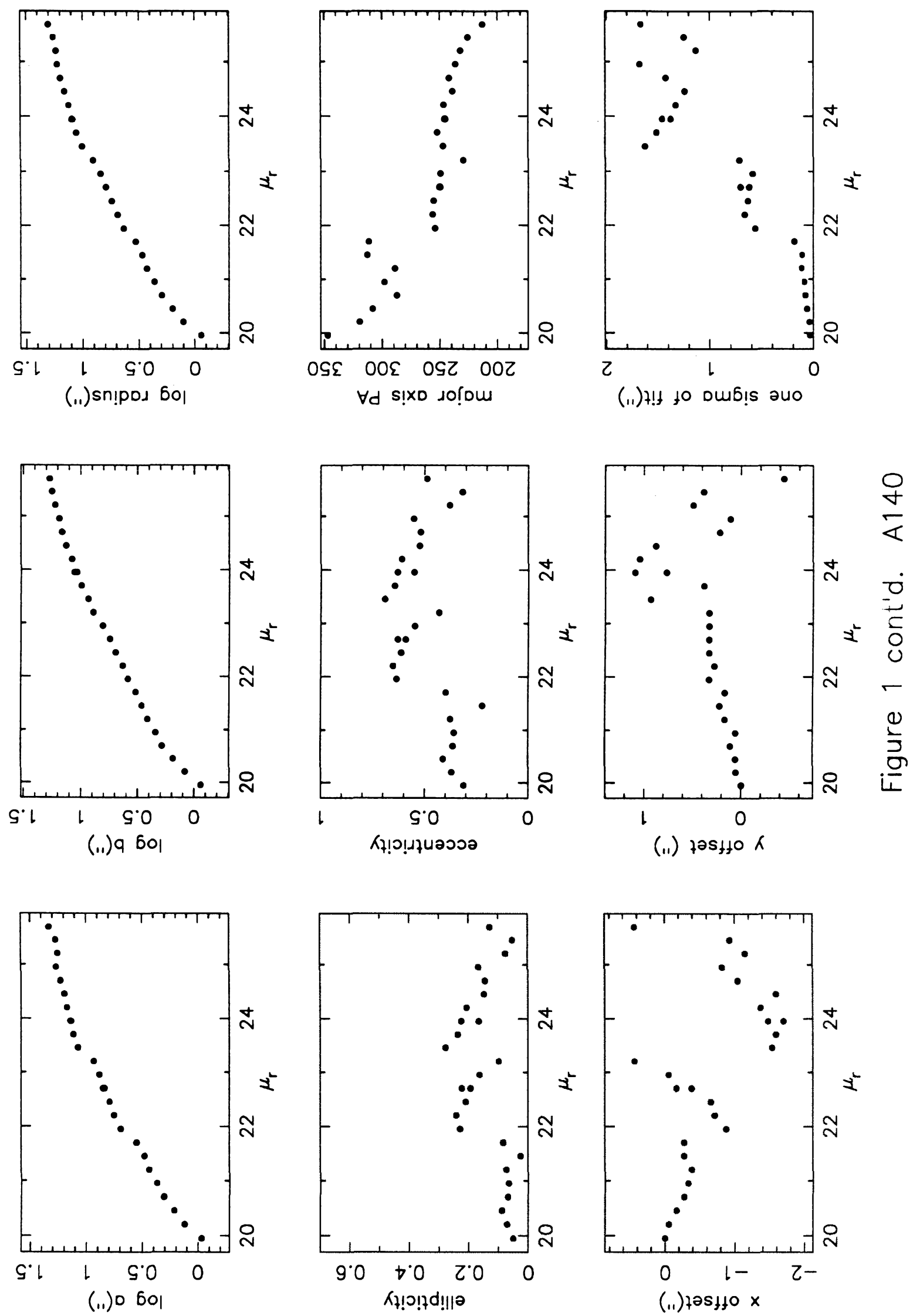


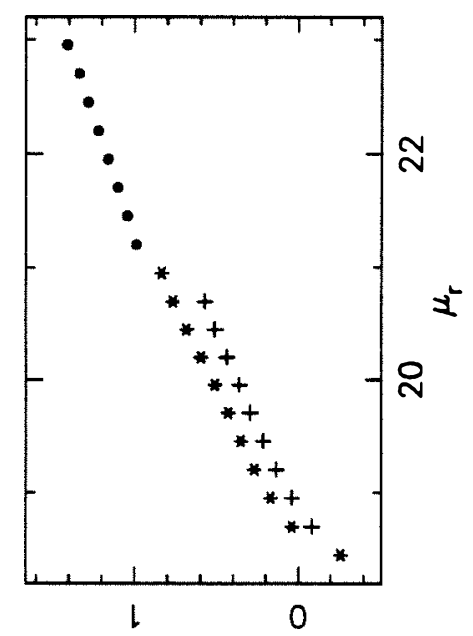

(.1)sn!pos 6ol

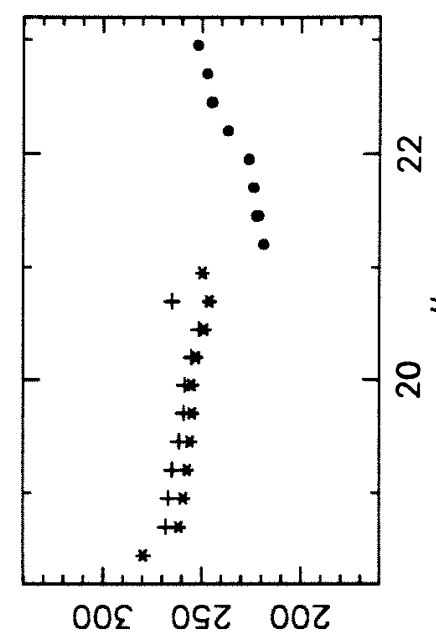

$\forall d$ s!xo so!om

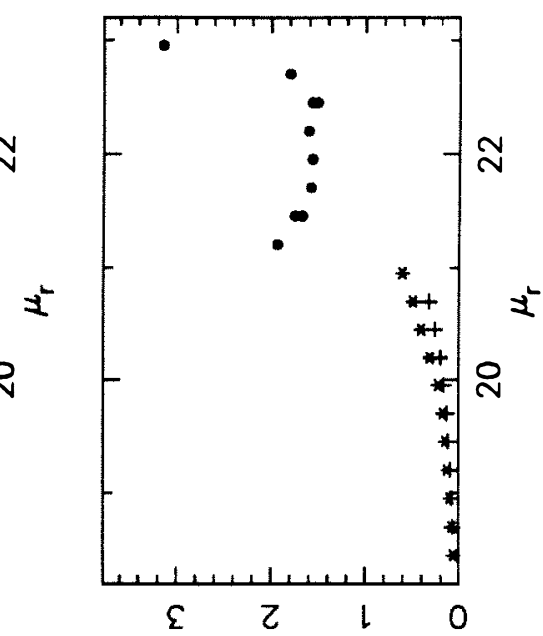

(11) 4 !t 10 oub!s əuo

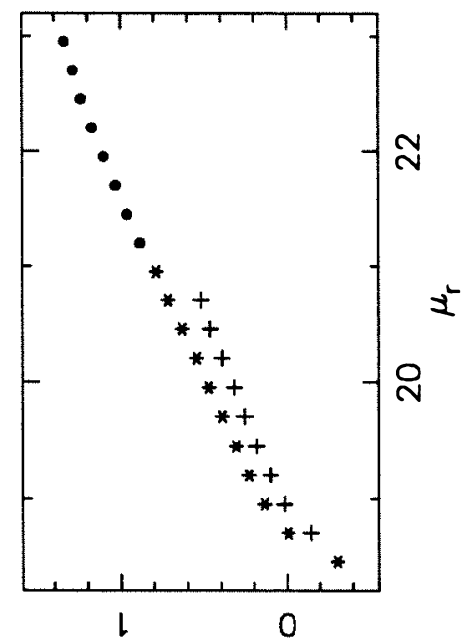

(.1) 9 6ol

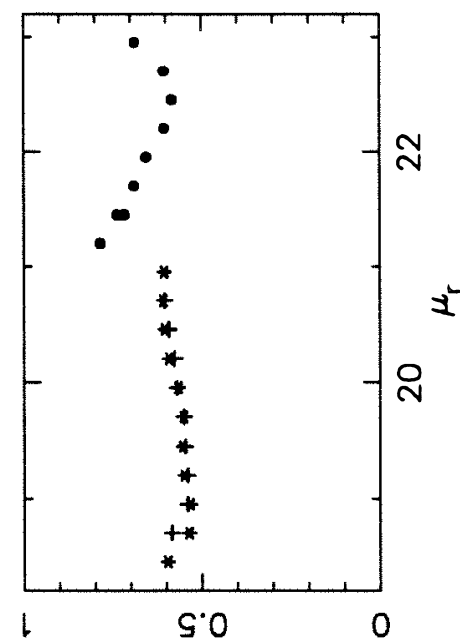

Кұ!ว!นในวววа

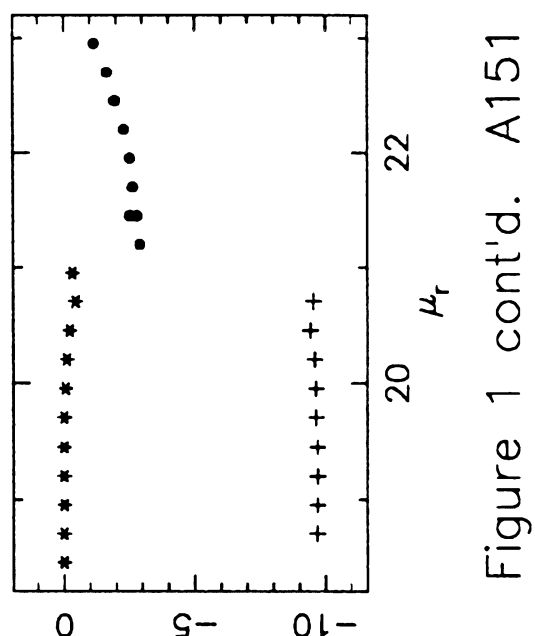

(ii) zas $150 \mathrm{~K}$

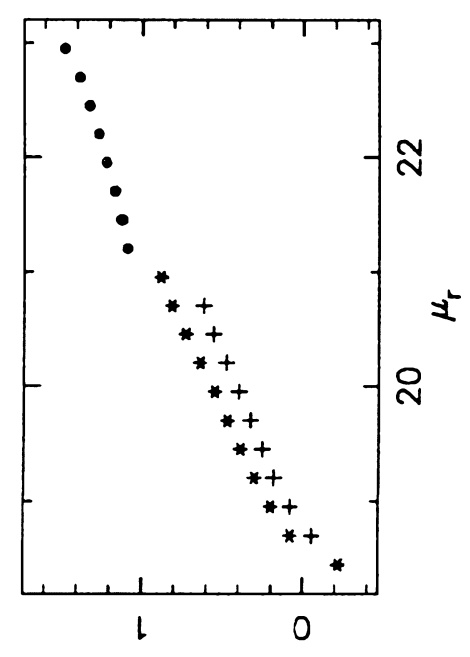

(..) 0 6ol
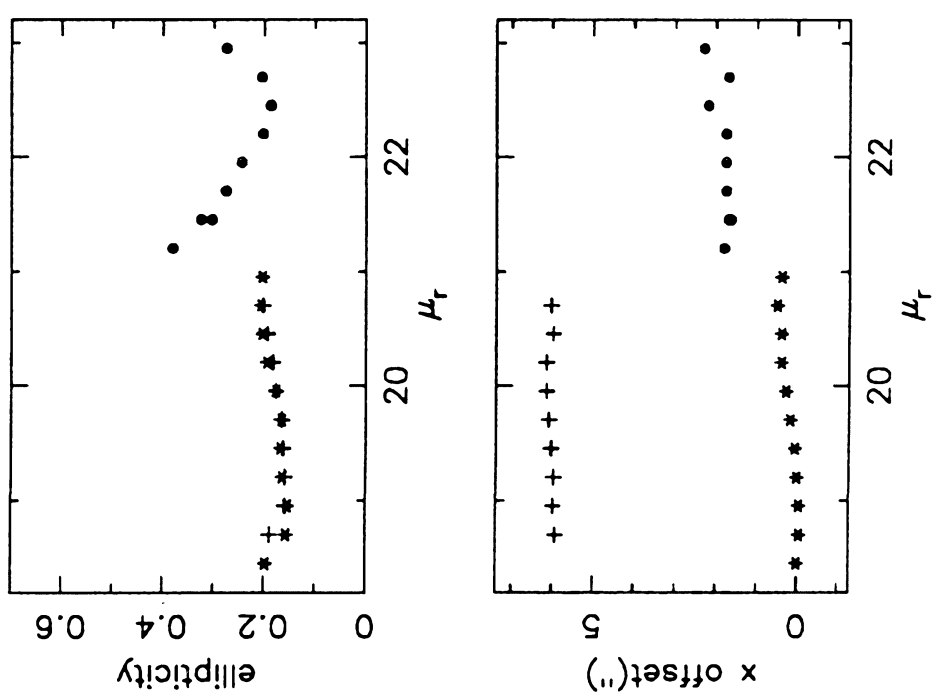
$-268-$
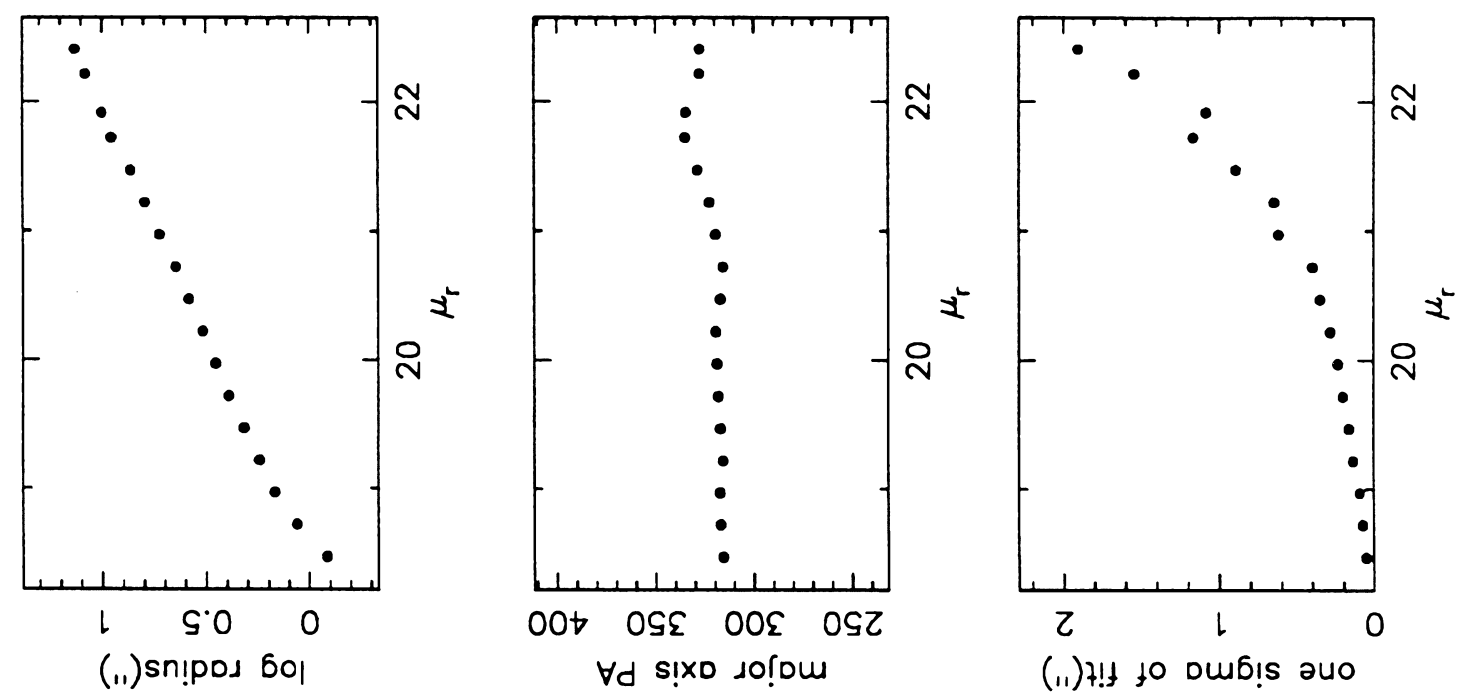

(11) $7 !+$ to oub!s auo

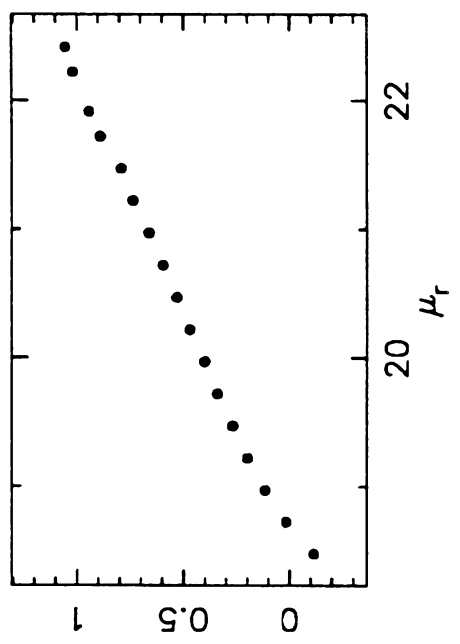

(.1) 9601

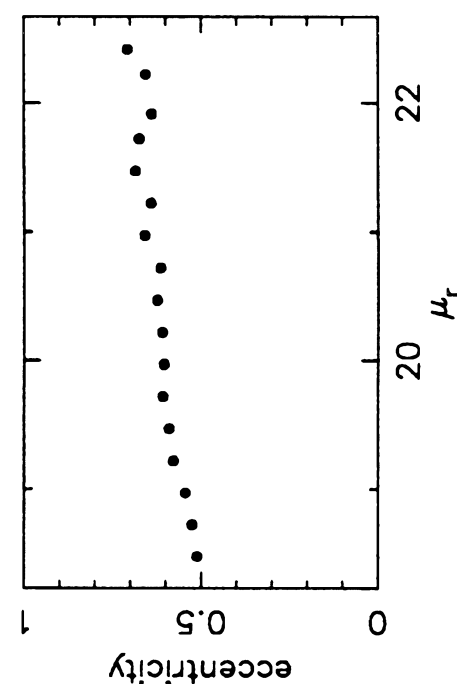

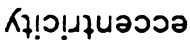
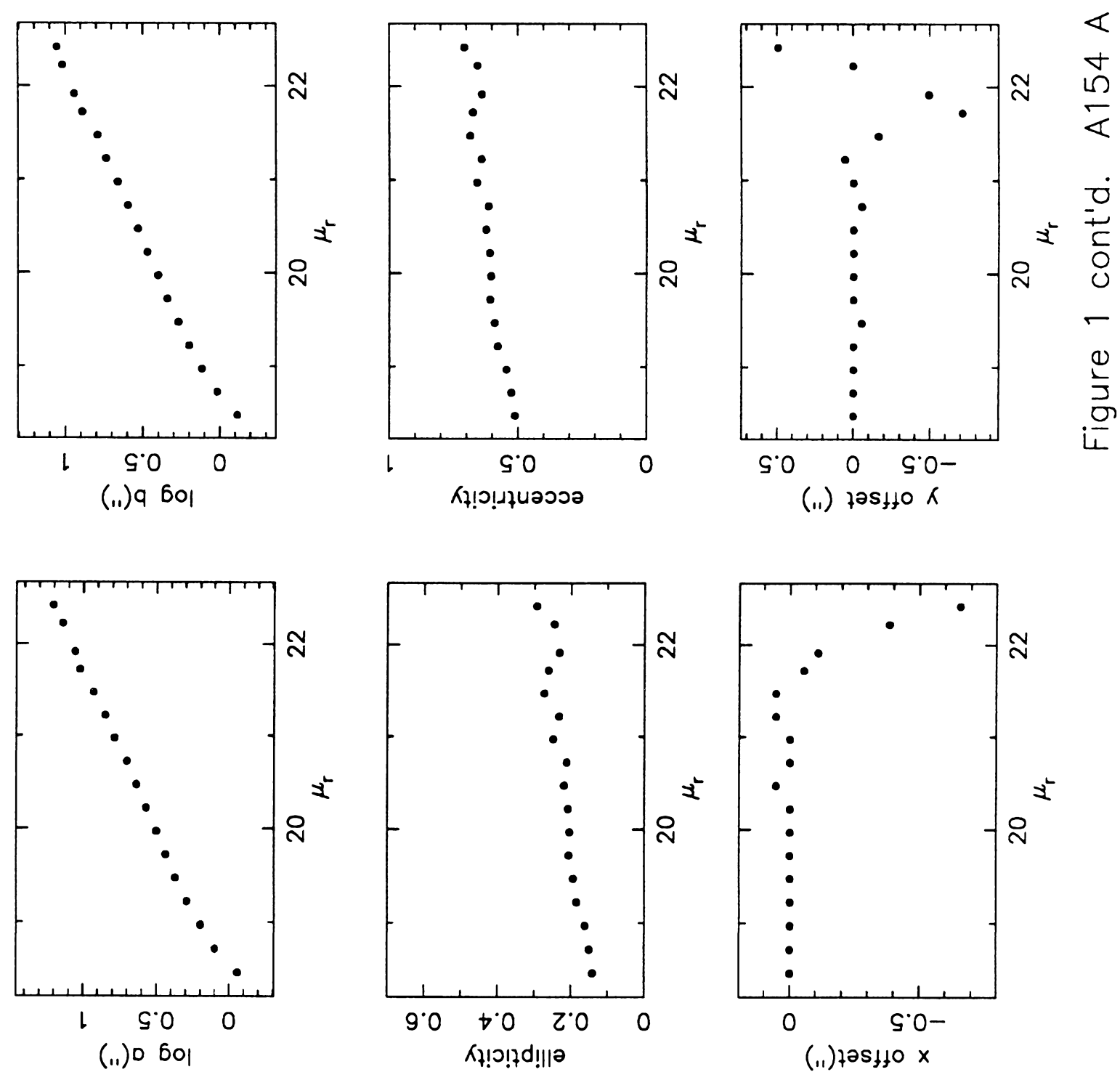

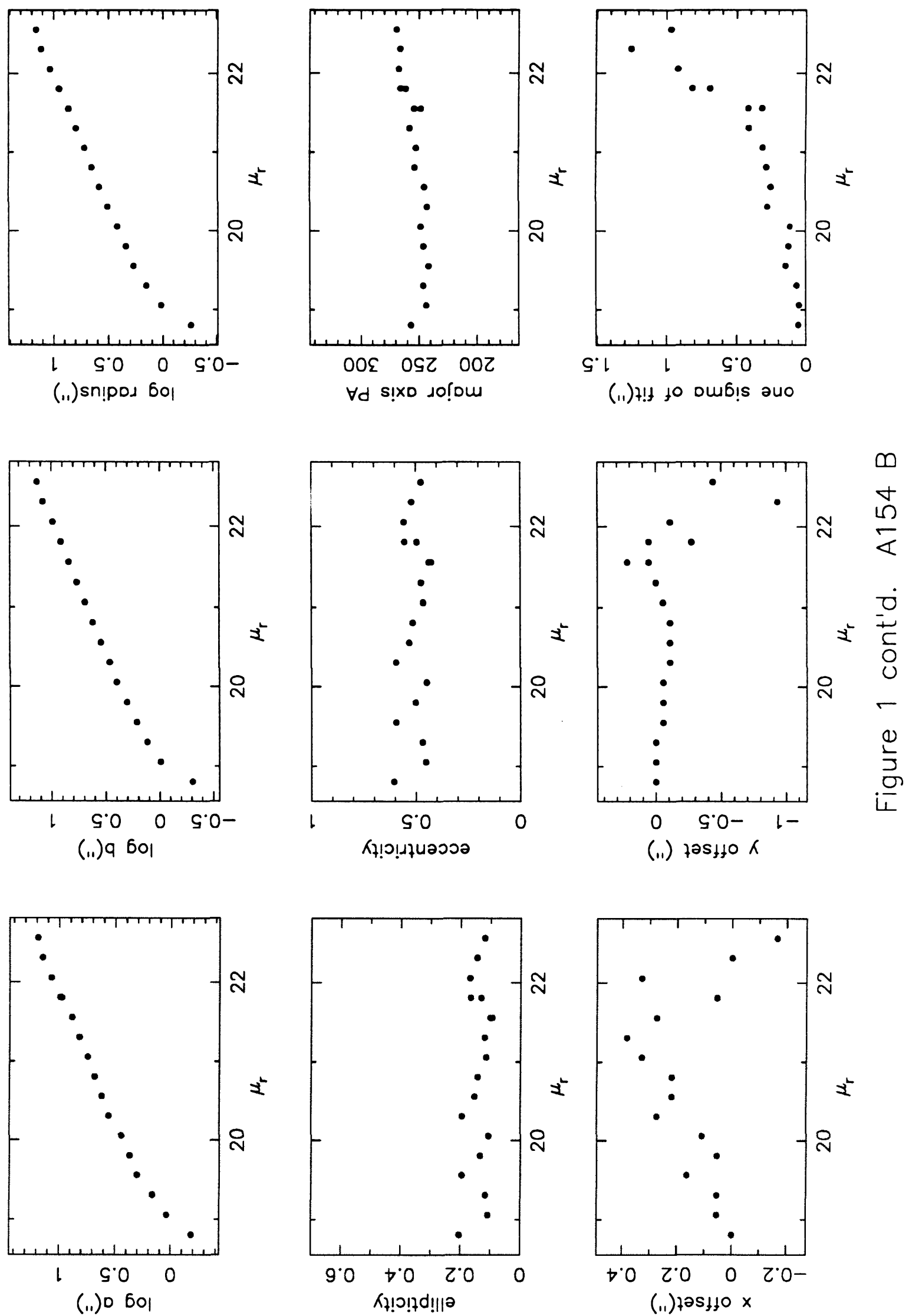


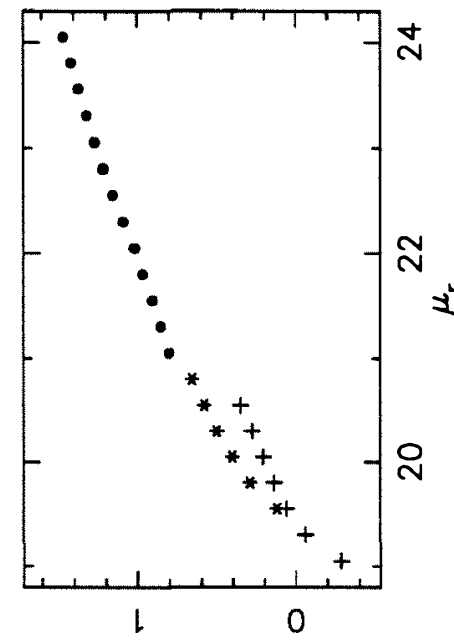

(.1) sn!pod 601
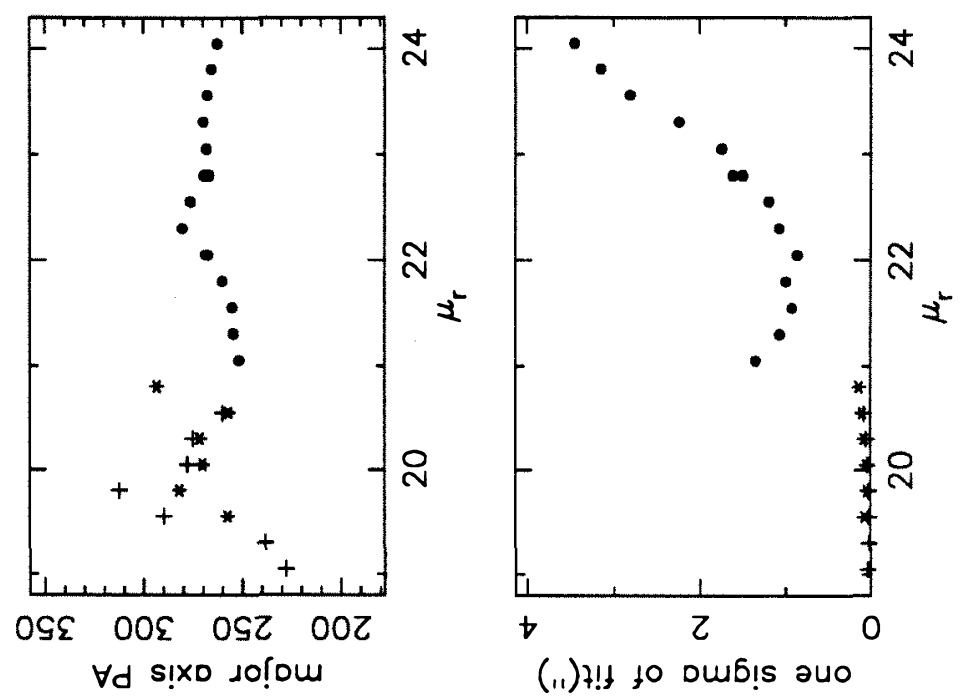

(11) $+!+$ to Dub!s auo
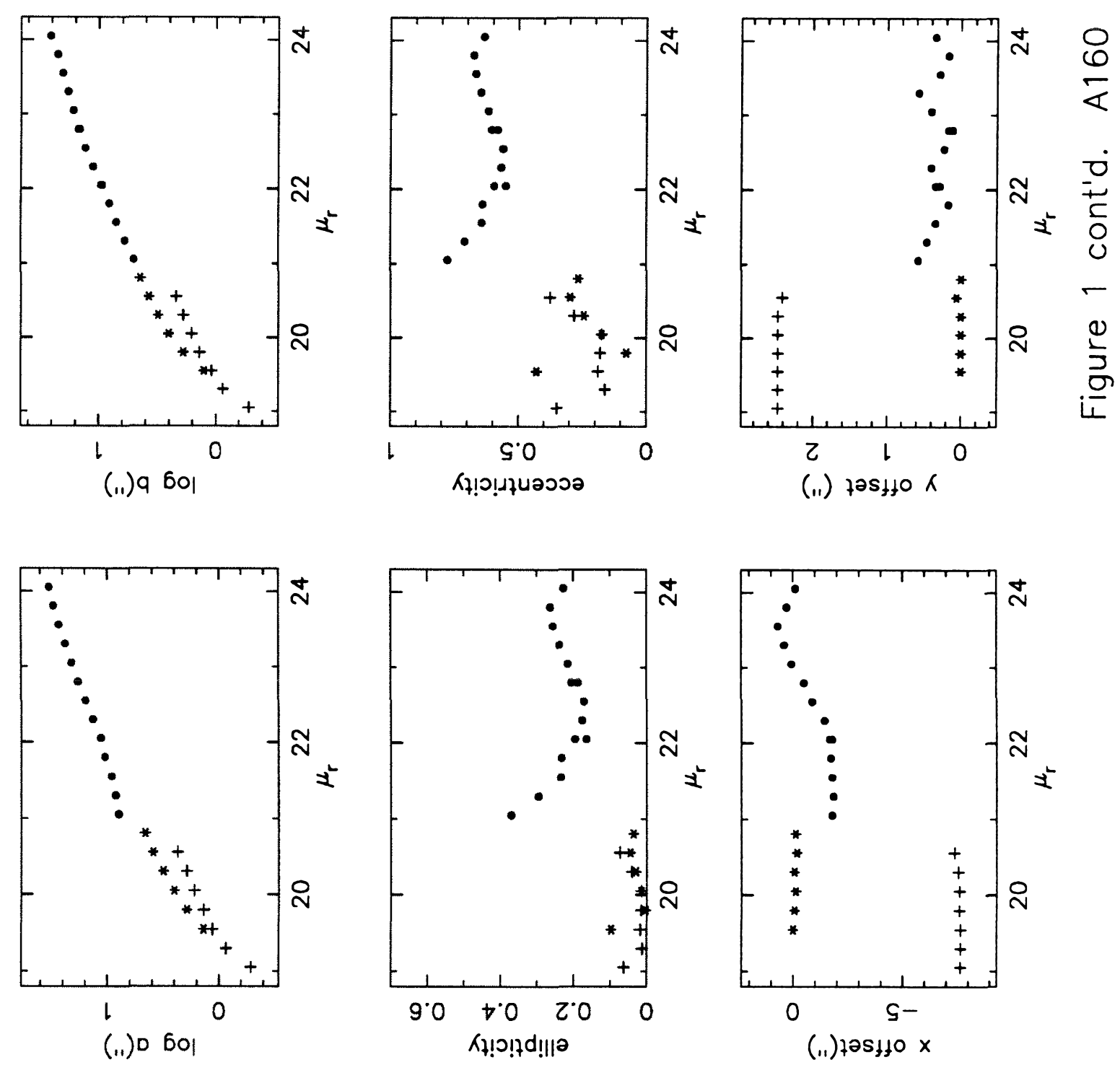

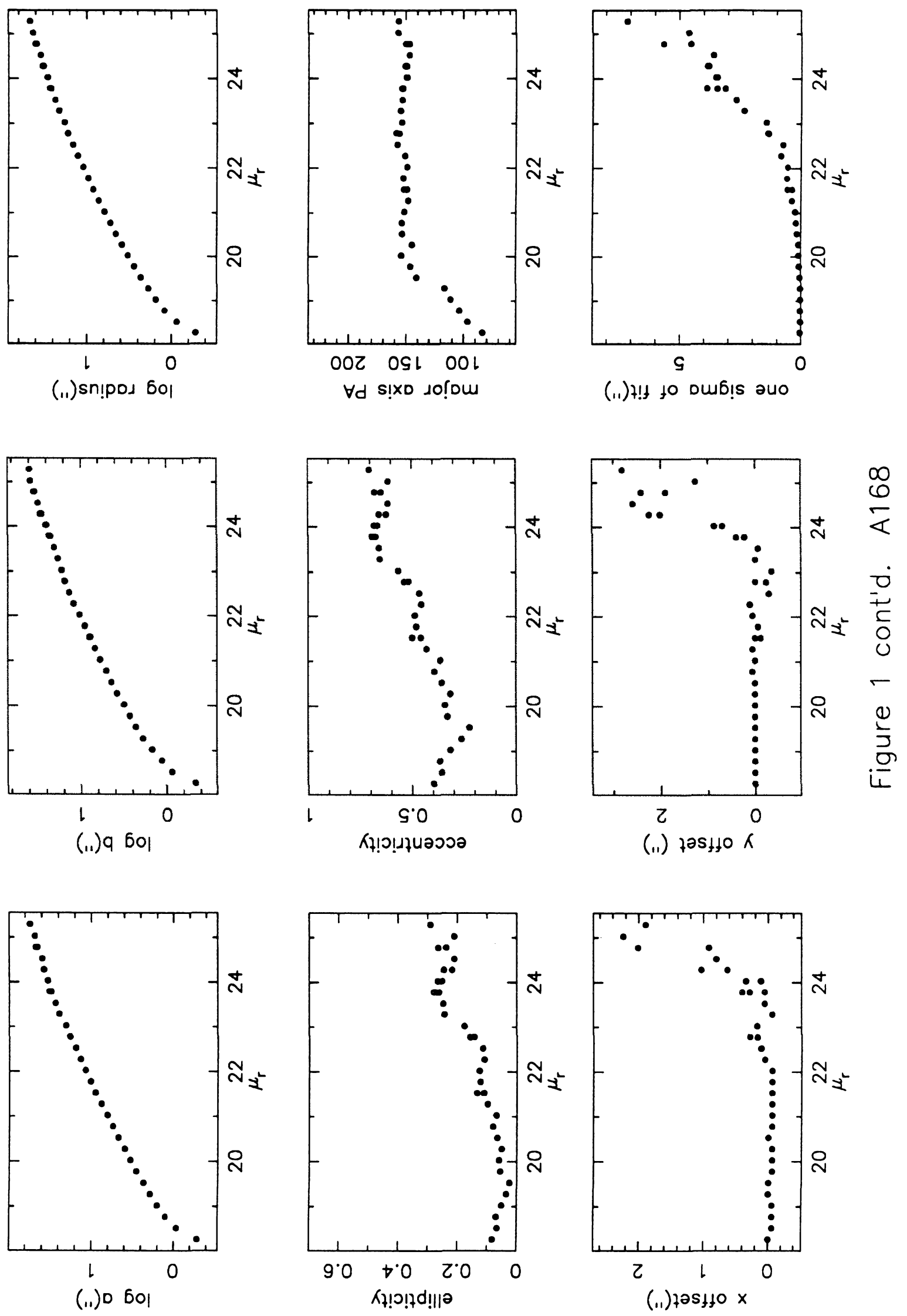


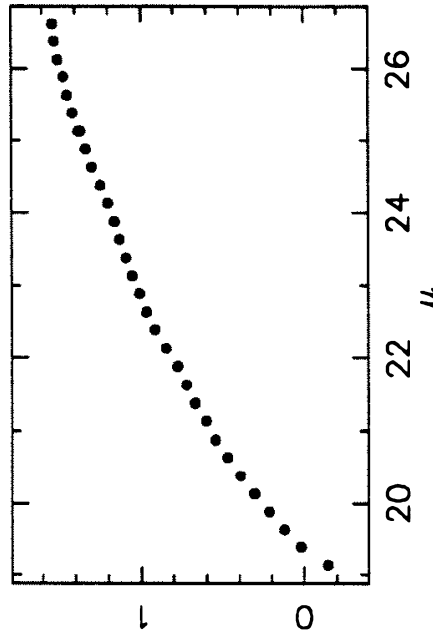

(..)sn!pos 6ol

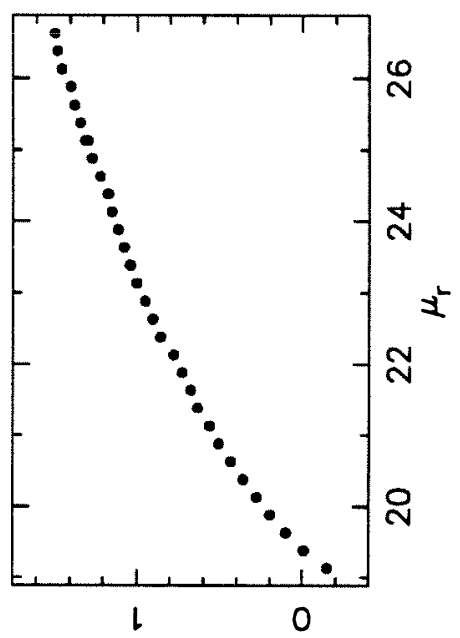

(.1) 9 bol

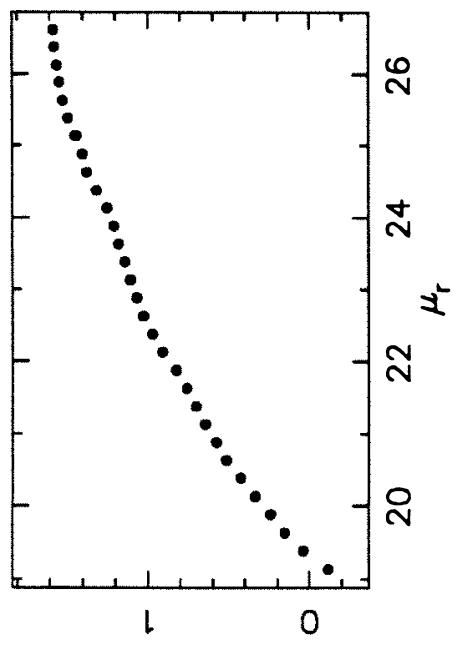

(.1) 0 bol

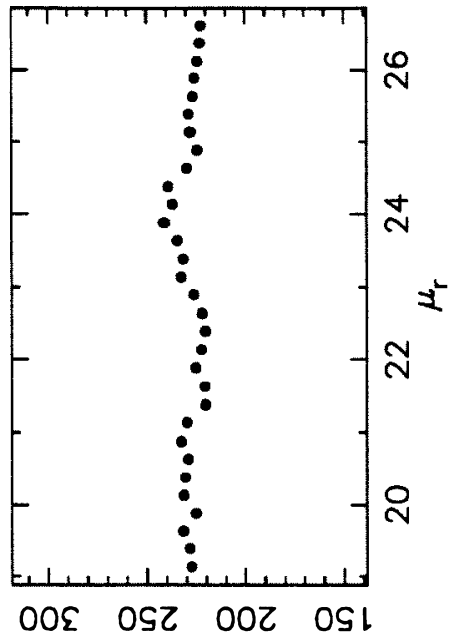

$\forall d$ s!xo دo!̣u

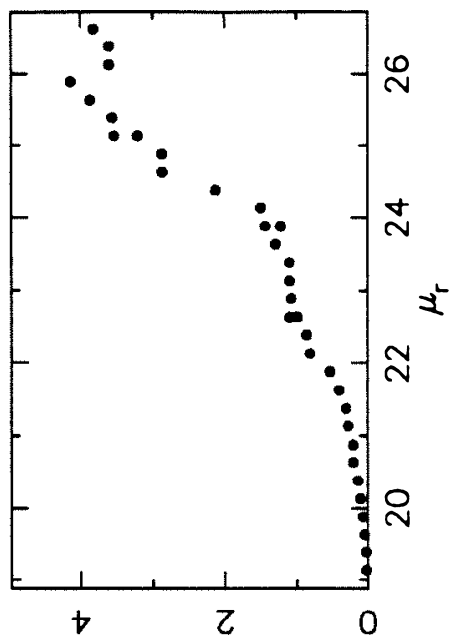

(.1) $+! 1$ to oub!!s au०

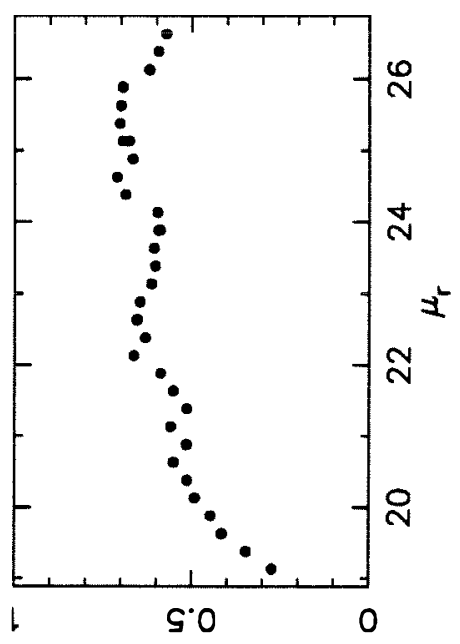

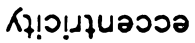

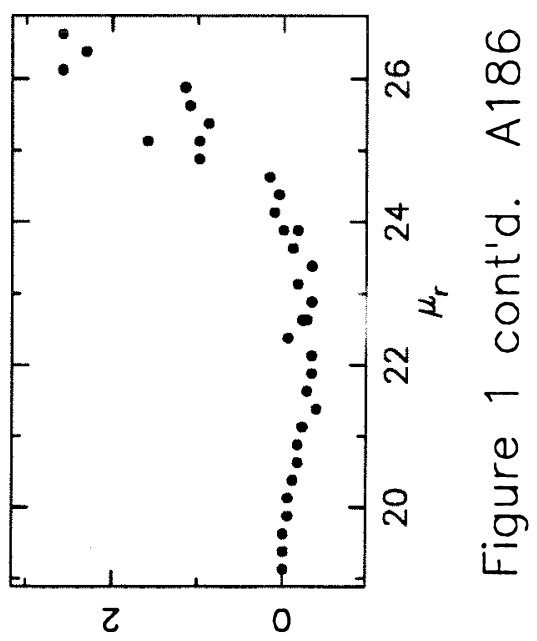

(11) $705 \% 10 \mathrm{~K}$

$$
\begin{aligned}
& 0 \\
& 0 \\
& \frac{0}{1} \\
& 0 \\
& 0 \\
& 0 \\
& 0 \\
& 0 \\
& \frac{1}{3} \\
& \frac{9}{2}
\end{aligned}
$$

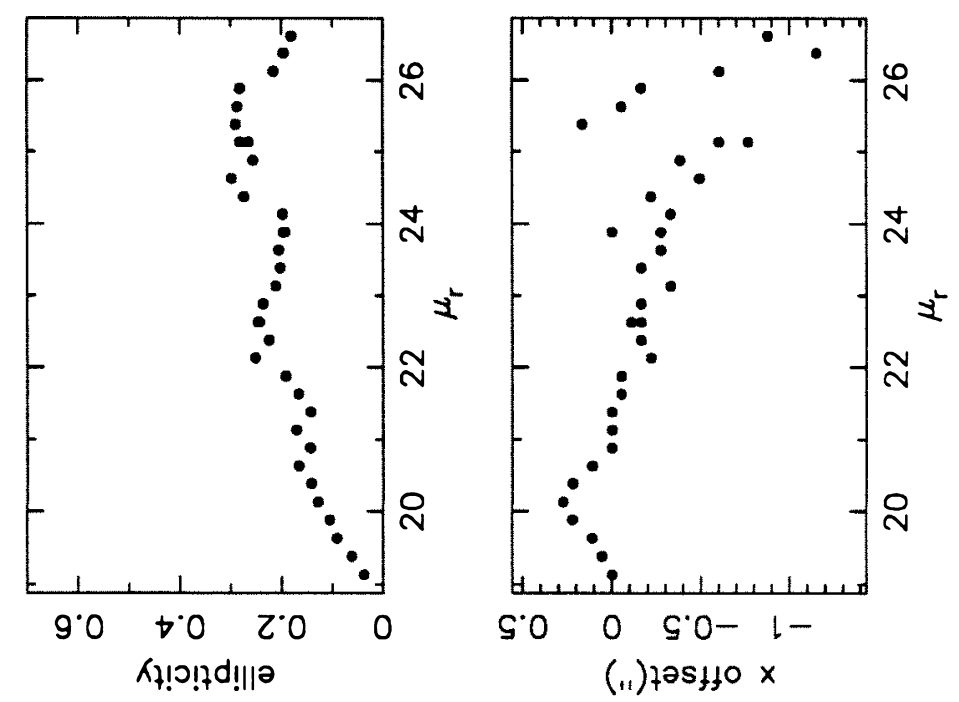




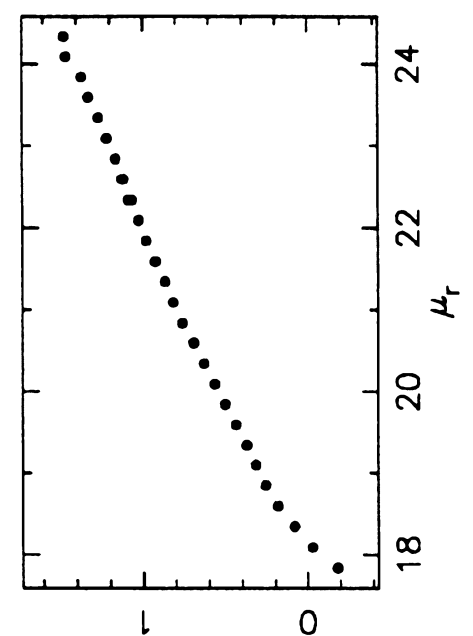

(.1) sn!pod 601

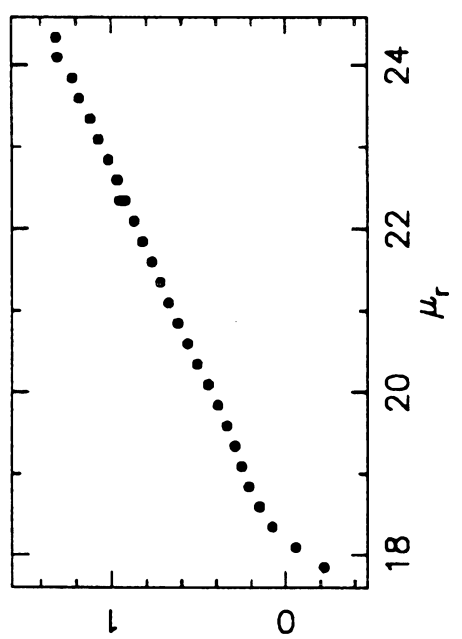

(.1) 9 bol

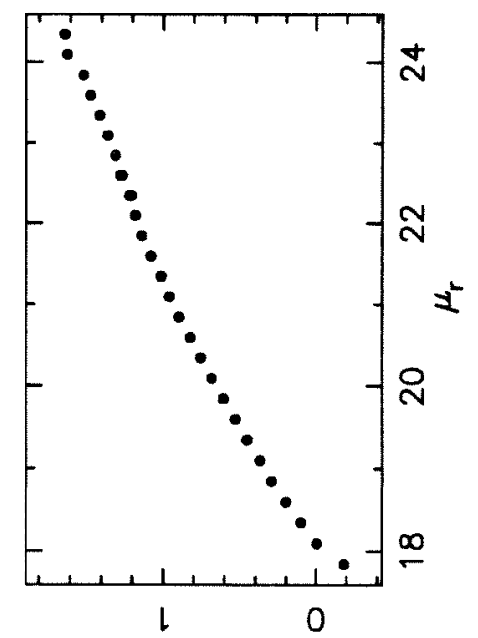

(.1) 0 6ol
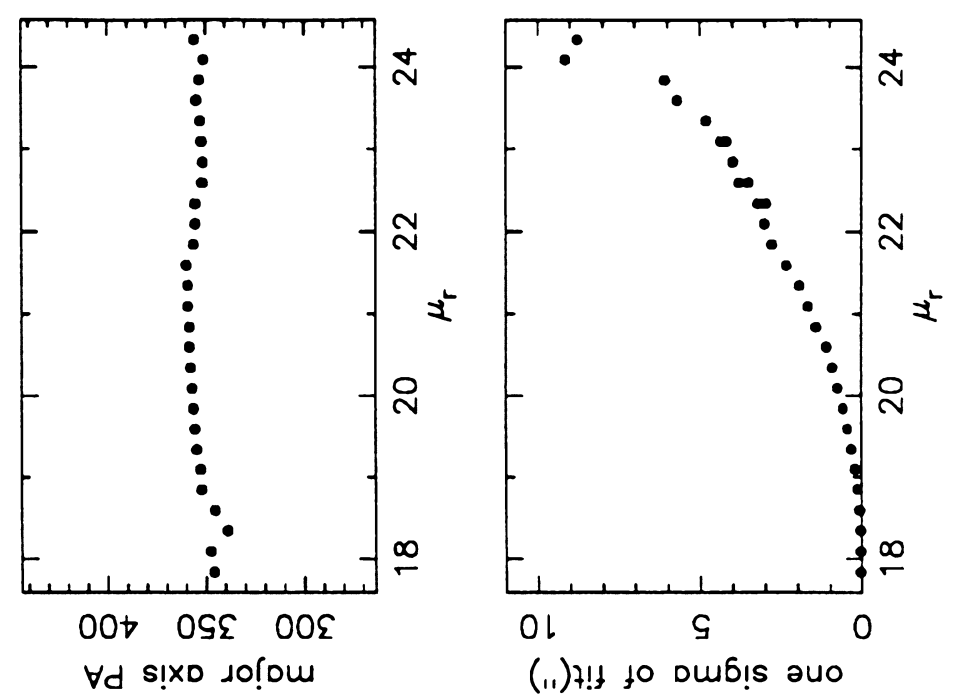

(11) 4 ! to Du6!s auo
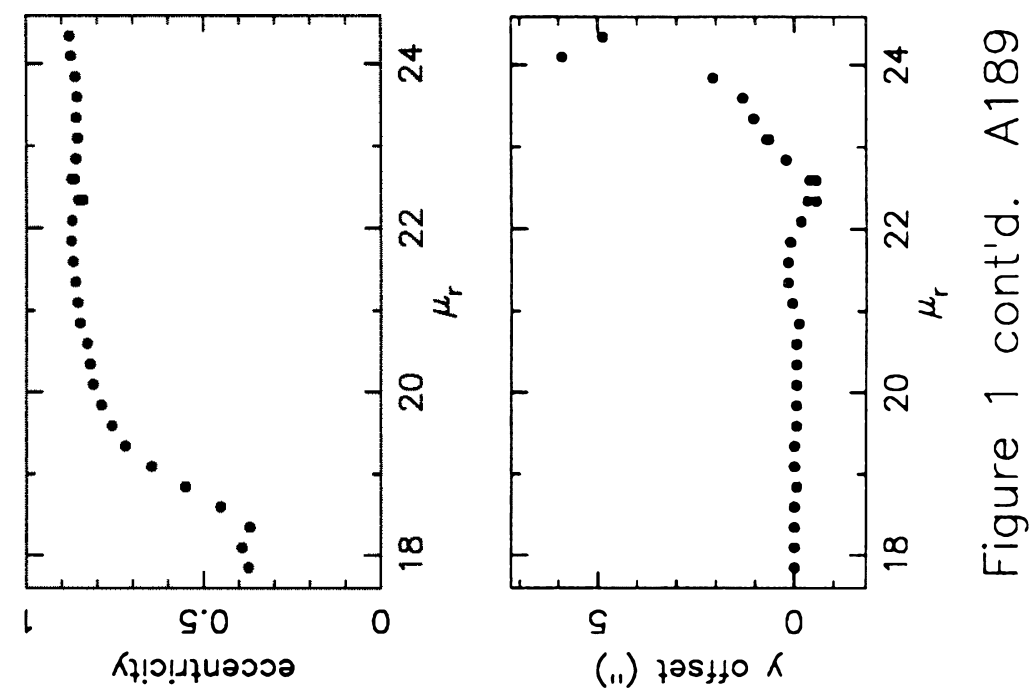

(ii) $725110 \mathrm{~K}$
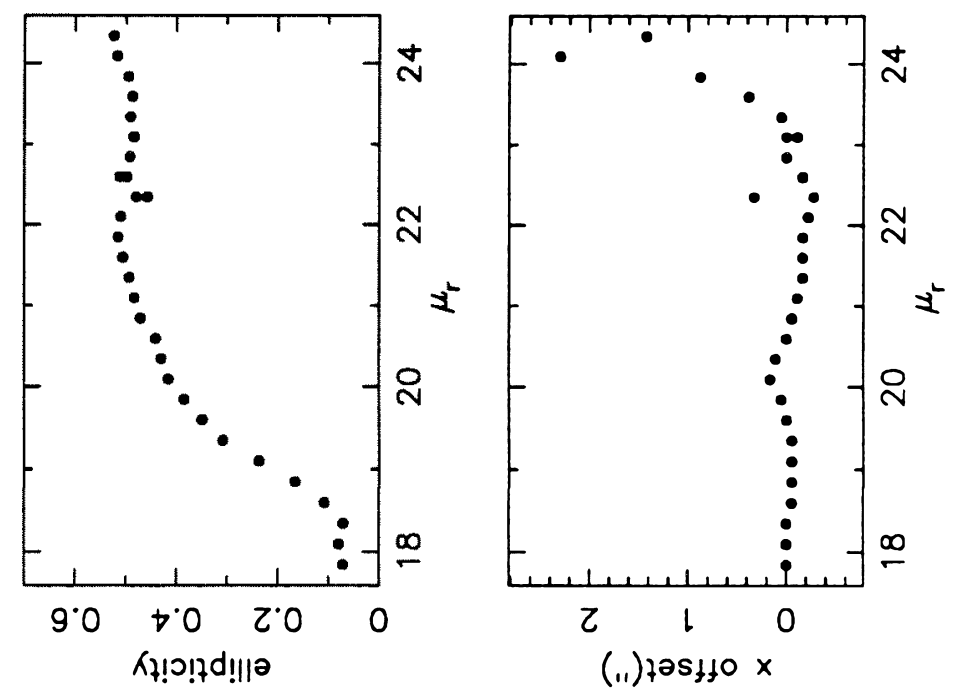

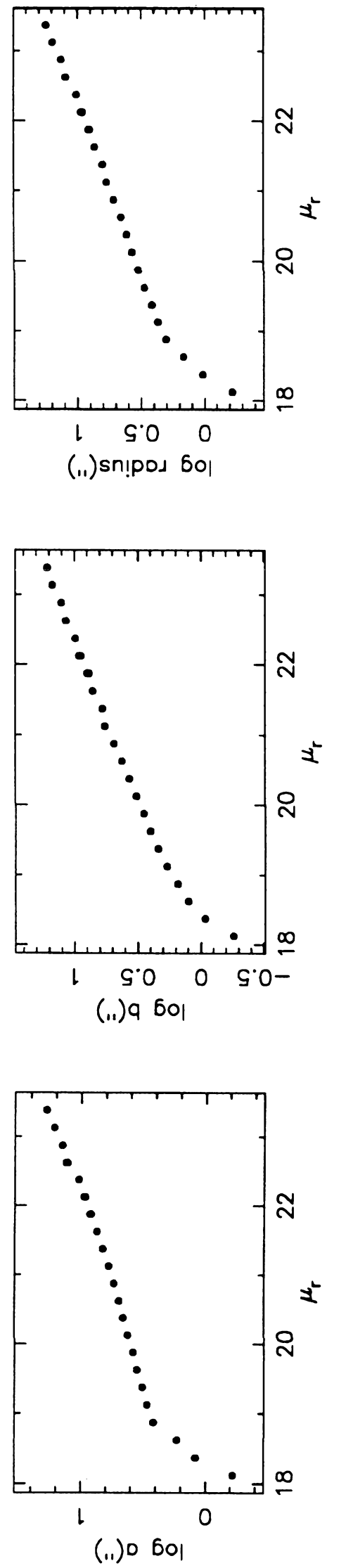

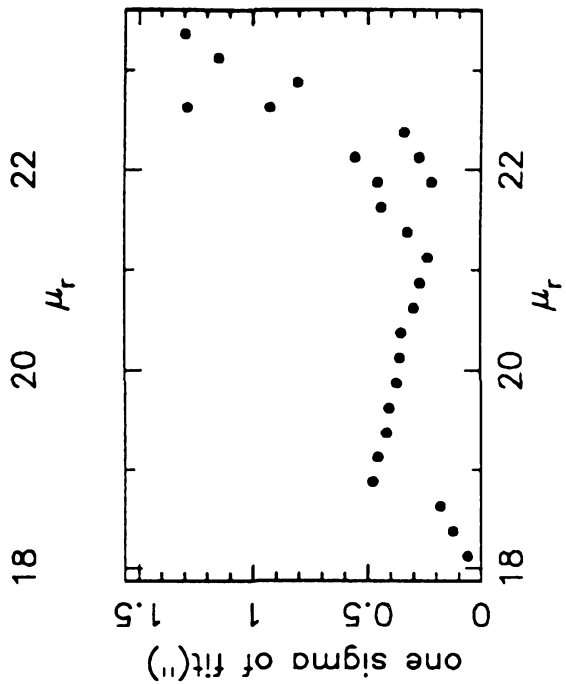

$\forall d$ s!Xo دo! lom
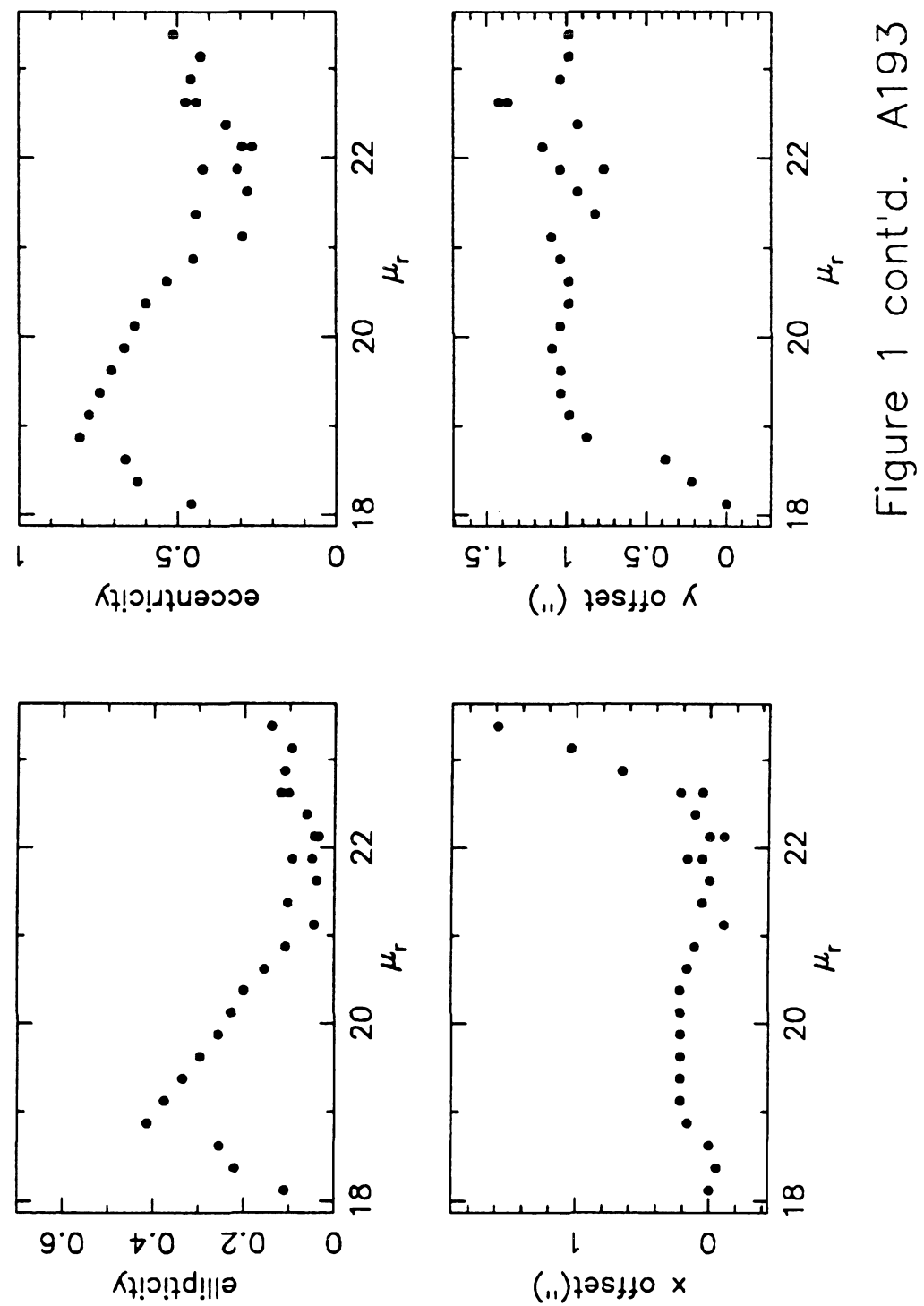


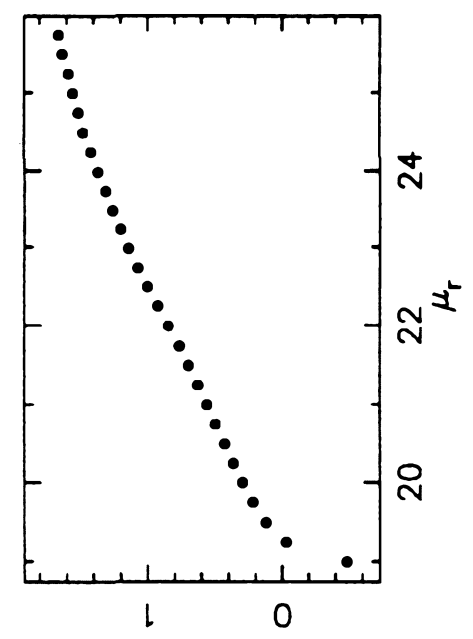

(.1) sn!pos 6ol

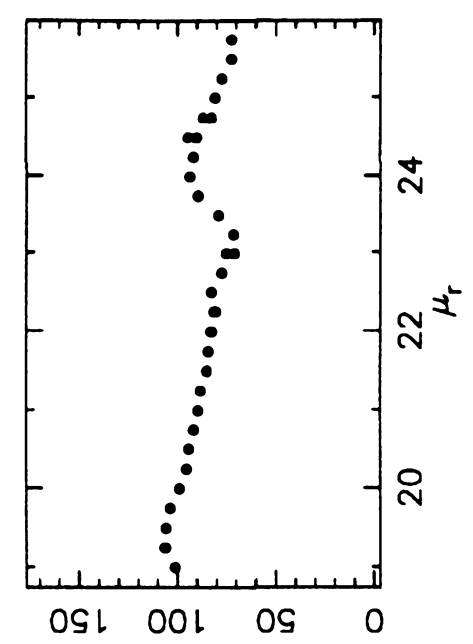

$\forall d$ s!xD so!ous

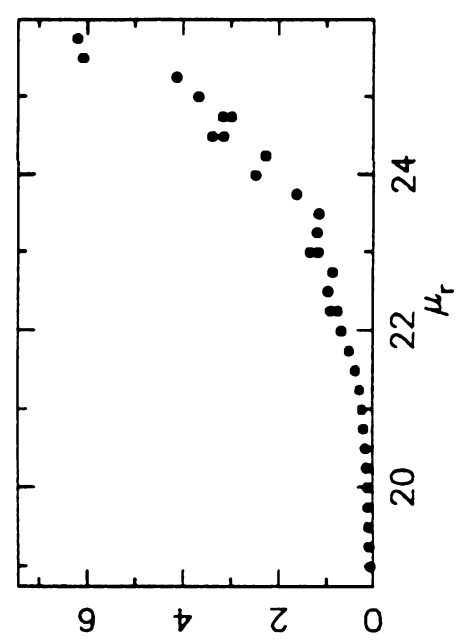

(11) $7 !+$ to oub! !s auo

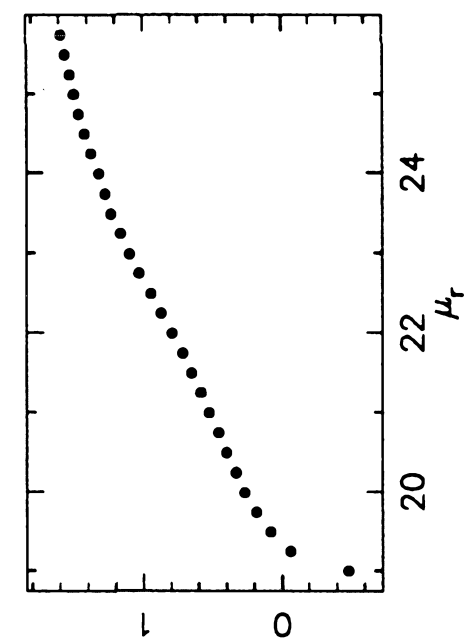

(.1) $9 \mathrm{bol}$

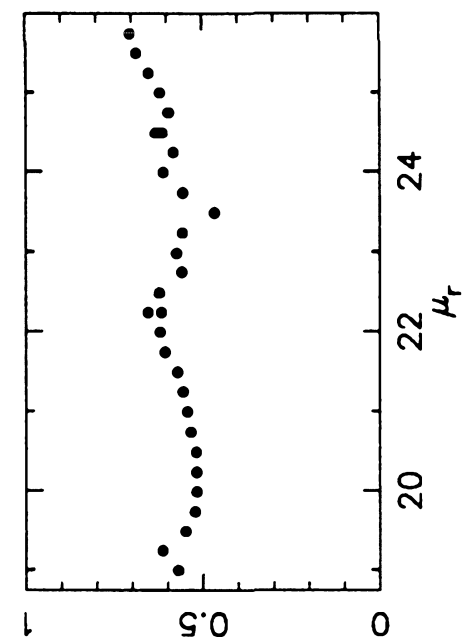

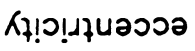

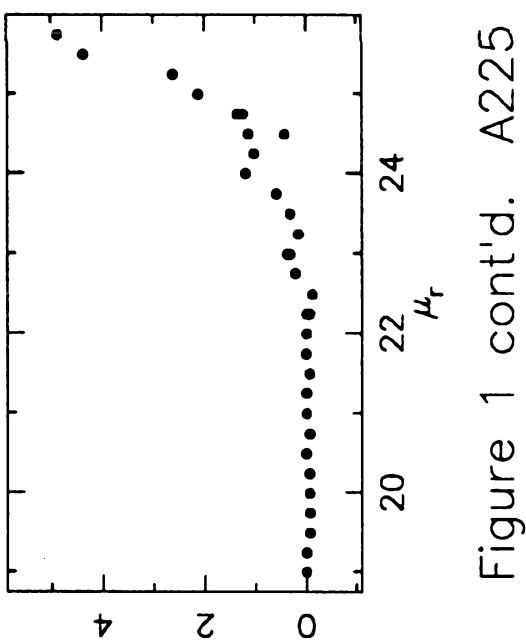

(11) $725150 k$

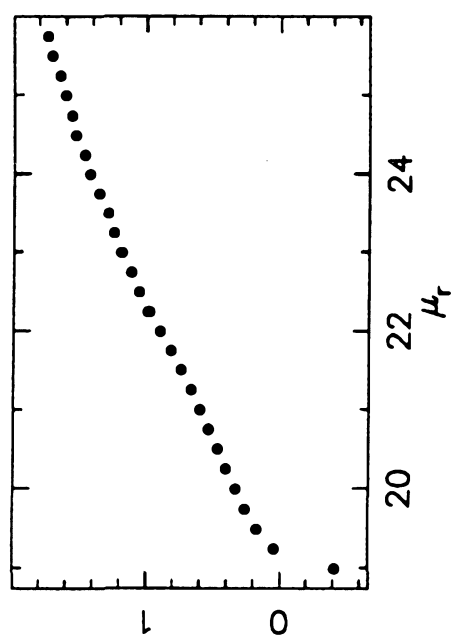

(.1) 0601
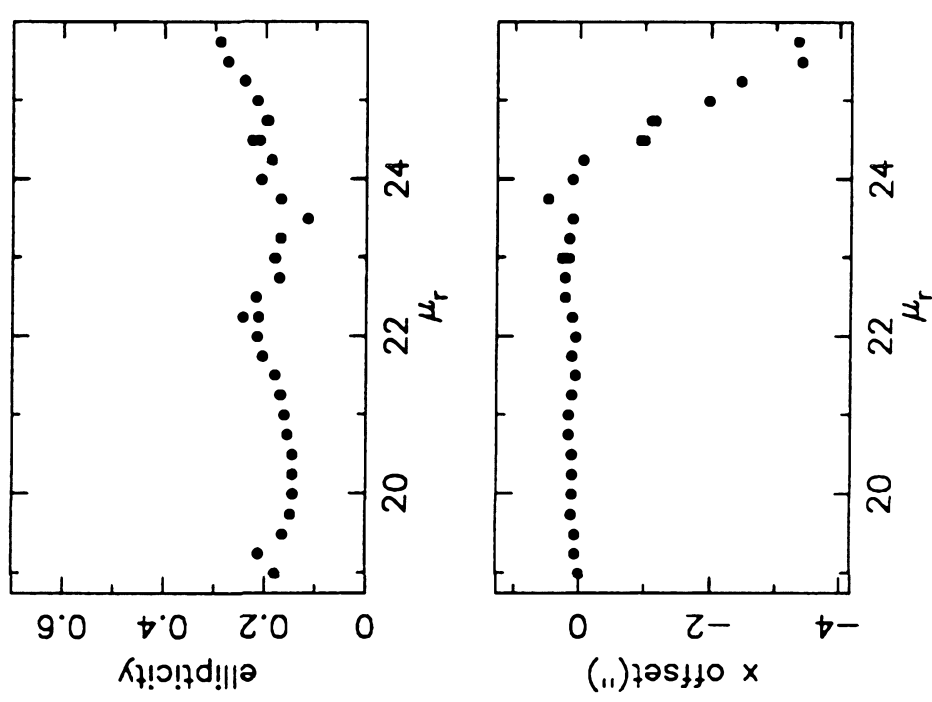


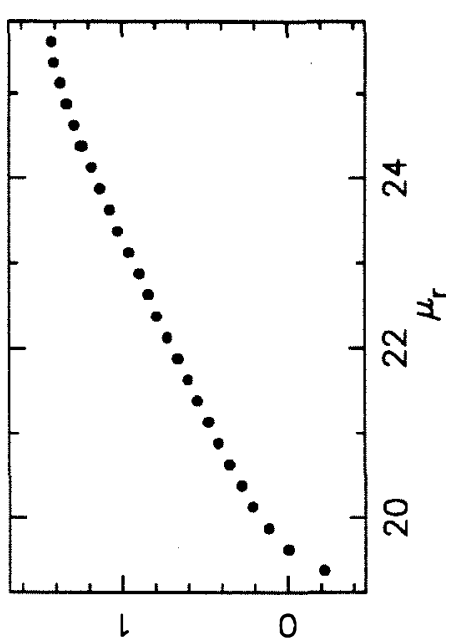

(.1)sn!pod 6ol
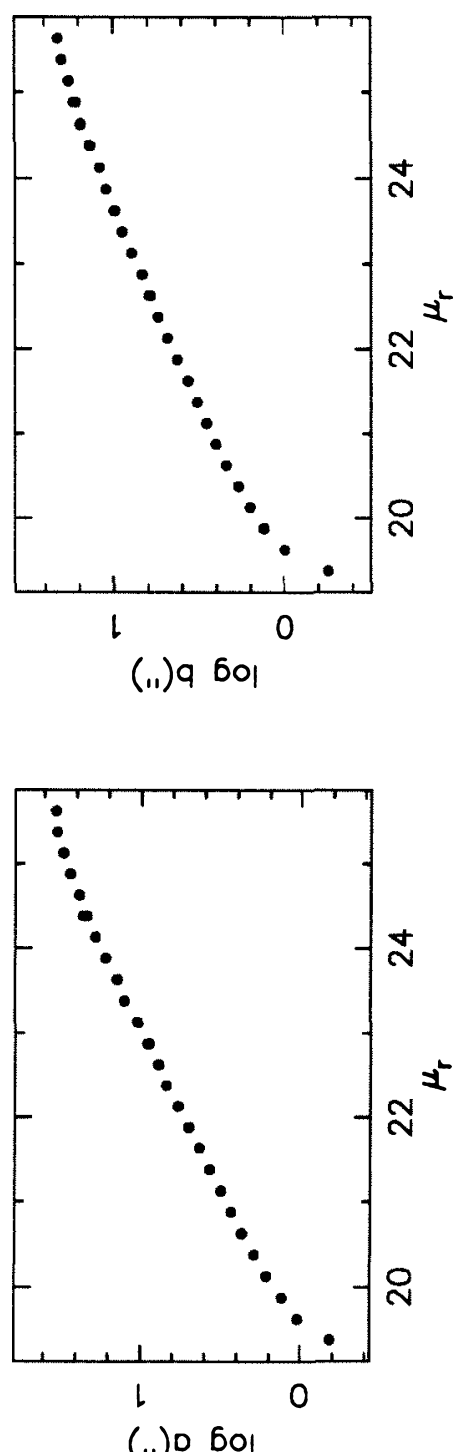

(11) 0 60이

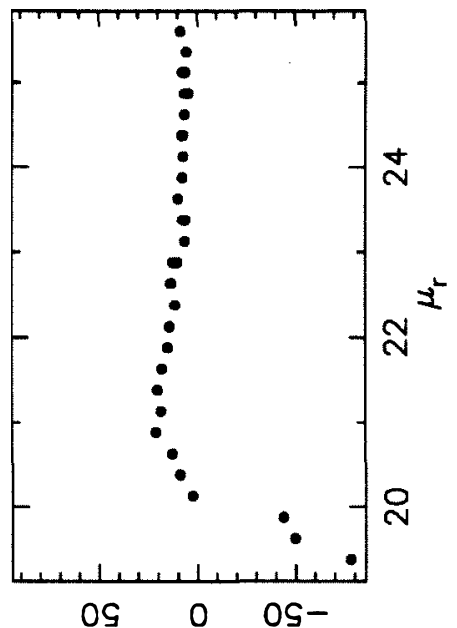

$\forall d$ s!xo so!lom
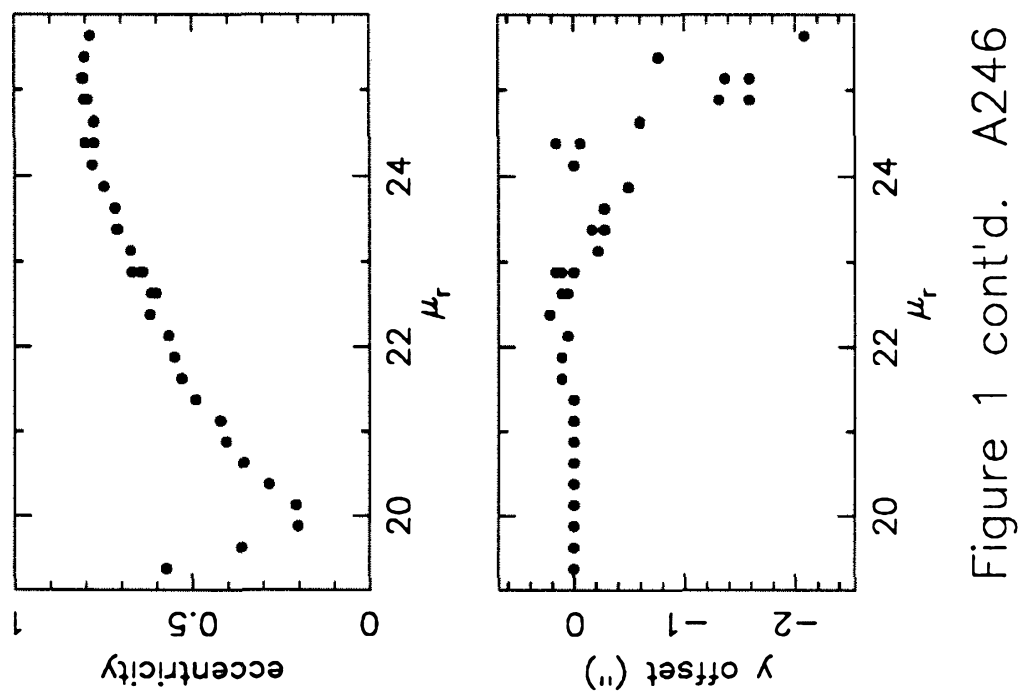
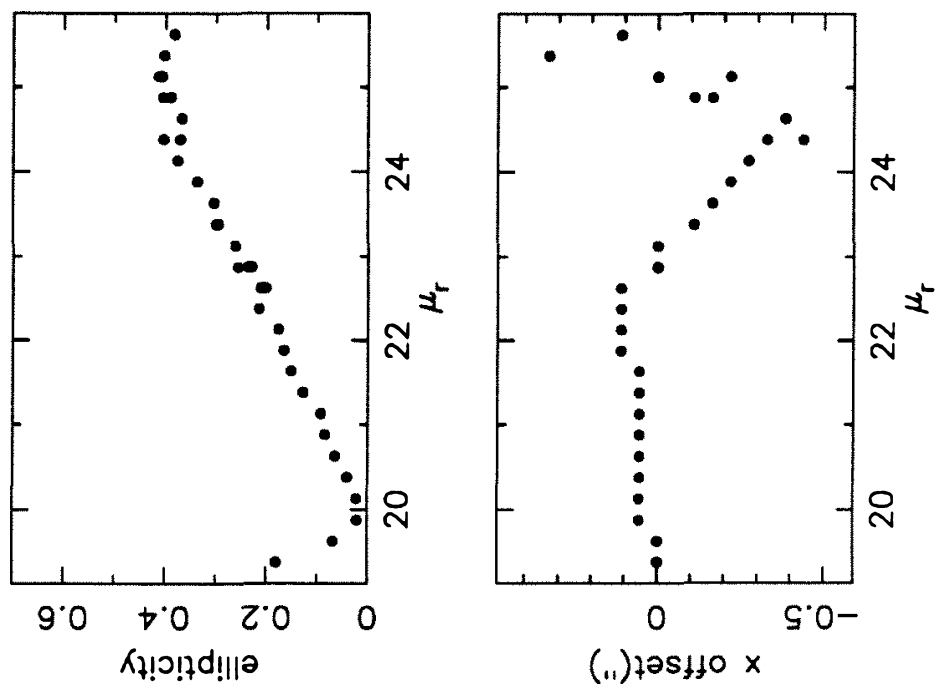


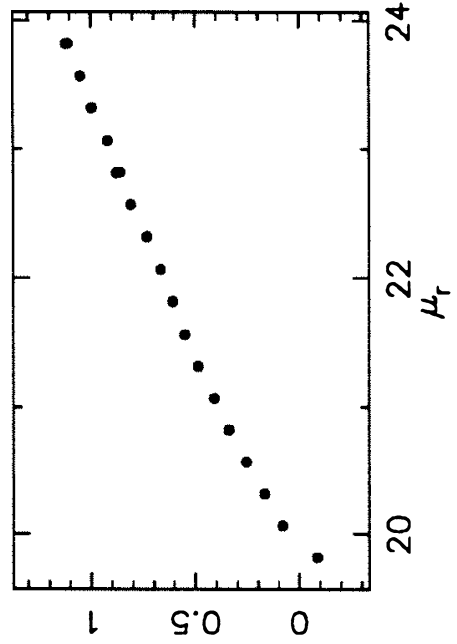

(.1) sn!pod 60।
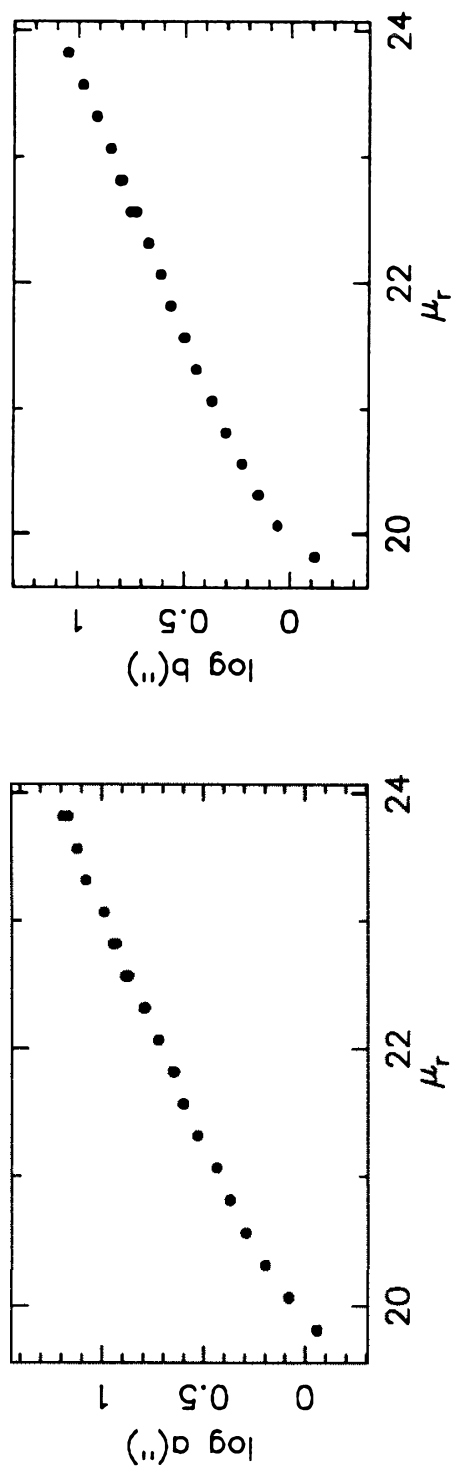

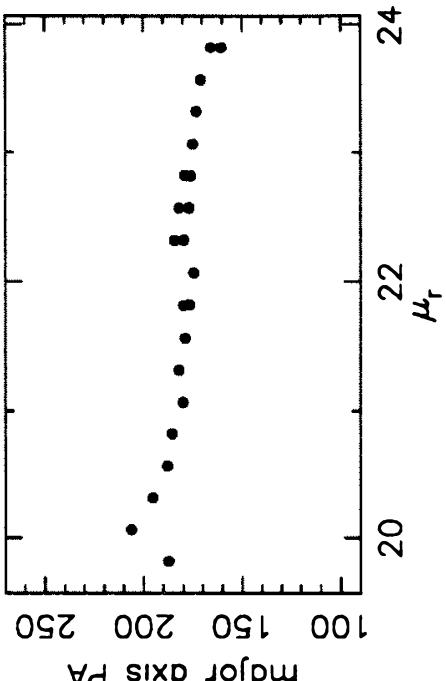

$\forall d$ s!xo so!om

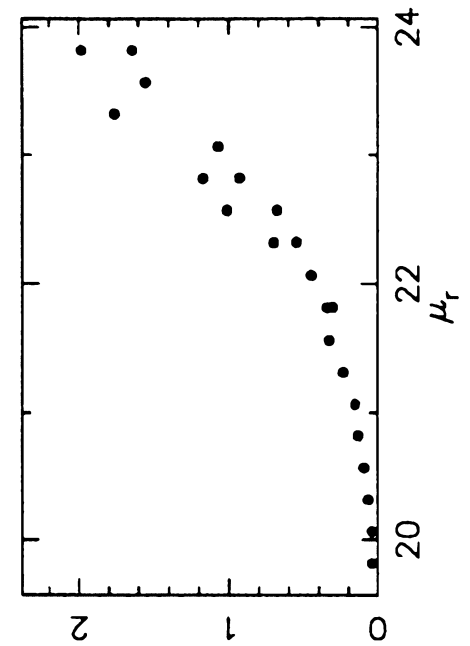

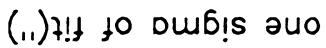
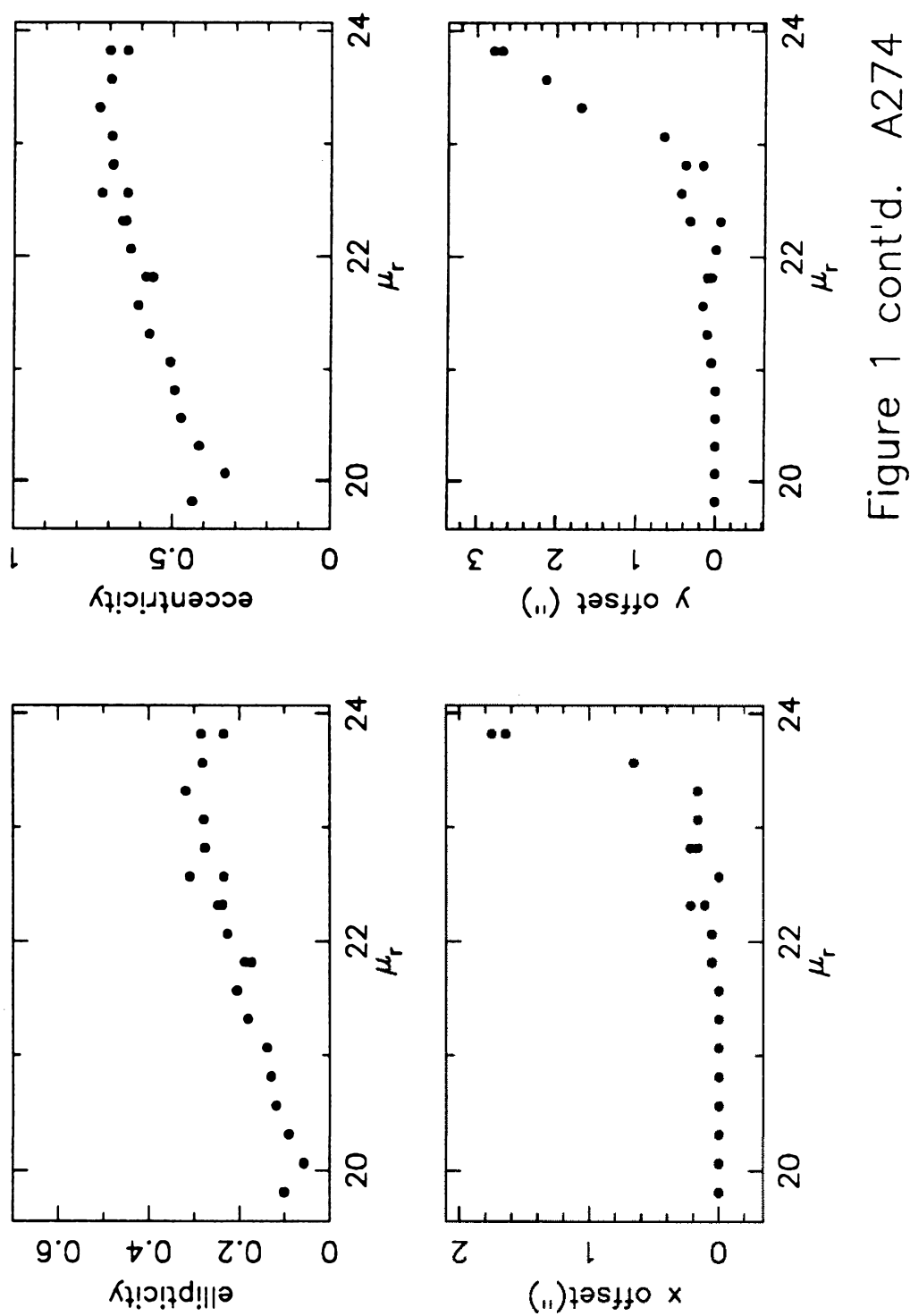
$-278-$
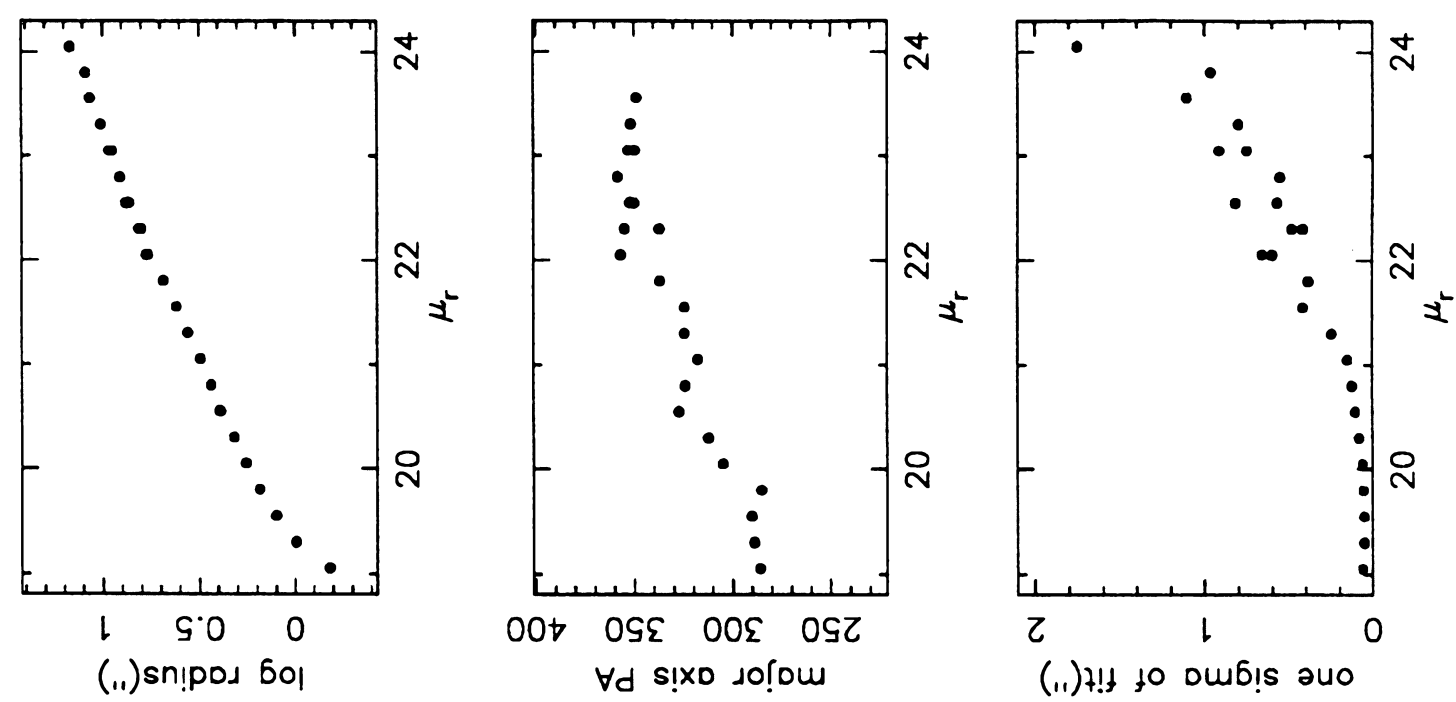

(.1)sn!pod 60।

(11) 4 !t to oub!s auo
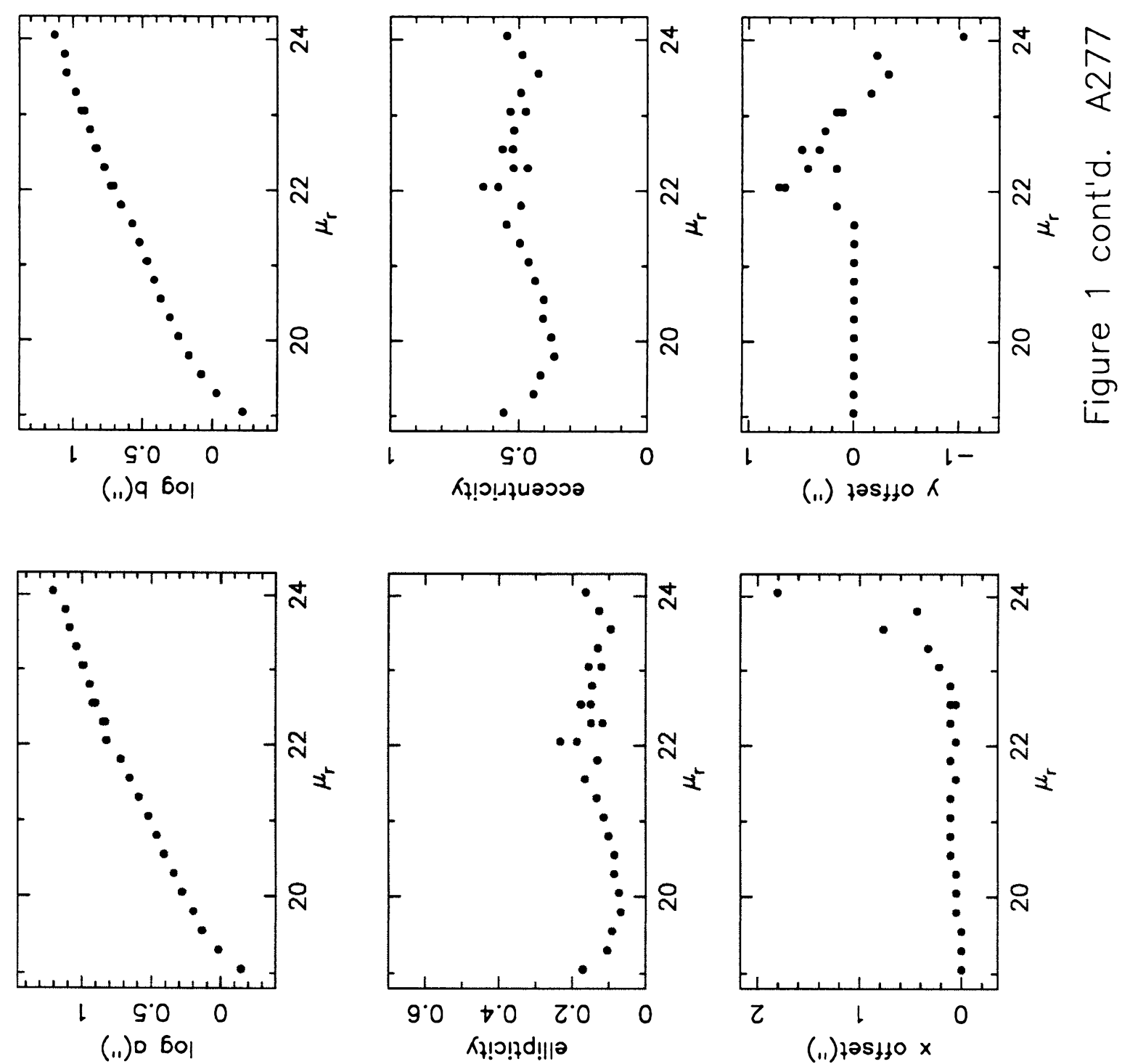


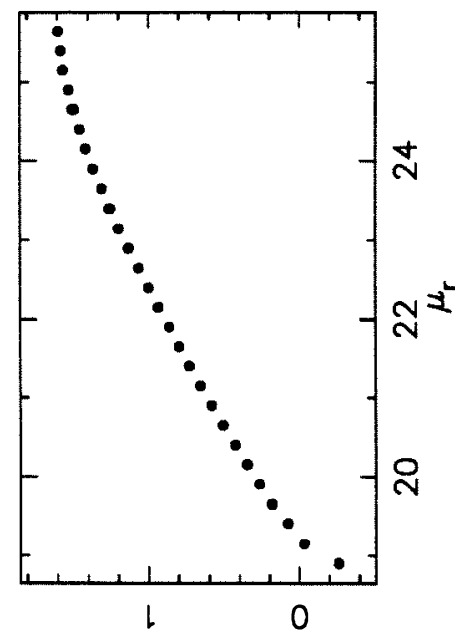

(11)sn!pos 6ol

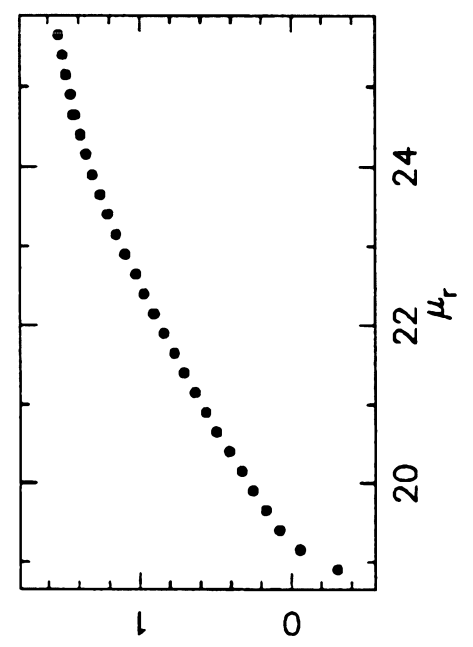

(.1) 9 60।

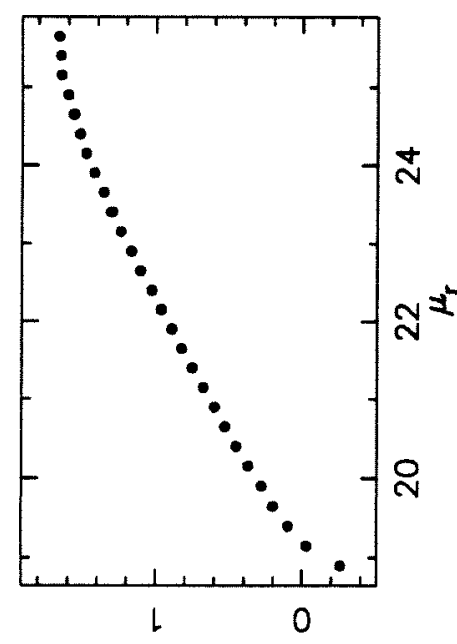

(.1) 0501
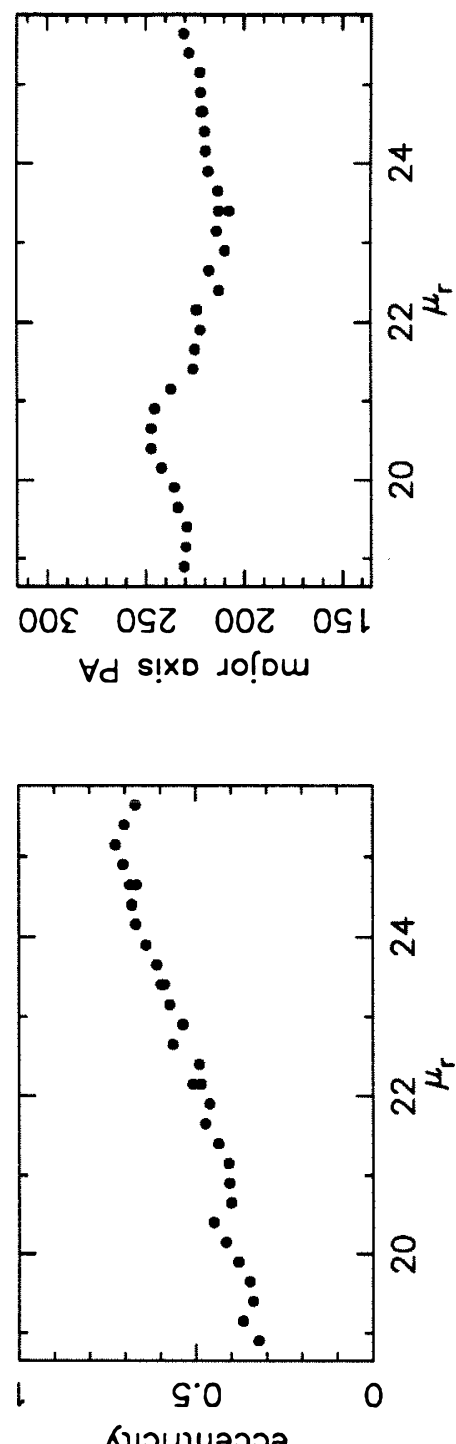

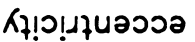

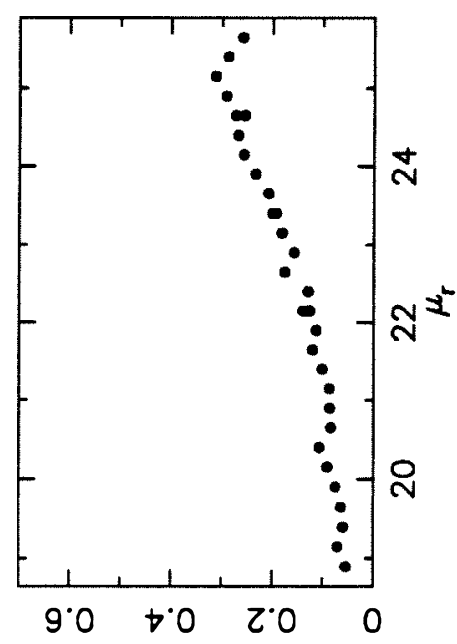

Kł!O!?d!||ə

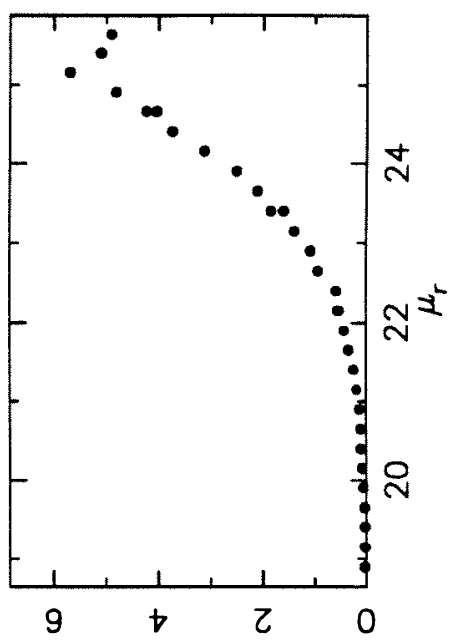

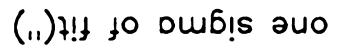

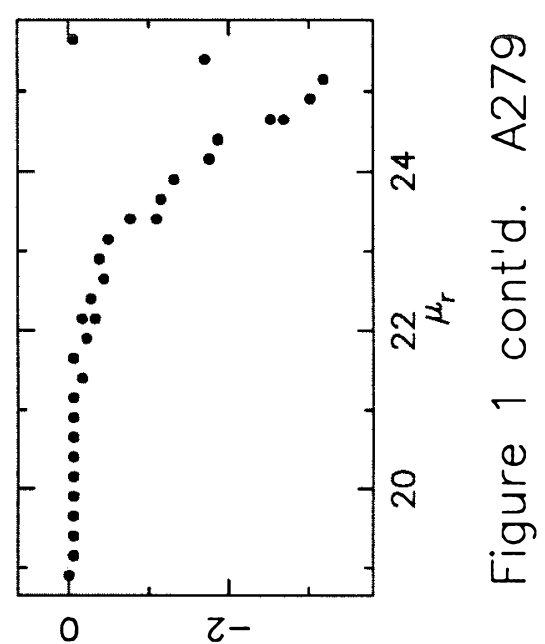

(11) $725+150 \mathrm{~K}$

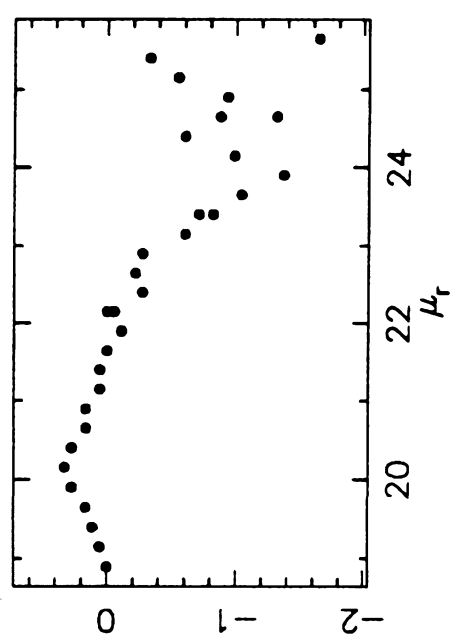

(11) tos $750 \times$ 


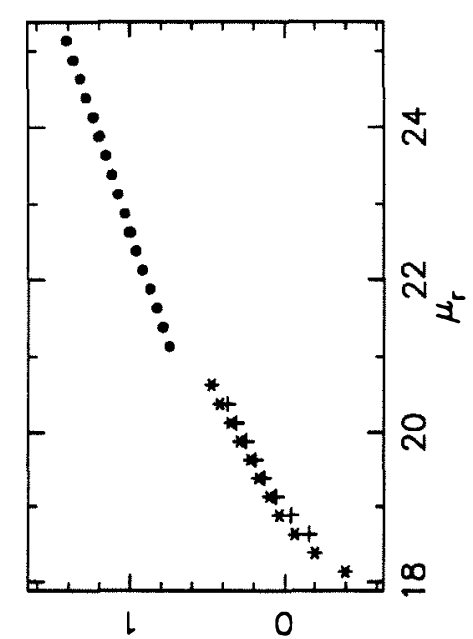

(.1) sn!pos 50|

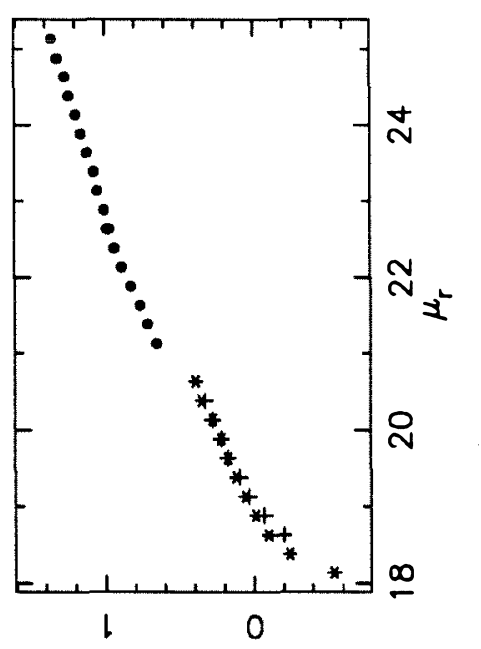

(.1) 9 bol

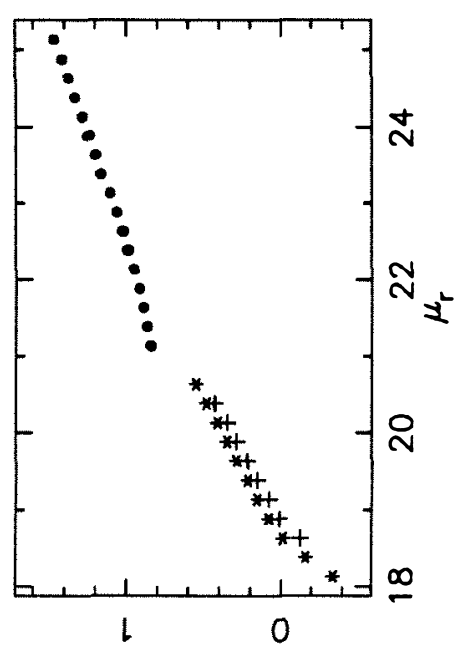

(.1) $\mathrm{D}$ 6이

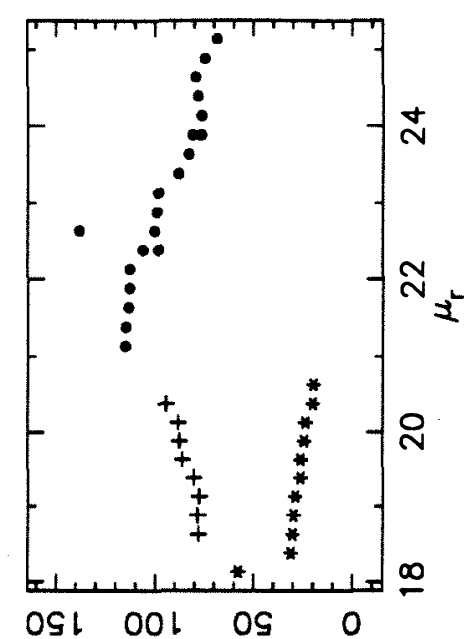

$\forall d$ s!xo Jo!́u

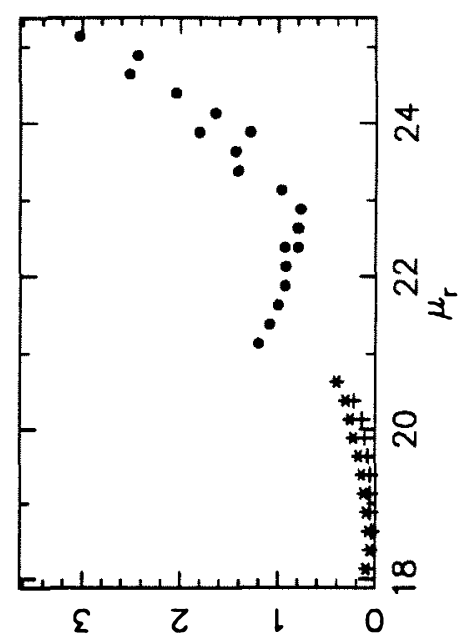

(i1) + !t to Dub!s auo

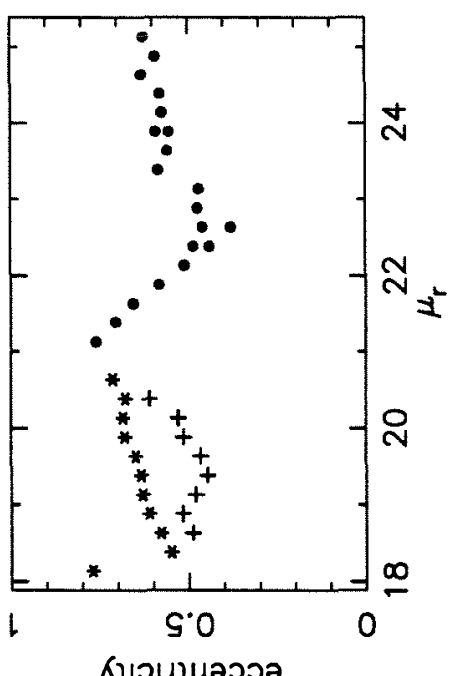

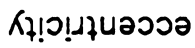

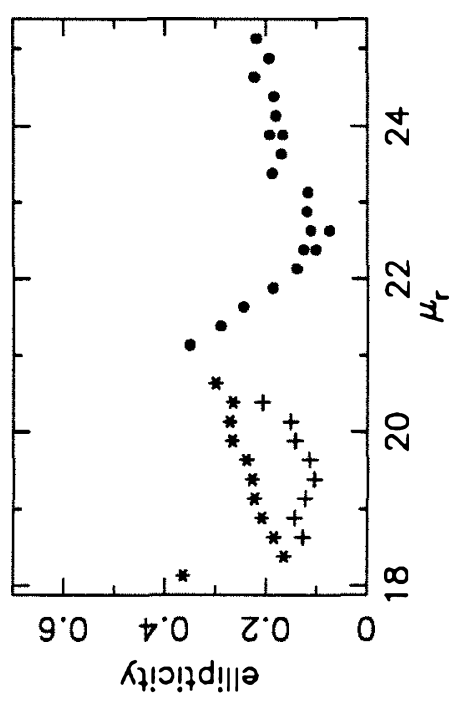

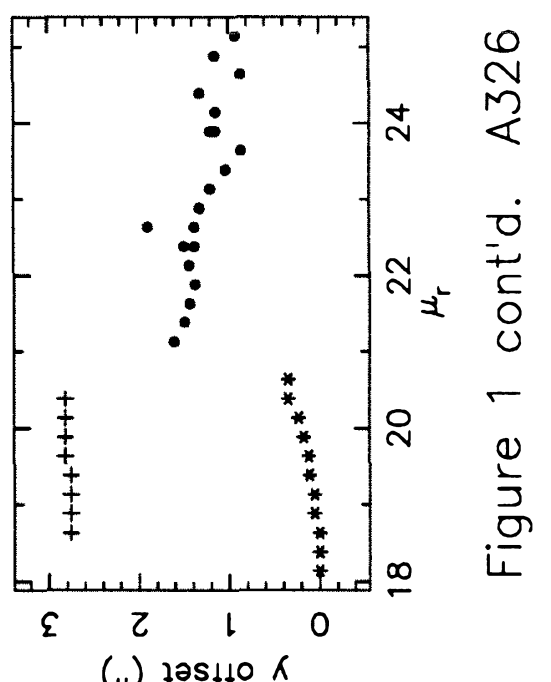

(ii) $725410 \pi$

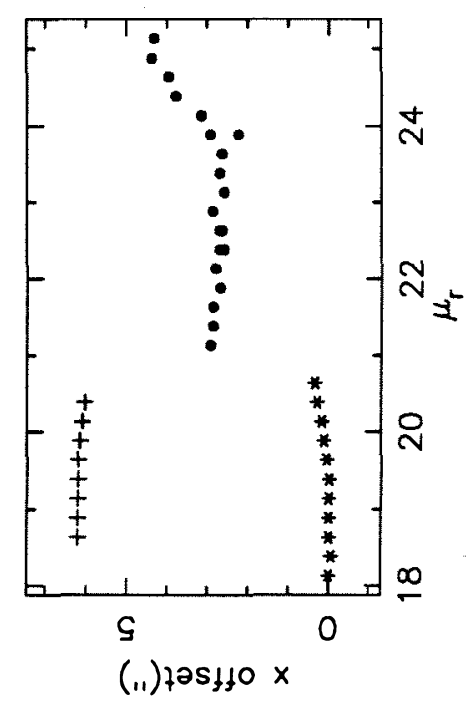




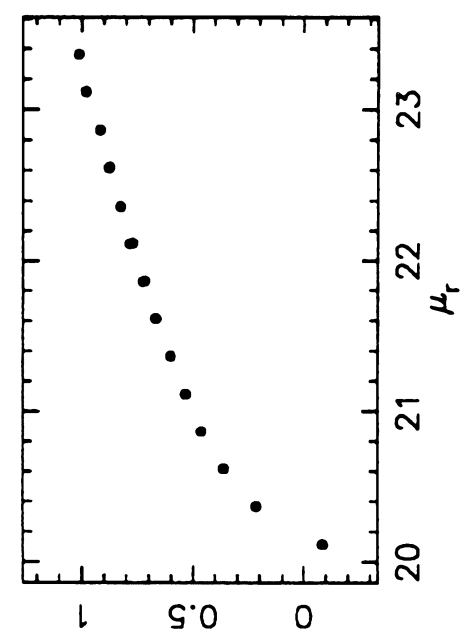

(.1)sn!pos 6ol

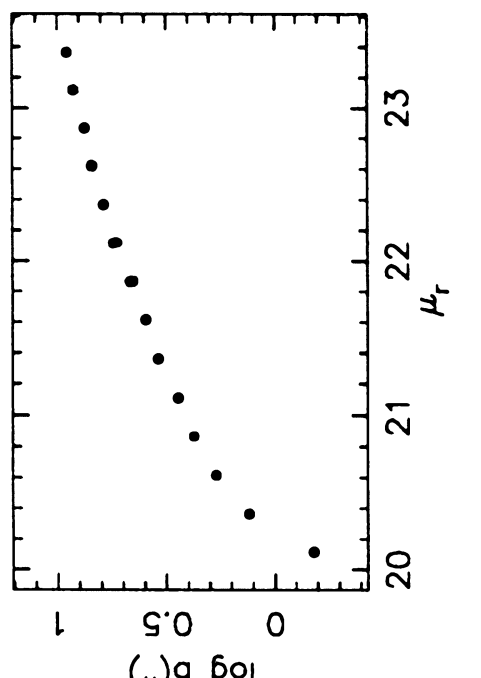

(.1) 9 6ol
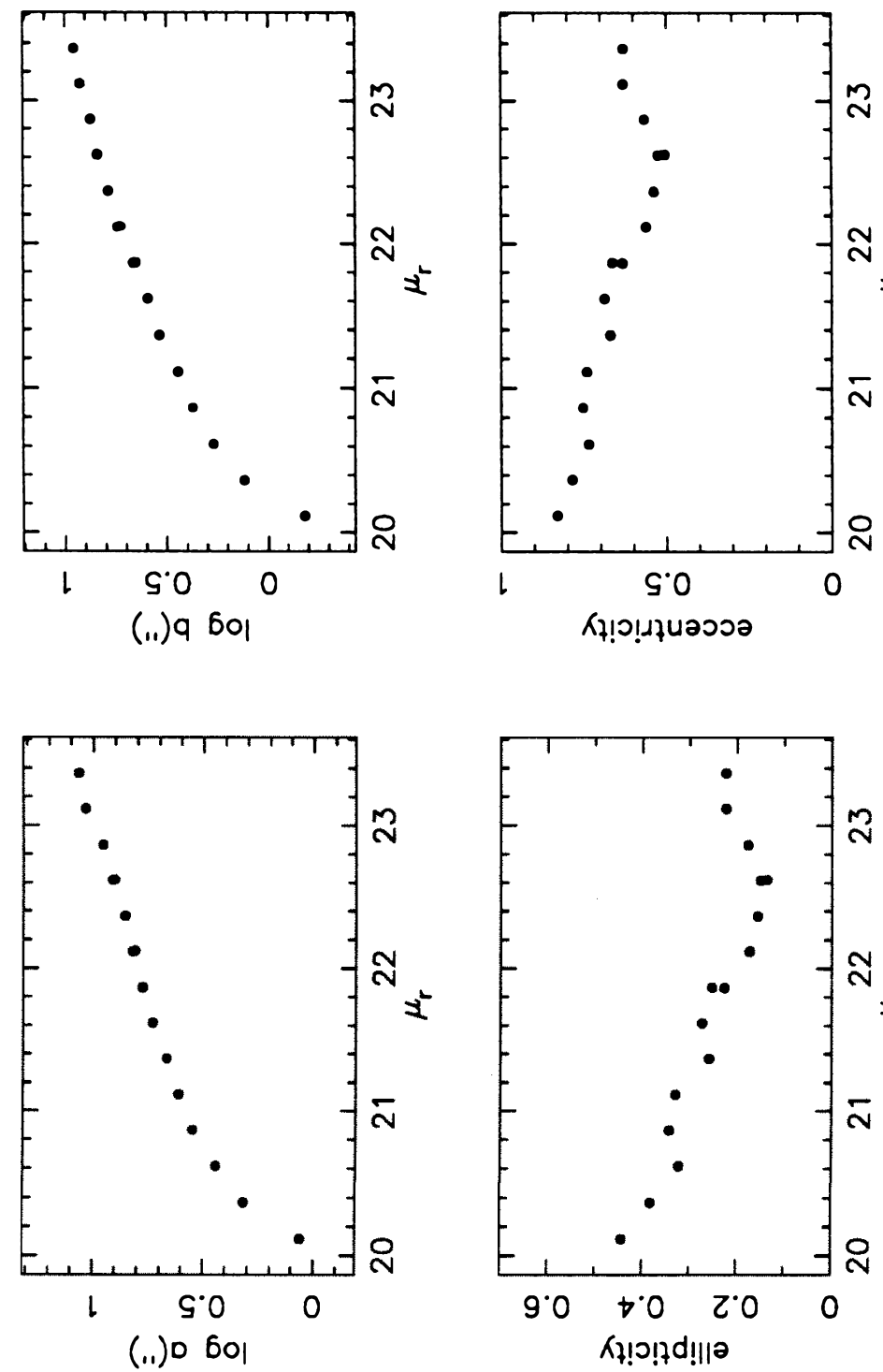

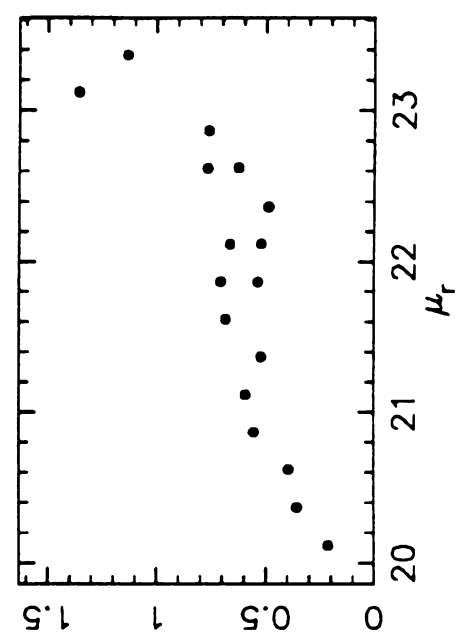

(.1) $+! t$ to oub! auo
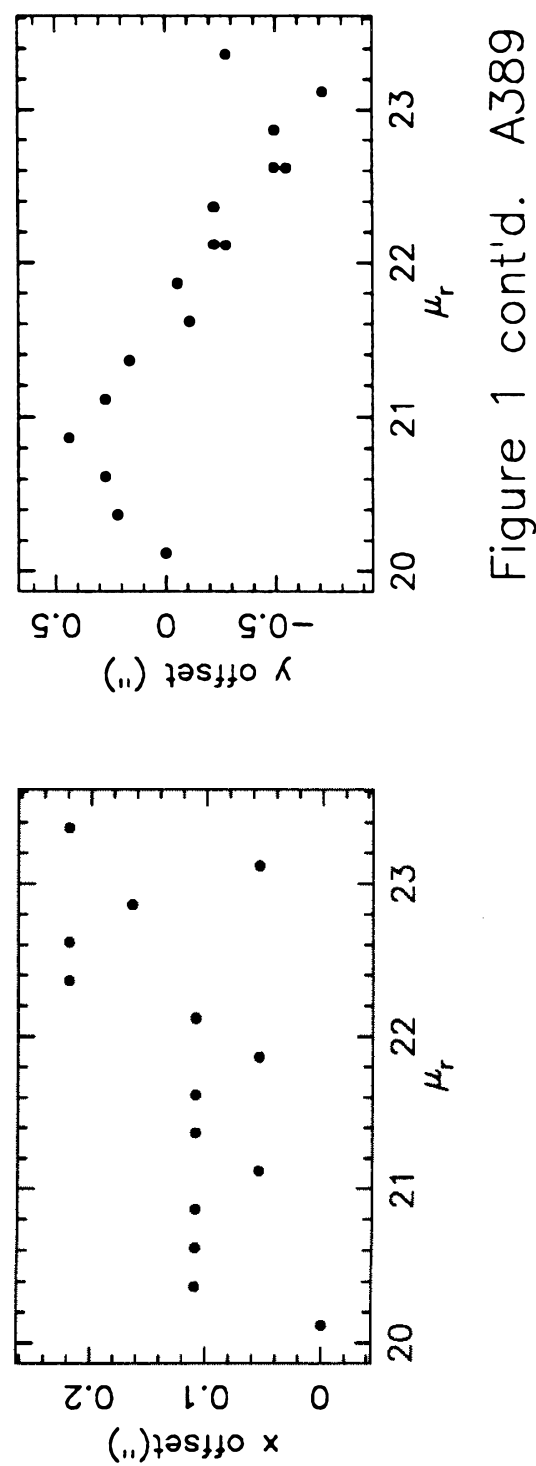


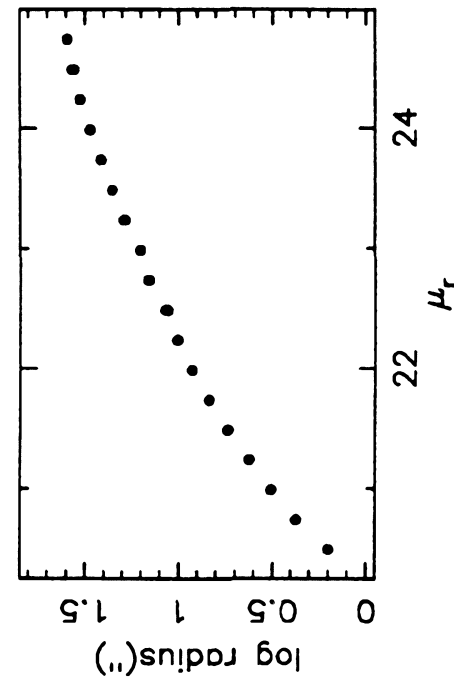

(.1)sn!pDs 60।

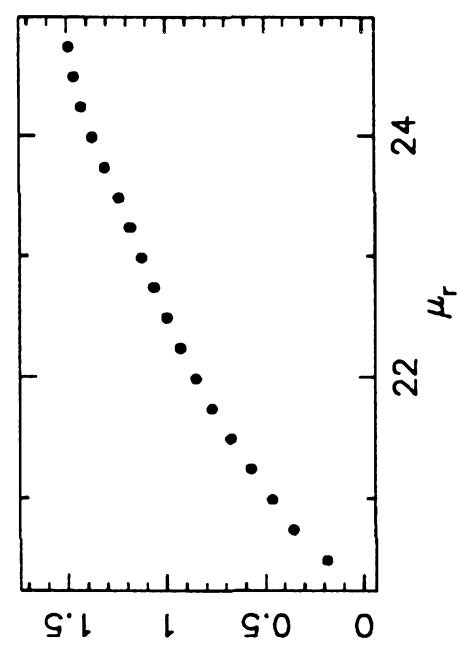

(.1) 9 60।

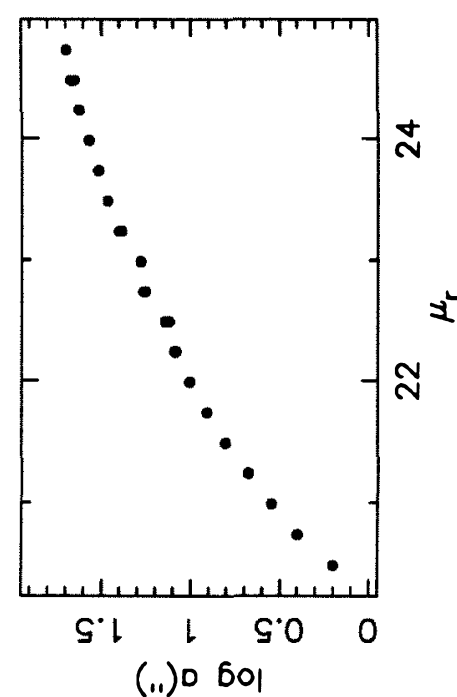

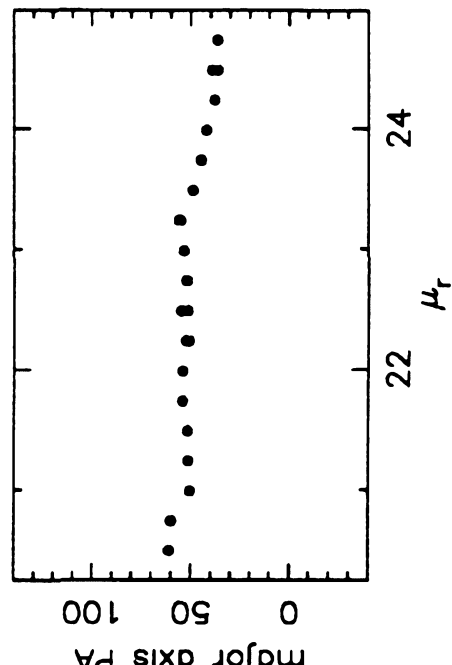

$\forall d$ s!xo so!́m

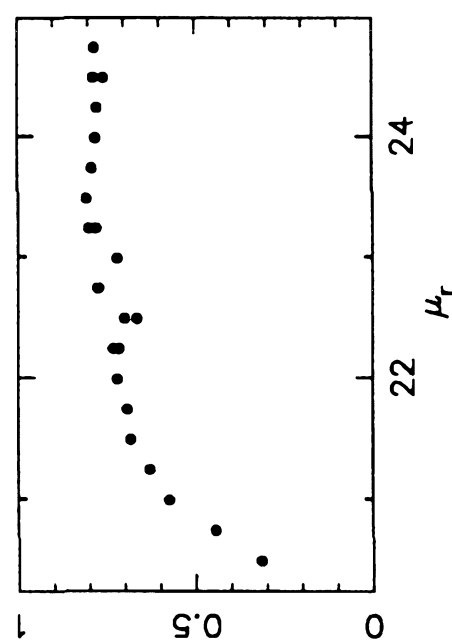

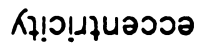

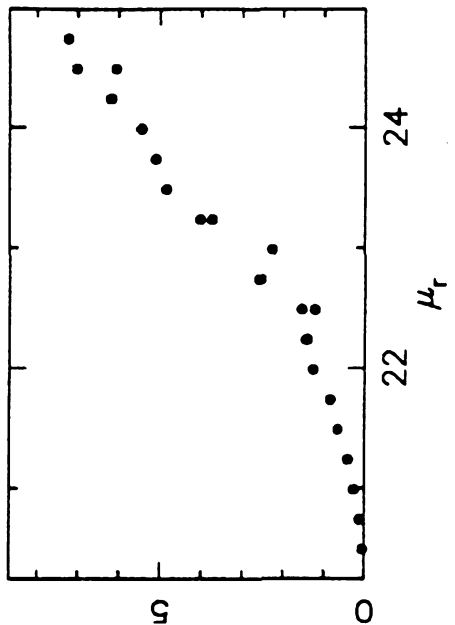

(11) + !! to ow6!s avo

(1)

\section{2}




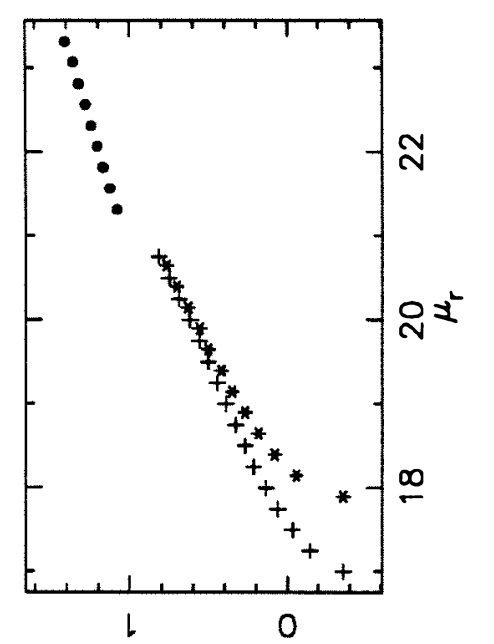

(.1) sn!pod 6ol

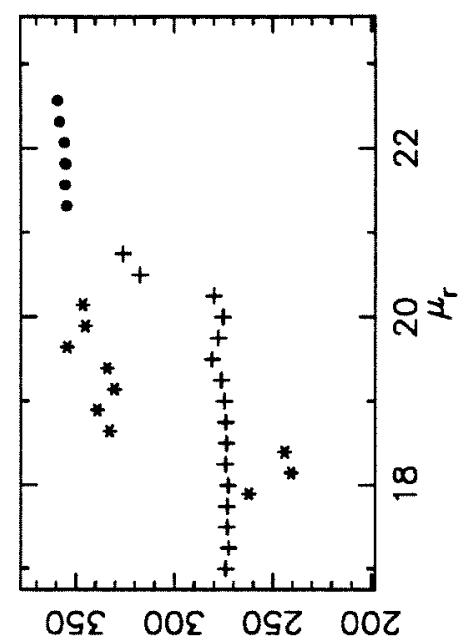

$\forall d$ S!Xo so!́m

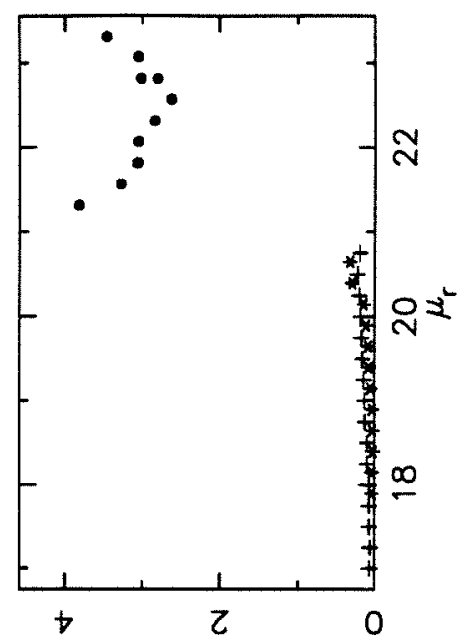

(11) 4 !t to Dub!s auo
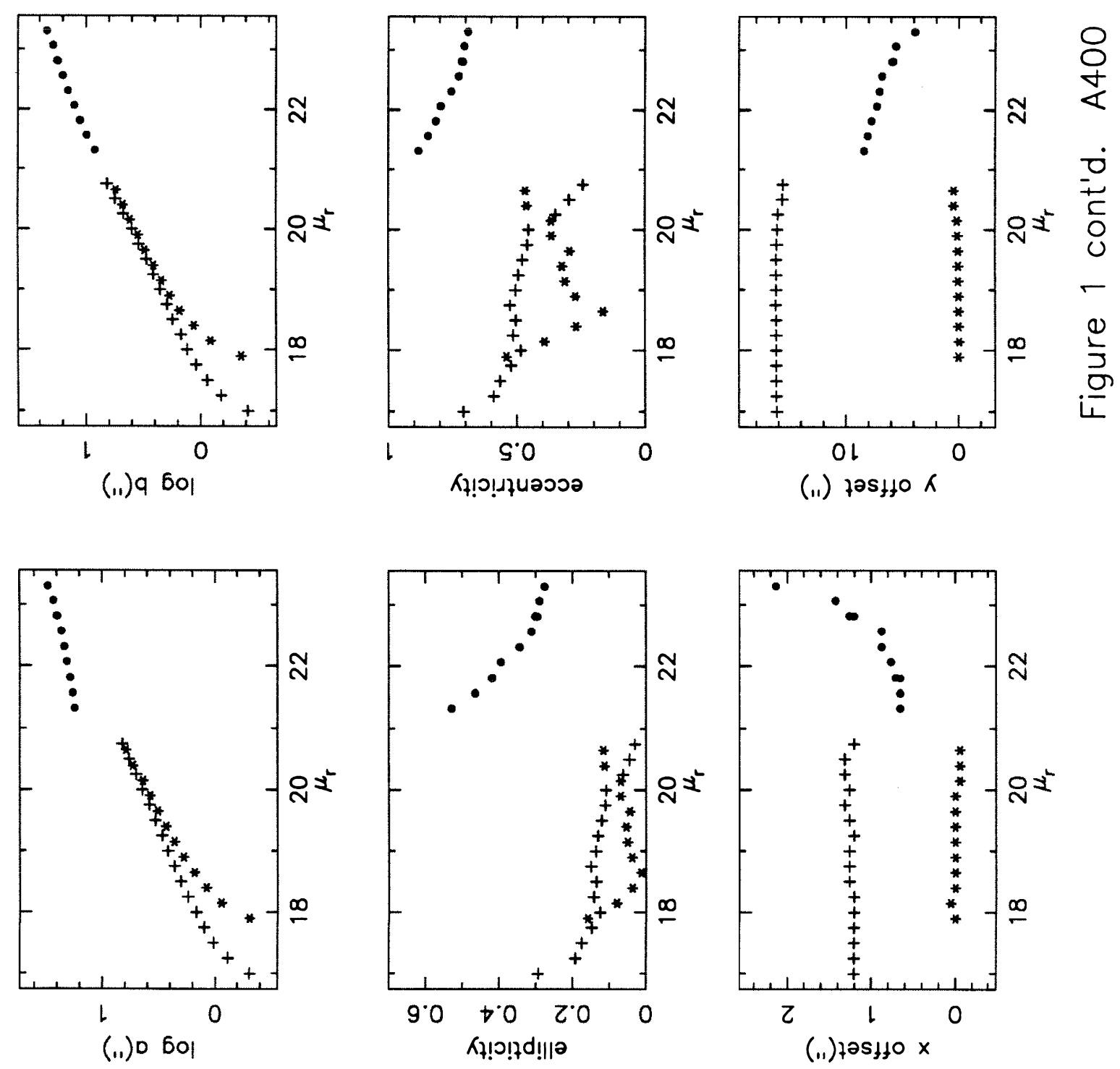


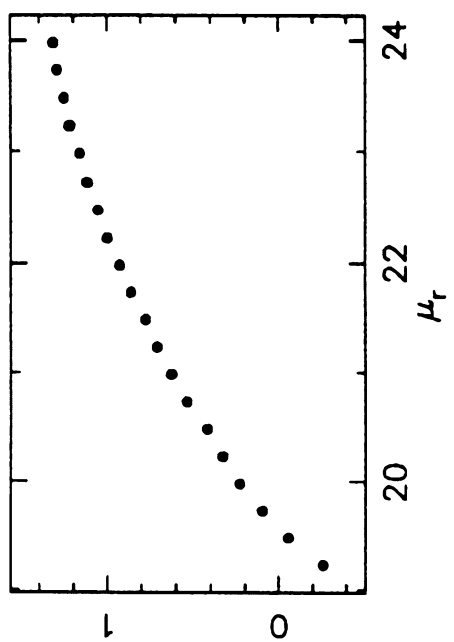

(11)sn!pod 601

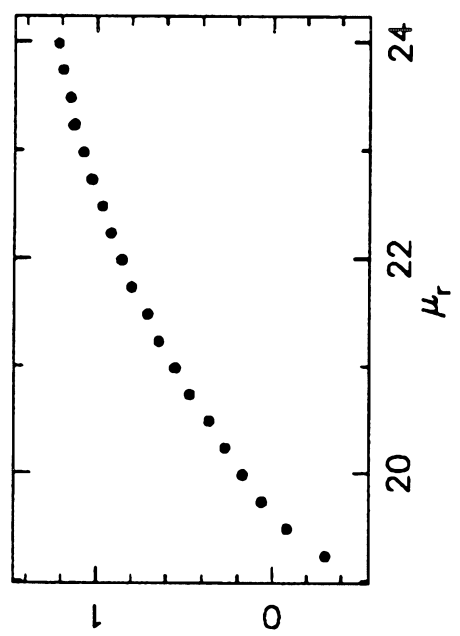

(.1) $9 \mathrm{bol}$

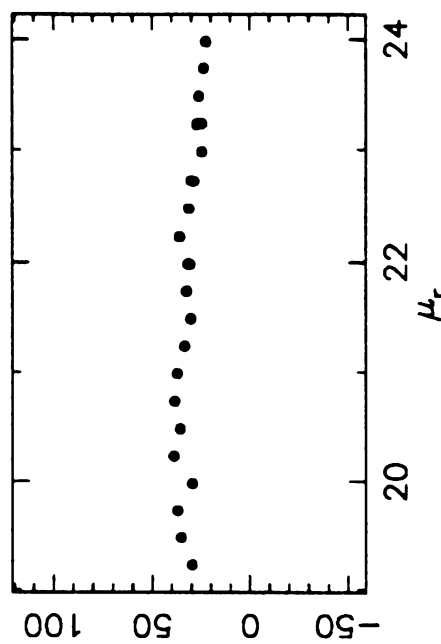

$\forall d$ s!xo so!om

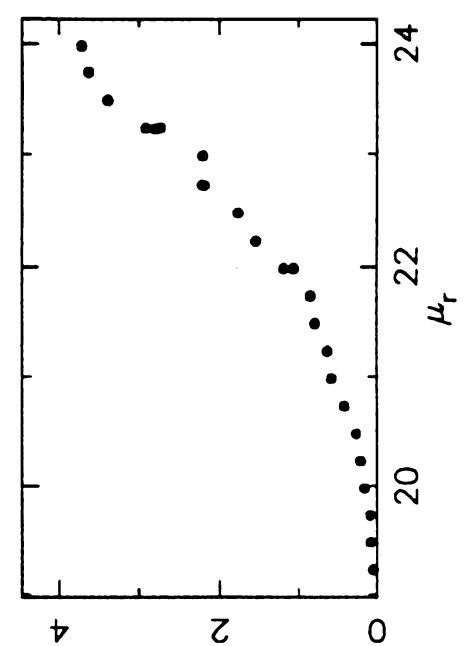

(11) 4 !t to Dub! a a
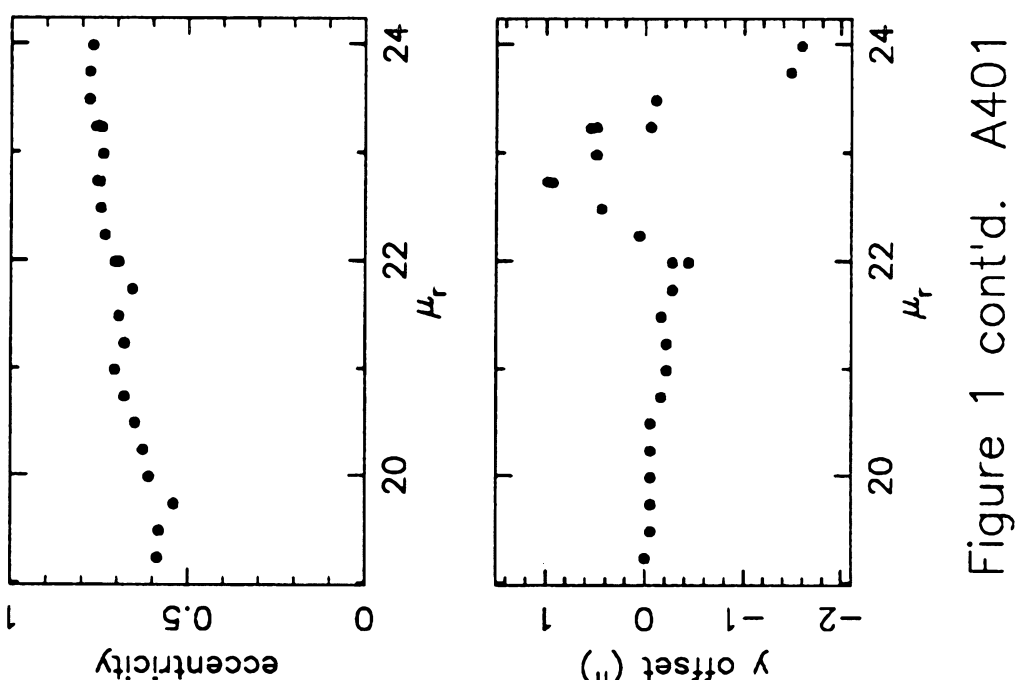

(11) $725 \% 10 \mathrm{~K}$

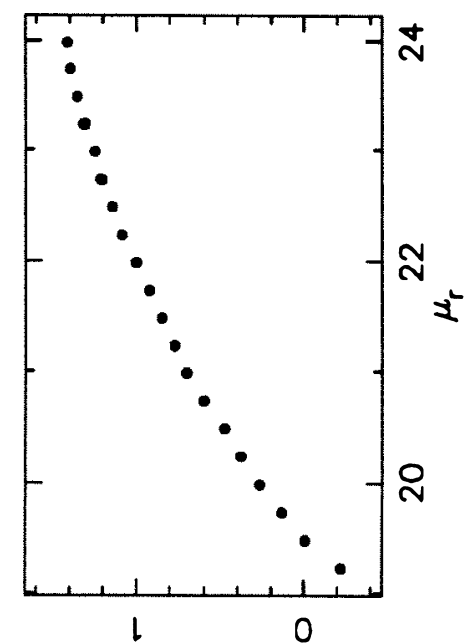

(.1) 060
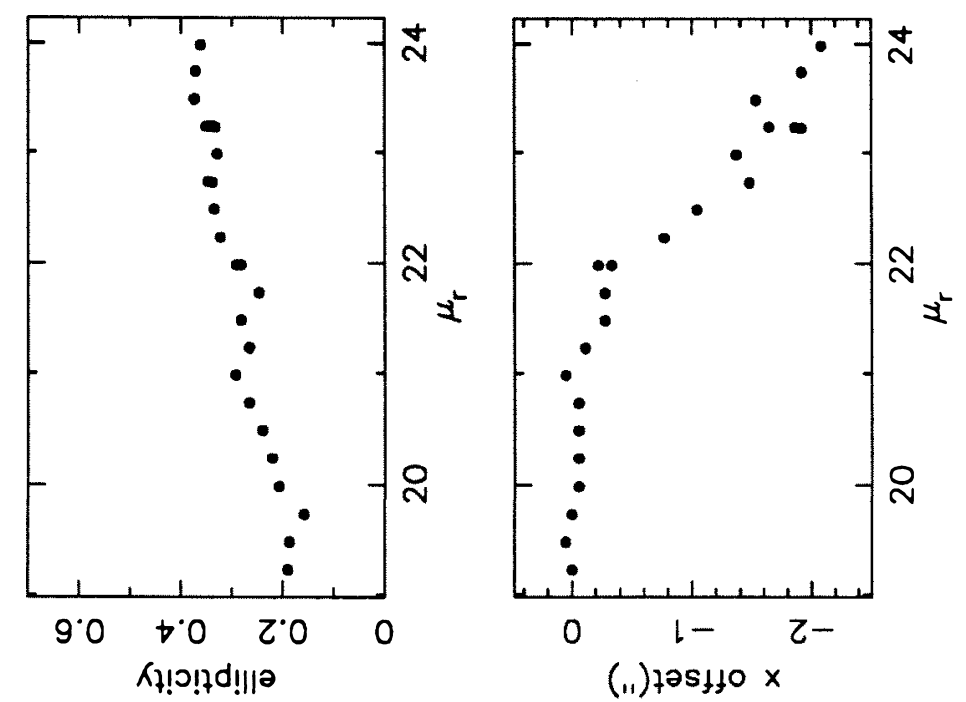


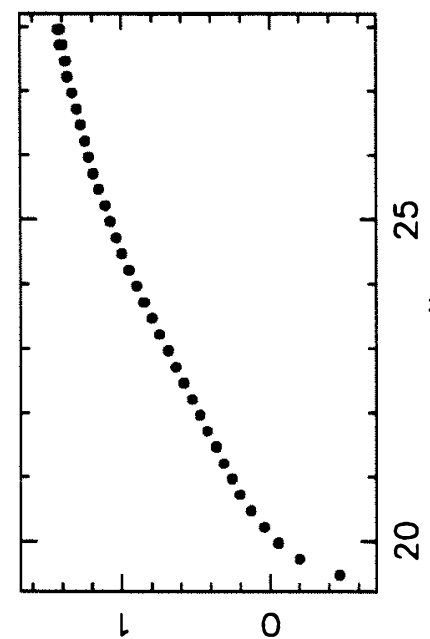

(.1) sn!pos 6ol
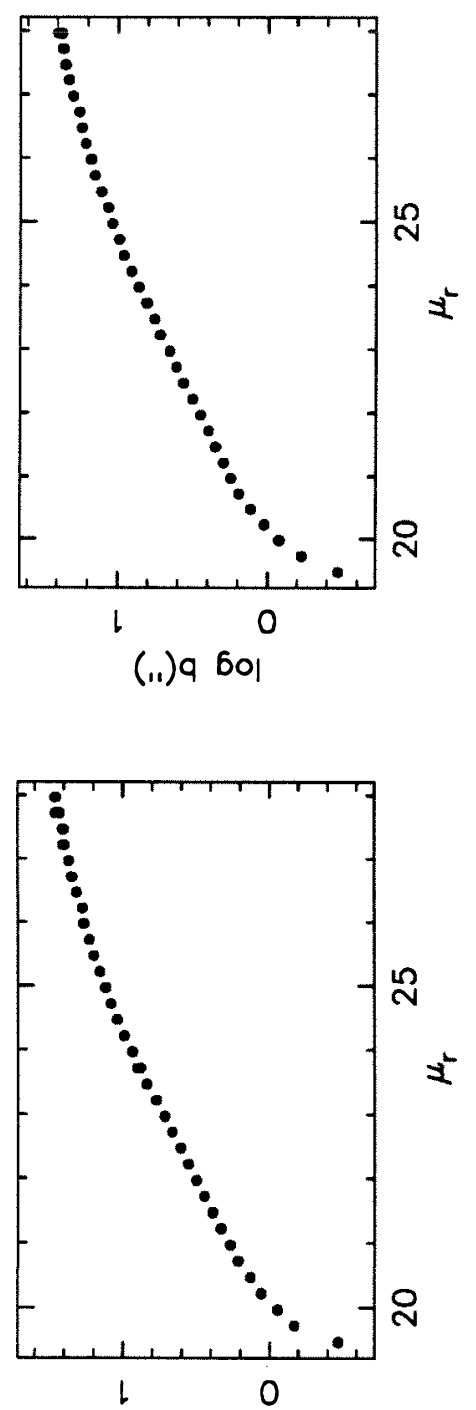

(1.) 0 60।

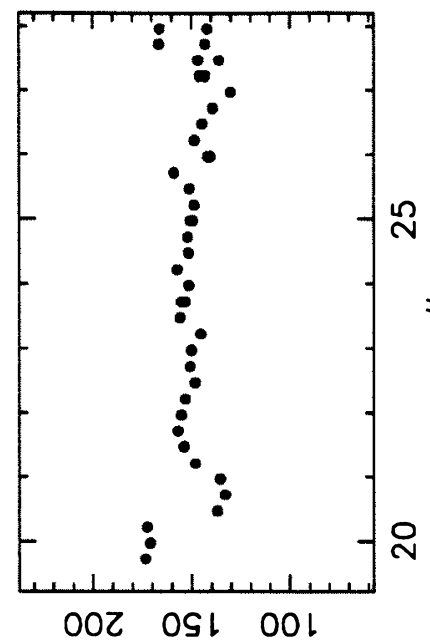

$\forall d$ s!xo so!̣u
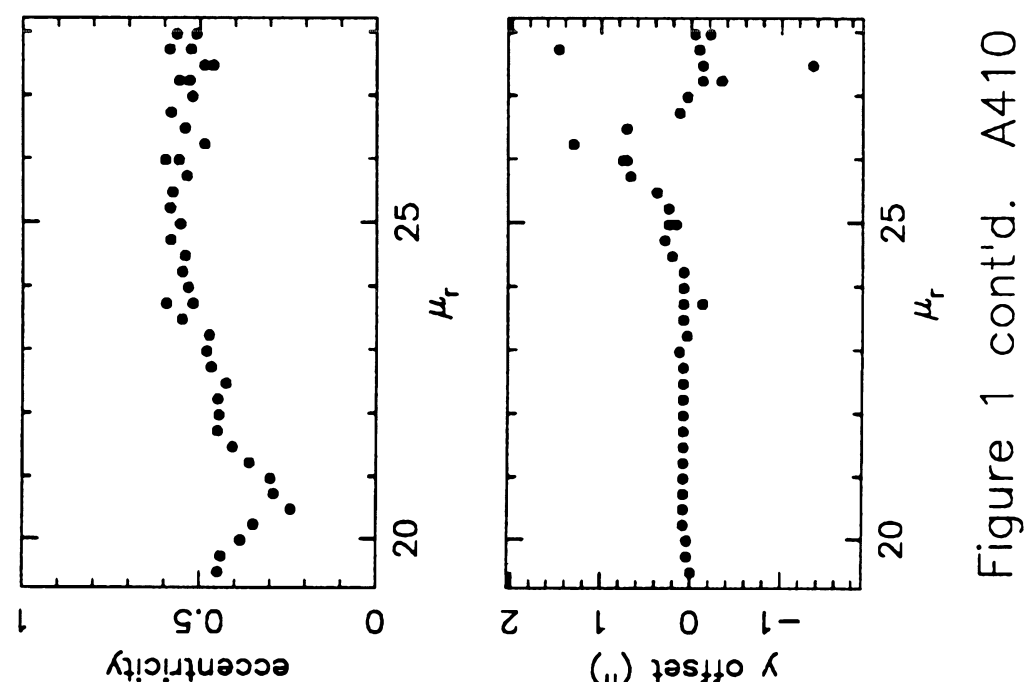

(.1) zas $\$ 10 \mathrm{~K}$

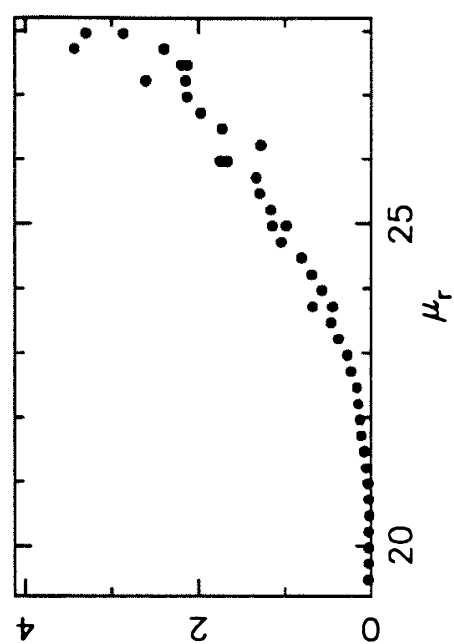

(.1) $) !+10$ oub!s ano
$\Sigma^{2}$

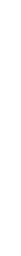

$\frac{0}{4}$
$\frac{8}{4}$
0
0
$\frac{5}{0}$
0
-
0
$\frac{1}{3}$
$\frac{0}{1}$

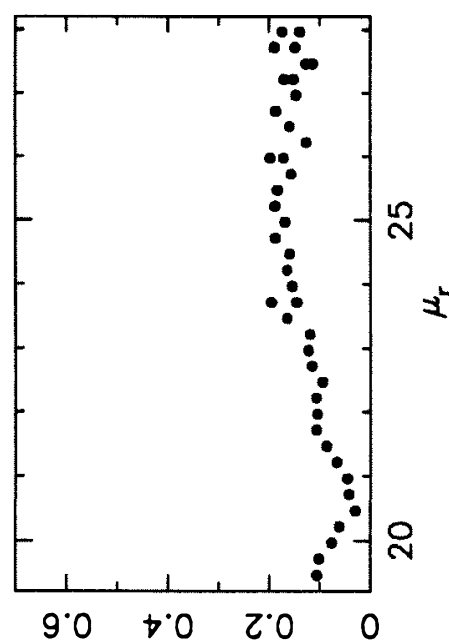

रł!?!子d!!|

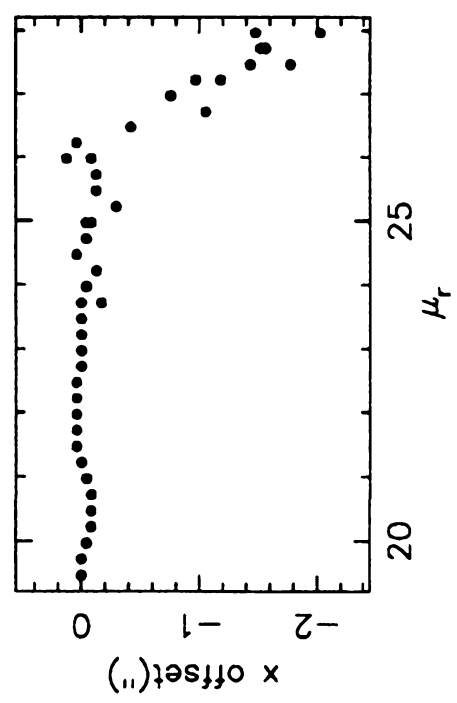



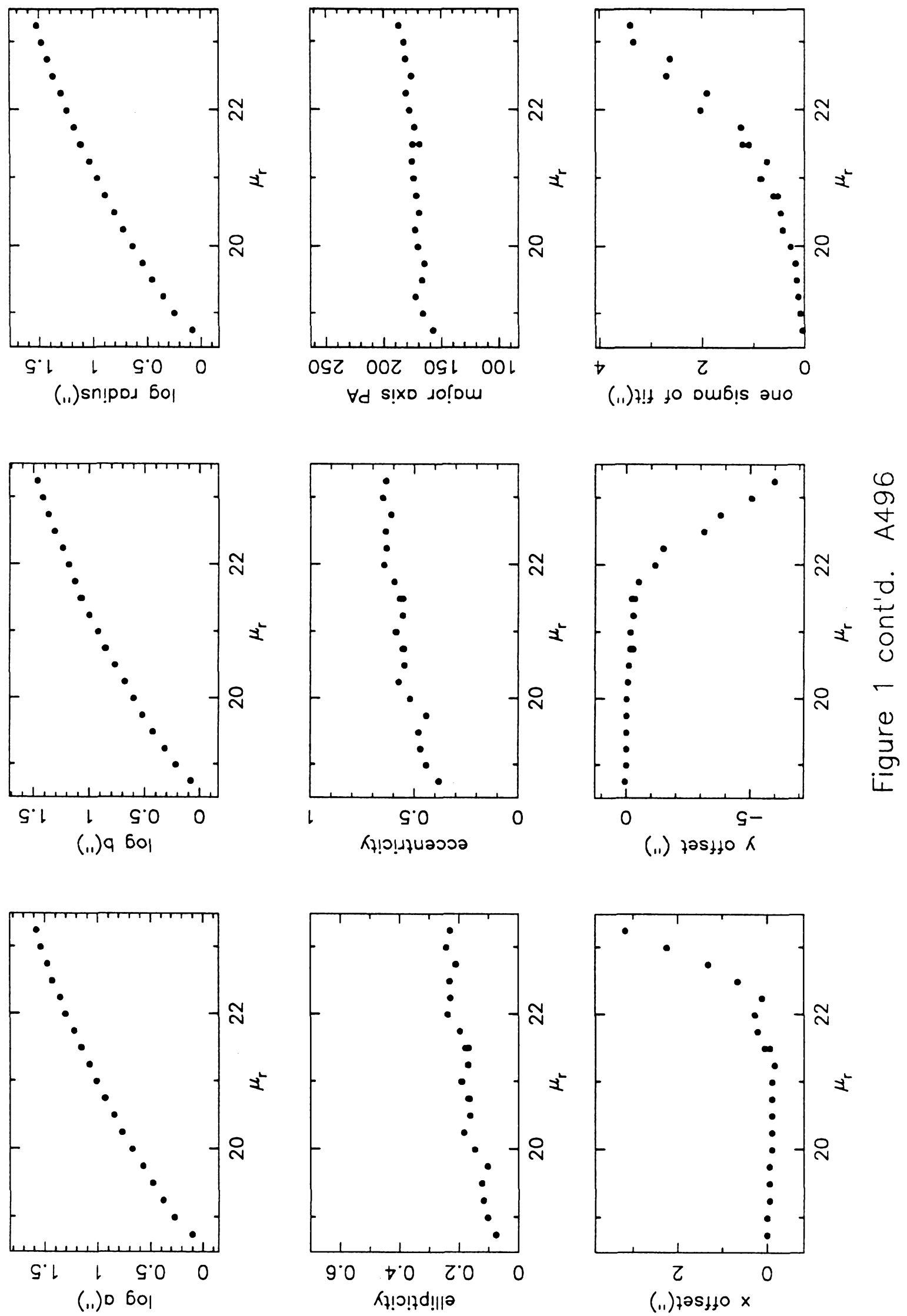


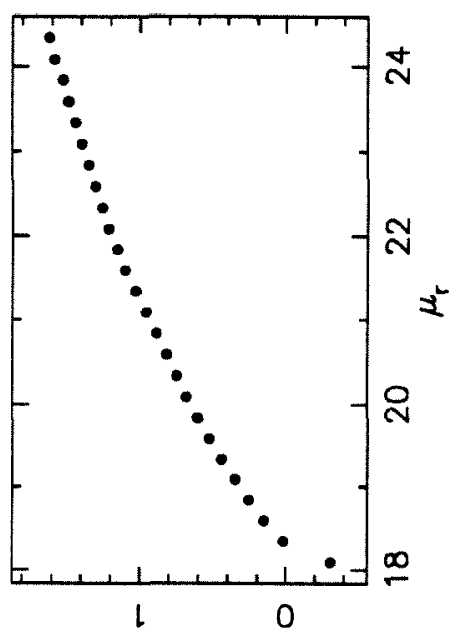

(.1)sn!pos 60l

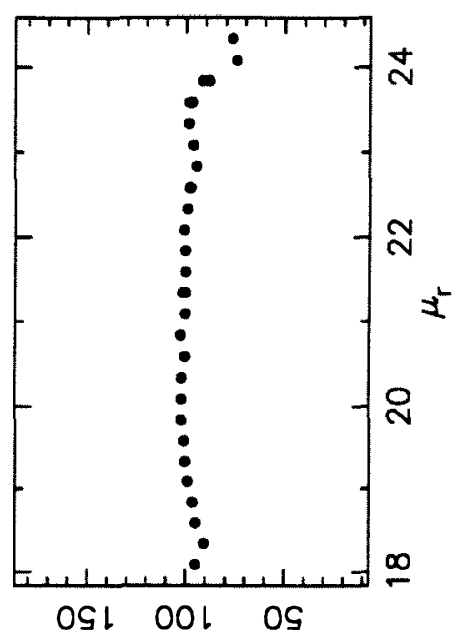

$\forall d$ s!xo so!́m

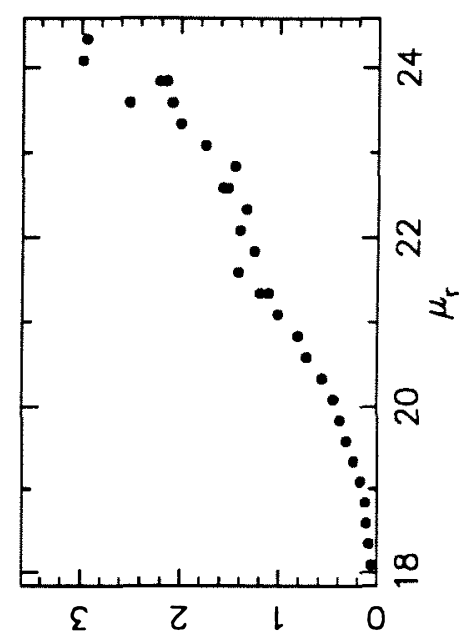

(11) $7 ! t$ to Dub! ano

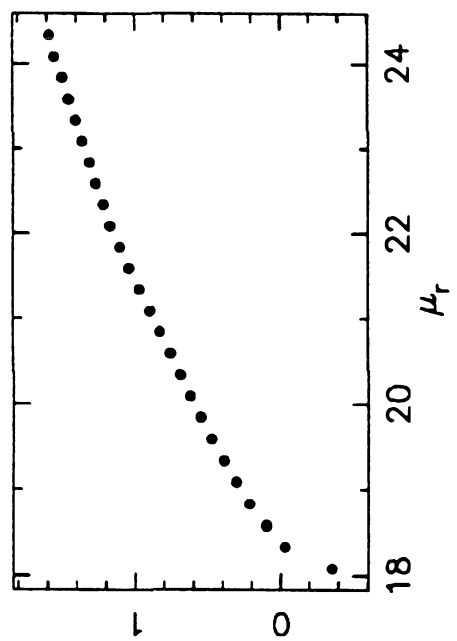

(.1) $9 \mathrm{bol}$

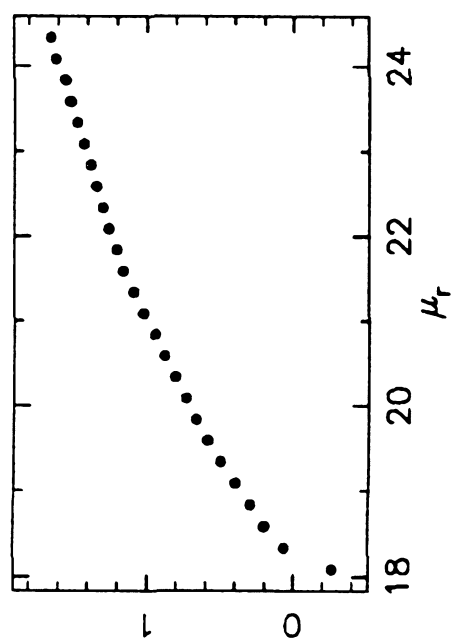

(i1) 0601

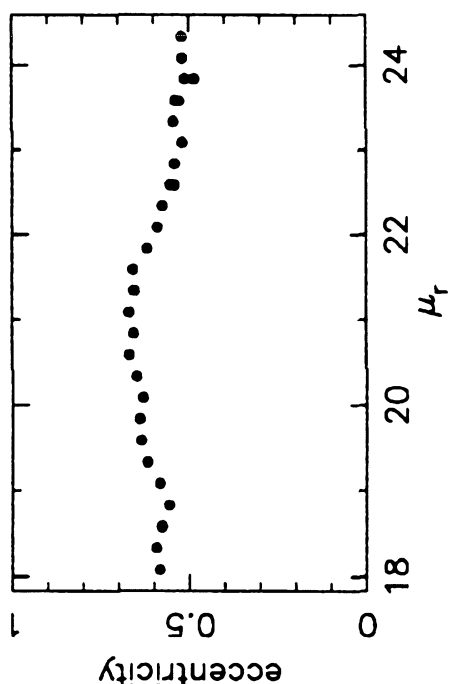

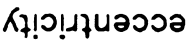

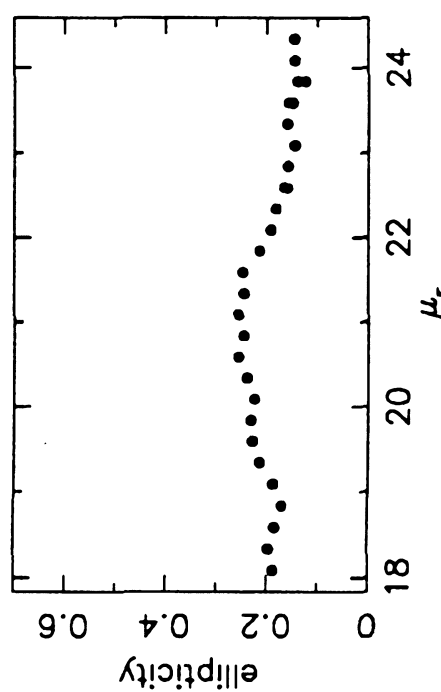

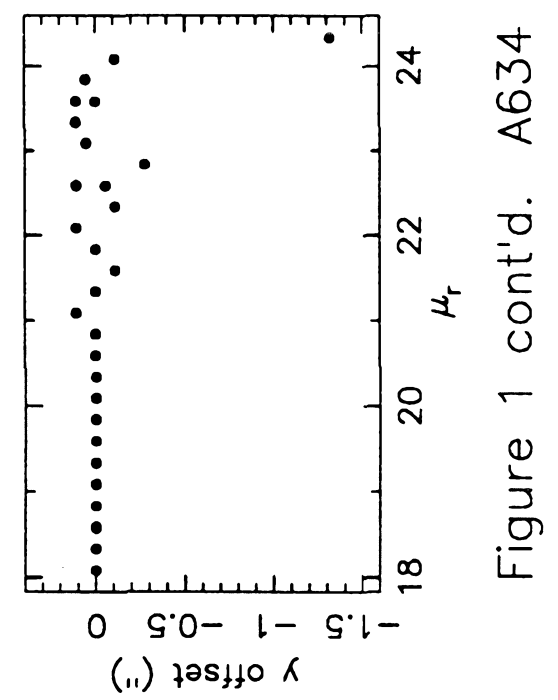

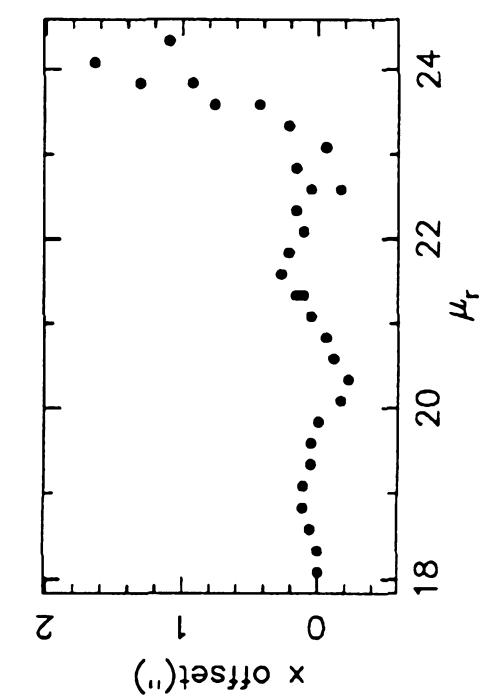




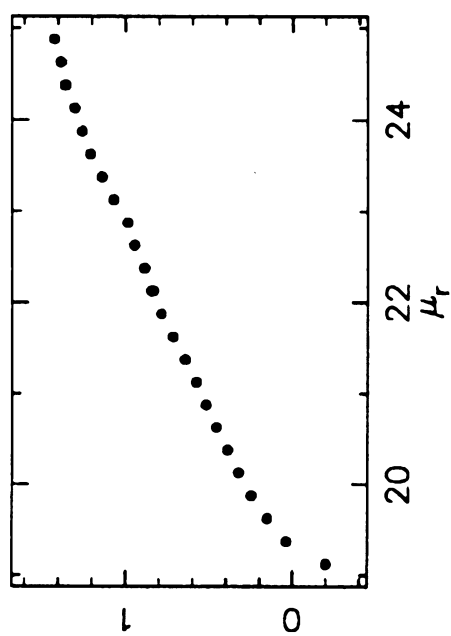

(.1) sn!pos 60।

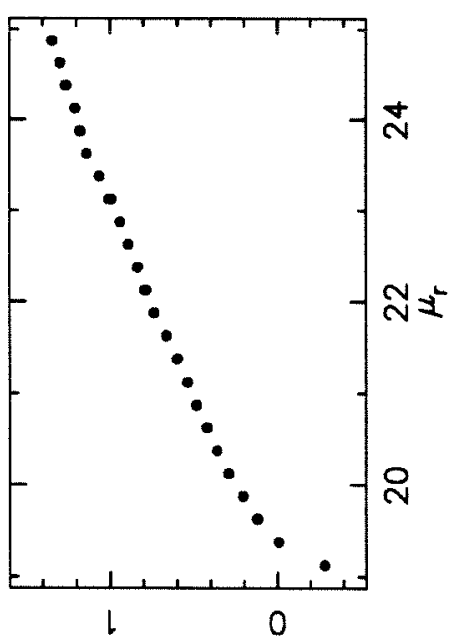

(.1) 9 bol

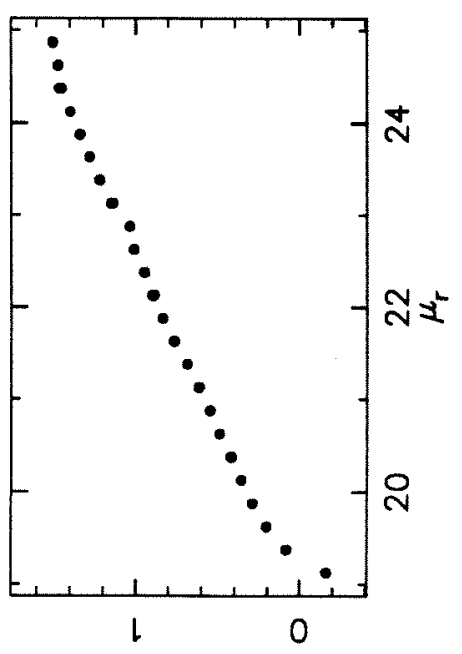

(i) 0 50이

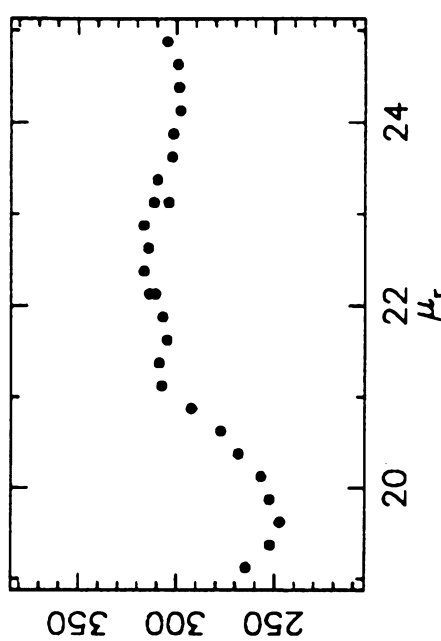

$\forall d$ s!xo دo! ow

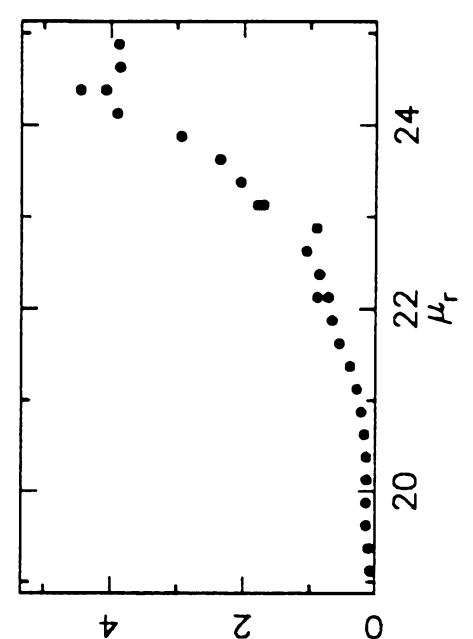

(i1) $4 !+$ to Dub!s au०

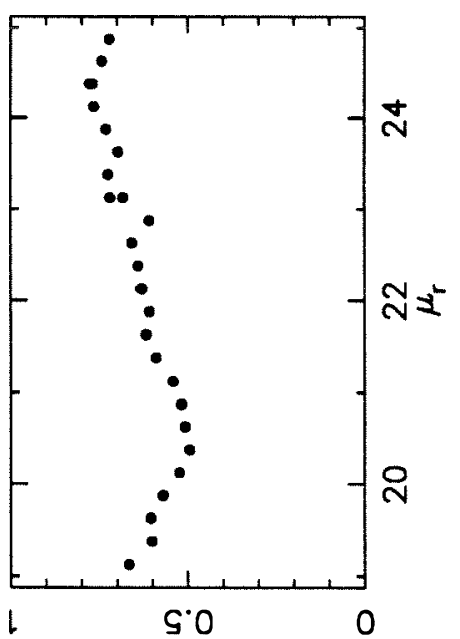

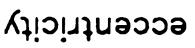

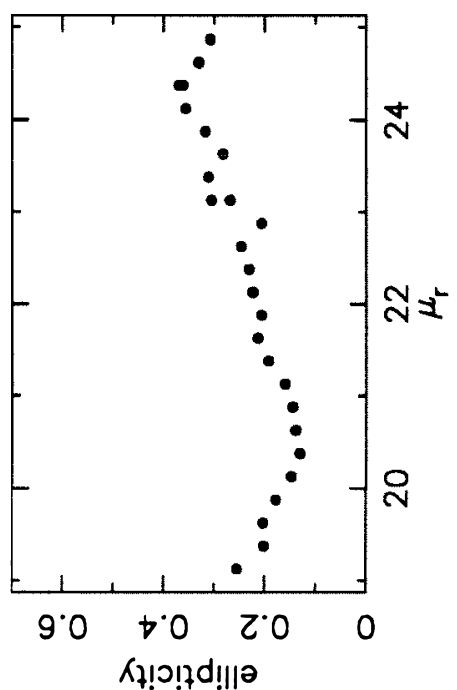

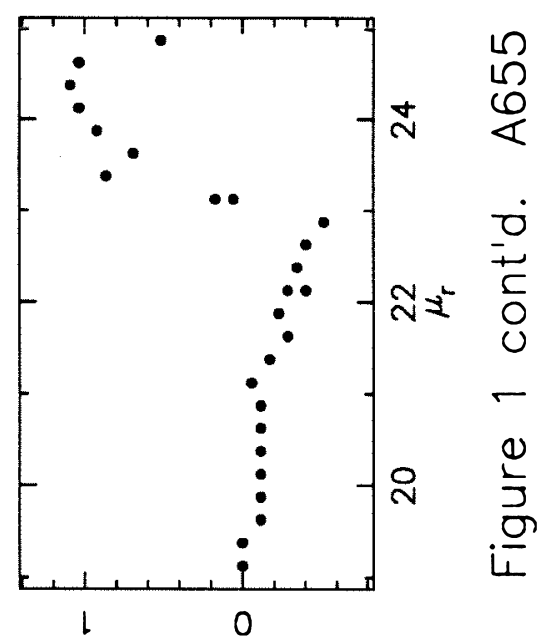

(i1) 7os $150 \mathrm{~K}$

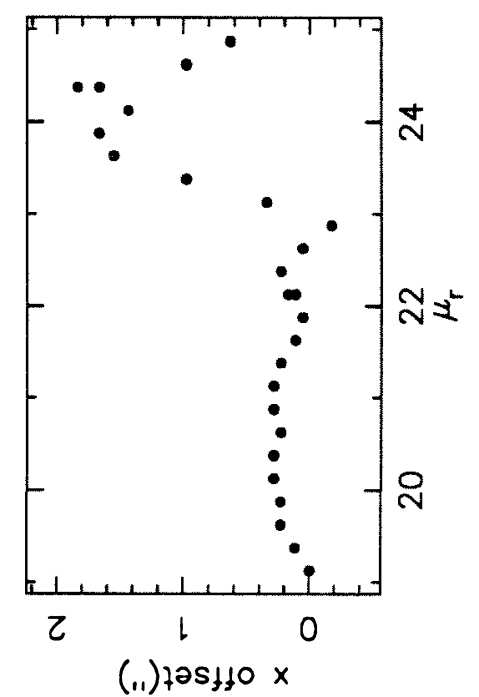



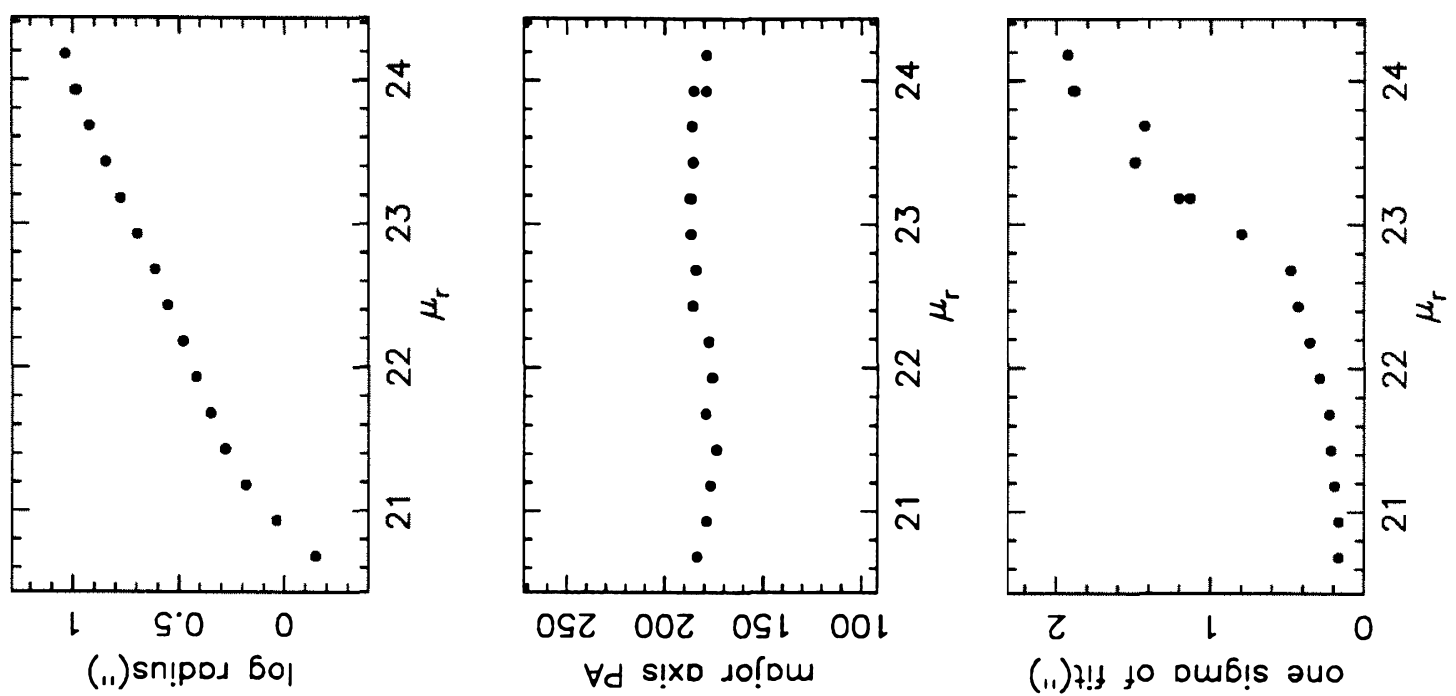

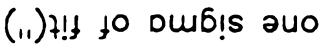

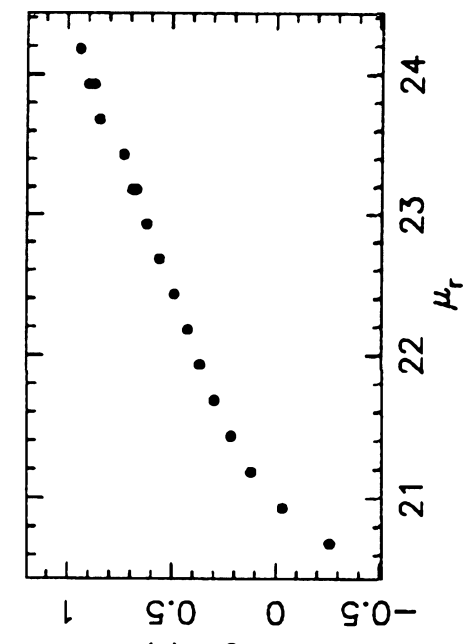

(.1) 9601

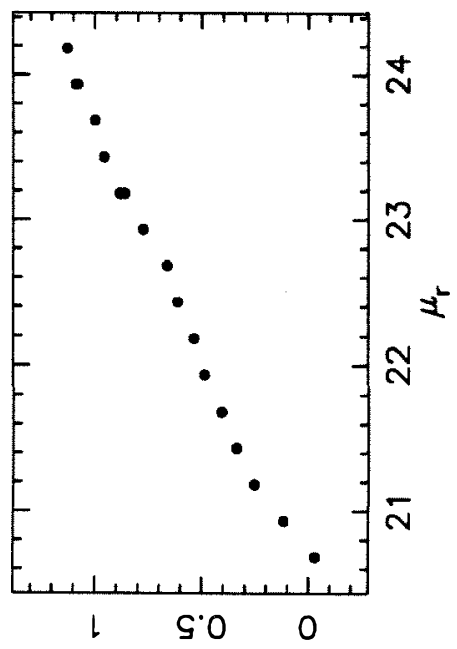

(.1) 0 6ol
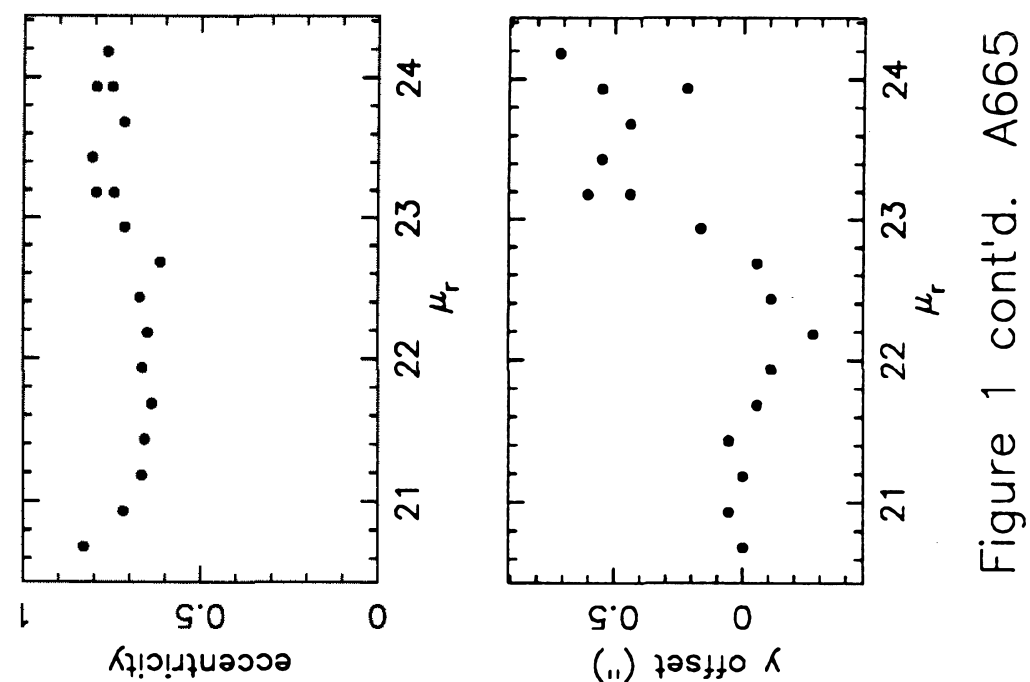
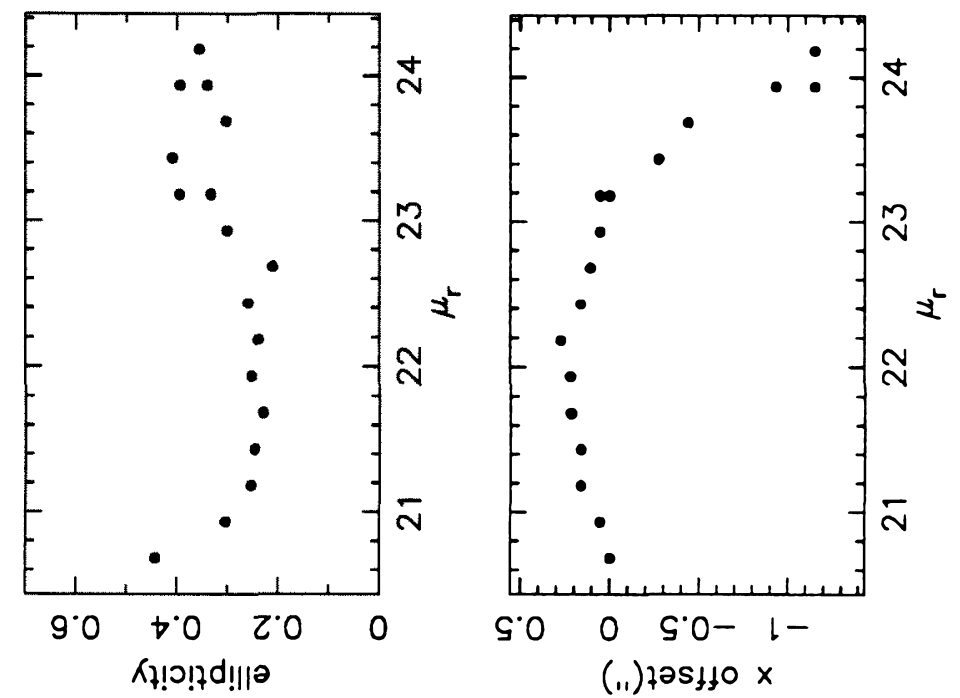

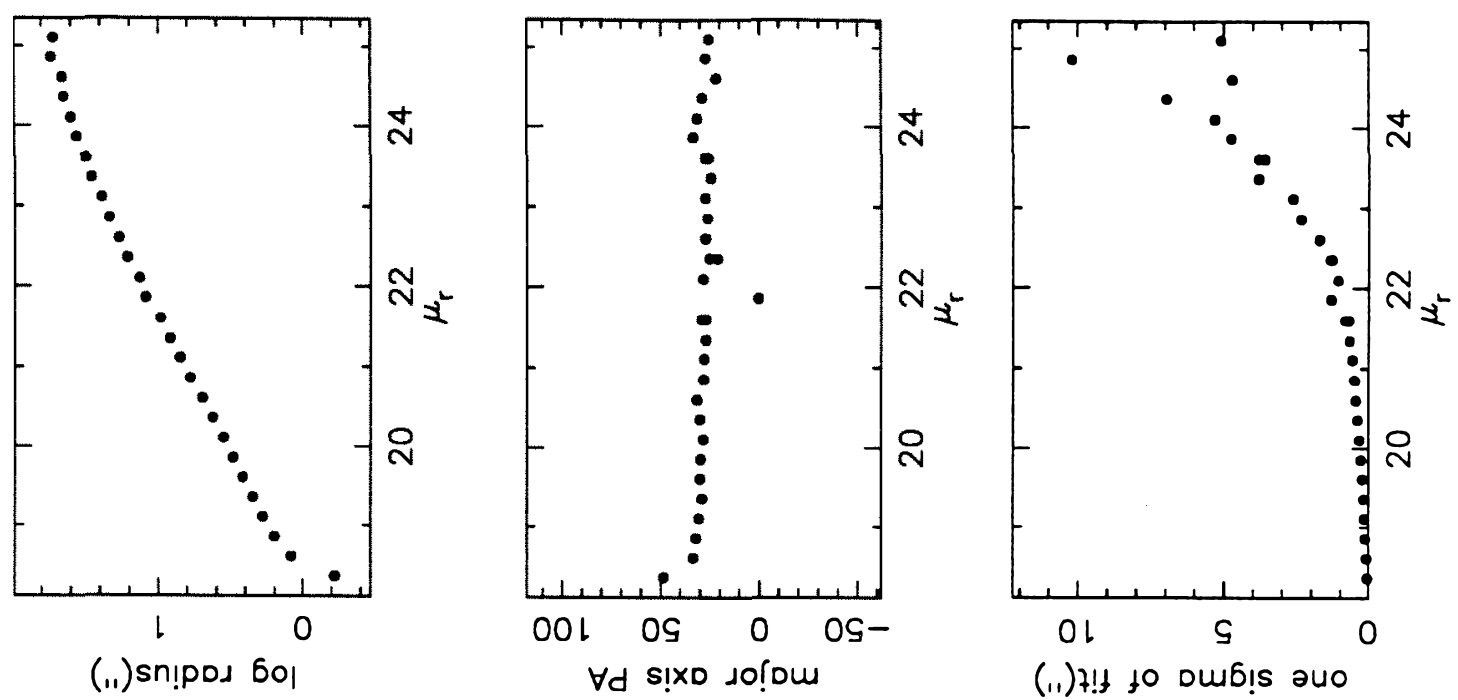

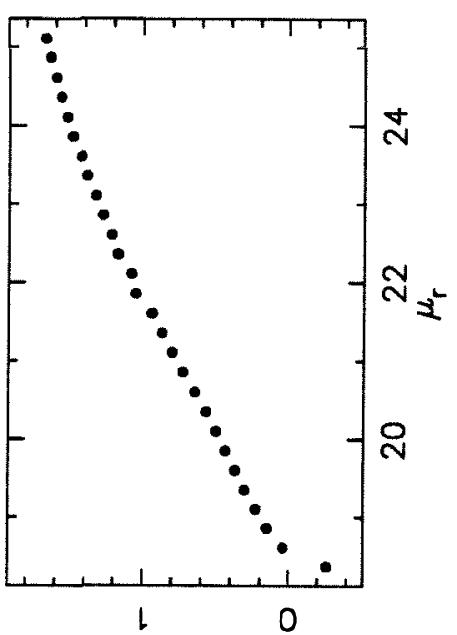

(.1) $9 \mathrm{bol}$
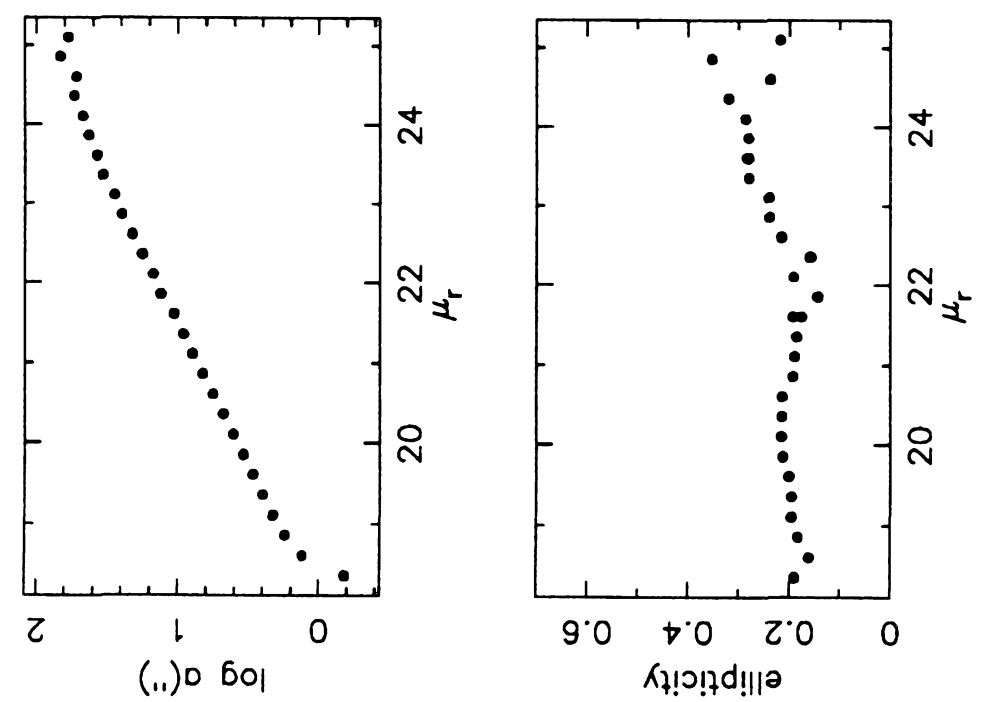

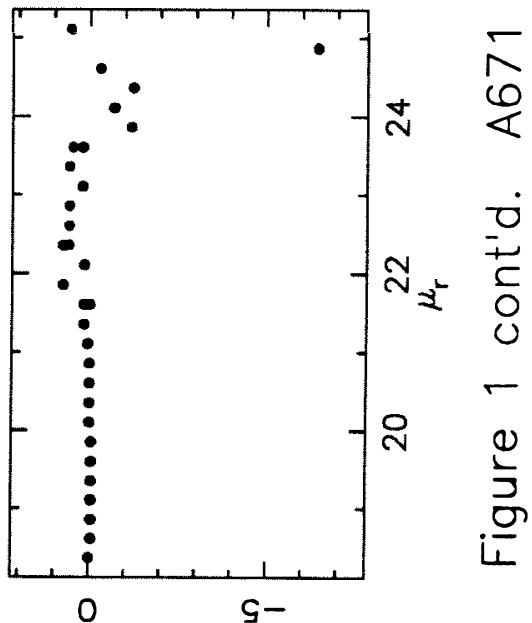

(i1) $725+10 \mathrm{~K}$

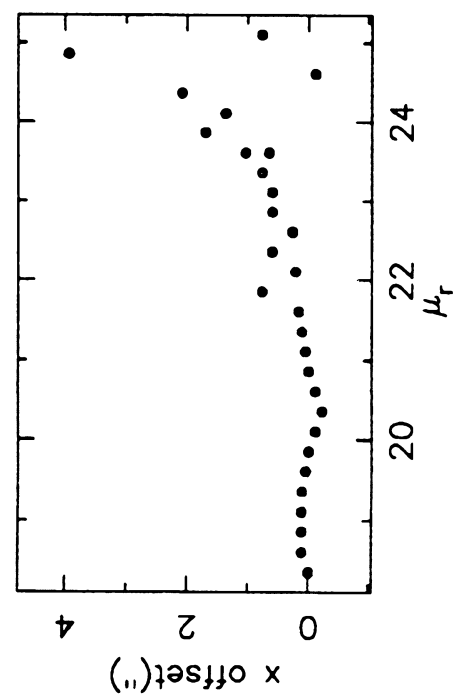




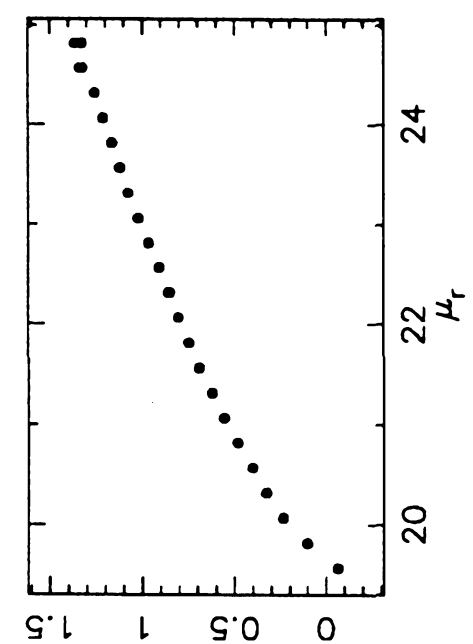

(.1)sn!pos 6ol
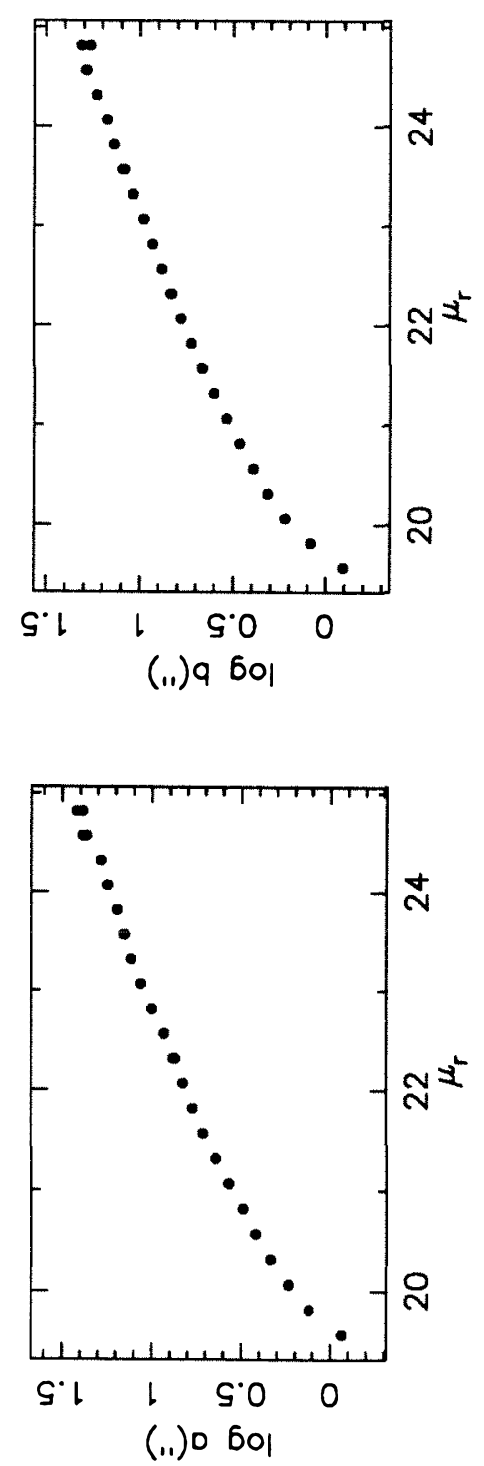

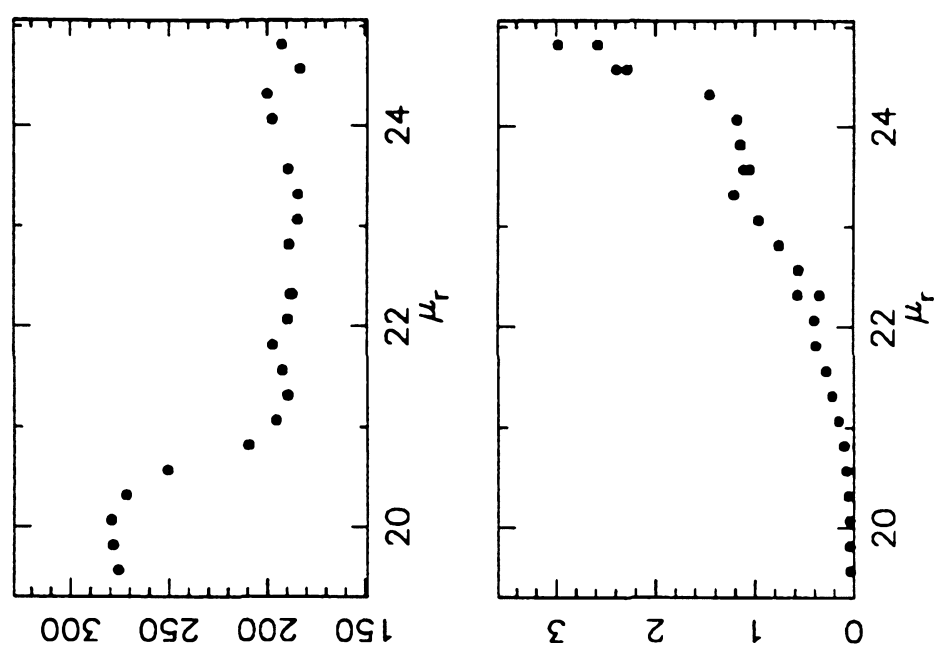

$\forall d$ S!Xo so!

(i1) 7 !t to oub!s auo
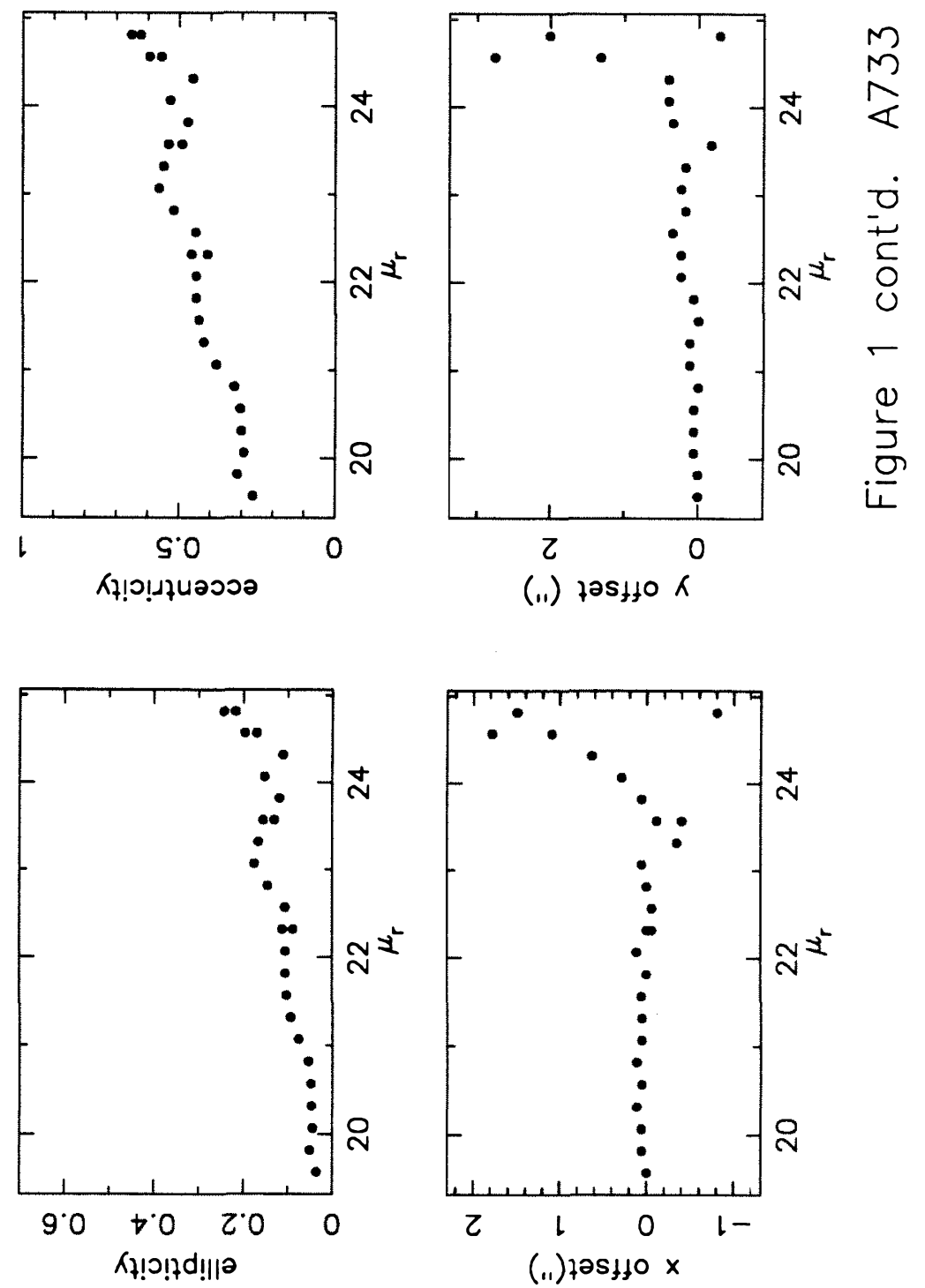


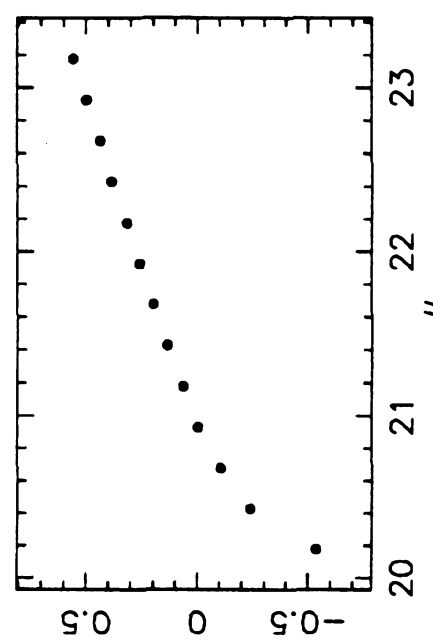

(.1) sn!pod 6o|
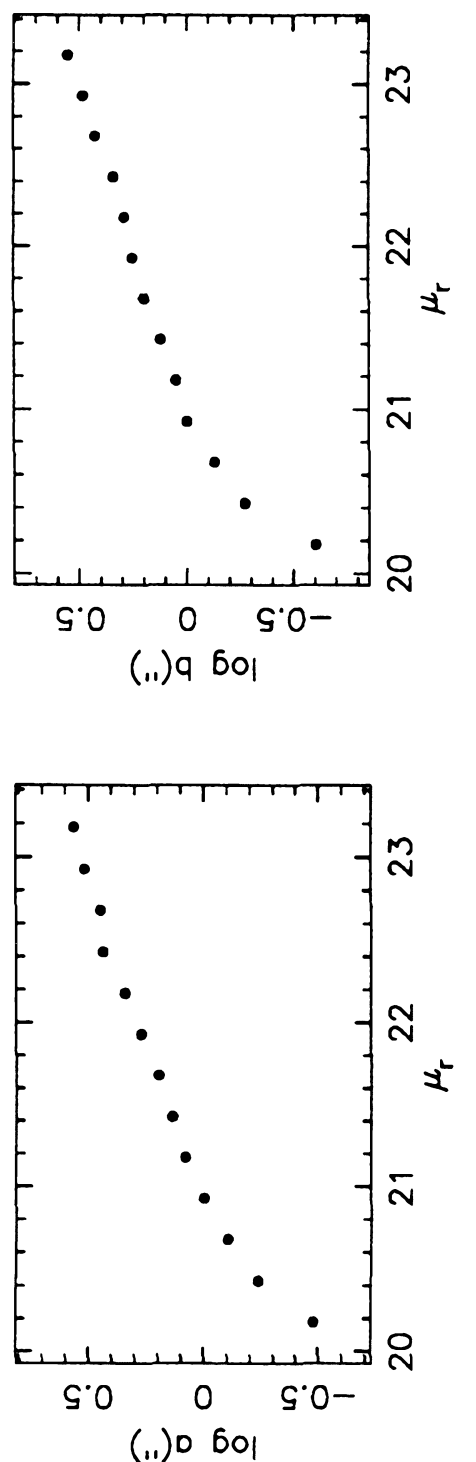

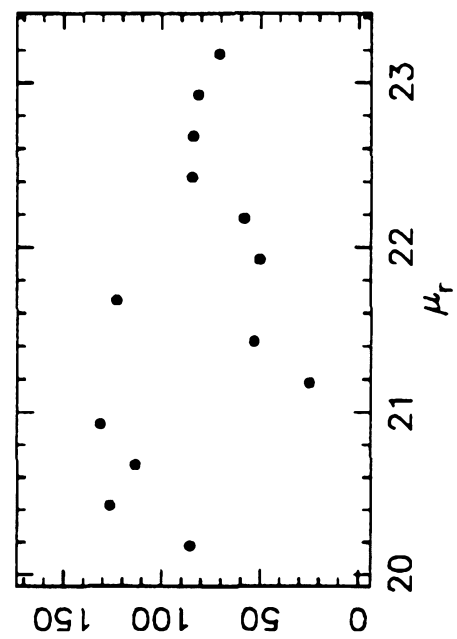

$\forall d$ s!xo so!ou

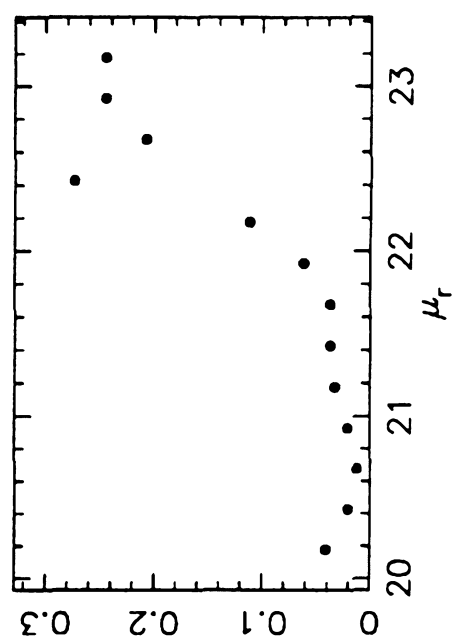

(i1) $7 !$ t to oub!s auo
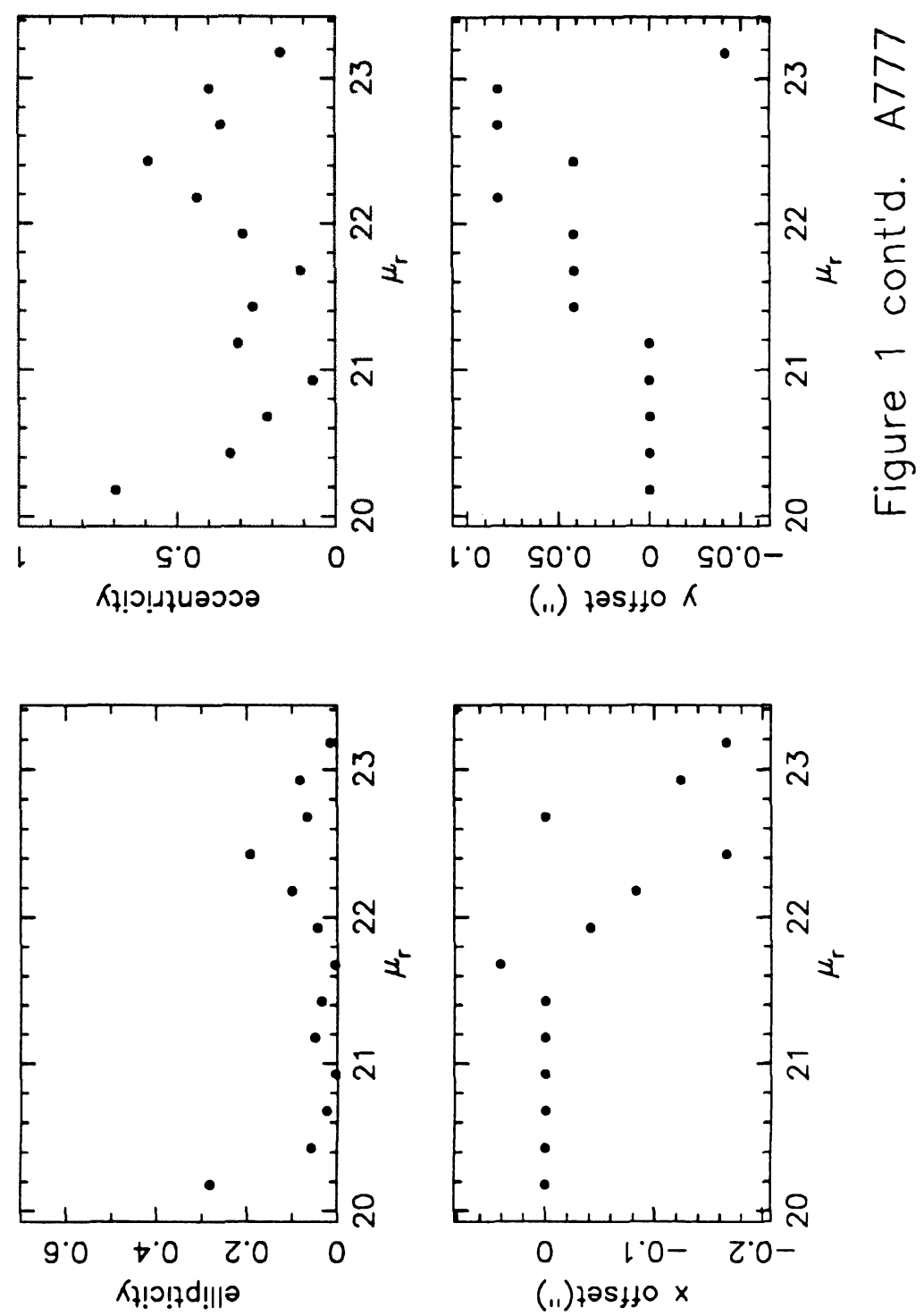

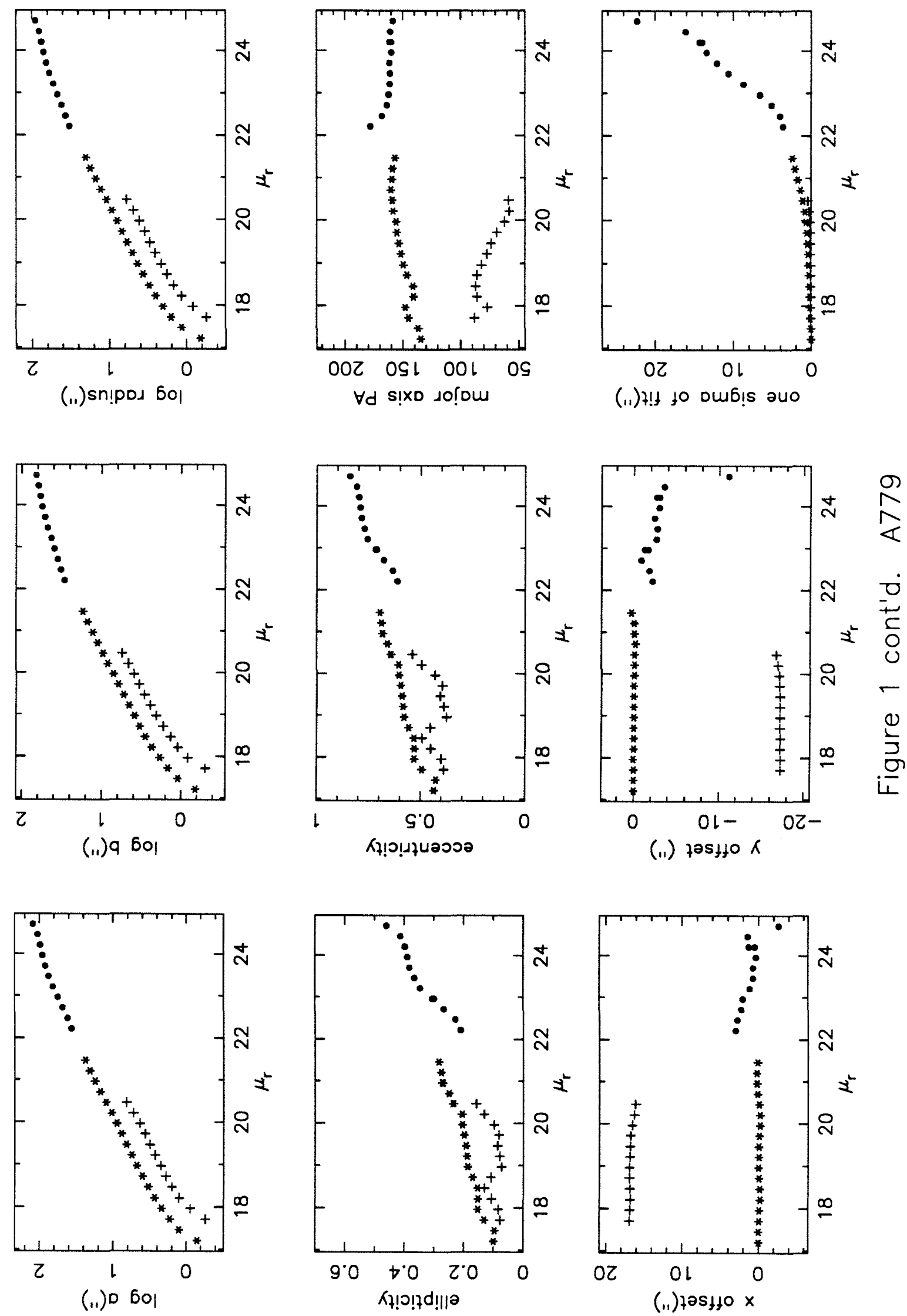


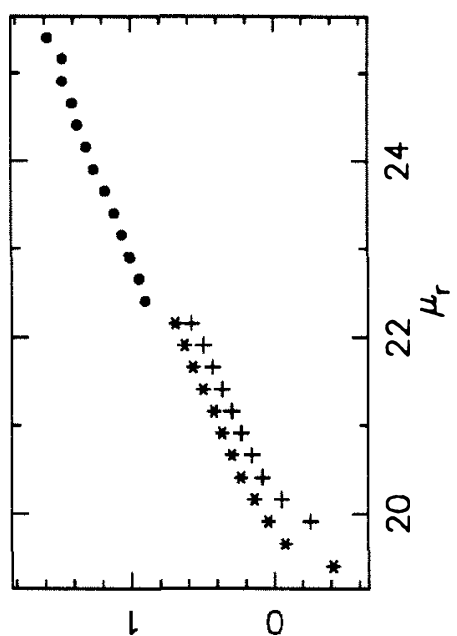

(11)sn!pod 6ol

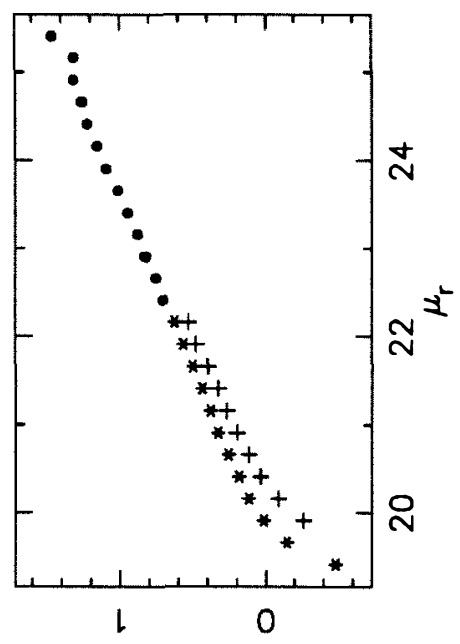

(.1) 9 5ol

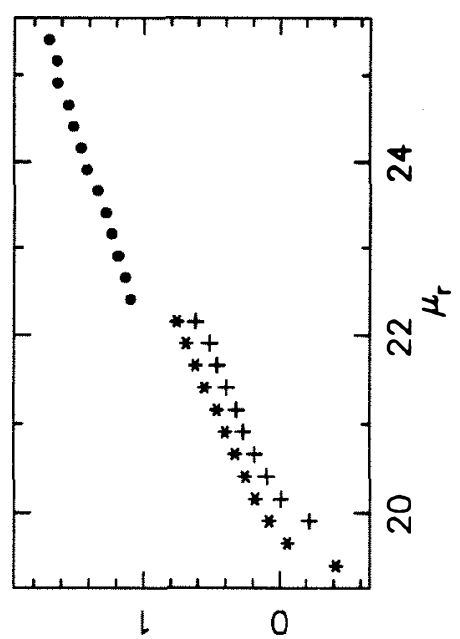

(.1) $0 \mathrm{60}$

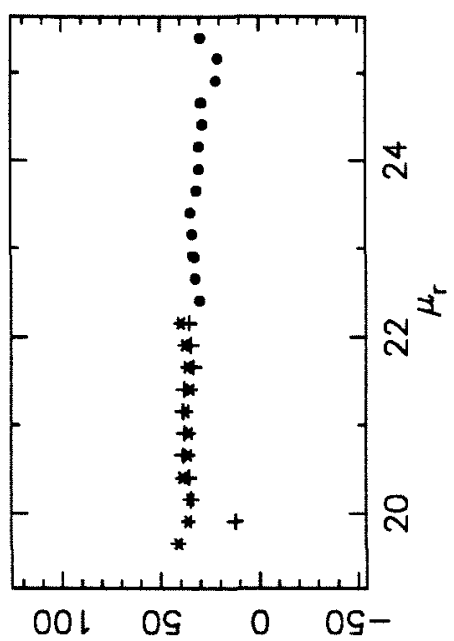

$\forall d$ s!xo do!̣u
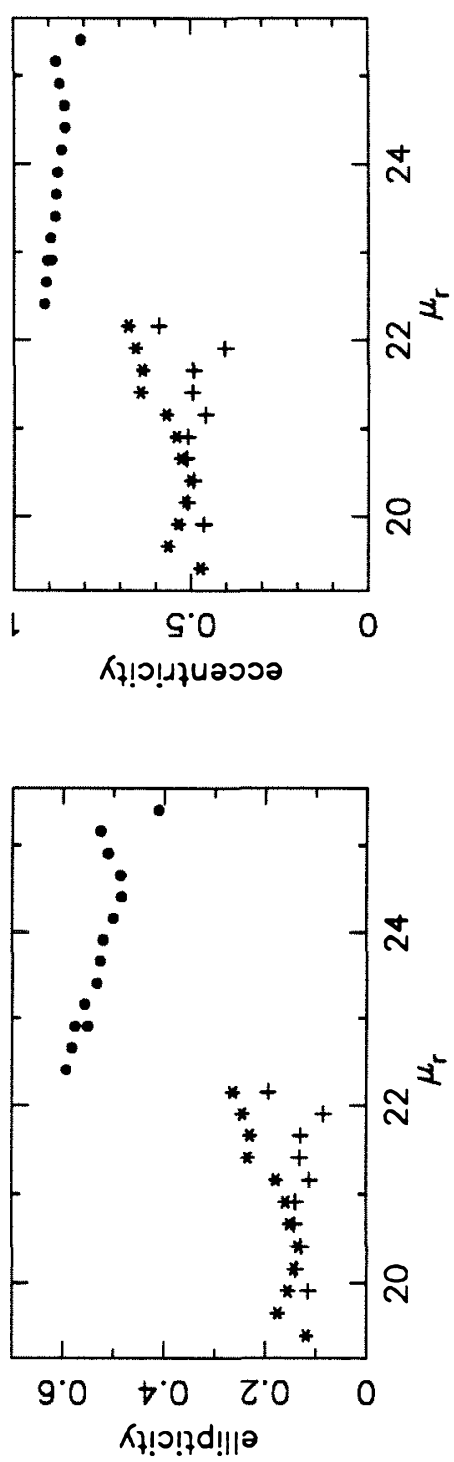

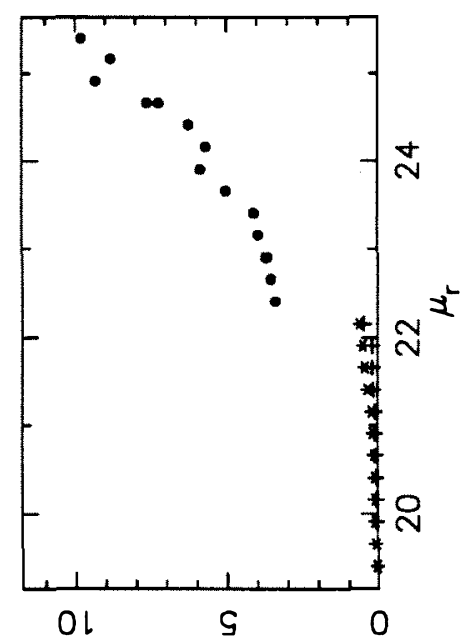

(.1) $+! t ~ t 0$ oub! avo 


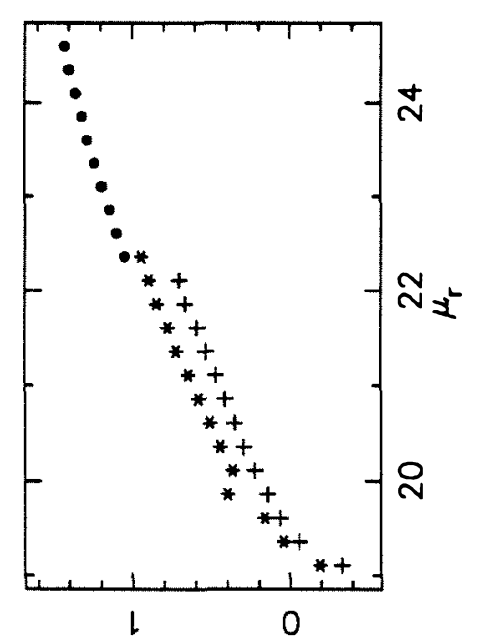

(.1)sn!pos 60।
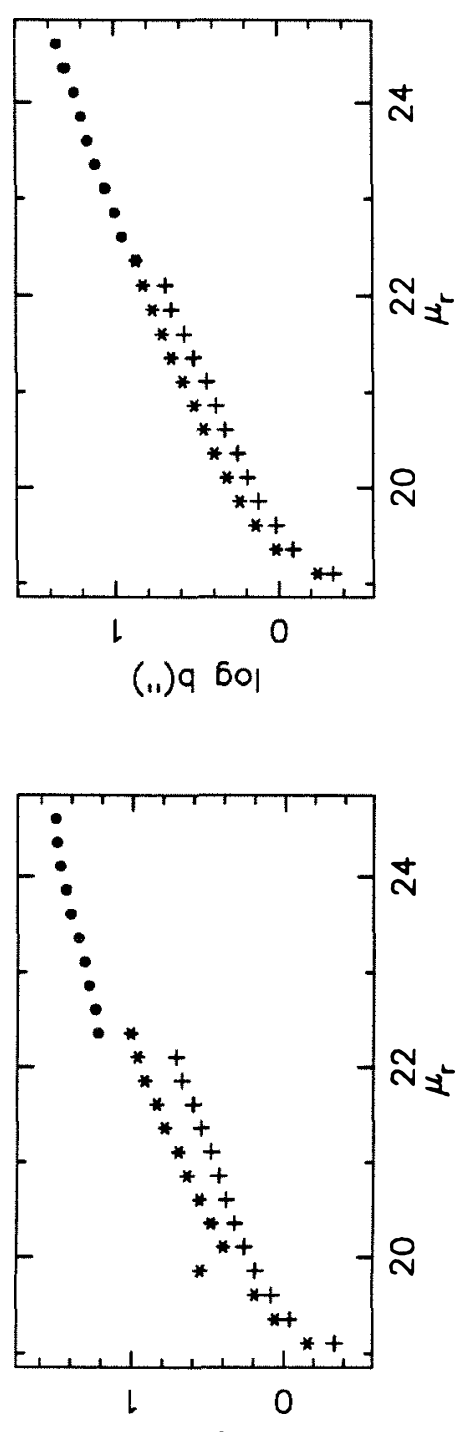

(.1) 0 601

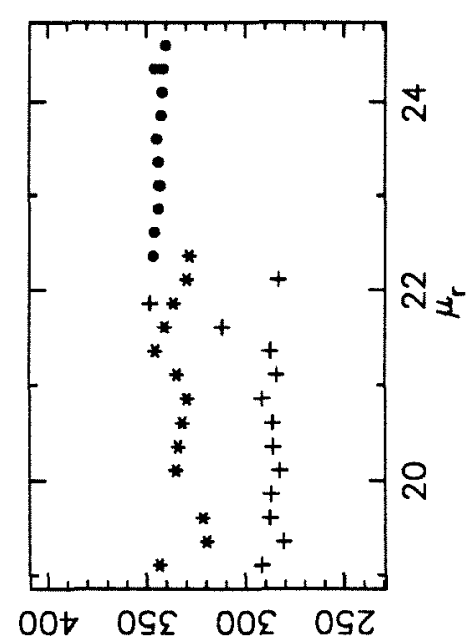

$\forall d$ s!xo jo! om

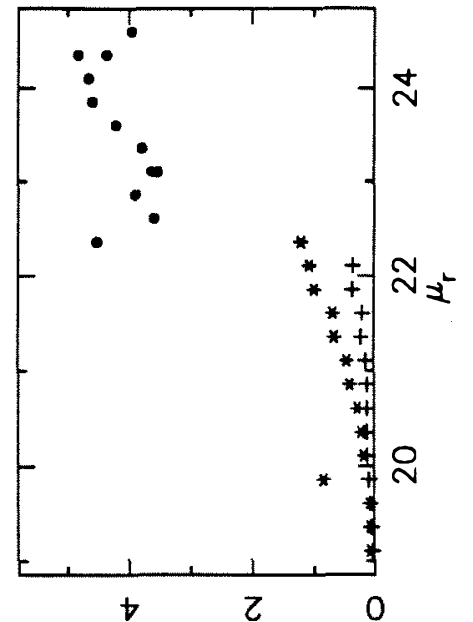

(11) t! to oub!s auo
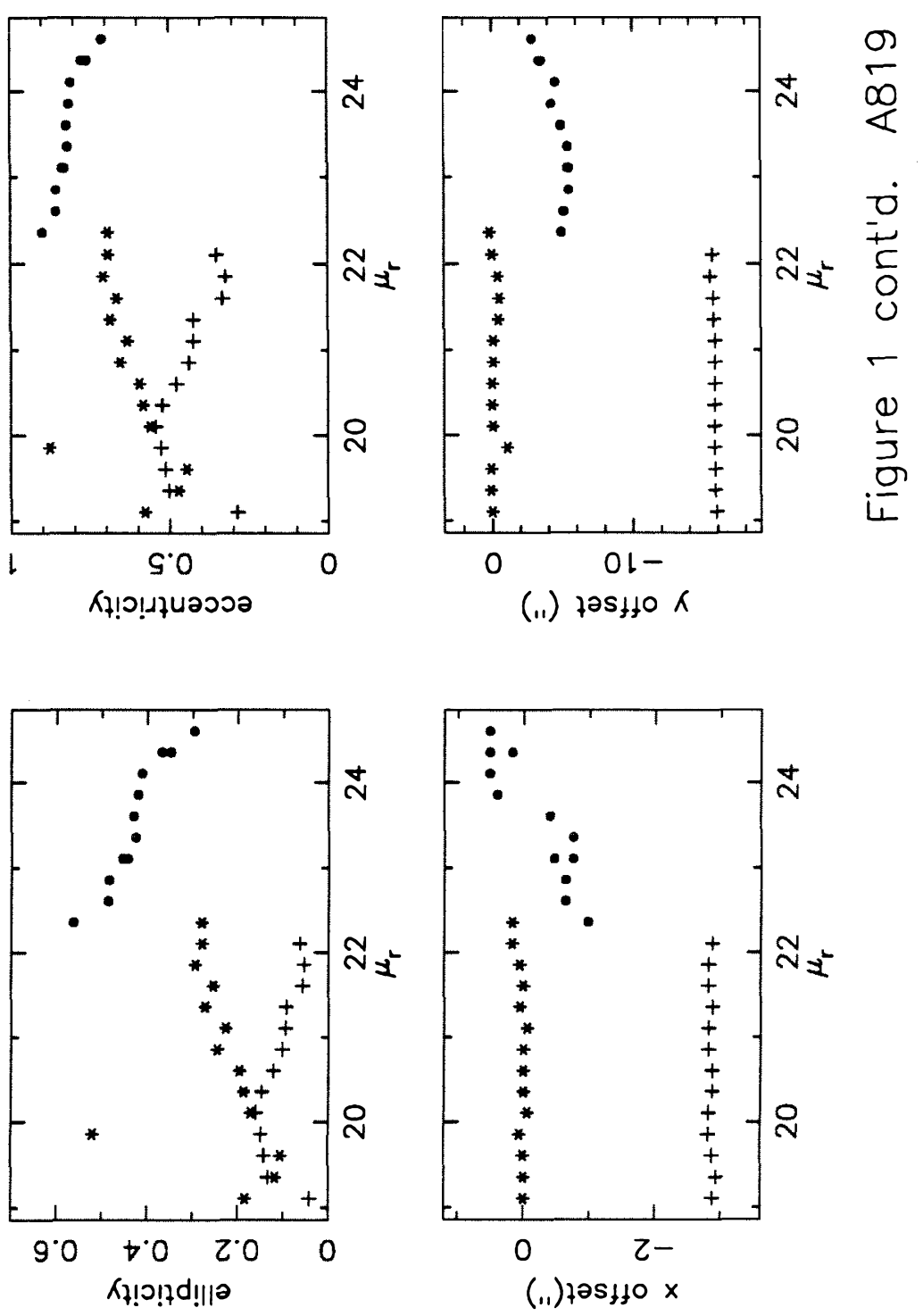


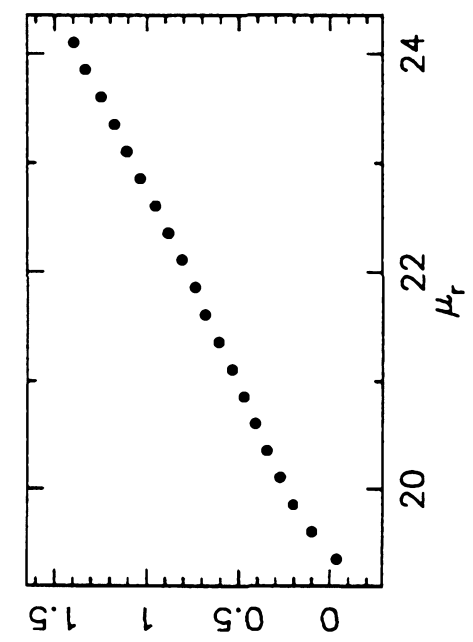

(.1)sn!pod 6ol

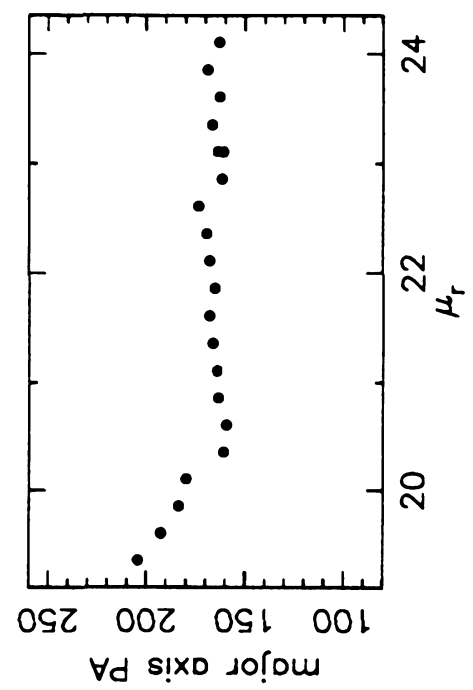

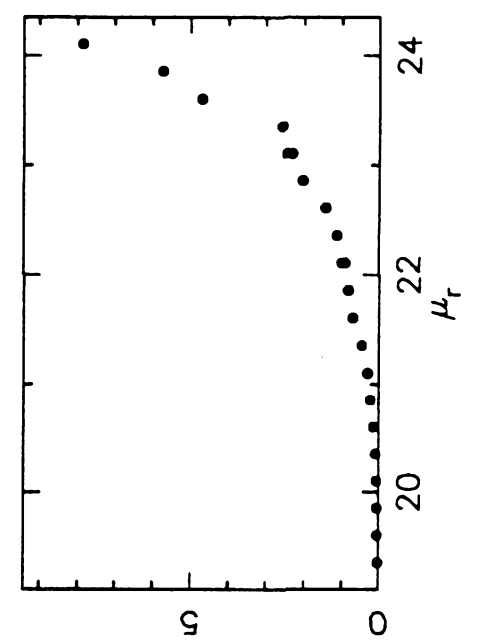

(11) $4 ! t$ to oub!s au०
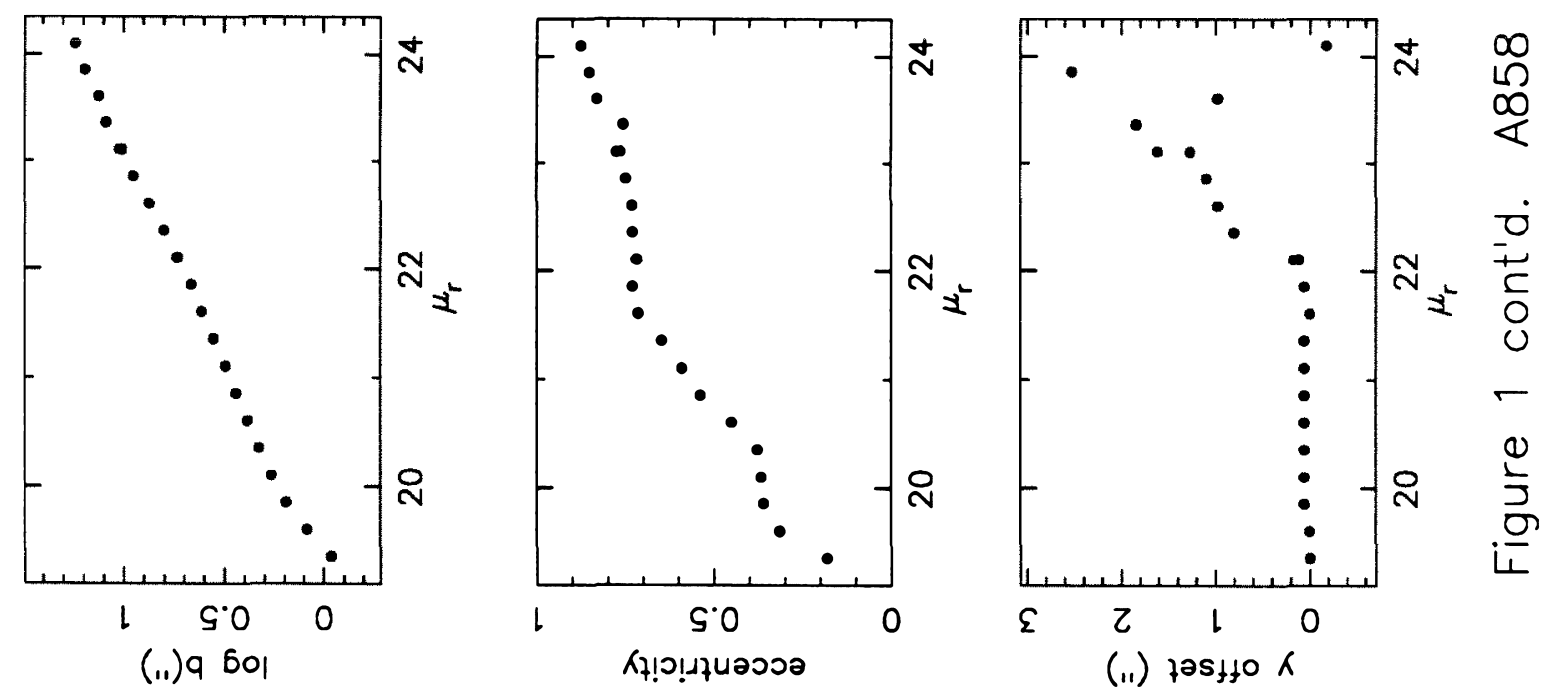

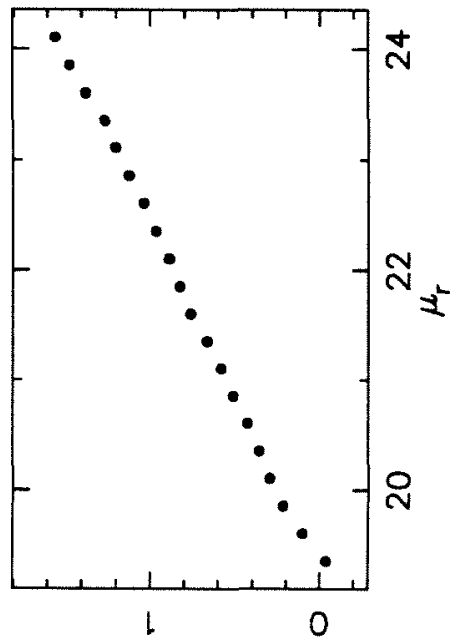

(i.) $\mathrm{D}$ 50
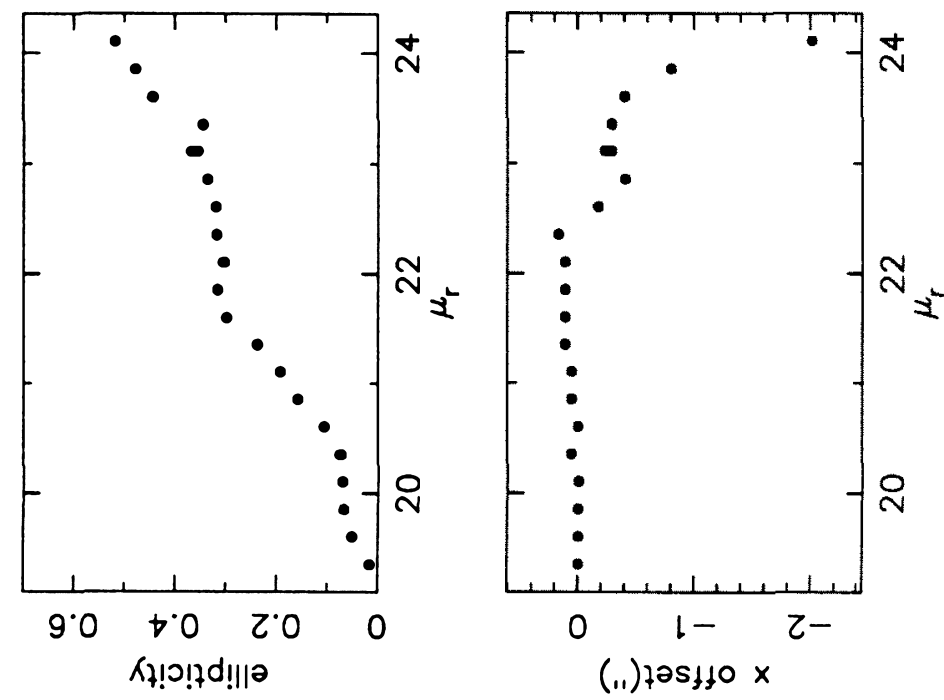


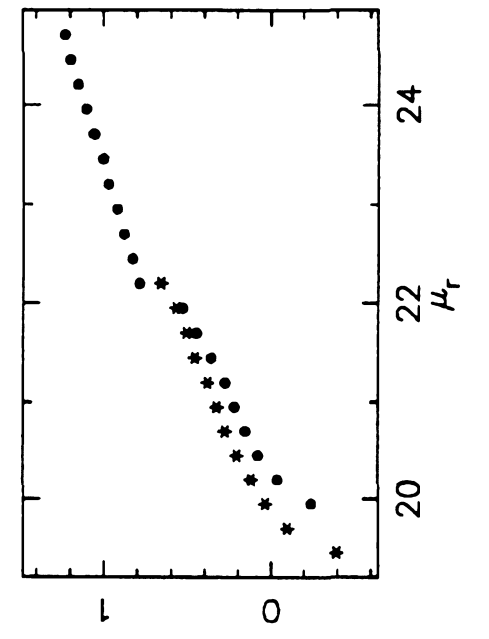

(.1)sn!pod 6ol
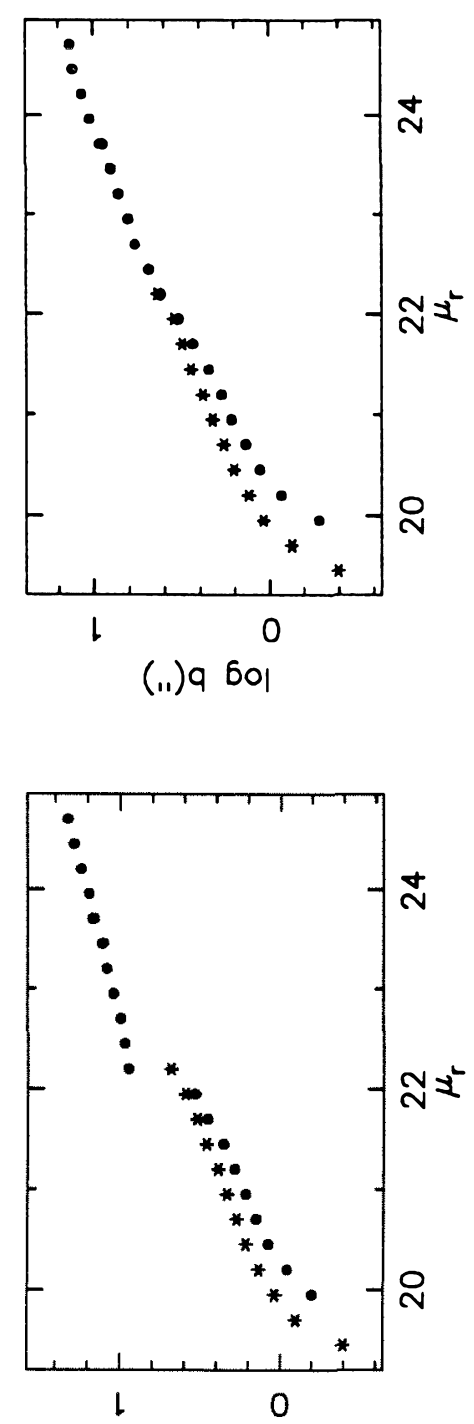

(.1) 0 6ol

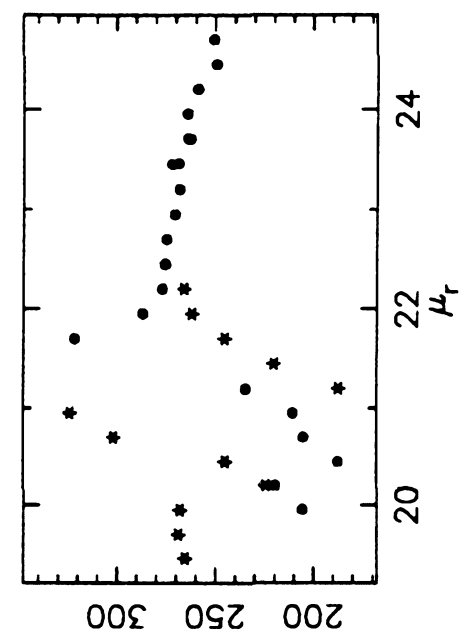

$\forall d$ s!xo so!

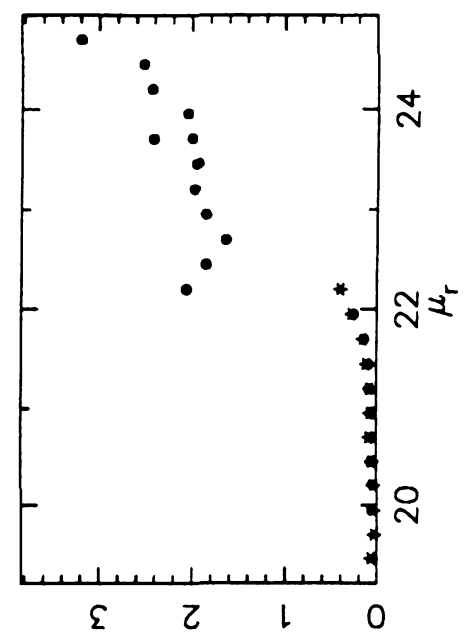

(11) $7 !+10$ oub!s auo
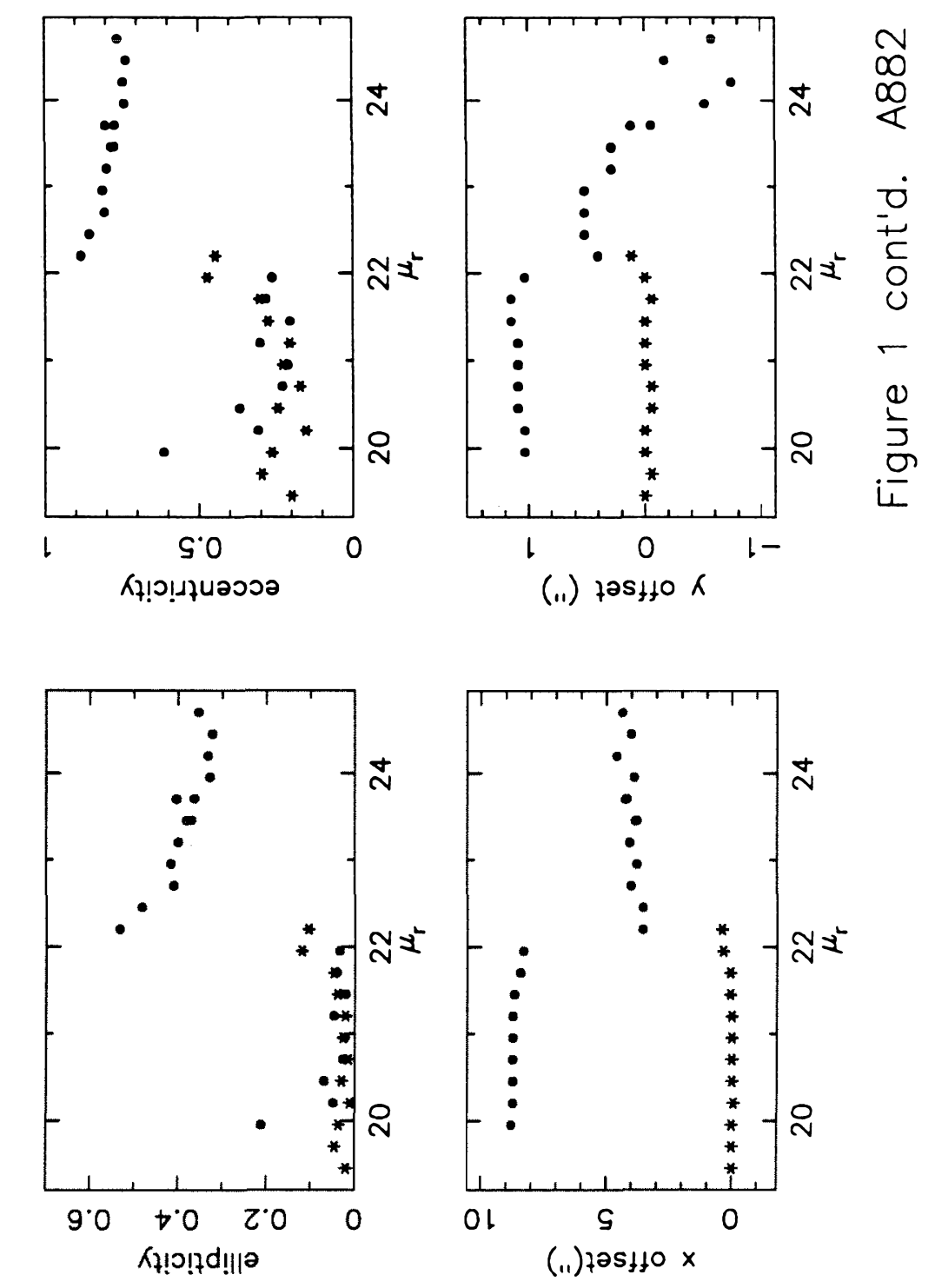


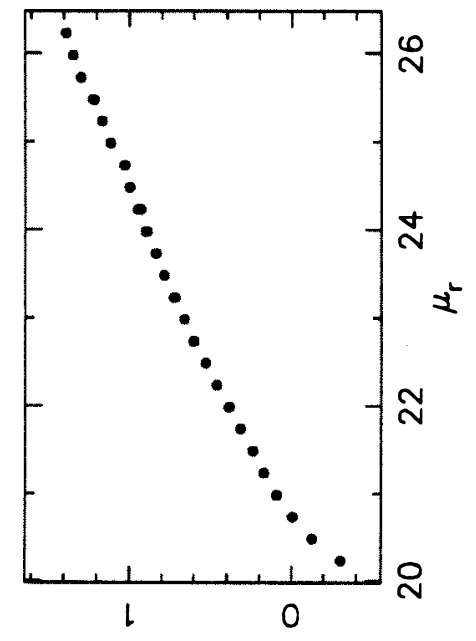

(.1)sn!pod 6ol

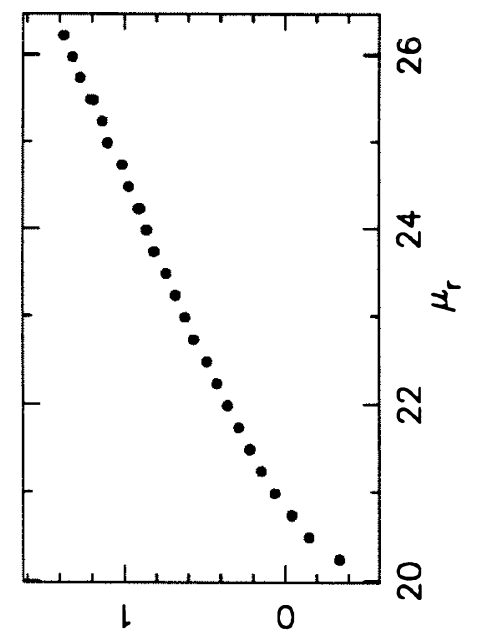

(.1) 960
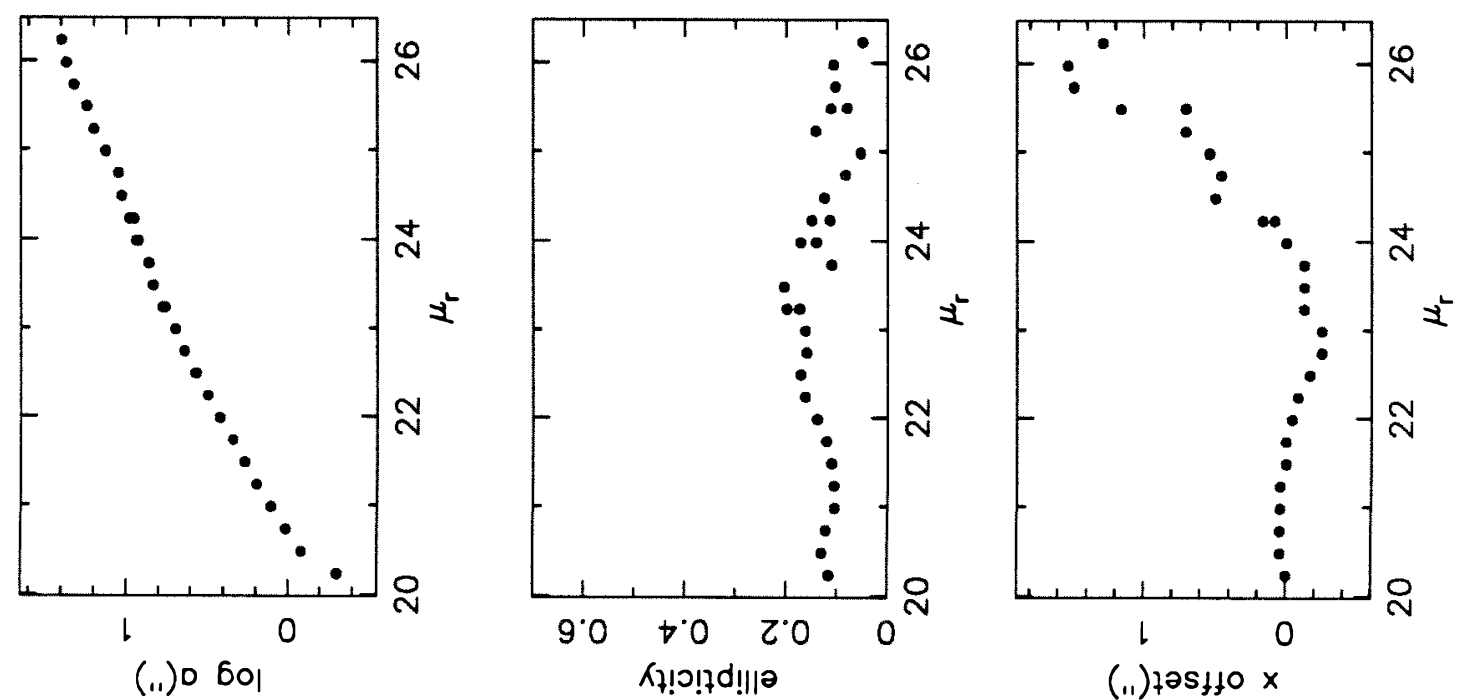
$-299-$
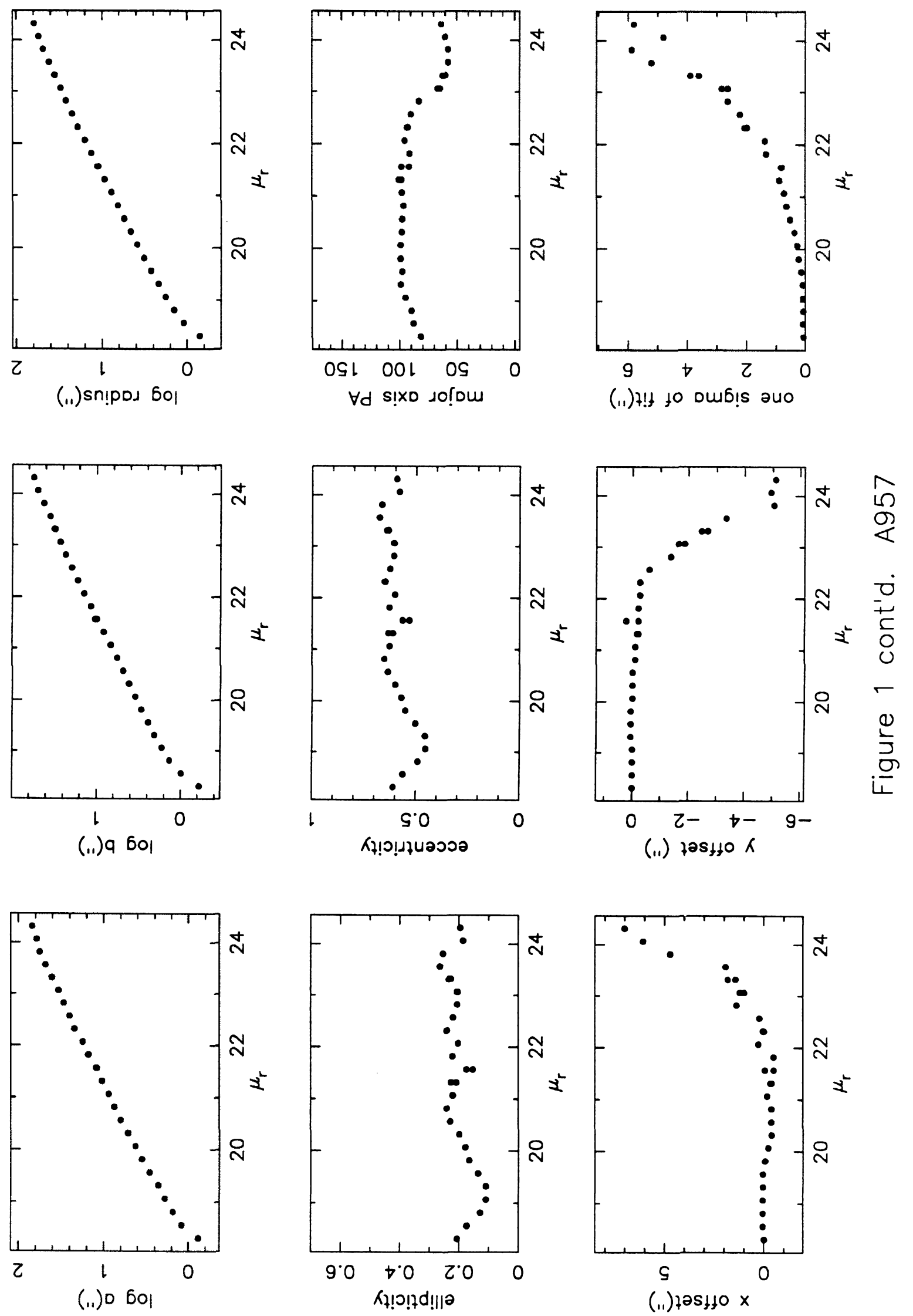


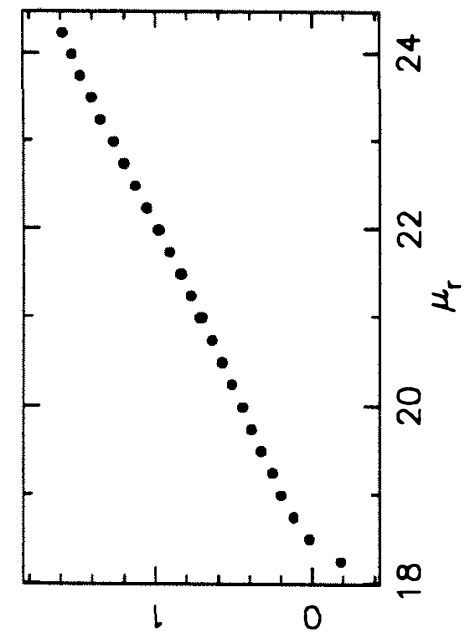

(.1) sn!pod 6 ol

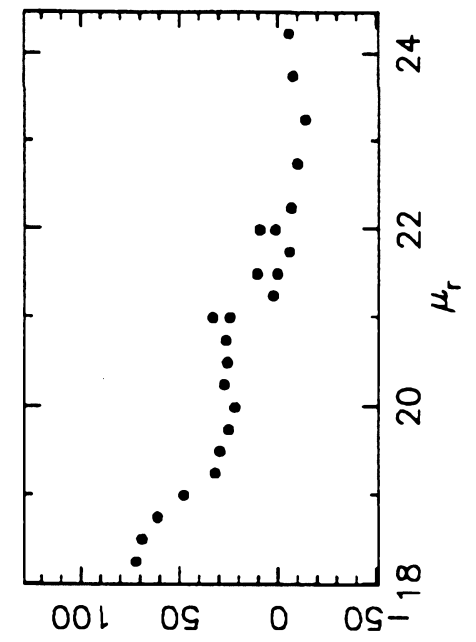

$\forall d$ S!xD dofom

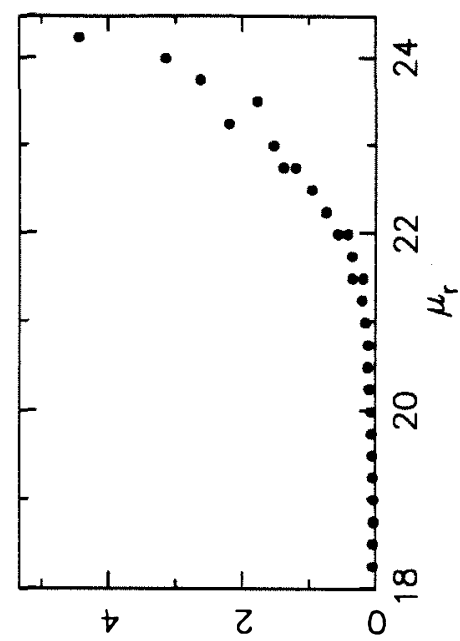

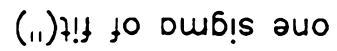

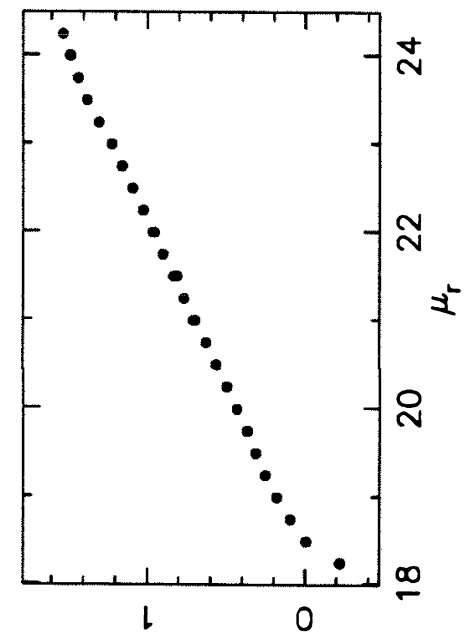

(..) 9 60이

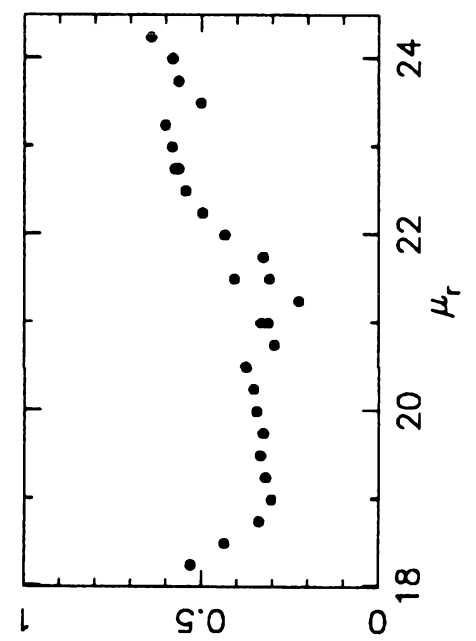

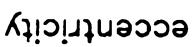

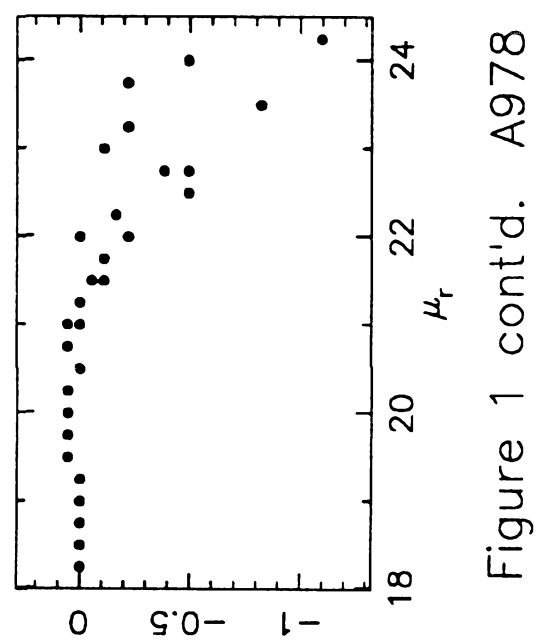

(i.) $725450 \mathrm{~K}$

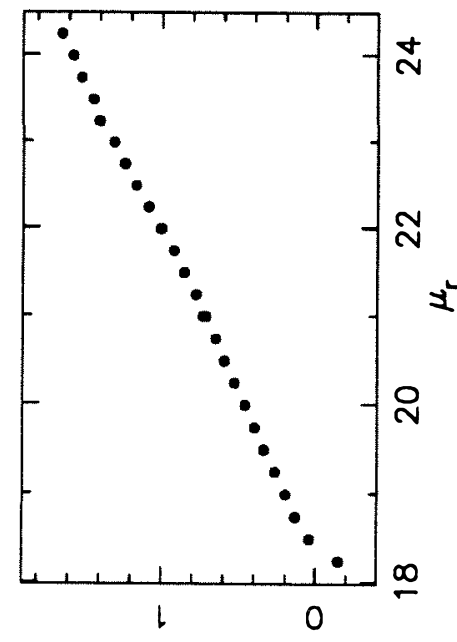

(..) 0 bol

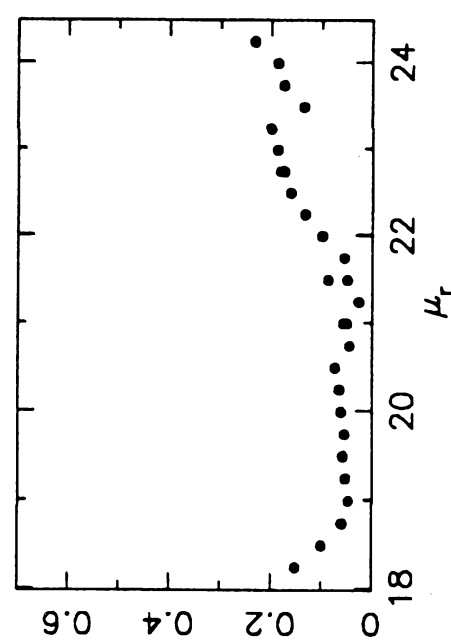

रฤ!ก!ฺd!||ว

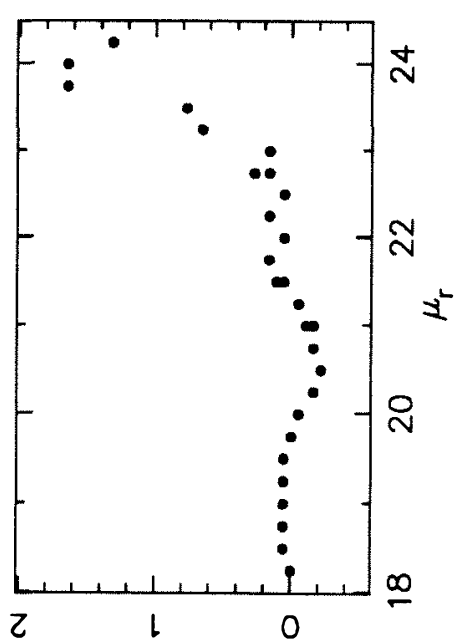

(.1) $725150 \times$ 


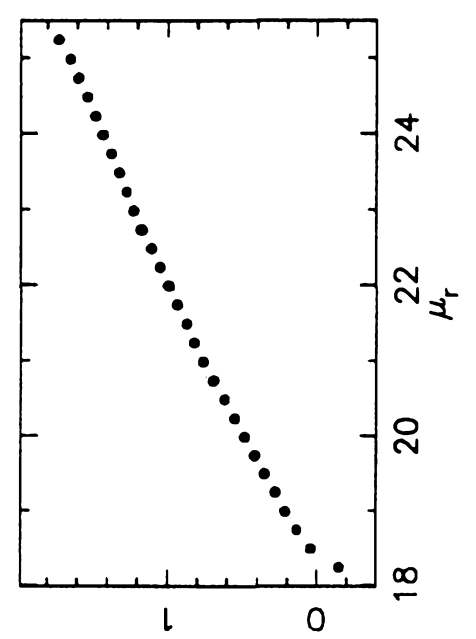

(.1) sn!pod 6ol

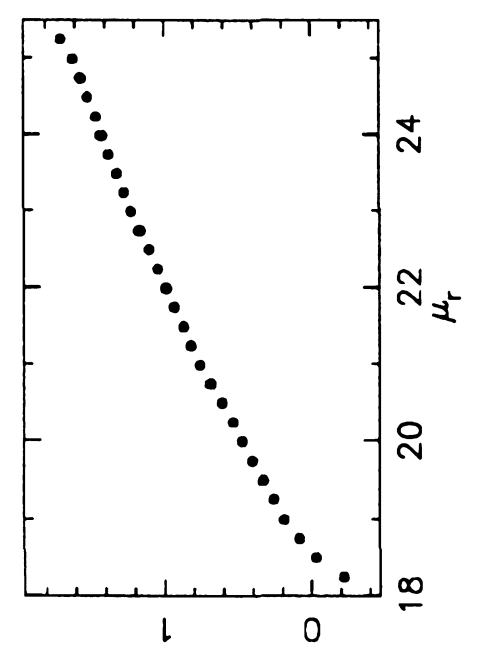

(..) 9 bol

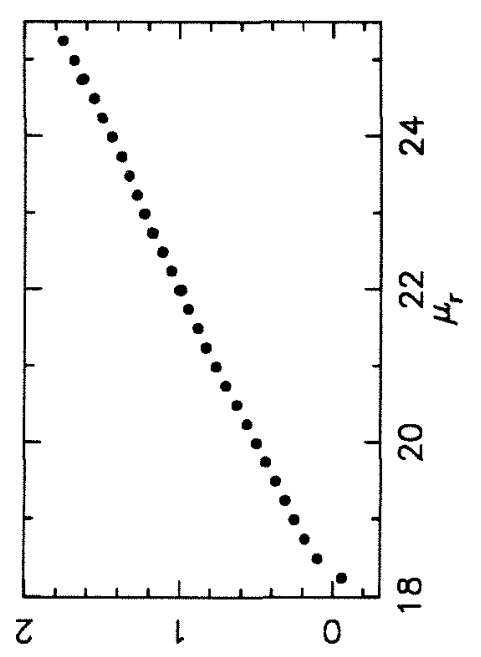

(..) 0 601

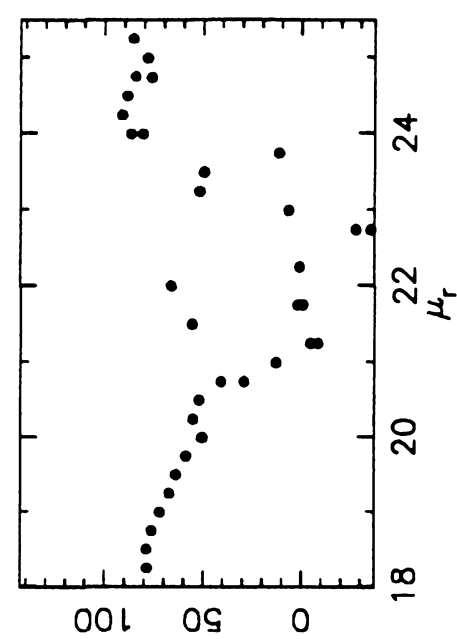

$\forall d$ s!xo do!́om

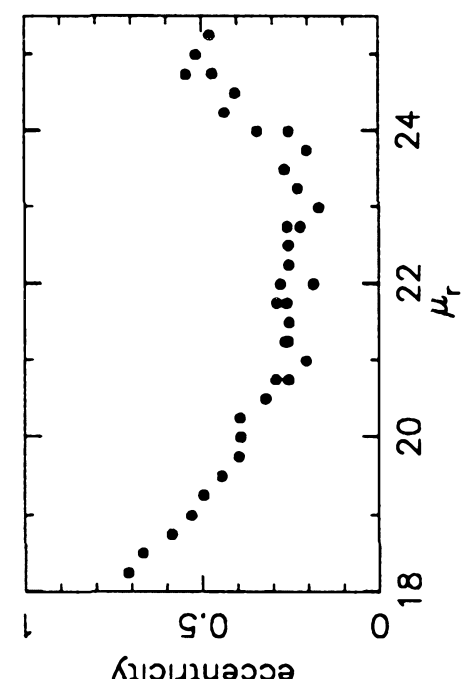

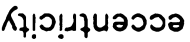
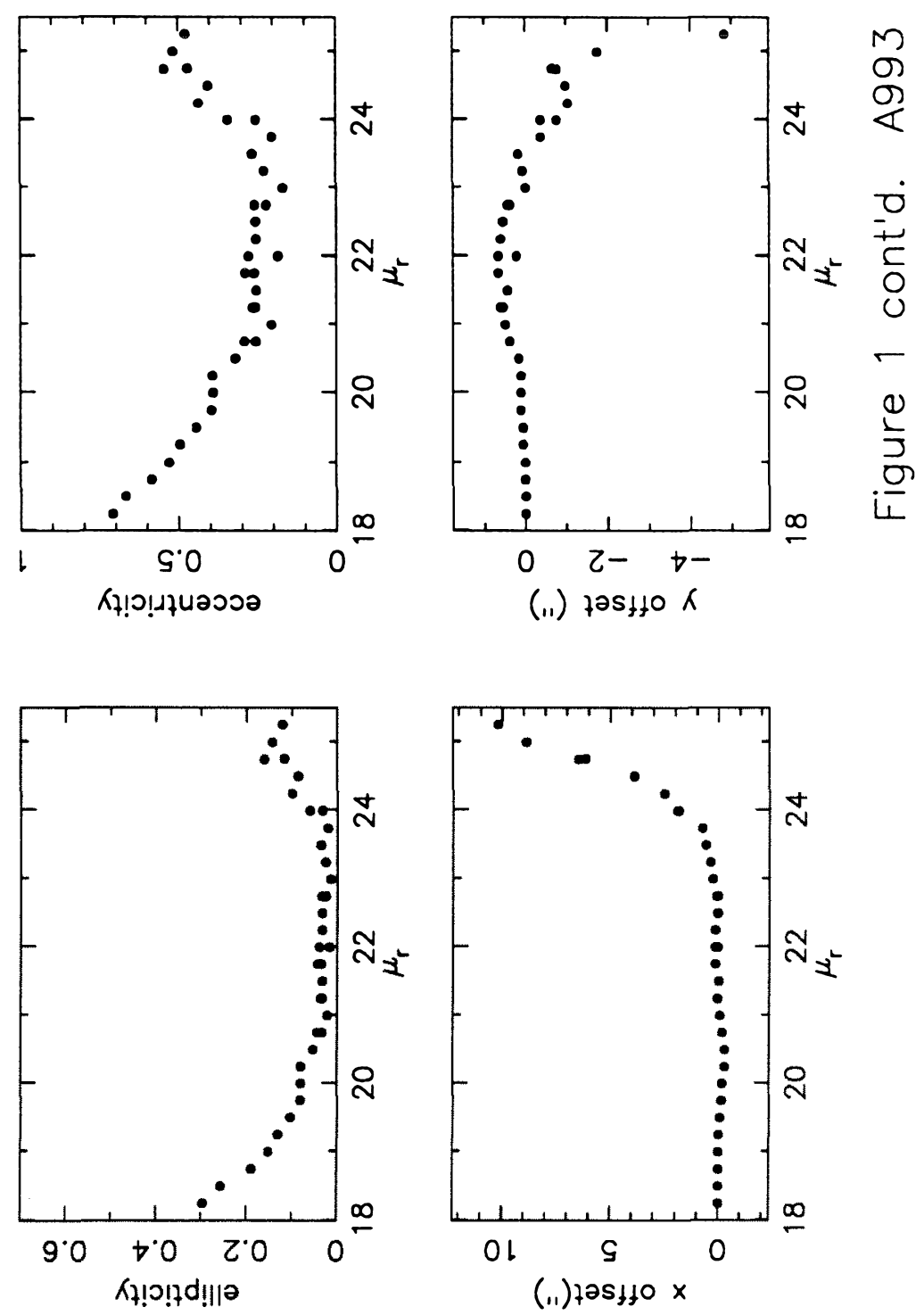

(11) $7 ! 1$ to om6!s auo

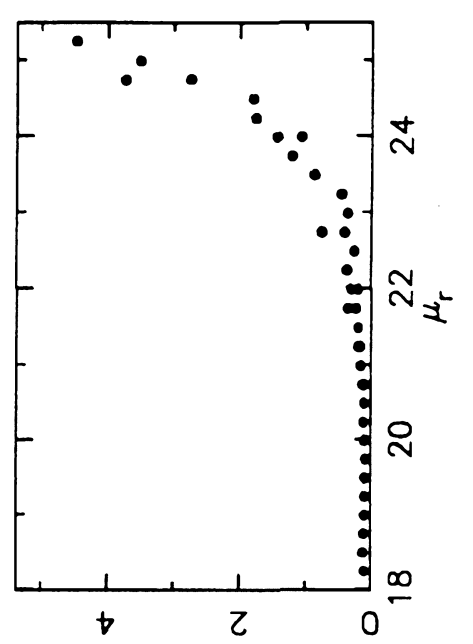




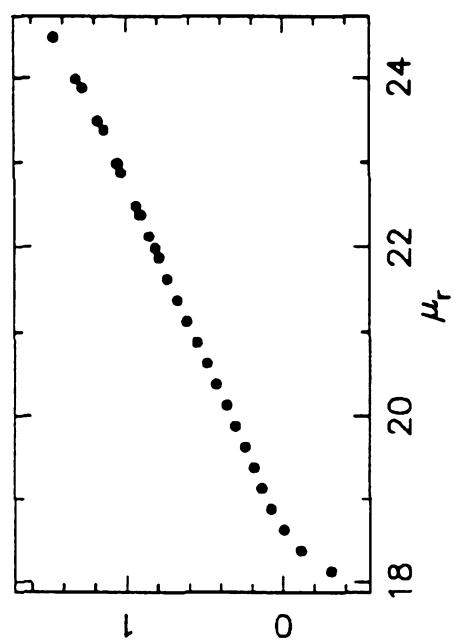

(.1) sn!pod 6ol

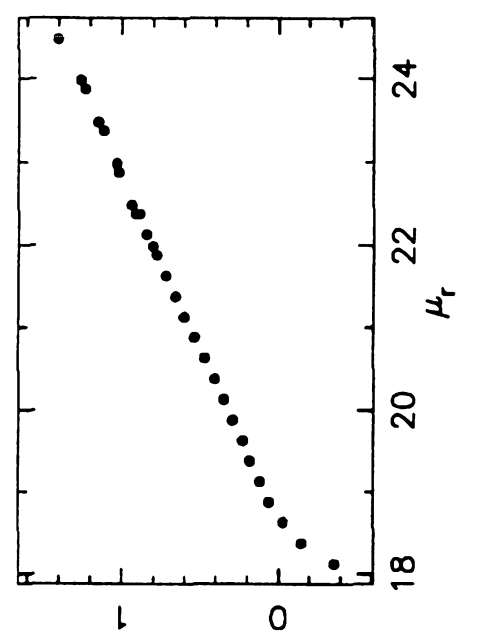

(..) $q$ bol

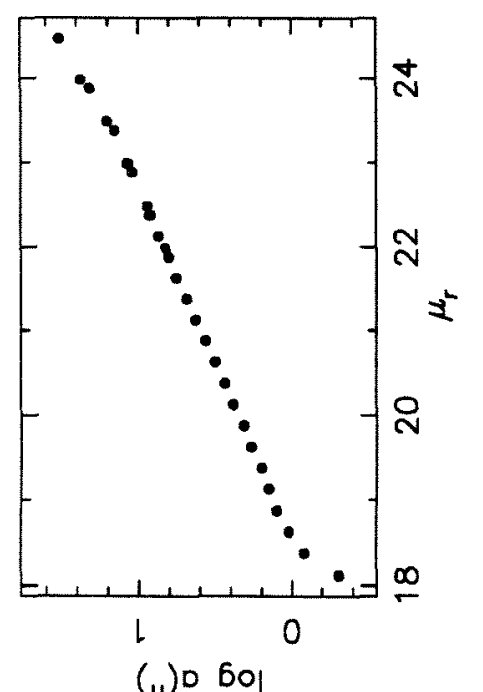

(.1.) $\mathrm{D}$ bo
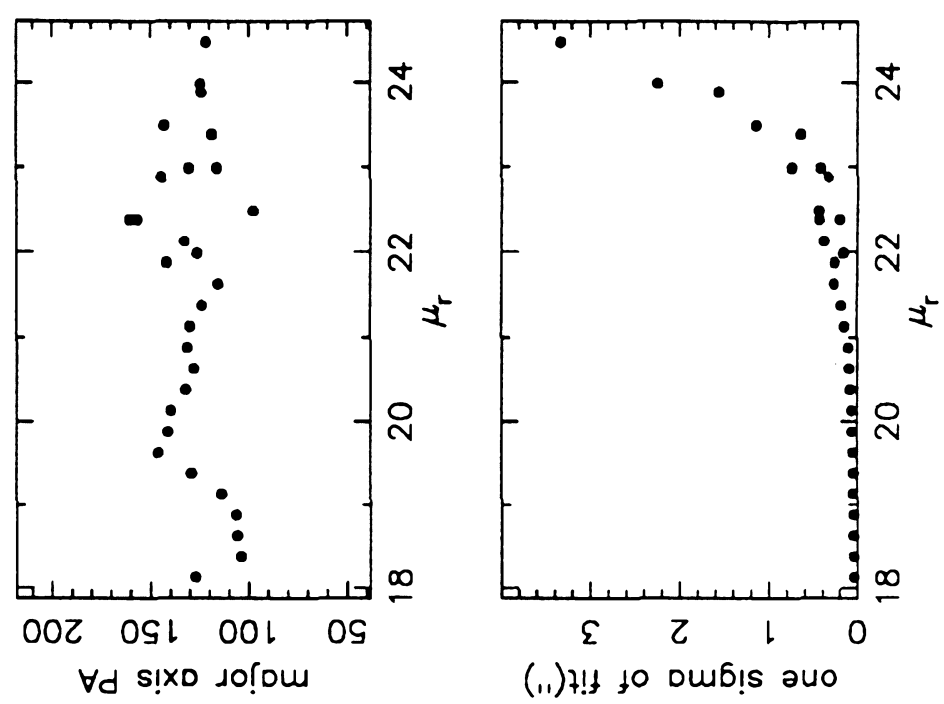

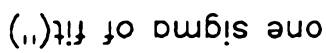
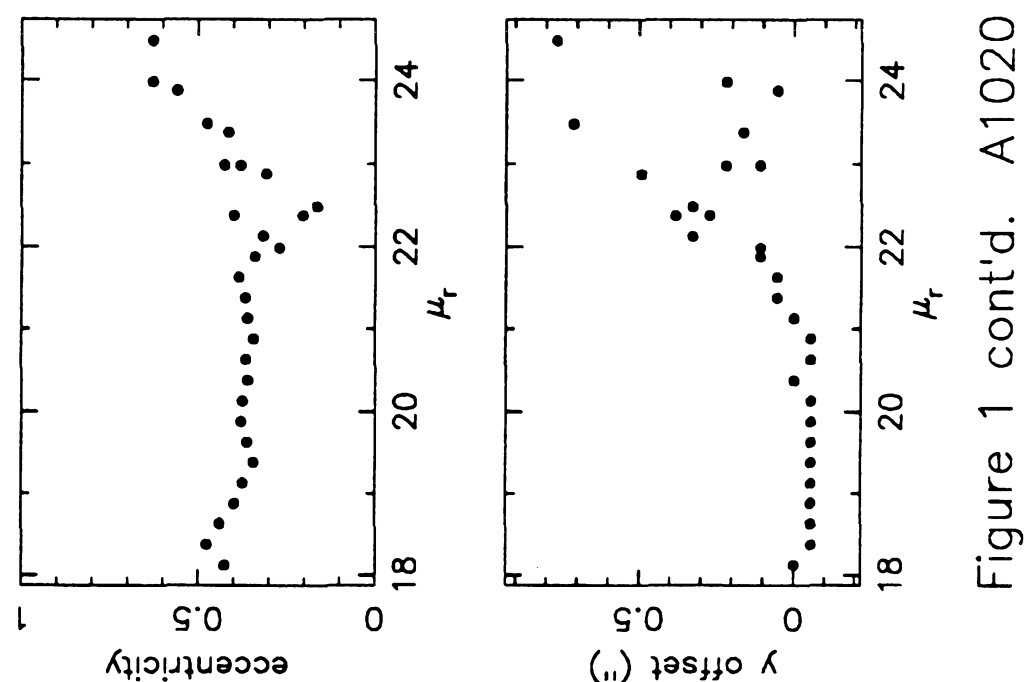

(.1) $725 \$ 10 \mathrm{~K}$
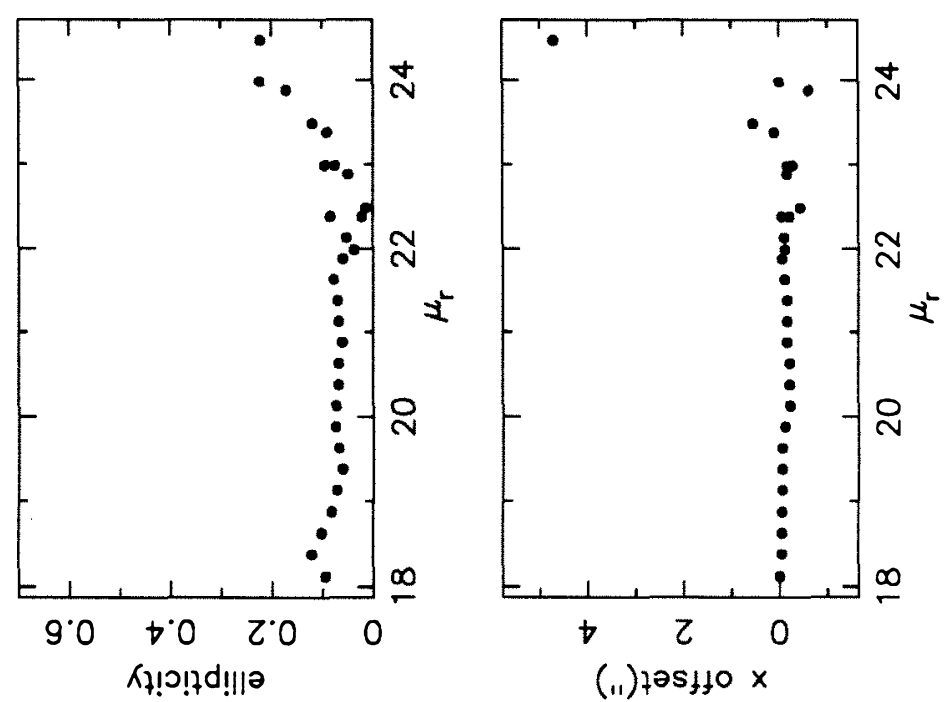


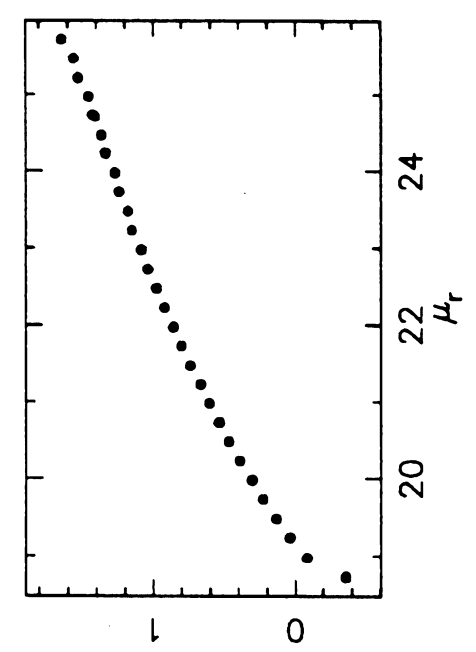

(.1) sn!pod 6ol

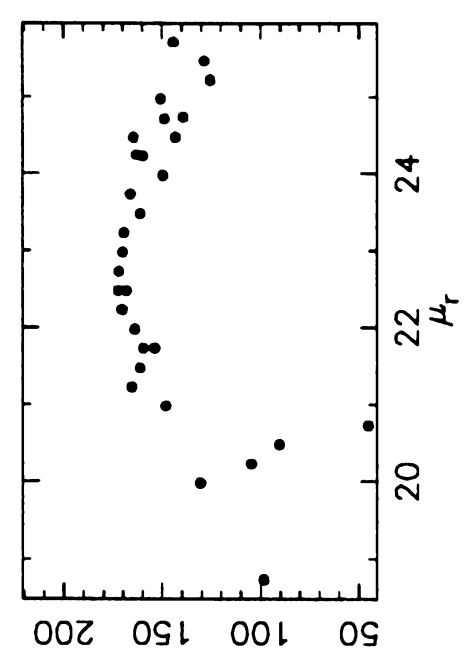

$\forall d$ s!xo so!́m

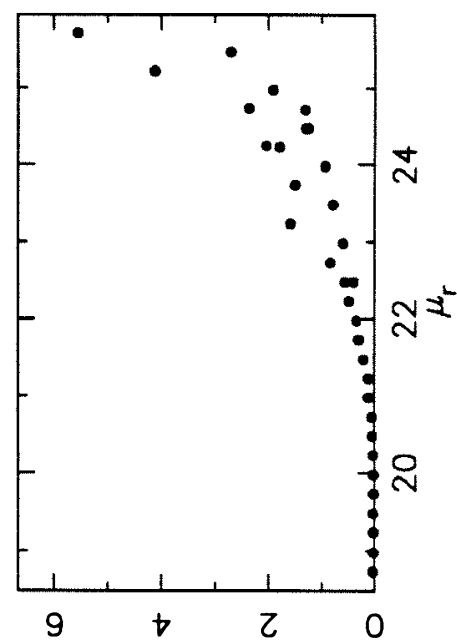

(11) $4 !+$ to Dub!s auo
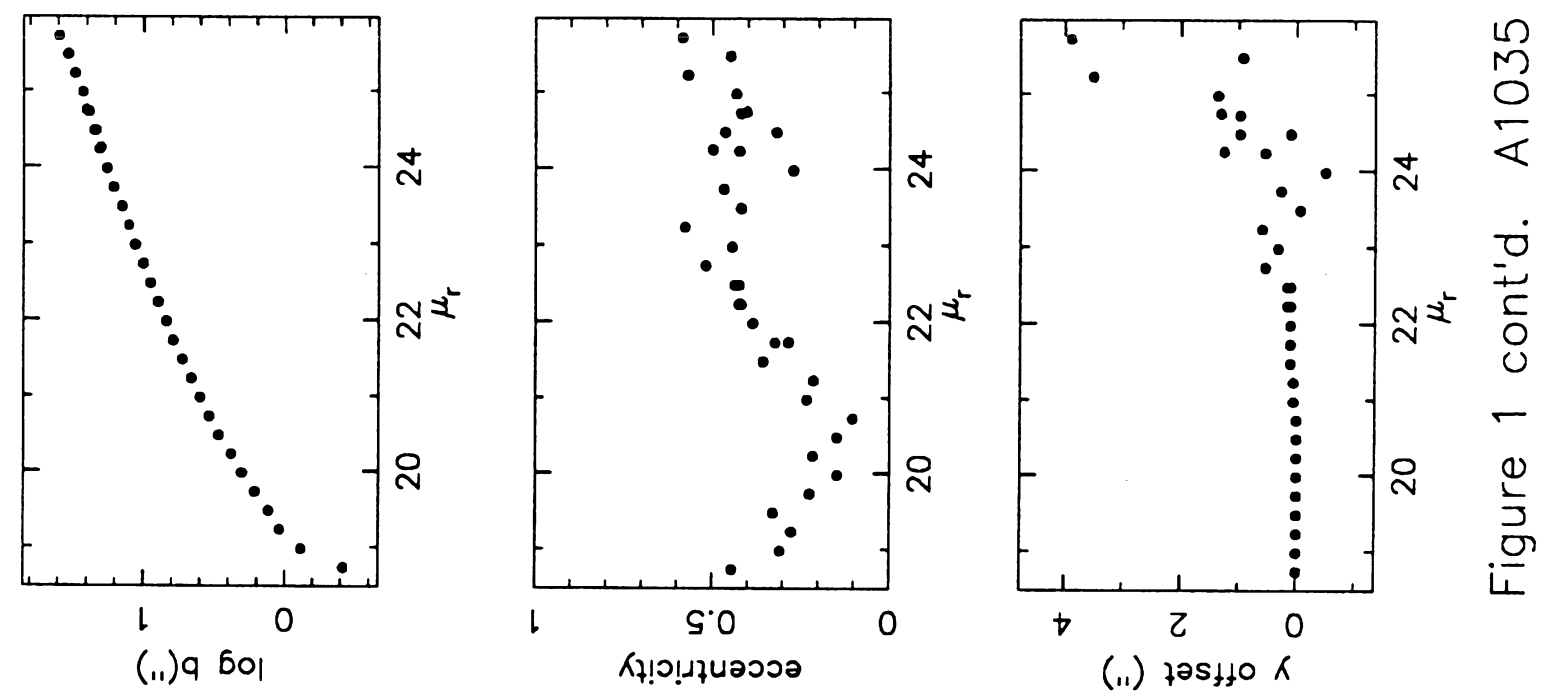

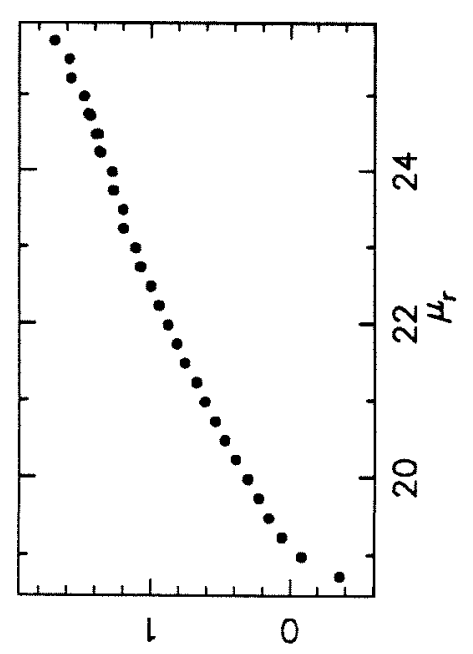

(.1) 0601

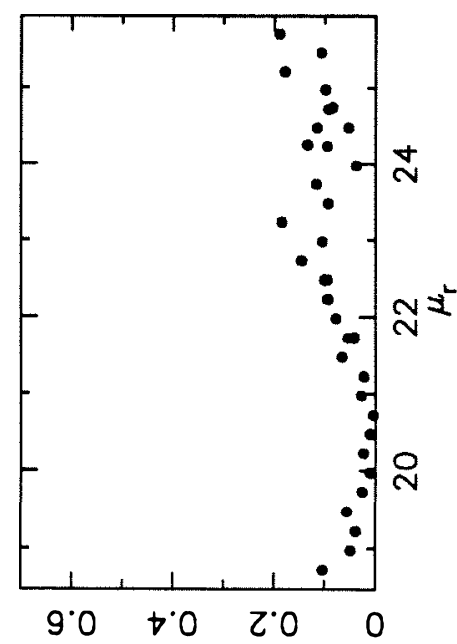

रł!อ!

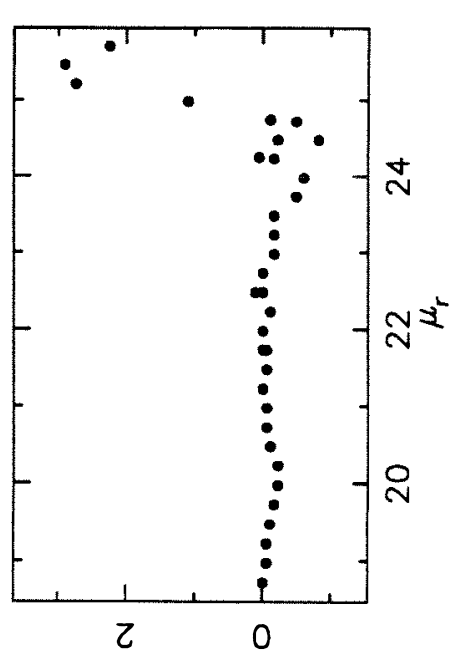

(ii) tos $740 \times$ 

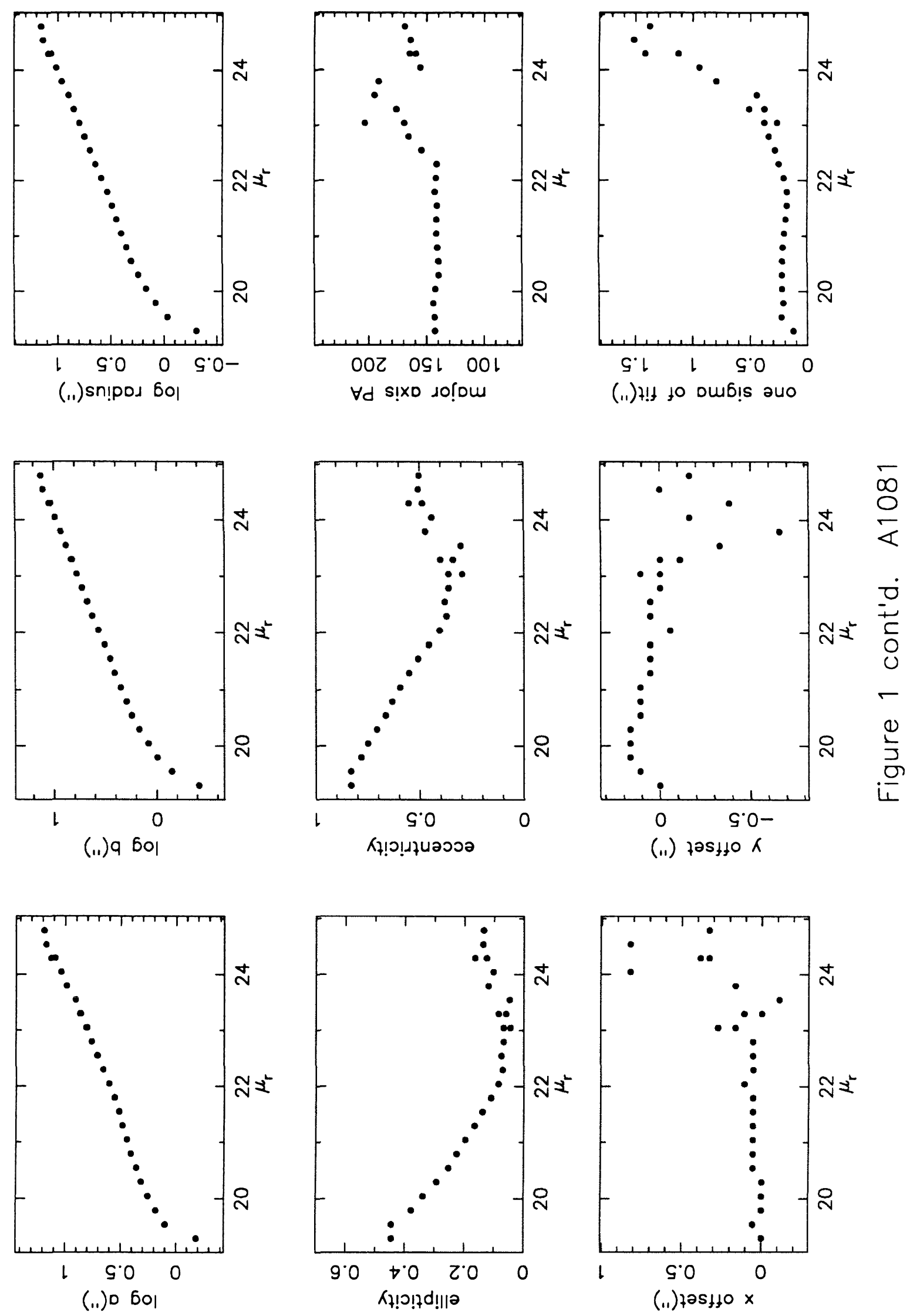

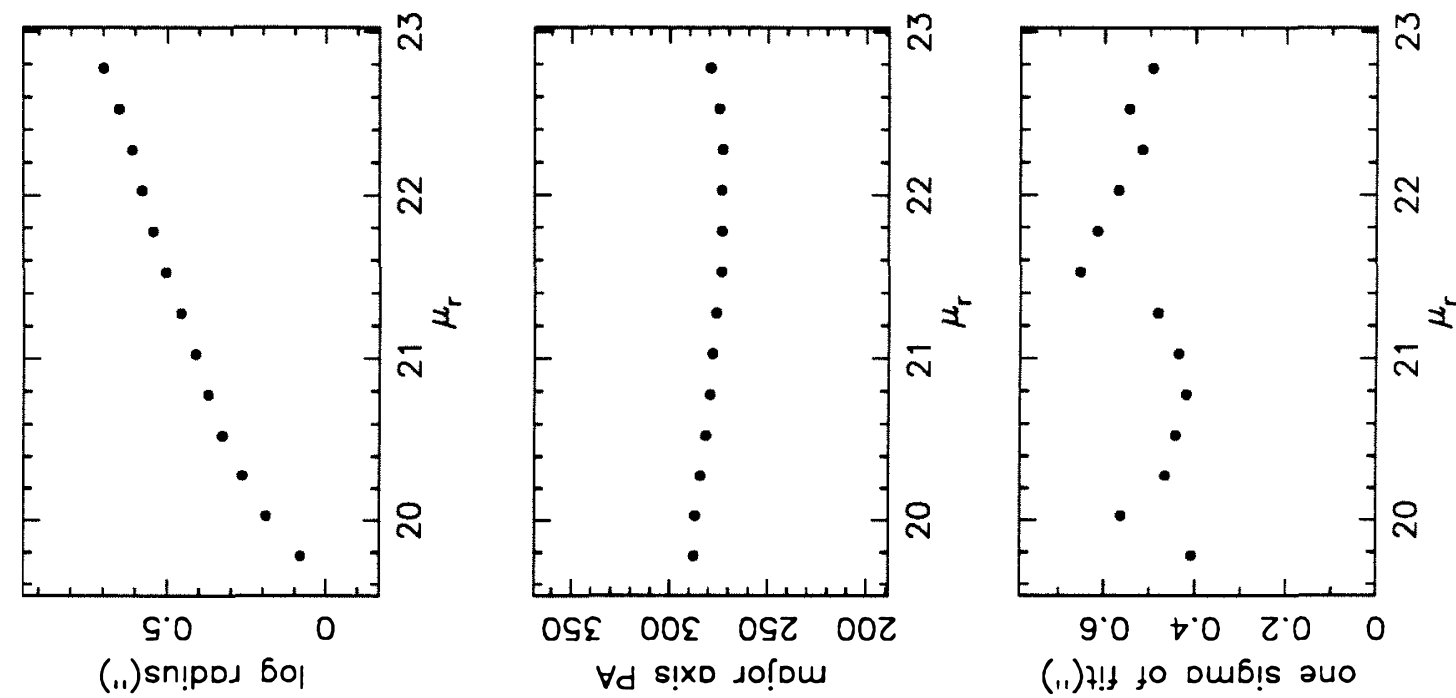

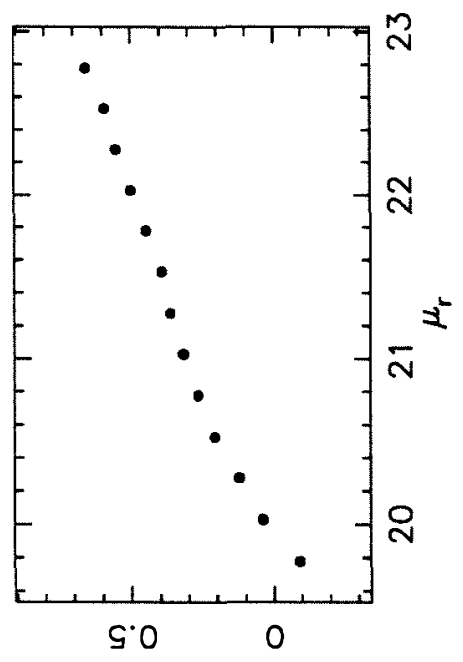

(..) 9 bol

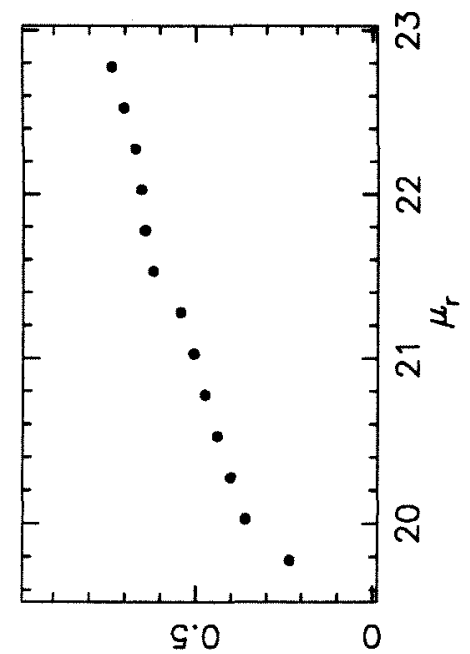

(..) 0 60

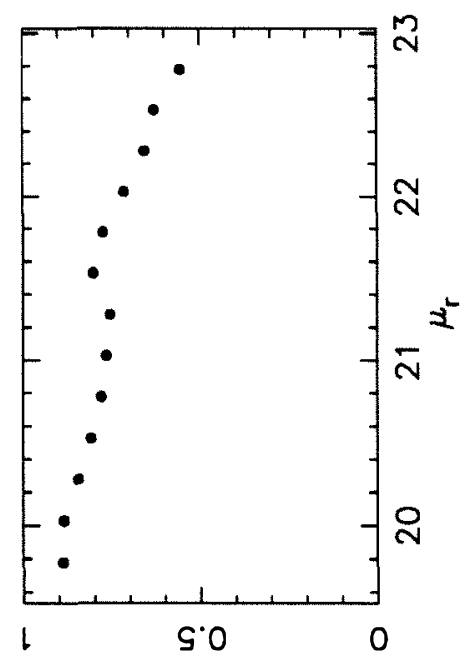

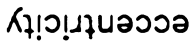
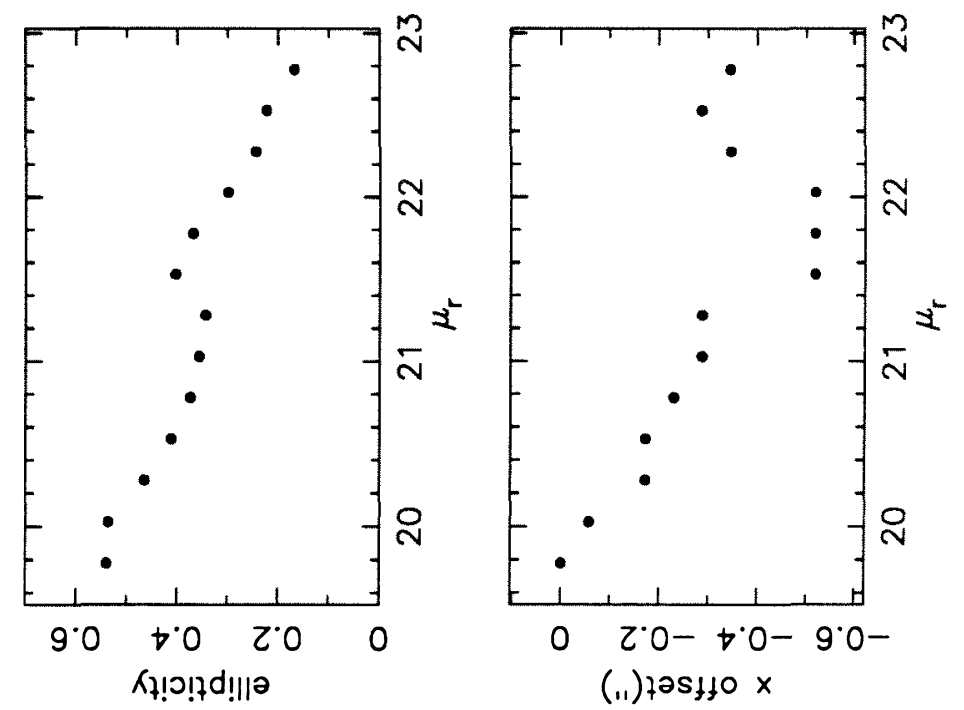

(.1) tas $1,0 \mathrm{~K}$

$\frac{m}{2}$

7

$\bar{v}^{ \pm} \frac{7}{c}$

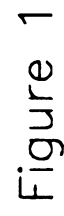




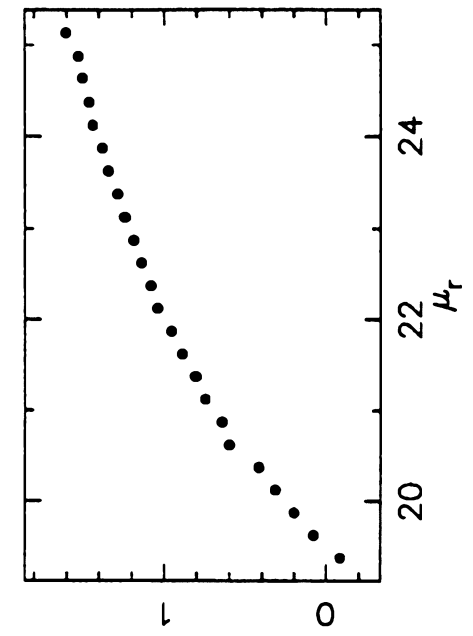

(.1)sn!pod 60।

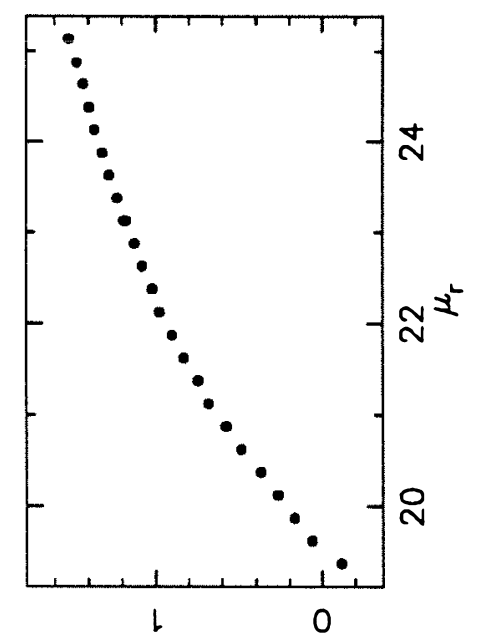

(.1) $9 \mathrm{bol}$

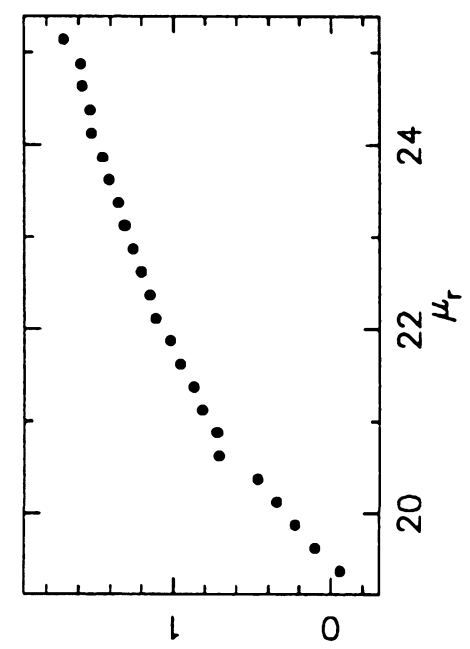

(.1) 0 6ol

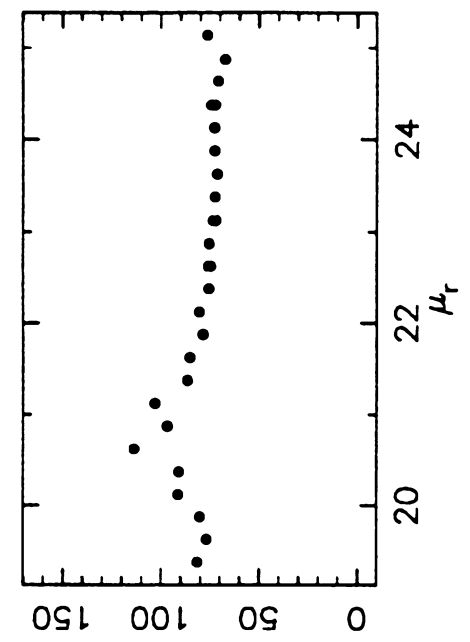

$\forall d$ s!xo so!ou

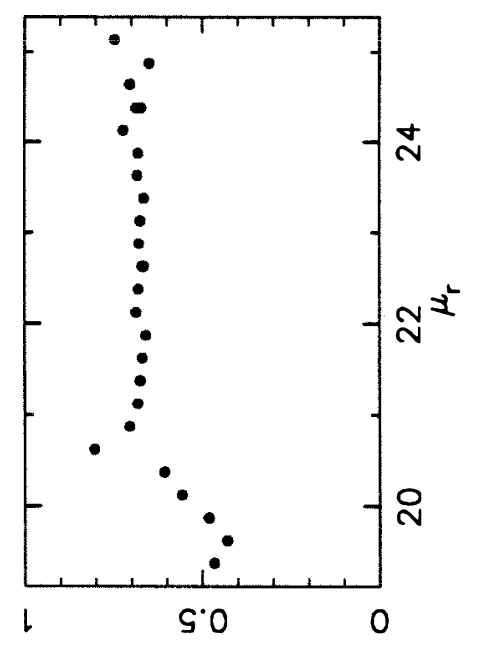

К!ว!นในวววа

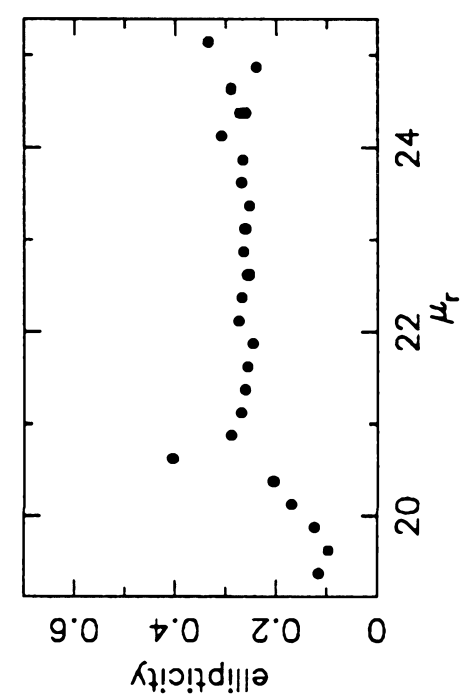

रł!อ!?d!||a

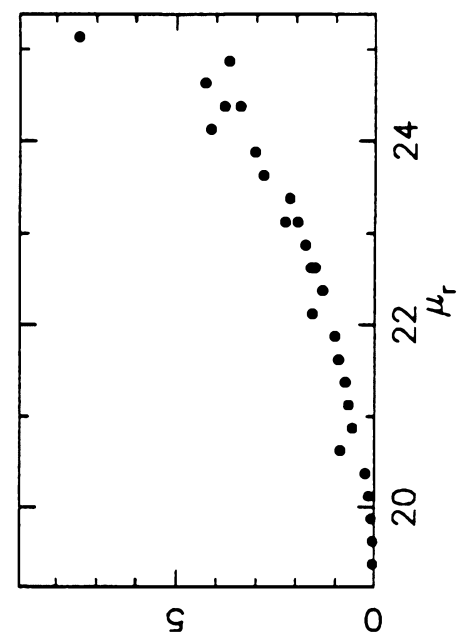

(11) $7 ! 1$ to Dub! an
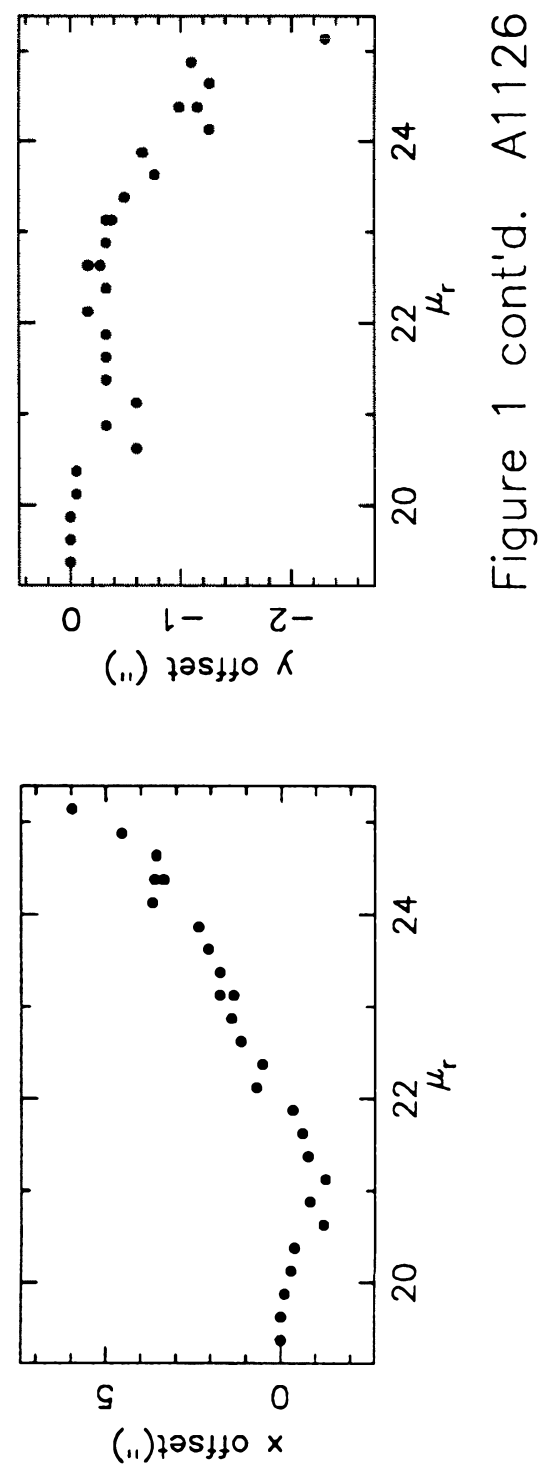

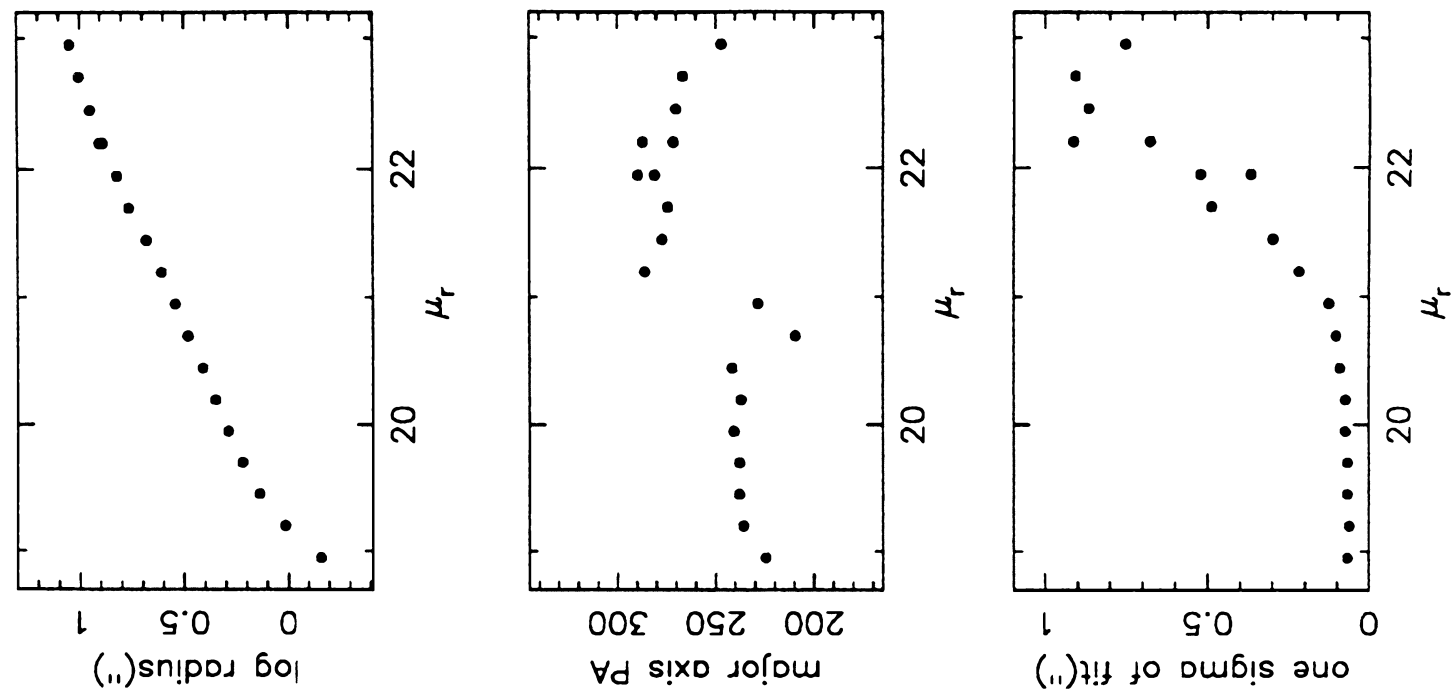

(11) $+!$ to Dub!s auo

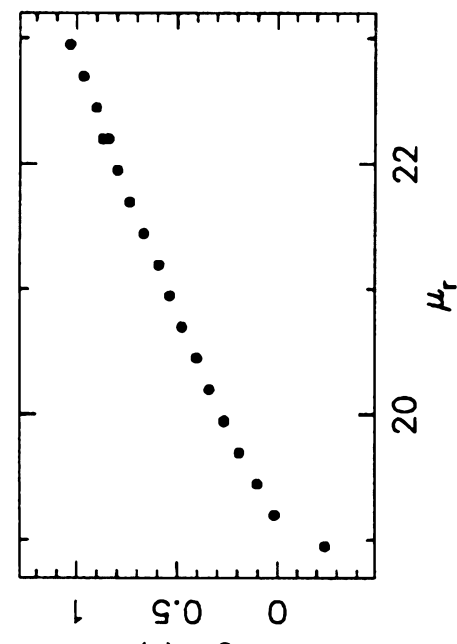

(.1) 960

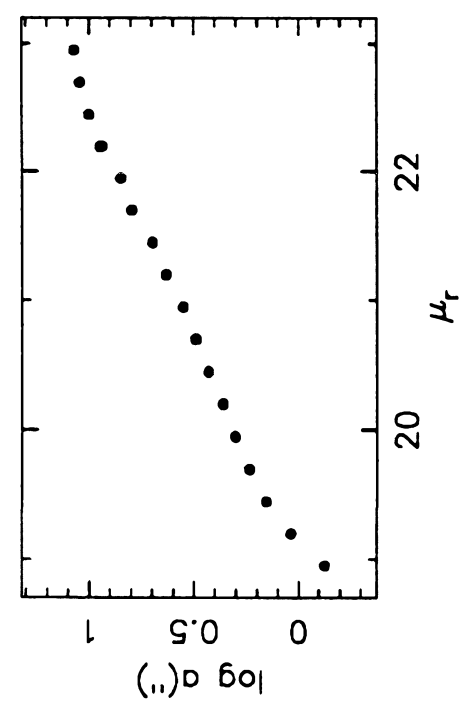

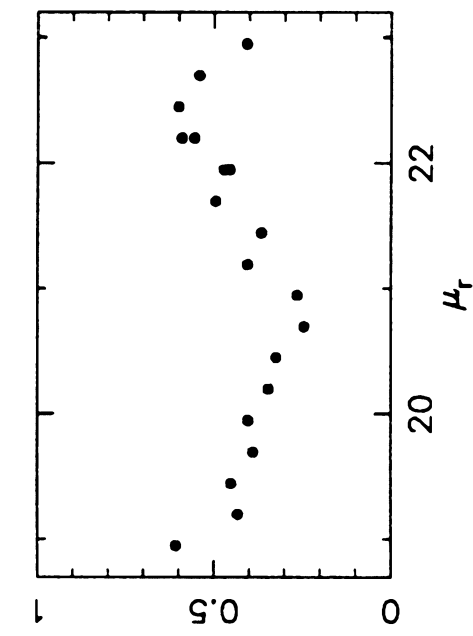

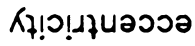

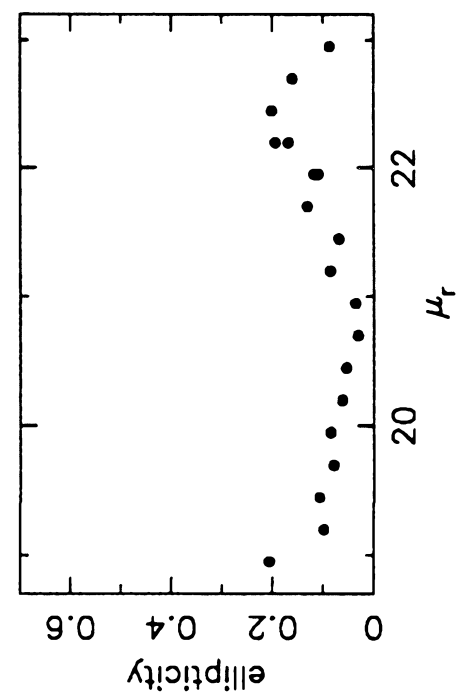

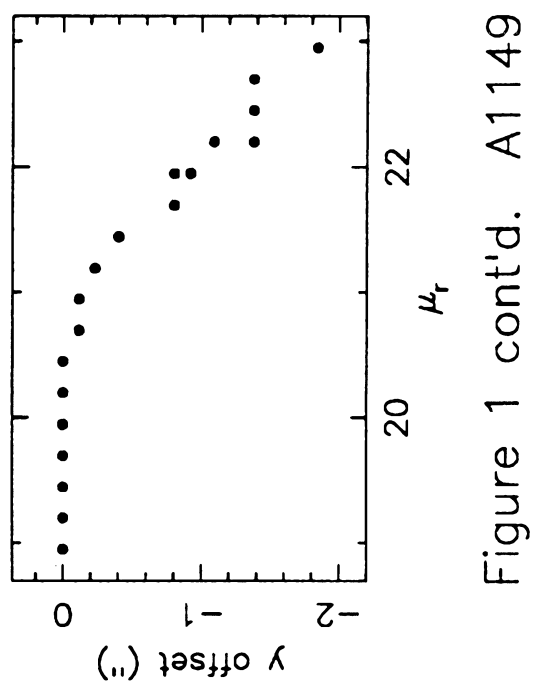

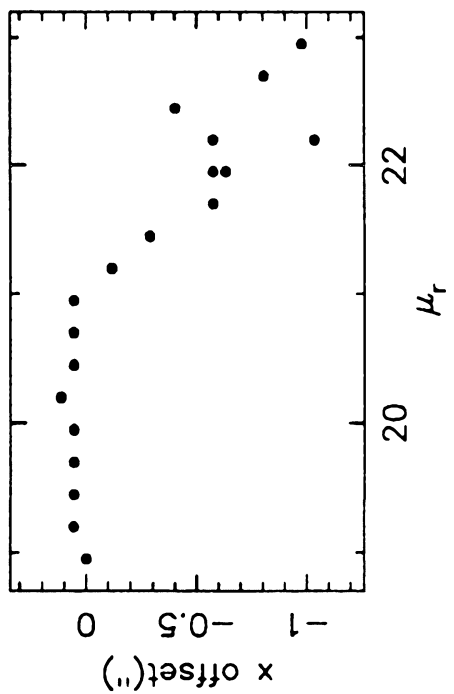



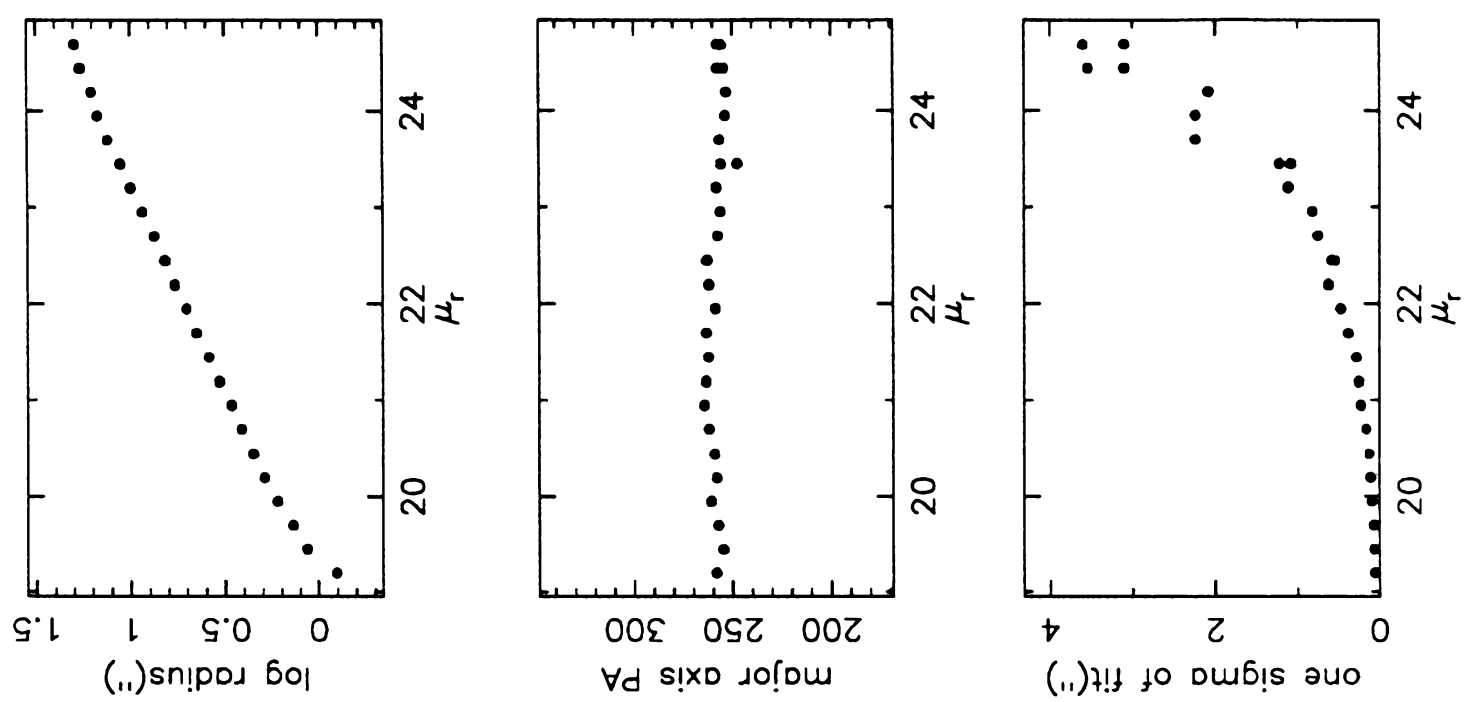

(11) + !! to Du6!s auo

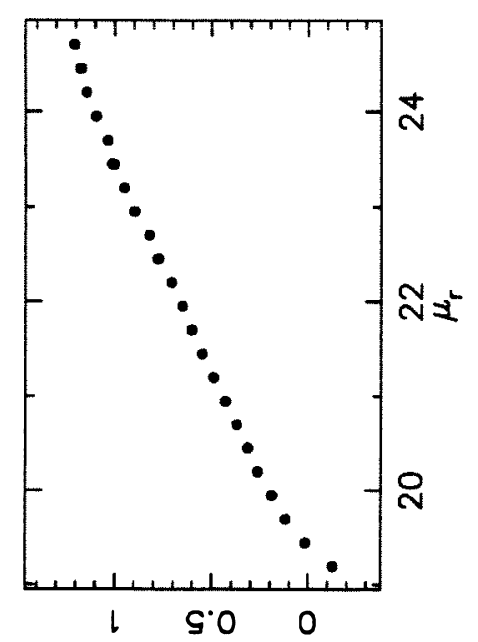

(.1) 9601

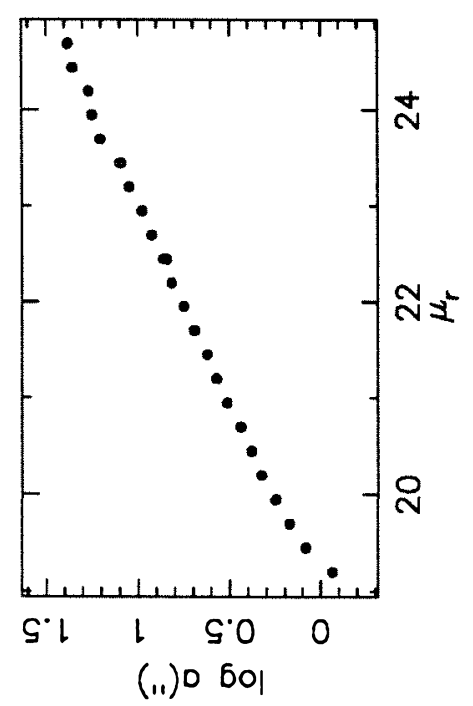

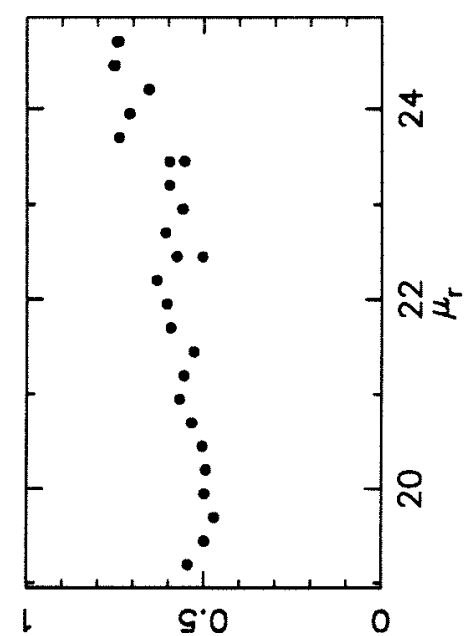

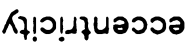

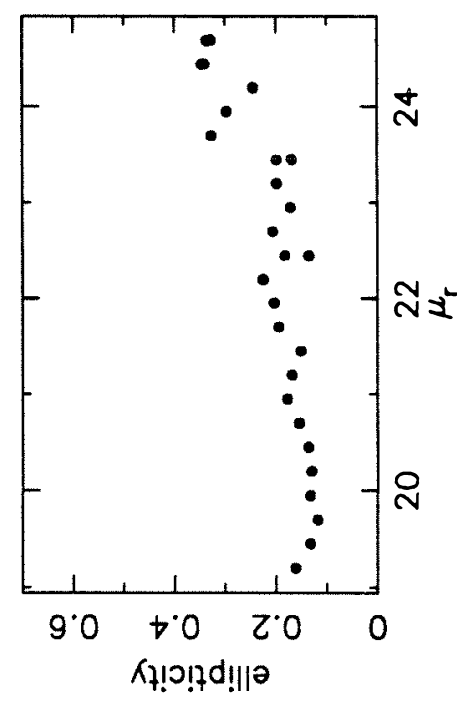

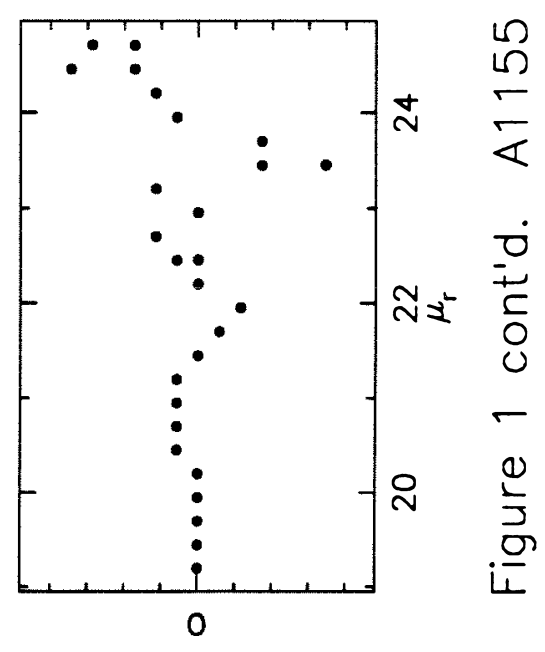

(ii) $725+10 K$

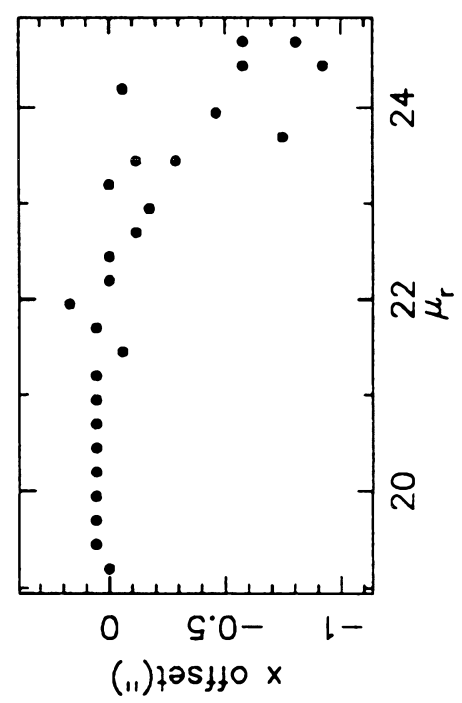




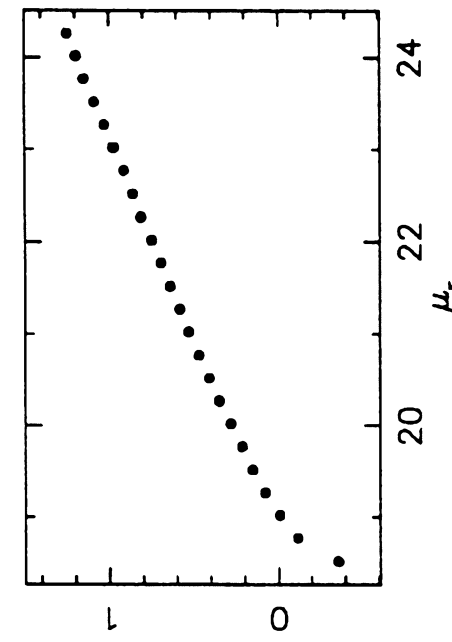

(11)sn!pod 6ol

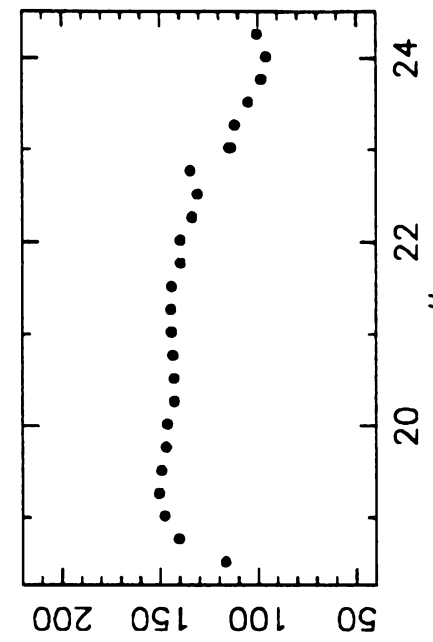

$\forall d$ s!Xo so!ou

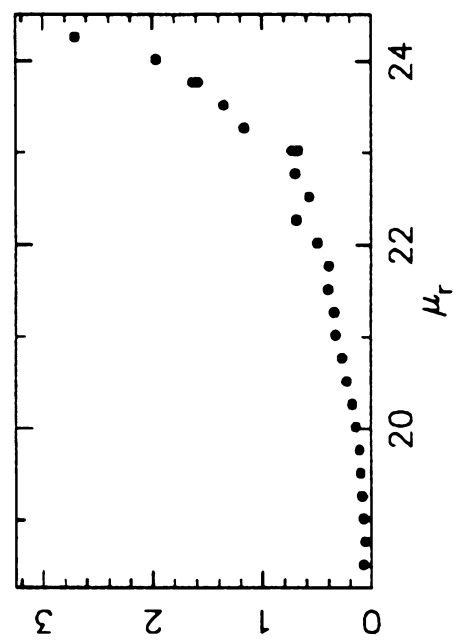

(11) $7 ! 1$ to oub!s 2u०

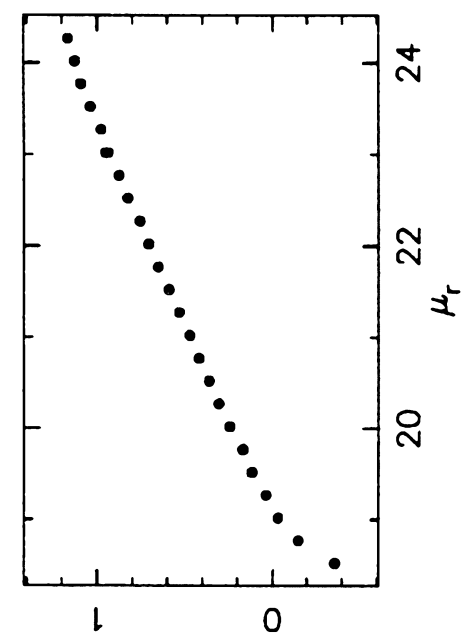

(.1) 9601

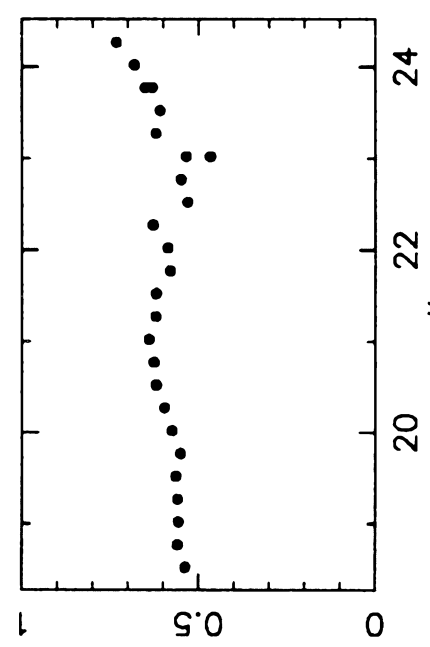

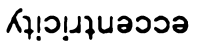

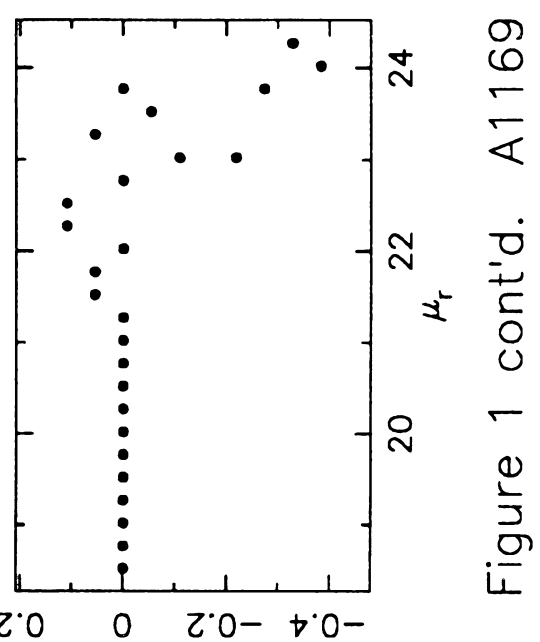

(11) $725 \% 10 \mathrm{~K}$

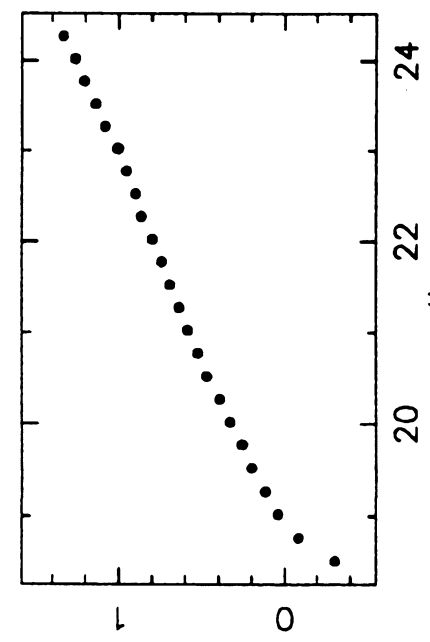

(.1) $0 \mathrm{bo}$ 이
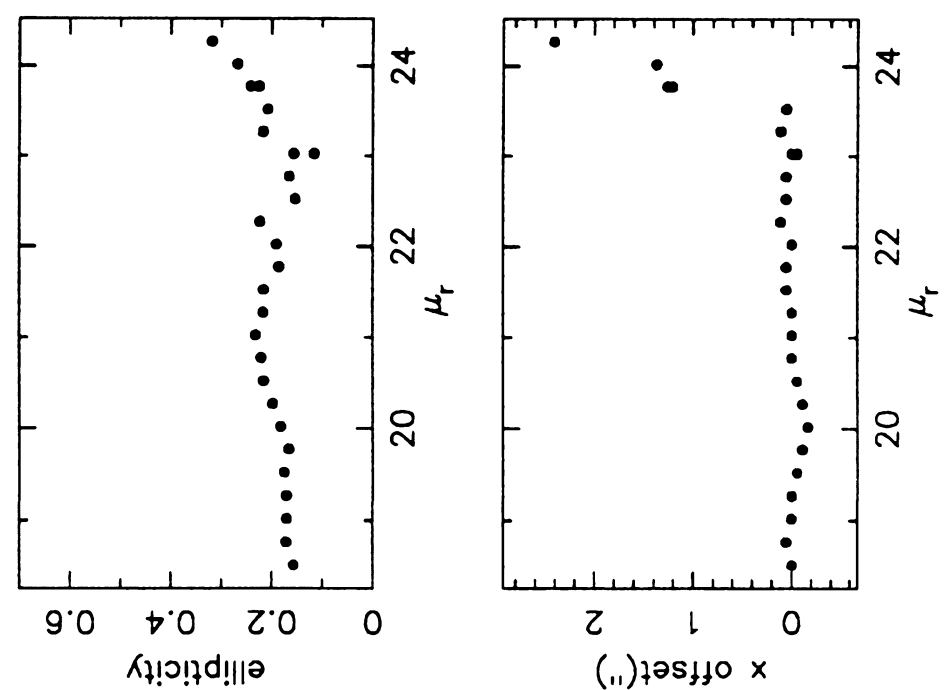


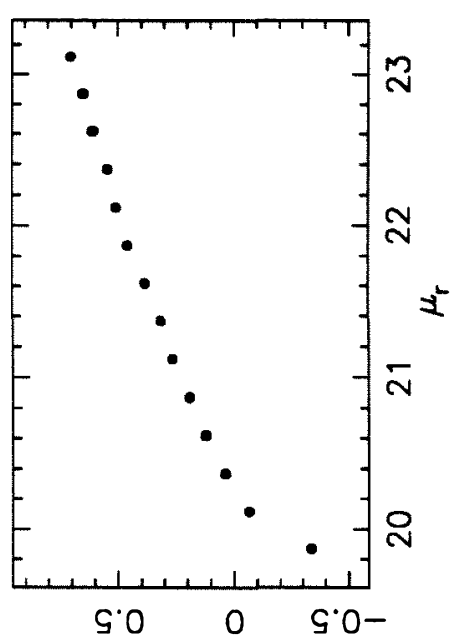

(.1) sn!pod 6ol

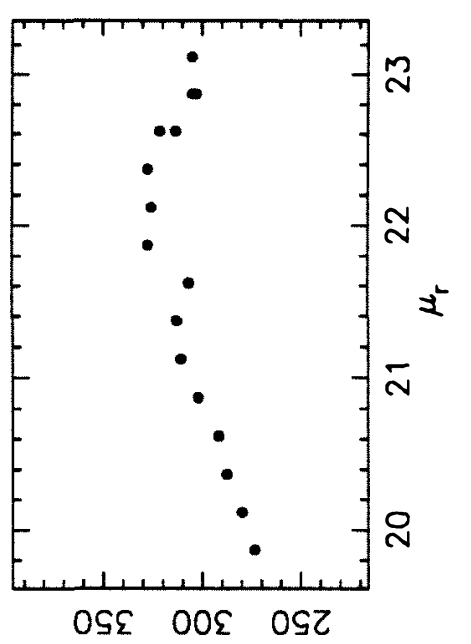

$\forall d$ s!xo so!ou

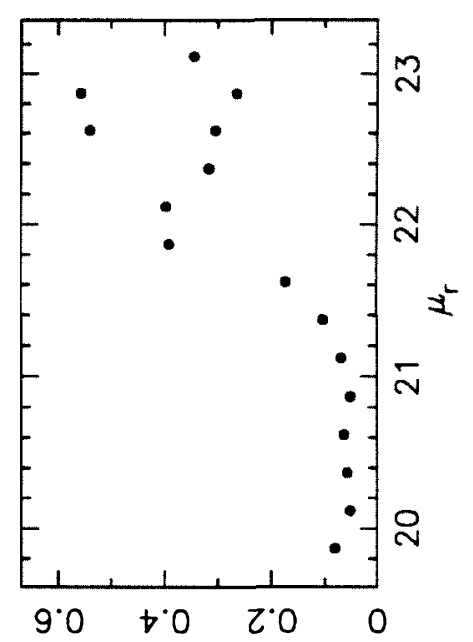

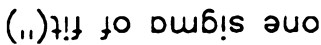
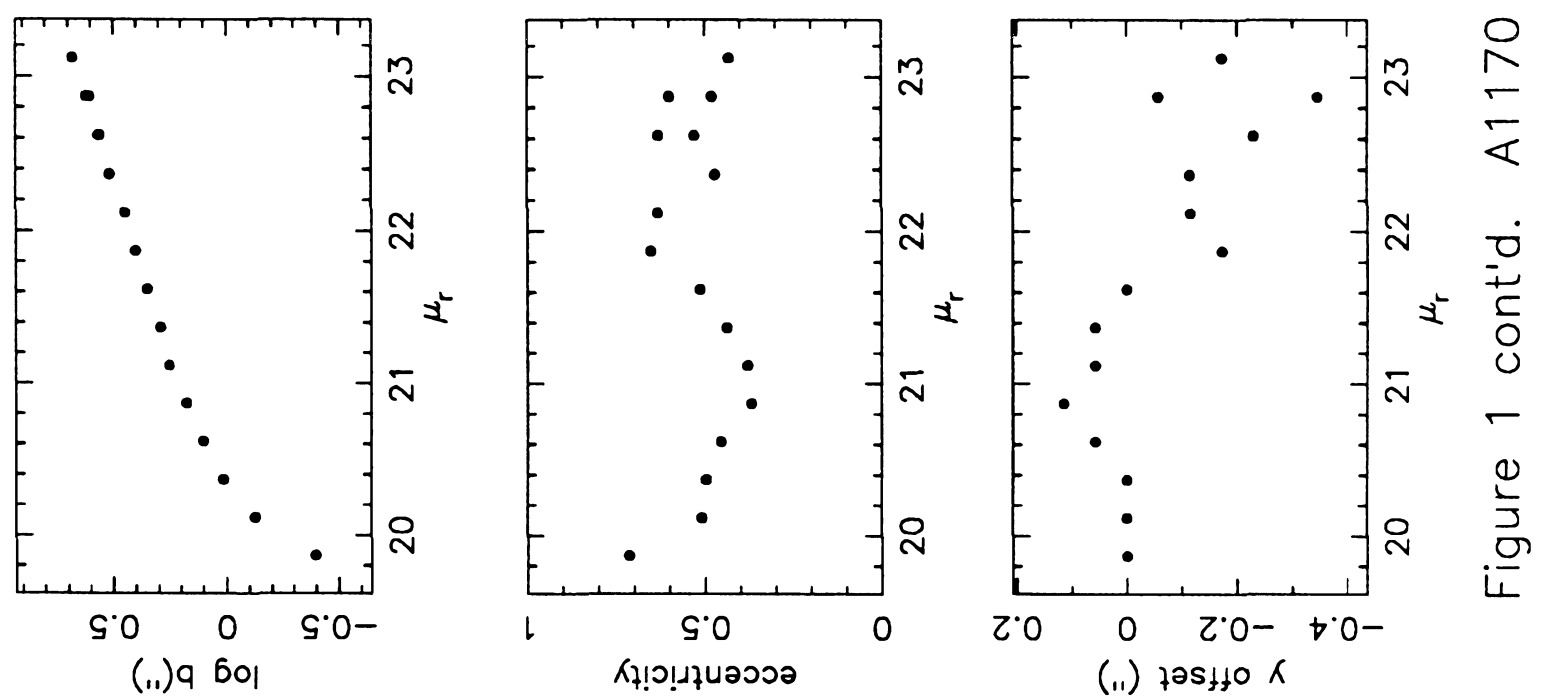

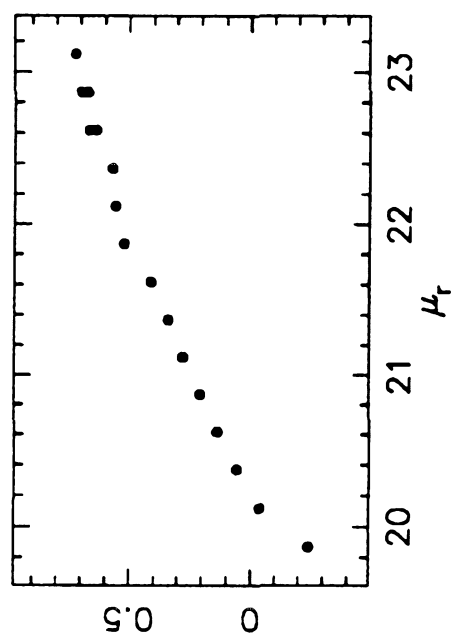

(.1.) $0 \mathrm{bol}$
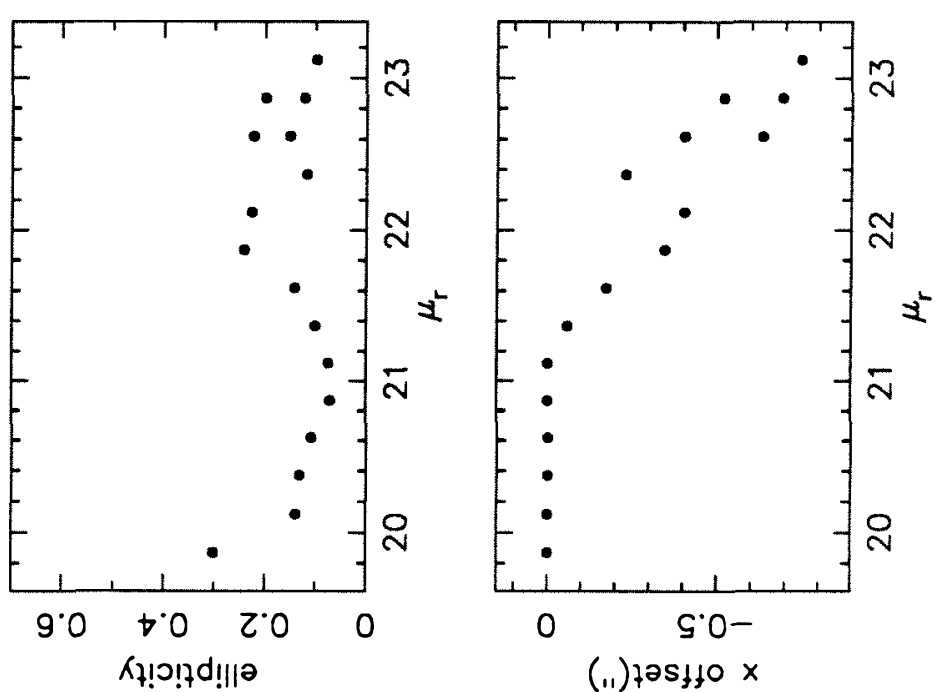

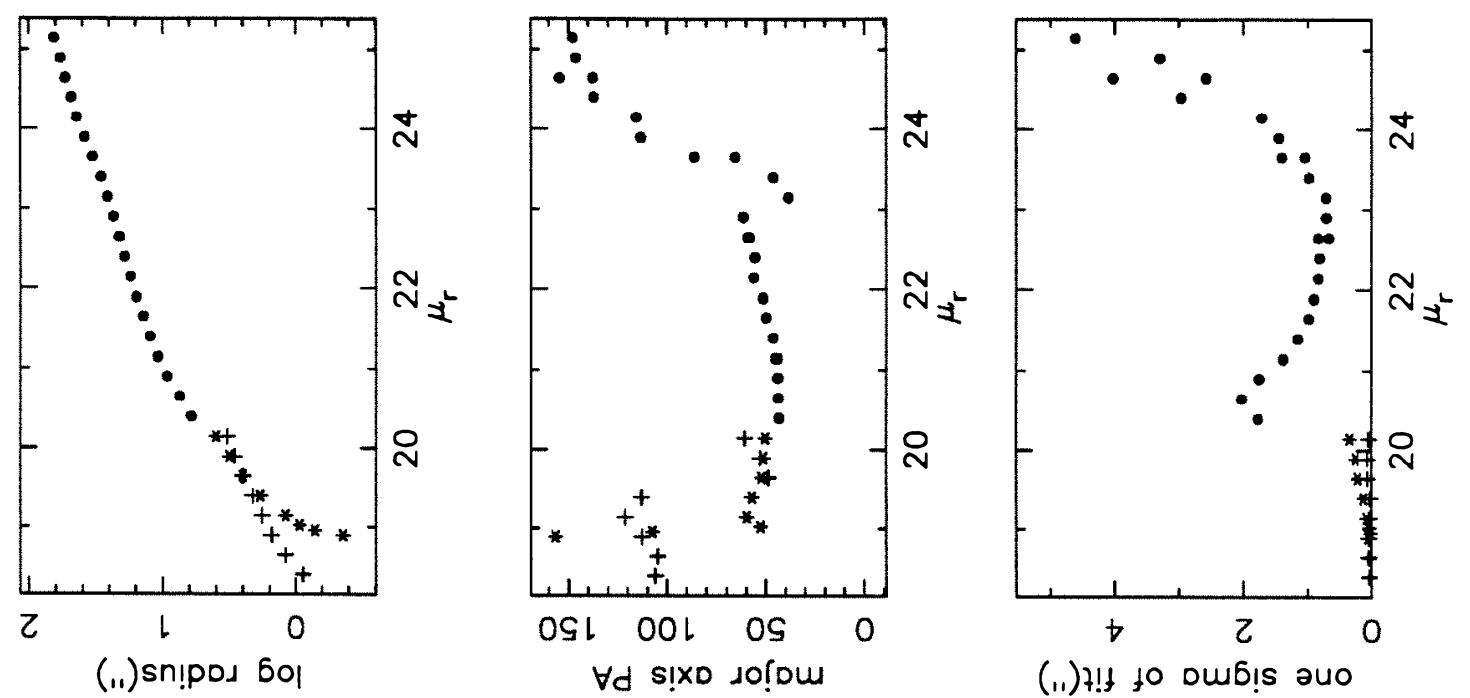

(11) 4 !t to oub!s auo
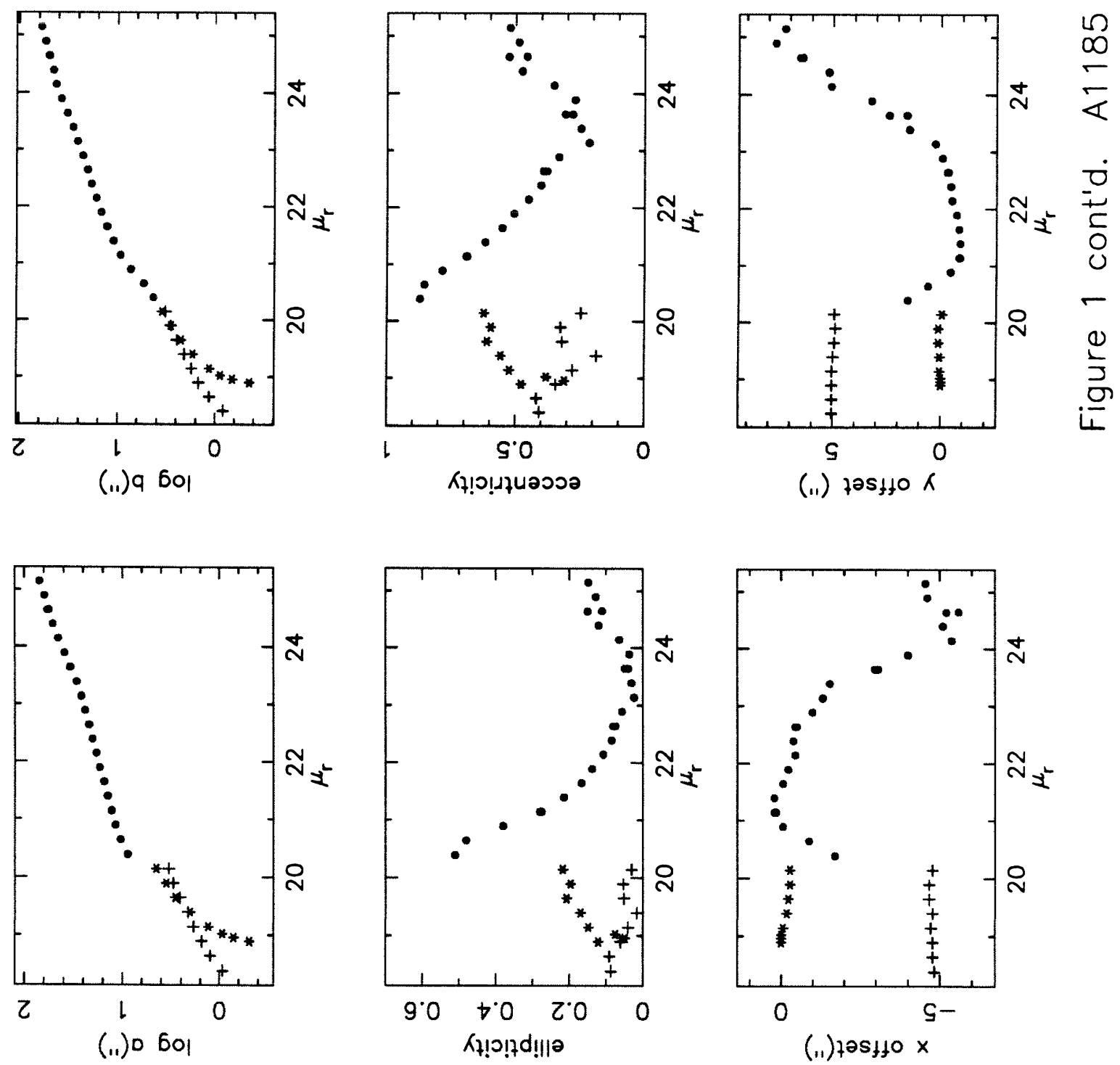

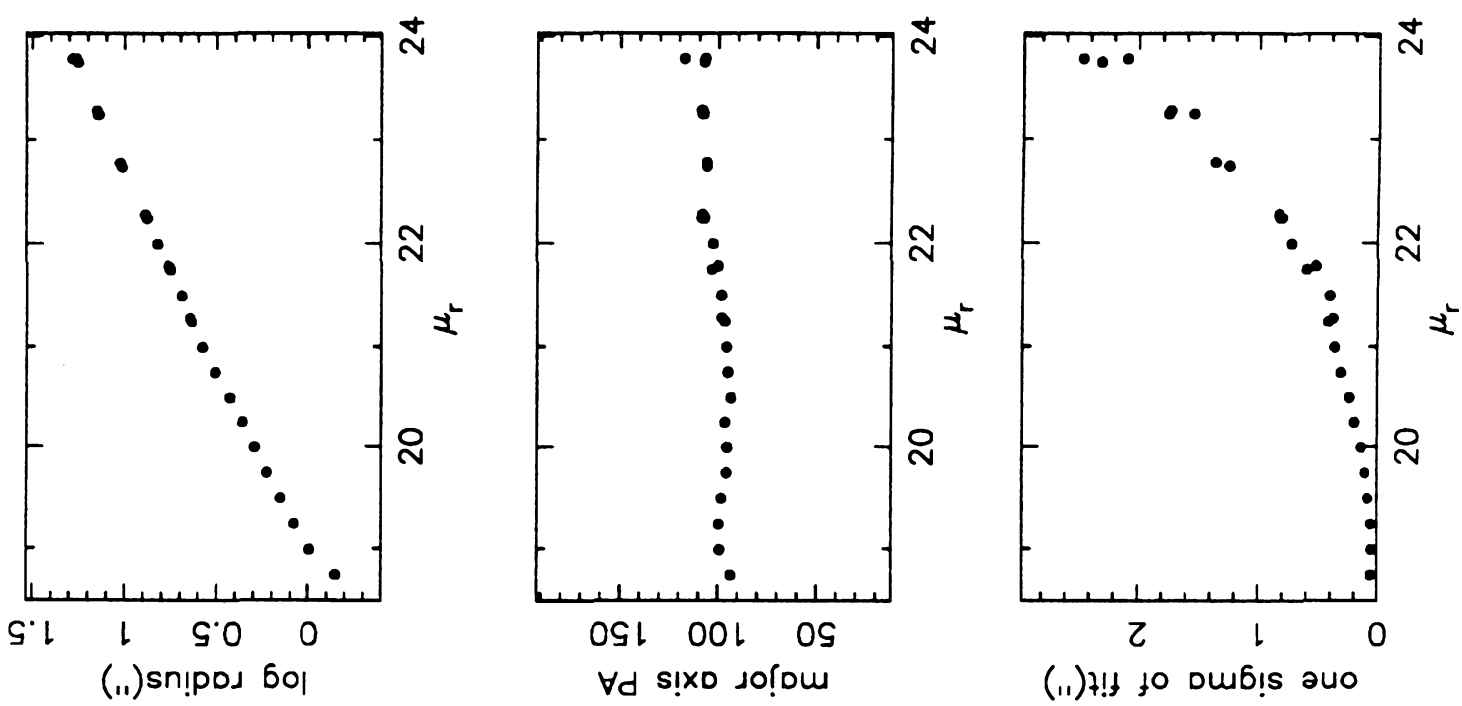

(11) 4 ! t to Dub!s auo
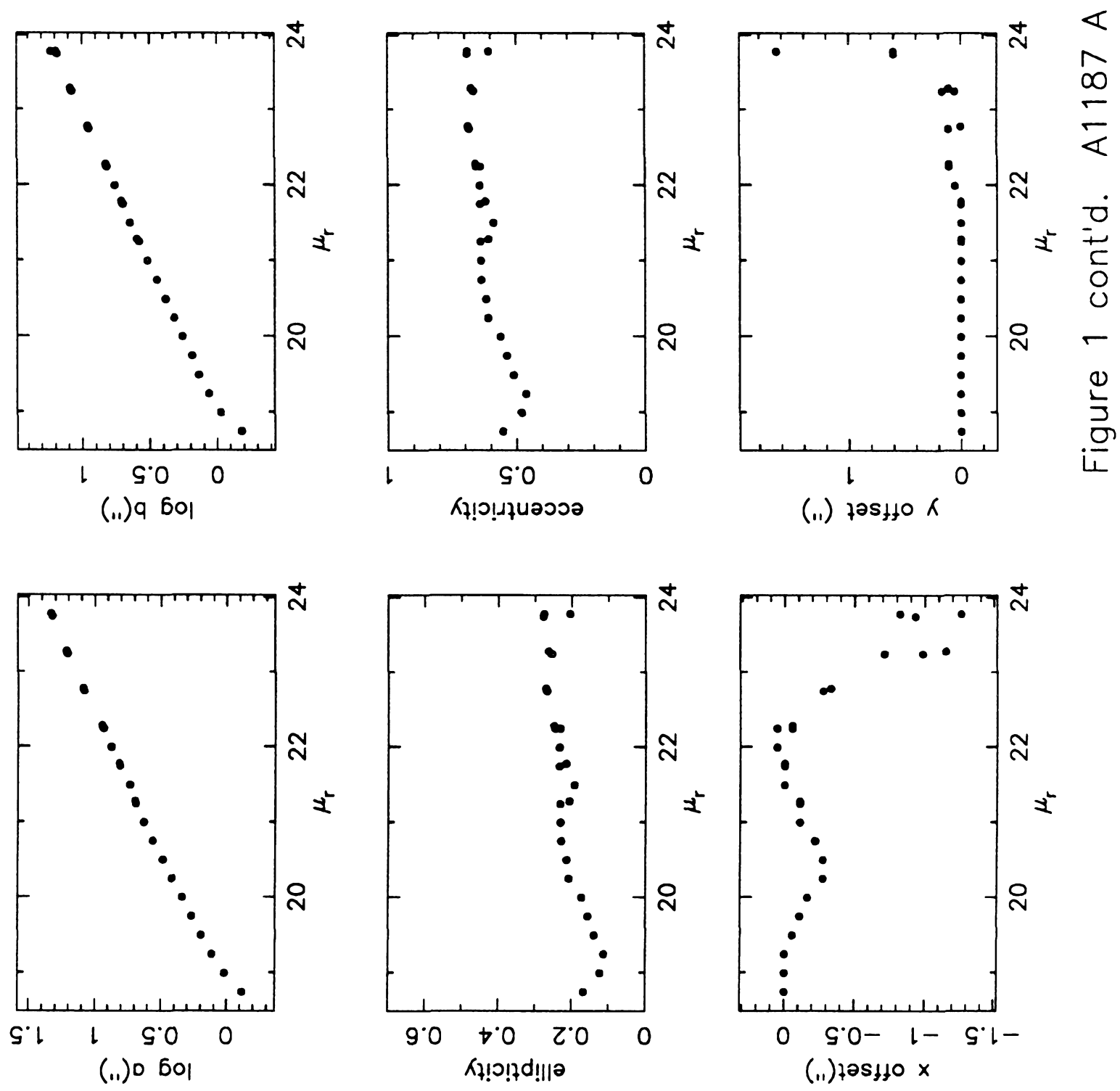

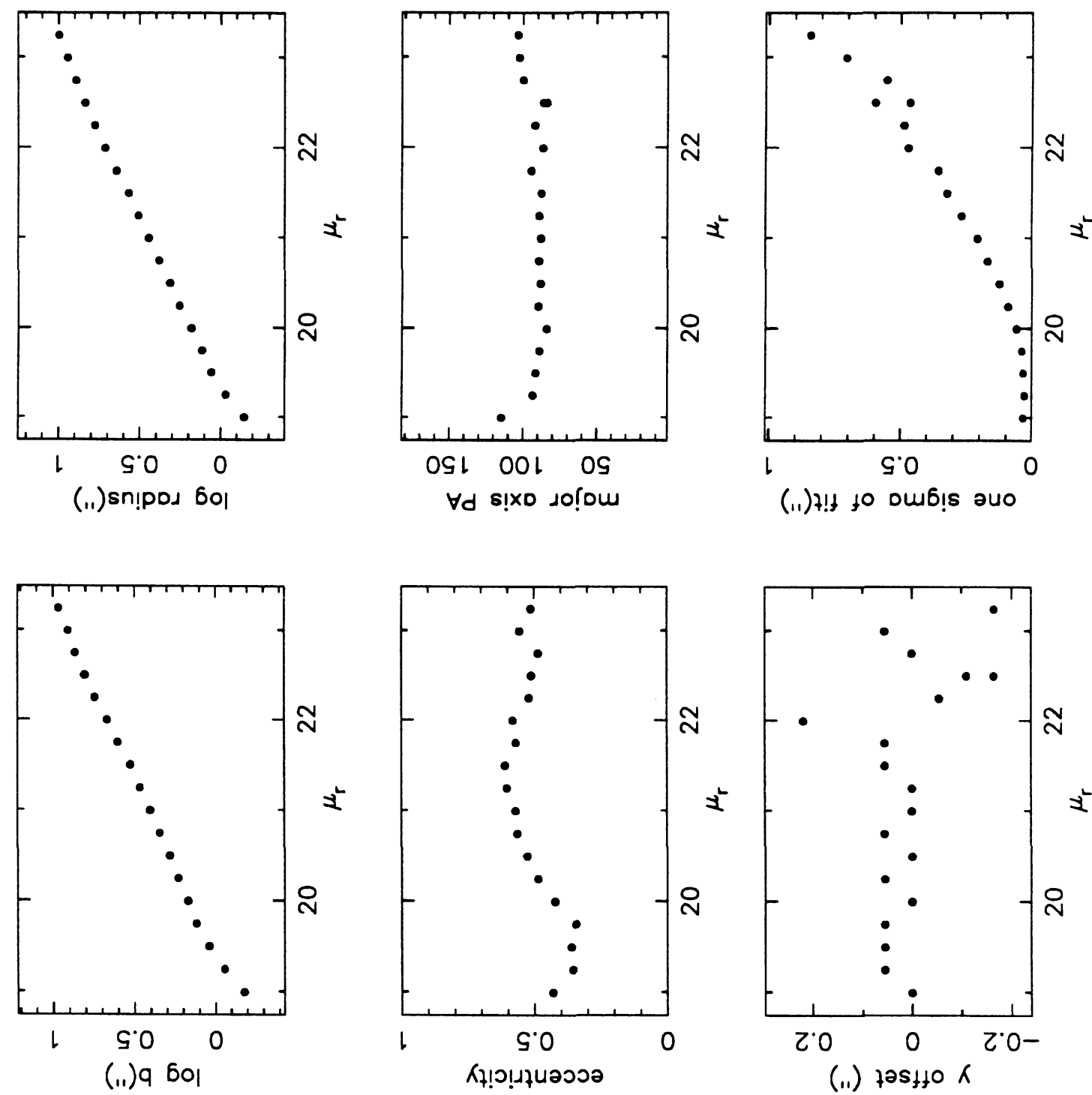

(11) $7 !+$ to oub!s əu०

2
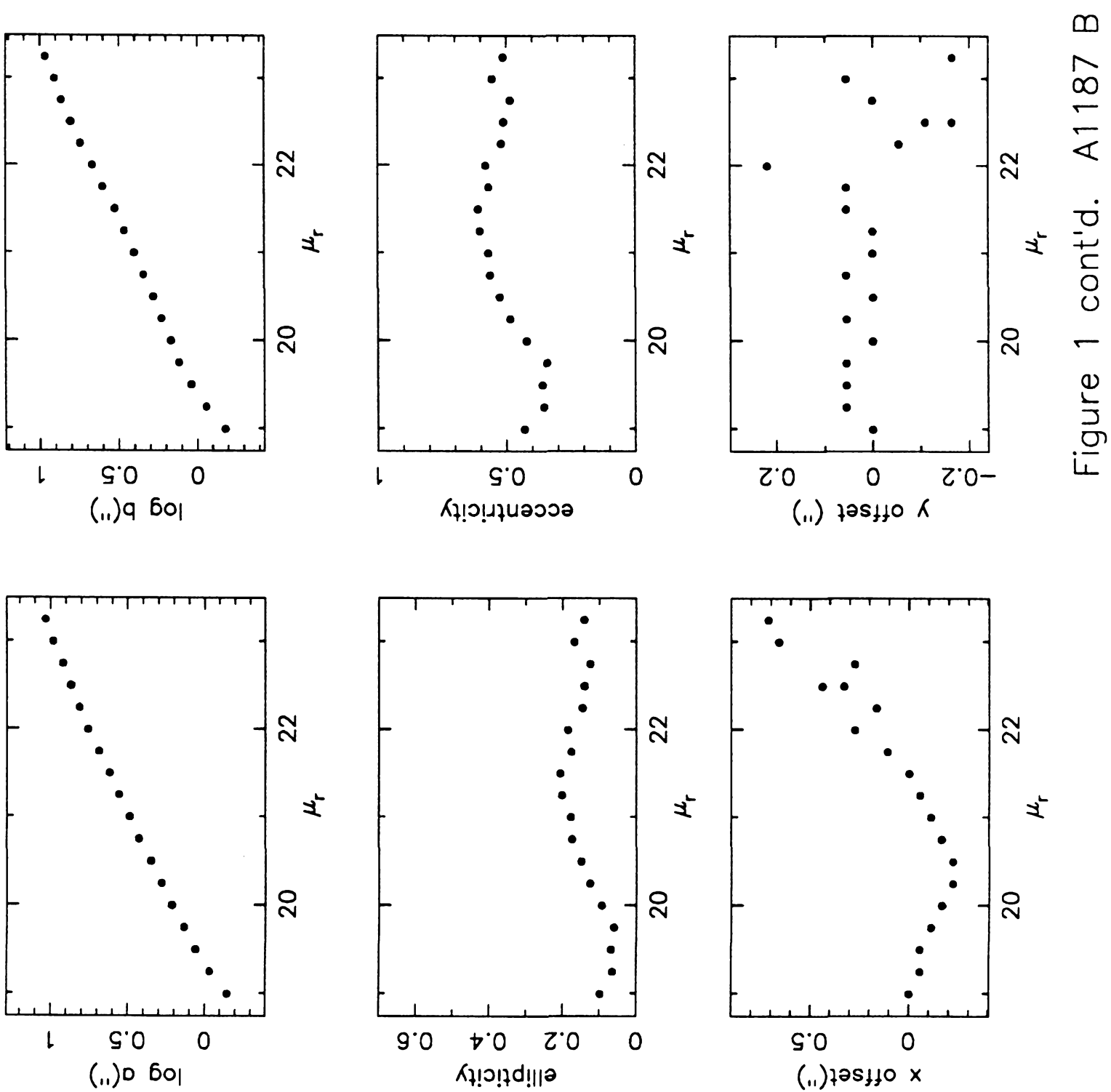

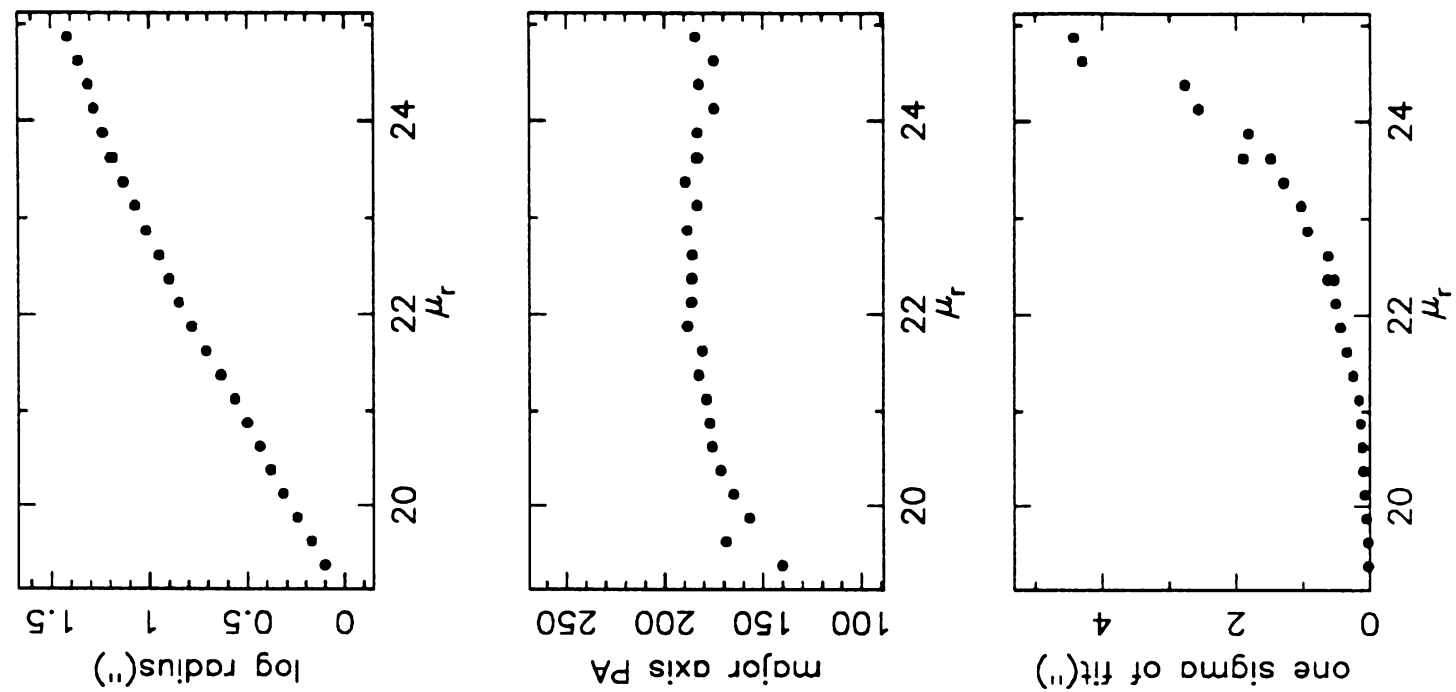

(11) 4 !t to oub!s auo

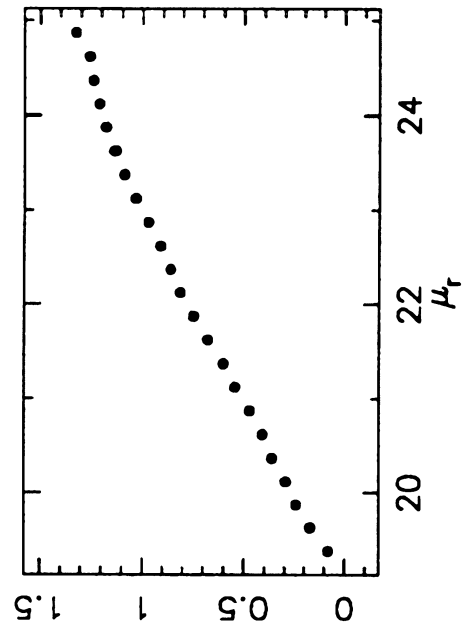

(.1) $\mathrm{q} \mathrm{bol}$

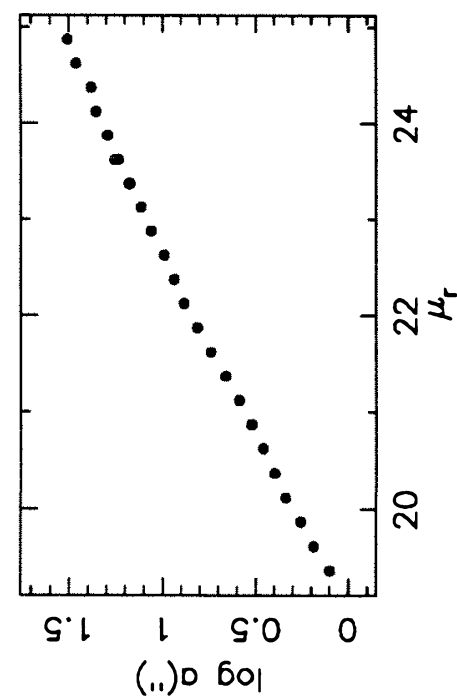

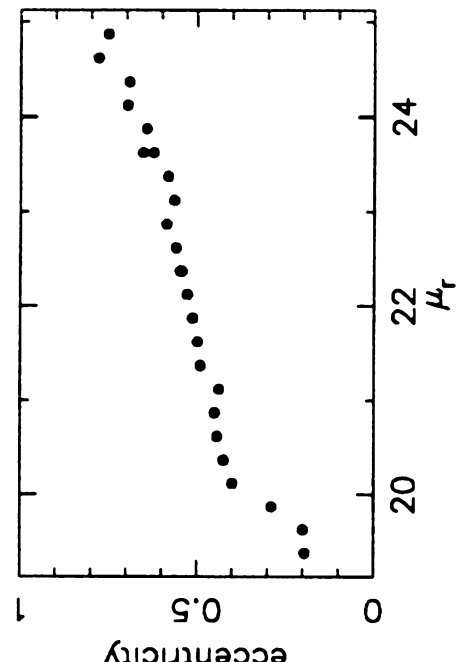

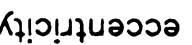

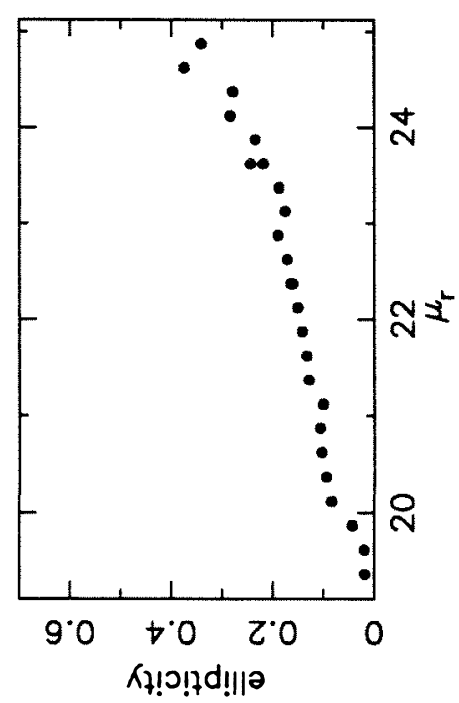

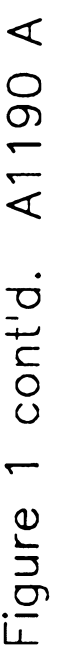

(11) tost10 K

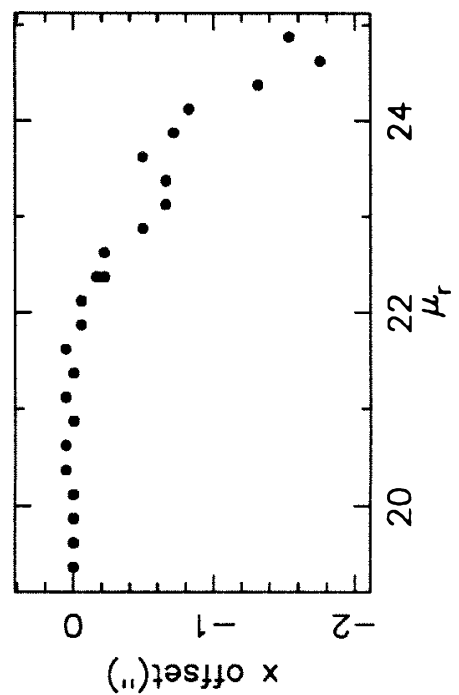




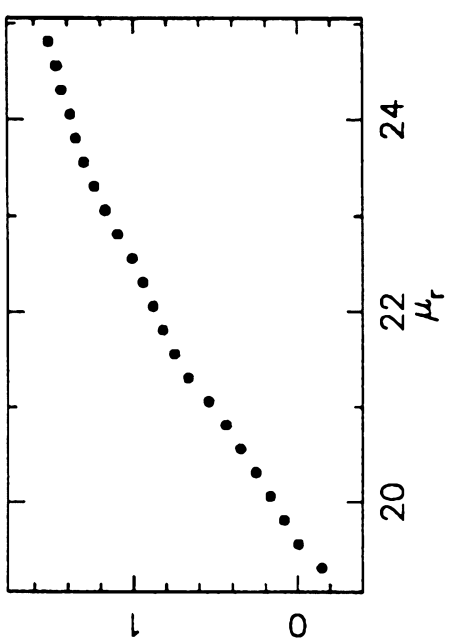

(.1) sn!pod 601

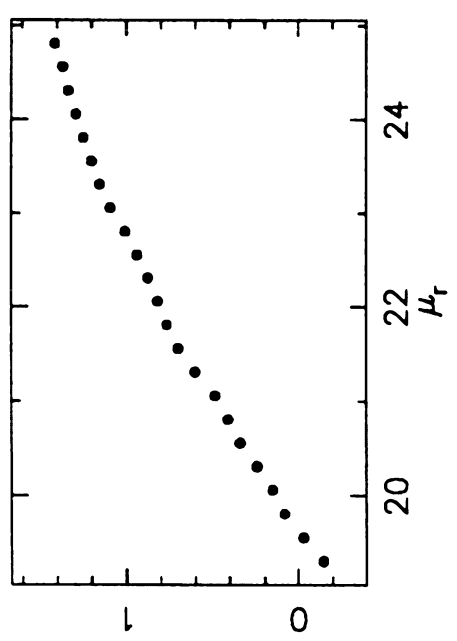

(.1) 9 60।

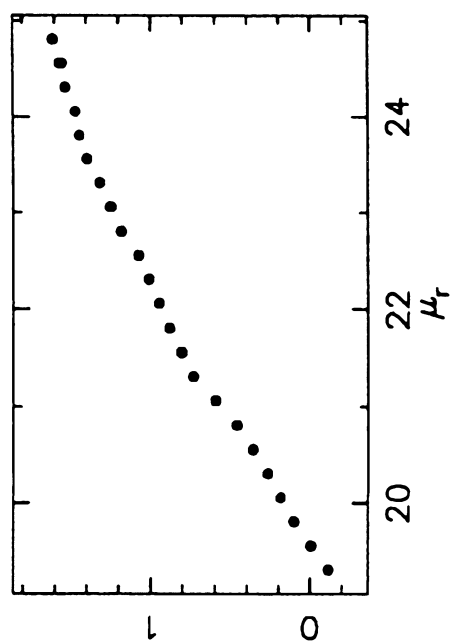

(.1) $\mathrm{D}$ 60l

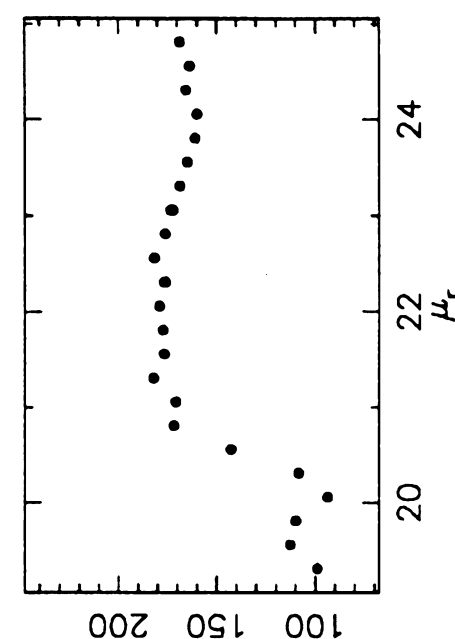

$\forall d$ s!xo so!nom

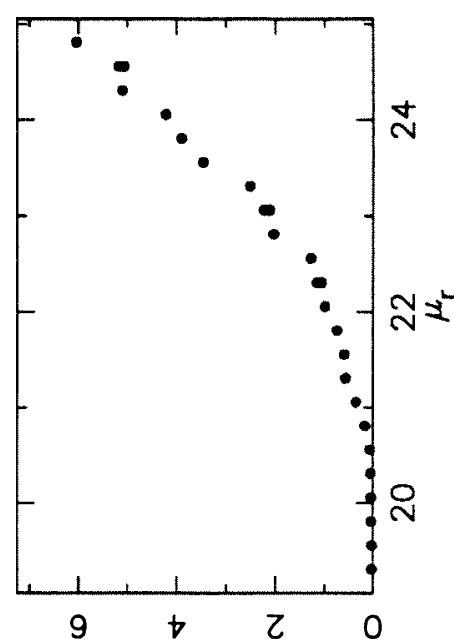

(11) 7 ! t 10 Dub! วu०

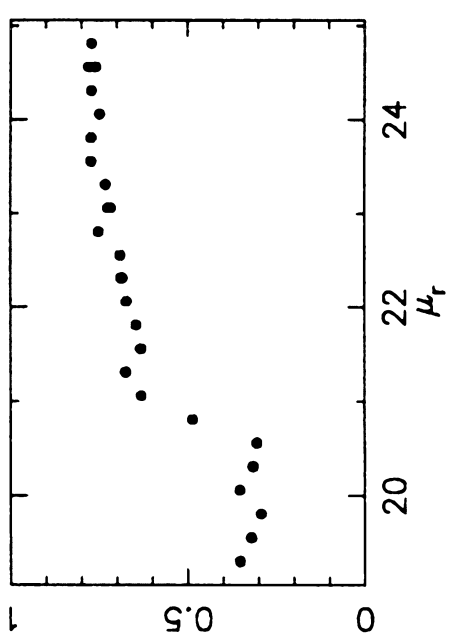

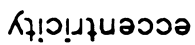

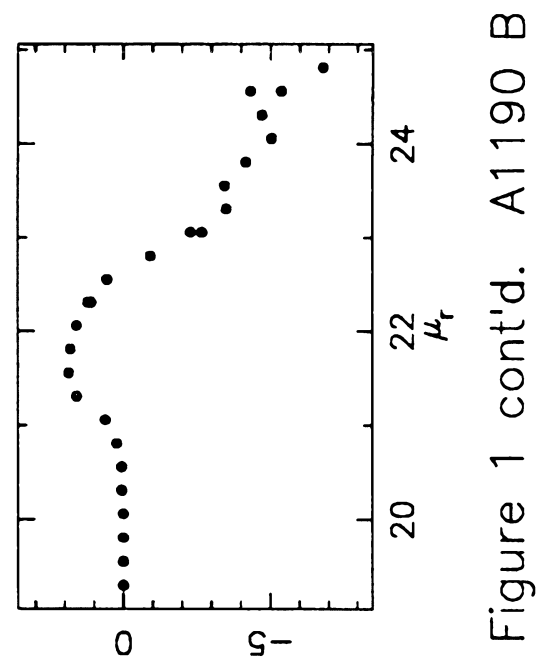

(ii) $705410 \mathrm{~K}$

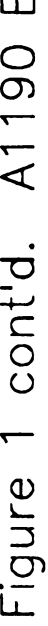
0 

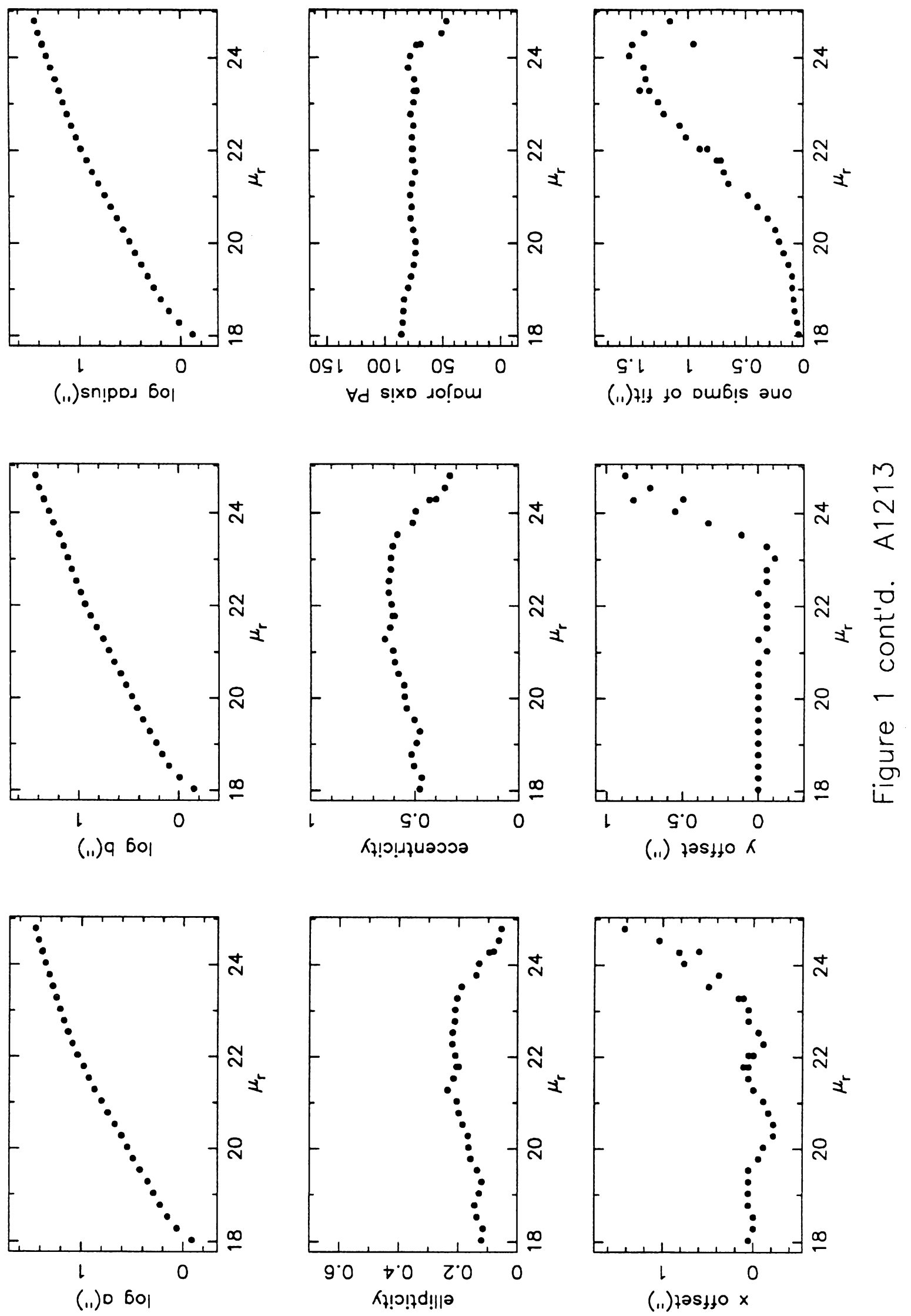

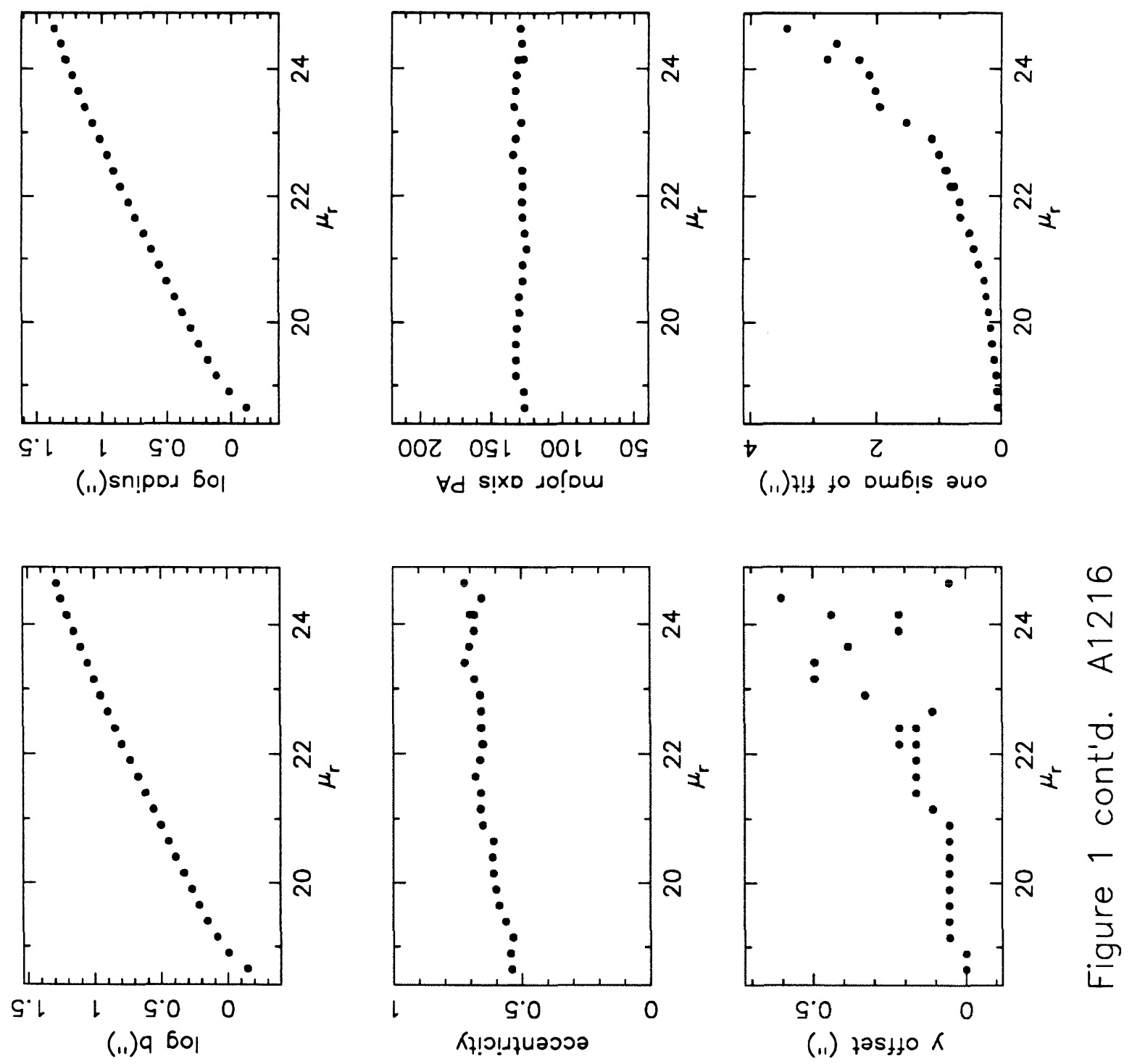

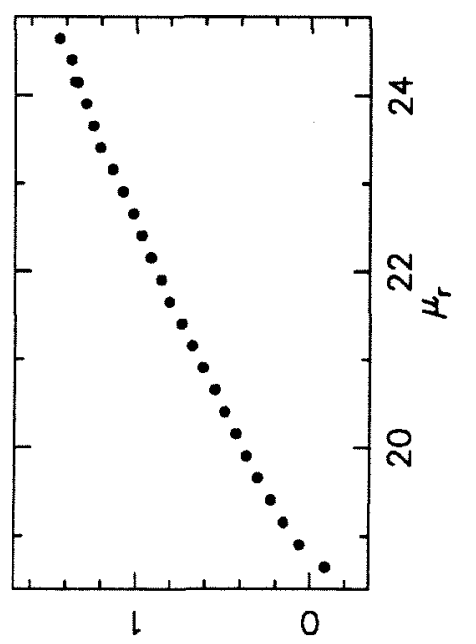

(11) 0 6ol
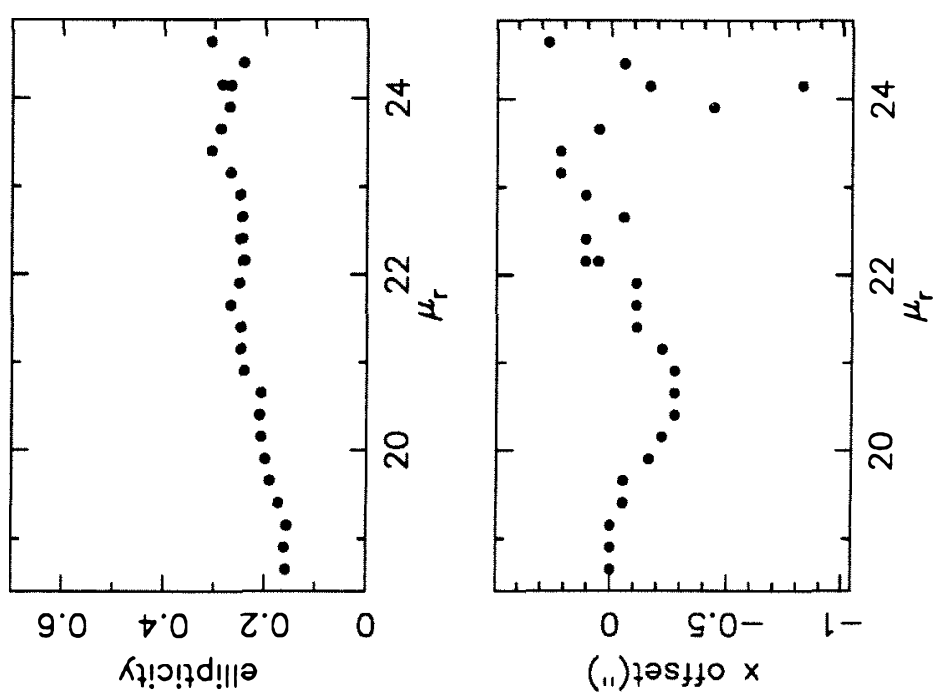


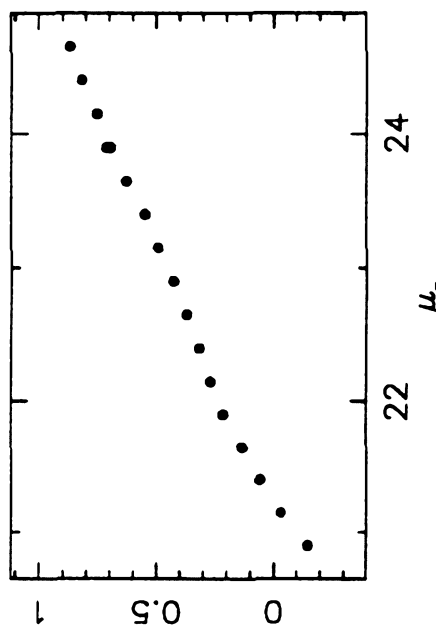

(.1) sn!pod 60।

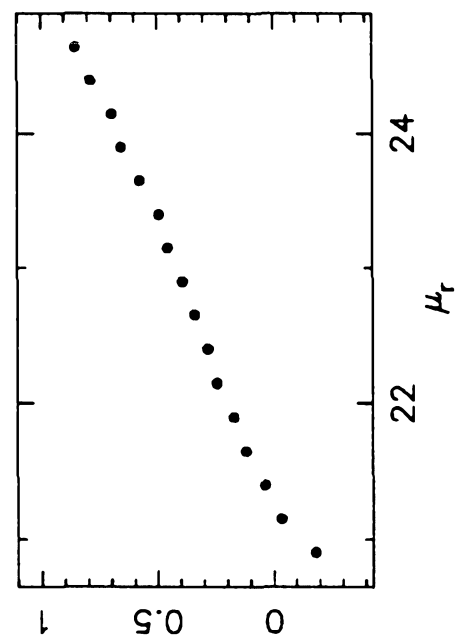

(.1) 9 601

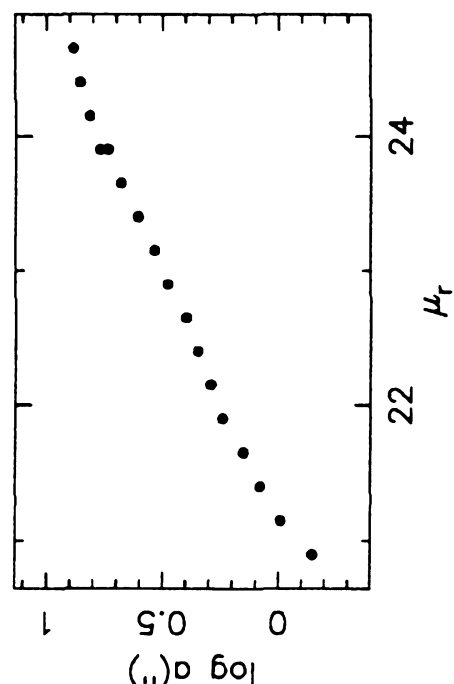

(.1) 0 601
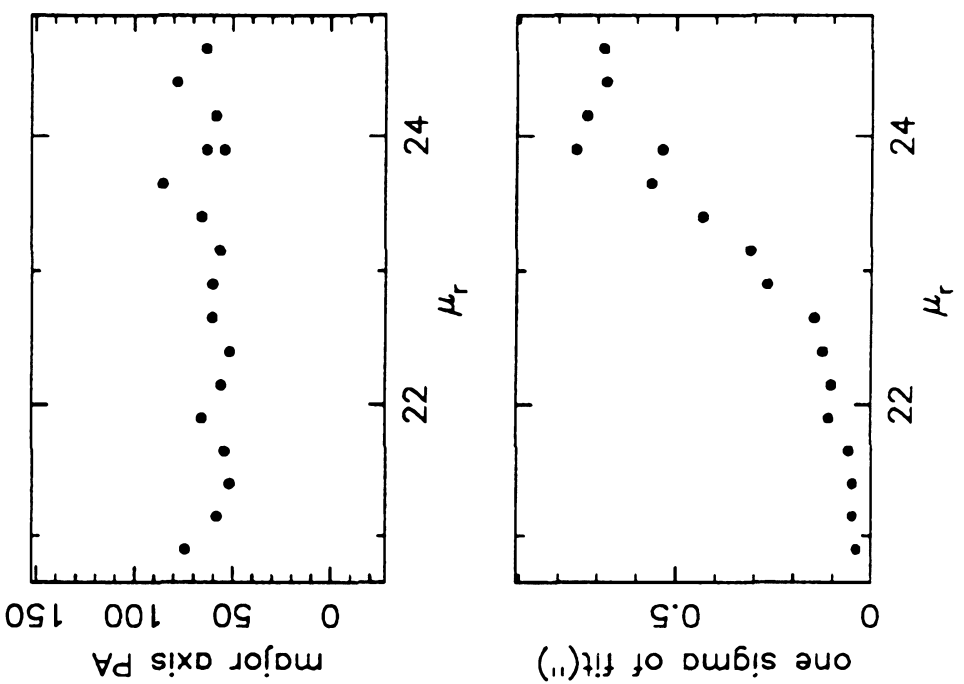

(.1) $+! 5$ to Dub! a a
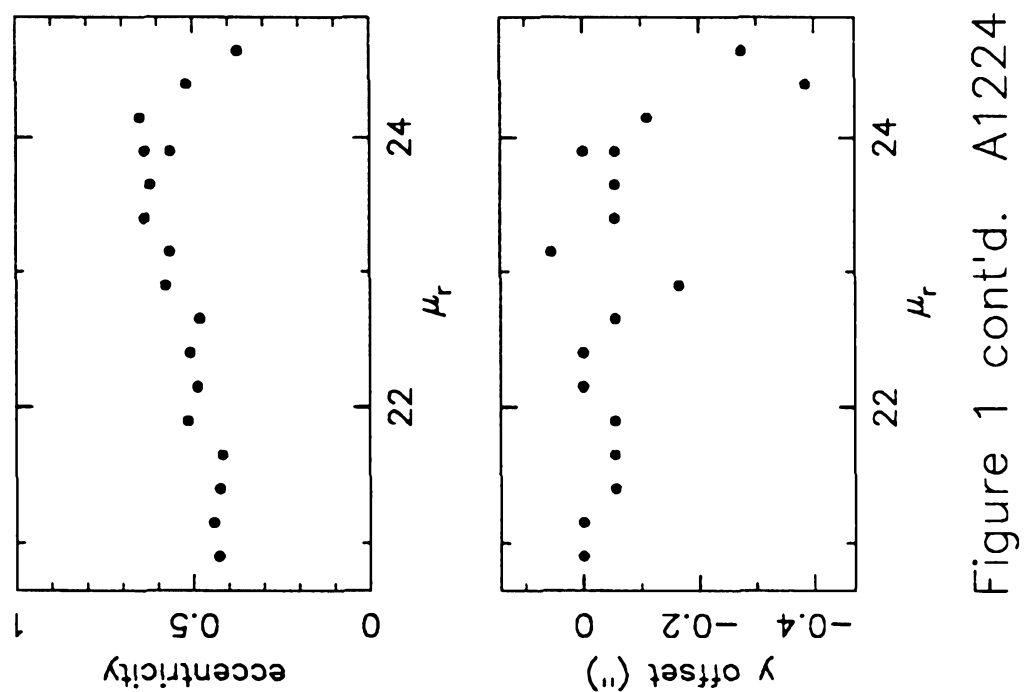

(i1) $725+10 \mathrm{~K}$

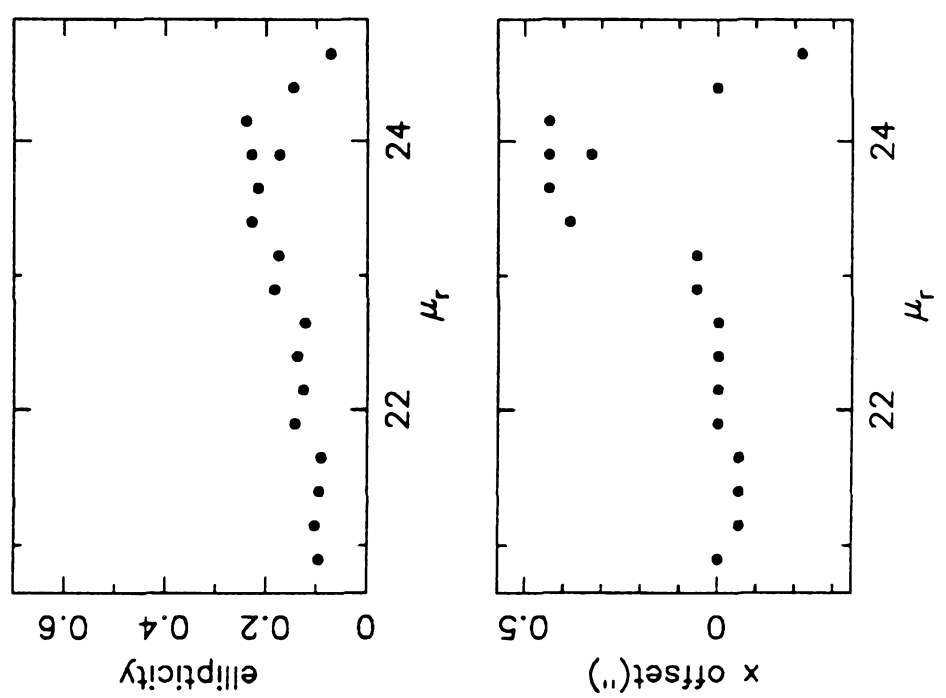




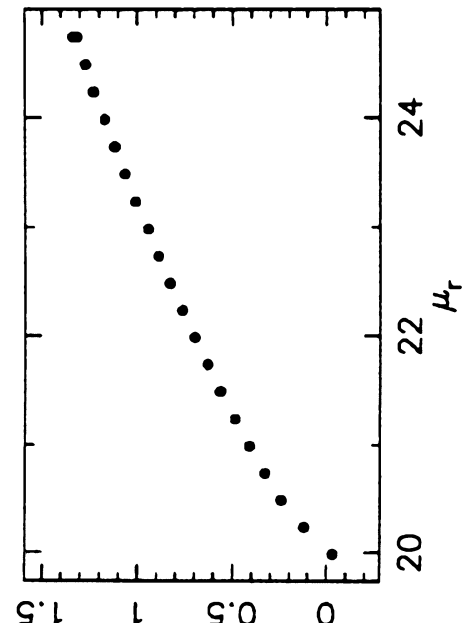

(.1) sn!pod 6ol
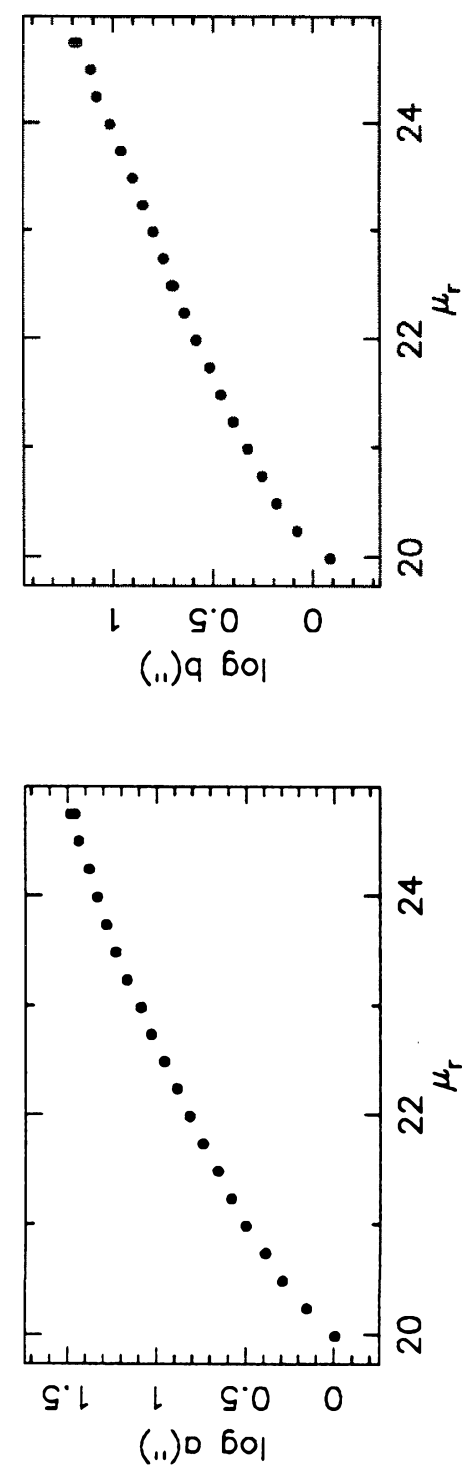
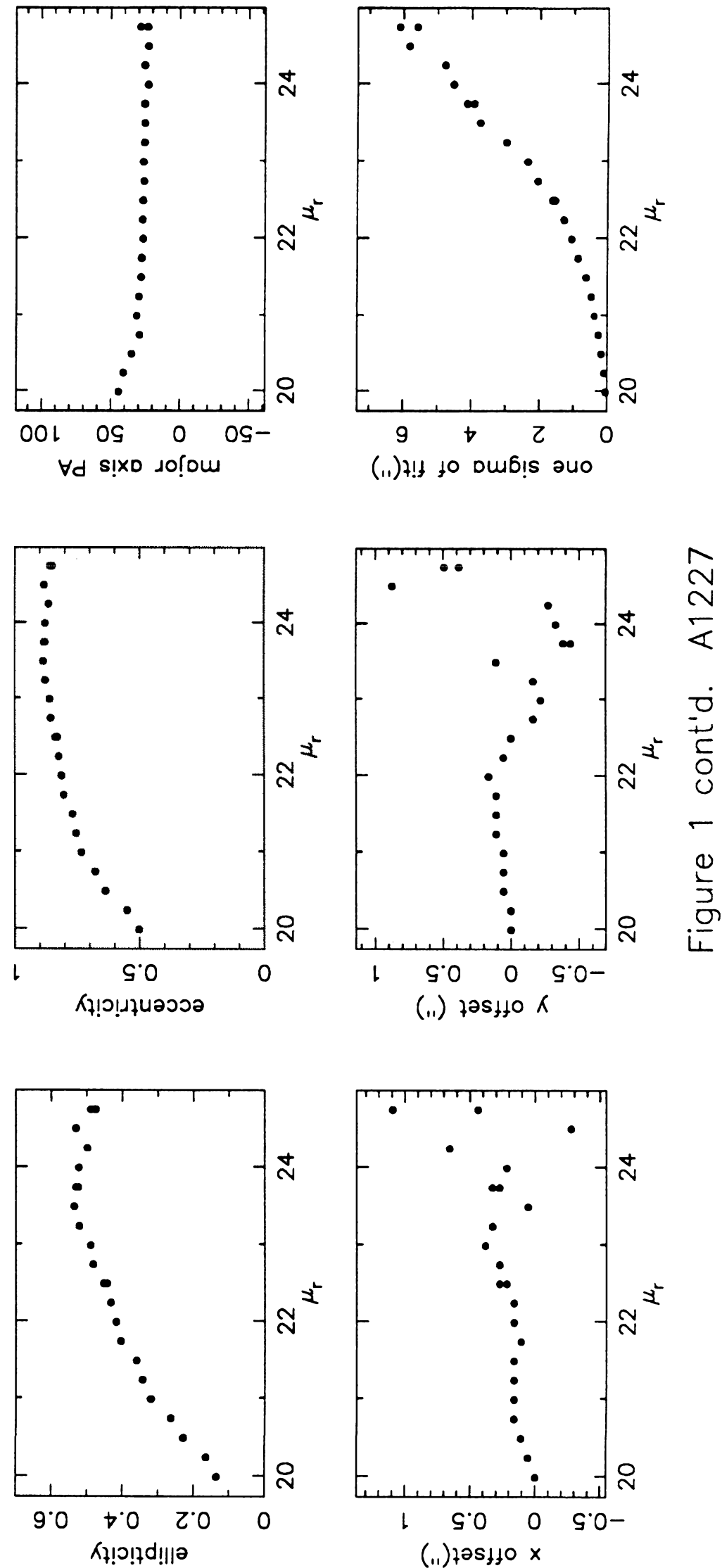

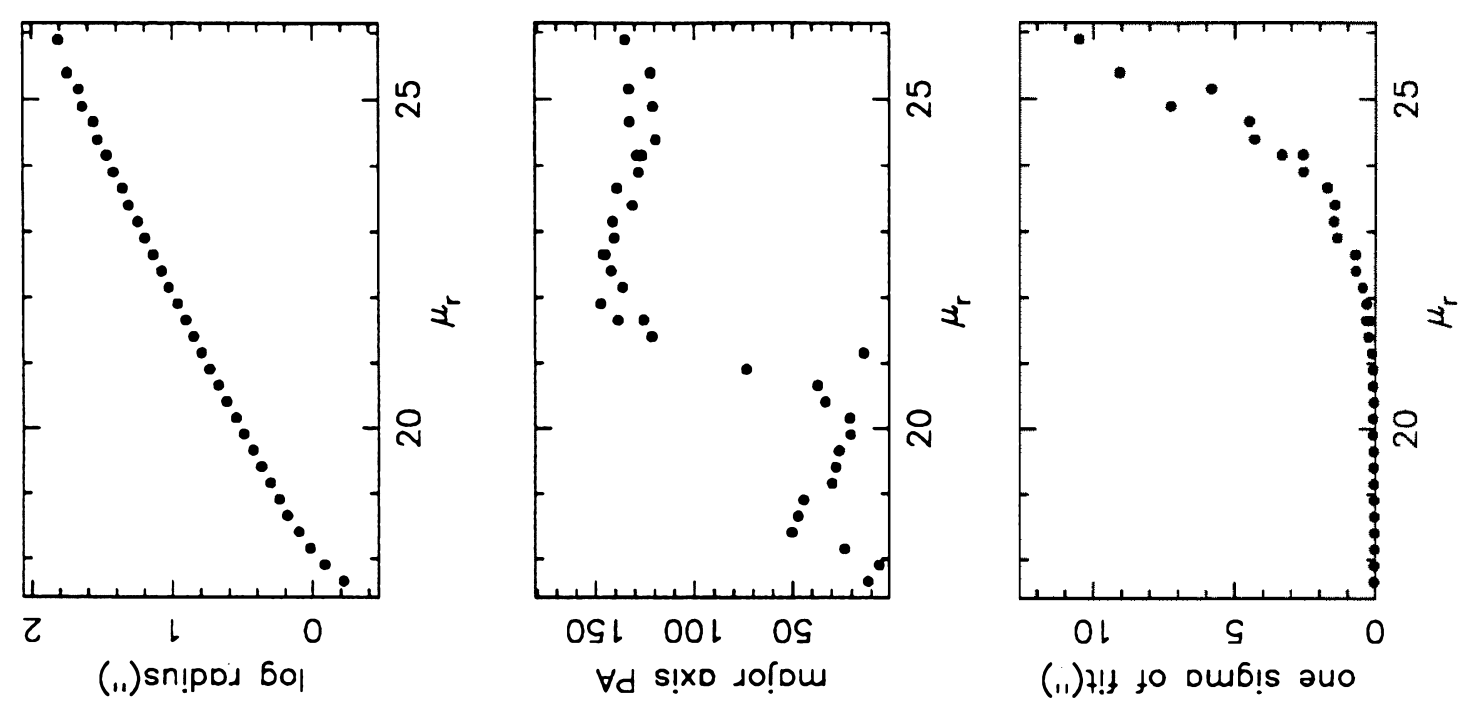

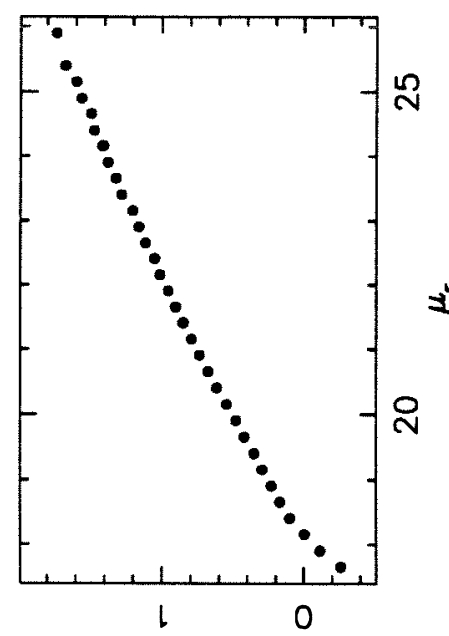

(i1) 9 6ol

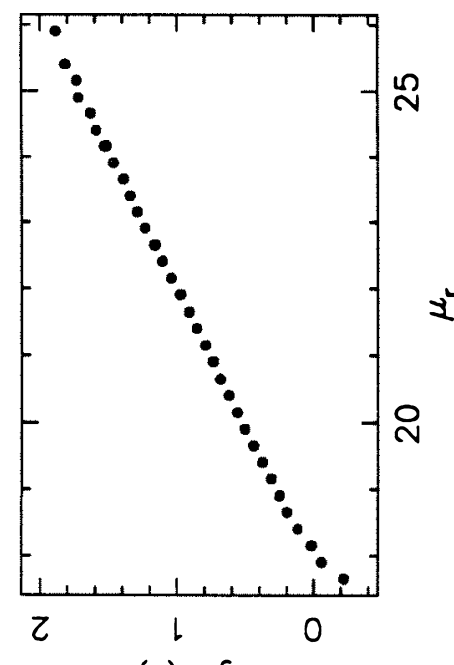

(..) 0 bol
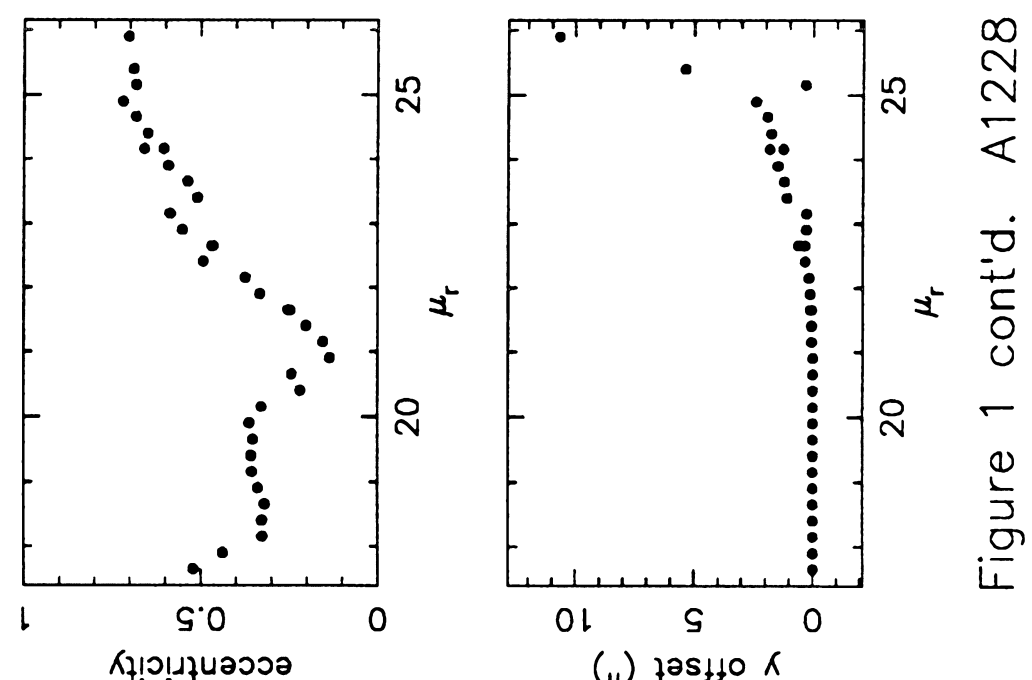

(.1) $705+50 \mathrm{~K}$

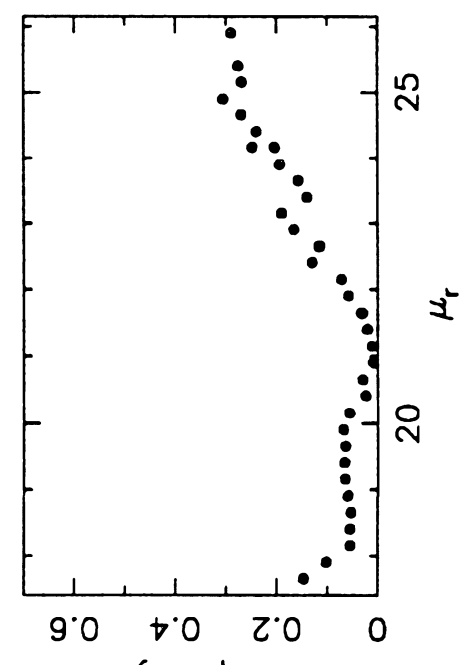

रł!ว!?d!||ə

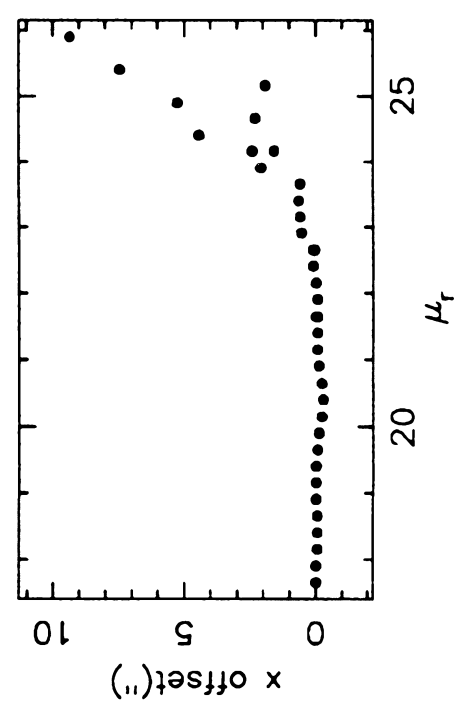




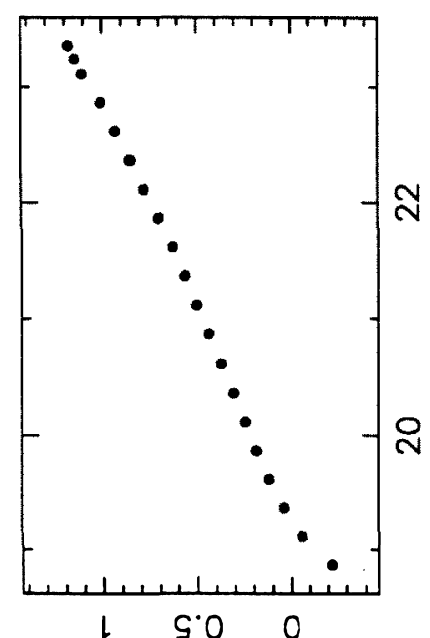

(.1) sn!pod 6ol
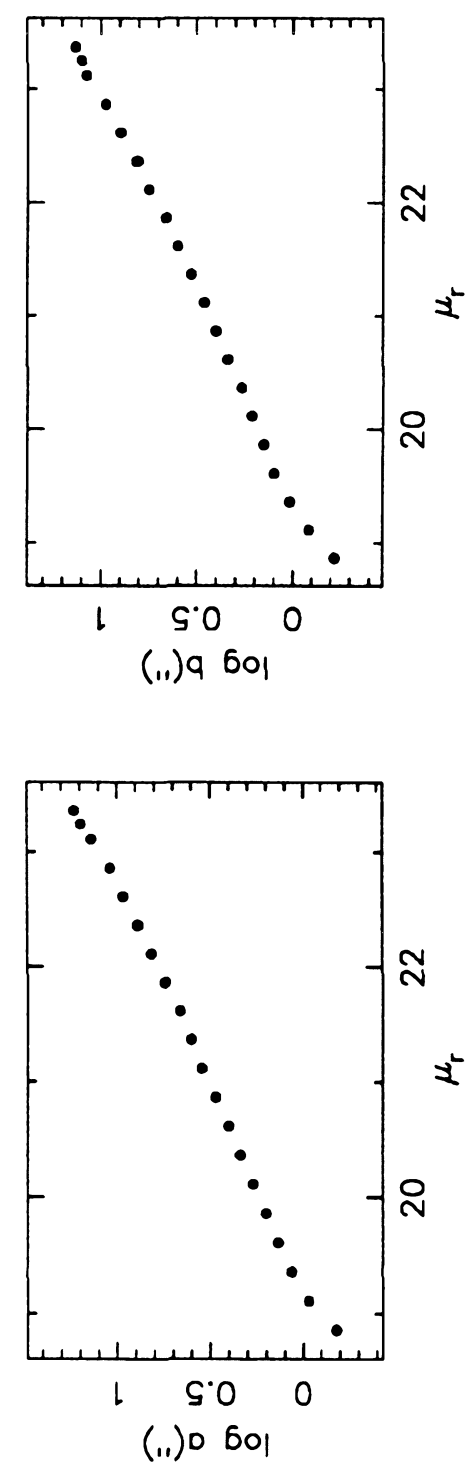

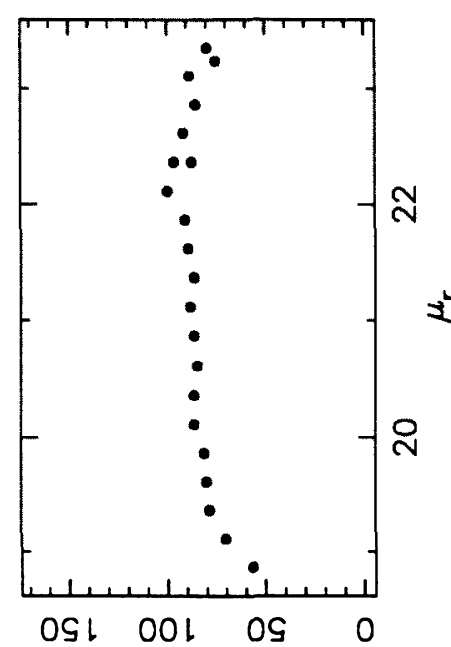

$\forall d$ s!xo so!́m
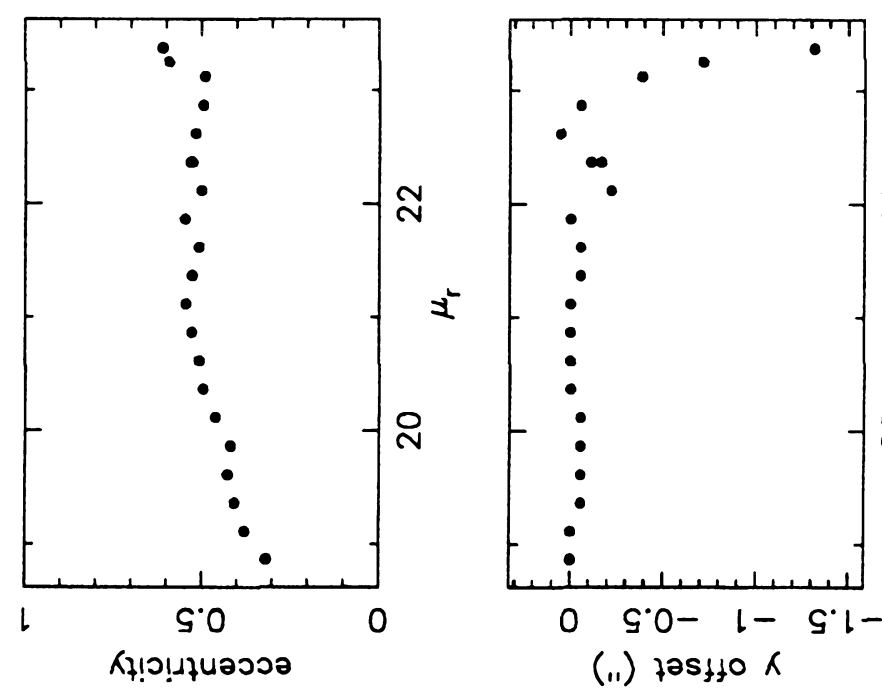

(.1) tas t $10 \mathrm{~K}$
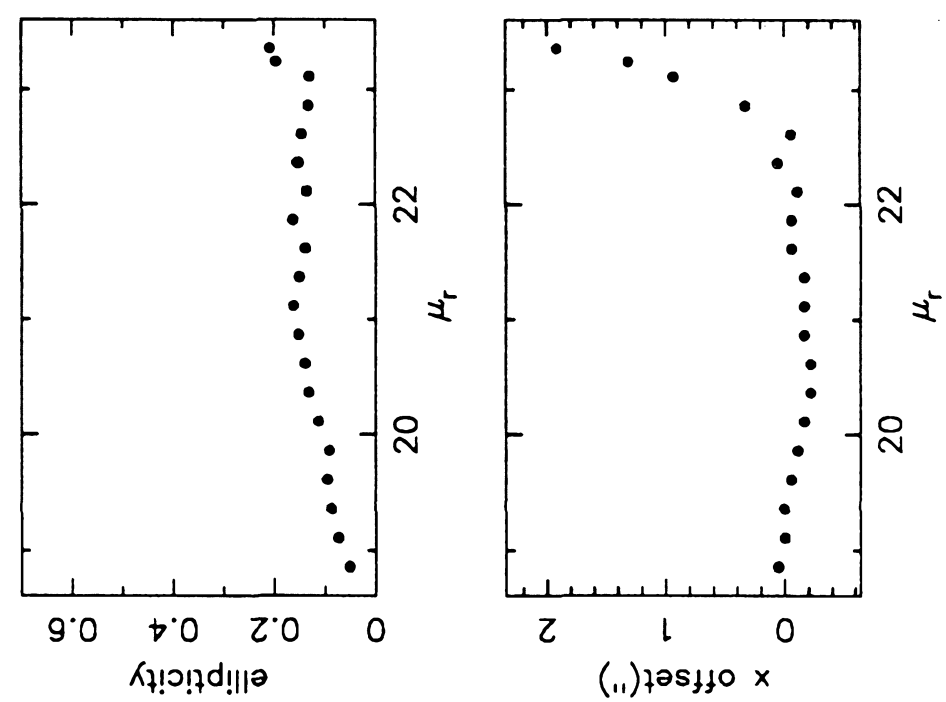

$\infty$

$\frac{N}{4}$

ล

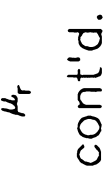

오

皇 


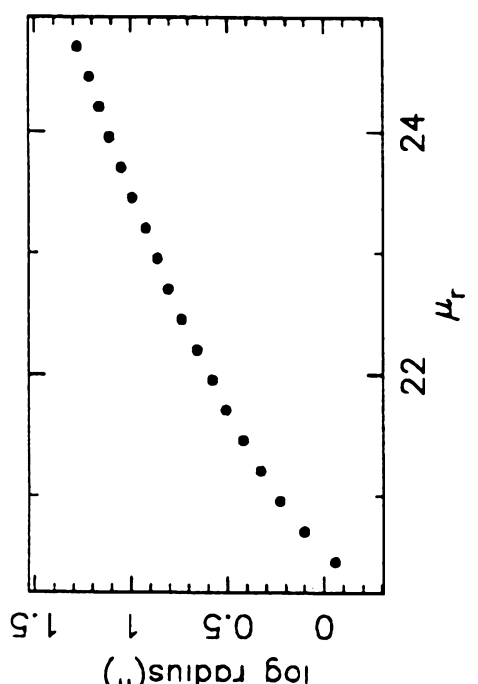

(11) sn!pos 6ol

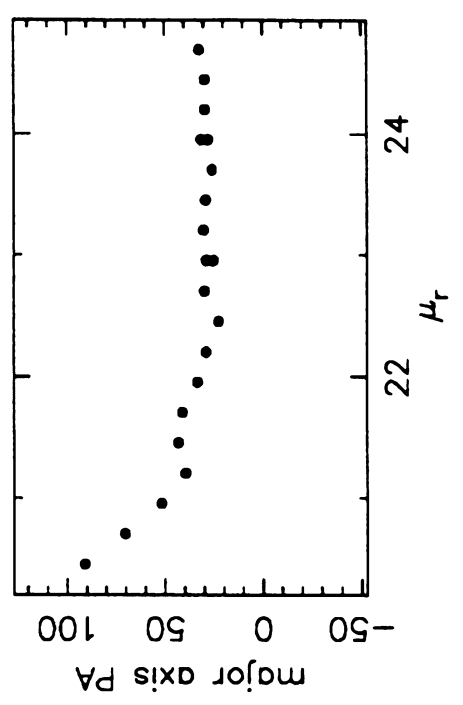

$\forall d$ s!xo so!

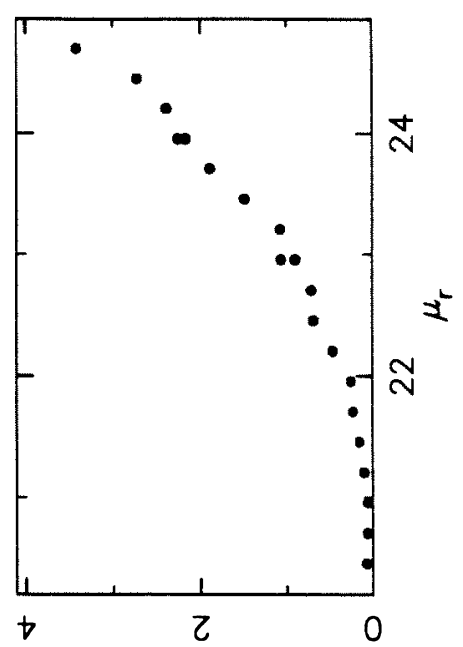

(11) $7 !+10$ Dub! ano
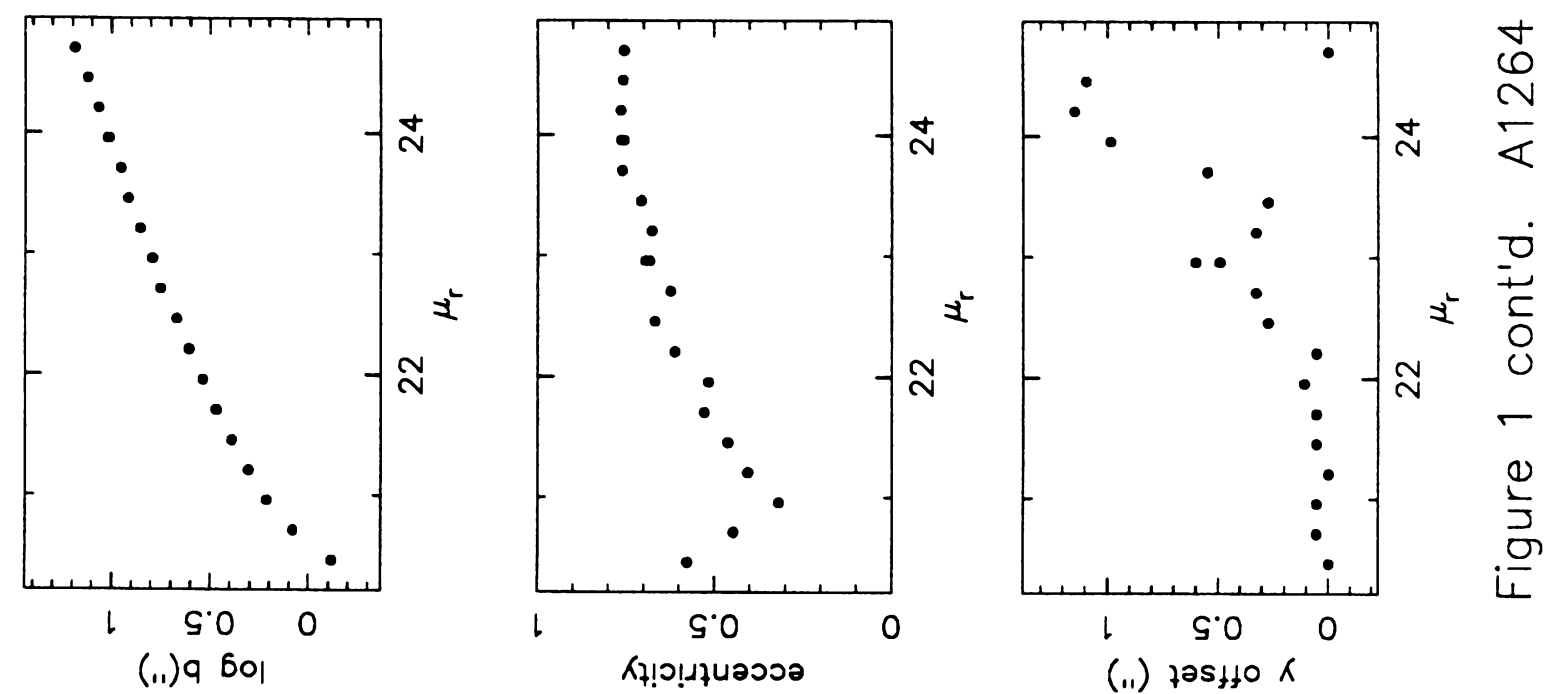

(ii) zos $110 \mathrm{~K}$
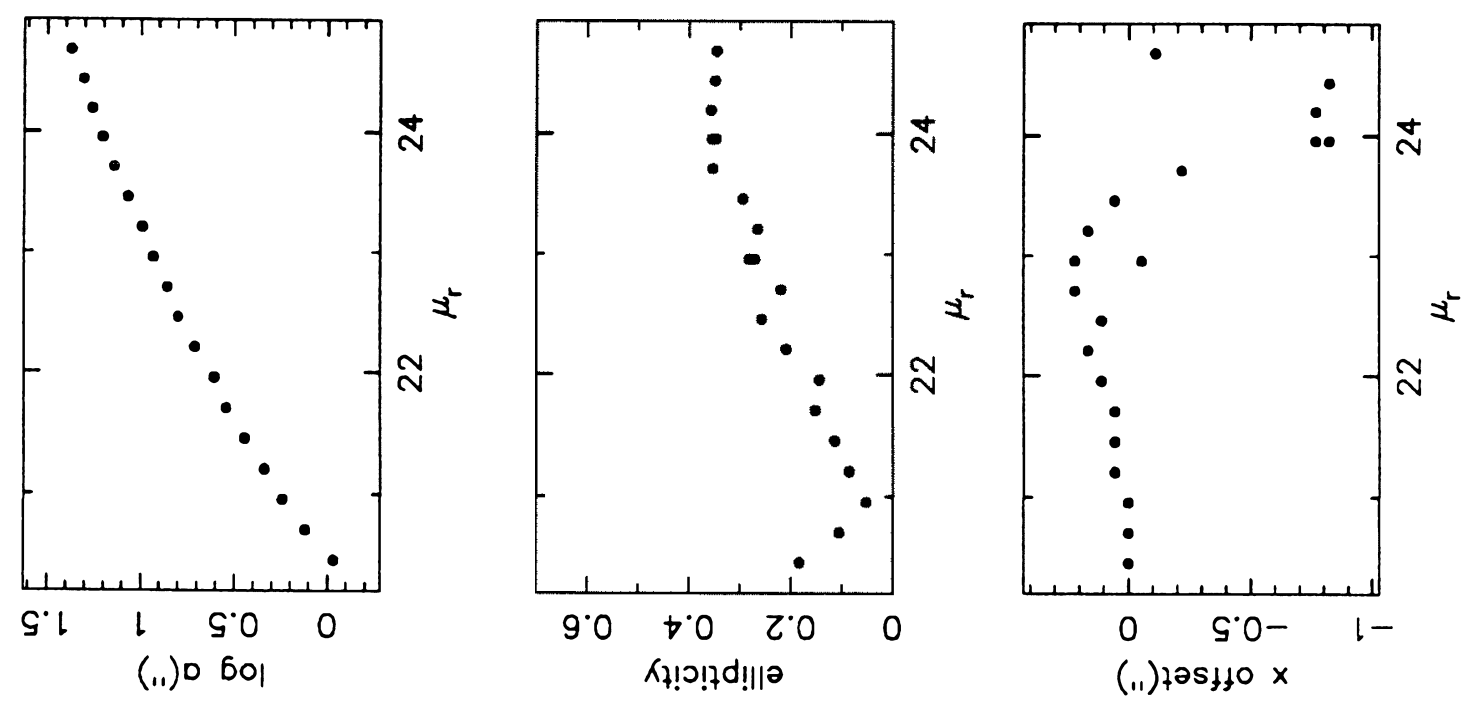


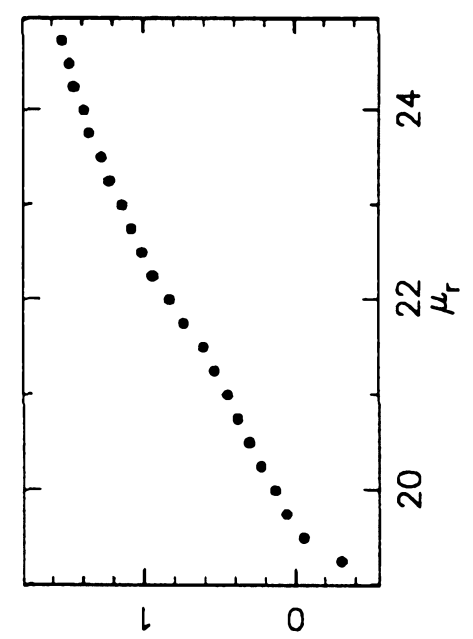

(.1)sn!pos bol

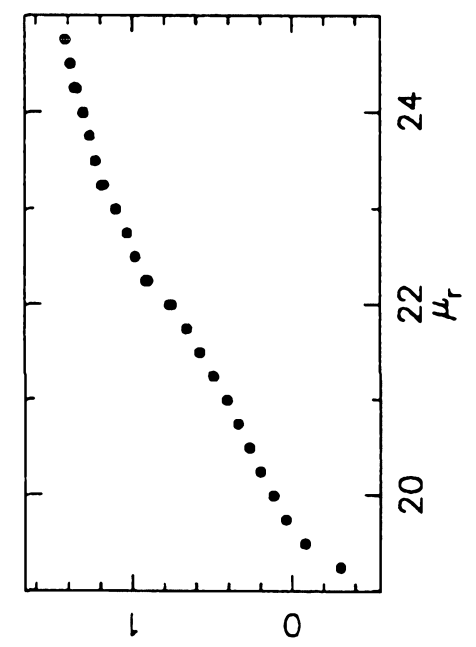

(.1) 9601

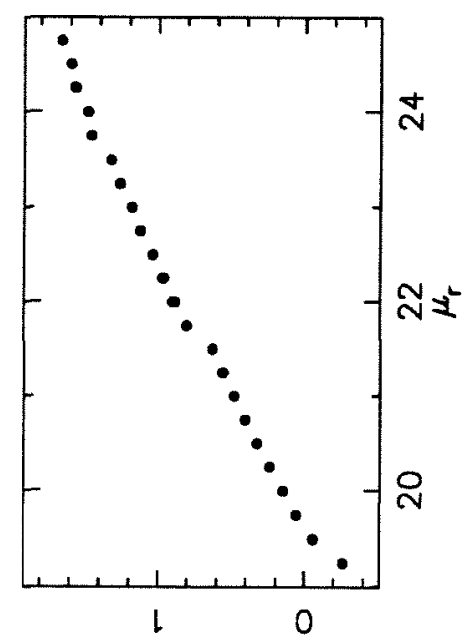

(.1) 6 이

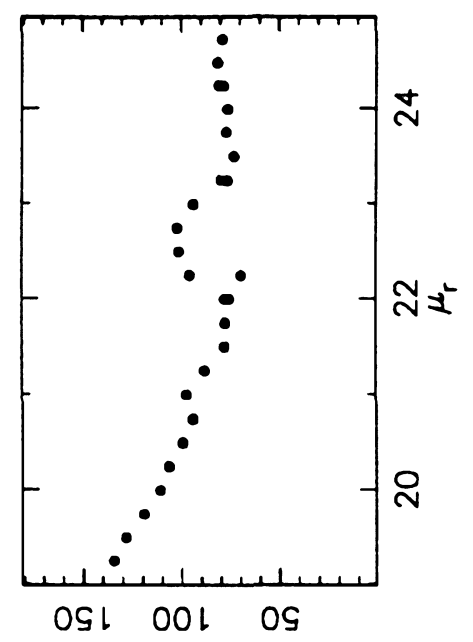

$\forall d$ s!XD Jo!ou

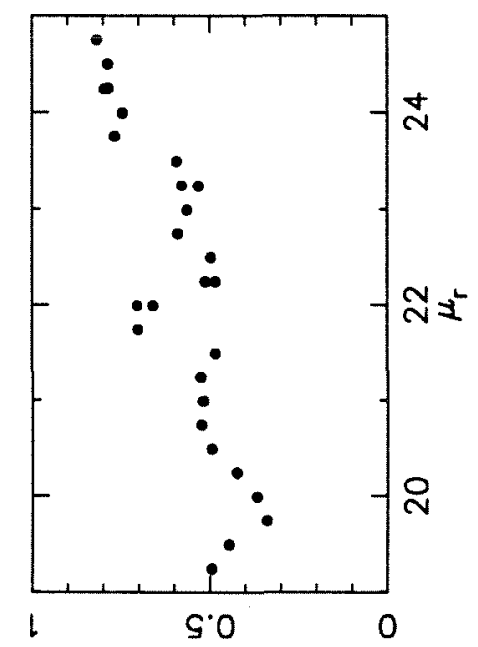

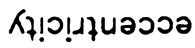
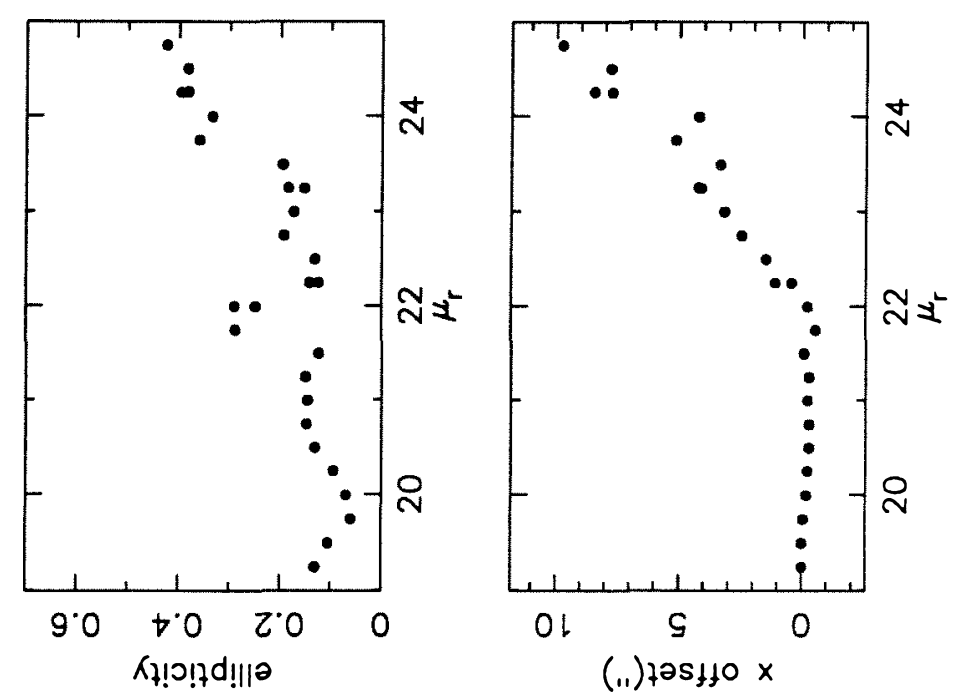


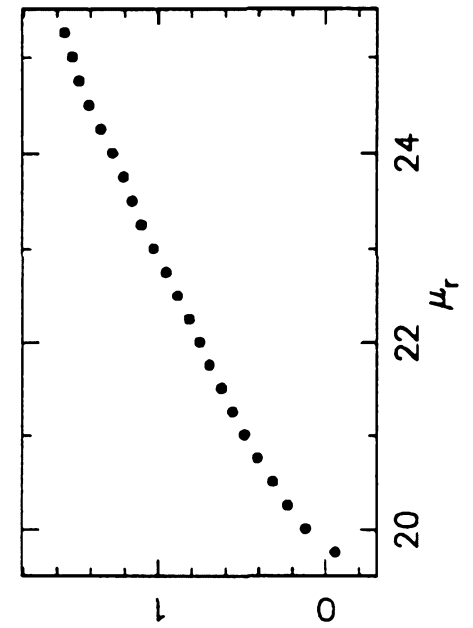

(.1)sn!pos 6ol

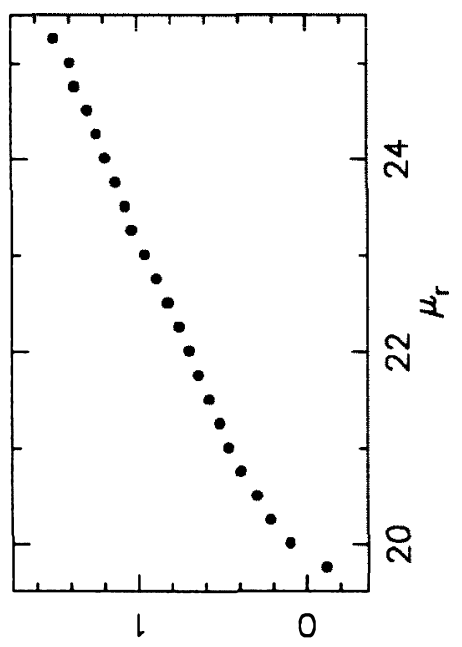

(i1) 9601

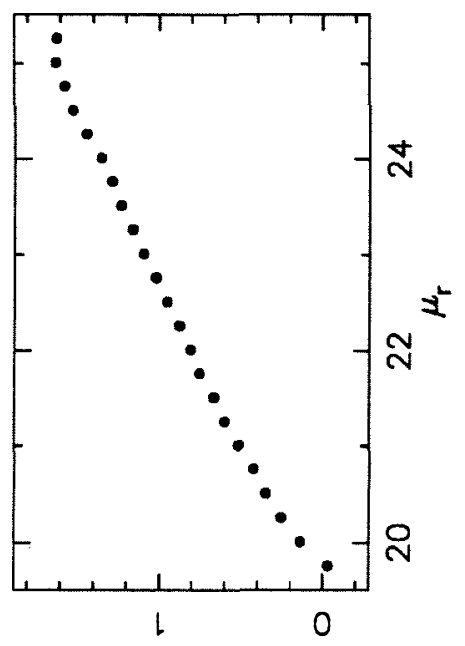

(.1) $0 \mathrm{bo}$

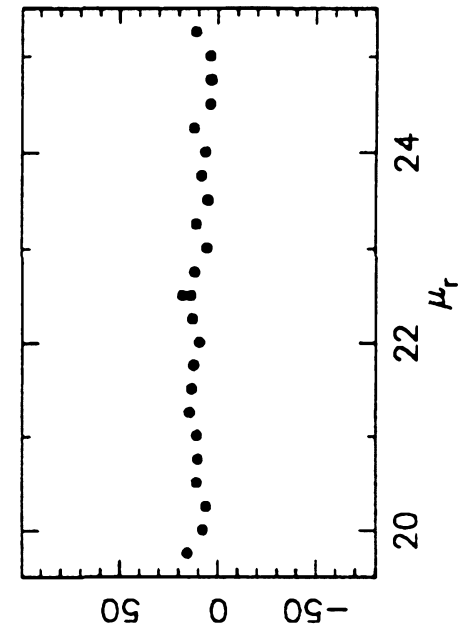

$\forall d$ s!xo so! ou

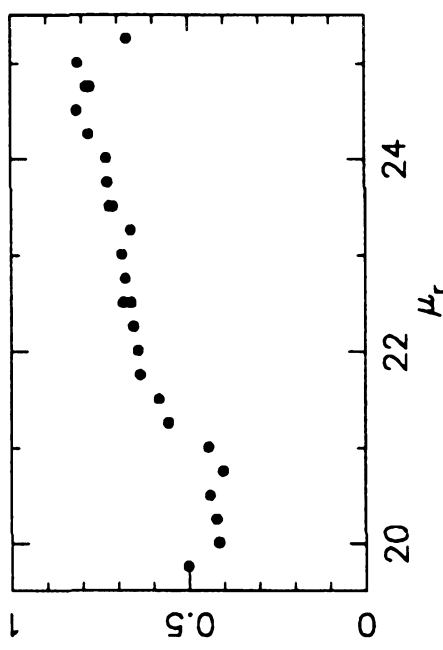

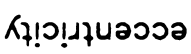

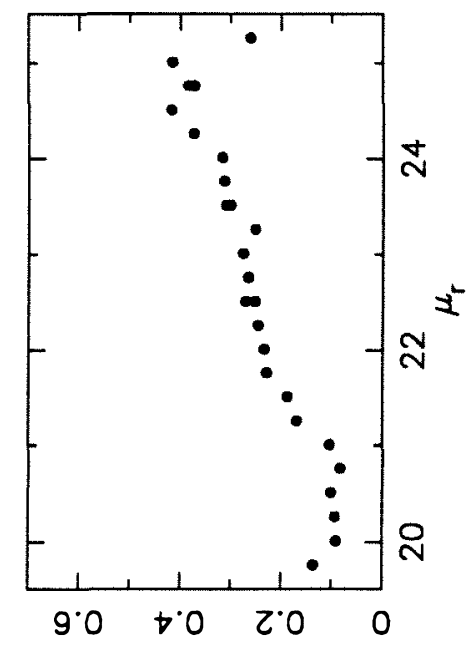

रł!O!†d!||ә

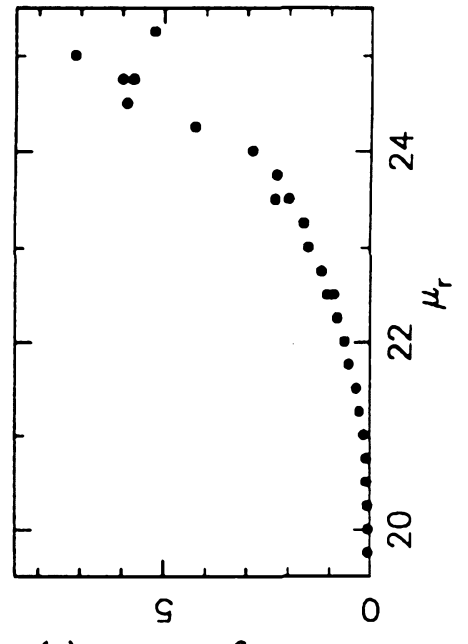

(11) $7 ! 1$ to Dub!s aиo

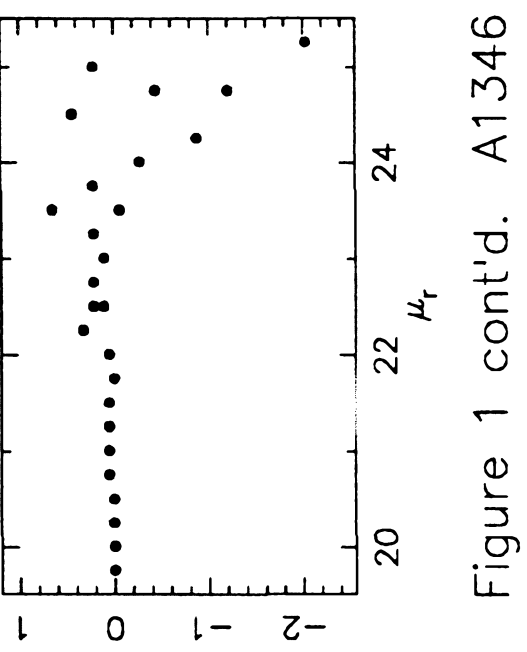

(11) tastlo $\mathrm{K}$

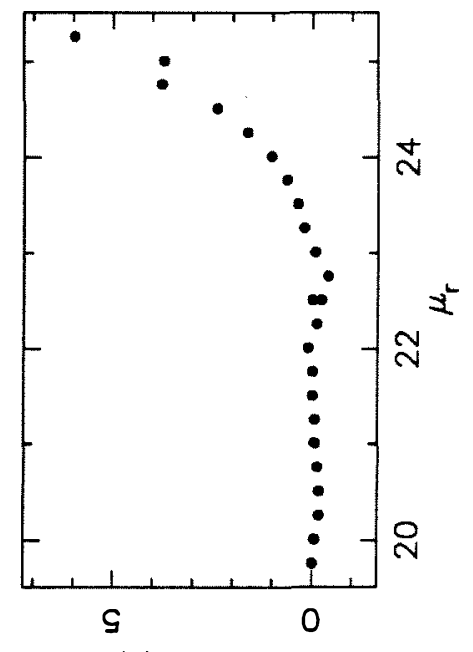

(11) tas $150 \times$ 


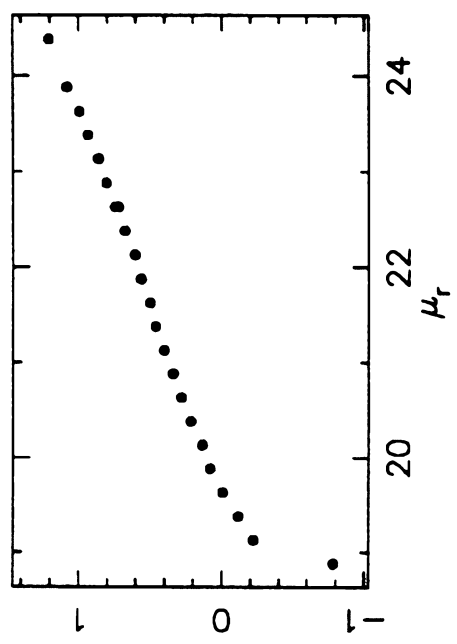

(11)sn!pod 6ol

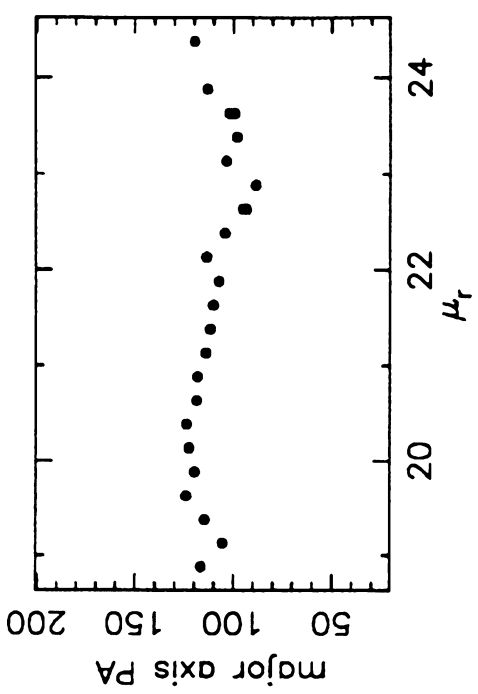

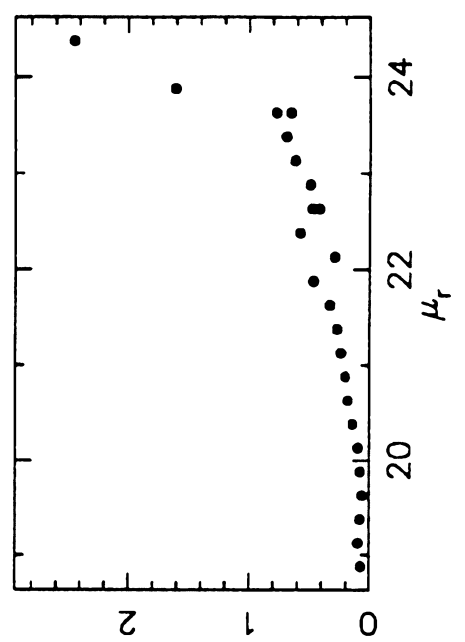

(11) $+!+~ 10$ oub!s au०

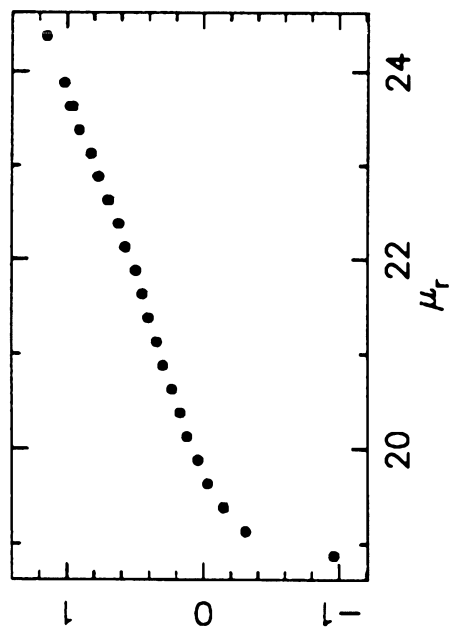

(.1) 9 bol

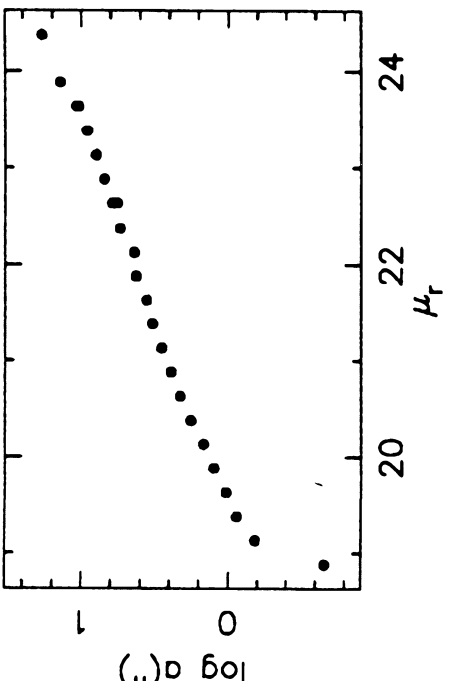

(.1) $0 \mathrm{bol}$

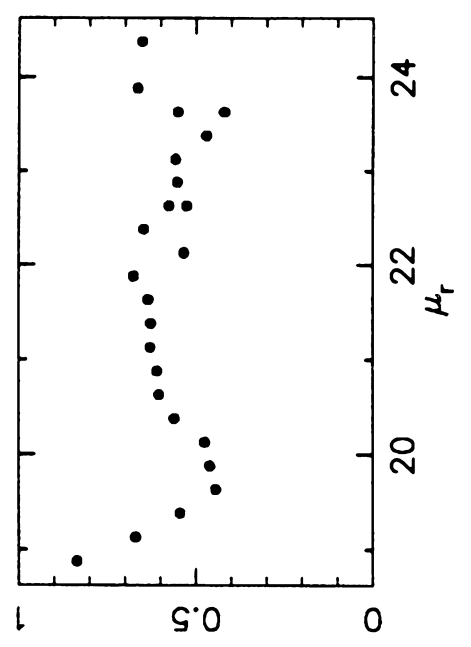

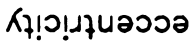

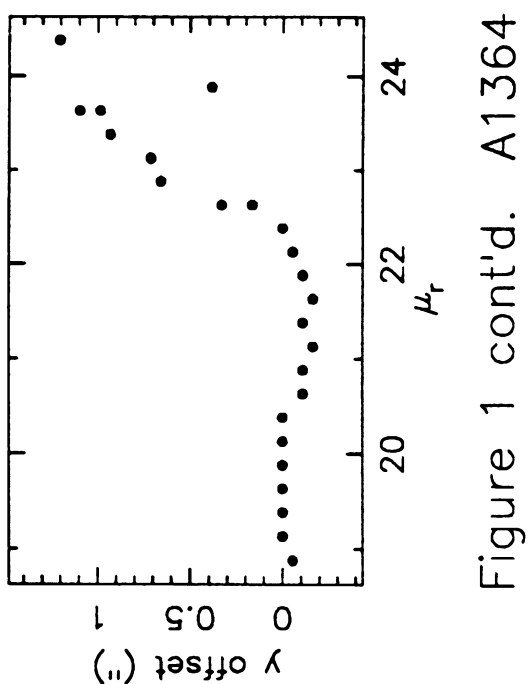

(ii) $725150 \mathrm{~K}$
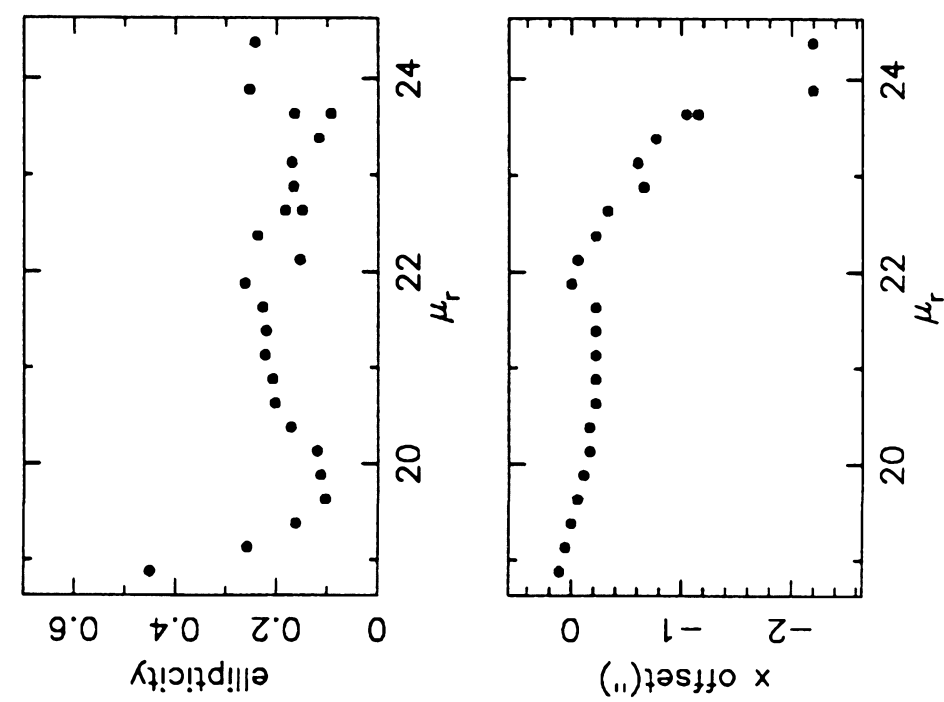


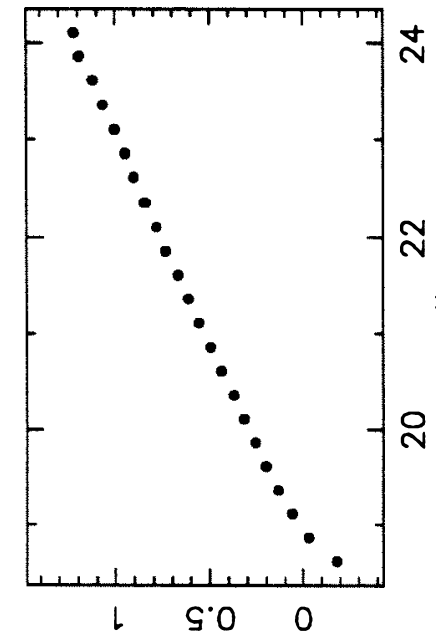

(.1) sn!pod 60l

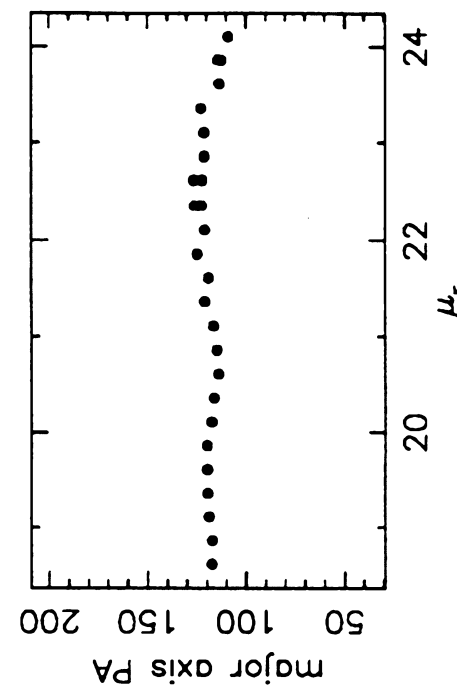

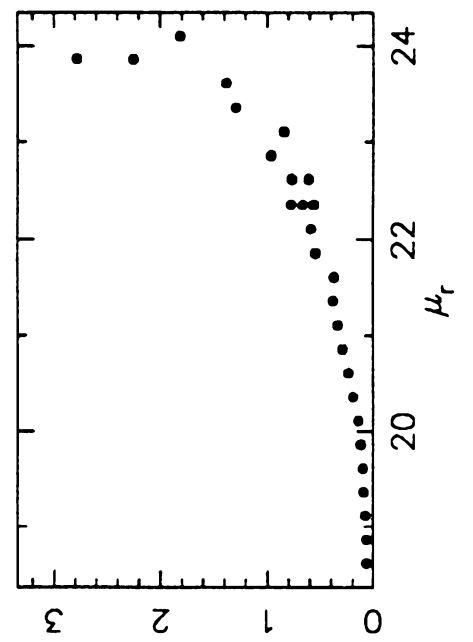

(i1) $)$ !! to Dub! auo
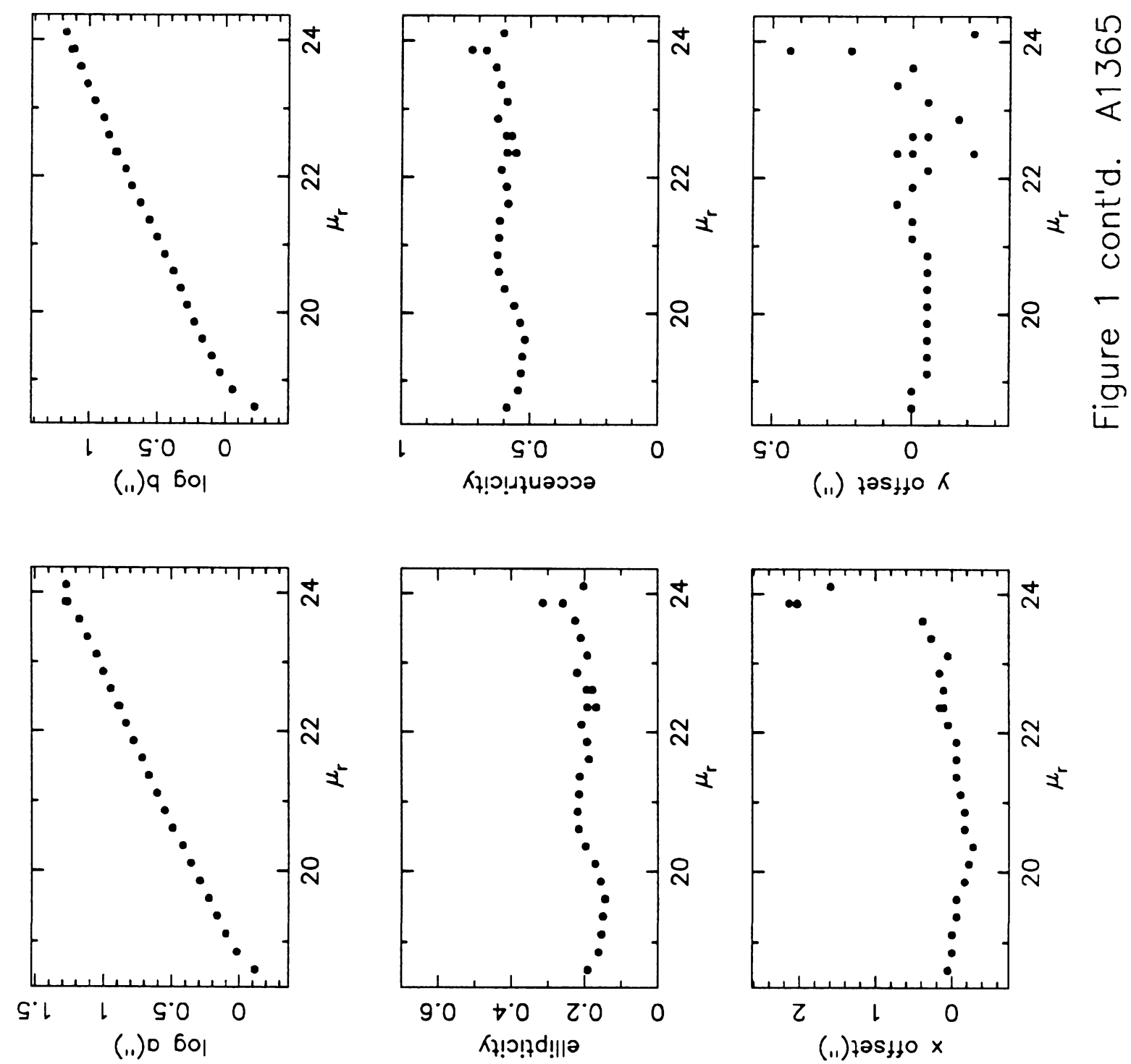


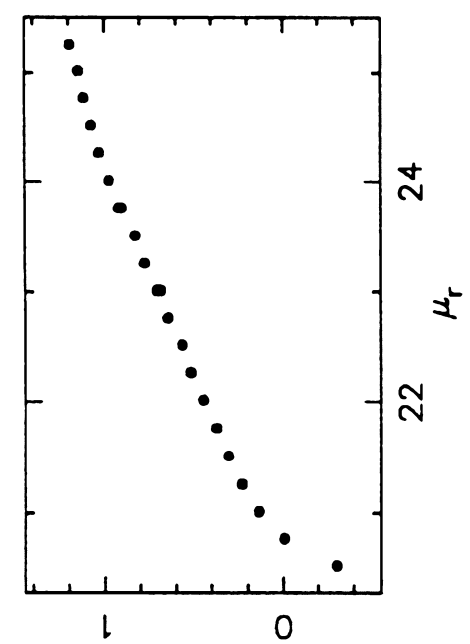

(11)sn!pod 6ol
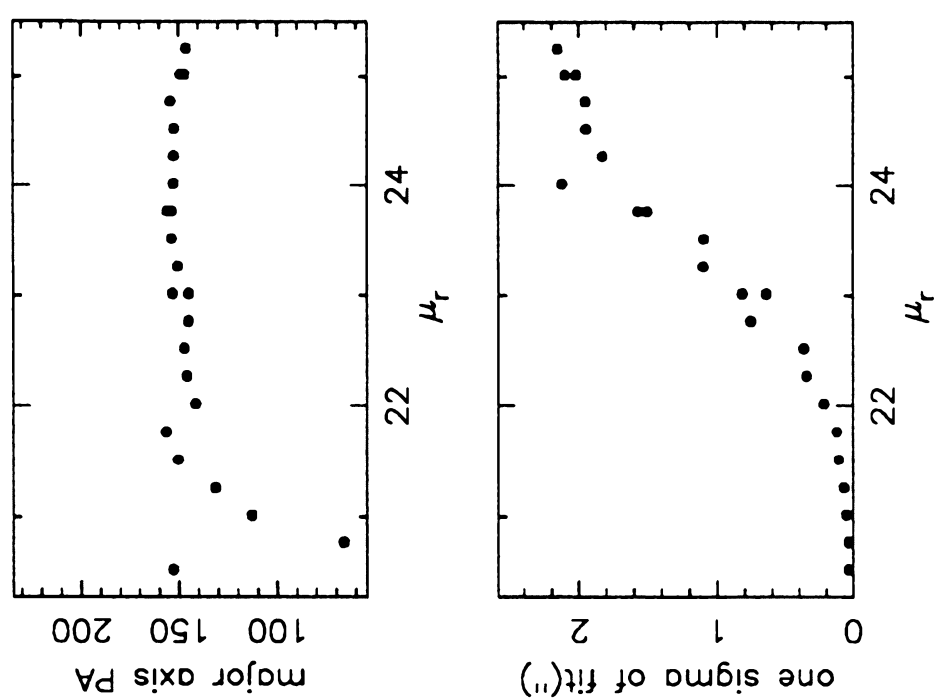

(i1)t!! to Dub!s au०

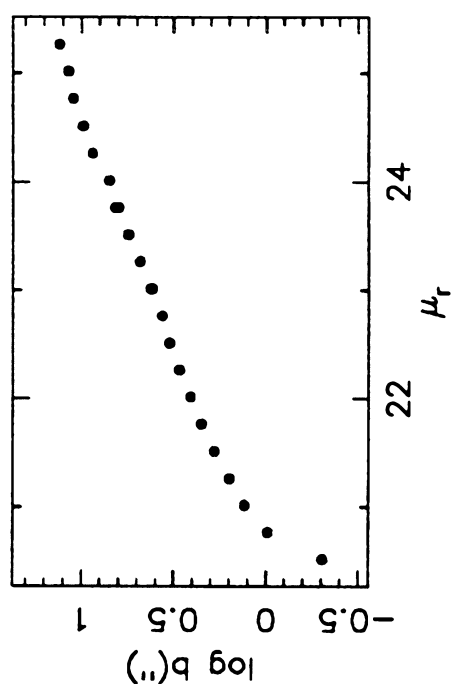

(.1) 9601

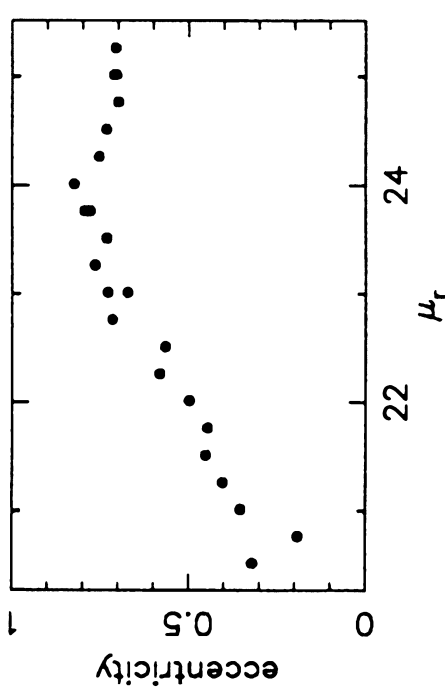

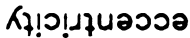

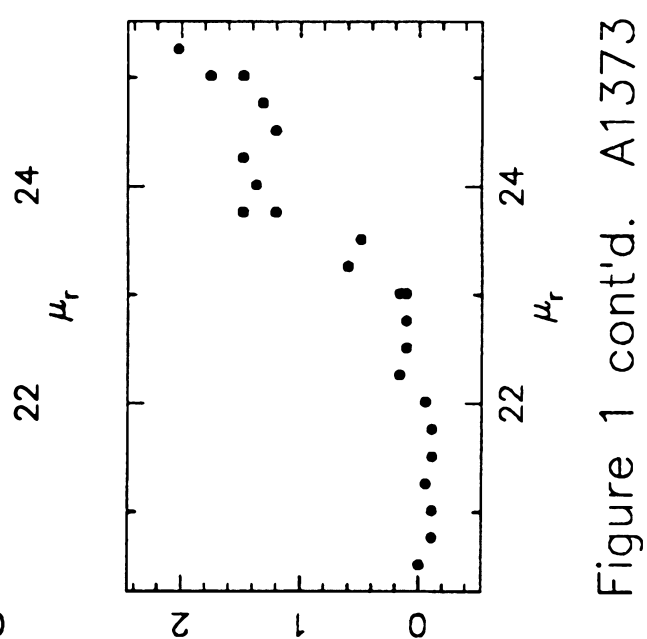

(II) $725450 \mathrm{~K}$

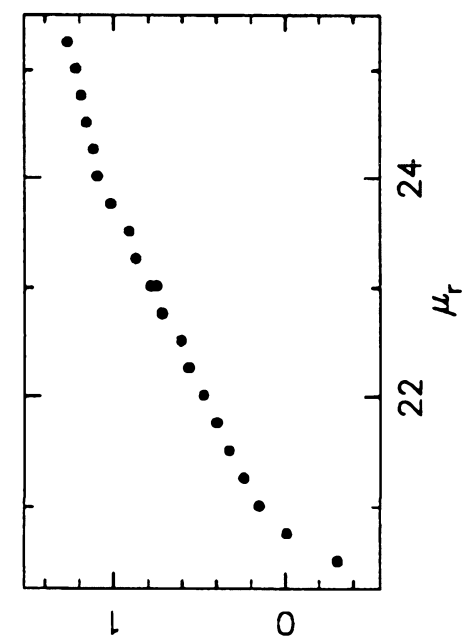

(.1) 060 이

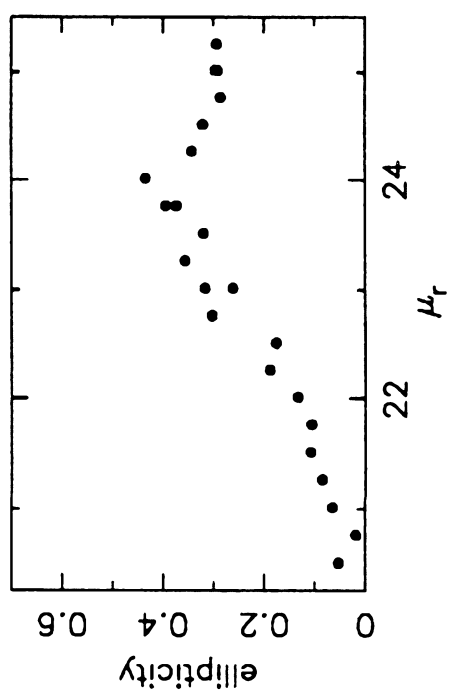

Kł!O!子d!!|a

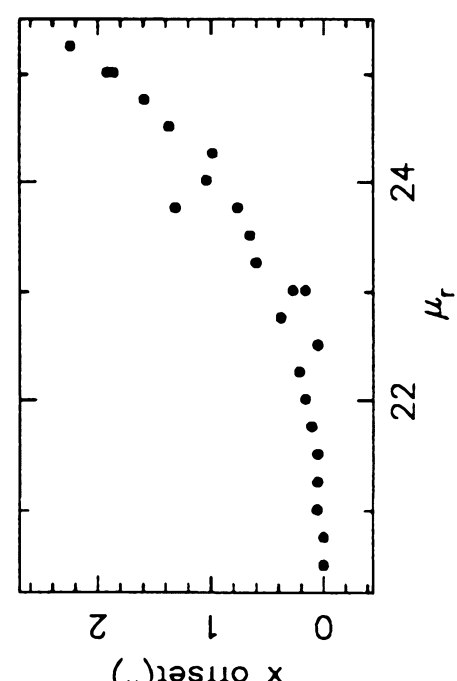

(.1) tas $130 \times$ 

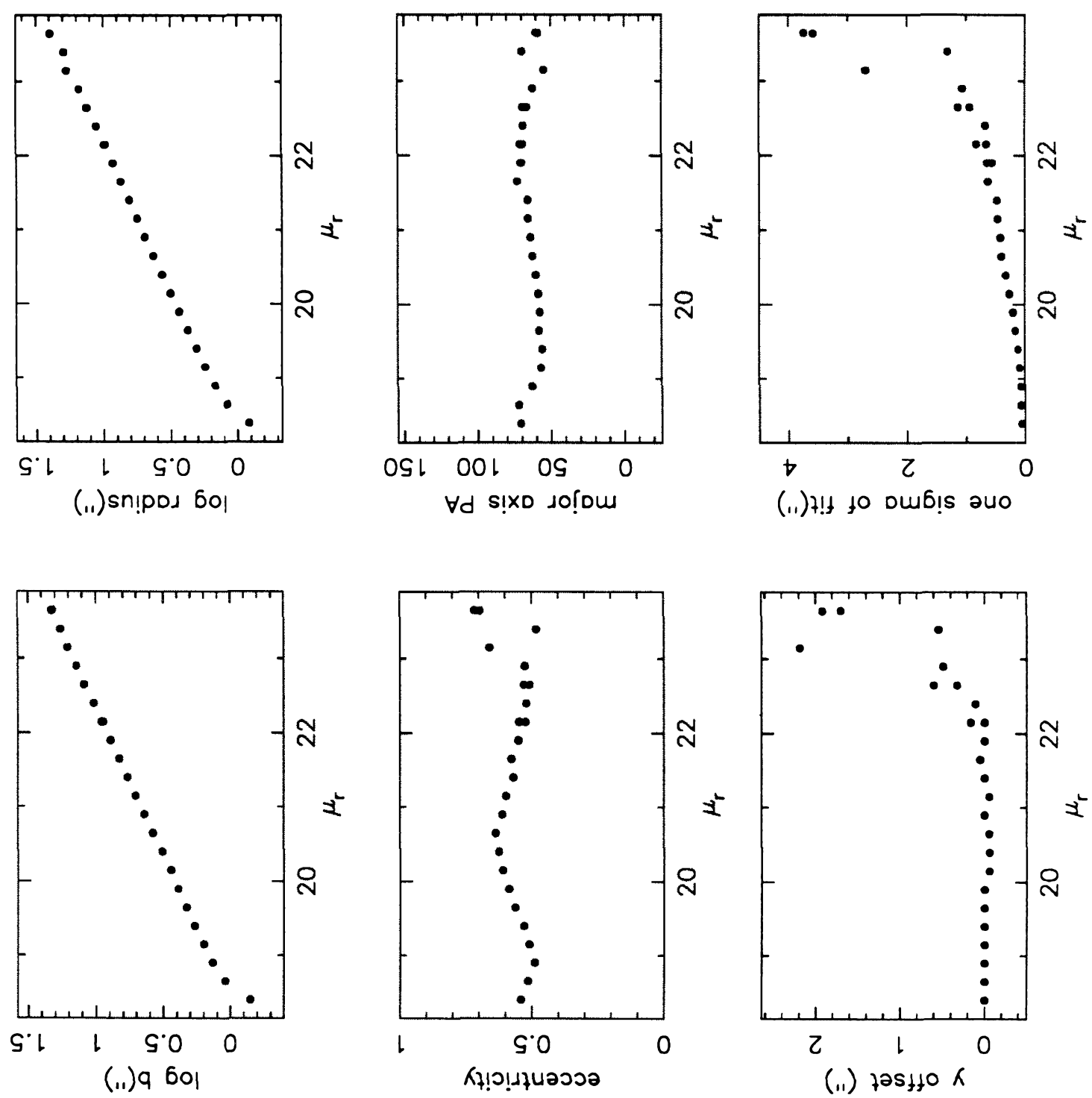

(11) $7 !+$ to ow6!s avo $x^{2}$ 오

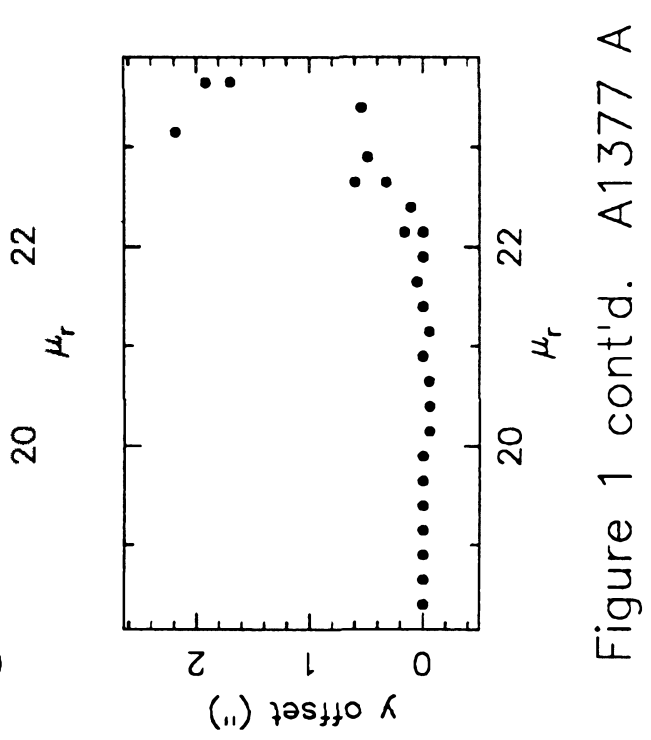

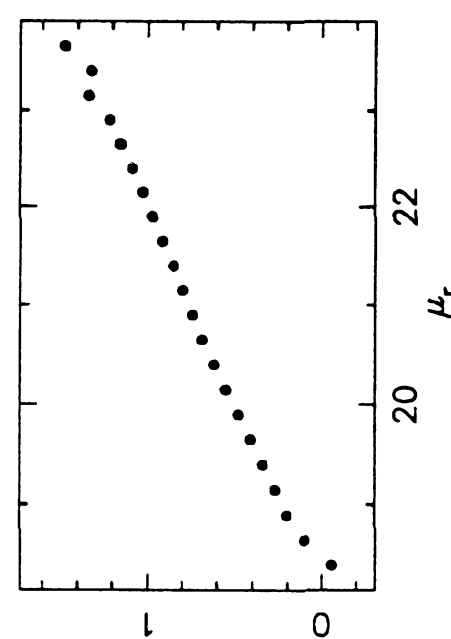

(.1) $0 \mathrm{bol}$

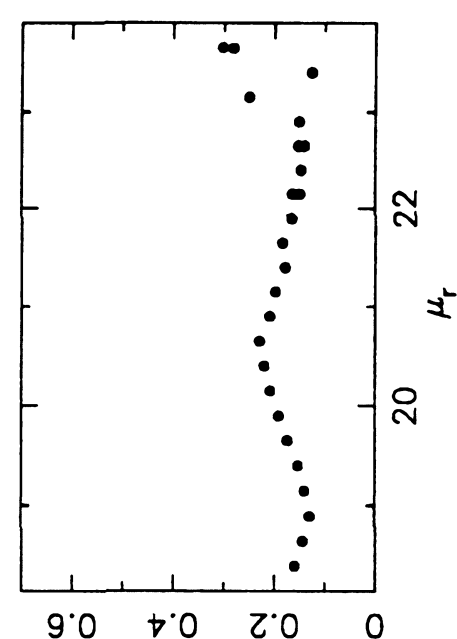

रł!ग!?d!||ә

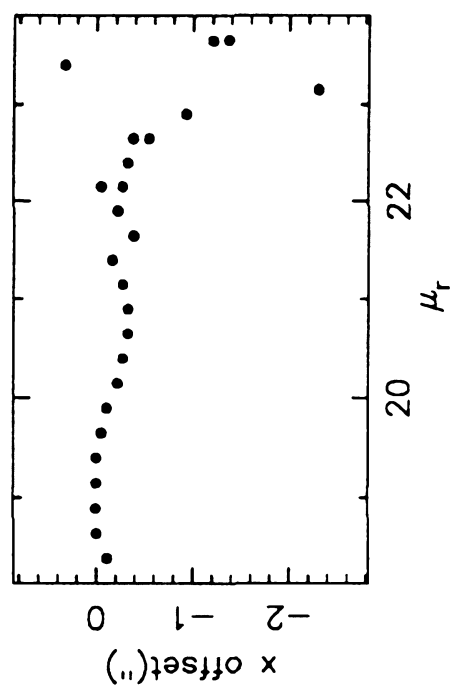



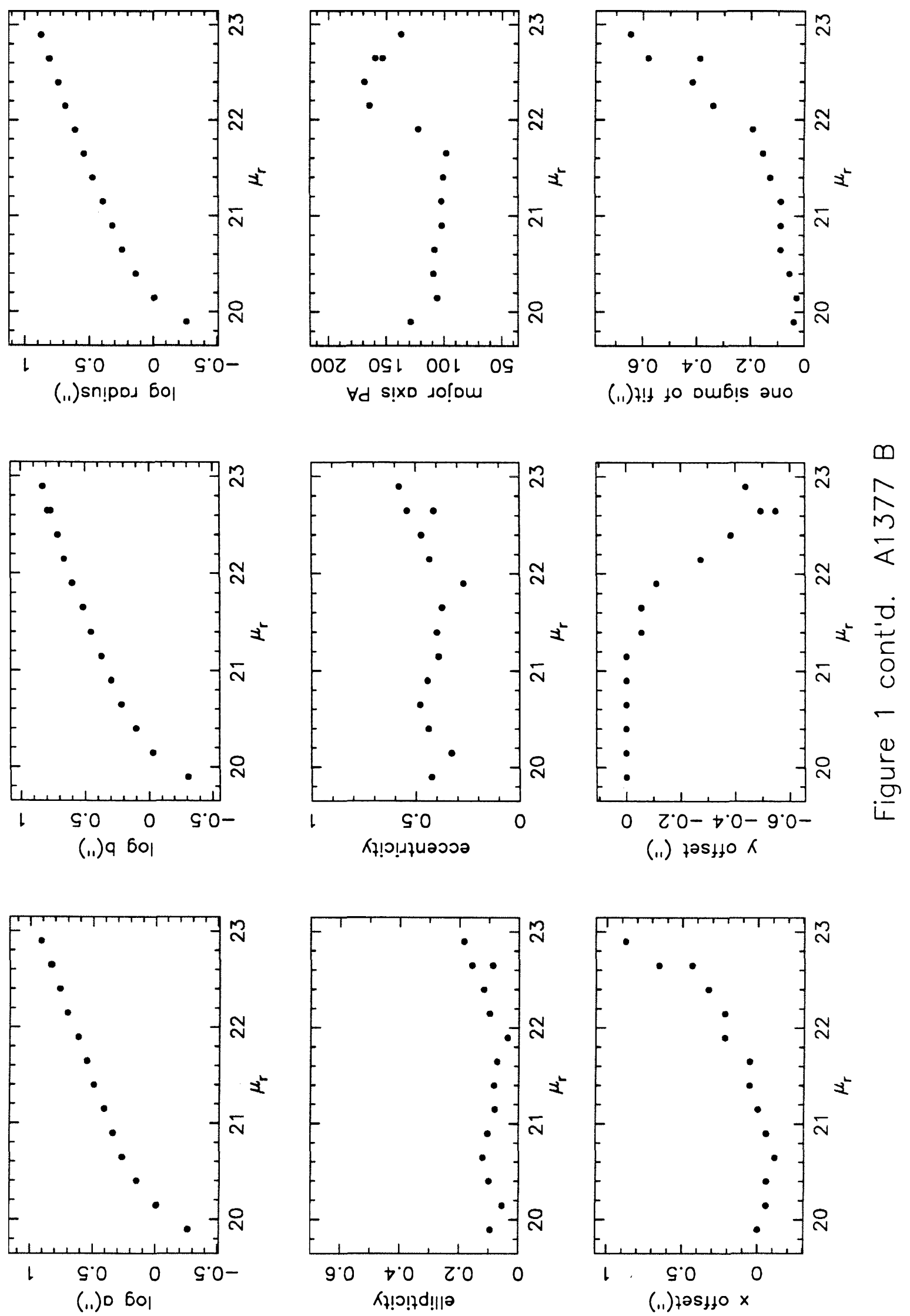


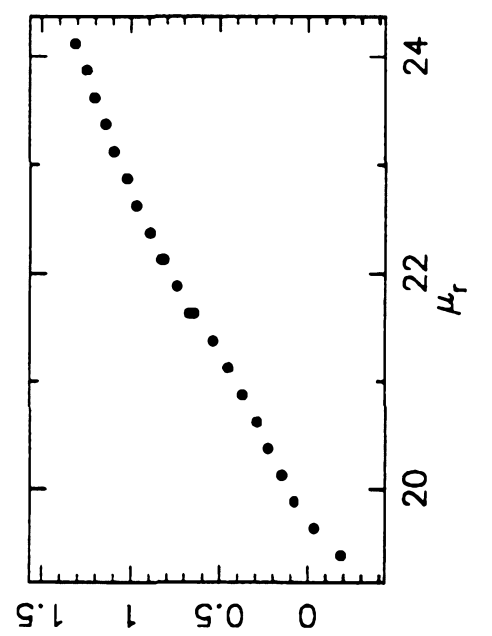

(.1)sn!pod bol

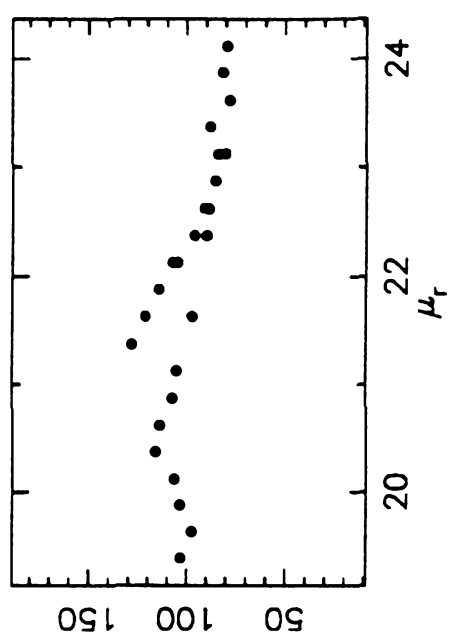

$\forall d$ s!xo so! lom

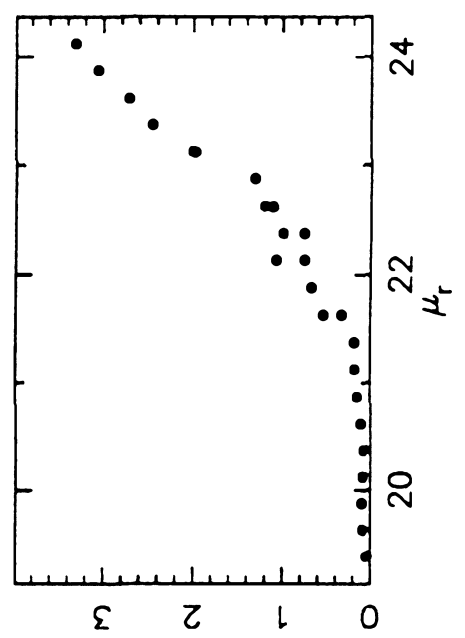

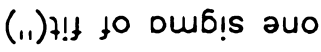
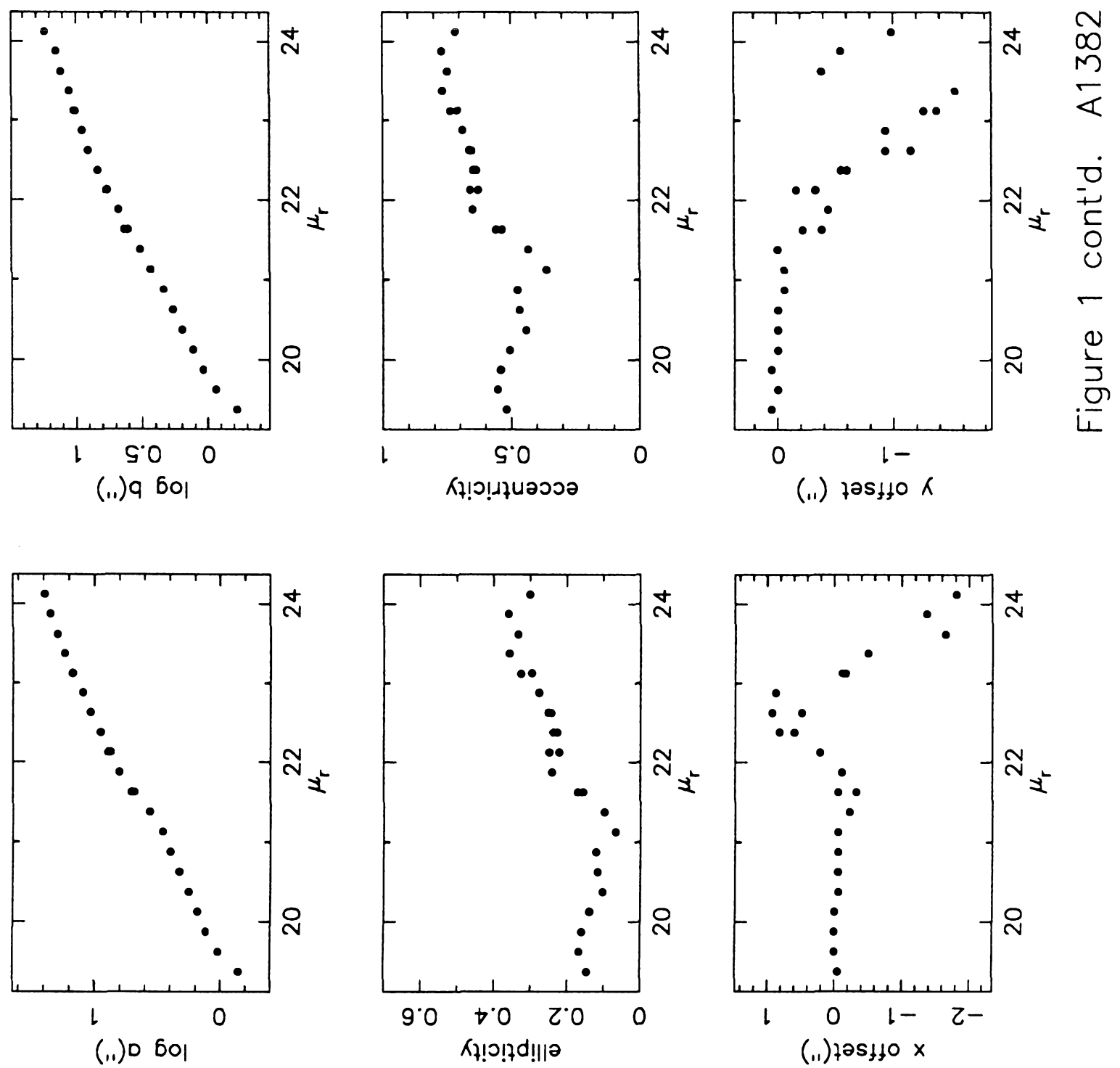


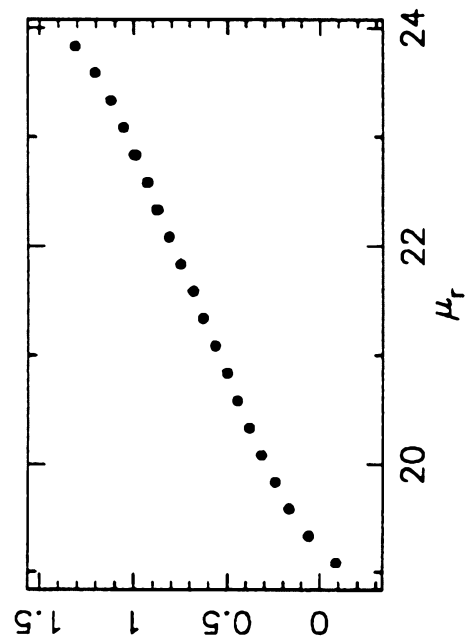

(.1) sn!pod 60।

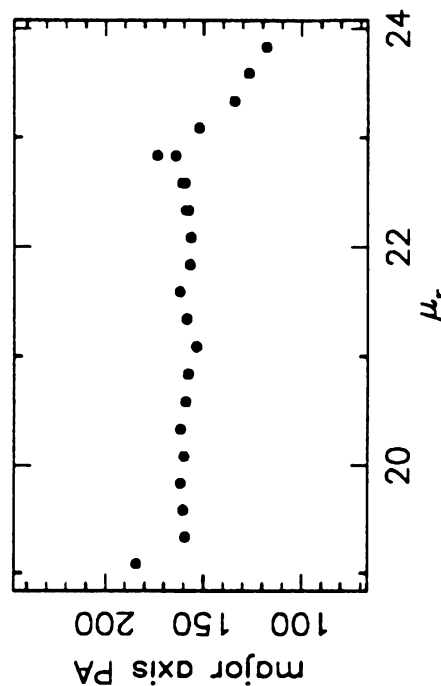

$\forall d$ s!xo Jo!́um

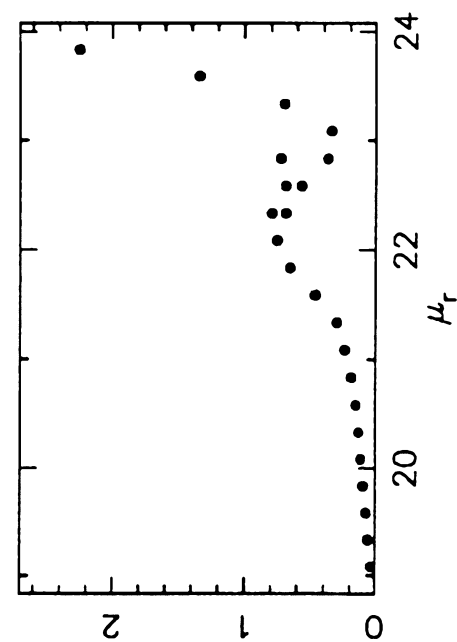

(11) 4 ! to Dub!s auo
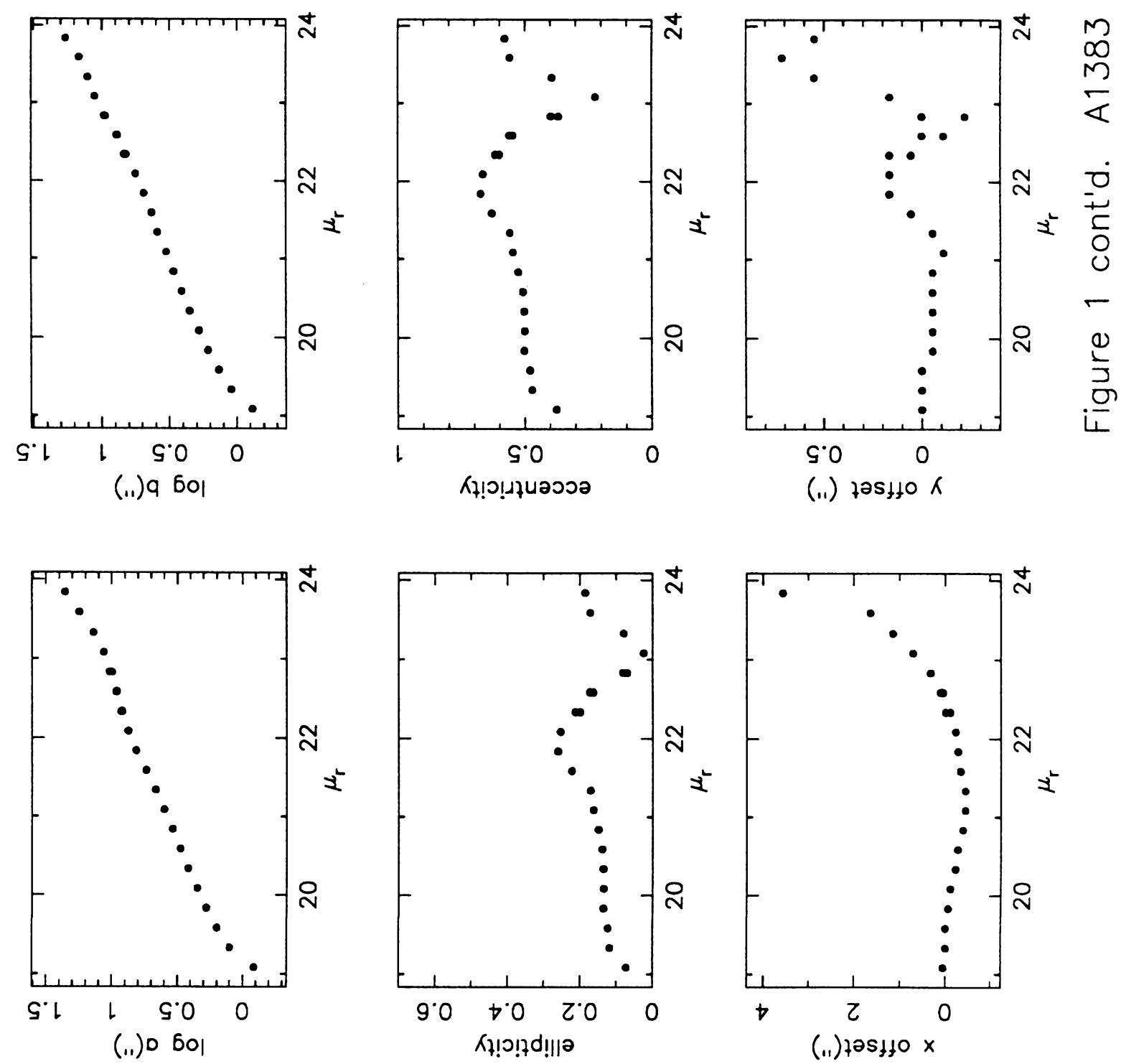

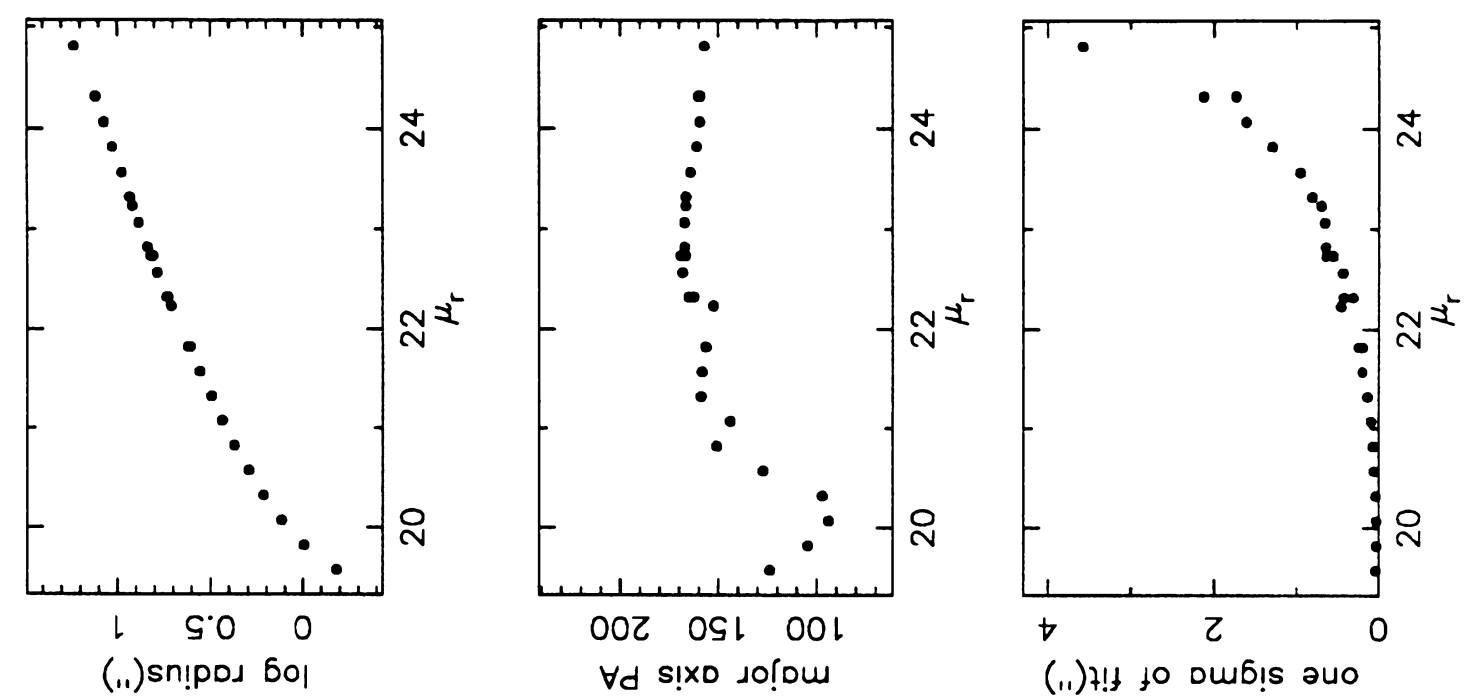

(11) 4 ! to oub!s auo

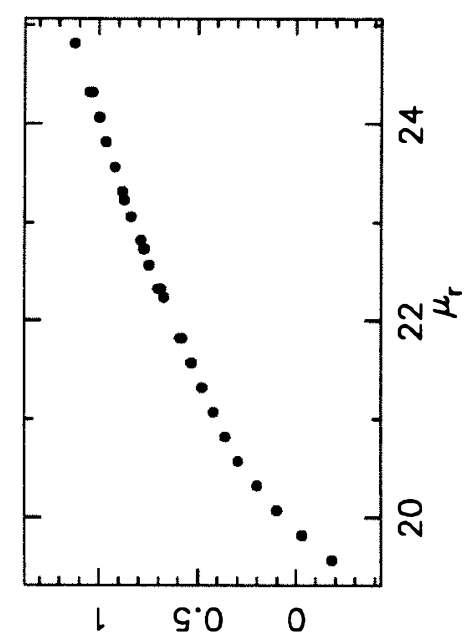

(.1) 960

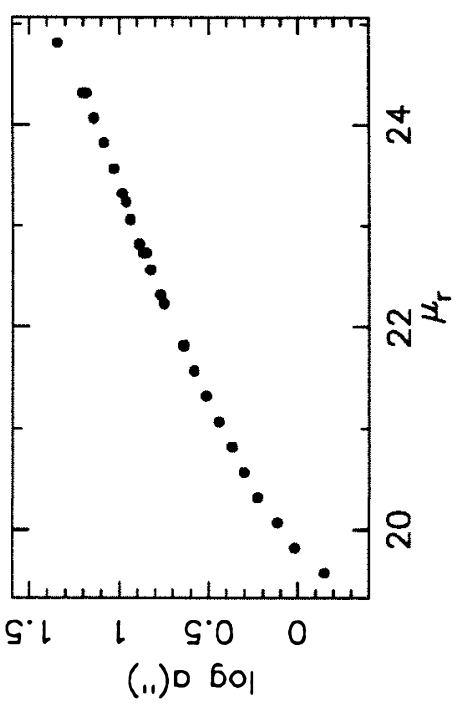

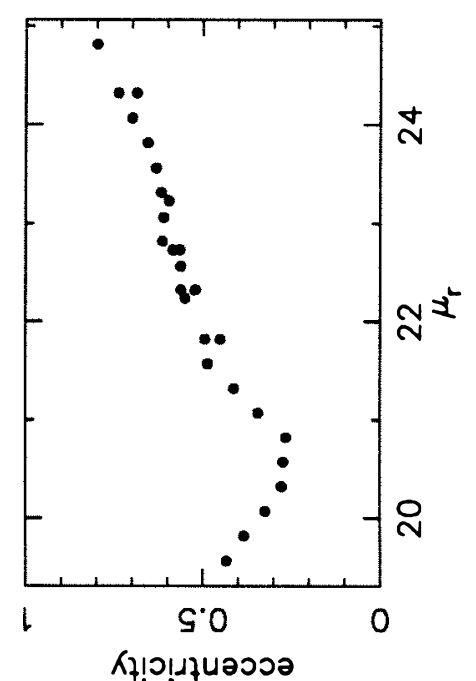

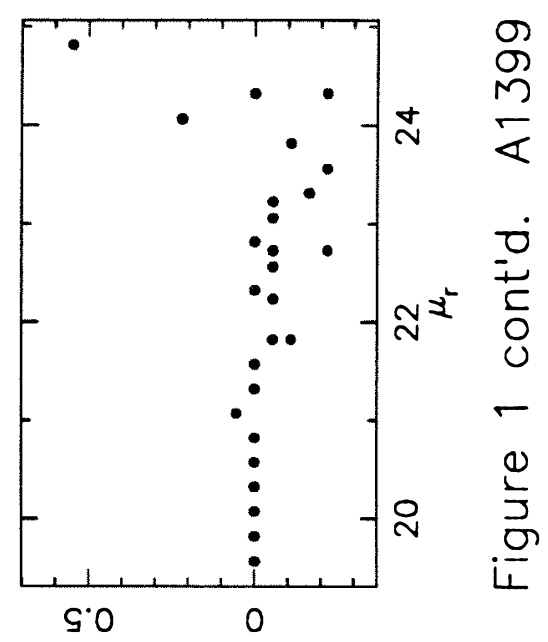

(.1) $725+150 \mathrm{~K}$
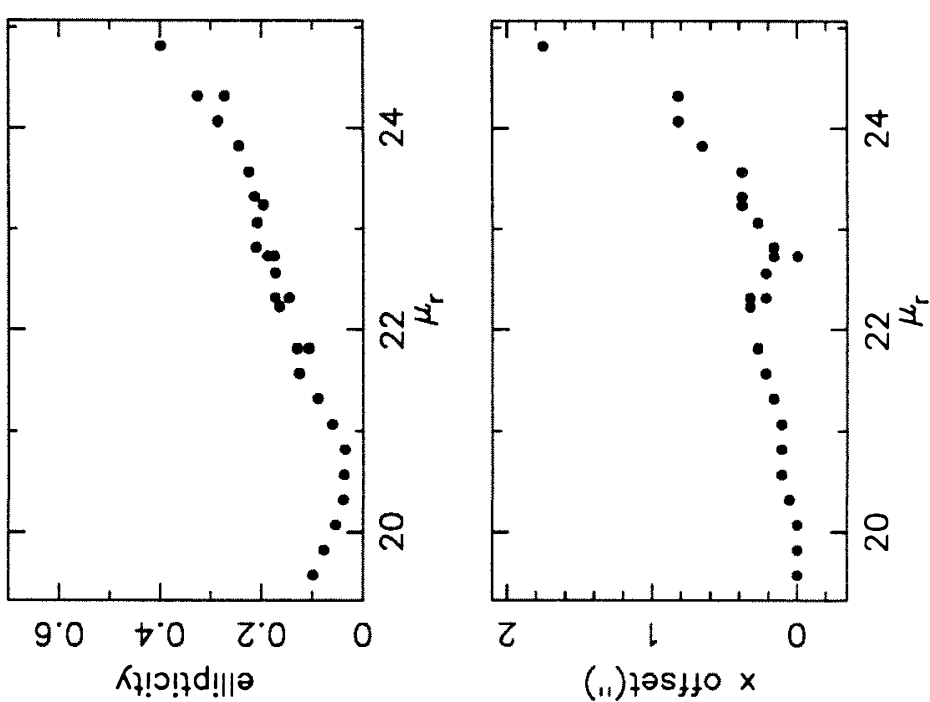

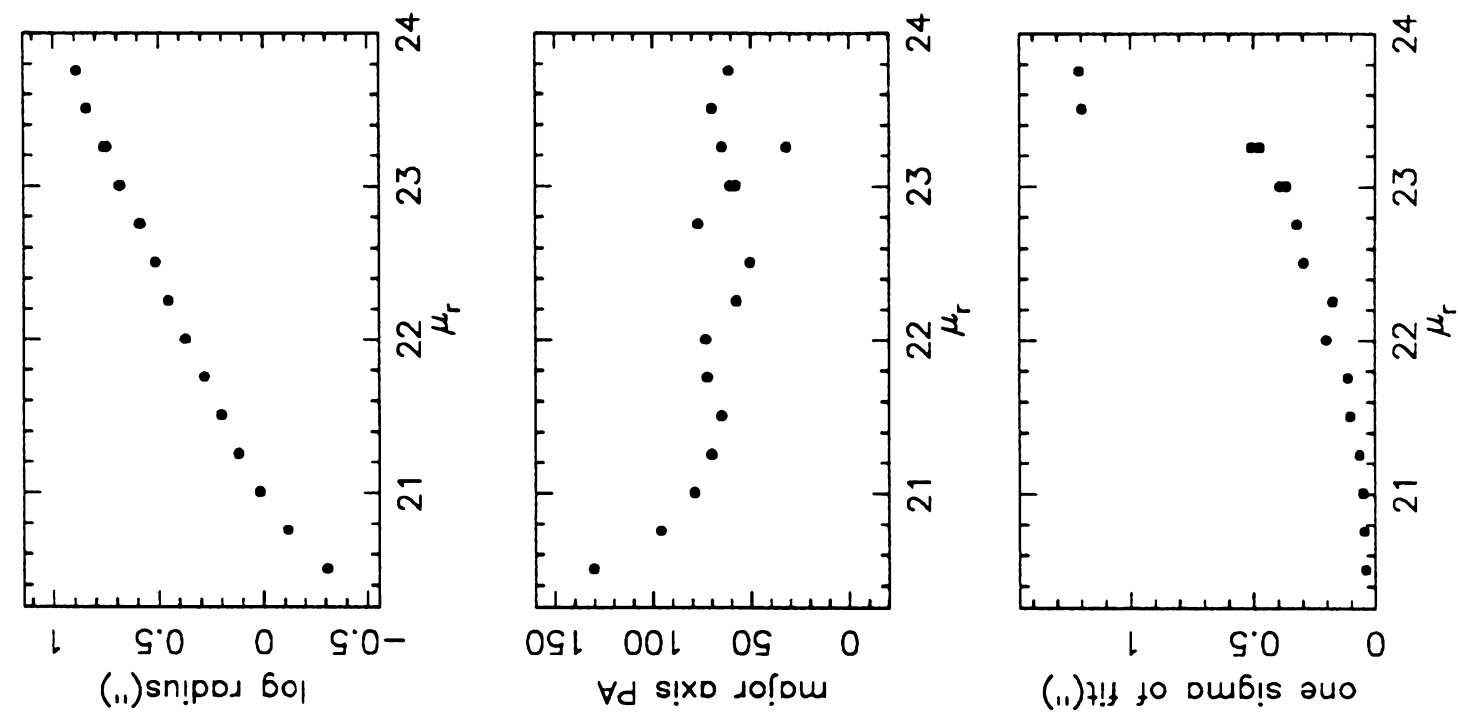

(11) $+! t$ to oub!s auo

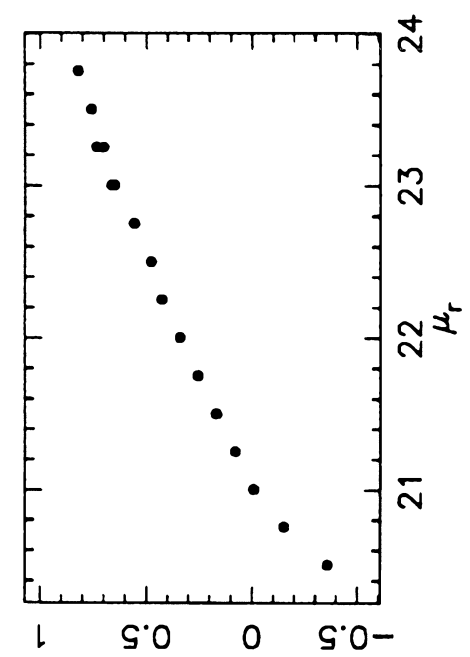

(.1) 9601

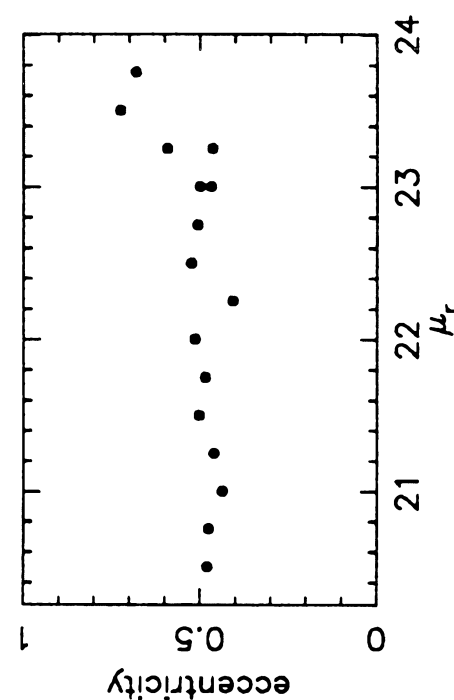

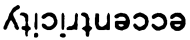

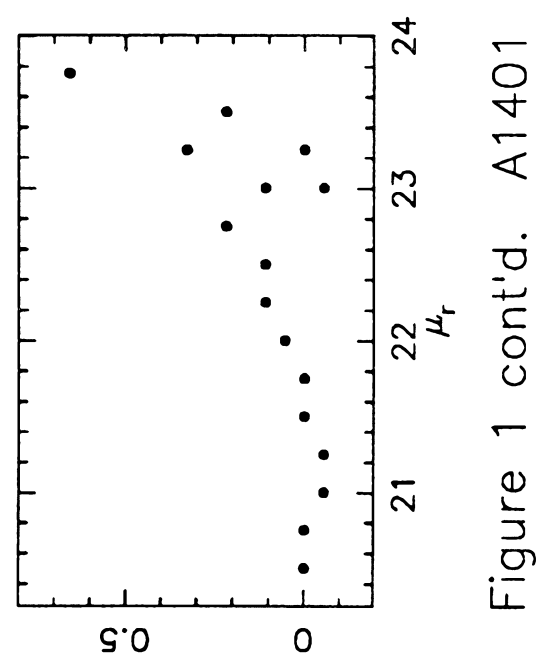

(.1) $725410 \mathrm{~K}$

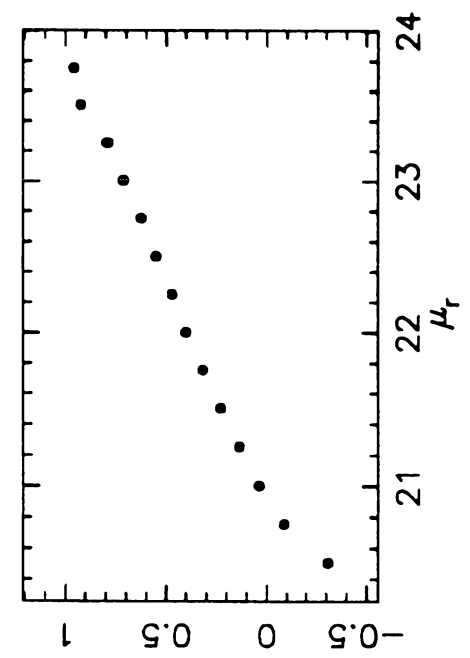

(.1) 060 이

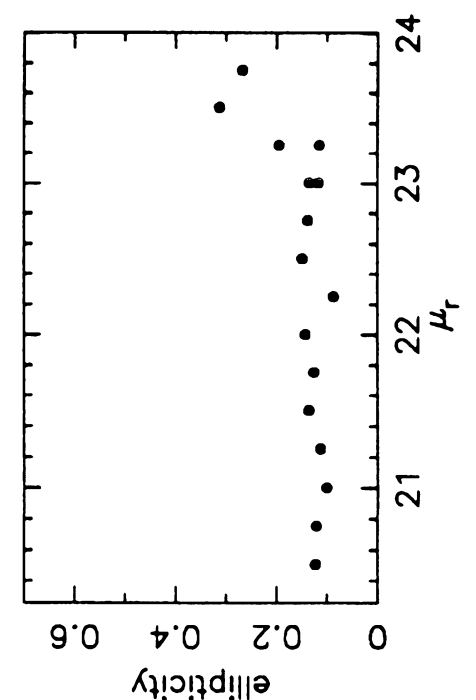

रł!O!子d!||a

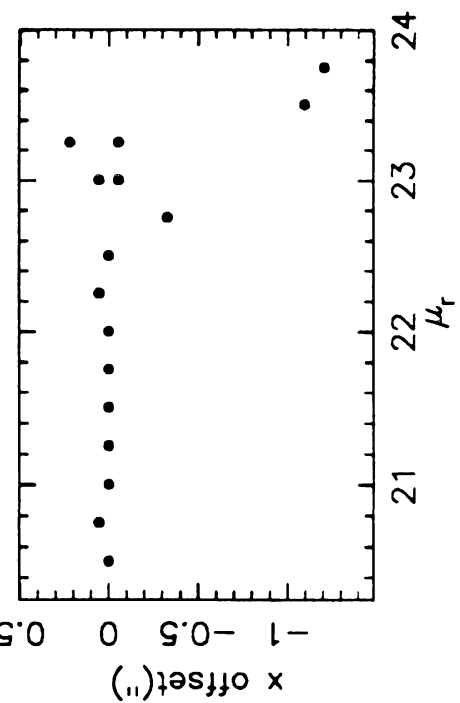




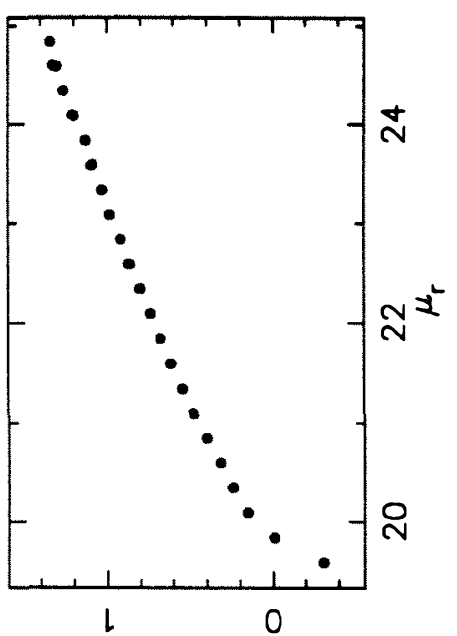

(.1)sn!pos 601

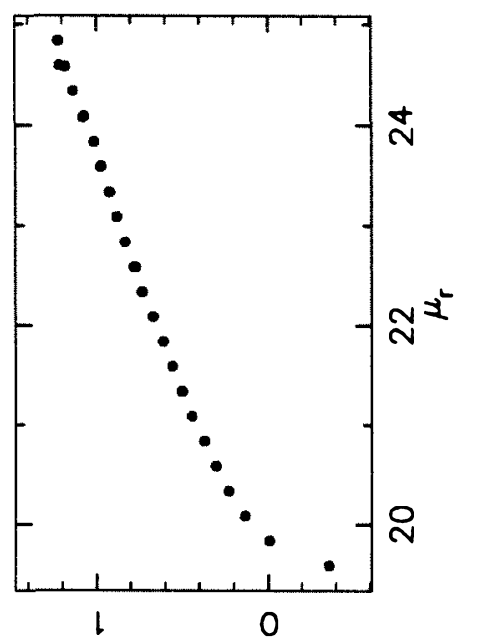

(.1) 9 6ol

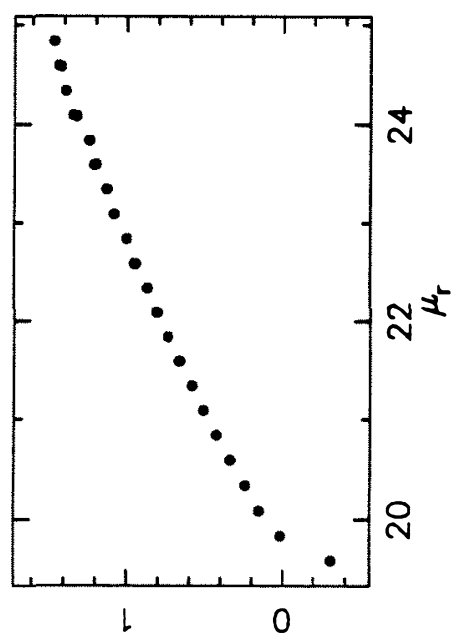

(11) $0 \mathrm{bol}$

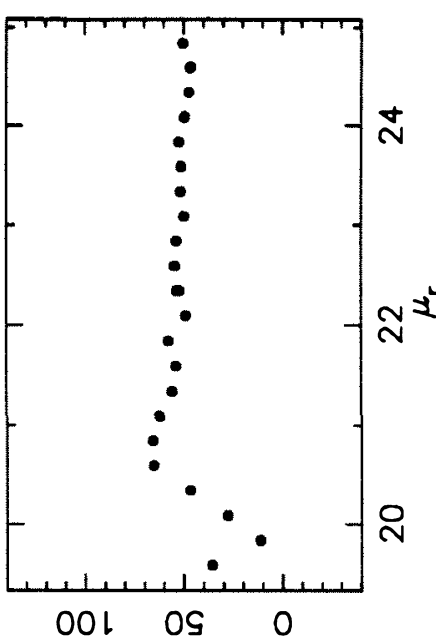

$\forall d$ s!Xo $د o$ !om
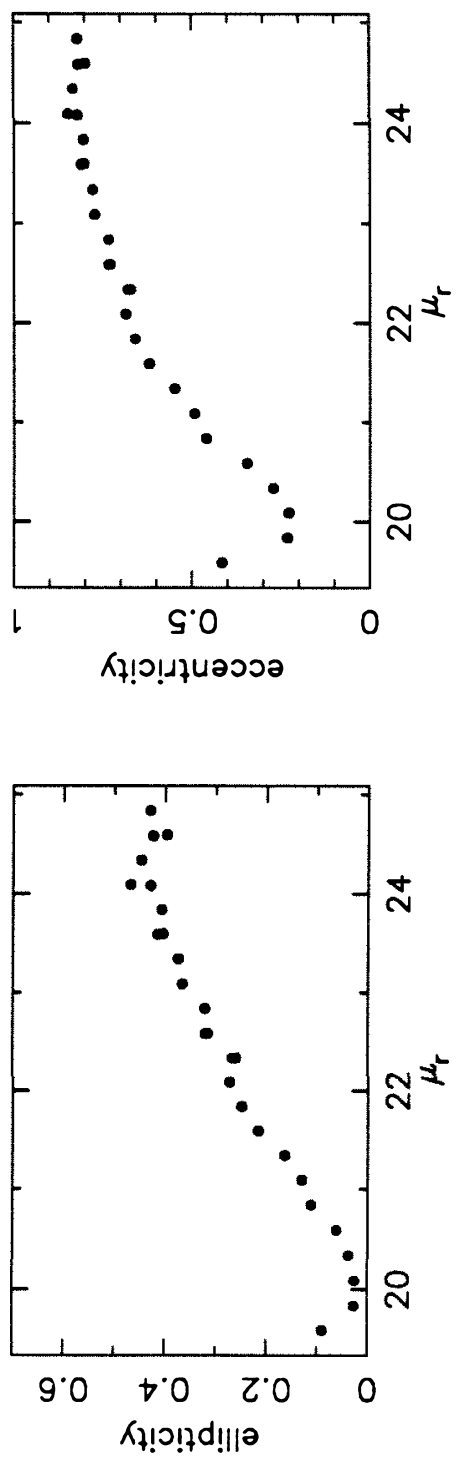

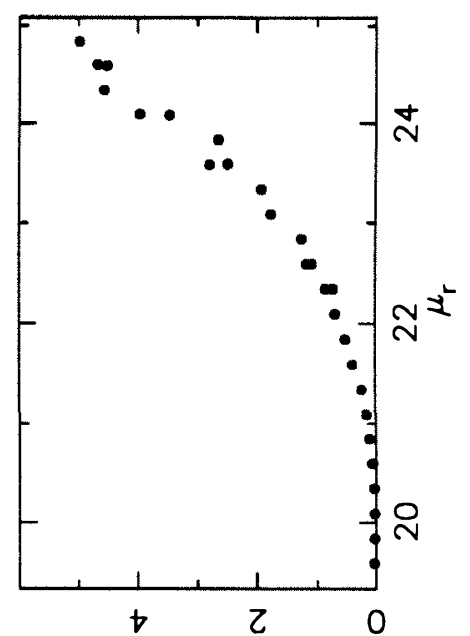

(11)+!t to Dub!s auo
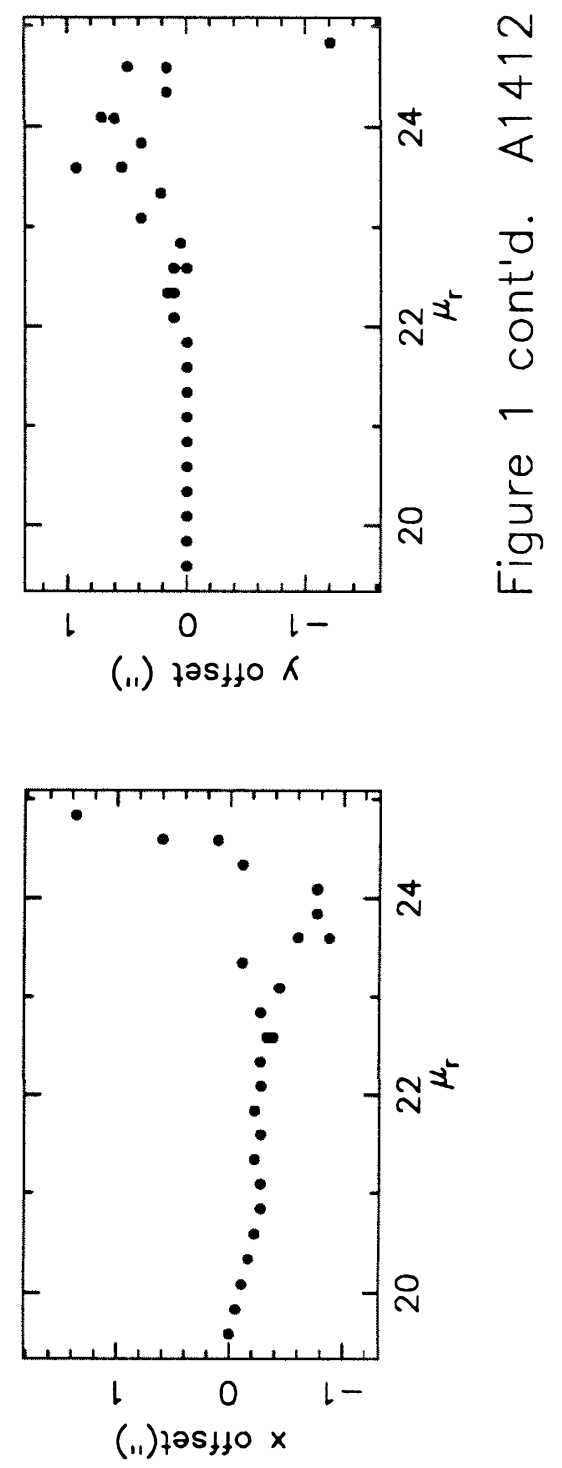


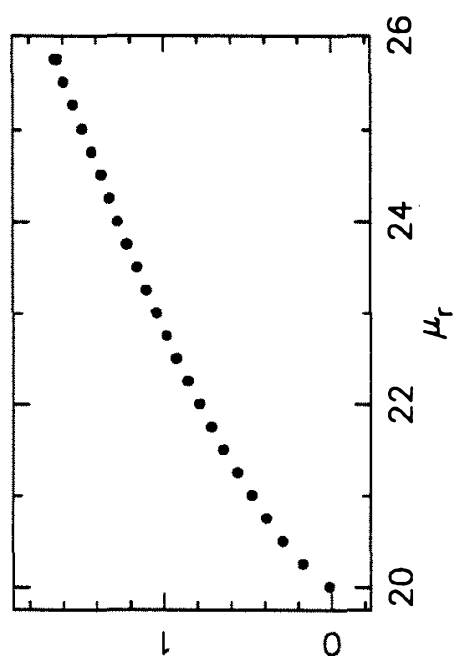

(.1)sn!pod 6ol

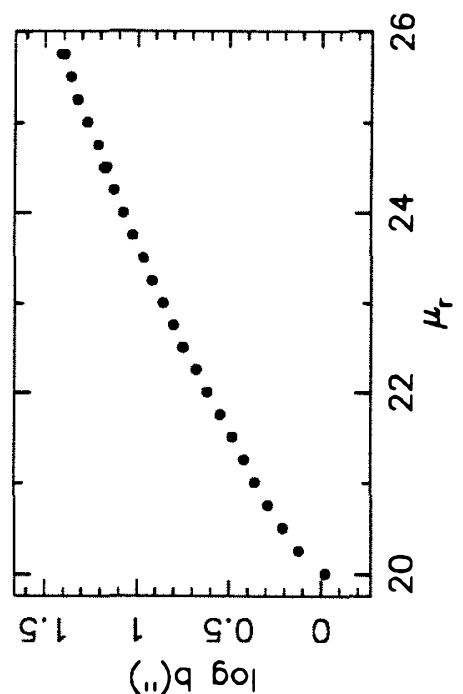

(.1) 9 6ol

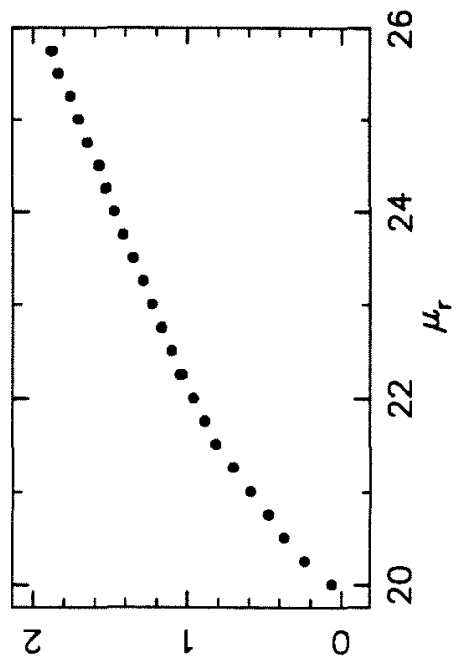

(.1) 0601
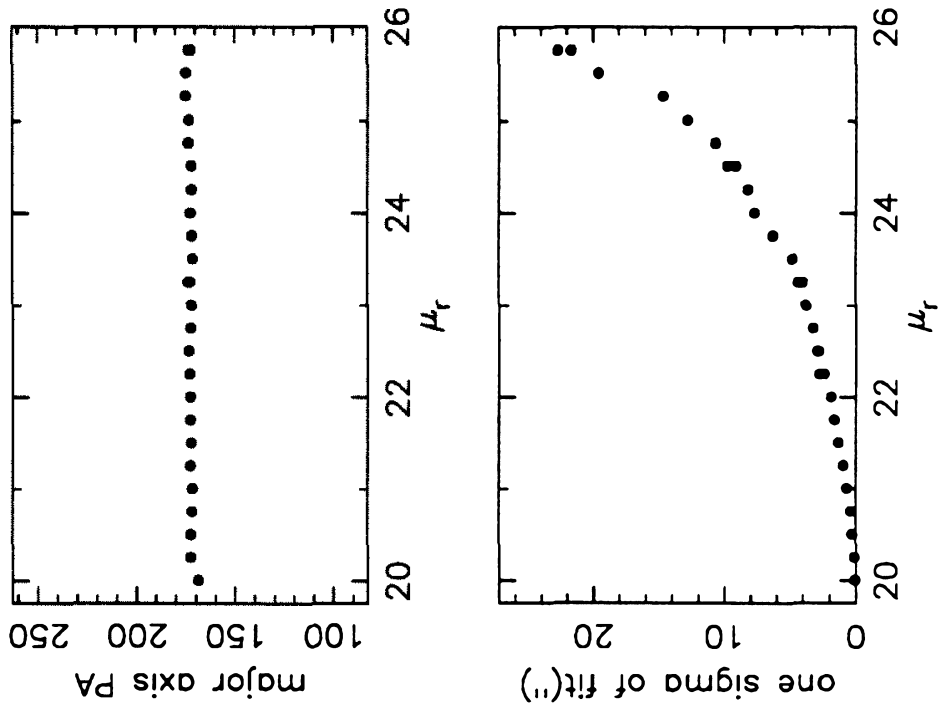

(11) $+! ! ~ 10$ oub!s auo
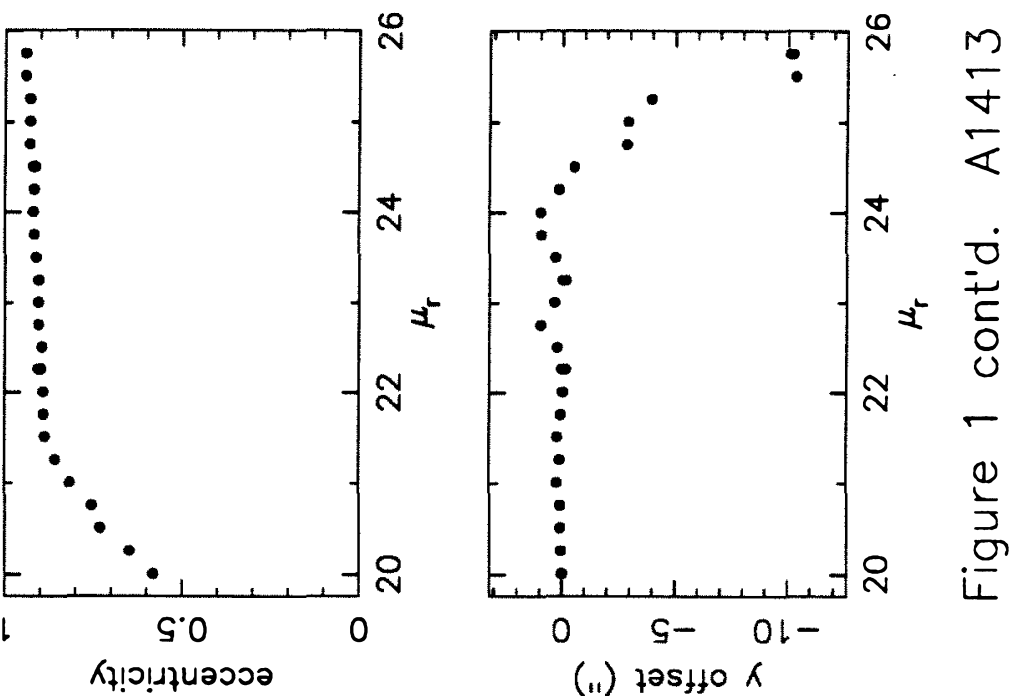

(ii) zəs $750 \mathrm{~K}$
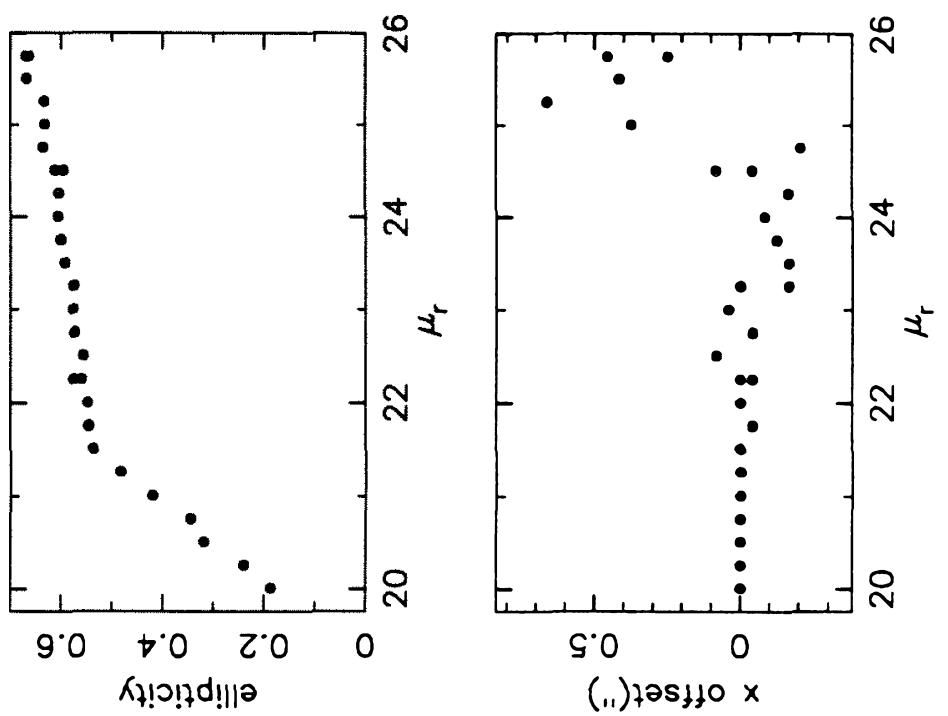


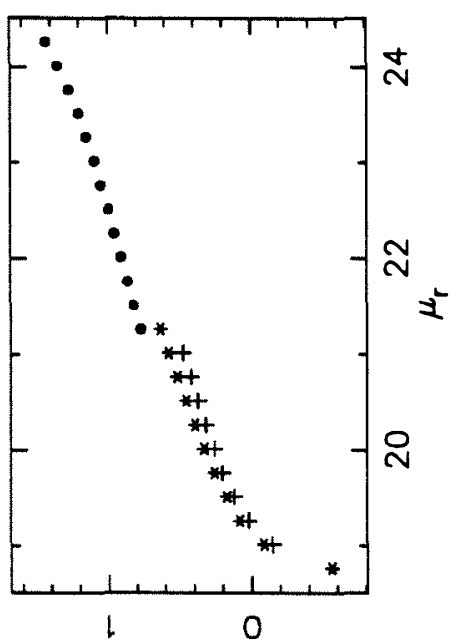

(.1) sn!pos 6ol

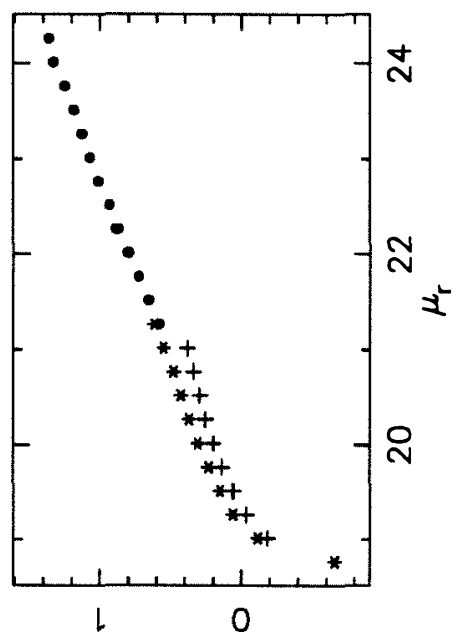

(.1) 9601

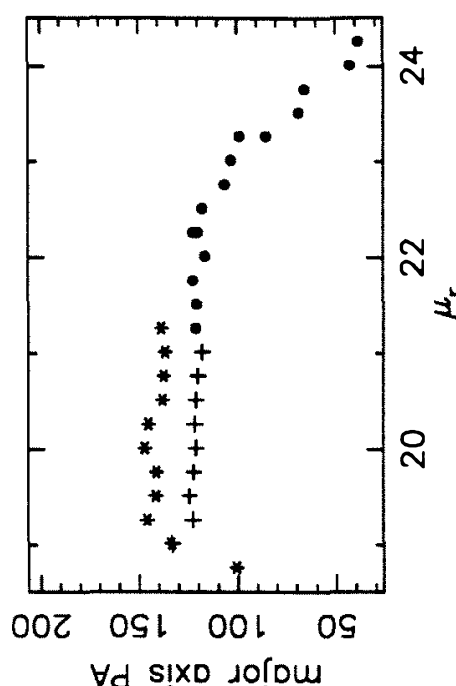

$\forall d$ s!Xo 10 !

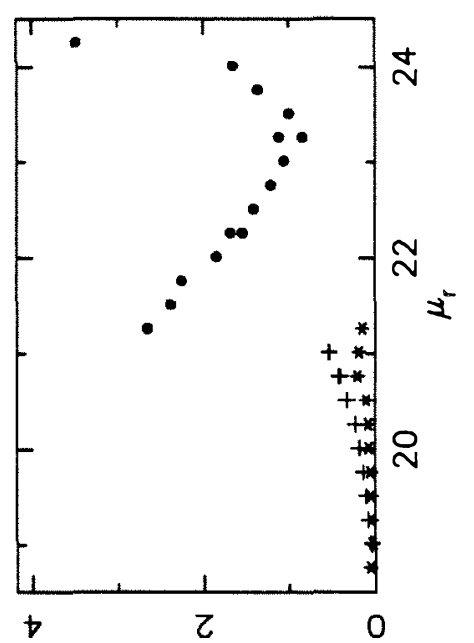

(11) $)$ !! to Du6!s auo
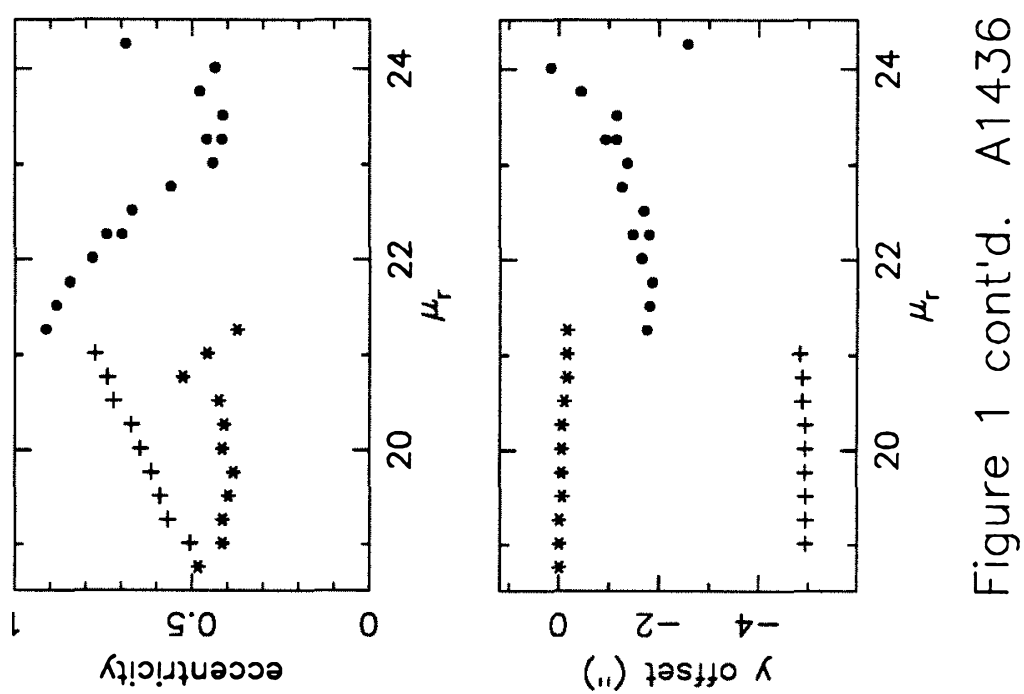

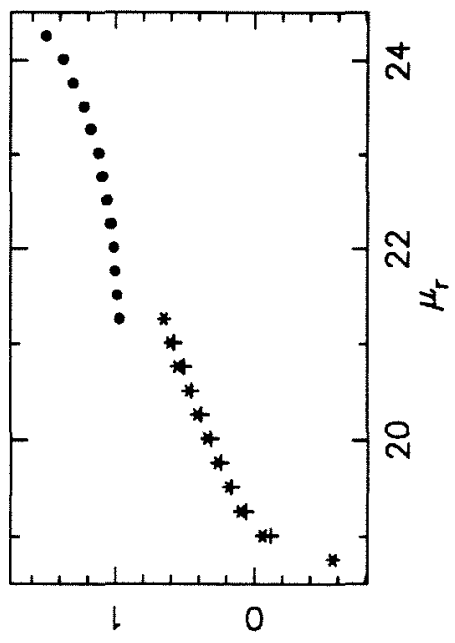

(.i) $\mathrm{O}$ 601
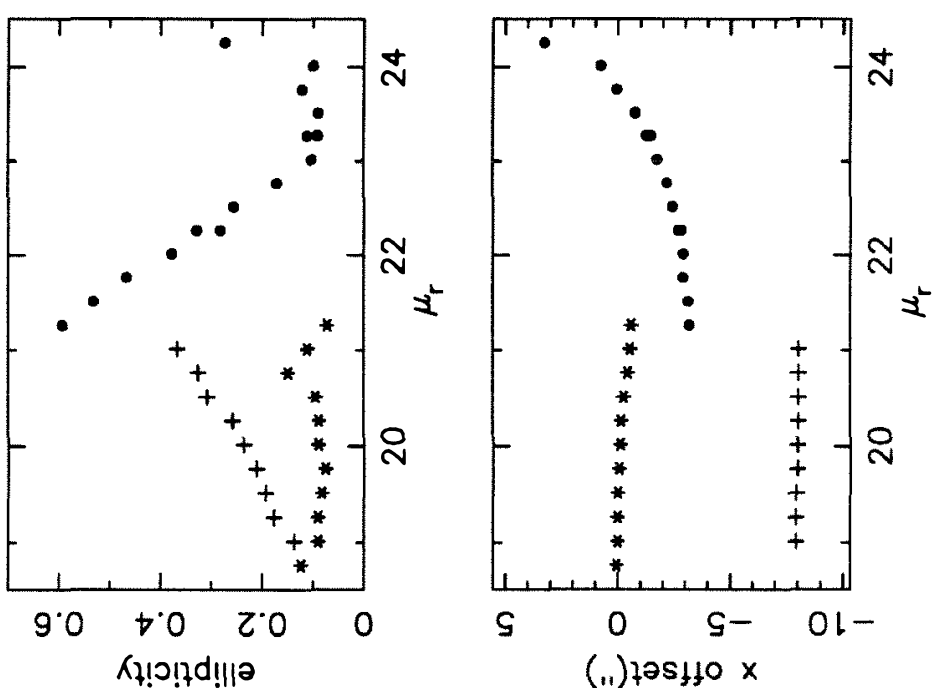


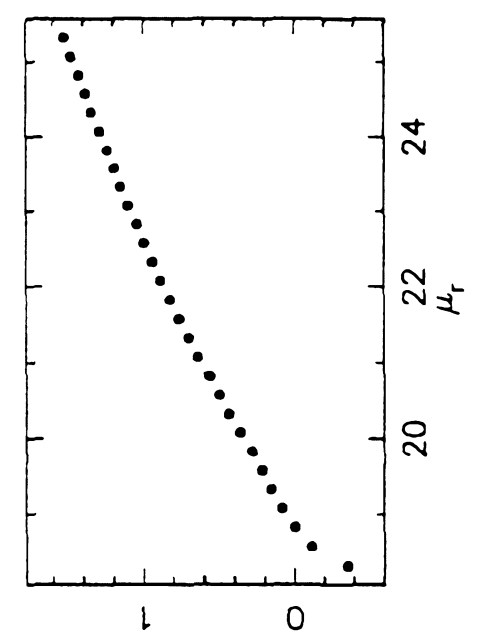

(.1) sn!pos 60।
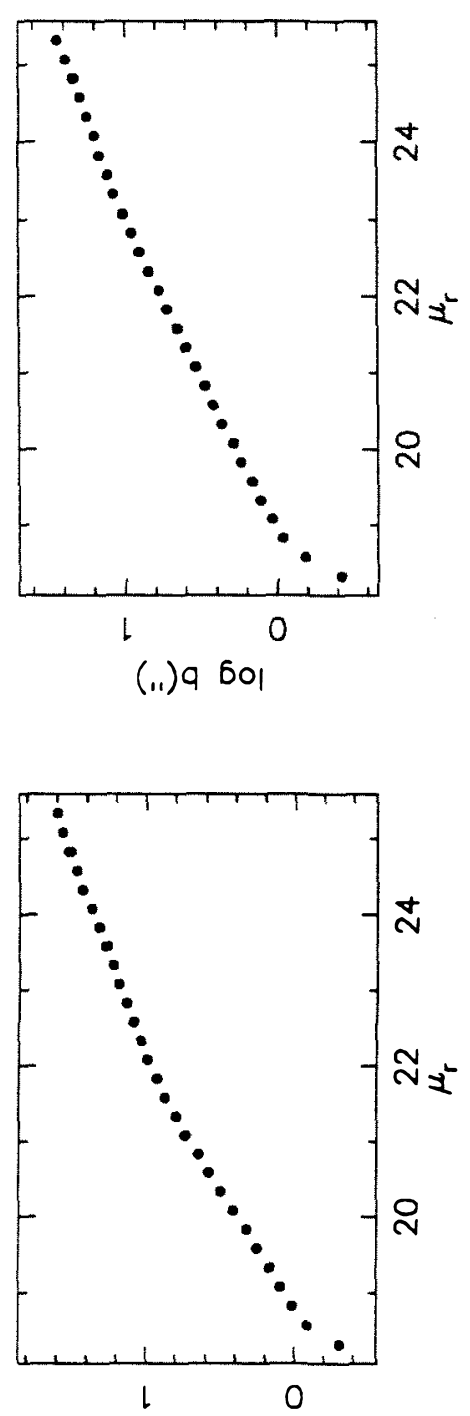

(..) $0 \mathrm{60}$
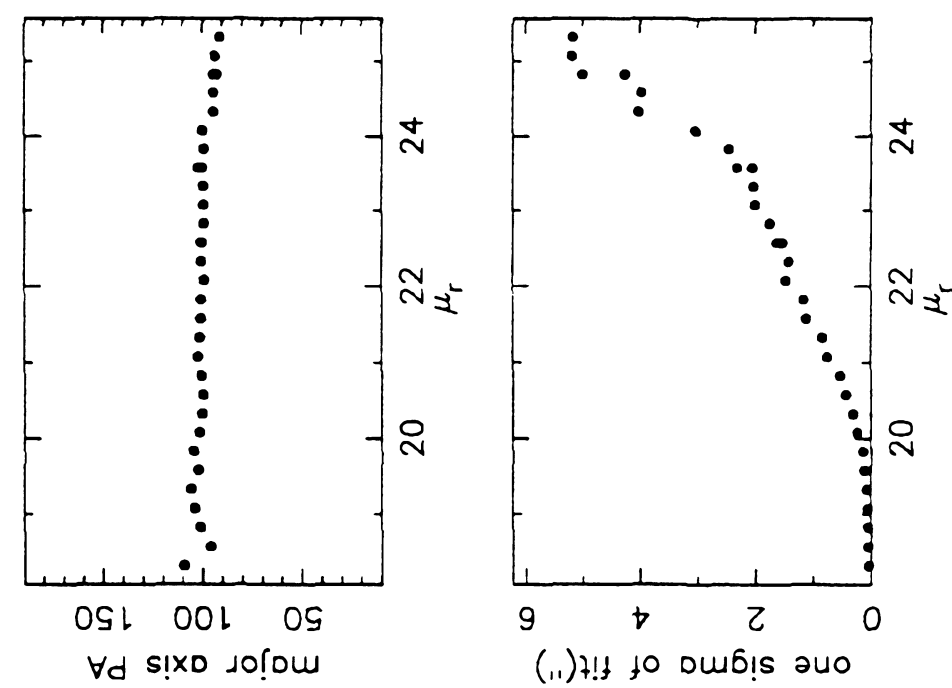

(11) $7 !+$ to Dub!s avo
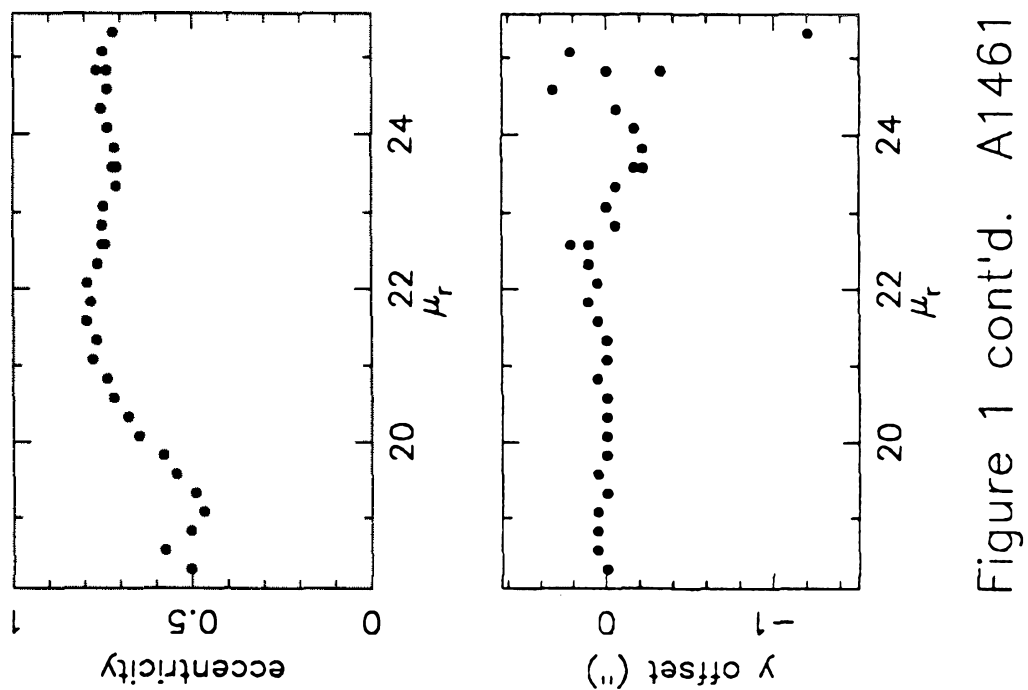
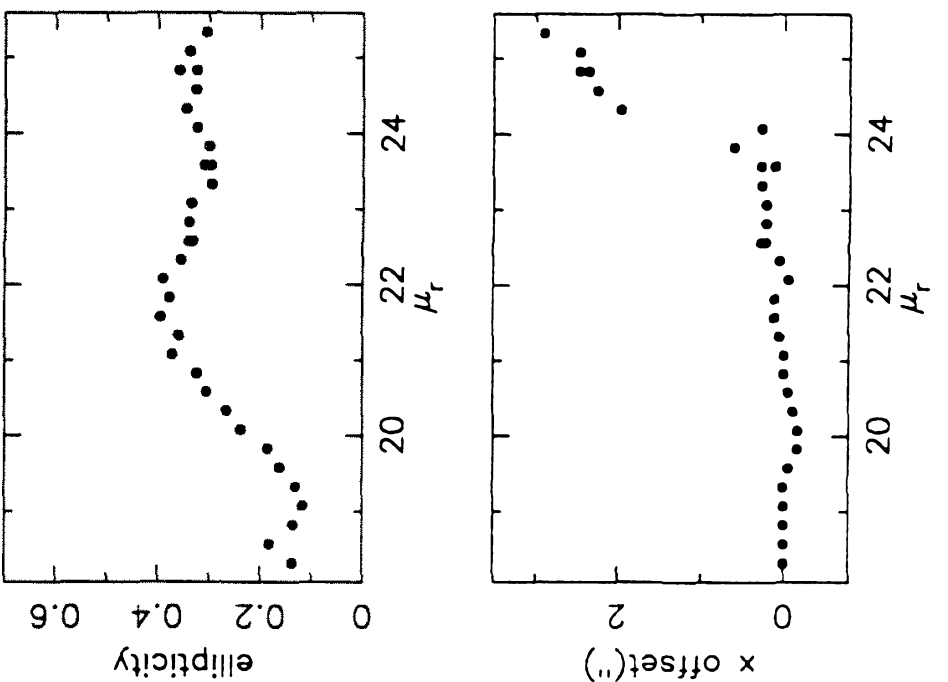
$-338-$
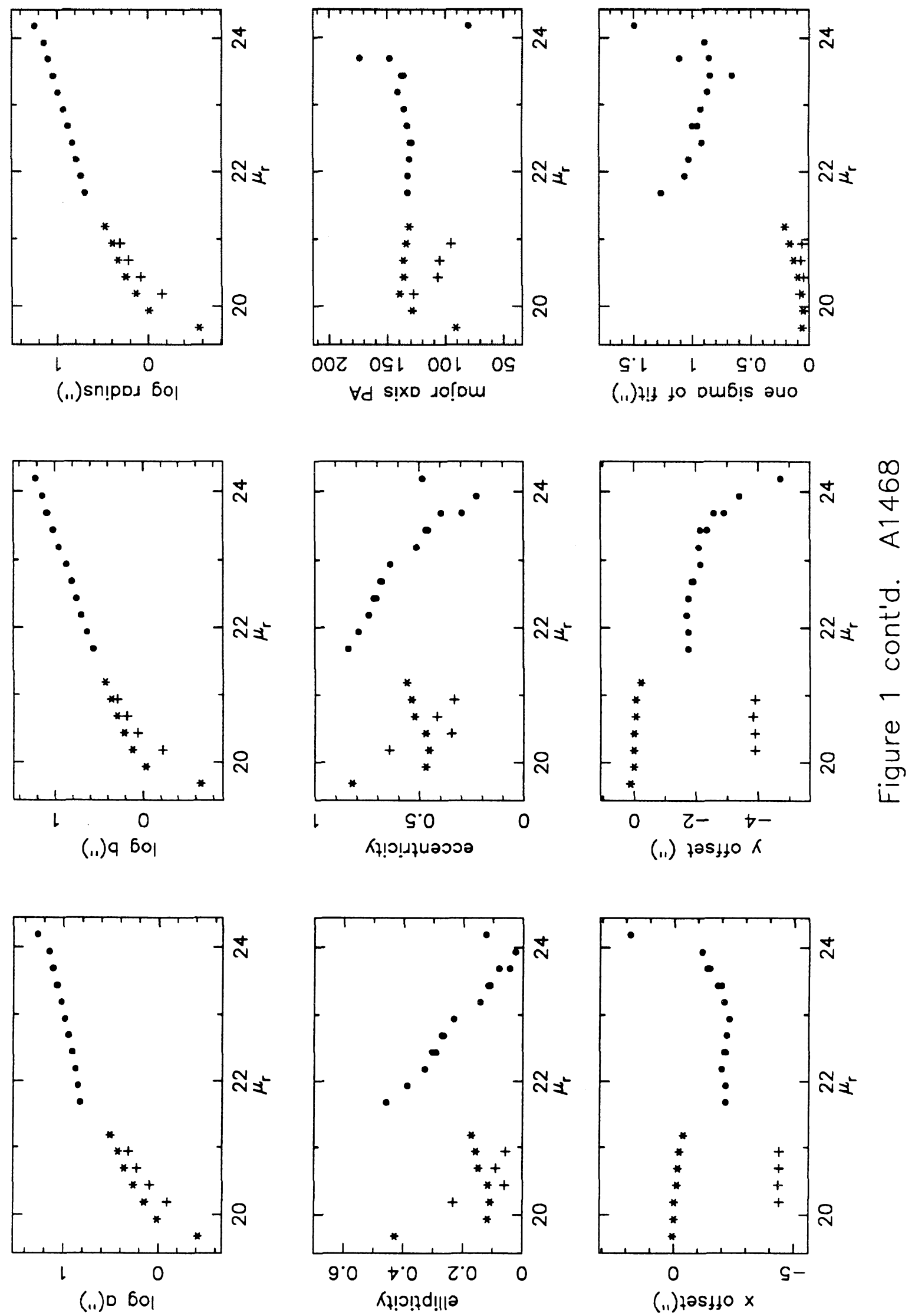


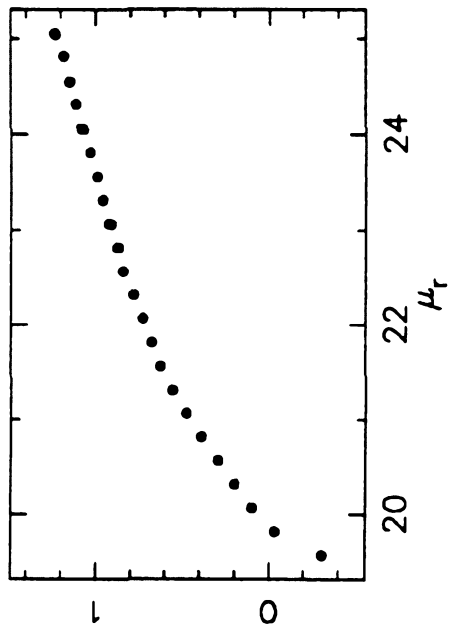

(.1) sn!pos 60।

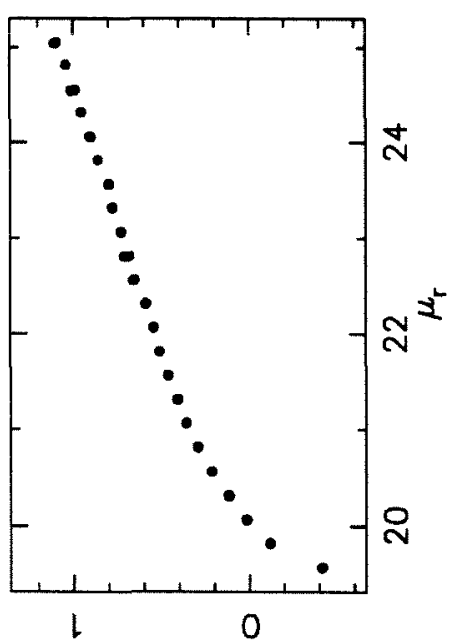

(11) 9 bol

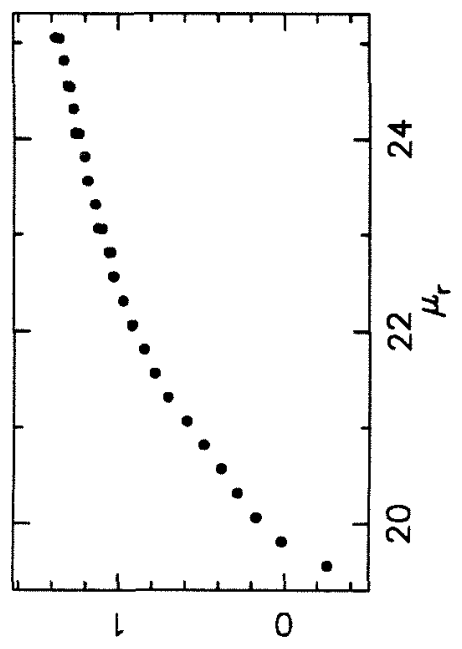

(.1) 0 bo이

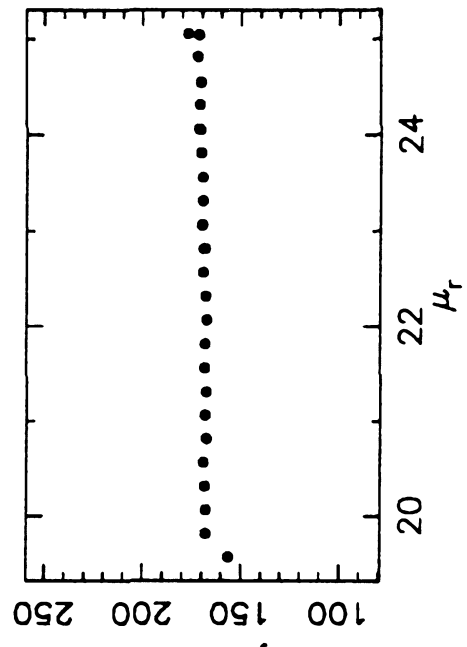

$\forall d$ s!xo so! om

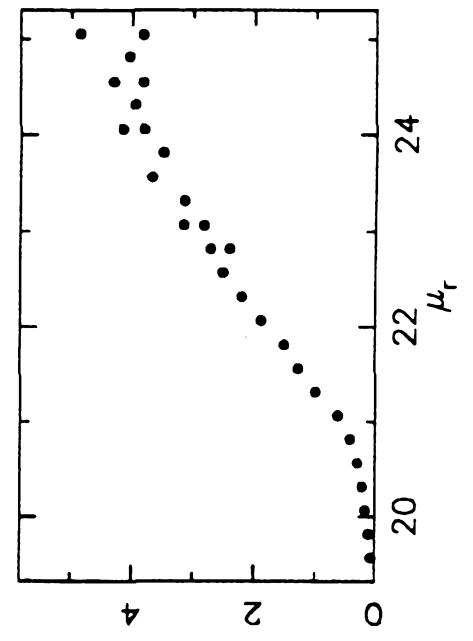

(11) $4 !+$ to oub!s aนo

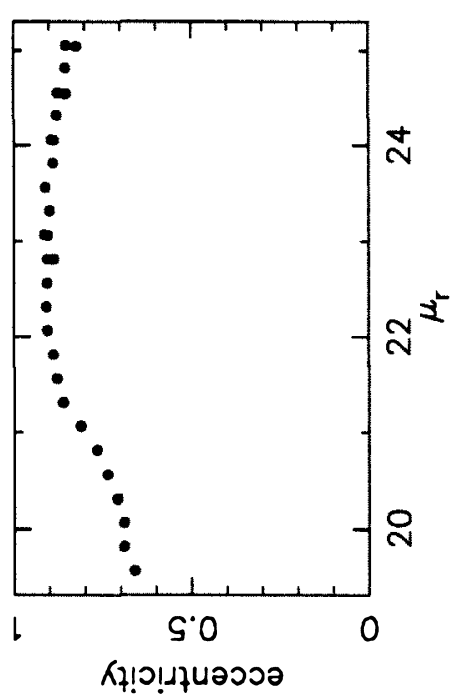

$\varangle$
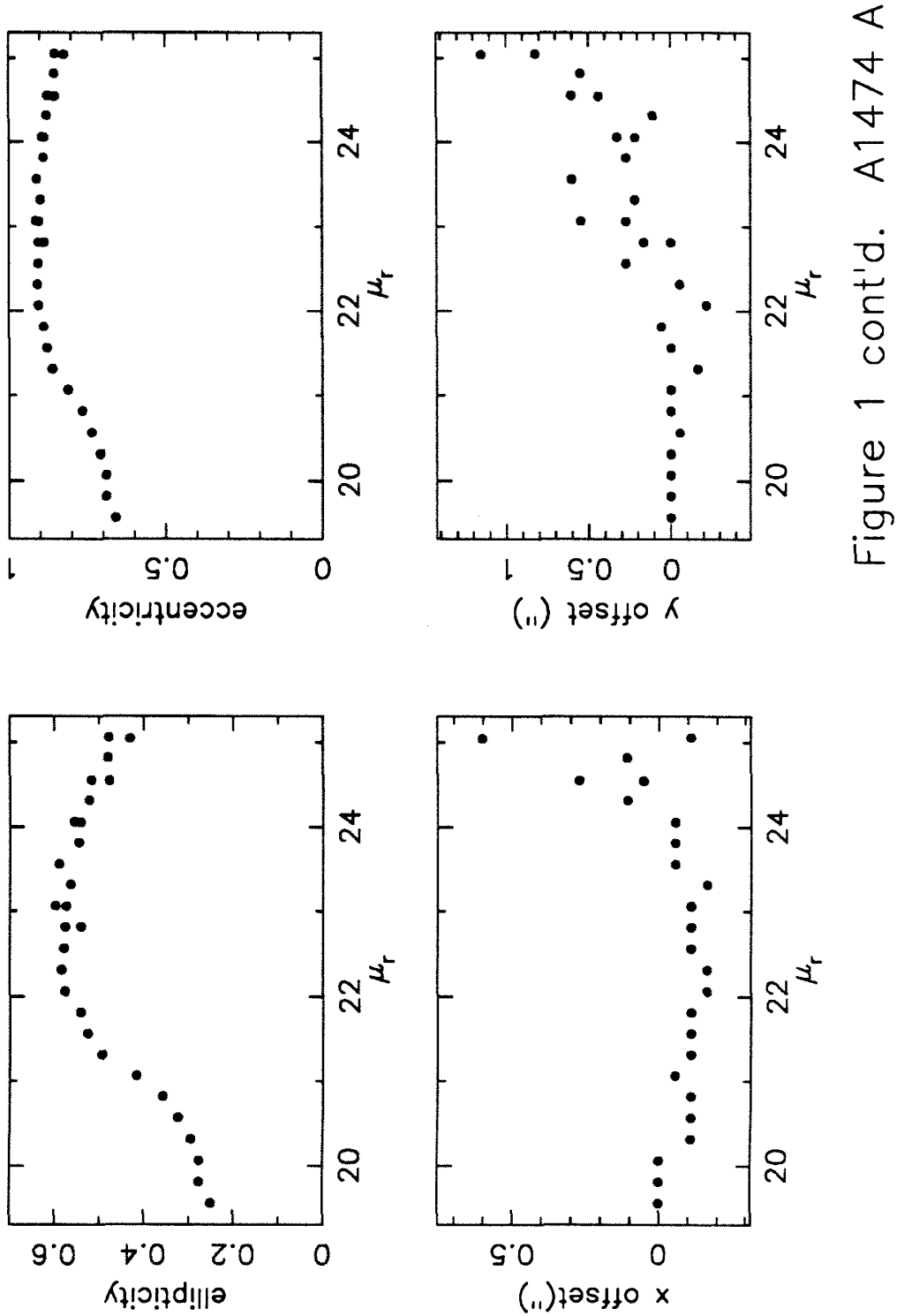


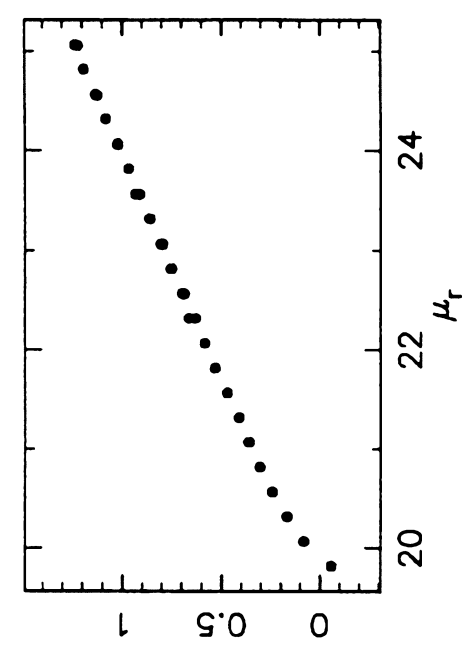

(.1)sn!pod 6ol

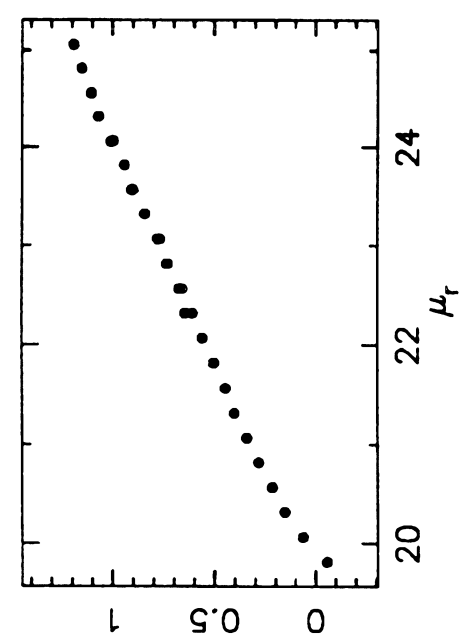

(i1) 9601

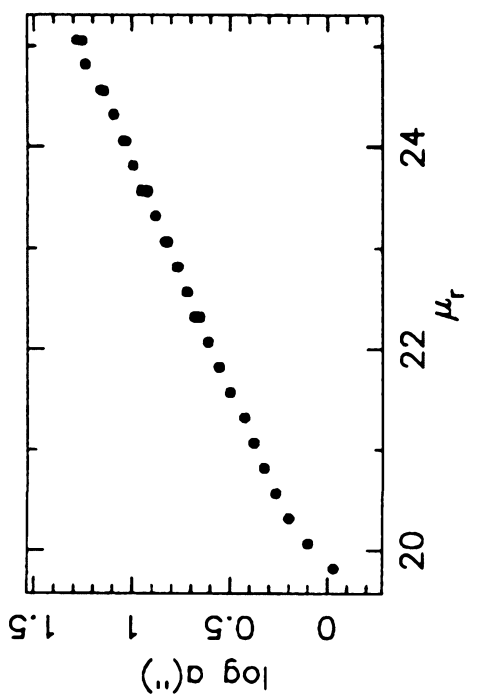

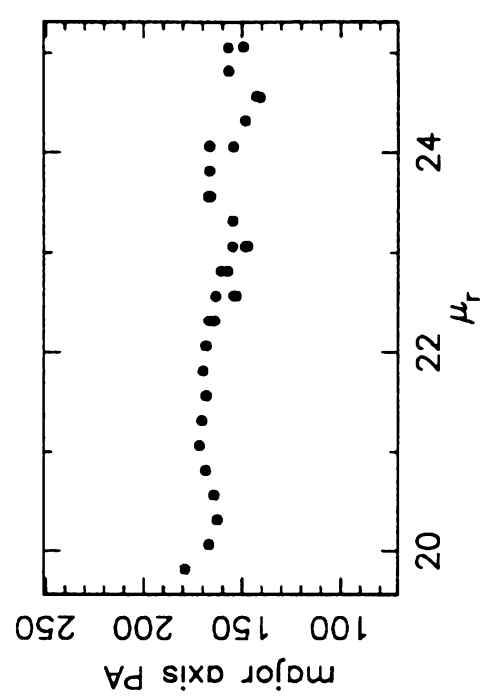

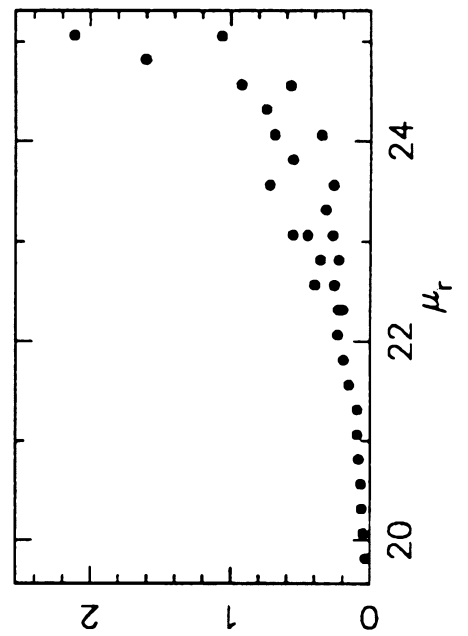

(11) $7 ! 1$ to Du6!s auo

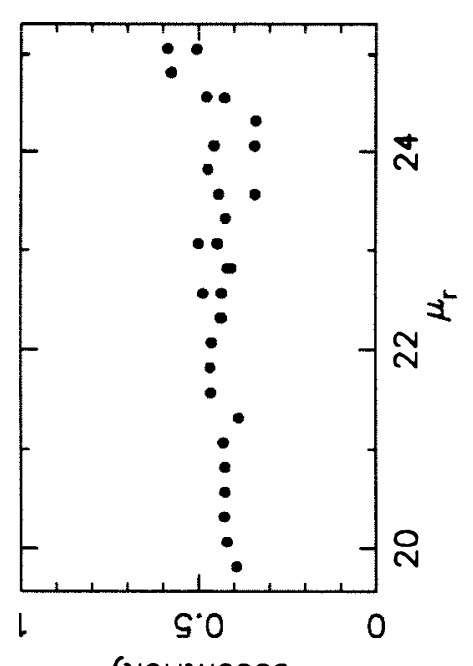

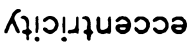

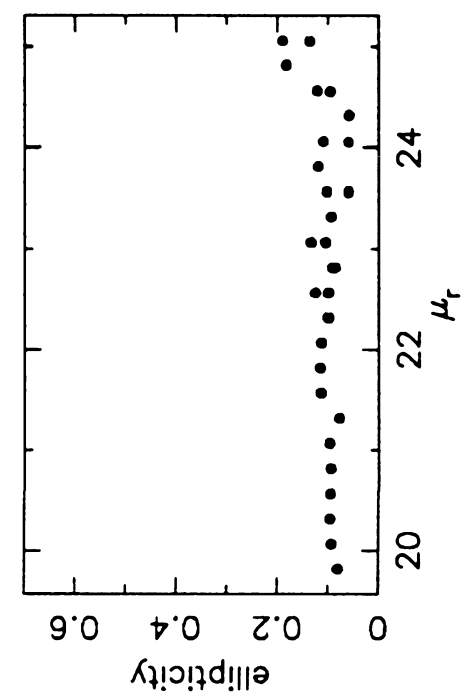

$m$

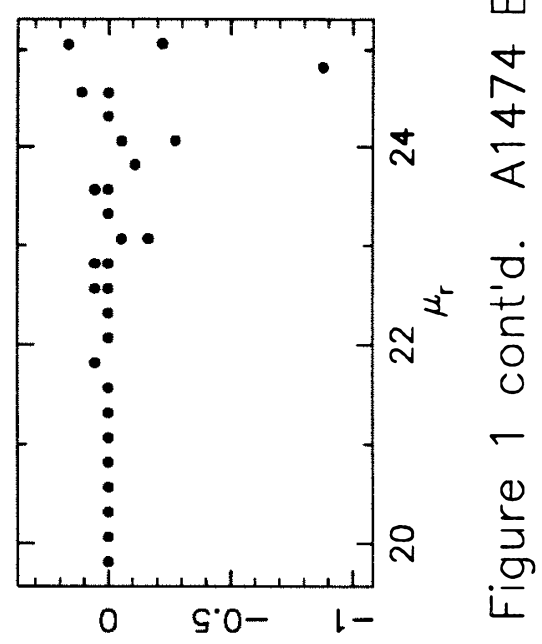

(ii) $725+10 k$

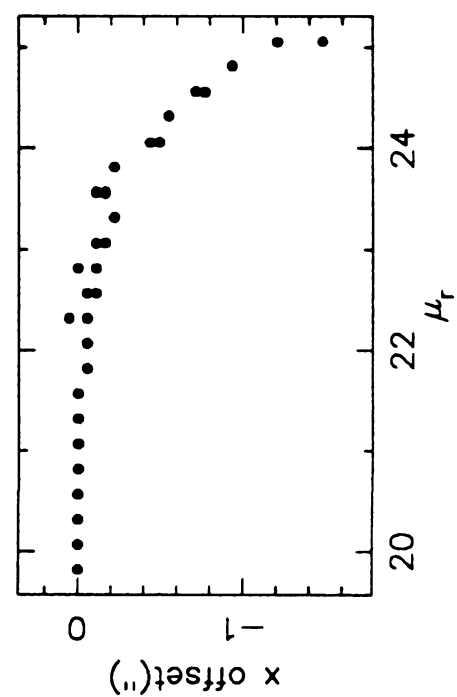




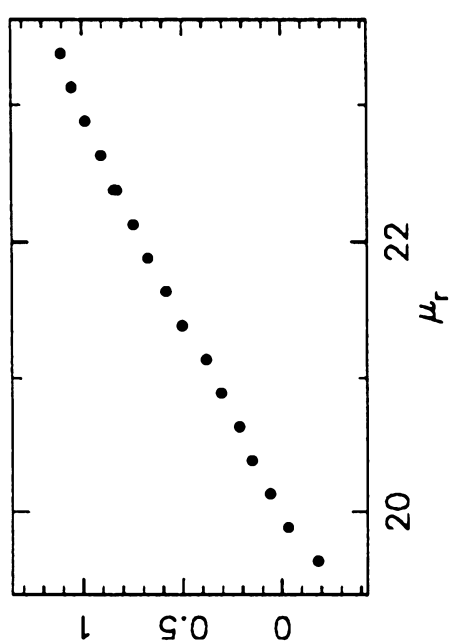

(.1) sn!pod 60।

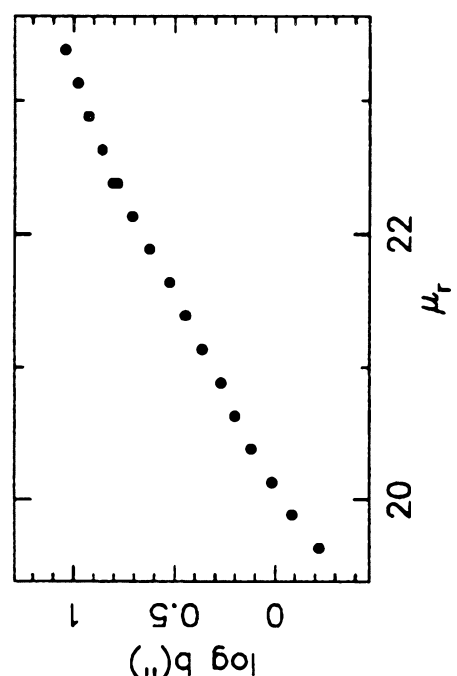

(.1) 960

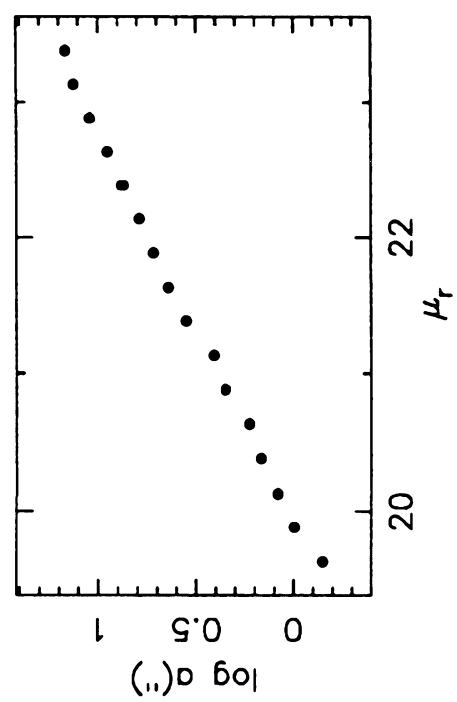

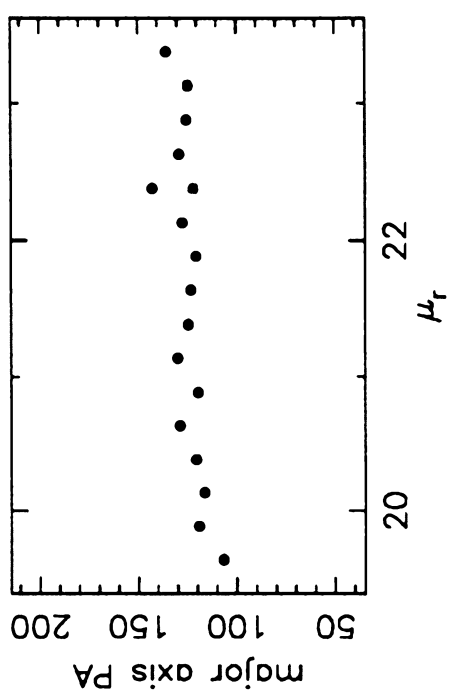

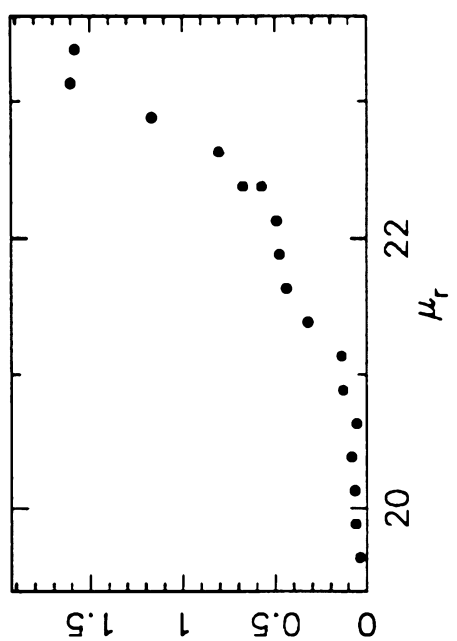

(11) $7 !+$ to Dub!s au०

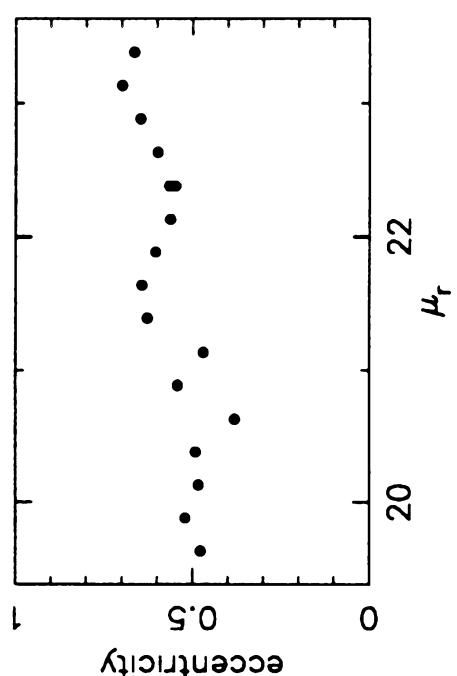

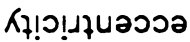

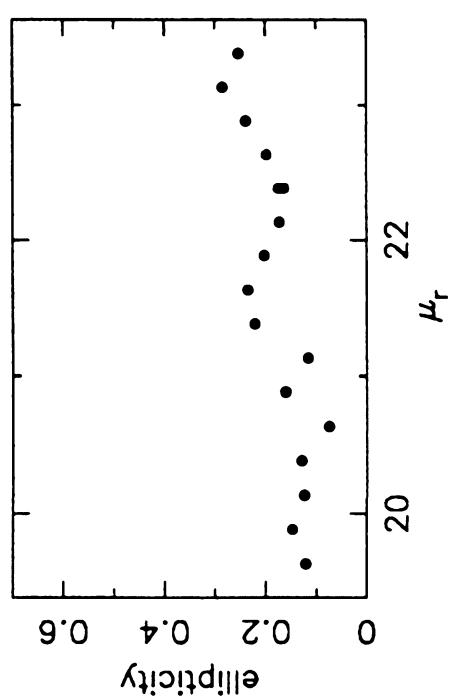

$\frac{5}{\frac{5}{6}}$

- - ก

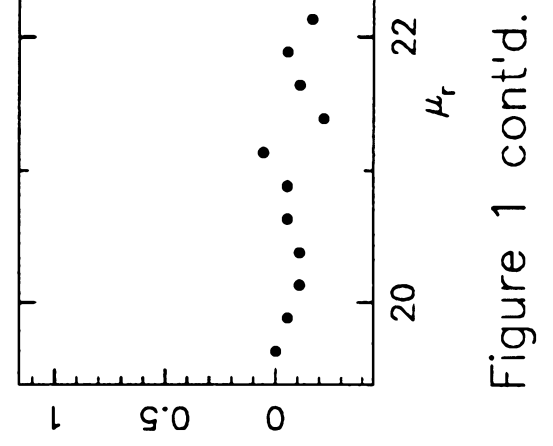

(11) $205 \% 10 \mathrm{~K}$

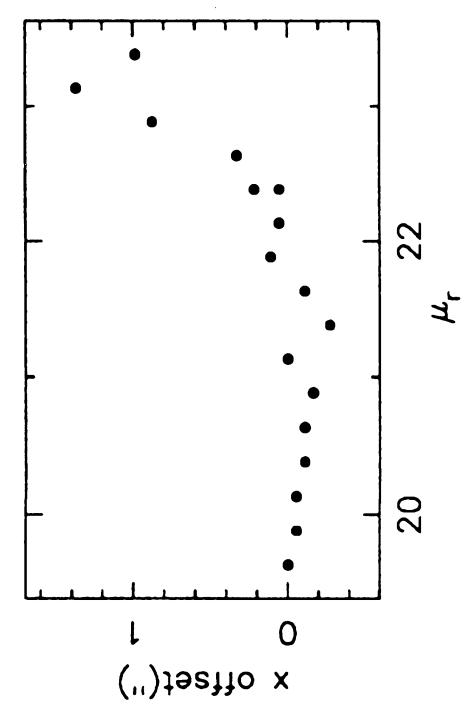




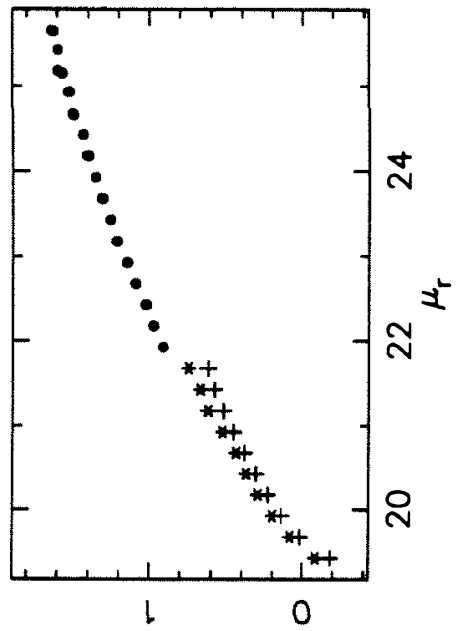

(.1)sn!pos 601

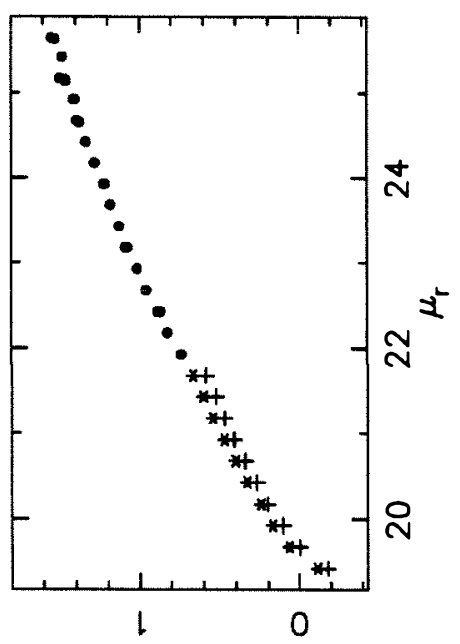

(i.) 9 60।

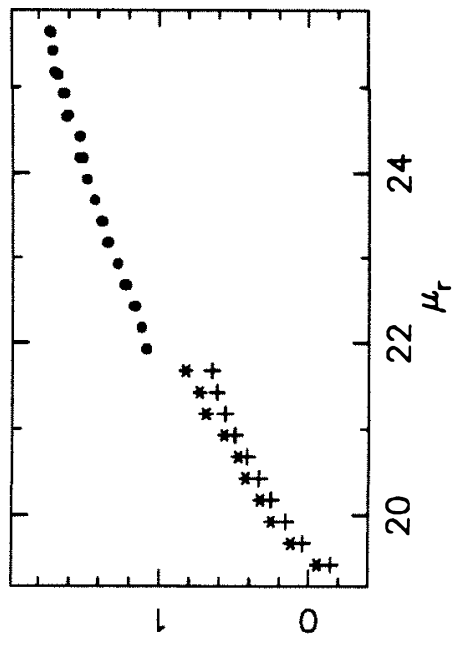

(.1) $\mathrm{D} \mathrm{bo}$

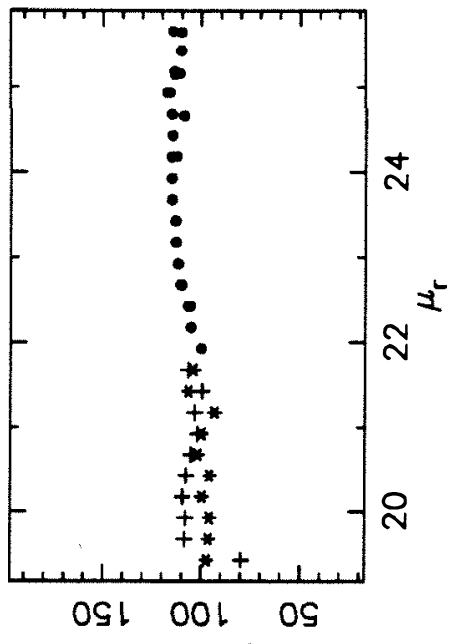

$\forall d 8$ !xo so!rom
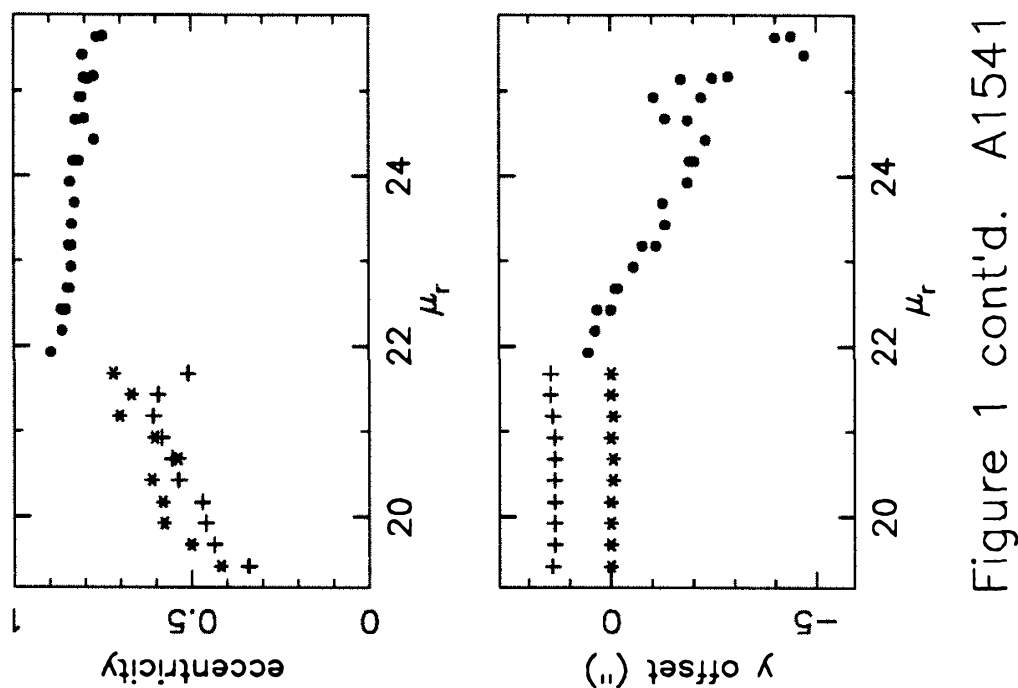

(ii) $725450 \mathrm{~K}$
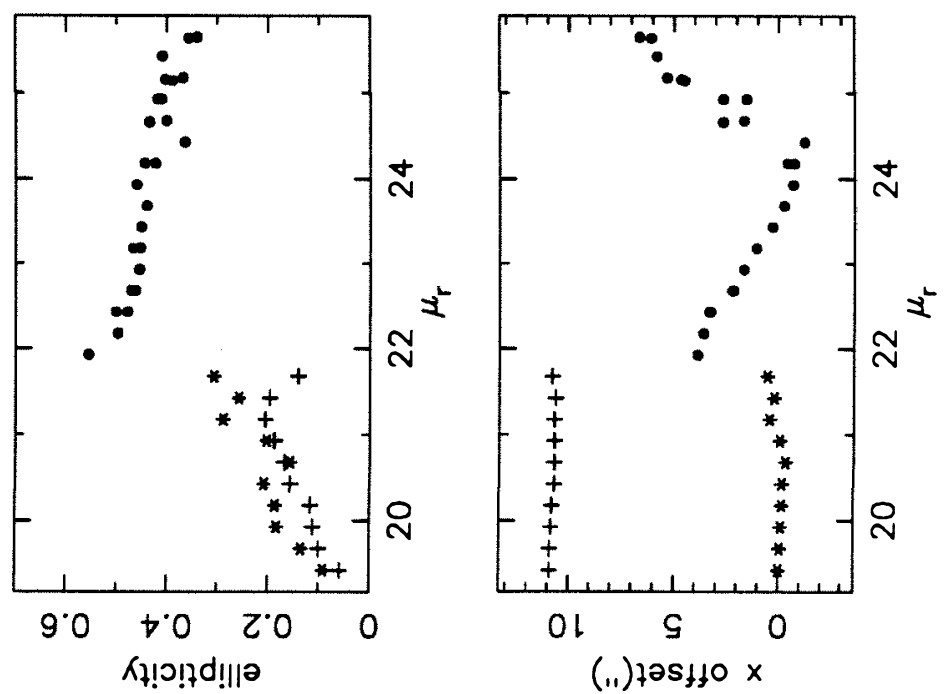


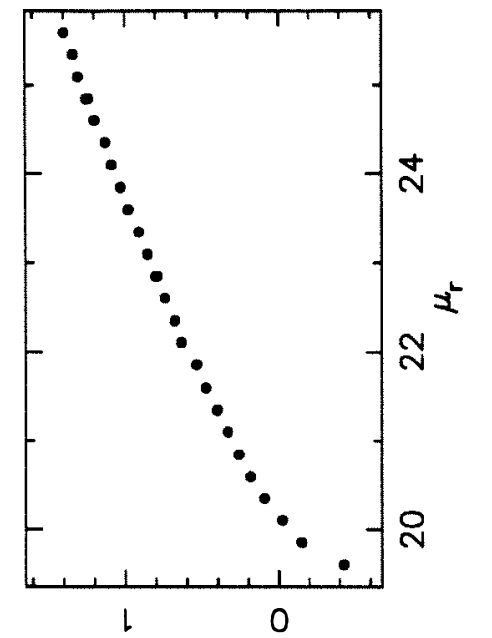

(.1)sn!pos 60।
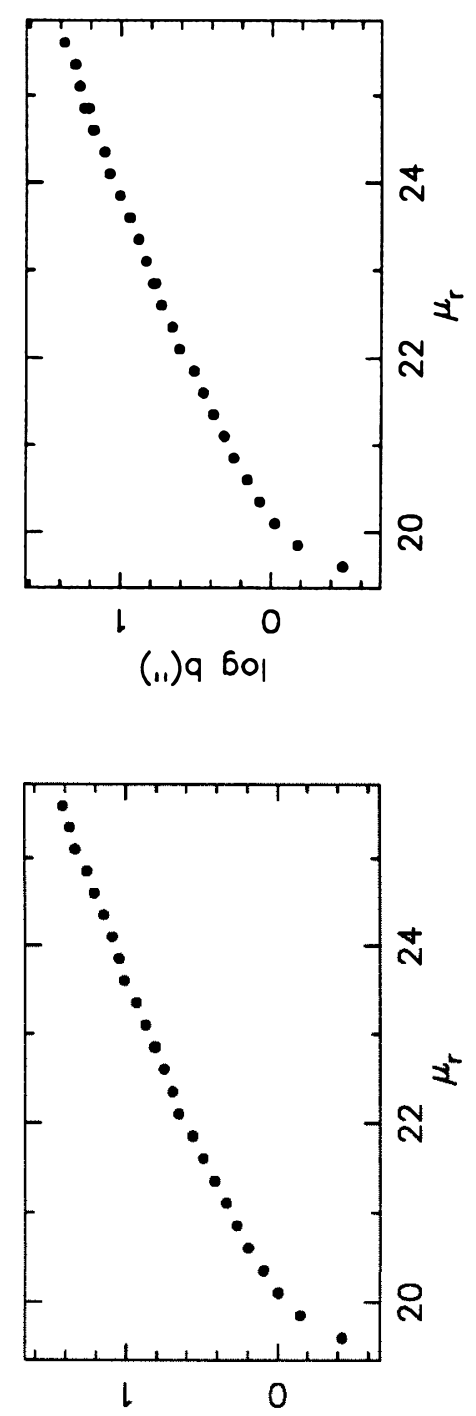

(.1) 0501

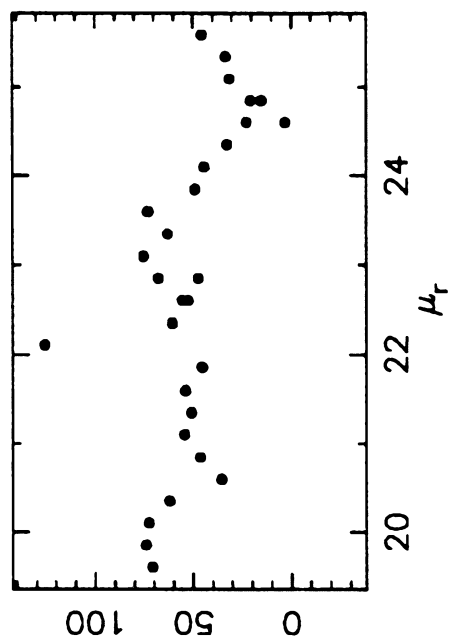

$\forall d$ s!xo so!nom

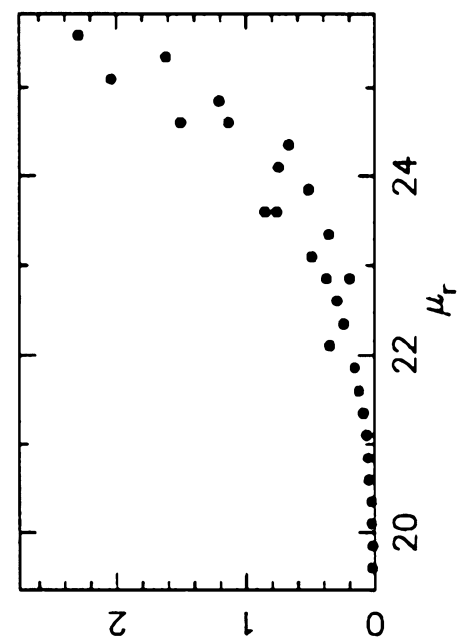

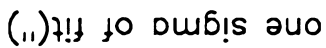
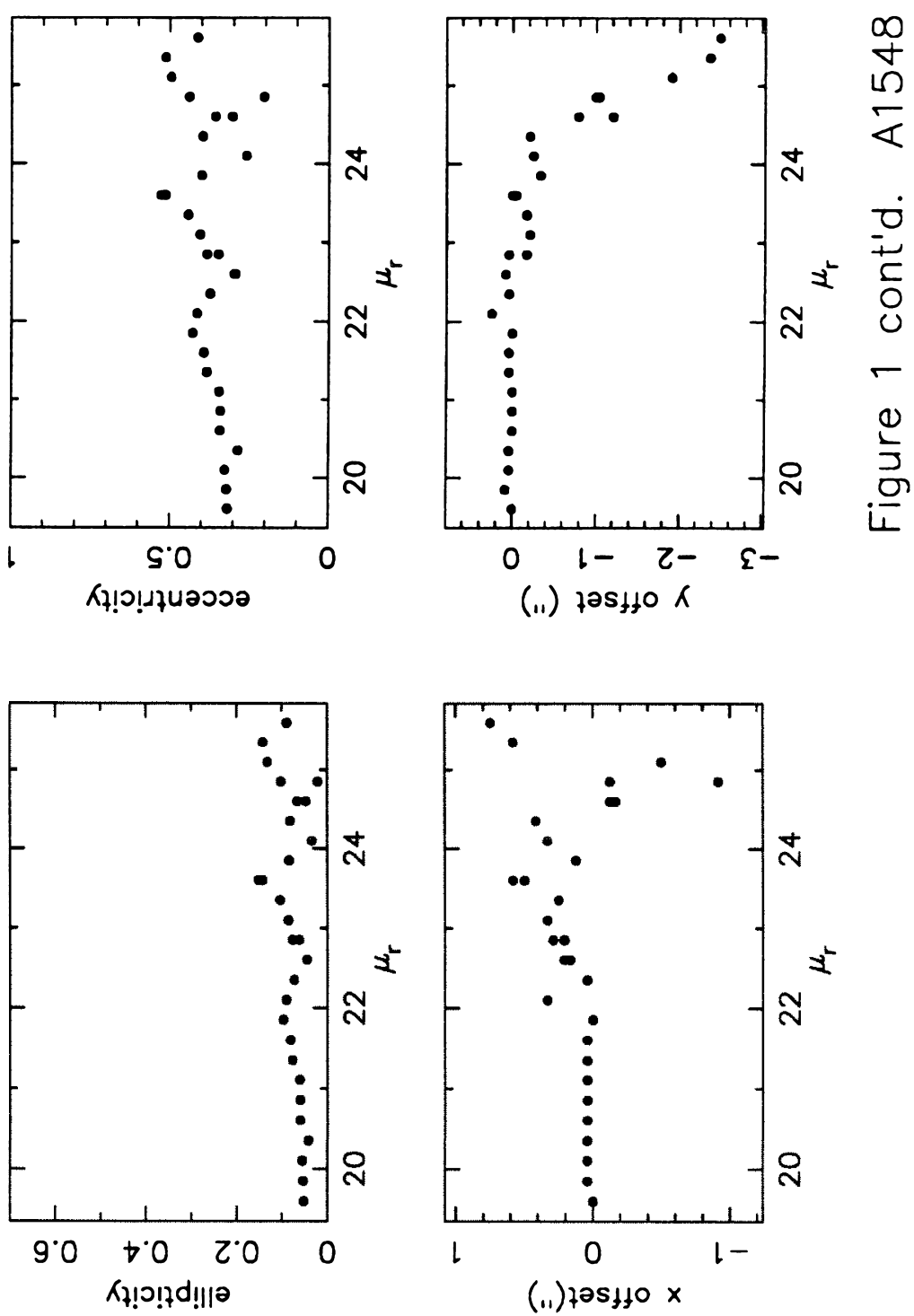


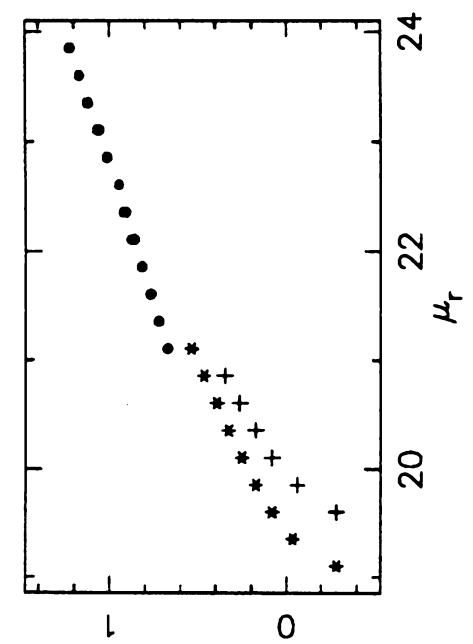

(.1) sn!pod 60।

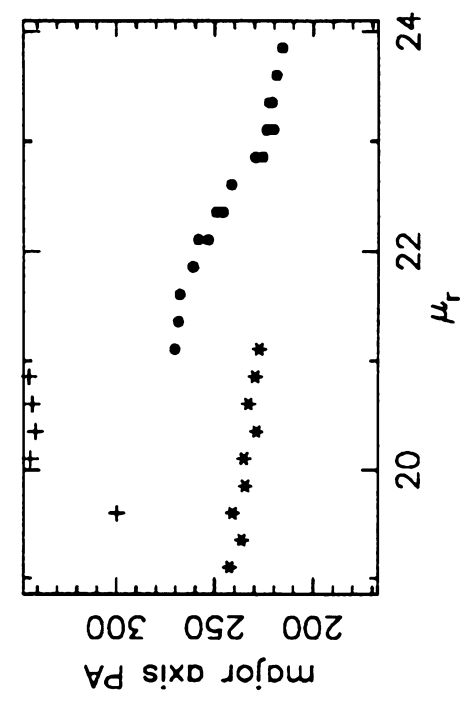

$\forall d$ s!xo so!

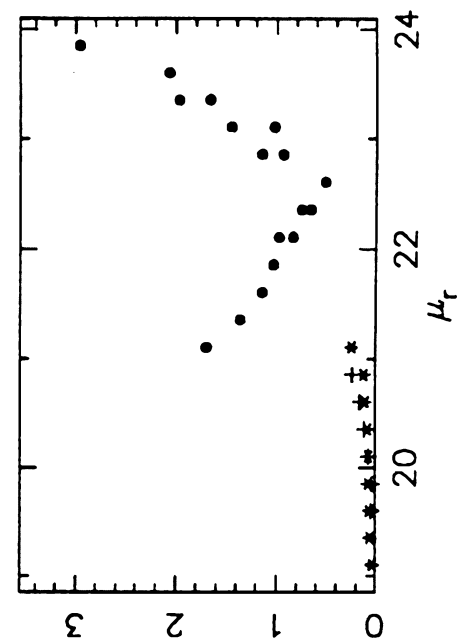

(11) 4 ! t to oub! 2uo

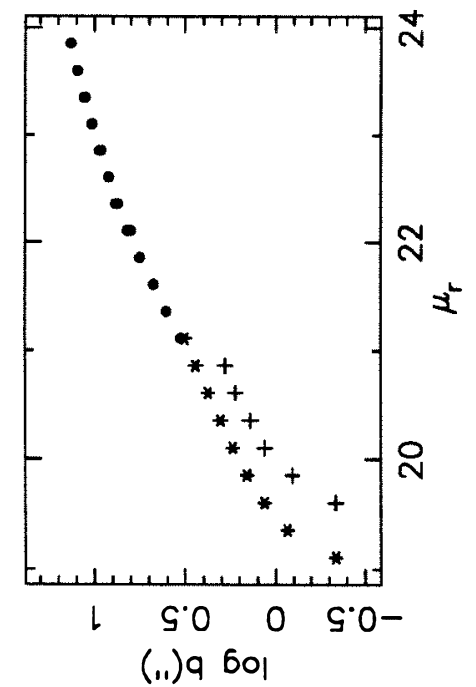

(.1) 960

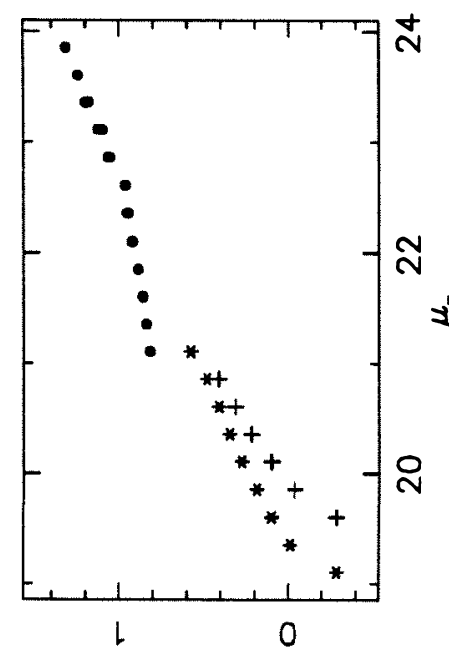

(.1) $\mathrm{D}$ bol
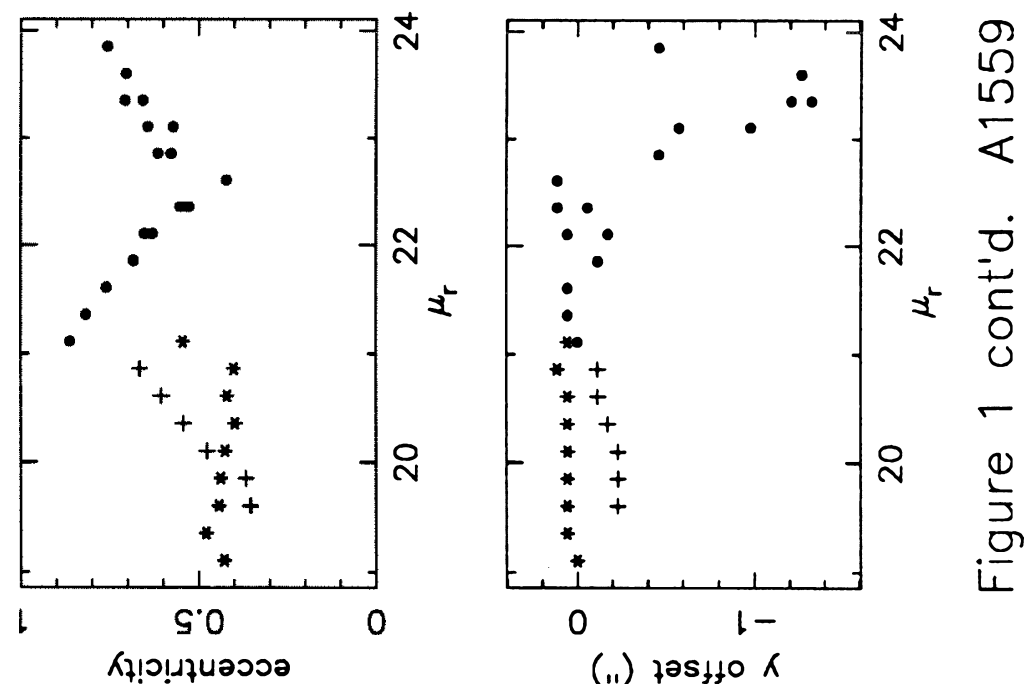

(i1) $725+10 \mathrm{~K}$
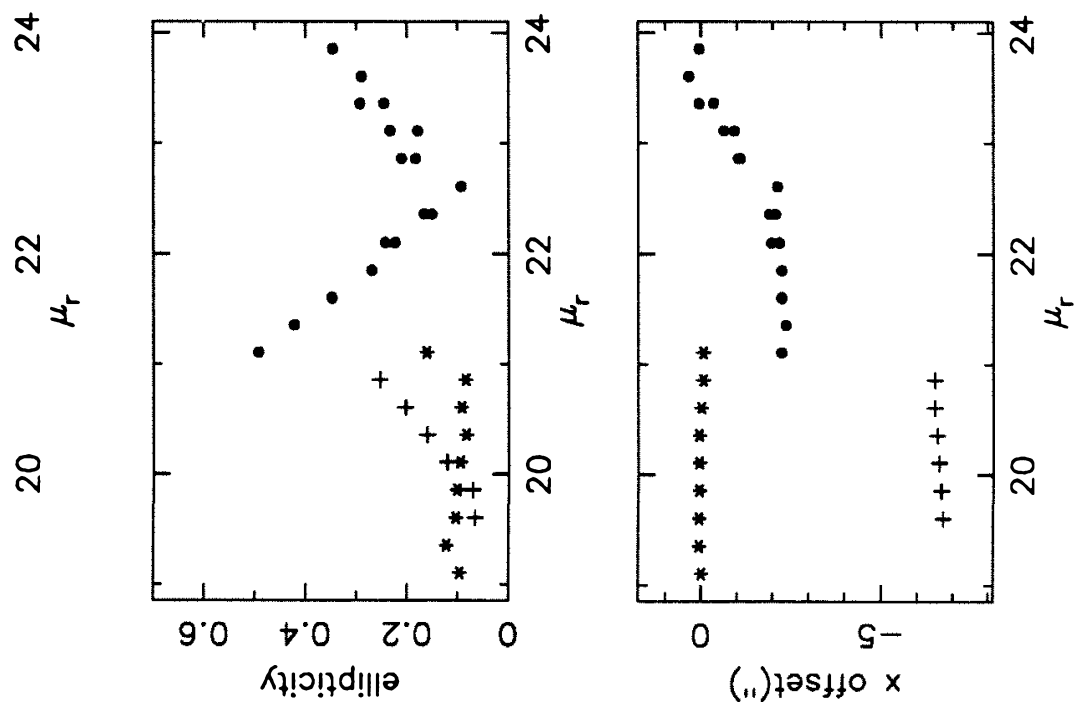


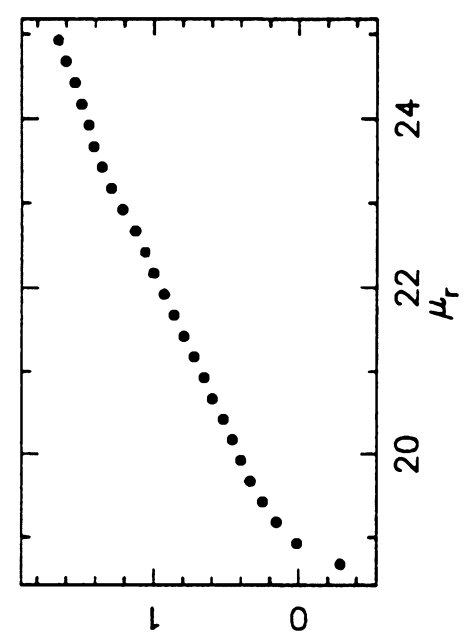

(.1) sn!pod 601

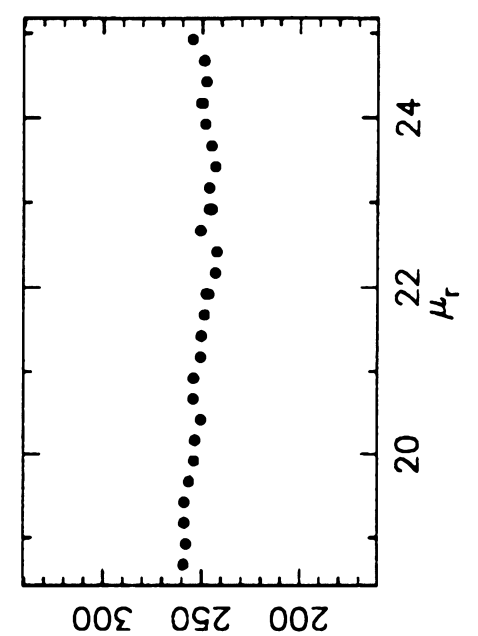

$\forall d$ s!xo دo!́om

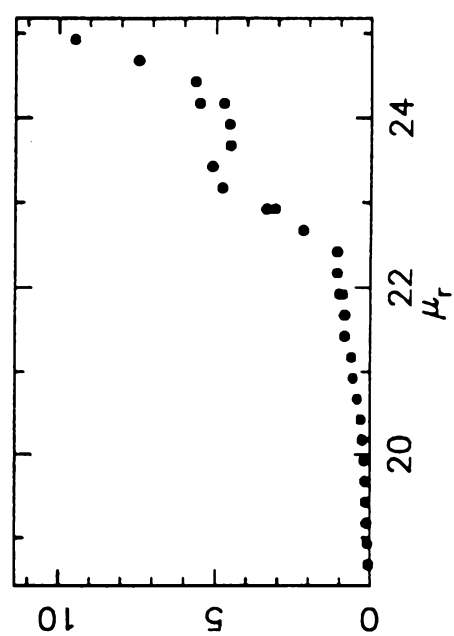

(11) 7 !t to oub!s auo

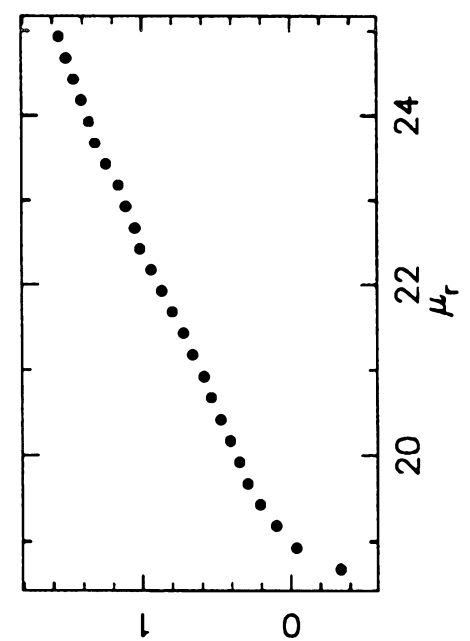

(.1) 9 6ol

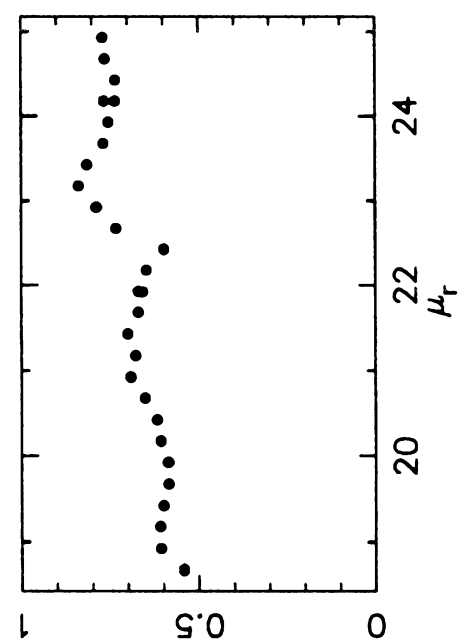

Кұ!ว!นในววว

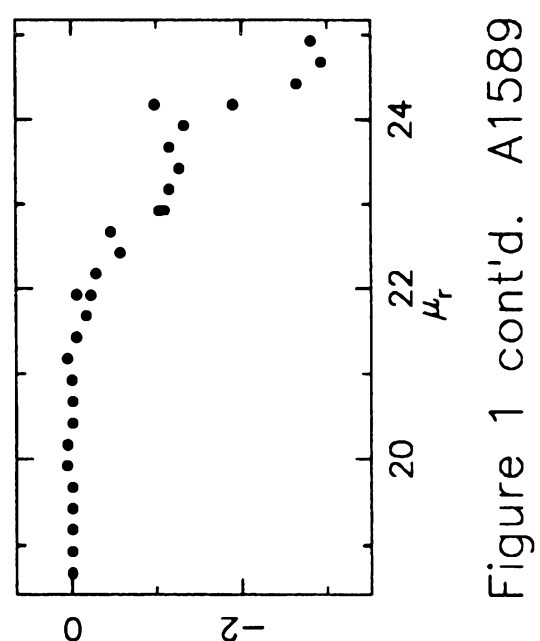

(i1) $725+10 K$
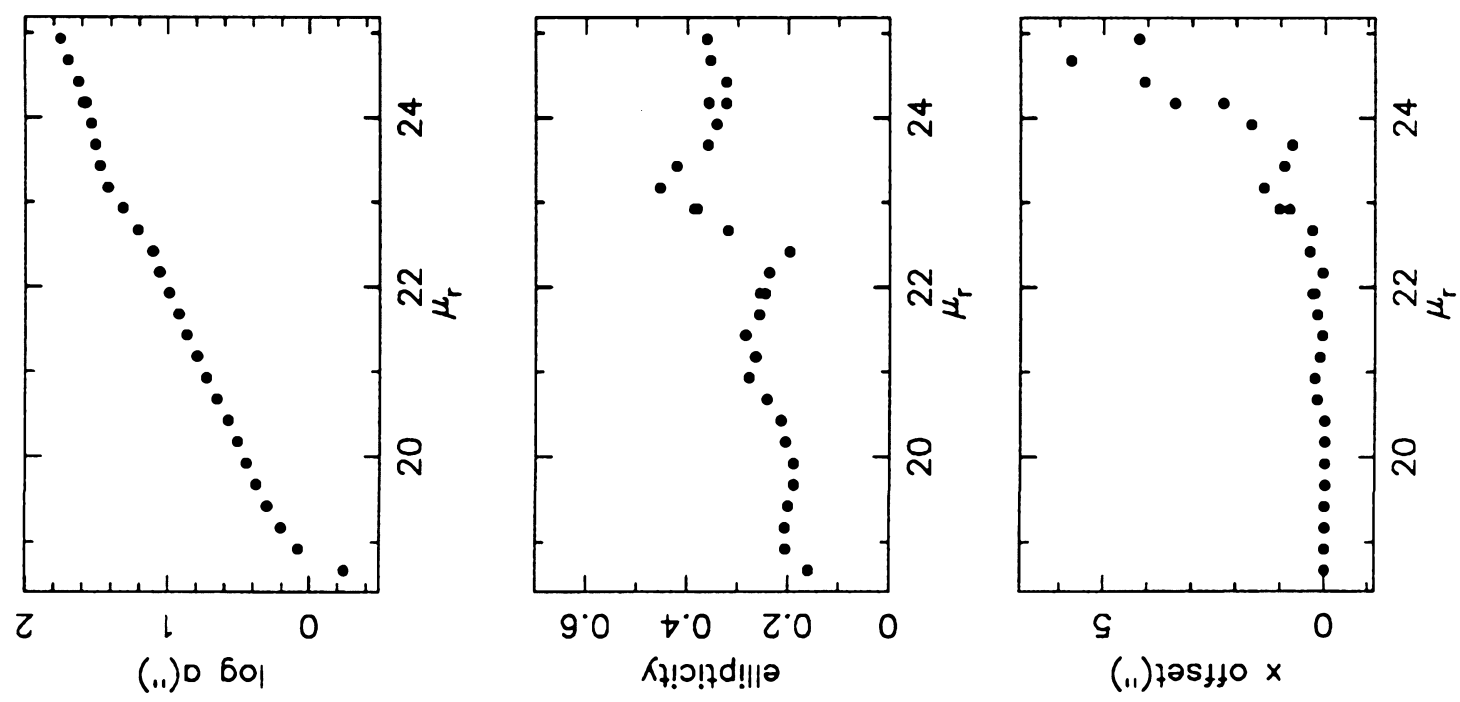

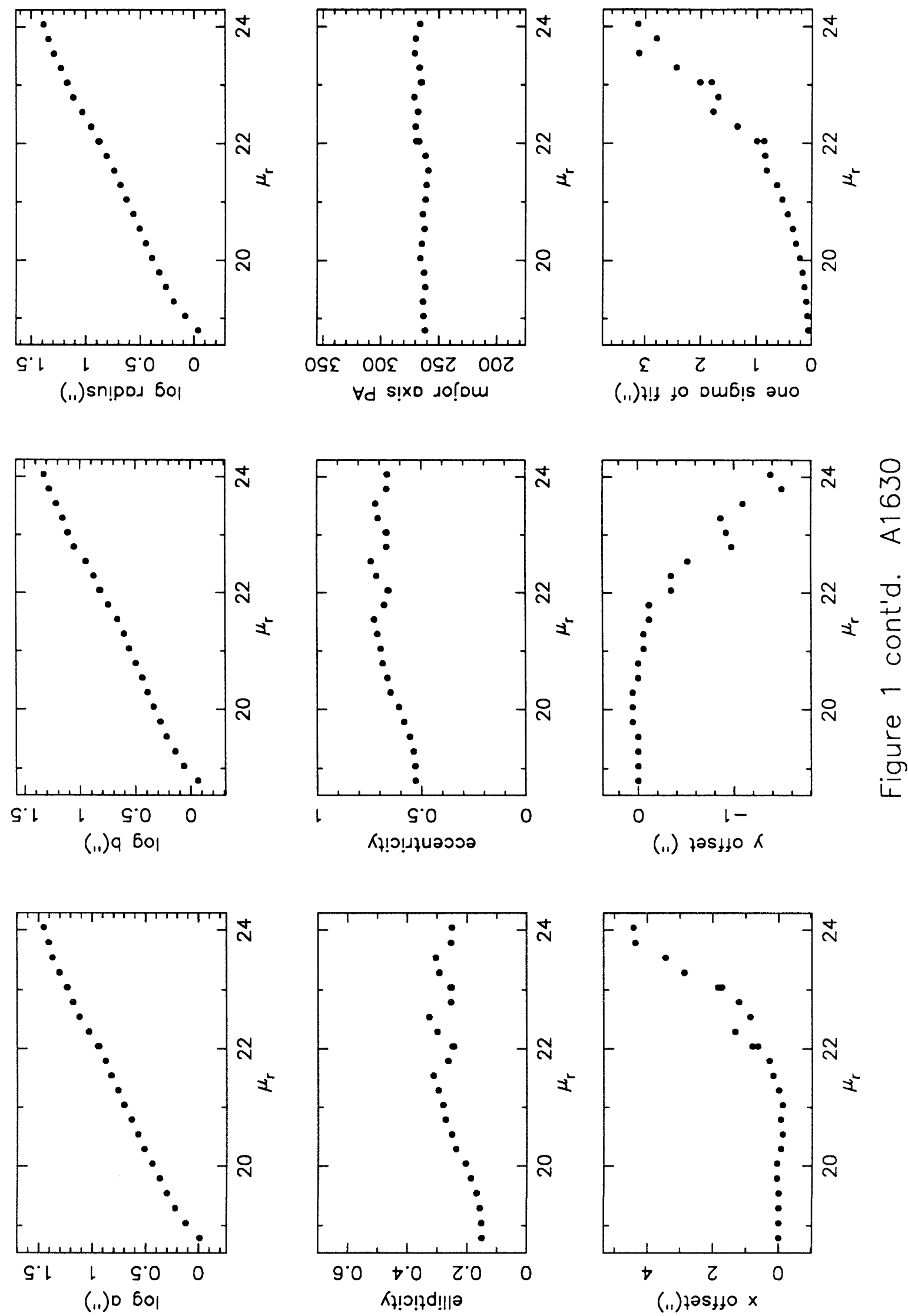


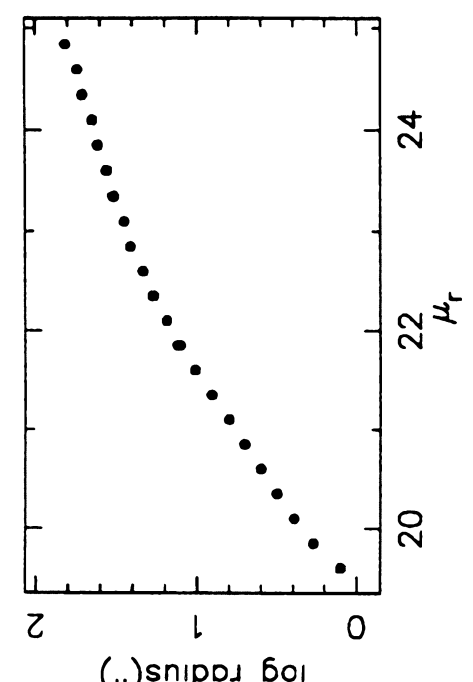

(.1) sn!pos 6ol

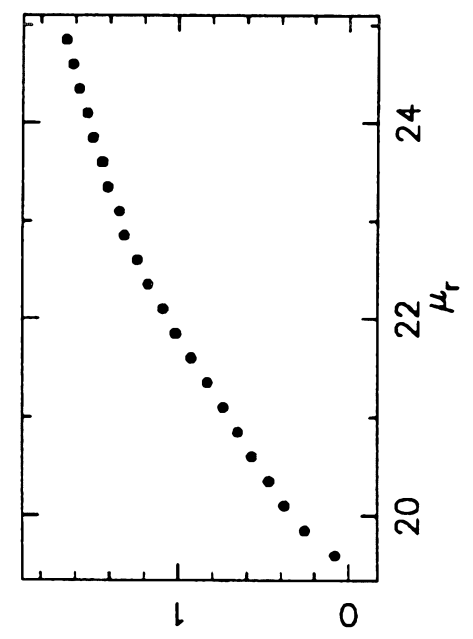

(.1) 9 6ol

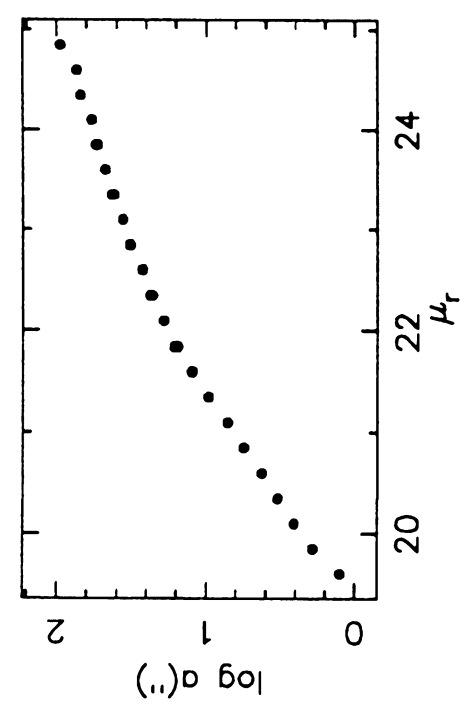

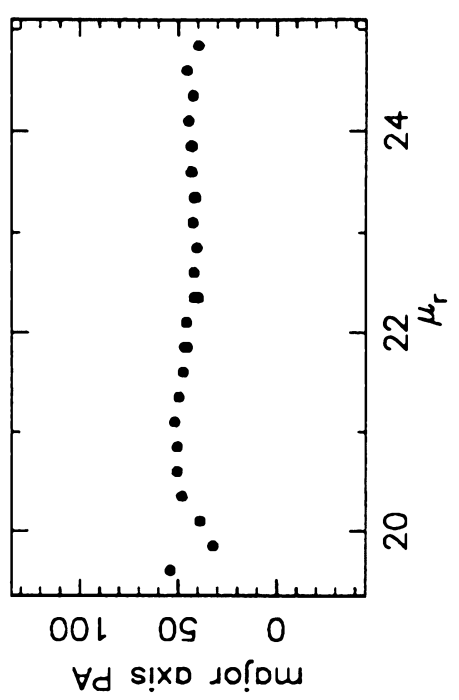

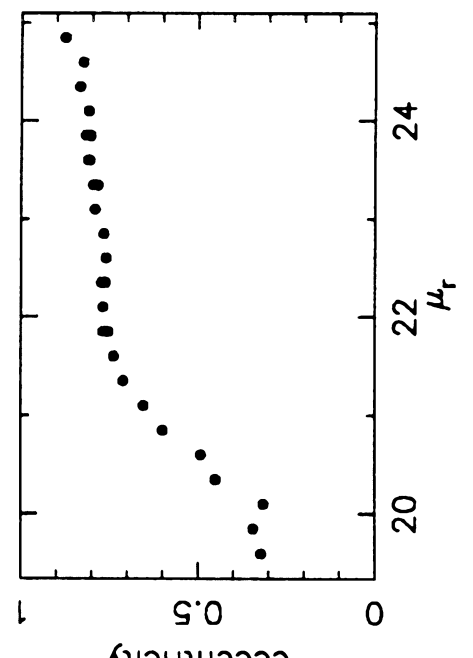

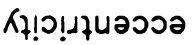

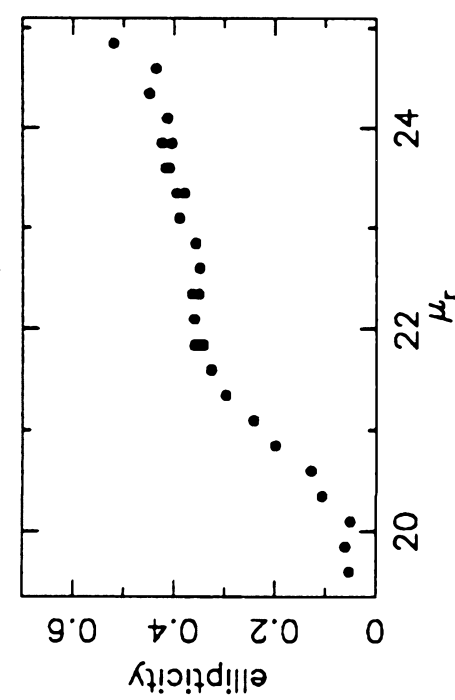

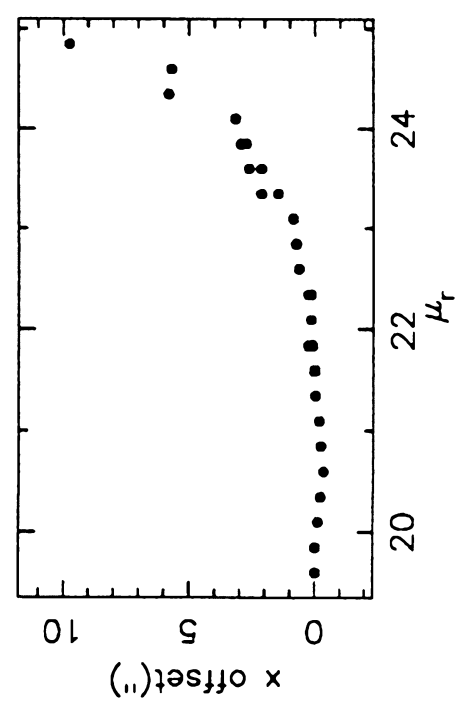

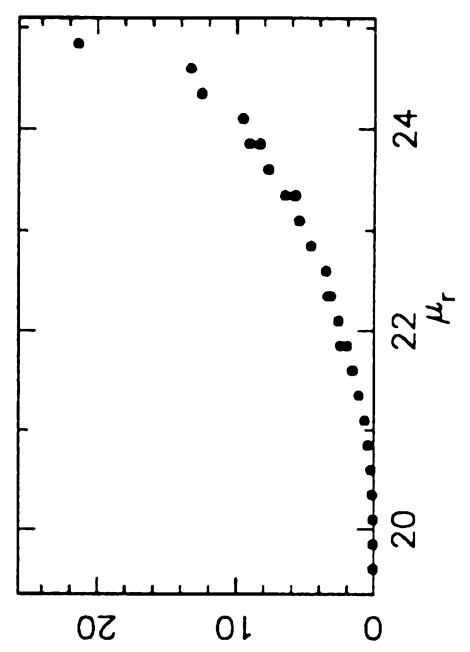

(.1) $7 !+$ to oub!s 2u०

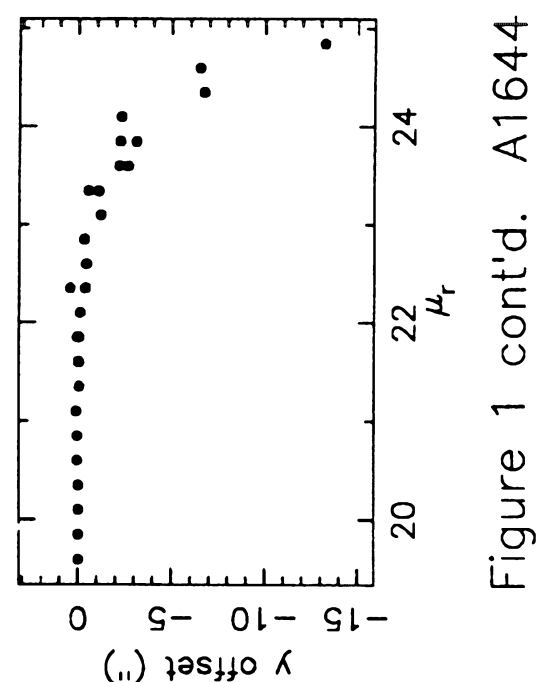

(.1) $725+10 K$

(ii) $1205 y 0 x$ 

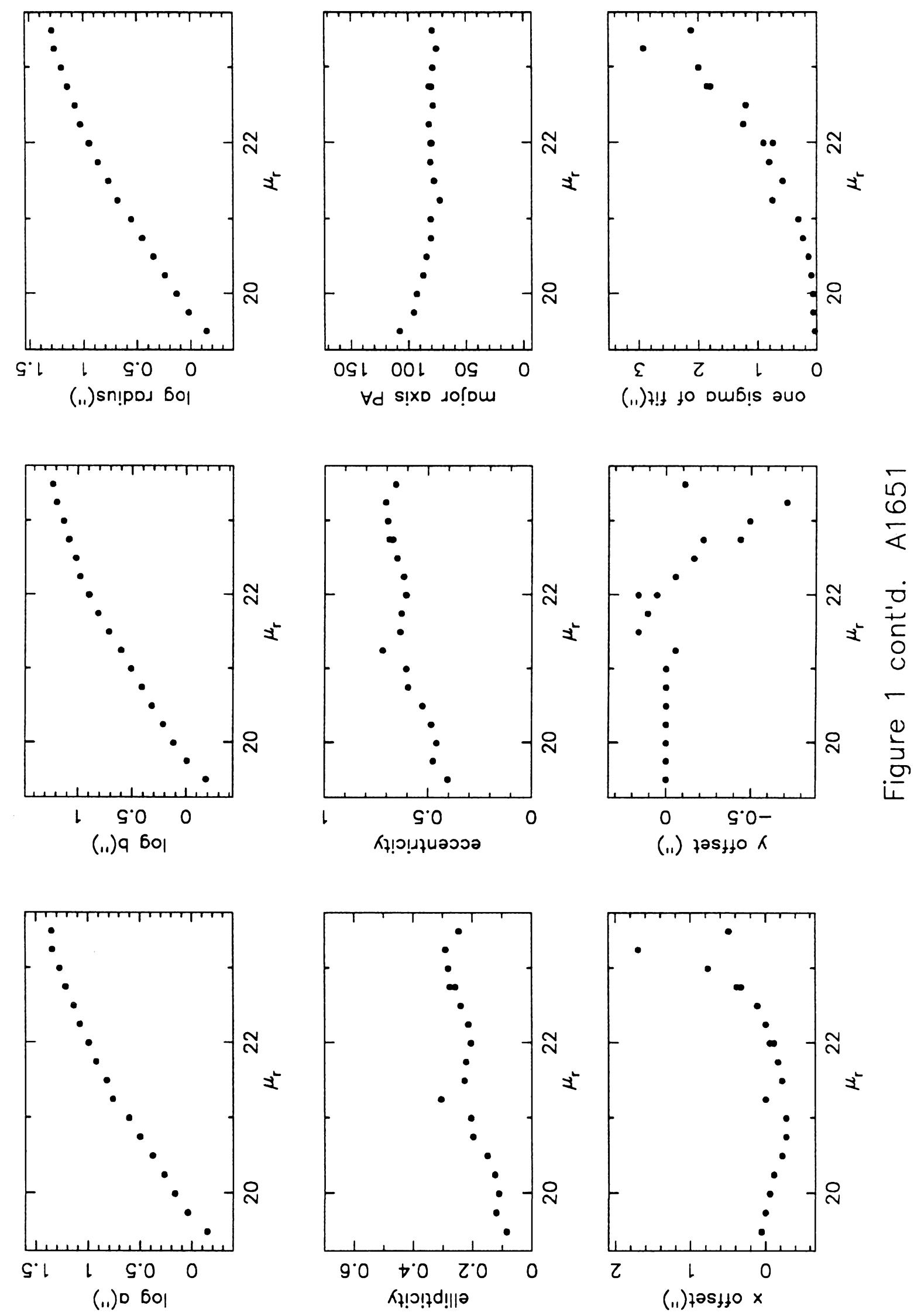

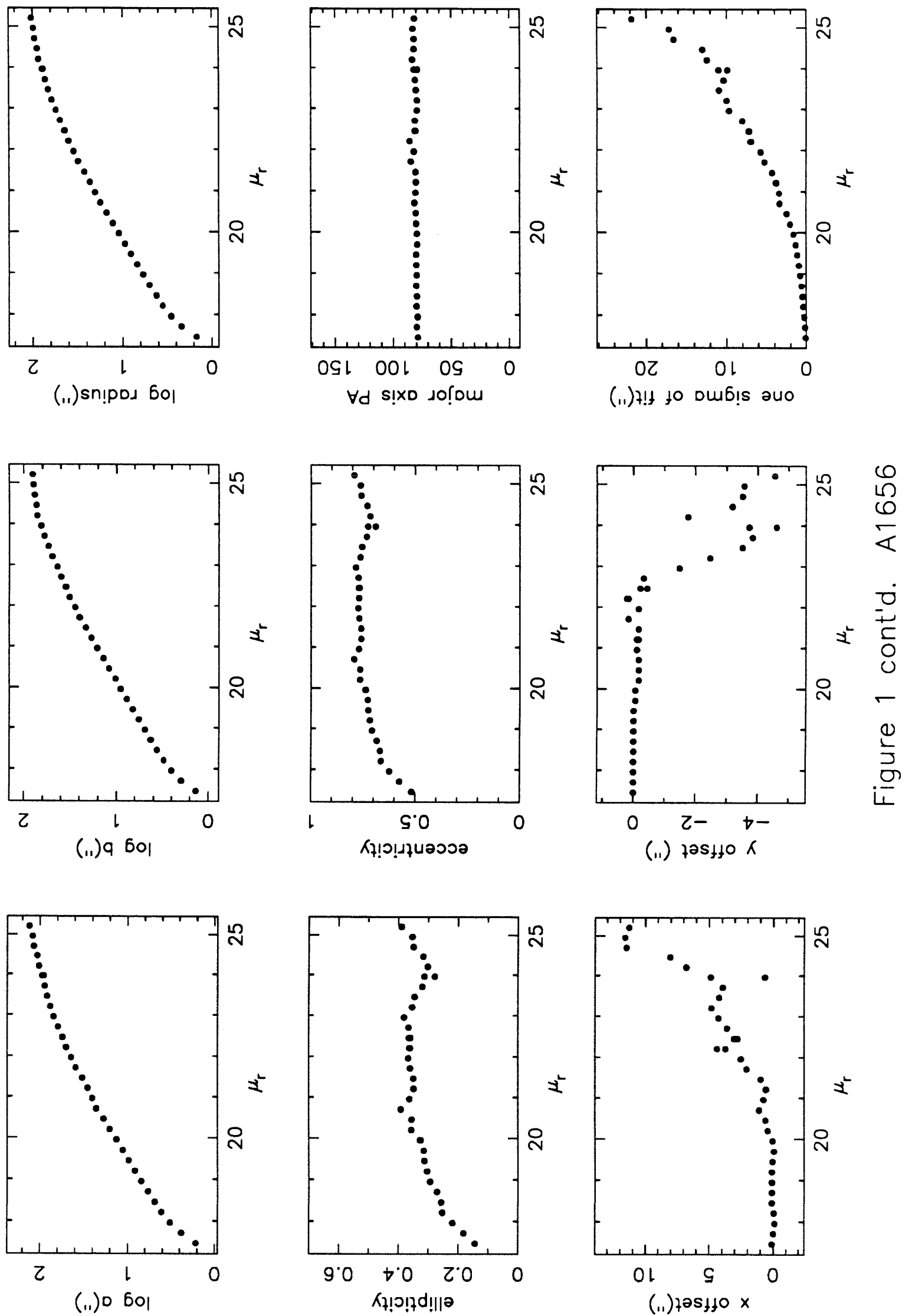


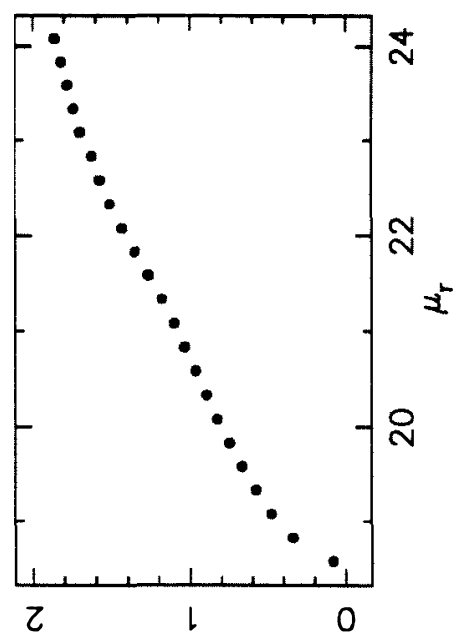

(.1) sn!pos 6ol
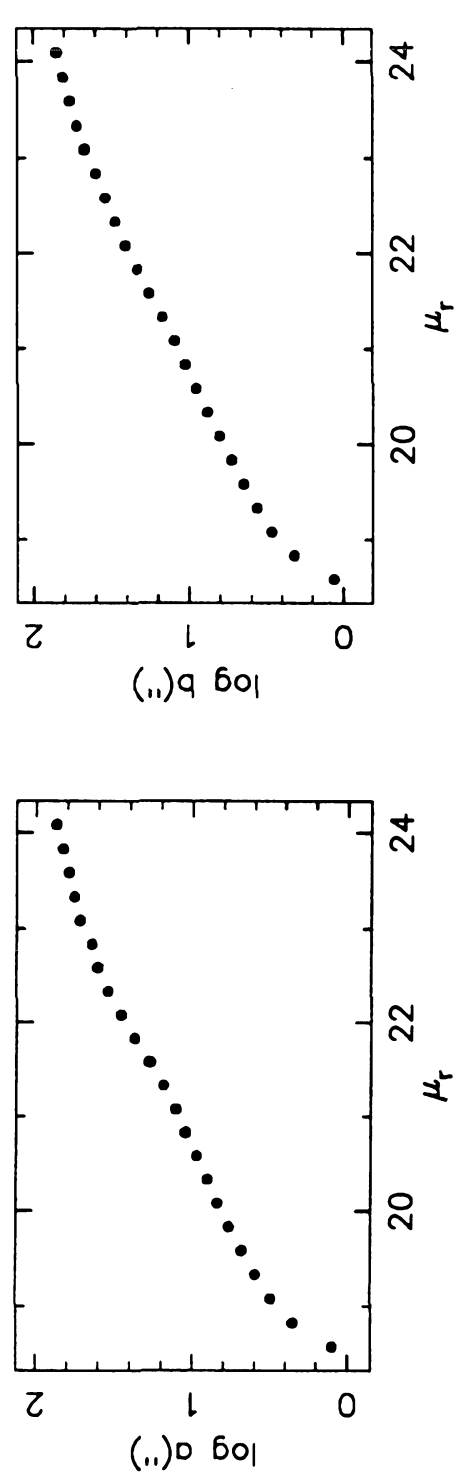
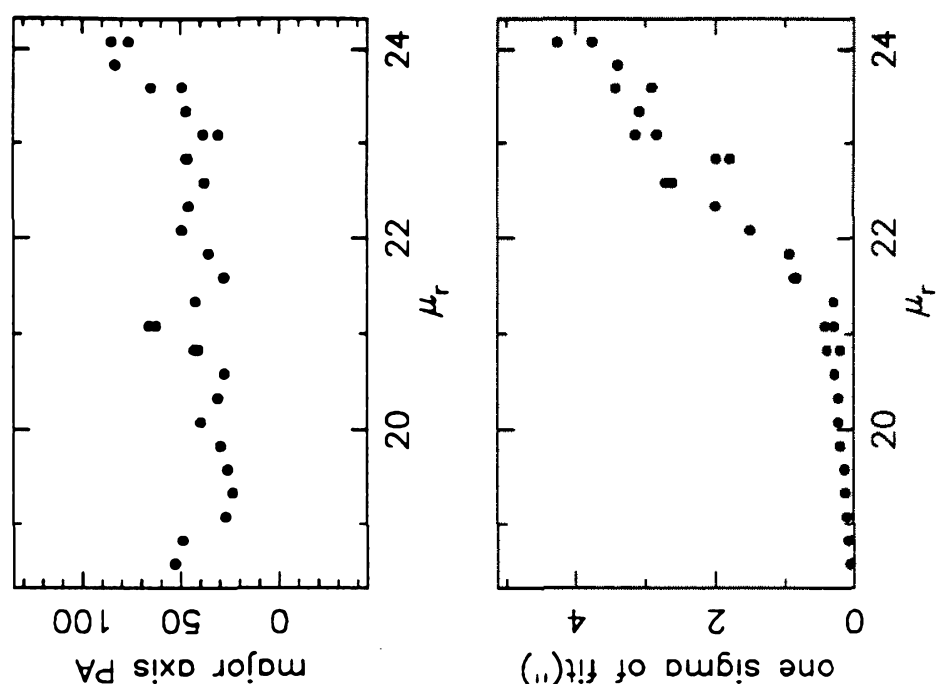

(i1) $+!+10$ oub!s au०

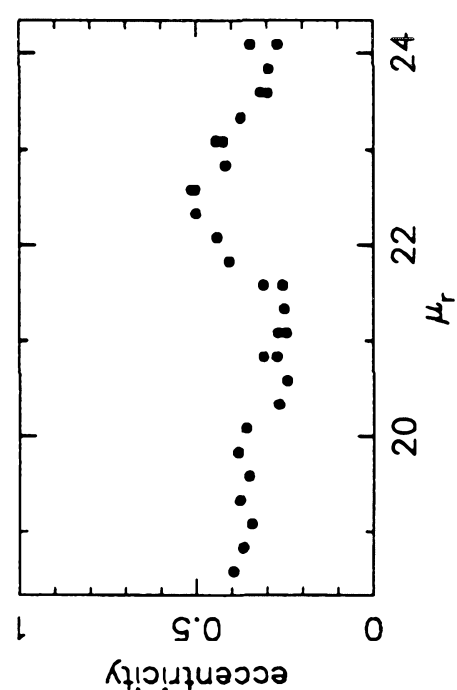

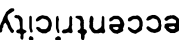

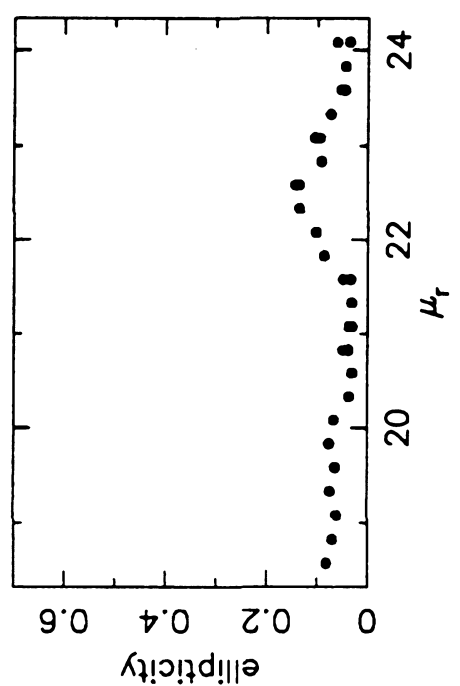

$\infty$

0 6

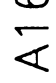
ن U

(ii) tast10 $\mathrm{K}$

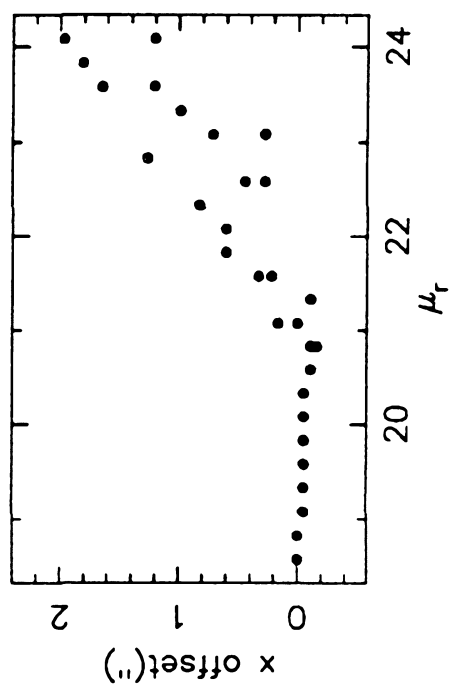




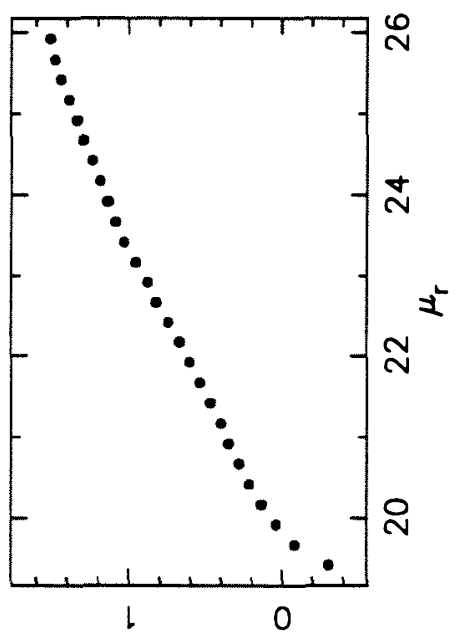

(.1)sn!pod 6ol

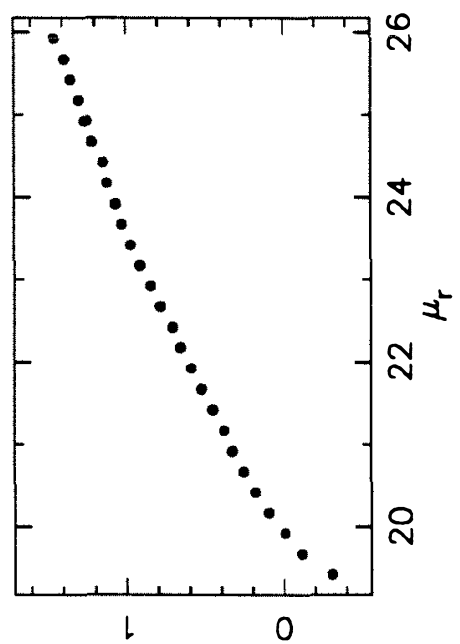

(.1) 9601
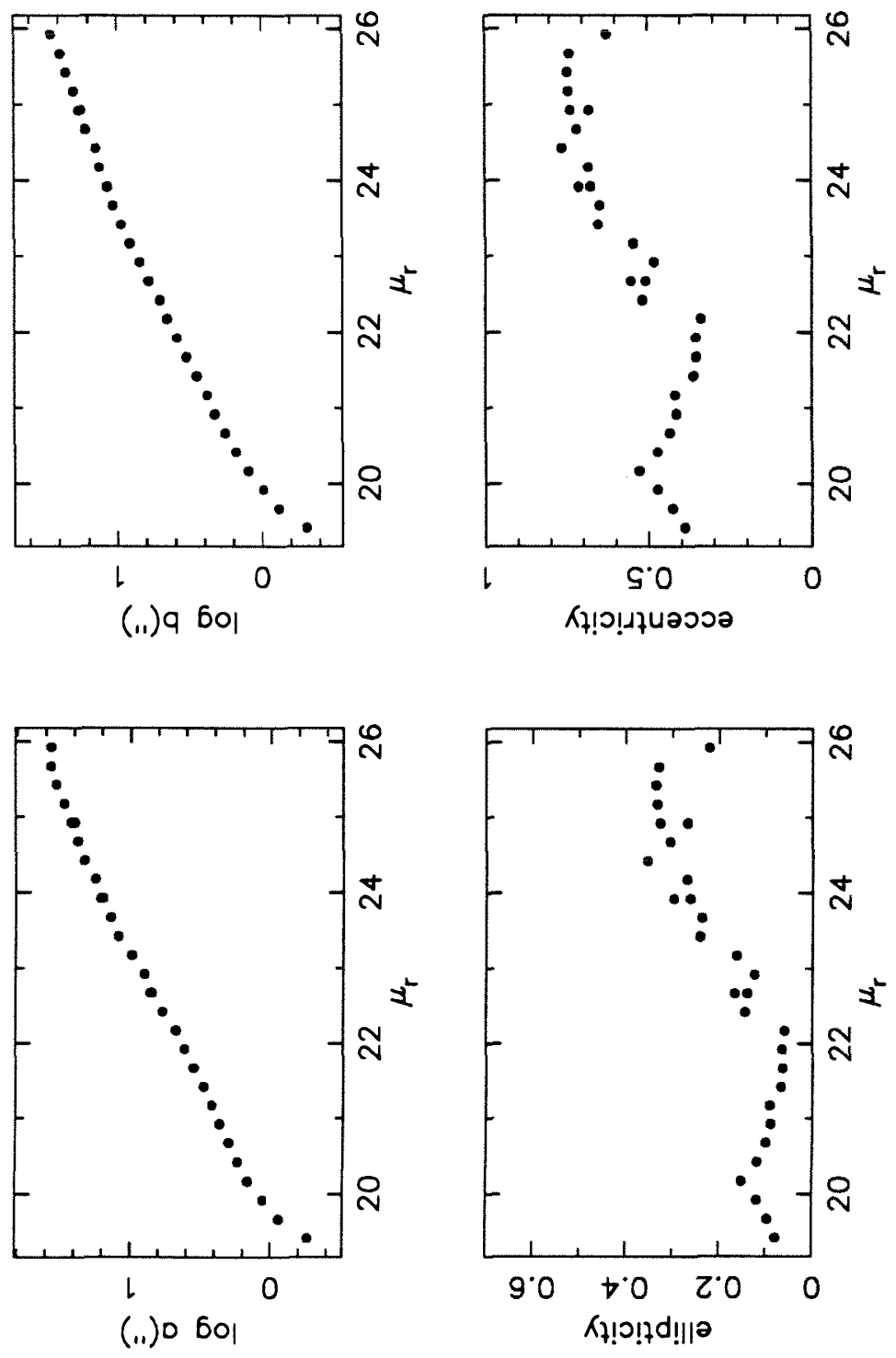

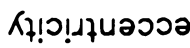

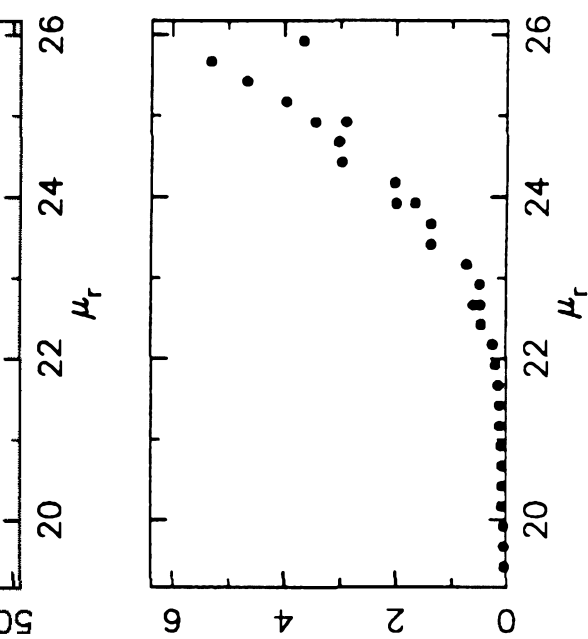

(11) $7 !+$ to Dub!s auo

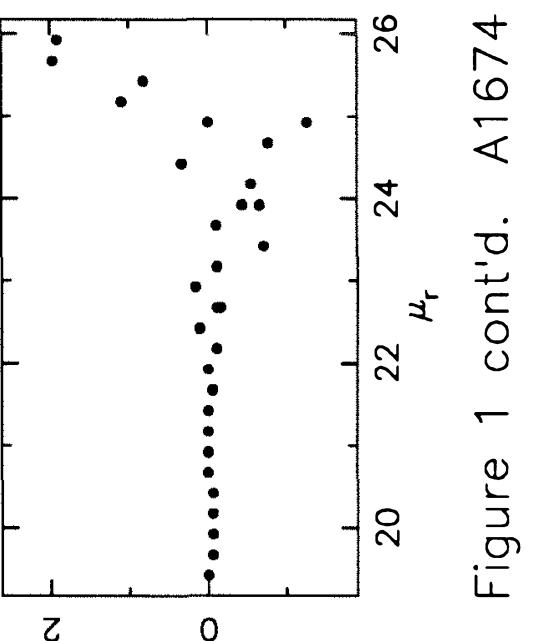

(ii) zastro $\mathrm{K}$

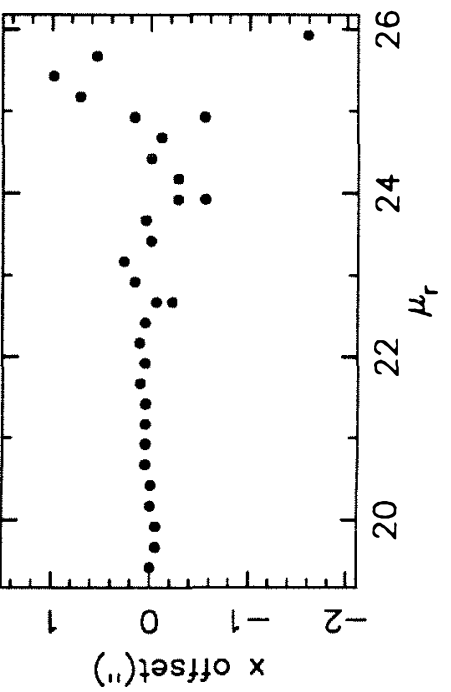



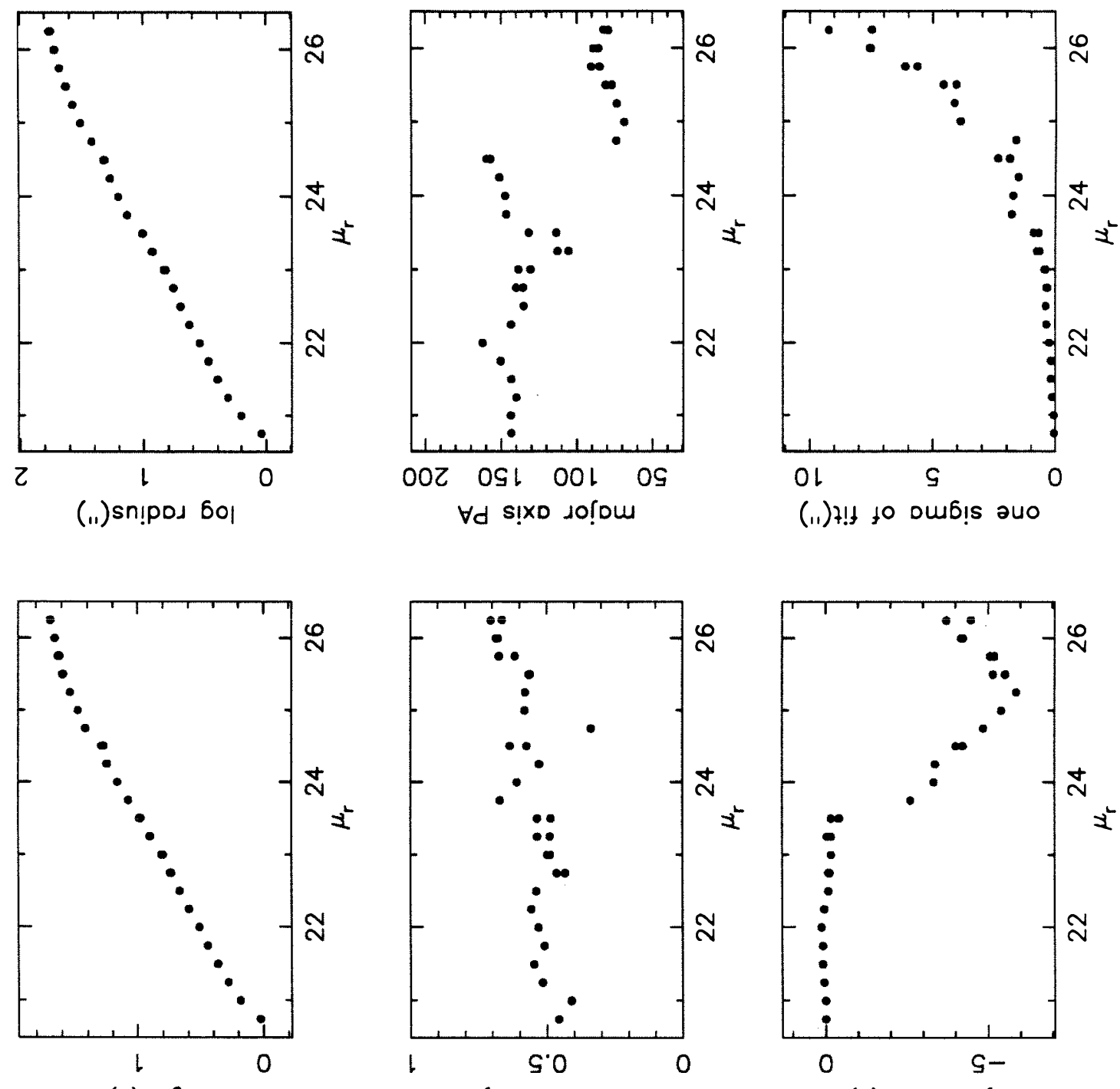

(.1) $9 \mathrm{bol}$
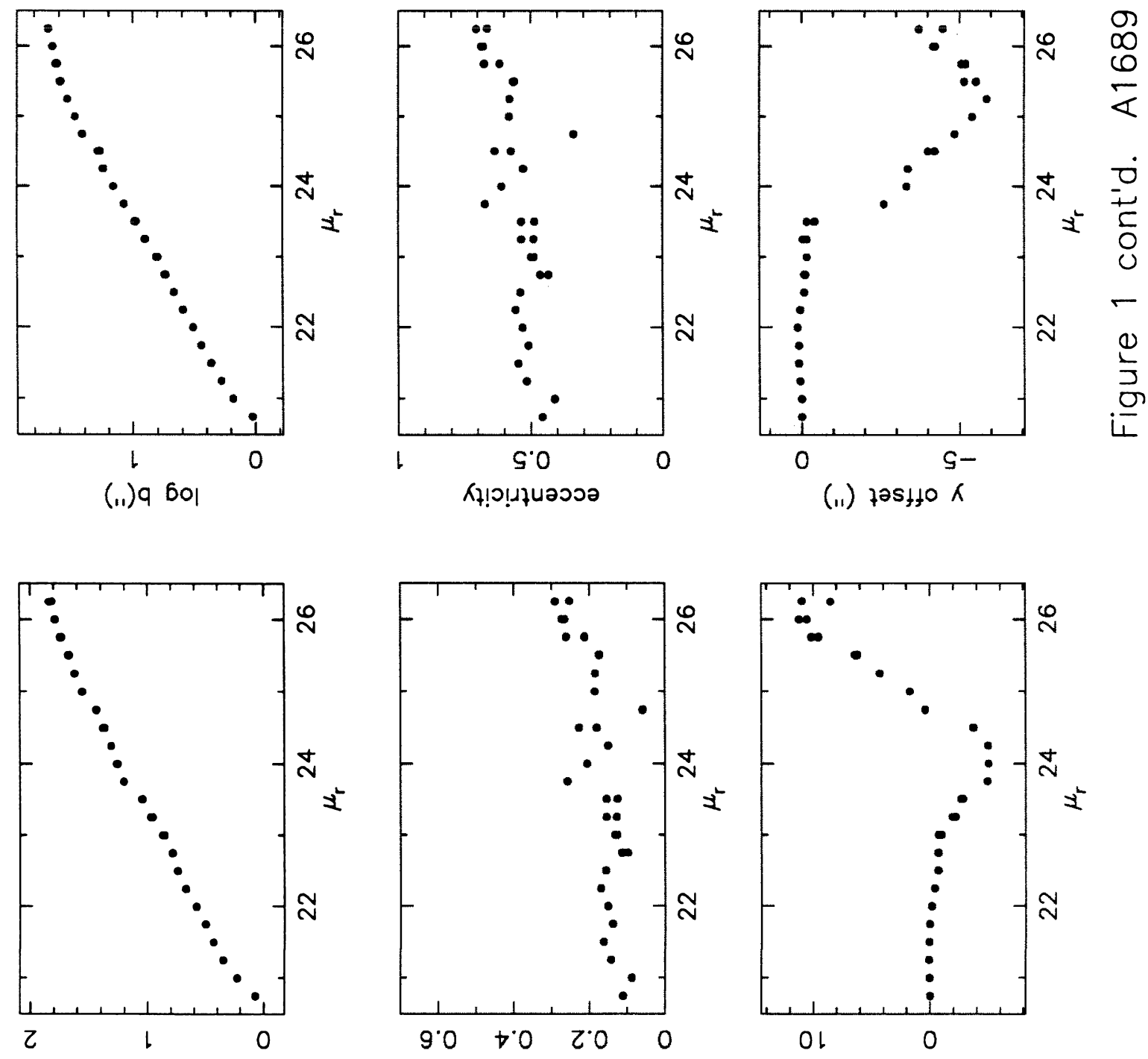

(.1) $\mathrm{D}$ 6ol
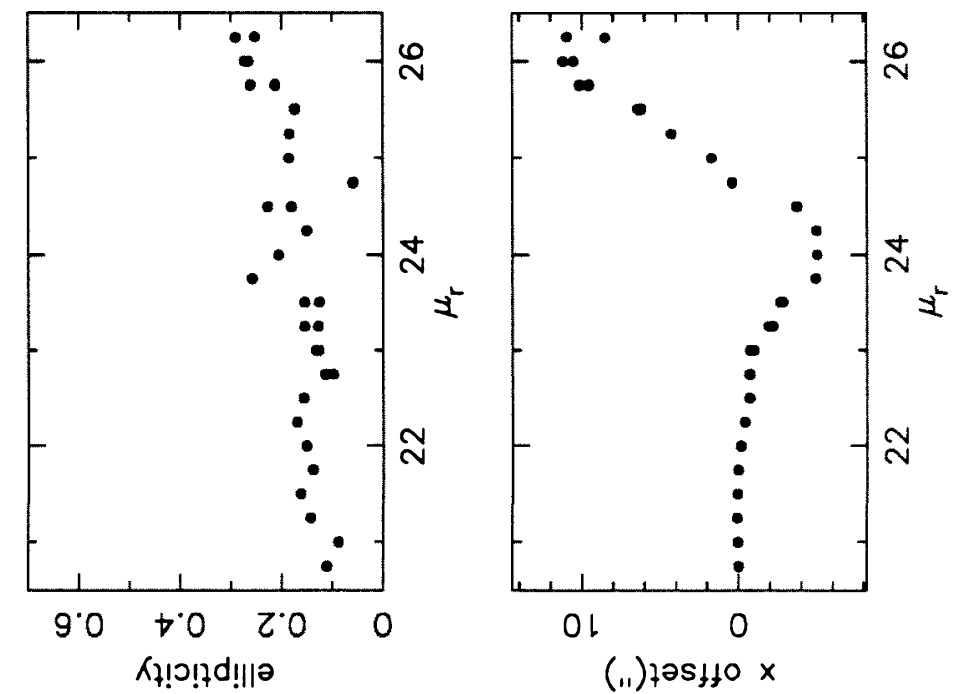


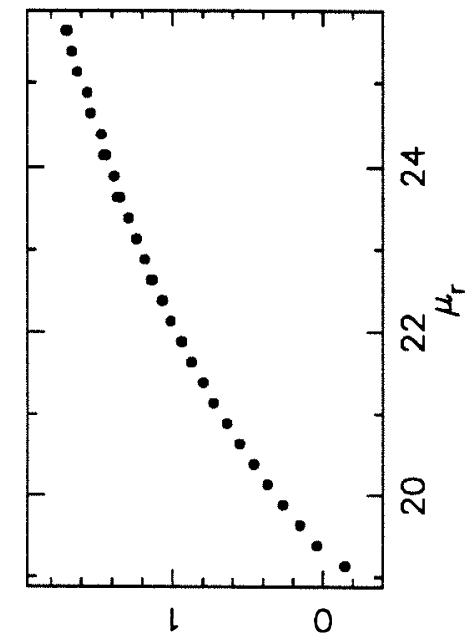

(.1) sn!pos 60।

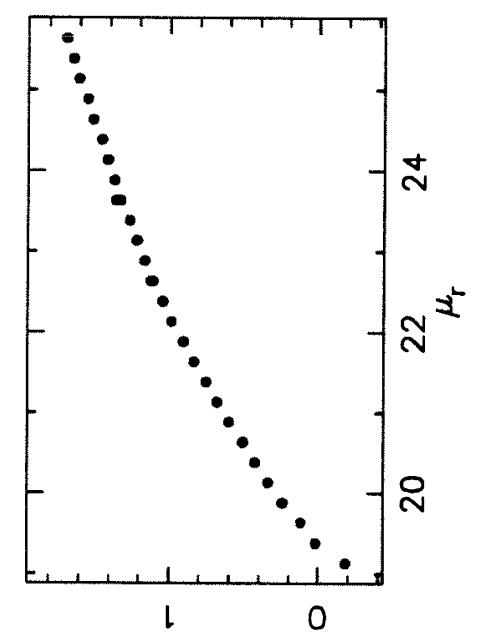

(11) 9601

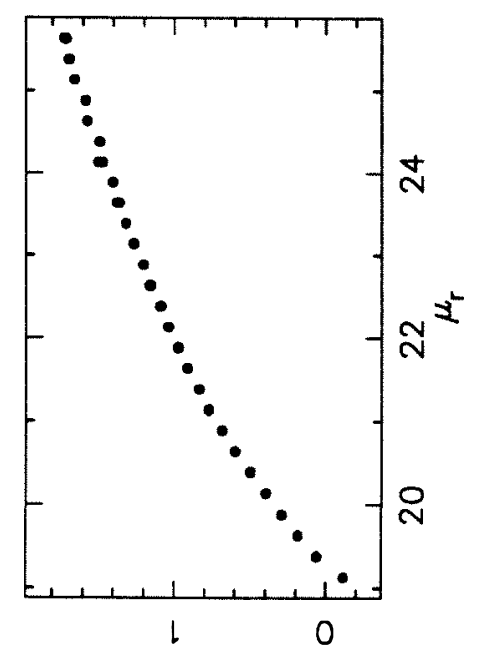

(.1) $0 \mathrm{bol}$
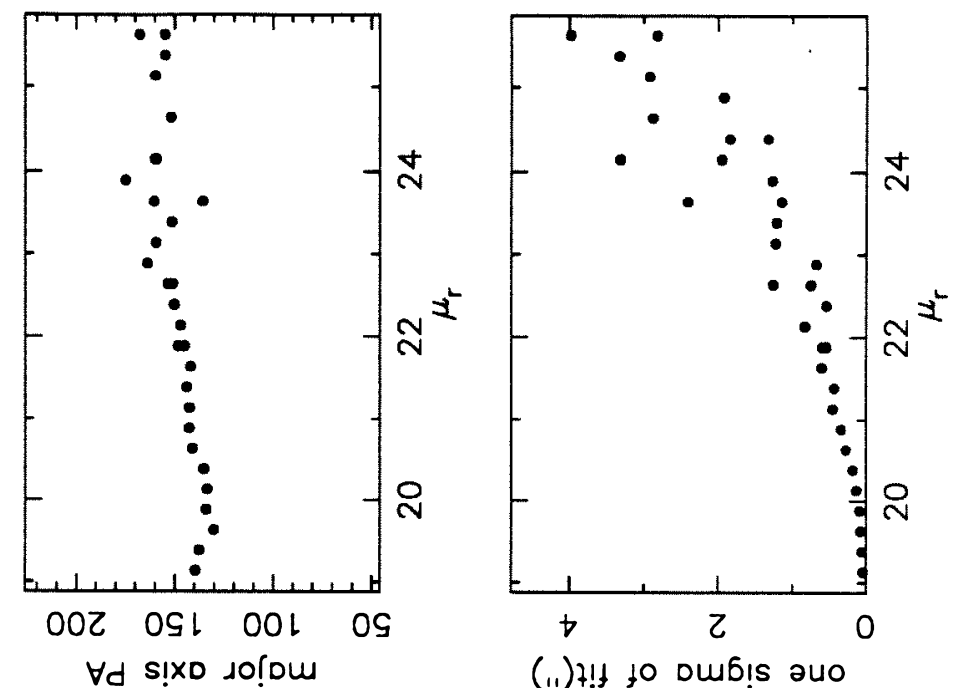

(i1) $+!+$ to Dub!s aนо

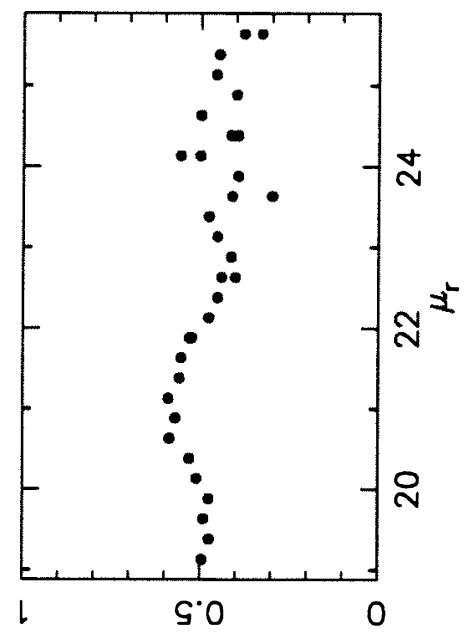

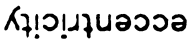

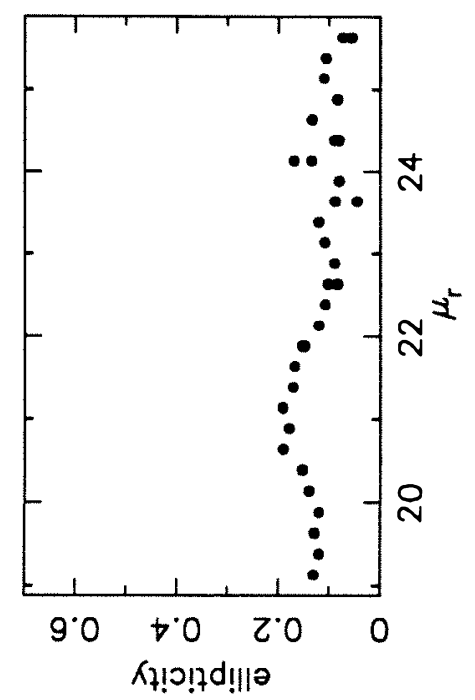

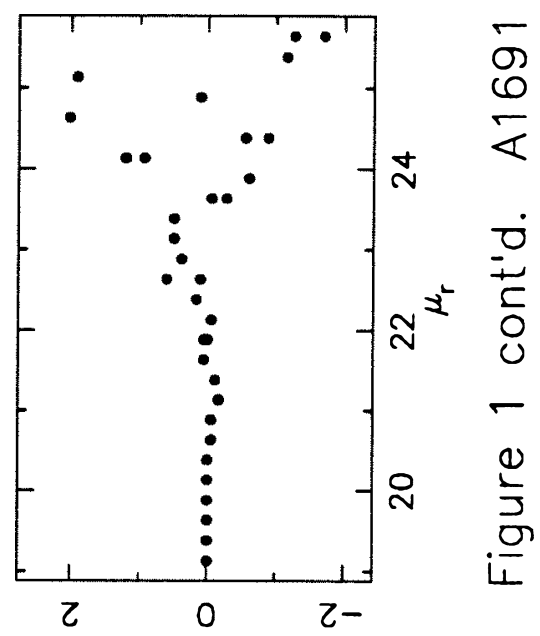

(i1) $725410 K$

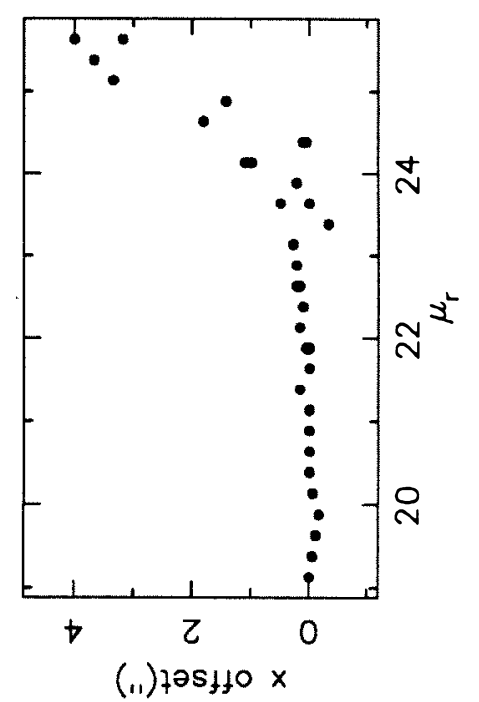




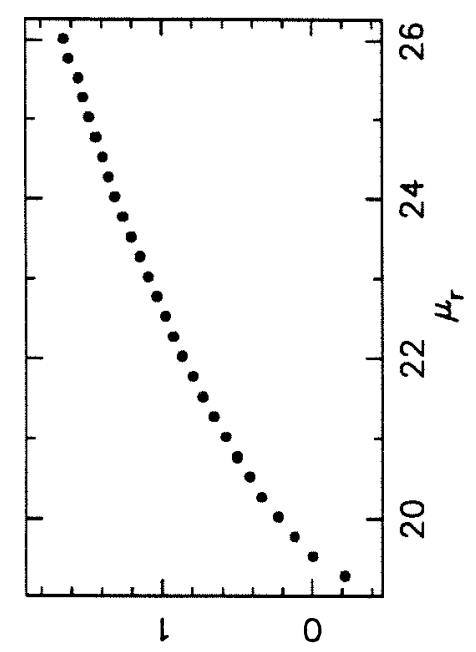

(.1) sn!pod 6ol
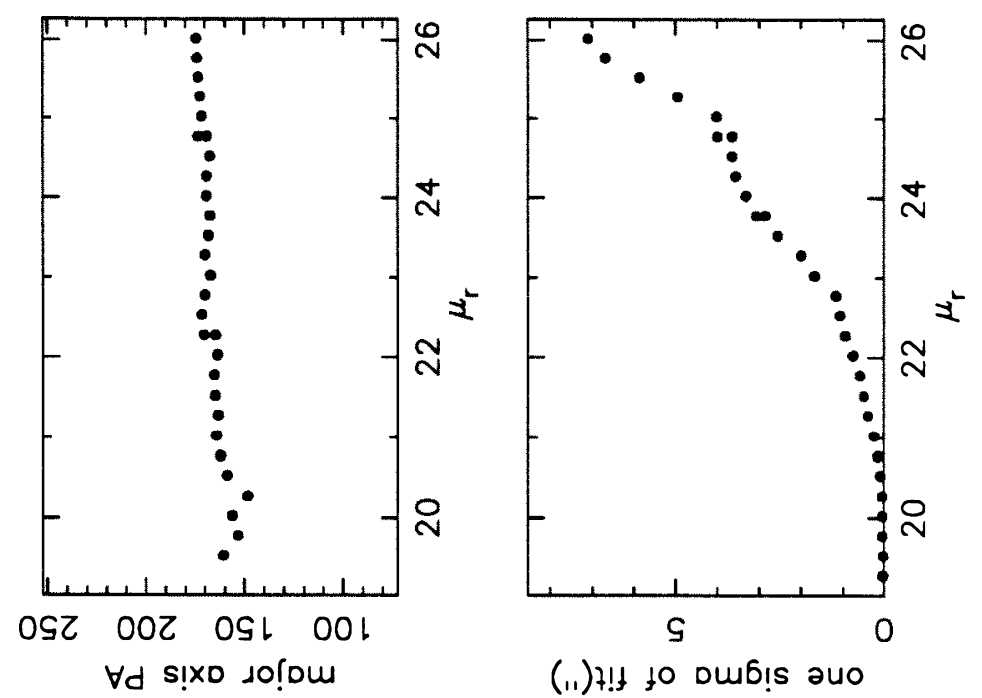

(11) $7 ! \downarrow$ to Dub!
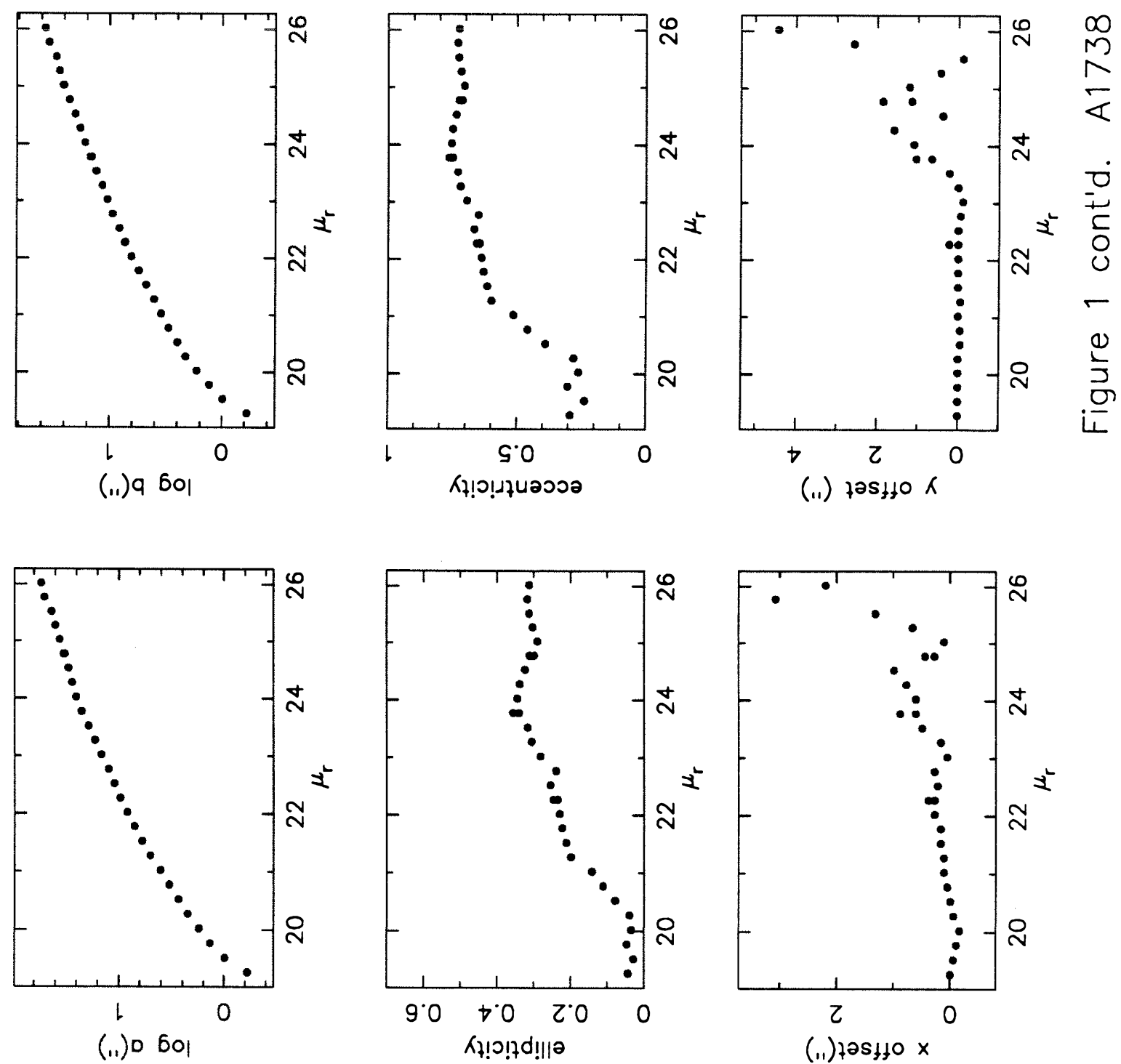


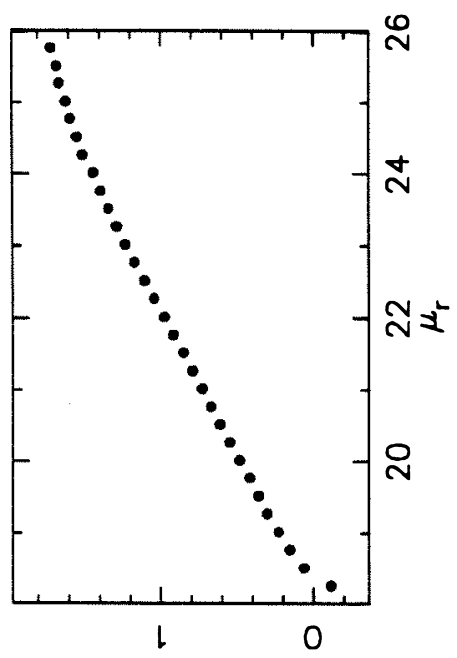

(.1)sn!pod 6ol

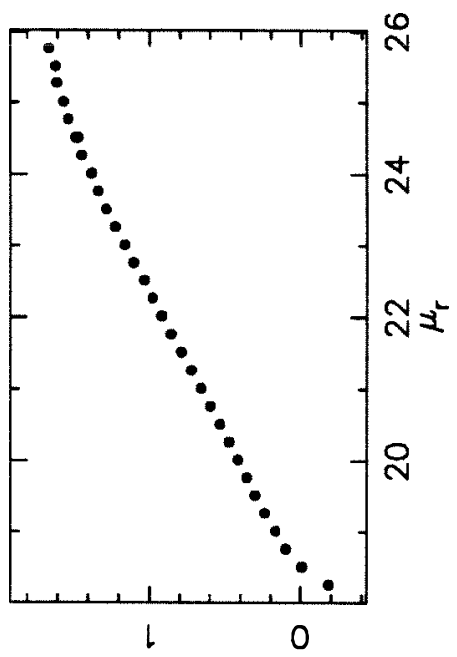

(.1)q 6 ol

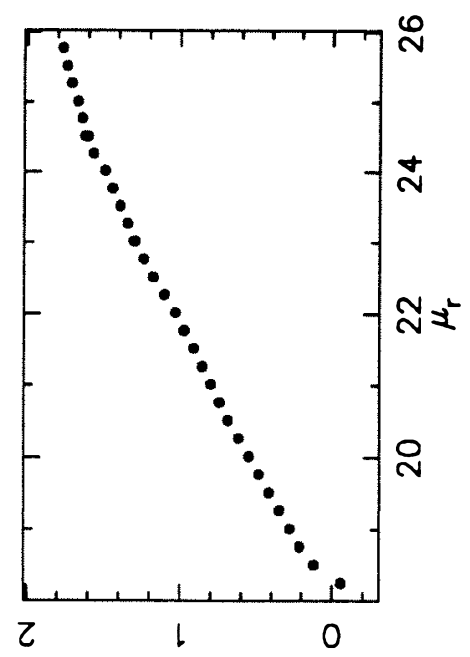

(..) 0 6ol

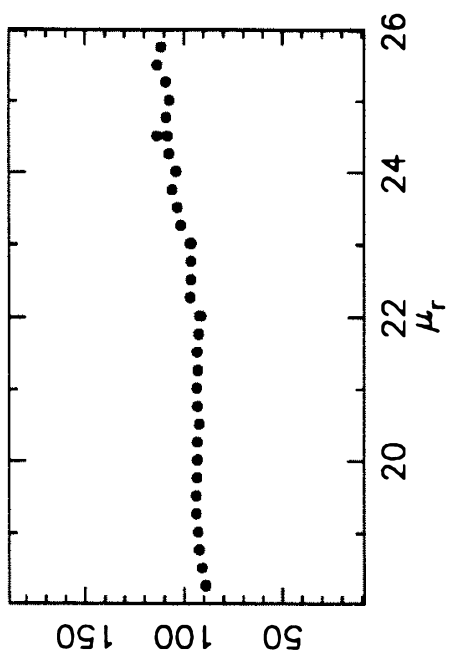

$\forall d$ s!xo solom

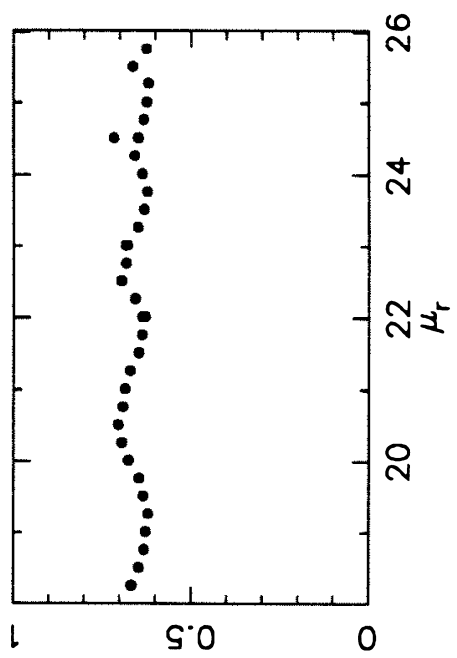

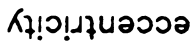
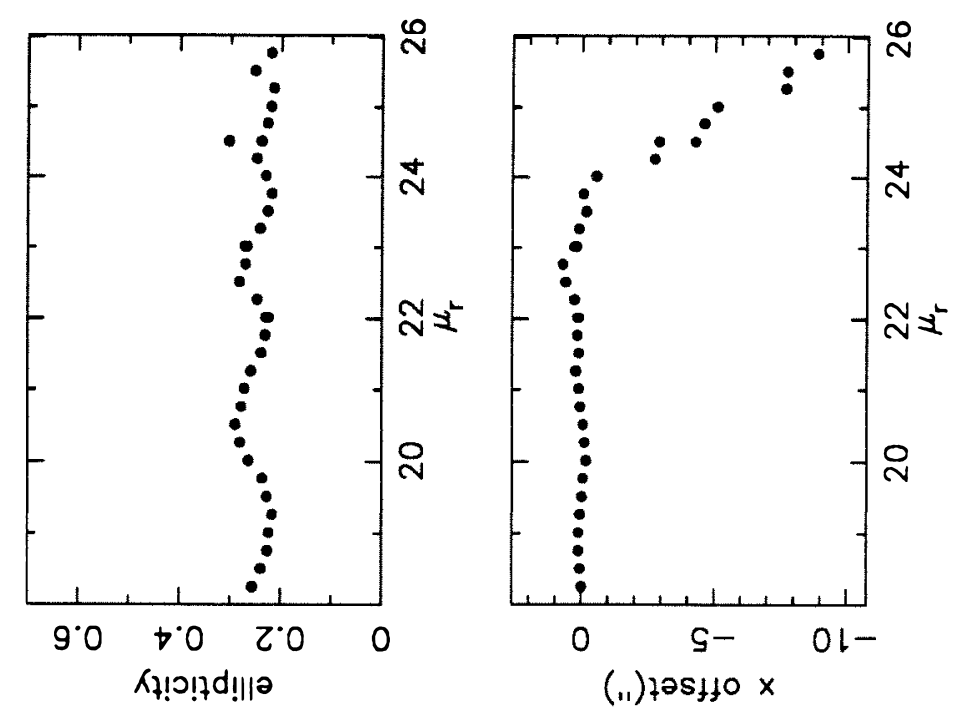


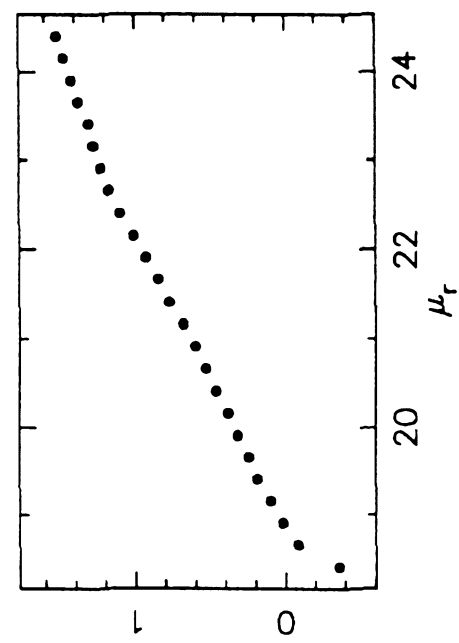

(.1) sn!pod 6ol

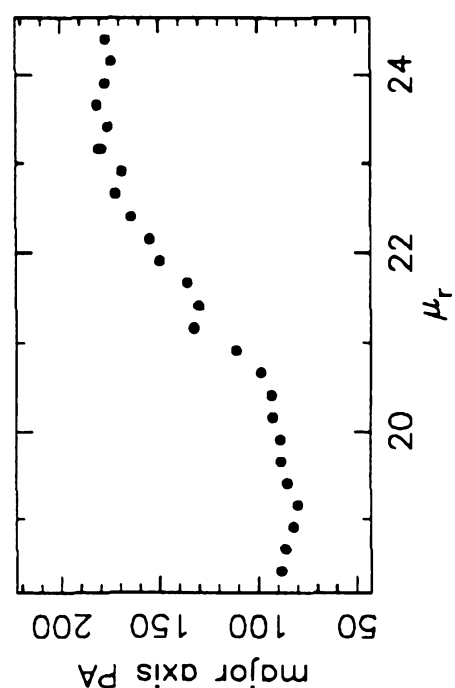

$\forall d$ s!xo solom

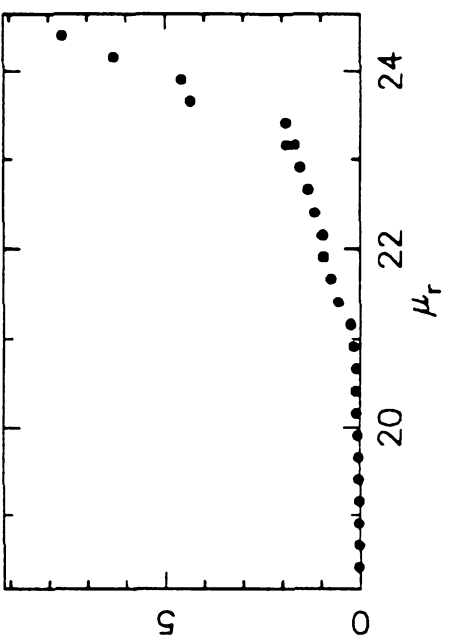

(11) $+1 !$ to oub!s aน०
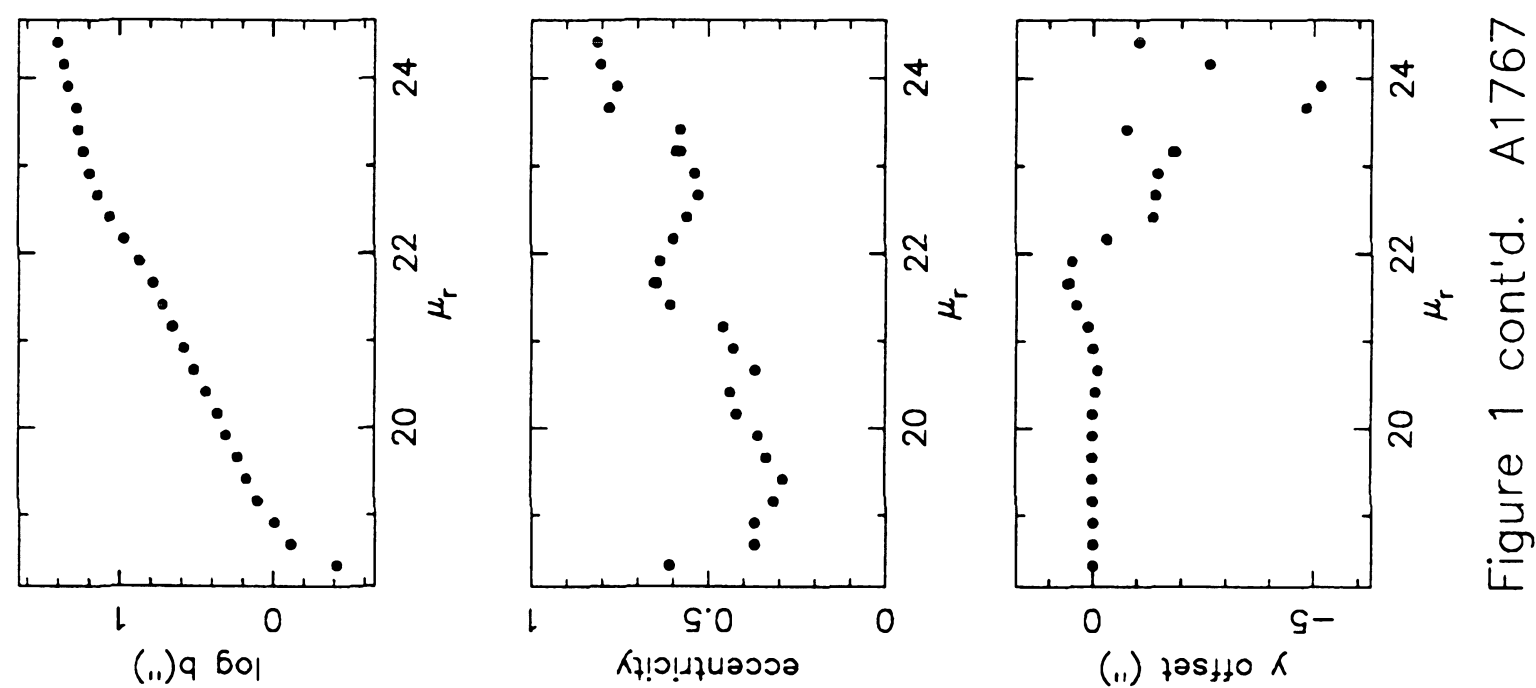

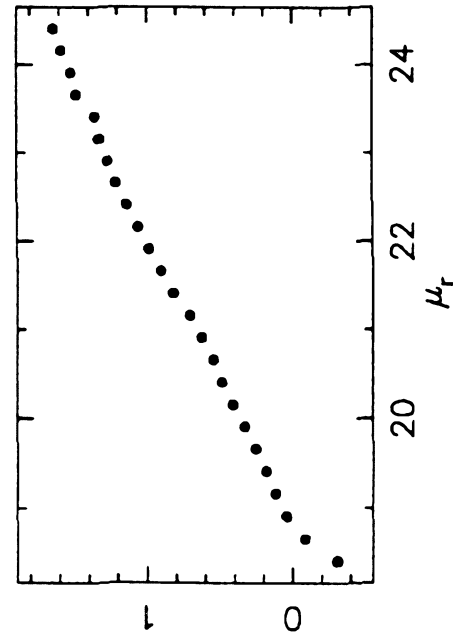

(..) $\mathrm{O}$ 6ol

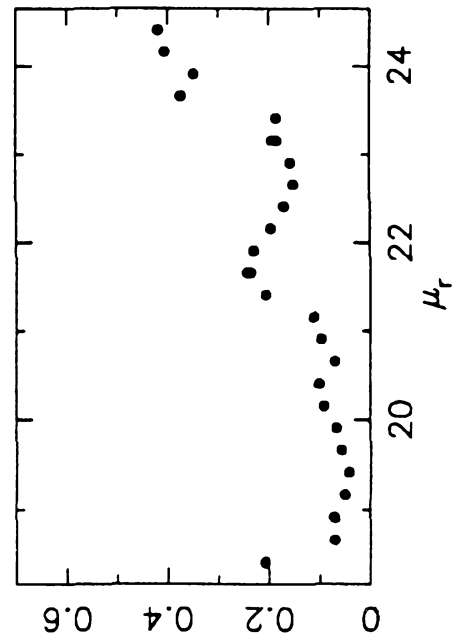

रł! !! d!||

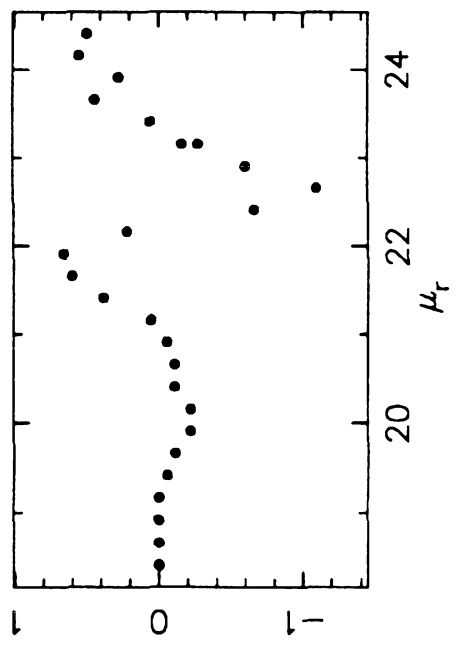

(11) $\operatorname{tas} 410 \times$ 

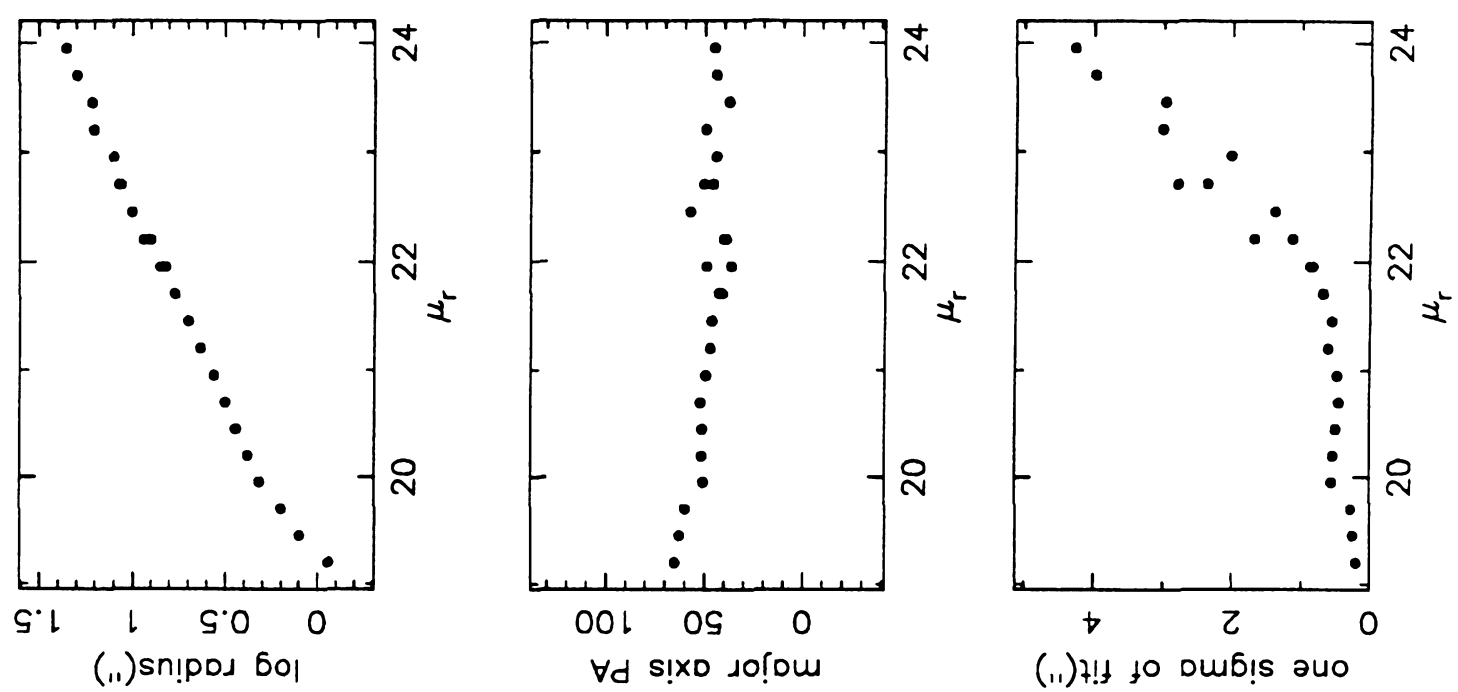

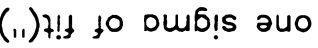
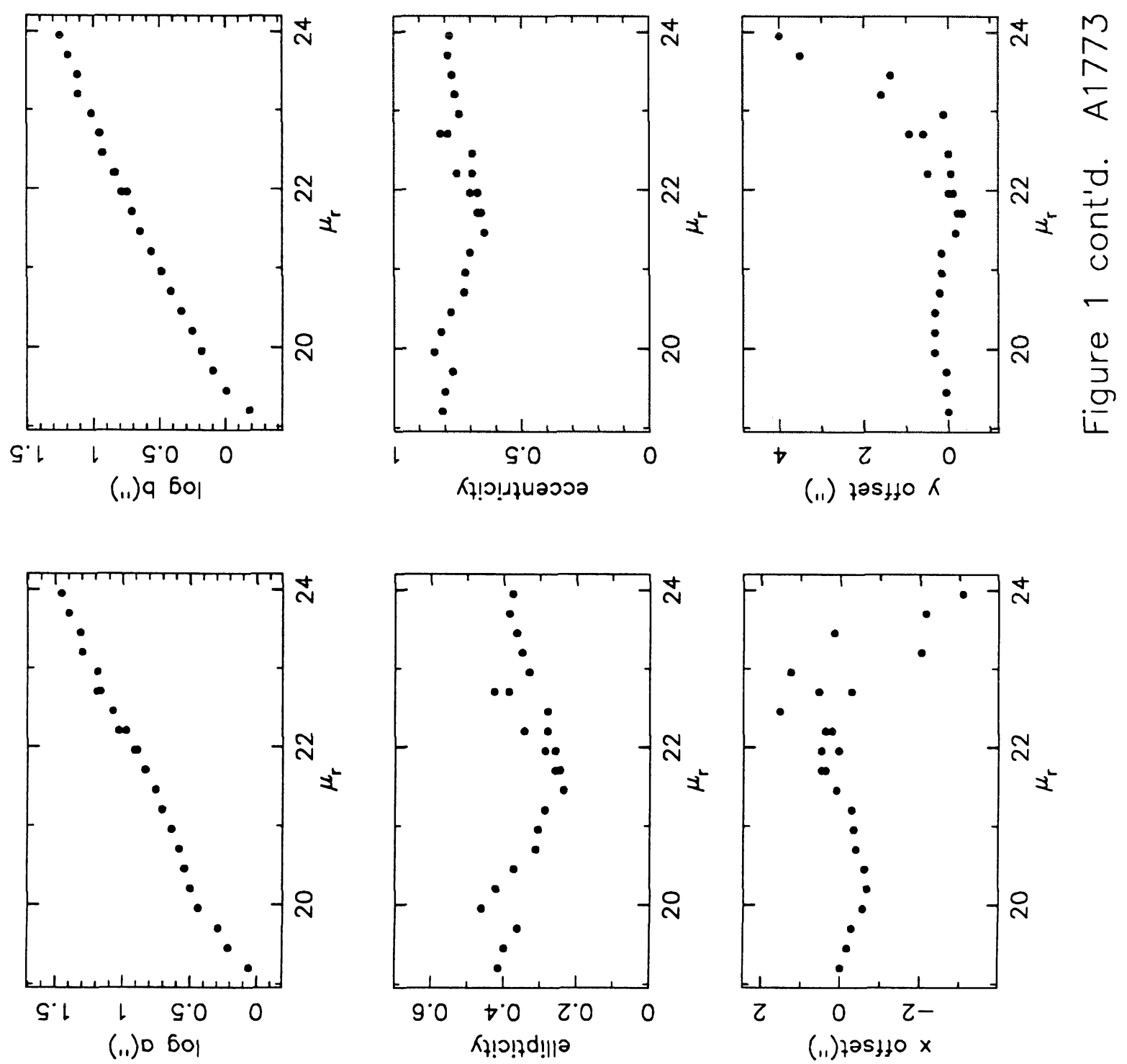


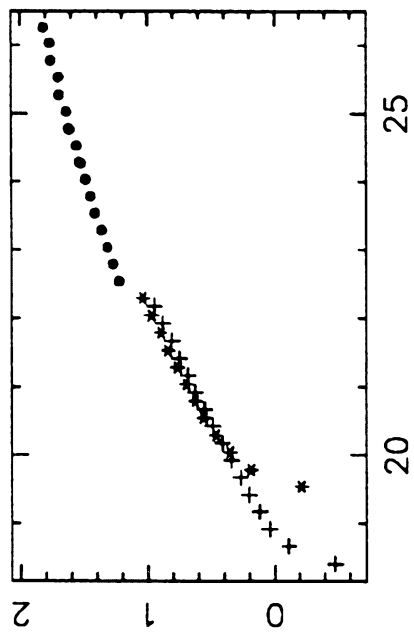

(.1) sn!pod 6ol

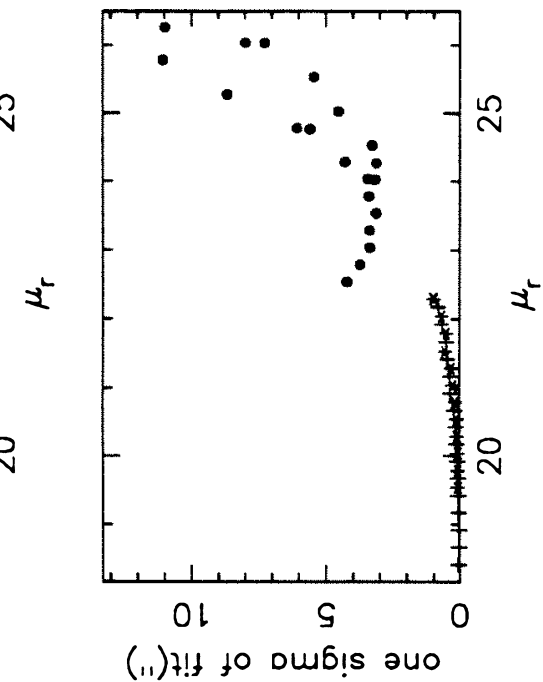

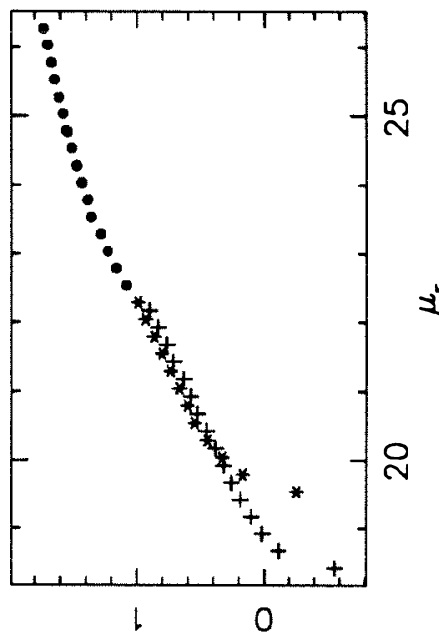

(.1) $a$ bol
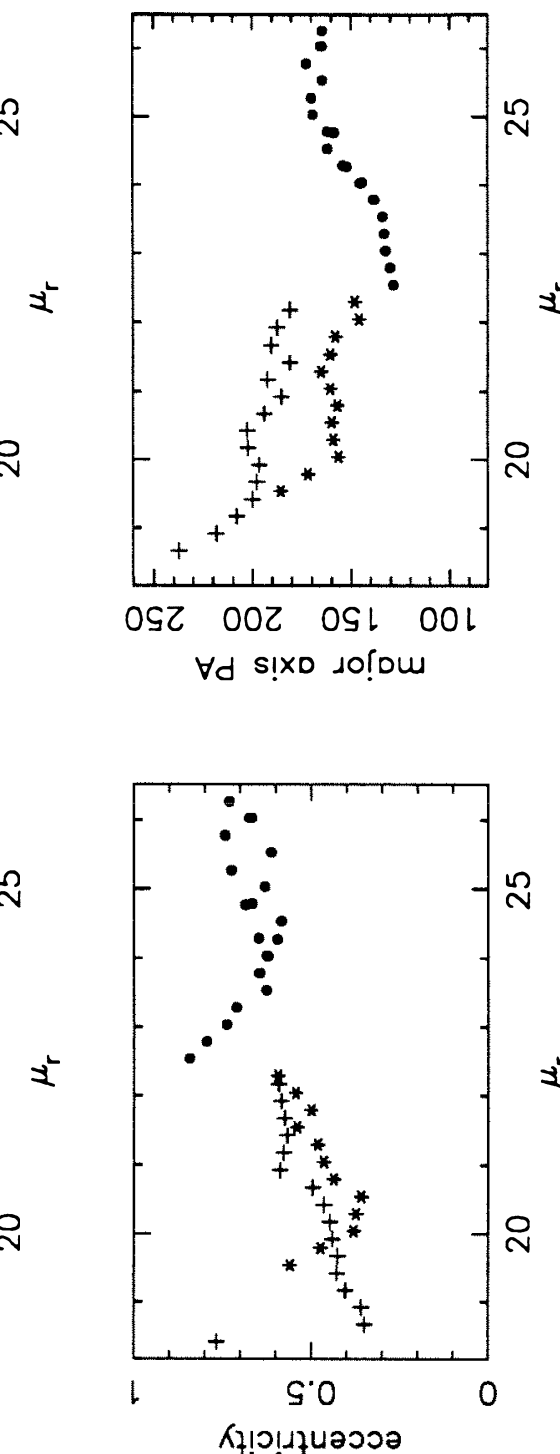

K\{!ว!นในวัวว
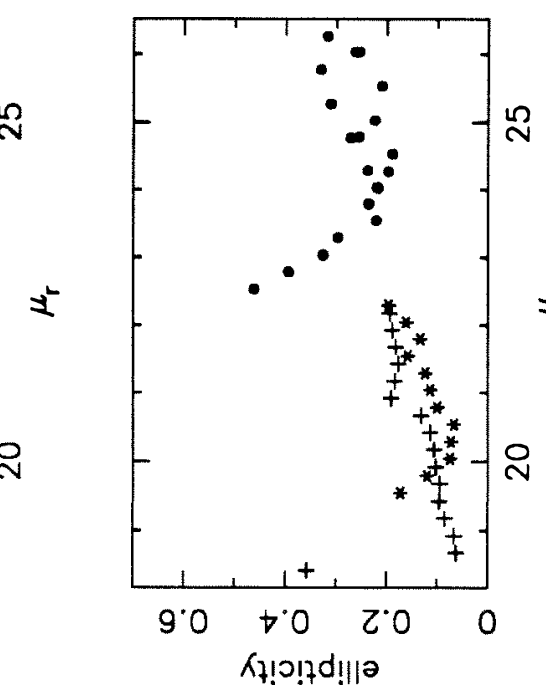

K₹!?!?d!||
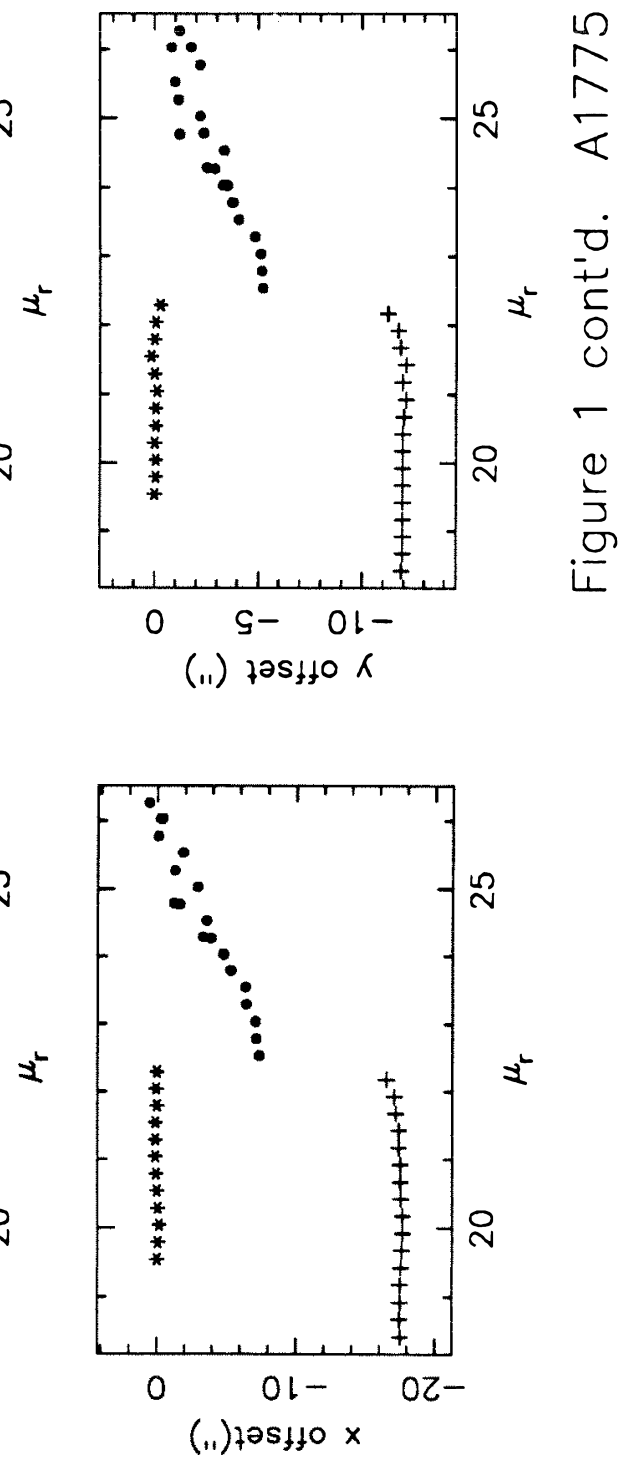

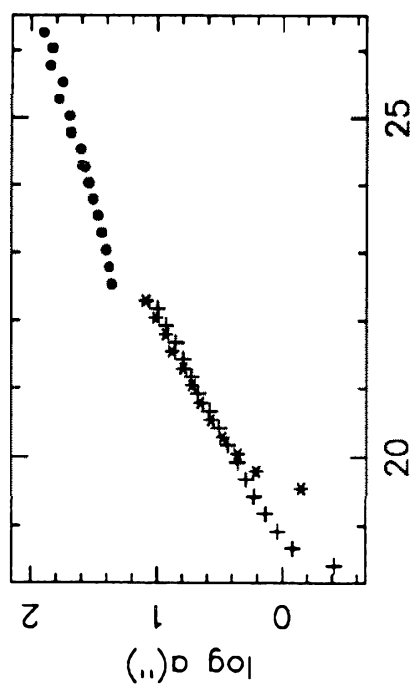




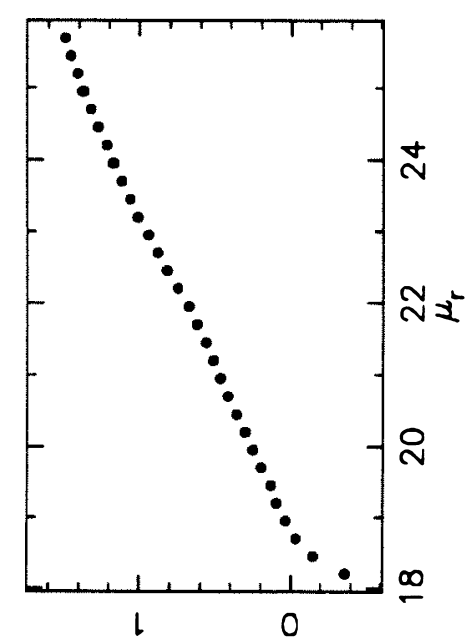

(.1) sn!pos 601

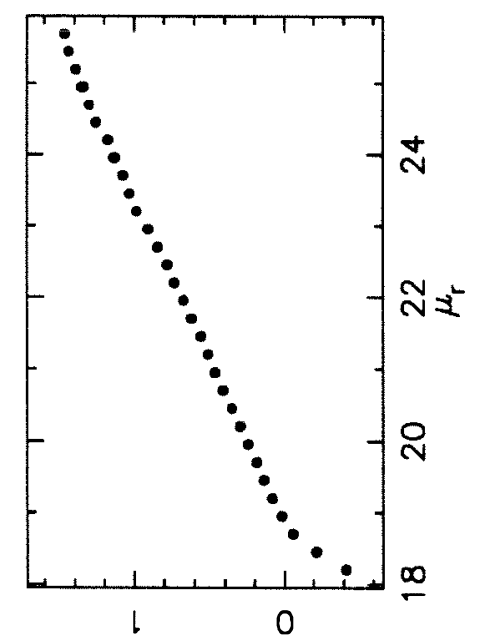

(.1) 9501

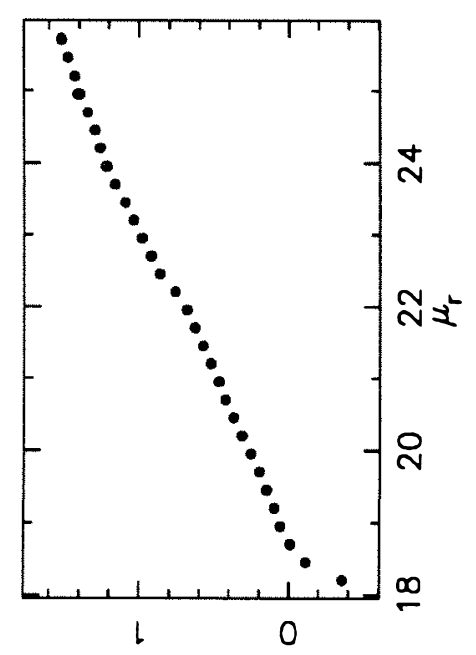

(.1) 0 601
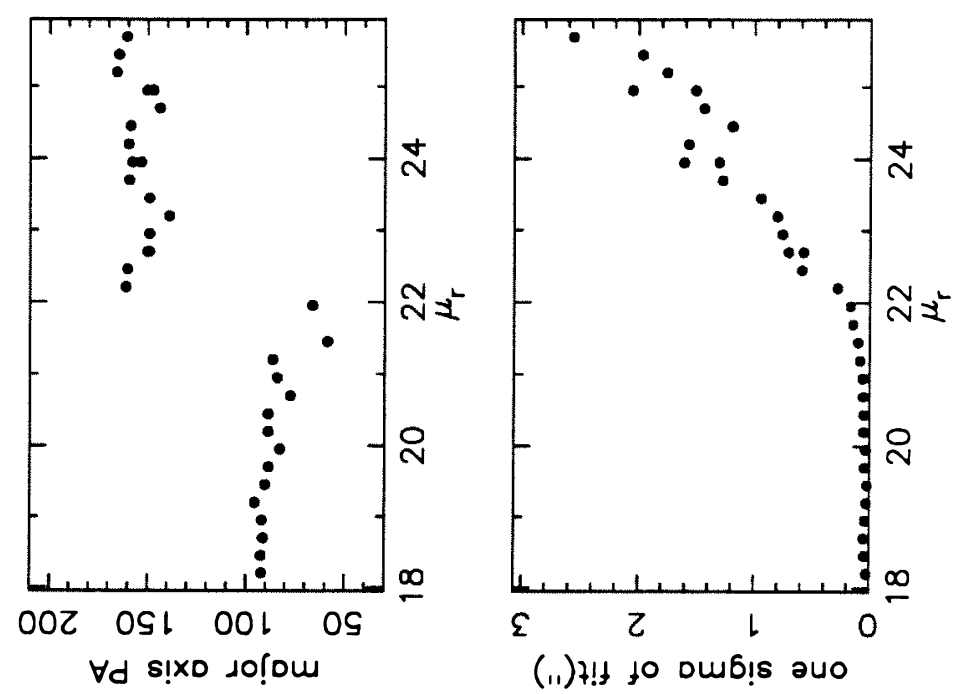

(.1) $7 !+$ to Dub!s au०

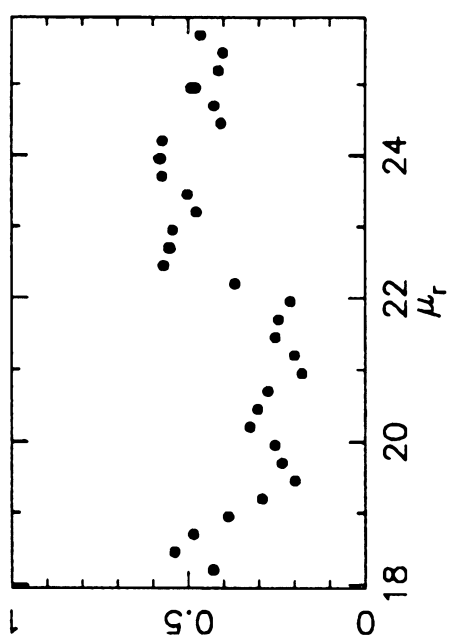

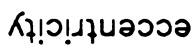

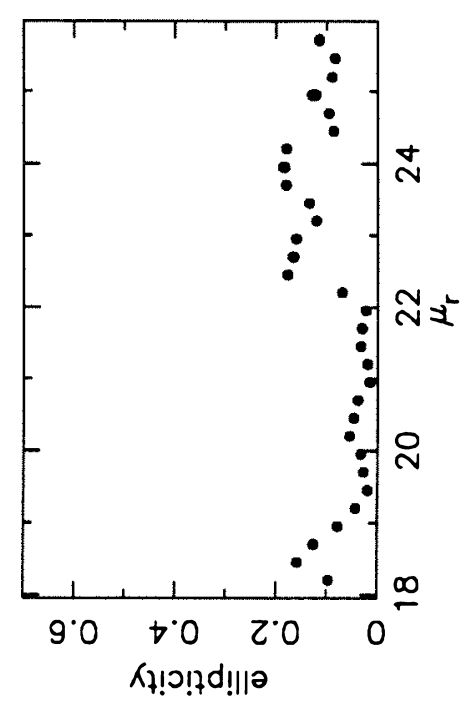

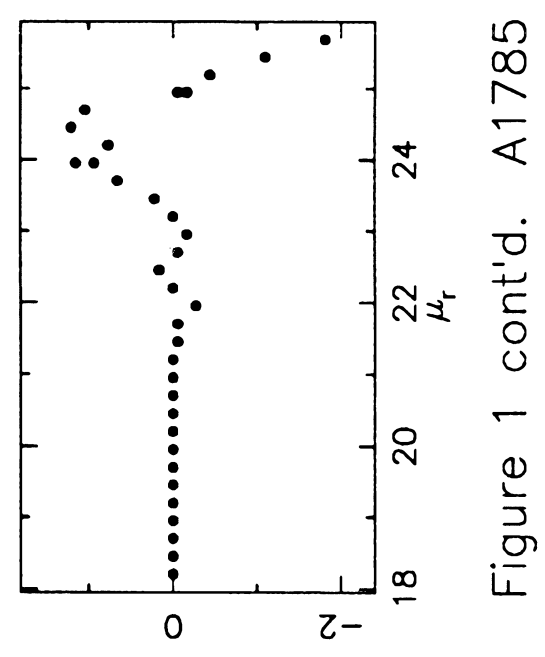

(ii) $725+10 \mathrm{~K}$

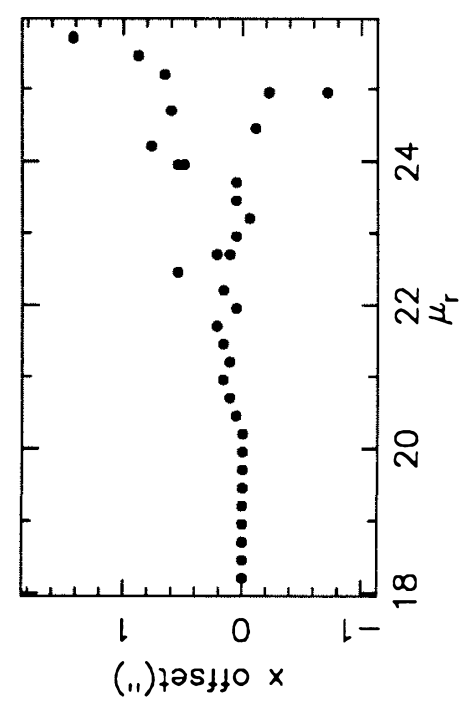




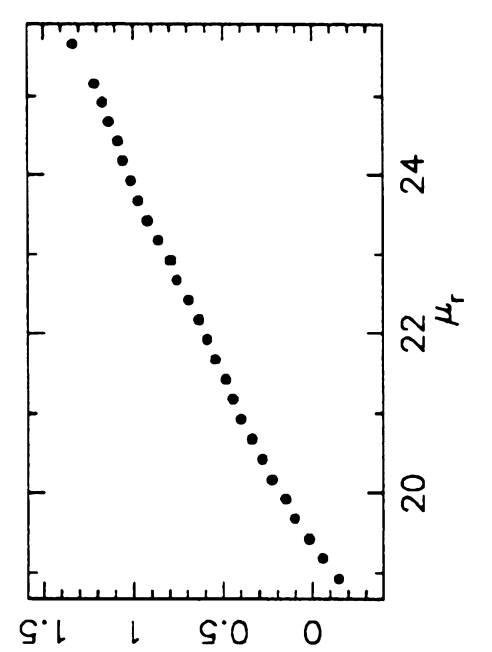

(.1) sn!pos 60।
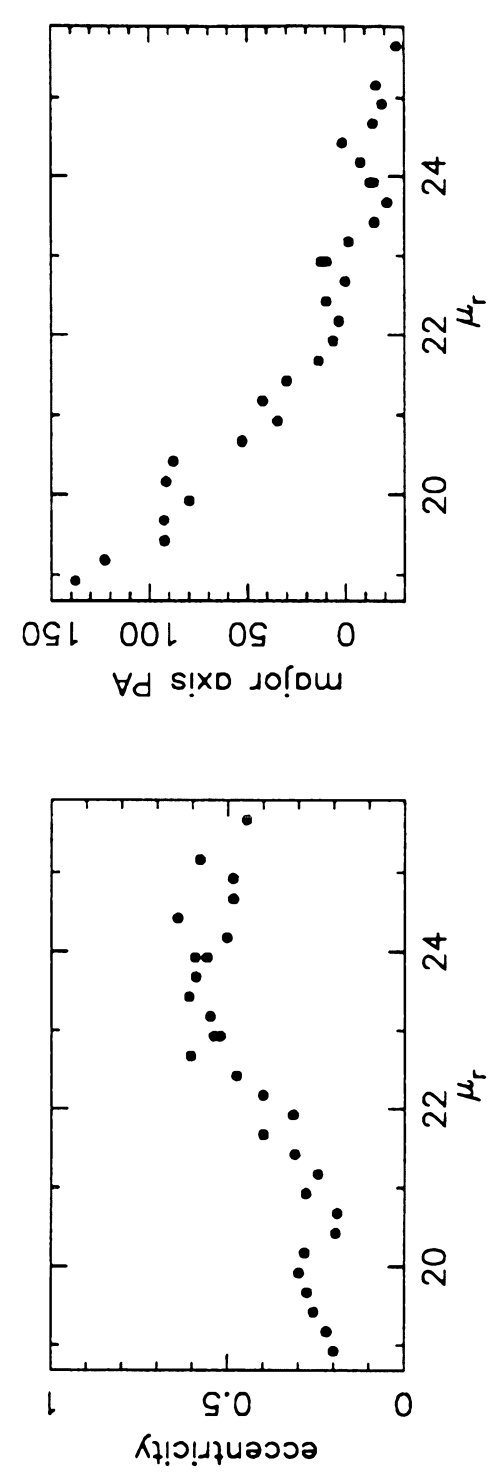

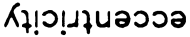

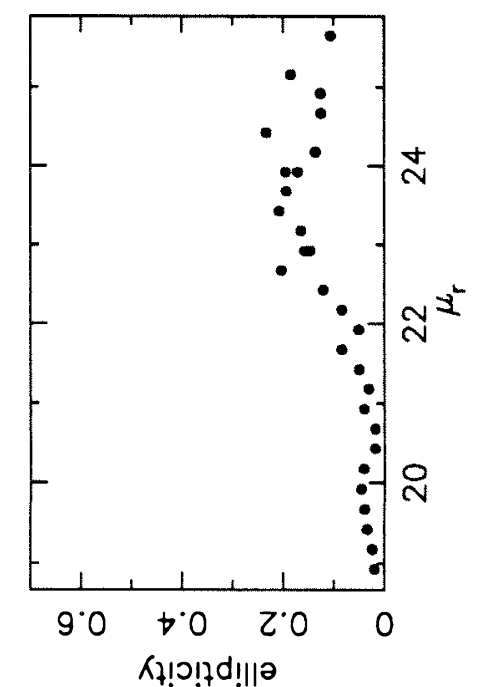

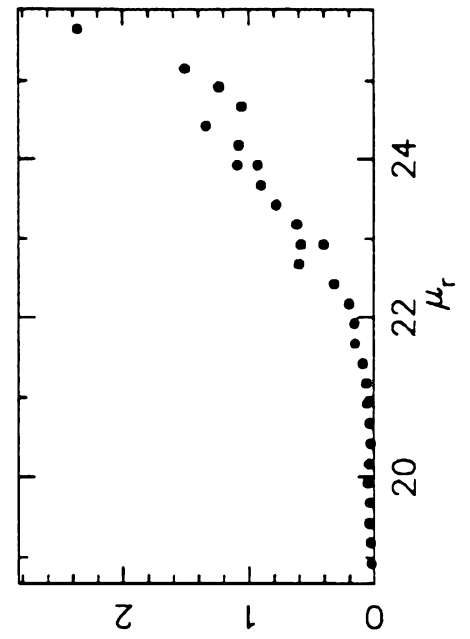

(11) 4 ! to Dub!s 2u०

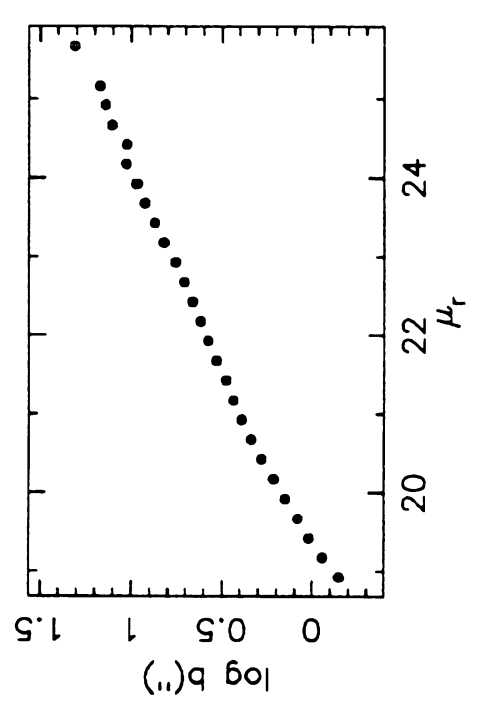

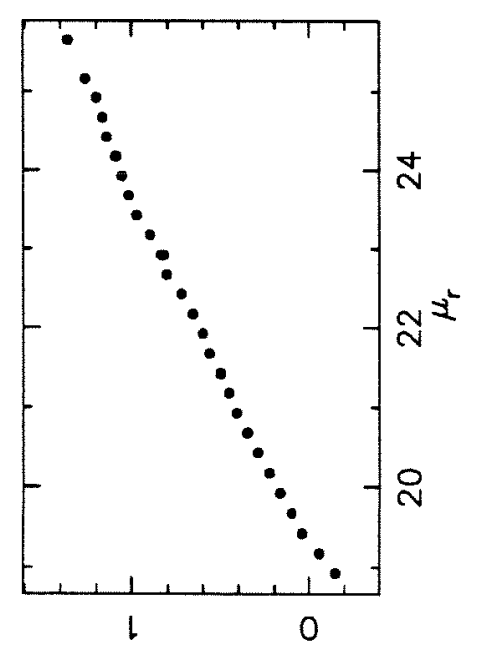

(1.) $0 \mathrm{60}$

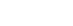
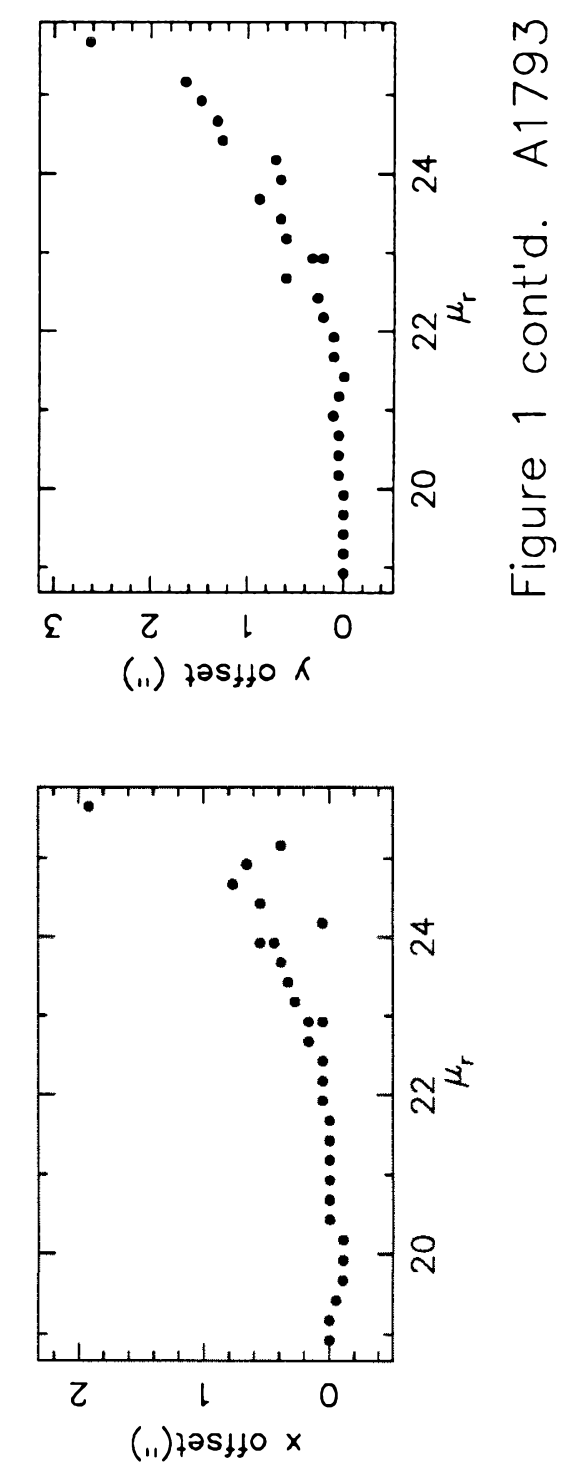


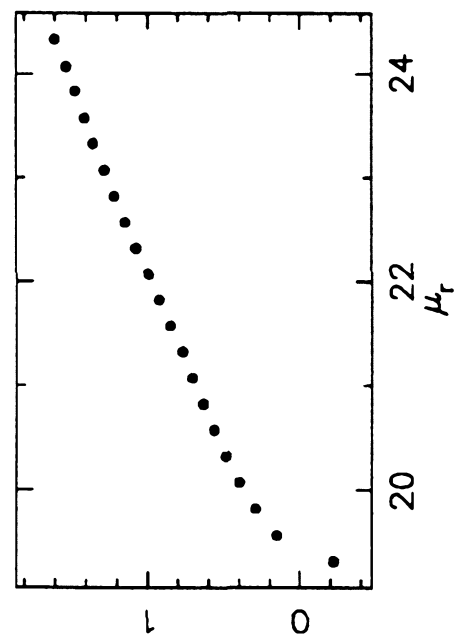

(.1) sn!pod 6ol
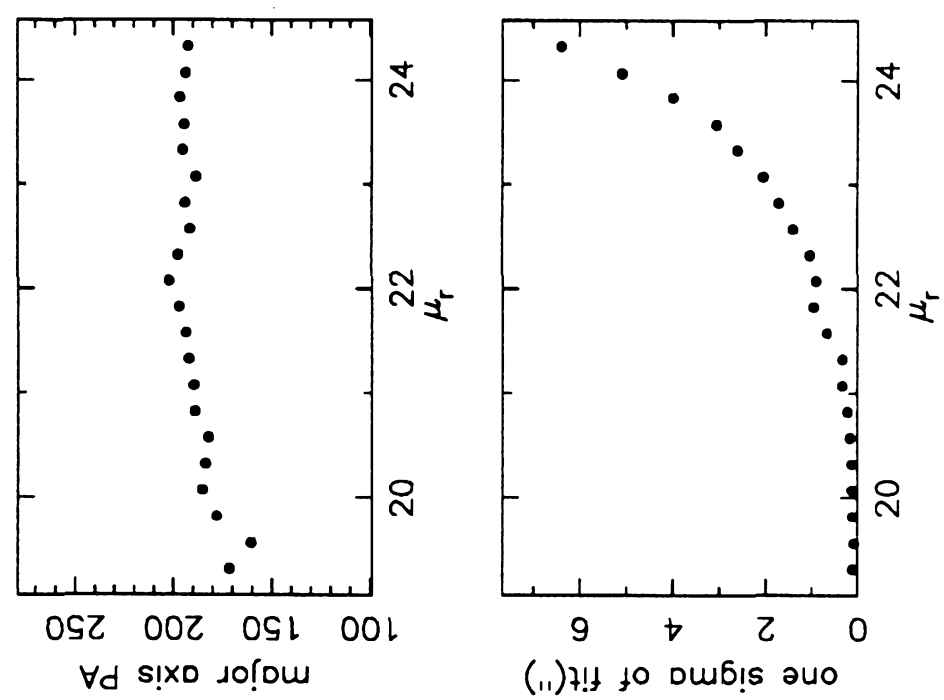

(11) $7 !+10$ Dub!! aน०

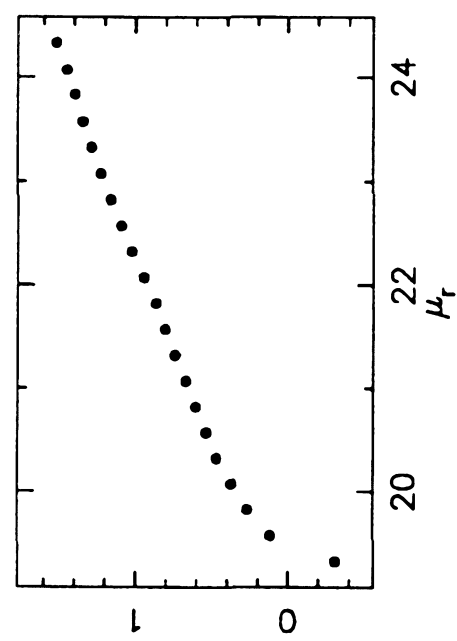

(.1) 9 60이

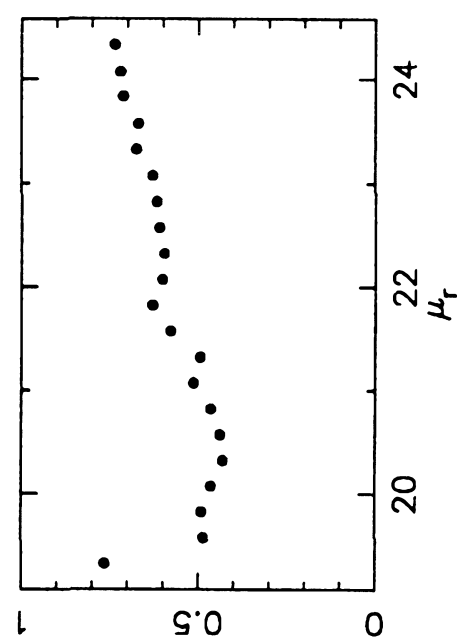

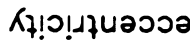

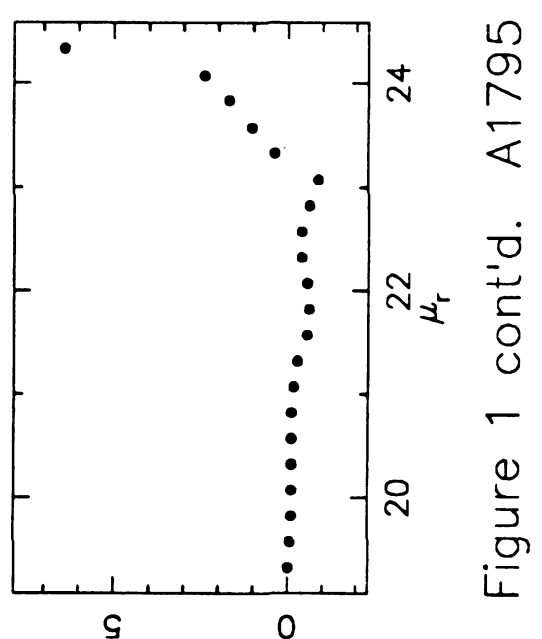

(i.) $725150 \mathrm{~K}$

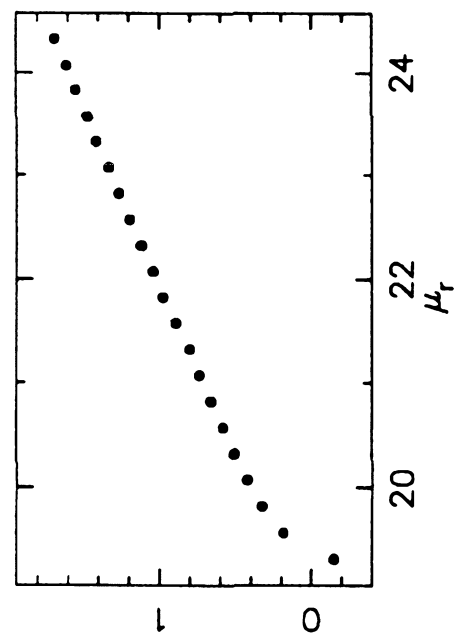

(11) 0 6ol
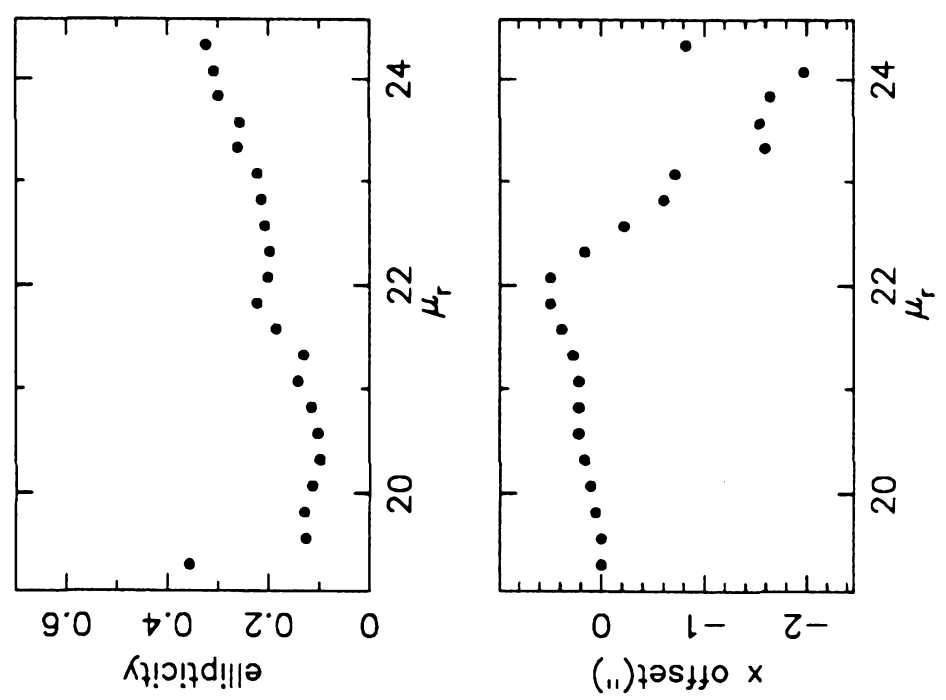

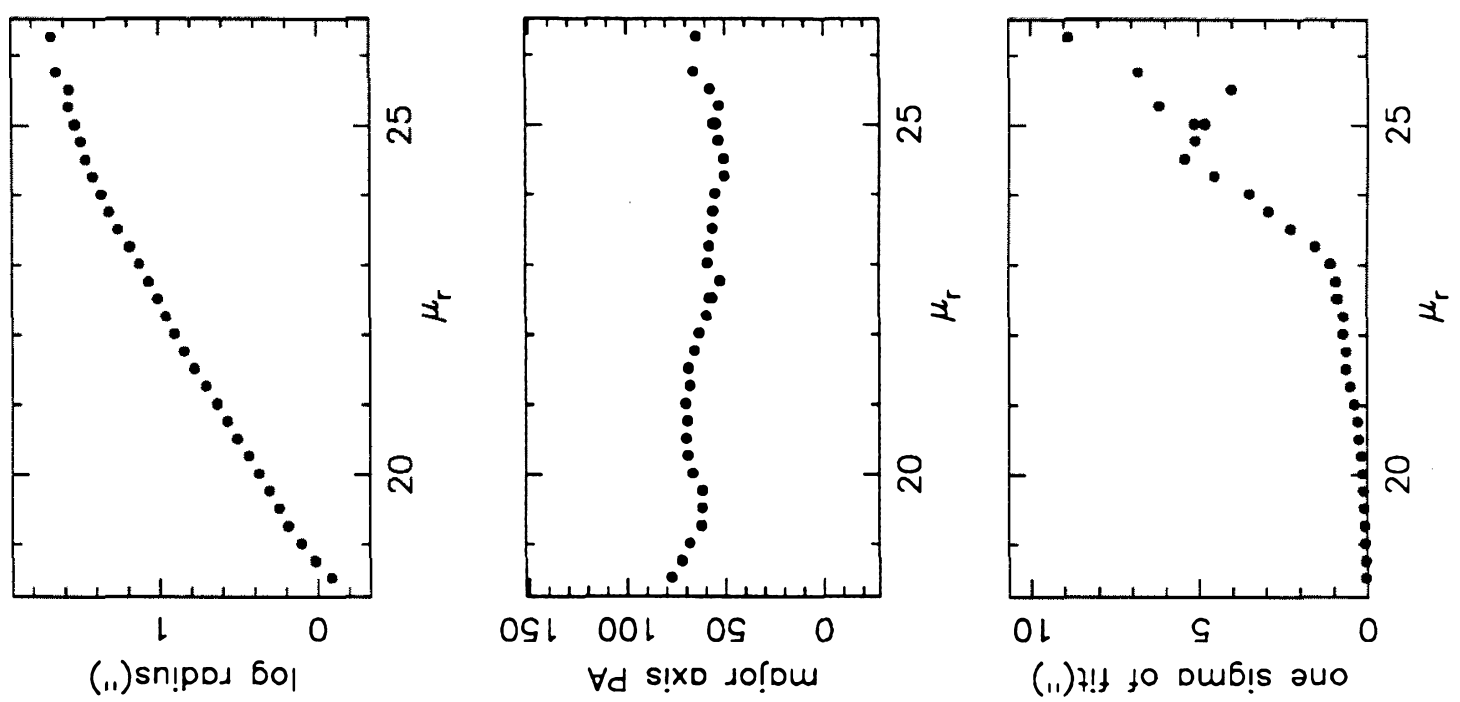

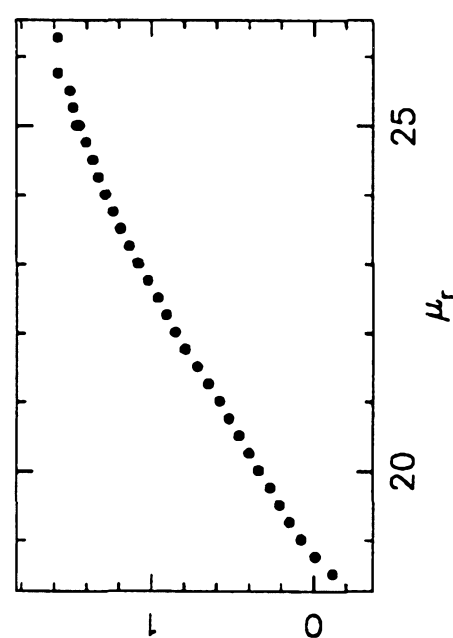

(11) 9601

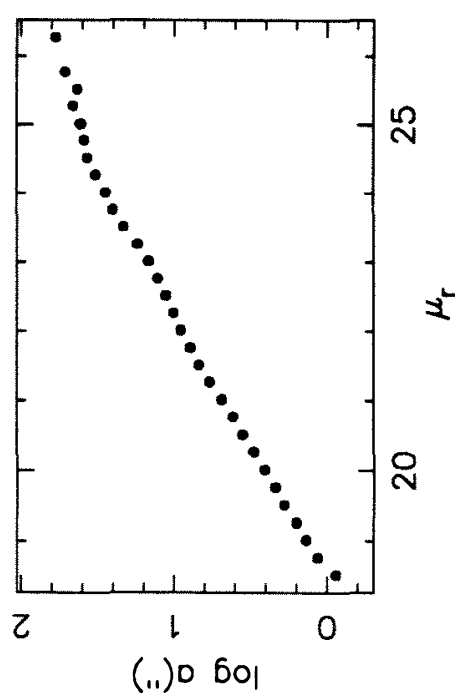

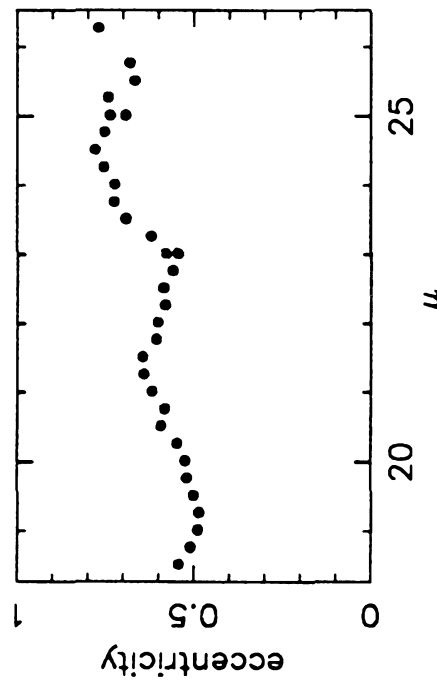

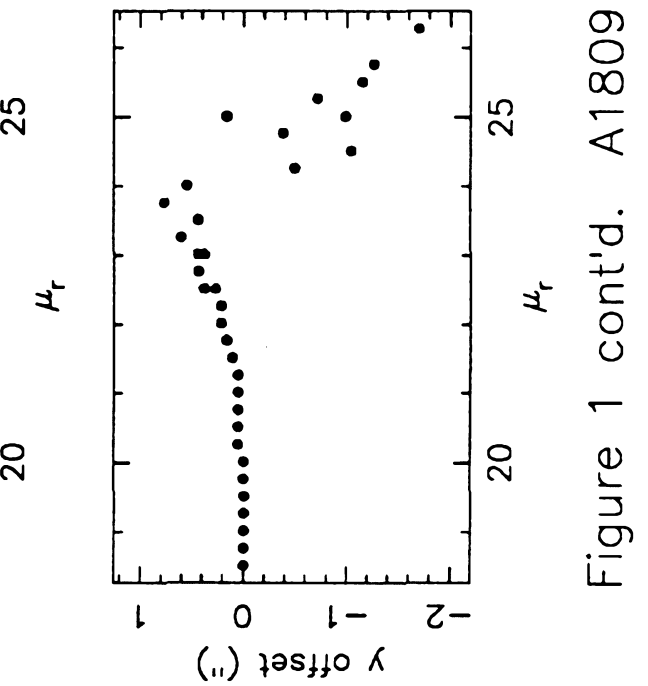

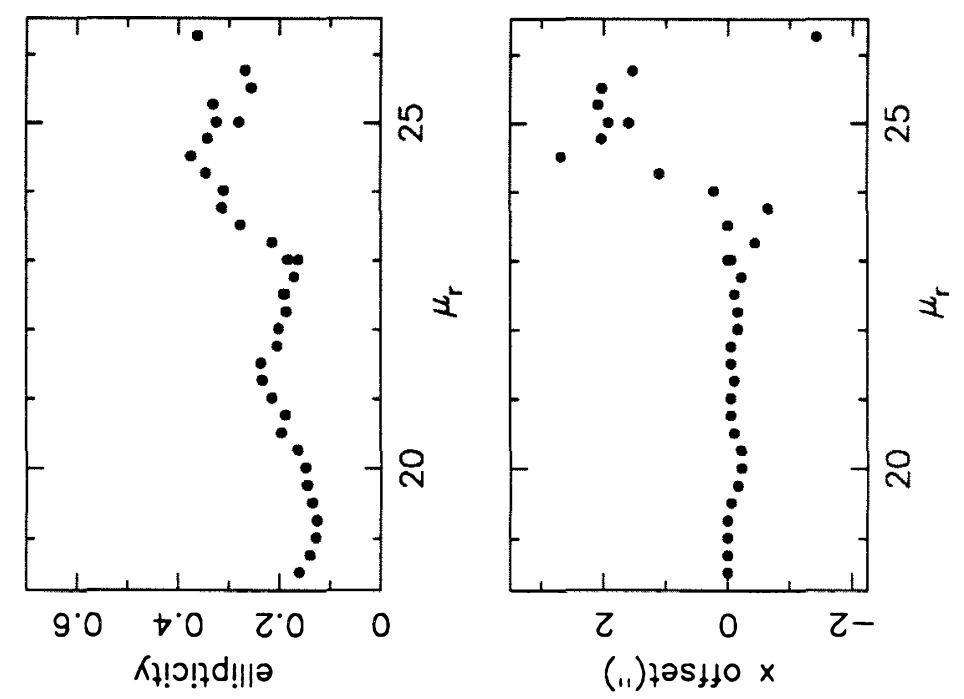




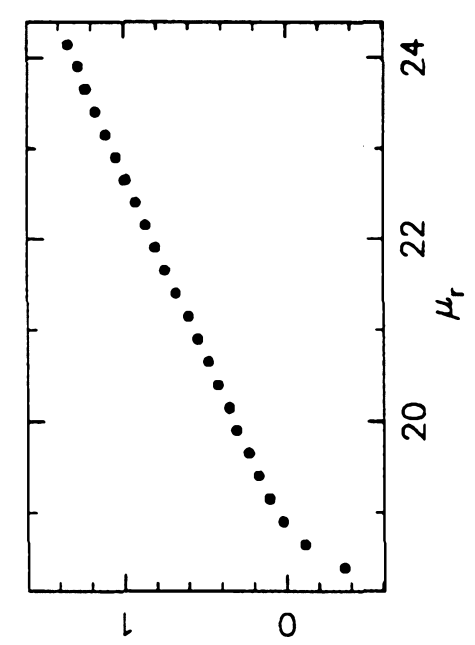

(.1) sn!pod 6ol

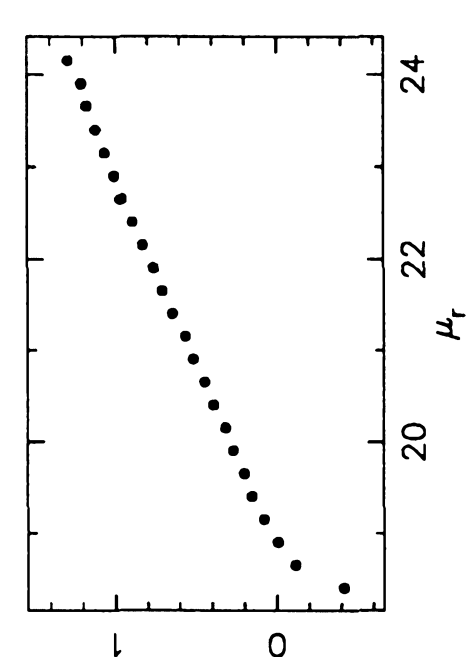

(.1) 9 6ol

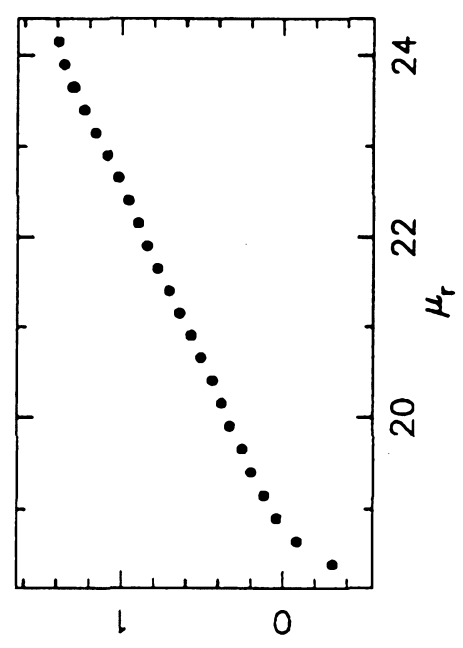

(..) 0 601

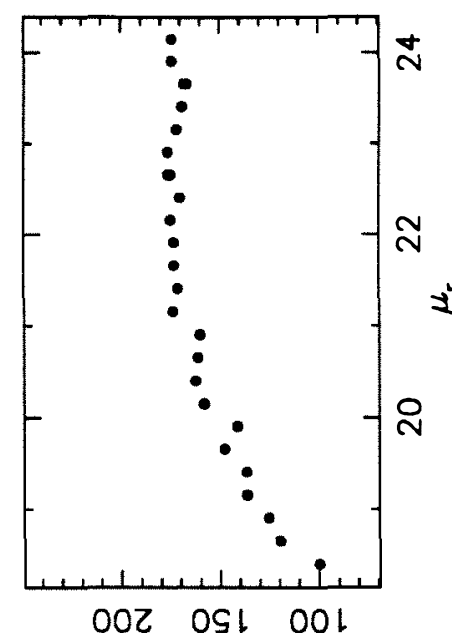

$\forall d$ s!xo so!́om

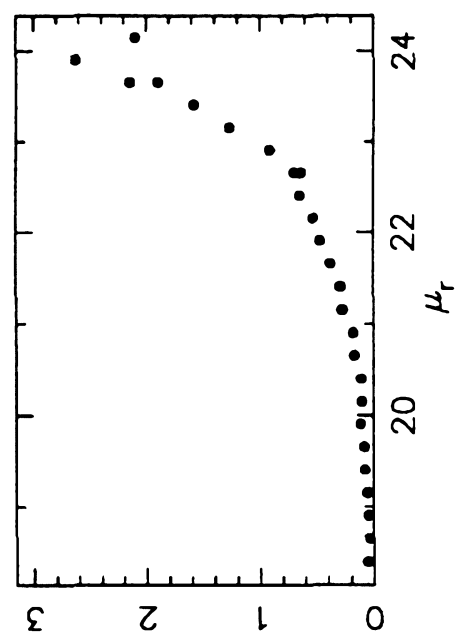

(.1) $7 ! !$ to Dub! ano
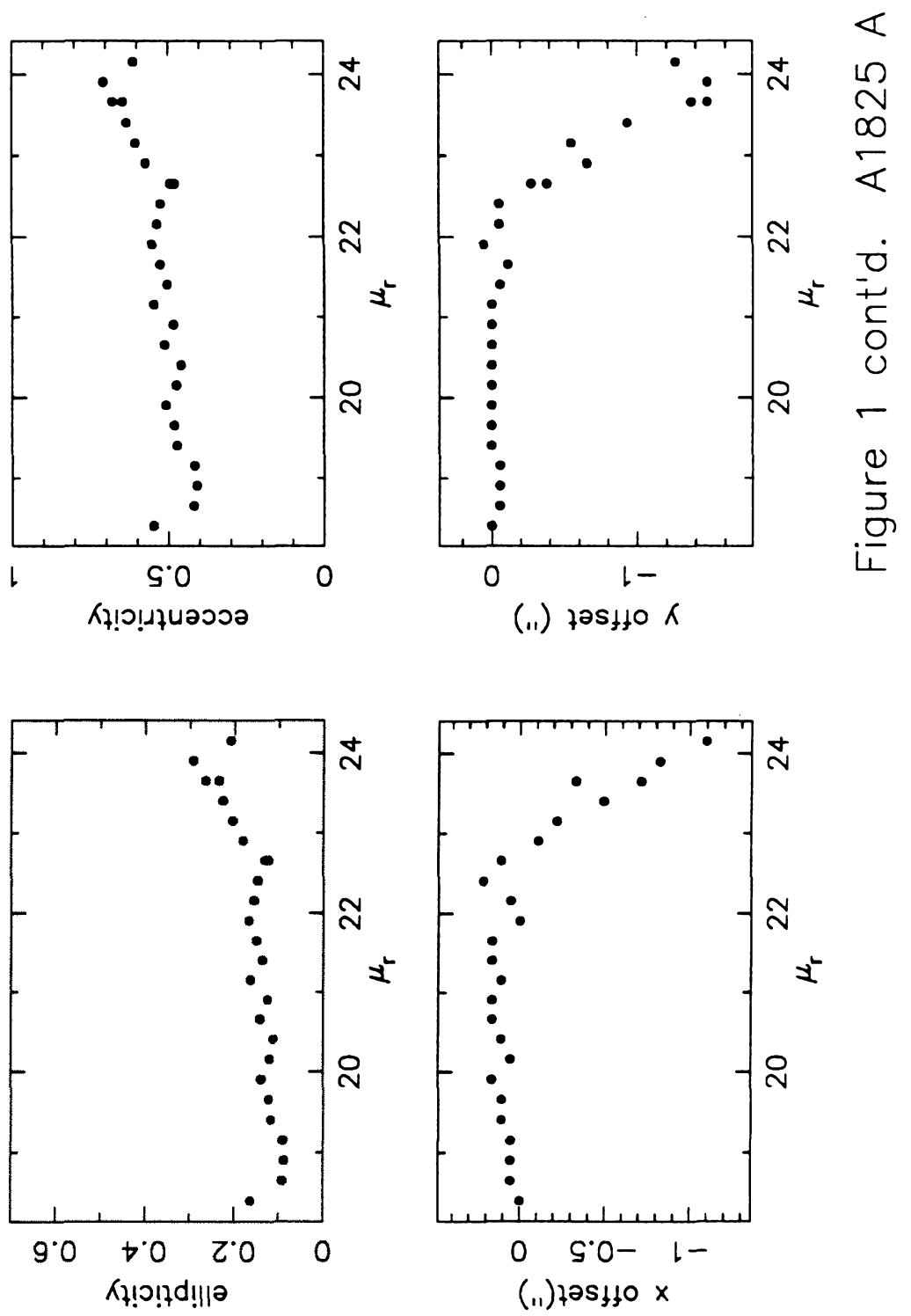

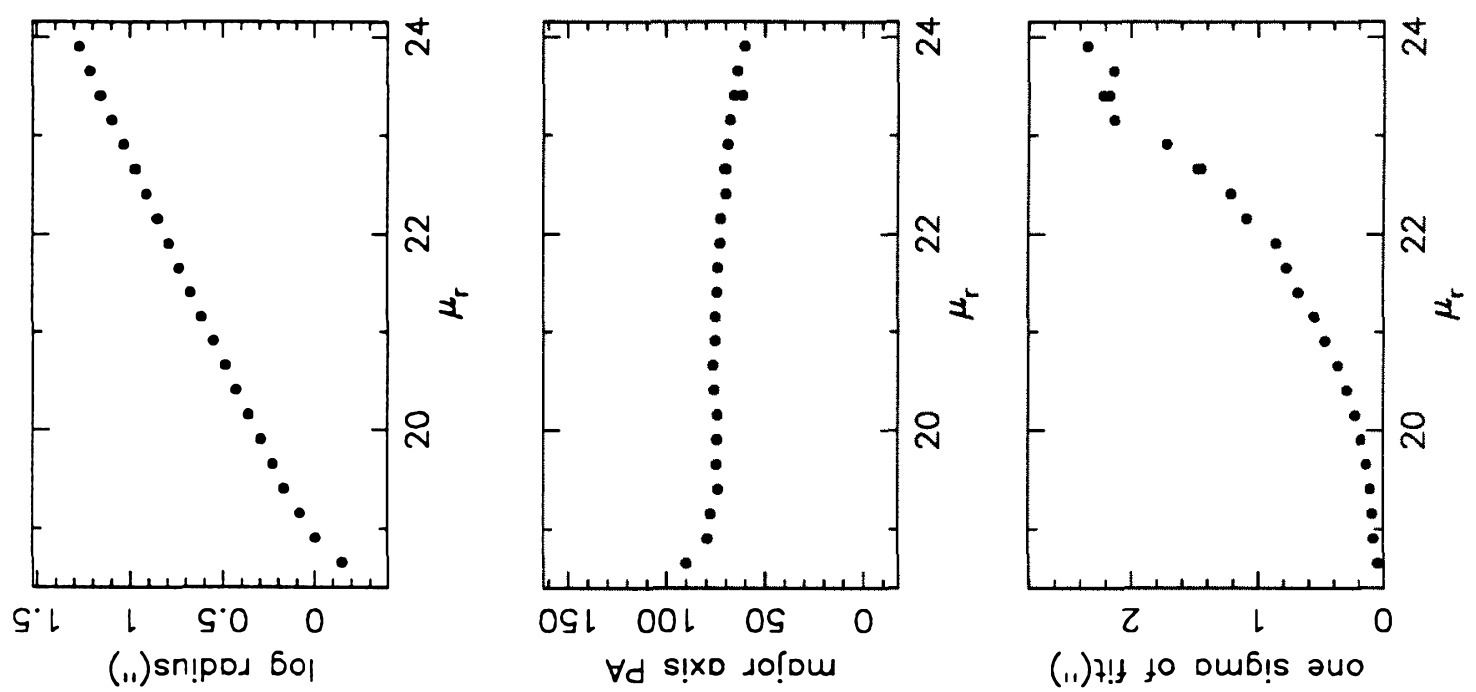

(11) $7 !$ t to oub!s auo
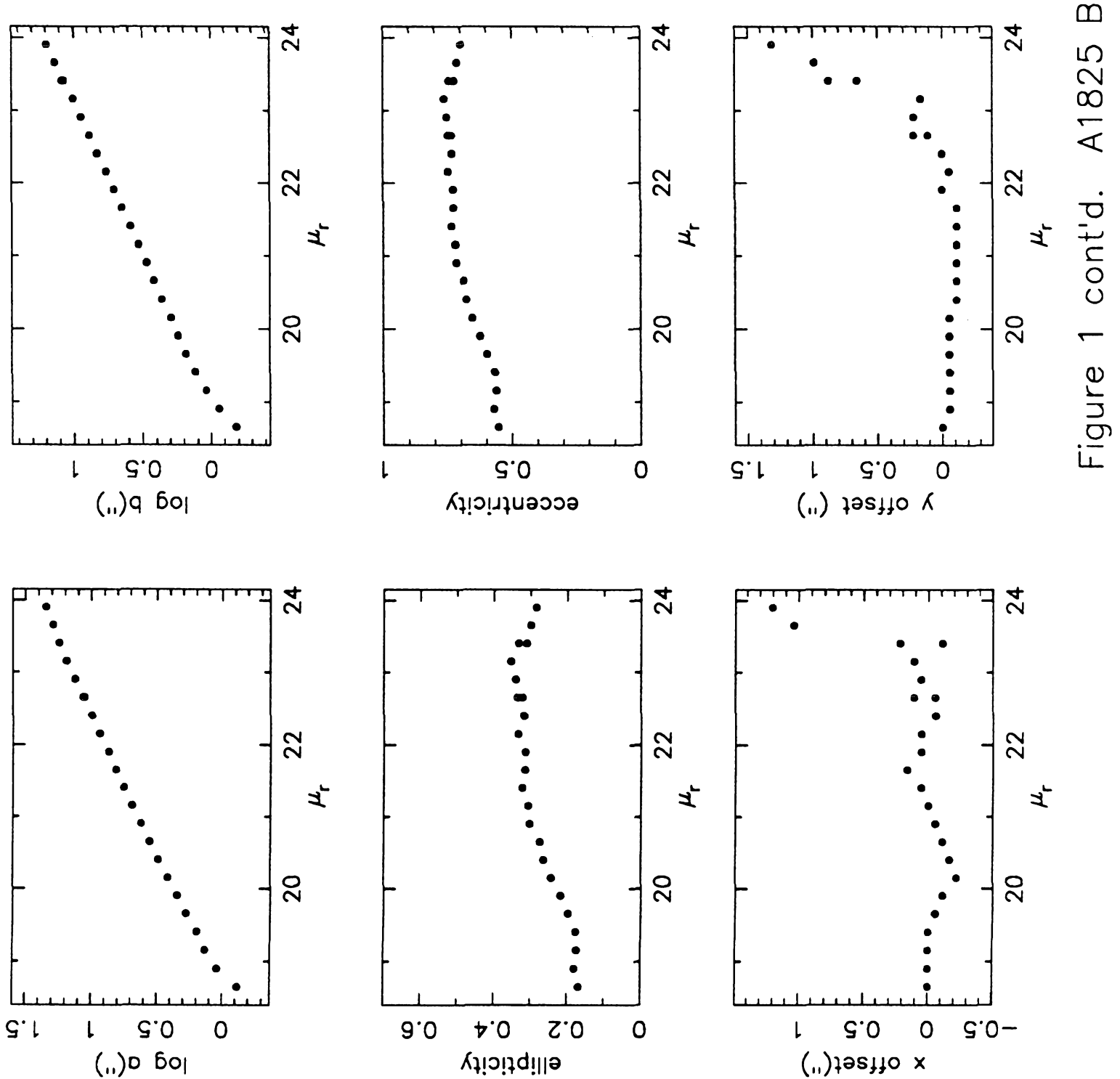


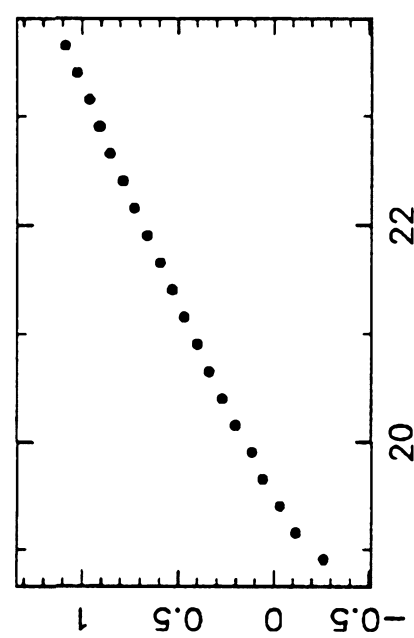

(.1) sn!pos 6ol

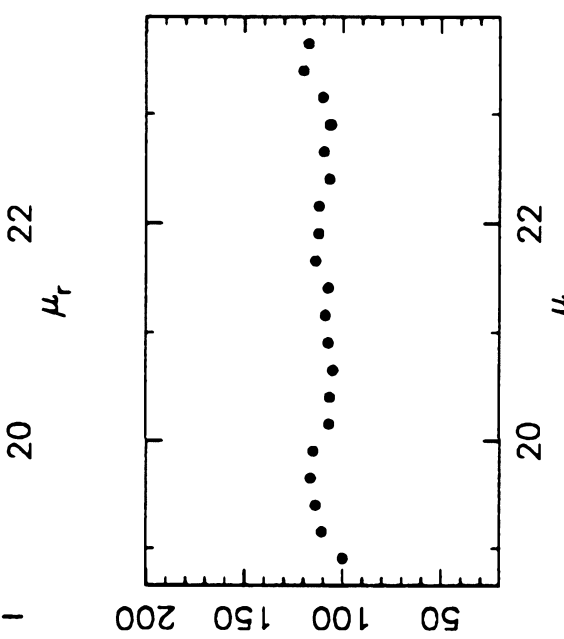

$\forall d s$ !xo so!

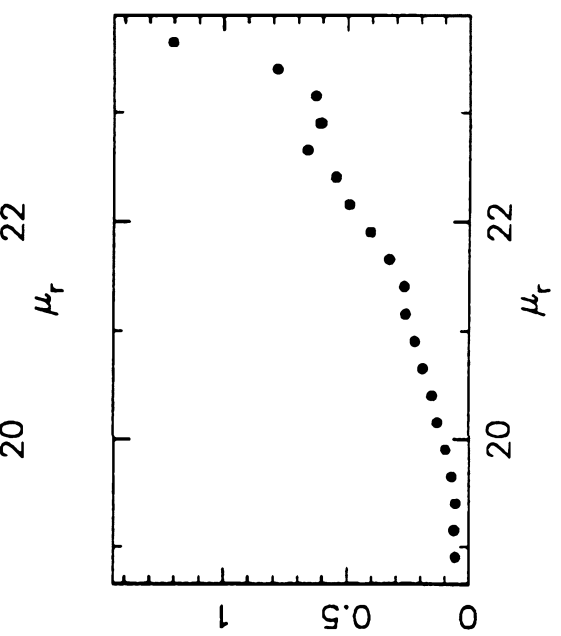

(11) $7 !+$ to oub! a a

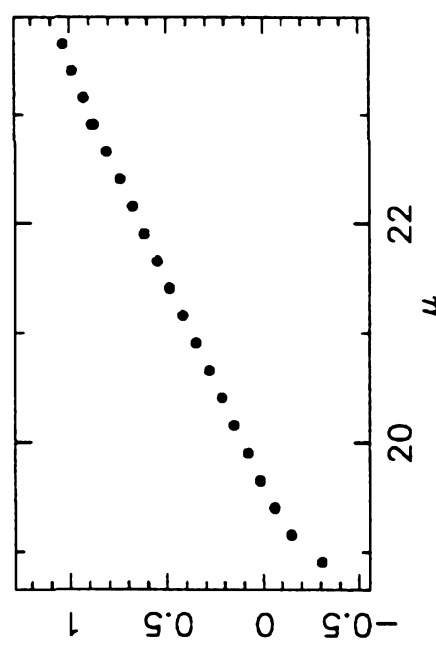

(.1) 9 bol

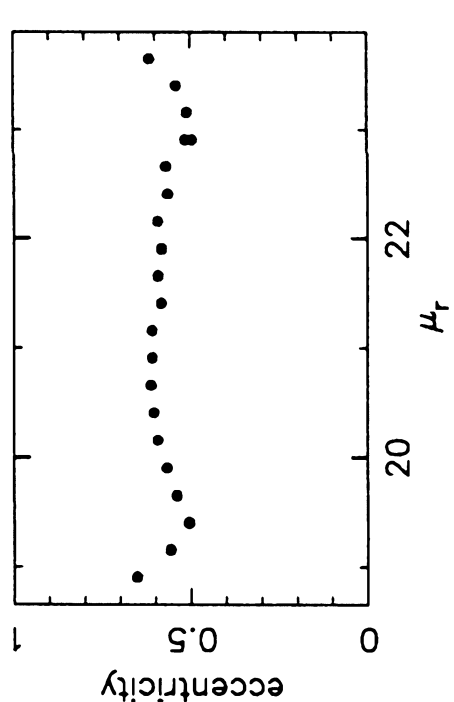

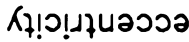

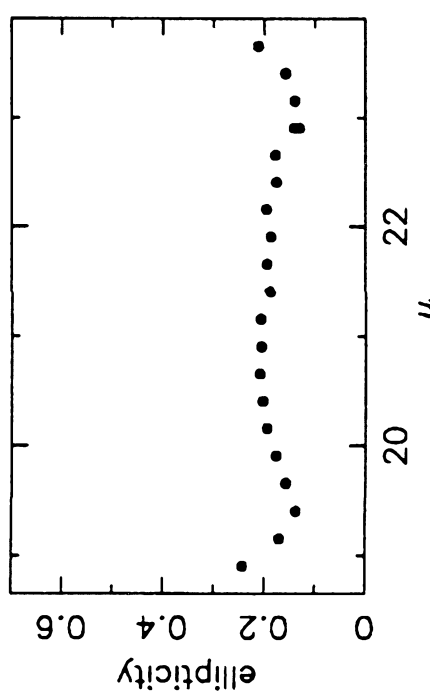

$\circlearrowleft$

น

$\infty$

$\frac{\infty}{<}$

ลี $\approx \frac{0}{7}$ 유 选
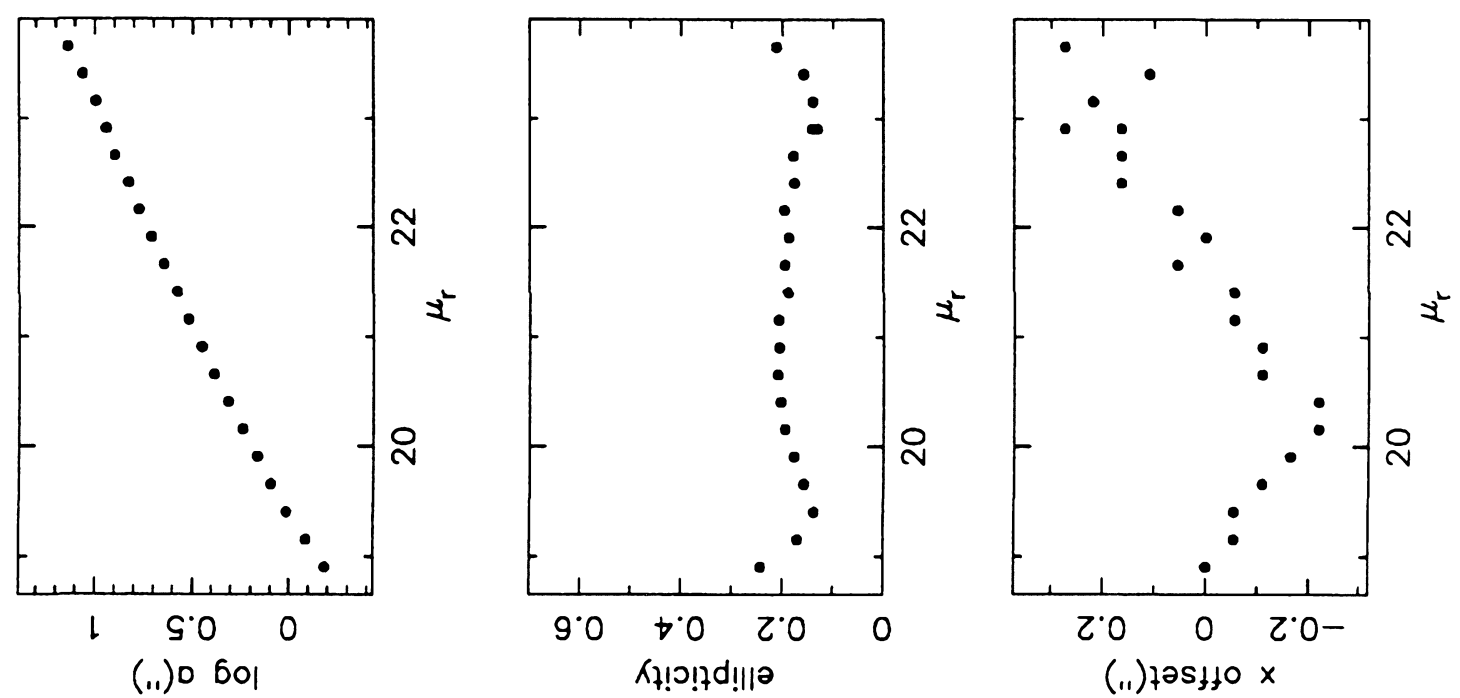


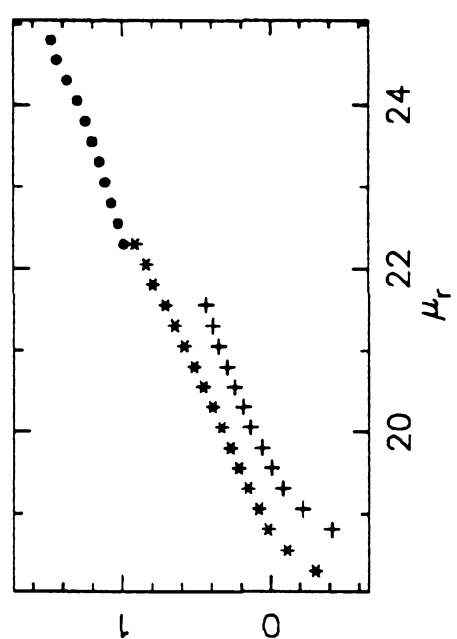

(11)sn!pos 6ol

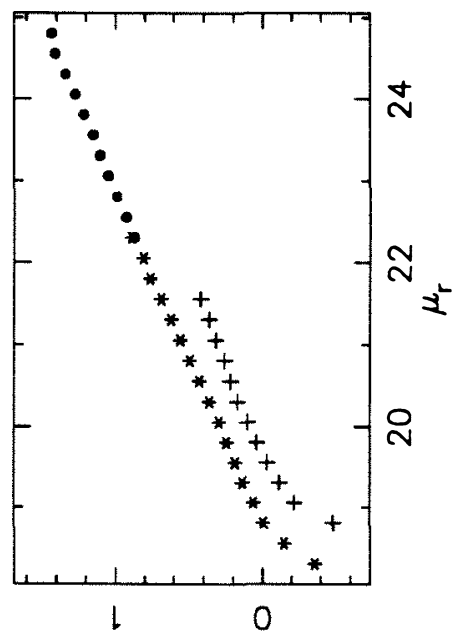

(.1) $\mathrm{q}$ 6ol

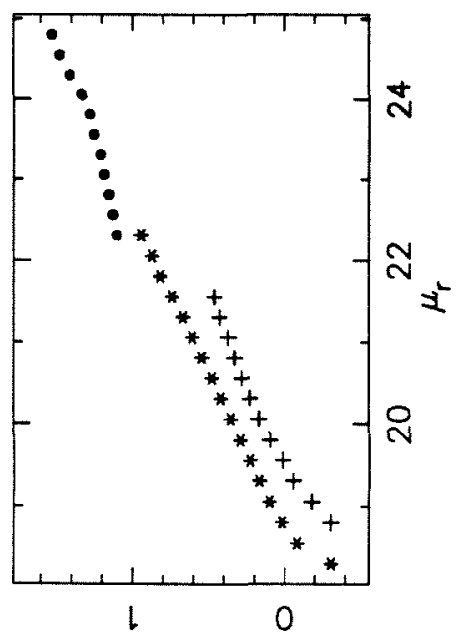

(.1) 0 501

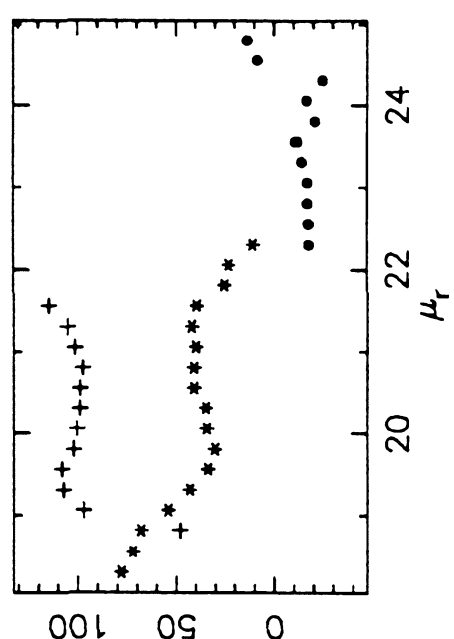

$\forall d$ s!xo so!om
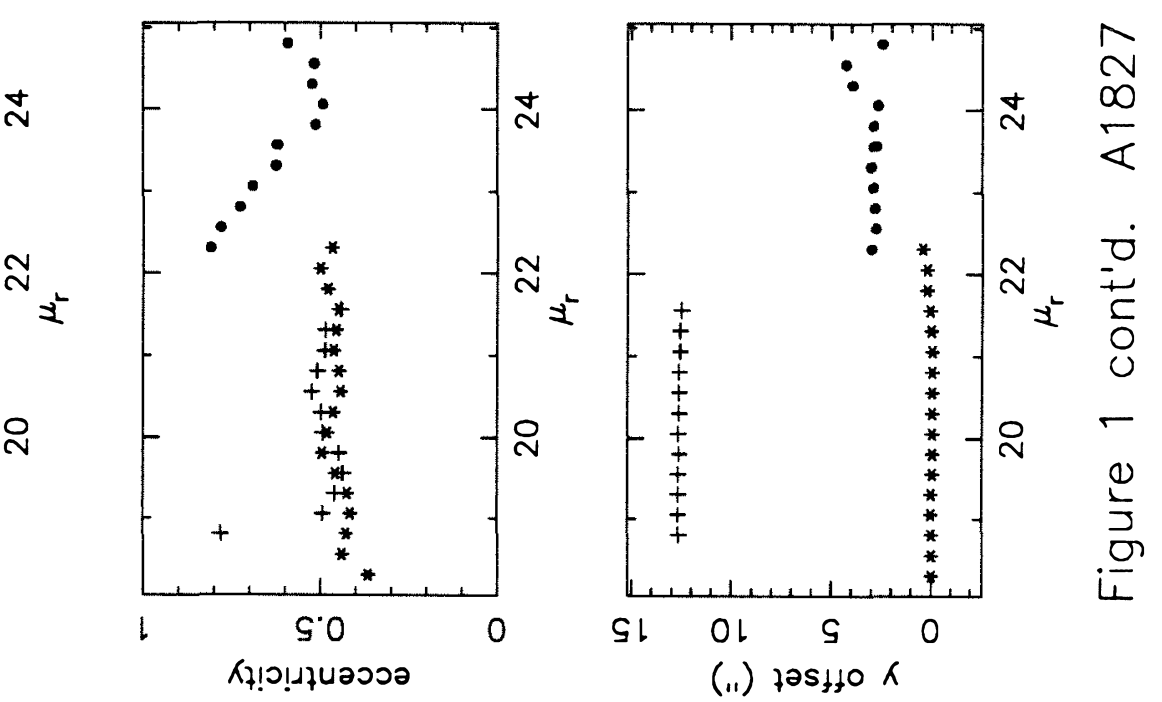

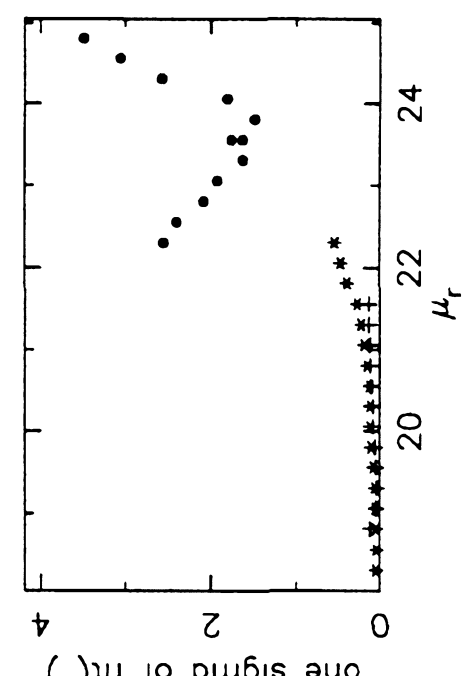

(11) $+!+$ to Dub!s auo
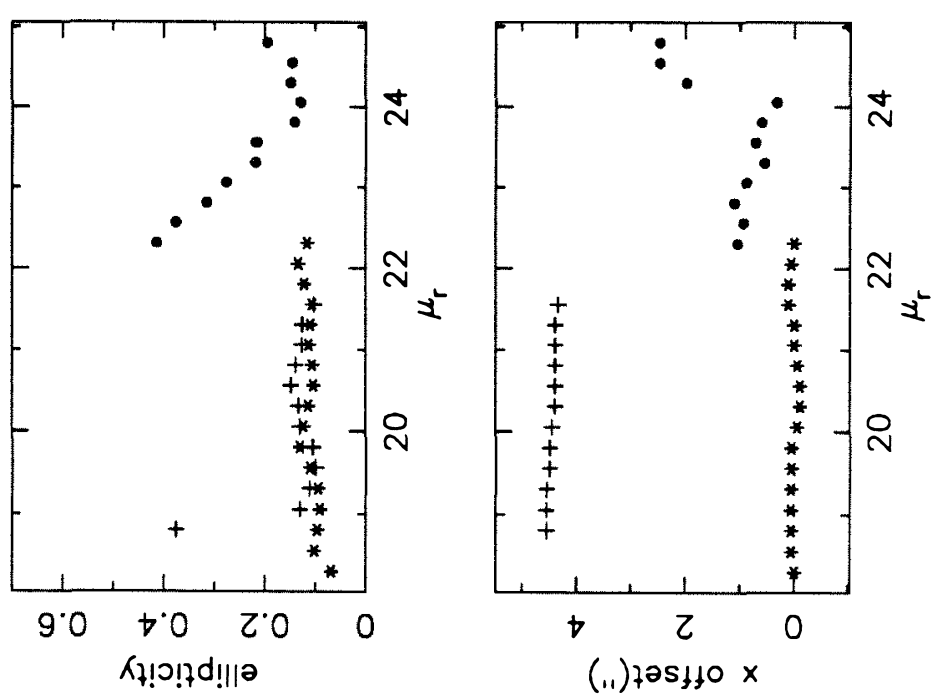

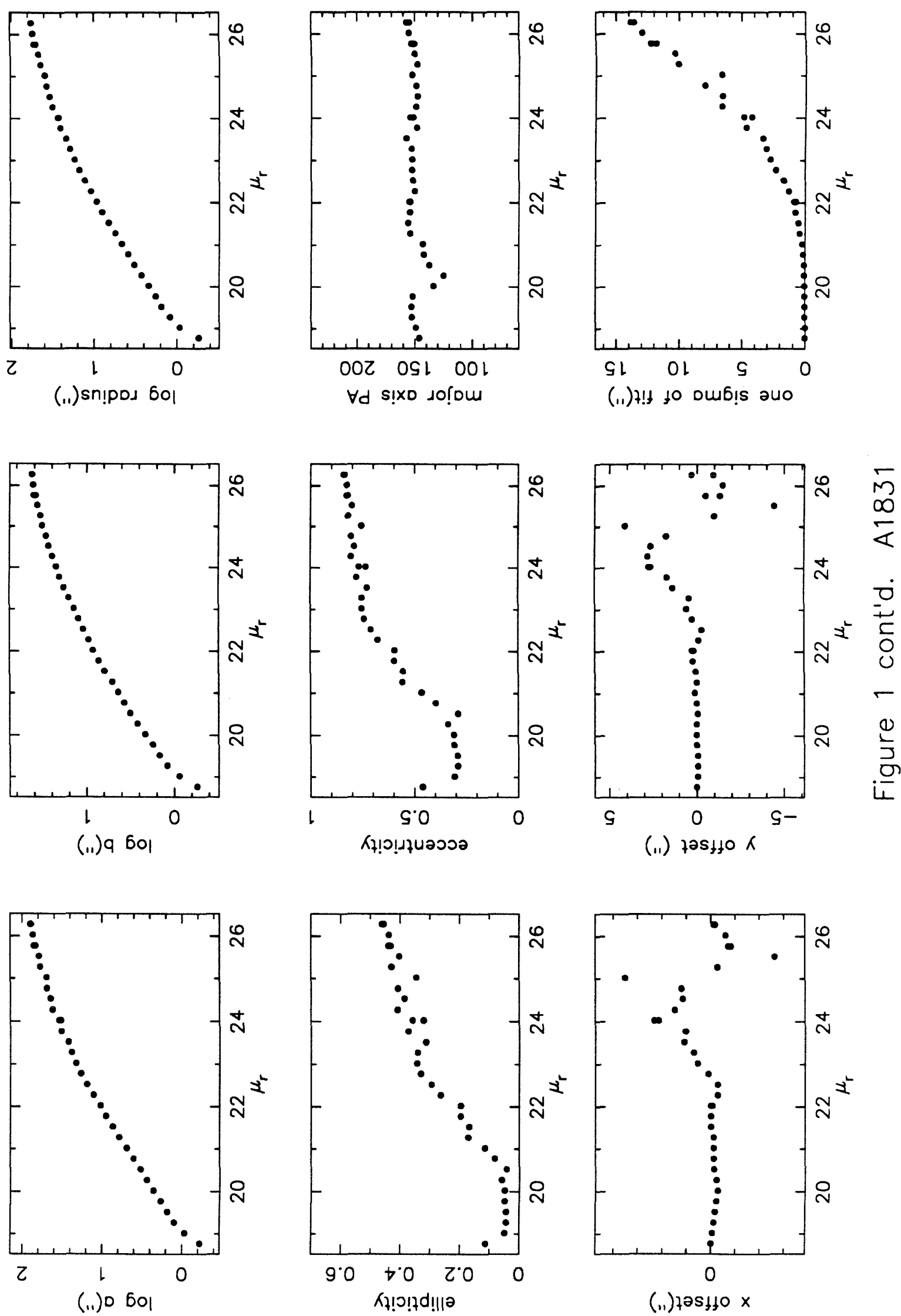

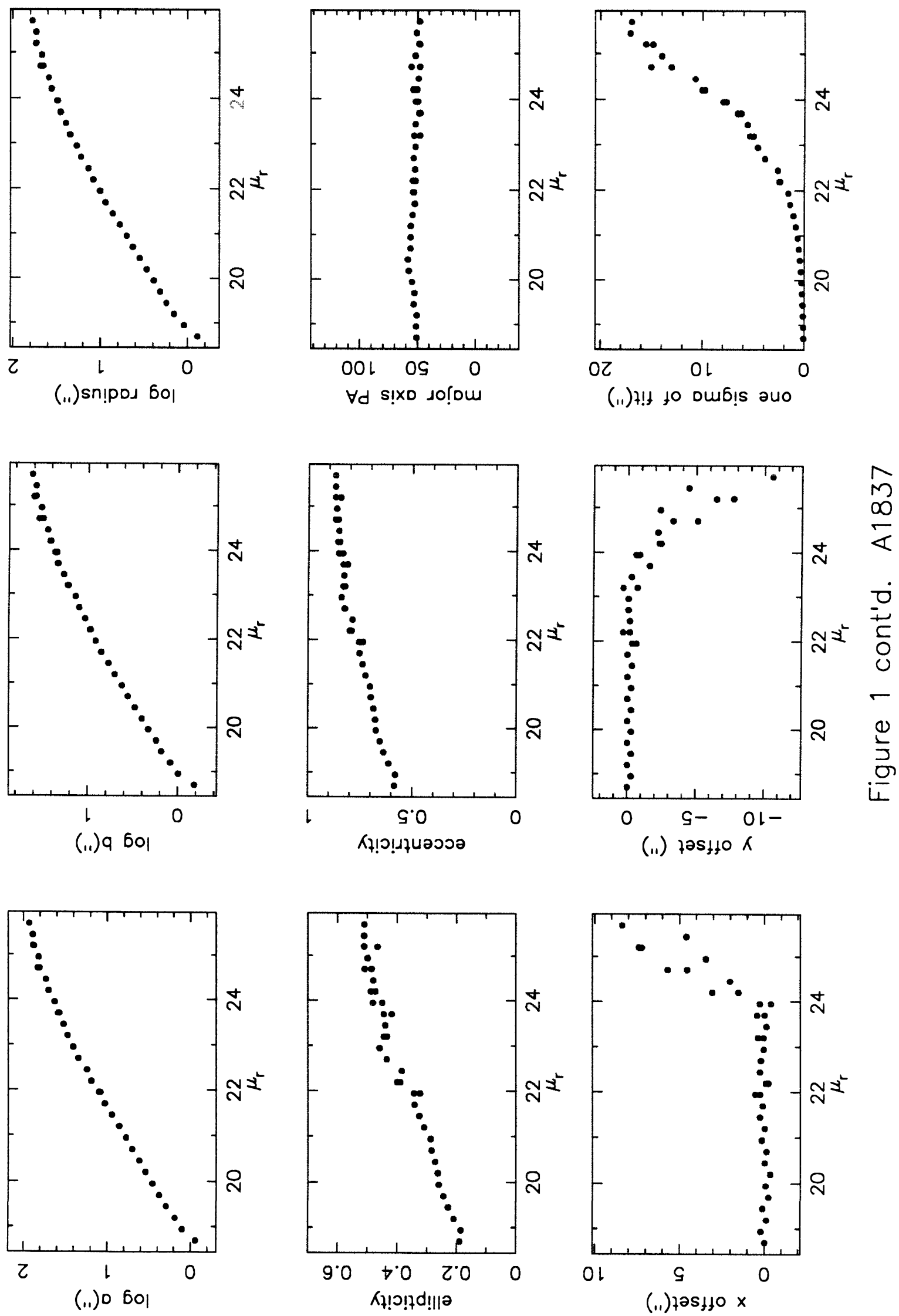


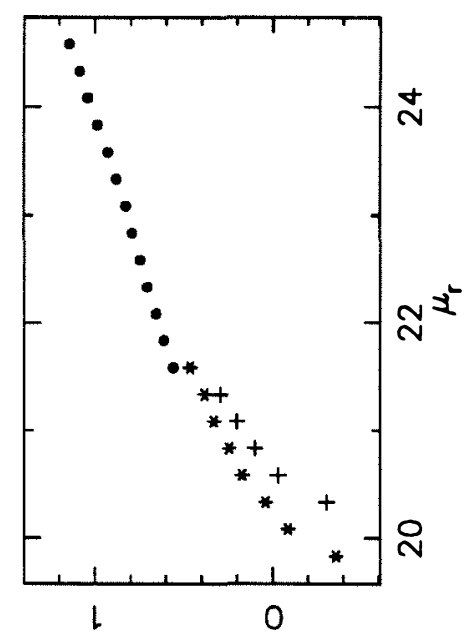

(.1) sn!pod 60|

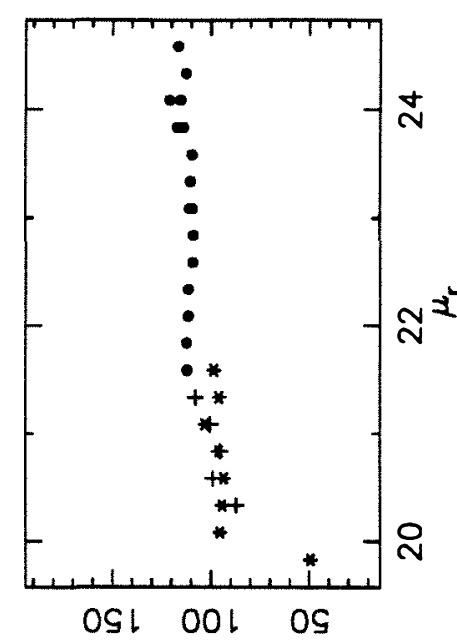

$\forall d$ s!xo $J 0$ !

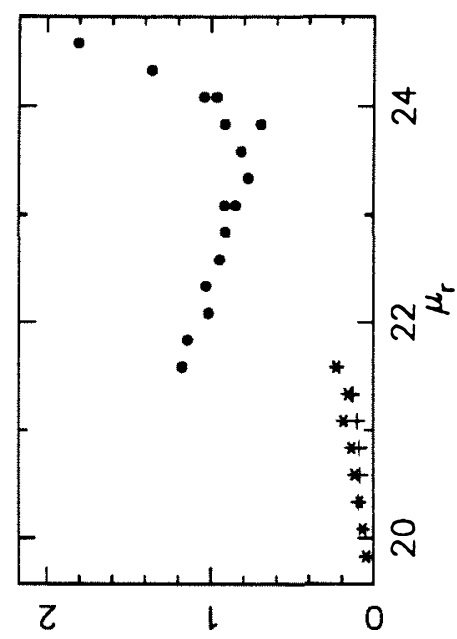

(11) $7 !+$ jo Dub! auo

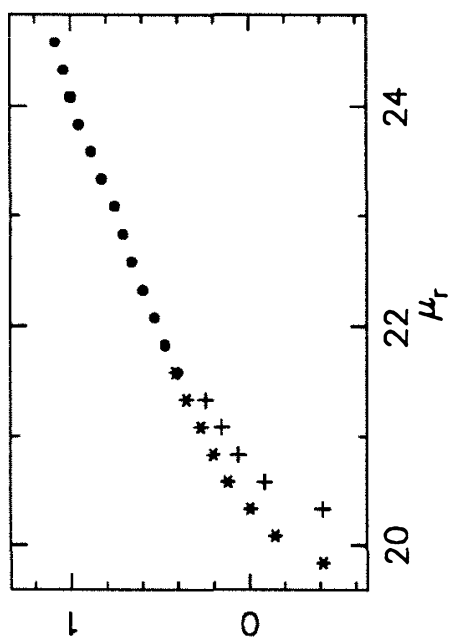

(.1) 9601

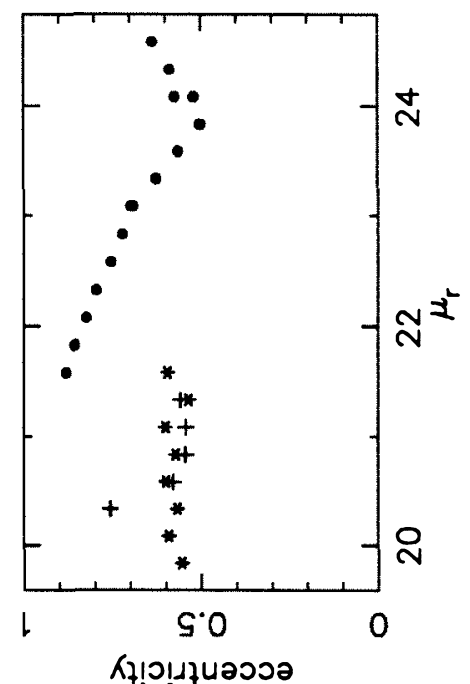

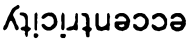

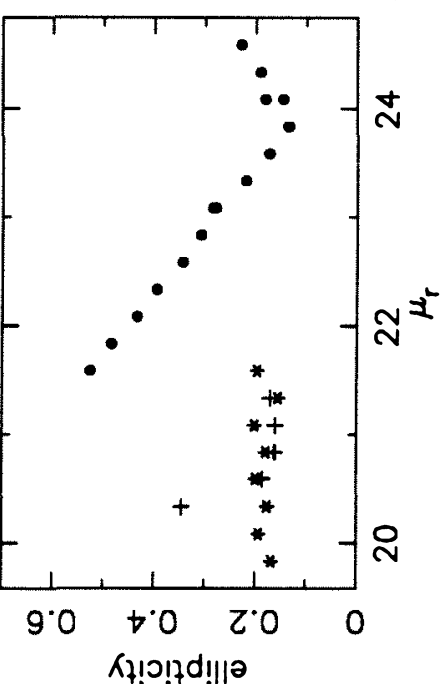

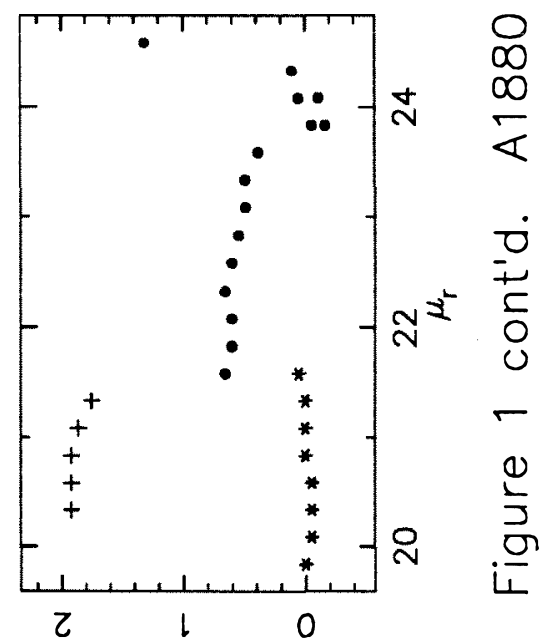

(iI) $725450 \mathrm{~K}$
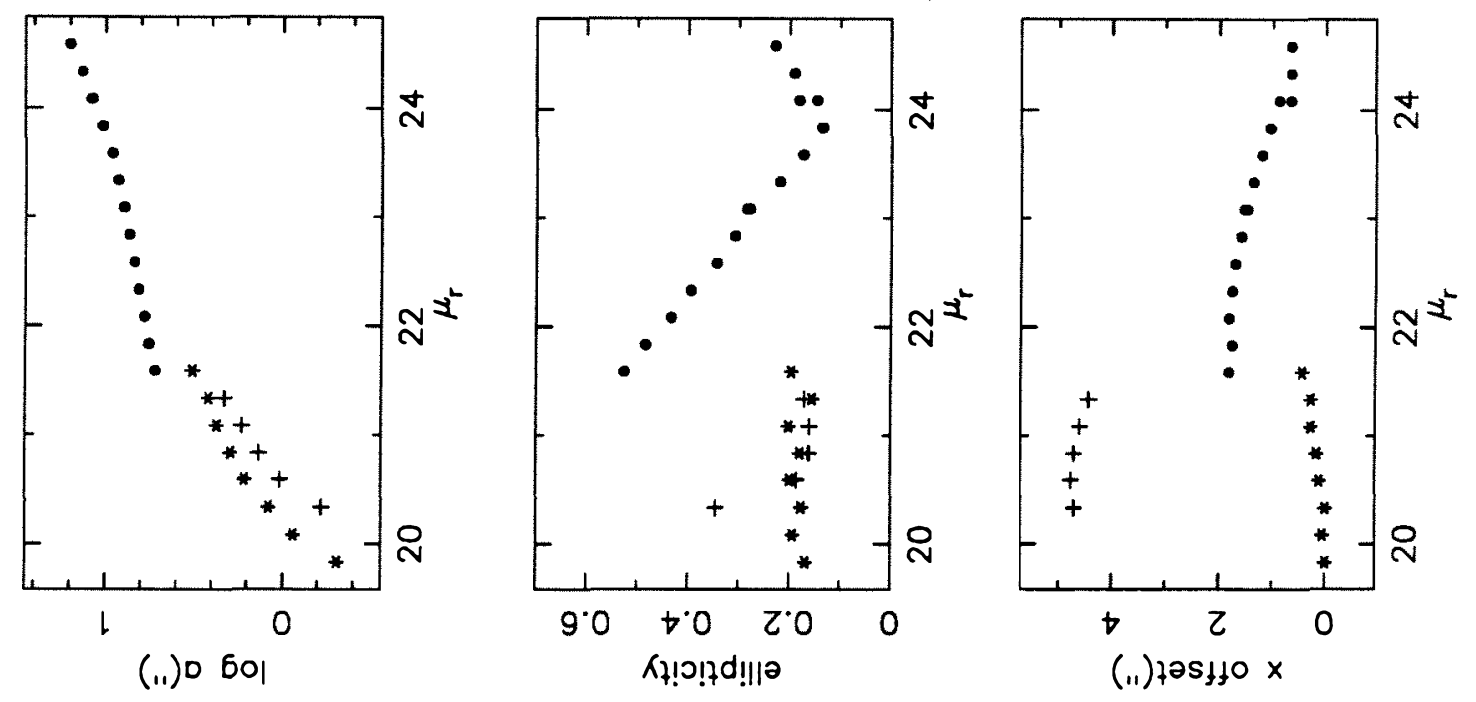


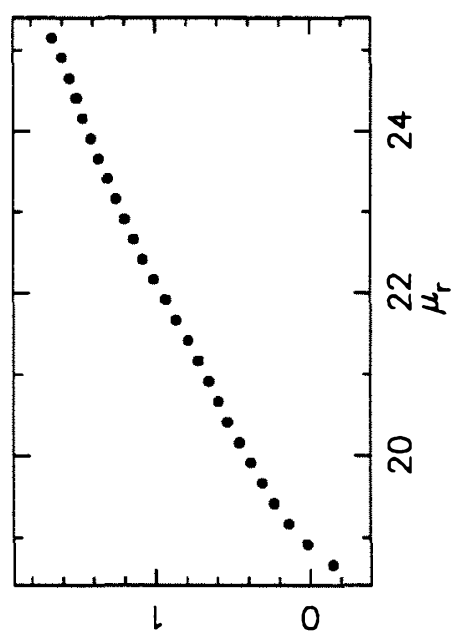

(II)sn!pos 6ol

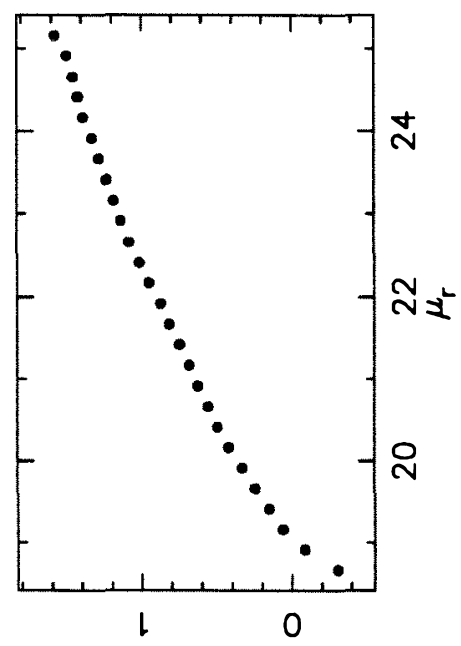

(.1) 9 6ol

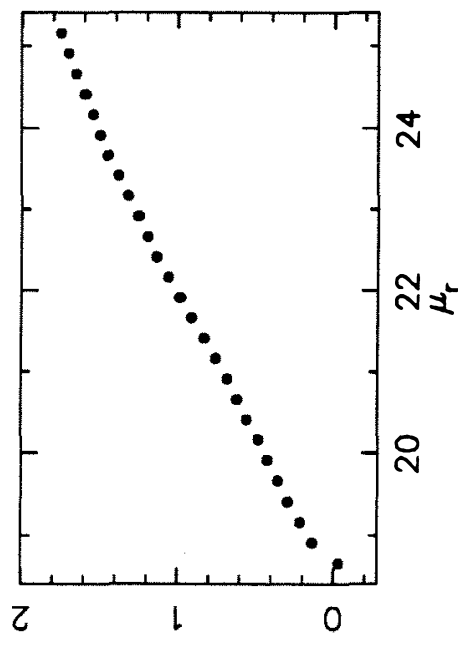

(i1) 0601
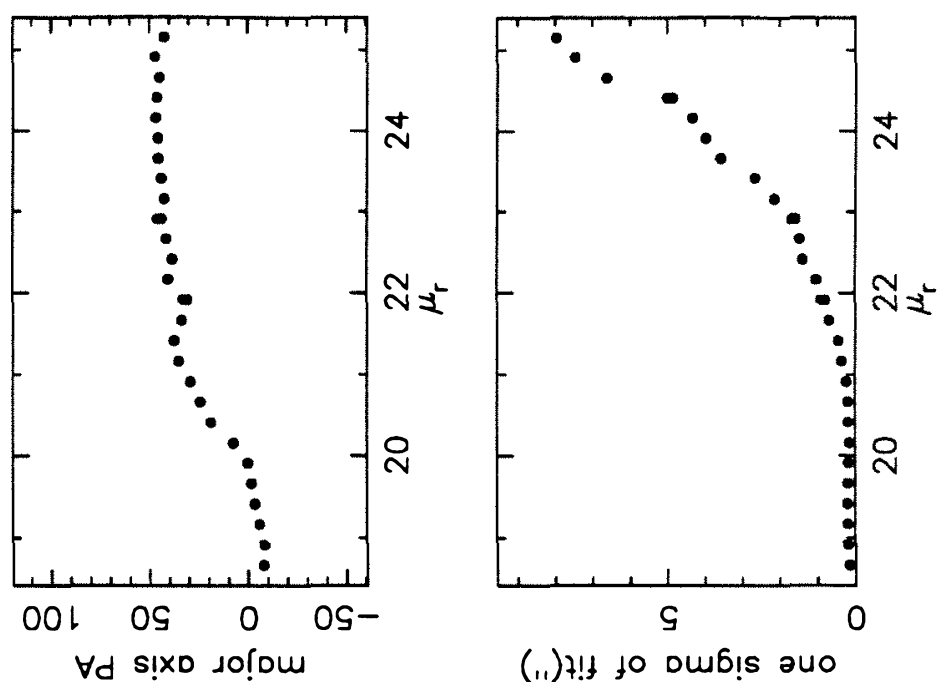

(11) $+! 1$ to oub! a

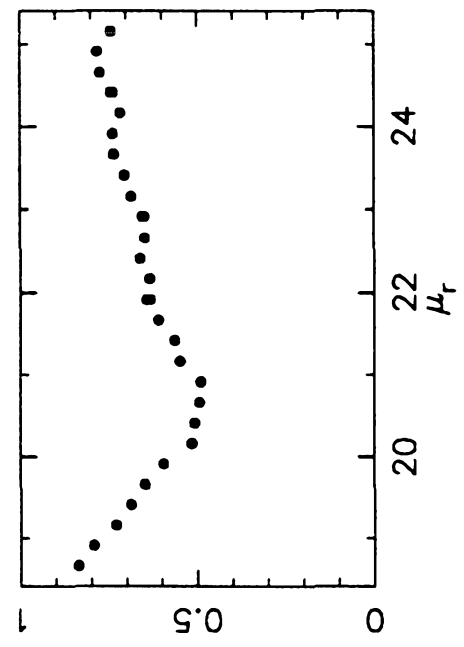

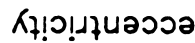

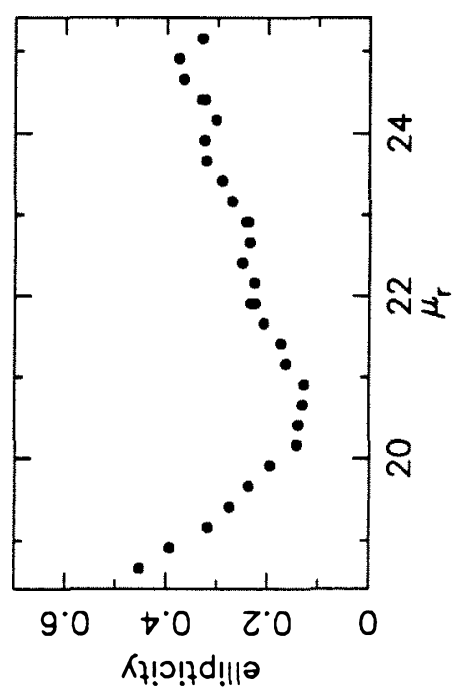

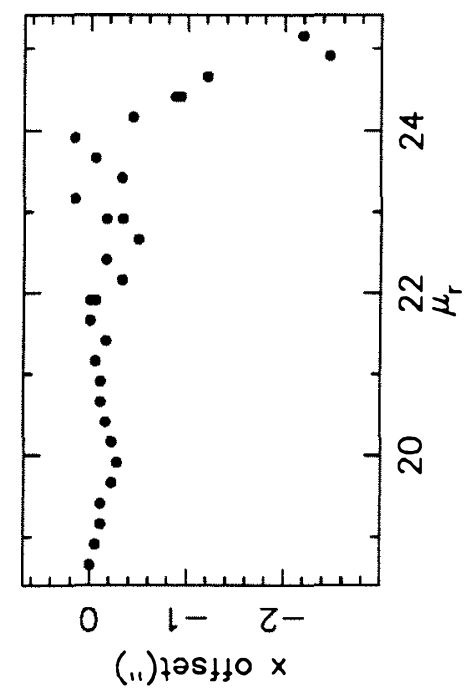




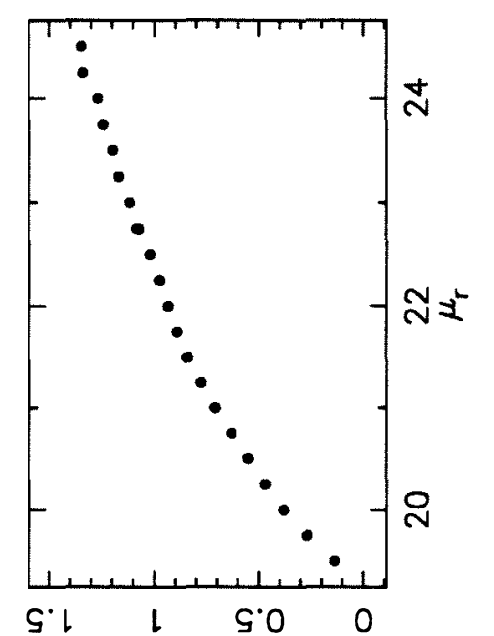

(11)sn!pos 6ol

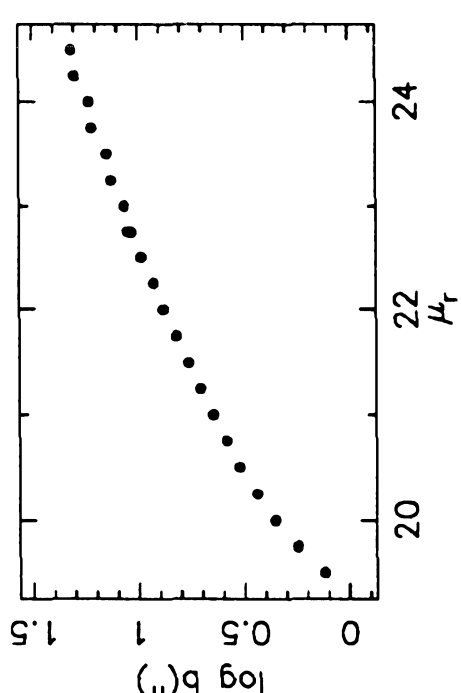

(.1) 9 6ol

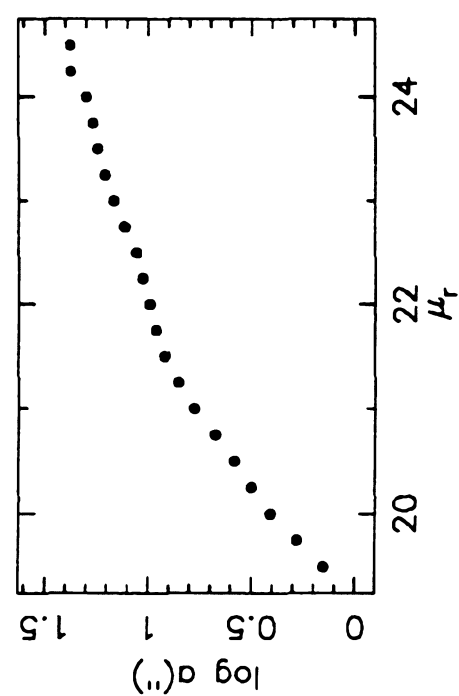

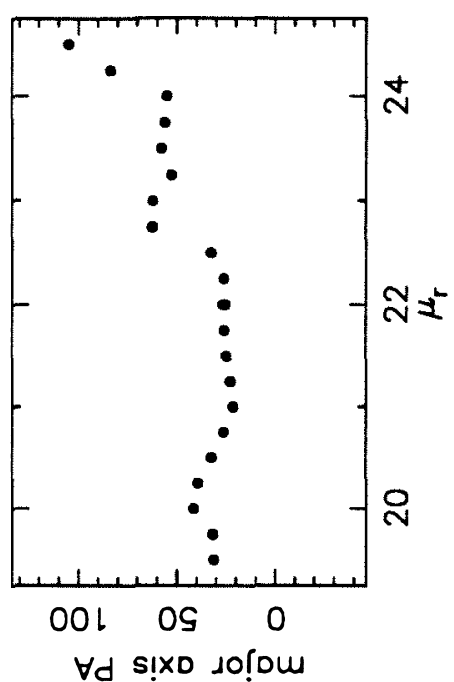

$\forall d$ s!xo so!nom

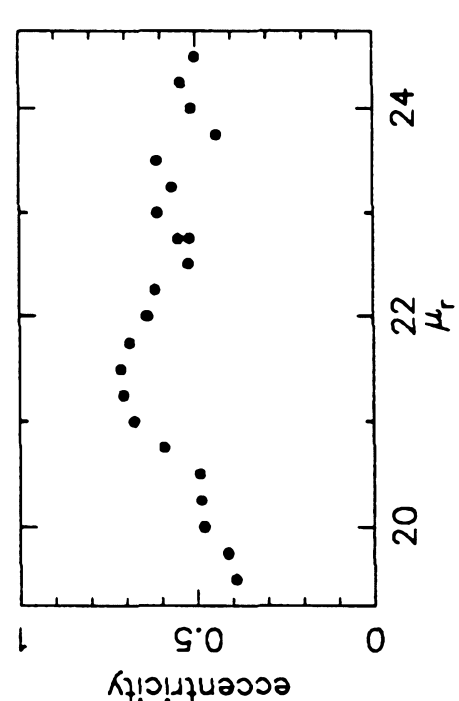

(11) $725 \% 10 \mathrm{~K}$
(.1) $+! ! ~ j 0$ oub! au०

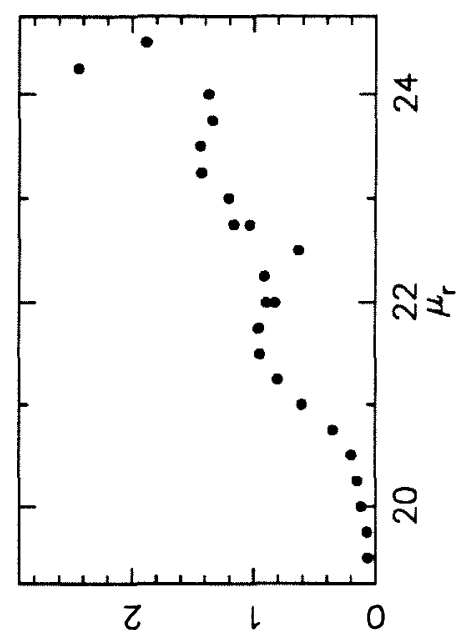

$<$
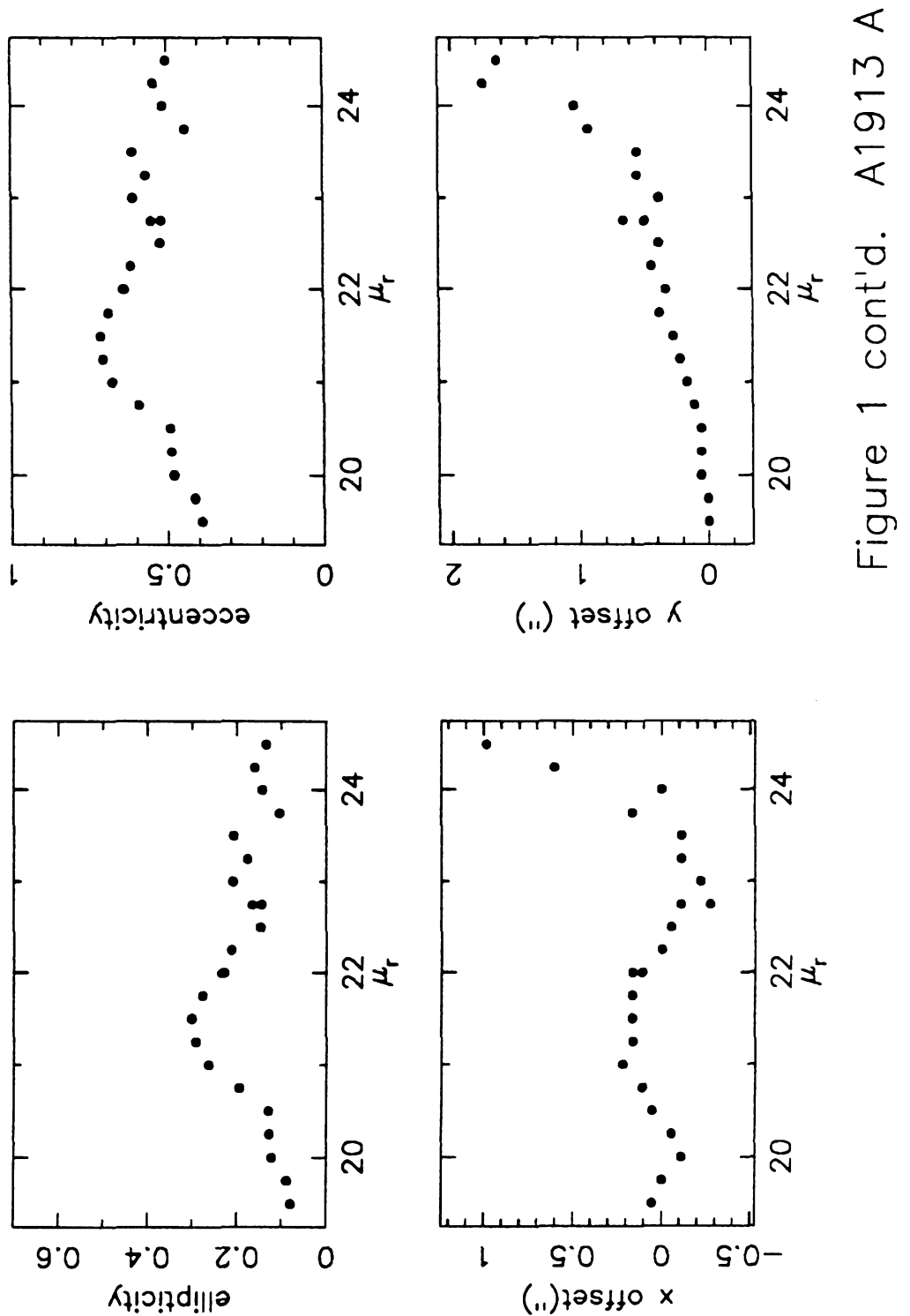

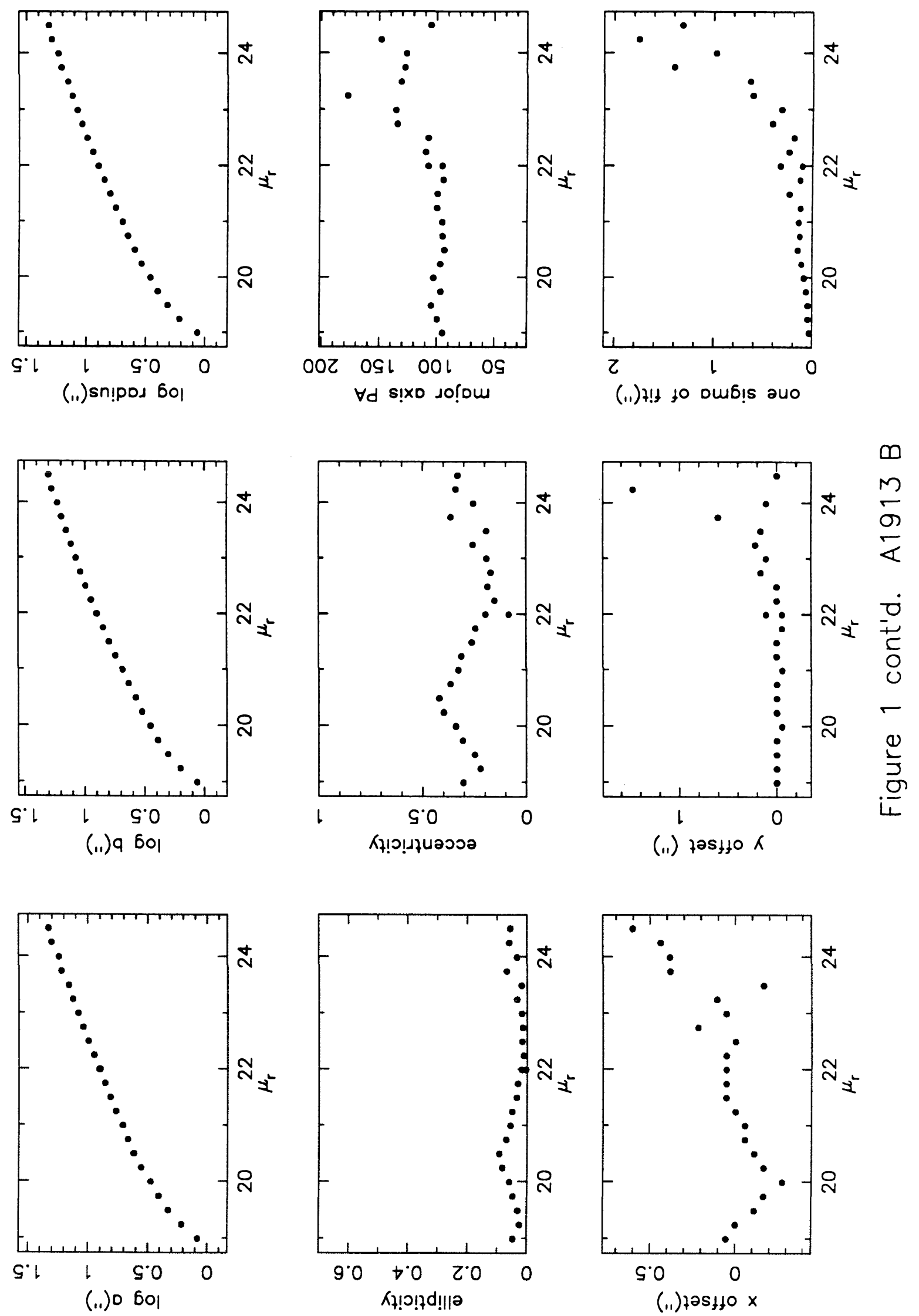


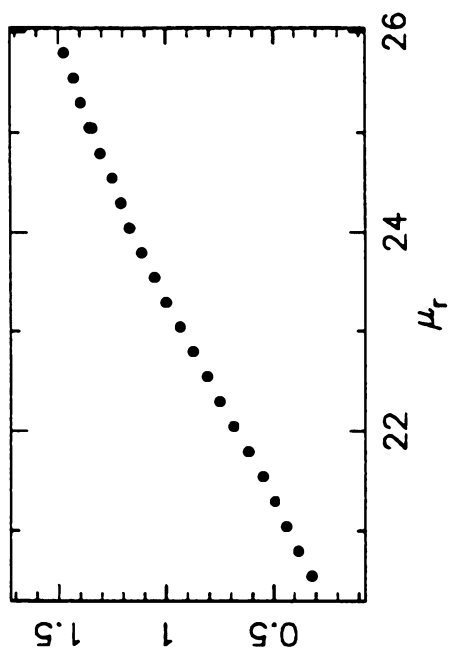

(.1) sn!pod 601

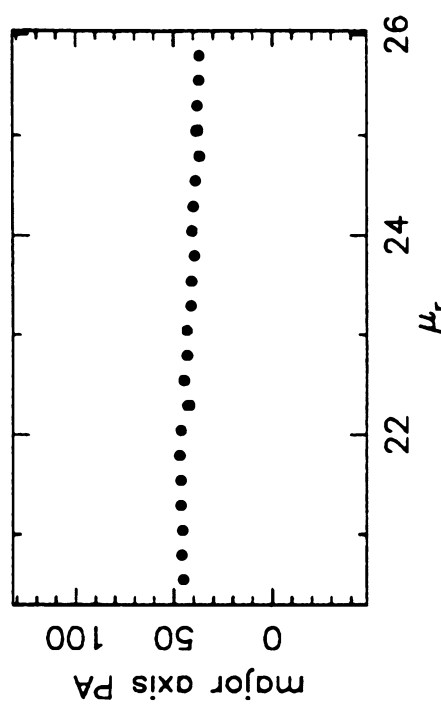

$\forall d$ s!xo so! lom

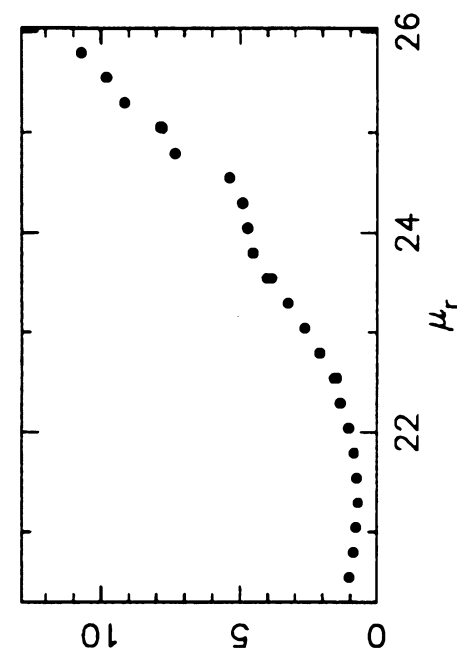

(11) $+! t$ to Du6!s auo
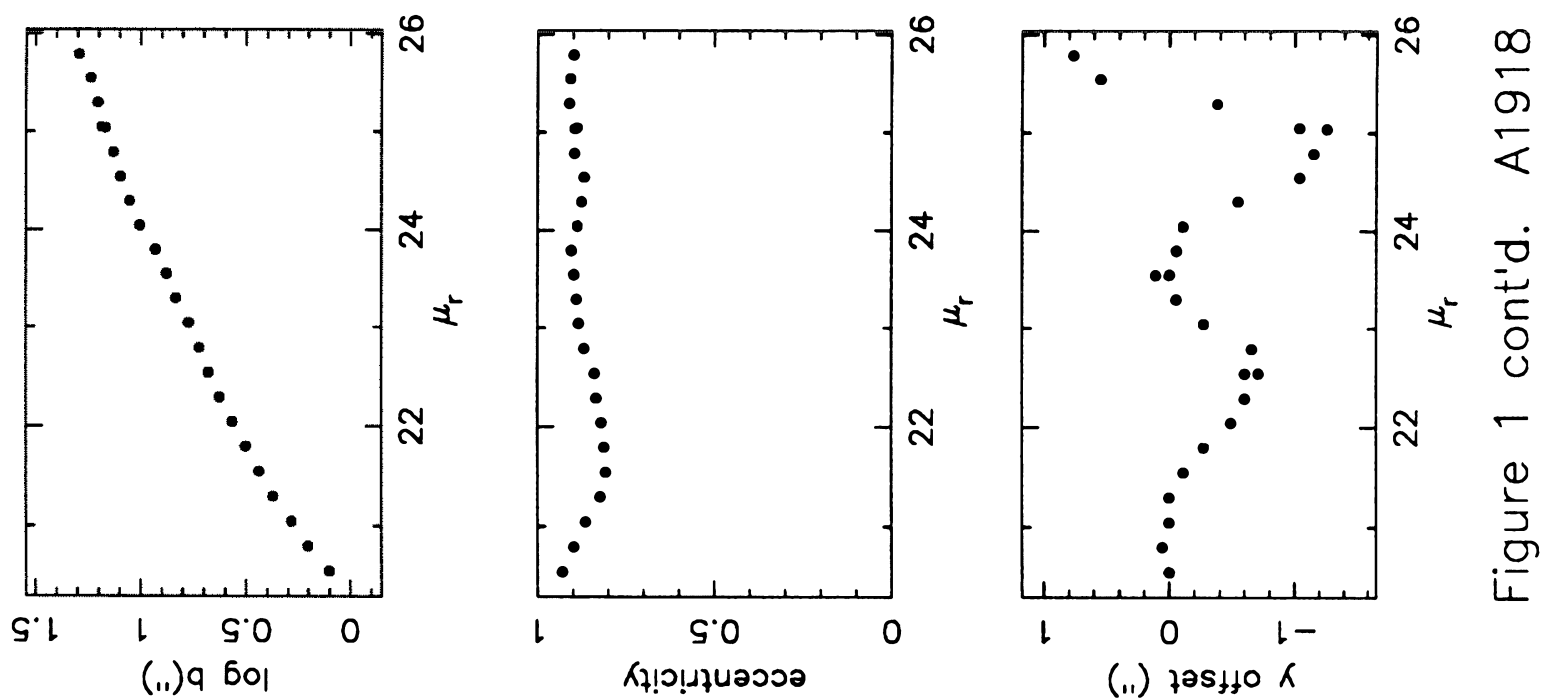

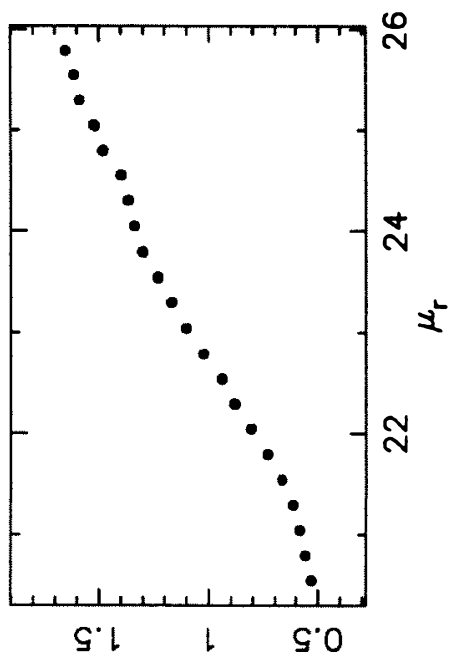

(.1) 0601

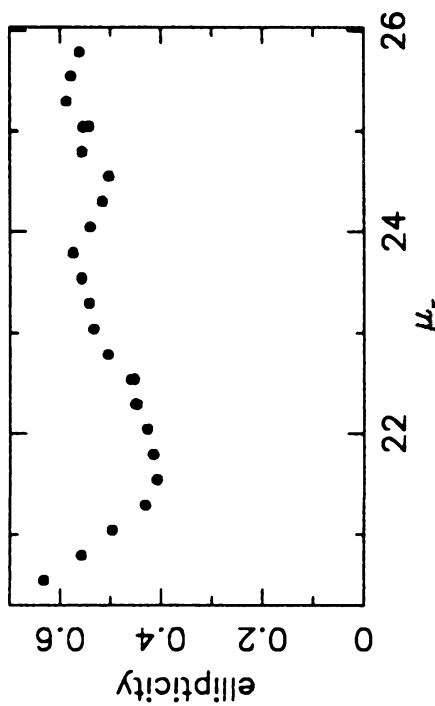

रł!ว!?d!||ə

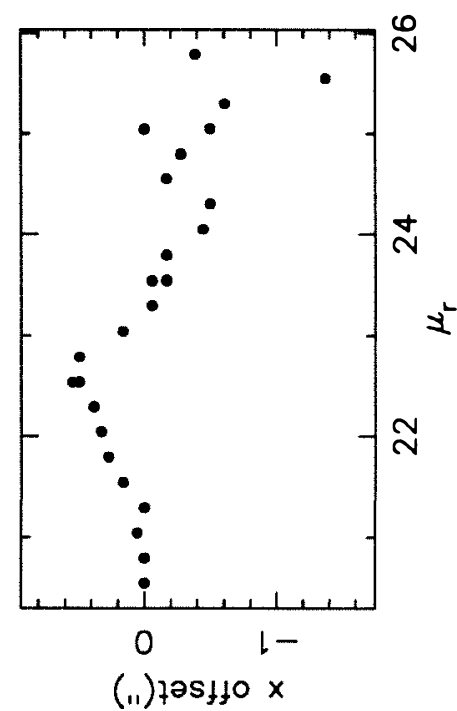




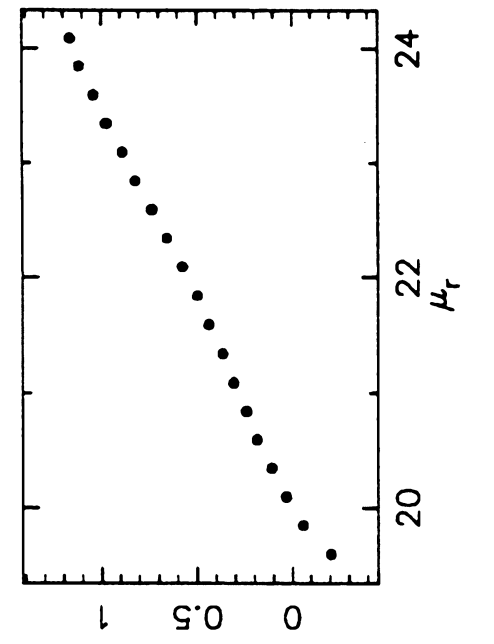

(.1) sn!pod 6ol

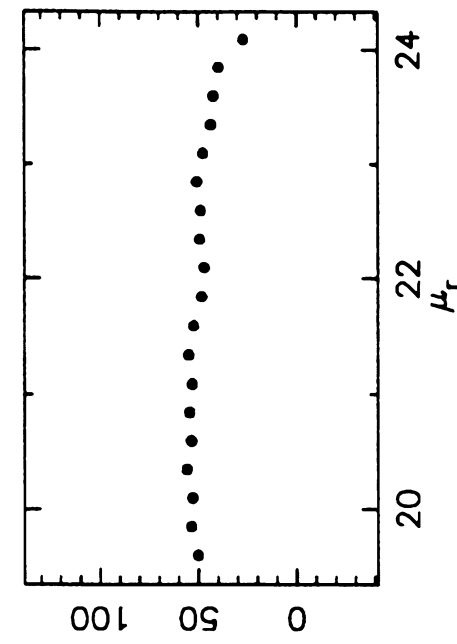

$\forall d$ s!xo 10 ! ou

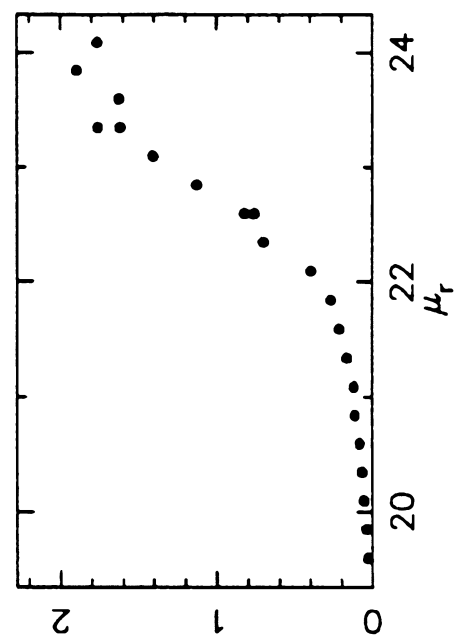

(.1) $7 ! \$$ to Dub!s auo
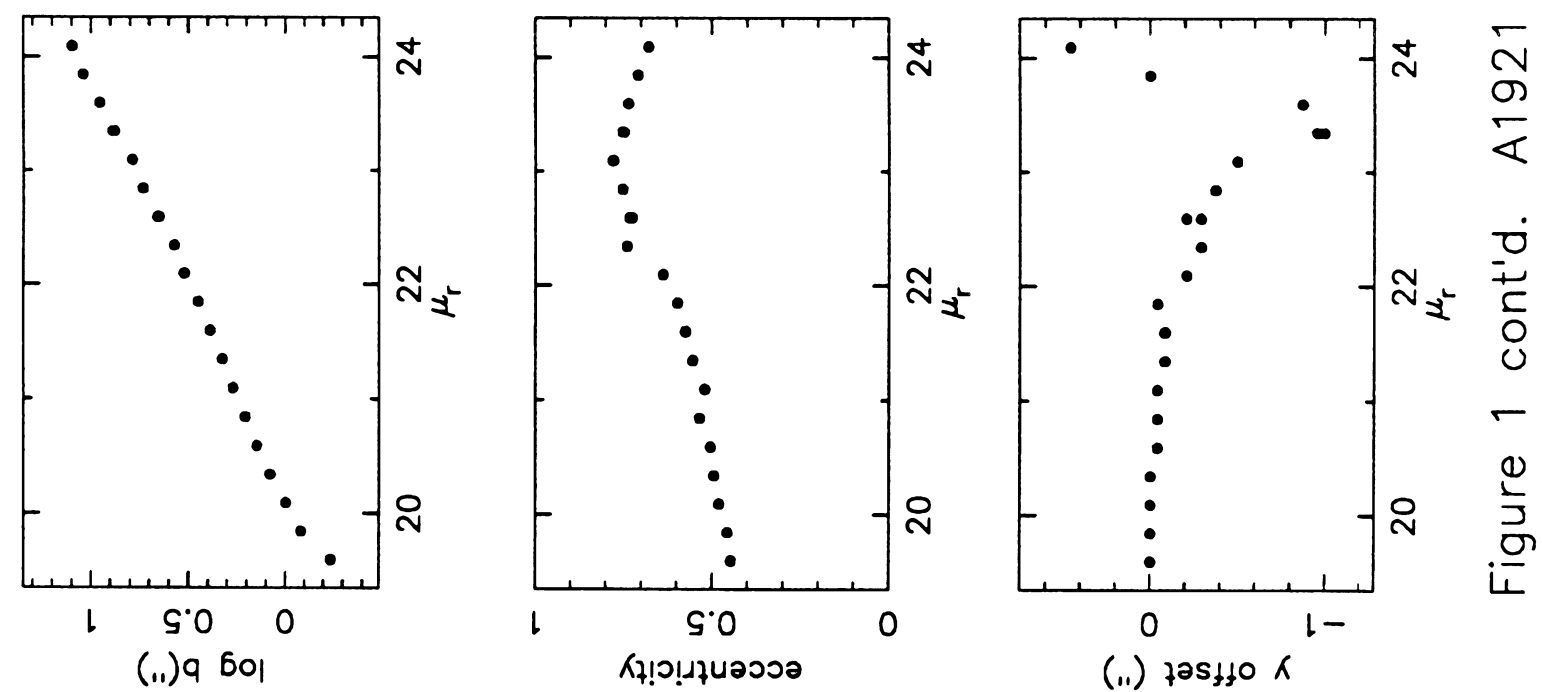

(ii) $725450 K$
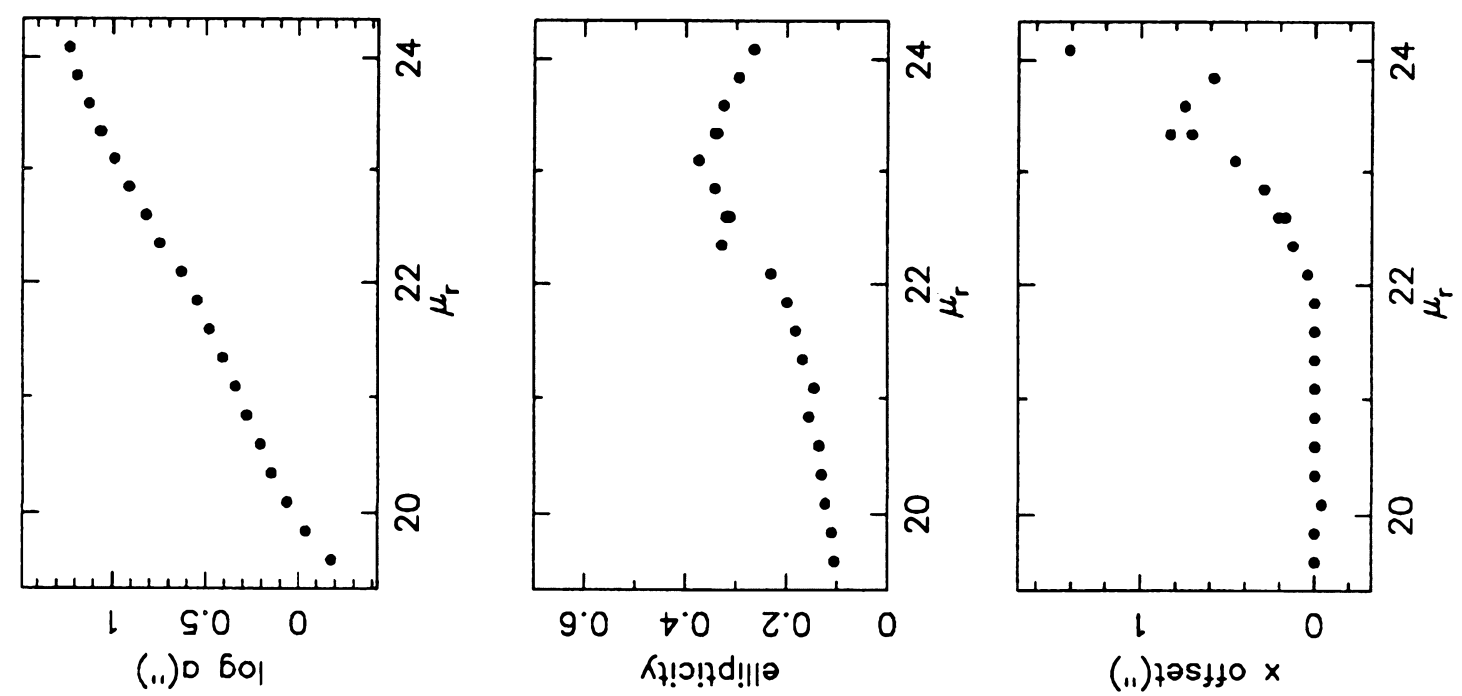
$-375-$
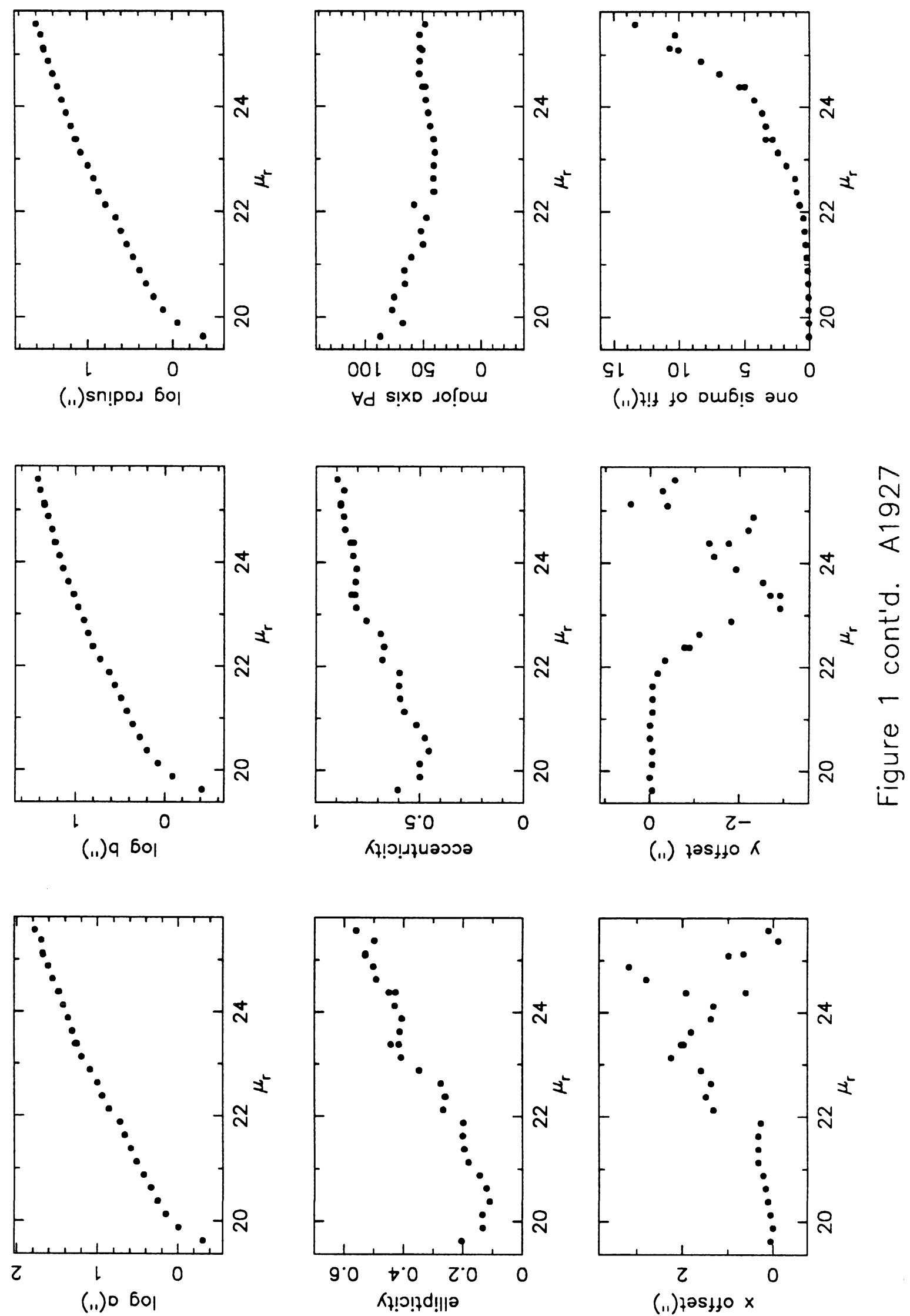

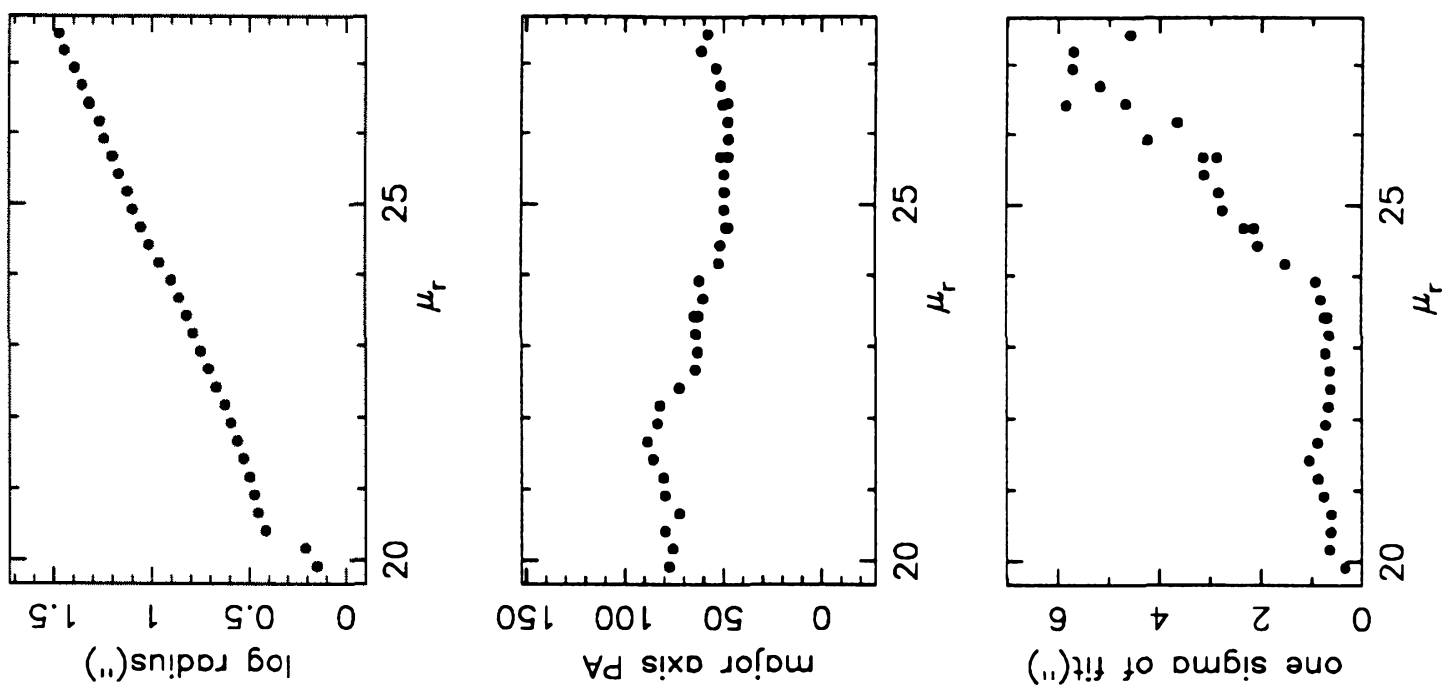

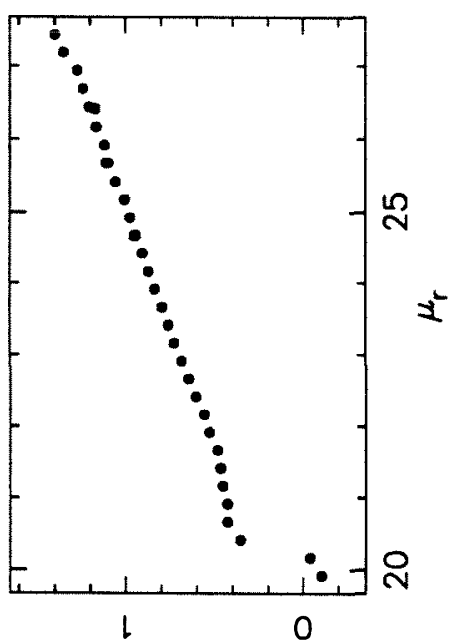

(11) 9 bol

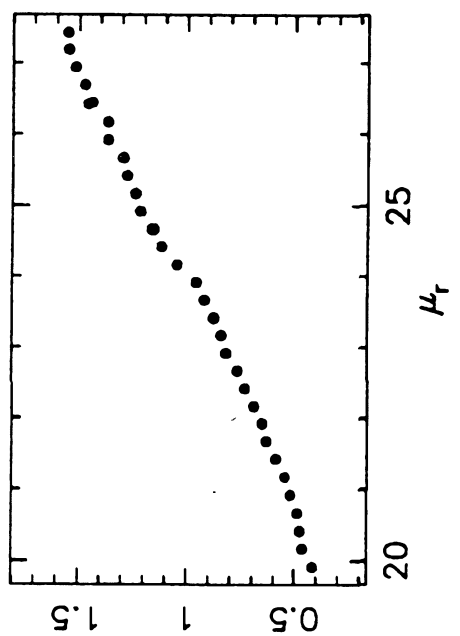

(.1) $0 \mathrm{60}$
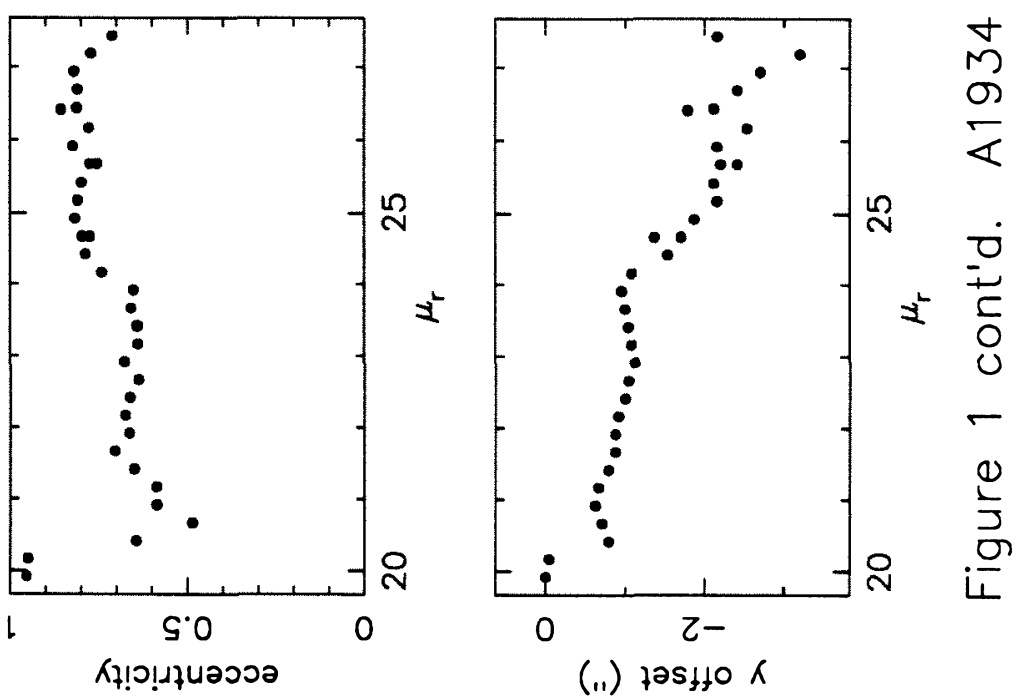

(11) $125 \% 10 \mathrm{~K}$
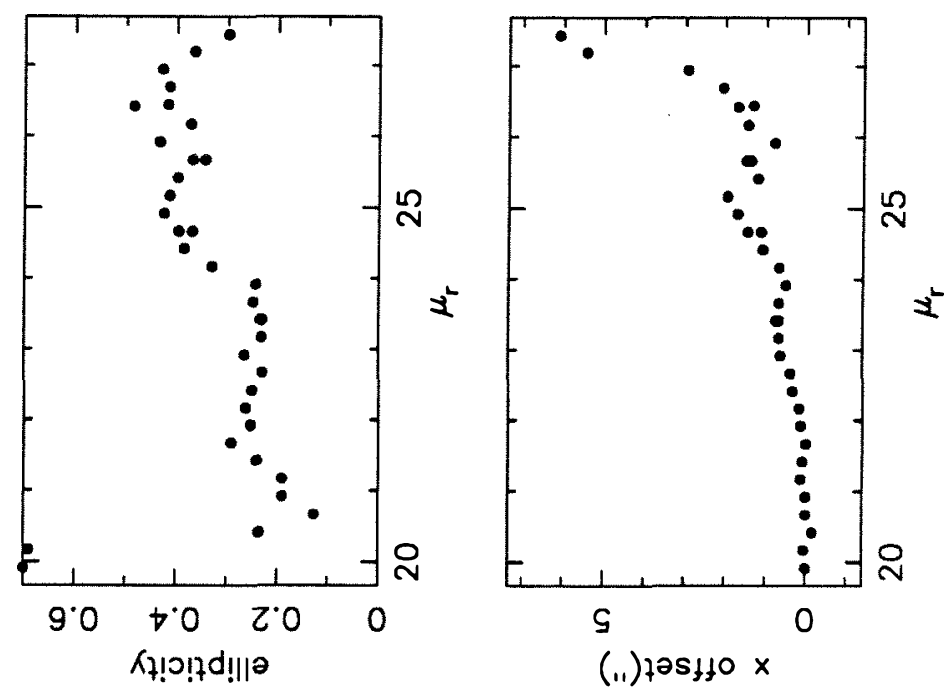


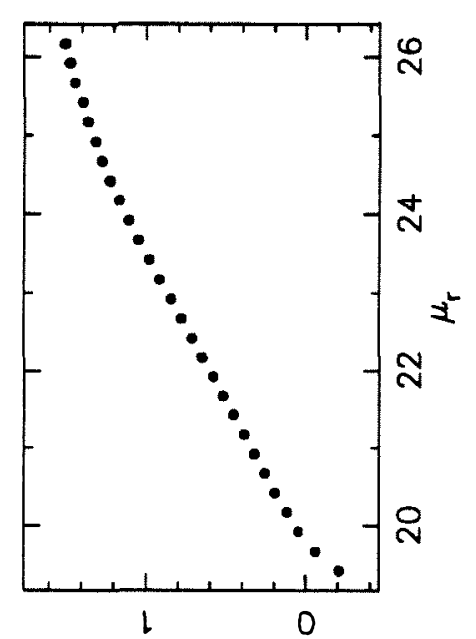

(.1)sn!pod 6ol

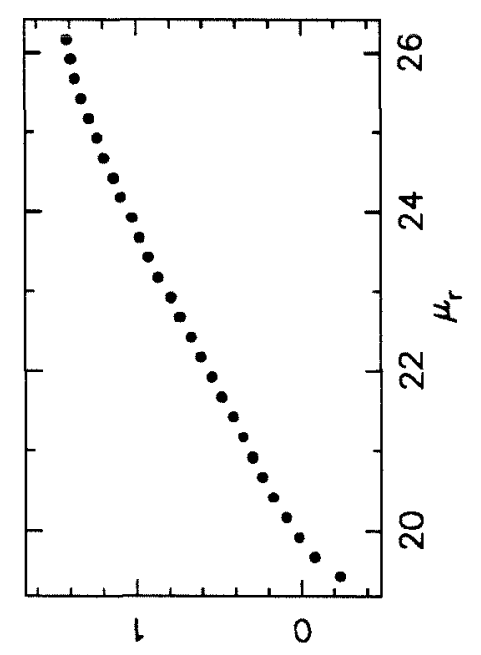

(.1) $9 \mathrm{60}$

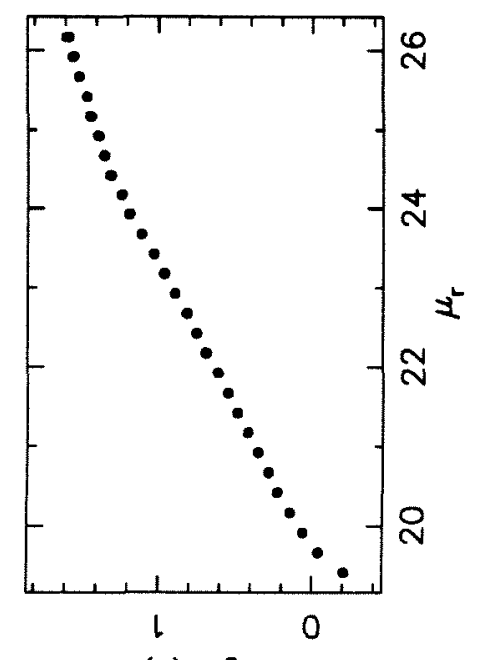

(.1) 0 601

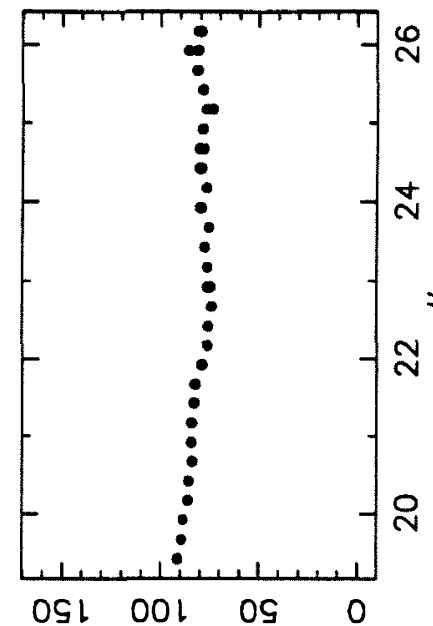

$\forall d$ s!xo so!̣om
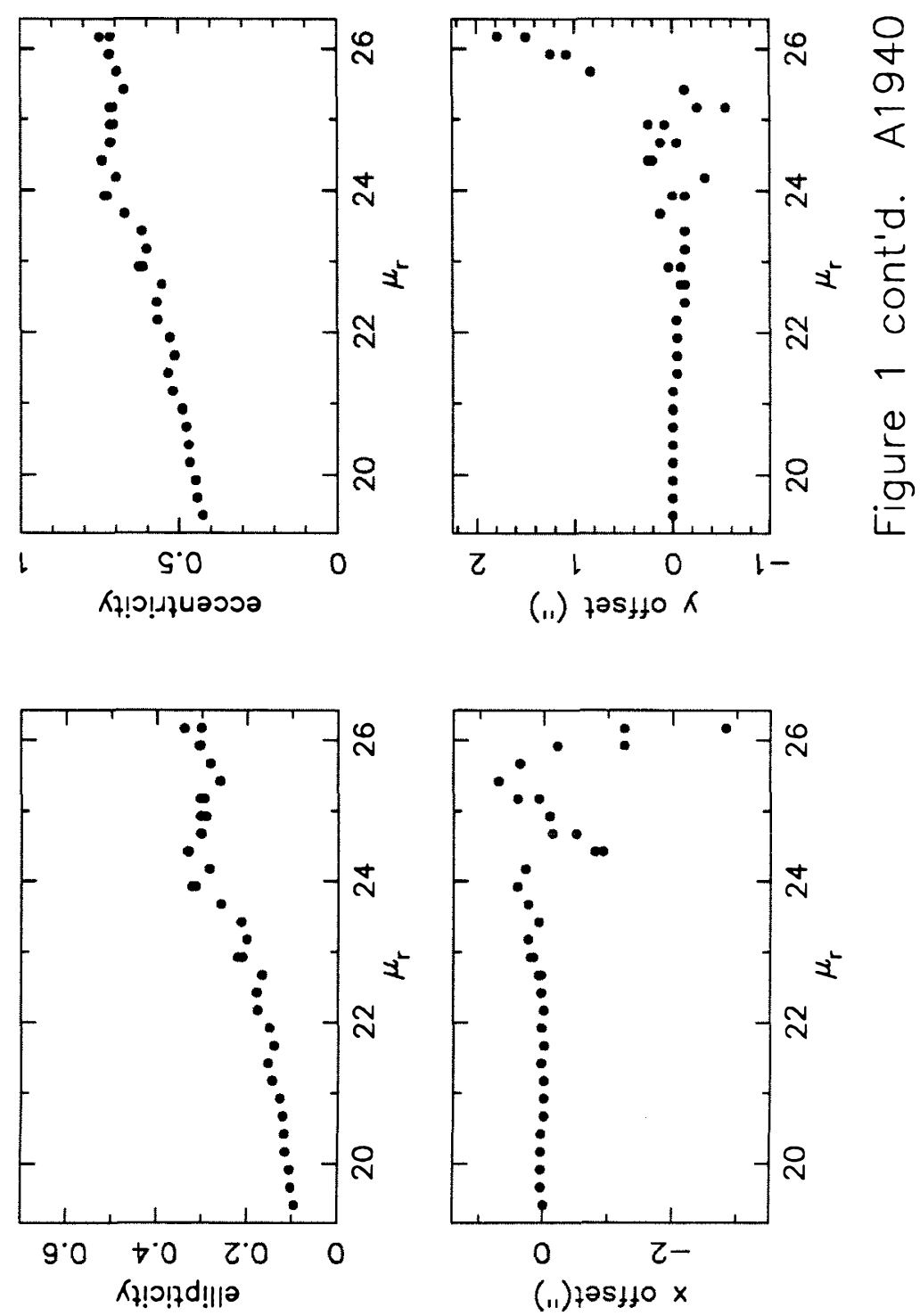


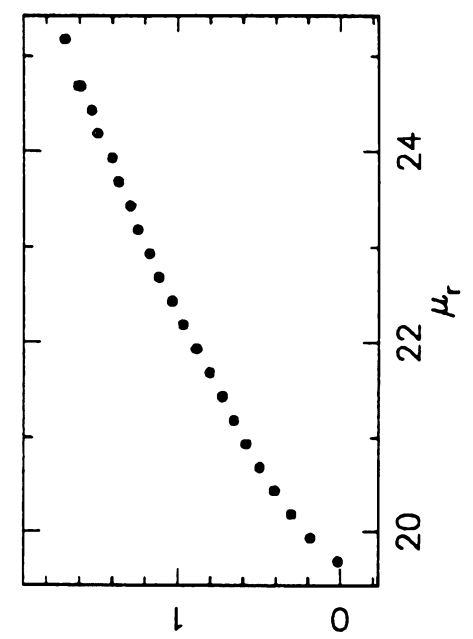

(.1) sn!pos 6ol

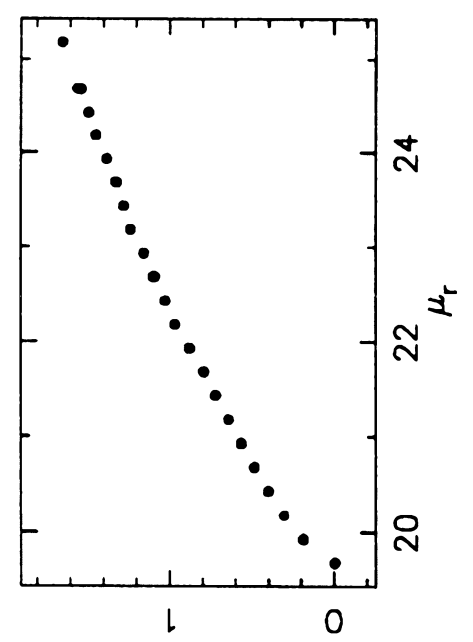

(.1) $9 \mathrm{bol}$

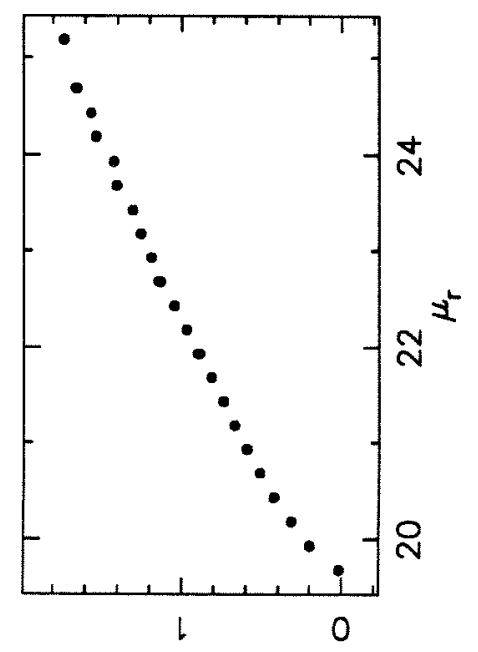

(.1) 0601

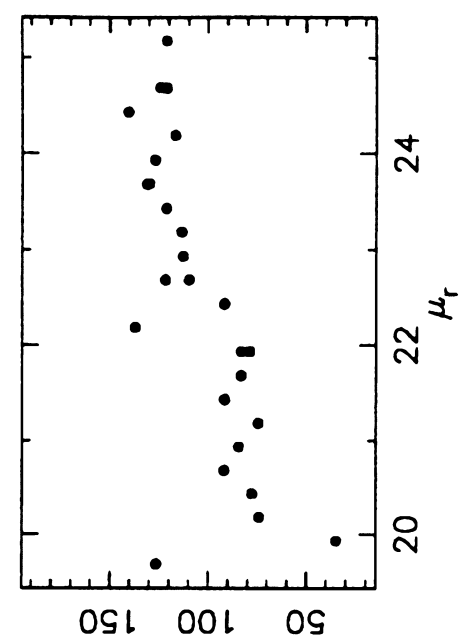

$\forall d$ s!xo so! om

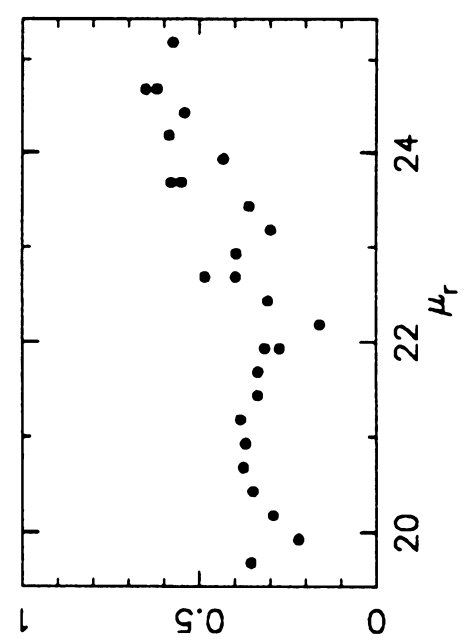

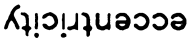

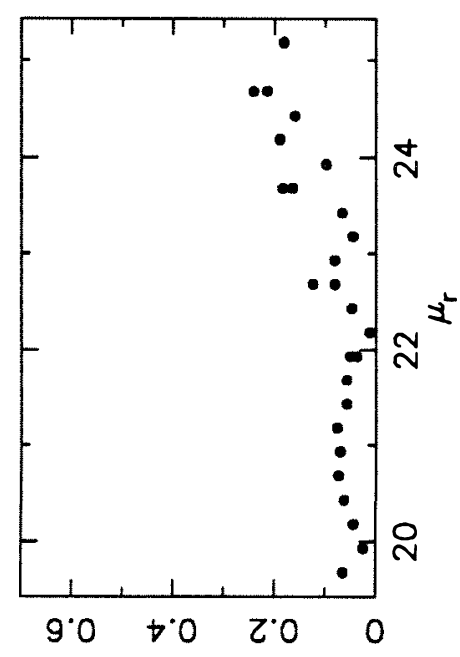

रł!?!子d!!|

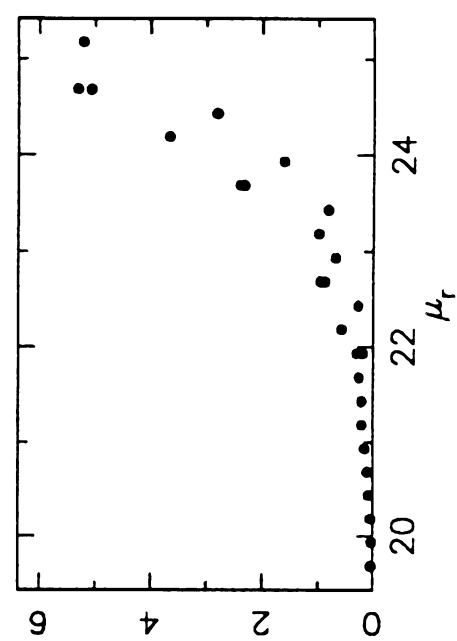

(11) $7 !+$ to oub!s auo

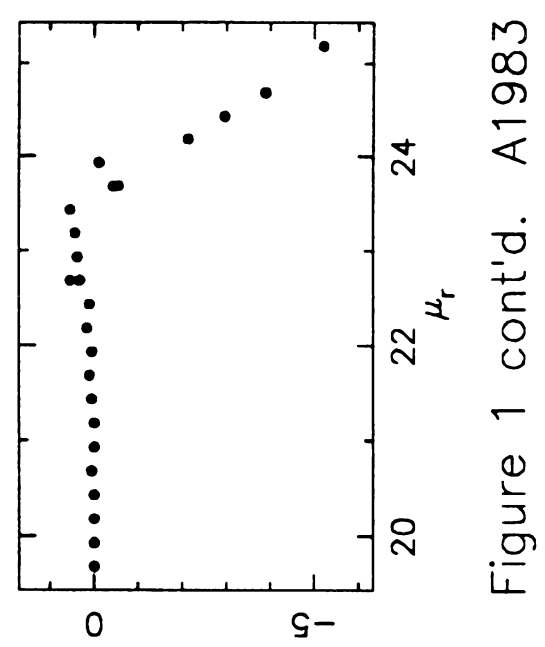

(ii) zos $110 \mathrm{~K}$

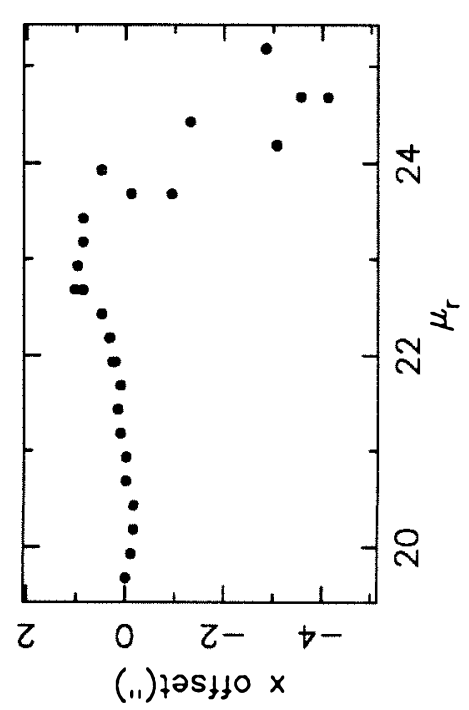




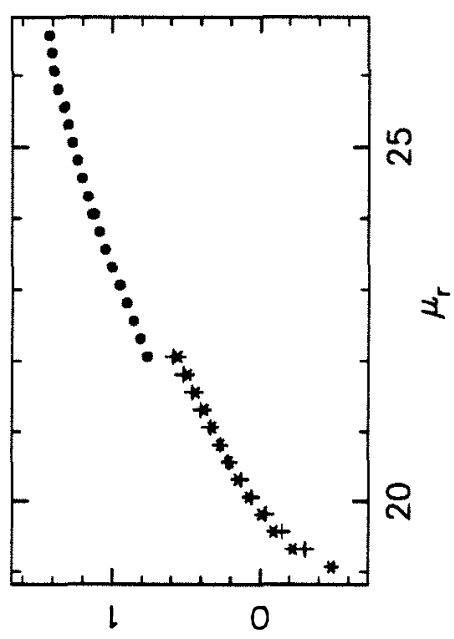

(.1)sn!pos 6ol

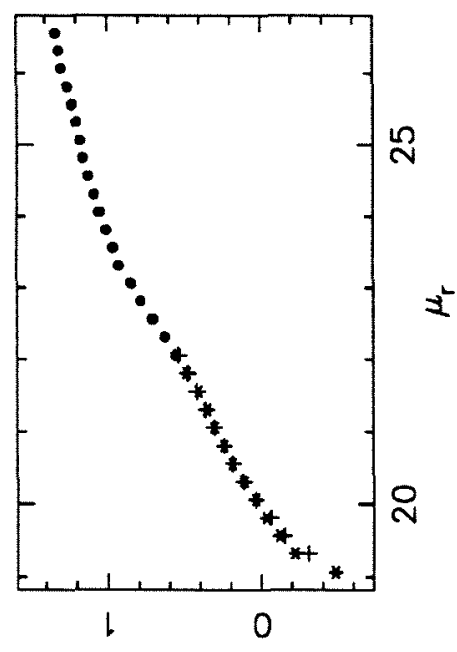

(i.) 9 601

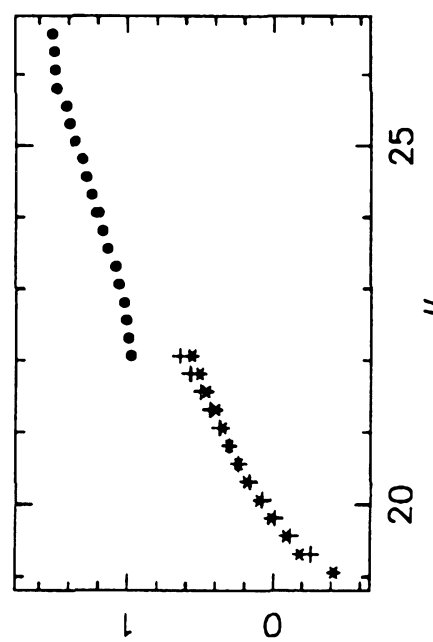

(.1) 0601

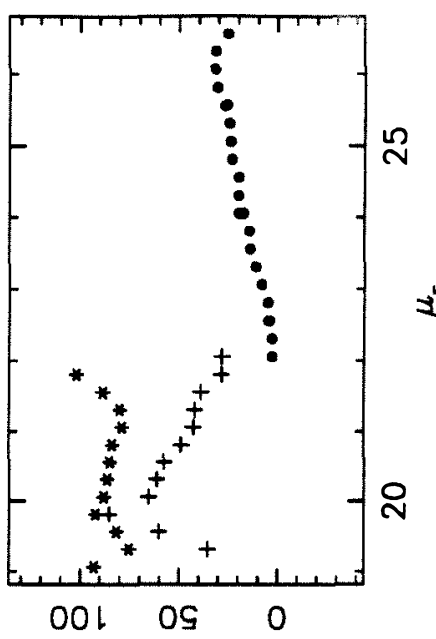

$\forall d$ s!xo do!́om
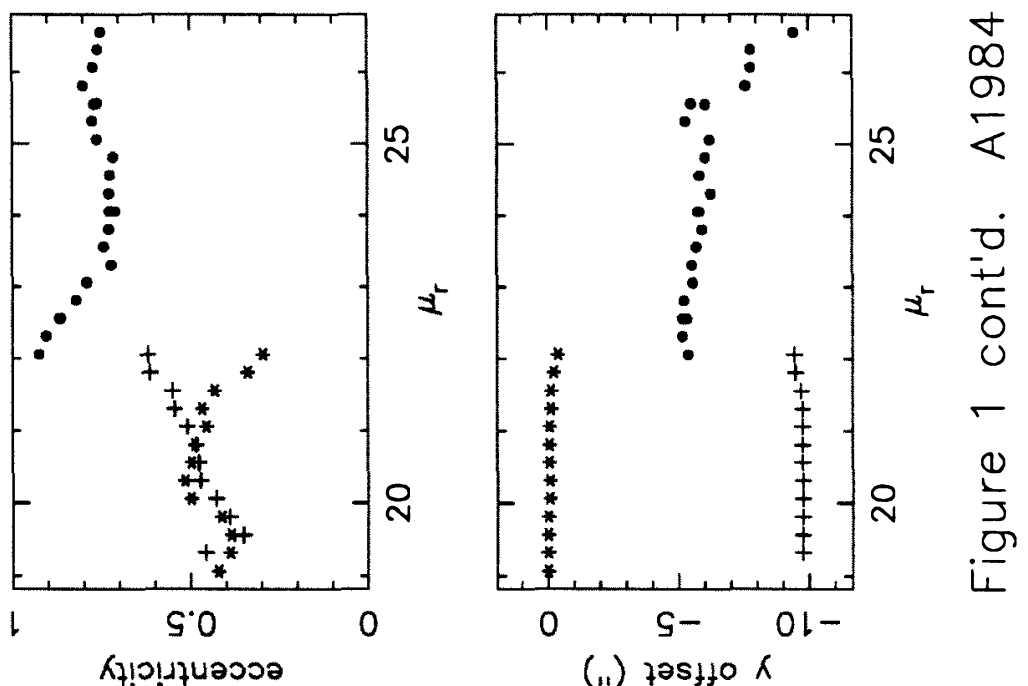

(11) $7 ! 1$ to Dub!s ano

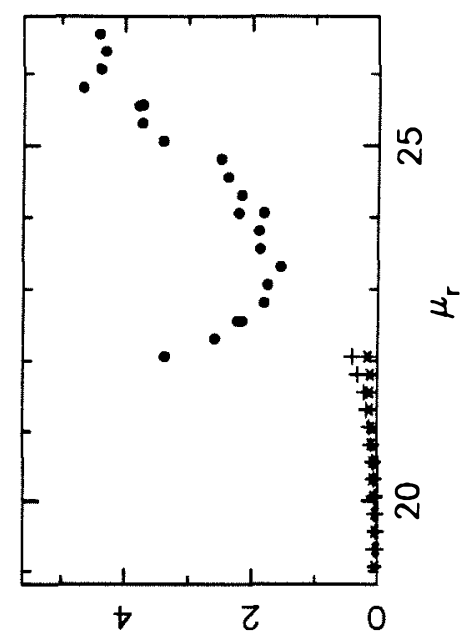

fossyo $x$
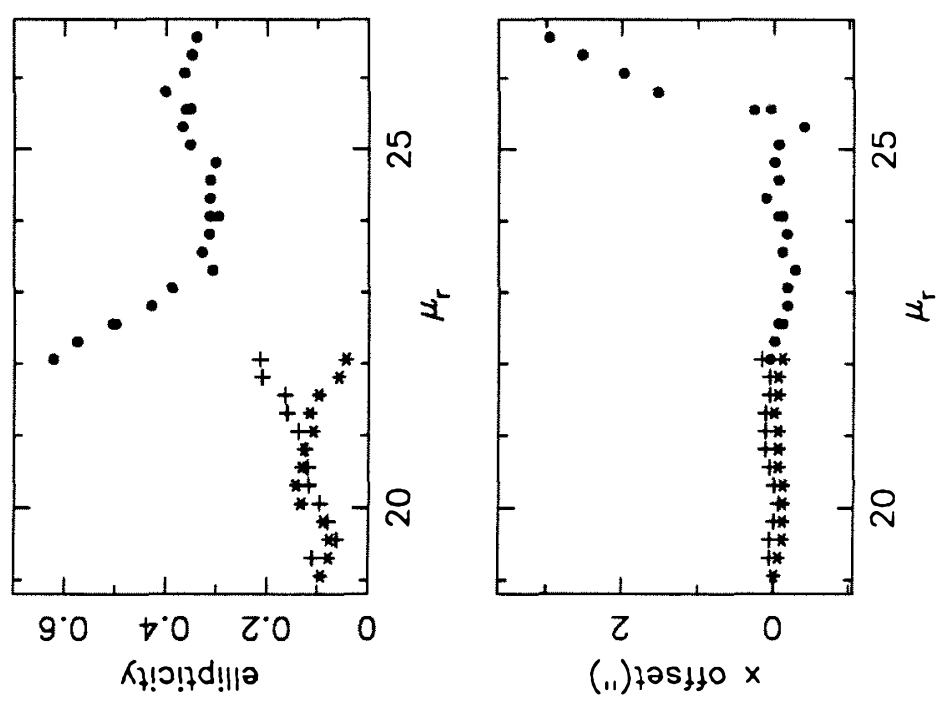


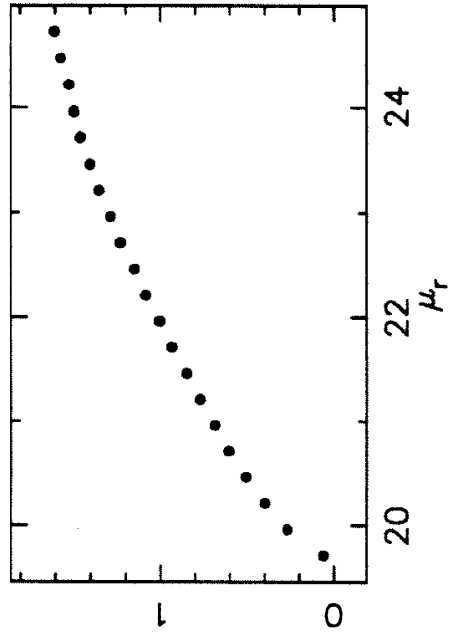

(11)sn!pod 60이
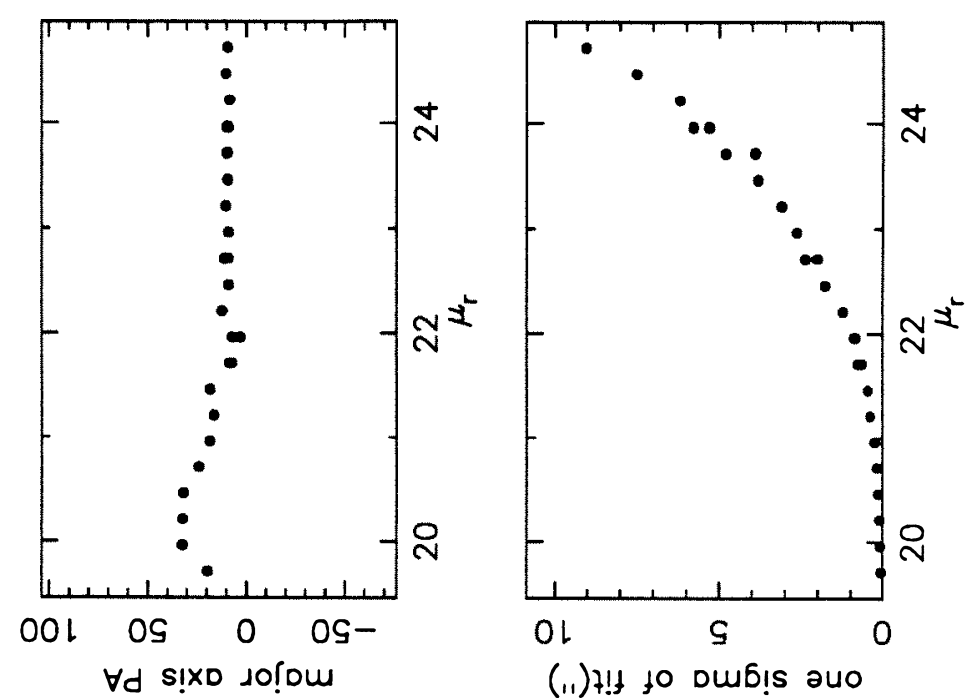

(11) $7 !+$ to Dub!s auo

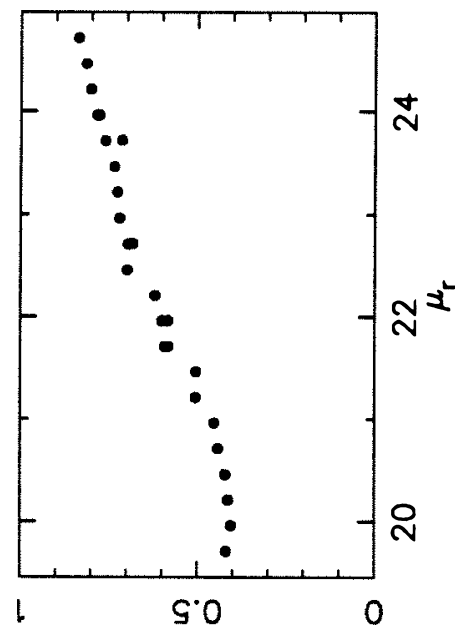

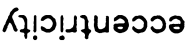

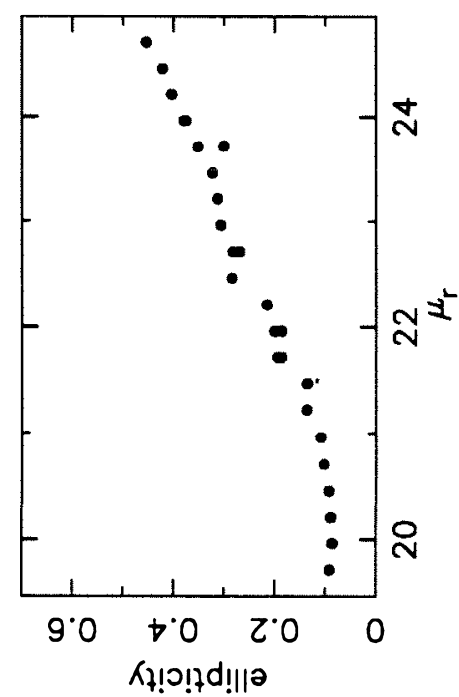

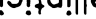
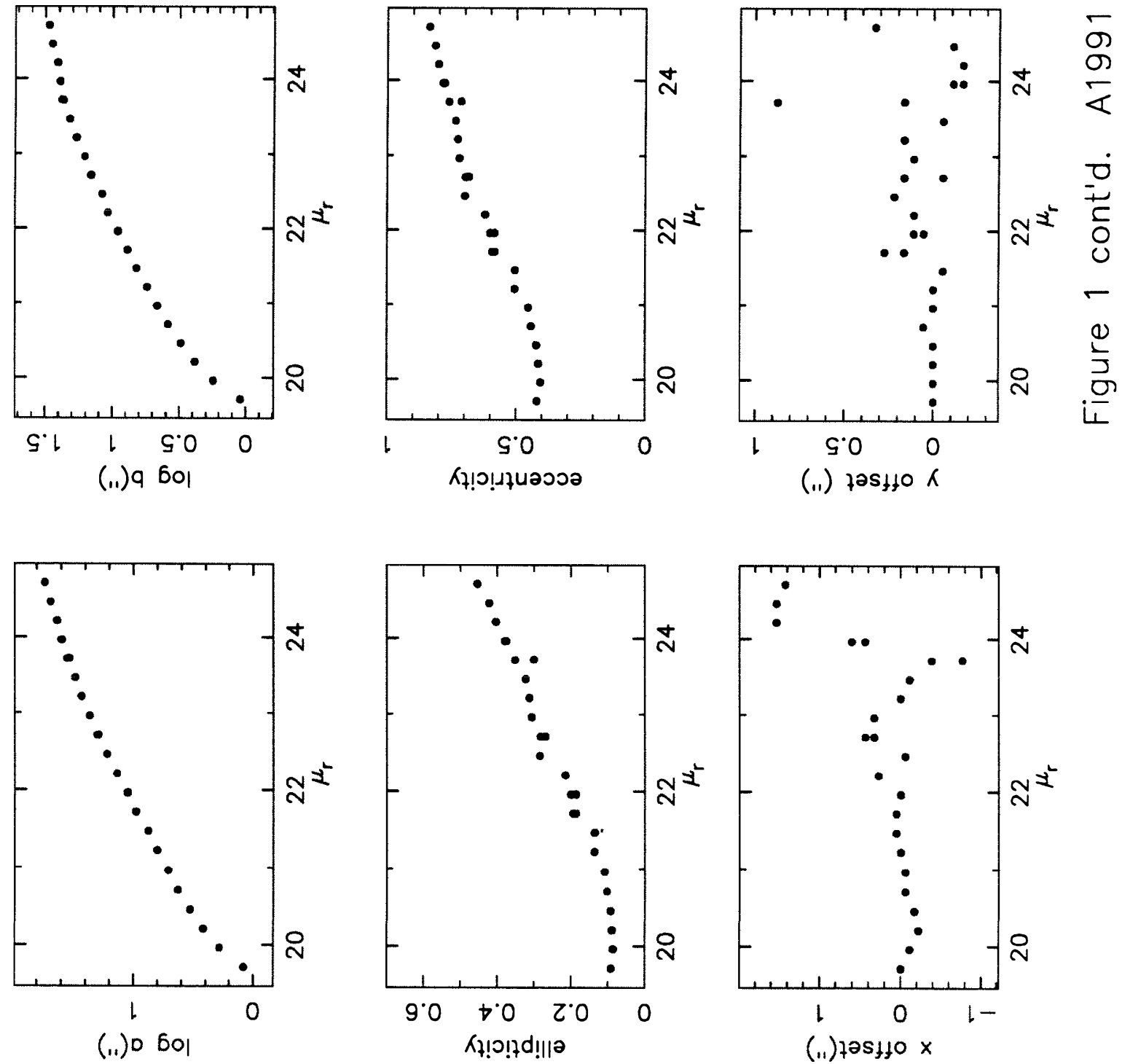

(.1) $725110 \mathrm{~K}$

(ii) tos $410 \times$ 

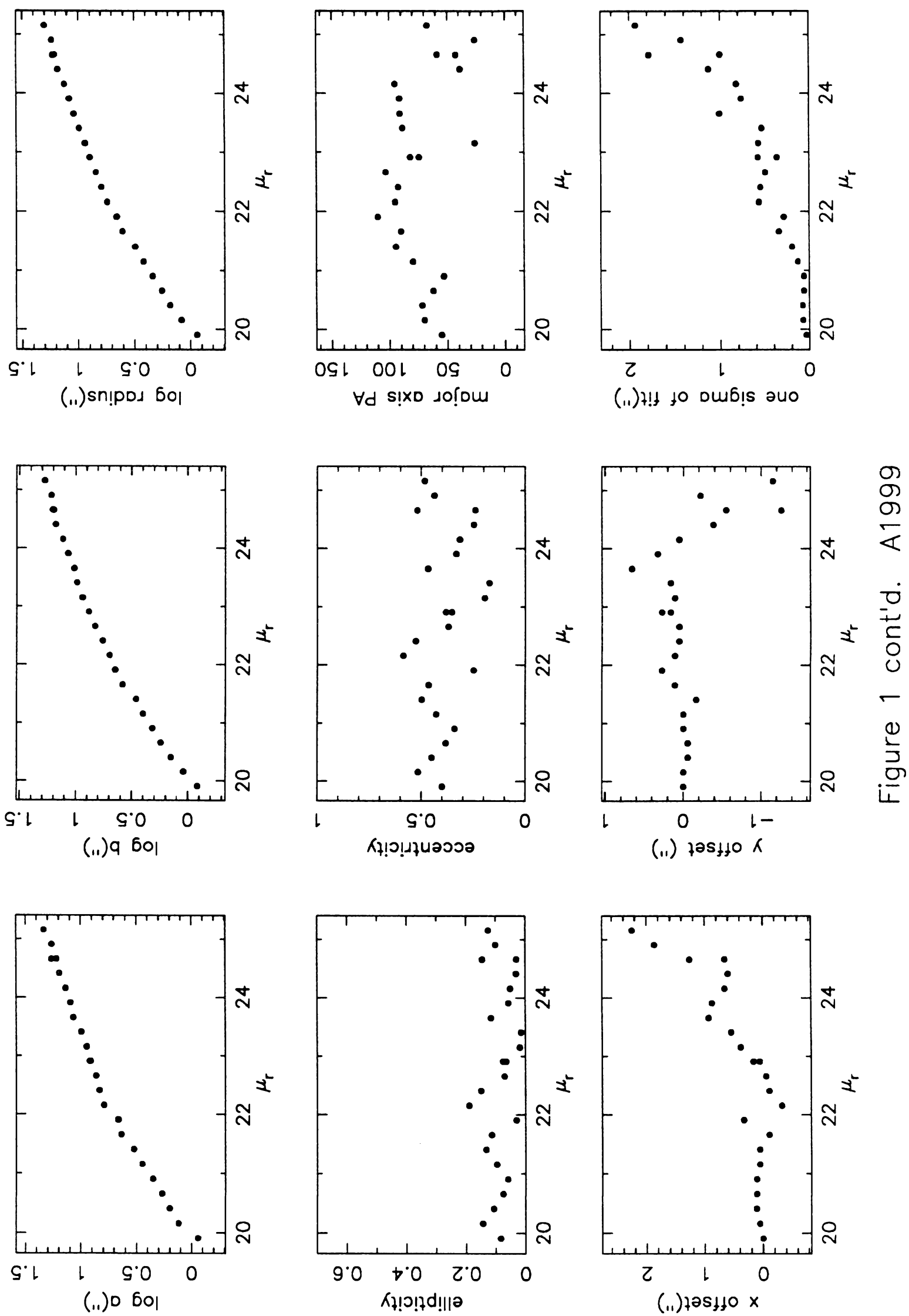


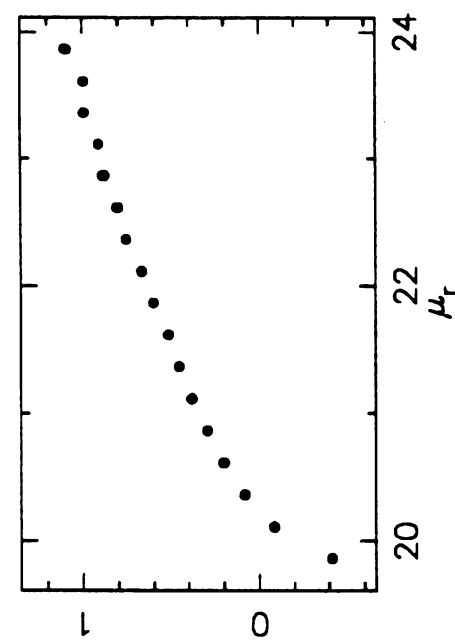

(.1)sn!pos 6ol

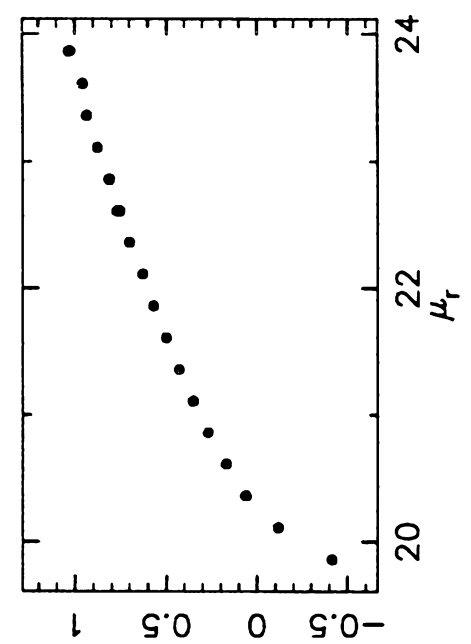

(.1) 9 6ol

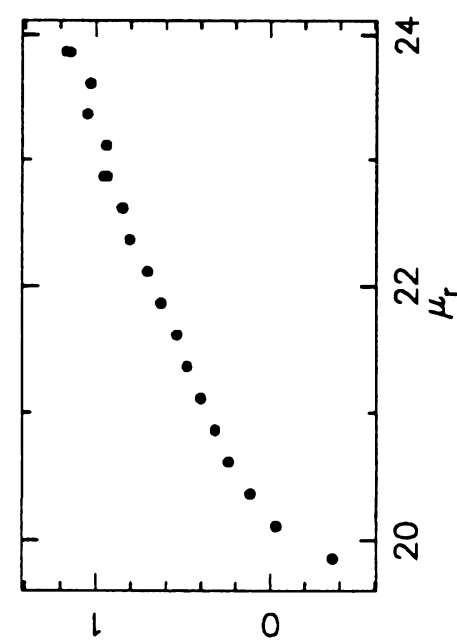

(11) $\mathrm{D} 6 \mathrm{OO}$

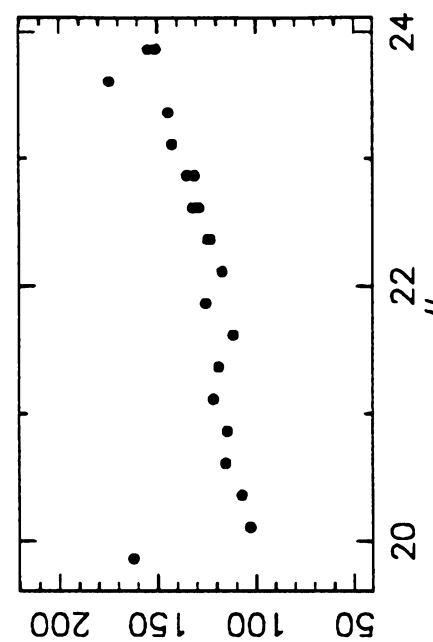

$\forall d$ s!xo do!̣om

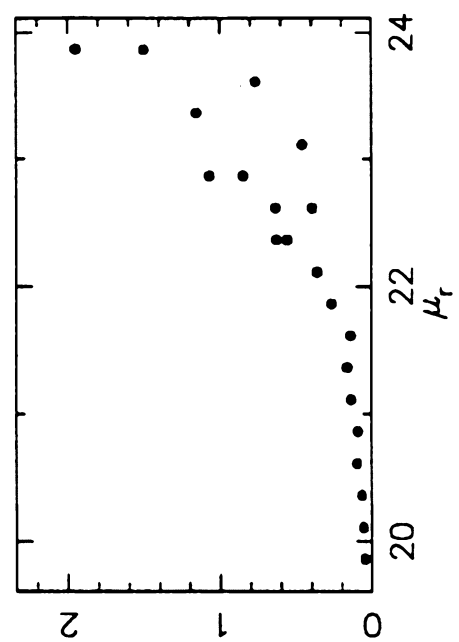

(i1) $7 ! !$ to Du6!s au०

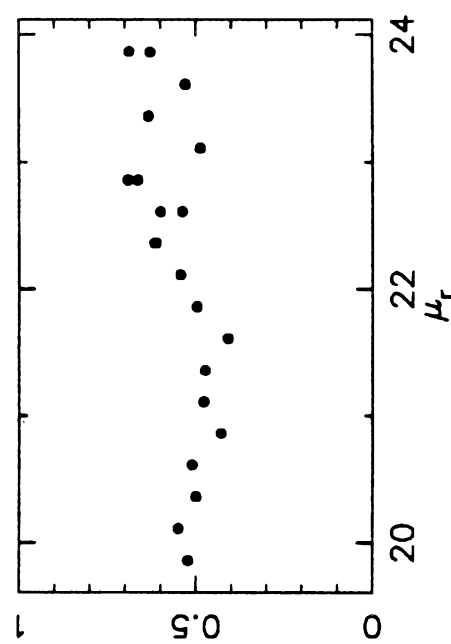

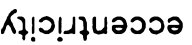

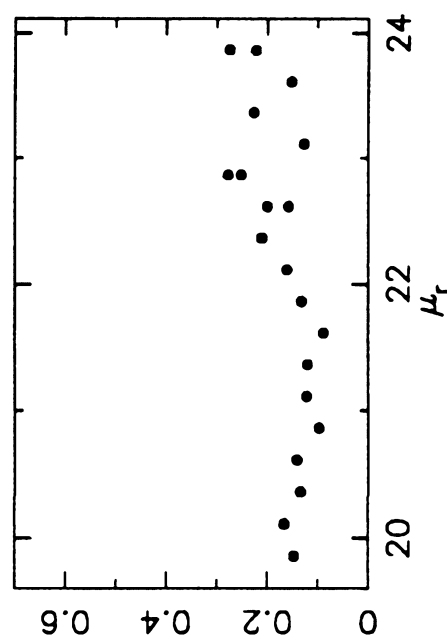

रł!?!子d!||ว

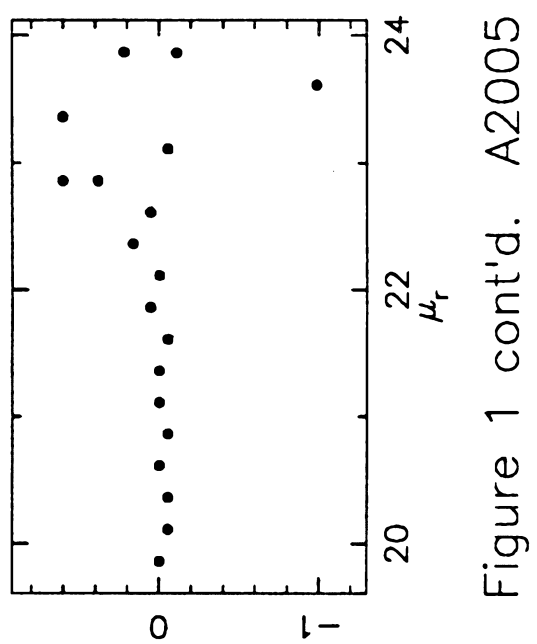

(11) tos $110 \mathrm{~K}$

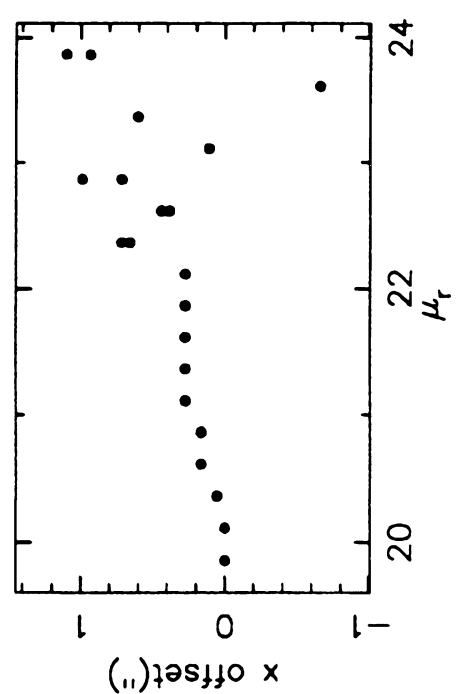



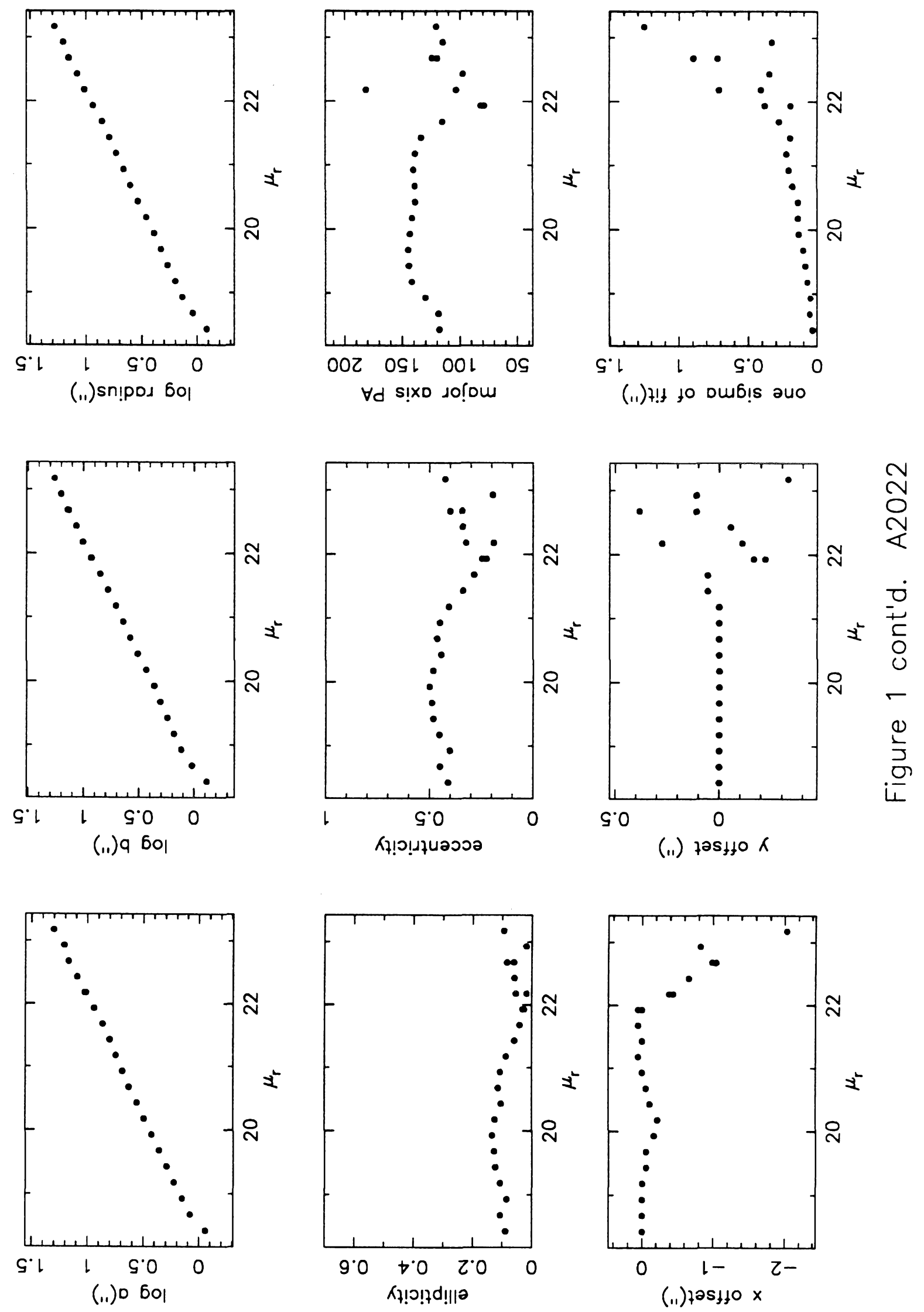


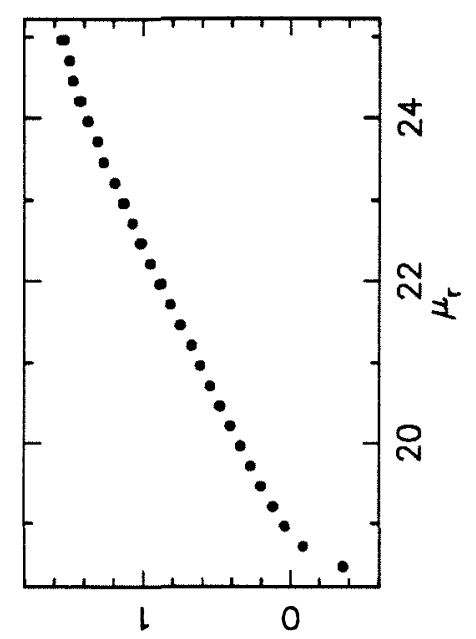

(.1) sn!pos 6ol

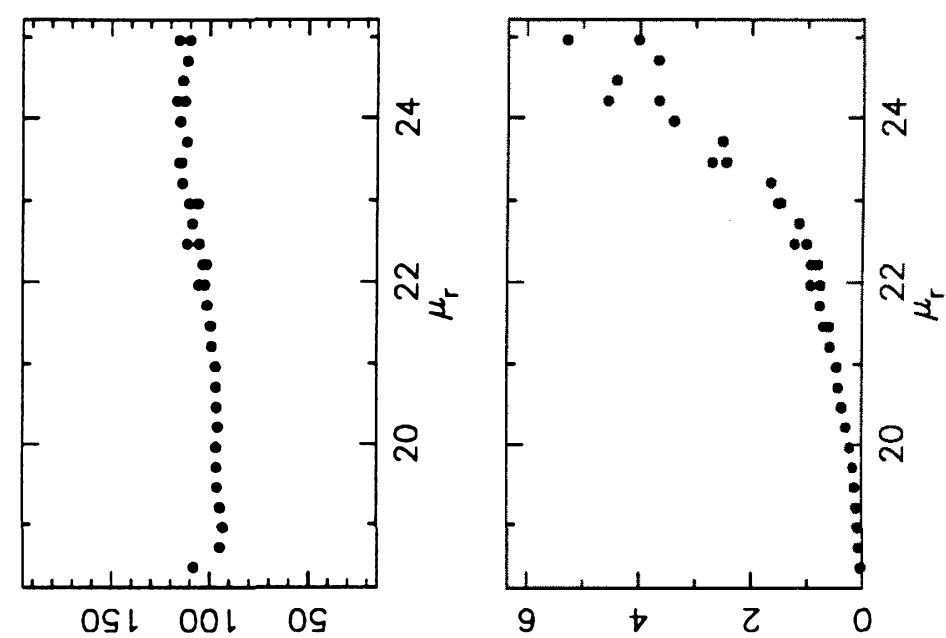

$\forall d$ s!xo دo! om

(11) 7 ! t to Dub!s avo

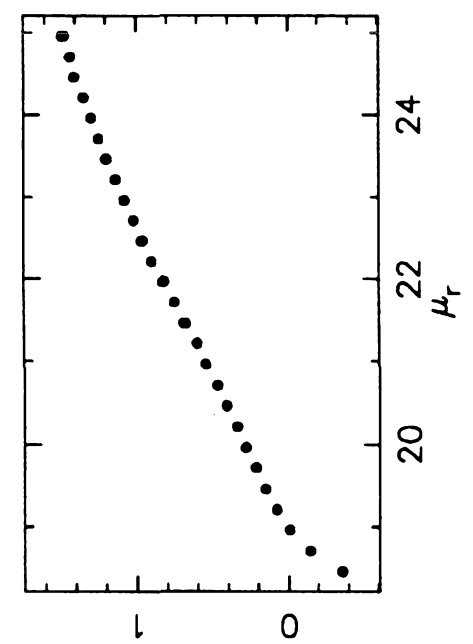

(.1) 9601

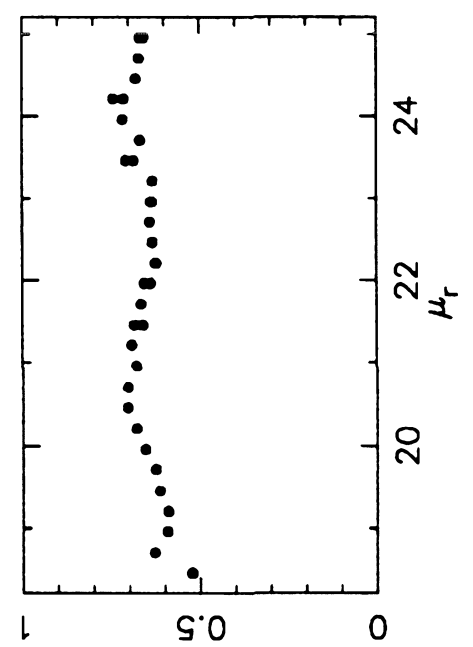

Kұ!ว!นใบวัวа

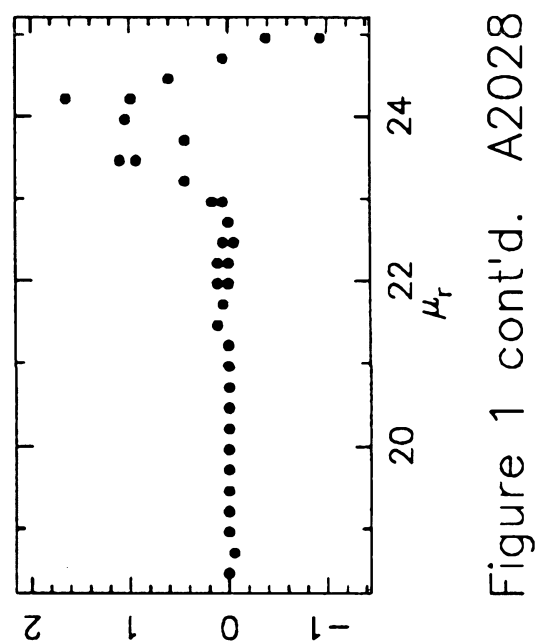

(ii) $725110 \mathrm{~K}$

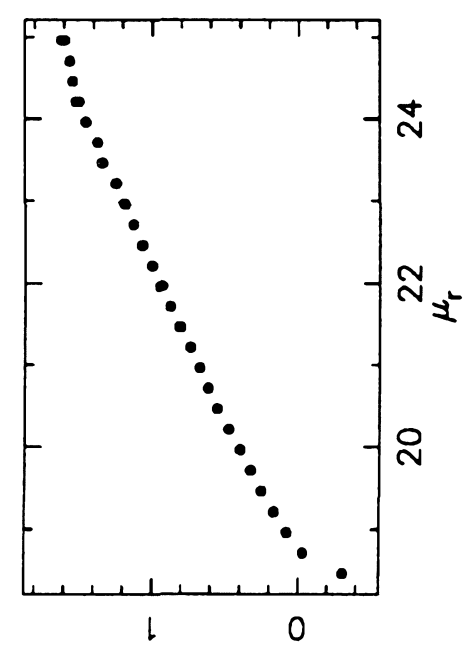

(..) 0 601
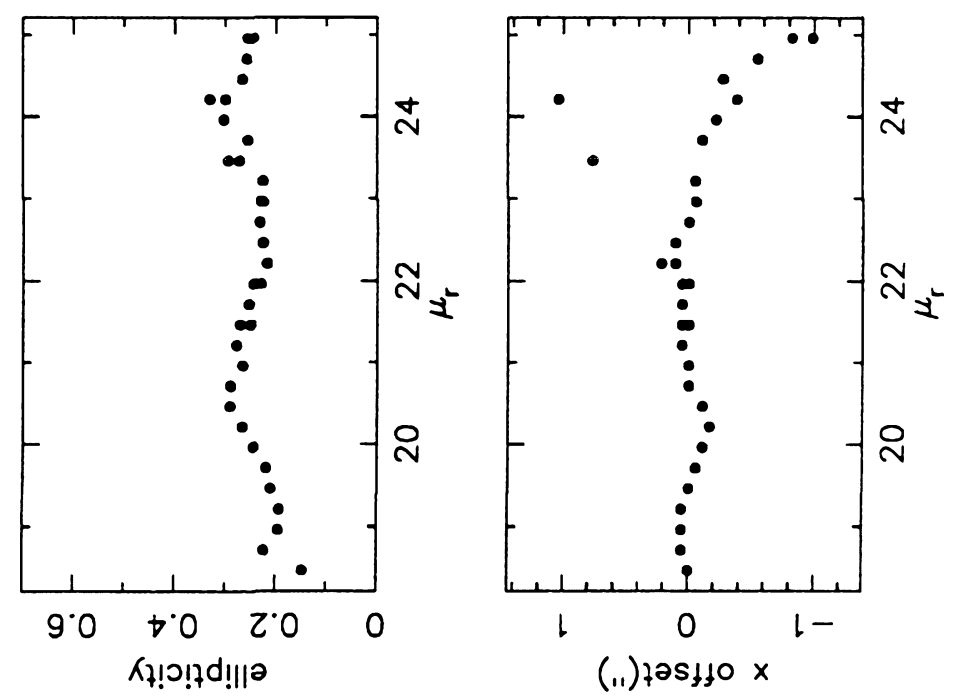

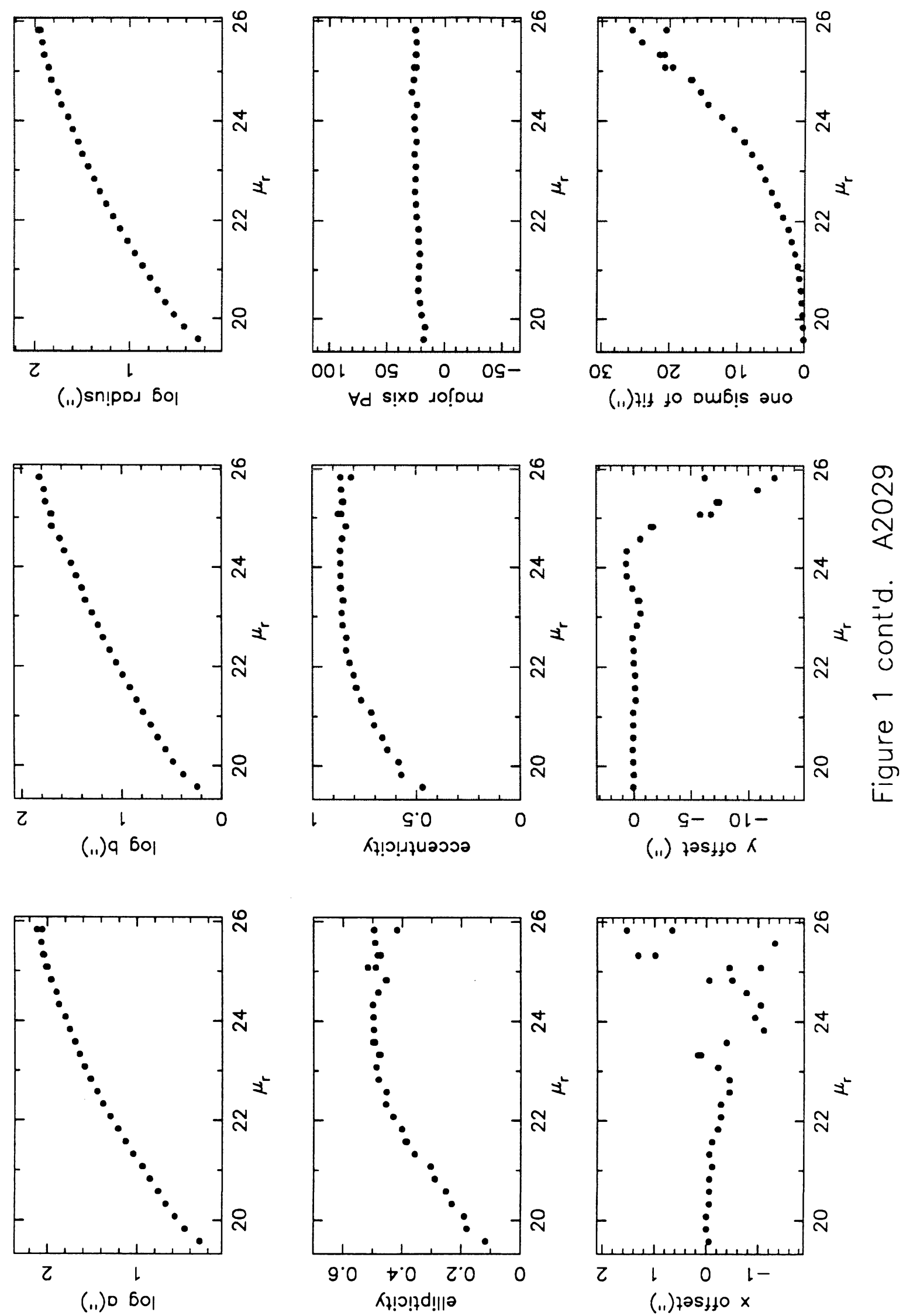

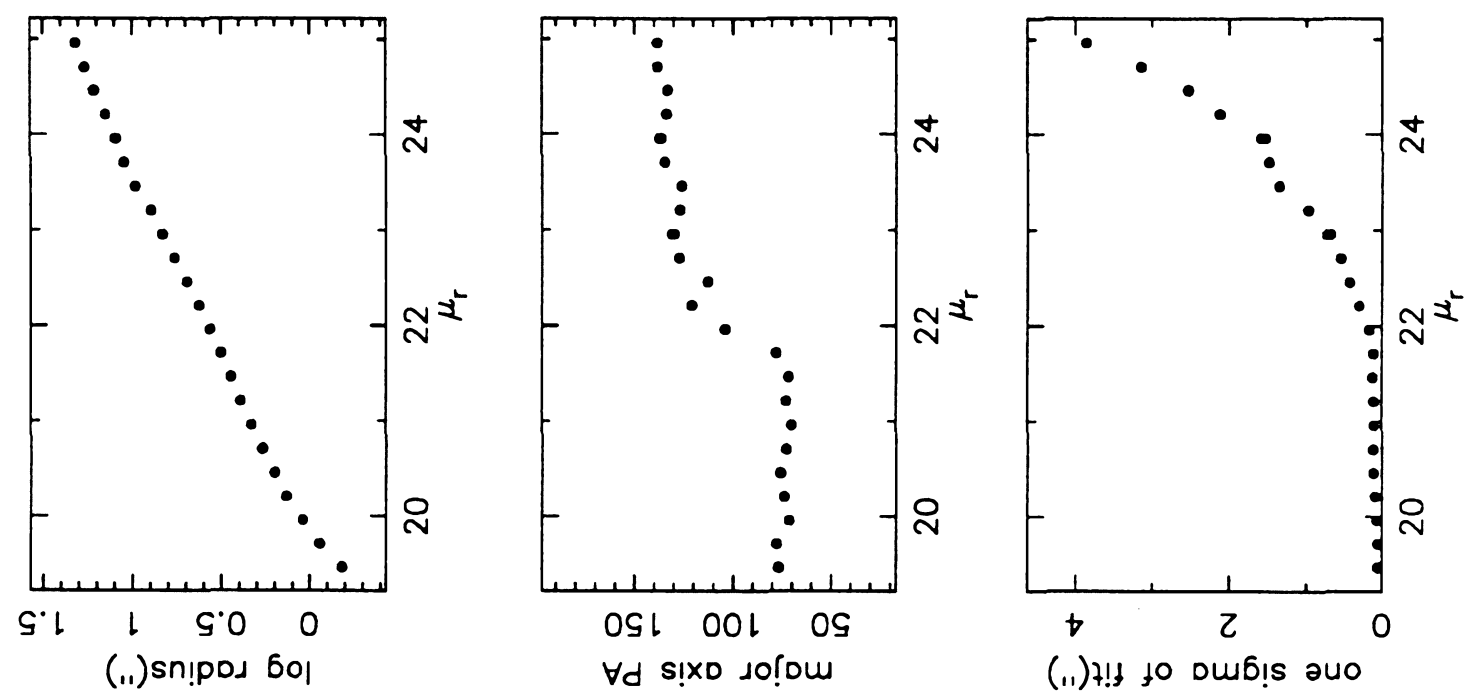

(.1) $7 ! \$$ to oub!s auo
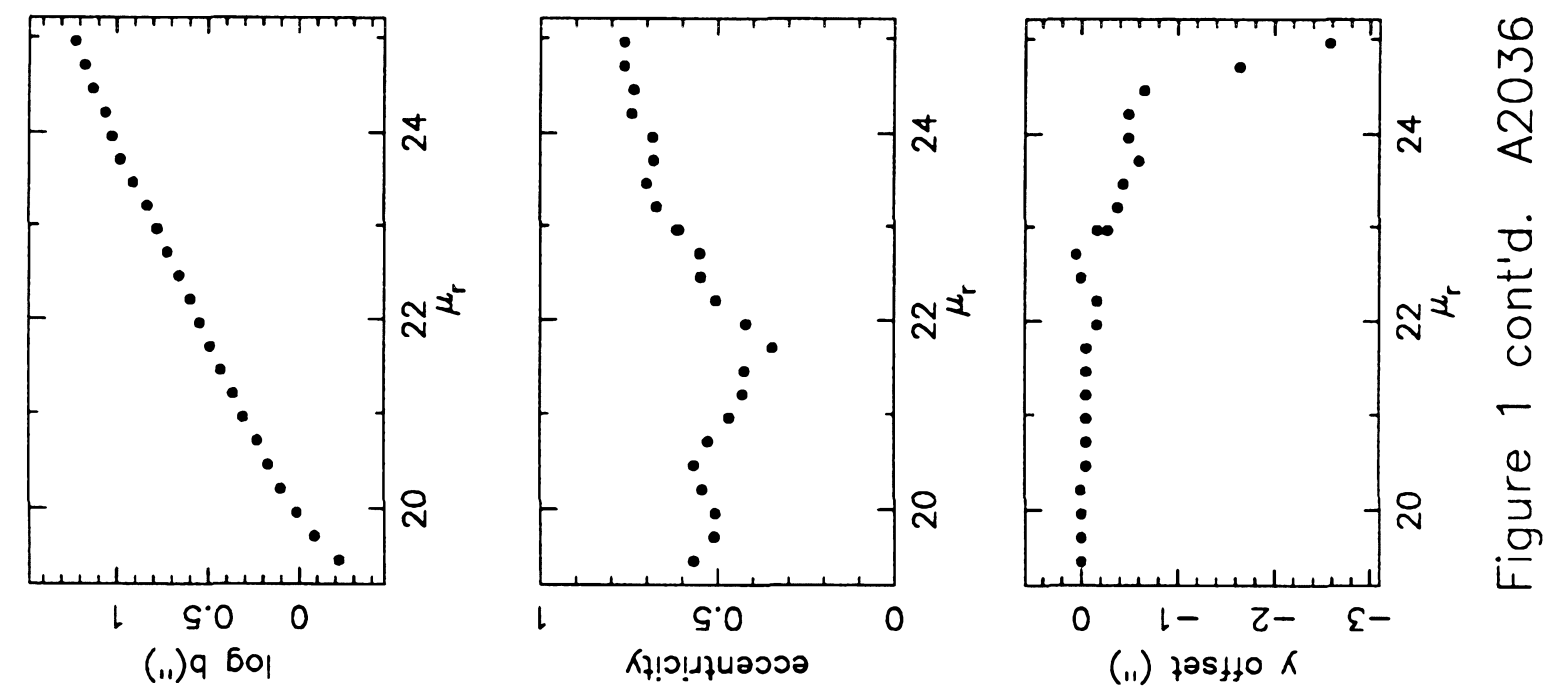

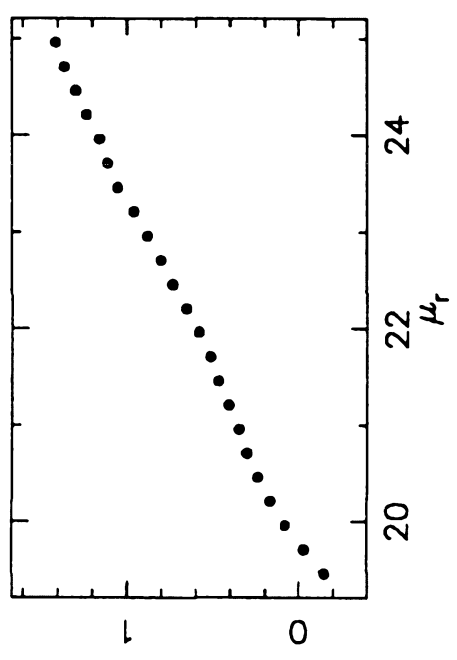

(.1) $0 \mathrm{bol}$
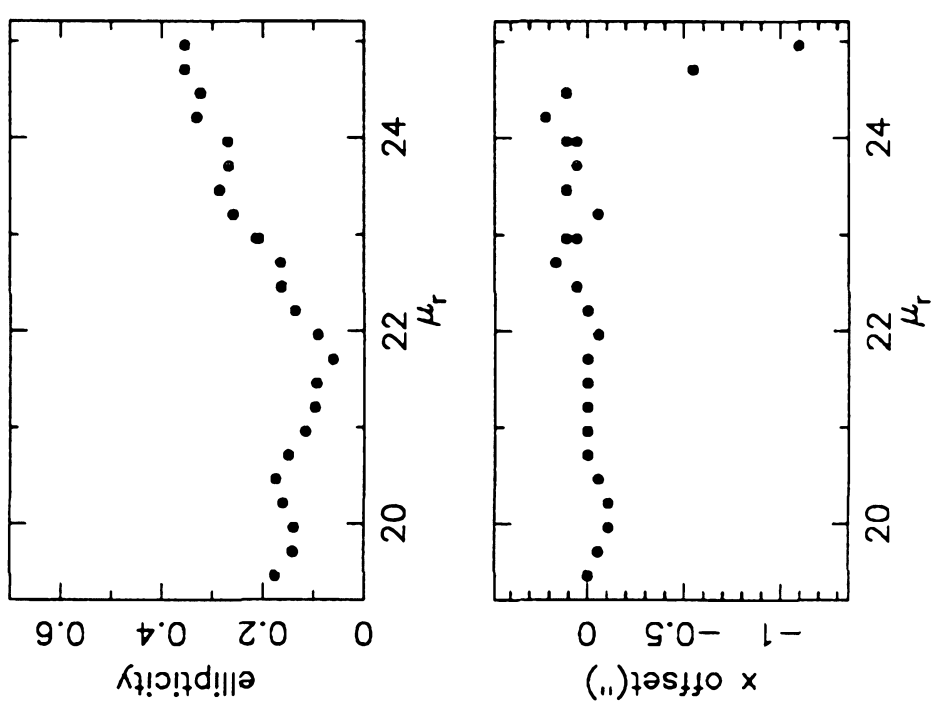


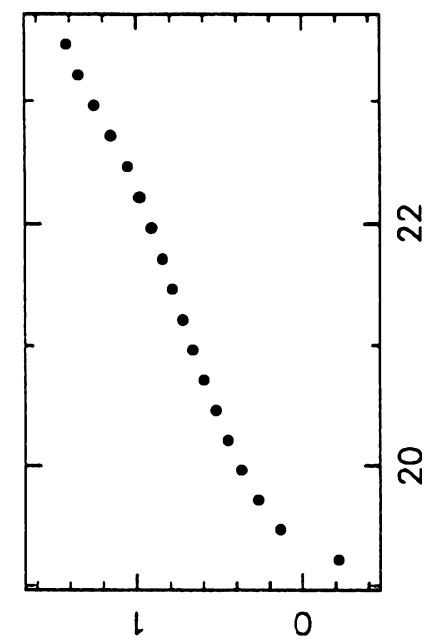

(.1) sn!pod 60।

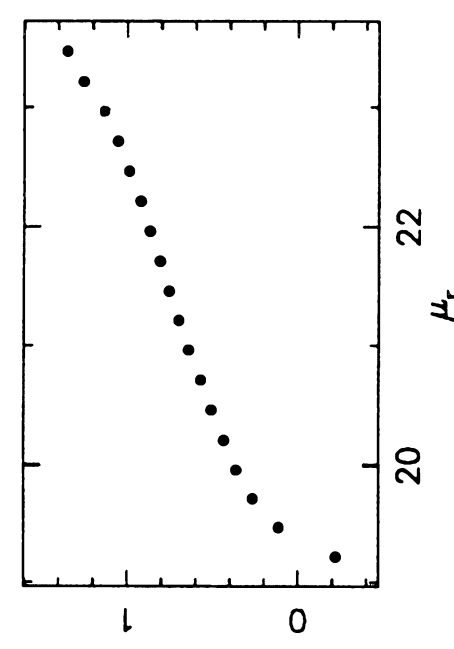

(ii) 9601

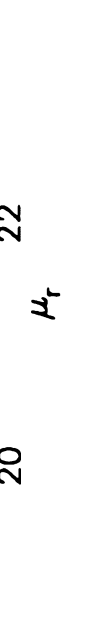

$\forall d$ s!xo so!om

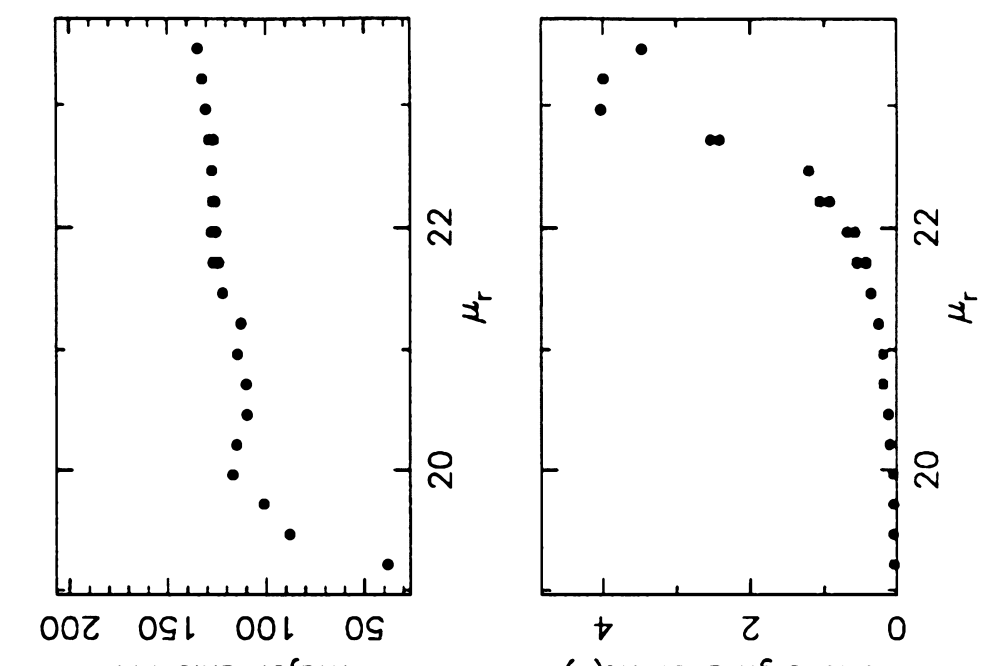

(i1) 4 !! to ou6!s aน०
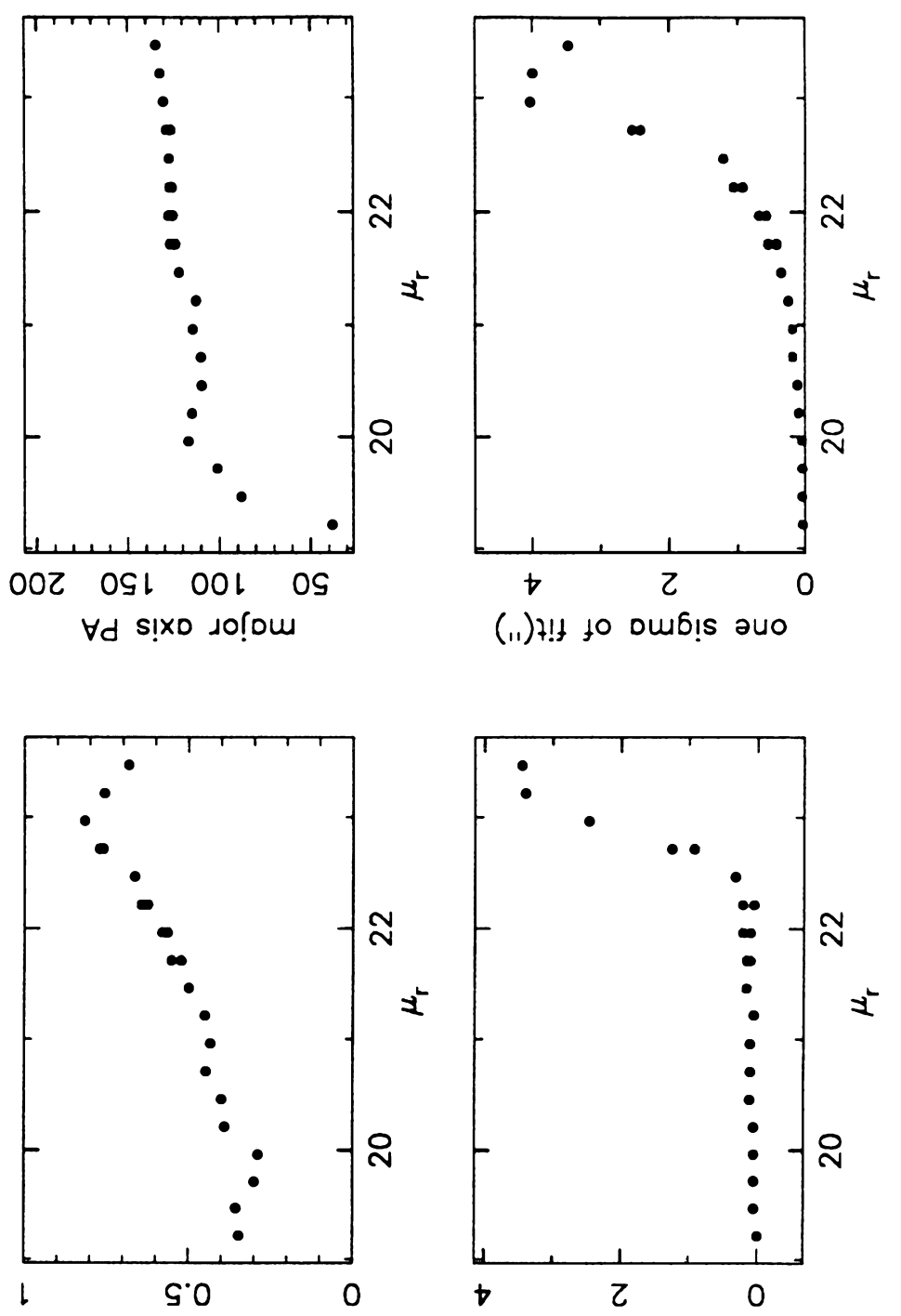

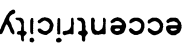
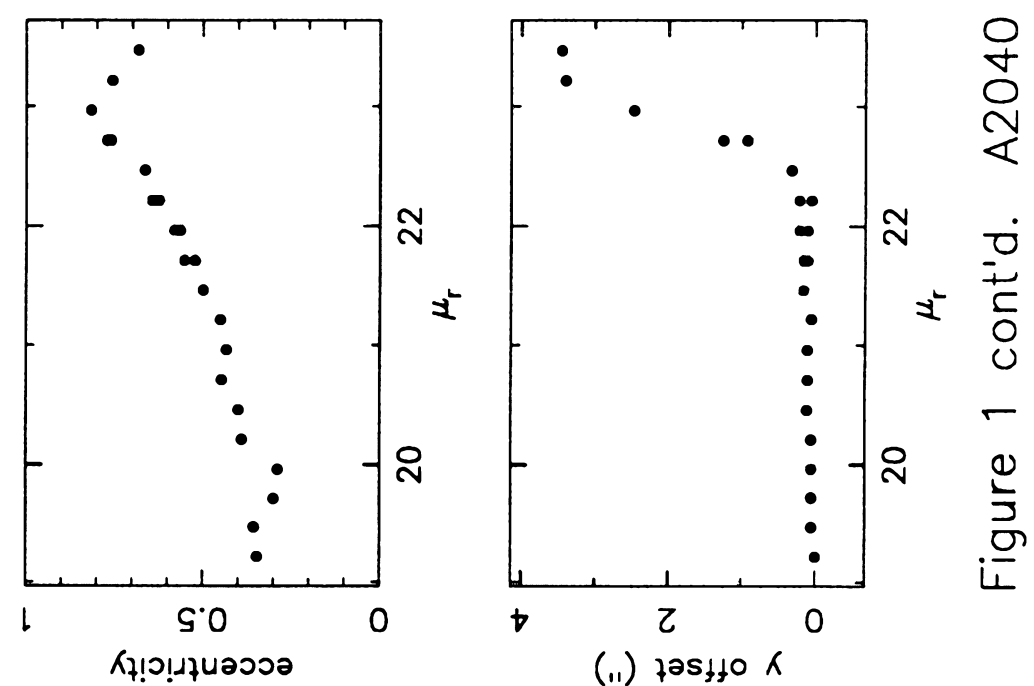

(.1) $725 \% 10 K$

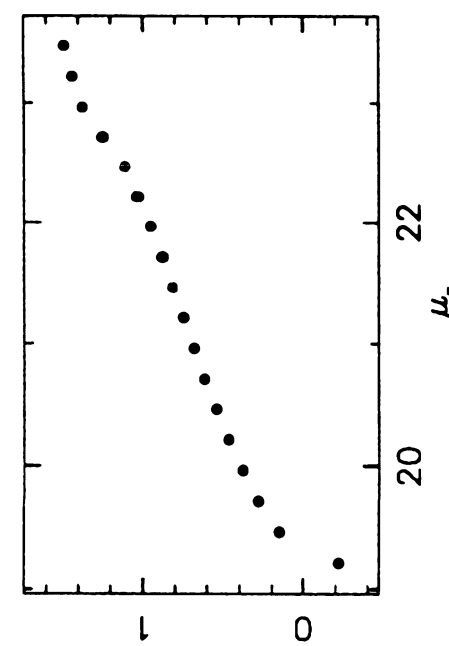

(11) $0 \mathrm{bol}$
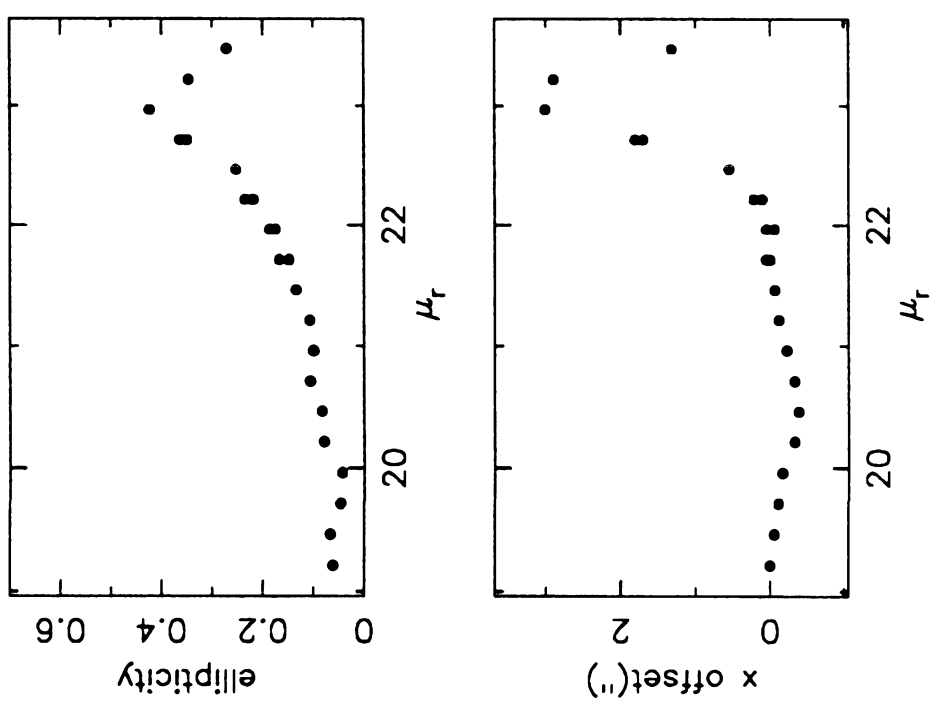


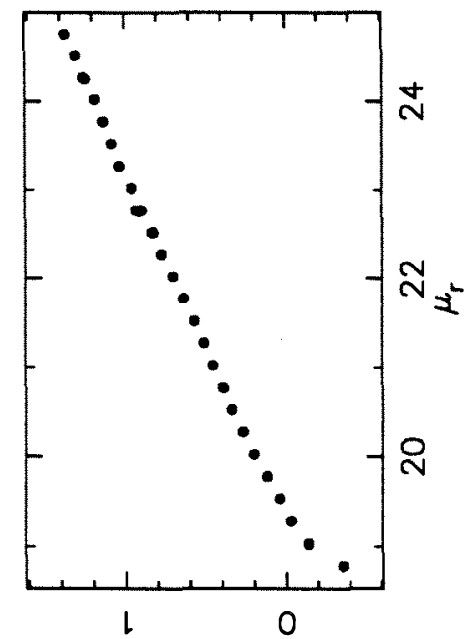

(.1)sn!pod 6ol

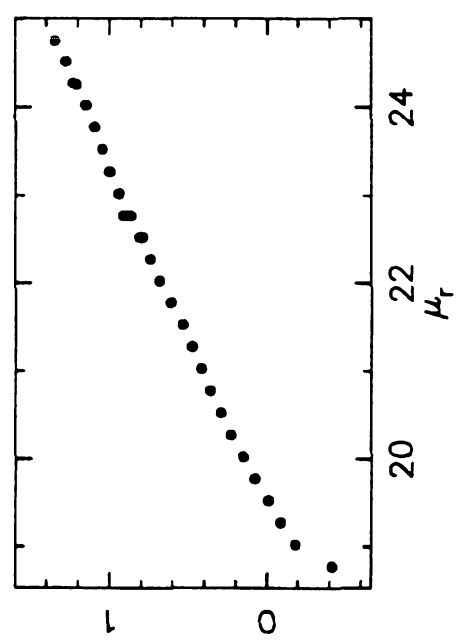

(.1) $9 \mathrm{bol}$

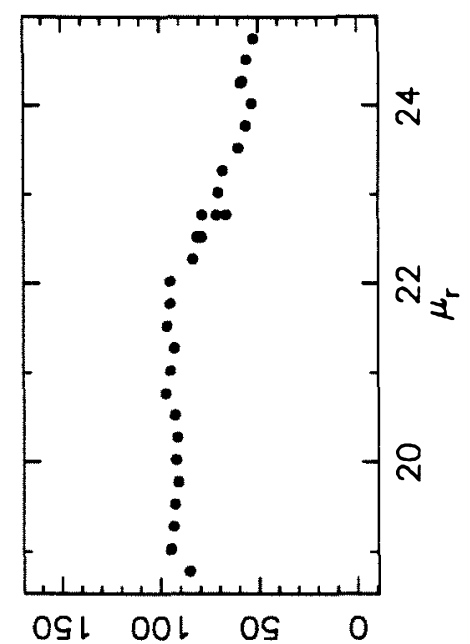

$\forall d$ s!xo do!̣um

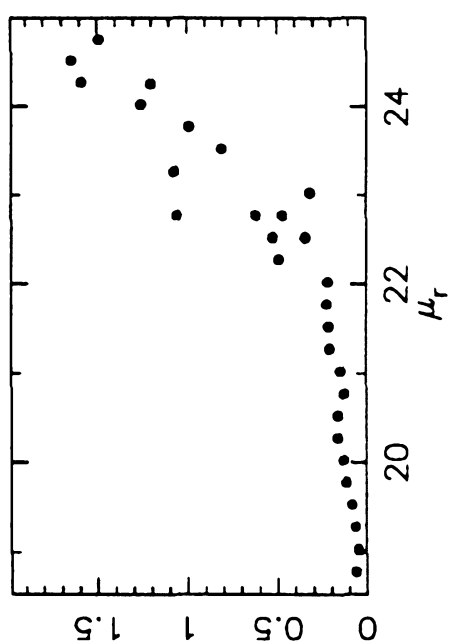

(i1) 4 !t to Dub!s auo

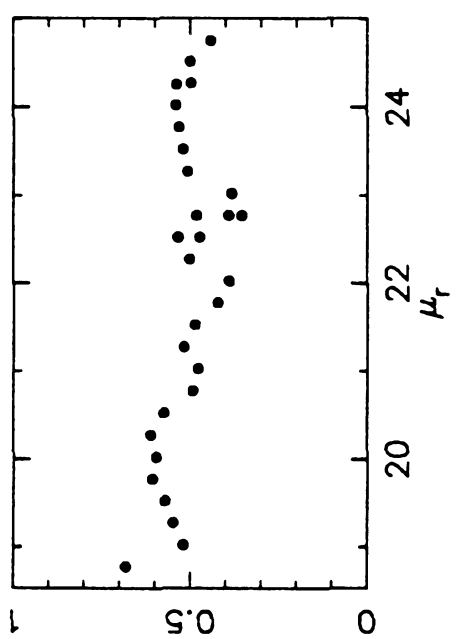

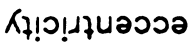

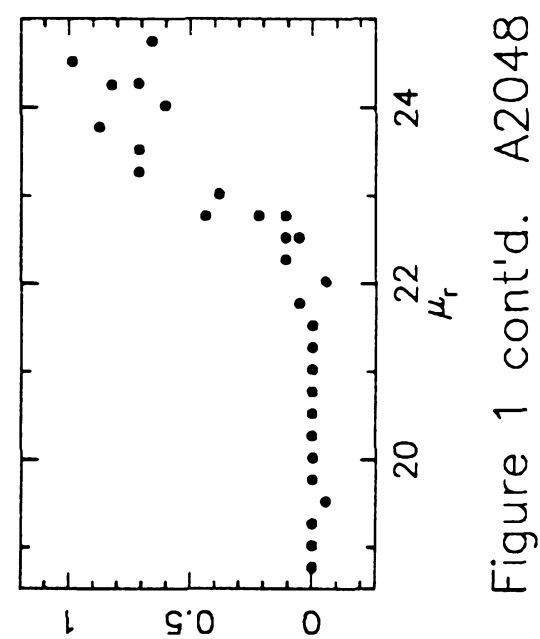

(ii) tos $750 \mathrm{~K}$

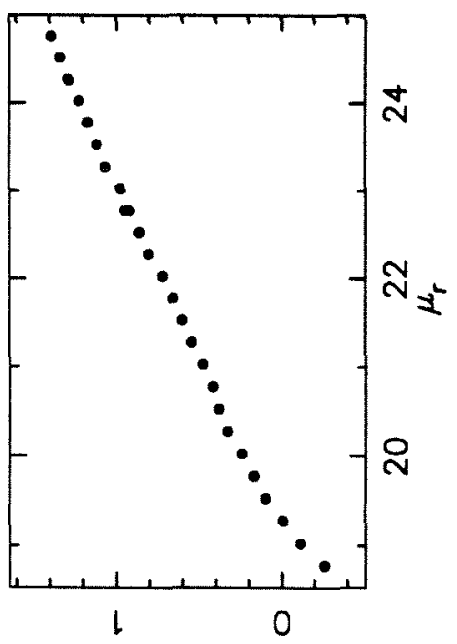

(ii) $\mathrm{b}$ 601
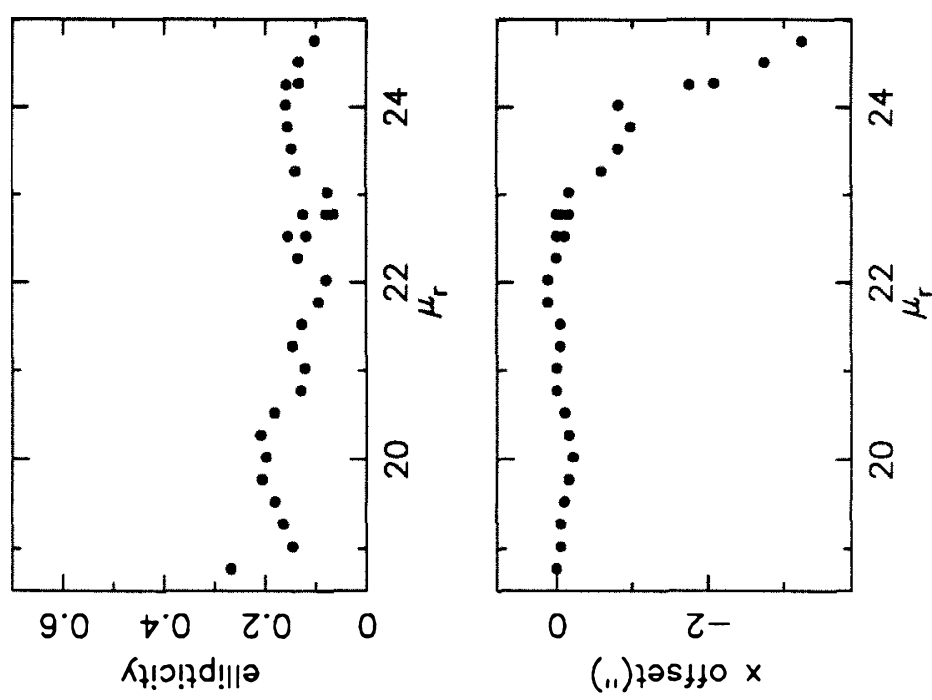

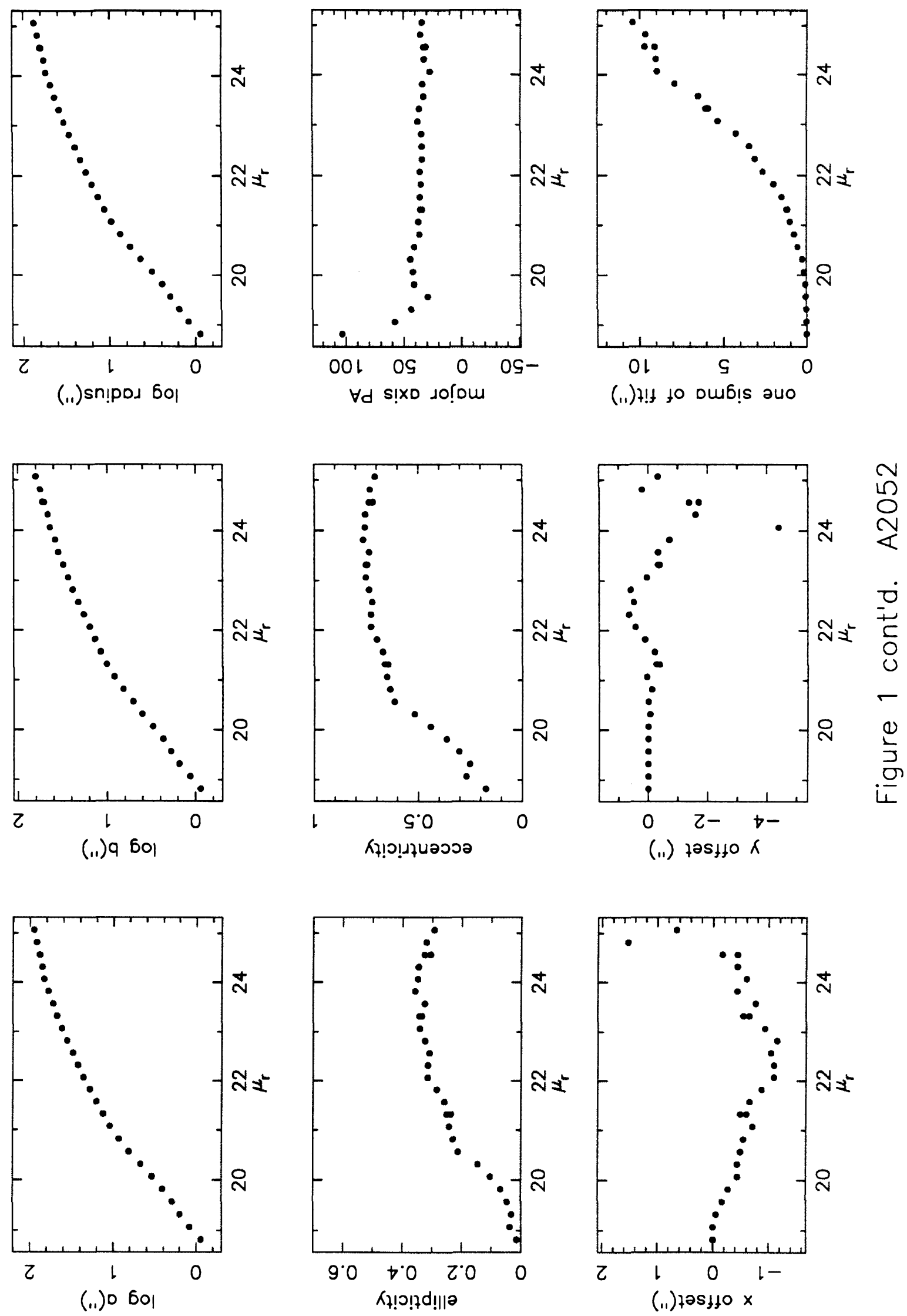


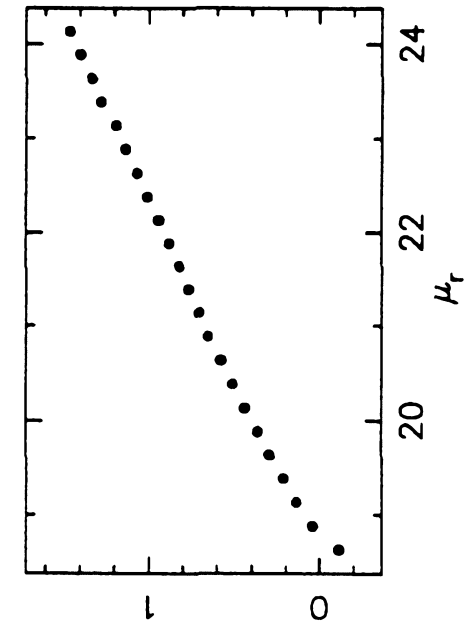

(.1)sn!pos 60।
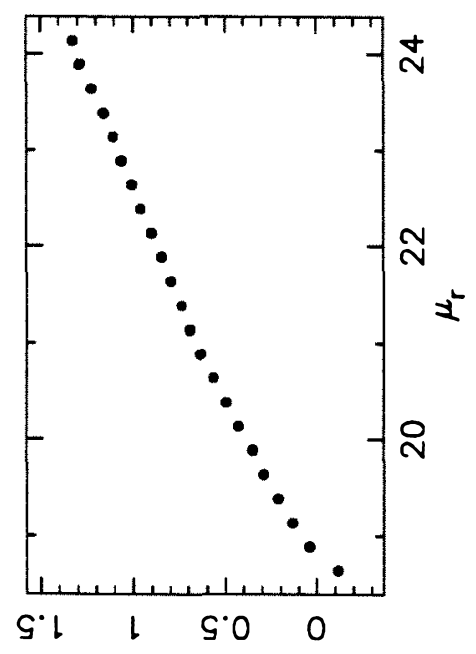

(.1) 960

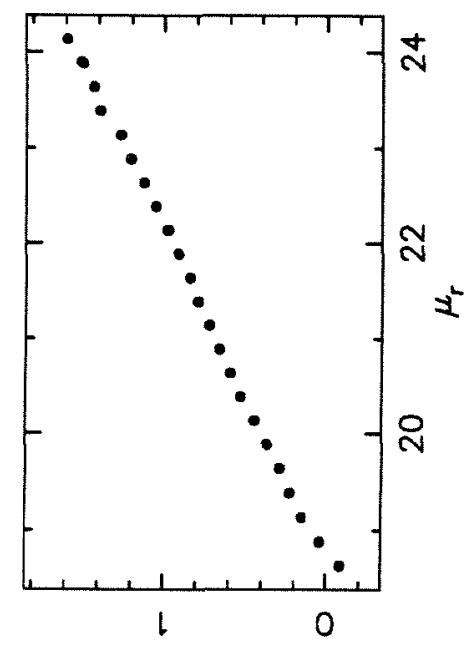

(i1) $\mathrm{D}$ 60
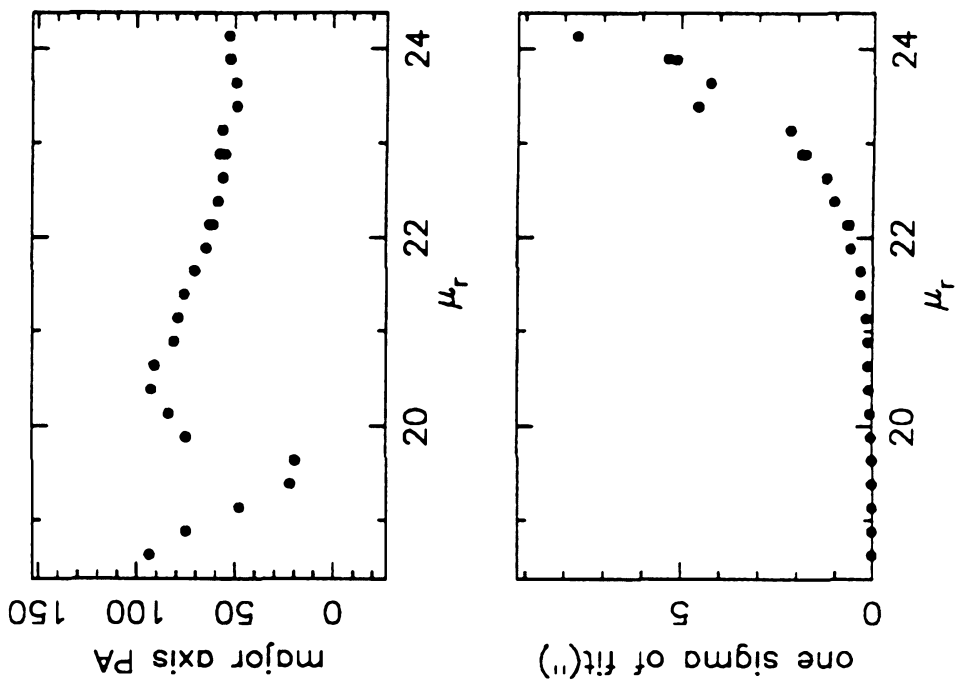

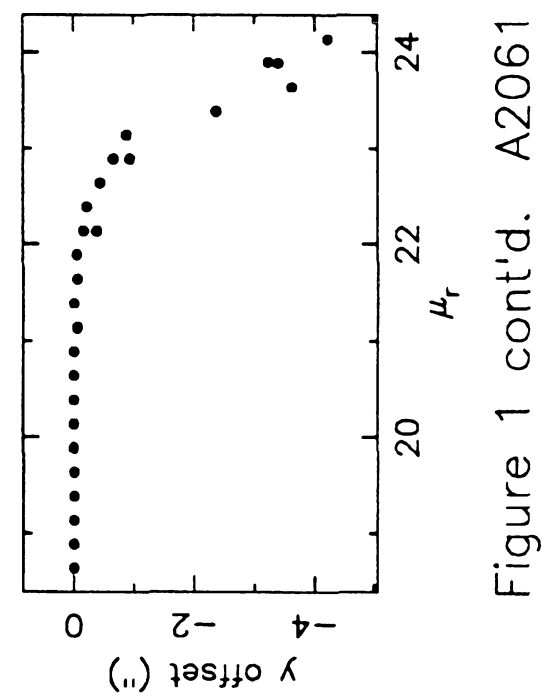

К์!อ!กษนวววว

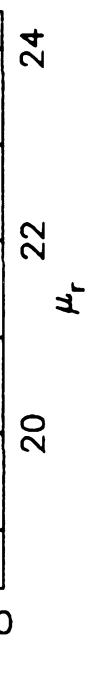

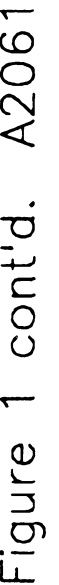
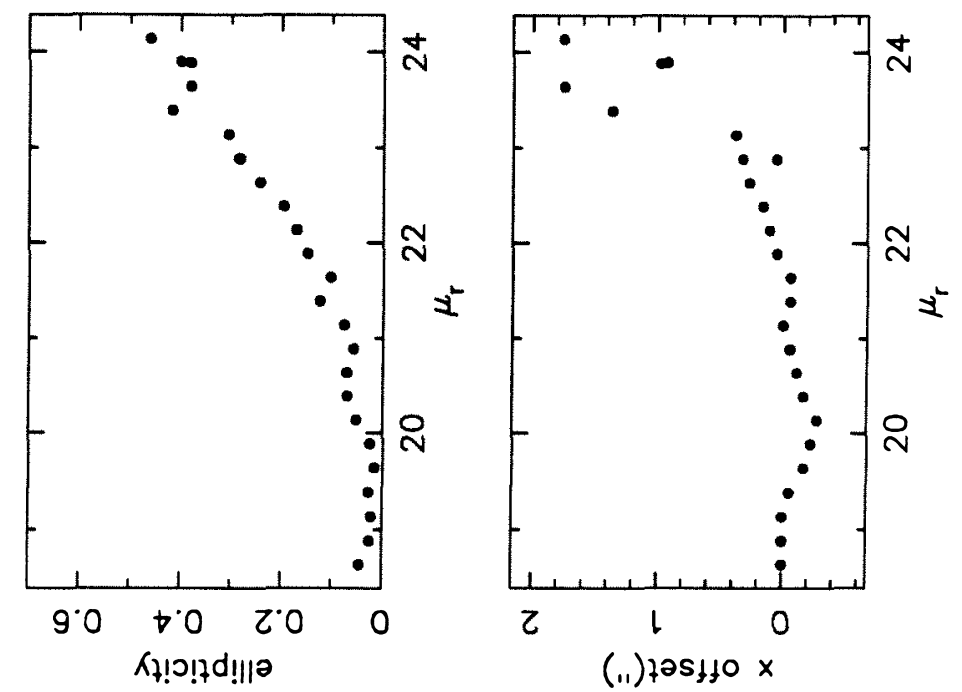


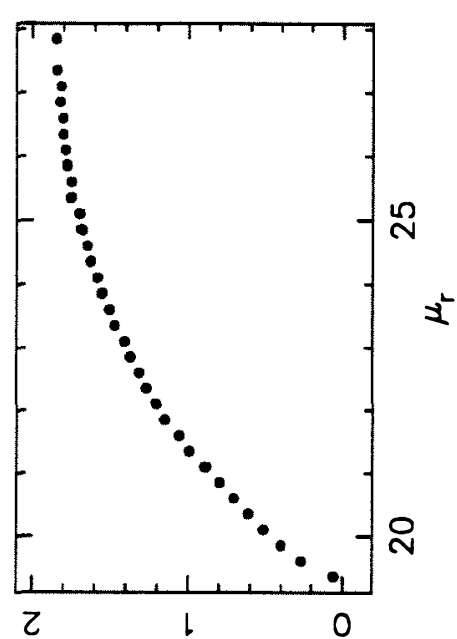

(.1)sn!pos 60।
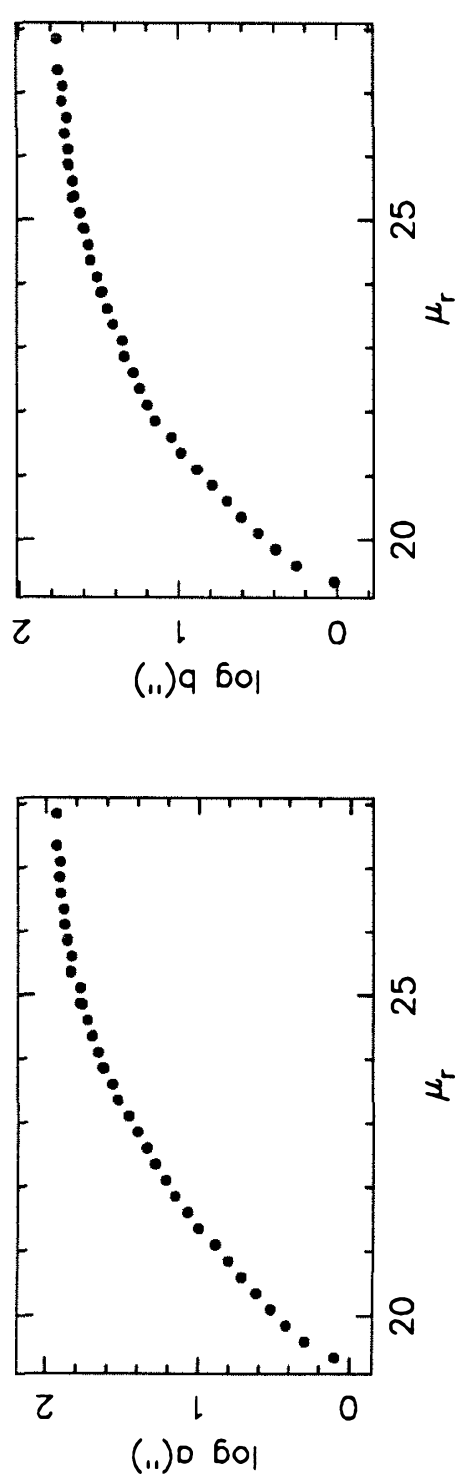

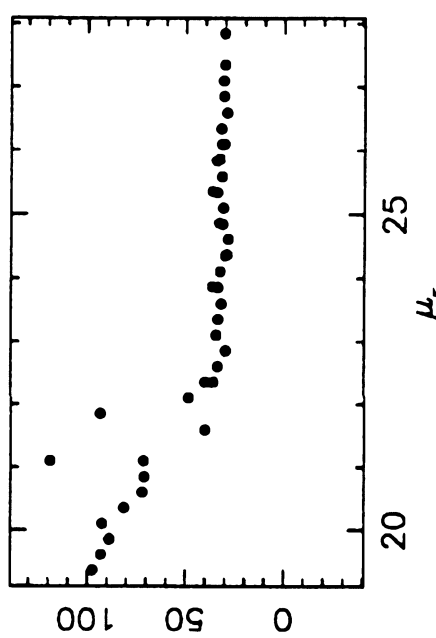

$\forall d$ s!xo so! ou
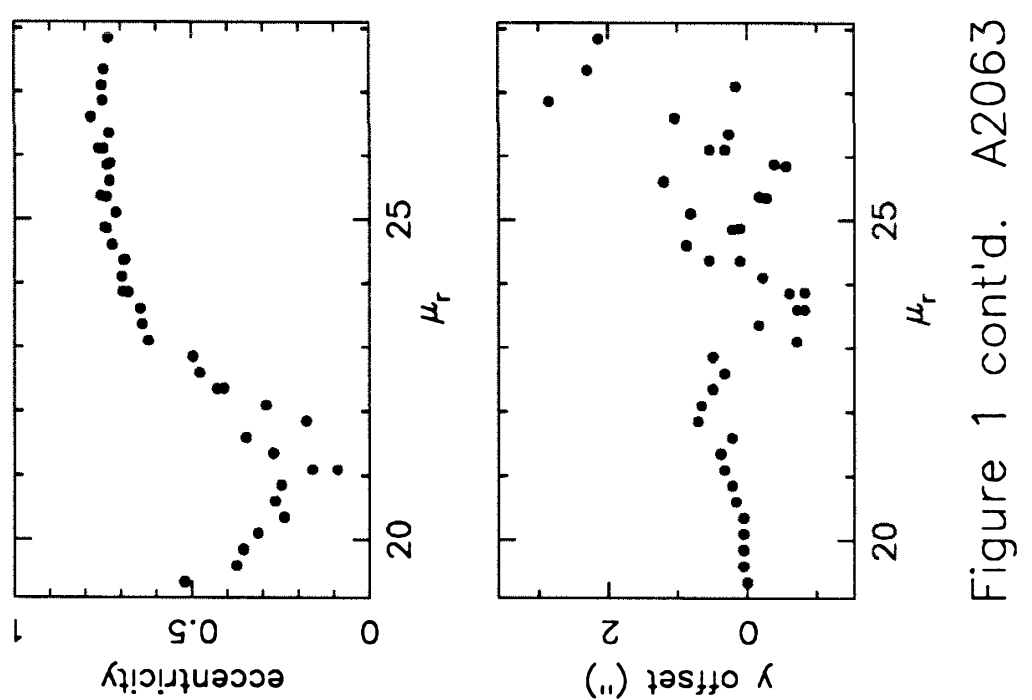

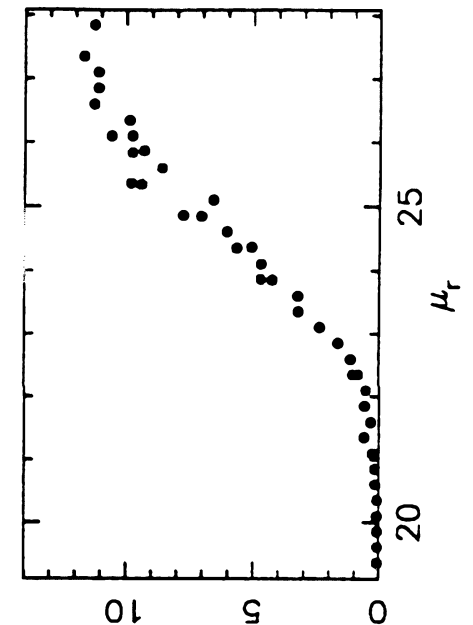

(11) t!! to Dub!s auo
(11) $725150 \mathrm{~K}$
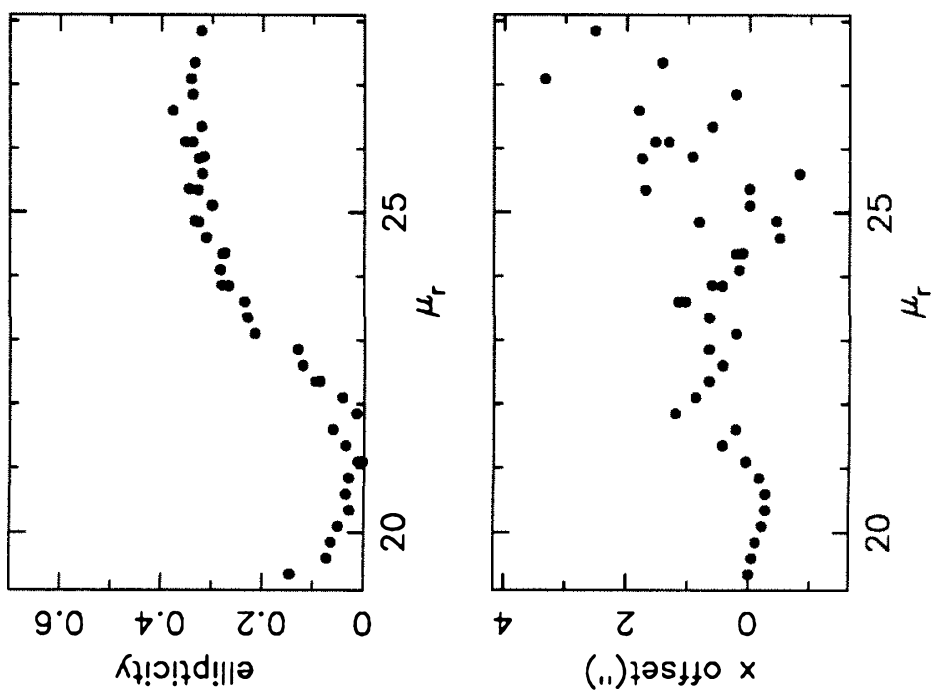


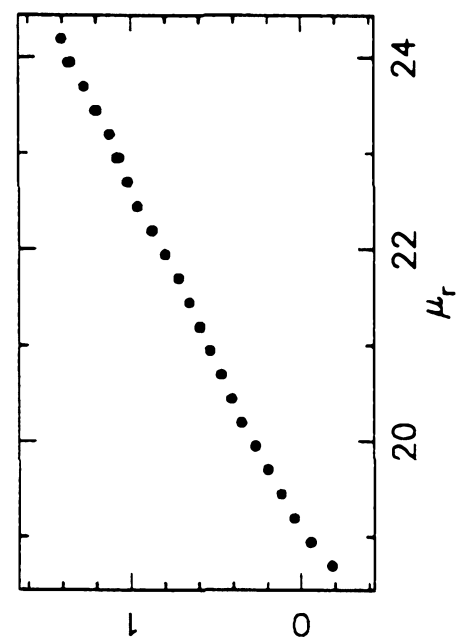

(.1)sn!pos 60।

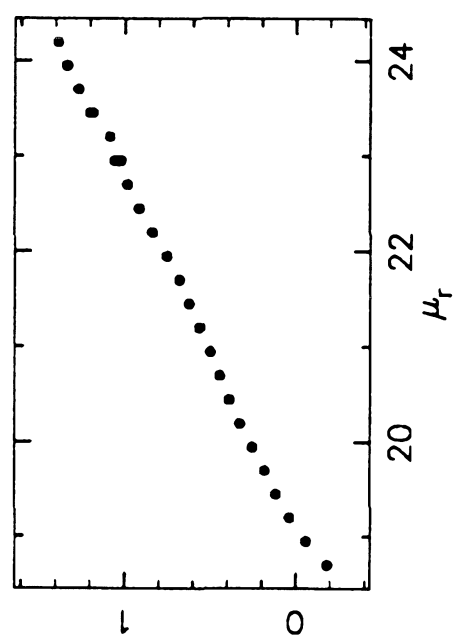

(.1) 950 이

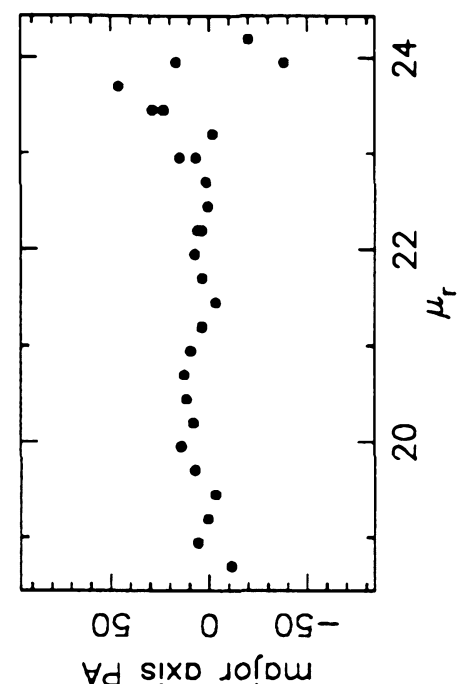

$\forall d$ s!xo so! om

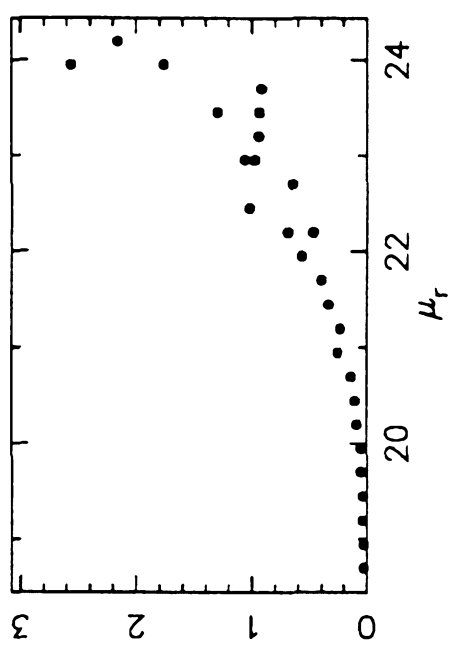

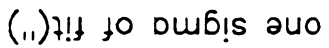

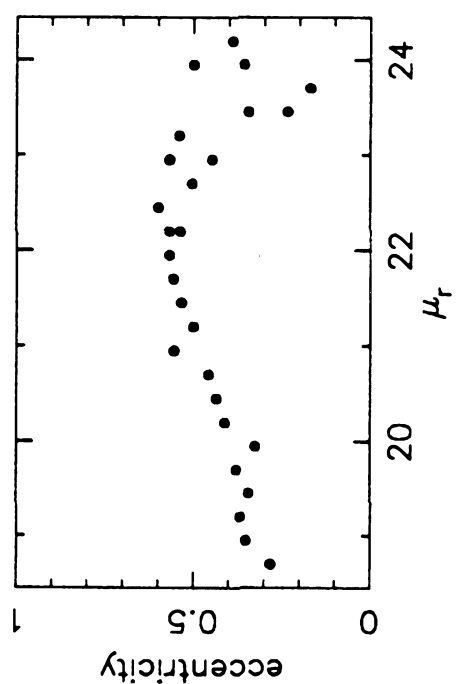

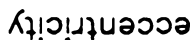
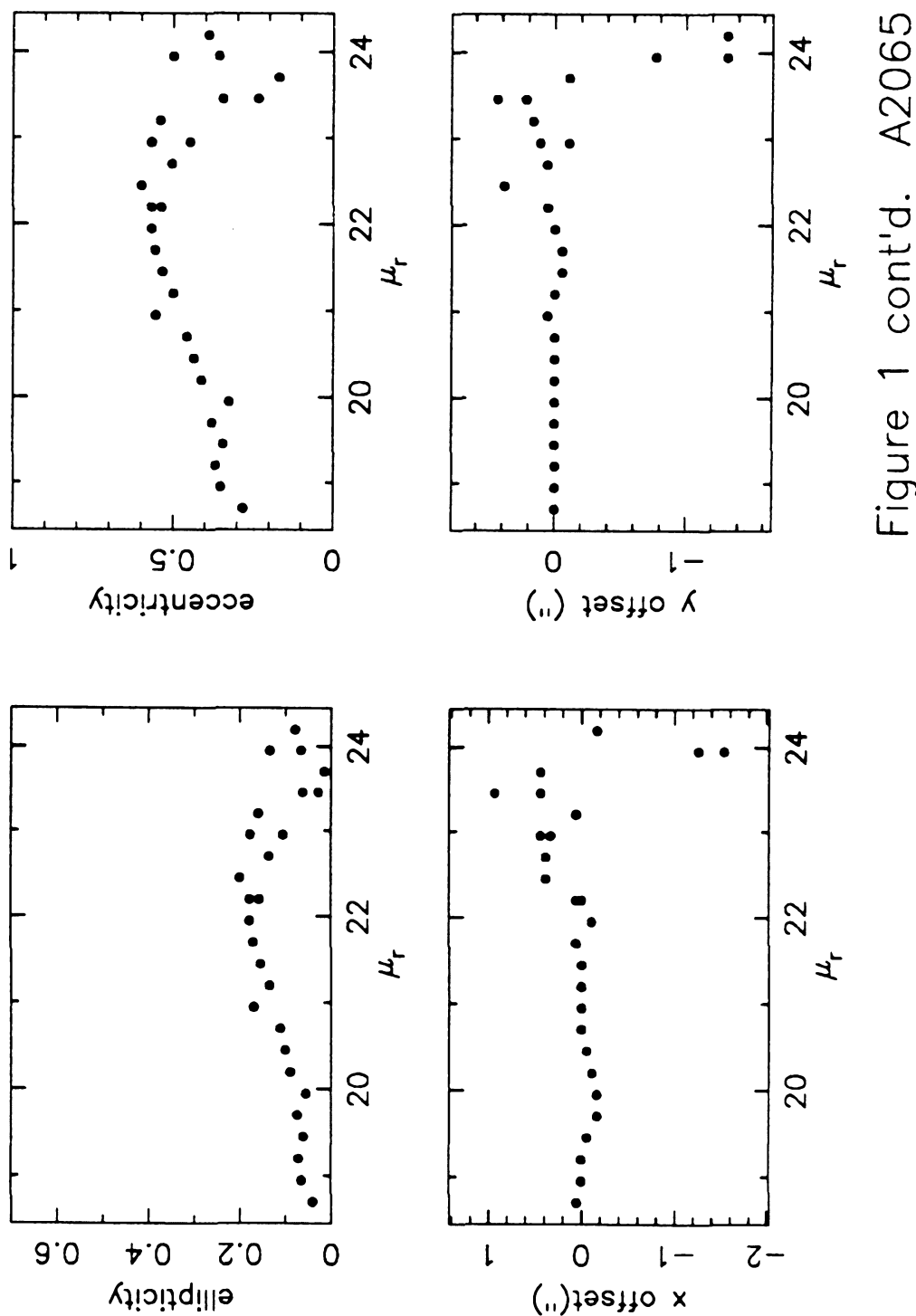

(11) $725 \% 10 \mathrm{~K}$ $x^{2}$

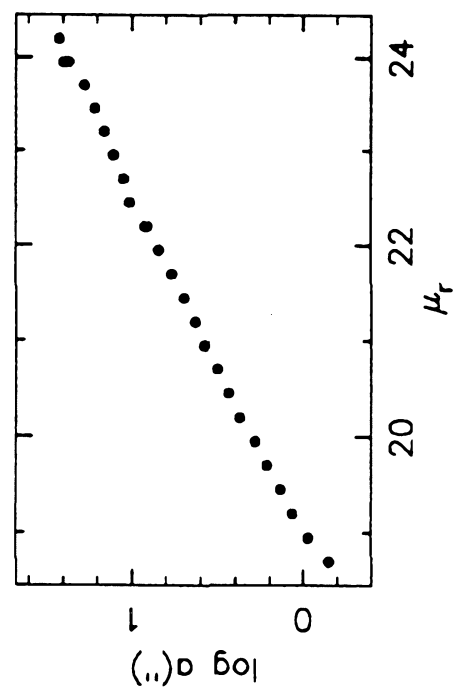

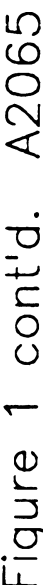




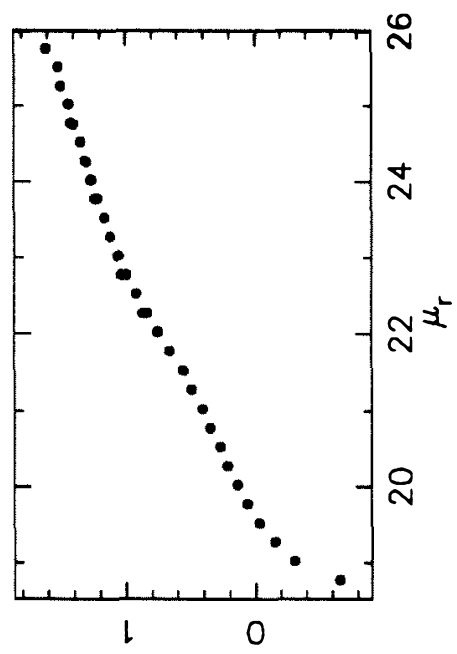

(.1) sn!pod 601

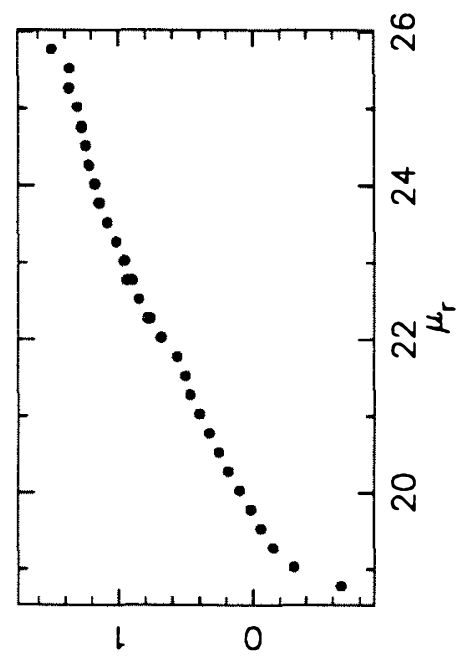

(.1) 9 601

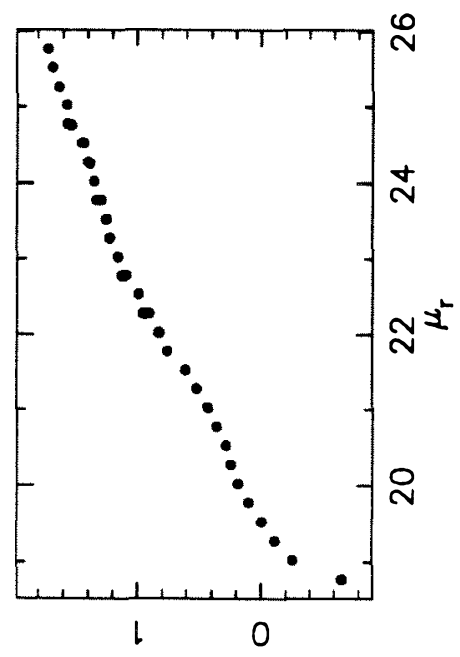

(.1) 0601

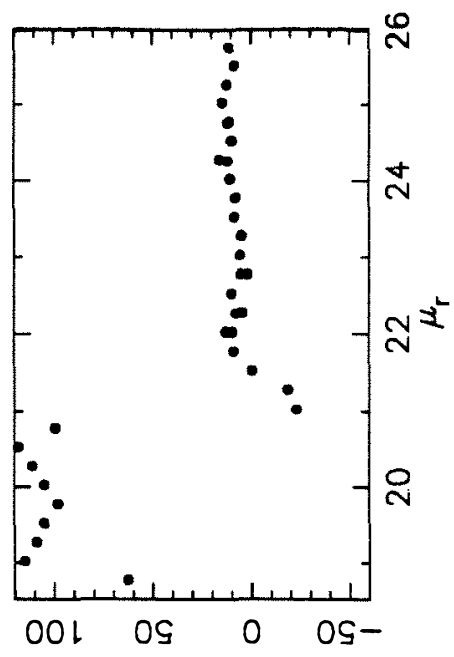

$\forall d$ s!xo solom

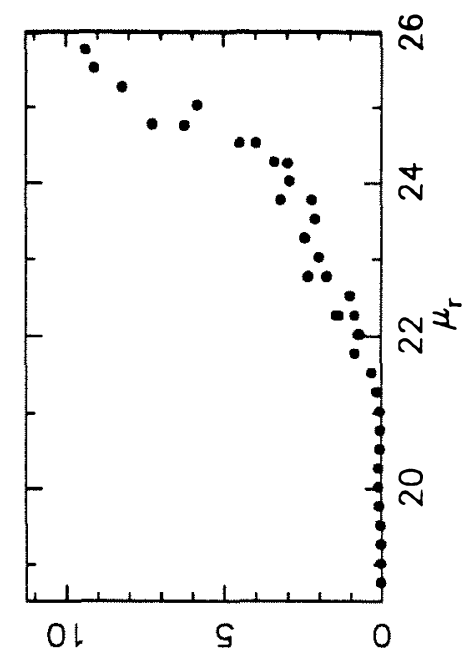

(.1) H!l to omb!s avo

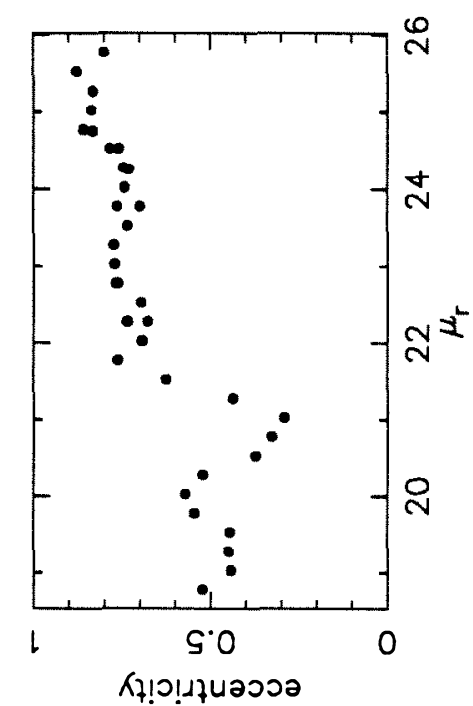

Kұ!ว!นในวววa

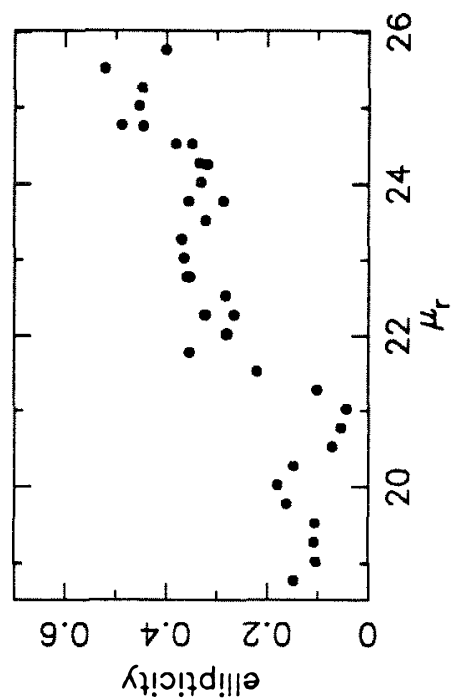

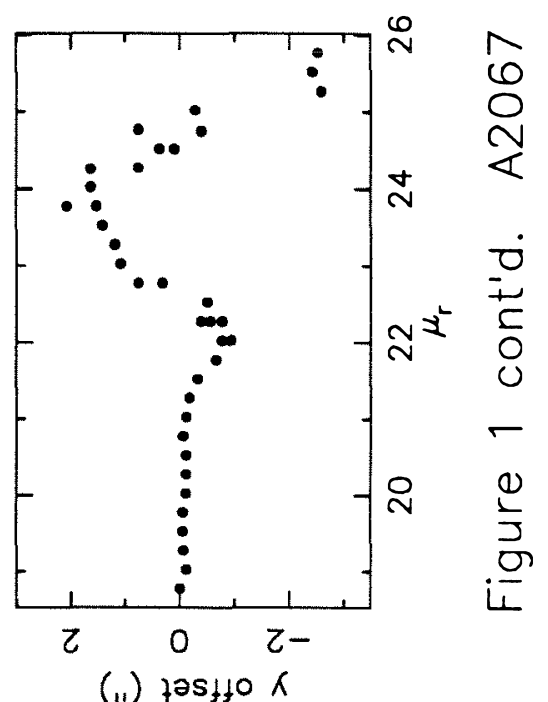

(i) zas t10 $\mathrm{K}$

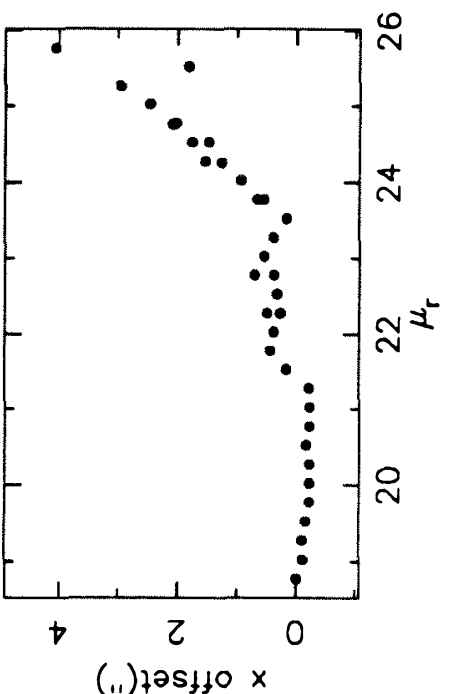




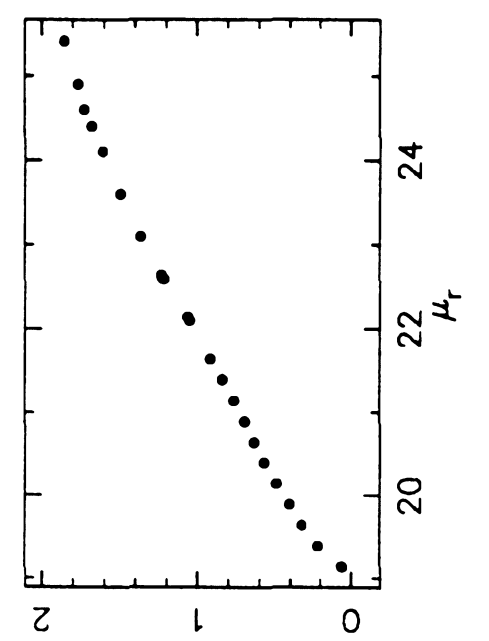

(.1) sn!pod 6ol

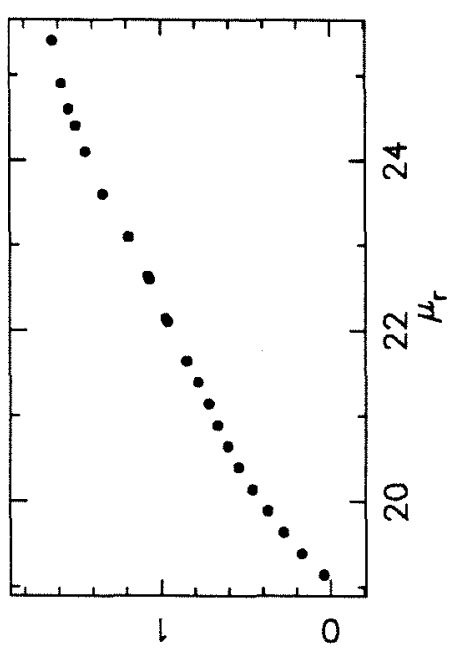

(.1) $q \mathrm{bol}$

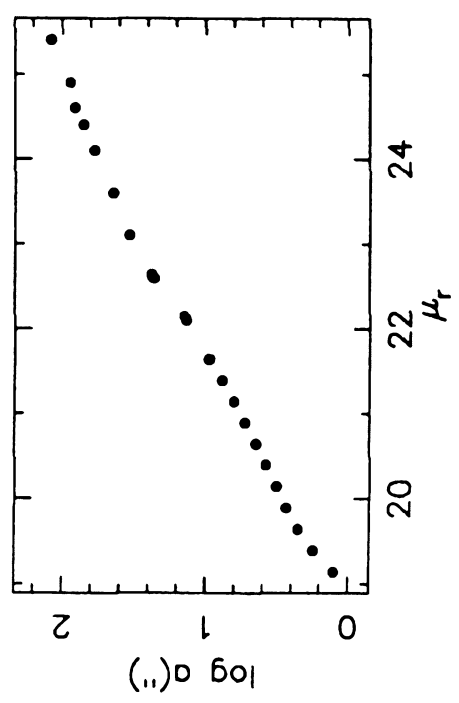

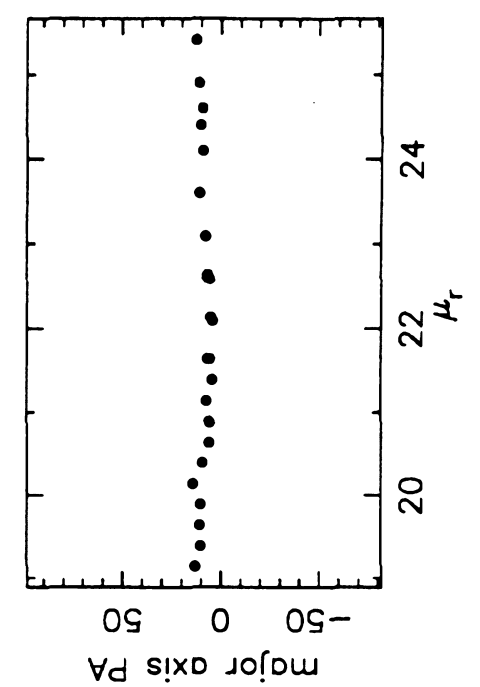

$\forall d$ S!Xo Jo! ou

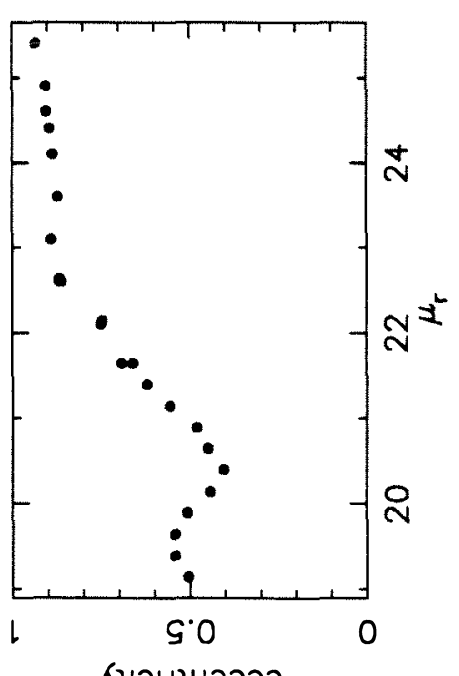

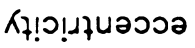

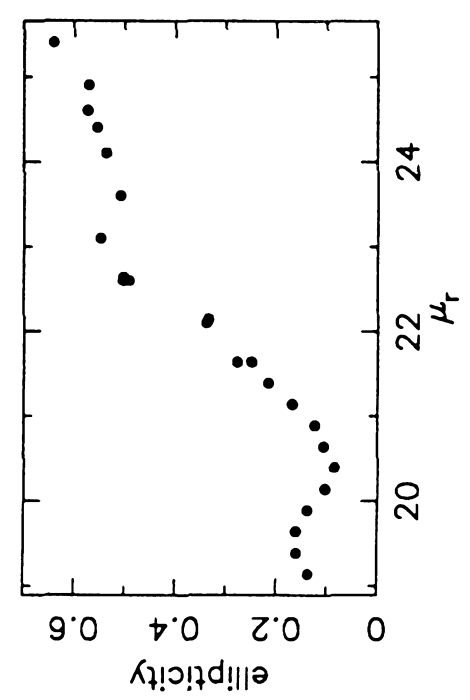

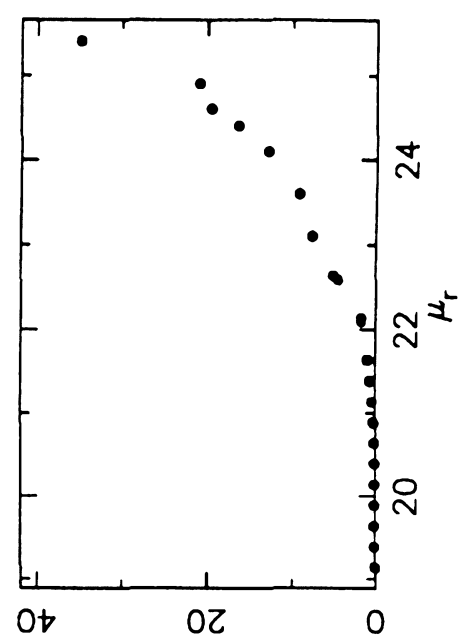

(.1) $) !+$ to oub!s auo

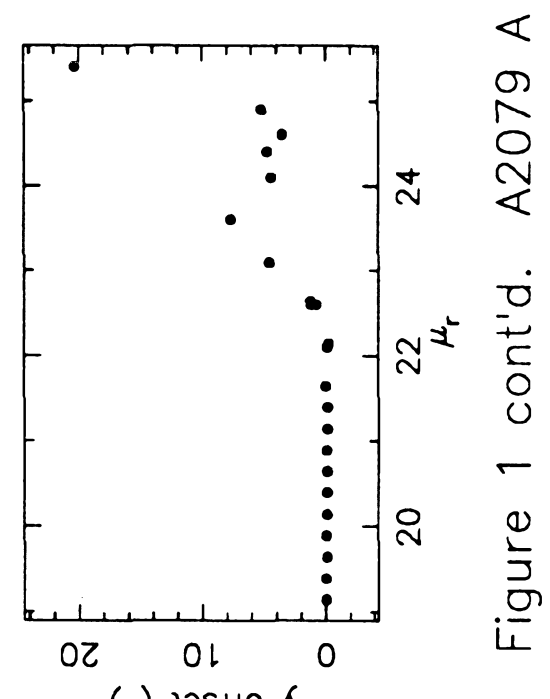

(i1) zast10 K

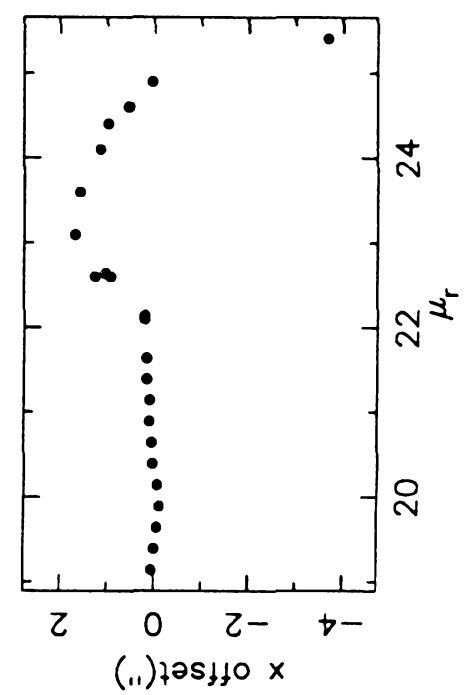




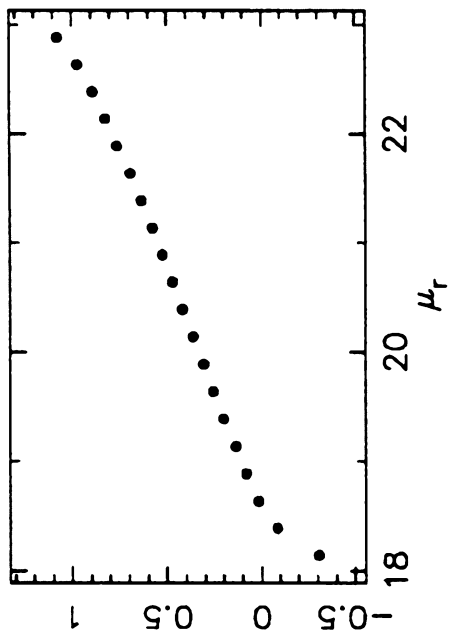

(..) sn!pod 6ol

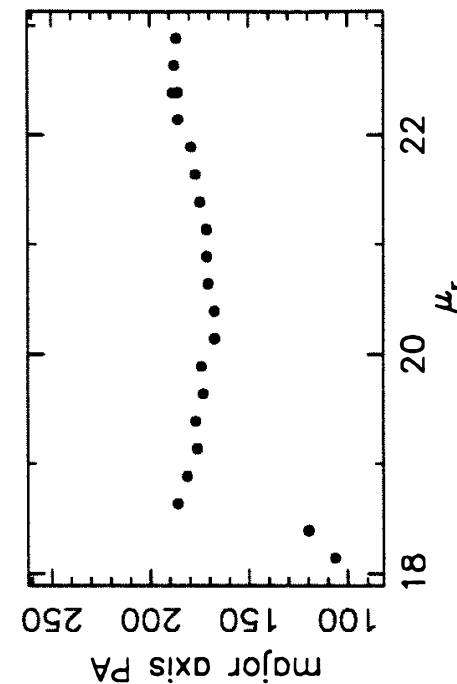

$\forall d$ S!XD do!om

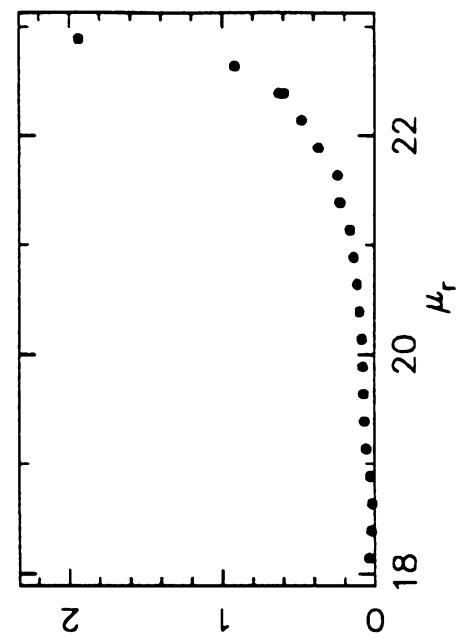

(.1) $7 ! 1$ to omb! ano

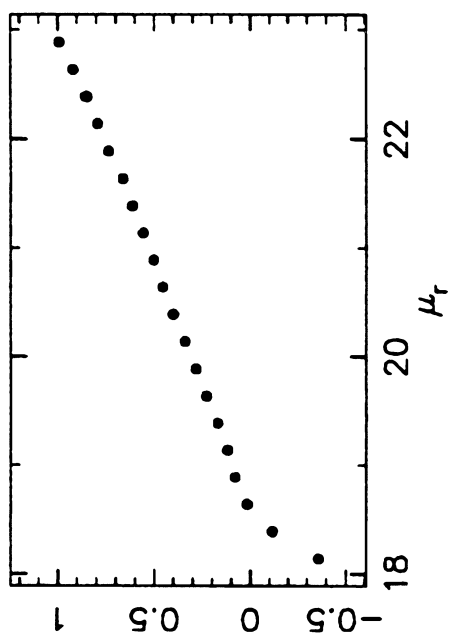

(.1) 9 6ol

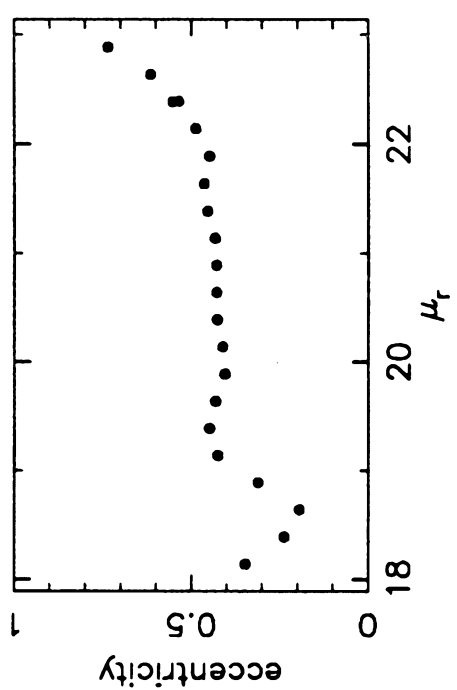

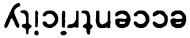

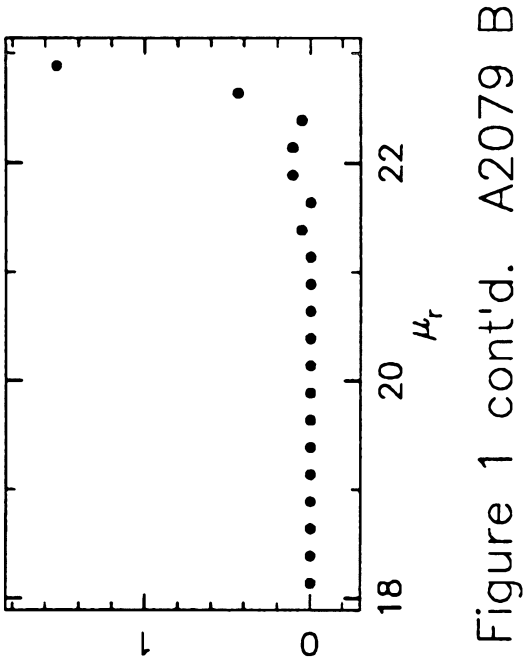

(11) zas $150 \mathrm{~K}$
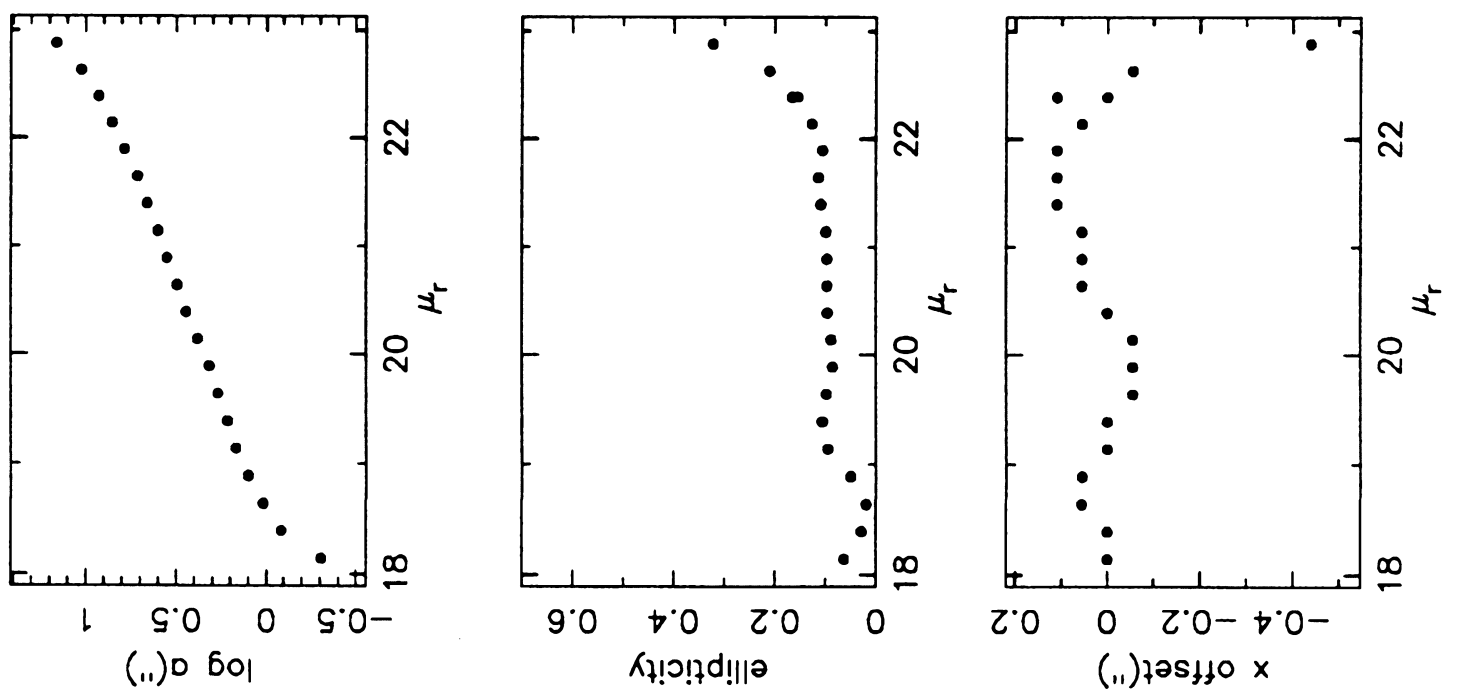

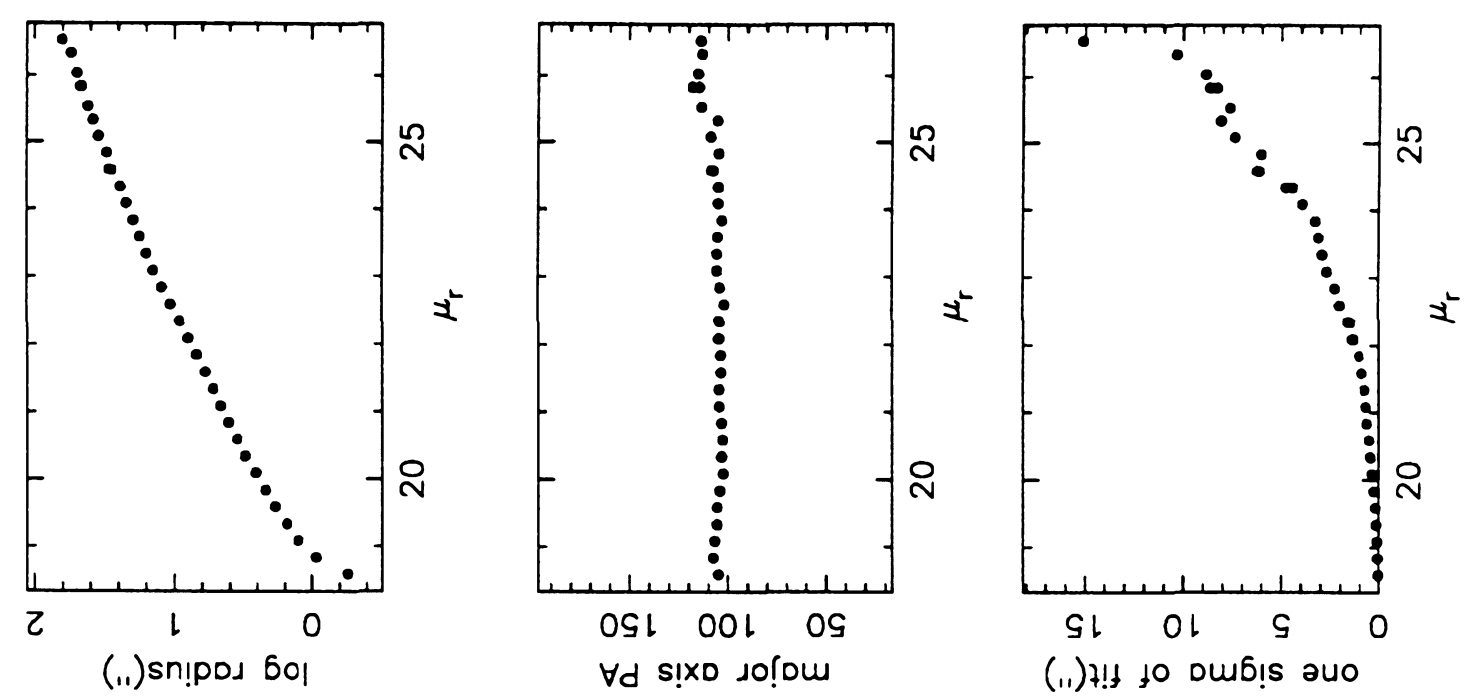

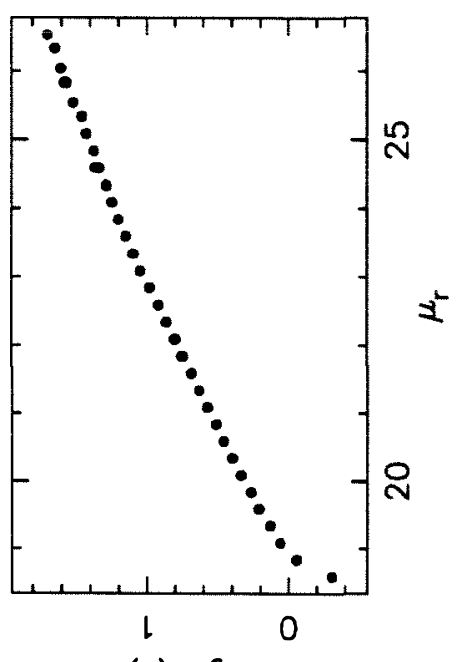

(11) 9601

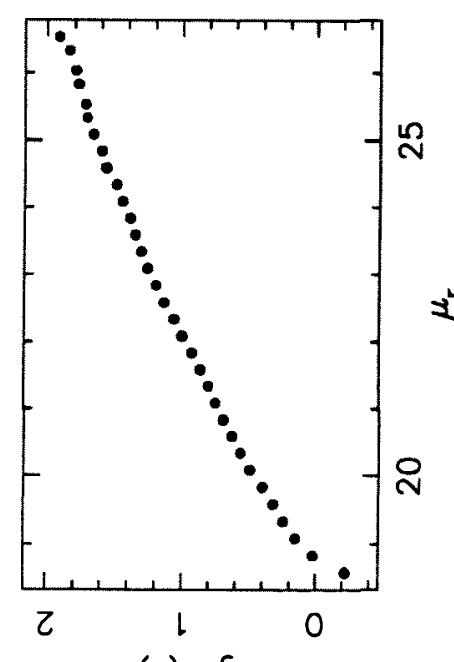

(..) $0 \mathrm{bol}$
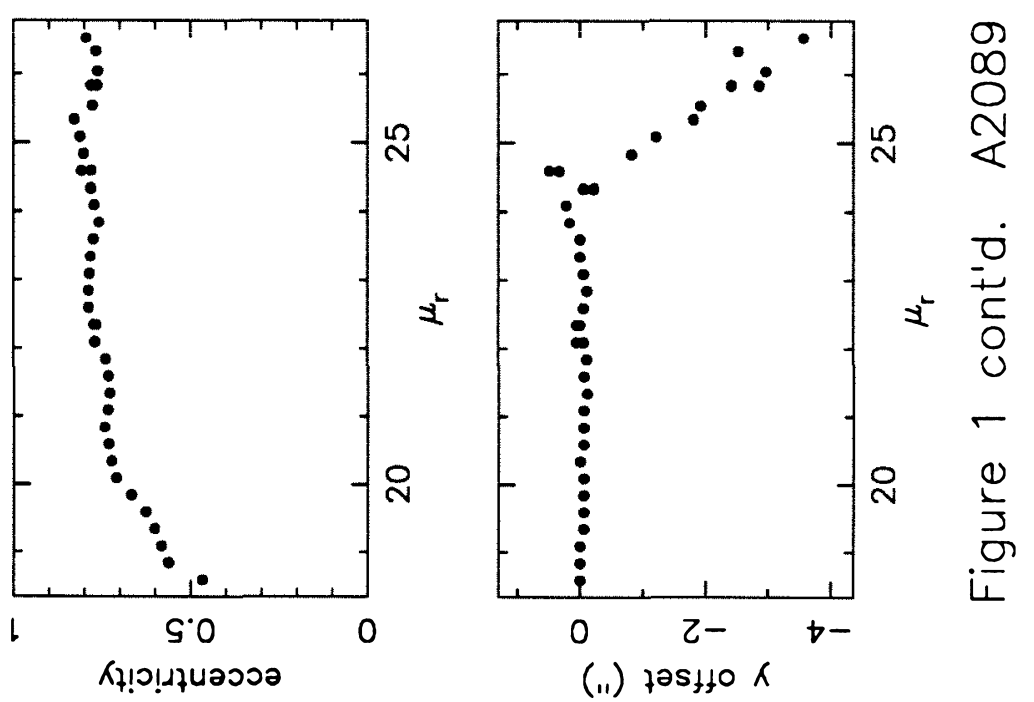


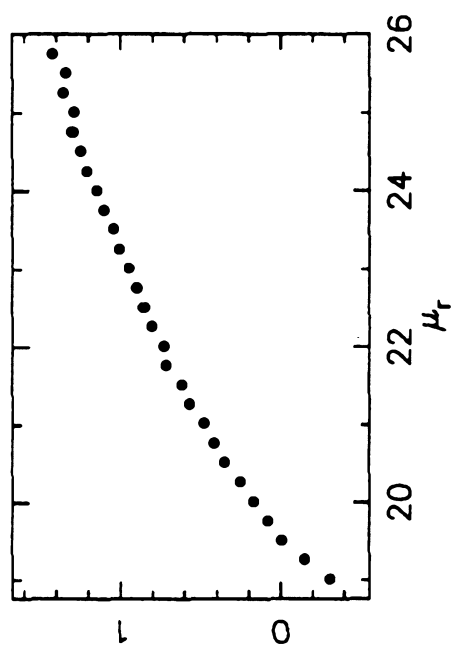

(.1) sn!pos 6ol

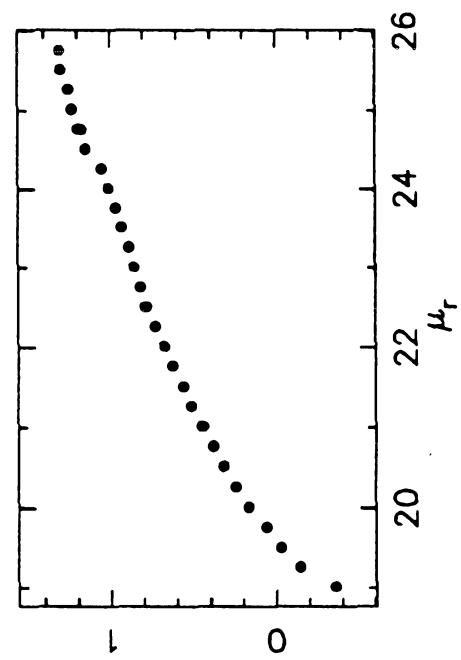

(.1) 9601

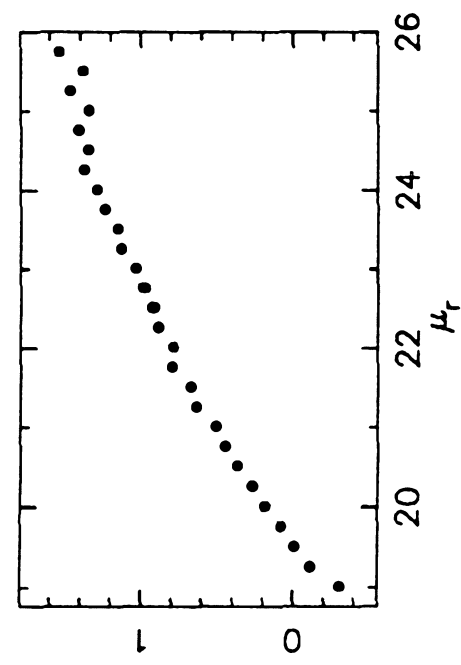

(.1) $\mathrm{D} 60 \mathrm{O}$

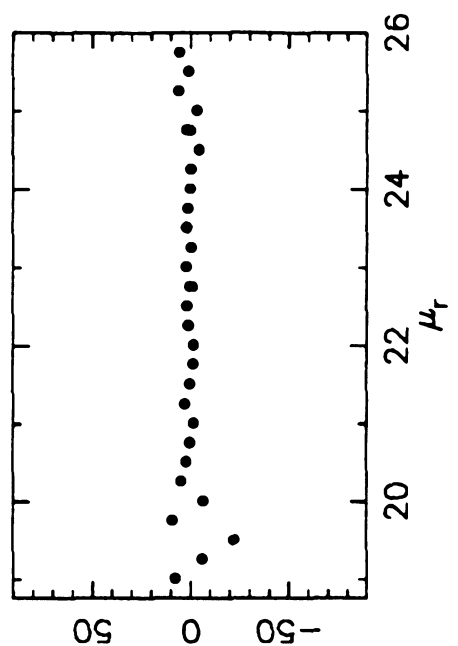

$\forall d$ s!xo so!nom

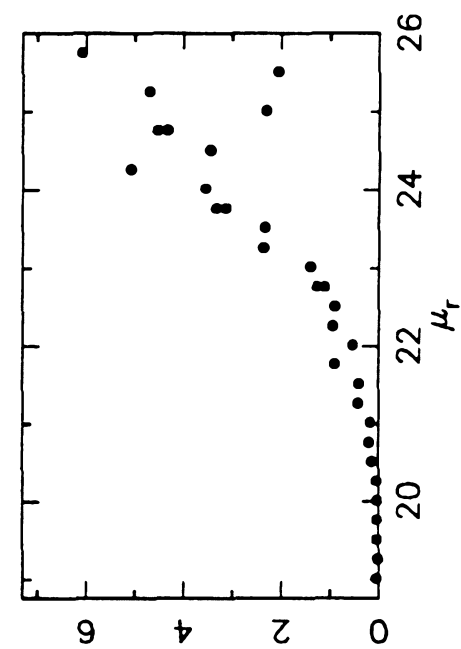

(11) 4 ! t to Dub!s auo

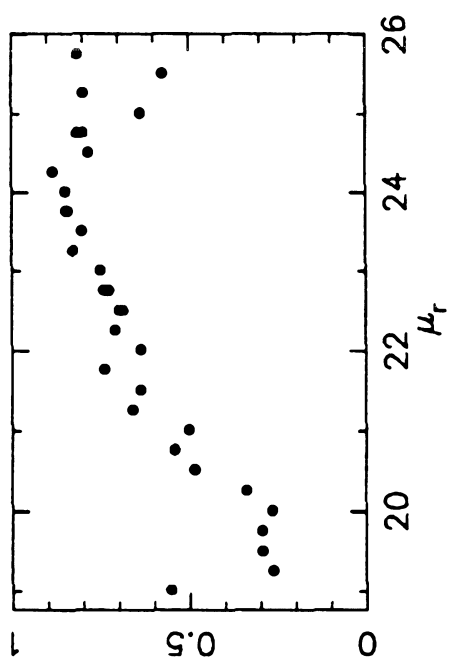

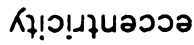

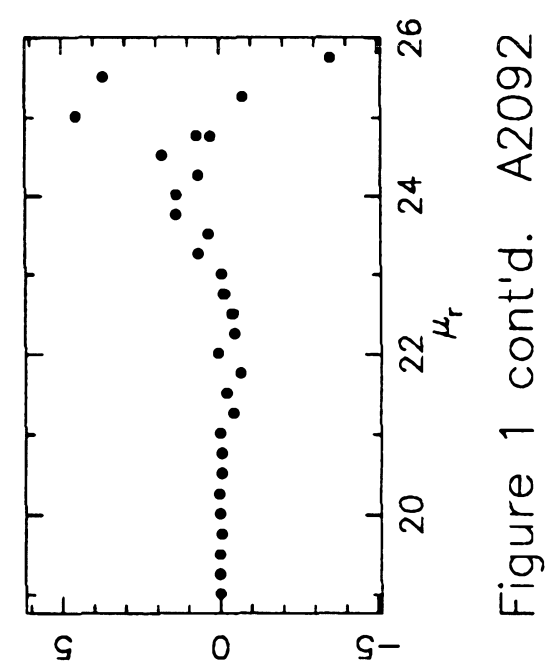

(11) $725+10 K$

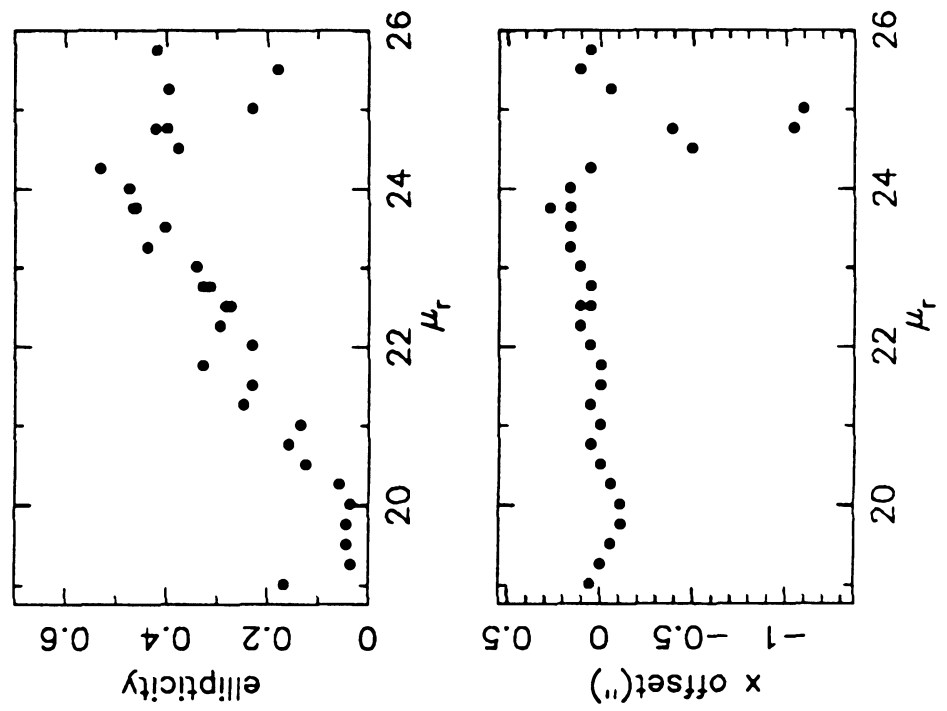




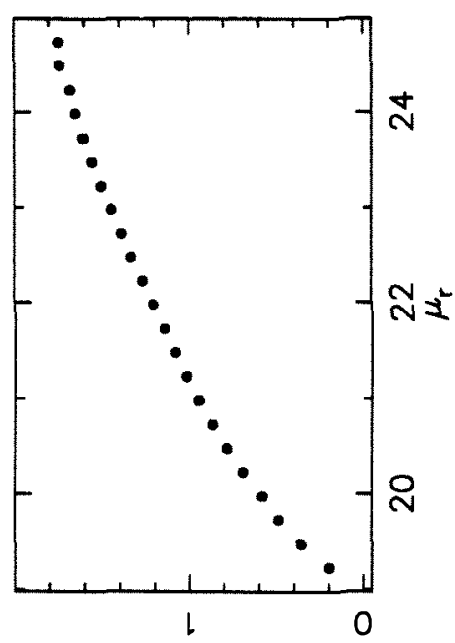

(11) sn!pos 6ol

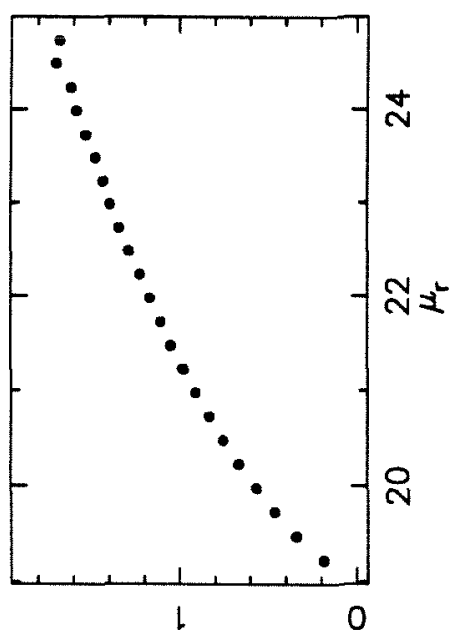

(.1) $q \mathrm{bol}$

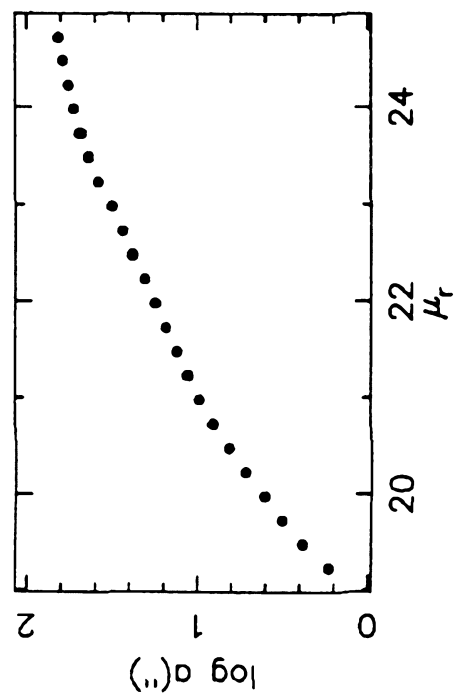

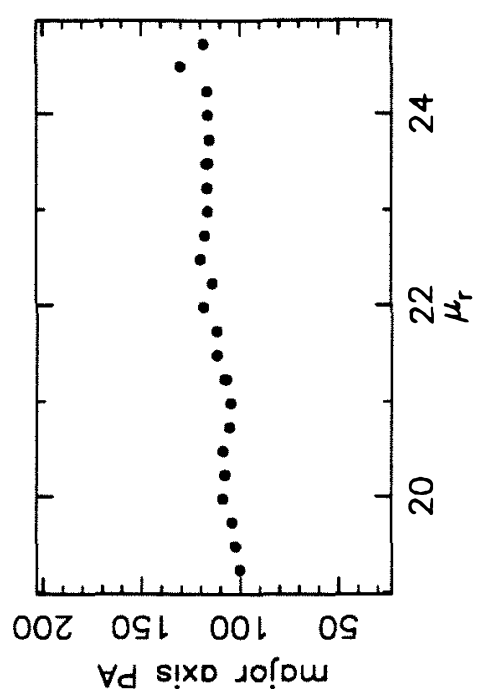

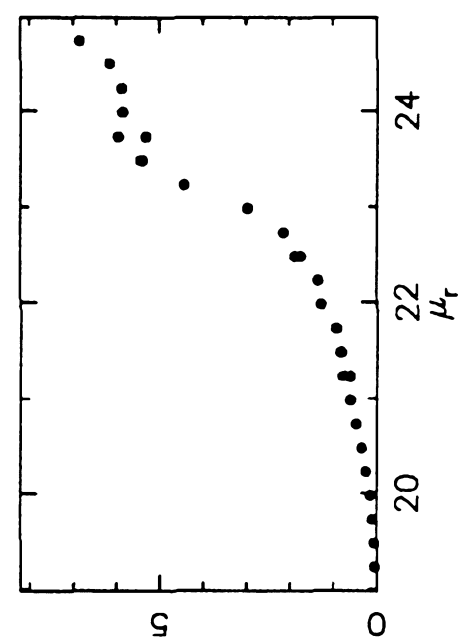

(11) $7 ! 1$ to Dub!
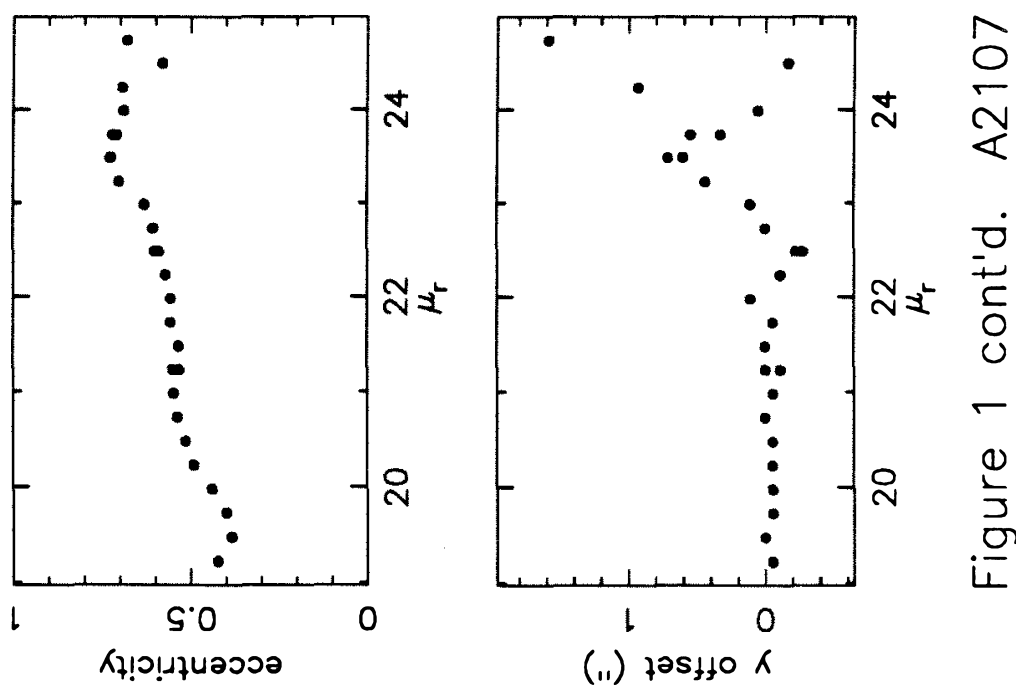

(i1) tast $70 \mathrm{~K}$

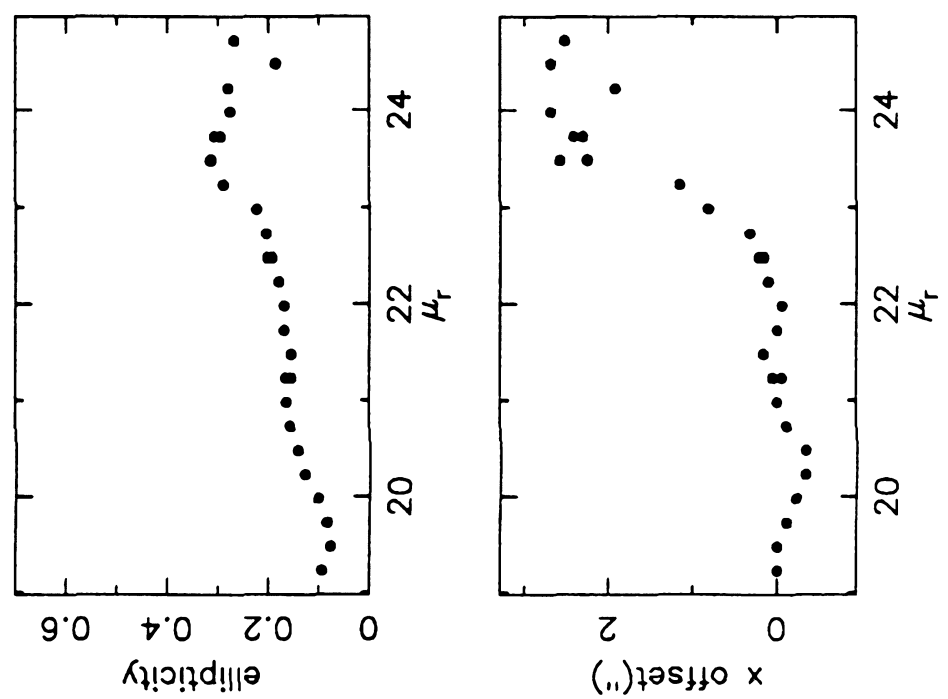




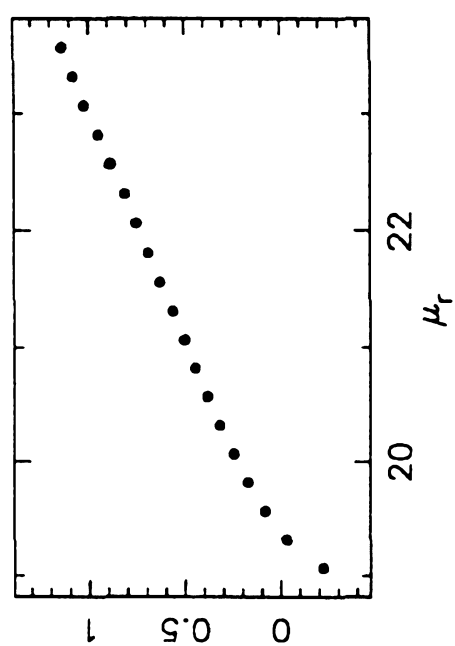

(11)sn!pD 60।

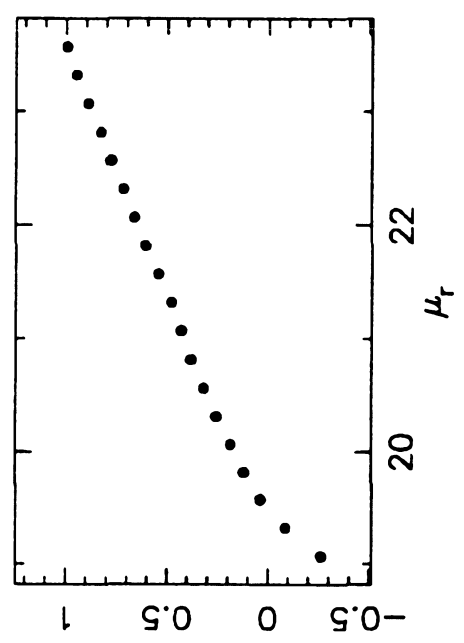

(.1) 9 6ol

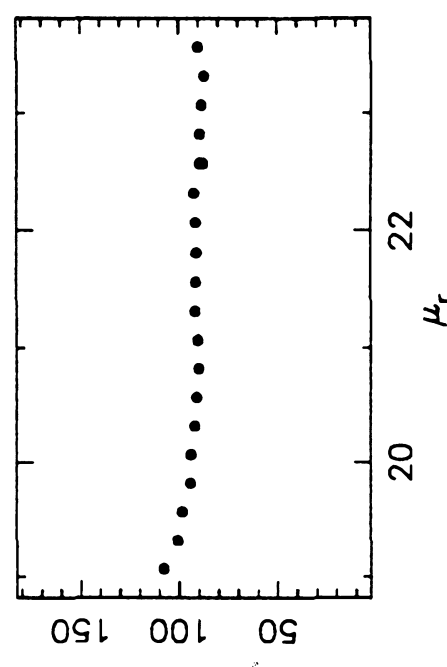

$\forall d$ s!xo do

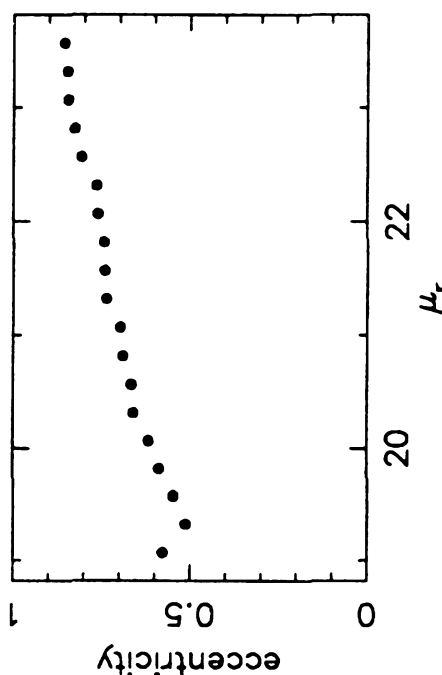

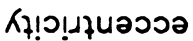

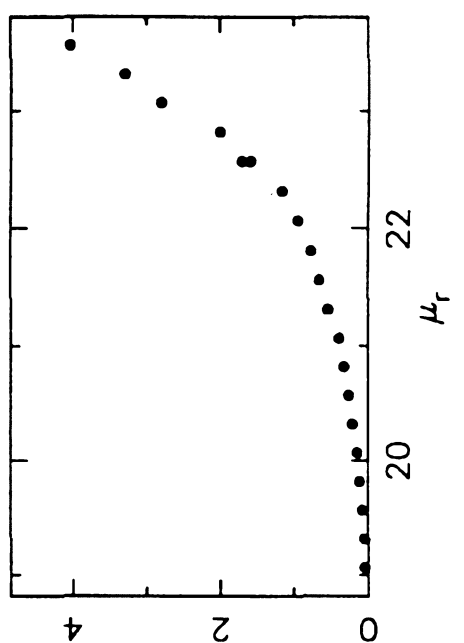

(11) $7 !+10$ Du6!s әи०
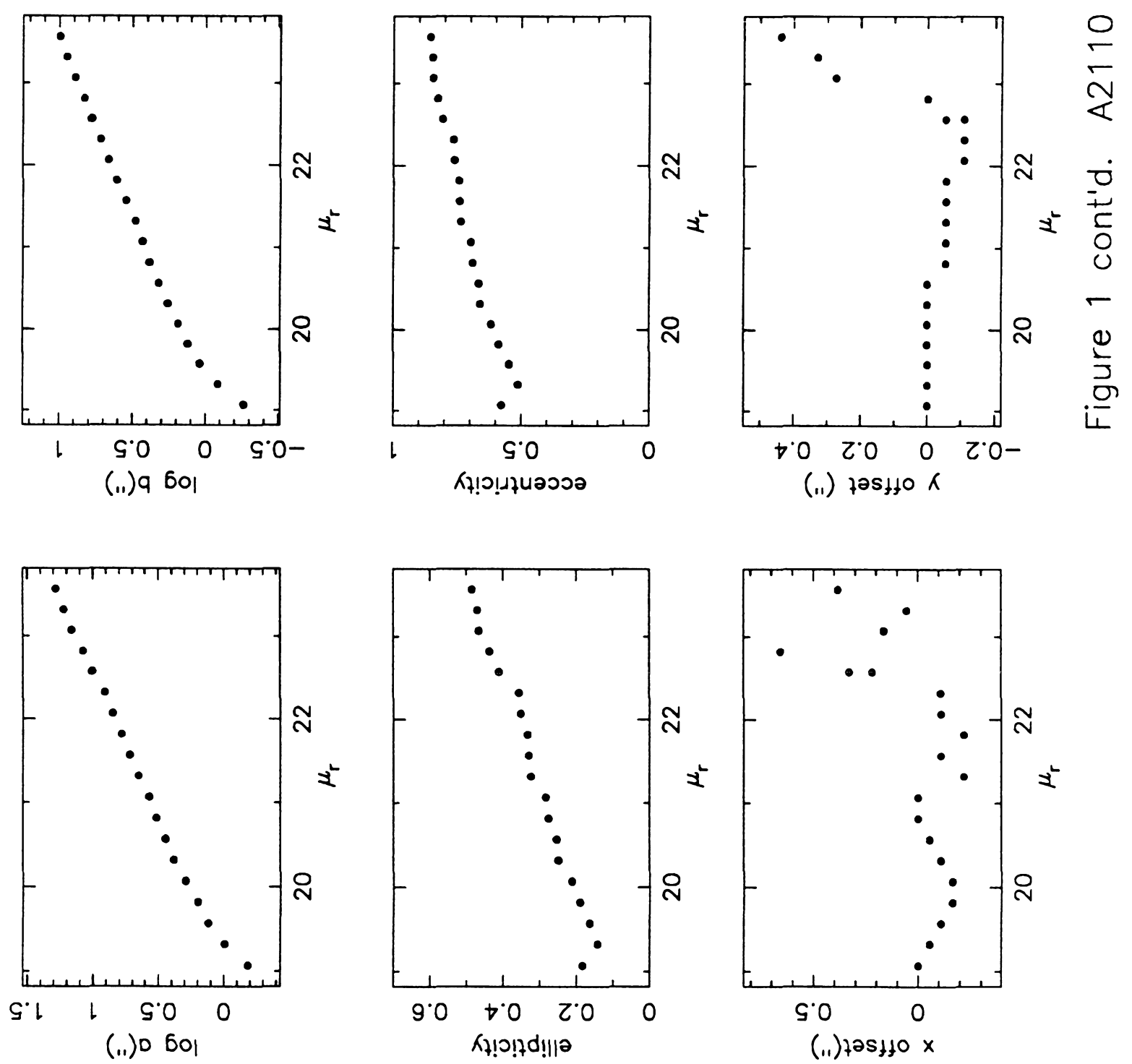

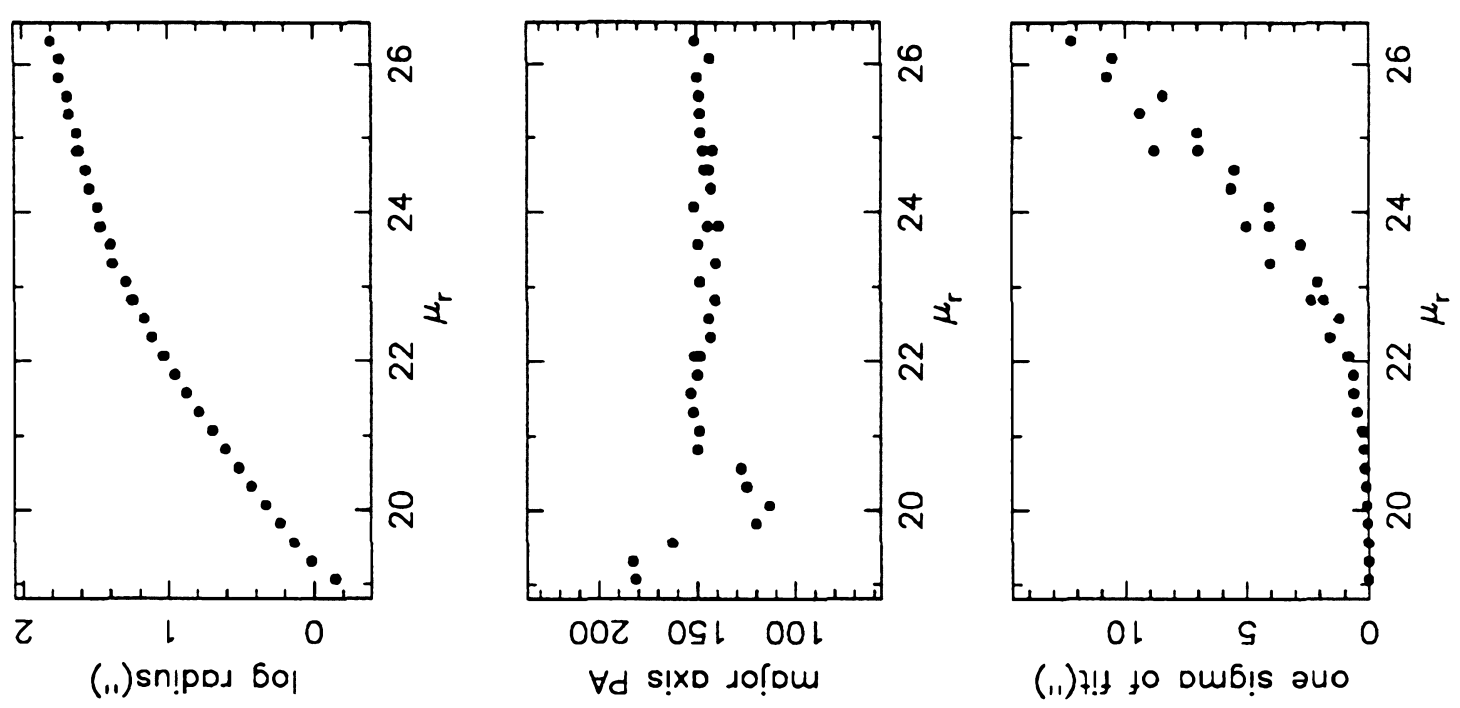

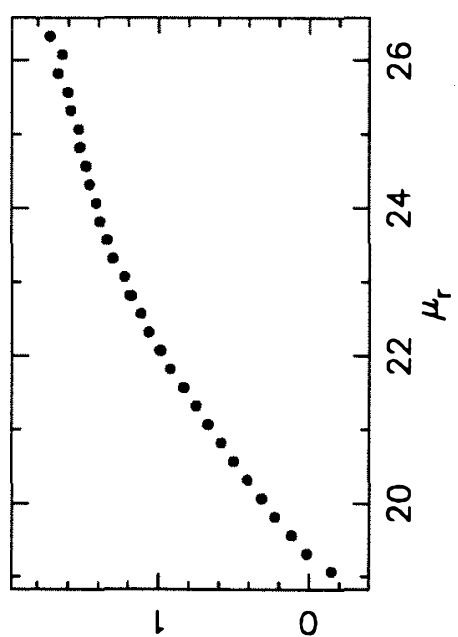

(.1) $9 \mathrm{60}$

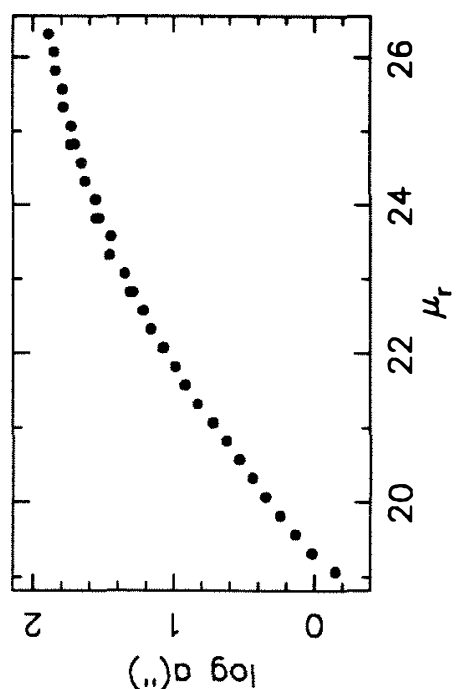

(..) $\mathrm{O}$ 60

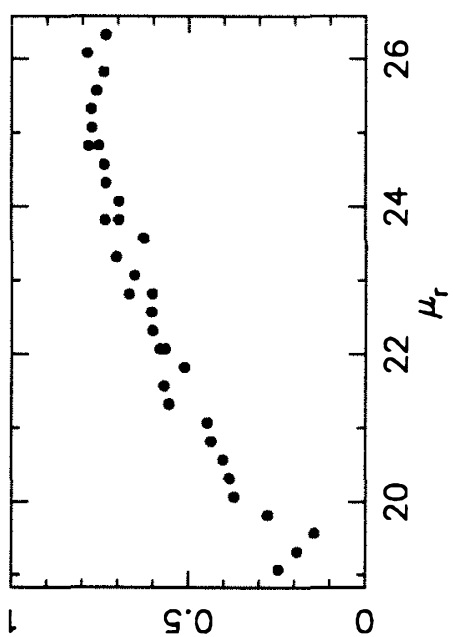

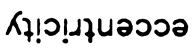

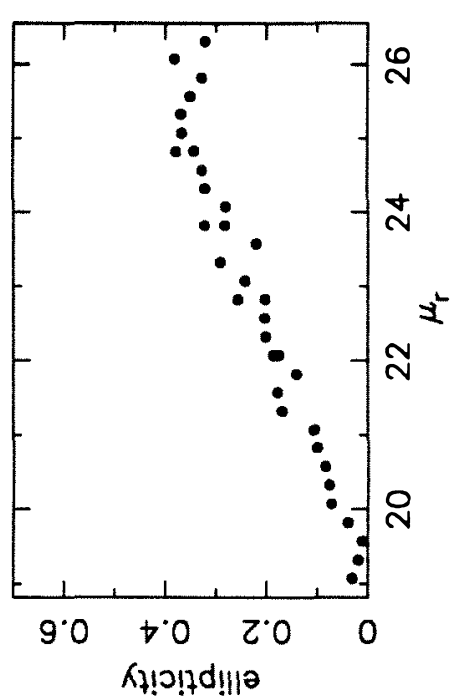

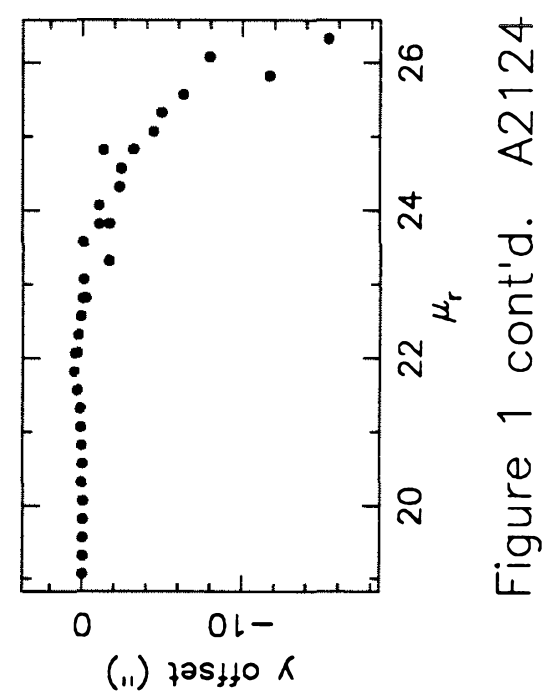

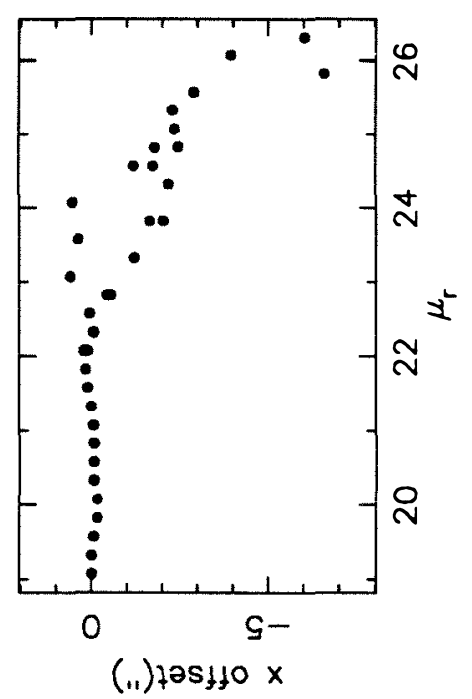




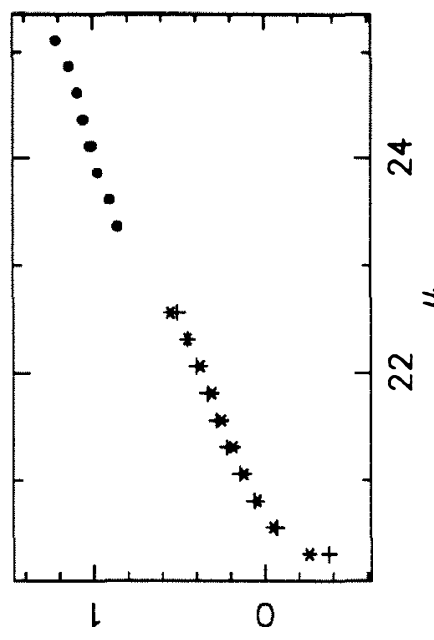

(.1) sn!pod 5ol

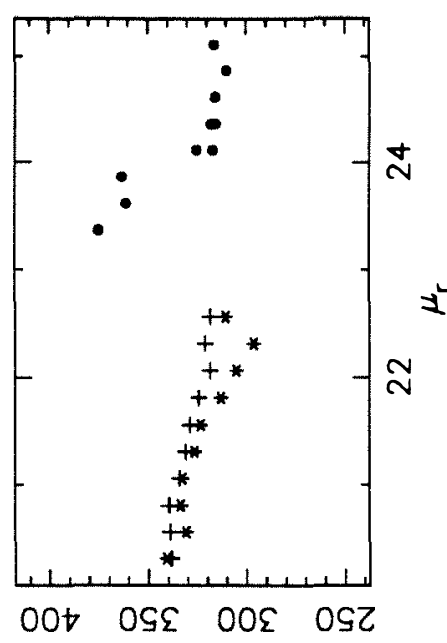

$\forall d$ S!xo so!̣u

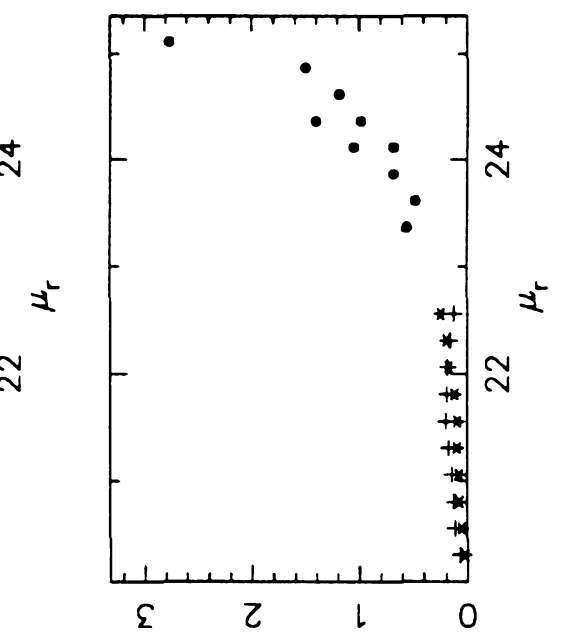

(11) $+! t$ to Dub!s au०
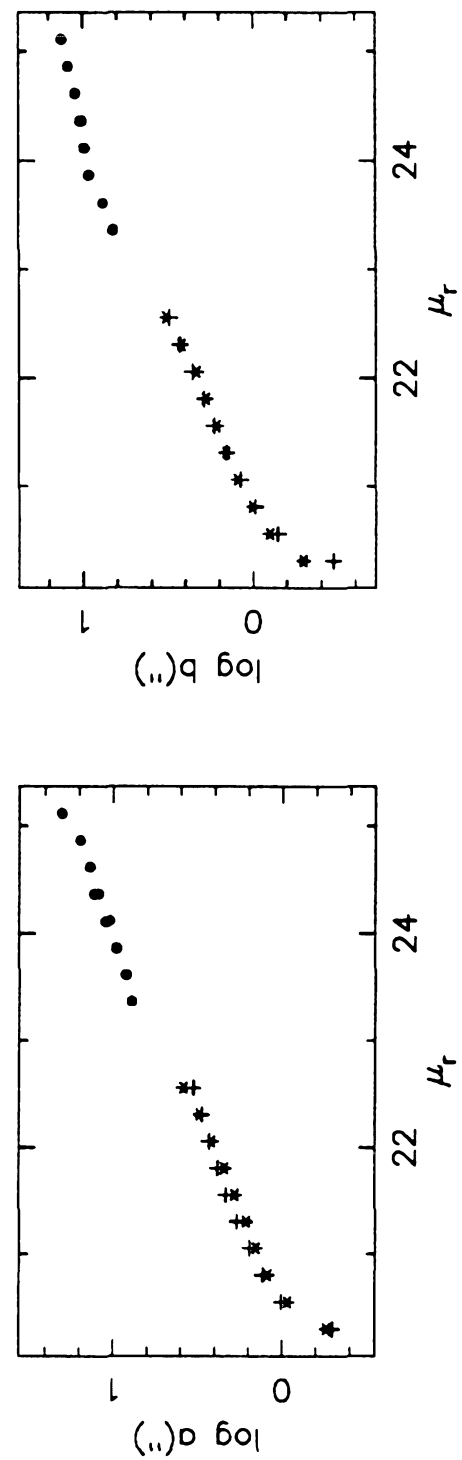
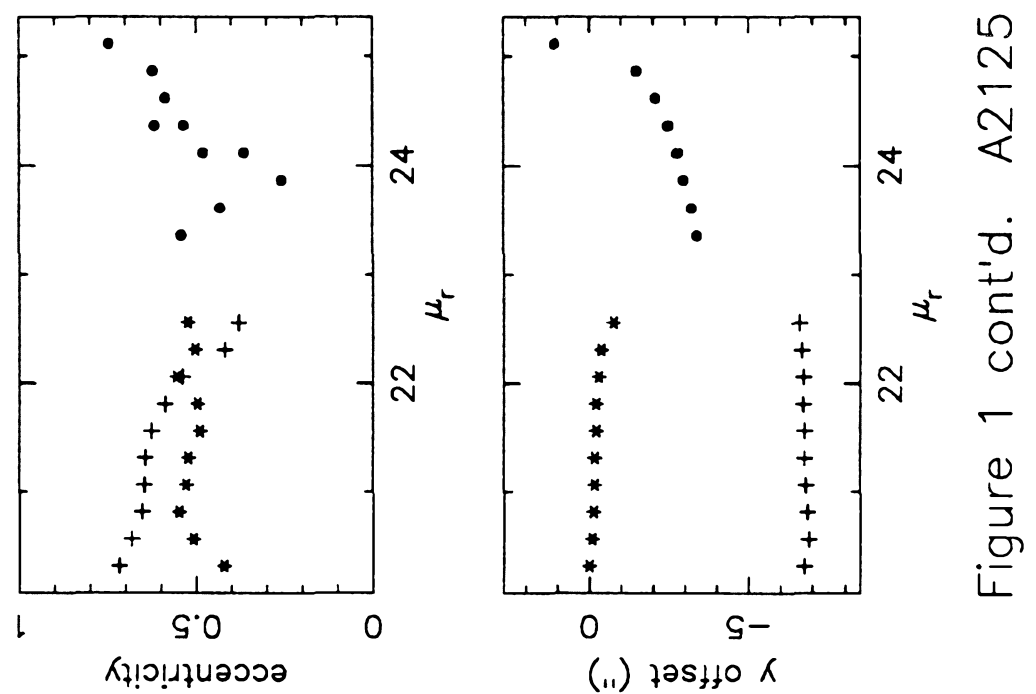

(11) $725 \% 10 \mathrm{~K}$
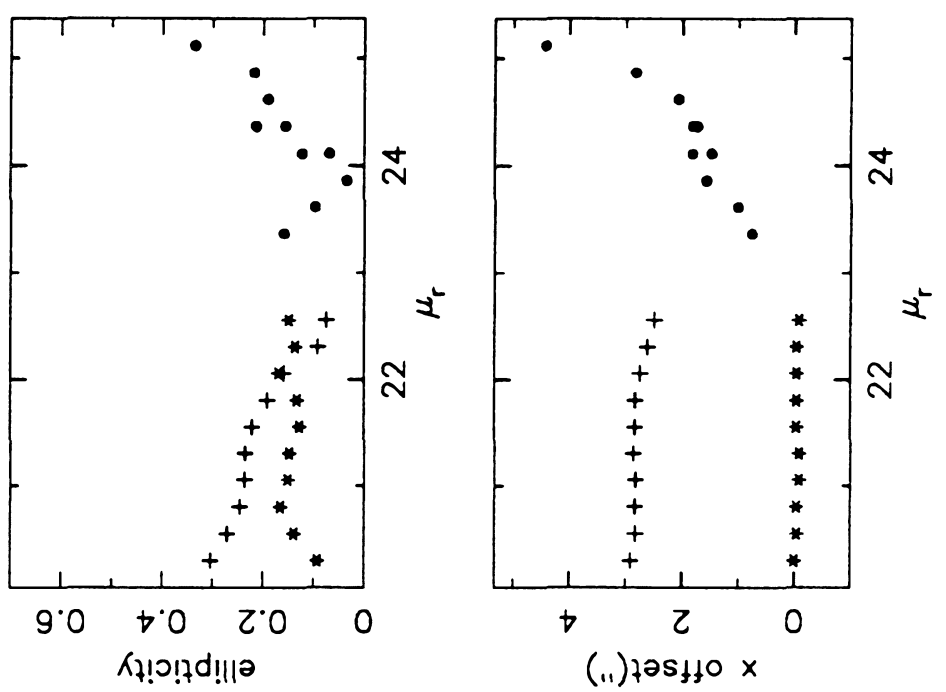

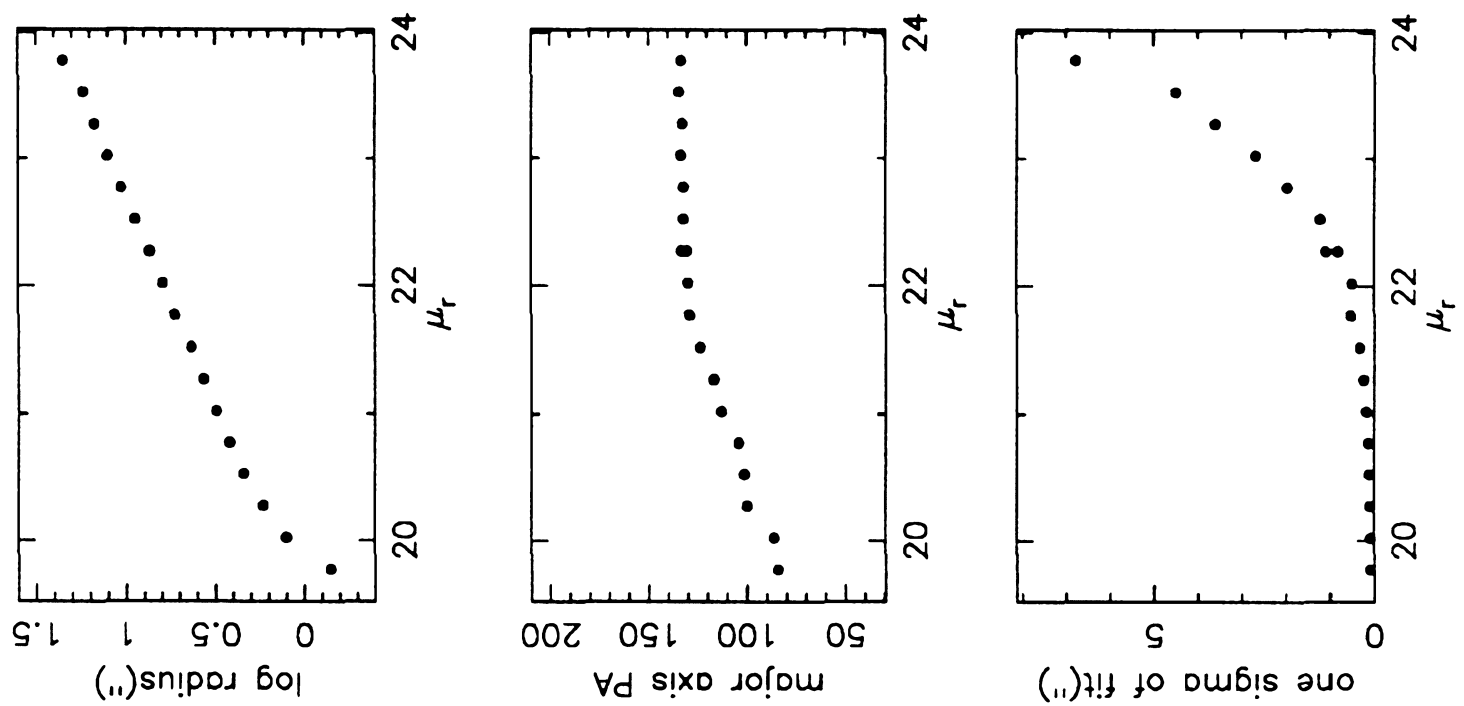

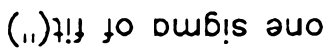
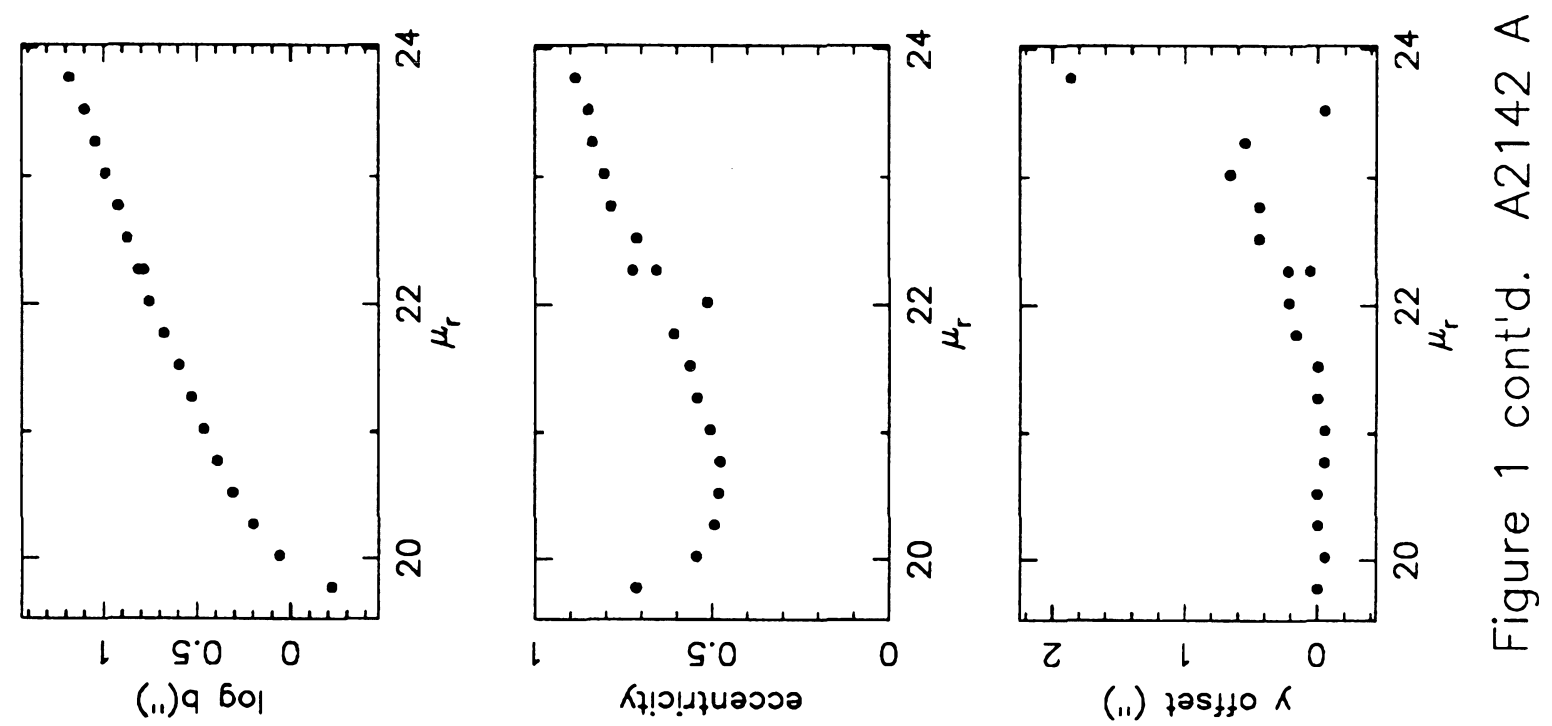

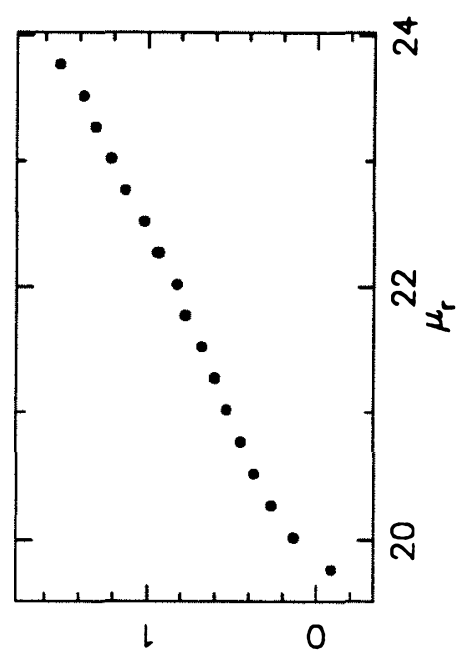

(ii) $\mathrm{D}$ 601
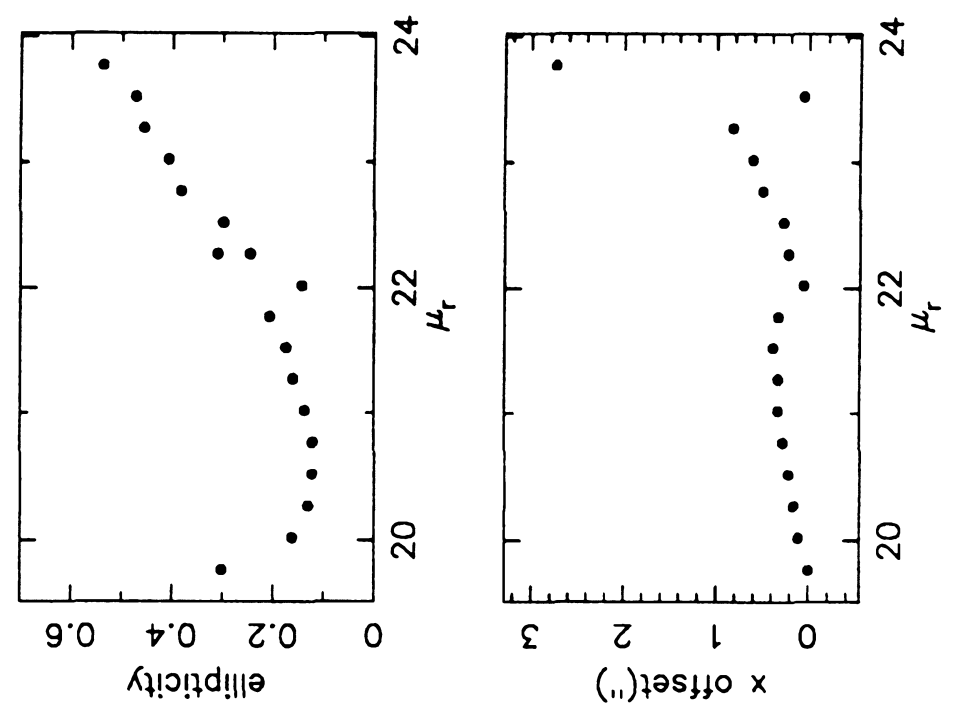


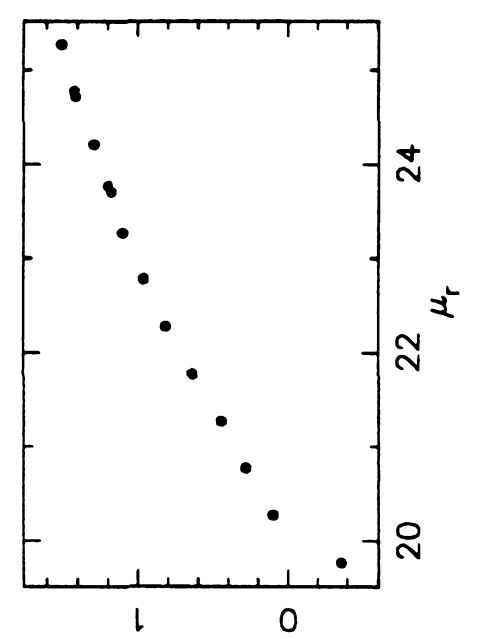

(.1) sn!pos 6ol

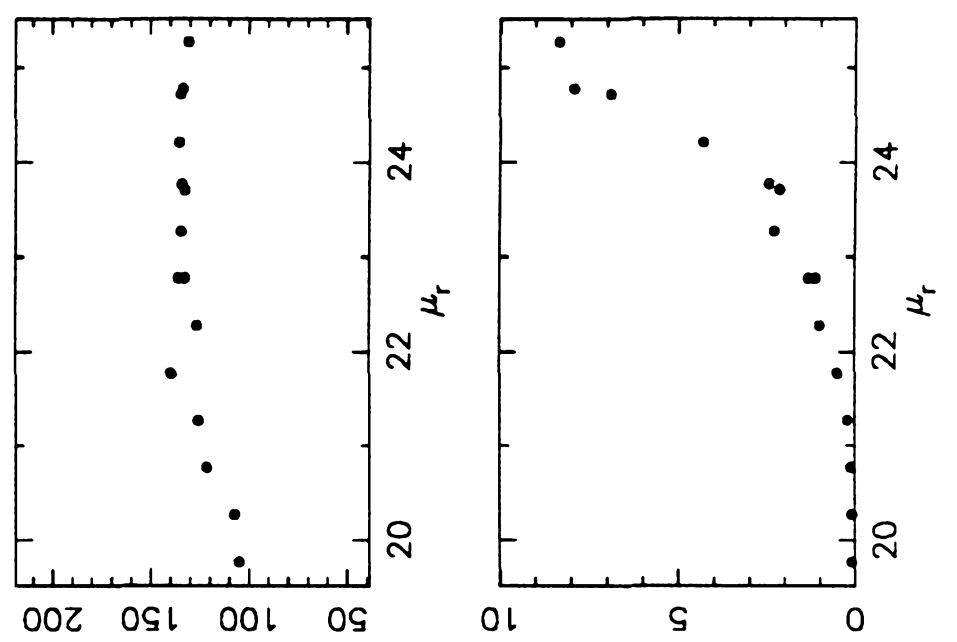

$\forall d$ s!xo so!ou

(11) $4 !+$ to Dub!s auo

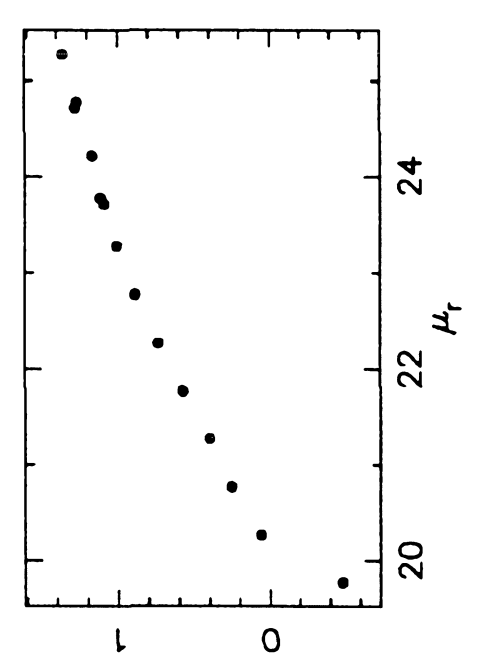

(ii) 9601

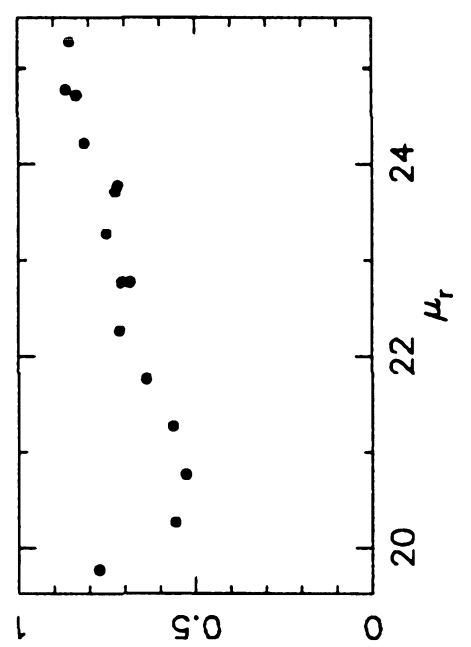

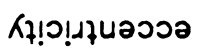

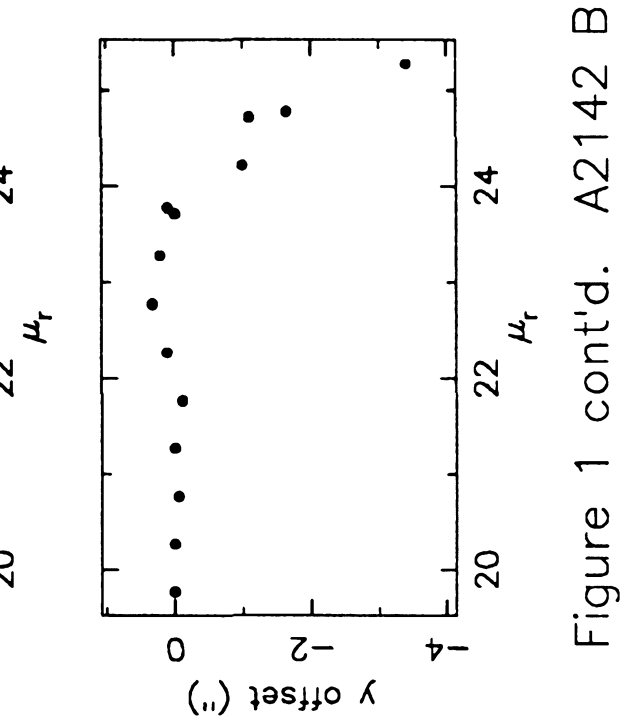

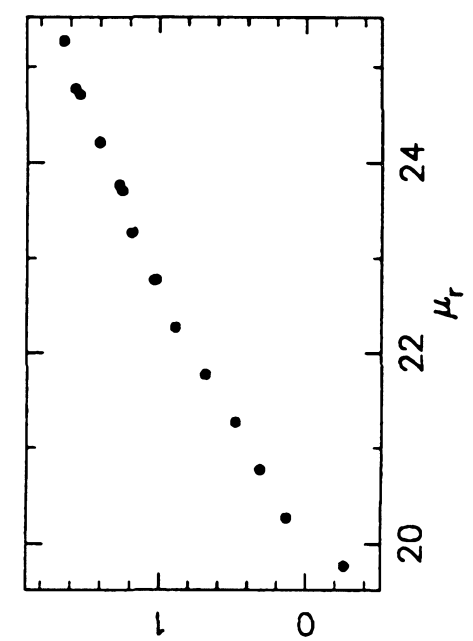

(.1) 0601

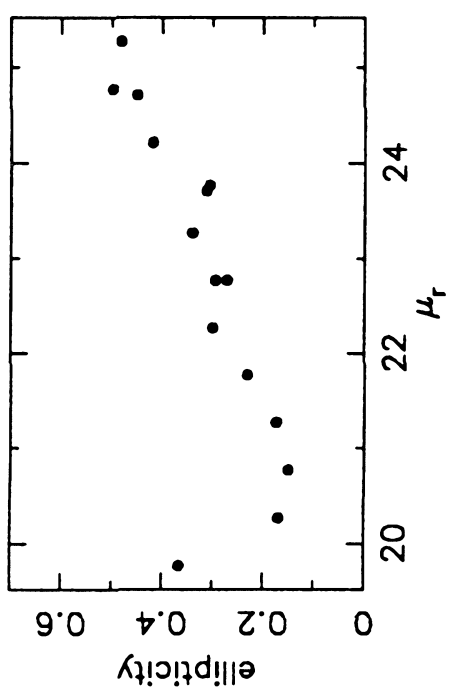

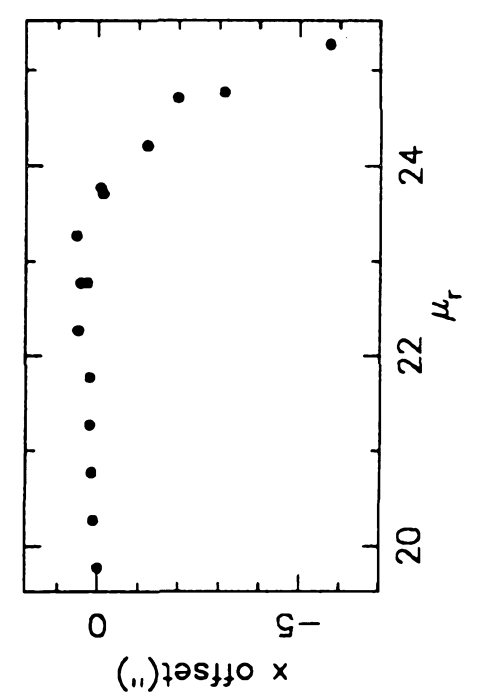




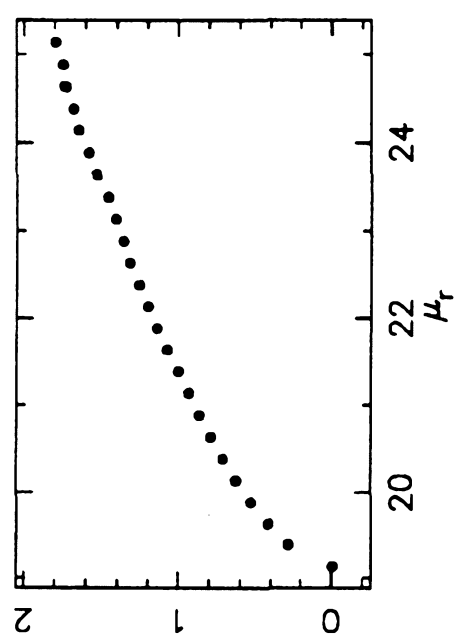

(..)sn!pod 6ol

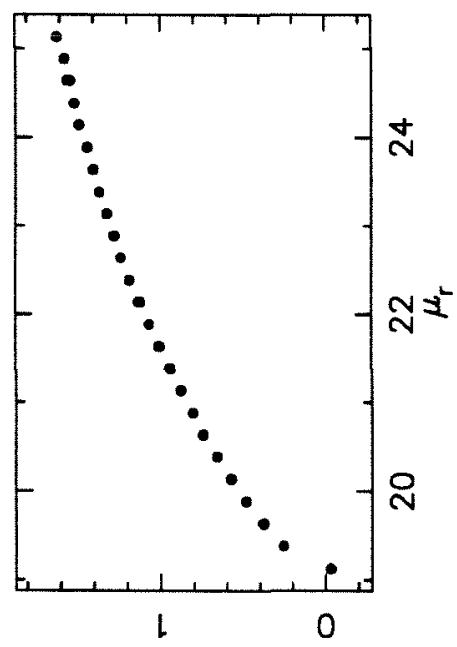

(.1) 9 601

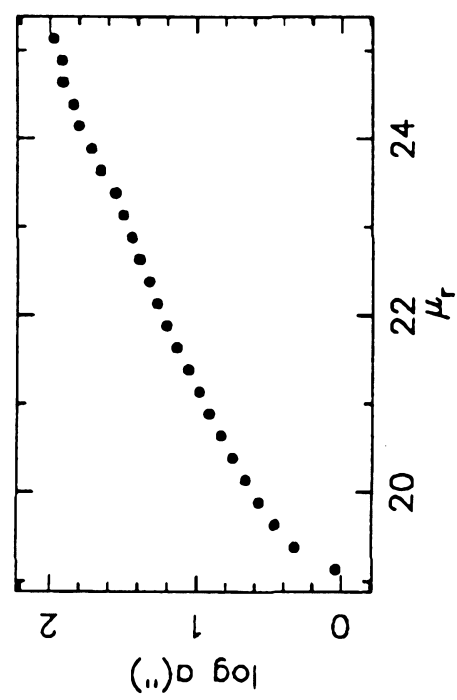

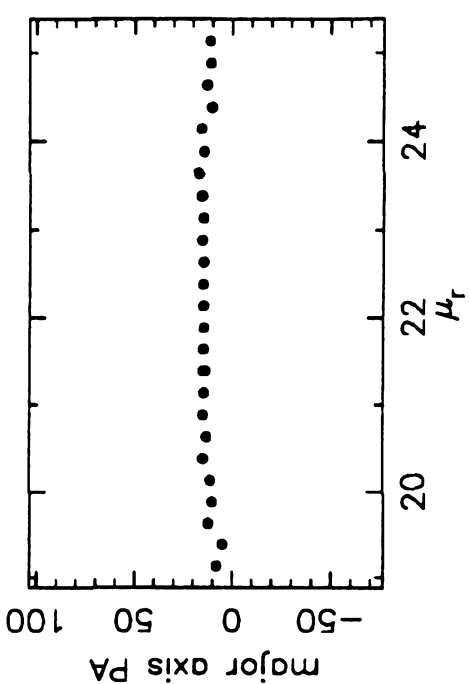

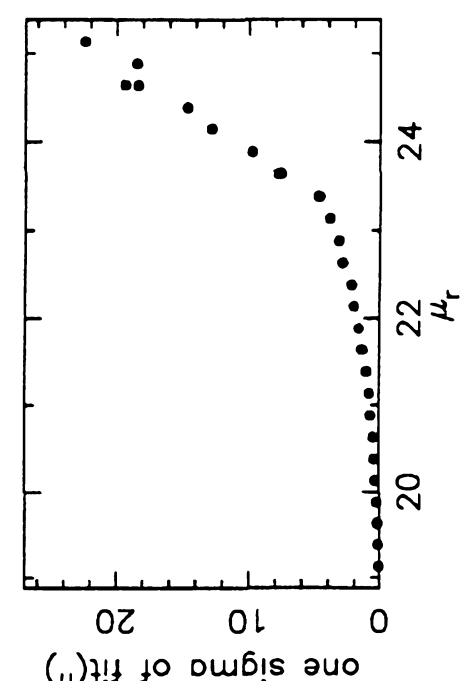

(.1) $+1 !$ to oub! ano

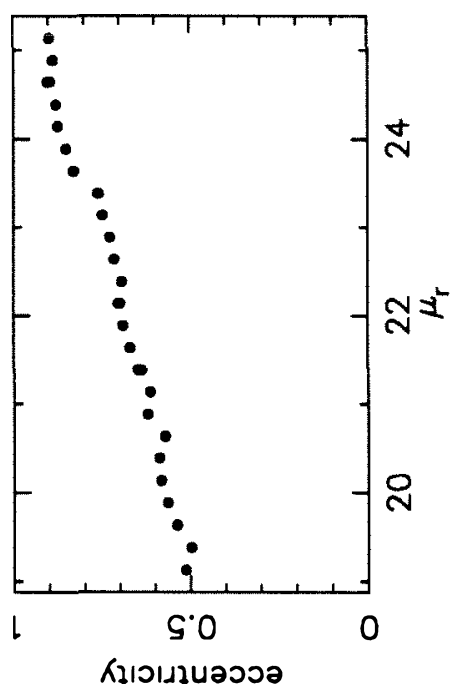

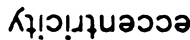

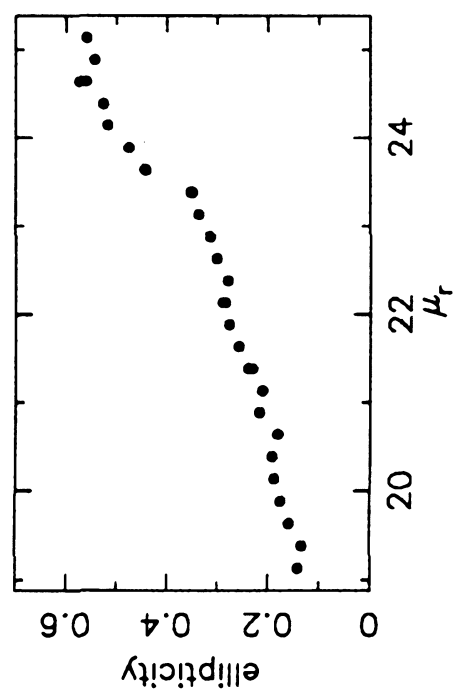

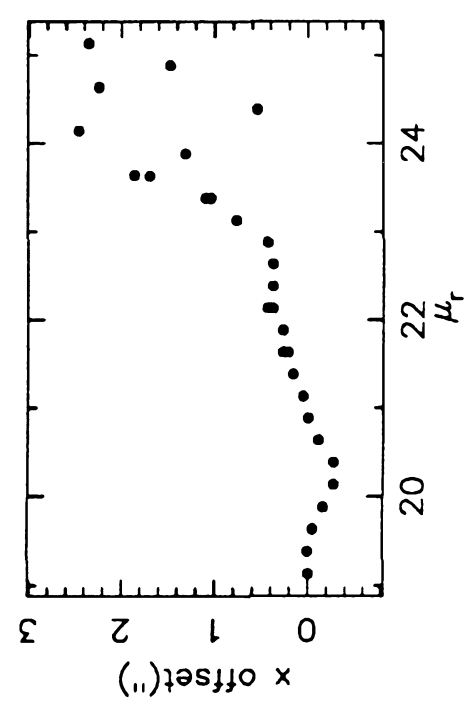



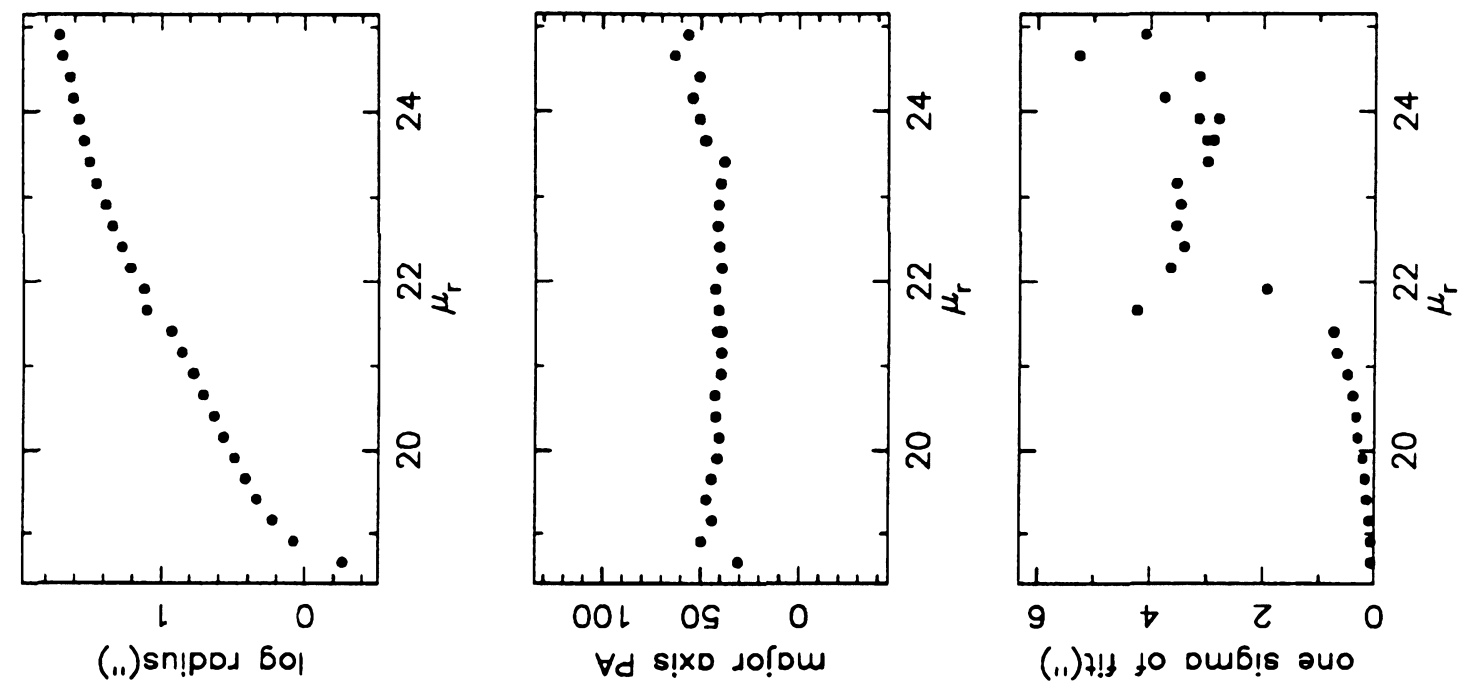

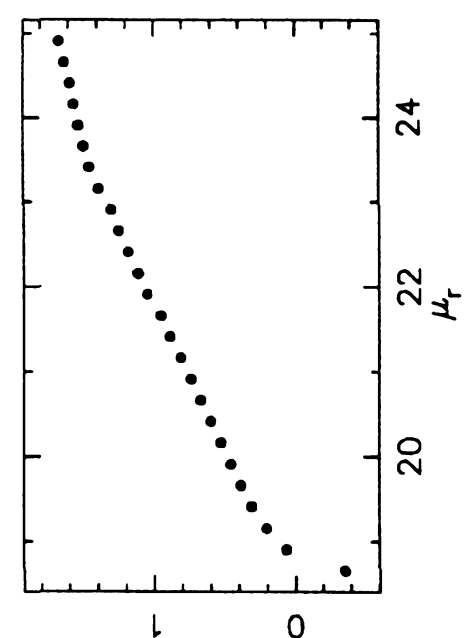

(.1) 9 6ol

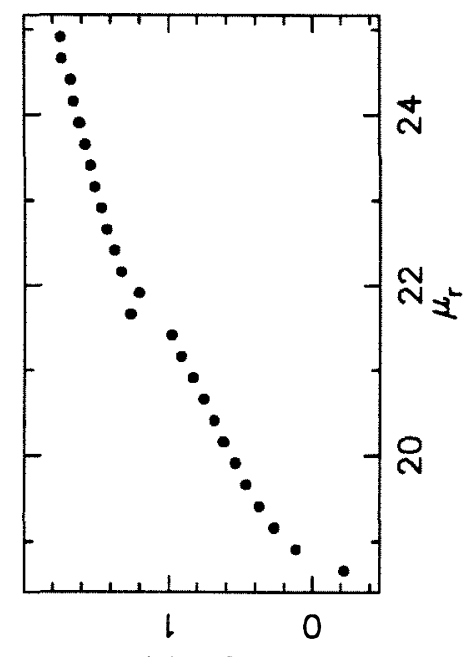

(..) $\mathrm{D}$ 6ol

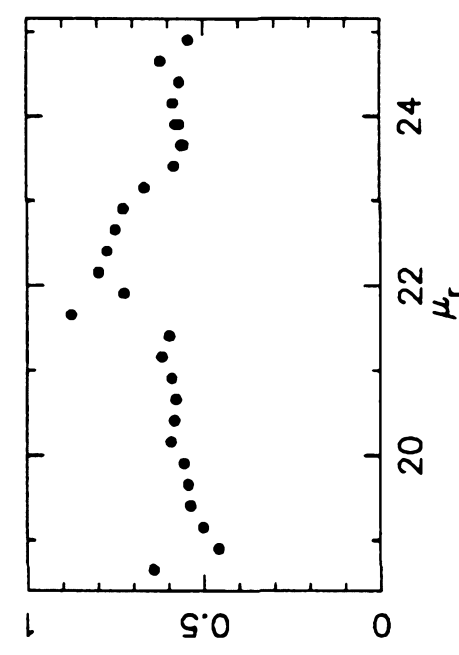

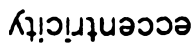

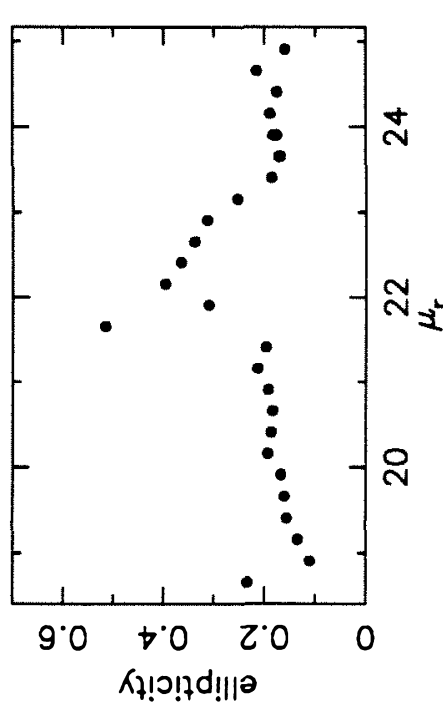

ธิ

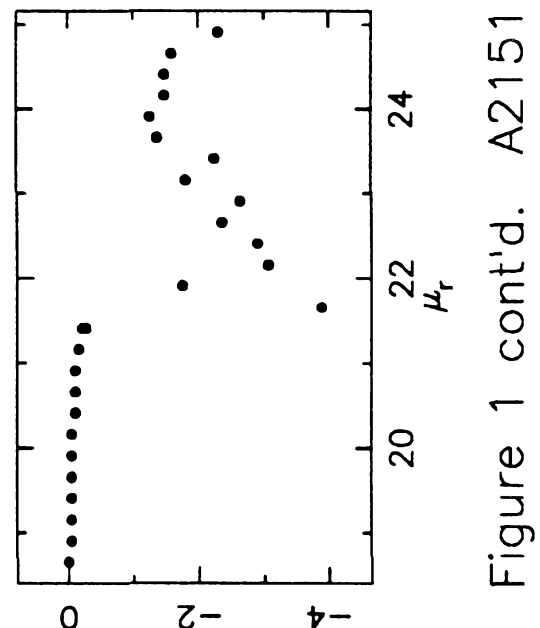

(i1) $725150 \mathrm{~K}$

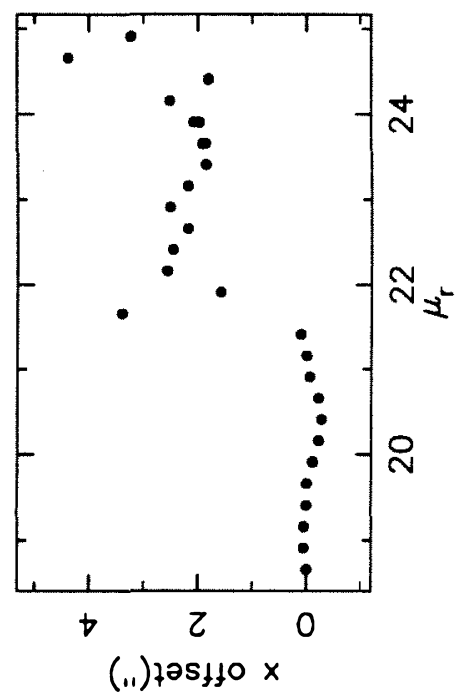




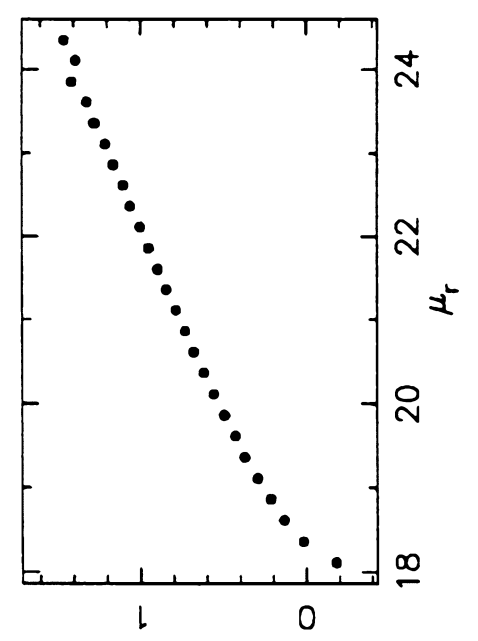

(.1) sn!pod 6ol

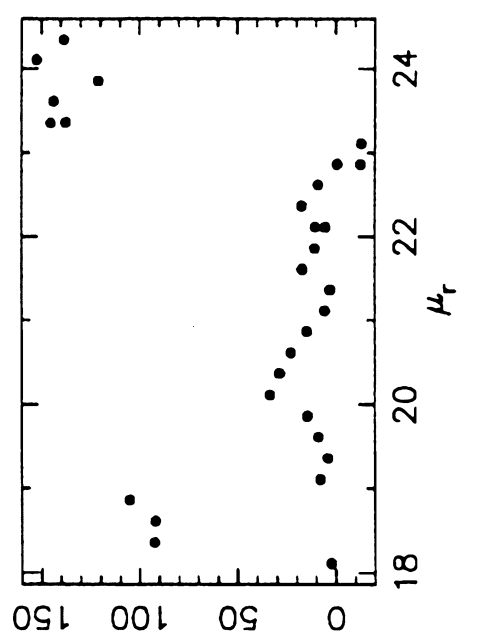

$\forall d$ s!xo do!́om

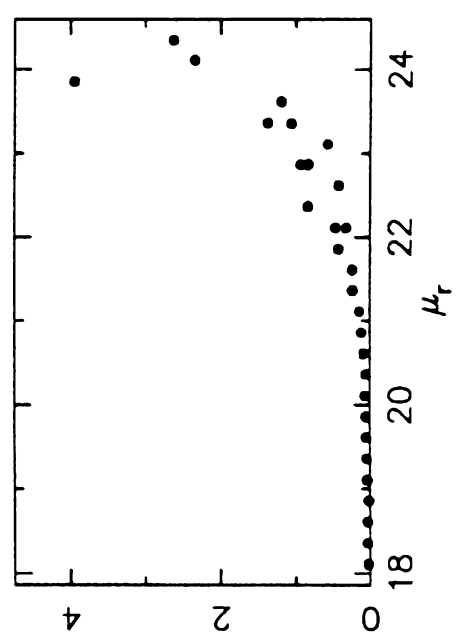

(11) $7 !+$ to omb!s au०

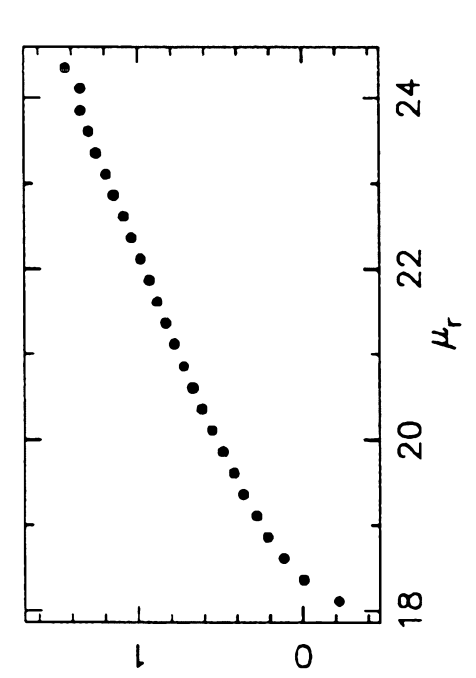

(.1)q 6ol

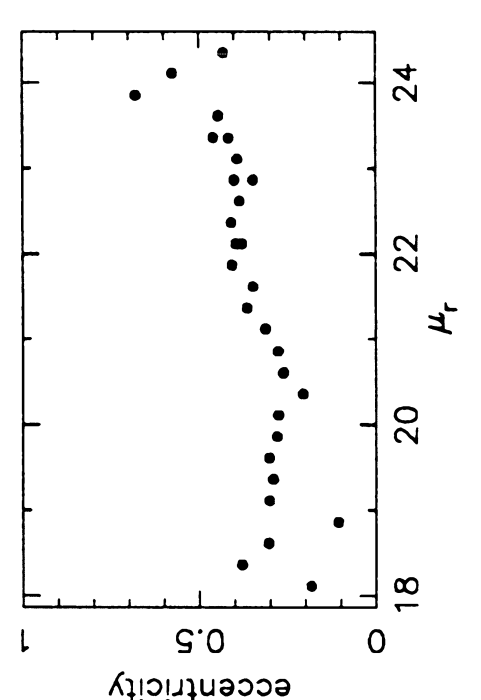

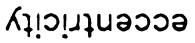

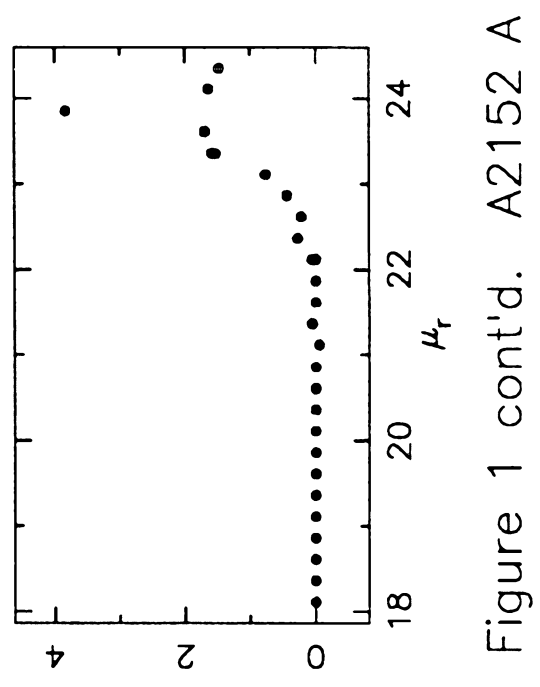

(in) $725 \%$

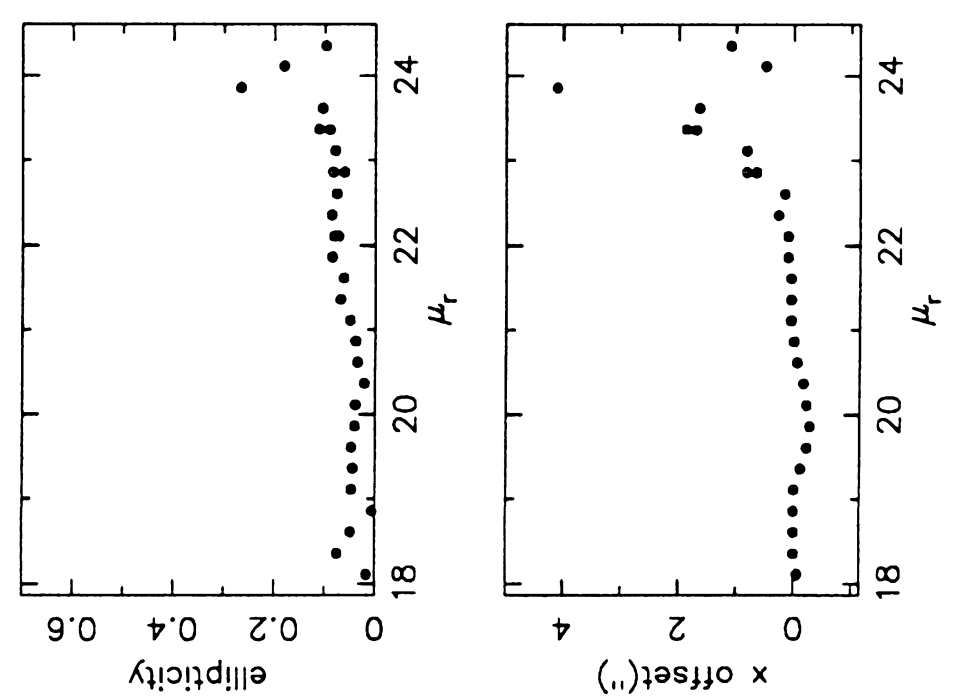




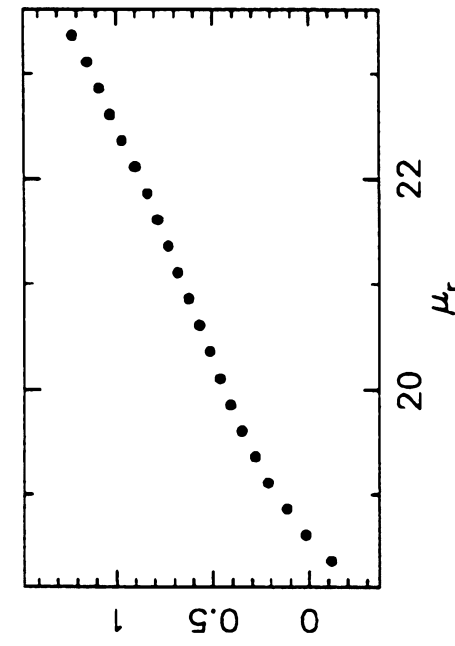

(.1) sn!pod 6ol
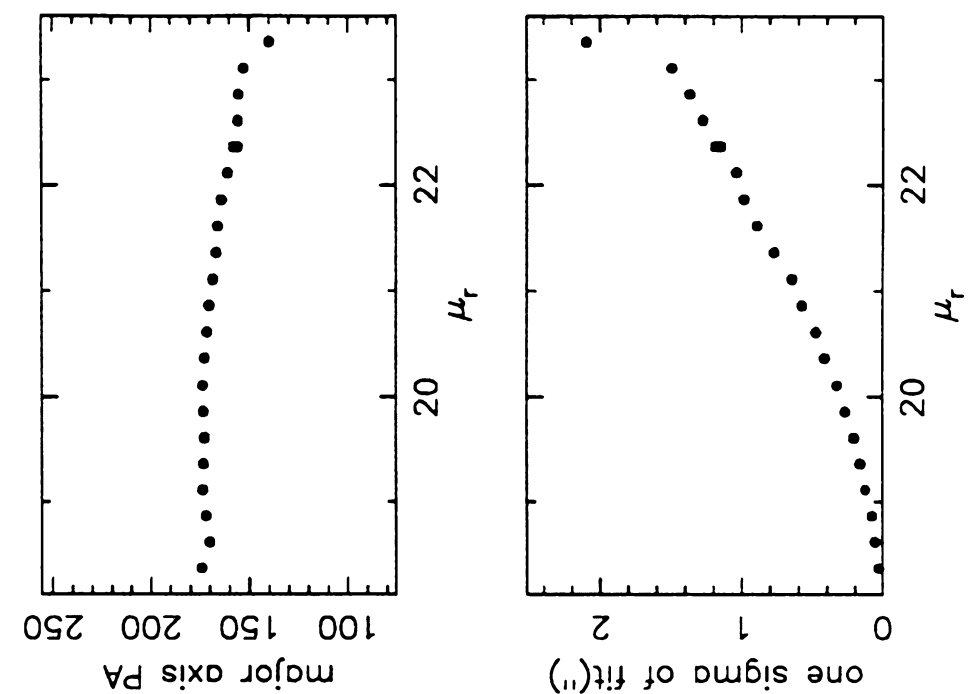

(11) $7 ! t$ to Dub! a
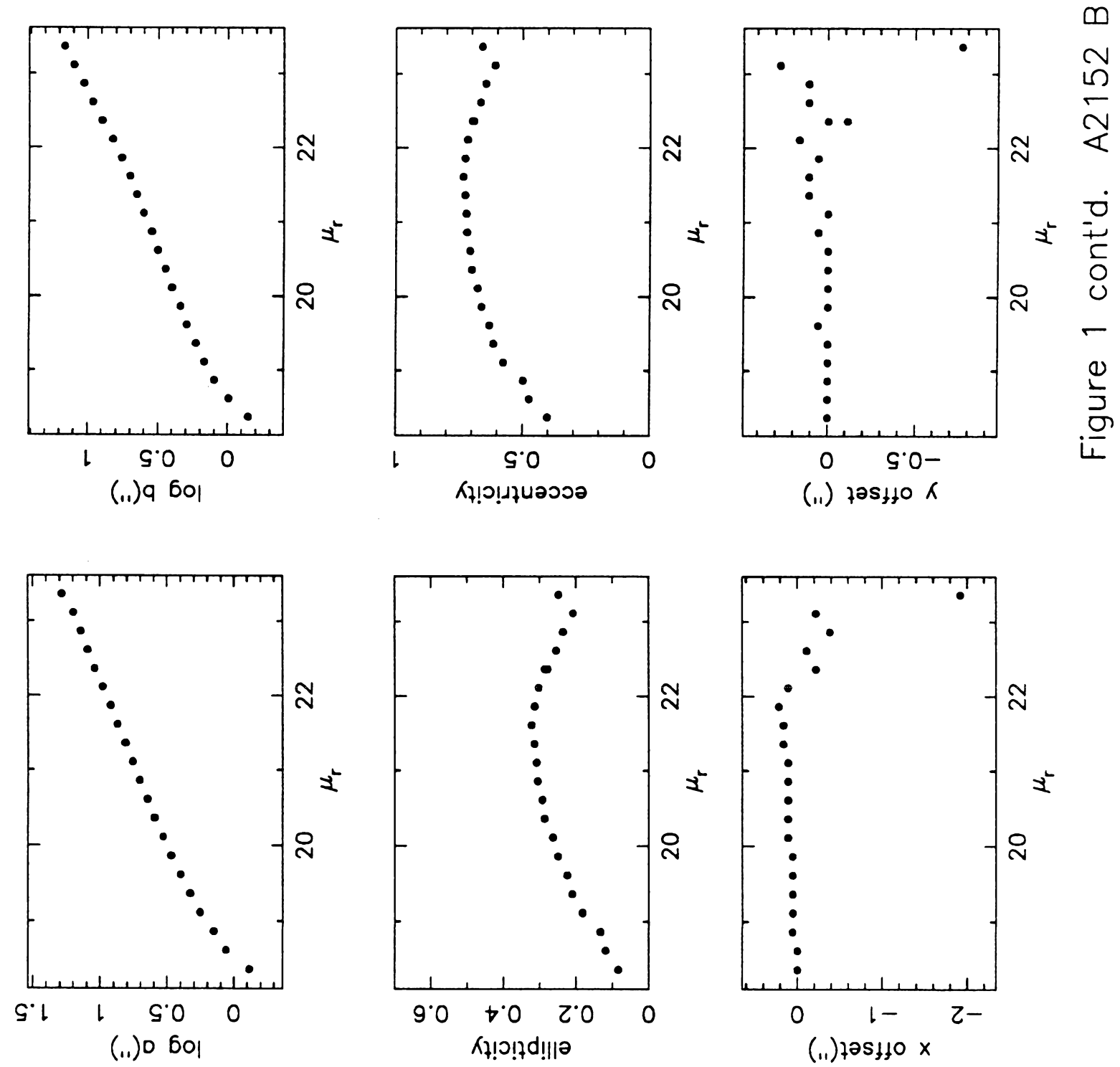


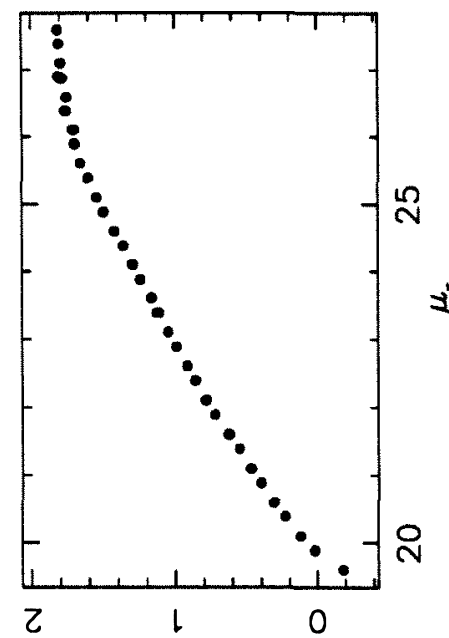

(11) sn!pod 60।

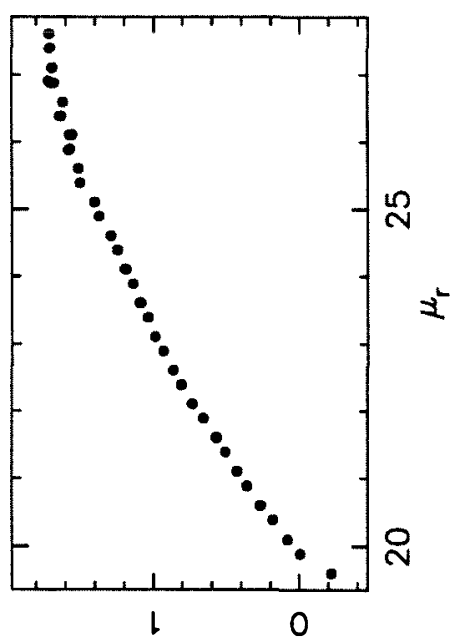

(i1) 9601

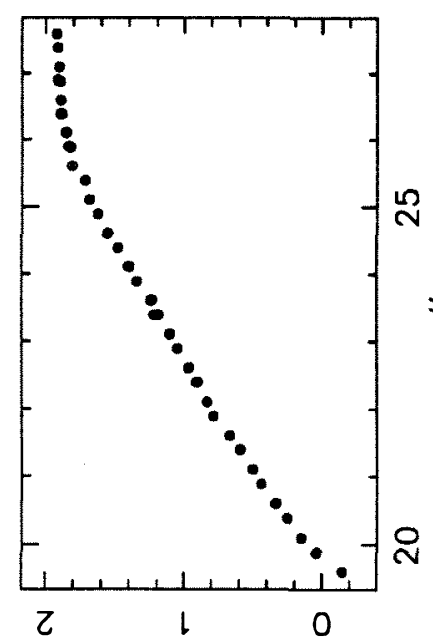

(.1) $0 \mathrm{60}$

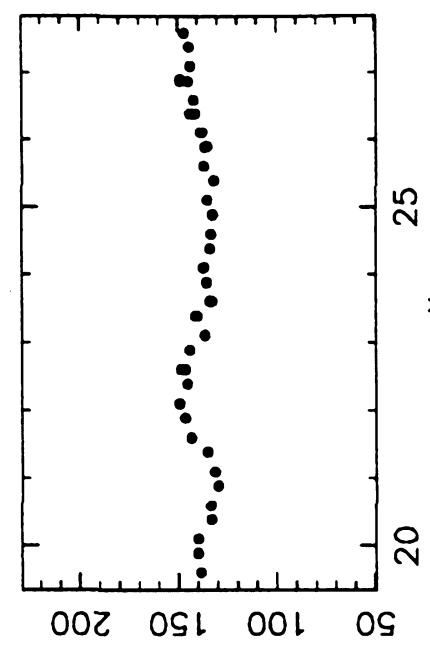

$\forall d$ s!xo so!̣u

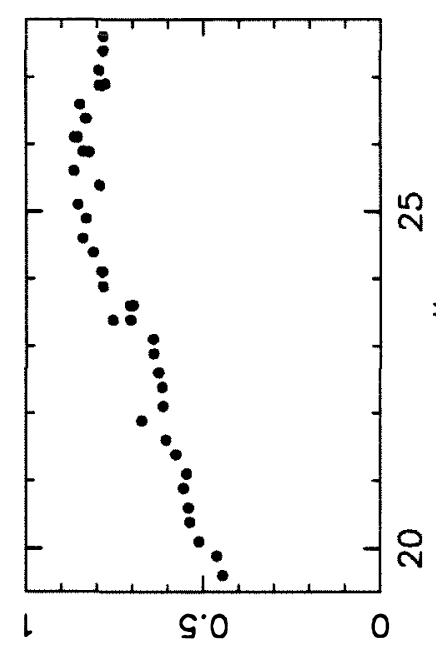

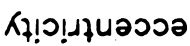

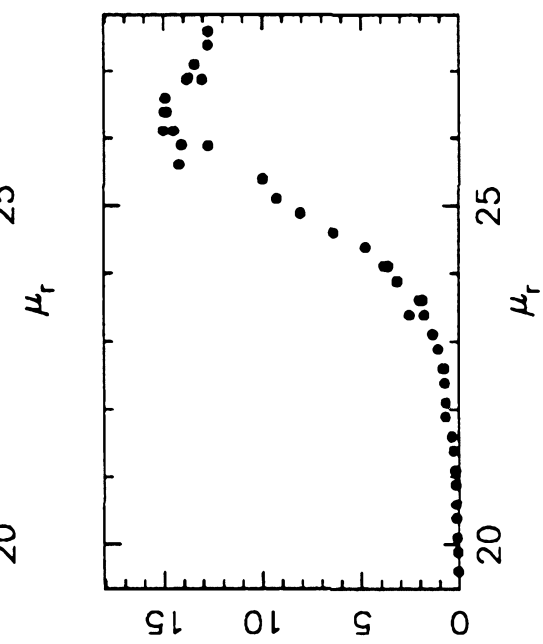

(11) $+!+$ to oub!s auo

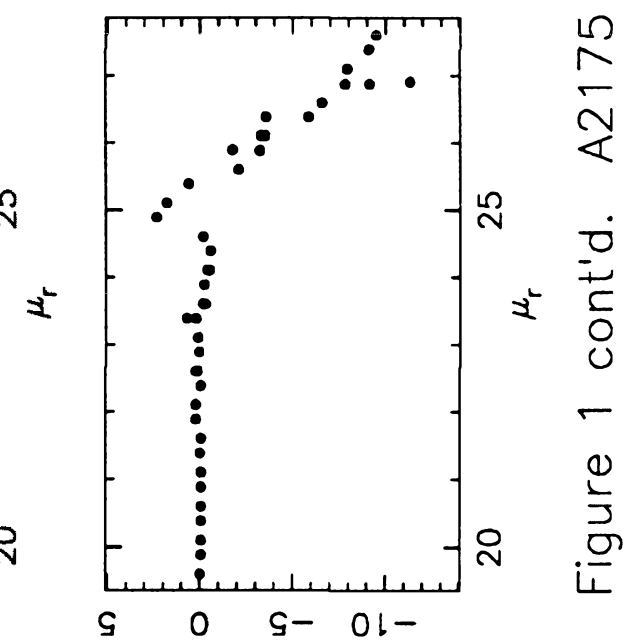

(.1) $725410 \cdot \mathrm{K}$
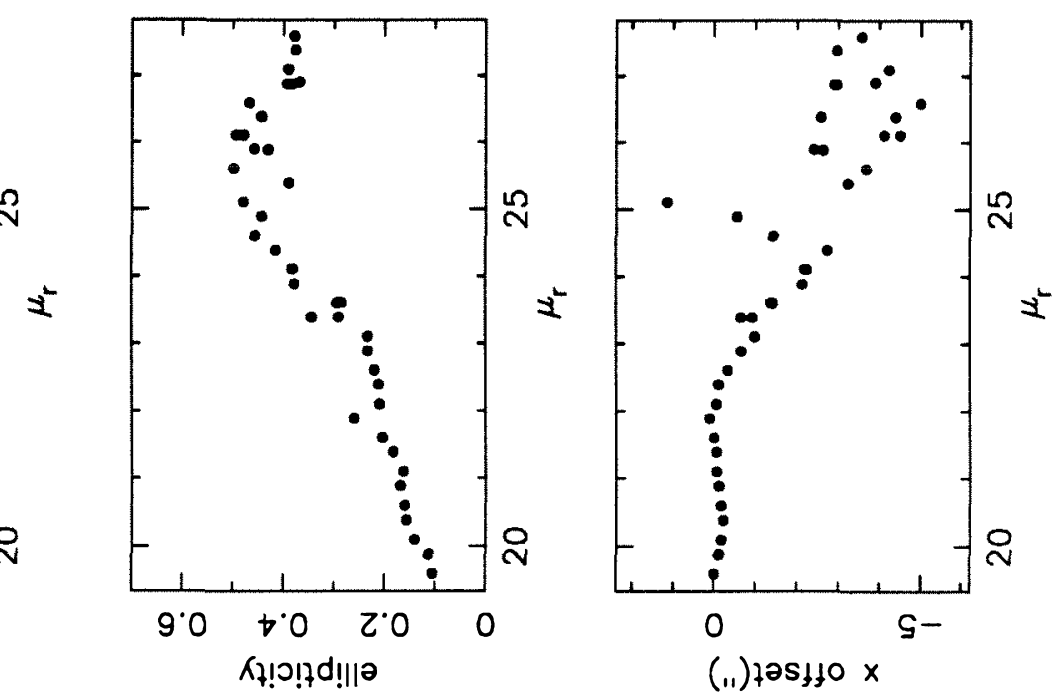


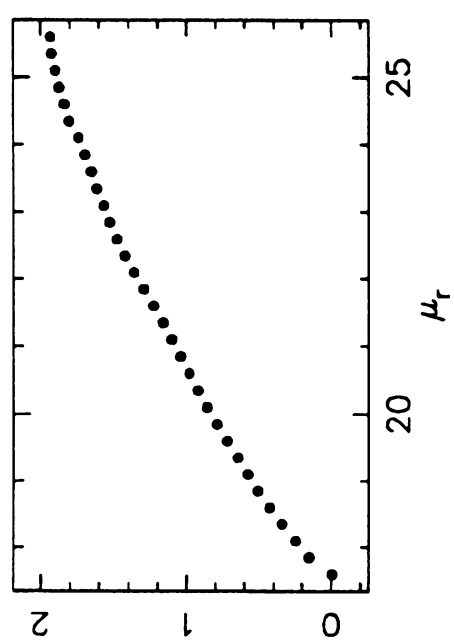

(.1) sn!pod 601

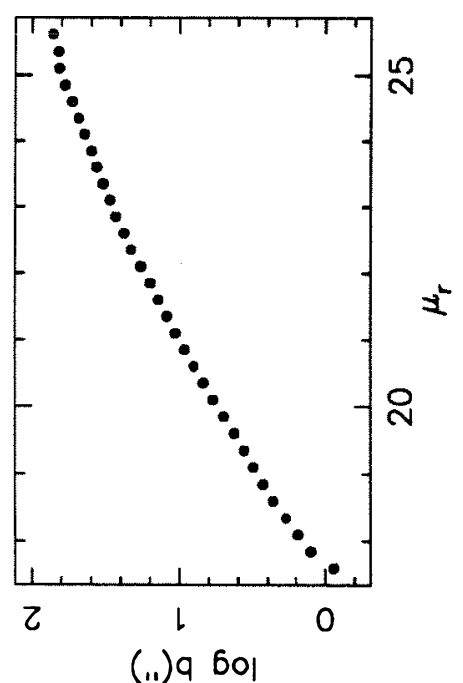

(.1) 960

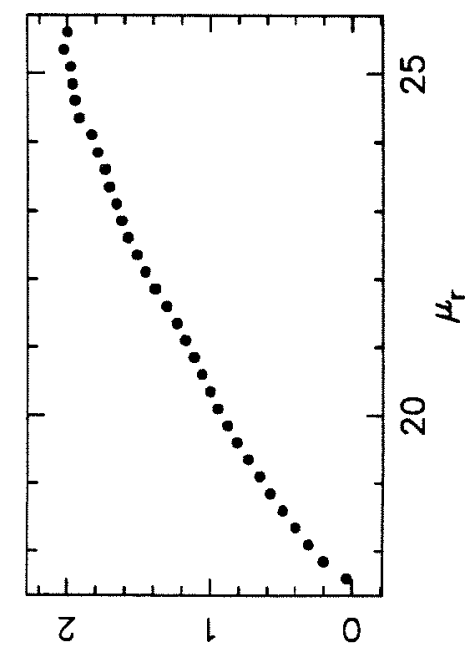

(.1) $\mathrm{O} 6 \mathrm{OO}$
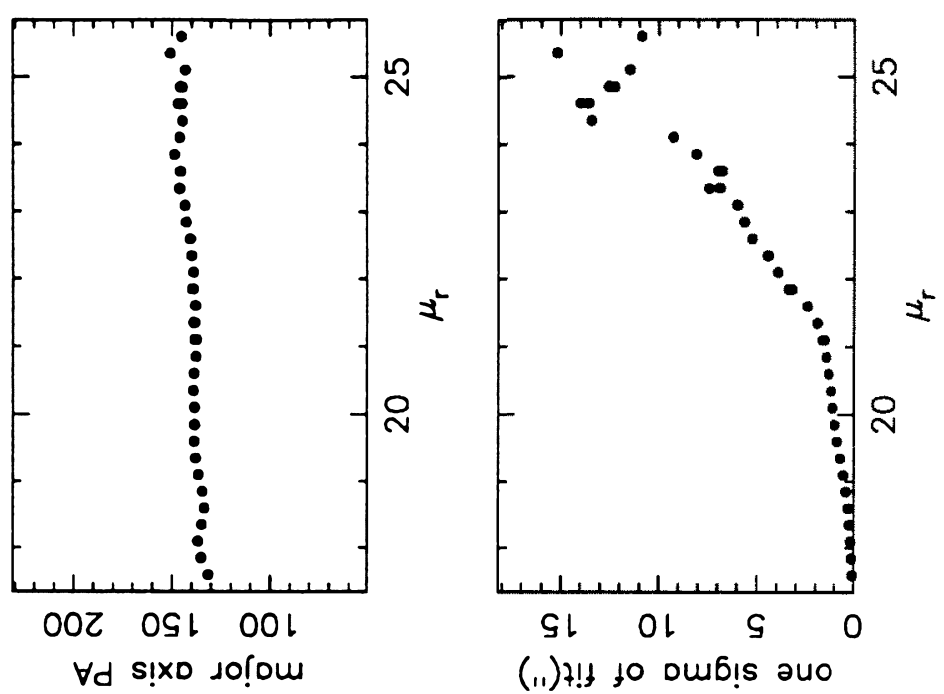

(11) $7 !+10$ oub!s ano
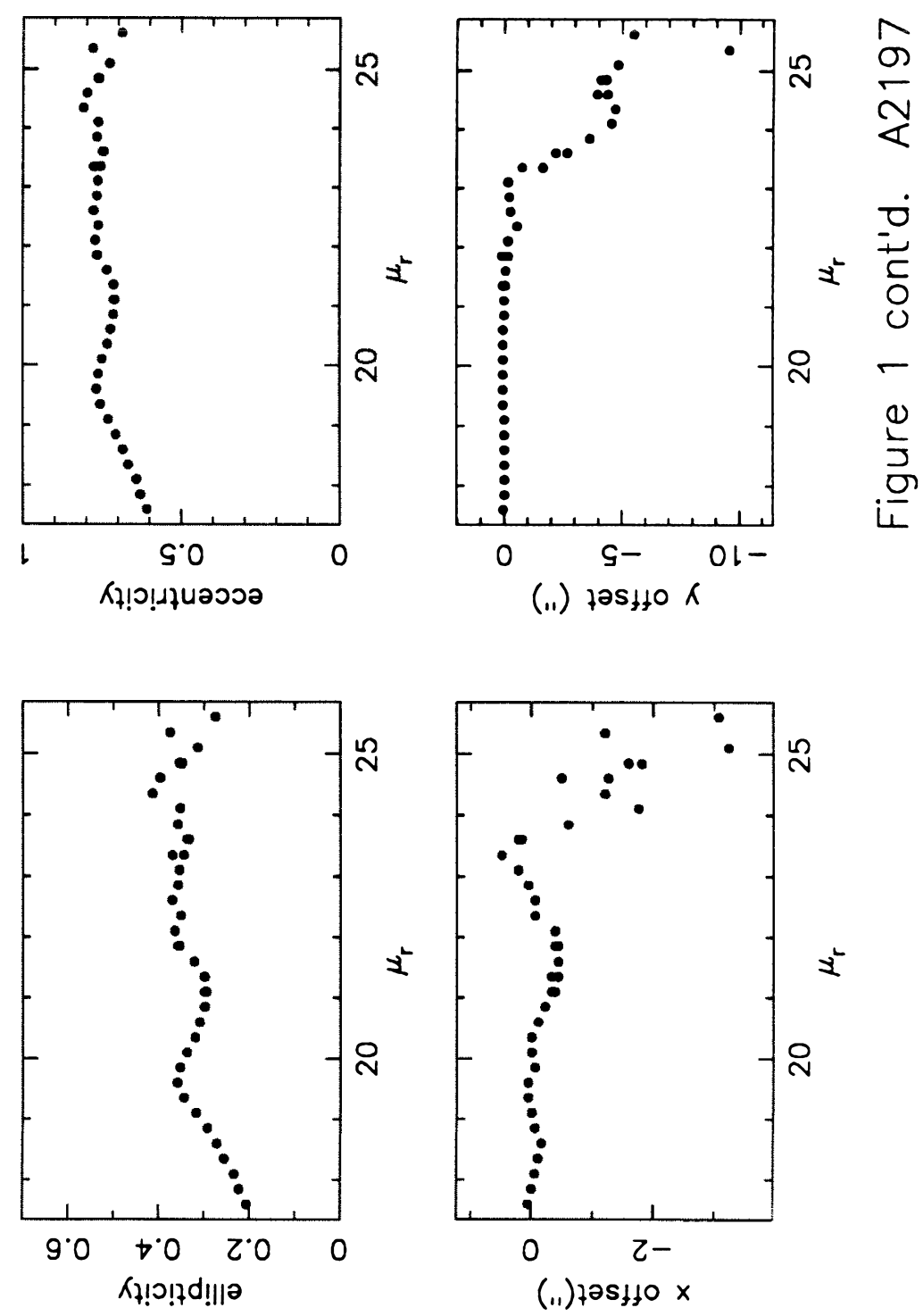

$\Sigma^{2}$ 


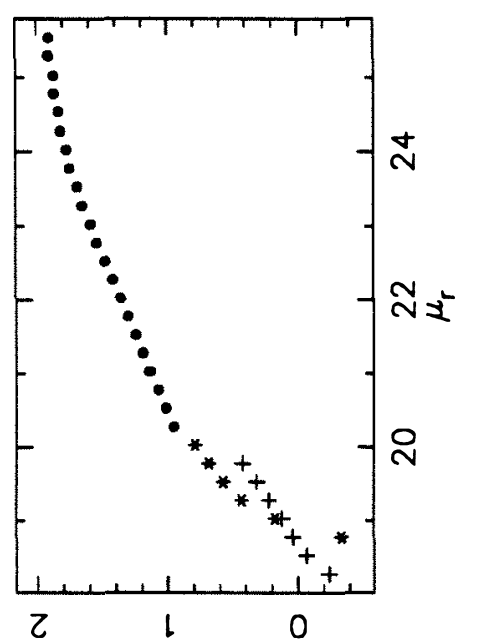

(.1) sn!pos 60।

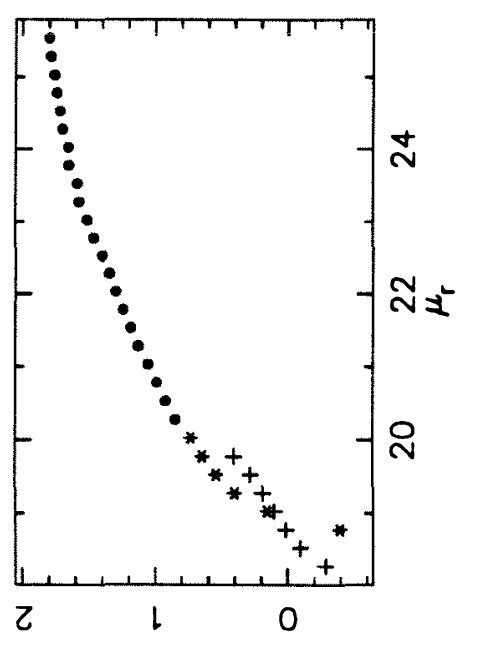

(i1) 950

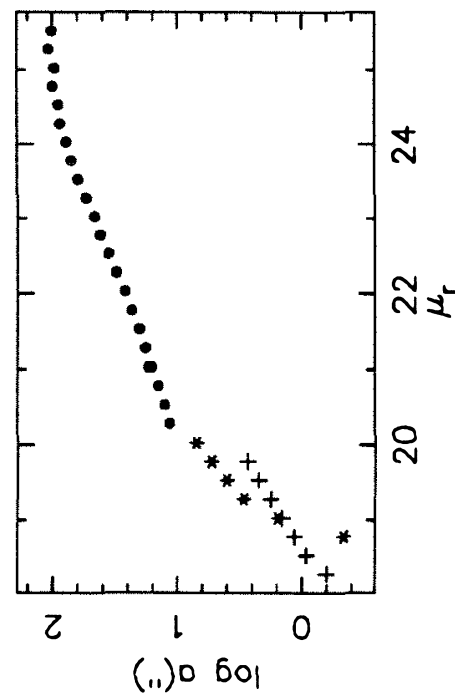

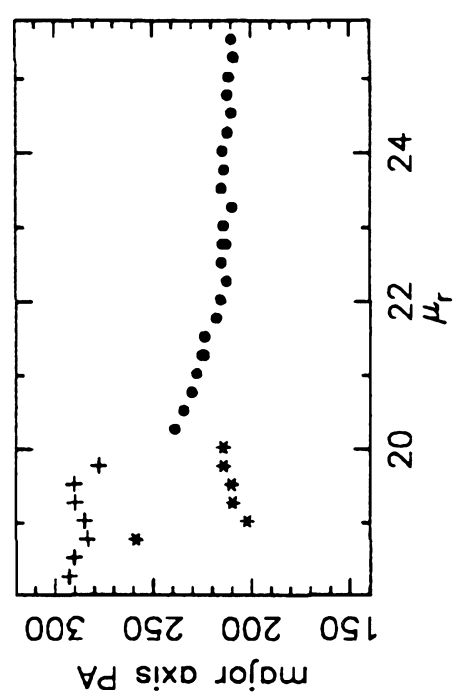

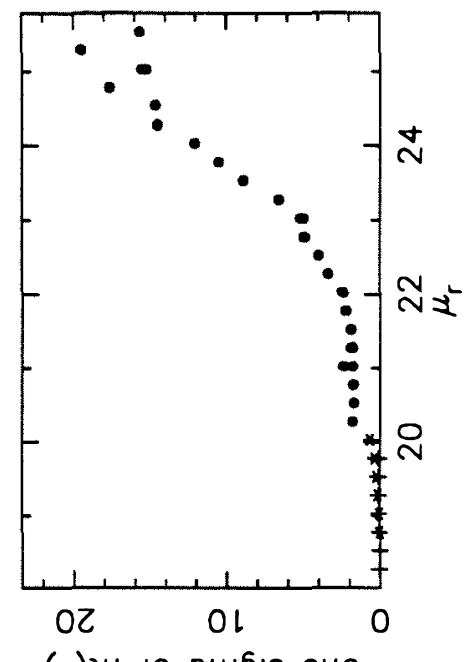

(11) $7 !+$ to oub!s auo
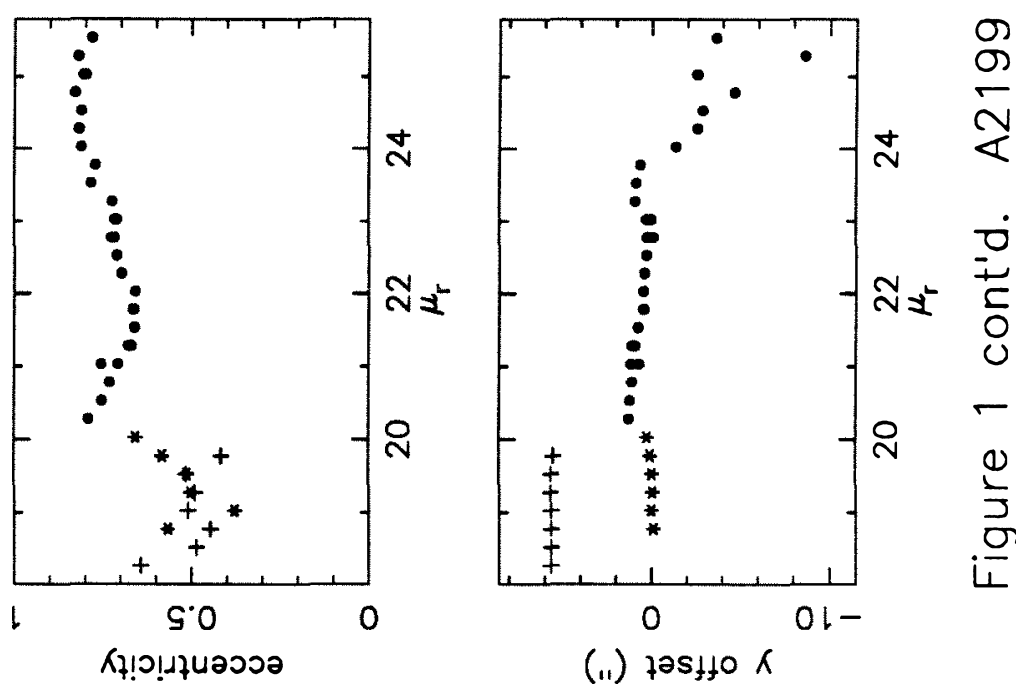

(11) $725150 \%$

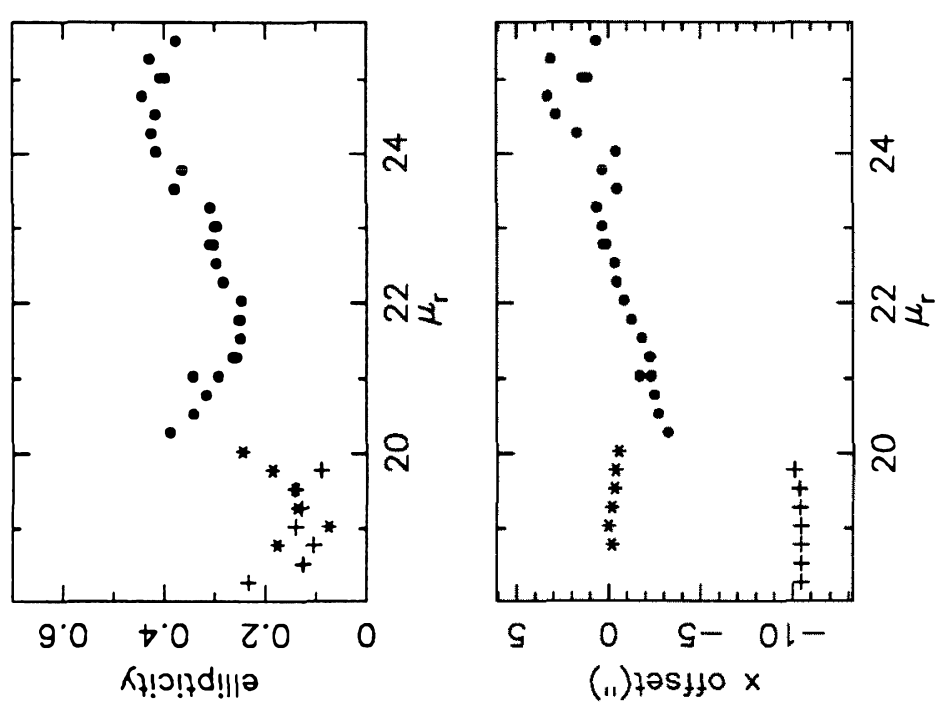



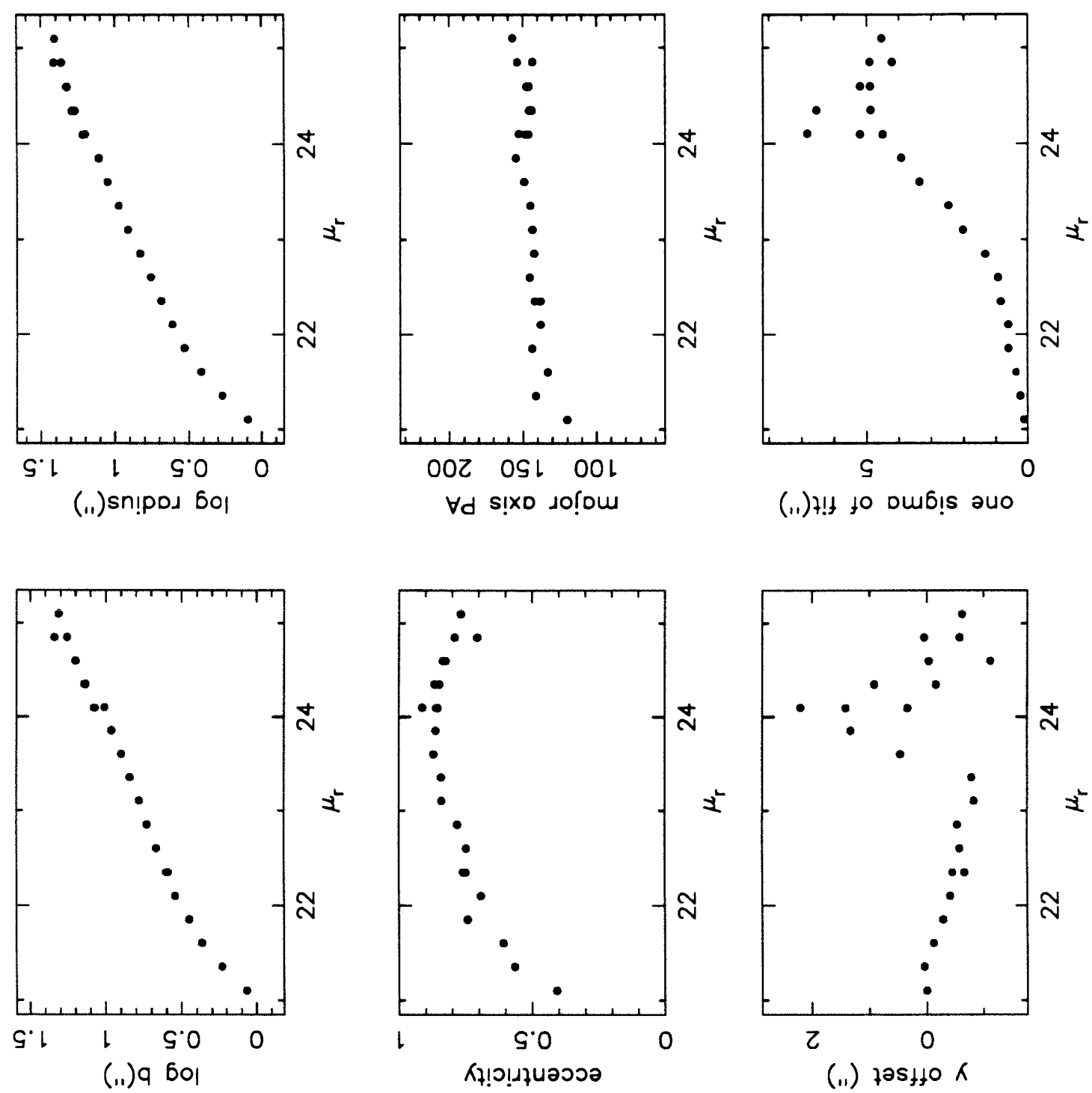

(11) $+! !$ to oub!s auo 2 


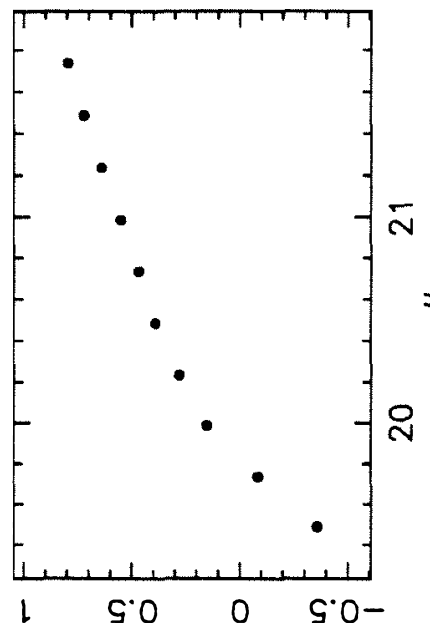

(i.) sn!pos 501

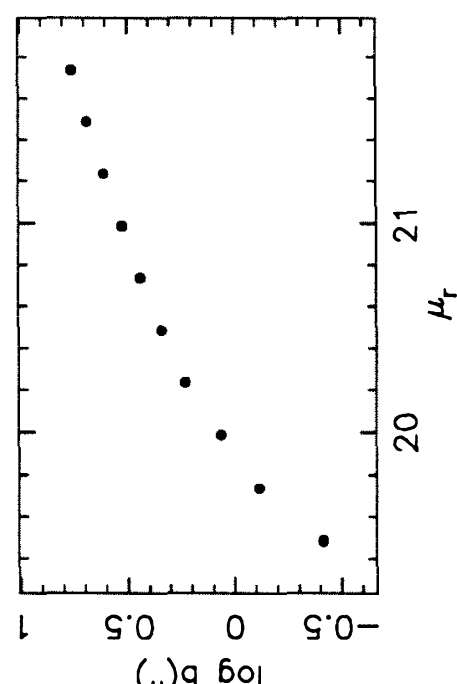

(.1) $9 \mathrm{bol}$

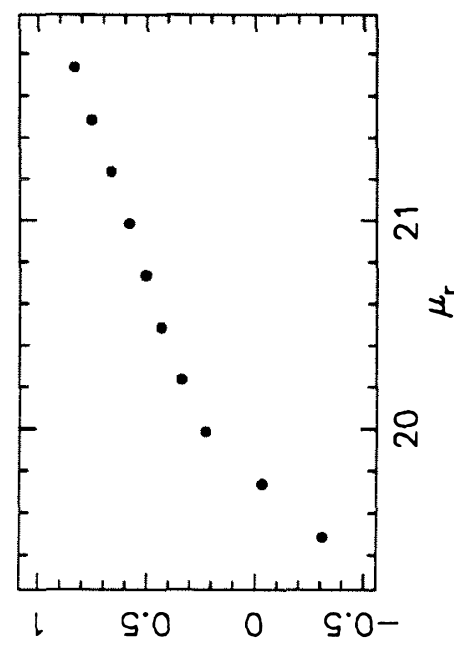

(.1) $0 \mathrm{60}$
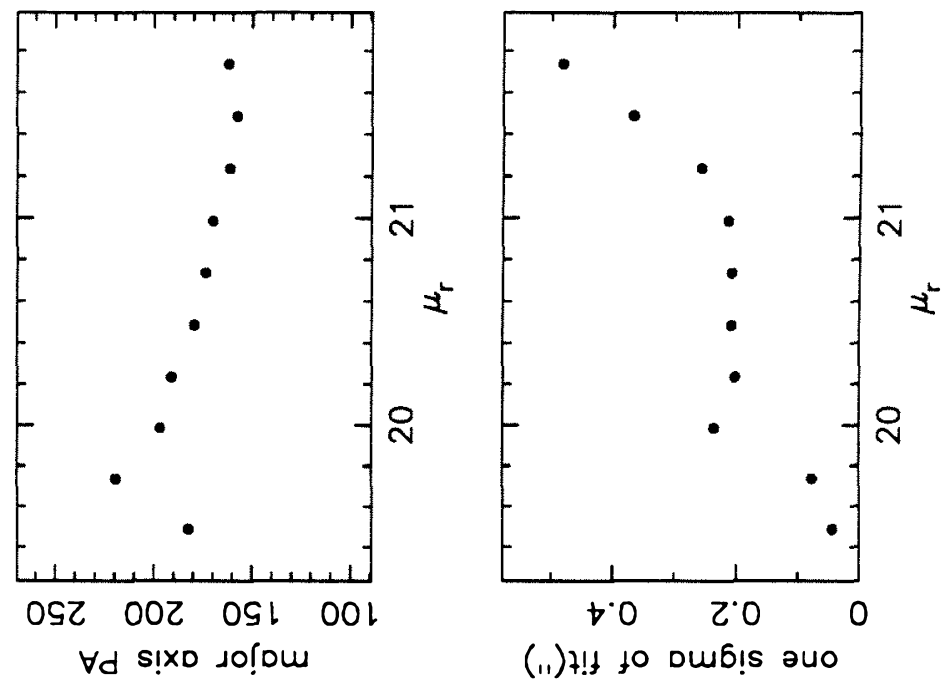

(11) $+!+$ to Dub!s auo
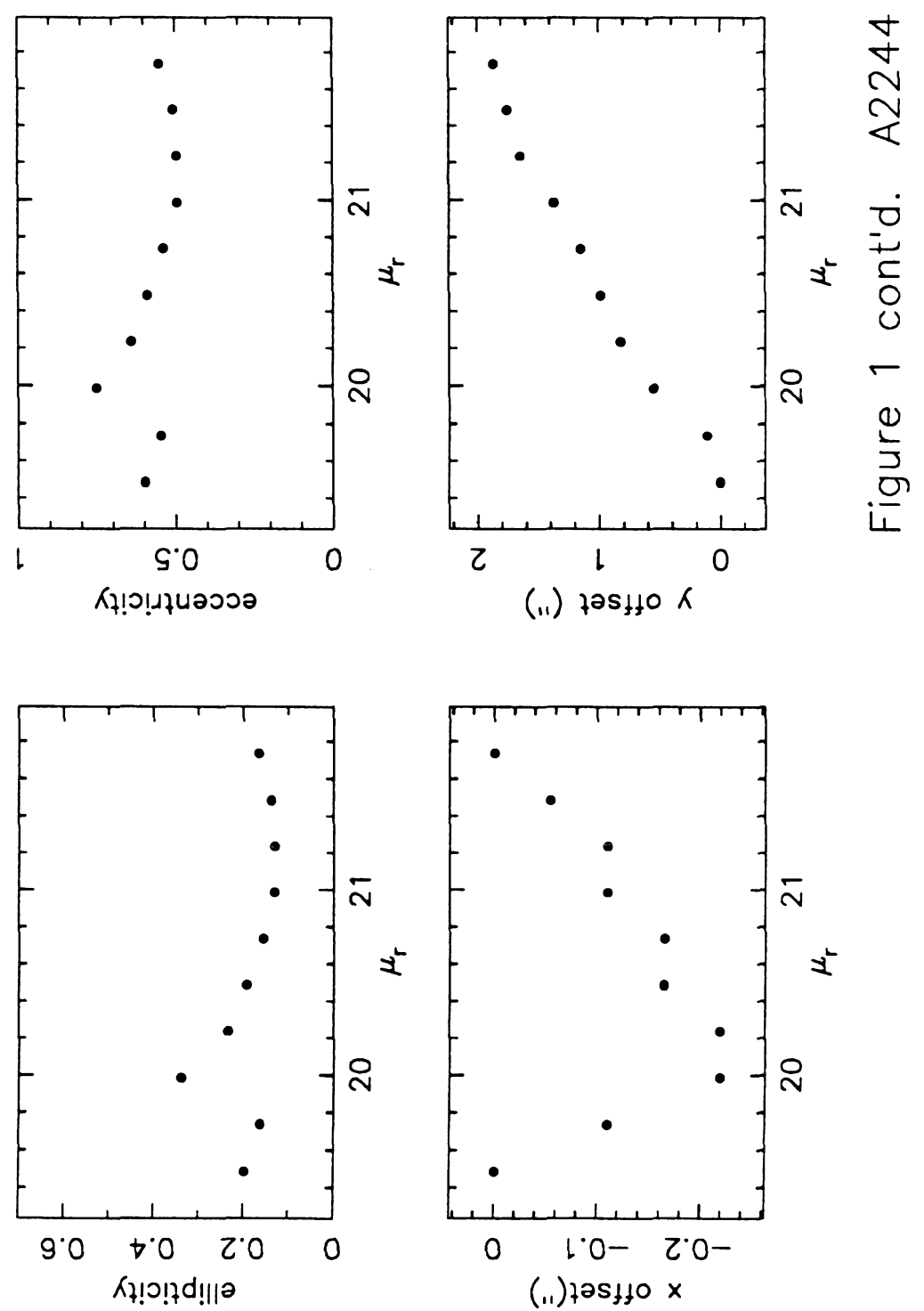


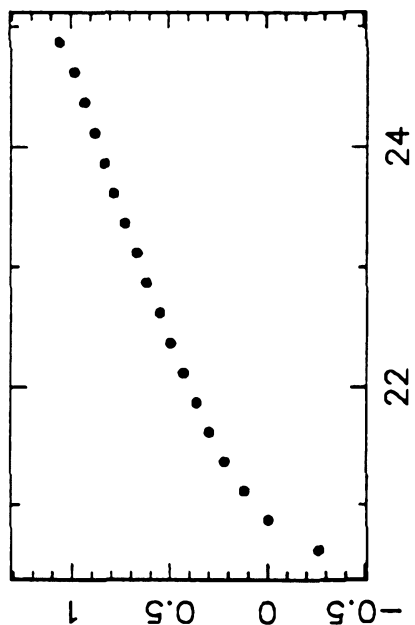

(.1) sn!pos 601
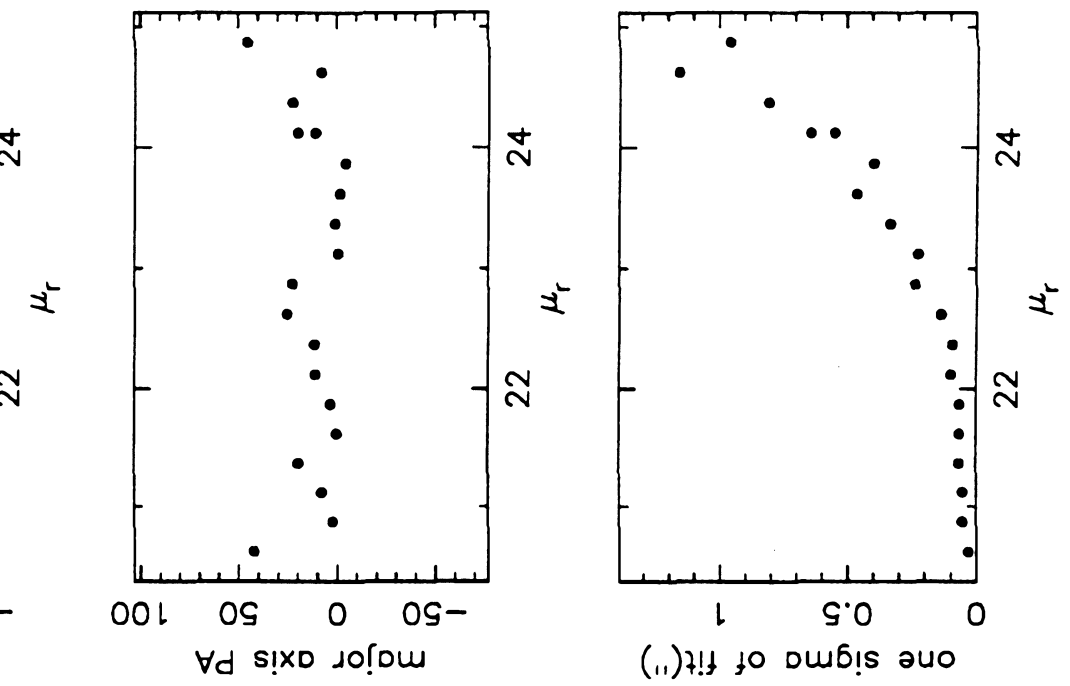

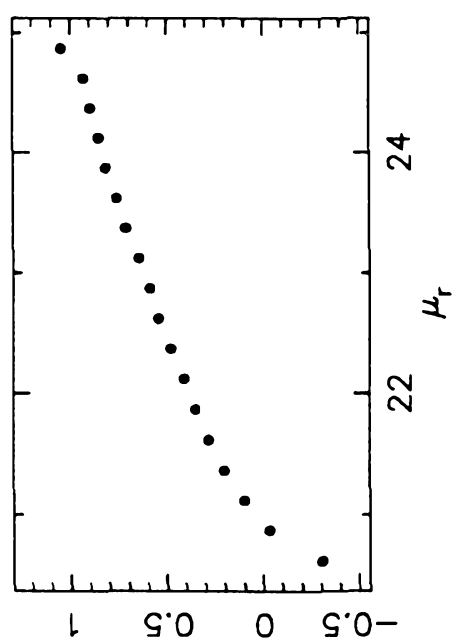

(i1) 9 6ol

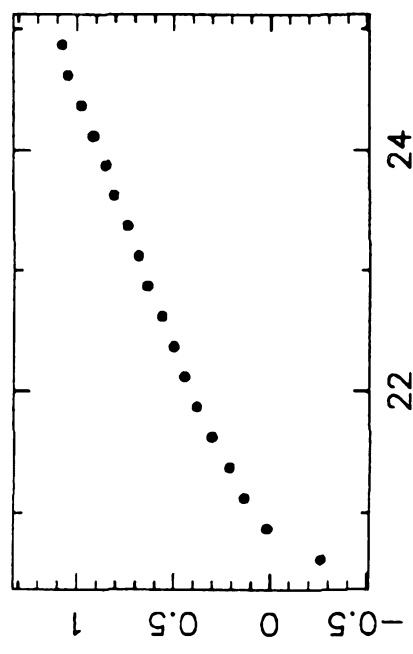

(..) 0 601

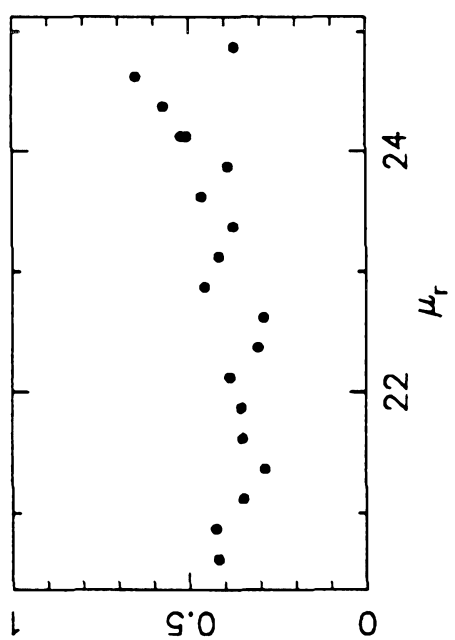

Кұ!ว!นในวววә

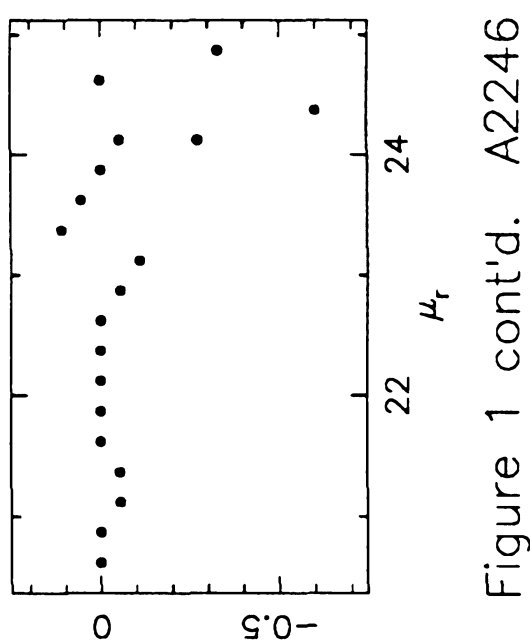

(ii) $72540 \mathrm{~K}$

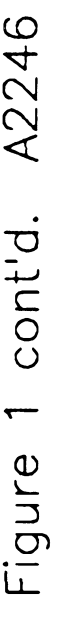




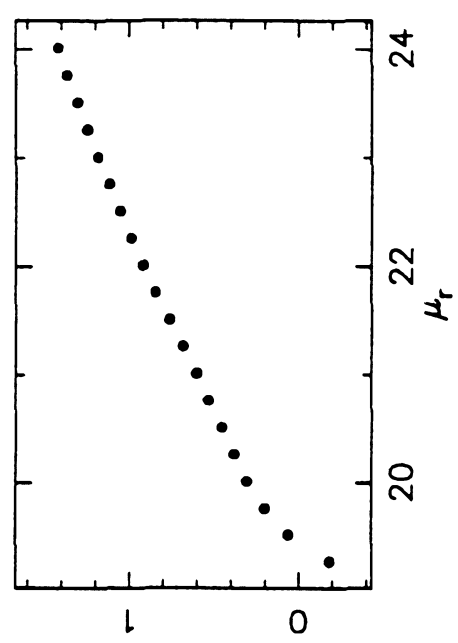

(.1) sn!pos 6ol

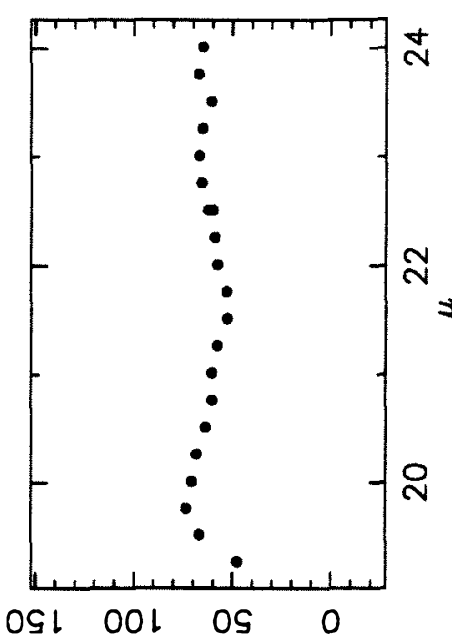

$\forall d$ s!xo so!̣om

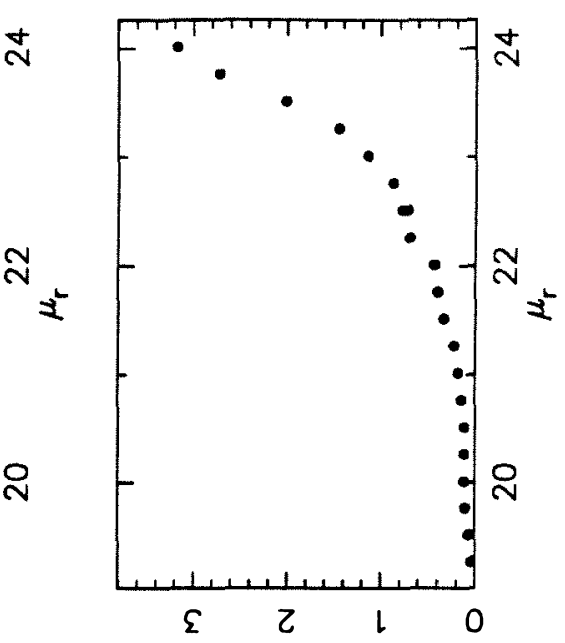

(11) 7 !t to oub!s ano

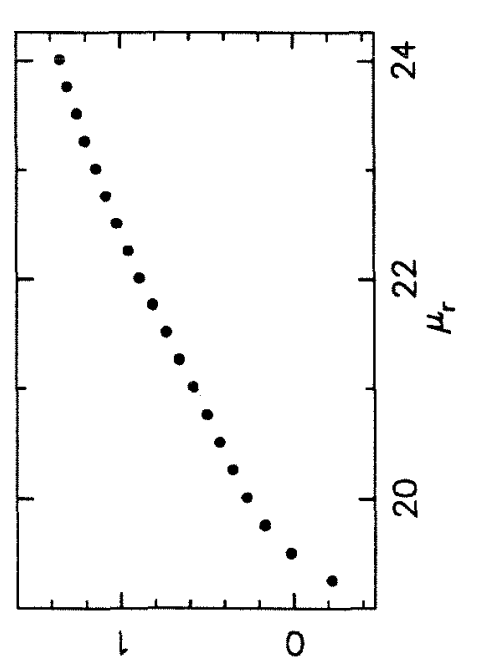

(.1) $q \mathrm{bol}$

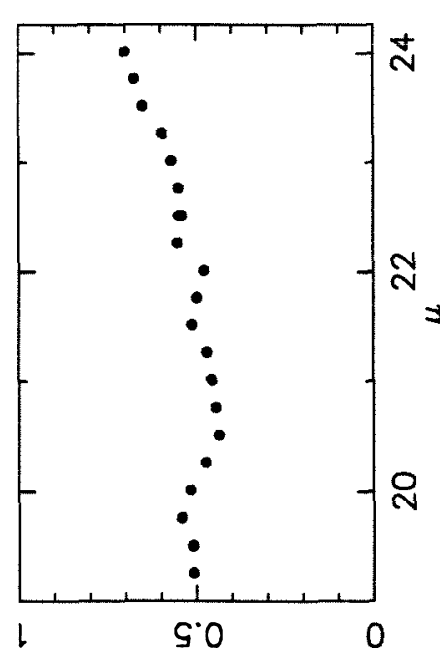

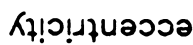

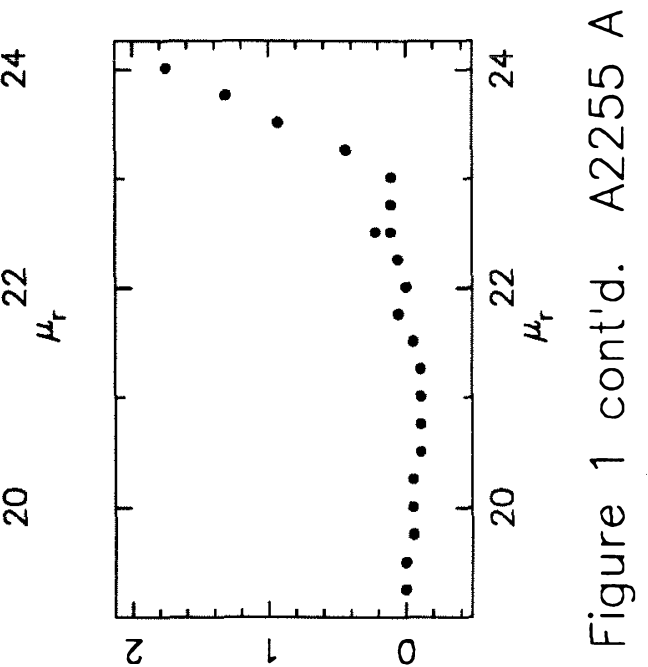

(.1) tast $150 \mathrm{~K}$

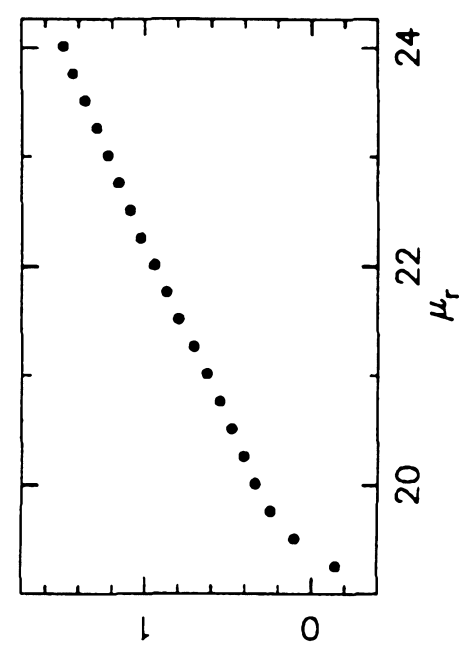

(.1) 0601
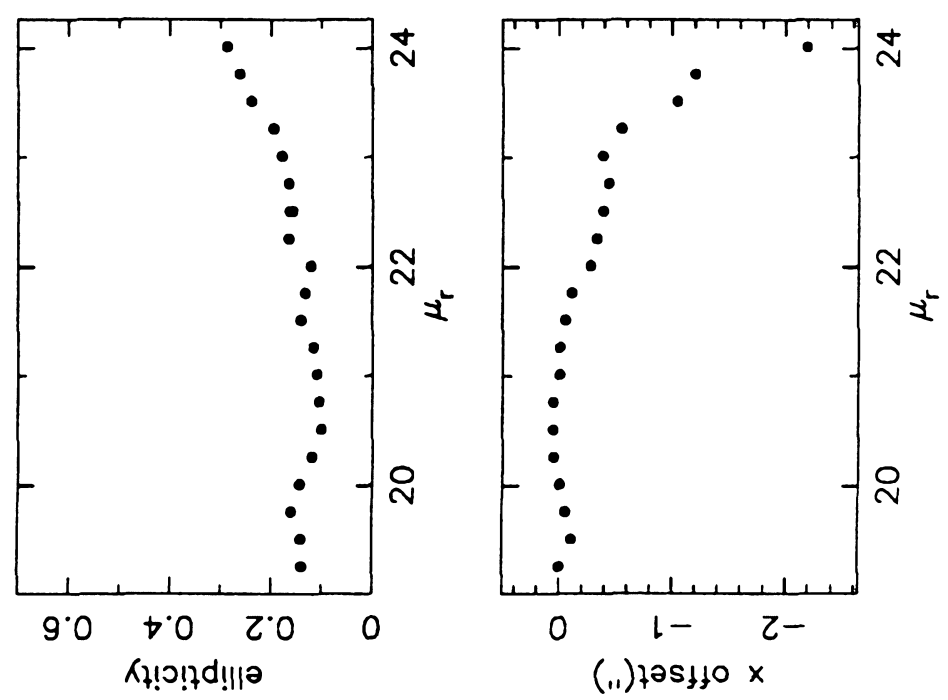

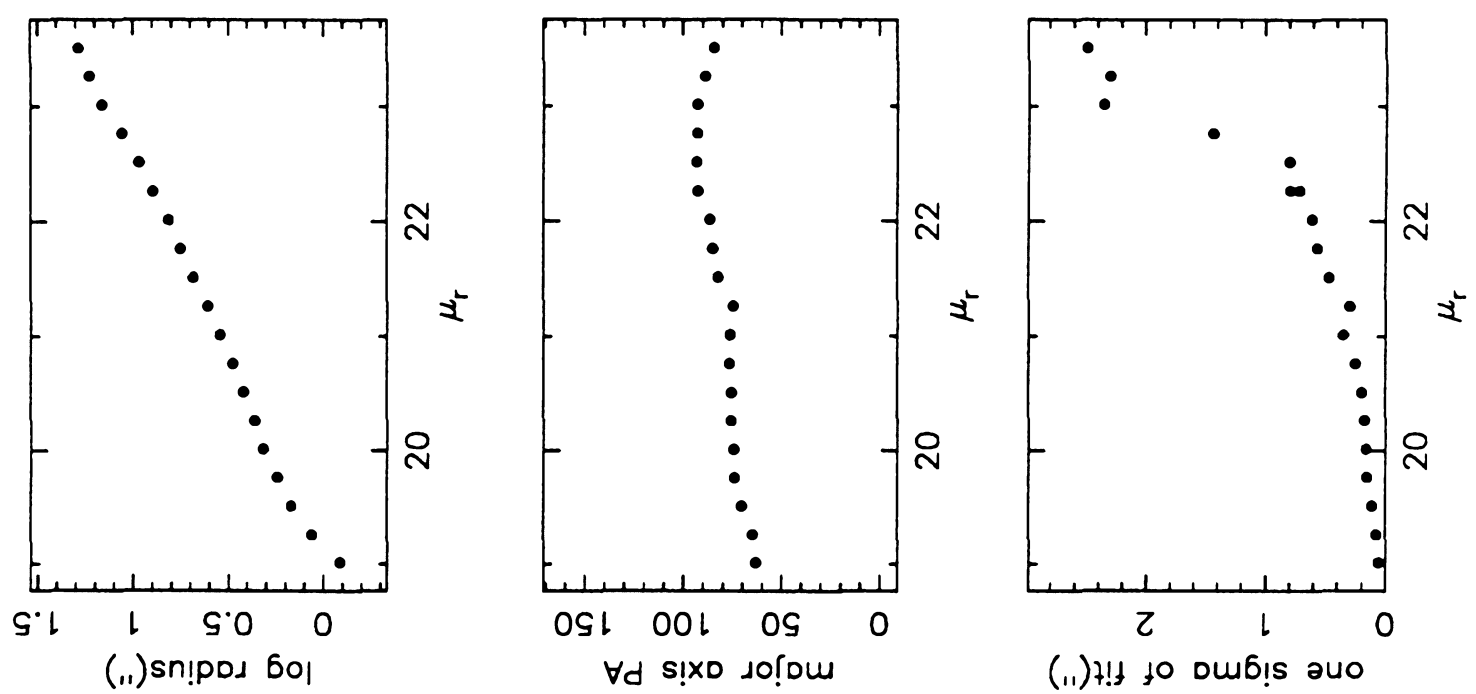

(.1) $+!+10$ oub!s avo
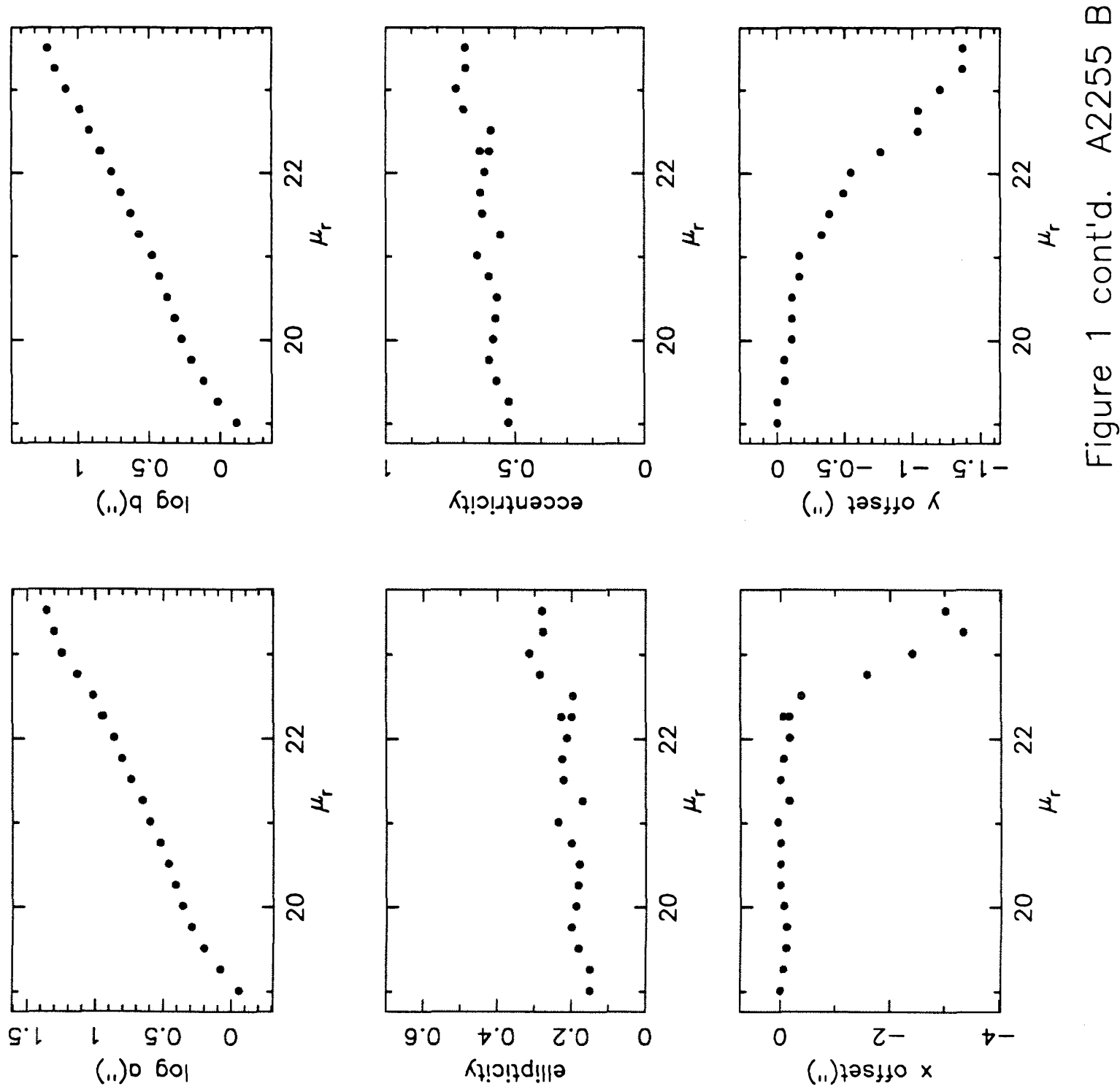

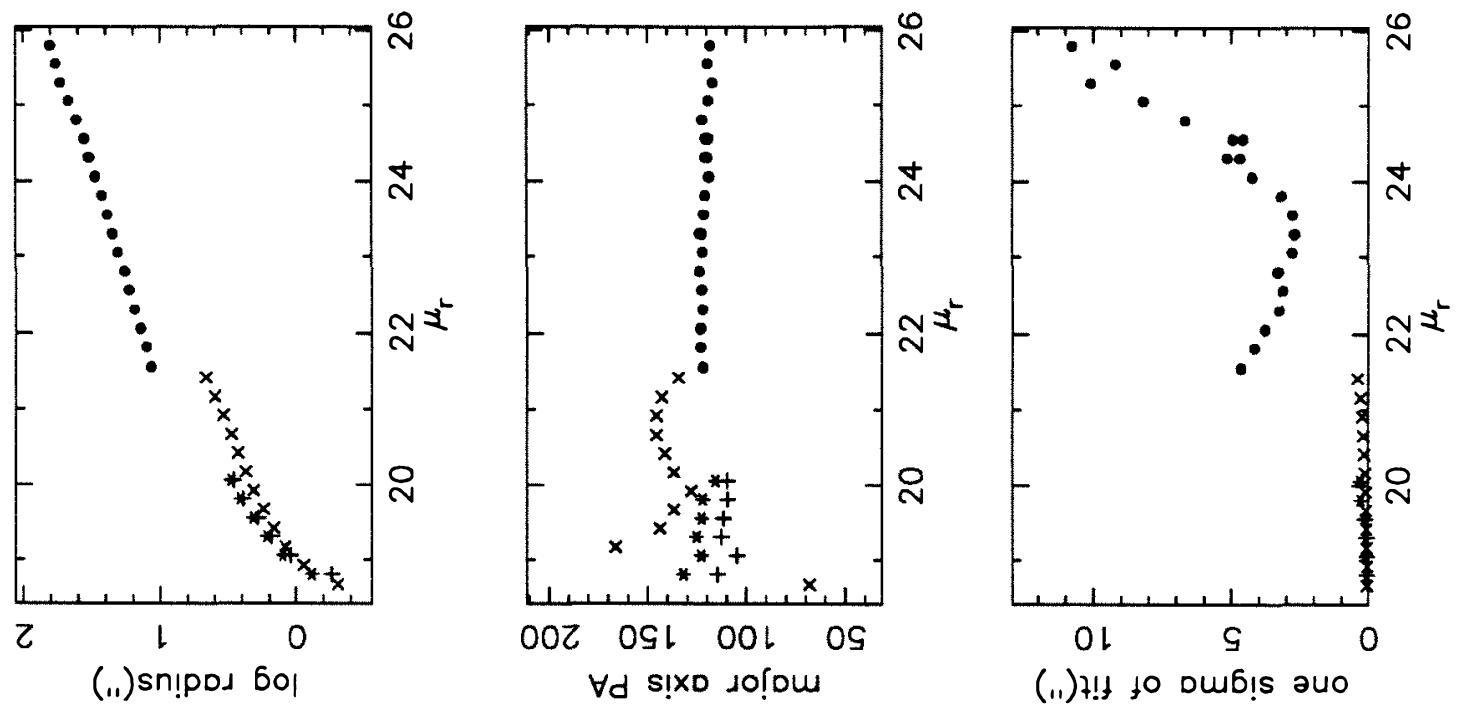

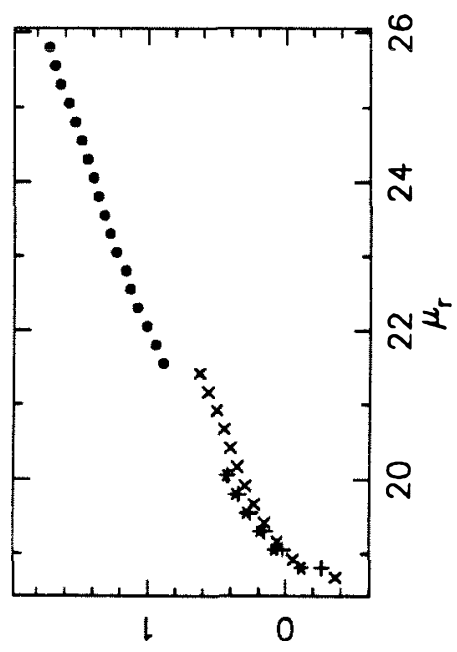

(.1) $9 \mathrm{60}$

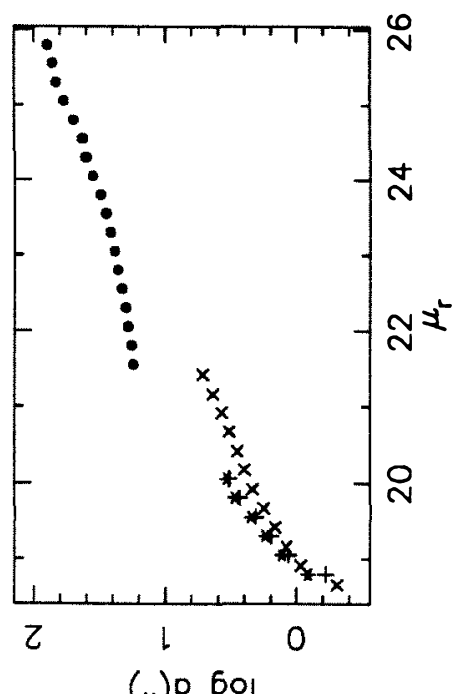

(.1) 0601

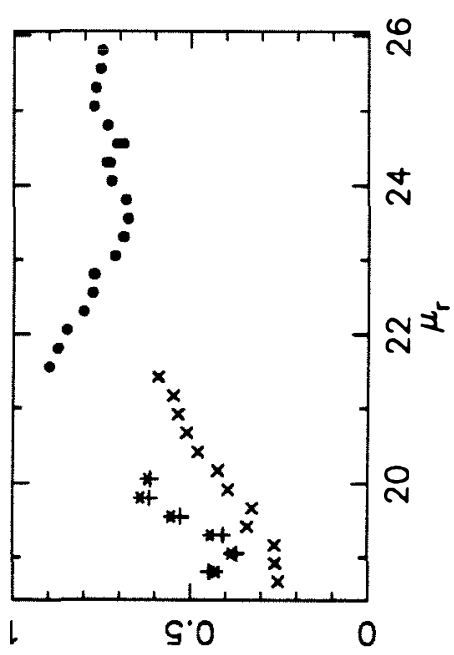

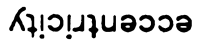

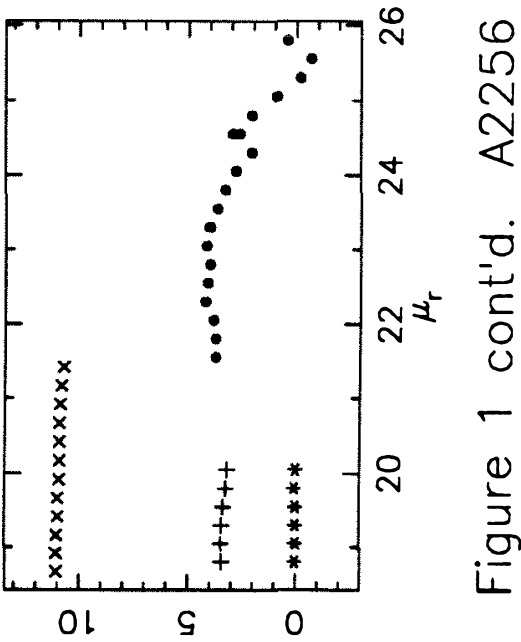

(ii) $725450 \mathrm{~K}$
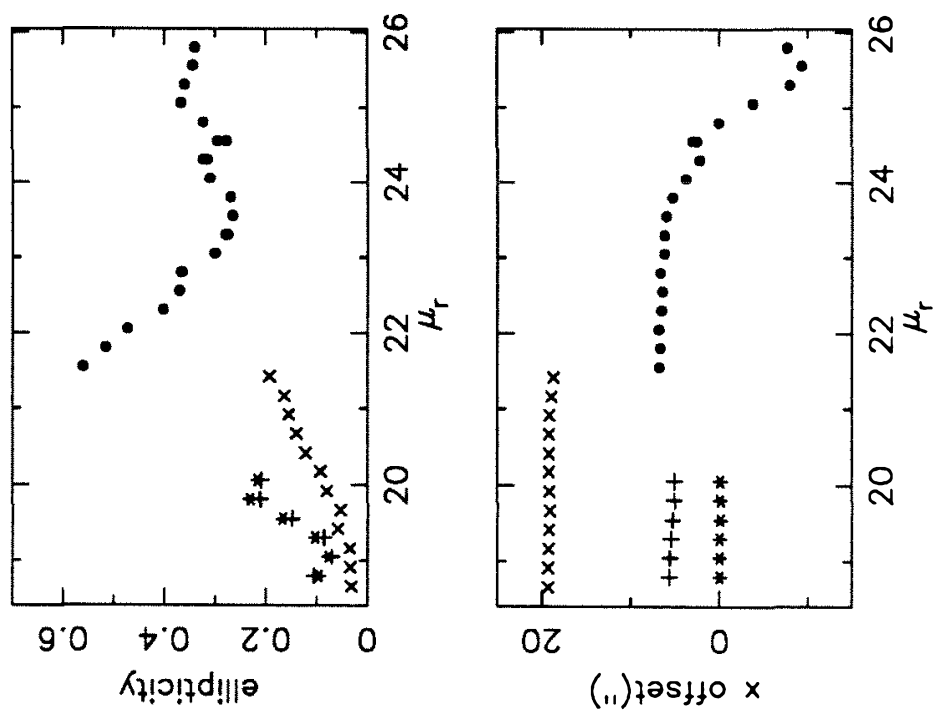

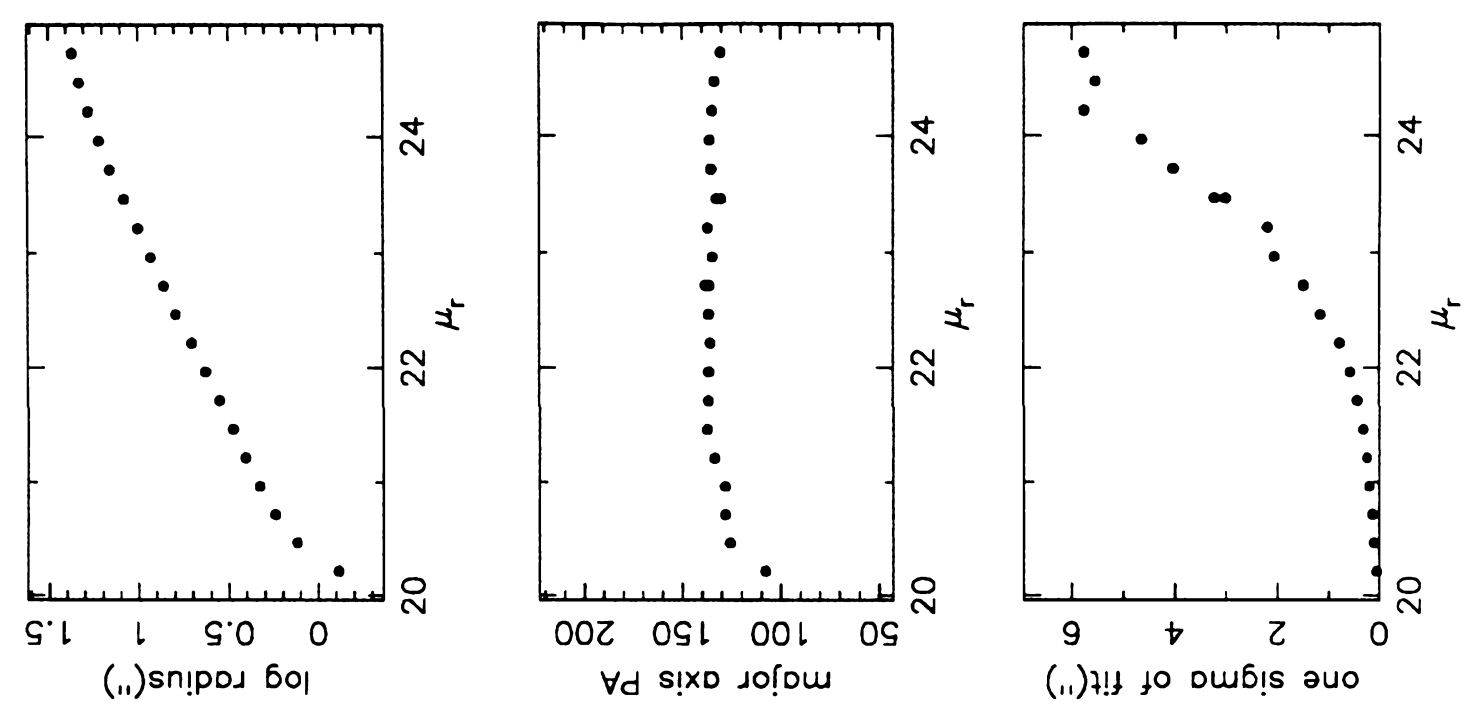

(11) $7 !+$ to oub!s auo
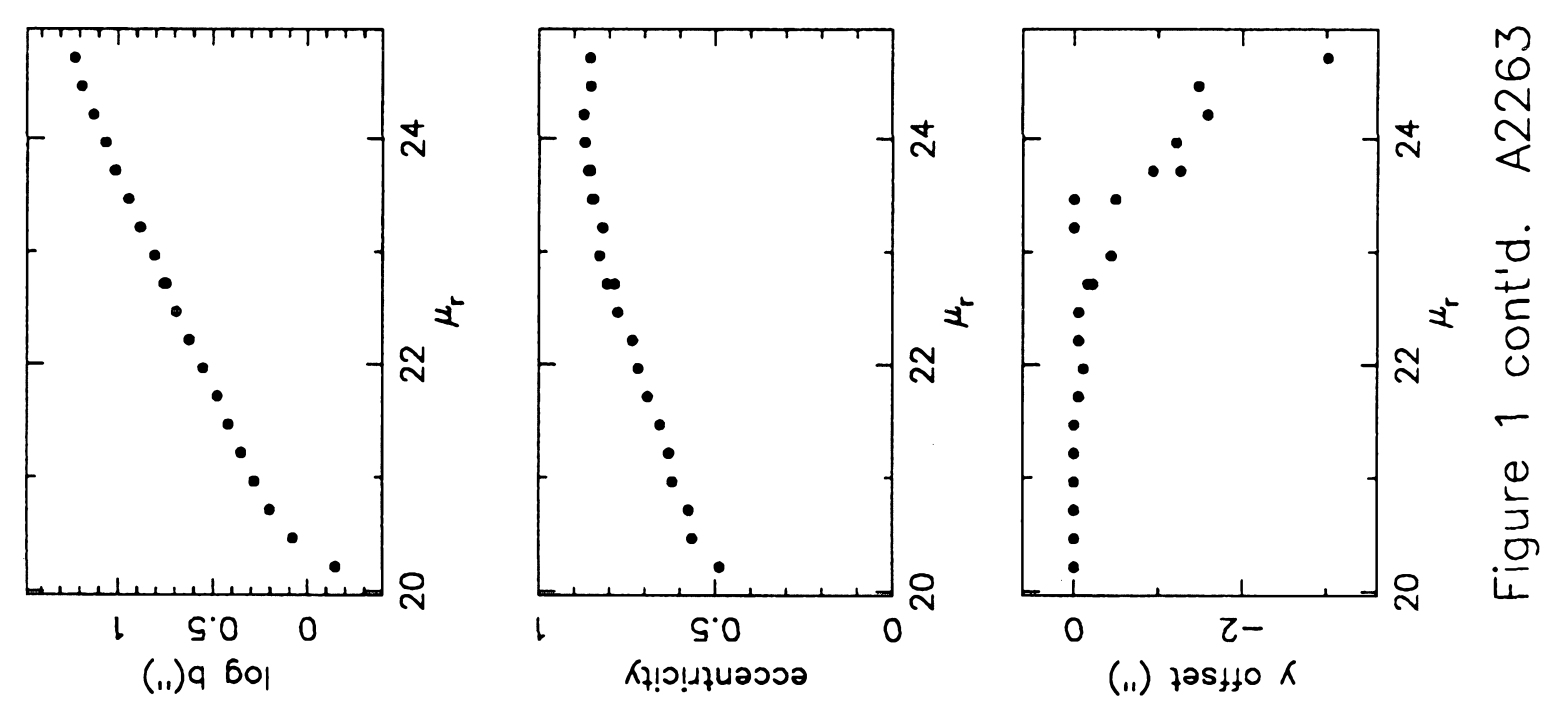

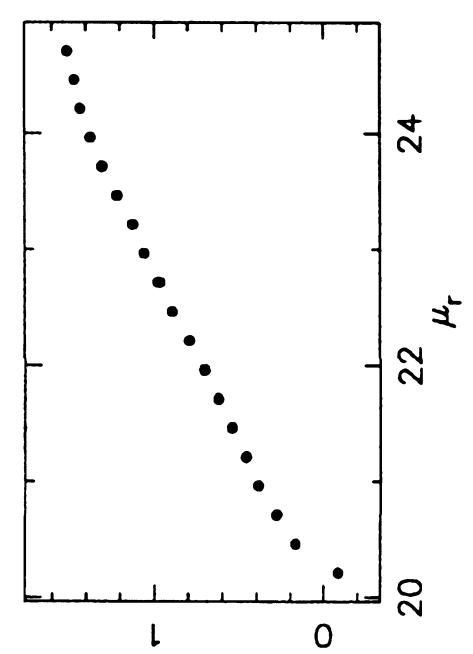

(.1) 0 601
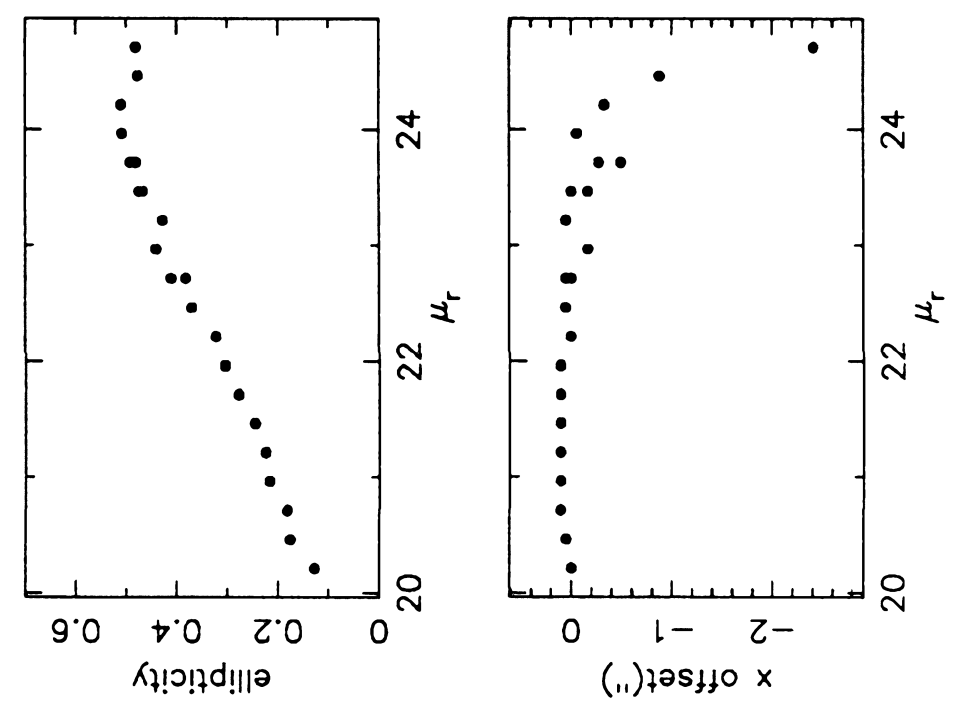


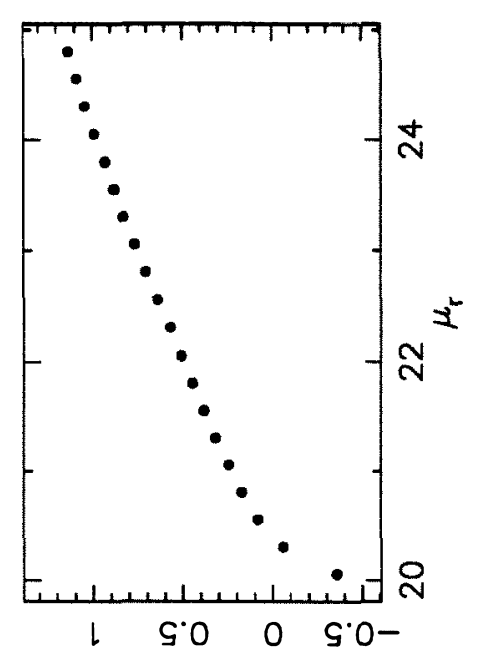

(.1)sn!pod 6ol
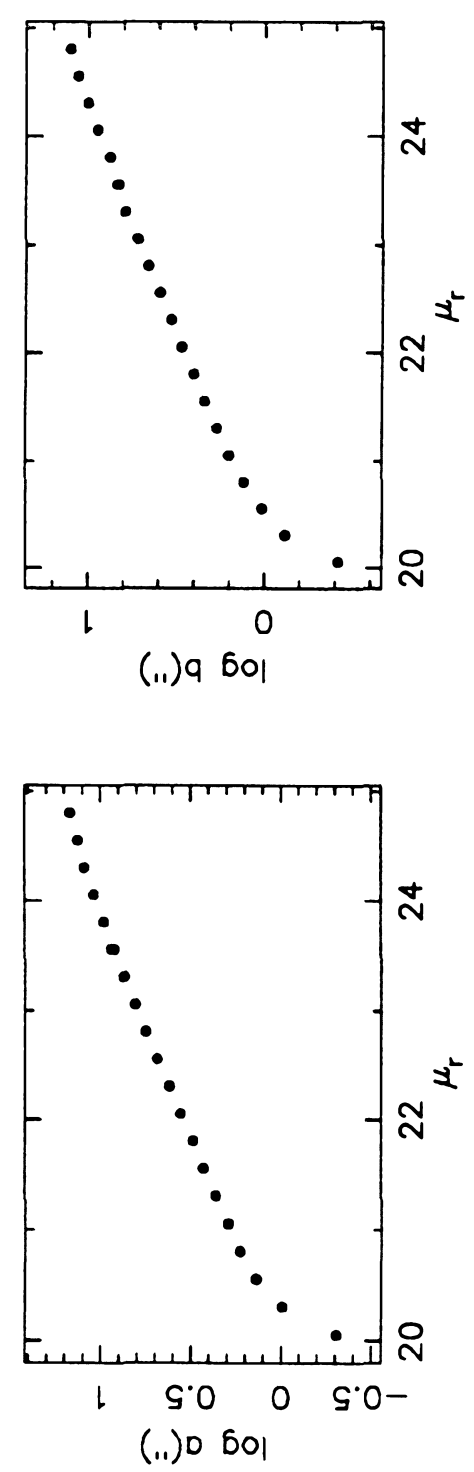

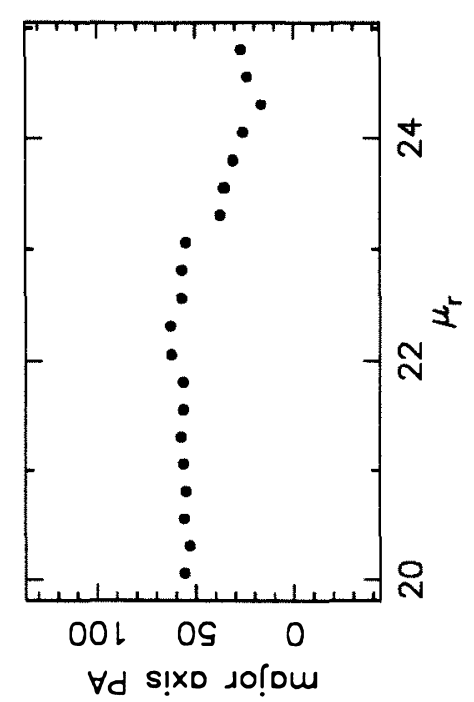

$\forall d$ s!xo do!

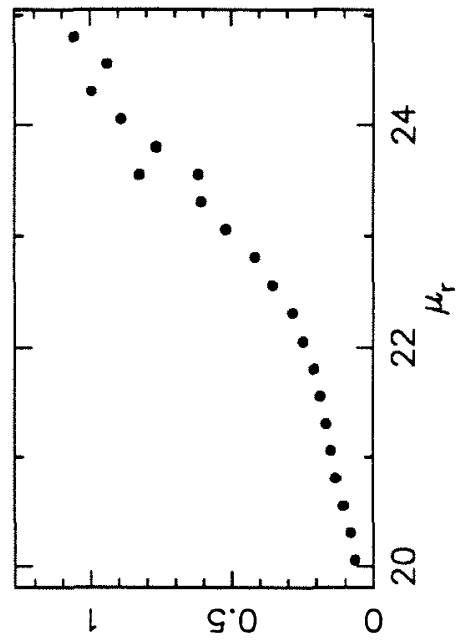

(11) $7 !+$ to Dub!s aน०
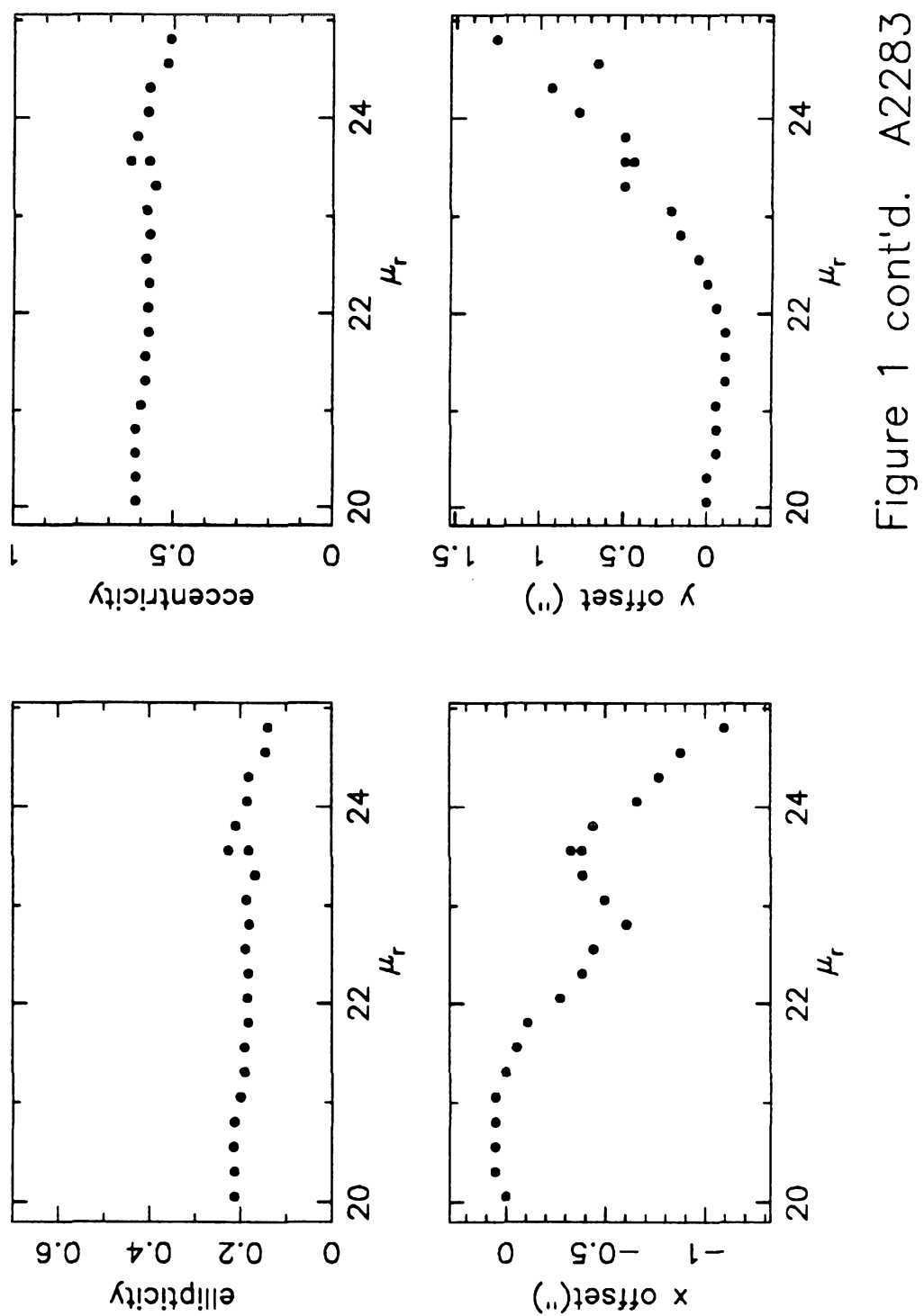


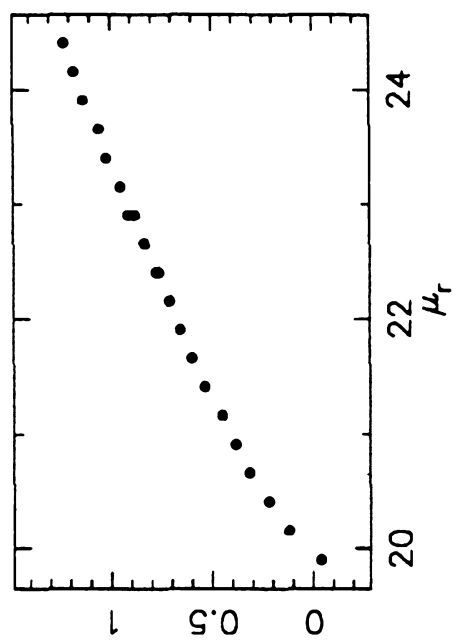

(.1) sn!pod 6ol

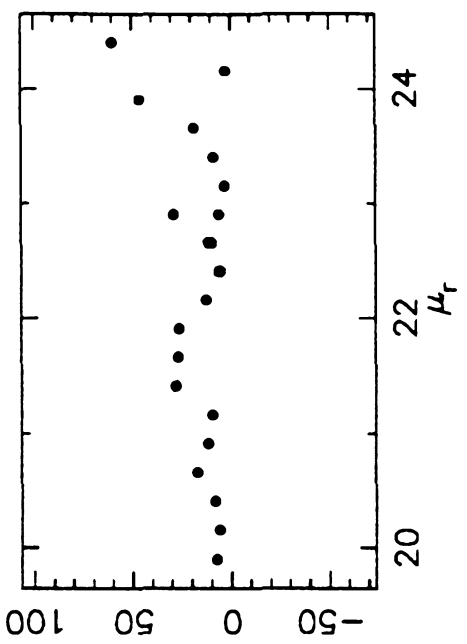

$\forall d$ s!xo 10 !

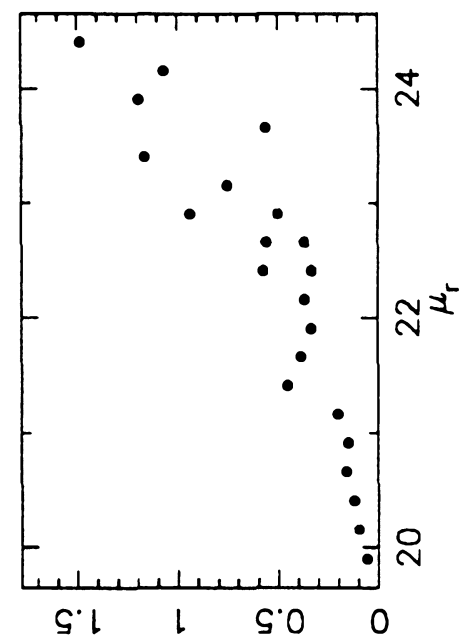

(11) $7 ! t$ to Dub!s auo
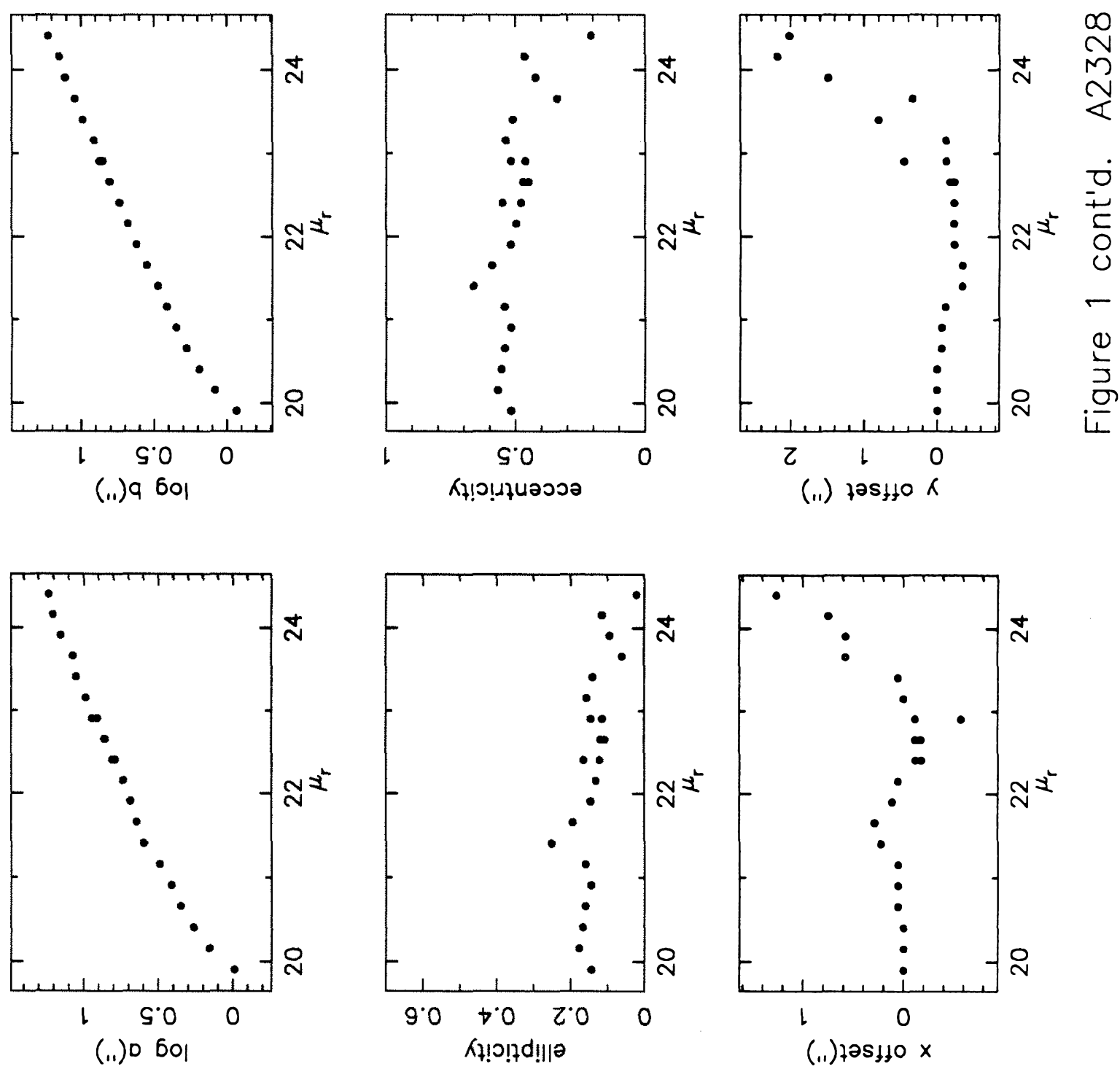

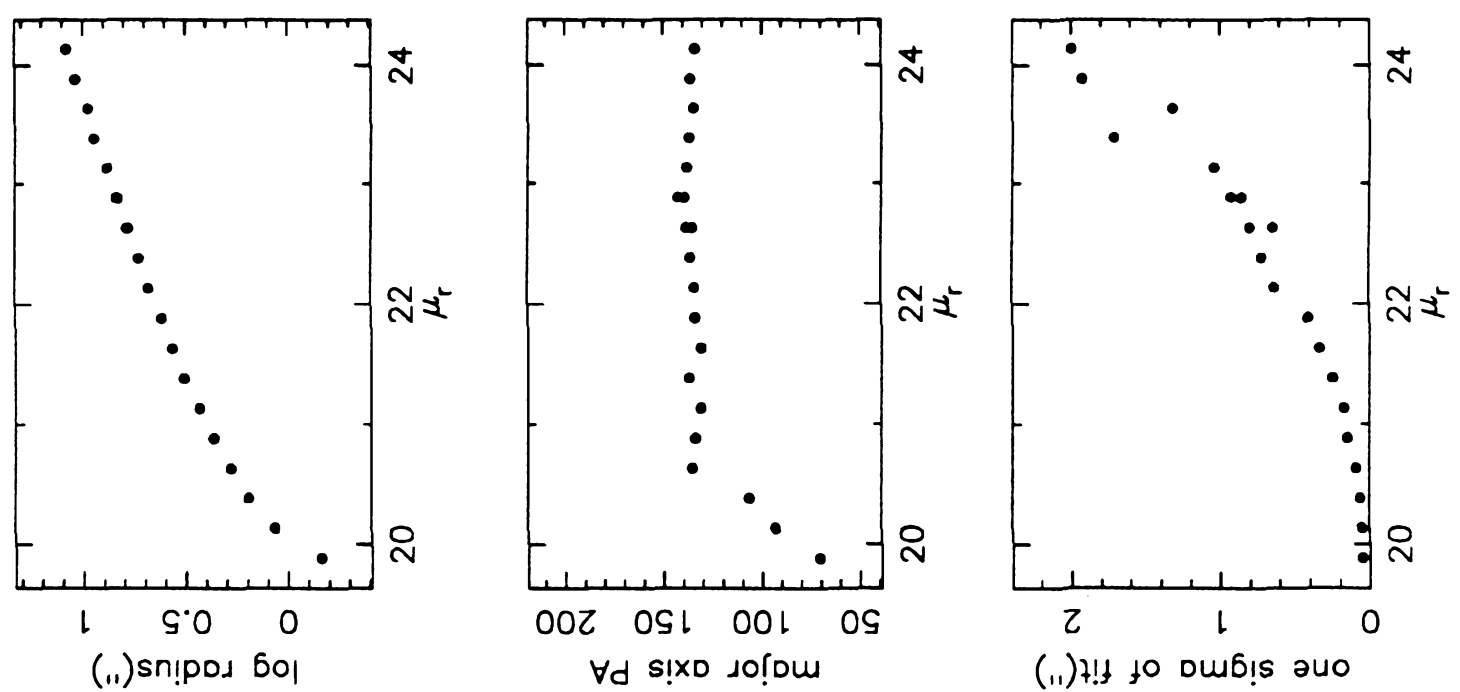

(11) $7 ! !$ to Dub!s auo
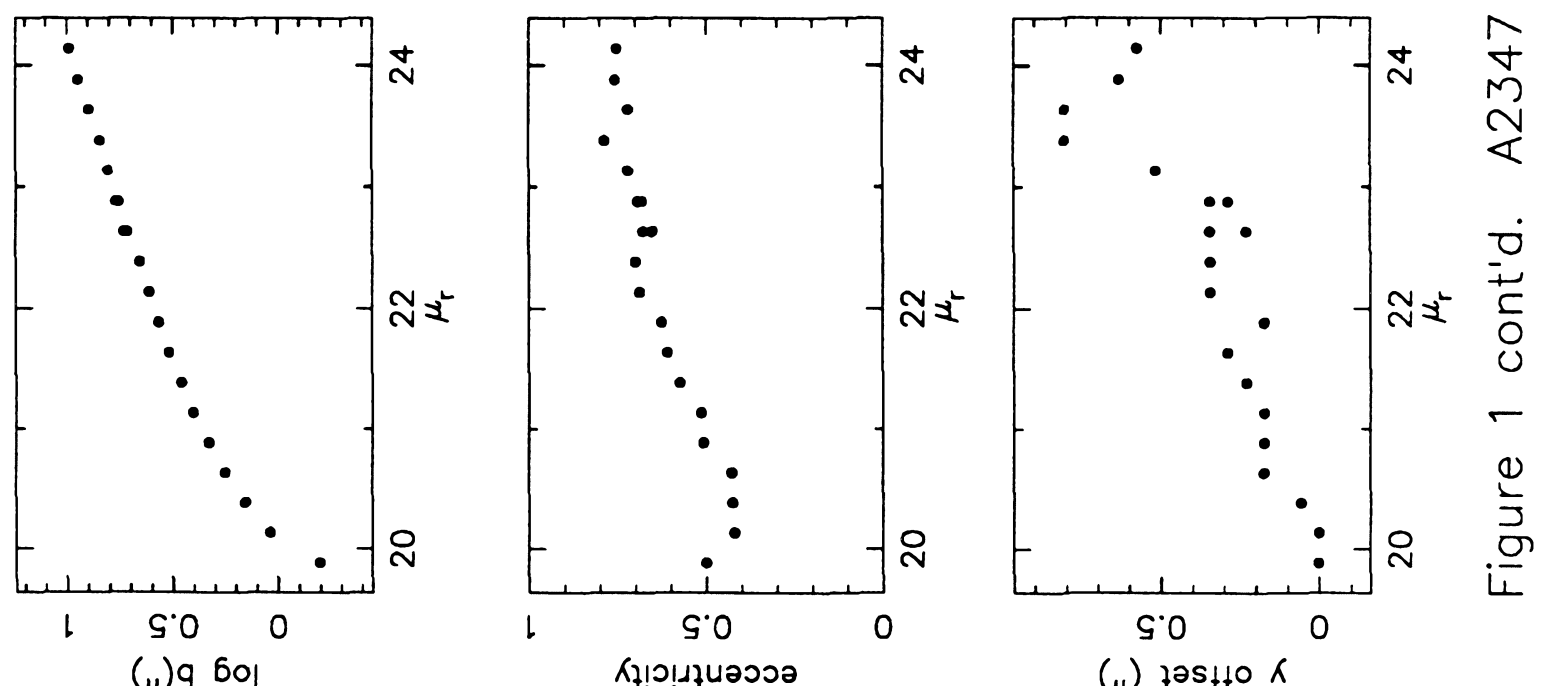

(.1) 9 6ol

(iI) $725110 \mathrm{~K}$
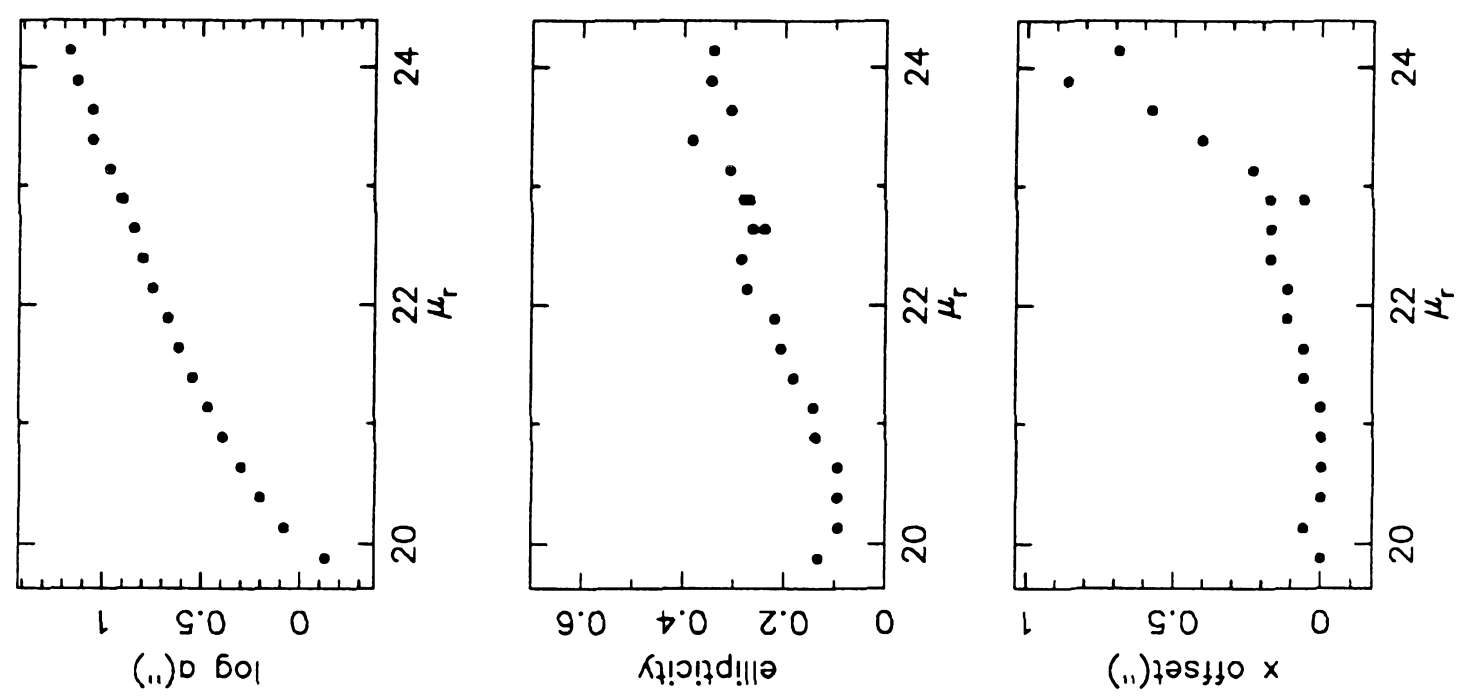

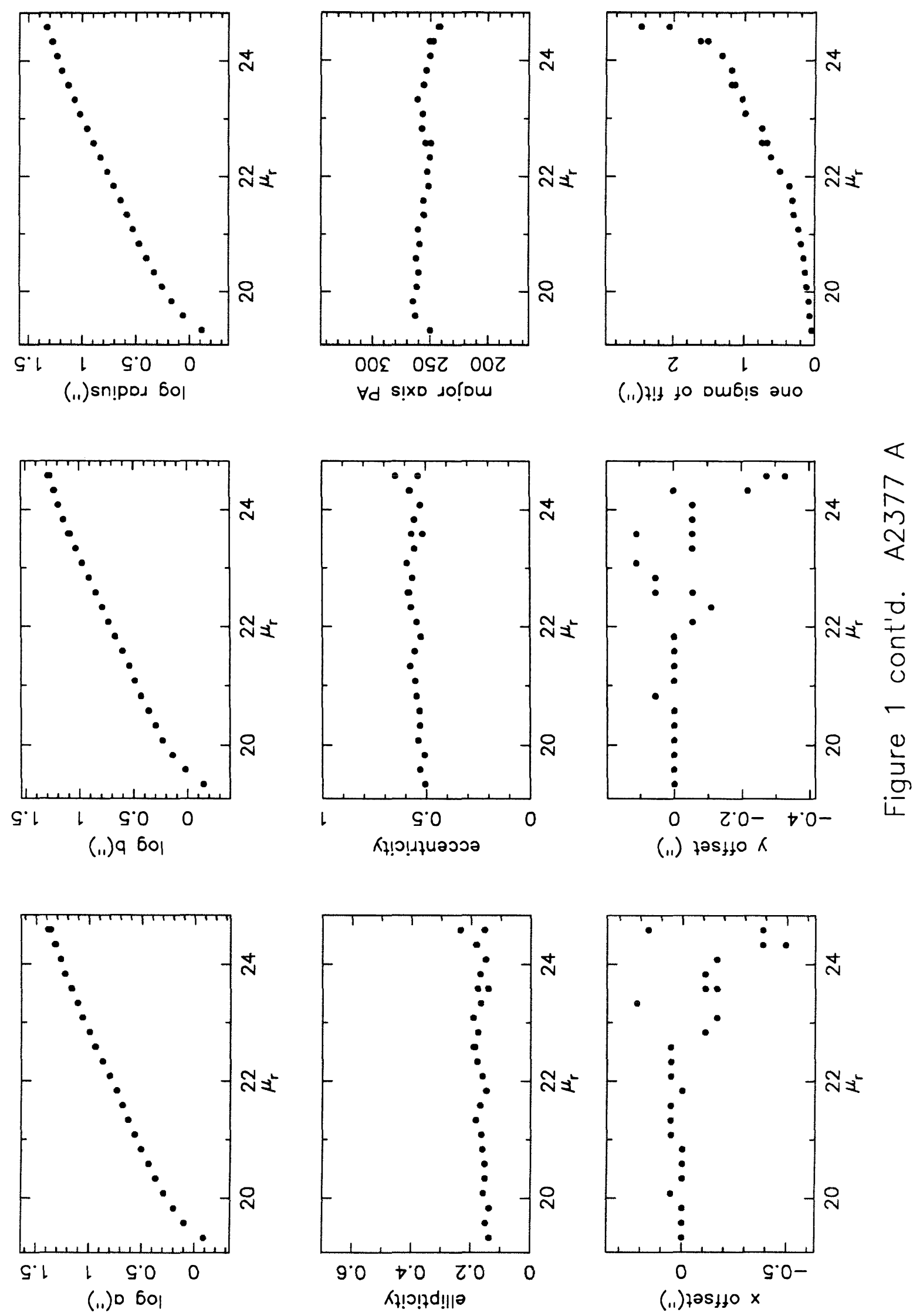

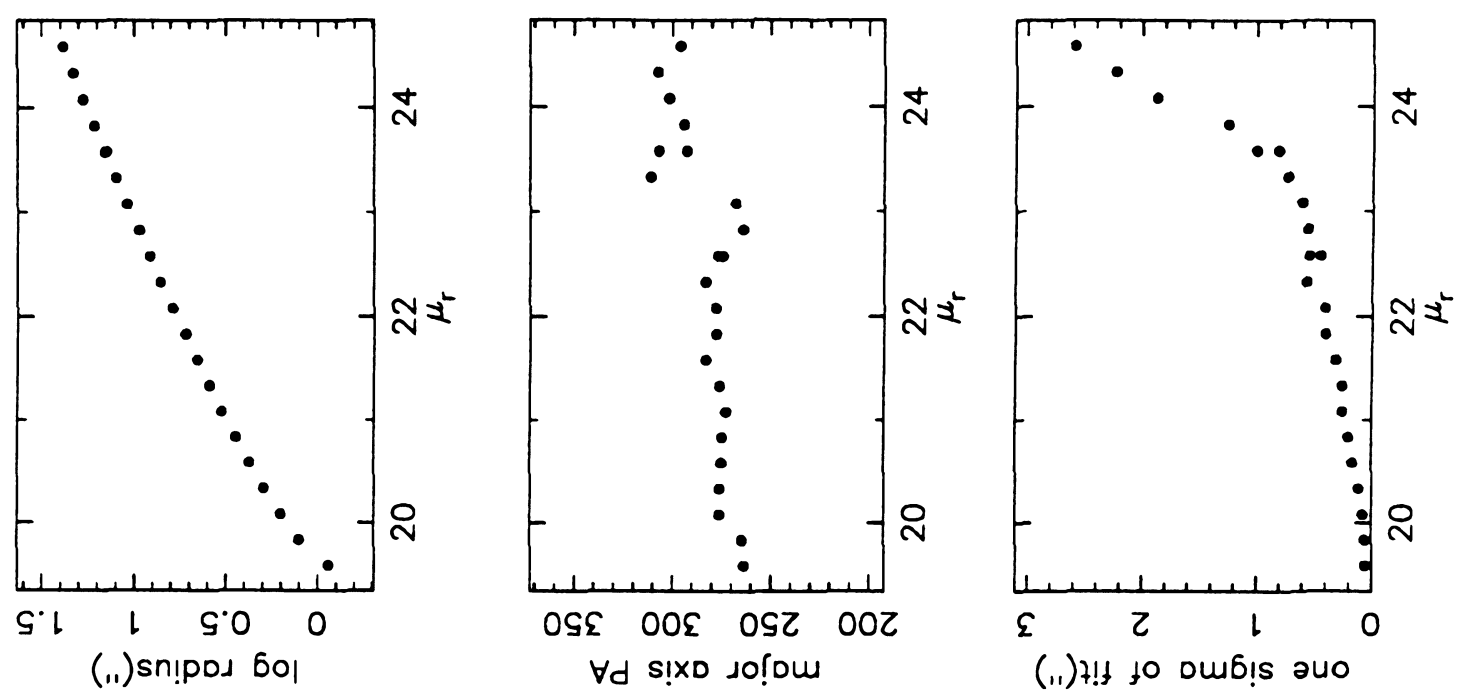

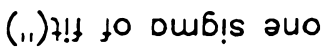
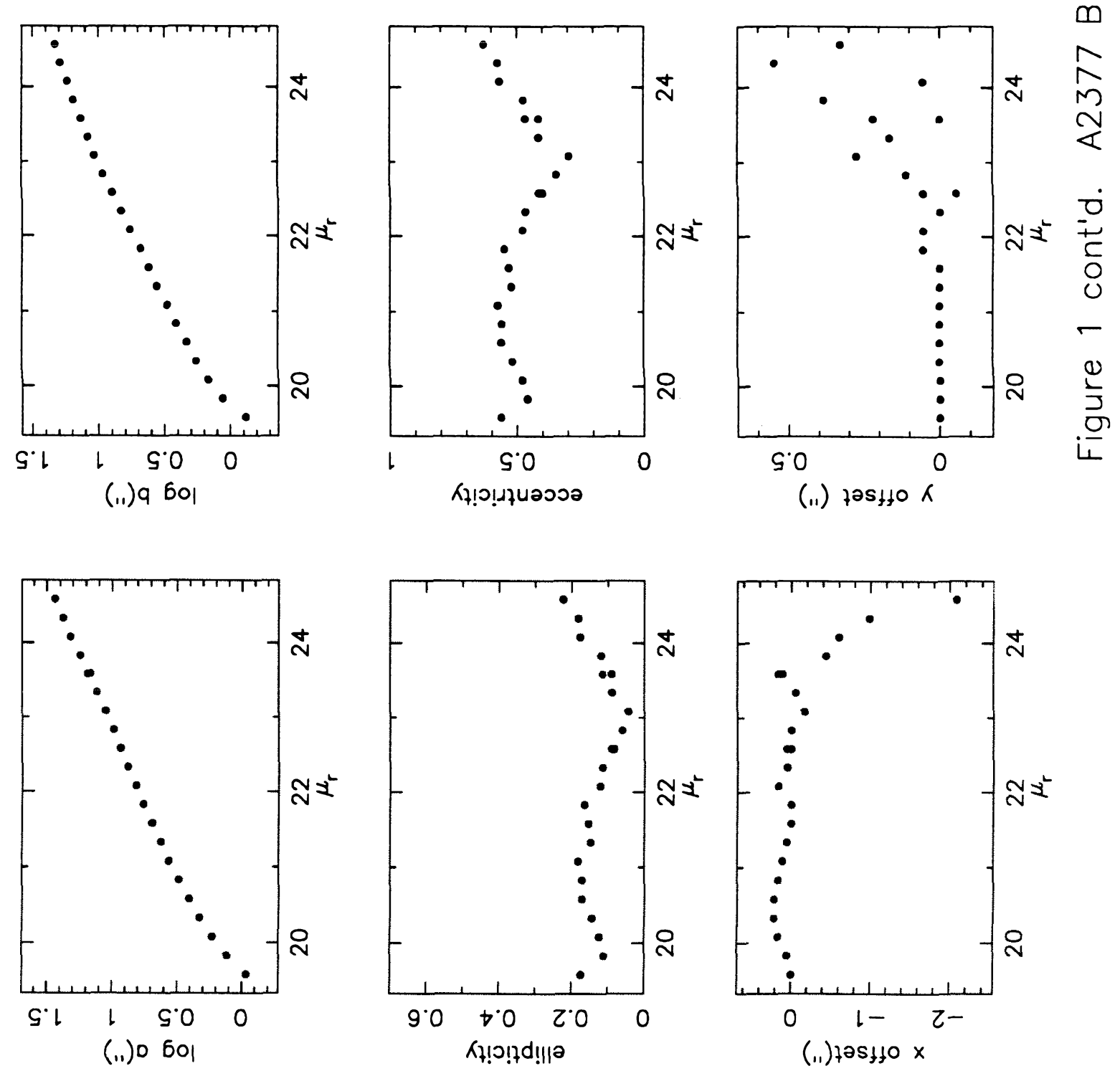


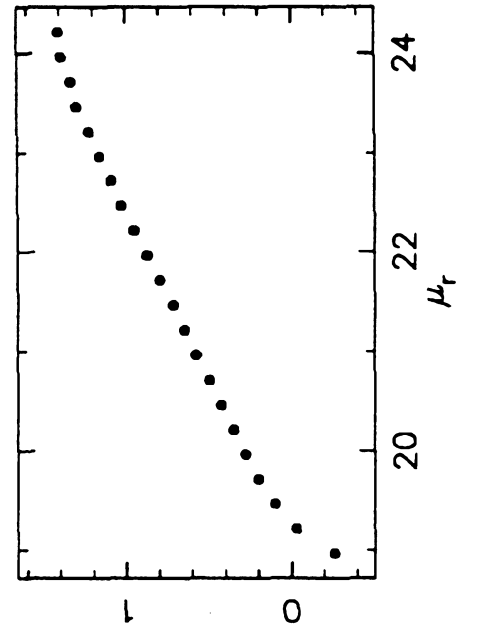

(11) sn!pod 6ol
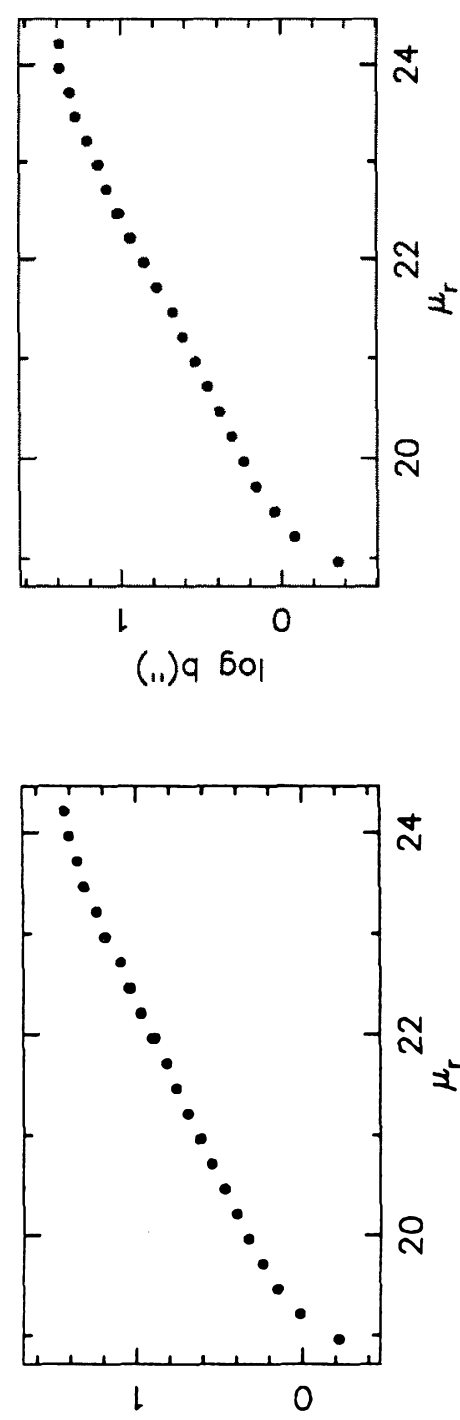

(.1) $0 \mathrm{bol}$

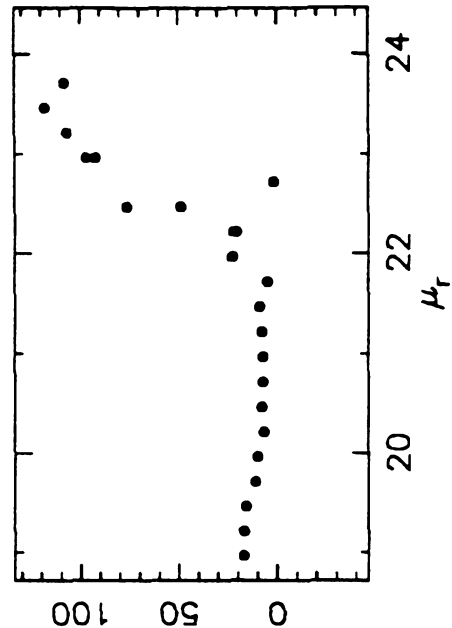

$\forall d$ s!xo دo!̣u

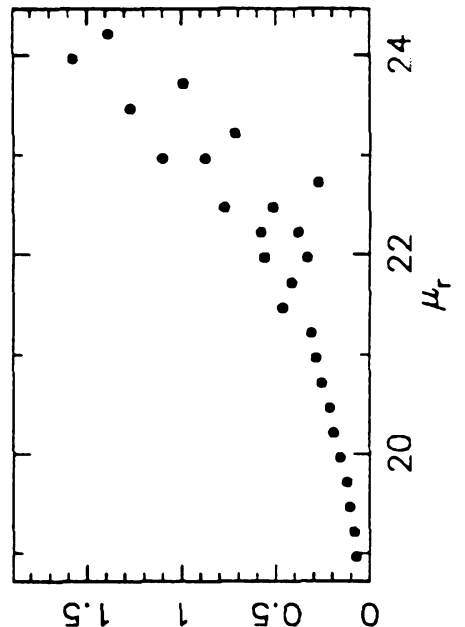

(11) $+!+10$ oub!s auo
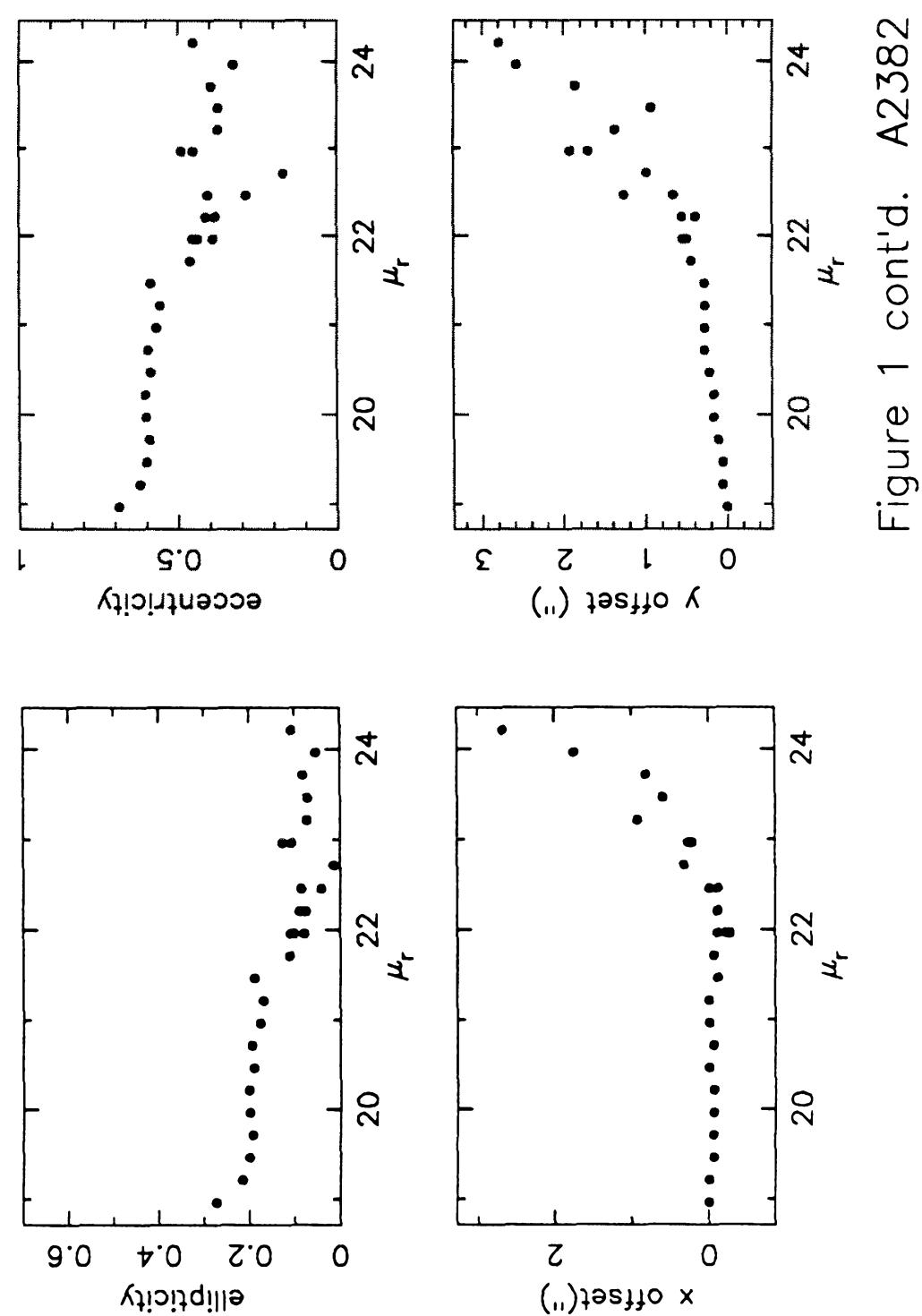


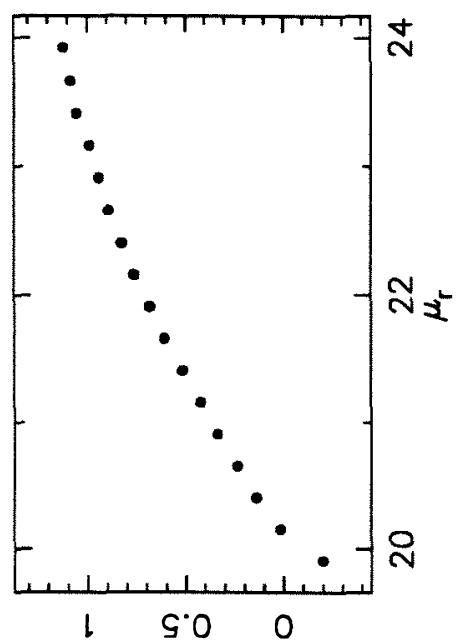

(.1) sn!pos 6ol
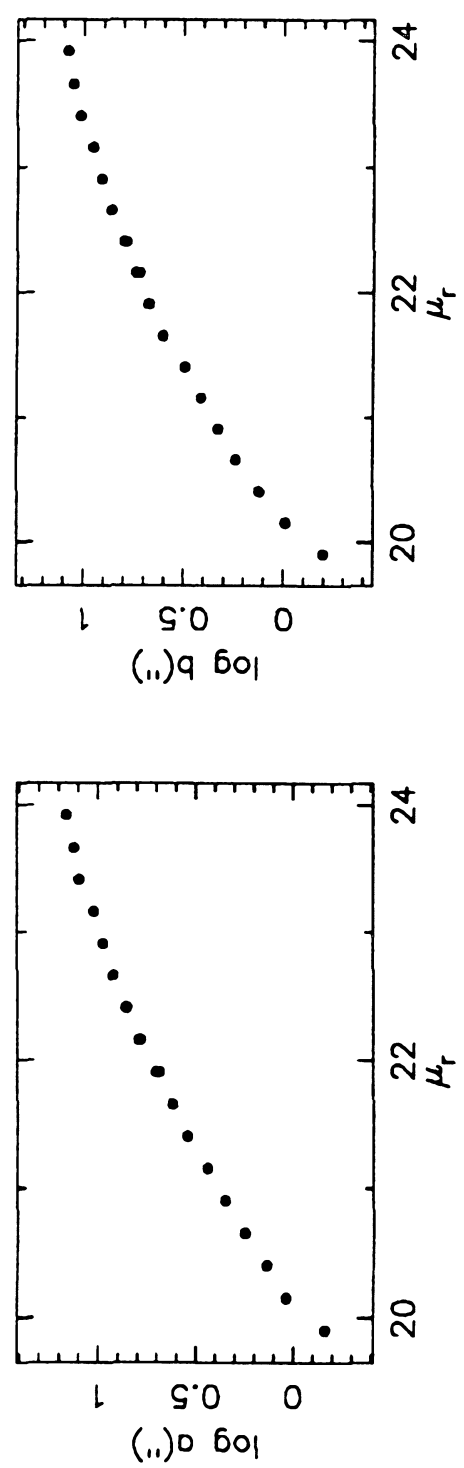

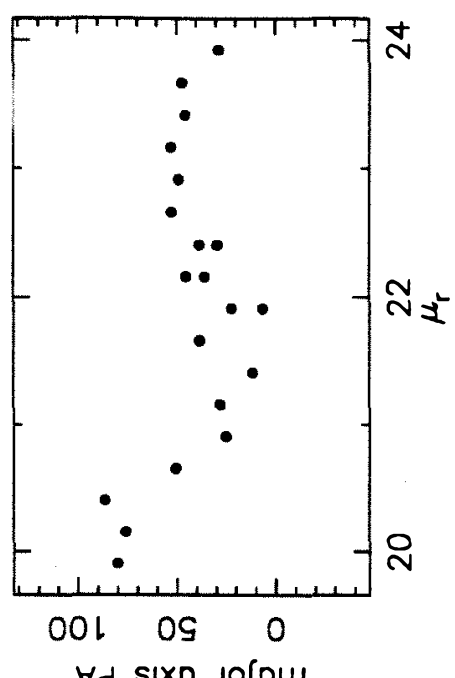

$\forall d$ s!xo so!̣us
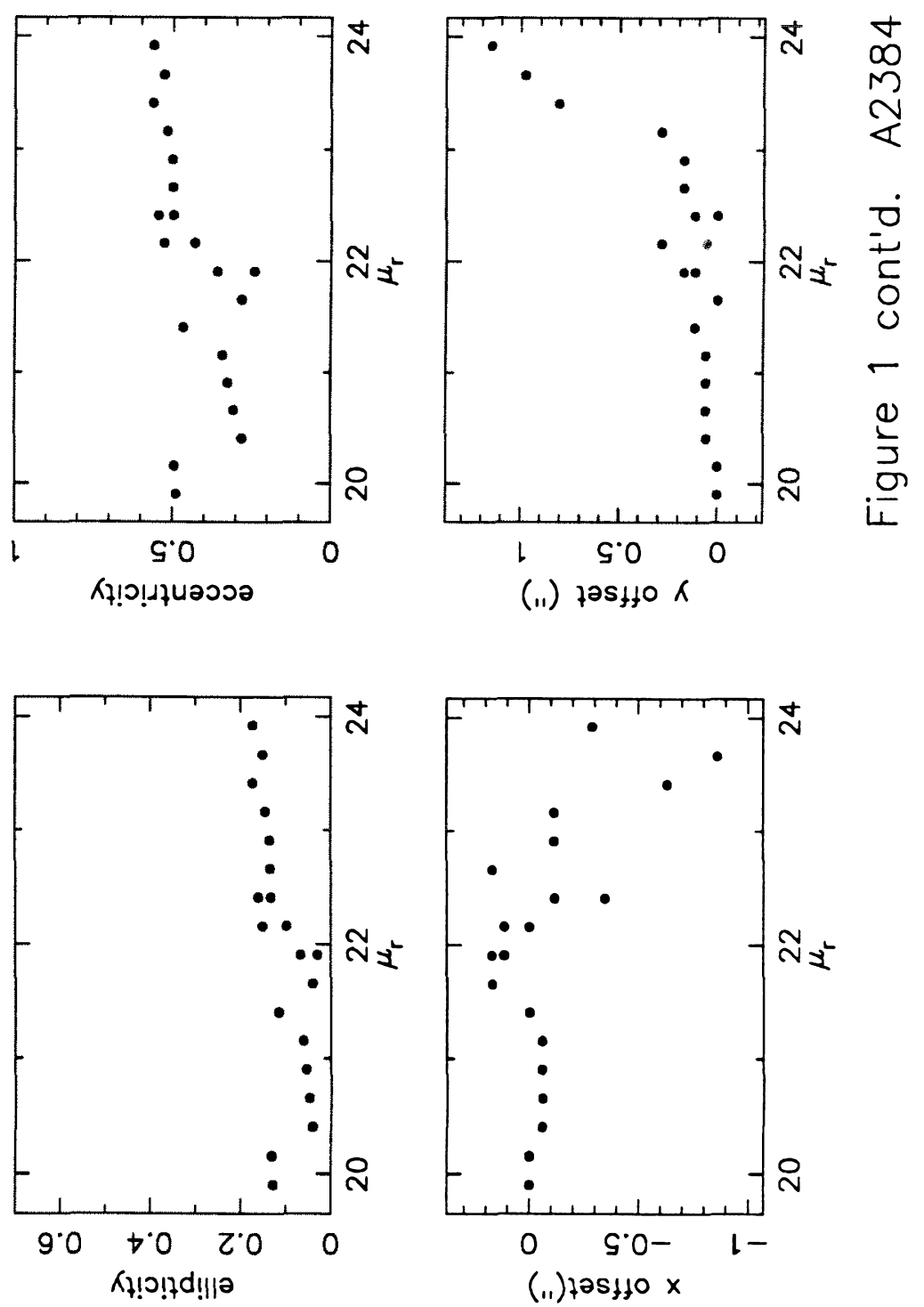


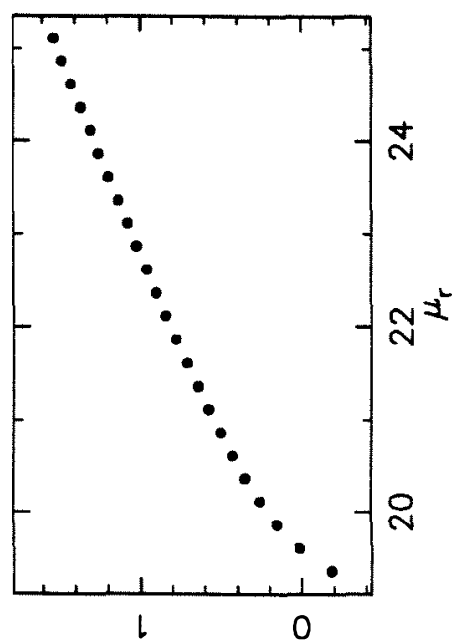

(.1)sn!pod 601

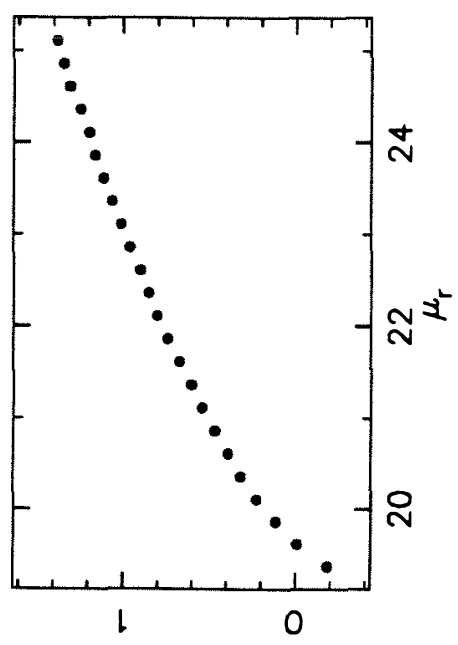

(.1) $9 \mathrm{bol}$

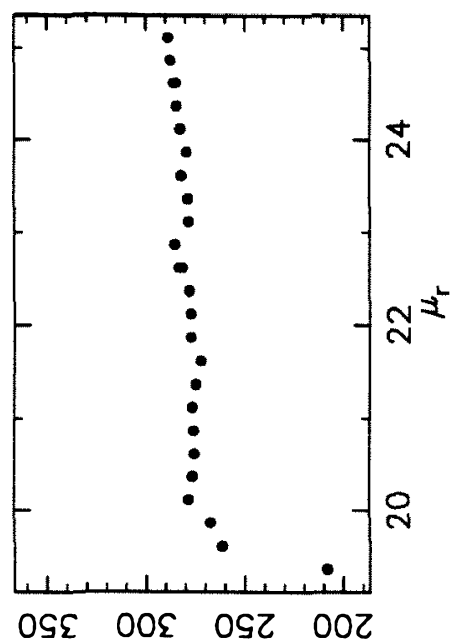

$\forall d s ! \times 0$ so!ou

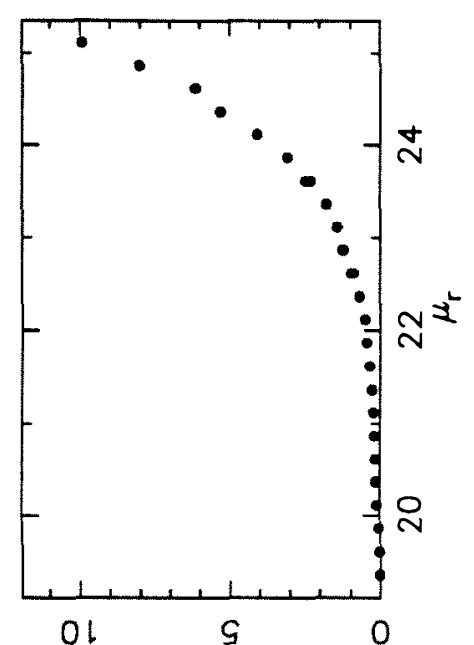

(i1) + !t to oub!s auo

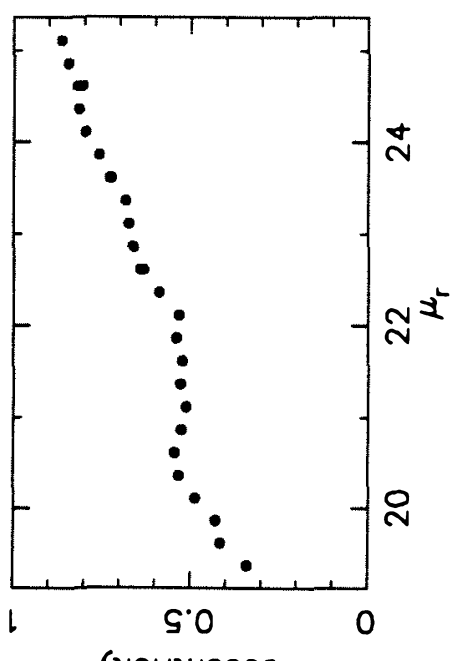

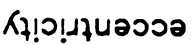

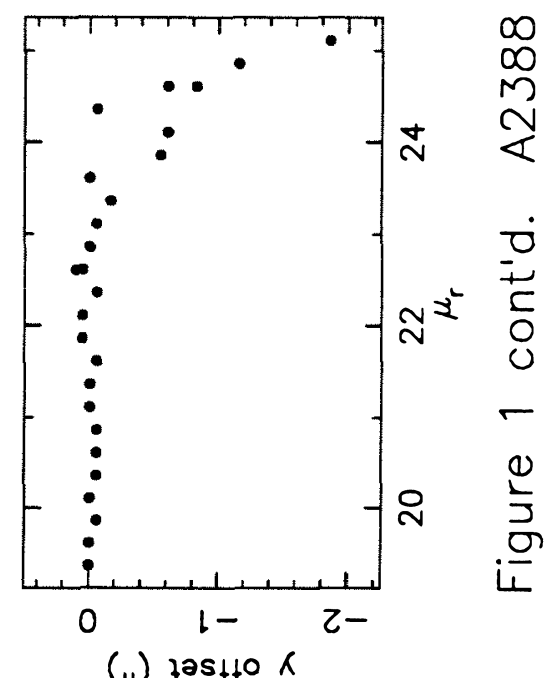

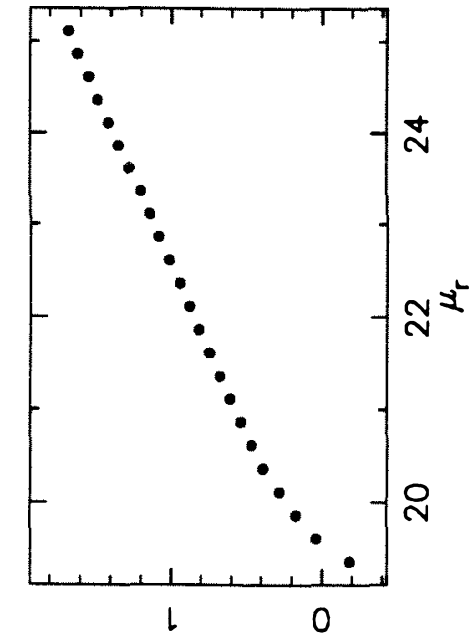

(ii) $\mathrm{O}$ 6ol
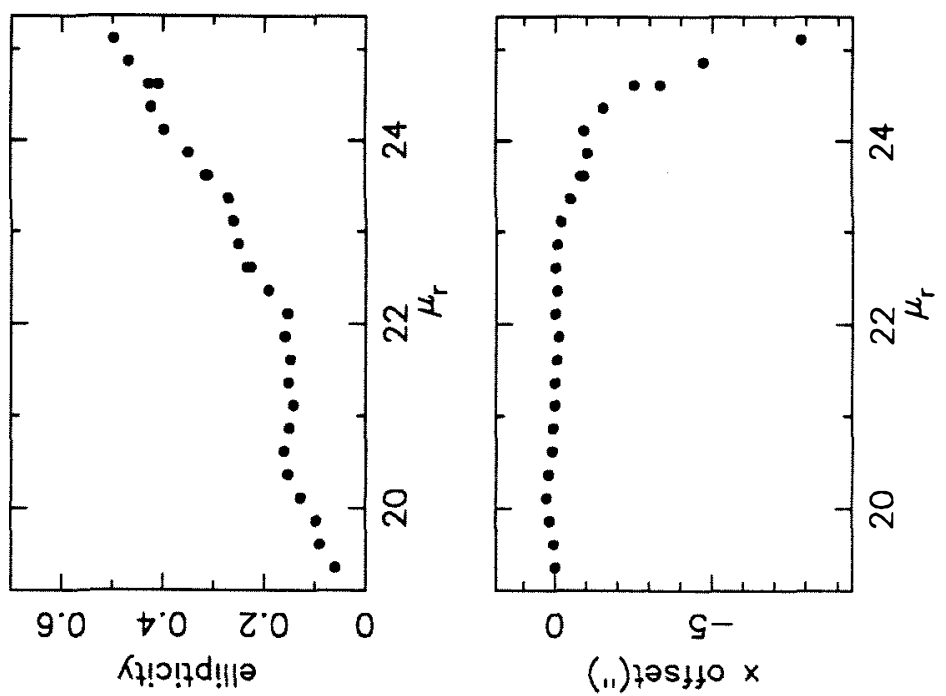


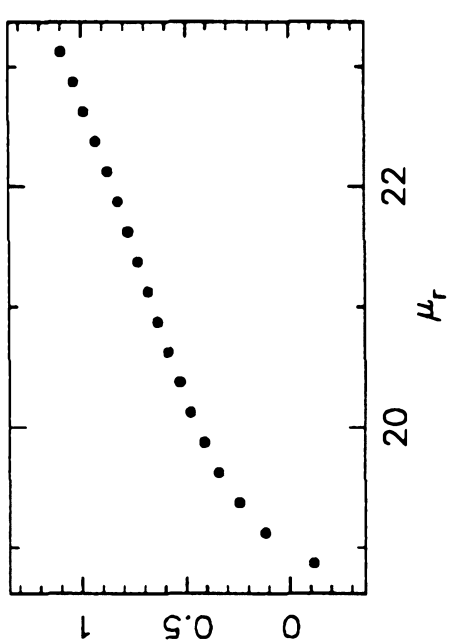

(.1)sn!pod 6ol

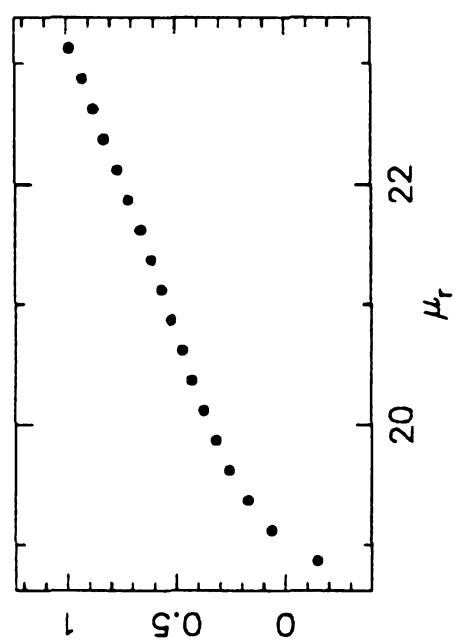

(.1) $9 \mathrm{bol}$

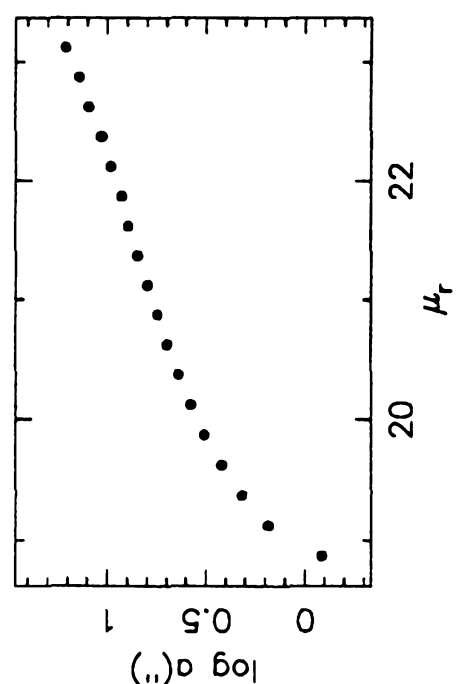

(.1) $0 \mathrm{bol}$

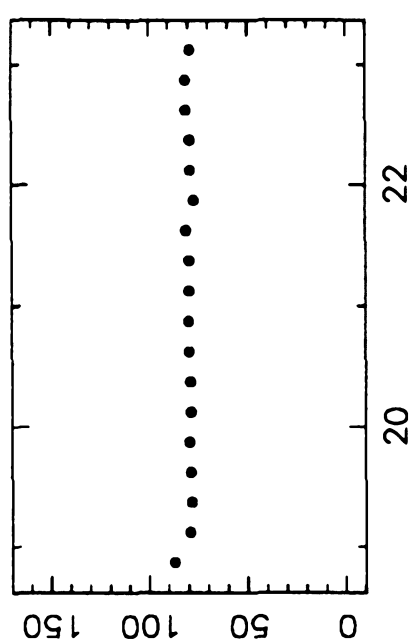

$\forall d$ s!xo so!now
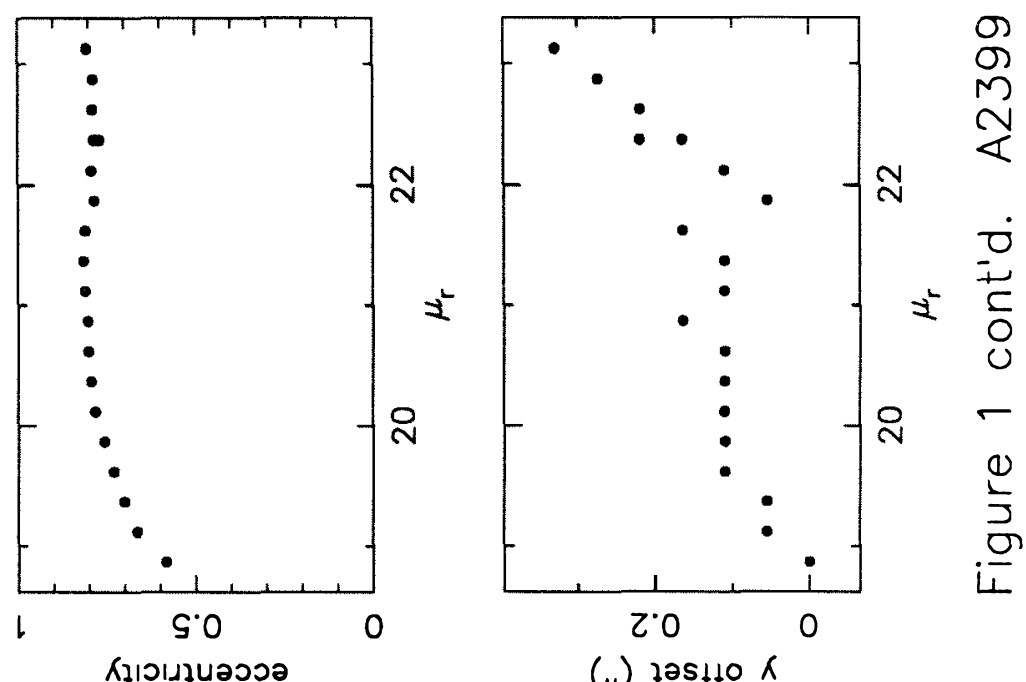

(i) $725110 \mathrm{~K}$
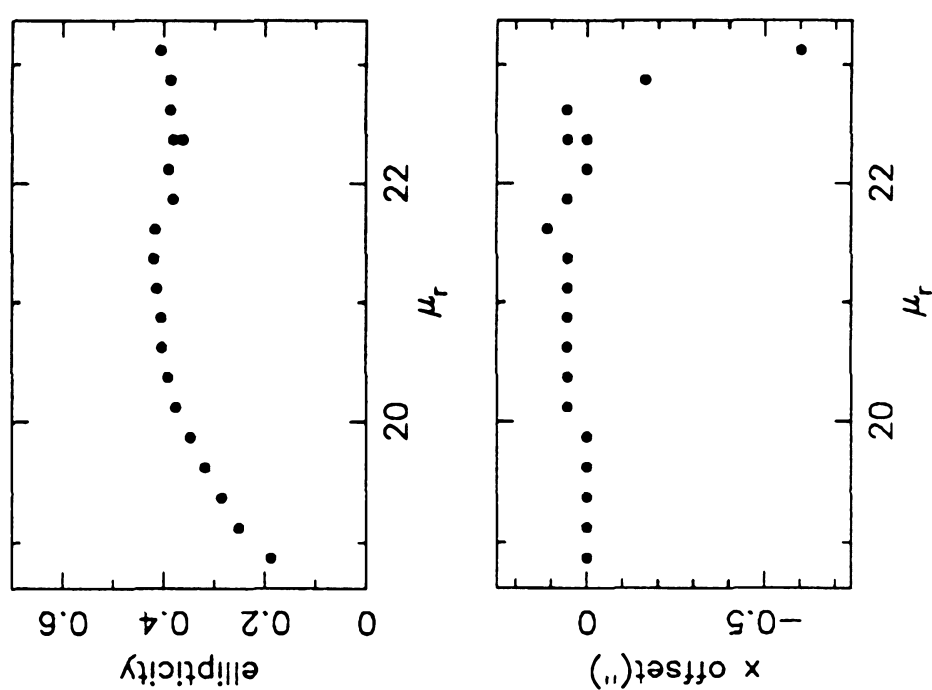


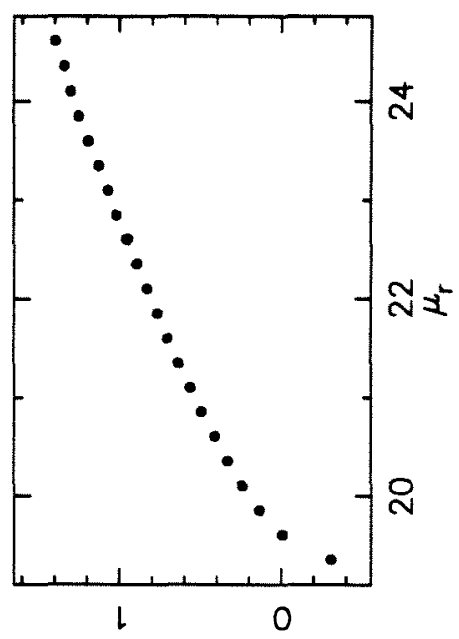

(.1) sn!pod 6ol
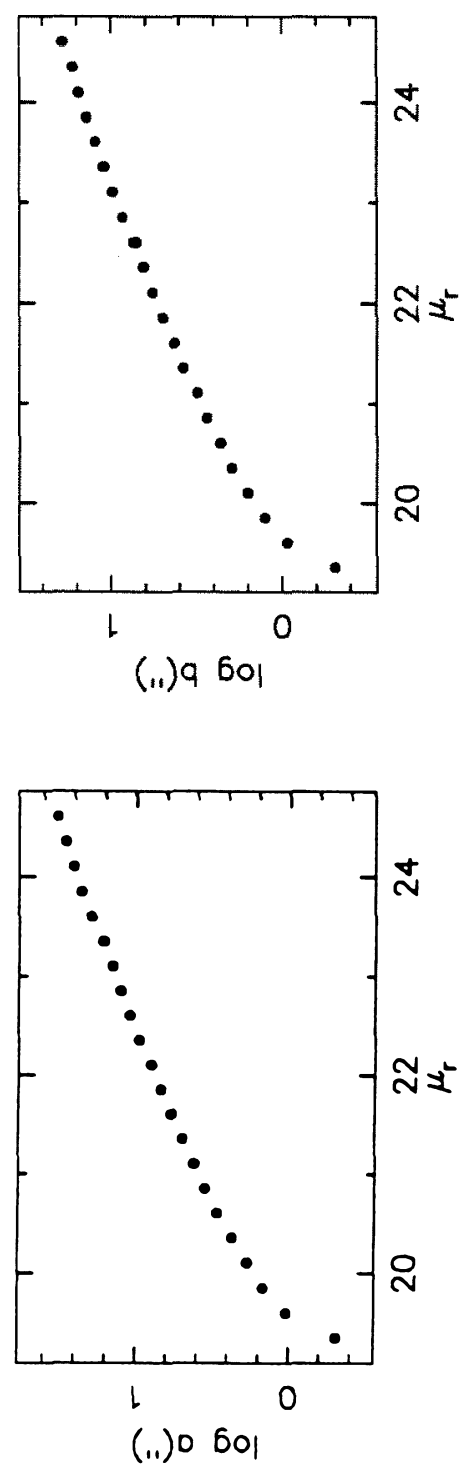
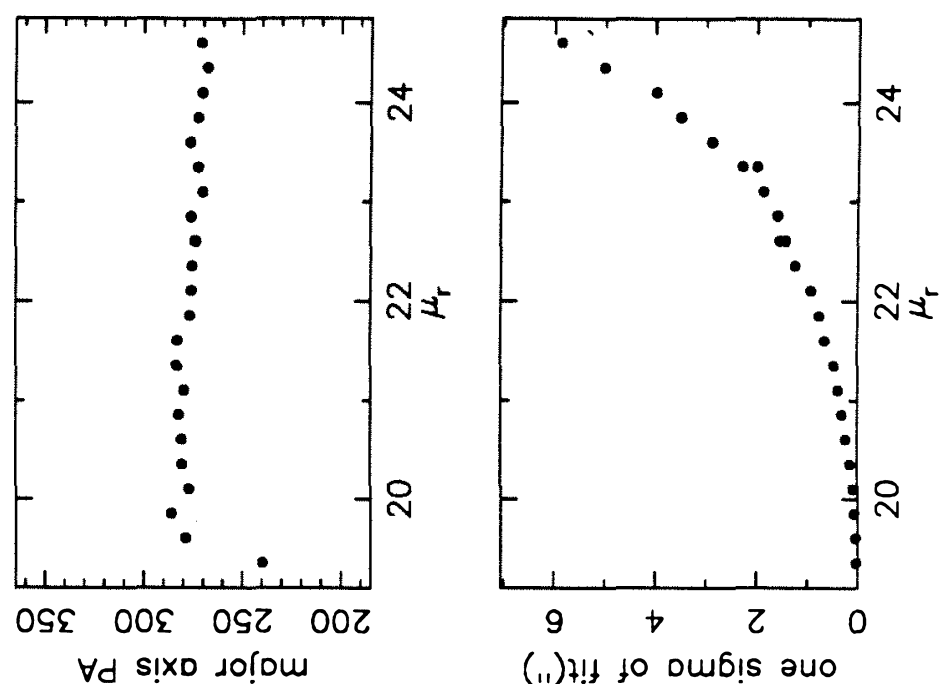

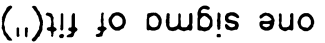
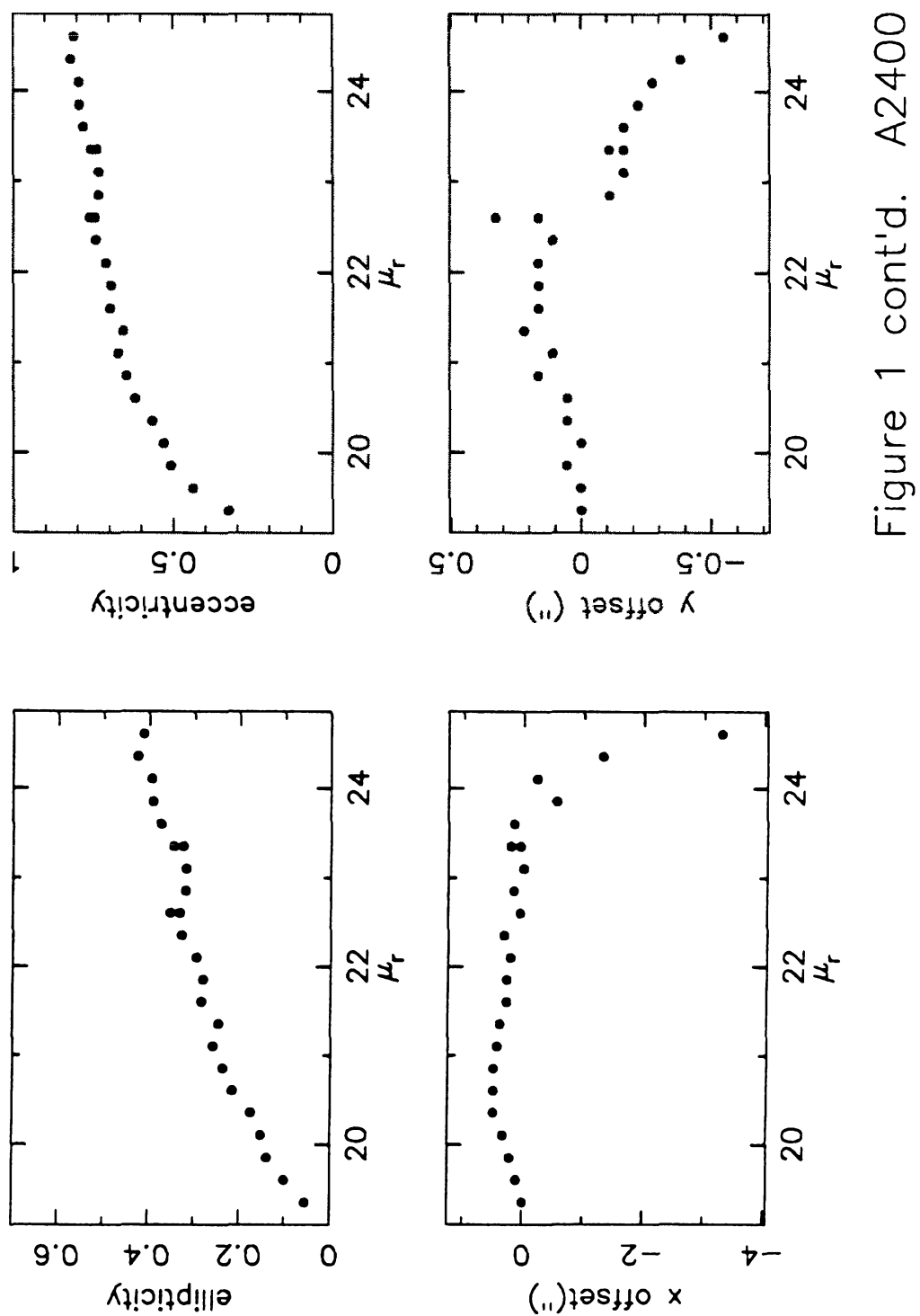


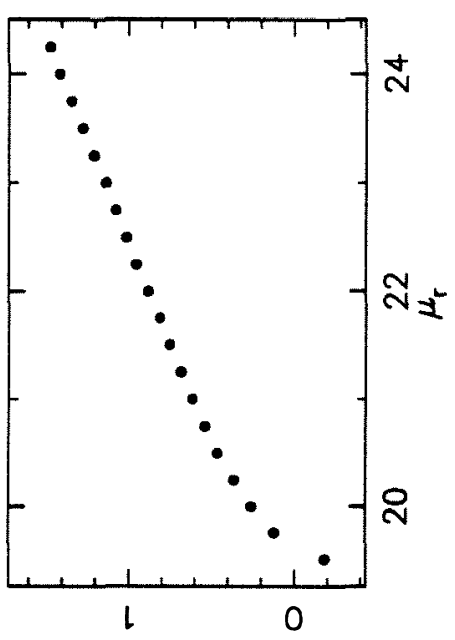

(.1) sn!pod 601

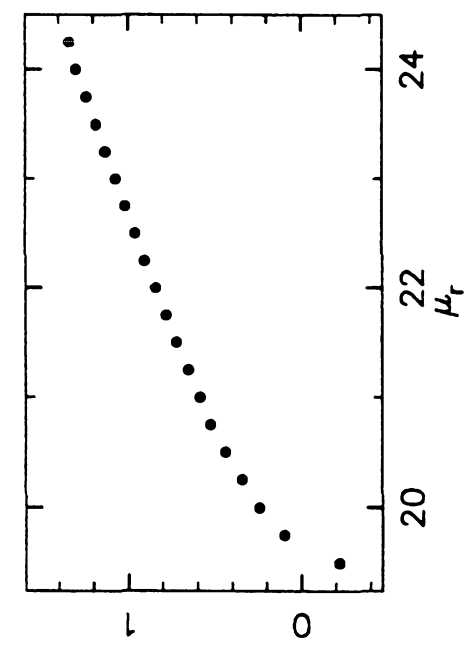

(.1) $9 \mathrm{bol}$

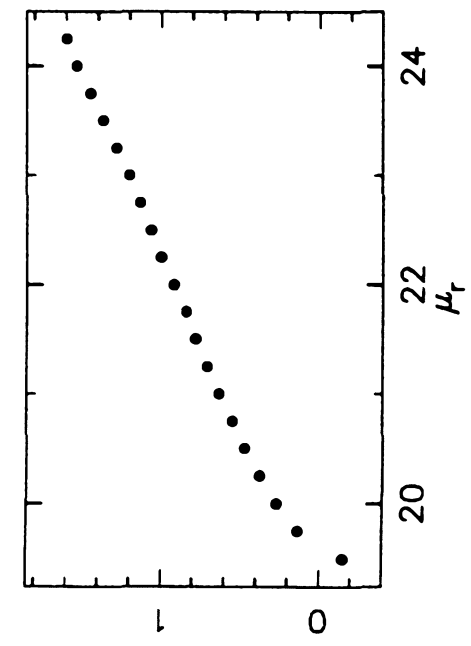

(11) 0601

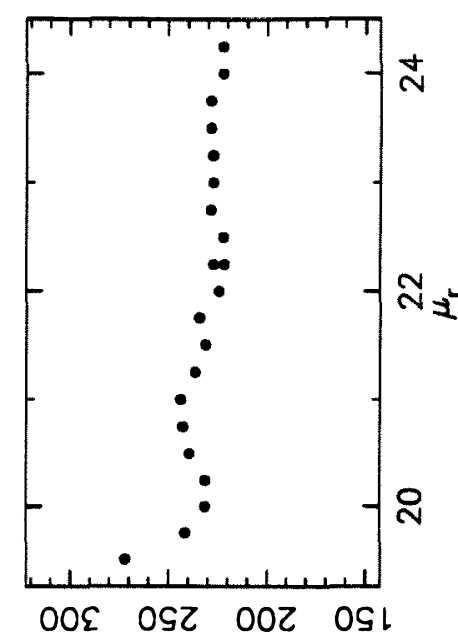

$\forall d$ s!xo so!̣om

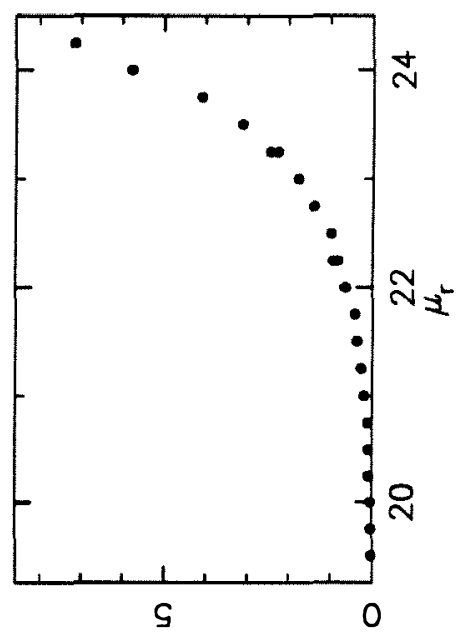

(11) $+!+10$ oub!

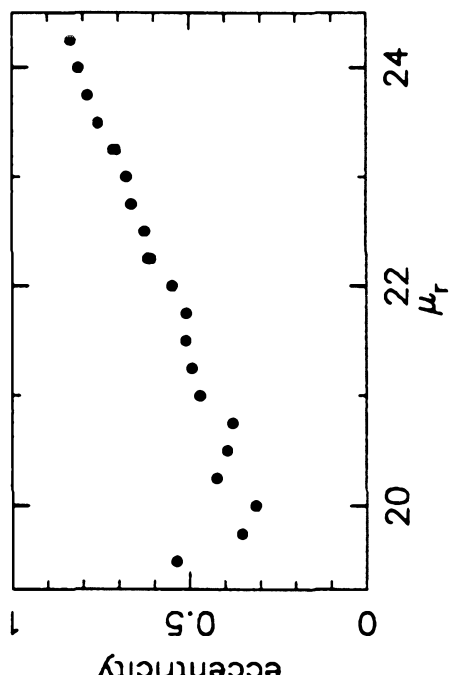

Kł!ว!น\}บวั0ว

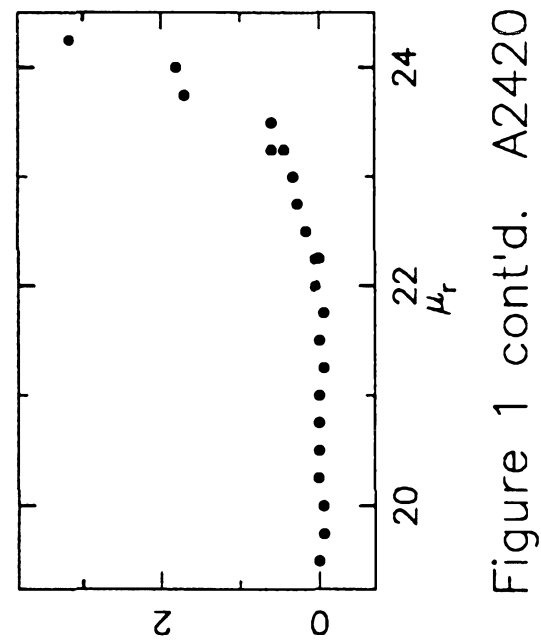

(i1) $725+10 K$

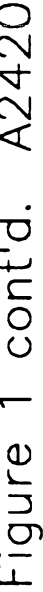
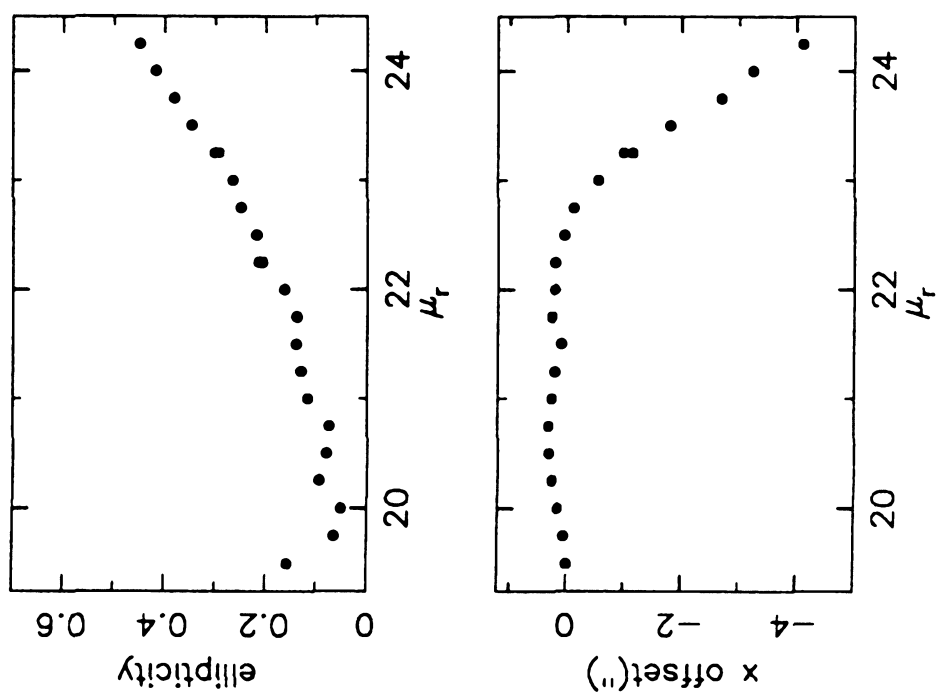


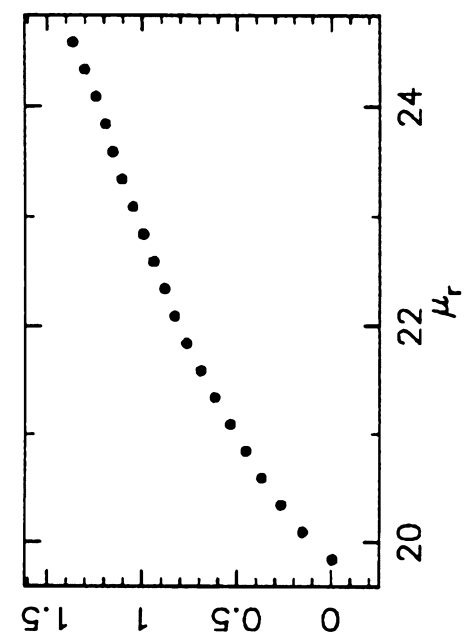

(.1) sn!pod 6ol
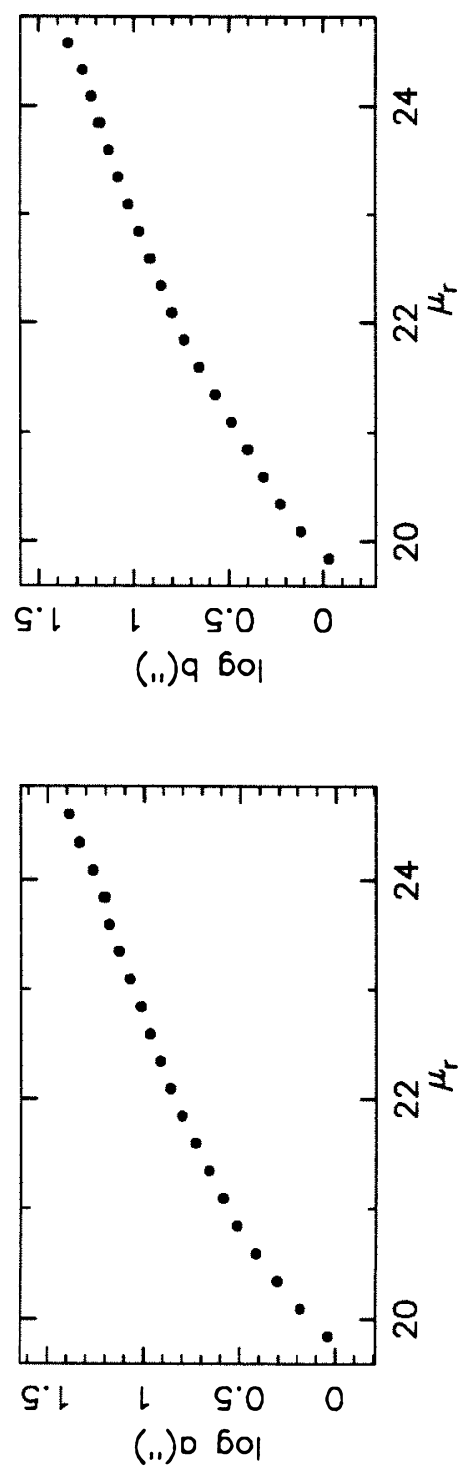
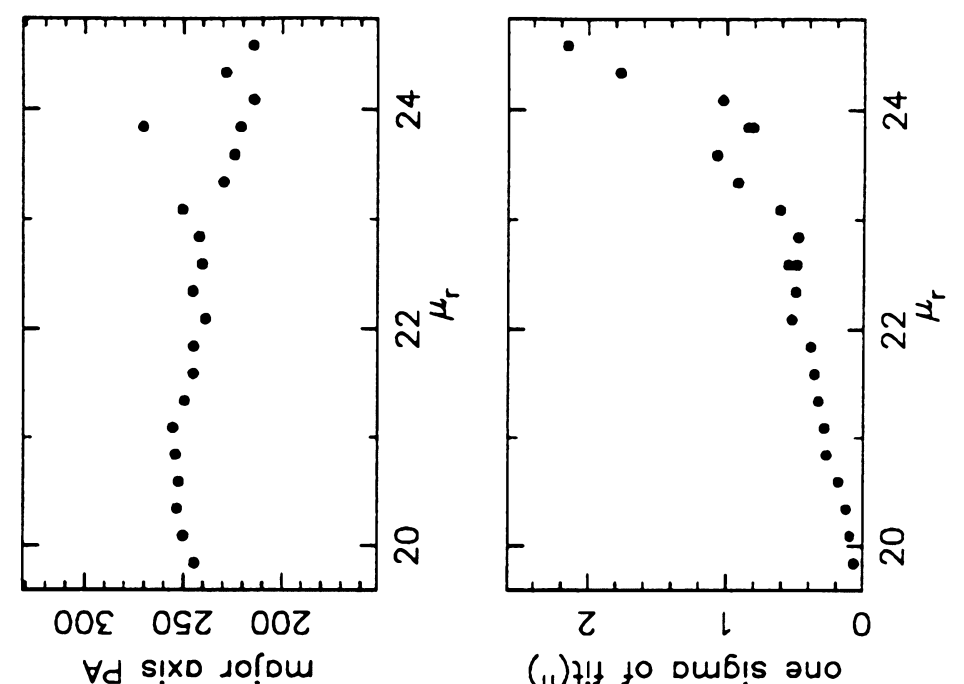

(.1) $+! 1 ~ t 0$ oub!s auo
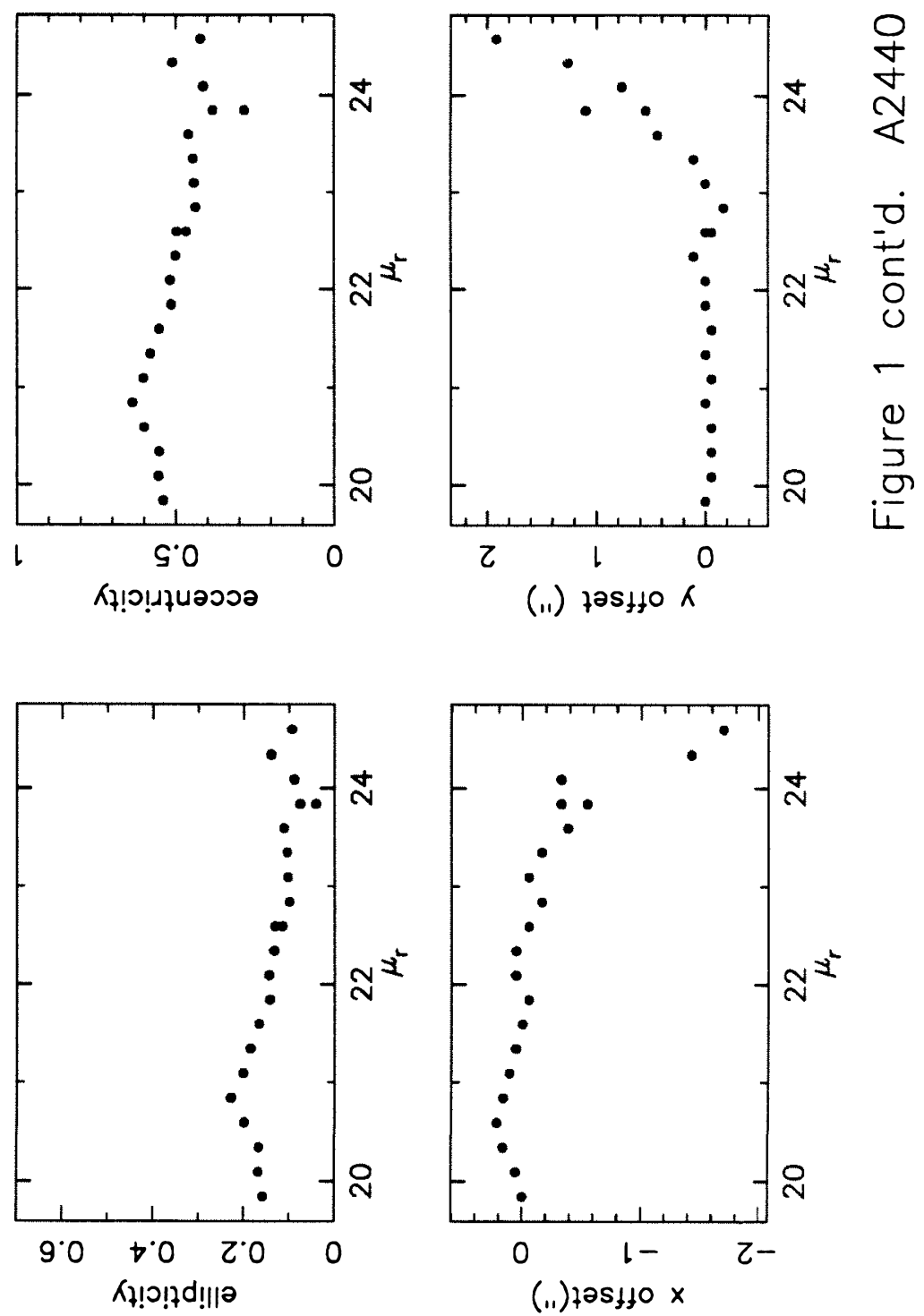

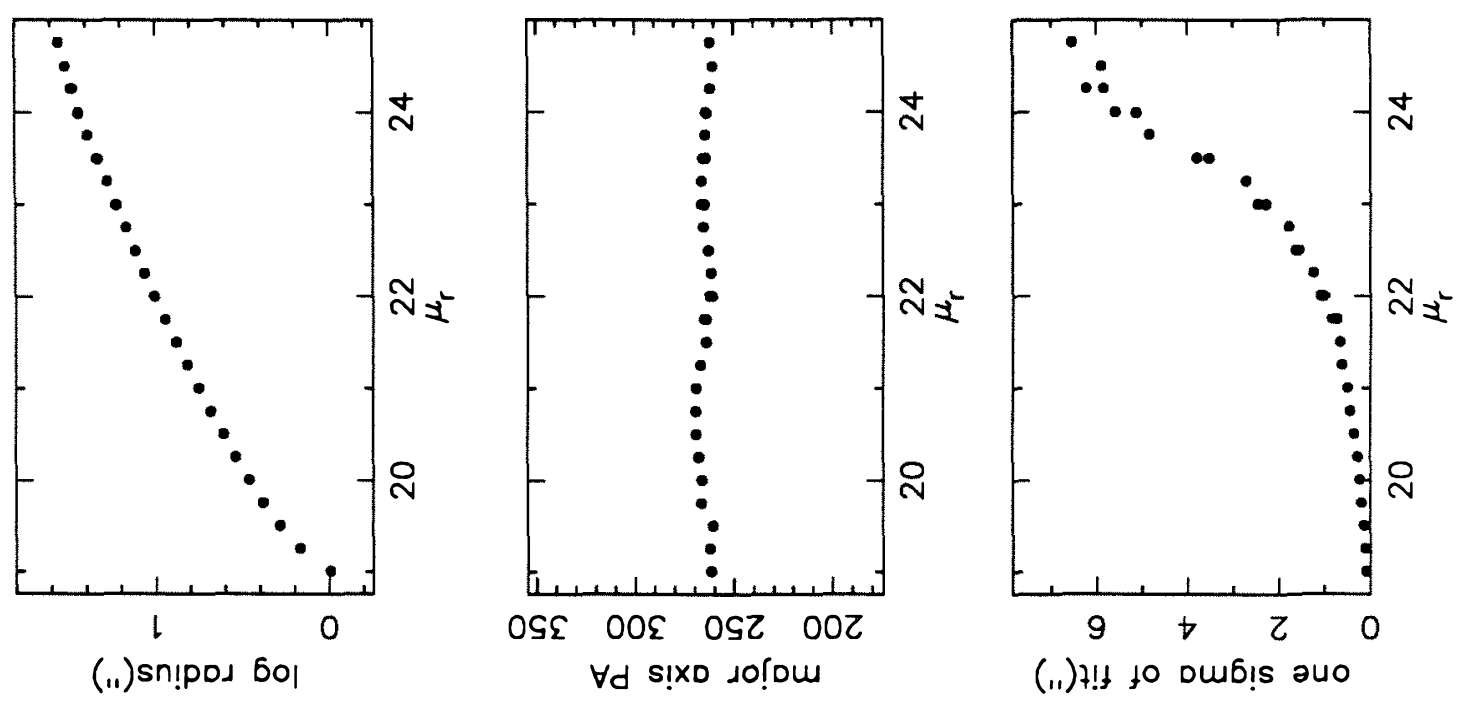

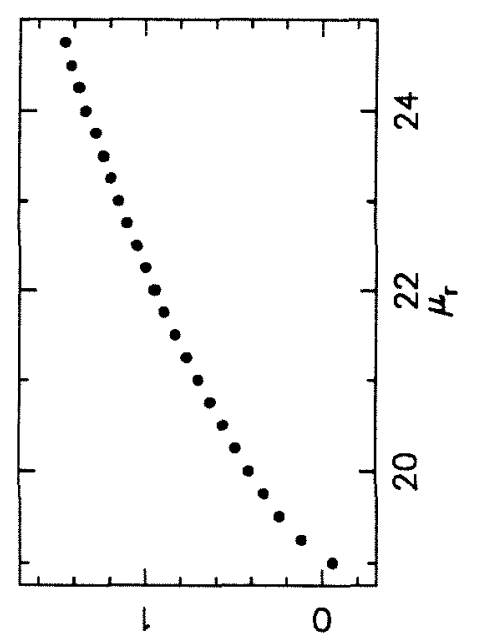

(.1) 9601

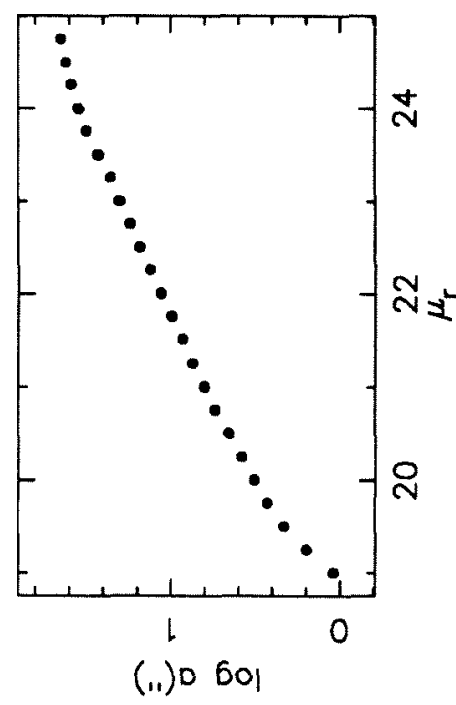

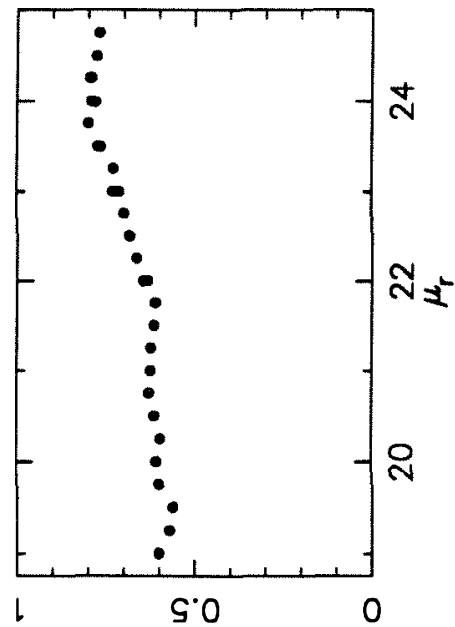

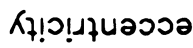

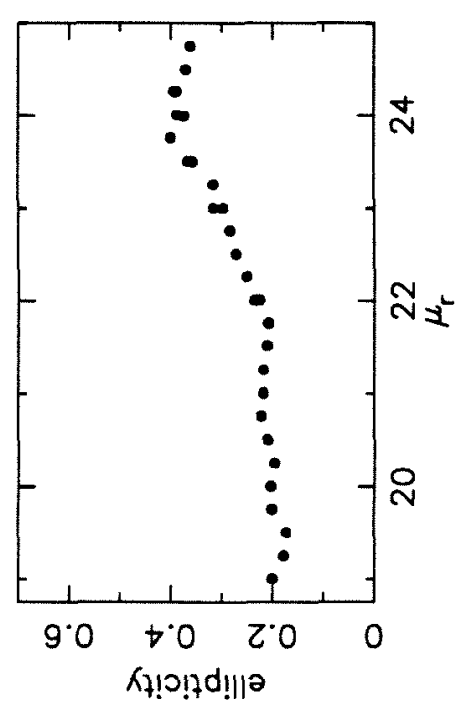

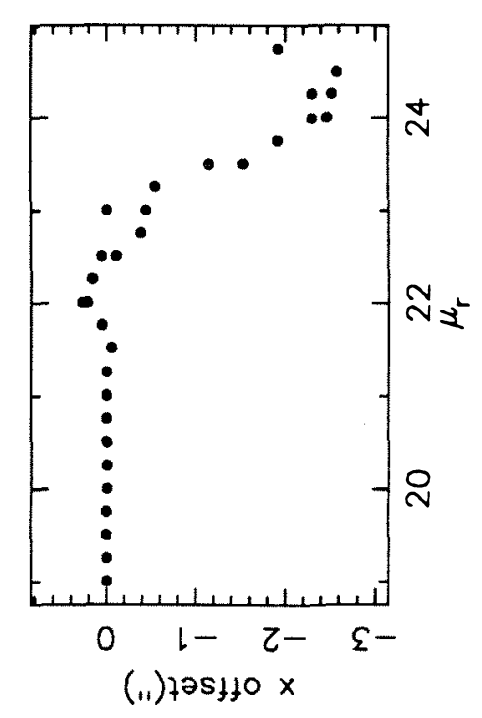

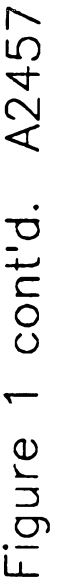

(i1) $725+10 \mathrm{~K}$

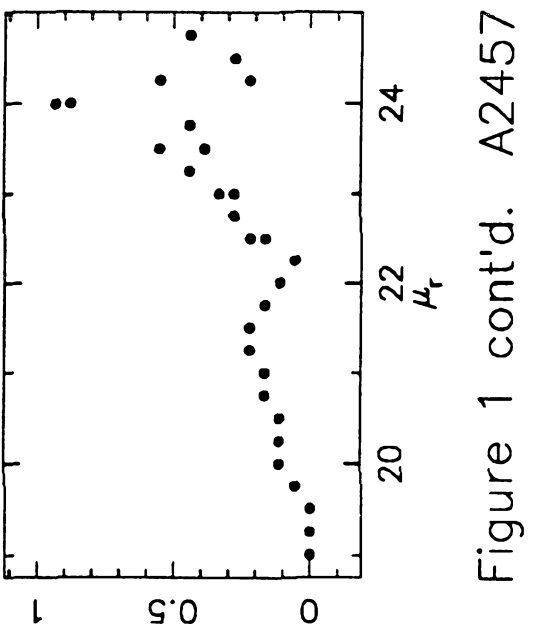




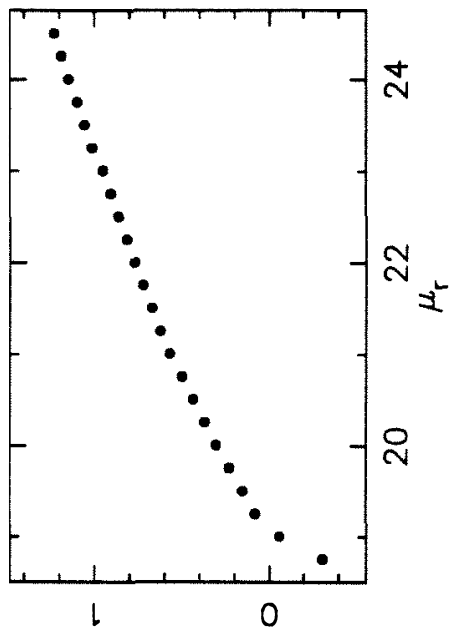

(.1)sn!pod 6ol

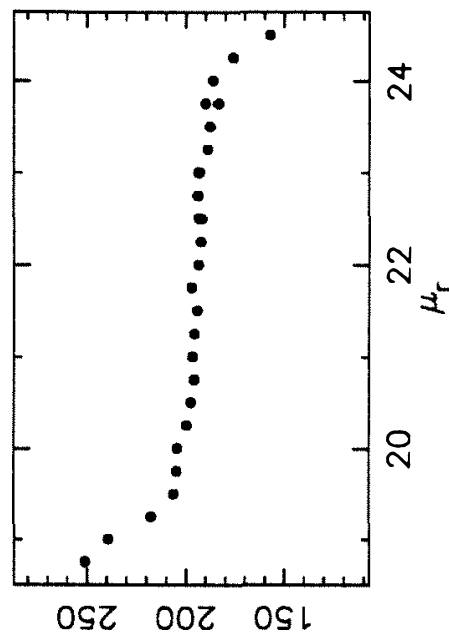

$\forall d$ s!xo Jo!́om

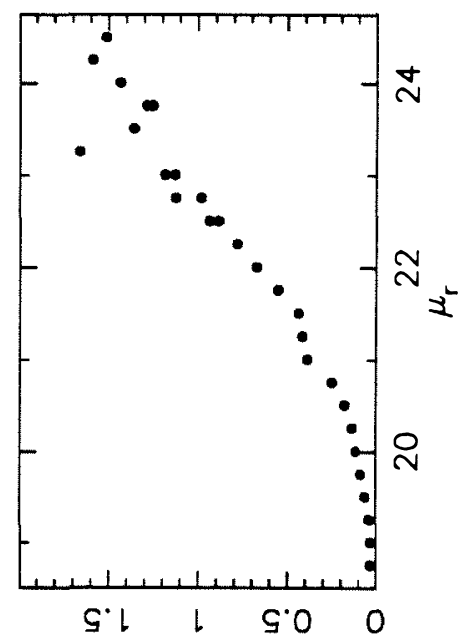

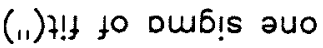
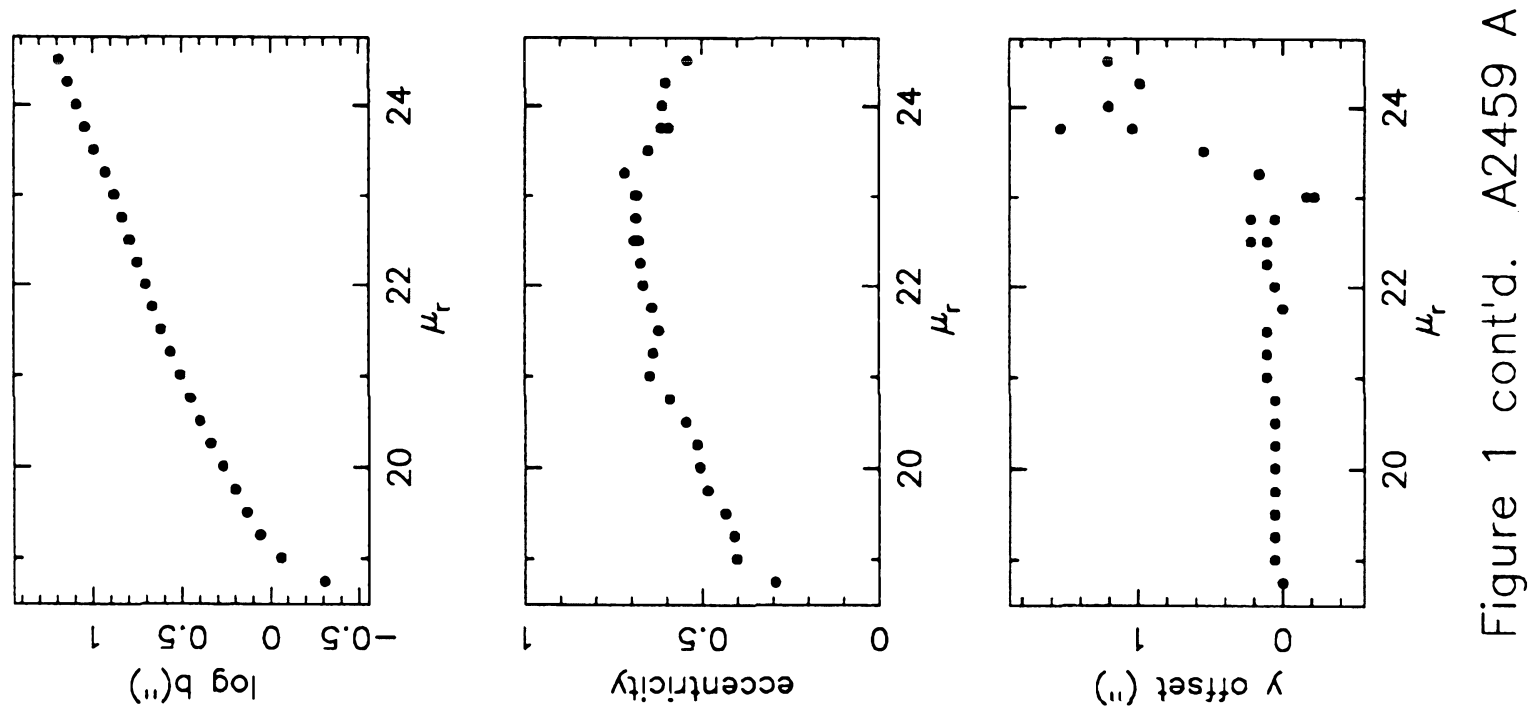

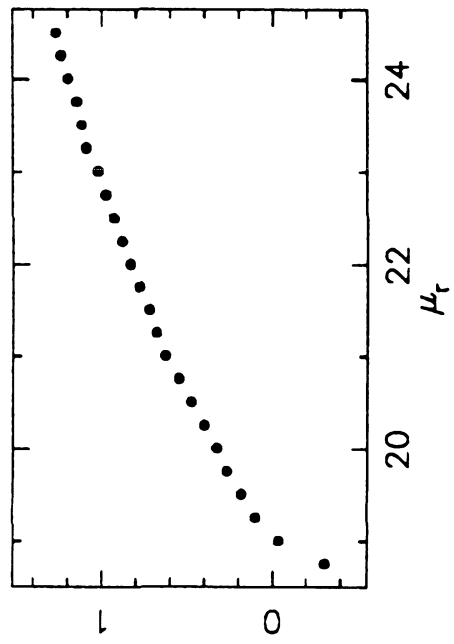

(..) 0 bol
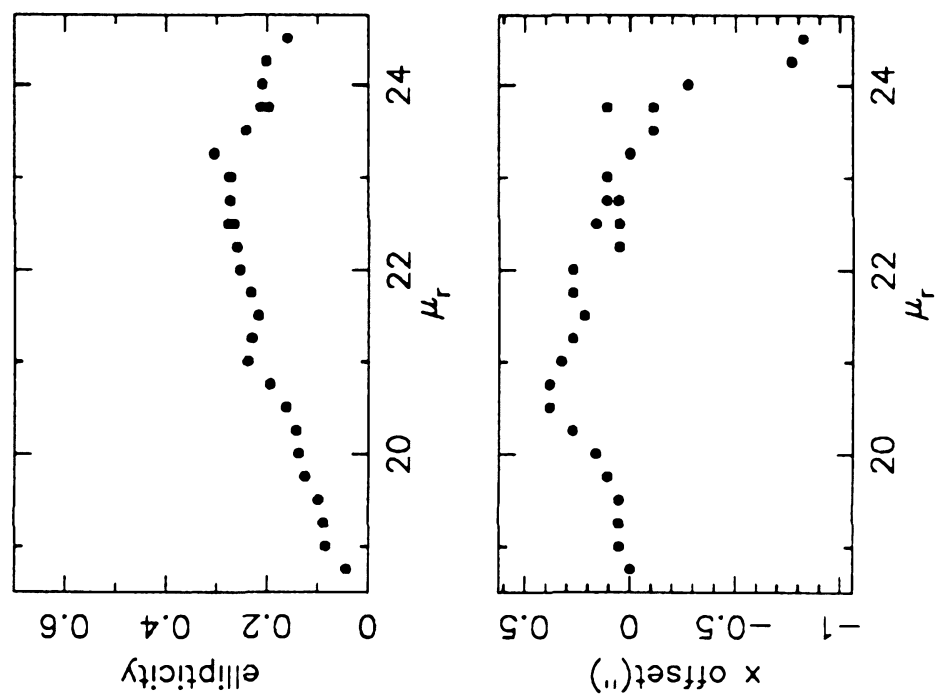


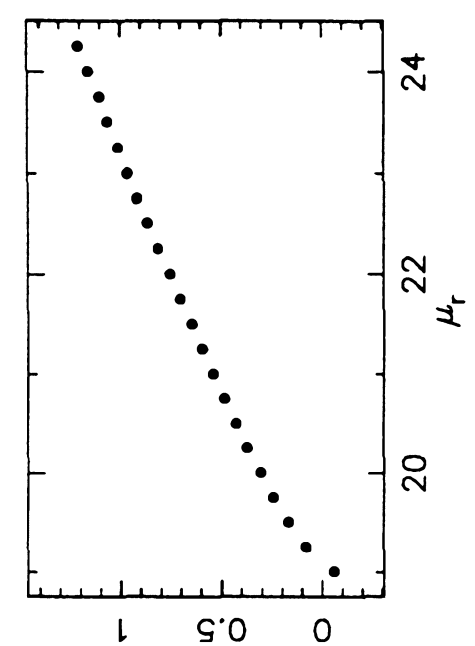

(.1) sn!pod bol

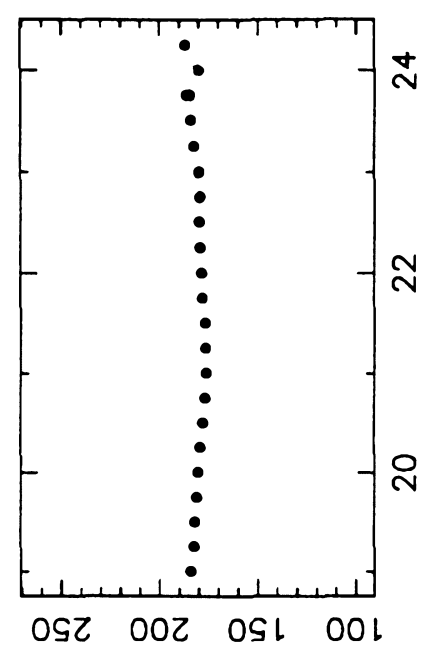

$\forall d$ s!xo so!lom

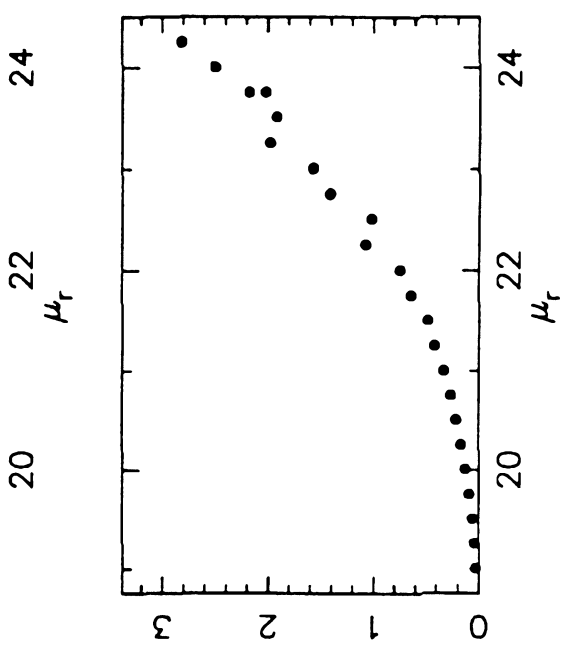

(11) $7 ! 1$ to oub!s auo

(ii) 11 go ow

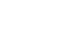

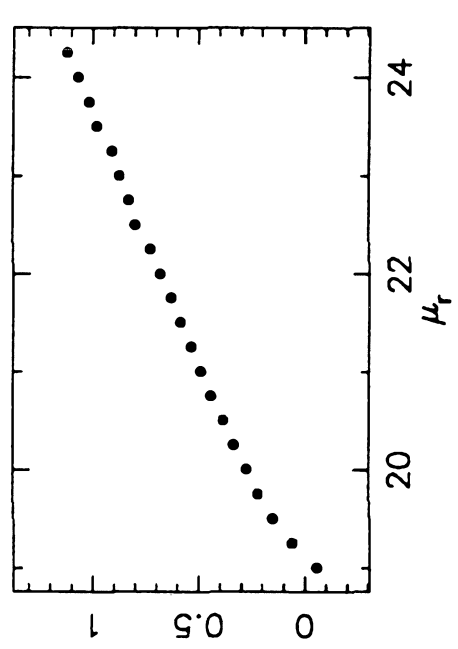

(iI) 9601

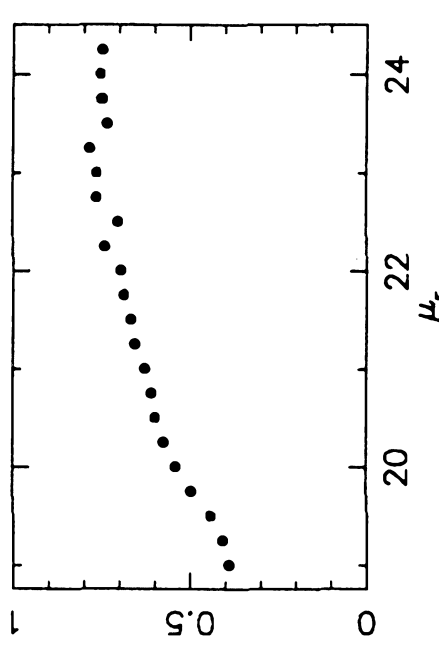

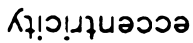

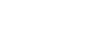




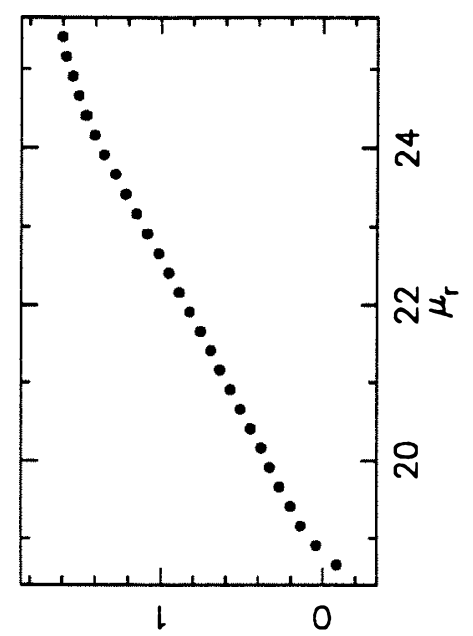

(.1) sn!pod 60।

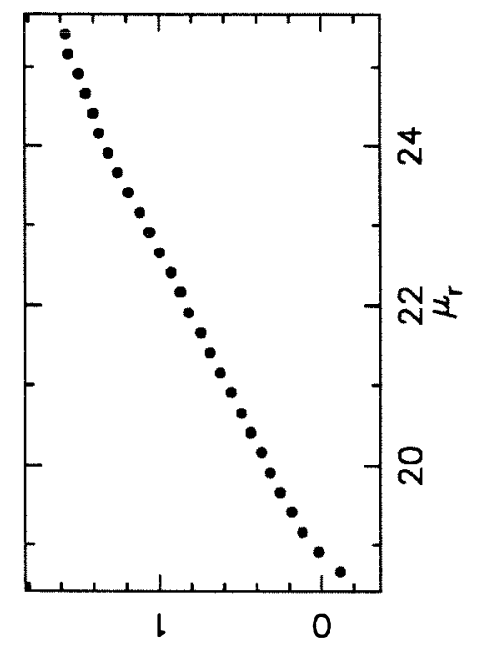

(i1) 9 bol

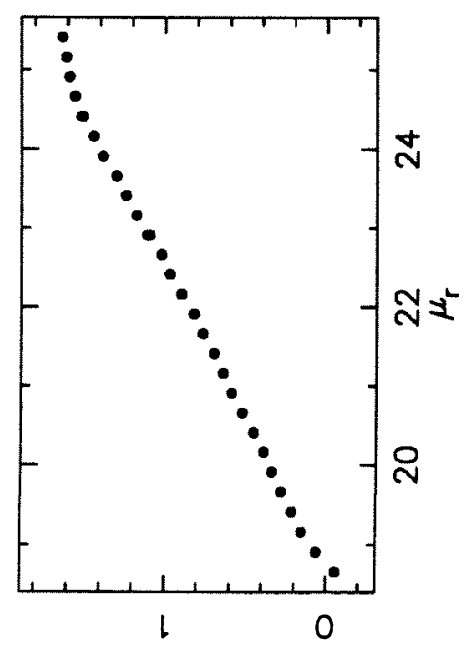

(.1) 0601

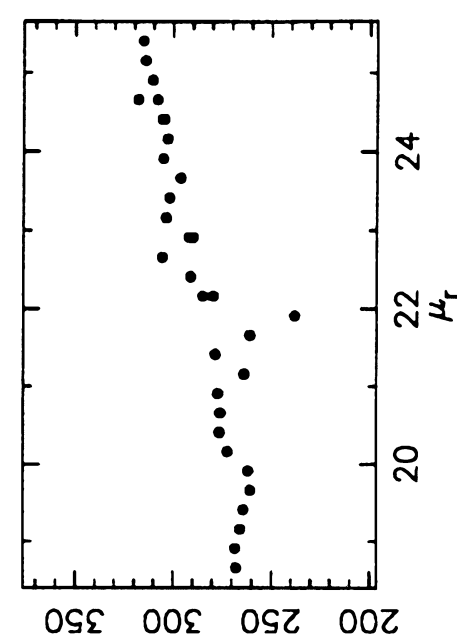

$\forall d$ s!xo دo! lou

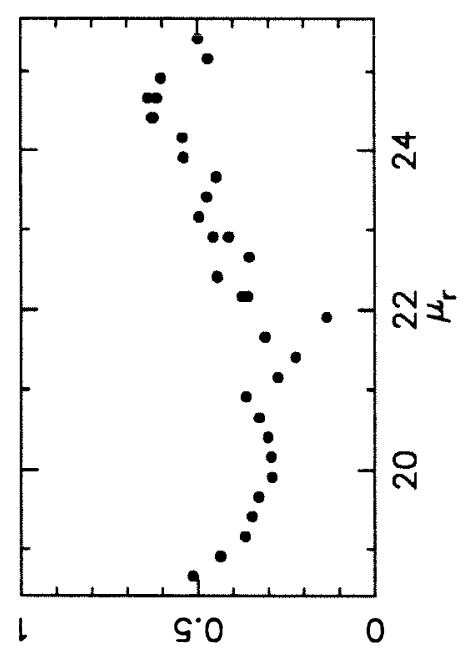

रห!ว!นาบวั0ว

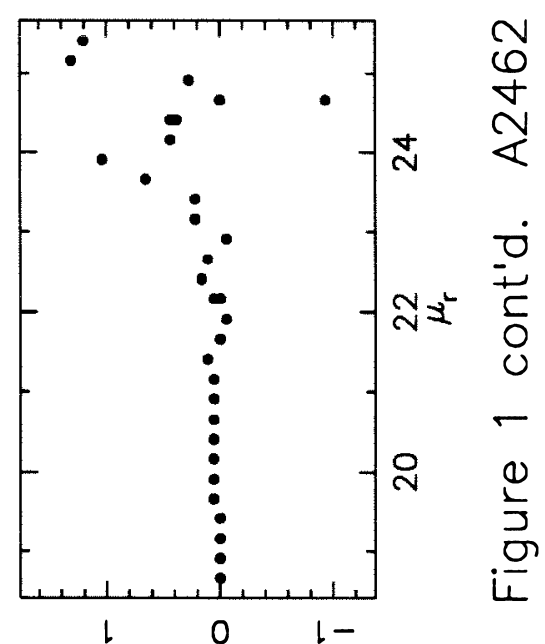

(ii) $725150 \mathrm{~K}$
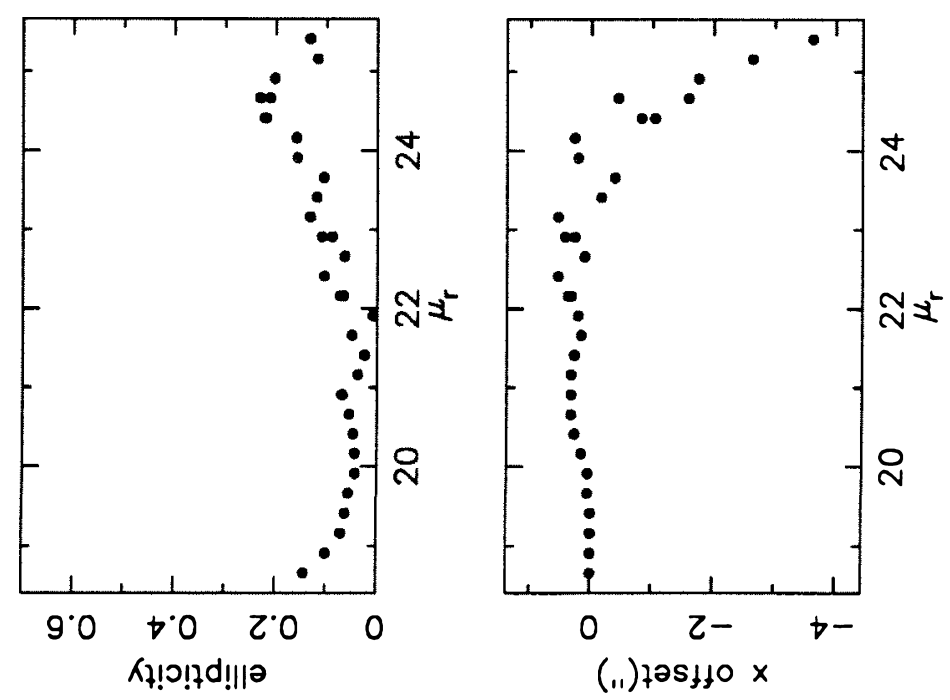


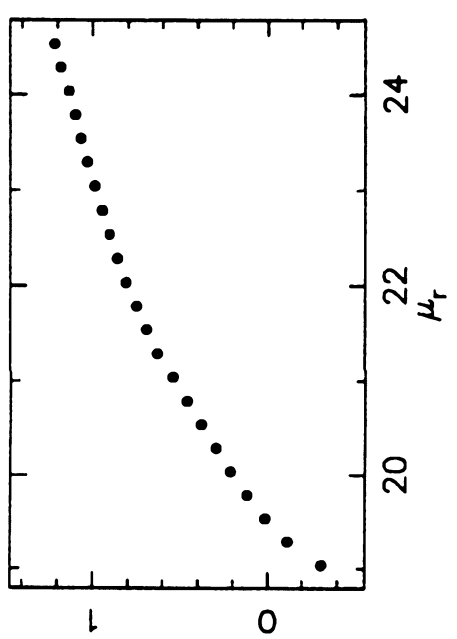

(.1) sn!pos 6ol

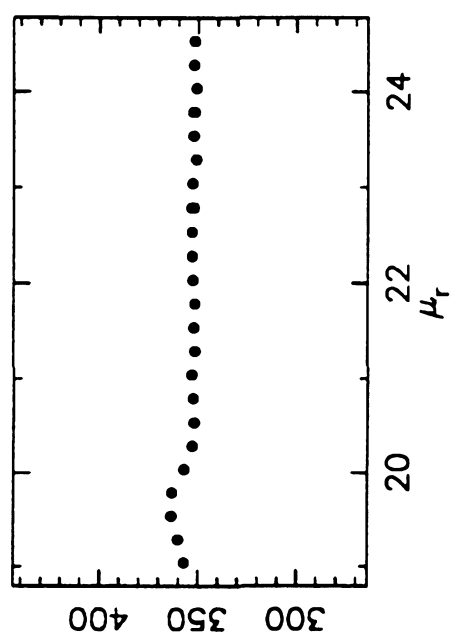

$\forall d$ s!xo so! ow

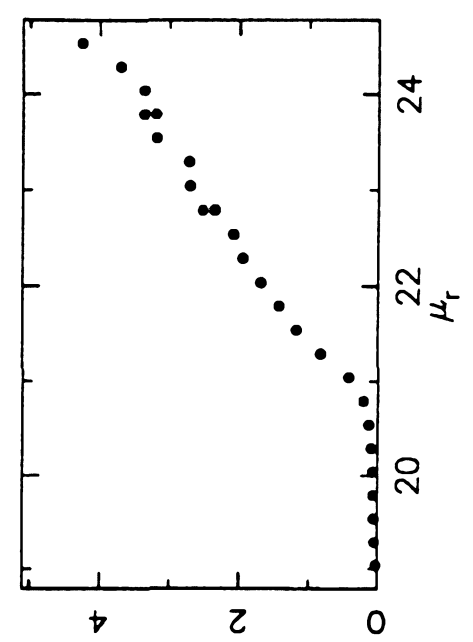

(i1) $4 ! \downarrow$ to Dub!s auo

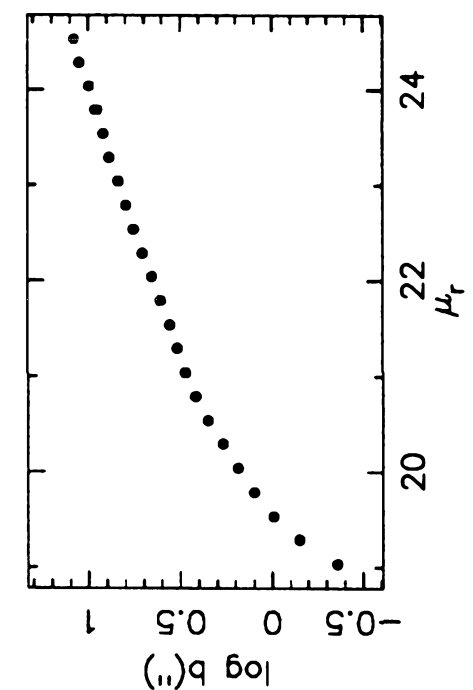

(.1) 960

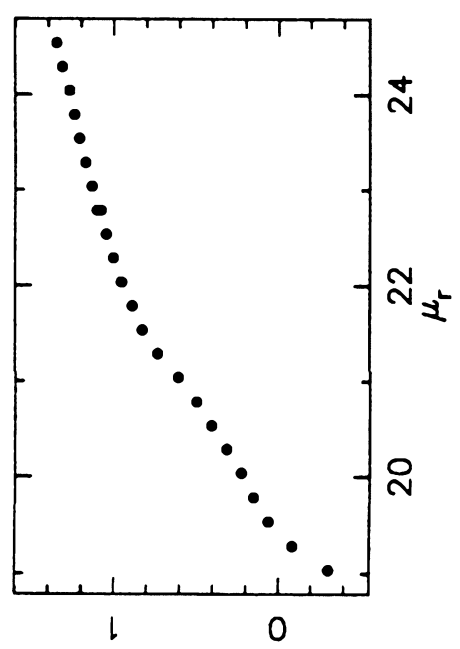

(.1) 0601
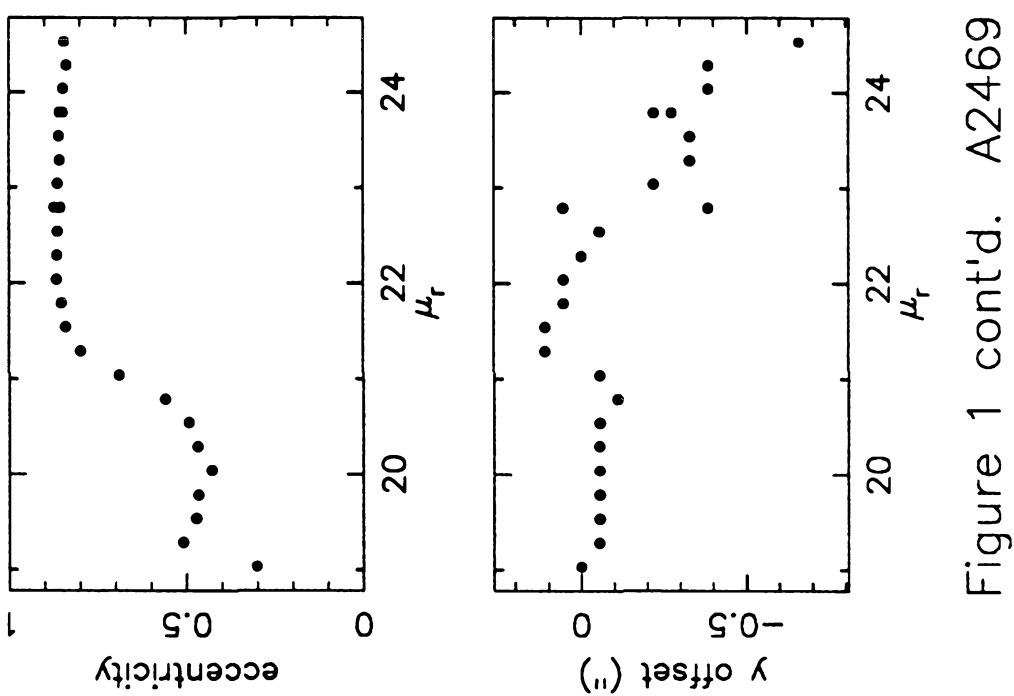

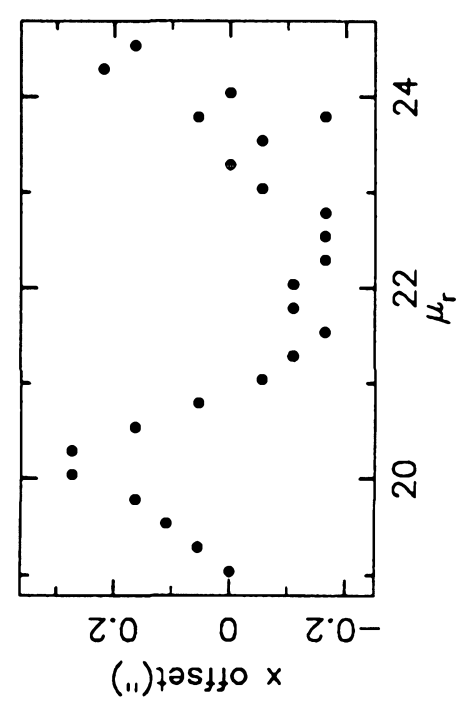



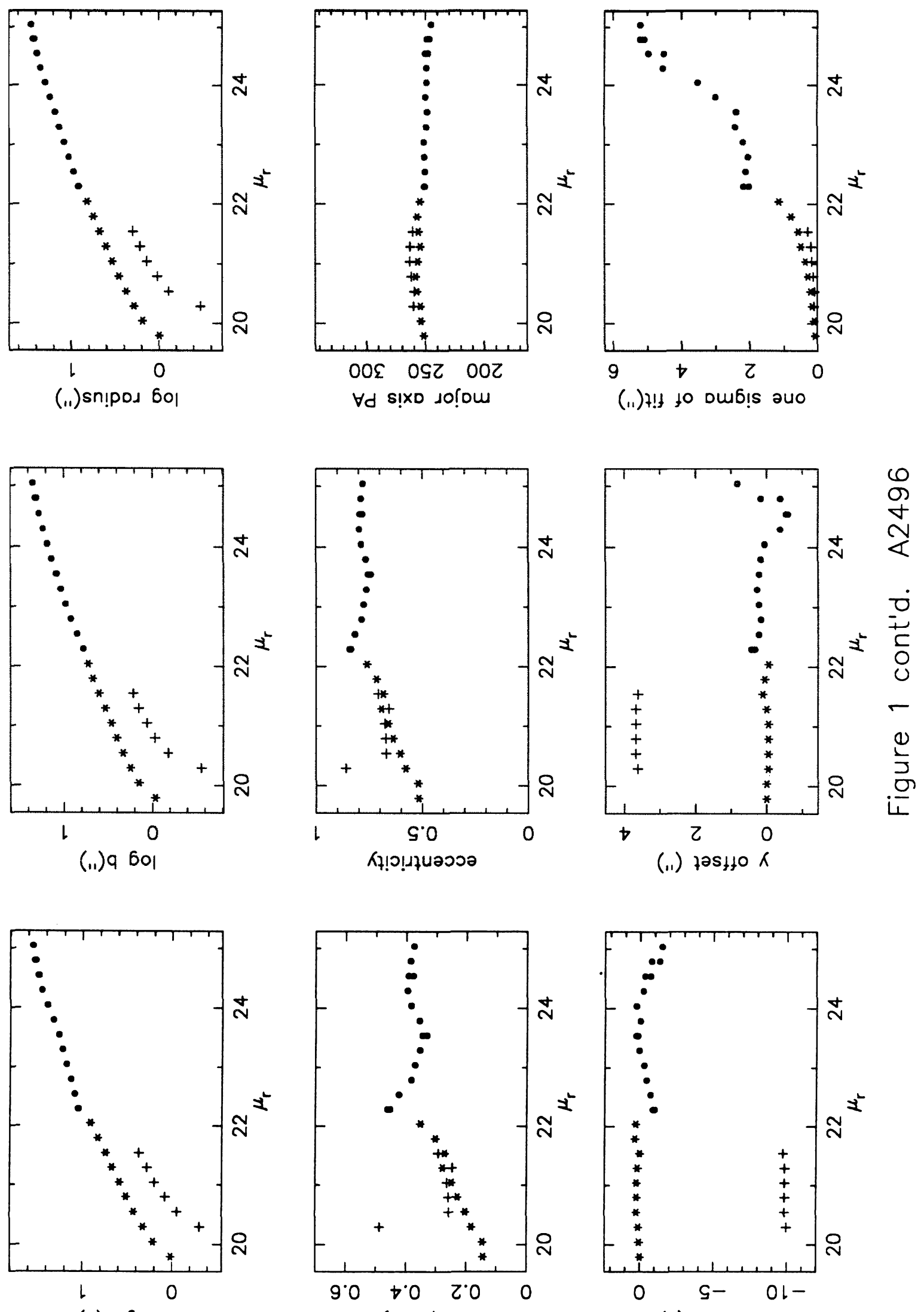

(.1) 0 6ol
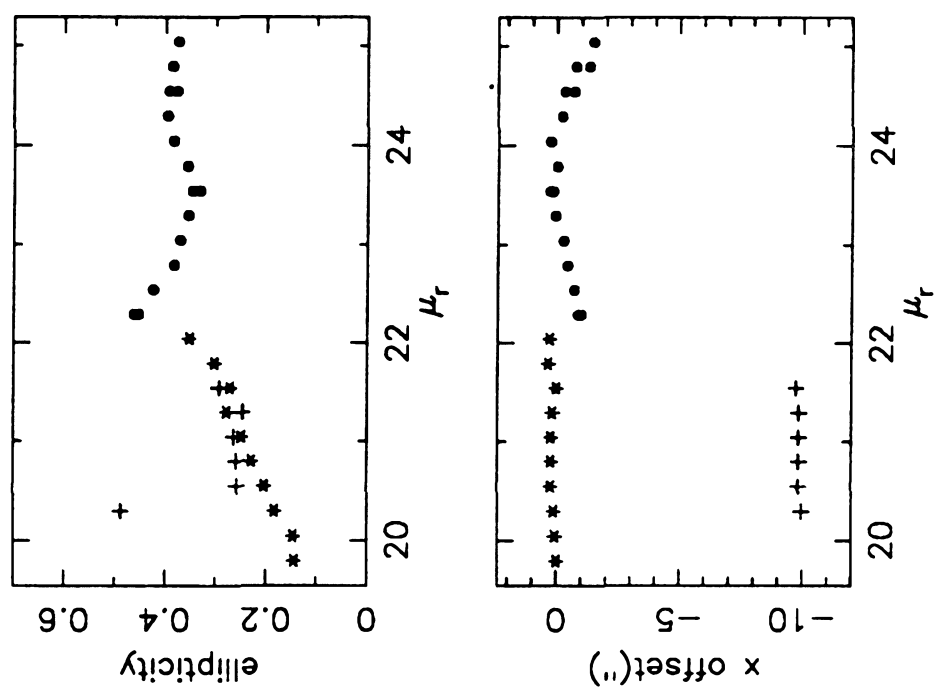

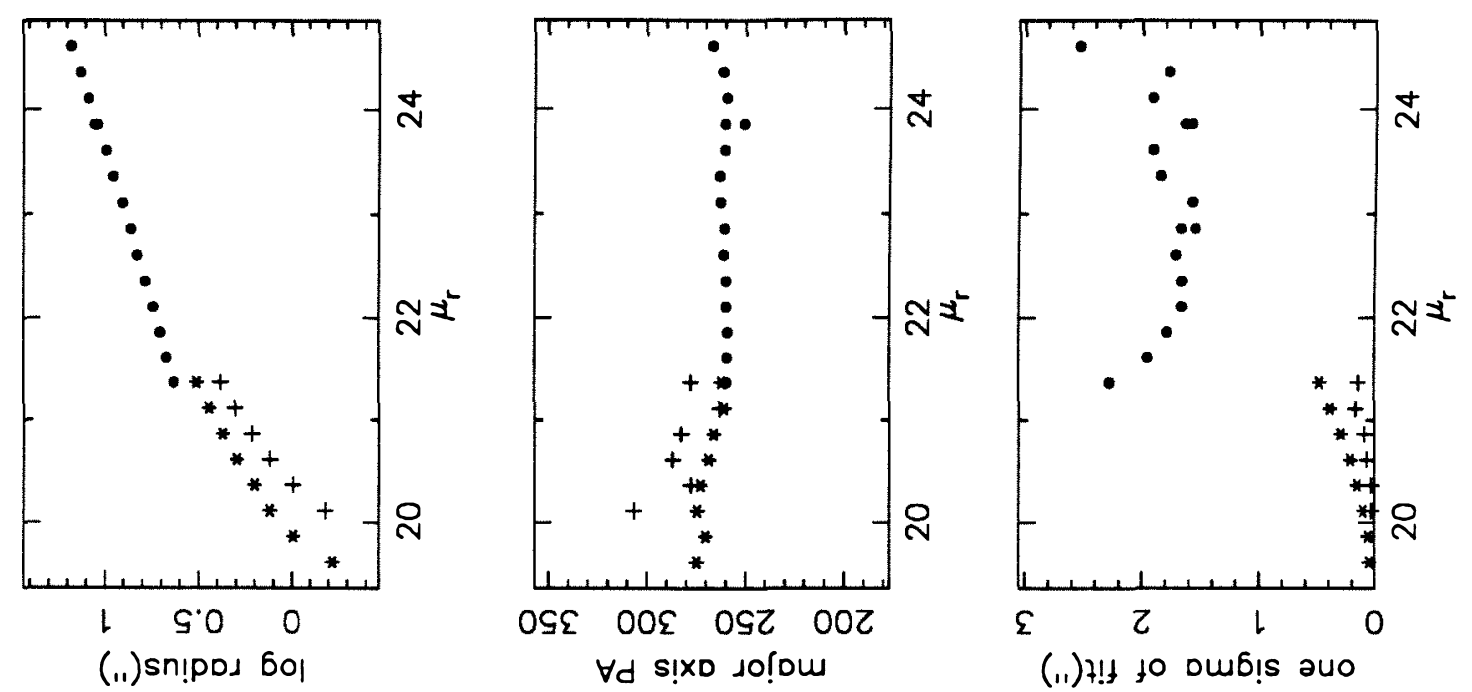

(11) $+! 1$ to oub!s auo
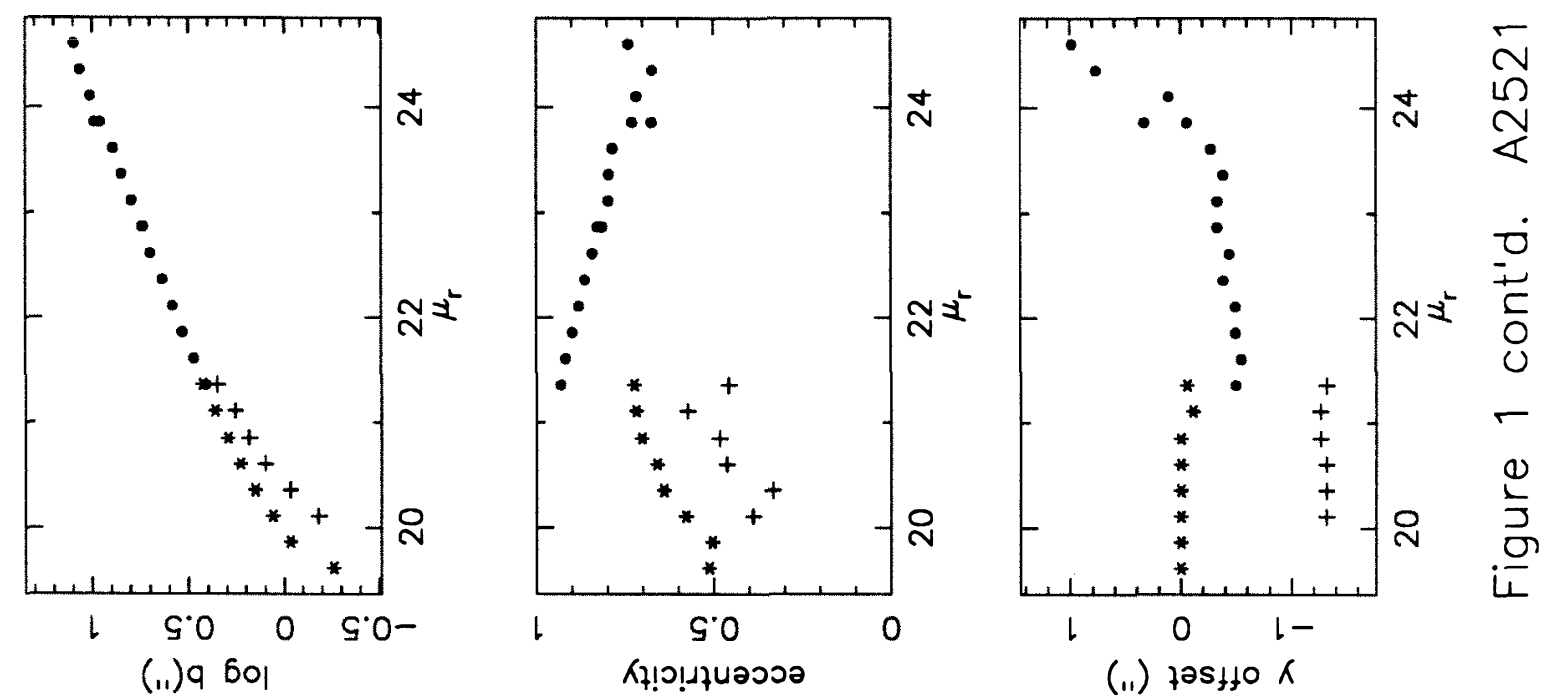

(i1) tos $110 \mathrm{~K}$
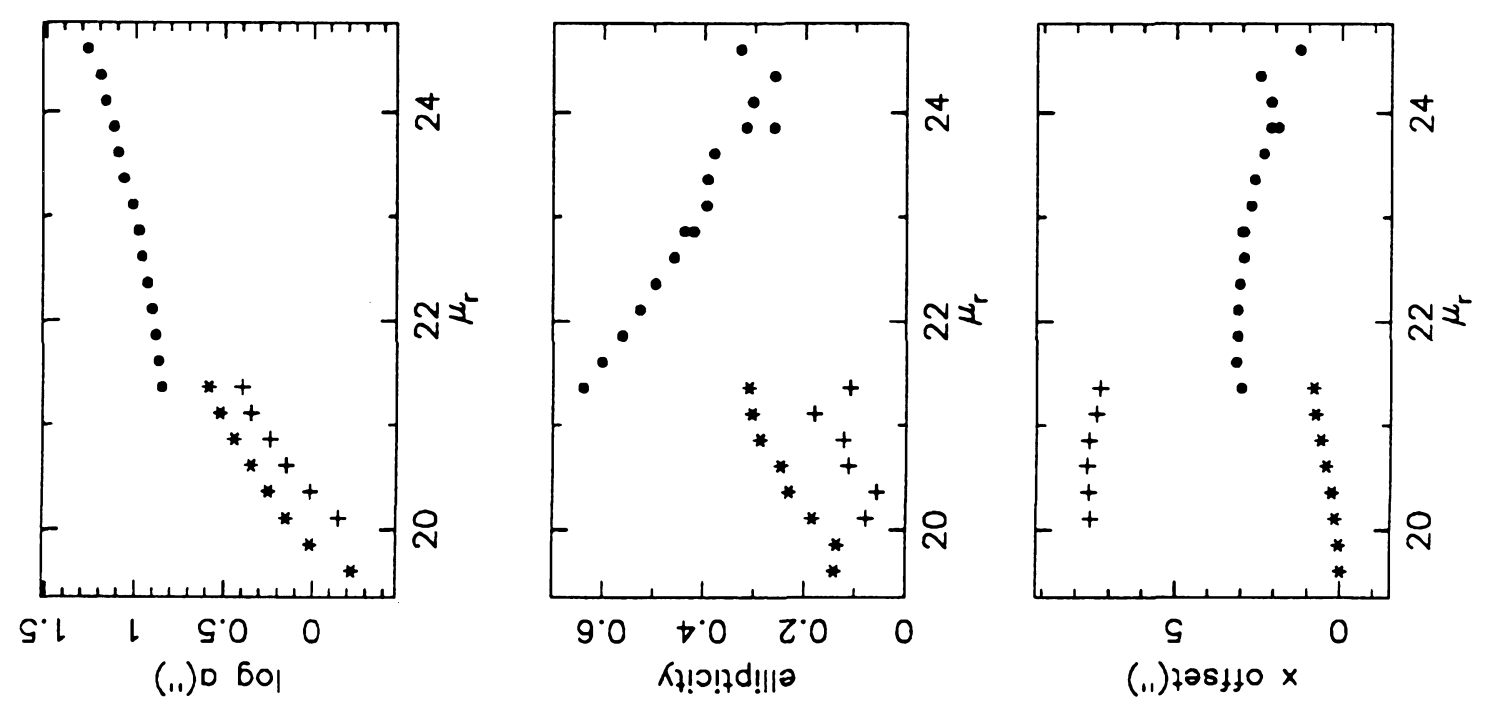


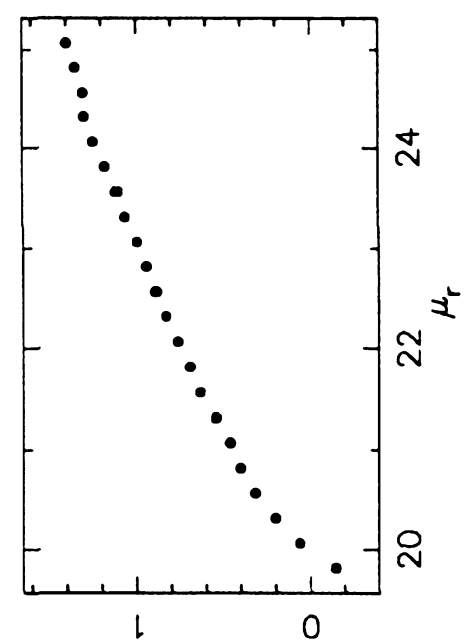

(.1) sn!pd 60।
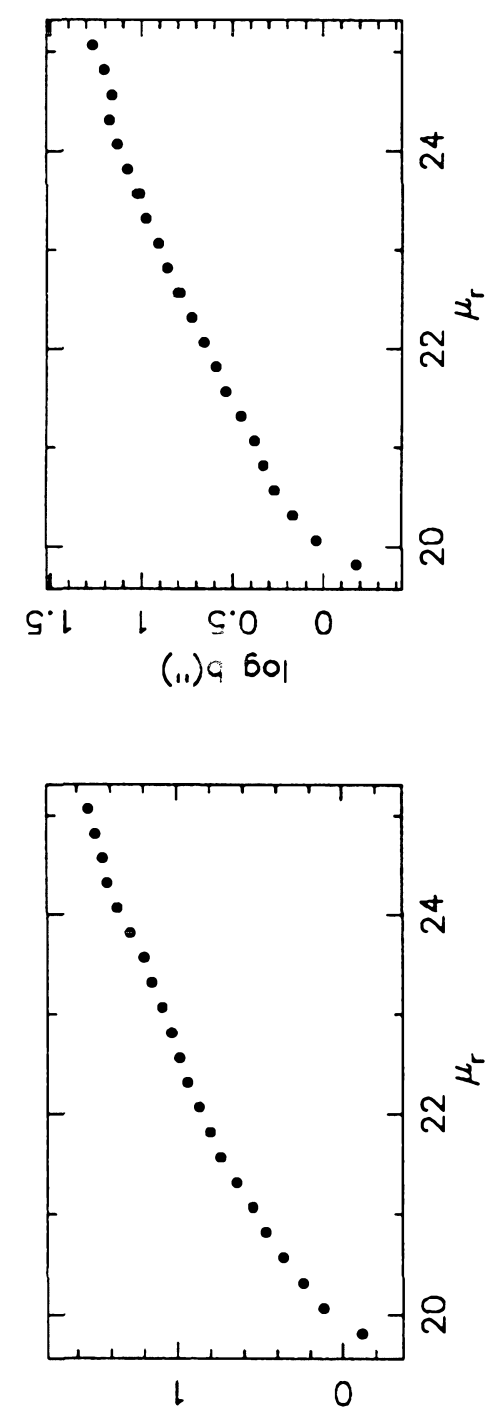

(.1) 0601

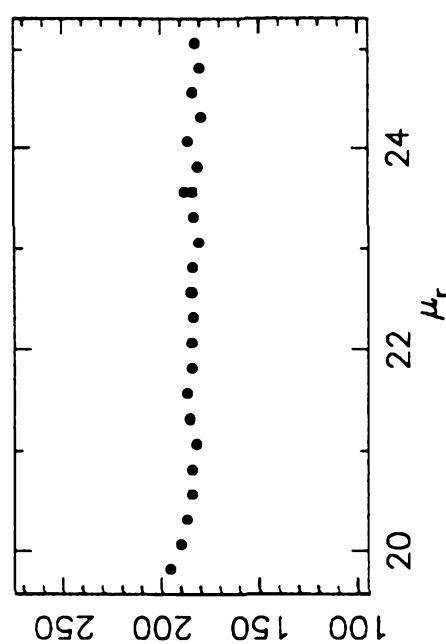

$\forall d$ s!xo so!̣us

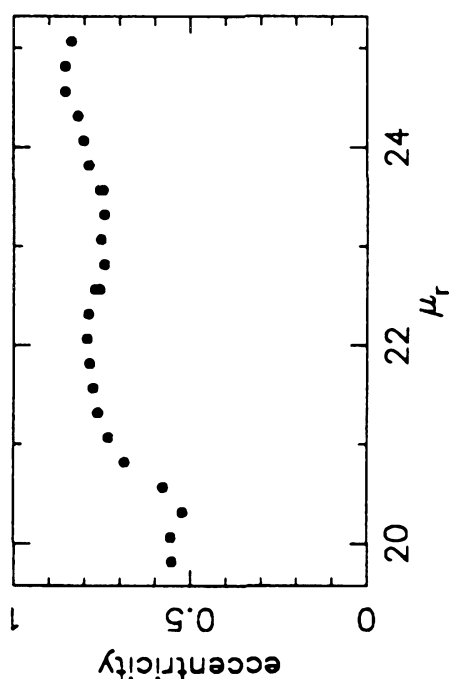

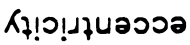

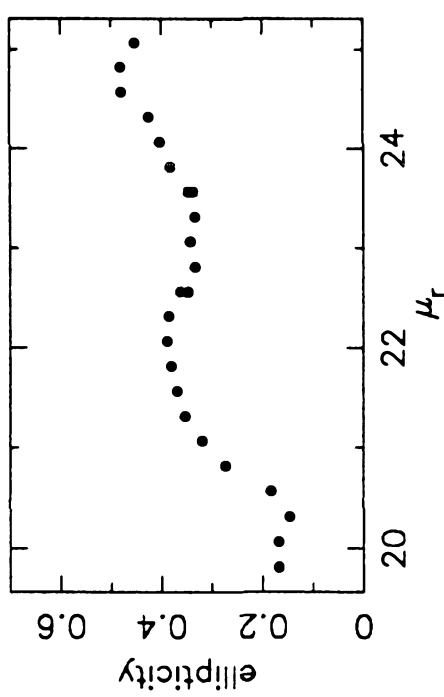

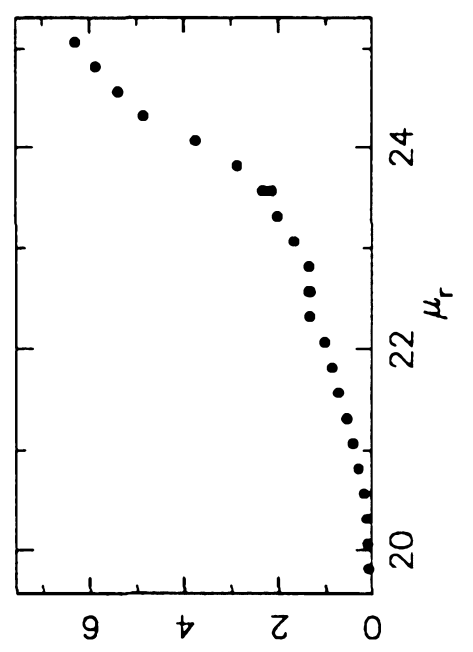

(i1) + !! to Dub!s auo
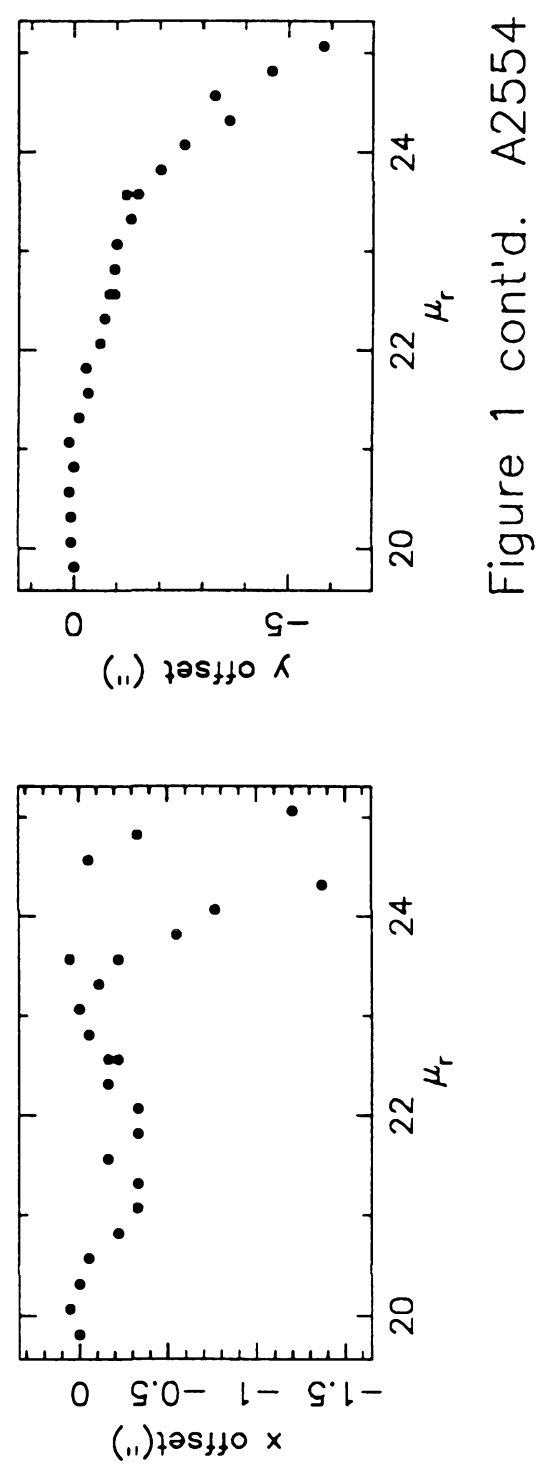


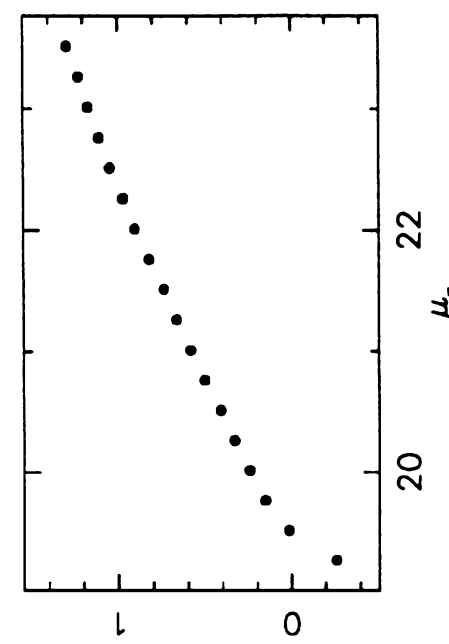

(.1)sn!pDd 601
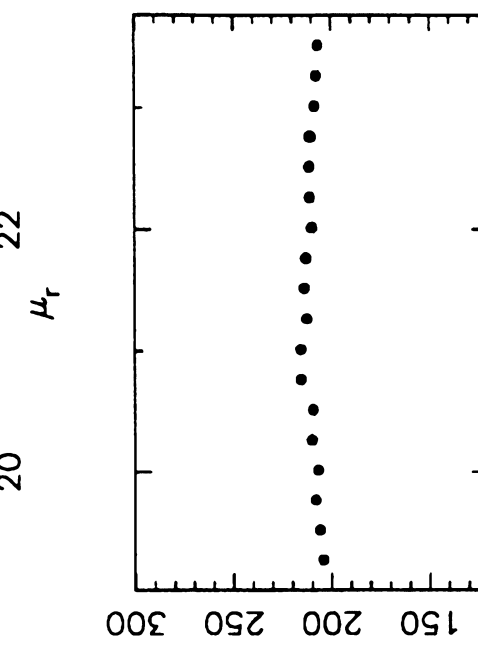

$\forall d$ s!xo so!́lom

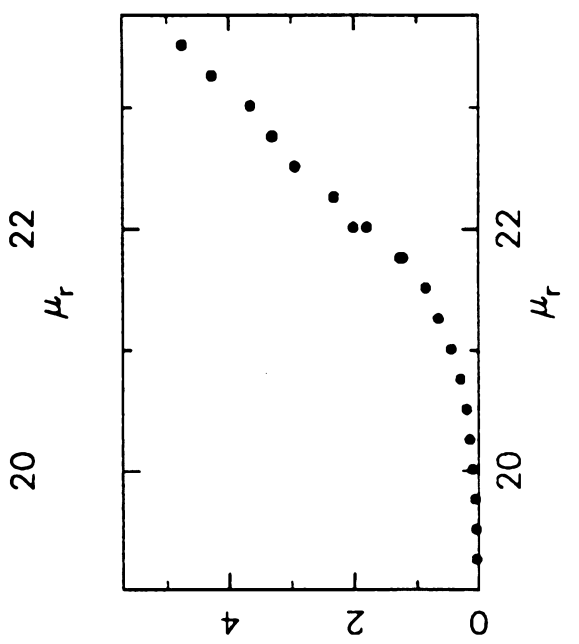

(11) $4 !+10$ Du6!s aиo

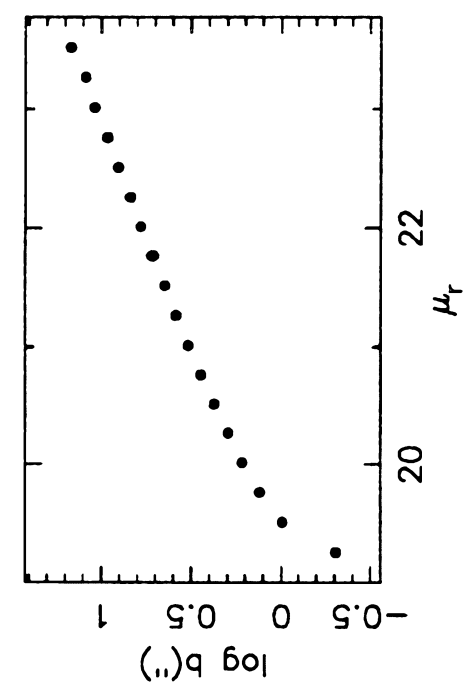

(.1) $9 \mathrm{bo}$

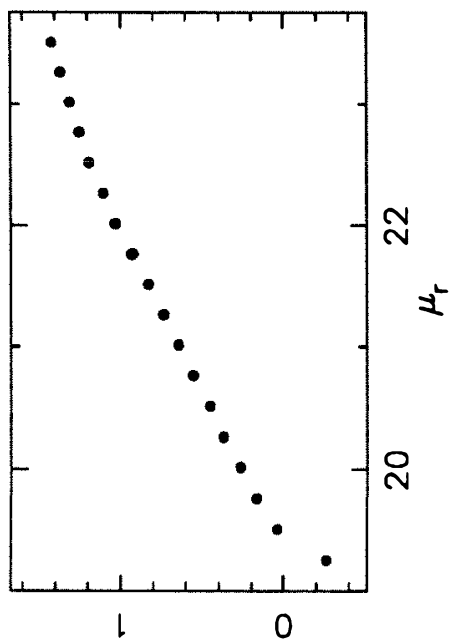

(i1) 0601
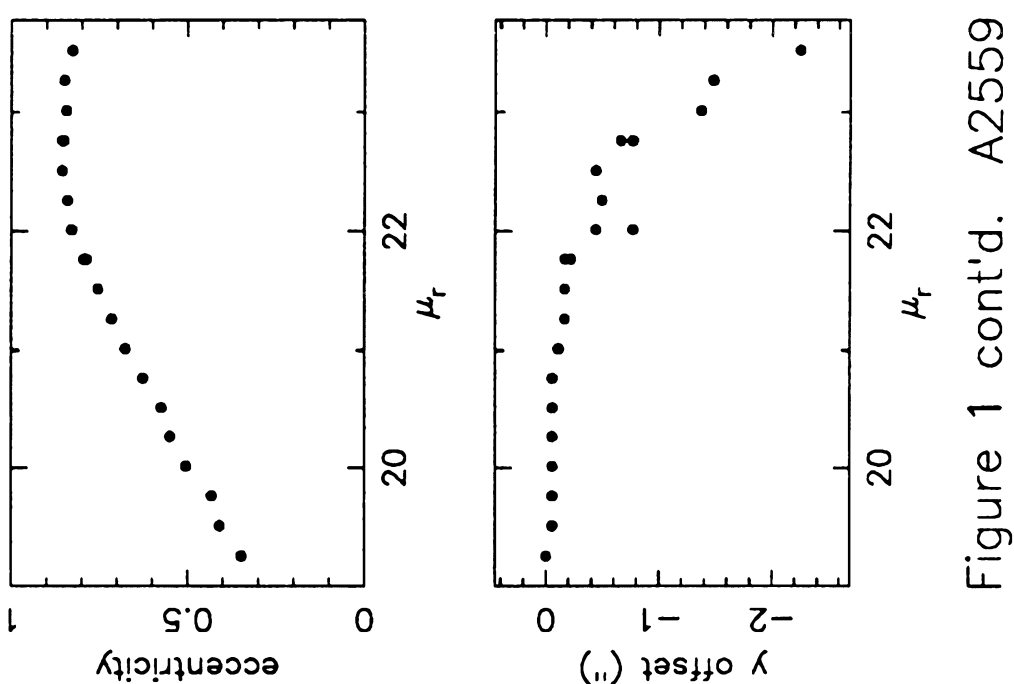
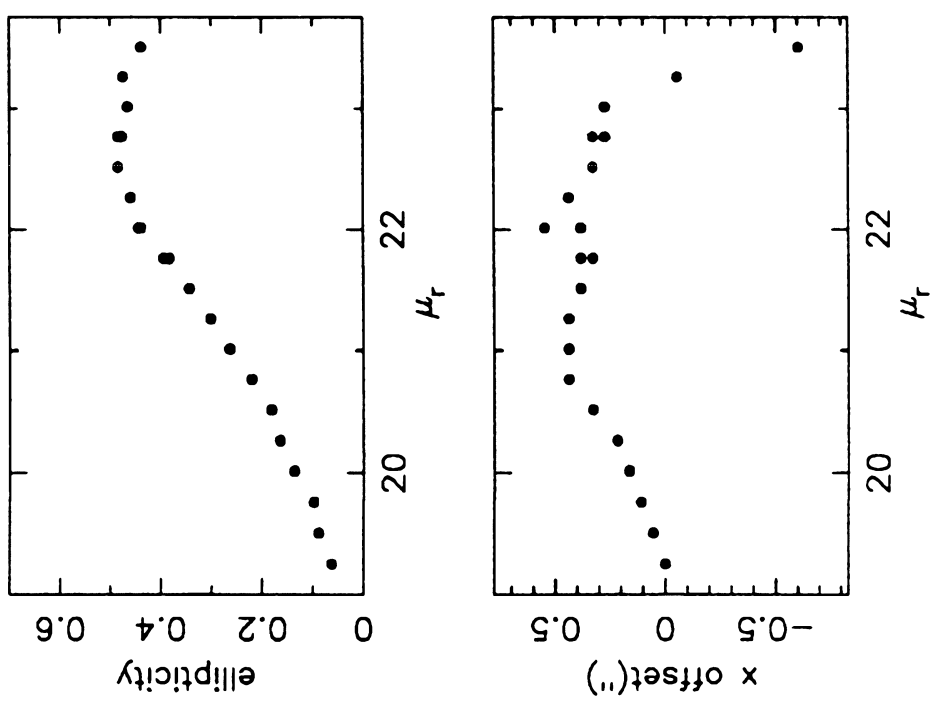


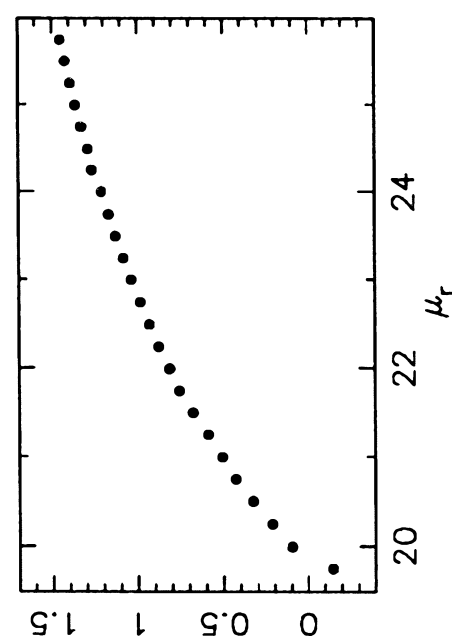

(.1) sn!pos 60।

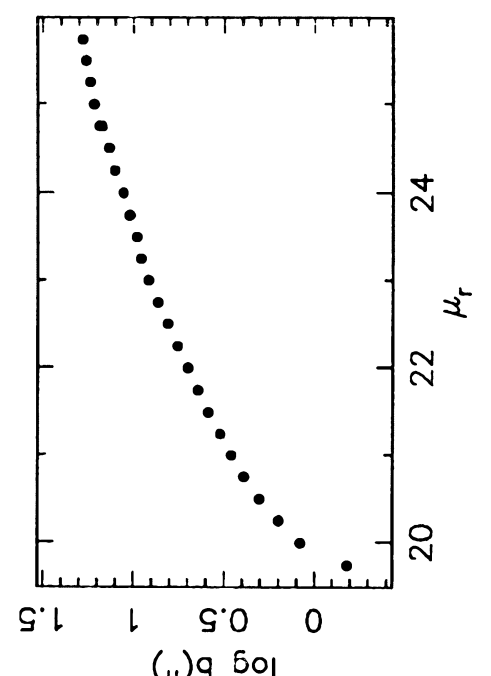

(ii) 9501

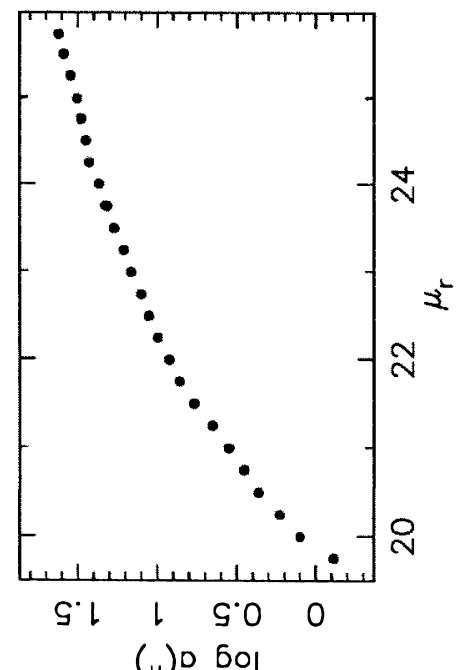

(i) 0 6ol
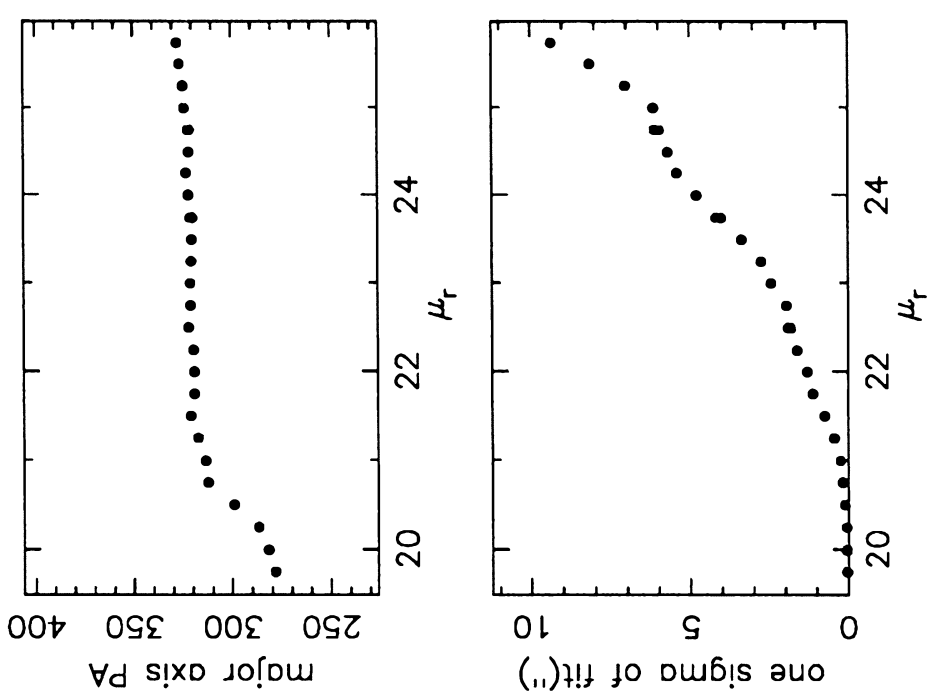

(.1) $) !+$ to ou6!s au०

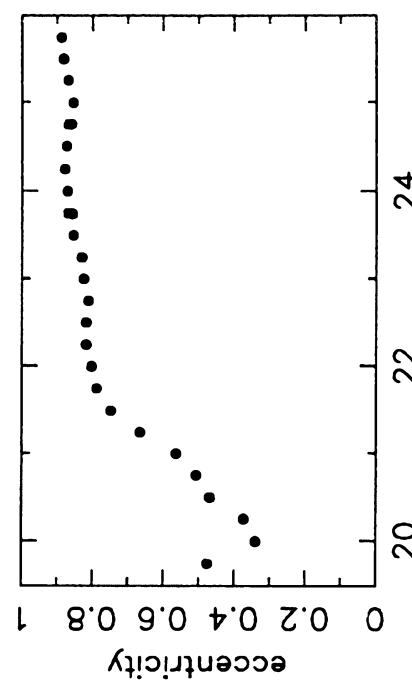

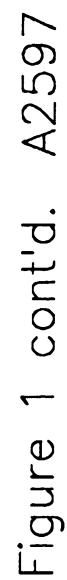

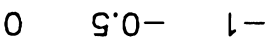

(.1) zas
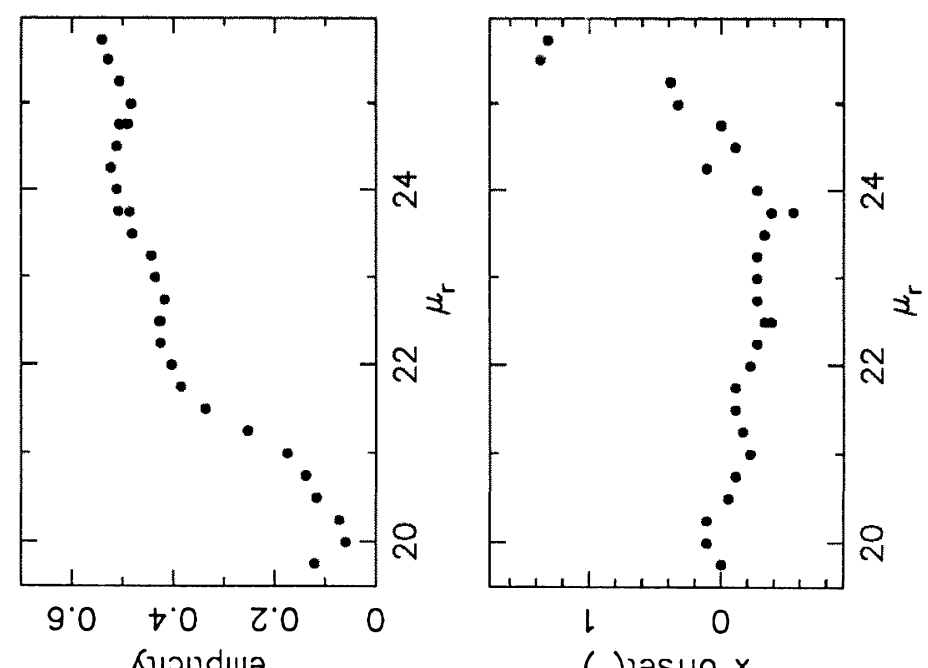

(11) tastyo $\mathrm{x}$ 

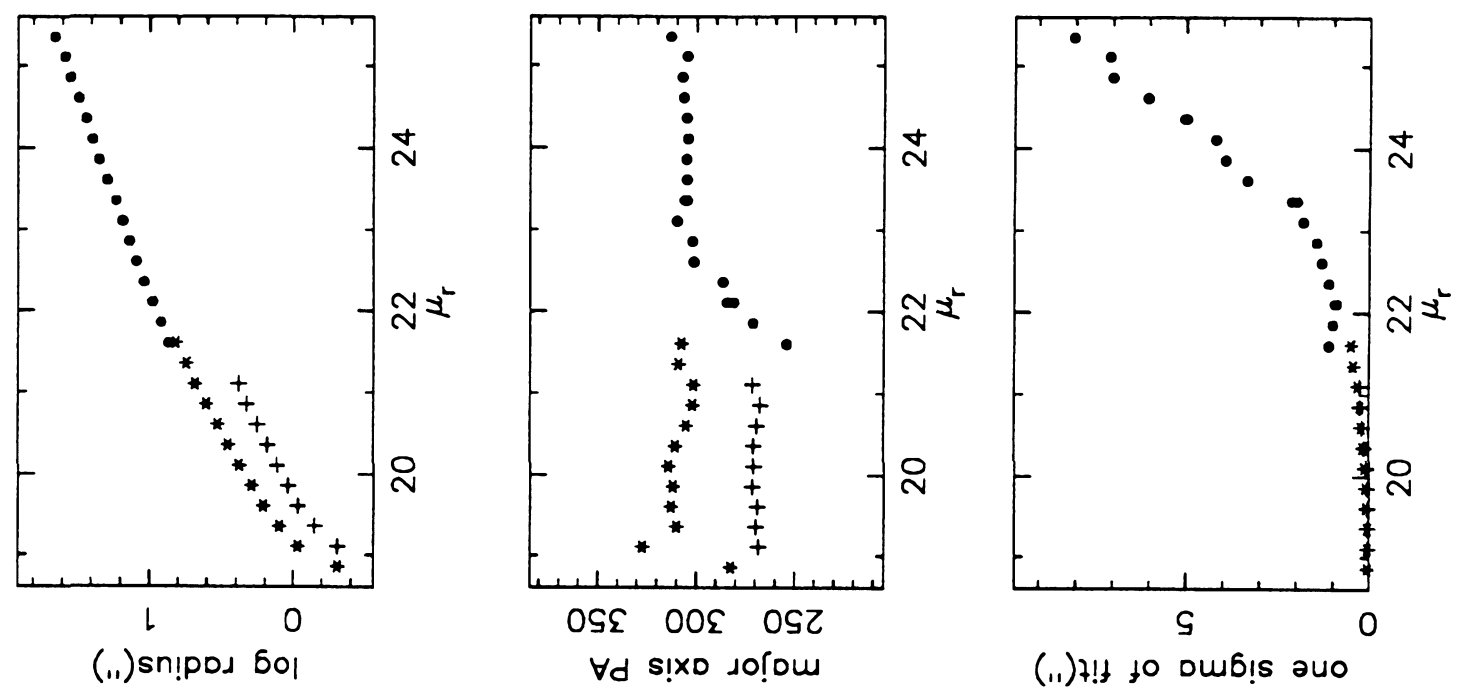

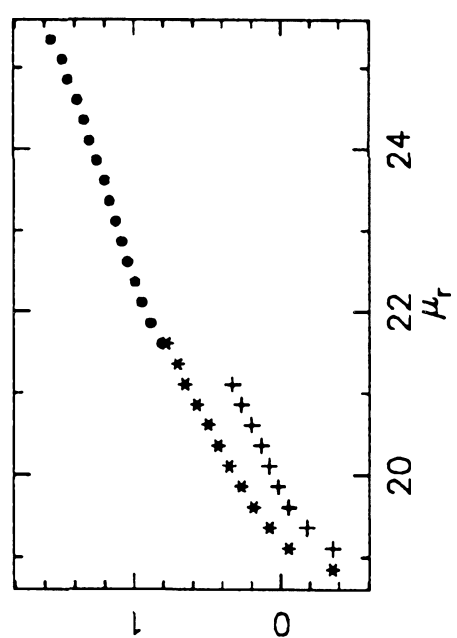

(11) 9501

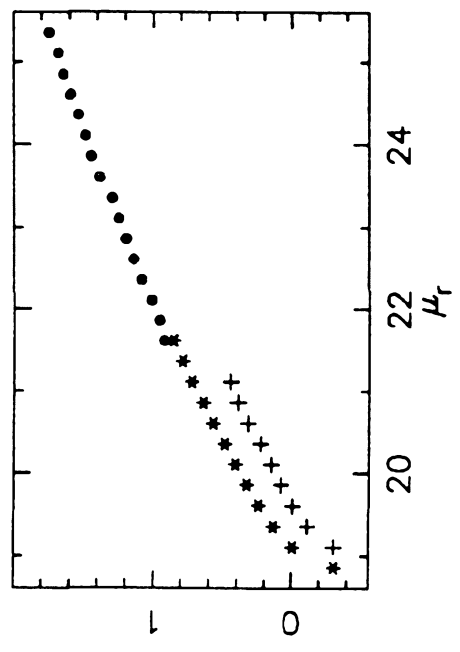

(.1) $\mathrm{O} \mathrm{bo}$
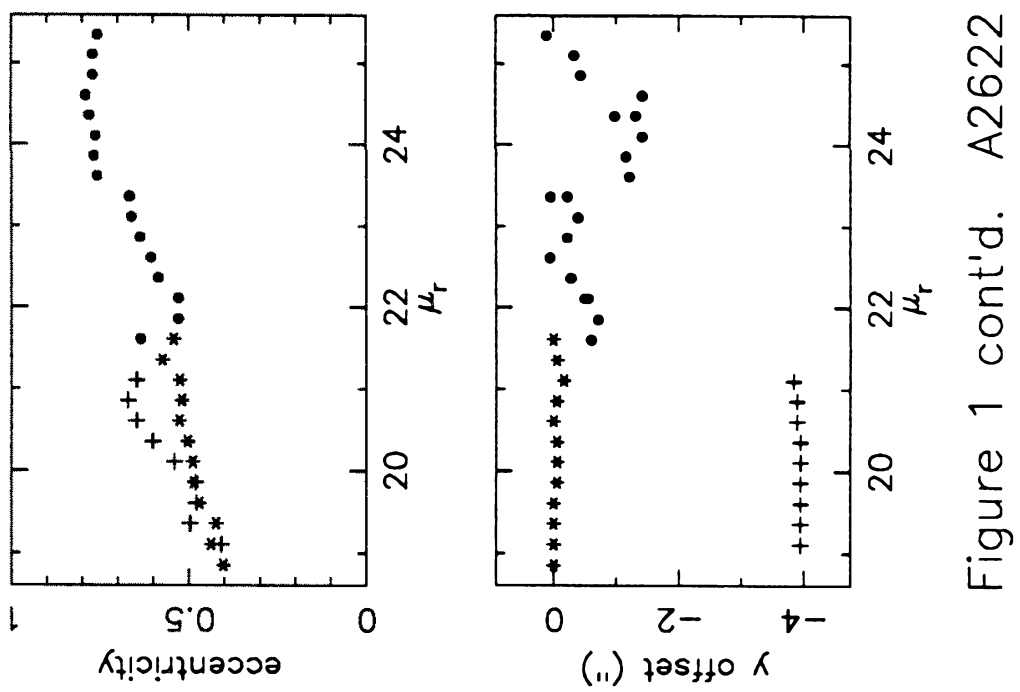
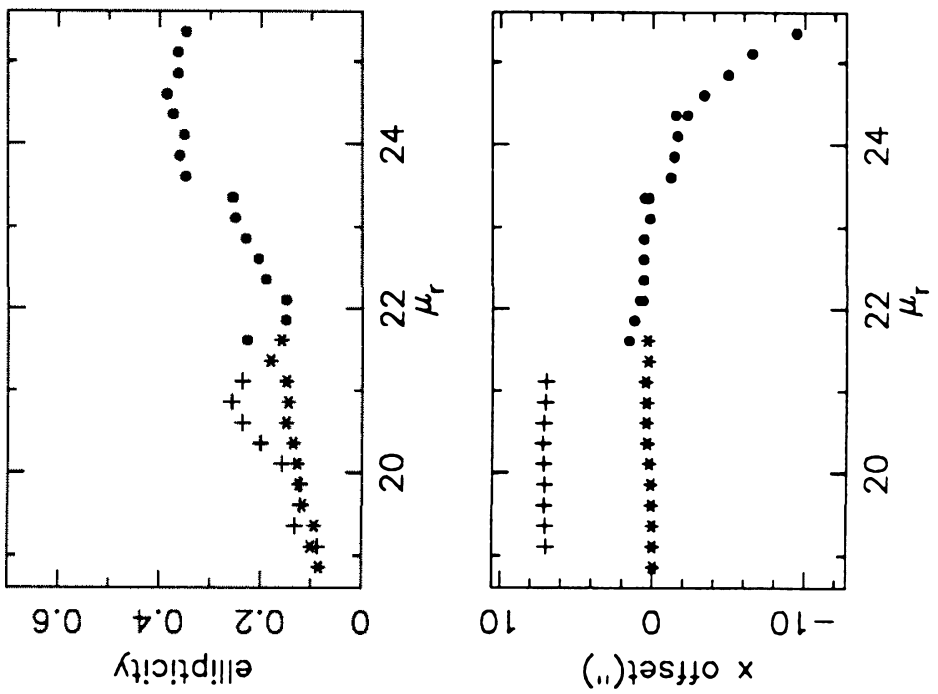


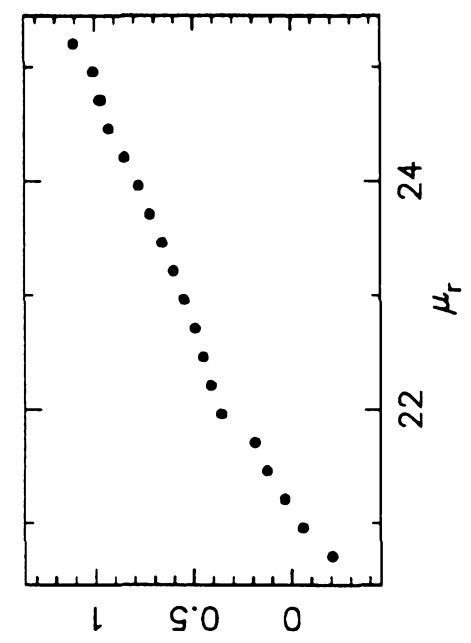

(.1) sn!pos 6ol

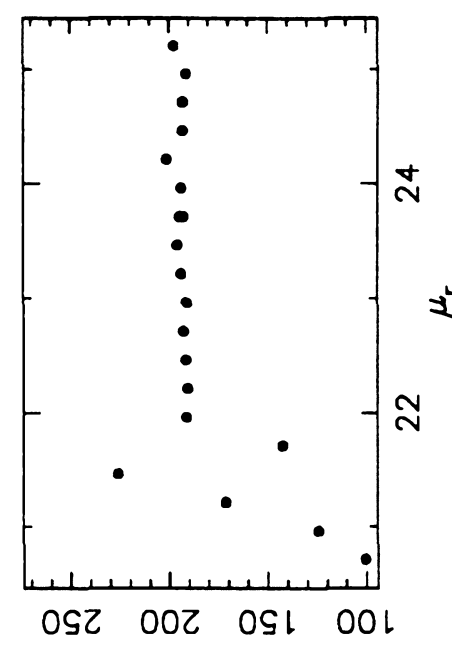

$\forall d$ s!xo so!nom

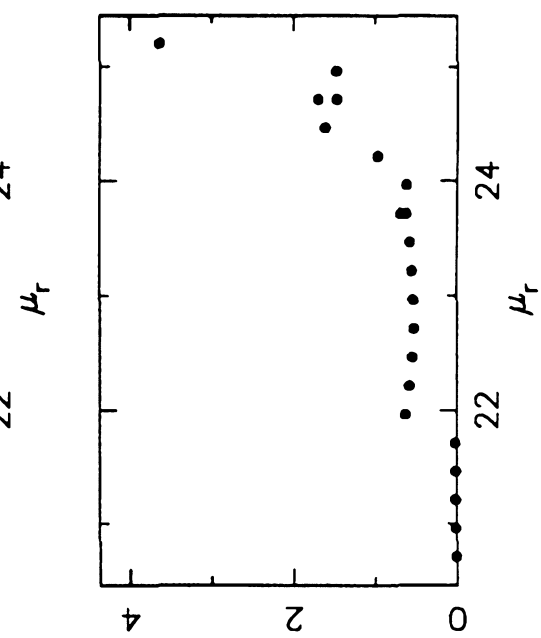

(11) 1 ! to oub!s ano
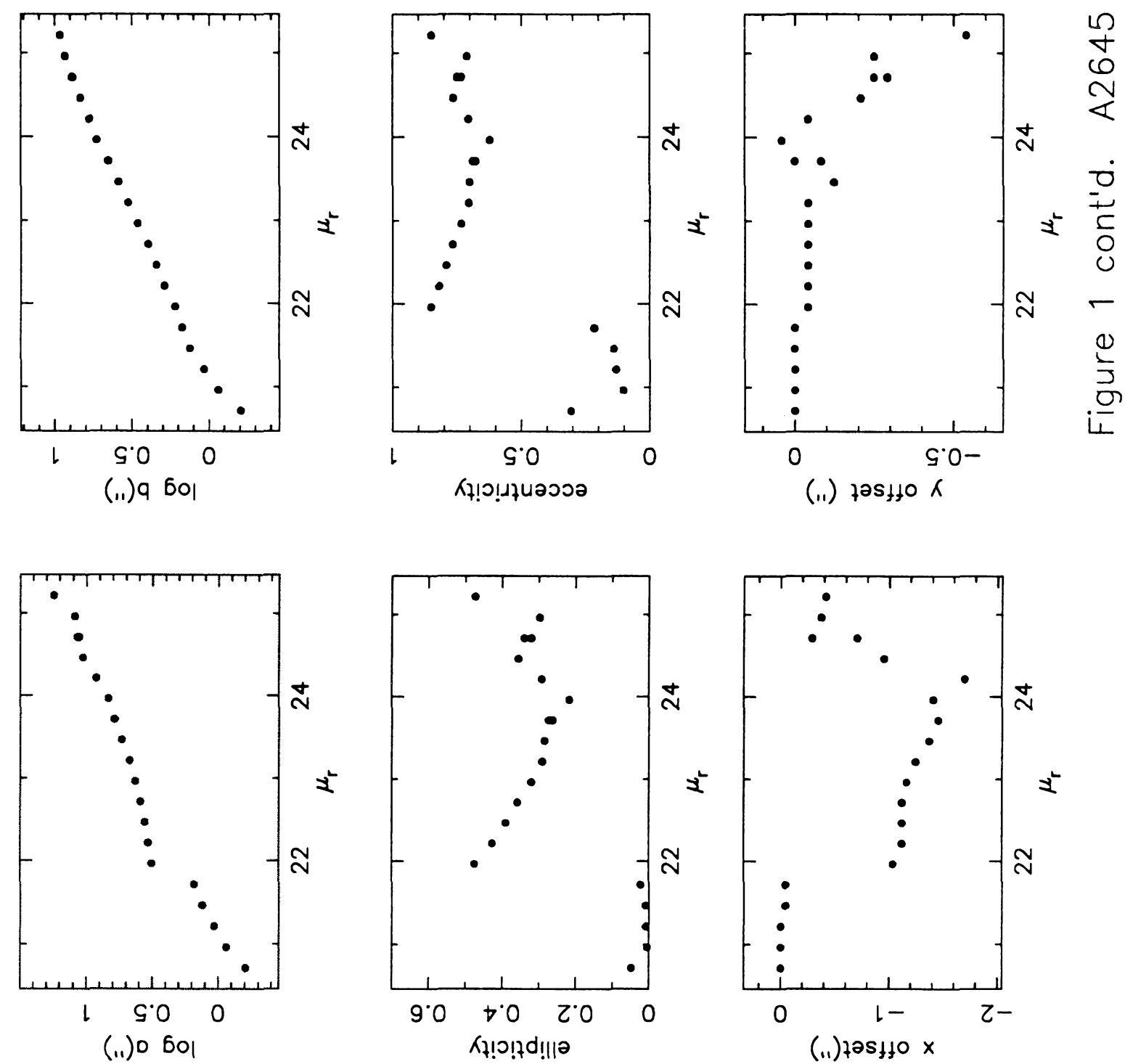


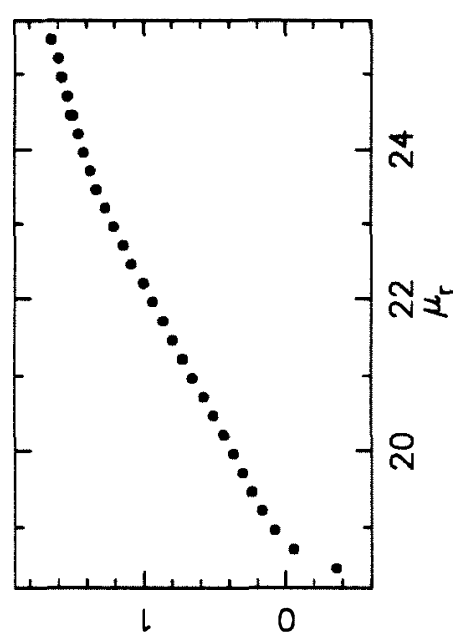

(11)sn!pod 601

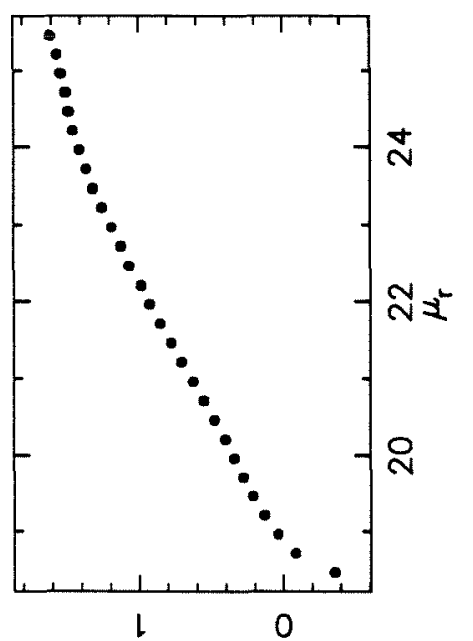

(.1) 9 601

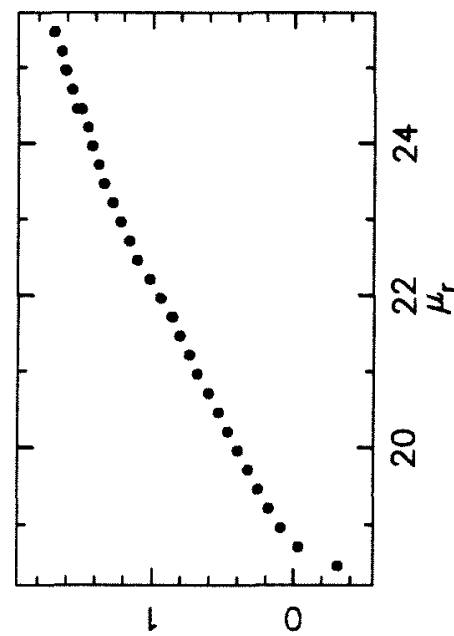

(.1) 0 bol
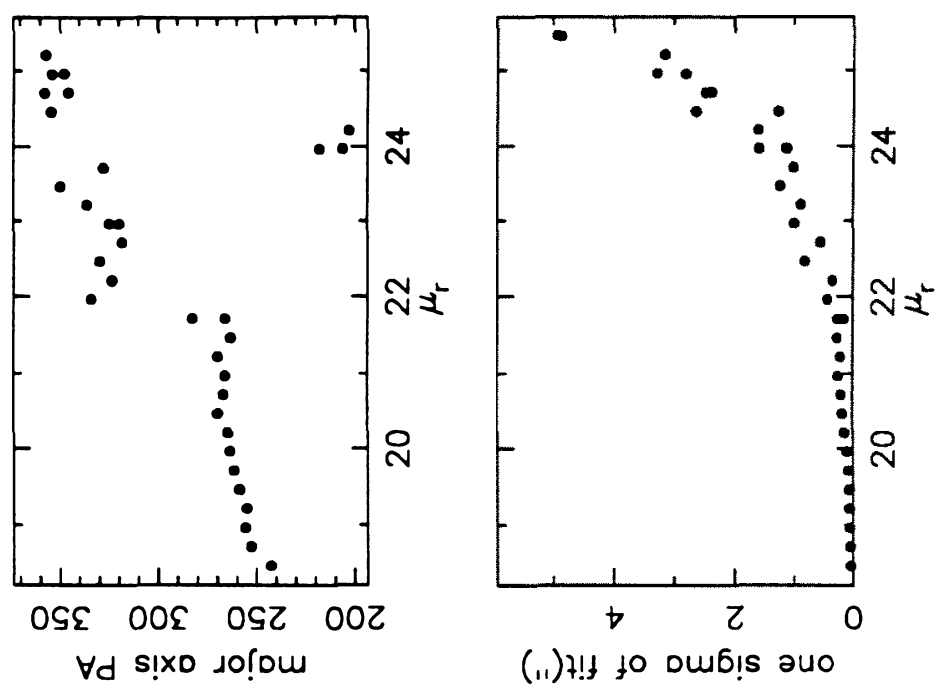

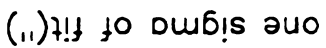

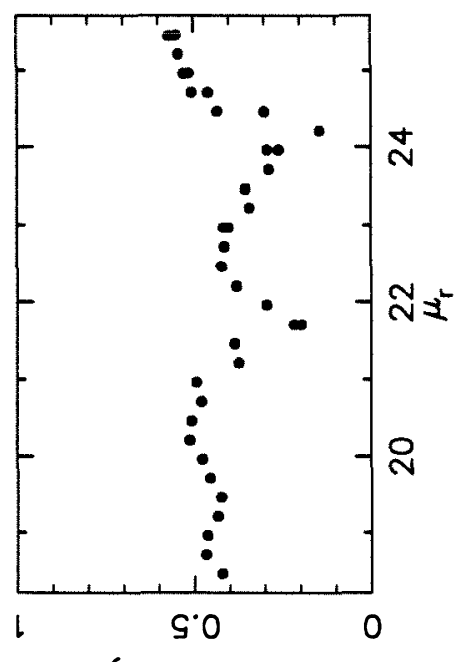

Кใ!ว!นนวววа

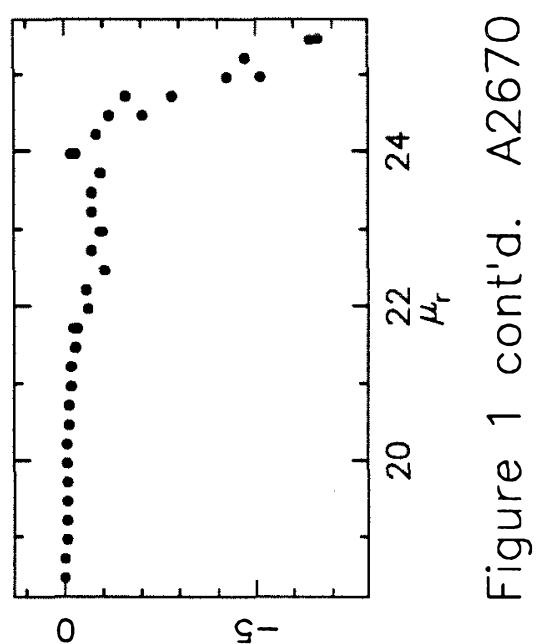

(i1) 7ast10 K
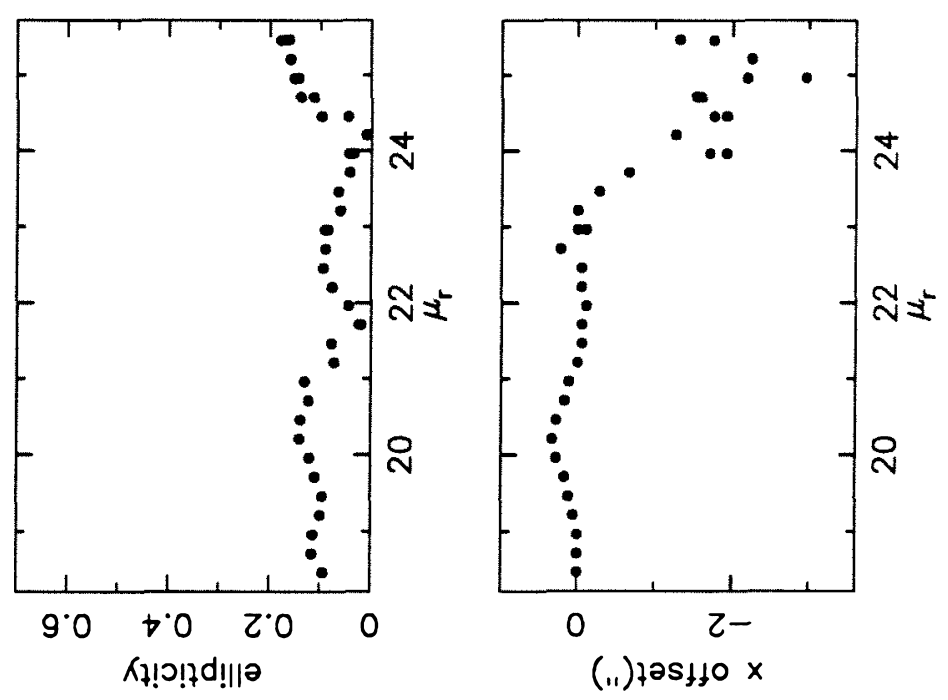


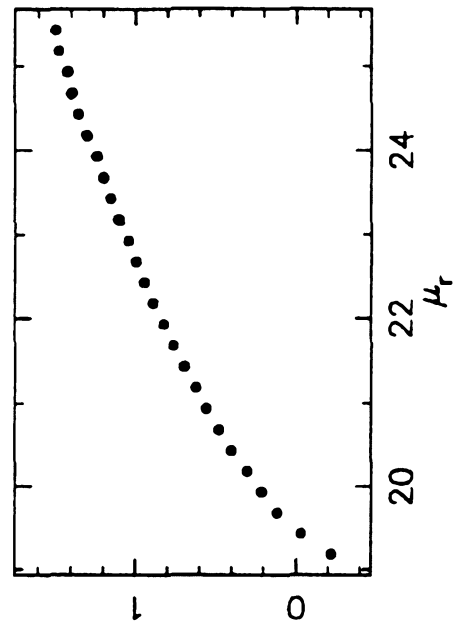

(.1) sn!pos 6ol

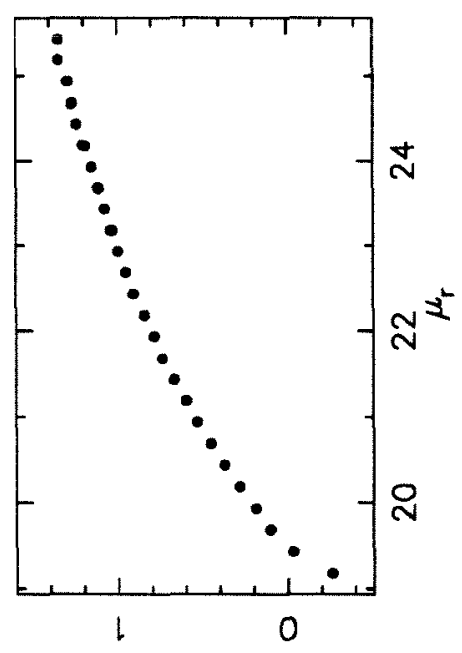

(.1) 9601

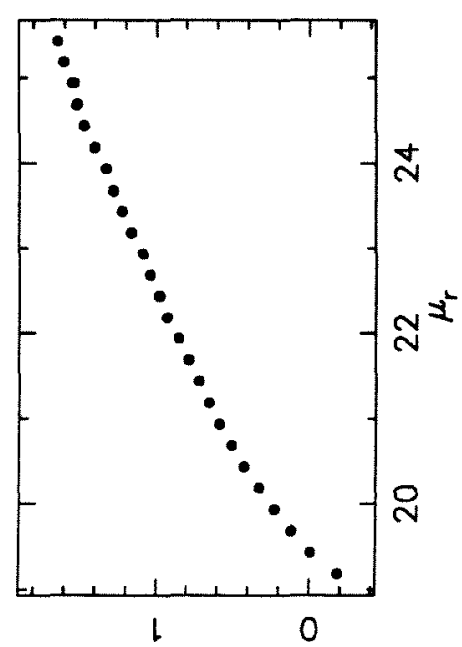

(.1) 0 6ol

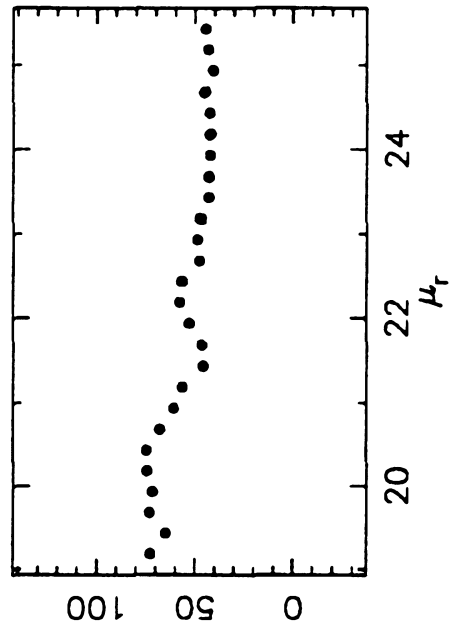

$\forall d$ s!xo so!̣om

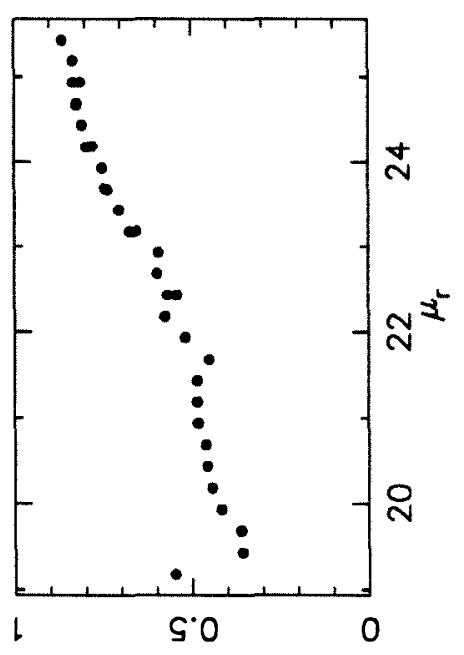

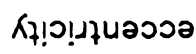
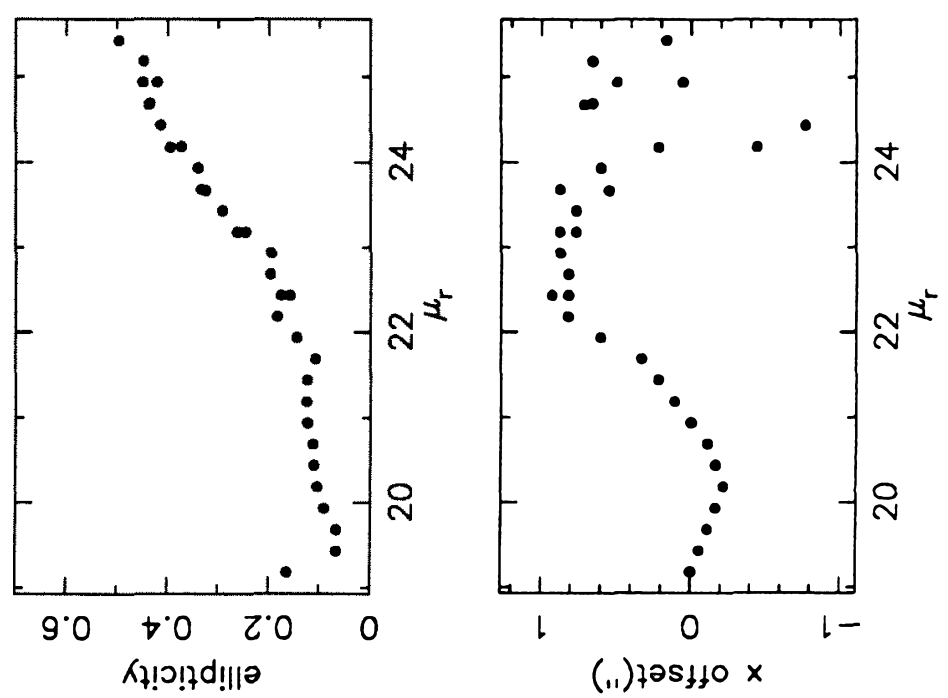


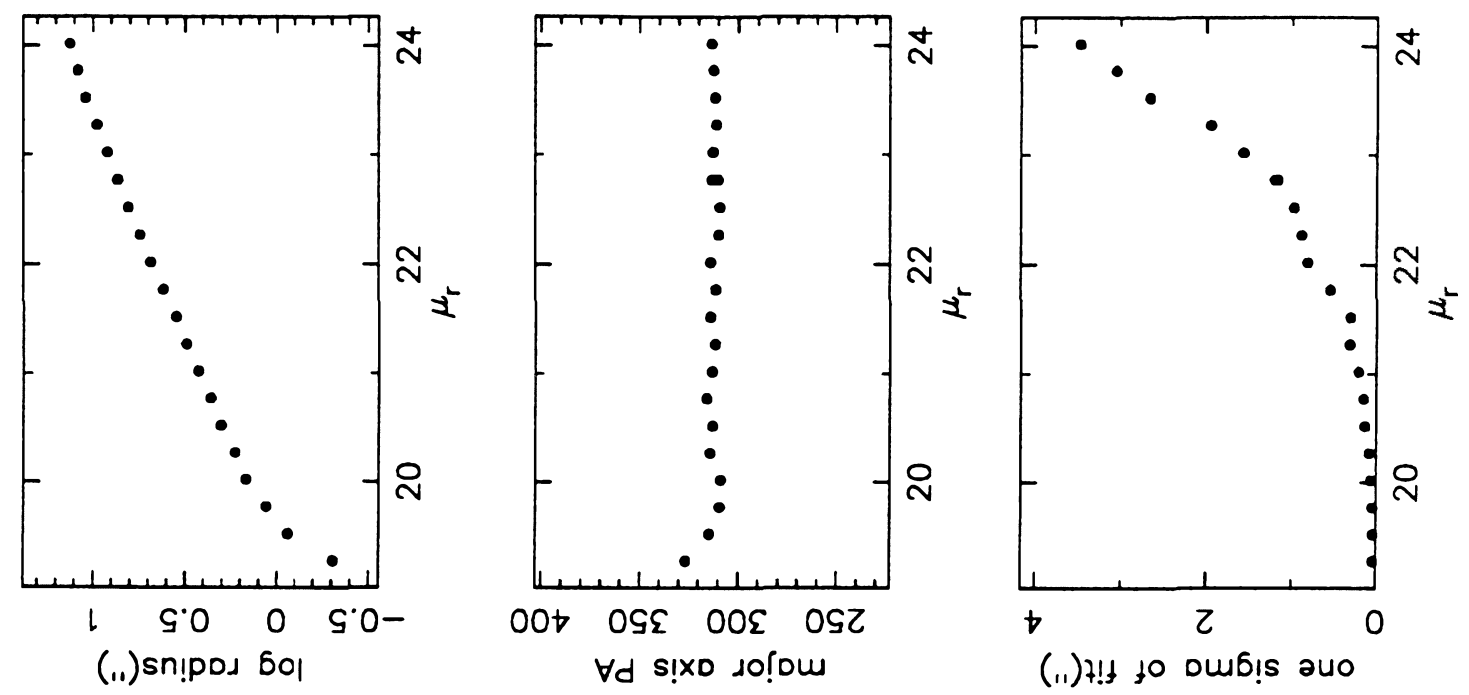

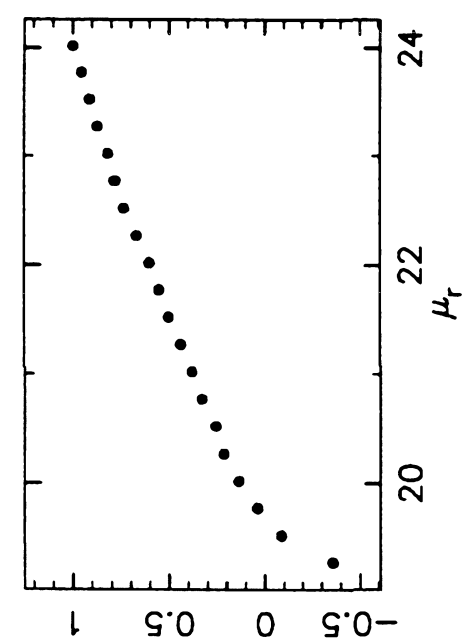

(.1) $9 \mathrm{bol}$

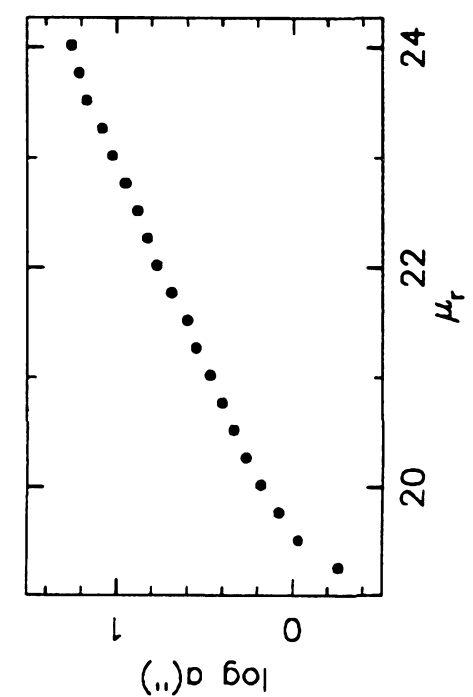

(..) $0 \mathrm{bo}$ 이

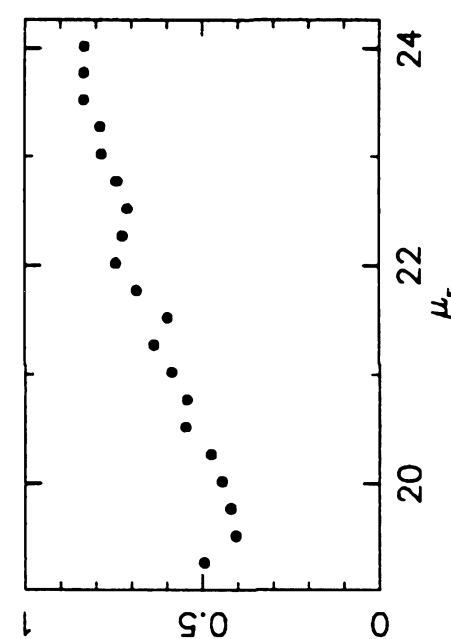

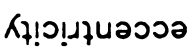

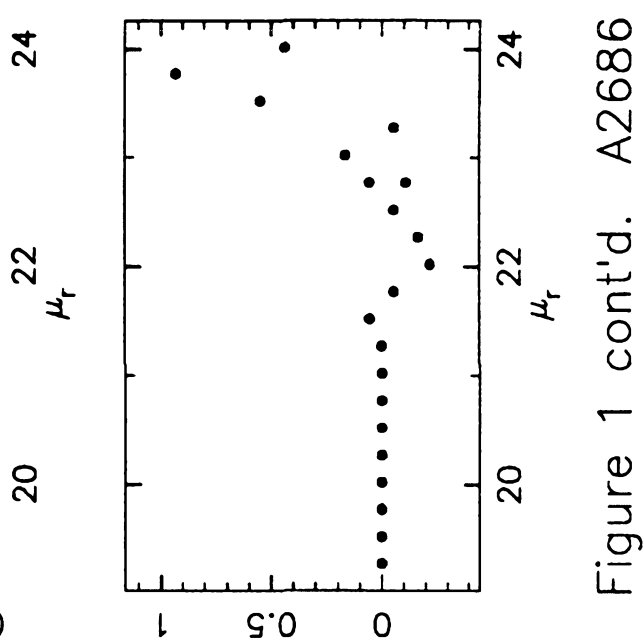

(ii) $725+10 \mathrm{~K}$

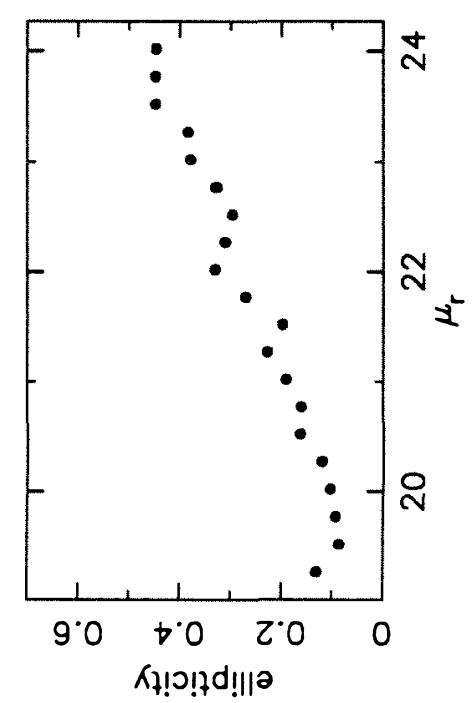

रł!ว!|d!||ว

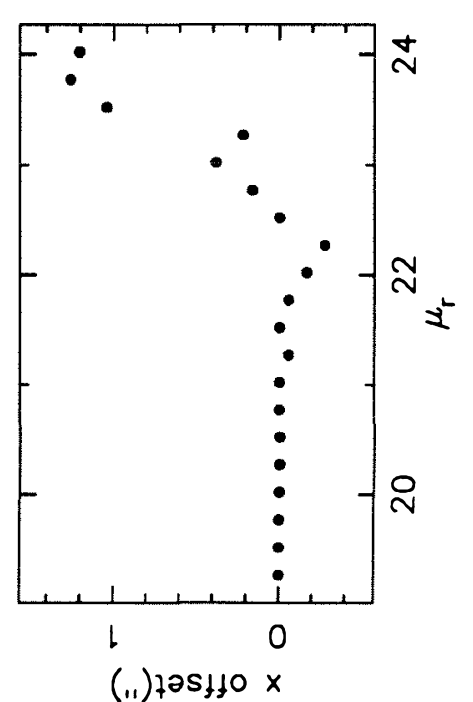




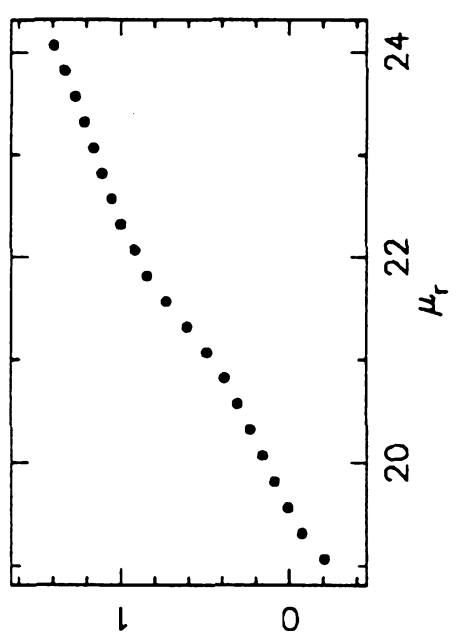

(.1) sn!pod 50।
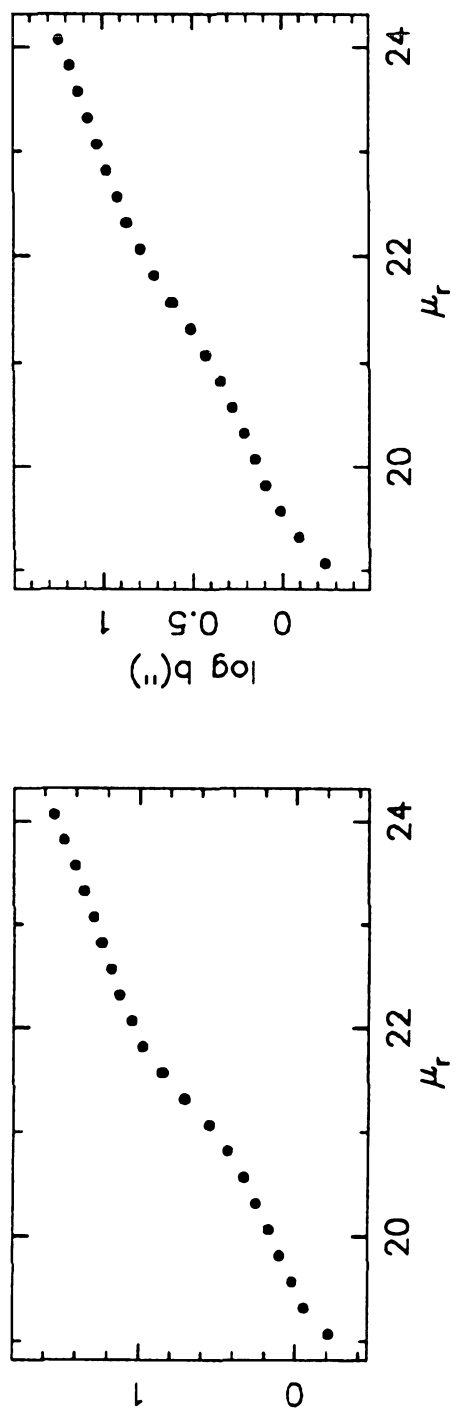

(i) 0601

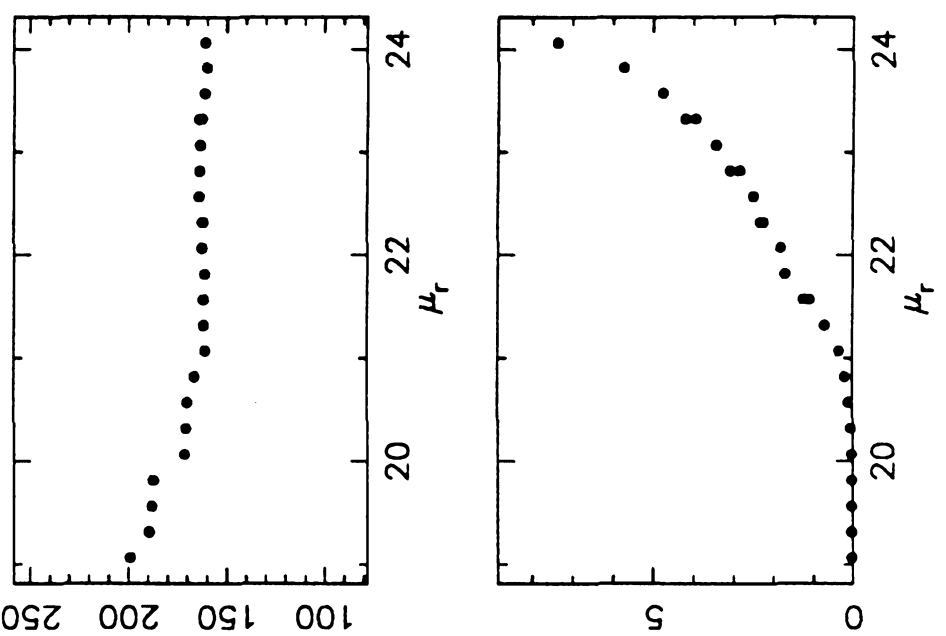

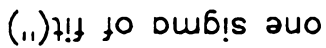
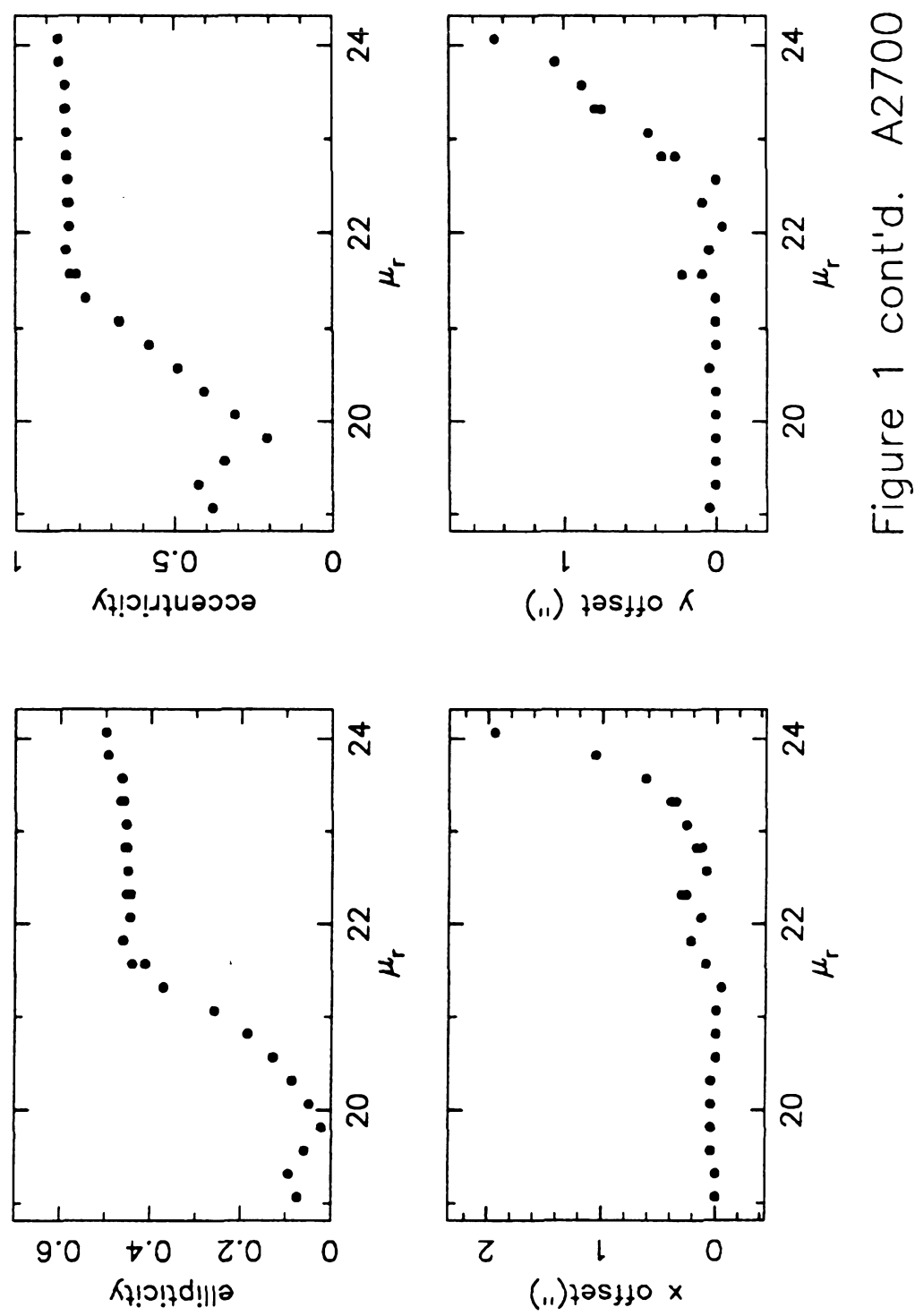


\title{
Remarks on Individual Clusters
}

\begin{abstract}
A21 This system is extremely complex. There are two bright galaxies in the inner $10^{\prime \prime}$, superimposed on a broad, low surface brightness object whose isophotes are not even approximately elliptical. Two more nuclei lurk within $15^{\prime \prime}$ of the center. The profile shown is of the brightest nucleus out to $a=3^{\prime \prime}$ and of the broad component outside that. This is one of the most likely candidates for an ongoing merger in the sample.
\end{abstract}

A85 This galaxy is well known to have an enormous scale length, although it shows no $\mathrm{cD}$ halo. The central ellipticity minimum is not a seeing artifact.

A98 This cluster has two X-ray surface brightness peaks. The galaxy in this study is near the southern, weaker peak.

A104 The BCE is ringed by 4 companions, which disturb the profile near $\mathrm{a}=10^{\prime \prime}$.

A140 This galaxy has companions very near the center.

A151 This is a close pair: the $\mathrm{BCE}$ has a large companion $11^{\prime \prime}$ away in $\mathrm{PA} 211^{\circ}$. The isophote twisting is marginally believable. This picture is severely mispointed, and the envelope profile is cut off by the frame edge.

A154 The northern member of this well-known pair is itself a close (2") pair. The center of the southern galaxy also appears slightly irregular.

A168 The central ellipticity decrease is a tracking artifact. The ellipticity increase in the outer isophotes appears real to the eye, but the image is not entirely flat. 
A189 This galaxy has a remarkably bright, round nucleus. The behavior of the outer isophotes (decreasing ellipticity, rotation, and centroid offset) may be due to contamination by companions.

A193 The central decreasing ellipticity profile and other complex structure are something unresolved. The seeing in this image is $1 . " 7$.

A225 There is a ring of several companions between $15^{\prime \prime}$ and $25^{\prime \prime}$ from the BCE. These cause the ellipticity dip between $22.5<\mu_{r}<24$. The isophote rotation at smaller radii is real and quite obvious to the eye.

A246 The isophote twisting outside $3^{\prime \prime}$ is visible to the eye. The interior eccentricity decrease is a tracking error.

A277 The large isophote twist is real and intrinsic to BCE. There are numerous small companions in vicinity.

A389 This galaxy's decreasing ellipticity profile with strong isophote rotation is intrinsic but appears to be due to a complex (mottled) interior structure observed in poor $\left(2 \prime^{\prime \prime}\right)$ seeing.

A399 The seeing in this image is $1 . " 8$, so the strength of the central ellipticity minimum is uncertain. A399 and A401 are neighbors in space. The twist in the outer isophotes appears real, though they look somewhat bean shaped.

A400 This is a close overlapping double. The southern galaxy has the greater extent. The central ellipticity decrease of the northern component is a tracking error artifact. See Porter (1988b) and Lauer (1988) for more detailed discussion. 
A401 The outer isophotes are probably severely contaminated by scattered light from a nearby bright star but appear significantly boxy. A399 and A401 are neighbors in space.

A496 Porter (1988a) confirms the southward centroid drift at large radii.

A634 The ellipticity decrease in the outermost isophotes is real. The companion galaxy about $2^{\prime}$ to the south of the $\mathrm{BCE}$ is extremely interesting: it has a bright nucleus, a bar, and an almost square edge!

A655 The central ellipticity decrease and centroid offset are not due to tracking error and probably indicate a complex nucleus. The seeing in this image is 1.'6. Some isophotes are visibly boxy.

A671 The companion $12^{\prime \prime}$ from the BCE nucleus in PA $329^{\circ}$ is fairly compact and should not be responsible for the ellipticity minimum at that radius in the profile.

A779 There is a large, round companion $24^{\prime \prime}$ from the $\mathrm{BCE}$ in PA $220^{\circ}$. There is significant overlap between their light profiles, but the companion is tidally truncated, and its outer isophotes rotate toward the BCE. The BCE also shows significant isophote rotation and has been called a $\mathrm{cD}$ on the basis of its light profile (Schombert 1988). See Porter (1988b) and Lauer (1988) for a complete discussion.

A787 There is a close (13") equal companion in PA $208^{\circ}$ and a significant amount of excess light on the opposite side of the $\mathrm{BCE}$.

A819 The central ellipticity structure is probably due to undersampling.

A858 There is a patch of light a few seconds north of the nucleus. 
A910 There is a compact companion 10" almost due north of the BCE nucleus, and a definite swath of excess light northwest of the BCE. See Porter (1988b) for more detailed discussion.

A957 The centroid shift and PA twist at large radii are real and intrinsic.

A978 There is a companion $20^{\prime \prime}$ away in PA $129^{\circ}$, but it is not causing the increased ellipticity below $\mu_{r}=22$. The PA change and ellipticity minimum at the same level appear real to the eye, and an inflection is also visible in the surface brightness profile. This suggests genuine two-component structure. The ellipticity and PA changes in the first few isophotes are probably artifacts of undersampling.

A993 The central ellipticity decrease may be mostly due to tracking error. There is a convincing centroid drift in declination in the region $\mathrm{a}=10^{\prime \prime}$, however, and the center may be an unresolved double or otherwise complex.

A1020 The isophote twisting in the inner $3^{\prime \prime}$ is marginally believable.

A1035 The ellipiticity decrease in the first $3^{\prime \prime}$ is probably due to tracking error.

A1081 The central sharp ellipticity decrease is quite genuine: there is bar or edgeon disk there.

A1126 This galaxy has a double nucleus (separation 5", PA of secondary $122^{\circ}$ ).

A1149 The central ellipticity decrease is a tracking error artifact, and the outer isophotes are contaminated by large nearby companions.

A1185 This is a very complicated triple system. See also Lauer (1988) and Porter (1988). The ellipticity decrease at intermediate radii is real, although $\sigma$ is large there. I also trust the centroid offset at large $r$; the picture is flat, and there are no large companions. The galaxy is about $3^{\prime}$ north of the X-ray center of the cluster. 
A1187 The centroid jogs in right ascension ( $\mathrm{x}$ ) are tracking errors.

A1190 The outer ellipticity increase corresponds to a truncated minor-axis surface brightness profile.

A1213 The turnover in the ellipticity curve is real, and the galaxy is not coincident with the X-ray center of the cluster. It may not be the true $\mathrm{BCE}$ or dynamically dominant galaxy.

A1216 The centroid jog in right ascension ( $\mathrm{x})$ is due to tracking error.

A1224 This high redshift $(z=0.3) B C E$ appears to have a halo.

A1228 The ellipticity minimum and PA twist look quite real and are in range $5-10^{\prime \prime}$. The inner $\mathrm{PA}, 50^{\circ}$, points toward a small companion. The outer PA is perpendicular to it. I think this is not tracking error.

A1291 This is a complicated multiple system: the envelope of the BCE overlaps 3 companions. The reality of isophote the twist in the BCE is hard to establish.

A1364 The center appears to be complex but unresolved. It is bean shaped. The isophote twist near $10^{\prime \prime}$ is due to a close companion.

A1365 The PA twists are real.

A1377 This galaxy has an increasing, then decreasing ellipticity profile. The most elliptical isophotes are boxy. The centroid drift toward the companion (26" away in $\mathrm{PA} 54^{\circ}$ ) is real, as is the isophote rotation.

A1382 The bright compact nucleus of this galaxy is not centered in its "halo," and the $\mathrm{X}$-ray center of the cluster lies $3^{\prime}$ to the east. The picture is not cosmetically good, but the centroid offset occurs at $\mu_{r}=21$ and is certain. Note the ellipticity minimum with a small PA twist. 
A1383 The complex structure all looks real: centroid offsets, local ellipticity increase with boxy isophotes, and ellipticity minimum with PA rotation. The general appearance of this $\mathrm{BCE}$ is very similar to that of the BCE in A1382.

A1399 The initial ellipticity decrease is probably due to tracking error. The isophote twist appears real to the eye.

A1412 This image has noticeable tracking error, and the image of the $\mathrm{BCE}$ is "dented" in the inner 2 " of the northeast quadrant. This might be a dust lane, but requires confirmation. The ellipticity becomes constant at large radii as the slope of the minor-axis surface brightness profile decreases.

A1413 This is a prototype $c D$. The southward centroid drift appears to be an artifact of improperly cleaned satellite galaxies in that region. See Porter (1988a) for a more detailed discussion.

A1461 The ellipticity decrease and increase beyond 15", though slight, appear real to the eye.

A1468 This apparently double nucleus BCE is in fact a chance projection (Smith et al. 1985).

A1474 The "BCE" of Paper IV is in fact an edge-on disk system. The elliptical 2' to its northwest was also photometered. It appears to have excess light in the outer parts of its southeast quadrant.

A1541 This BCE is an almost equal pair 11" apart along their major axes. It is tempting to believe the centroid offset at large radii, since it is on the side of the $\mathrm{BCE}$ away from the component, but the $\mathrm{BCE}$ is close to the edge of the $\mathrm{CCD}$ frame, and the offset requires confirmation. 
A1548 This profile has severe contamination from companions in most regions outside $10^{\prime \prime}$.

A1630 The inner few seconds are patchy; the isophotes, nonelliptical.

A1644 The centroid drift at large radii is due to contamination by companions and truncation by the edge of the frame.

A1651 The centroid drift in right ascension (x) is tracking error.

A1656 This is NGC 4889. The centermost ellipticity dip is due to $2^{\prime \prime}$ seeing. The large companion elliptical $63^{\prime \prime}$ away in PA $309^{\circ}$ affects the profile in the range $23<\mu_{r}<24.5$. The edge-on disk on the west side of NGC 4889 was well edited and does not greatly affect the profile. The centroid drift at large radii is an artifact of imperfect flat fielding.

A1656B This is NGC 4874 , a cD. The ellipticity increase at a $=25^{\prime \prime}$, forming a major axis bulge, is well within the ring of satellite galaxies at $1^{\prime}$.

A1691 The ellipticity maximum and PA twist are visible to the eye. The most elliptical isophotes appear boxy.

A1749 Note the unique waves in the ellipticity profile. There is a circlet of 5 satellites at about $35^{\prime \prime}$ from center. There are no companions overlapping the $\epsilon$ wave, and it looks real to the eye. The centroid drift at large radii is due to the companions.

A1767 This BCE has a clear shoulder of light to the northwest at about a $=10^{\prime \prime}$. It was classified as a $\mathrm{cD}$ by Schombert. The isophotes remain fairly elliptical despite sharp twists and centroid jogs. 
A1773 This image is extremely poor cosmetically because of a very bright star just outside the field, and the $\mathrm{BCE}$ is a complicated multiple system as well. There are at least 3 components in the inner $5^{\prime \prime}$, and two more large companions within $15^{\prime \prime}$, one to the northeast and one to the southwest. Specifically, the ellipticity decrease in the first $10^{\prime \prime}$ cannot be trusted.

A1775 The second galaxy in this well-known close pair is a strong radio source with a large velocity relative to the BCE (v. Hintzen 1979). See Porter (1988b) for an analytic decomposition of this image. The inner ellipticity decrease is a tracking error, but the isophote rotations at intermediate radii appear real to the eye.

A1793 There are small companions $4^{\prime \prime}$ due north and $8^{\prime \prime}$ in PA $335^{\circ}$. These are probably the cause of the nonzero $\epsilon$ below $\mu_{r}=22$ and the stabilization of the major-axis angle. The central PA is undersampled, so the large isophote twist may well not be real.

A1795 The ellipticity, PA, and centroid breaks at $\mathrm{a}=10^{\prime \prime}\left(\mu_{r}=22\right)$ are associated with a small companion in $\mathrm{PA} 216^{\circ}$, but it appears compact. The breaks are more likely real perturbations than errors due to improper picture editing. This galaxy is a strong radio source and has many optical emission filaments.

A1809 The isophote twist at $a<3^{\prime \prime}$ is probably due to undersampling. The twist at larger radii and centroid drift at large radii are probably real. The $\epsilon$ minimum at $10^{\prime \prime}$ is visible to the eye. See the discussion of seeing tests in Section III.

A1831 There is a medium-sized companion $39^{\prime \prime}$ away in PA $136^{\circ}$. The centroid jogs near this radius are not convincing.

A 1837 The PA twist at $\mathrm{a}=6^{\prime \prime}$ is visible to eye. The centroid displacements beyond $40^{\prime \prime}$ appear real, due to excess light southwest of $\mathrm{BCE}$. This image is fairly flat. 
A1904 The center of the galaxy is complex. The ellipticity, PA, and centroid changes appear real. The seeing was $1 . " 3$. The cluster X-ray emission is also patchy, and is centered about $3^{\prime}$ south of the BCE.

A1913 There is clearly some tracking error in this picture, and it is probably responsible for the observed ellipticity peak in the profile of the second-ranked galaxy in this cluster. The ellipticity peak and isophote twisting at $10^{\prime \prime}$ in the $\mathrm{BCE}$, however, are obviously real. It looks to the eye like a good candidate for a thick disk or bar. The separation of the two galaxies is $49^{\prime \prime}$. They lie about $8^{\prime}$ north-northwest of the cluster's X-ray center.

A1918 The BCE is flanked by companions only 2".5 to the northeast and southwest. The profile plotted is of their common envelope and is meaningful only outside about $8^{\prime \prime}$.

A1921 The halo outside 5" appears real and intrinsic: the seeing in this image is 1:'2. Its isophotes appear boxy. The center does show structure, however.

A1927 This is a quadruple system (Lauer 1988), with considerable contamination of all profiles outside $6^{\prime \prime}$.

A1940 The major-axis halo appears real: the seeing is $1 . " 1$.

A1983 Isophotes larger than $\mathrm{a}=30^{\prime \prime}$ are severely contaminated by companions to the southeast.

A1984 This is a close overlapping double. See Porter (1988b) for more detailed discussion. The ellipticity decrease of the northern component appears to be real.

A1991 The isophote rotation within first $10^{\prime \prime}$ appears real to the eye. This profile is limited by a bright star $50^{\prime \prime}$ to the east. 
A1999 There are four components within $4^{\prime \prime}$ of $\mathrm{BCE}$ center. One is quite faint, and one appears to be an edge-on disk, but the profile outside 6 " in no way represents a single galaxy.

A2005 This profile is severely limited by a relatively bright star $15^{\prime \prime}$ northwest of the nucleus of the BCE.

A2022 There is a bright, extended companion only $28^{\prime \prime}$ away in PA $100^{\circ}$. The ellipticity decrease of the BCE is convincing. See Porter (1988b) for an analytic decomposition.

A2028 The profile beyond $\mathrm{a}=15^{\prime \prime}$ is severely contaminated by a ring of 4 companion galaxies, but the ellipticity decrease outside $3^{\prime \prime}$ is convincing.

A2029 Note the lack of any cD halo. This is confirmed by Schombert (1977) and Porter (1988a).

A2036 The entire inner 5" of the profile-ellipticity decrease, isophote twist, and major-axis brightness profile dimple, are contaminated by a severe tracking error.

A2040 The seeing in this image is $2 . " 8$, and the profile is cut off by a saturation track from a very bright star to the south. However, the asymmetric halo appears to be real.

A2048 The BCE is not that much bigger or brighter than half a dozen other galaxies in the field. Many of these other galaxies have remarkably complex internal structures. The centroid offset in the largest isophotes of the $\mathrm{BCE}$ is probably due to contamination by small companions, and the isophote twisting beyond $5^{\prime \prime}$ is marginal, but the ellipticity decrease is real. 
A2052 The apparently compact companion at $14^{\prime \prime}$ in PA $36^{\circ}$ is probably at least partly responsible for the centroid drift at small radii, but perhaps not entirely. The ellipticity changes are certainly intrinsic to the $\mathrm{BCE}$.

A2063 The strongly truncated profile is confirmed by Schombert (1987). The centroid offsets look convincing, but may be artifacts of a saturation track.

A2065 This cluster is odd: there are about a dozen edge-on disks in this picture! They do not look particularly aligned or concentrated. Three or four of those in the south are within $20^{\circ}$ of east-west alignment, and the $\mathrm{BCE}$ has a major axis aligned north-south. This is one of the BCEs with turnover in the ellipticity profile, and it appears real to the eye. The X-ray center of the cluster coincides with a pair of ellipticals $2^{\prime}$ away from the BCE.

A2067 This galaxy is especially interesting for two reasons: it has a protrusion of some sort in its eastern side and a companion $13^{\prime \prime}$ to the north, that appears compact, but is probably associated with a halo of light which is displaced several seconds to the northwest and not entirely smooth.

A2079 This extremely interesting system has a second bright galaxy $71^{\prime \prime}$ away in PA $194^{\circ}$ and a broad swath of extra light on the opposite side of the BCE. The isophotes of both galaxies show significant departures from ellipticity.

A2089 The central ellipticity minimum may be an artifact of the 1"'5 seeing.

A2092 The BCE has an extremely compact companion $5^{\prime \prime}$ south of its nucleus. With its collinear distribution of bright galaxies, this cluster resembles A2079, although it does not appear to have as much loose light. The northward centroid displacement and minor-axis halo of the BCE look real to the eye, although they are somewhat noisy. 
A2107 The seeing in this image is poor: 2"'6.

A2124 The isophote twist between $2^{\prime \prime}$ and $5^{\prime \prime}$ looks real to the eye. Note the steep surface brightness profile, as in A2063.

A2142 The initial slight decrease in $\epsilon$ and PA twist are probably tracking errors.

A2147 This is a $c D$ with a bright major-axis halo.

A2151 This is the Hercules cluster. This is a poor-seeing (2!'4) image of a difficult triple-nucleus $\mathrm{BCE}$. The profile beyond $10^{\prime \prime}$ is strongly contaminated by the nucleus $19^{\prime \prime}$ from the primary in PA $220^{\circ}$. See Lauer (1988). This cluster has a cD galaxy (NGC 6034) in its outlying regions.

A2152 This BCE is so round that the scatter of measured isophote PAs between $0^{\circ}$ and $90^{\circ}$ is probably due to pixellation. The profiles of the two large galaxies in this cluster merge about $30^{\prime \prime}$ from the center of the $\mathrm{BCE}$. The turnover of the ellipticity profile of the second galaxy at E3.5 is a good counterpoint to the lack of this kind of behavior in BCEs.

A2175 The isophote twist, ellipticity break, and centroid jog between $5^{\prime \prime}<a<15^{\prime \prime}$ are due to contamination by a companion. The ellipticity decrease in the largest isophotes is also probably contamination. See also Figure 2.

A2197 There appears to be a faint extension along the major axis, at a $=90^{\prime \prime}$. This results in the increased $\sigma$ in that part of the profile (the isophotes are boxy), and perhaps the centroid offset. These features are weak and require confirmation. A2199 This is the prototype of the multiple-nucleus $\mathrm{cD}$. The broadest nucleus is aligned with the outer halo. See Porter (1988a) for a more detailed discussion, and Lauer (1988) for a simultaneous ellipse fit to the nuclei. 
A2218 The central structure is complex: the isophotes are ragged. The seeing is good: 1.2 . The ellipticity profile turns over because the major axis profile ends.

A2256 This is a difficult triple system. The outer halo seems well centered on the middle of the three nuclei. The seeing is $2^{\prime \prime}$.

A2283 The very small ellipticity decrease is too slight to be convincing.

A2328 The ellipticity decrease is marginally credible. The image is cosmetically poor, and there is a very faint companion $3^{\prime \prime}$ to the southwest of the BCE nucleus.

A2382 This is the most believable instance of a steady ellipticity decrease in the entire sample. There is a compact companion $15^{\prime \prime}$ south of the BCE, near the detection limit of the BCE's profile. The profile at this level appears to the eye to be flattened east-west (as opposed to the north-south alignment of the interior), but this requires confirmation.

A2384 This image is so poorly flattened, and the galaxy so round, that the shape profiles are not very trustworthy.

A2440 The ellipticity maximum and PA twist are easily visible by inspection.

A2457 The isophote twisting is marginally visible to the eye. The outer isophotes appear somewhat diamond shaped.

A2459 The ellipiticity decrease in the outer isophotes of the BCE is genuine and is associated with a minor-axis halo.

A2462 The interior ellipticity decrease is a tracking error. The ellipticity minimum near $\mathrm{a}=10^{\prime \prime}$ appears to be due to a diffuse companion south of the nucleus.

A2469 The ellipticity increase from E1 to E5 is almost discontinuous. Check tracking. 
A2521 This is a close, equal, overlapping pair. The eastern component has a definite extension on its northwest side.

A2670 This is one of the roundest galaxies in the sample. The ellipticity minima are probably cleaning artifacts.

A2675 This picture has severe tracking error, which is the cause of the $\mathrm{x}$ centroid offset, and probably also the behavior of the inner $10^{\prime \prime}$ of the ellipticity and PA profiles.

A2700 This galaxy has an extremely bright, round nucleus. It appears to increse the central surface brightness by about two magnitudes. The PA of the isophotes outside this nucleus is probably essentially constant. 
I linger yet with Nature, for the Night Hath been to me a more familiar face Than that of man; and in her starry shade Of $\operatorname{dim}$ and solitary loveliness, I learned the language of another world. 